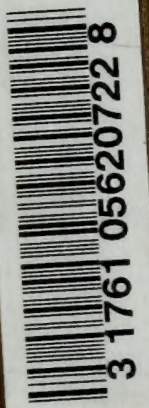

Univ. of TonaสT0 LIORAW 

BLNBING LIST DEC 11921 
Digitized by the Internet Archive in 2010 with funding from University of Toronto 


\title{
THE DISTRIBUTION OF VEGETATION IN THE UNITED STATES, AS RELATED TO CLIMATIC CONDITIONS
}

\author{
BURTON E. LIVINGSTON \\ AND \\ FORREST SHREVE
}
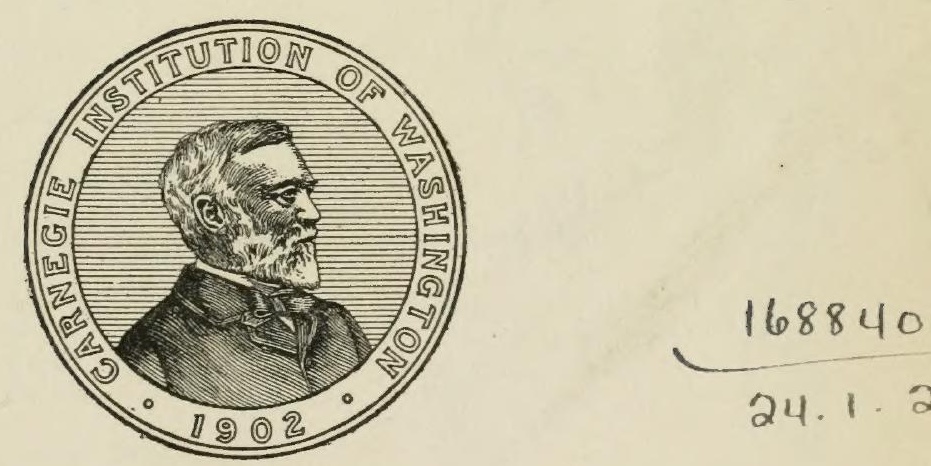

Published by the Carnegie Institution of Washington 1921 
CARNEGIE INSTITUTION OF WASHINGTON

Publication No. 284

PRESS OF GIBSON BROTHERS, INC.

WASHINGTON, D. C. 


\section{CONTENTS.}

PAGE.

Preface

IX

\section{Part I. The Vegetation of the United States.}

INTRODUCTION:

I. The distribution of vegetation in general, as related to climatic conditions. . 3

II. Study of the distribution of individual species..................

III. Manifold operation of environmental conditions ..................

IV. Growth-forms of plants.

TABLE 1.-Analysis of Drude's criteria for distinguishing

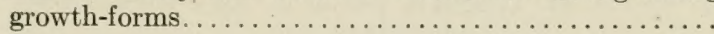

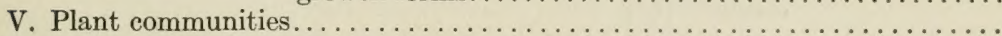

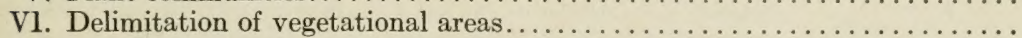

Distribution of Vegetation in the United States:

I. Methods used in securing and presenting the distributional data ........

II. Leading vegetation types of the United States and their geographical areas

III. Distributional areas of conformic groups of plants................

IV. Distributional areas of selected individual species..................

Part II. Environmental Conditions.

INTRODUCTION

General Influence of the Environment on Plant Life:

1. External and internal conditions and plant activity $\ldots \ldots \ldots \ldots \ldots \ldots$

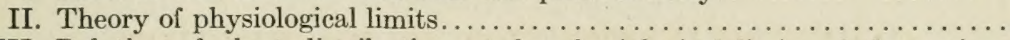

III. Relation of plant distribution to the physiological limits of the various

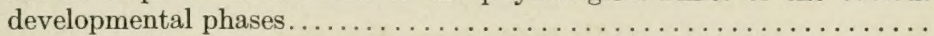

IV. Genetic continuity of protoplasm and its cyclic activities, in connection

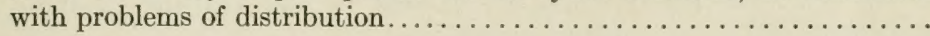

Chief Environmental Conditions and the General Nature of Their Effects Upon Plants:

I. General classification of environmental factors...................

II. Moisture:

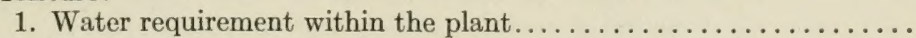

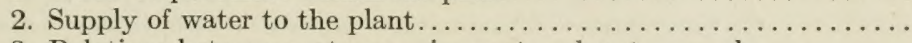

3. Relations between water-requirement and water-supply .........

III. Temperature:

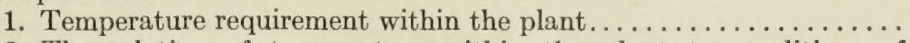

2. The relation of temperature within the plant to conditions of

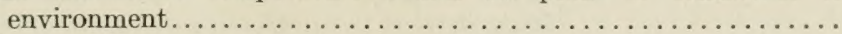

IV. Light:

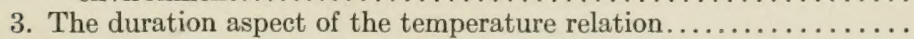

1. General nature of light........................... 135

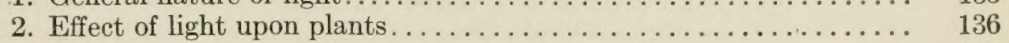

3. Duration aspect of light relation of ordinary plants............ 137

V. Chemical conditions:

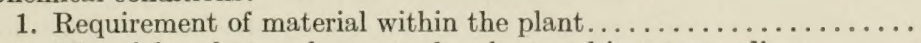

2. Material exchanges between the plant and its surroundings........

3. Chemical environment in nature $\ldots \ldots \ldots \ldots \ldots \ldots \ldots \ldots \ldots \ldots \ldots$

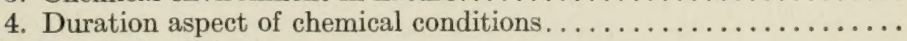

VI. Mechanical conditions:

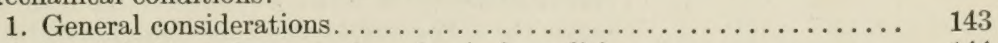

2. Destructive influences of mechanical conditions............... 144

3. Favorable influences of mechanical conditions................. 145

VII. Interrelations of the environmental conditions................. 146

VIII. Experimental determination of relations between plant activity and

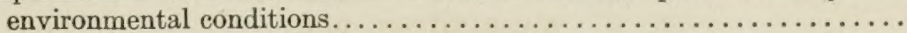


The Chimatic Conditions of the United States:

PAGE.

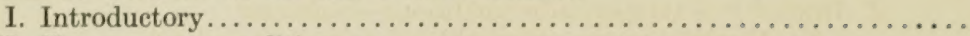

II. Temperature conditions.

1. Duration of temperature conditions.

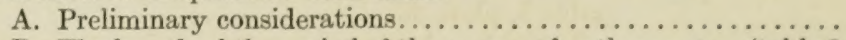

B. The length of the period of the average frostless season (table 2,

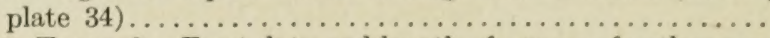

TABLE 2.-Frost data and length of average frostless season for 1803 stations in the United States ..........

C. Length of period of average frost season...............

D. Length of period of high normal daily mean temperatures

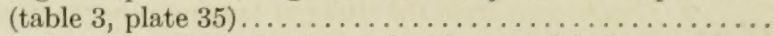

TABLE 3.-Length of period with normal daily mean temperatures of $68^{\circ} \mathrm{F}$. or above, and of period with similar

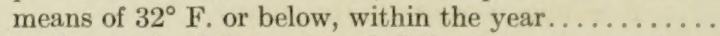

E. Length of period of low normal daily mean temperatures

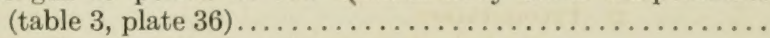
2. Intensity of temperature conditions.

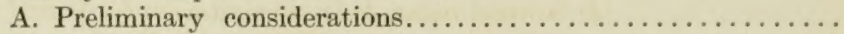

(1) Direct indices of temperature efficiency for plant growth

(2) Remainder indices of temperature efficiency for plant growth, Merriam's chart (plate 37 )............

(3) Exponential indices of temperature efficiency for plant

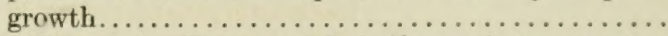

TABLE 4.-Exponential indices of temperature efficiency for plant growth, based on a coefficient of 2.0 for each rise in temperature of $18^{\circ}$ above $40^{\circ} \mathrm{F}$, for each temperature from $41^{\circ}$ to $100^{\circ} \mathrm{F}$.

(4) Physiological indices of temperature efficiency for

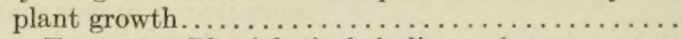

TABLE 5.-Physiological indices of temperature efficiency for plant growth, based on Lehenbauer's 12-hour exposures with maize seedlings.

B. Summations of direct indices of temperature efficiency for

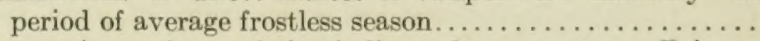

C. Summations of remainder indices of temperature efficiency for period of average frostless season (table 6, plate 38)

TABLE 6.-Summations of normal daily mean remainder indices of temperature efficiency for plant growth, for period of average frostless season, the daily indices being derived by subtracting $0,32,39$, or 50 , from the values of the normal daily mean temperature on the

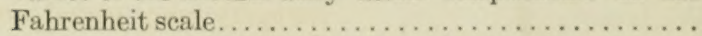

D. Summations of exponential indices of temperature efficiency for period of average frostless season (table 7 , plate 39)...

TABLE 7.-Summation of normal daily indices of temperature efficiency for plant growth, for period of average frostless season, the mean daily efficiency indices being derived from the corresponding temperature indices, (1) by the exponential equation of chemical reaction velocities and (2) by the empirical growthrate coefficients for maize seedlings as found by Lehenbauer for a 12-hour exposure to maintained temperature. The temperature efficiency for $40^{\circ} \mathrm{F}$, is taken as

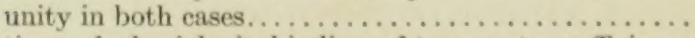

E. Summations of physiological indices of temperature efficiency for period of average frostless season (table 7 , plate 40 , and

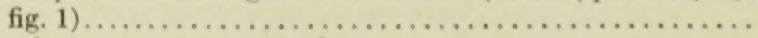

F. Absolute temperature $\operatorname{maxi} a \ldots \ldots \ldots \ldots \ldots \ldots \ldots \ldots$ 
The Chimatic Conditions of the United States-continued.

PAGE.

II. Temperature conditions-continued.

2 . Intensity of temperature conditions-continued.

G. Absolute temperature minima (plates 41 and 42 ).........

H. Average daily normal temperature for coldest 14 days of year

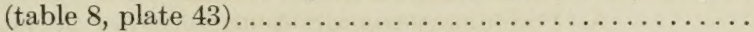

TABLE 8.-Average normal daily temperatures for coldest 14 days of the year...................

I. Merriam's mean normal temperature for hottest six weeks of

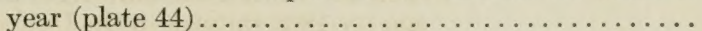

J. Normal mean annual temperature, U. S. Weather Bureau

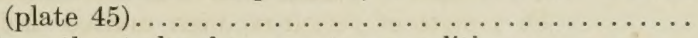

3. Conclusions from the study of temperature conditions........... III. Moisture conditions.

1. Introductory . . . . . . . . . . . . . . . . . . . . . . .

2. Supply of water to the plant.

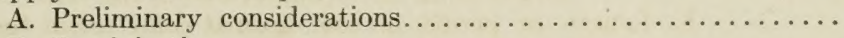

B. Precipitation.

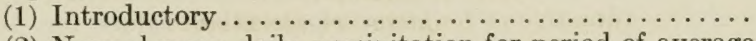

(2) Normal mean daily precipitation for period of average frostless season $(P / S)$ (table 11, plate 46 , and fig. 2)

TABLE 11.-Precipitation and evaporation data for the period of the average frostless season......

(3) Total normal precipitation for period of average frostless season plus preceding 30 days, divided by number of days in average frostless season $(\pi / S)$ (table 11$)$. .

(4) Number of normally rainy days in period of average frostless season (table 13, plate 47)............

TABLE 13.-Number of days in period of average frostless season with normal precipitation of more than 0.10 inch and with normal precipitation of 0.10 inch or less, the latter also expressed as percentage of the number of days in the average frostless season.....................

(5) Number of normally dry days in period of average frostless season (table 13, plate 48)............

(6) Percentage of days in period of average frostless season that are dry days (with normal daily precipitation of 0.10 inch or less) (table 13, plate 49) .............

(7) Length of longest normally rainy period in period of average frostless season (table 14, plate 50 ).........

TABLE 14.-Beginning, ending, and duration of each normally dry period and of longest normally rainy period within period of average frostless season........................

(8) Length of longest normally dry period in period of average frostless season (table 14, plate 51 )...........

(9) Normal annual precipitation, after Gannett (plate 52)

(10) Conclusions from study of precipitation conditions.... 3. Removal of water from plant.

A. Introductory.

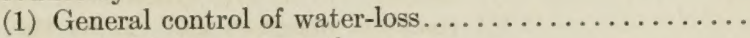

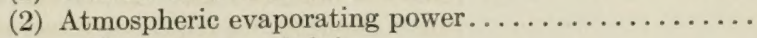

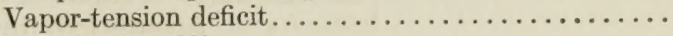

Relative humidity.......................

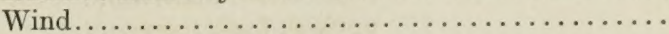

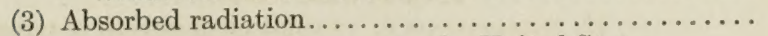

B. Atmospheric evaporating power in the United States.

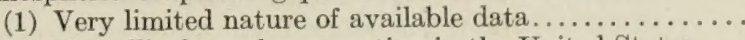

(2) Russell's data of evaporation in the United States.... 
The_Climatic Conditions of the United States-continued.

PAGE.

III. Moisture conditions-continued.

3. Removal of water from plant-continued.

B. Atmospheric evaporating power in United States-continued.

(2) Russell's data of evaporation in United States-continued.

Evaporation intensities for the period of the average frostless season (table 11, plate 53, and fig. 14)...

Annual evaporation intensities (table 15, plate 54). .

Evaporation intensities for the three summer months (table 15, plate 55$) \ldots \ldots \ldots \ldots \ldots \ldots \ldots \ldots \ldots \ldots$

TABLE 15.-Precipitation and evaporation data for the year and for the three summer months. .

(3) Evaporation studies in 1908.

Presentation of data......................

TABLE 16.-Weekly precipitation $(P)$ and weekly rates of evaporation $(E)$, the latter from cylindrical porous-cup atmometers, summer of 1908 .

Summer march of evaporation at selected stations. .

Mean evaporation values for 5 -week periods and for 15-week season (table 17, plate 56 )......... TABLE 17.-Summary of precipitation and evaporation for summer of 1908 , with averages and precipitation-evaporation ratios $(P / E)$ for the 15 weeks, May 26 to Sept. $7 \ldots \ldots \ldots \ldots \ldots$

Comparison between plates 55 and $56 \ldots \ldots \ldots \ldots$.

Summer evaporation, 1908, as shown by geographic

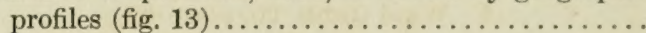

(4) Conclusions from study of evaporation conditions(fig.14)

C. Ratios of precipitation to evaporation.

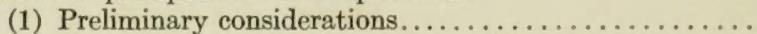

(2) Ratios of total precipitation, for the period of the average frostless season, to total evaporation for the same period, July 1887 to June $1888(P / E)$ (table 11, plate 57 , and fig. 16) ......................

(3) Ratios of total precipitation to total evaporation, for the period of the average frostless season, July 1887 to June $1888(P / E)$ (table 18 plate 58$) \ldots \ldots \ldots \ldots$

TABLE 18.-Data of precipitation and evaporation for the period of the average frostless season, for the year July 1887 to June 1888, and corresponding ratios of precipitation to evaporation, together with similar ratios derived by employing normal data of precipitation instead of those for this single year.....................

(4) Ratios of normal total precipitation, for the period of the average frostless season plus 30 days, to total evaporation for the same period, July 1887 to June $1888(\pi / E)$ (table 11, plate 59)................

(5) Ratios of normal total annual precipitation to total annual evaporation, July 1887 to June 1888

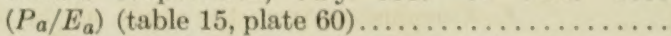

(6) Ratios of normal total precipitation for the three summer months, June to August, to total evaporation for July and August 1887 and June $1888\left(P_{s} / E_{s}\right)$

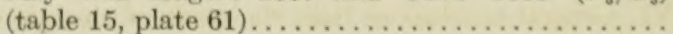

(7) Ratios of total precipitation for 15 weeks, summer of 1908 , to total evaporation for the same period and year $\left(P_{s} 1908 / E_{s} 1908\right)$ (table 17 , plate 62$) \ldots \ldots \ldots$

(8) Conclusions from study of precipitation-evaporation ratios (fig. 16) $\ldots \ldots \ldots \ldots \ldots \ldots \ldots \ldots \ldots \ldots \ldots \ldots \ldots$ 
The Chmatic Conditions of the United States-continued.

PAGE.

III. Moisture conditions-continued.

3. Removal of water from plant-continued.

D. Aqueous-vapor pressure,

(1) Preliminary considerations.

(2) Normal mean aqueous-vapor pressures for the period of the average frostless season (table 19, plate 63)..

TABLE 19.-Normal mean relative humidities, for year and for period of average frostless season, mean relative humidities for the three summer months, 1908, and normal mean vapor-pressures for the year and for period of average frostless

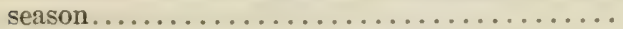

(3) Normal mean aqueous-vapor pressure for the year

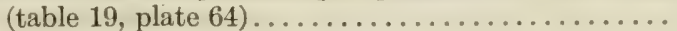
E. Relative air humidity.

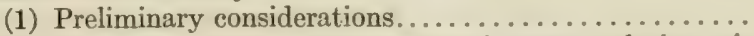

(2) Percentages representing normal mean relative air humidity for period of average frostless season (table 19, plate 65 and fig. 17)..............

(3) Percentages representing normal mean relative air humidity for the year (table 19, plate 66 ).........

(4) Percentages representing mean relative air humidity for June, July, and August 1908 (table 19, plate 67).

(5) Generalizations from the three charts of relative humidity values (plates 65 to 67 and fig. 17)..........

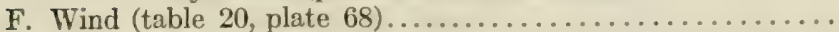

TABLE 20--Average wind velocities for the year and for the period of the average frostless

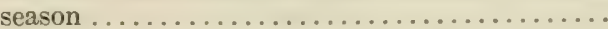

G. Sunlight as a condition influencing water-loss from plants

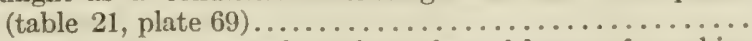

TABLE 21.-Normal total number of hours of sunshine within the period of the average frostless season....

IV. Moisture-temperature indices.

A. Introductory . . . . . . . . . . . . . . . . . . .

B. Moisture-temperature indices based on temperature summation-indices obtained by the remainder method (above $39^{\circ}$ F.), for the period of the average frostless season (table 22,

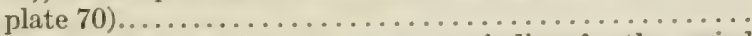

TABLE 22.-Moisture-temperature indices for the period of the average frostless season, by remainder (above $39^{\circ} \mathrm{F}$.), exponential, and physiological methods.....

C. Moisture-temperature indices based on temperature summation-indices obtained by the exponential method, for the period of the average frostless season (table 22, plate 71)

D. Moisture-temperature indices based on temperature summation-indices obtained by the physiological method, for the period of the average frostless season (table 22, plate 72,

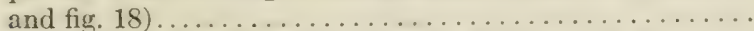

E. Conclusions from the study of the three forms of moisture-

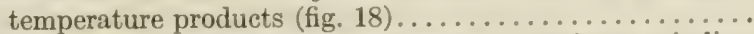

V. Cartographical combination of temperature and moisture indices

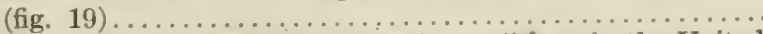

VI. General conclusions from the study of climatic conditions in the United

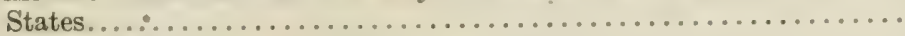

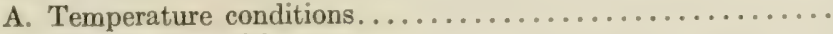

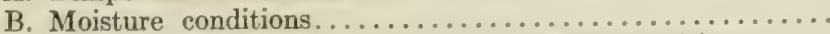

C. Combinations of temperature and moisture conditions...... 
Part III. The Correlation or Distributional Features witu Climatic

Conditions.

PAGE.

Introduction . . . . . . . . . . . . . . . . . . . . . . . . . . . 389

Presentation of the Correlation Data.

II. Climatic extremes for each of the various vegetational features........ 398

Discussion and Preliminary Interpretations of the Correlation Data.

1. Correlations as indicating controlling conditions............. 487

II. Comparative climatic features of the nine general vegetational areas.... 490

III. Conditions that probably determine the general vegetational areas.

1. Observations from the charts...................... 498

2. Discussion of the observations..................... 515

IV. Conditions that probably determine the life-zones of Merriam.

1. Observations from the charts..................... 519

2. Discussion of the observations...................... 526

V. Conditions that probably determine the distribution of growth-forms and the ecological distribution of individual species.

1. Growth-forms................................ 529

2. Species........................................ 537

VI. Correlation of vegetational areas with generalized climatic provinces.

1. Introductory .................................. 570

2. Temperature provinces............................. 571

3. Moisture provinces............................... 572

4. Temperature-moisture provinces based on product index....... 576

5. Two-dimensional climatic provinces.................. 578

Conclusion

Literature References........................................ 587 


\section{PREFACE.}

The differences in plant life which exist between distant or even between nearby localities must have come under the notice of man in the earliest semicivilized stages of his existence. Human dependence upon the products of the regetable kingdom has served to maintain throughout all historic time a vivid realization of the vegetational differences encountered with changes of latitude, altitude, and proximity to the sea. The later stages of modern civilization have done extremely little to liberate man from his dependence upon plants, although the development of methods for the preservation and transportation of food has given him greater freedom of movement into the jungle, the polar regions, the desert, and the modern city.

Many of the activities of the last 150 years have been such as to increase our interest in the distribution of plants and in the nature of the plant populations which characterize different regions, different soils, or different topographic situations. Innumerable bands of explorers and collectors have penetrated all parts of the world, bringing back materials upon which we have been able to base a knowledge of the flora and the larger aspects of the vegetation of all but the most inaccessible portions of the globe. The extensive introduction of economic and ornamental plants into new regions and even into new continents has awakened an interest in the possibility of still further introductions and in a study of the causes of the success or failure of such as have been made. The increasing value of all the products of the forest has led to the planting of trees on a large scale within their native regions and to the experimental introduction of trees from distant countries, as well as to attempts to improve natural forest stands. The increasing population of the world has augmented the value of its agricultural lands and has given importance to the study of the properties of the soil in their relation to plants. The search for new agricultural regions and for crop plants adapted to the conditions in newly settled areas has led to an interest in natural plant growth as an index of the most promising soils or of the most suitable crops to be cultivated.

Hand in hand with this widening utilization of the plant products of the world has gone a rapid development of the scientific study of plants in relation to their natural environment. During the first half of the nineteenth century there was a rapid accumulation of facts regarding the composition of the floras of the outlying portions of the earth, and these facts were almost as rapidly marshaled into an ordered knowledge of the great floristic regions. In this immense task the names of Humboldt, Schouw, Grisebach, de Candolle, Hooker, and Engler are intimately associated with the greatest accomplishments. The interests of plant geography in this stage of its development were 
largely confined to the distribution of species. Each species was of importance, because its distribution threw light upon the floristic affinities of the region in which it grew. Only a historical interest now attaches to the discussions of "centers of creation" which occupied de Candolle and his contemporaries. The inevitable questions as to the origin and significance of the floral regions of the world were immediately given a new trend by the publication of The Origin of Species. Two features of the Darwinian conceptions and method were destined to be of the most fundamental influence upon the further development of plant geography and its later outgrowths. The first was the demonstration-which it is now difficult for us to realize as so recent-that the present features of plant distribution have grown out of the distributional features of the past, and the second was the emphasis which it laid upon the importance to the plant of the entire complex or constellation of its environmental conditions.

In the hands of Darwin the great store of distributional facts served as a source of material for aiding in the demonstration of his new principle. The multifarious structures of plants, as yet incompletely investigated by physiologists and anatomists, assumed a new significance as having an important rôle in the existence of the individual and the race.

For 20 years after the appearance of The Origin of Species there was keen activity in the reinterpretation of distributional facts and in the fresh interpretation of plant structures as related to environmental conditions. The study of plant distribution and of the differences between the great floristic areas was now carried on as a dynamic subject, correlated with our knowledge of the geological past, and interpreted in terms of the importance of areas and periods of evolutionary activity, of paths of migration, of barriers, of the importance of isolation, and of relict forms. This path of investigation was traversed up to the point at which it became obscure and difficult. Its culminating achievements are recorded in Engler's Entwickelungsgeschichte der Pflanzenwelt, a work which would even now admit of only minor revisions, 39 years after its first appearance. The fresh interpretation of plant structures, to which stimulus was given by the work of Darwin, was due to a new appreciation of the importance of these structures in relation to environmental conditions; but it was unfortunately the course of events for many years that the structures themselves received attention, to the great neglect of the enviromment. The principal attempt of the outdoor workers who had become imbued with the Darwinian conceptions was to attach a signifieance to every structure and habit in plants, no matter whether such significance had been experimentally demonstrated or merely scemed to be highly. plausible. One of the most energetic and ingenious of these workers was Kerner yon Marilaun, to whom we owe many acute observations, 
interpreted by an imagination of unrivaled vigor. It was characteristic of this epoch that chief stress was laid upon the living environment, or "biological factors," while little or no attention was given to the fundamental physical factors. Much careful work was done relative to the importance of insects for pollination-the structures in plants which serve to attract insects of a beneficial character or to repel harmful insects, mammals, snails, or toads. It is impossible, however, to overestimate the value of this period, in which travel and outdoor observation received such a great stimulus. Many facts were assembled, and the value of these was by no means vitiated through the frequently wrong interpretations that were placed upon them.

The rapidly approaching completion of our knowledge of the floras of the world, and the inevitable slowness of all further investigations as to their origin and geologic history, have led to a great growth of interest in the natural assemblages of plants-in those plant communities, large and small, which we designate as vegetation. For 25. years there has been an increasing interest in the study of vegetation. This has been partly an outgrowth of the relatively finished condition of the science of floristics and partly a result of the readjustment of the principles of the interrelation of the plant and its environment. The study of vegetation has already passed through the descriptive phase which ushers in every new branch of science, into its period of greatest fruitfulness, and has brought its leading problems to the point at which they demand for their solution a precise knowledge of the functional activities of the plant and an equally precise knowledge of the environment. The subject of plant distribution and that of the relation of the plant to its environment are inseparable, and the study of vegetation during the past 20 years has been marked by a rapid coalescence of these two fields. In short, the old field of plant geography and the post-Darwinian field of environmental study have been brought together, and have pushed their problems to a point at which physiological facts and methods are of first importance for the next steps in their solution. The modern study of plant ecology may be looked upon as plant geography which has drawn its major outlines and has begun to give attention to details, or it may be regarded as a study of the relation of plant and environment in which the plant is viewed as a functioning organism and the environment as a physical complex.

The study of the environmental control of the activities of a single species of plant has many differences from the study of the control of a plant population, in so far as concerns the more general features of the controls in each case, but in final analysis the two problems merge into each other. It is precisely this fact that has come to be generally recognized and has resulted in the coalescence that has been alluded to as forming the subject-matter of ecology. 
Both communities and species may be studied with respect to the phylogenetic relationship of the species concerned and their places in the natural system of classification. The phylogenetic study of individual species has formed the sharply defined field of taxonomy, but the study of communities from the standpoint of the phylogenetic relationship of their component individuals has long been such a prominent part of plant geography that it has remained as a large element in ecological activities. The investigation of the causes which determine the distribution of plants and plant communities is essentially a physiological task, in which it is necessary for us to regard the plant as a functioning organism and to give little attention, for the time being, to the fact that it has a descent-kinship with other plants. We must keep the plant in mind as an aggregation of coordinated physiological processes, continually controlled by a complex of environmental conditions. It is only by a sharp separation of the phylogenetic and the physiological considerations of the plant that we can hope to investigate with success the relation of plants to their environmental controls. The physiologist has thus far been mainly interested in the individual processes of the plant as affected by the environmental conditions acting singly. The ecologist is interested in the collective activities of the plant, as controlled by the entire set of envirommental conditions and as measured by the dispersal, establishment, growth, reproduction, and survival of the plant in a state of nature. He is further interested in the assemblages of plants which occupy the same natural situations or habitats, which appear to be subjected to closely similar sets of environmental conditions and appear to meet these environmental complexes by closely similar or dissimilar types of physiological behavior. In short, the physiologist has mainly investigated the absolute value of conditions by the prearranged and controlled methods of experiment, while the ecologist investigates the relative value of these conditions as they cooperate to influence the plant, endeavoring to determine which of them are effective in determining habitat and distribution, and what intensities are of eritical importance in this comnection. He is also especially interested in the combination of the many different kinds of environmental conditions, as these form the infinite variety of environmental complexes furnished by nature.

While plant geography is an old science, with a large literature, and while the newer science of ecological plant distribution already possesses numerous monographs that present types of regetation, plant associations, ete., as related, in at general way, to environmental conditions, yet these studies have usually heen primarily deseriptive of the vegetation itsolf, and but little has yot been accomplished in the way of eorresponding deseriptions of the emironmental conditions that are observed to be concomitant with the rarions forms of regeta- 
tion. Much less has it been possible to discover quantitative relations between vegetation characters on the one hand and environmental conditions on the other. Before such relations can be looked for it is obvious that environmental conditions must be described in more or less quantitative terms, and similarly quantitative descriptions of the corresponding vegetational forms must also be available. The present publication is a first attempt to bring these two kinds of descriptive knowledge together for the geographic area of the United States. Because of the newness of the point of view, if not of the subject, but little detailed discussion of the reasons for the actual quantitative relations that exist between plants and their surroundings is here attempted. We have generally been content to point out the kinds of observations that appear to be needed and to bring together such observations and descriptive deductions as we have been able to obtain, both with reference to the vegetation and with reference to those of the environmental conditions that are measurable, and for which measurements are at hand.

It is obvious at once that the subterranean conditions of plant habitats have not yet received enough attention from the present point of view to make even a tentative description of these conditions possible; the soil studies that have been made are either not sufficiently quantitative or else they deal with features that are not directly related to plants, or the relation of which to plants is not yet clear. This being the case, the main environmental conditions that thus far lend themselves to quantitative study, albeit in a very superficial way, are those that are effective above the soil surface. These features comprise those conditions that are generally termed climatic. Therefore our study has dealt almost wholly with climatic features, and the relationships between vegetation and climate are the main relationships with which we have been constrained to deal. It is almost certain that the causal relationships between plants and their environments can not be satisfactorily discussed in the majority of cases until subterranean conditions are given at least as thorough treatment as we have been able to give to the aerial conditions, so that any apparently definite conclusions that seem to emerge from our comparisons must be held tentatively until suitable methods for the quantitative study of soil conditions have been devised and generally applied.

Another aspect of the causal relations that obtain, or have obtained, between plants and the environmental complexes of their habitats brings what has been termed the historic factor into prominence, and this factor involves conditions of the remote past, both aerial and subterranean. With this aspect we do not deal seriously in the present publication.

On the whole, then, our aim has not been to discover true causal relationships between the two categories of observations here con- 
sidered, but, rather, simply to describe some of the vegetational and climatic features of the country, in such a way as to emphasize the desirability of pushing this sort of study forward, and to make clear what sort of observations and what sort of deductions therefrom seem to give promise in this direction. Our work is primarily descriptive, as most ecological work must be for a long time to come, and the discovery of simple concomitancy is our nearest approach toward the establishment of causal relations. We have been led to the view that ecological science can be most rapidly advanced through this general method of quantitative comparison and by the placing upon record of such cases of concomitancy (between plants and their surroundings) as this method is able to bring forth.

Our attitude toward plants has been that of the physiologist, and we have tried to bear constantly in mind the conception that vegetational characters are simply expressions of the activities of individual plants. We maintain that all discovery of true causal relations in ecology must depend finally upon this point of view. Our attitude toward climatic conditions has been somewhat, though not wholly, like that of the climatologist; with meteorology and the causes of climatic features we have had nothing to do. Attention has been centered, as far as possible, upon those particular climatic features that directly affect plant activity. Thus most of our climatic discussions bear either upon temperature or moisture conditions. We have tried to consider climate in relation to plant growth in much the same way as the experimental physiologist considers the relations between his cultures and their surrounding conditions.

Many features of the vegetation and many climatic conditions have been omitted or have received scant consideration in the present publication. Those have been more seriously considered which seemed to give the greatest promise and for which the needed data were most readily available. The work has grown from very unpretentious beginnings made over a decade ago, and its ramifications into aspects not at first thought of have been controlled partly by a priori judgment as 10 what appeared more or less promising, partly by arailability of the requisite observations and partly by our own limitations as to time and energy as well as ability. Many other features or dimensions of elimate and of vegetation might have been dealt with, and the reader will find here many suggestions for investigations, the carrying out of which would require from a few hours to many years. In short, emphasis should be laid on the fact that the present study is to be regarded only as a beginning along a line that holds forth very great promise. The real conclusions from our work are to be drawn by others as this kind of study is pushed forward.

It may be in place here to give a little space to our reasons for attributing to guantitative physiological plant geography such great 
importance and promise for the future as we frankly do. Ecological science has won its way in a comparatively short time, and now finds itself in the front rank of those lines of intellectual effort that constitute biology in the broad sense. Following Warming and Schimper, the biological world has rapidly become very thoroughly interested in the occurrence and behavior of organisms under natural conditions and in the reasons for this occurrence and behavior. This widespread interest may be taken as evidence that ecological study is now generally regarded as fully as worth while as are taxonomy and phylogeny.

Since ecological problems are dynamic ones by their very nature, the quantitative aspect of ecological description and the dynamic relation of different sets of conditions within and without the plant must receive the main attention as soon as a superficial acquaintance with the field has been attained. Plant geography can progress but little farther by qualitative observational methods, and the physiological and quantitative point of view must, of necessity, finally prevail. Our aim has been largely to make some planned preparation for this newer development, which has already gained considerable headway.

Another and more obviously practical reason for regarding the physiological ecology of animals and plants as of very great promise lies in the fact that the art of animal and plant production (agriculture) rests almost wholly upon this branch of biological science. The problems with which the physiological ecologist deals are the same problems as have to be solved by the agriculturist. One may study natural vegetation or the distribution and environmental relations of wild animals and the other may give his attention wholly to agricultural crops and the rearing of domestic animals, but the problems and the general methods by which solution may be obtained are the same in both cases. The interpretation of crop production in terms of climatological conditions has already attained to great importance. The government of Russia long maintained an organization for the study of agricultural climatology and the results warranted great expectation. During the years in which we have been engaged upon the present investigation, the Canadian government has copied many features of the Russian organization, and this branch of the Dominion Meteorological Service is now well established. Finally, the obvious importance of climatology in agriculture has been emphasized in the United States through the establishment of a special division of agricultural meteorology in the United States Weather Bureau.

Also, it should be remarked that much of the art of forestry rests upon the science of physiological plant geography; so much so that students of forestry already clearly realize the need for studies of the kind suggested by this publication. 
The studies here reported were carried out under the auspices of the Department of Botanical Research of the Carnegie Institution of Washington. They were begun when both the authors were located at the Desert Laboratory. We are greatly indebted to many persons who collaborated and assisted in various ways. Especially should be mentioned Grace J. Flanders, who carried out most of the climatologieal computations upon which the work rests and who assisted very much in the preparation of the elimatic charts. Others who helped with computations are J. IT. Shive and H. E. Pulling. The cooperators in our series of evaporation observations, whose names are given in table 13, should also be mentioned here. It is interesting and significant to remark that this series of studies, in which these individuals so kindly assisted, formed the point of departure from which the whole study, as here presented, has been developed. 


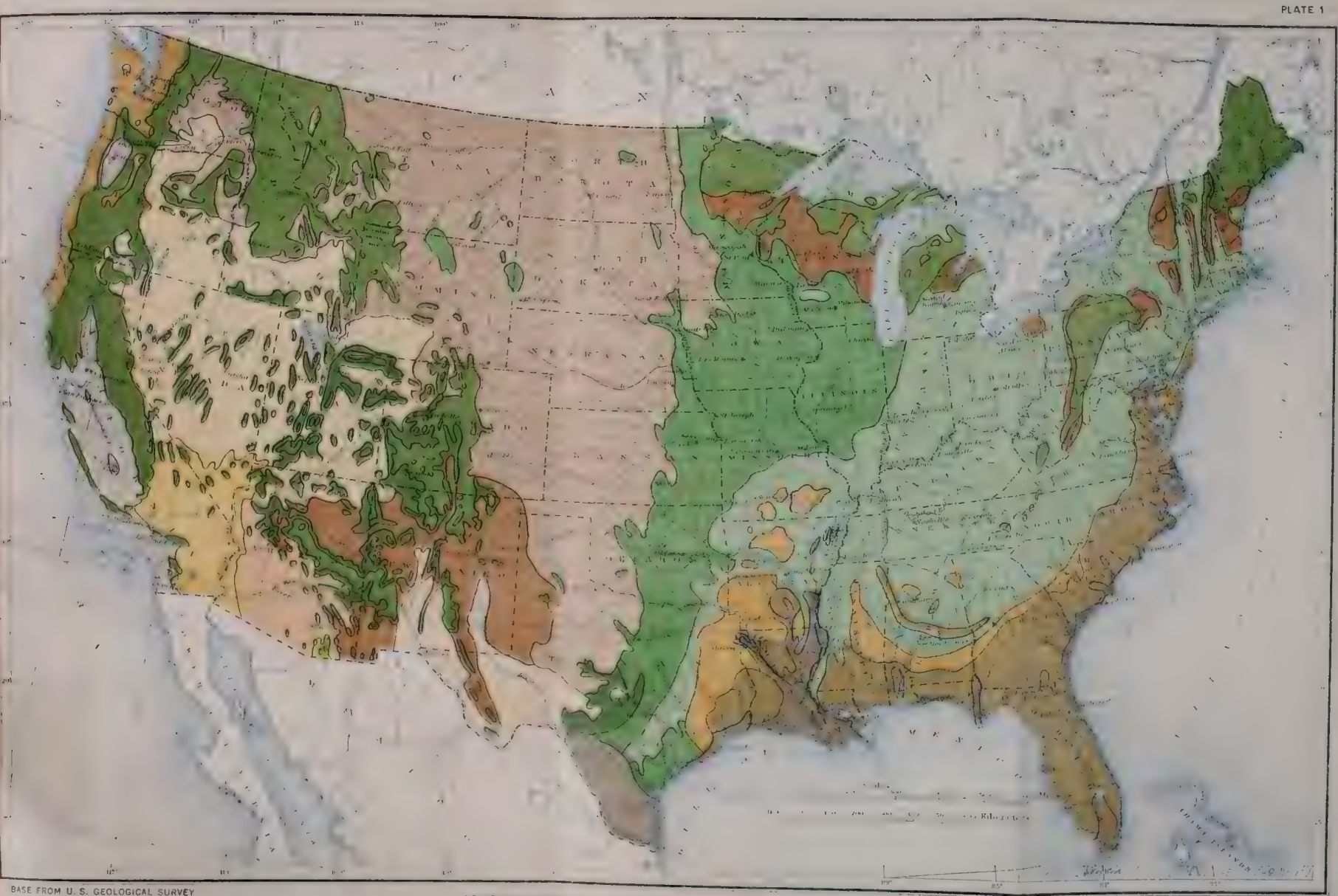

VEGETATION AREAS OF THE UNITED STATES Compiled by FORREST SHREve.
Scale 1: $9,600,000$.
EXPIANATION

$\square$

California Microphyll Desert.

Great Basin Microphyll Desert.

revas Semi-Deeer.

$\square$

Arizona Succulent Deager.

D.

Texas Succulent Desert.

Paoifio Semi-Deser $\square$

Desart-Graseland Traneition.

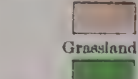

Grasaland-Deciduous-Forest.

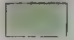

Dividuoun Forest.

\lceil]

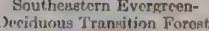

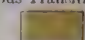

Houtheastern Mosonhytic
Evergereen Forest.

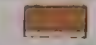

Northrastron Everartobn
Deciduous Transition Foreat.

$$
\square
$$

Northern Mesophytic

Evergreen Forest.

Weatern Xerophy

$\square$

Northwestern Bygrophytic

Evergreen Forest.

Npine Summit

$\square$

Swamps and Merohes. 

PART I.

THE VEGETATION OF THE UNITED STATES. 



\section{INTRODUCTION.}

\section{THE DISTRIBUTION OF VEGETATION IN GENERAL, AS RELATED TO CLIMATIC CONDITIONS.}

This publication constitutes an attempt to correlate the distribution of the vegetation of the United States with the distribution of some of the climatic conditions that appear to be most important to plants. It has long been a matter of common information that such a correlation exists, and some of its most obvious features have commanded popular attention from the earliest settlement of the country. The influence of a low and uncertain rainfall in inhibiting the occurrence of trees in certain portions of Kansas and Nebraska, for example, and the influence of the high winter precipitation of the Pacific Northwest in permitting the occurrence of a heavy forest in that region, are matters that have come to the attention of every one familiar with those regions. It has been the aim of our work to make a somewhat thorough investigation of such correlations as these by bringing together a carefully elaborated set of climatological data and a representative set of data with respect to the occurrence of certain characteristic species of plants, in addition to the facts of the distribution of typical vegetations. We have sought, by appropriate means, to ascertain the extremes of each climatic feature for each of the vegetational or distributional areas. In short, we have determined the maximum and minimum values of each climatic feature for such well-known regions as the Great Plains, the Gulf pine-belt, or for such well-known species as the Sitka spruce (Picea sitchensis), the sage-brush (Artemisia tridentata), and the small cane (Arundinaria tecta).

Our desire is not only to set forth the basal facts upon which we have worked and such features of correlation as we have been able to discover, but also to clarify some of the conceptions fundamental to such work and to stimulate a greater interest in it. It is particularly desirable that our work should be regarded as a preliminary and extremely general investigation of this subject for the United States, and that more exact studies of smaller areas should be carried out in order to study more thoroughly the relations with which we have dealt. It will of course be possible to use the climatological data which we have gathered for the study of other subdivisions of the vegetation than those that we have used and for the determination of the climatic controls for other species than those we have selected. It is also to be hoped that the United States Weather Bureau and other agencies will make it possible, at no distant date, to draw other climatic maps than those we have been able to construct from the data now available. 
While the determination and delineation of climatic conditions is capable of being given mathematical precision in a number of different ways (as will be shown in Part II), the classification and geographical delimitation of vegetation requires a preliminary discussion of the point of view from which such work may be done and of the conceptions on which it may be based. The term "vegetation," meaning the total plant population of an area viewed from the anatomical and physiological rather than the taxonomic and floristic standpoint, is easy of definition and clear in its meaning. So complex, however, are the natural plant assemblages of almost every locality that an attempt to classify them, even in the most preliminary manner, requires at once the use of personal judgment as to criteria of classification and as to the relative weight to be accorded to characteristics that are totally distinct in kind.

Several workers have devised methods for giving mathematical definiteness to the description of plant assemblages in terms of floristics, by count or estimation of the number of individuals of each species involved. To give this same assemblage, however, a definition which might serve to compare adequately the physiological characteristics of its component individuals with those of the individuals in another near or distant assemblage, is at present very far from possible. We are merely able to define such an assemblage as being "salt marsh," "arctic tundra," "coniferous forest," or the like. These categories are sufficiently definite in their meaning to give us a mental picture of the size, gross anatomy, density of stand, seasonal activities, and other features of the plants concerned; they are not adequate, however, for a strict comparison of the salt marsh and the arctic tundra in physiological terms, nor for the comparison of two salt marshes that are widely distant.

The study of vegetation as such has been, on the whole, greatly obscured by the fact that it has never been completely diroreed from the study of the flora. Too much emphasis can not be laid, at the present time, on the radical distinctness of the work of physiological plant geography, on the one hand, which attempts to relate the occurrence and distribution of species as physiologieal entities, to the factors of environment, and the work of floristic plant geography, or phytogeography, on the other hand-which attempts to reveal the geological history, the movements, and vicissitudes of species as phylogenetic entities. The floristic flavor which plant geography and ecology have always possessed may be largely aceounted for by the fact that all plant-geographical interest has sprung historically out of floristics, and by the fact that we are in the position of not being able to mention a plant of particular identity without using its technical Iatin name, which is solely an abbreviated expression for denoting the place we believe it to oceupy in the phylogenetie seheme. No one will deny 
that the Latin name is a convenient means of expressing genetic relationship, that it is a convenient designation for speaking about species in any connection whatever, and that it will continue to have these uses even after the student of genetics has been driven to use some numerical scheme of designation for the forms in which he is particularly interested. Nevertheless, in order to come squarely to face with the problems of physiological plant geography, we shall have to lay aside much that floristics has taught us, and shall have to ignore phylogeny, except in so far as it shows us that plants of close kinship often have the same or similar anatomical and physiological characteristics. The first writer to insist upon the physiological point of view in plant geography was A. F. W. Schimper, whose monumental Plant Geography, which appeared 22 years ago, has done much to stimulate interest and activity in what we may designate as causational or etiological plant geography.

We have approached our problems in plant geography with the mental conception that they are merely problems in physiology, with all of the environmental conditions fluctuating and uncontrolled, but nevertheless measurable, and with all the activities of the plant in normal performance and also measurable, not by auxograph and balance, but by such features as distributional extent, habitat occurrence, communal behavior, relative abundance, size, seasonal behavior, etc.

The observation, description, and classification of the innumerable types of vegetation which clothe the earth have been carried on in great detail for some of the areas of Europe, Africa, and North America, and have been outlined for the whole globe. These observations and descriptions range from the hasty and incomplete work of pioneer explorers, who were perhaps making many kinds of observations at the same time, to the most painstaking charting of the location of individual plants over larger or smaller areas. The classification of plant communities from non-floristic standpoints has been made, in connection with their description, by numerous workers in many countries, and almost innumerable schemes of vegetational classification and nomenclature have been proposed for general application.

The fundamental defect of these attempts at the classification of such a complex body of material is that they are all largely subjective in character. As a result of this, the total amount of disagreement among students of vegetation is at least as great as the amount of agreement. Perhaps no ten workers could be found all of whom would place the same plant community in the same general vegetational category or would propose less than 6 or 8 different technical designations for it. There is a strong desire among all classes of plant geographers to come into closer agreement in these matters, but it may well be asked whether, in default of fundamental and universal criteria of classification, such agreement is possible among men of different 
observational experiences, and, indeed, whether such agreement would actually yield substantial advantages.

The classification of vegetations is thus seen to be by no means an easy task, and it is no marvel that there is lack of agreement among those interested in it. Plant communities are usually made up of many species, and these species are usually of distinct floristic relationship, or dissimilar geographic range, and of varied physiological requirements and behavior. Furthermore, a particular community does not range far without the acquisition of new members and the loss of old ones. The classification of such a complex material requires the adoption of arbitrary standards, and consequently leads to an unnatural system or else to one which is only of local applicability.

Physiographic ecology affords a genetic basis for the classification of vegetation, and the logic of such a system has much to recommend it. Physiographic criteria are not, however, of universal applicability for the classing of vegetation, and lead to unnatural interpretations of regetational phenomena in many regions, particularly in the tropics, in deserts, and in regions with diversified geological and soil conditions. Furthermore, physiographic ecology is inclined to lead to a false sense of satisfaction with the assembled results of the study of a series of successions, and has often failed to stimulate a study of the physical causes which underlie the observed successional phenomena.

Several Scandinavian and German botanists have rightly urged that the classification of plant communities should be based upon the recognition of certain distinctive types or forms of plants. These "biological types," "life-forms," "vegetation forms" or "growthforms" are recognized without regard to phylogenetic relationships, and serve to distinguish such groups as deciduous broad-leaved trees, evergreen coniferous trees, perennial grasses, sclerophyllous shrubs, etc. Several systems of growth-forms have been proposed, each more elaborate and complete than the next preceding. In so far as these systems represent an attempt at a physiological classification of plants they are highly commendable, and must lie at the foundation of physiological plant geography.

Unfortunately, however, our knowledge of the physiology of plants is chicfly based on the behavior of a small number of kinds of plants, mostly cultivated forms growing under sets of conditions at best only partially controlled or measured, and the existing classifications of growth-forms have been based on the inference that the gross anatomical features of plants are an index of their major physiological characteristies. We have not yet secured enough evidence to test this inferenee in more than a general way. We know that there are marked differences between the annual mareh of transpiration and photosynthesis in crergreen conifers and in deciduous broad-leaved trees. and that there is no contradietory significance in the fact that such 
dissimilar trees often grow side by side. We know that arborescent cacti and microphyllous trees differ markedly in the character and activity of their root-systems, and that the annual course of absorption and transpiration in the two types is very unlike, in spite of the fact that the two are constant associates. In view of the incompleteness of the knowledge which might afford a basis for the classification of life forms, it behooves us to recognize a limited number of such forms and to extend the list only on securing evidence of the divergent behavior of groups of plants.

The difficulties inherent in the classification of vegetation have led some of the German and British botanists to use the physical characteristics of the habitat either as the sole criterion for classification or else as a secondary criterion, employed together with the characteristics of the plants themselves. Such a procedure may be looked upon as an indirect method of securing evidence of the physiological character of the plants involved and is logically allowable only on such ground. In any case in which plants of identical growth-form occur in two situations of very unlike physical conditions, an excellent opportunity is afforded to investigate the comparative physiology of the plants. If, as is very unlikely, the two groups should be found to be physiologically alike, there would then be no ground whatever for the separation of the two vegetations in classification. If physiological distinctions should be discovered, these, rather than the unlikeness of the habitats, should then form the basis for separating the two vegetations.

Our deepest concern for the development of plant geography is that its activities may be diverted from the description and classification of vegetation on subjective grounds and that they may be directed toward experimental work so planned as to yield an actual physiological basis for the classification of vegetation. Starting with the small body of experimental data which now makes it possible to recognize certain groups of plants with a general coherence of physiological behavior, it will become possible in the course of time, and of much hard work, to extend our knowledge far enough to actually understand the different types of vegetation which we now photograph, map, list, and name.

\section{STUDY OF THE DISTRIBUTION OF INDIVIDUAL SPECIES.}

The investigation of correlations between climatic conditions and vegetation has been extended, in our work, to the relations between the climate and the distribution of certain individual species of plants. We are here treading upon fresher ground, on which extremely little work has been done. To attempt to define the factors which are responsible for the geographical distribution of any one plant species 
is, indeed, to face a problem of considerable complexity. Eren in the presence of the results of our own work we are not prepared to maintain that the distribution of all species is at the present time strictly controlled by the complex of physical conditions for which we have tried to derive numerical values. We are in the position, however, of being convinced that certain species are thus controlled, the evidence in this direction being partly our own and partly due to an analysis of the work of others.

It is very generally maintained that the distribution of many plants is due to certain "historical factors," which is merely to say certain physical conditions which have operated in the past, or the conditions which determined the distribution of the ancestral stock of the plant in question. When we speak of the historical factors and the present factors operative in determining plant distribution, we must bear in mind that we are embracing in the former term two very dissimilar things which have registered a combined effect. We must look to evolutionary history and to paleobotany to tell us where a particular genus originated, at what epoch, and from what stock. We must look to paleobotany and paleoclimatology to tell us what have been the movements, the extensions, and the retreats of this genus. The initiation of a species is, from our standpoint, purely an evolutionary event; the history and fate of the species after its initiation are considered as dependent upon the changes in orography or climate which it may encounter, always with the possible cooperation of physiological changes in the stock which are unaccompanied by morphological modifications of diagnostic value. It is conducive to clearer thinking, therefore, to distinguish between the evolutionary factors and the paleoclimatic factors which compose the historical factor. This distinction has its principal value in compelling us to regard the present distributional phenomena of the earth as merely a momentary stage in the prolonged and incessantly active procession of change due to secular or sudden changes of climate, or to destructive or constructive events in surface geology, and discontinuously marked by evolutionary activity. We are made to realize that there is no gulf between the climate of the past and that of the present, and that there has been no sudden, extensive, or unaceountable readjustment of distributions.

The rôle of the erolutionary factor can not be escaped in any consideration of distribution. For example, we owe it to facts in the history of the great plant stocks that Yucca arborescens occurs in the Mohave Desert of Southern California and that . lloe dichotoma, somewhat similar to it in form, grows in the deserts of southwest Africa. It is likewise a part of phylogenetic history that very many plants were formerly confined to partioular regions, whereas they now have been introdued overseas, into elimates which prove to be wholly congenial to them. However, the reasons for the distributional ranges 
of Yucca and Aloe in their respective continents are to be sought in the operation of the environment, and the spread of an introduced plant in a new continent must be controlled by environmental conditions just as it was controlled in its native continent.

Evolutionary activities may be thought of as supplying the raw materials out of which the physical environment has made the present distributional complex of the earth's surface. We can not hope at present to understand why a strong development of the genus Yucca has occurred in the southwestern United States and a rich development of Aloe in South Africa, and the answer to such a question, if forthcoming, would have only a remote relation to the study of the present influences which are limiting the distribution of the members of these two stocks in their respective continents.

The rôle of paleoclimatic factors is also an immanent one in the determination of plant distribution. Many species have a known ancestry and a known ancestral range, at the same time that they now inhabit areas of such small size that it is difficult to believe that any present physical conditions are restricting them. The history of these plants has been one of extinction and retraction, due to changes in their old extended environment. They are now unable to regain their old ranges, or even to spread over relatively short distances. These species may well be looked upon as physiologically and genetically decadent, and either decadent because they are geologically old or else old because they have failed to change their requirements to that slight extent which might permit a greater extension, or to that greater extent that would have reacted upon form in such a manner as to make us regard the resulting plants as distinct species from the old ones.

In addition to the old and restricted, or relict, species we have another class of plants of limited range, those which are apparently just appearing on the scene and have not yet had time to occupy the entire area in which they might be expected to find congenial conditions. These plants are generally members of large genera, such as Opuntia, Antennaria, and Cratoegus, or else of genera which have been recently subdivided, whereas the relict species are usually members of small genera. It is not always easy to decide whether a given restricted distribution is of the relict type or of the "novitiate" type, as we may call the emerging species. Our decision, in the lack of other evidence, is apt to be based upon the existence or absence of fossil records of the species or its allies, or else upon the size and phylogenetic position of the genus or family. Such a restricted conifer as Pinus mayriana may well belong in either class, and the members of many small or monotypic genera of Mexican Compositæ may also stand in a doubtful position.

A very considerable number of our plants belong neither to the relict nor to the novitiate class. They are of such age that they have had 
time to extend themselves as far as the subtle factors of their environment, the crude power of great barriers, or the operation of suitable agents of dispersal have permitted, and their course has not run so far that climatic and orographic calamities have overtaken and restricted them.

This great class of physically controlled plants can not be specifically enumerated at the present time. There are many good reasons for believing it to contain the bulk of the species which form the dominant element in all vegetation, whether these are plants of extended range and frequent occurrence, plants of more restricted range, or plants whose occurrence is determined by complexes of conditions which are themselves rare. We may find areas of vegetation in which a relict plant is dominant, as is true of the groves of Cupressus macrocarpa near Monterey, California, or others in which a novitiate plant is very abundant, as is true of the Cababi Hills in southern Arizona, where an undescribed Opuntia, known nowhere else, is extremely abundant. Although cases of this sort are fairly common, they are usually readily detected by a more thorough study of the adjacent regions. Such trees as Liriodendron, Taxodium, and Liquidambar are known to have undergone distributional recessions, but they occupy such large areas at present that they can scarcely be classed as relict species, and can surely be placed among the plants whose distributional controls are worth looking for among factors at present operative. Whether the physically controlled plants form a large or a small percentage of our flora, we can at least state with some assurance, based upon the correlation of distribution and climatic conditions, that they form the predominant part of our regetation. The great bulk of the trees, shrubs, grasses, root-perennials, and other plants which make up the dominant natural regetation of the world may safely be held to have had their present distributional limits imposed by physical factors which are either now operative or were operative in very recent time. Such factors may be acting directly through the conditions of climate or may be acting indirectly through soil conditions, through geographic or physiographic changes, through the influence of associated plants, through animal, fungal, or bacterial enemies, through fire or mechanical agencies, or through the means of animate or inanimate agents of dispersal. Since these indirect factors of environment can affect plants only through the same kinds of physiological influences as are exerted by the direet factors of the climate, they are at bottom of the same nature, whether we allude to them as "biotic" factors, "mechanical factors," or what not.

In the isolated desert mountains of southem Arizona the great bulk of the species are physically controlled in their distribution, as is abundantly shown by the universality of each of these species throughout a given altitudinal range or a given set of habitats, and by the definiteness with which it is limited by rather sharply drawn lines. 
When one of these mountains is compared with another the floras are found to be closely similar but not identical. Certain species range over the western cordillera of Mexico or over the central Rocky Mountains and have reached certain of the isolated desert mountains without having reached all of them. At least a few cases are known in which a given species is common throughout several mountains and is known from only one or two restricted localities in another mountain, although it is there surrounded by an area possessing a favorable environment for it. In view of the universality of the distribution of most of the mountain species, these cases appear to be the opening invasions by which new components are being added to the flora. They are in a way analogous to the novitiate species of larger areas, and their presence in no way vitiates the evidence for the physical control of the distribution of the major portion of the mountain flora.

In determining the limits of the types of vegetation which are shown in plate 1, no consideration whatever has been given to the ranges of individual species of plants. It is true, nevertheless, that each of these great vegetations possesses many species, particularly among its dominant forms, which are roughly confined to the area occupied by the vegetation itself. Picea sitchensis, for example, is nearly coincident in distribution with the Northwestern Hygrophytic Evergreen Forest; Pinus treda extends very little beyond the range of the Southeastern Mesophytic Evergreen Forest, and Bulbilis dactyloides extends over nearly the same area as the Grassland and the Grassland-Deciduous Forest Transition. Other forms are nearly coincident with groups of areas, as is the case with Covillea tridentata, which is found in the California Microphyll Desert, the Arizona Succulent Desert, and the Texas Succulent Desert. Since the limits of groups of dominant plants have been unconsciously and necessarily taken into account in delimiting the vegetational areas, it is to be expected that there should be many cases in which the physical conditions concomitant with the distribution of a given type of vegetation and those concomitant with the distribution of some of its dominant species should prove to be nearly identical.

We regard the view that vegetation is determined in its distribution by complexes of physical conditions, as established beyond all cavil by the work of a large number of men, if indeed it is not almost axiomatic. That the distribution of individual species is also controlled by physical conditions is equally well demonstrated, so long as we confine our attention to the common forms which are important elements of the vegetation. There are doubtless many of the novitiate and relict species of plants which find the physical conditions of the present time a barrier to their spread, but such cases have not yet been demonstrated. We have, therefore, confined our consideration of individual species to forms which are extremely abundant, except in a few cases which will be noted. 


\section{MANIFOLD OPERATION OF ENVIRONMENTAL CONDITIONS.}

Our knowledge of the modes in which the nature of the environment may affect the activities of plants, and thereby affect or determine their distribution, is sufficiently great to give a profound impression of the complexity of the problem of the physical control of distribution. The experiences of agriculturists and horticulturists, the work of plant physiologists, and the observations and deductions of ecologists, have all combined to give us a very large body of facts relative to the manifold features of the physical environment which may be of critical importance for the spread or survival of particular plants under particular conditions. We are very well informed, both empirically and experimentally, with regard to many of the conditions which are harmful to cultivated crops and the climatic conditions which make it impossible to cultivate them beyond certain well-defined boundaries. A large part of this information relates to the ability or inability of plants to withstand frost or freezing temperatures, and to their ability to survive and grow under given limitations of water-supply.

Enough has been done in the selection and breeding of economic plants to show that closely related forms may often exhibit great differences in ability to withstand low temperatures, low soil-moisture content, great ranges of soil texture, and the like. Enough is known of the distributional limits of plants which are associated in given regions to indicate that these are not limited by the same sets of conditions; they range to different distances and into regions of diverse character in such manner as to indicate that they have very dissimilar distributional controls.

In spite of the manifold nature of the environmental controls and the well-known diversity among plants with respect to the nature and intensities of the conditions which control them, it is possible, nevertheless, to distinguish certain classes of controlling conditions. The broadest line that may be drawn is the one separating the simple or direct factors of a climatic character and those which are not directly attributable to the climate. Later pages will be deroted to an elaboration of some of the major types of direct climatic conditions, but little will be said hereafter regarding the non-climatic conditions, such as the nature of the soil, such "biotic factors" as competing plants and preying animals, and such mechanical factors as the influence of wind (in causing mechanical injury), lightning, fire, landslips, inundations, active erosion, and other agencies of very real importance but usually of local or eomparatively infrequent oceurrence. So much is known regarding the importance of the character of the soil that it is customary to speak of "elimatic and soil eonditions" as if the two were of eoordinate importance. The rôle of the soil in mantaining a watersupply for plants is of vastly greater importanee to them than any of 
the other rôles which it plays. Although the texture of the soil is of prime importance with relation to the penetration, movement, and conservation of a water-supply for plants, it is fundamentally the climatic elements of rainfall and evaporation that determine what the soils of a given texture are able to do in presenting a moisture-supply of a given amount. We are compelled, therefore, to regard the soil as a medium through which the climate acts upon plants. The supply of moisture to the plant, due primarily to climatic conditions, is secondarily determined by the soil, just as the loss of moisture from the plant is determined through the medium of the atmosphere. The soil is a medium which differs from place to place independently of the climate, while the atmosphere is alike in all places, except in so far as it is directly affected in its movements, temperature, and moisturecontent by the primary conditions of climate.

Such a view of the rôle of the soil in forming a portion of the environment of plants takes no account of the cases in which the chemical nature of the soil and the amount and character of the salts and other solutes in the soil-water become factors of great moment. In the consideration of saline and alkaline areas, and certain limestone and serpentine regions, it is necessary to do more than investigate the texture of the soil in its rôle as a stabilizer of the climatic moisture conditions.

It has been customary to speak of competition as if it were a distinct condition of elemental character, capable of admitting or excluding a given plant to a given area in much the same manner as that in which a purely climatic condition would operate. The results of competition are registered upon a plant, however, in exactly the same manner as the results of a given climatic condition or set of conditions. Competition may exclude light, may restrict water-supply, or may operate in any one of a number of ways. The end-effects upon the processes of the plant are exactly such as might be exerted through climatic agencies, except it be in those cases in which there is an addition of toxic root-excretions to the soil. Even in such cases, the toxic substances act as chemicals and the plants producing them are not directly effective. Competition may be of importance in determining the composition of small areas of vegetation, but even then the competing plants must be regarded as struggling not with each other, but with physical conditions which are of precisely the same general nature as the conditions due in other places solely to climatic causes. The cases in which plants grow so closely as to exert an effect on the environmental conditions are similar to the cases in which the major plants modify the climate for the minor plants. Both of these cases must be left out of consideration in an attempt to determine the larger features of the rôle of climate in relation to vegetation.

It is possible to lay down a program for the study of distribution and its controlling conditions, applicable almost equally well to a given 
species or to a given type of vegetation, and idealistic only in that it would require extension or modification to fit the necessities of any given case. Such a program, briefly outlined, is as follows:

A. The securing of the distributional facts.

1. Securing an exact knowledge of the geographical range of the given plant.

2. Determining the ecological distribution of the plant.

a. Its region of greatest abundance.

$b$. Its region of greatest size.

c. Its region of most rapid growth.

$d$. Its region of greatest productivity.

$e$. Its region of greatest catholicity of habitat.

3. Determining the behavior of the plant at the limits of its range.

a. The character of its limital habitats.

$b$. The evidences for its limitation.

B. Ascertaining the apparent climatic controls on a correlational basis.

1. Determining the isoclimatic lines which follow nearest to the geographical limits of the plant form considered.

2. Determining its habitat behavior with respect to climatic elements discovered in 1 .

3. Determining its comparative behavior at different portions of the periphery of its geographical range.

C. Ascertaining the actual climatic controls by experimentation.

\section{GROWTH-FORMS OF PLANTS.}

When we undertake to regard the vegetable kingdom from the ecological standpoint, and to investigate the importance of the physiological characteristics of plants as related to their distributional features, it is clear that considerations of phylogenetic relationship become of little importance. If we attempt to arrange the multifarious plant-forms of the earth in a series of groups according to their physiological affinities, so as to bring together the plants which have solved the same problems of environmental adjustment in the same manner, we shall have to depart very far from the families and genera of the natural system of classification.

This is very obvious from a consideration of the diversity that exists in some of the large plant families. In the Compositæ, for example, we have trees, shrubs, and herbs, terrestrial plants and aquaties, large-leaved, small-leaved, and leafless perennials, matforming or cushion plants, slender climbers, etc. In the southwestern United States we have, conversely, a group of small-leaved or leafless woody perennials which are green-stemmed and richly branched and bear a close resemblance to each other, in so far as regetative structures are concerned, in spite of the fact that they may belong to entirely different families. Among these plants are Thamnosma montanum (Rutaces), Kaberlinia spinosa (Kioberliniacex), IIolacantha emoryi (Simarubacese), Canotia holacantha (Celastracese), and Parlinsonia microphylla (Leguminosæ).

Among the criteria used in the phylogenetic classification of plants are: the structure of the flower, the developmental history of the floral 
organs, the systems of arrangement of leaves (phyllotaxy), the stelar anatomy of the stem, the existence and character of vestigial structures, and the recapitulation of ancestral features in the early life history of the plant. The facts which fall under these categories have been of long-standing use in phylogenetic classification, but have no direct bearing upon the present relation of the plants to their environmental conditions. Therefore these are not the criteria that would be useful in classifying plants for the purposes of ecology and plant geography. For these purposes we require a classification which shall give first attention to the vegetative rather than the reproductive organs of the plants, and to those features and structures which have to do most obviously with their relation to the conditions of the soil and atmosphere.

When plant geographers first began to break away from floristic considerations and commenced to consider plants collectively, as vegetation, they felt the need of a means by which it would be possible to express physiological relationships. It was a very difficult thing to depart from the point of view by which plants could be placed in such definite categories as the Saxifragaceæ or the Liliaceæ, or in such groups as "arctic circumpolar" or "littoral pantropist." It was still more difficult to attempt, for example, to arrange the plants of heath, moor, tundra, and alpine meadow, in a series of groups that would bring out their physiological affinities. The very instant that we distinguish between the vegetation of any two areas we have taken into account, consciously or unconsciously, certain features of difference between the plants of these areas. We notice the difference between the soft carpet of short grass which lies just above mean high tide in a brackish marsh and the tall, coarse grass which inhabits the quiet shallows below the high-tide line. We notice the difference between the forests of the southern Alleghenies and those of the Gulf Coast. In each case we have had our attention called to certain differences in the gross anatomy of the plants involved. In spite of the fact that the plants which characterize the two areas of marsh are both grasses, we recognize in them plants of different type, just as we distinguish the pines of the Gulf Coast and the oaks and chestnuts of the Alleghenian region. It is these obvious differences between plants, conspicuous even to the man who knows no Latin names for them, that form the basis for all the distinctions between vegetational areas.

Considerable attention has been given, from time to time, to the definition of these anatomically and physiologically distinctive types of plants which are best designated as growth-forms. These attempts have a very fundamental importance to plant geography, for, although many of them have been extremely crude, they represent an attempt to express an ecological similarity that exists between many plants of distant phyletic relationships. They represent an effort to establish 
categories in which we can place the rich variety of types that the plant organism has assumed. They constitute the beginnings of an ecological classification of plants from a physiological standpoint. Everyone must be aware that such a classification should have its beginnings in physiological work and not in the descriptive work of plant geography. It is not strange, however, that need for it should arise in geographical work and should be felt more by plant geographers and ecologists than by most physiologists.

In a brief review of the attempts that have thus far been made to establish systems of growth-forms that will be of service in plant geography, it will suffice to mention only a few. The first was proposed by Humbold $t^{1}$ in 1805 , in connection with his effort to determine the features that give distinctive character to the vegetation of different altitudes in tropical America. Humboldt saw, in the types which he recognized, the distinctive vegetational units that serve to bring about the physiognomic diversity of the different regions of the earth, rather than groups of possible physiological affinity. His list of 1 ? types included the coniferous tree, the palm, the cactus, the tamarincllike tree, grasses, aroids, and the like. Grisebach ${ }^{2}$ described 60 regetative forms, and his classification, like that of Humboldt, had to do largely with the conspicuous types of plants which determine the physiognomy of vegetation and aid in differentiating the great floral regions of the earth. Following upon these early classifications have come the systems of Drude, ${ }^{3}$ Krause, ${ }^{4}$ Pound and Clements, ${ }^{5}$ Raunkiär, ${ }^{6}$ and Warming. ${ }^{7}$ The systems proposed by these men are far more elaborate than the earlier ones; they embrace all crrptogamic as well as phanerogamic plants; they include aquatics as well as land plants; they take into account seasonal behavior as well as form and differentiation, and, what is best of all in an attempt to derise a natural system, they introduce subordinate categories.

The system of growth-forms most widely used at the present time, and the one that seens to have attracted the most attention to this subject, is that proposed by Raunkiär. His system is based entirely on the character of the perennating organs of plants and their position with respect to the substratum. His five groups are as follows:

\footnotetext{
${ }^{1}$ Humboldt, Alexander von., Essai sur la Geographie des Plantes, Paris, 1805.

'Grisebach, A. R. H., Die Vegetation der Erde, Leipzig, 1872.

${ }^{3}$ Drude, O., Deutschlands Pflanzengeographie, 1596 , and earlier papers.

"Tirause, E. II. L., Die Eintheilung der Pflanzen nach ihrer Dauer., Ber. d. deut. Bot. Ges. 9: $233-237,1891$.

${ }^{5}$ Pound, R., and F. E. Clements, The phytogeography of Nehraska, Lincoln, $189 \mathrm{~S}$.

${ }^{6}$ Raunkiür, C. Types biologiques pour la géographie hotanique, Bull. Acad. Roy. Sc. Danemark, Copenhague, 1905.-Livsformernes Statistik som Grundlag for biologisk Plantegeogrufi. Botan. Tidssk., 29, Kjohenhavn, 1905 (translation in Beih. Bot. Centralhl. 87, 1910).-Formationsundersögelse or Formntionsstatistik. Botan. Tidssk., 30, Kjobenhavn, 1909 (English abstract in Bot. Centralbl. 113:(662, 1910).

${ }^{7}$ Warming, E., Om Planterigets Livsformer. Festekr. ugd. af Universifet, Kjobenhavn, $190 \mathrm{~s}$.
} 
Phanerophytes: Trees and shrubs with buds exposed on branches.

Chamæphytes: Plants with their dormant buds on the surface of the soil or just above it $(30 \mathrm{~cm}$.).

Hemicryptophytes: Plants with buds in the surface layer of the soil.

Cryptophytes: With, subterranean dormant buds.

Therophytes: Perennating as seeds; annuals.

This classification expresses the physiological diversities of the vegetable kingdom in a very inadequate manner. It lays stress upon the resting organs, with total disregard of what we may term the "working organs."

Its author has more recently ${ }^{1}$ proposed a subdivision of his group "phanerophytes," based on the size of the leaves. These six "sizeclasses" make it possible to use somewhat more definite terms in descriptive plant geography, but they do not satisfy the requirement for a more precise knowledge of the physiological significance of leafsize. The fact that the transpiring power of leaves is not definitely related to their size is one of the considerations which makes this criterion of doubtful value even for a preliminary classification of growth-forms.

Raunkiär, Paulsen, and other workers have used the above system of growth-forms to derive what they have designated as "biological spectra." By this method the entire flora of a given region is apportioned among the five classes of the system, and the values are thus secured for the percentage of the total flora which is formed by each class. These spectra possess little value to the student of vegetation, inasmuch as they are based upon a consideration of the flora rather than the vegetation. The biological spectrum of a pine forest with 175 species of root perennials growing in its shade would be very slightly changed by the removal of the pines, although this would effect a very profound change in the character of the vegetation. The securing of the biological spectrum for a given number of the commonest plants of an area, as has been done by Taylor ${ }^{2}$ for Long Island, gives results of some value, but their ecological importance is still limited by the inadequacy of the classification.

The most carefully elaborated system of growth-forms is that of Drude, proposed in his Oekologie der Pflanzen. ${ }^{3}$ This system is thoroughgoing and complete at the same time that it is eminently natural, in the sense that it comprises almost no subjective or phylogenetic distinctions. The principal subdivision of the vegetable kingdom is into terrestrial, aquatic, and non-vascular plants, and the total

\footnotetext{
${ }^{i}$ Raunkiär, C., Om Bladstörrelsens Anvendelse i den biologiske Plantegeografi, Bot. Tidssk 33:225-240, 1916. - Translation by G. D. Fuller and A. L. Bakke in The Plant World 21:25-37, 1918.

${ }^{2}$ Taylor, Norman, Flora of the vicinity of New York, a contribution to plant geography, Mem. New York Bot. Gard., v. vi +683 p. New York, 1915.

${ }^{3}$ Drude, Oscar, Die Okologie der Pflanzen, 305 p., 80 figs., Braunschweig, 1913.
} 
number of growth-forms recognized is 5.5. The number of growthforms apportioned to each of the various clasises of plants is shown by the following table:

I. Terrestrial plants (38):

Trees.................. 7

Shrubs................ 9

Climbers.................. 4

Parasites and saprophytes..... 2

Grasses................. 3

Suerulents............. 3

Small perennials........... 7

Annuals.
II. Aquatic plants (6):

Amphibious.............. 3

Submerged............... 2

Floating................ 1

III. Non-vascular plants............ 11

The importance for us of a carefully elaborated and natural system of growth-forms such as that of Drude lies not so much in its details as in the criteria on which it is based. Some of the gross anatomical or physiognomic criteria are of profound and obvious physiological importance, such as the major distinction between terrestrial and aquatic plants, the distinction between perennials and annuals, and that between succulent and non-succulent forms. Other criteria are of known physiological importance, such as the distinction between saprophytic, parasitic, and autonomous plants, or between the deciduous and perennial habits of leaves. When, however, we approach such distinctions as those between broad and narrow leaves, between porerty and richness of branching, and between the possession of rhizomes and that of bulbs, we are on extremely controversial ground.

There is much evidence to indicate that the form and size of leaves has been overestimated as a criterion of importance in the ecological classification of plants. Paleobotanical evidence shows that many unusual forms of leaf, such as those of Liquidambar, Platanus, and Artocarpus, have persisted through long periods of time. The fact that these trees have undergone extensive migrations and recessions, undoubtedly encountering substantial changes of environment, affords some basis for a belief that leaf-form is often as conservative as the structure of the floral organs ${ }^{1}$. The importance of mere leaf-size in relation to water-loss has also been overestimated, as it has been shown that the transpiring power of a leaf bears no invariable relation to its size. This explains the existence, side by side in the deserts of sunthern Arizona, of such plants as Franseria ambrosiondes, with leaves from 25 to $40 \mathrm{sc} . \mathrm{cm}$. in area, and such plants as Hymenoclea monogyra and Baccharis cmoryi, with leaves from 1 to $2 \mathrm{sq}$. $\mathrm{cm}$. in area; or the concomitant occurrence, in relatively dry habitats in the mountains of Jamaica, of Bocconia frutescens, with leares often $200 \mathrm{sq}$. cm. in area, and Mirromeria obotata, with leaves less than $0.25 \mathrm{~cm}$. in area.

${ }^{1}$ For a disenssion of this topic from the palcohotanical standpoint, see: Berry, Edward W., The Lower Eocene Floras of Southeastern North America, U. S. Geol. Surv.. P'rofessional Paper 91, $351 \% ., 1916($ (1) 73). 
The nature of the growth-forms recognized by Drude has been examined with a view to determining what features of plant structure have been used by him as criteria for his subdivisions. The chief of these criteria are listed in table 1, together with the divisions based upon these criteria, and the environmental conditions to which these features seem, in the present state of our knowledge, to be most closely related. An examination of this table will show that Drude has used, in the main, criteria to which a definite physiological importance can be attached, or to which, in some cases, several lines of importance can be ascribed. The definitions which Drude has given some of his growth-forms employ the words "dicotyledonous" and "monocotyledonous." It is difficult to decide whether these words indicate a recognition of phylogenetic divisions or whether they are used as a brief and convenient means of distinguishing types of stem, of leaf, and of branching, which may have a physiological as well as a phylogenetic significance.

TABLE 1.-Analysis of the criteria used by Drude in distinguishing growth-forms.

\begin{tabular}{|c|c|}
\hline Criterion and subdivisions based upon it. & $\begin{array}{c}\text { Environmental conditions to which it is } \\
\text { related. }\end{array}$ \\
\hline Size: Trees: shrulss. & General favorableness of all conditions. \\
\hline $\begin{array}{l}\text { Length of life: Perennial (or biennial); an- } \\
\text { nual. }\end{array}$ & General favorableness of all conditions. \\
\hline $\begin{array}{l}\text { Status: Autonomous; climbing; epiphytic; } \\
\text { parasitic, saprophytic (?). }\end{array}$ & $\begin{array}{l}\text { Source of food materials. Ratio of material } \\
\text { expended in mechanical tissues to extent } \\
\text { of leaf surface. }\end{array}$ \\
\hline Stem: Caulescent; acaulescent.. & $\begin{array}{l}\text { Ratio of material expended in mechanical } \\
\text { tissues to extent of leaf surface. Exposure } \\
\text { to atmospheric factors. }\end{array}$ \\
\hline Habit of stem: Erect; procumbent. & $\begin{array}{l}\text { Ratio of material expended in mechanical } \\
\text { tissues to extent of leaf surface. Exposure } \\
\text { to atmospheric factors. }\end{array}$ \\
\hline Type of stem: Woody; succulent; herbaceous & $\begin{array}{l}\text { General favorableness of all conditions. Sea- } \\
\text { sonal incidence of water-supply. }\end{array}$ \\
\hline Leaf: Leafy; leafless. & General water-relations. \\
\hline Shape of leaf: Broad; needle-like.. & General water-relations (phylogeny). \\
\hline Type of leaf: Deciduous; perennial......... & Seasonal distribution of rainfall. \\
\hline $\begin{array}{l}\text { Branching: Absent (palms); poor (serew- } \\
\text { pines); rich (polster plants). }\end{array}$ & $\begin{array}{l}\text { General water and temperature conditions } \\
\text { (phylogeny). }\end{array}$ \\
\hline $\begin{array}{l}\text { Arrangement of foliage: Generalized; uni- } \\
\text { centric. }\end{array}$ & General water and light conditions. \\
\hline $\begin{array}{l}\text { Type of subterranean organs: Rhizome; } \\
\text { woody root; bulb. }\end{array}$ & Incidence and duration of cold or dry seasons. \\
\hline
\end{tabular}

We are here brought to face the difficult question as to whether the distinction between the dicotyledonous and monocotyledonous types of stem should be maintained in a classification of this kind. Is the distinction to be regarded as a purely phylogenetic one, or is there sufficient difference between the physiological efficiency of these very dissimilar organs of conduction and leaf display to warrant separating them? A similar question is raised as to the physiological importance of the parallel-veined and net-veined condition of leaves. Again, 
should the unicentric foliage of a Yucca be distinguished from the similar leaf arrangement of Echeveria, in which the leaves are separated by internodes? Should the sessile foliage of Agave be regarded as performing its functions in precisely the same manner as the similar leafrosette of Aloe, which is raised well above the ground on a stout stem? It is only to future investigations that we can look for knowledge that will enable us to draw a line between the structural features that are of physiological or ecological importance and those that are due to what we might designate as evolutionary inertia. It is still impossible for us to distinguish between structures that are vestigial, in the sense that they no longer perform an office that they were able to perform in the early history of their race, and structures or structural features that arose fortuitously and never served a vital function, at the same time that they were not of such a nature as to be eliminated by selection.

It does not require an examination of the physiological significance of the criteria used in any of the classifications of growth-forms to discover the fact that the water-relations of plants have done far more to influence their external form than have any other set of relations to environmental conditions. Anyone familiar with the cultivation of plants could predict with great certainty the relative water requirements of Parosela spinosa, a hoary, small-leaved tree of the Colorado Desert, and such a tree as the red maple. It is not an invariable rule, however, that the water requirements are obvious, as witness the close similarity of Baccharis scoparia of the Jamaican mountains and Baccharis emoryi of the Colorado Desert, or the general similarity of the grasses of dunes and swamps. The water requirements of a plant may, however, be much more commonly read from its outward form than may its temperature requirements. There is nothing, for example, in the appearance of Pinus diraricata and Pinus cariboca to indicate that the former grows in the cold taiga of Canada and the latter in the IVest Indian Islands and Florida.

Schimper recognized the importance of giving equal weight to the water and temperature requirements of plants in grouping them for ecological purposes. He accordingly divided each of the general classes of plants which are recognized on a basis of their water-relationsxerophytes, mesophytes, and hydrophytes-into three classes based on temperature requirements-microtherms, mesotherms, and megatherms. In this manner nine eategories were secured, in which it was possible to place plants only after securing some knowledge of their habitat requirements.

The only logical basis on which we can proceed to a classification of the vegetation of the world is one in which we take account of the nature of the regetation itself, and give no weight whatever to any of the natural conditions or circumstances by which regetation is affected. It is for this reason that importance attaches to the study of growth- 
forms. If we wish to understand vegetation we must understand the individual species of which it is composed. If we wish to understand the relation of each plant species to its environment we must understand the nature of its functions and the character and rôle of each of the organs through which they are carried on. Whatever features of the gross anatomy of plants may be discovered to have no apparent importance in any aspect of their adjustment to environment will have no place in shaping our ultimate system of growth-forms. Progress toward such an ultimate system is beset by two dangers: that which would lead us to be satisfied with a system which is too simple, and that which would lead us to adopt a system in which anatomical features of questionable importance would be recognized along with those of demonstrated importance.

Vegetational units have been grouped or classified by various workers according to the nature of the habitats in which they are found, according to their floristic make-up, and according to their successional relation to one another. A large body of work has been done by these methods, giving us a substantial part of our knowledge of the vegetation of the globe. The only one of these methods which is purely vegetational is the last. If it were possible to demonstrate changes of vegetation in a state of nature which were not accompanied by changes of environmental conditions, it would indeed be necessary to give strict attention to the stages of succession in making any attempt to correlate vegetation and conditions. If it were true that identical conditions might sometimes present different vegetations, our problem of correlation would be made still more complicated than it already is. It has been amply shown, however, that successional changes of vegetation are both preceded by and accompanied by changes of environment. The well-known work of Cowles has shown the importance of the changes of soil-moisture which accompany physiographic development, and the work of Fuller, of Gates, of Weaver, and of Cooper has shown the importance of other conditions of both soil and atmosphere. In addition to the physiographically initiated changes in the environment are those initiated by the vegetation itself, supplying conditions favorable for invasion by a new group of species. Although a large amount of work has been done in describing successions and in relating successional stages to each other, it is only recently that the workers just cited have made a beginning in the investigation of the physical conditions which underlie the separate stages. As soon as we begin to study the relation of physical conditions to successional stages, the relation of these stages to each other sinks to a position of minor importance, and our work emerges upon the broad field of causational plant geography.

The imperfections of our present knowledge of the physiology of plants and the consequent imperfections of our system of growth- 
forms are carried on into our classification of vegetation. The fact that the water-relations of plants are more easily known from external criteria, and the fact that they have been more thoroughly investigated, have not only influenced our prevailing system of growth-forms, but have determined the nature of our vegetational units.

The classification of growth-forms and the classification of vegetation are like all other scientific efforts to reduce natural phenomena to a logical system, in that the classification possesses its chief value as a concise expression of the results of research. I classification of growth-forms which had been highly perfected by our present methods and knowledge would still be roughly made from the point of view of the ecologist and physiologist of tomorrow. It is perhaps idealistic, and is surely premature, to hope that we may one day have an ecological classification of the vegetable kingdom on a physiological basis. Such a classification will merely be the perfecting of the beginning which has been made by Drude and his predecessors. It will not be possible without a great deal of physiological work that is not yet so much as planned, and it will not be of more than academic interest unless it is constructed from a broad ecological point of riew.

\section{PLANT COMMUNITIES.}

The study of vegetation is essentially a study of plants which are growing together in a state of nature, it is an investigation of all the phenomena which these plants exhibit as an aggregation, as distinguished from the behavior of any one of them when considered alone. The natural assemblages of plants which characterize given areas have been assiduously studied by a very large number of workers in all portions of the world. The contrast between the small aggregations of local character, the larger ones of more general oceurrence, and the still larger ones of very wide distribution has given rise to the recognition of various ranks of aggregation or association and to the study of the relationship existing between aggregations of different rank. By common consent among plant geographers and ecologists. the term "eommunity" has been adopted as a general designation for any assemblage of plants regardless of its rank in the formal schemes of classification.

In our work with the regetation of the United States we have had to do with the climatie enditions influeneing certain of the plant communities, and we have been under the necessity of deciding unon the criteria to he used in differentiating the conmunities, as well as under the need of disregarding, for our immediate purpose, certain other communities which stand in definite relation to climatie conditions, as woll as the communities which are affected by climate chiefly through the medium of the soil. We have, perhaps, been some- 
what informal in our handling of this eminently formal subject, upon which so much has been written and so much has been enacted by botanical congresses.

As already intimated, the study of vegetation has resulted in the recognition of different degrees of communal existence among plants. These degrees have been designated by names, among which may be found the words formation, region, zone, society, association, district, consocies, group, belt, strip, and a score of others. Scarcely any two workers have used the same term in precisely the same sense, and few of the workers have defined their terms in such a manner as to enable a botanist to recognize one of the communities in case he should find himself in its midst. There has been an organized effort in recent years to secure a general and international agreement regarding the classification and nomenclature of plant communities. The extensive areas such as the sagebrush plains of the Great Basin, the grasslands of Nebraska and Kansas, or the pine forests of the Atlantic Coastal Plain are designated as formations. The smaller and less markedly differentiated areas within a formation are designated as associations, as, for example, the forests of shortleaf pine in New Jersey, those of loblolly pine in Maryland and Virginia, and those of longleaf pine in the Gulf States, all lying within the Coastal Plain formation. The smallest units of vegetation are termed societies, and these are of small area and represent portions of the association in which a definite aggregation of species is to be found.

This classification of communities is simple and natural and has much to commend it for general use in describing vegetation. It is to be noted that, just as the formation is defined in terms of growthforms which are found to be most common and characteristic in that community, so the association is defined partly in terms of the growthforms present and partly in terms of species, while the society is defined chiefly by the species which it contains.

The most important criterion to be employed in the distinguishing of communities is always the kind or kinds of growth-forms which are present, and this is a criterion which can be used for societies as well as for formations. A community may present a single growth-form, represented by a single species or by a group of species, as is true of many saline marshes and of very many forest areas. It may present an intermingling of two or three growth-forms, as is true of those saline marshes that contain grasses, the succulent Salicornia and the largeleaved Statice. In certain localities there occur communities which are made up of a very large number of growth-forms, as, for example, in the Karroo Desert of South Africa and in the deserts of Tehuacan, Mexico.

Communities may be of such a character as to contain only plants of a single order of size, as the short-grass prairies of Nebraska, or they 
may contain individuals of a particular height, together with other smaller individuals, as is true of the fresh-water marshes of the tributaries of Chesapeake Bay. By far the most common condition is that in which several successive stages of height are found together, so that the regetation is said to possess a stratification with respect to the foliage of the plants concerned. This is found in every forest and reaches a splendid climax in the tropical rain-forests. In the communities which possess several strata of plants, the highest one has been designated the "facies," but its members will here be alluded to as the "major" plants of the community and the several lower strata as the "minor" plants.

$A$ further salient feature of communities depends upon whether the plants cover the ground closely or stand in an open formation. Te may discover open or closed communities among plants of every stature, from the smallest grasses to trees of a height of 50 or 60 feet. The greatest density of stand is reached by trees only under conditions which favor the attainment of a greater size than this. In open communities there may be plants of different heights, but in such cases the low plants are not dependent on the large plants for the conditions which render their existence possible. Even in open communities there may be a certain degree of stratification under the largest individuals as is observable in southern Arizona, where many small annuals occur abundantly beneath the trees as well as away from them.

Last among the criteria of the community is the number of species comprised in it. Even if there is a uniformity of growth-form throughout the community, there is importance, from our standpoint, in knowing whether this uniformity is caused by the existence of a single species or of many. It is customary to designate the species which is most common in a community as the "dominant" one and the other species as "subordinate."

The salient features by which we distinguish types of regetation are, then, to sum up: the growth-forms involved, the presence of one or many strata of plants, the open or closed condition, and the degree of simplicity or complexity of the specific content. These are all features which must be looked upon as products of the environmental conditions just as truly as are any of the structures or physiological reactions of the individual plants themselves. The presence of a single growth-form or of many, the existence of a low carpet of plants or a lofty forest, the openness or density, and the dominance of a single species or the successful association of many, are all features which are determned by the environmental complex just as truly as is the rate of growth or that of photosynthesis for an individual plant or species.

The only other criteria that have been used in defining communities are the physical nature of the habitat and the specifie identity of the 
plants concerned, each of which has been sufficiently discussed to indicate that they are counter to our purpose.

In making a general study of the relation of climate to vegetation for as large an area as the United States, it has been necessary for us to disregard the small communities which are unlike the general vegetation of the surrounding region, such as the local prairies of Arkansas and Mississippi and the bands of trees that border the rivers of the western plains. These require special treatment, in which soil conditions can be given a thorough investigation. It has also been necessary for us to leave out of reckoning all of the minor plants in the stratified communities, inasmuch as the conditions under which they live are unlike those for the major plants. The climatic conditions under which the major plants exist are modified by them in such a manner as frequently to give the minor plants a very different environmental complex.

By means of these omissions we have done a great deal to simplify our problem from the standpoint of the demarcation of vegetational areas. It has been desirable, furthermore, to consider only the most general features of the vegetation, because we have only very general data as to the distribution of the climatic factors. The subsidiary communities of every region are so largely controlled through the soil conditions that it would have carried us beyond our investigation of climatic controls to have entered upon a consideration of them.

The statement that the occurrence and geographical range of all plant communities is controlled by the physical, and rarely the chemical, characteristics of the environment is almost axiomatic. The operation of these controls has been observed from time immemorial by men of no technical training but of keen powers of observation, and the knowledge of them has become more exact among the practical pioneers in the agriculture and forestry of many lands where the natural vegetation has been used as an index of the cultural capabilities of given situations. Any skepticism regarding the physical control of communities would be dissipated by an extended course of travel in diversified regions, or equally well by a careful reading of Schimper's Plant Geography, to say nothing of an examination of the many scattered papers which give proofs in regard to particular instances of such control.

We can, in brief, put it down as a law of plant geography that the existence, limits, and movements of plant communities are controlled by physical conditions. The conditions that control the movements of the community are those of the soil; the conditions that control the broader geographical limits are almost solely those of the climate. The existence of the community and the extent of the area occupied are, of course, controlled by conditions of both soil and climate. 


\section{DELIMITATION OF VEGETATIONAL AREAS.}

The botanical areas that have formed a basis for the correlations discussed in the following pages have been outlined in such manner as to show either the distribution of particular types of vegetation or else the ranges of individual species or groups of species. The determining of the distributional area of a given species is a relatively simple matter, depending for its accuracy only on the exploration that has been carried out and the records of occurrence that are arailable. The delimitation of regetational areas, however, demands a careful scrutiny of criteria and methods such as we have attempted to give above. "We have endeavored primarily to classify and map the vegetation of the United States upon a basis which is purely vegetational, without regard to floristics, climate, topography, or other features, however closely these may seem to be associated with the regetation.

The effort to observe this requirement, for the sake of the logical soundness of our work, is nevertheless far from removing all of the difficulties which liecet an attempt to classify and map the regetation of a large area. It is difficult for the worker to avoid a subjective treatment of his material and to escape the bias which his own particular experiences or field of observation may have given him. I more tangible set of difficulties arises in deciding where to draw the lines of demarcation in subdividing a set of intergrading vegetations, and where, on the map, to place the lines of separation between regetations that merge into each other over areas of great extent. This difficulty has been met by drawing lines on the chart through all transitional regions, these lines being so drawn as to be regarded as connecting the points that exhibit the same stage in transition, after the manner of isotherms and other isoclimatic lines.

After a series of regetational areas has been distinguished and delimited, and each has possibly been subdivided, we must refrain from regarding these divisions or subdivisions as of coordinate value. for there is no means of putting the degree of their relationship to a test. The subdivisions of the forest areas and those of the desert areas appear, on the printed page, to be of the same rank in classification, but we have no actual knowledge upon which we can base such a supposition.

We have already seen that the features of outward configuration which are considered in distinguishing growth-forms have to do, to a predominant extent, with the water-retations of plants. When wo examine into the other features which we use in distinguishing reget:ations, such as the height of the dominant plants, the density of stand. and the simplieity or eomplexity of the stand. we are impressed with the fact that these features stand also in dependence upon water. relations. 
These considerations force us to realize that the most commonly used and most natural subdivisions of regetation are based upon criteria which have to do with the relations of the communities to watersupply and water-loss. (It is quite true that the water-relations of plants have more to do with the control of the local and general distribution of vegetation than have any other conditions. This is not true of the local and general distribution of the species themselves, for we here find temperature relations playing a strong rôle. 'For the purposes of our investigation into the correlations existing between regetation and climate it is therefore significant that we are under the necessity of using a classification of vegetation which rests so largely upon a basis of the water-relations of plants. We might foresee from this fact that strong correlations would be discovered between vegetation and water conditions and weak correlations between regetation and temperature conditions.

In spite of the efforts of Schimper and of others to give the temperature-relations of plants a place in vegetational distinctions, by the recognition of microtherms, mesotherms, and megatherms, we are as yet unable to place a given species in its proper thermal category without possessing facts which are still lacking for all but a rery few plants. The can not tell a megathermous plant from a microthermous plant when we see them growing side by side, and it follows that we can not go into the field in Georgia or Texas and pick out the plants in each habitat which have great or small temperature requirements, as we can rather satisfactorily distinguish those of great or small water requirement. These categories are consequently of no present use in delimiting vegetational areas.

The system of life-zones which was worked out by Merriam $^{1}$ for the United States, and has been elaborated by members of the United States Biological Survey, constitutes an effort to delimit biological areas primarily with respect to the influence of temperature. With such a classification of biological areas in hand it is not possible, however, to make an impartial effort to determine which of several climatic factors is primarily concerned in conditioning the existence and limits of the areas. This attempt would indicate a very close correlation of biological areas and certain temperature conditions, because the climatic maps showing these temperature conditions were used as a basis in the original form of the life-zone map.

If we were to make a map of the mean rainfall of the growing-season in the United States it would be found to possess certain isohyetal lines which corresponded closely with the distribution of certain plants or vegetations. If we were, then, to modify the rainfall map in slight

\footnotetext{
${ }^{1}$ Merriam, C. Hart. Laws of temperature control of the geographic distribution of terrestrial animals and plants, Nat. Geog. Mag., 6:229-238, 3 maps, 1894.-Life zones and crop zones of the United States. U. S. Dept. Agric., Biol. Surv. Bull. 10, 1898.
} 
particulars so as to make it conform more closely to the distribution of vegetation, using vegetation rather than additional rainfall data as a basis, we would secure a map of great interest and value as a delineation of the vegetation of the United States. This map would be of no value for a correlational study, however, since it would be in inherent and foreseeable agreement with the map of mean rainfall of the growing season, which is very similar to all other maps of moisture conditions. The drawing of this map would resemble in all particulars the construction of the life-zone map of the United States, both with respect to the manner in which it was made and with regard to its unsuitability for our purposes.

It may also be emphasized in this connection that, although the temperature conditions and the moisture conditions of climate are considered as distinct in analytical studies, yet they are not truly independent of each other. Since evaporation and precipitation are so largely influenced by temperature, neither the chart of rainfall nor that of temperature is wholly without indications of the influence of one condition upon the other. It frequently happens, for example, that a region of low temperature is one of high soil-moisture content, low evaporation, etc. These considerations will receive more attention in Part II. 


\section{DISTRIBUTION OF VEGETATION IN THE UNITED STATES.}

\section{METHODS USED IN SECURING AND PRESENTING THE DISTRIBUTIONAL DATA.}

The botanical data on which we have based the correlations that are to be discussed in the succeeding pages are presented in cartographic form in plates 1 to 33 . A detailed map of the vegetation of the United States (plate 1) has been executed as a basis for our correlations of climate with the vegetational areas of the country as a whole. ${ }^{1}$ The features of the map will be discussed in a succeeding section, together with a general account of the vegetation of the 18 subdivisions which it recognizes. This map is of very uneven merit for the different parts of the United States, owing to the fact that there is an abundance of literature for certain portions of the country, while there are very few descriptive treatments or maps of the vegetation for other portions. As it stands, however, this map is somewhat too detailed for use in correlation with the climatological data that we have been able to secure. For this reason we have deemed it desirable to make a generalized map based upon the detailed one (plate 2). The latter contains 18 vegetational areas, whereas in the former the number has been reduced to 9 by a combination of the areas which are most similar in character. Even after this is done there are some of the vegetational areas of the United States for which we have only a very small number of climatological stations.

In order to investigate the correlations between climatic conditions and the distribution of certain common growth-forms, a series of 7 maps has been drawn, showing the cumulative occurrence of these forms (plates 3 to 9). The maps have been prepared by the method used by Transeau in his investigation of the forest-centers of the eastern United States. ${ }^{2}$ It consists merely in indicating on a single map the distributional limits of all of the plants involved. The area in which all of them are found together represents the region of maximum development of the particular group which has been selected. In some cases these maps have been drawn for all of the species of a particular growth-form, whereas in other cases they have been drawn for a representative group of the most common species of a particular growthform. These maps are of value, not only in showing the center of development of a particular form or group of species and in showing the extreme limits of the form, but also in showing the manner in which the abundance of the given form shades off in different directions from the center.

\footnotetext{
${ }^{1}$ Shreve, Forrest, A map of the vegetation of the United States, Geographical Rev. 3: 119125. With map. 1917. This map is reproduced here as our plate 1 .

${ }^{2}$ Transeau, E. N., Forest Centers of Eastern America, Am. Nat., 39: 875-889. 1905.
} 
Three maps have also been prepared showing the ecoloxical distribution of individual species of plants (plates S, 9, 10). On these maps an effort is made to show the features of distribution in such a way as to indicate the regions of greatest abundance and greatest catholicity of habitat, the resions of frecuent omenrrence, the regions of rare occurrence, and the extreme geographical limits of the species. Maps of this character afford a picture of the distribution of a single species which is similar to the picture afforded by the majos of cumulative distribution of groups of related growth-forms.

As a basis for the correlation of climatic conditions with the ranges of individual species, 70 plants were selected, the distributional areas of which are shown by groups on plates 13 to 33. Some of the species selected for this purpose were chosen because they are common and dominant elements in important regetations of wide extent. Others were selected because their geographical ranges seem to be typical of those exhibited by a large number of species. Certain other species were selected because of the interest which attaches, from our point of view, to the character and particular direction of their distribution. Several of these plants extend across the continent from the Atlantic to the Pacific, either in the northern or in the southern part of the United States, in such a manner that they cross the principal boundaries between regetational areas. Distributions of this character seem to indicate that the plants in question are probably controlled by temperature conditions rather than moisture conditions. Sereral plants of this character and of more limited range were selected, because they are commonly found in swamps or marshes and may therefore be thought of as growing through a wide range of atmospheric conditions, at the same time that they are subjected to a relatively uniform set of soil-moisture conditions. It is to be anticipated that plants of this character differ markedly in their distribution from those whose range is greatly influenced by soil-water conditions.

The construction of the regetational maps has involved the examination of a large body of ecological, floristic, and geographical literature. It was originally our plan to publish a complete list of the sources that were used in the compilation of these maps, but the appearance of IIarshberger's Phytogeographic Survey of North America has sinee made it superfluous to do so, inasmuch as this author has given a very thorough bibliography of the literature of Ameriean vegetation, including nearly all of the publications that we have used. ${ }^{1}$

In the construction of the maps of vegetation we have been heavily indebted to the maps published by the United States Forest Service, to the maps of grazing-lands published by the United states Bureau of Plant Industry, and to the detailed maps which have been published

\footnotetext{
1Harshberger, J. W., Phytogeographic survey of North America, Die Vegetation der Erde,
} 13: 863 p., 32 figs, 18 pls., 1 map, Loipzig, 1911. 
for sereral States. We have not only drawn upon ecological literature and the publications of the United States Geological Survey, but have been greatly aided by consultation of the photographic illustrations in numerous works of a non-botanical character, and we are particularly indebted to many of our colleagues, who have generously given us the information at their disposal.

In the preparation of the maps showing the cumulative distribution of growth-forms and the distribution of individual species we have used all of the manuals, floras, and local lists that it was possible to secure. We have not consulted the specimens that are to be found in any of the large herbaria, but have depended solely upon published statements of occurrence. We have done this because it is so frequently possible to secure, in ecological literature or in the publications of the United States Biological Survey, statements regarding the ranges of plants which would in all probability be represented in the herbaria by only a few collections, on the labels of which the information regarding the ecological occurrence would be extremely meager.

Some of the ranges of individual species are naturally outlined much more accurately than others. The distribution of trees is in general much better known that that of herbaceous plants, and the distribution of grasses is better known than that of plants whose economic importance is not so great. Some of the ranges exhibited in our maps have been based on extremely few stations, as is true particularly of stich plants as Florkea occidentalis and Trautvetteria grandis. It may be taken for granted that all of our distributional areas which are represented by smooth and wide-sweeping lines are in general based upon less precise information than are the areas limited by very sinuous lines. The limitations of our method of mapping have required that the range of numerous mountain plants occurring in the Western States be exhibited by passing a bounding-line around the entire region in which they are found locally at their appropriate elevations. In similar manner several of the aquatic and palustrine plants have been plotted as if growing continuously throughout extensive stretches of country in which they are really of very local occurrence. This is notably true for Sium cicutcefolium and for Cephalanthus occidentalis, which is not actually known in New Mexico and is very uncommon in Arizona, although it reappears in great abundance in interior California. The scale of our maps has made it necessary to include in the ranges of very many of our plants certain extreme coastal locations in which they are actually absent. This matter is mentioned only because several important climatological stations are located on the coast in areas which differ very markedly from the adjacent mainland in the character of their regetation. This is true of Key West, Cape Hatteras, and Point Flattery. Further details regarding the vegetational maps will be given in the succeeding pages. 


\section{LEADING VEGETATION TYPES OF THE UNITED STATES AND THEIR GEOGRAPHICAL AREAS.}

In subdividing the vegetation of the United States we have used primarily the old distinction of desert, grassland, and forest. The distinguishing of these formations involves practically all of the criteria that have been discussed in previous pages. Our ultimate units have resulted from a subdivision of the desert and the forest, but we have not attempted to subdivide the grassland, partly because of lack of descriptive literature for all parts of the grassland region, and partly because of the extreme complexity exhibited by this region, particularly in its central portion.

The desert areas have fallen into two groups which may be designated as Continental Desert and Coastal Desert. The latter include the semidesert regions of coastal California and of extreme southern Texas, each of them regions in which truly desert areas are found together with areas which scarcely merit this designation. The Continental Desert falls naturally into two regions, one of which is dominated by sclerophyllous shrubs and the other by a mixture of such shrubs and succulent or semisucculent plants. The sclerophyllous desert has been subdivided into the Great Basin Desert and the California Desert, while the succulent desert has been subdivided into the Arizona and Texas areas. The two bodies of sclerophyllous desert are adjacent and merge into each other, whereas the succulent deserts are separated by regions of dissimilar character.

The Grassland area is bordered on the southwest by the DesertGrassland Transition and on the east by the Grassland-Deciduous Forest Transition.

The forested portion of the United States has been subdivided into the Deciduous Forest and 4 areas of Evergreen Needle-Leaved Forest. Two of these evergreen-forest areas are mesophytic in character, one of them xerophytic, and one of them hygrophytic. The have included in the Northern Mesophytic Evergreen Forest all of the needle-leared forests of the northeastern States as well as of the Rocky Mountain and Pacific regions, with the exception of the extreme Northwest. Although this large forest region exhibits marked differences in its floristic make-up and in minor features of its physiognomy and ecological characteristies, there are nevertheless more reasons for maintaining it as a single area than for separating it into minor subdivisions, so far as the purposes of our work are concerned. The Southeastern Mesophytic Evergreen Forest, the Western Xerophytic Forest, and the Northwestern Hygrophytie Evergreen Forest are all regions of distinctive character which are neither similar to the Northern Mesophytic Evergreen Forest nor to each other. 'Two transitional areas have also been outlined, connecting the Deciduous Forest with the Evergreen Forests to the north and to the south of it. 
We have also recognized as alpine summits all of the areas lying above timber-line, and as Swamps and Marshes the areas with saturated soil in the Atlantic Coastal Plain.

In selecting names for the vegetational areas thus distinguished we have felt it desirable to use designations which have to do with the ecological character of the plants found in these areas. We have avoided the use of such words as coniferous, which alludes to a morphological feature, and have also avoided using any designations which would imply that the regetation of a particular area is controlled by a particular physical condition, as, for example, the use of such terms as "monsoon forest" or "pinelands of the oölitic limestone." We are well aware that the names that we have used are neither brief nor convenient, but they have been selected with the cautions that have been mentioned, for purposes of ecological consistency.

An effort has been made to draw all of the vegetational boundaries on the map shown as plate 1 with relation to the original plant covering. This primeval condition has been so greatly disturbed over large portions of the country, particularly in the Northeast, that it is now very difficult to be certain as to the limits between the virgin forest formations. Our map is therefore undoubtedly much less accurate for the northeastern portion of the United States than it is for the Western States, in which the vegetational differences are more marked, the country is less disturbed by human activities, and the published ecological descriptions of vegetation are much fuller and more accurate.

The following paragraphs serve to give a brief characterization of each of the vegetational areas found in our detailed map, plate 1:

California Microphyll Desert.- - The southernmost part of Nevada and the interior portion of California form an area in which the vegetation is closely related to that of the Great Basin by reason of the fact that the dominant plants in each are microphyllous shrubs. In the California Desert the creosote-bush (Covillea tridentata) and the sandbur (Franseria dumosa) are the most common plants and the dominant ones over extensive areas. A large number of deciduous shrubs are found, large semisucculents occur throughout the more elevated portions of the desert (Yucca brerifolia, Y. arborescens), and stem-succulents (Opuntia spp.) are found in small numbers. In both the Great Basin and the California deserts the plants which perennate by underground parts are very rare and grasses are uncommon, while shortlived annuals are abundant in the spring months.

Great Basin Microphyll Desert.-This desert occupies the floor and low mountains of the Great Basin from southern Washington to southern Nevada and eastward to Colorado, lying at elevations of 1,000 to 5,000 feet. Throughout practically the whole of this area the vegetation is dominated by a single species, the sagebrush (Artemisia tridentata), a small-leaved evergreen shrub which sometimes 
occurs in very open stand with a height of a few inches or at other times grows so thickly as nearly to cover the ground, reaching a height of 4 feet or more. Together with the sagebrush are to be found several other microphyllous shrubs of similar size and distribution (Sarcobatus vermiculatus, Grayia spinosa, Coleogyne ramosissima, Kunzia tridentata, Tetradymia glabrata, etc.). The Great Basin desert is distinctively a region of microphyllous shrubs, in which succulent plants are rare and confined to the highest mountain-slopes. The simplicity of the vegetation and the uniformity in the character of the several shrubs which play a secondary rôle in it are features very unlike the other desert areas about to be described.

Texas Succulent Desert.-The central valley of the Rio Grande and the valley of the Pecos River form a desert area in which extensive tracts are dominated by evergreen shrubs in open stand, while other areas are chiefly occupied by deciduous shrubbery (Acacia, Flourensia, Brayodendron). Large areas are covered by the low leaf-succulent lechuguilla (Agave lechuguilla), but not to the exclusion of the shrubbery. Other areas are dominated more conspicuously by the sotol (Dasylirion texanum), a plant with perennial foliage and with a store of water and reserve materials in its stout stem. Succulents are abundantly represented in this desert, but chiefly by small species which do not play an important part in the physiognomy of the vegetation. Perennial bunch-grasses are common in certain portions of the Texas Desert, and the number of plants perennial by underground succulent or semisucculent parts is larger than in the Arizona Desert. This is distinctively a region of leaf-succulents and semisucculents as contrasted with the Arizona region of stem-succulents.

Arizona Succulent Desert.-This area comprises the southern portion of Arizona drained by the Bill Williams and Gila Rivers and lying below 4,000 feet. In this desert the regetation is largely made up of microphyllous shrubs, but there is everywhere a rich commingling of other types of non-succulent plants and of sereral types of succulents. The regetation is open and low, but of irregular height. The sclerophyllous shrubs comprise the evergreen creosote-bush (Corillea tridentata), deciduous acacias (Acacia pancispina, A. constricta), and bitter bush (Flourensia cermua), the drought-deciduous ocotillo (Fouquicria splendens), several small-leaved or leafless trees and shrubs with green bark and stems (Parlinsonia, Canotia, Holacantha, Kaborlinia, Ephedra), as well as the columnar giant eactus (Curnegiea gigantea) and numerous species of flat-jointed and round-jointed carti $(O$ puntia). This desert is by no means poor in peremnial grasses, and the seasonal rains are followed by the appearance of earpets of amnual grasises and other herbaceous plants. The leafy sueculents are rare in this desert. except at its upper elges around the higher mountains, and the plants with subterranean water-storing organs are very infrequent. 
Texas Semidesert (Mesquital-Grassland Complex).-The valley of the Rio Grande below the Balcones Escarpment and the River Neuces presents a region in which the deciduous compound-leaved leguminous shrubs and trees form the dominant vegetation, interspersed with areas of more or less open grassland. The shrubs and trees form a more or less closed scrub from 3 to 6 feet high where the shrubs predominate and a more open one from 15 to 25 feet high where the trees are most abundant. The commonest of the trees is the mesquite (Prosopis glandulosa), which has spread somewhat over adjacent areas since the advent of the white man. With it the evergreen broadleaved live-oak (Quercus virginiana) is a minor component of the vegetation. In the scrub the dominant species are huisache (Acacia farnesiana), guajillo (Acacia berlandierei), and other microphyllous forms (Momesia, Parkinsonia, Condalia, Sesban, etc.), while the succulents are confined to the frequent occurrence of a prickly-pear cactus (Opuntia lindheimeri) and a yucca (Yucca treculeana). Ephemeral herbaceous plants are also present here.

Pacific Semidesert (Chaparral-Encinal-Desert Complex).-The Pacific semidesert region is best designated thus in spite of the fact that it comprises many small areas which are far from being desert. There is no other portion of the United States in which such profound differences of vegetation exist within such small areas, and in which it would be more difficult to map on a small scale the complex allocation of these areas. Over the low hills and around the bases of the Coast Ranges is to be found chaparral, varying from place to place in height and density; in the valleys and on the north faces of some of the hills are to be found groves of evergreen or deciduous oaks; while in other valleys, particularly the broad valleys of the Sacramento and San Joaquin Rivers, and on the interior hills of the Coast Ranges, are to be found some of the most truly desert areas in the United States. The chaparral is sometimes a very low aggregation of shrubs, or sometimes reaches a height of 6 or 7 feet. It is also variable in its density, but is commonly so close-set that it can be traversed only very slowly. It is made up predominantly of evergreen shrubs (Ademostoma, Arctostaphylos, Heteromeles), but partly of deciduous shrubs (Ceanothus spp.). The encinal is made up of evergreen oaks (Quercus agrifolia) and deciduous oaks (Q. lobata, $Q$. wislizeni), with the digger pine (Pinus sabiniana) an occasional component of it. In the desert of the interior hills and the great valley is to be witnessed a region almost totally devoid of perennial plants, in which the only cover of vegetation is due to the herbaceous annuals that appear in the spring.

Desert-Grassland Transition.-This transition region comprises the Llano Estacado of Texas and certain plateau lands in Arizona and New Mexico above 5,000 feet in elevation. It is a region that is intermediate in all important respects between the Grasslands to the east 
and the Desert regions to the west. It is essentially a very open stand of perennial grasses, together with the herbaceous annuals or perennials of the Desert and an extremely scattered stand of succulent or semisucculent plants. In the Llano Estacado and in southern New Mexico and Arizona the latter group comprises sotol (Dasylirion) and bear-grass (Nolina), while farther north in Texas and New Mexico the commonest succulent or semisucculent forms are a yucca (Yucca glauca) and a round-jointed cactus (Opuntia arborescens). In northern Arizona and northwestern New Mexico there are low, shrubby sages (Artemisia), Mormon tea (Ephedra), and other scattered bushes, and small cacti (Opuntia hystricina, O. whipplei). Throughout the Transition region the grasses are omnipresent, sometimes forming nearly as dense a carpet as they do in the Grassland itself. There is a considerable variety in the grass flora, but the commonest forms are species of Bouteloua, Hilaria, Bulbilis, and Aristida.

Grassland.-The Grassland region extends from central Texas to the Canadian boundary, merging on the east into the transition region which separates it from the Deciduous Forest, and on the west either merging into the Desert-Grassland Transition or else terminating at the eastern base of the Rocky Mountains. Smaller detached areas of Grassland also surround the northernmost salients of the desert.

Throughout the Grassland region the vegetation is dominated by a more or less continuous cover of perennial grasses -in some localities by a dense sod, in others by an open sod, and in still others by an open stand of bunch-grasses. The types of grasses which form the grassland are varied, both in the region as a whole and in any small portion of it. A score of grass species form the great bulk of the regetation, several of them being of very widespread occurrence throughout the region, as Bouteloua oligostachya, Bulbilis dactyloides, Koleria cristata, and species of Andropogon, while others are confined to different portions of the area or to particular soils, as the species of Hilaria in Texas, the species of Sporobolus and Stipa in Kansas and Nebraska, and the speries of Agropyron in the northwestern part of the area. In addition to the score of commonest species, there are a hundred or more that are either frequent over large areas or common over smaller portions of the area. From a floristic standpoint the Grassland presents two gradations, one encountered in going from the eastern edge toward the Rocky Mountains, the other encountered in going from south to north through its entire length of over 1,200 miles. From a regetational standpoint, however, this is all a region of great uniformity. Its principal variations are in the relative density or opennese of the grassy cover, in the character of the areas in which grasses are sparse or absent, and in the frequence of plants other than grasses. It may be said, in general, that the carpet of grasses is most evenly closed along the eastern edge of the area and in the central portion. In central Texas 
and in the extreme north much of the Grassland is relatively open, particularly in the sandhills of Nebraska, where Andropogon scoparius is predominant, and in the portions of the region lying in Washington and Oregon, where Agropyron spicatum and Poa sandbergii are predominant. In the highly eroded "bad lands," such as occur in southern South Dakota, low, shrubby perennials are predominant, as the rabbitbrush (Chrysothamnus graveolens), the white "sage" (Eurotia lanata), and greasewood (Sarcobatus vermiculatus). Throughout the portion of the Grassland which lies nearest the Rocky Mountains there is a complicated patchwork of vegetation, in which closed grassland, open grassland, and open stands of low bushes, with or without grasses, are found to alternate in habitats of different character. The shrubby perennials found in such areas are chiefly those which have just been mentioned, together with species of true sage (Artemisia tridentata, A. frigida). The Grassland is locally invaded by plants of the types which are dominant in all of the surrounding regions. In the southwestern portion of the area arborescent round-jointed cacti (Opuntia arborescens) are sporadic, and also yucca (Yucca glauca), which is likewise common in the sandhills of Nebraska. Throughout the area a low prickly pear (Opuntia missouriensis) is abundant on coarse soil, particularly in the Bad Lands.

In the vicinity of the mountains the Grassland is invaded by shrubs (Cercocarpus, Quercus, Symphoricarpus), and in some localities even by coniferous trees (Pinus ponderosa), while the bottomlands of the rivers are the westernmost localities for many eastern broad-leaved deciduous trees.

In every portion of the Grassland there are to be found very many types of low annual or root-perennial plants other than grasses. Among these certain composites are perhaps the most abundant, as Grindelia squarrosa and Chrysopsis villosa, although there are many plants of other types. The seasonal habits of the grasses and of these associated non-gramineous plants are such as to give rise to one of the most striking characteristics of the Grassland, namely, its different aspect in different portions of the growing-season, and the difference in the conspicuously predominant plants in different months.

Grassland-Deciduous Forest Transition.-This is the broadest and most extensive of the transition areas, but is so purely transitional in its character that it does not merit recognition on any other basis. Its eastern limit has been fixed along the line at which the Deciduous Forest ceases to be an unbroken formation and begins to exhibit the islands of grassland locally known as "oak openings." The western limit has been placed where timber ceases to occur on the upland. The transition area is seen, therefore, to increase in the amount of timber found on going east and to increase in the extent of grassland exhibited on going west. 
In the northern part of the Transition belt the commonest trees are black oak (Quercus velutina) and bur oak (Q. macrocarpa); in the southern part the commonest are post oak (Quercus minor) and blackjack (Q. marilandica). The grasses originally most abundant in the central part of the belt were Andropogon furcatus, Sorghastrum nutans, Andropogon virginicus, and Sporobolus cryptandrus.

Deciduous Forest.-The Deciduous Forest formerly occupied the lower elevations of the Northeastern States, the summits and slopes of the southern Allegheny Mountains, the Piedmont region, and the valleys of the Ohio, Cumberland, and Tennessee Rivers, with extensions into southern Texas and into northern Michigan and the Dakotas, and an attenuated edge that merges into the transition area toward the west. There is no one of the vegetational areas of the United States that has been more completely and profoundly altered by man than has this one. In fact, it is difficult at the present time to secure reliable information as to the exact original extent of this type of forest in the Northeastern States. The virgin stands of deciduous forest were made up solely of deciduous broad-leaved trees over extensive areas, and these forests were both dense and of a stature as great as 100 feet, or even more. At the western edge of the Deciduous Forest its continuity becomes interrupted by open areas, while in the Texan extension of it the stand of trees is even, but very open. In all of the mountainous or hilly portions of the area the needle-leared evergreens frequently become components of the Deciduous Forest, and on steep bluffs, rocky slopes, and limestone ledges the needle-leaved trees are sometimes predominant. The floor of the Deciduous Forest is sometimes thickly covered with shrubbery and young trees, or is often open and more conspicuously occupied by herbaceous perennials, among which the chief regetative activity takes place in the spring, before the complete unfolding of the foliage of the trees.

The number of tree species participating prominently in the make-up of the Deciduous Forest is large, and very many of the commonest ones are found almost throughout the area, as the white oak (Qucrcus alba), black oak (Quercus relutina), pignut hickory (Hicoria glabra), beech (Fagus formaina), and tulip-tree (Liriodendron tulipifera). The Appalachian region is the place in which the Deciduous Forest reaches its finest development, both in respect of density and stature of the stand and with regard to the number of tree species participating in its composition. The most common trees of that region are the widespread ones which have just been mentioned, and also chestnut (Castanea dentula), chestnut oak (Quercus primus), searlet oak (Qucrus cocrinca), shaghark hickory (II icoria ornlu), Spanish oak (Quereus digitula), sugar maple (Acri succharum), and red maple (Acer rubrum). On passing northward from the center of the Deciduous Forest, the number of tree species becomes smaller, as many are left behind and 
few new ones are met, as birch (Betula lutea) and popular (Populus balsamifera). On passing southward, the commonest forms of the Alleghenian region are found to be confined to the mountainous dis tricts, while their place is taken on the Piedmont and in the Mississippi Valley by a still larger group of species, many of which are found not only on the upland, but in the half-swampy areas of the level regions. Prominent among these trees are Spanish oak (Quercus digitata), water oak (Quercus nigra), willow oak (Quercus phellos), black gum (Nyssa sylvatica), red gum (Liquidambar styraciflua), and blue-jack oak (Quercus brevifolia).

The most southwesterly portion of the Deciduous Forest in central Texas is made up almost solely of post oak (Quercus minor) and blackjack oak (Quercus marilandica), while the most northwesterly islands of deciduous forest in the Dakotas are made up chiefly of bur oak (Quercus macrocarpa).

Southeastern Evergreen-Deciduous Transition Forest.-This transition lies between the coastal Evergreen Forest and the interior Deciduous Forest, occupying hilly and broken land, except at the extreme western end. Throughout this area there are small bodies of pure evergreen needle-leaved forest and other bodies of pure deciduous forest, but the vegetation consists in the main of an admixture of the two types of trees in such percentages that neither dominates strongly over the other. As in all other transition regions, the local conditions of soil and topography often determine the precise composition of the forest.

East of the Mississippi the Evergreen-Deciduous Transition is formed chiefly of the loblolly pine and the species of deciduous oaks that will be mentioned in connection with the inner portion of the Southeastern Evergreen Forest. West of the Mississippi the loblolly pine occurs near the Gulf coast and is chiefly replaced by the shortleaf pine (Pinus echinata) above the Neches River, while the same species of deciduous oaks accompany each of the pines.

Southeastern Mesophytic Evergreen Forest.-This region stretches from Long Island to Louisiana along the Coastal Plain, with an extension into peninsular Florida, and with outlying areas in central Alabama and in Arkansas, Louisiana, and Texas. This area is dominated by evergreens, and a secondary rôle in the vegetation is played by deciduous broad-leaved trees and by evergreen broad-leaved trees. The forest stands of this region are nowhere dense in the same sense as are some of the evergreen stands of Montana or Maine; indeed, many of the pine stands in all parts of the area, and particularly in Florida, are rather open. Some of the heaviest stands are found in Louisiana and Texas and the lightest are those of Florida and New Jersey. As a rule the pinelands which lie nearest the coast, particularly in the Gulf States, are the purest, while those of Maryland and the Carolinas, as well as the interior areas of Alabama and Arkansas, 
are much richer in deciduous broad-leaved associates. In the pine forests of New Jersey the floor is extremely shrubby, and this is often the case as far south as South Carolina. In southern Georgia and the Gulf region the floor is much more open. In both of these cases it is difficult to decide how much the normal conditions may have been disturbed by fire and by clearing. Under present conditions, at least, the Gulf pinelands present a very clean floor, closely carpeted by grasses, palmetto, pitcher-plants, and a multitude of other herbaceous species. Along the branches and other depressions there is a dense stand of shrubbery and a slightly different type of forest.

The half dozen species of pine which dominate the different sections of the Southeastern Mesophytic Evergreen Forest are very similar in their appearance, and there is consequently a general resemblance between the pinelands of the entire Coastal Plain. In Long Island and New Jersey the scrub pine (Pinus rigida) is the dominant species, and is scarcely found elsewhere in this forest. In Maryland, Virginia, and North Carolina the loblolly pine (Pinus toeda) is the leading species, while in peninsular Florida the Cuban pine (Pinus caribea) is the chief form. Throughout the remaining major portion of the forest the longleaf pine (Pinus palustris) is always the dominant tree, or at least abundantly represented in company with the loblolly pine. Throughout the Gulf region the slash pine (Pinus heterophylla) is found in moist soils and the pond pine (Pinus elliottii) in wet soils. Throughout the interior portions of this forest the shortleaf pine (Pinus echinata) is a characteristic tree, seldom found in company with the longleaf pine.

In the northern and interior portions of the Southeastern Forest, the broad-leaved deciduous oaks are frequent associates of the pines. The post oak (Quercus minor) and the Spanish oak (Quercus digitata) are common from Maryland to Texas, and the blue-jack oak (Quercus brevifolia) and turkey oak (Quercus catesbcei) from Georgia to Mississippi. The red gum (Liquidambar styraciflua), the black gum (Nyssa biflora), and the red maple (Acer rubrum) are also common broadleaved deciduous elements of this forest, although usually most common in poorly drained soil. From South Carolina to Texas the branches and moist depressions of the forest are characterized by many evergreen broal-leaved trees. Some of these are always evergreens, as the magnolia (Magnolia grundiftora) and the live oak (Qucrcus virginiana), while others are evergreen in the Gulf region and deciduous further north, as the water-oak (Quercus nigra) and the laurel oak (Quercus laurifolia).

Throughout the portions of the area which are poorest in associated deciduous trees there are a number of evergreen broad-leaved shrubs which form a conspicuous clement of the regetation. The most notable of these are the gallherry (lex glubra), the red bay (P'crse carolina), the waxherry (Myriea carolinima), the ti-ti (Cliflomia momophylla), 
and the sweet illicium (Illicium floridanum). In the extreme coastal region and in peninsular Florida the saw palmetto (Serenoa serrulata) is more conspicuous than the shrubs, although there is an increasing number of species of the latter, including two dwarf oaks (Quercus pumila and Quercus minima).

Northeastern Evergreen-Deciduous Transition Forest.-This type of forest fringes the Northern Mesophytic Evergreen Forest from Minnesota to Maine and southward along the Alleghenies. It is sometimes well marked as a nearly equal admixture of deciduous and evergreen needle-leaved trees, but on its southern and northern edges it merges into the larger types of forest, except where sudden changes of soil break the influence of climate. The most important deciduous constituents are sugar-maple (Acer saccharum), beech (Fagus atropunicea), birch (Betula spp.), and basswood (Tilia americana). The commonest evergreen trees are hemlock (Tsuga canadensis), white pine (Pinus strobus), balsam fir (Abies balsamea), and jack pine (Pinus divaricata).

Northern Mesophytic Evergreen Forest.-This forest occupies portions of the northern Pacific coast, all but the alpine portions of the Rocky Mountains above an elevation of 6,000 to 7,000 feet, and the higher summits of all the coastal and inland mountain ranges of the Western States. It also occurs in northern Minnesota, Wisconsin, and Michigan and extends from Maine over the higher elevations of the Appalachian region through New York, Pennsylvania, and West Virginia south to North Carolina.

This widespread forest is essentially similar in its physiognomy throughout. It is dominated in all portions by the needle-leaved evergreen tree, although it is by no means free of an admixture of broadleaved deciduous trees, and the latter are particularly common along the streams or as small trees beneath the canopy of evergreens. This forest is usually between 50 and 100 feet in stature, and it is commonly so dense that the entire ground is in shade, although this is notably untrue of the forests at elevations approaching timber-line on high mountains, and of those which approach the lower limit of timber on the desert mountain ranges of the interior. The heaviest stands of this forest are almost devoid of either shrubby or herbaceous undergrowth, but are carpeted by beds of moss. In the more open stands there is usually considerable shrubbery, and when this exists it is made up of deciduous plants.

In spite of the essential similarity of the Northern Mesophytic Evergreen Forest, throughout its extensive range of occurrence, it is made up of a large number of tree species, and its composition varies greatly from State to State, and especially when the eastern and western portions of the forest are compared. Owing to differences in habit of growth that exist between different coniferous trees there are some very striking differences in the physiognomy of the forest that 
are to be thus explained, as, for example, the contrast of the open yellow-pine forest with the very dense lodgepole-pine forest. In the mountains of the Western States there are also considerable differences in the coniferous forests due to altitude, and these differences are visible in the floristic composition as well as in the physiognomy.

The leading tree in the composition of all the western forests of this character is the western yellow pine (Pinus ponderosa), which often forms extensive pure stands at middle altitudes, is replaced by other species at the highest elevations, and is sometimes the tree of lowest range, as in the northern Rocky Mountains, or is mingled with the oaks, junipers, and nut pines of the Western Xerophytic Evergreen Forest at its lower range. The tree which most frequently replaces the yellow pine in the domination of this forest is the lodgepole pine (Pinus murrayana), which is extremely abundant in pure stands in Idaho and Montana, occupies a belt of dominance from 3,000 to 5,000 feet in portions of eastern Washington, is almost the sole forest tree in the Big Horn Mountains of Wyoming, grows with the Douglas fir (Pseudotsuga mucronata) on the western side of the Coast Range, and is locally dominant in the Rocky Mountains of Colorado.

The most hygrophilous portions of the Mesophytic Evergreen Forest are the coastward slopes of the mountains of Oregon and the western slopes of the Rocky Mountains in Montana and Idaho. In the former of these regions the lowest belt of forest is formed by yellow pine, Douglas fir, and lodgepole pine; above 5,000 feet the Douglas fir is the dominant tree, growing with yellow pine, sugar pine (Pimus lambertiana), white fir (Abies concolor), lodgepole pine, and noble fir (Abics nobilis); while the higher forested elevations are dominated by the alpine hemlock (T'suga pattonii), together with lodgepole pine, noble fir, white-bark pine (Pinus albicaulis), western white pine (Pinus monticola), and alpine fir (Abies lasiocarpa).

The moister forests of the northern Rocky Mountains lie between a pure stand of yellow pine at lower elevations and an open stand of alpine fir and white-bark pine above. The commonest trees of this forest are western white pine and western larch (Larix occidentalis). The Douglas fir is frequent and the alpine fir (Abies lasiocarpa), the lodgepole pine, and giant cedar (Thuja plicata) form a small percentage of the arborescent vegetation.

In the Sierra Nevada the lowest coastward fringe of forest is formed by an open stand of the digger pine (P'inus sabiniana), and the main body of the forest is formed of yollow pine, incense cedar (Libocedrus decurrens), sugar pine (Pinus lamberliana), Jeffrey pine (Pinus jefreyi) and white fir (Abies concolor). At the highest timbered elevations lodgepole pine, Jeffrey pine, and red fir (Abies magnifica) are the characteristic trees. In the Fan Bernardino and Fan Jacinto Mountains the forest helow 6,000 feet is composed almost solely of yellow pine, 
and above that elevation of an admixture of yellow pine, white fir, and sugar pine.

In the Rocky Mountains of Colorado the principal areas of forest are dominated either by yellow pine or lodgepole pine, or more rarely by an admixture of them. The higher elevations are characterized by Douglas fir (Pseudotsuga mucronata), Engelmann spruce (Picea engelmanni), and by the fox-tail and limber pines (Pinus aristata and $P$. flexilis) and the Parry fir (Picea parryana) and alpine fir (Abies lasiocarpa).

The forest in the Black Hills of South Dakota and in the desert mountains of Arizona and New Mexico is chiefly formed by yellow pine.

The Mesophytic Evergreen Forest of northern Minnesota is a composite of white pine (Pinus strobus) on the deeper soils; of Norway pine (Pinus resinosa) and jack pine (Pinus divaricata) on the lighter soils; with tamarack (Larix laricina), black spruce (Picea mariana), and white spruce (Picea canadensis) in wet soils; and arborvitæ (Thuja occidentalis) in the bogs. The deciduous broad-leaved trees are more conspicuous here than in any portion of the western half of this forest, bur oak (Quercus macrocarpa), basswood (Tilia americana), and sugar maple (Acer saccharum) being the commonest species.

In Maine the principal trees in the evergreen forest are spruce (Picea nigra), balsam fir (Abies balsamea), white pine (Pinus strobus), and hemlock (Tsuga canadensis). This group of trees is also characteristic of the coniferous areas of the other New England States, of the Catskill and Adirondack Mountains in New York, and of the mountains of Pennsylvania. The small coniferous areas on the mountains of North Carolina are chiefly composed of black spruce (Picea mariana) and the Fraser fir (Abies fraseri).

Western Xerophytic Evergreen Forest.-The Xerophytic Evergreen Forest is a dwarf and open form of "woodland" or "semi-forest" that characterizes the edges of the Mesophytic Evergreen Forest throughout the southern half of the western portion of that forest. The Xerophytic Forest seldom covers extensive areas, except in northern Arizona, and in all localities it becomes more open at the lower edges, where it meets the Desert or the Desert-Grassland Transition, and more closed at the upper edge, where it merges with the Mesophytic Evergreen Forest.

The Xerophytic Forest is similar to the desert in that its dominant plants are widely spaced, leaving much unoccupied ground. It is, again, similar to the desert and unlike the other forest areas in the small stature of its trees, which never exceed 50 feet and frequently attain less than 25 feet in height. The two types of tree which dominate the Xerophytic Forest are the nut pine (Pinus edulis, $P$. cembroides, $P$. parryana) and the juniper (Juniperus utahensis, $J$. californica, $J$. 
occidentalis, J. pachyplocu). Sometimes these two types are equally mingled, or more frequently one of the two is predominant. The species mentioned do not greatly overlap, but occupy different areas within the Xerophytic Forest. With these coniferous trees grow also certain evergreen broad-leaved oaks. In the Great Basin and in Colorado the rôle played by the oaks is a minor one, but in New Mexico it is more important, and in southern Arizona several arborescent species of evergreen oaks are frequently as common as the conifers, or more so. The Xerophytic Forest also contains numerous conspicuous shrubs of different types (Cercocarpus, Cowania, Artemisia, Ephedra, etc.), as well as such succulent and semisucculent plants as the yuccas and agaves, and conspicuous bunch-grasses and other perennials of intermittent or seasonal activity.

Northwestern Hygrophytic Evergreen Forest.-This forest occupies the coastal region of Washington, Oregon, and extreme northern California, and an isolated portion of it lies on the western slopes of the Cascade Range in Oregon. This area exceeds any portions of the Mesophytic Evergreen Forest in density of stand and in the stature of the trees, which very frequently exceed 100 feet in height. The heavily shaded floor of the forest is covered with fallen trunks and limbs, overgrown with mosses and hepatics, and underlaid by a deep bed of humus. The deciduous trees are few and small, but a number of evergreen ericaceous shrubs are common on the floor of the forest, as are also ferns and large-leaved herbaceous plants.

The density, tall stature, and vigorous activity of the Hygrophytic Forest give it a very distinctive physiognomy and betoken a set of environmental conditions unlike those of the Mesophytic Forest, in accordance with which it possesses a number of distinctive tree species. The tree which is of most general occurrence throughout the area is the Douglas fir (Pscudotsuga mucronata), which is also found far beyond the limits of this forest. It is accompanied in nearly equal admixture in many localities by the black hemlock (Tsuga mertensiana). Other species eommon in this forest are the Sitka spruce (Picea sitchensis), white fir (Abies grandis), giant cedar (Thuja plicata), amabilis fir (Abies amabilis), noble fir (Abies nobilis), redwood (Sequoia sempervirens), and western white pine (Pinus monticola). The highest oceanward elevations of the Coast Range are similar to other subalpine areas in the coniferous forests, and are eharacterized by an open stand of symmetrieal conifers, branching to the ground.

Alpine summits. - The principal alpine summits are those of the Cascade, Sierra Nerada, and Rocky Mountains, although small areas oecur elsewhere. 'Their vegetation is eomposed of such dwarfed or prostrate trees as may be able to exist above timber-line, together with low, matted, or polsterform perennial plants with large roots. Meadows or the margins of lakes above timber-line are the habitats of 
numerous herbaceous species, usually less highly specialized than those growing in rocky soil or crevices. On the highest mountains of the United States the vegetation is often limited to mosses and lichens or is locally absent.

Swamps and Marshes.-These terms comprise an extremely varied series of communities, partially dominated by trees and partially by coarse grasses, sedges, or other palustrine plants. The words "swamp" and "marsh" are both somewhat objectionable for use in the present connection because of their intrinsic reference to the nature of the habitat. The distinction between swamp and marsh has so long been drawn in popular speech and scientific writing, however, that the words are used here as terms descriptive of the vegetation rather than designations that imply the feature of the environment which determines the vegetation. The areas of swamp and marsh are so intricately interwoven that no effort has been made to separate them.

The greatest development of swamps and marshes is to be found along the shores of the Atlantic Coastal Plain, although there are smaller areas of marsh on the Pacific Coast and scattered areas of swamp throughout the glaciated region.

The saline marshes are dominated by nearly pure stands of halophytic grasses, while the fresh or brackish marshes are inhabited by very diverse populations of herbaceous perennials and annuals. The swamps of the Southeastern States are composed of a particularly rich assemblage of deciduous broad-leaved trees (Nyssa, Acer, Magnotia, and Quercus) or of nearly pure stands of the deciduous needleleaved bald cypress (Taxodium distichum).

Although the map of the vegetation of the United States which has just been described (plate 1) is not so detailed as might be possible or desirable, it was found, nevertheless, as has been noted, that many of its smallest areas and the sinuosities of many of its major boundaries would be meaningless when brought into comparison with the relatively small number of stations from which we were able to secure climatic data. We have therefore prepared a generalized map of the vegetation of the United States, shown as plate 2, which was executed with special reference to the number of stations represented in our accumulations of climatological data. The detailed map has been published and described for the sake of giving the basis upon which this more generalized map has been drawn. The number of areas has thus been reduced from 18 to 9 by a reduction of the four desert areas to one, by a consideration of the two semidesert areas as one, by the elimination of the transition areas from desert to grassland and from deciduous forest to the forest areas to the north and south of it, and by disregarding the alpine summits and swamps and marshes. We have chosen to separate our study of climatic correlations for the eastern and western portions of the Northern Mesophytic Evergrcen Forest. Although these two areas are ecologically alike, it seemed 
desirable to give them separate study, in view of the fact that they are so widely separated, at least within the geographical limits of the United States.

The generalized map (plate 2$)^{1}$ is, therefore, a simplification of the detailed regetation map, in addition to being a generalization from it in the sense that the lines between the plant formations have been smoothed, although their location has in no case been changed in such manner as to throw any of our leading climatological stations into vegetations other than those in which they actually belong.

The areas represented on the generalized map of the vegetation have been designated as follows:

1. Desert.

2. Semidesert.

3. Grassland.

4. Grassland-Deciduous Forest Transition.

5. Deciduous Forest.

6. Northwestern Hygrophytic Evergreen Forest.

7. Western Section of the Northern Mesophytic Evergreen Forest.

8. Eastern Section of the Northern Mesophytic Evergreen Forest.

9. Southeastern Mesophytic Evergreen Forest.

\section{DISTRIBUTIONAL AREAS OF CONFORMIC GROUPS OF PLANTS.}

Under this heading we desire to discuss briefly the groups of conformic plants (plants of the same growth-form) which we have used in the correlations dealt with on the following pages. Four groups of such plants have been charted and are exhibited in plates 3,4 , and 5 . It seems desirable to give here some of the detailed data upon which these maps have been based.

\section{CUMULATIVE DISTRIBUTION OF EVERGREEN BROAD.LEAVED TREES.}

The evergreen habit in broad-leaved trees is commonly regarded as one that has developed in moist, warm climates, and this view is confirmed by the predominance of trees of this type in the tropical rain-forests of both hemispheres. We have endeavored to define both the term "tree" and the term "broad-leaf" as definitely as possible for securing the list that we have used. We have regarded as trees only those woody plants which have a well-defined trunk and a height of 20 feet or more, and have regarded as evergreen all of those trees which retain some of their leaves throughout the year, at least holding the old ones until the time of appearance of the new leaves. The needleleaved evergreen trees have not been included in this class. The trees of this group merere into shrubs and in such a manner that it is extremely diffieult to draw a hard-and-fast line between them, and indeed some of the species which are arborescent in one portion of their

\footnotetext{
'On l'late 2, and also on plates $6,7,11,34-37,39,42-72$, tho description "Southenstern mesophytic forest" should read "Southeastern mesophytic evergreen forest."
} 


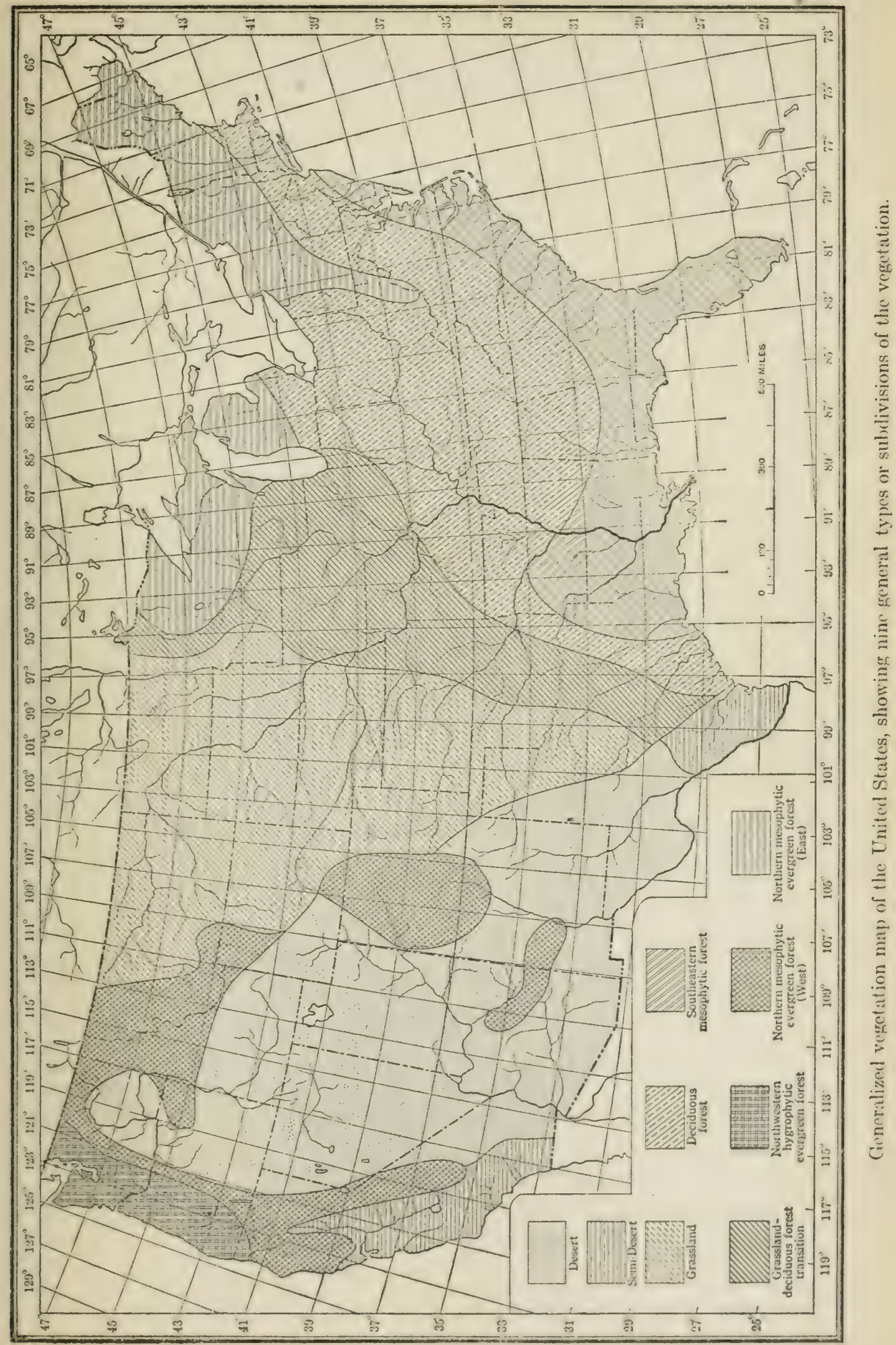



range are shrubs in another. It is also well known that a number of trees which are evergreen in southern latitudes are somewhat deciduous near the northern limits of their ranges.

Evergreen broad-leaved trees are found in the United States only on the Pacific coast, in the mountains of the extreme Southwest, and in the southeastern portion of the United States, reaching their greatest abundance in peninsular Florida. Those of the Pacific region are either confined to the Pacific coast of the United States or are found only in extreme northwestern Mexico. The evergreens of the southwestern mountains are largely trees which have the major portion of their range in the Sierra Madre region of Mexico. Those of the southeastern United States are partly peculiar to that region and partly trees of wide distribution in the West Indies, this being notably true of those found in extreme southern Florida. In the western states the greatest extension given the area of this growth-form, at least to the north, is due to the extended range of Arbutus menziesii. In the east the maximum extension of this type is due to the ranges of Ilex opaca and Magnolia glauca, which extend north in the Coastal Plain as far as Massachusetts. The member of the southeastern group which extends farthest west is Quercus virginiana, which is found in western Texas. The inclusion of the evergreen shrubs Rhododendron maximum and Kalmia latifolia would have extended the region of occurrence of this growth-form into the southern Alleghenies and farther into the Northeastern States. With the exception of these shrubs, the evergreen habit is rather poorly represented among the shrubs in the deciduous-forest region, although the evergreen broad-leaved habit again appears in the north as characteristic of numerous bog shrubs.

In the construction of the map of cumulative occurrence of broadleaved evergreens 129 species have been used. Of these species, 25 occur in California and the Southwestern States, 25 in the Eastern States exclusive of peninsular Florida, and 79 in the last-named region. The western and eastern groups do not overlap, except in so far as Quercus virginiana is sometimes found in western Texas in the same region with evergreen oaks characteristic of the Mexican Cordillera. Our eastern group of evergreens merges into the group for peninsular Florida in a manner which it is impossible to describe accurately on the basis of existing literature. Several of the evergreens of the Southeastern States do not range to the extreme southern end of Florida. Twelve of our 25 eastern species have been eliminated with certainty from the number credited to southern Florida. Out of the 79 species which we are listing for peninsular Florida, only 65 are found in the Everglade region exclusive of the keys, while 26 are confined to the keys and their adjacent shores. The complicated distribution of many of these trees in peninsular Florida has made it necessary for us to map that region in a somewhat conventional manner. 
Following is given a list of the species of evergreen broad-leaved trees which have been used in the compilation shown on plate 3 . In order to identify these with greater certainty, author names are given:

\section{Evfrgreen Broad-Lenved T'rees of the United States.}

Western group:

Arbutus arizonica (Gray) Sarg.

Arbutus menziesii Pursh.

Arbutus texana Buck.

Castanopsis chrysophylla (Hook) A. DC.

Ceanothus spinosus Nutt.

Ceanothus thyrsiflorus Esch.

Ehretia elliptica DC.

Fremontodendron californicum (Torr.) Cov.

Garrya elliptica Dougl.

Myrica californica Cham.

Prunus ilicifolia (Nutt.) Walp.

Quercus agrifolia Nee.

Quercus arizonica Sarg.

Quercus chrysolepis Liebm.

Quercus densiflora Hook. and Arn.

Quercus emoryi 'Torr.

Quercus engelmanni Greene.

Quercus hypoleuca Engelm.

Quercus oblongifolia Torr.

Quercus reticulata Humb, and Bonpl.

Quercus wislizeni A. DC.

Rhus integrifolia (Nutt.) Benth. and Hook.

Sophora sccundiflora (Cav.) DC.

Umbellularia californica (Hook. and Arn.) Nutt.

Vauquelinia californica (Torr.) Sarg.

Southeastern group:

Bumelia angustifolia Nutt.

Bumelia cassinifolia Small.

Bumelia lanuginosa (Michx.) Pers.

Bumelia lucida Small.

Bumelia tenax (L.) Willd.

Bumelia texana Buckl.

Cliftonia monophylla (Lam.) Sarg.

Cyrilla racemiftora L.

Gordonia lasianthus (L.) Ellis.

Ilex cassine Walt.

Ilex myrtifolia Walt.

Ilex opaca Ait.

Magnolia glauca $\mathrm{L}$.

Magnolia grandiflora $\mathrm{L}$.

Osmanthus americanus (L.) B. and II.

Persea borbonia ( $\boldsymbol{I}_{\text {L. }}$ ) Spreng.

P'ersea pubescens (Pursh.) Sarg.

l'runus caroliniana (Mill.) Ait.

Quercus laurifolia Michx.

Quercus nigra L.

Quercus virginianas lill.

Sabal palmetto (Walt.) R. and S.
Southeastern group-conlinued:

Symplocos tinctoria (L.) L'Her.

Vaccinium arboreum Marsh.

Xanthoxylum fagara (L.) Sarg.

Peninsular Florida group:

Alvaradoa amorphoides Liebm.

Amyris balsamifera L.

Amyris maritima Jacq.

Anamomis dicrana (Berg.) Britton.

Anona glabra $\mathbf{L}$.

Avicennia nitida Jacq.

Bourreria havanensis (R. and S.) Miers.

Bourreria virgata (Sw.) D. Don.

Bucida buceras L.

Bumelia angustifolia Nutt.

Calyptranthes pallens (Poiret) Griscb.

Canella winteriana (L.) Gaert.

Capparis jamaicensis Jacq.

Chrysobalanus icaco L.

Chrysophyllum oliviforme $\mathrm{L}$.

Citharexylon cinereum L.

Coccolobis laurifolia (Jacq.) Sarg.

Coccolobis uvifera (L.) Sarg.

Colubrina reclinata (L'Her.) Brongn.

Conocarpus erecta $\mathrm{L}$.

Cordia sebestina L.

Crescentia cujete $\mathrm{L}$.

Crescentia cucurbitina I.

Cupania glabra Sw.

Dipholis salicifolia (L.) A. DC.

Drypetes diversifolia Urb.

Drypetes lateriflora (Sw.) Urb.

Eugenia axillaris (Sw.) Willd.

Eugenia buxifolia (Sw.) Willd.

Eugenia confusa DC.

Eugenia longipes Berg.

Eugenia rhombea (Berg.) Iirug and Urban.

Exostem caribeum (Jacq.) Griseb.

Exotheca paniculata (Juss.) Radlk.

Ficus aureà Nutt.

Ficus brevifolia Nutt.

Genipa clusiifolia (Jacq.) Griseb.

Guaiacum sanctum $\mathbf{I}$.

(iuettarda elliptica Sw.

Guettarda seabra Vent.

Gyminda latifolia (Sw.) Urban.

Gymnanthes lucidn Sw.

Hippomane mancinella $\mathrm{L}$.

Hypelate trifoliata Sw.

Icacoren paniculata (Nutt.) Sudw.

Ichthyomethia piscipula (L.) A. S Hiteh. 


\section{Evergreen Broad-Leaved Trees of the United States-continued.}

Peninsular Florida group--continued: Ilex krugiana Loesn. Jacquinia keyensis Mez. Krugiodendron ferreum (Vahl.) Urb. Laguncularia racemosa (L.) Gaertn. f. Lysiloma bahamensis Benth. Mimusops parvifolia (Nutt.) Radlk. Ocotea catesbyana (Michx.) Sarg.

Oreodoxa regia $\mathrm{H}$. B. K.

Picramnia pentandra Sw.

Pithecolobium guadalupense Chapm.

Prunus sphærocarpa Sw.

Pseudophøenix sargentii Wend.

Psychotria undata Jacq.

Rapanea guianensis Aubl.

Reynosia septentrionalis Urb.

Rhacoma crossopetalum L.

Rhizophora mangle L.

Rhus metopium L.
Peninsular Florida group-continued:

Sapindus saponaria $\mathrm{L}$.

Schaefferia frutescens Jacq.

Schoepfia chrysophylloides (A. Rich.) Planch.

Sideroxylon foetidissimum Jacq.

Simaruba medicinalis Endl.

Swietenia mahogoni Jacq.

Terebinthus simaruba (L.) W. F. Wight.

Thrinax microcarpa Sarg.

Thrinax parviflora Sw.

Torrubia longifolia (Heimerl.) Britton.

Trema floridana Britton.

Xanthoxylum coriaceum A. Rich.

Xanthoxylum flavum Vahl.

Ximenia americana 1.

Zygia unguis-cacti (L.) Sudw.

\section{CUMULATIVE DISTRIBUTION OF MICROPHYLLOUS TREES. (PLATE 3.)}

This group comprises plants which are trees in form and reach a height of 15 feet or more, being characterized by leaves which are either simple and very small or have pinnate or bipinnate leaves with small leaflets. Several species have been comprised which have green stems and leaves which are of very short duration or wholly absent. The members of this group merge into the much larger class of shrubs in the southwestern United States which possess a similar character. Eight of the species which have been used are extremely common as shrubs, but frequently become trees within the limits of our definition.

Microphyllous trees are most strongly represented in the United States in southern Texas and southern Arizona. The maximum northward extension of individuals of this group reaches northern Texas and the southern portion of Nevada, due to the range of Prosopis glandulosa. The cumulative distribution of this group, as well as of the group just considered, is shown in plate 3 . The twenty-three species used in constructing this map are as follows:

\section{Microphyllous Trees of the United States.}

Acacia farnesiana Willd.

Acacia greggi iGray.

Acacia wrightii Benth.

Brayodendron texanum (Scheele) Small.

Canotia holacantha Torr.

Cercidium floridum Benth.

Cercidium torreyanum (Wats.) Sarg.

Condalia obovata Hook.

Holacantha emoryi Gray.

Koberlinia spinosa Zucc.

Leucæna glauca (L.) Benth.

Leucxna greggii Wats.
Leucæna pulverulenta (Schl.) Benth.

Olneya tesota Gray.

Parkinsonia aculeata $\mathrm{L}$.

Parkinsonia microphylla Torr.

Parosela spinosa (Gray) Heller.

Pithecolobium brevifolium Benth.

Pithecolobium flexicaule Coulter.

Porliera angustifolia (Engelm.) Gray.

Prosopis glandolosa Torr.

Prosopis pubescens Benth.

Prosopis velutina Wooton. 


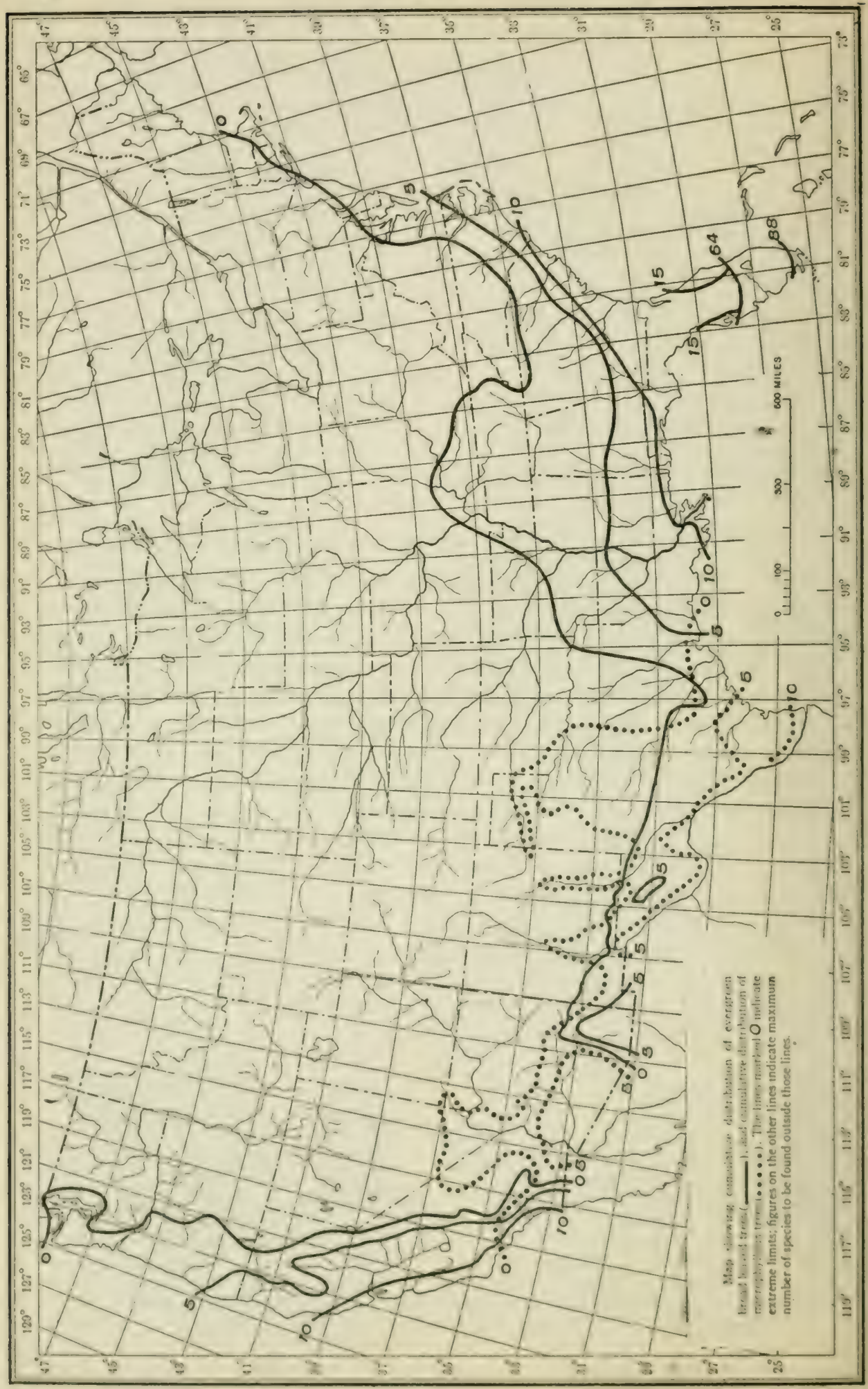




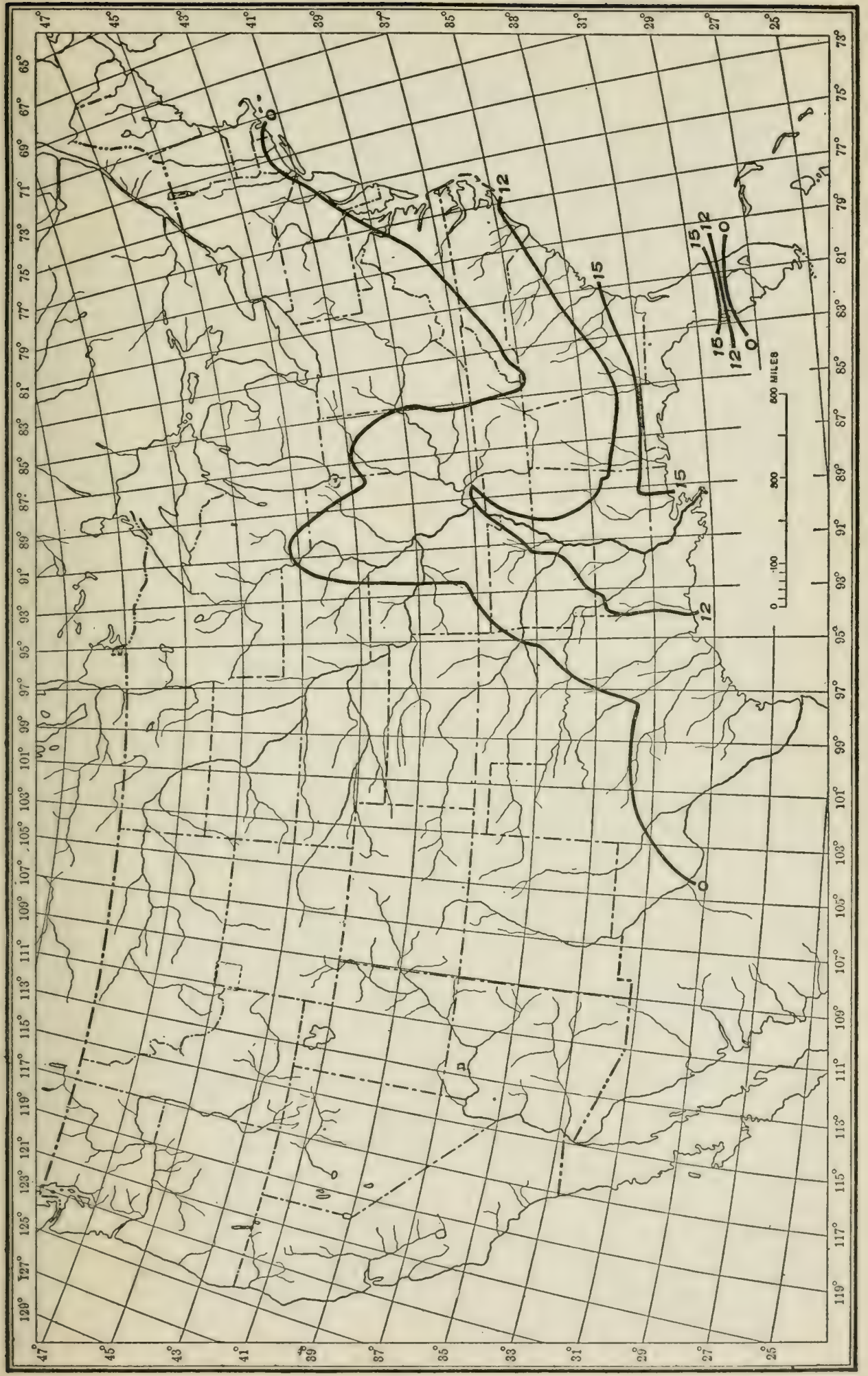

 
DECIDUOUS TREES OF THE SOUTHEASTERN UNITED STATES. (PLATE 4.)

On the basis of the literature descriptive of the vegetation of the southeastern United States, a group of fifteen deciduous broad-leaved trees has been selected as representative of this vegetation form in that section of the country. The cumulative distribution of the fifteen trees which have been selected is shown in plate 4 .

These trees are of interest because they are extremely common in the Atlantic Coastal Plain and are nearly all either infrequent in the Piedmont and Allegheny regions or are absent there. These trees are, in short, representatives of the deciduous habit which have their maximum cumulative occurrence as well as their maximum abundance outside the deciduous forest area and in the heart of the Southeastern Mesophytic Evergreen Forest. Five of the species used are palustrine and nearly all of them occupy other habitats than those in which the evergreen needle-leaved trees are dominant. The following is a list of the species which have been used in the construction of the map shown in plate 4 :

Acer drummondii Hook, and Arn.

Fraxinus caroliniana Mill.

Hicoria aquatica (Michx. f.) Britton.

Liquidambar styracifiua L.

Nyssa aquatica L.

Nyssa ogeche Marsh.

Planera aquatica (Walt.) Gmel.

Populus heterophylla $\mathrm{L}$.

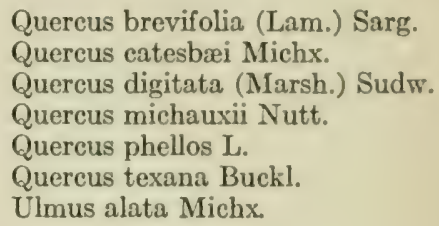

\section{CUMULATIVE DISTRIBUTION OF THE COMMONEST EASTERN DECIDUOUS TREES. (PLATE 5.)}

A selection has been made of the thirteen deciduous trees which are commonest in the Deciduous Forest area and are most widely distributed throughout it. These are all large forest trees which are wholly deciduous throughout their ranges and are commonly found in upland habitats. The maximum occurrence of this group is in the region extending from central New York to northern Alabama, comprising the entire extent of the Allegheny Mountains. From this region, in which 13 of the species are found, the abundance of this group shades off to the east, south, and west, so that the area in which from 12 to 8 speceies are found covers the Coastal Plain of Virginia and Carolina, extends routh to western Florida, and west as far as the eastern boundaries of Texals, Kansas, and Ninnesota. The following is a list of the thirteen species that have been used in the preparation of this map, shown in plate 5 :

Acer saccharum Marsh.

Carpinus caroliniann Walt.

Castanea dentata (Marsh.) Borkh.

Fagus atropunicea Ehrh.

liraxinus americana $\mathrm{I}$.

IIicoria glabra (Mill.) Britton.

Hicoria minima (Marsh.) Britton.
Juglans nigra $\mathrm{I}$.

Liriodendron tulipifera L.

Quercus alba L.

Quereus prinus I.

Quereus velutina Lam.

Ulmus americana $\mathrm{l}$. 
THE COMMONEST EVERGREEN NEEDLE-LEAVED TREES OF THE SOUTHEASTERN UNITED STATES. (PLATE 6.)

The four evergreen needle-leaved trees which are most widespread and most dominant in the Southeastern Mesophytic Forest are Pinus echinata, $P$. toeda, $P$. palustris, and $P$. caribcaa. The ranges of these 4 pines have been superposed on a single map (plate 6). Pinus echinata possesses the most northerly range of this group, and it and Pinus toeda exceed the distribution of the southeastern evergreen formation itself. The three most widely distributed species of this group reach their western limit at about the ninety-sixth meridian. The distribution of $P$. palustris is closely coincident with that of the southeastern evergreen formation, while that of $P$. caribca lies entirely within that formation. These four species are all found in southern Georgia and northern Florida and the extreme southern portions of Alabama and Mississippi. The region of maximum occurrence of this group lies, therefore, in the heart of the southeastern evergreen area.

\section{THE COMMONEST EVERGREEN NEEDLE-LEAVED TREES OF THE NORTHEASTERN UNITED STATES. (PLATE 7.)}

The ranges of the 4 evergreen needle-leaved trees which are most generally dominant in the eastern section of the Northern Mesophytic Evergreen Forest are plotted together and are shown in plate 7. These trees are Pinus strobus, Tsuga canadensis, Abies balsamea, and Pinus divaricata.

The region of cumulative occurrence of these trees corresponds closely with the distribution of the evergreen forest formation. The southernmost extension of this group is found in the case of Tsuga and the northernmost in the case of Pinus divaricata. All four of these trees are found together in northern New England, northern New York, and in Michigan, Wisconsin, and Minnesota.

The range of climatic conditions has been determined separately for each of these trees, owing to the fact that their regions of cumulative occurrence correspond so closely with the eastern section of the Northern Mesophytic Evergreen Forest. The same has been done with respect to the dominant trees of the Southeastern Evergreen Forest.

THE ECOLOGICAL DISTRIBUTION OF PINUS TEDA. (PLATE 8.)

It is rarely that data are available on the relative abundance of a plant within its area of geographical distribution. Owing to the excellent work of Mohr, ${ }^{1}$ we are able to use both the geographical and ecological distribution of the loblolly pine (Pinus torda). The map prepared by Mohr has been reproduced in plate 8 and shows three areas of varying abundance in addition to the region of scattered

\footnotetext{
${ }^{1}$ Mohr, Charles, Timber Pines of the southern United States, U. S. Dept. of Agric., Bur. For. Bull. 3, 1896.
} 


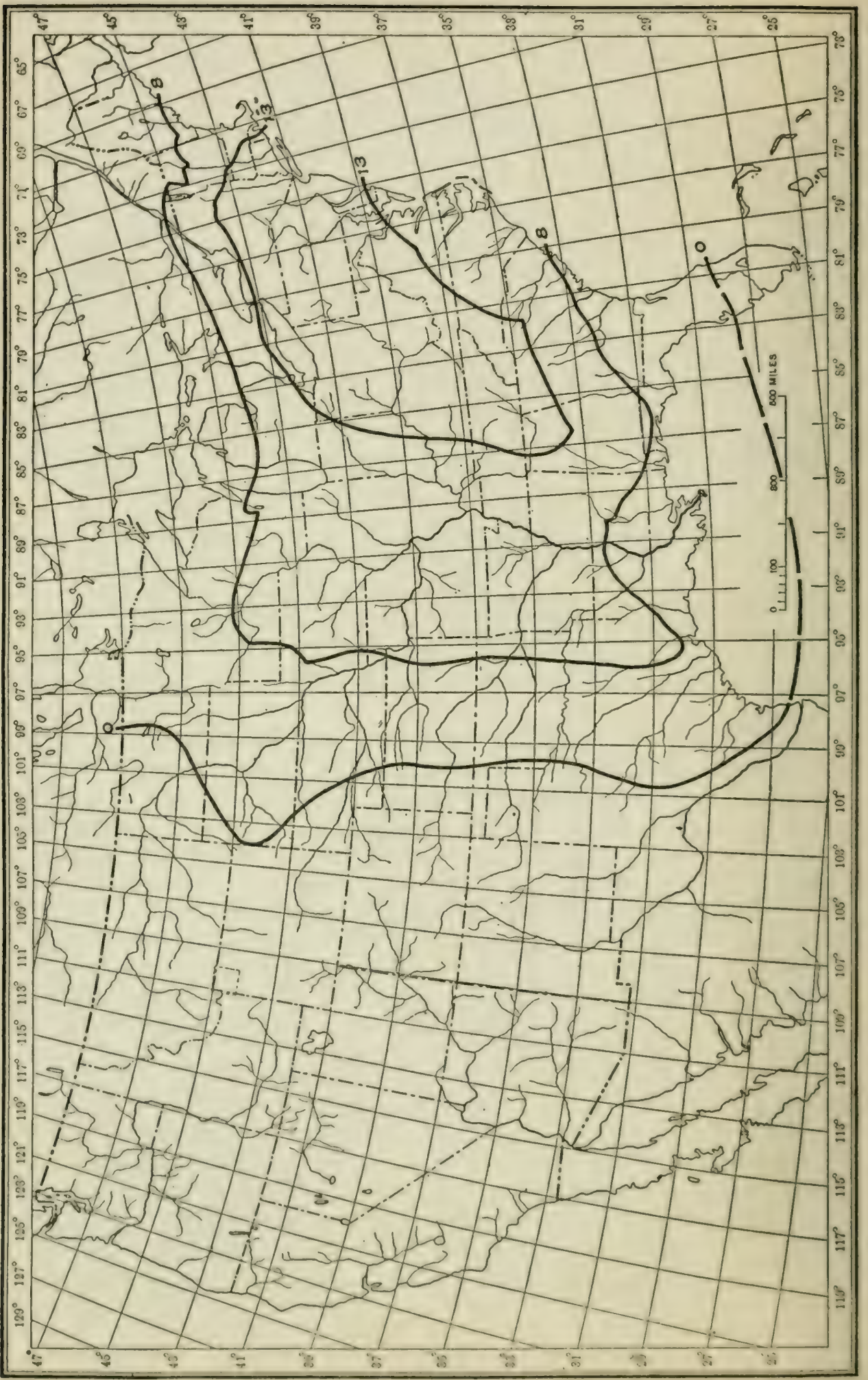

 


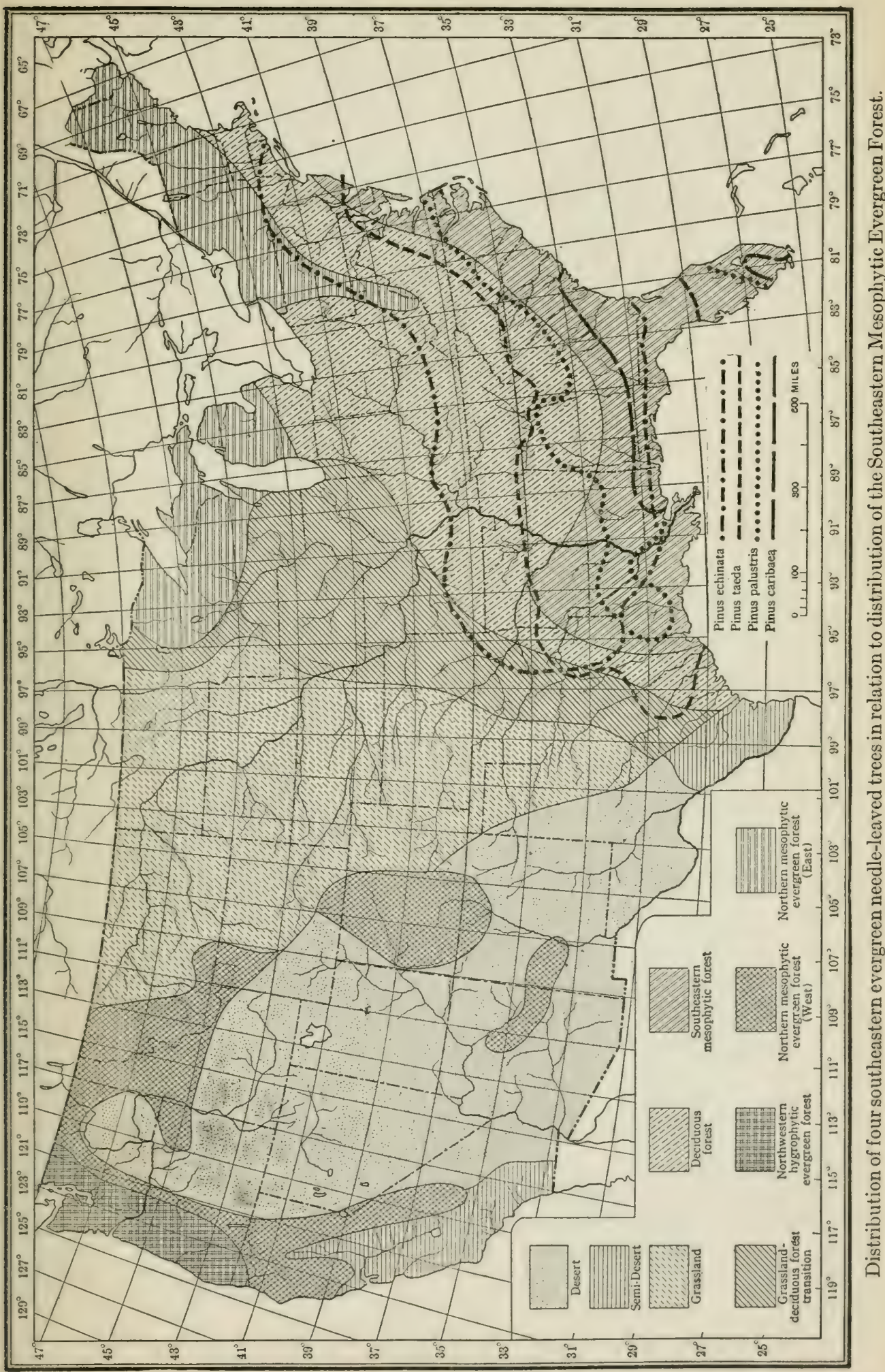




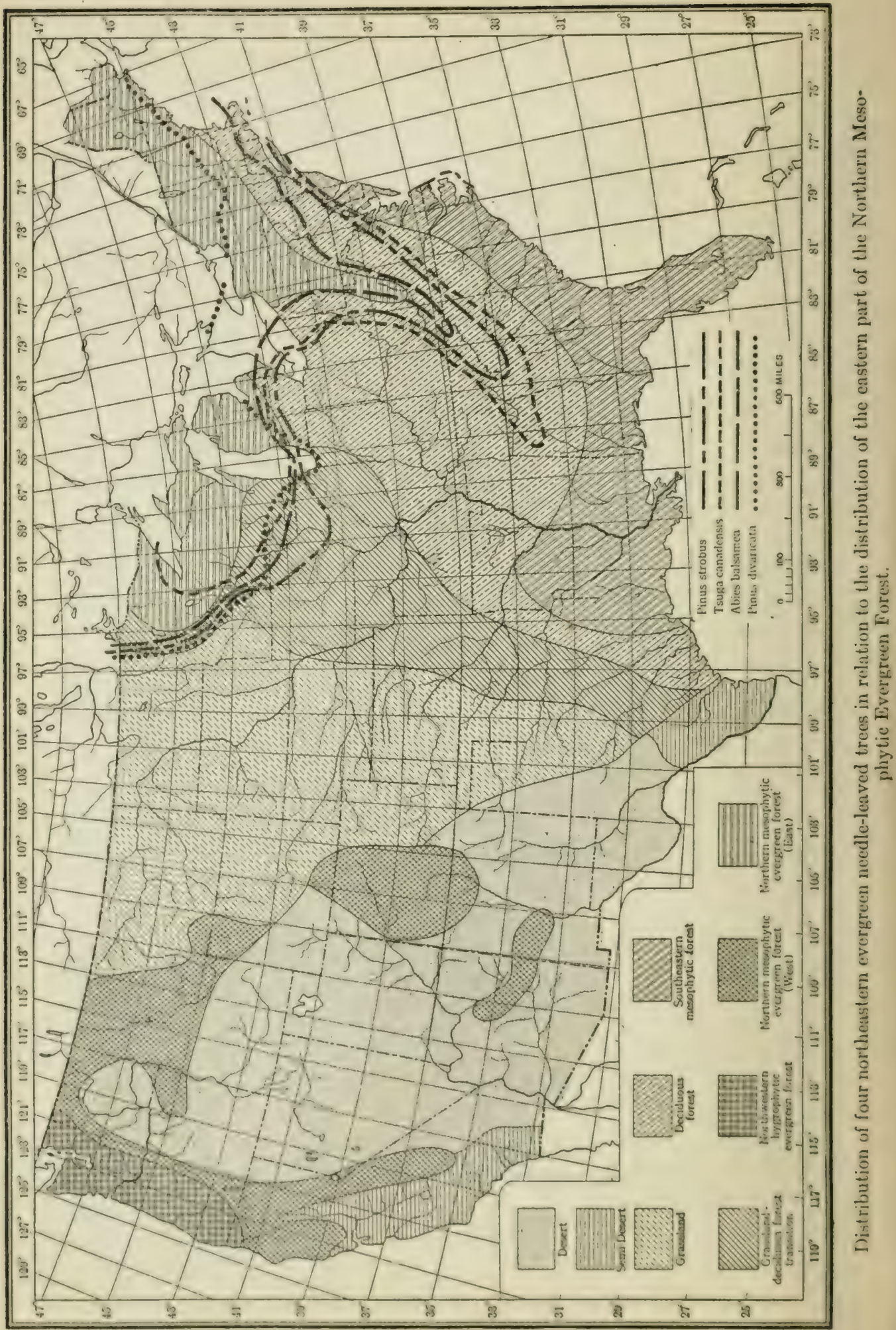




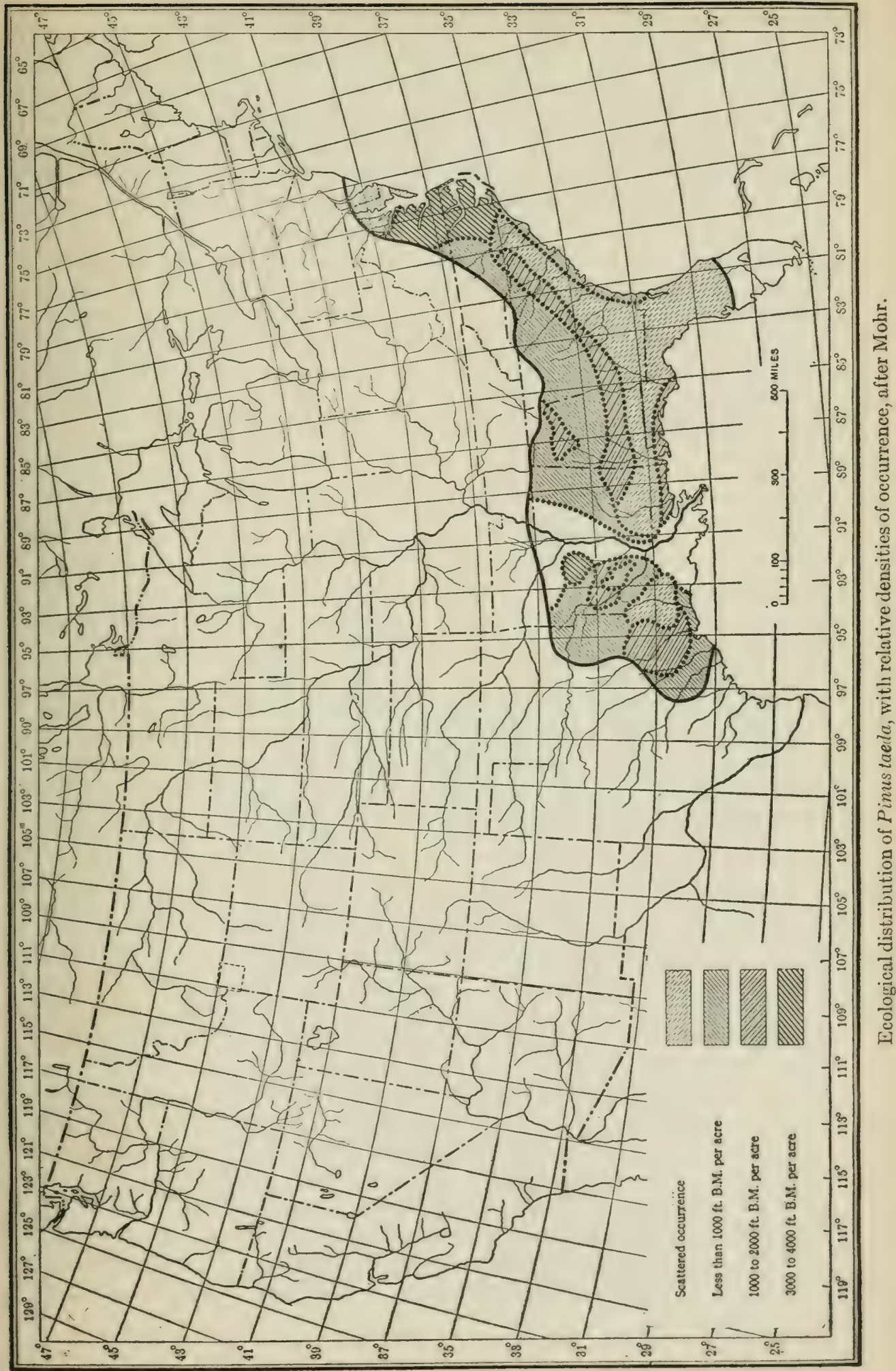


occurrence. The region in which this tree was formerly most abundant is characterized by stands of 3,000 to 4,000 feet board measure per acre, the area of next greatest abundance by stands of 1,000 to 2,000 feet board measure, and the third by stands of 1,000 feet board measure or less. It has been possible for us to determine the climatic conditions for each of these areas separately. The location of the areas of different abundance within the area of geographical range is so irregular for Pinus tada as to make our correlations difficult and to suggest the strong importance of soil influence in determining the stands of this tree in the different parts of its area. There is no other case, however, in which the ecological distribution of any plant has been so carefully worked out, and we are consequently unable to make use of other maps showing the distribution of plants over regions in which soil conditions are not so important in determining their relative abundance.

\section{THE ECOLOGICAL DISTRIBUTION OF LIRIODENDRON TULIPIFERA. (PLATE 9.)}

There are several deciduous trees for which the areas of commercial abundance have been determined, and we have selected one of these for use in our climatic correlations. Mr. George M. Lamb, of the United States Forest Service, has courteously given us the data for the map shown in plate 9 , indicating the geographical range and commercial range of the tulip tree (Liriodendron tulipifera). This map subdivides the range of the tree much less satisfactorily than the map of Mohr for Pinus tøda, since it indicates only the two regions of relative abundance. The simplicity of this map, however, makes it extremely useful for our purposes, especially in view of the relatively small number of climatological stations from which we have data.

\section{THE ECOLOGICAL DISTRIBUTION OF BULBILIS DACTYLOIDES. (PLATE 10.)}

In the absence of any previously published maps showing the ecological distribution of plants other than trees, we have endeavored to construct such a chart for buffalo-grass (Bulbilis dactyloides). This has been made on the basis of all arailable descriptive literature and has been submitted for eriticism to several botanists familiar with the Great Plains region, to all of whom we are greatly indebted for information. The map shown in plate 10 is designed to indicate the area in which buffalo-grass was formerly a very common element of the Grassland, the area in which it was of frequent occurrence merely, and the area in which it is of seattered or rare oceurrence. The geographical range of this species eoineides in a general way with the distribution of the Grassland regetation, although it does not range quite so far to the northwest and extends beyond its limits at the southeast. The area of its optimum oecurrence lies in fouth Dakota, Nebraska, western Kansas, and extreme western Oklahoma, in the heart of the Grassland area. 


\section{CUMULATIVE OCCURRENCE OF CHARACTERISTIC GRASSES. (PLATE 11.)}

On plate 11 have been laid the distributional limits of 4 grasses which are widespread and dominant in the Grassland region of the United States. These species are Bouteloua oligostachya, Bulbilis dactyloides, Bouteloua hirsuta, and Koleria cristata. The most widespread of these grasses is Køleria, which is found throughout the Northern States from Maine and Pennsylvania to Washington and California, extending southwest into Texas. Bouteloua oligostachya is also a plant of extensive range, having its limit in the GrasslandDeciduous Forest Transition on the east and occurring locally as far west as the northern Rockies and the desert plains of Utah, Arizona, and southern California. The other species are more nearly coincident in their distribution with the Grassland and Desert-Grassland Transition. The area of maximum occurrence in which all 4 species are found extends from the Canadian boundary to the Rio Grande, stretching approximately from the ninety-sixth to the one hundred and fourth meridian.

\section{EXTREME LIMITS OF TWO TYPES OF CACTI. (PLATE 12.)}

The most widely distributed genus of cacti in North America is Opuntia, in which a large diversity of types are to be found which have been roughly grouped in the two subgenera, Cylindropuntia and Platyopuntia. In plate 12 we have shown the extreme range of these two types of cacti in the United States. The limit of the range of the platyopuntias is the limit of the family Cactaceæ. This limit is carried from the southwestern United States, in which plants of this type are so abundant, eastward to the Atlantic coast and northward in the Coastal Plain to the Northeastern States. Members of this type are absent from the Allegheny region, but are found in eastern Kentucky, southern Michigan, and southwestern Minnesota. This limit is formed by the extreme ranges of Opuntia opuntia and Opuntia polyacantha. In the Western States the limit of this type is found at the base of the northern Rocky Mountains and at the eastern foot of the Cascade and Sierra Nevada ranges, being formed chiefly by the extreme occurrence of Opuntia polyacantha and its closely allied forms.

The arborescent cacti comprised in the group of Cylindropuntia are much more closely restricted to the desert region of the Southwestern States. The limit of this type is shown in plate 12 and is formed on the east by the extreme range of Opuntia arborescens, on the north, in Nevada, by Opuntia acanthocarpa and O. echinocarpa, and on the west by Opuntia parryi (bernardina), and $O$. prolifera. 


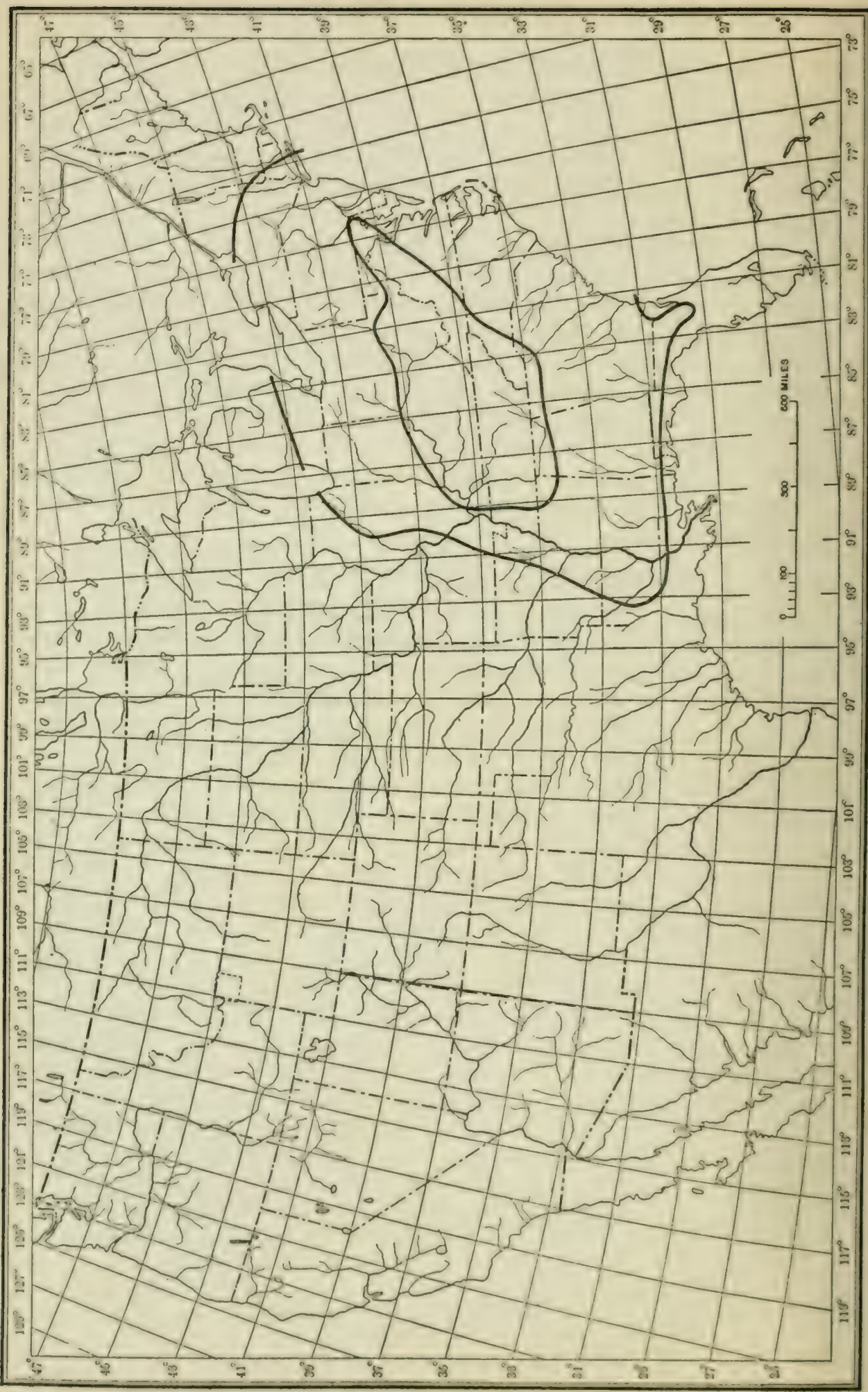




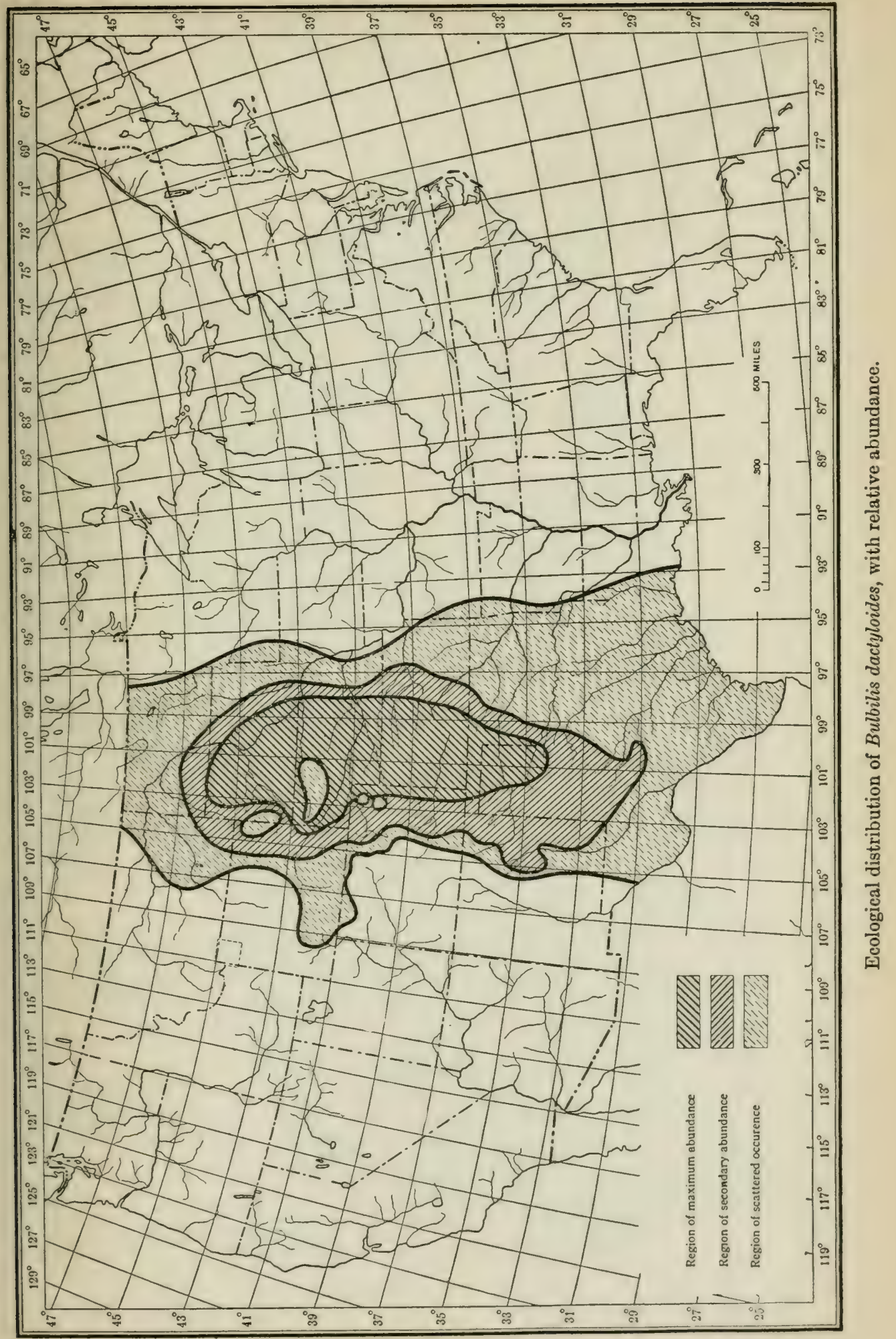




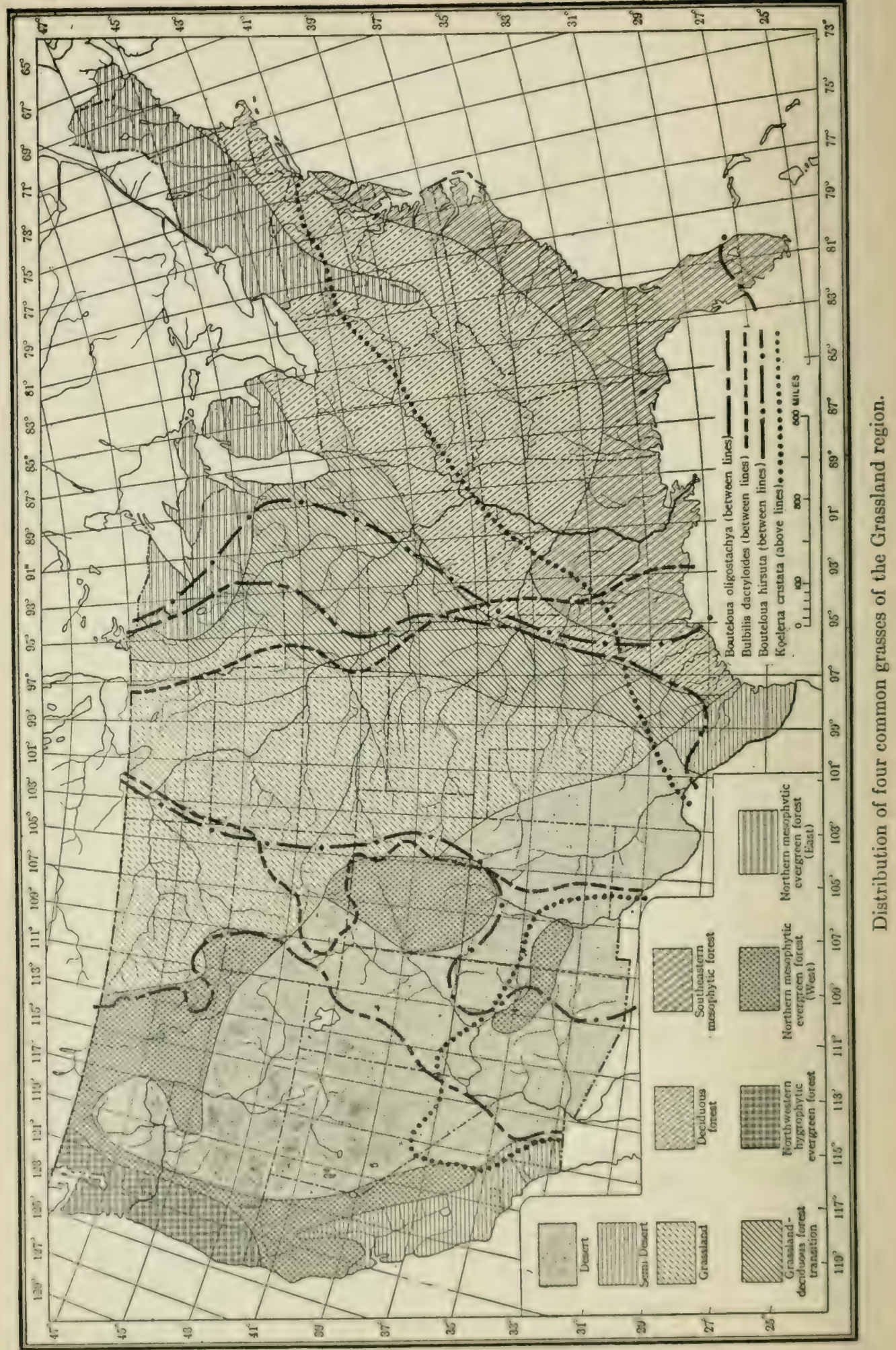




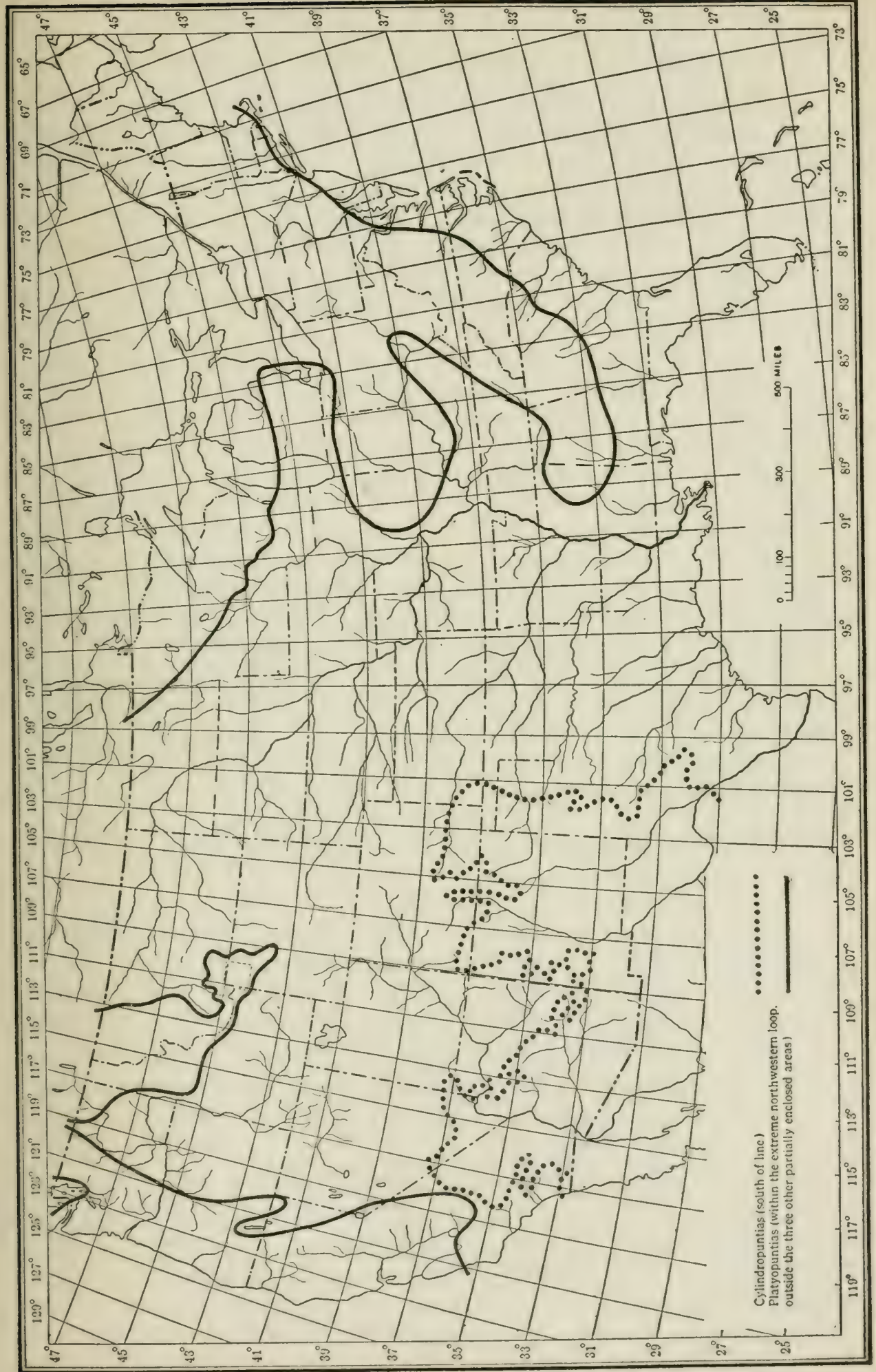

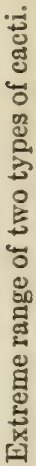




\section{DISTRIBUTIONAL AREAS OF SELECTED INDIVIDUAL SPECIES.}

$A$ relatively large number of individual species have been so selected as to include a few of the dominant plants in each of the leading vegetations. Many of the minor plants have distributional areas which coincide roughly with vegetational areas, as has already been shown for some of the northeastern and southeastern evergreen needle-leaved trees. Each of the dominant species that we have used for correlation with the climatic conditions is accompanied by numerous minor or subordinate species for which the same climatic controls must often be of importance.

The trees are predominant in the list of species which we have used, partly because they are the dominant element in so many of our types of vegetation and partly because it is easier to secure full and accurate distributional data for them than for plants of any other type. The distribution of most of the flowering plants of the United States would seem to be fairly well known if a list of the known occurrences of these species were examined. When, however, the attempt is made to plot the distribution on a map, the very great gaps which exist in our knowledge become very evident. We have depended largely on the invaluable data of the United States Forest Service, as given in various publications, for our maps of the distribution of trees.

The species which we have used fall into some 22 groups, which will now be enumerated. The distributional areas of these plants have been arranged on the maps so that their limits will intersect as little as possible and so as to economize space. The plates on which these distributions are represented are given in each case.

\section{Northwestern Evergreen Needle-Leaved Trees. (Plate 13.)}

Tsuga heterophylla (Raf.) Sarg.

Picea sitchensis (Bong.) Trautv, and Mayer.

These two trees are taken as typical of the numerous evergreen needle-leaved forms found in the hygrophytic forest of the Northwestern states. Tsugg ranges eastward to the Rocky Mountains of northern Idaho and northwestem Montana, but Picca is closely restricted in its range to the hygrophytic forest region itself.

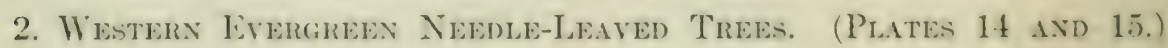

Pscudotsuga mucronata (Raf.) Sudw. (=P. taxifolia (Lam.) Britton = P. douglasii Carr).

Pinus ponderosa Laws. (including P. scopulorum (Engelm.) Lemmon).

Pinus contorta Loud. (including P. murrayana Oreg. Com.) Pinus edulis Engelm.

In this group are comprised four of the leading trees of the western portion of the Northern Mesophytie Evergreen Forest. It has heen possible to map the oceurrences of Pscudotsuga with considerable accuracy, in fact, in far more detail than our series of elimatologrieal figures would warrant. Pinus ponderosa is likewise widely distributed 


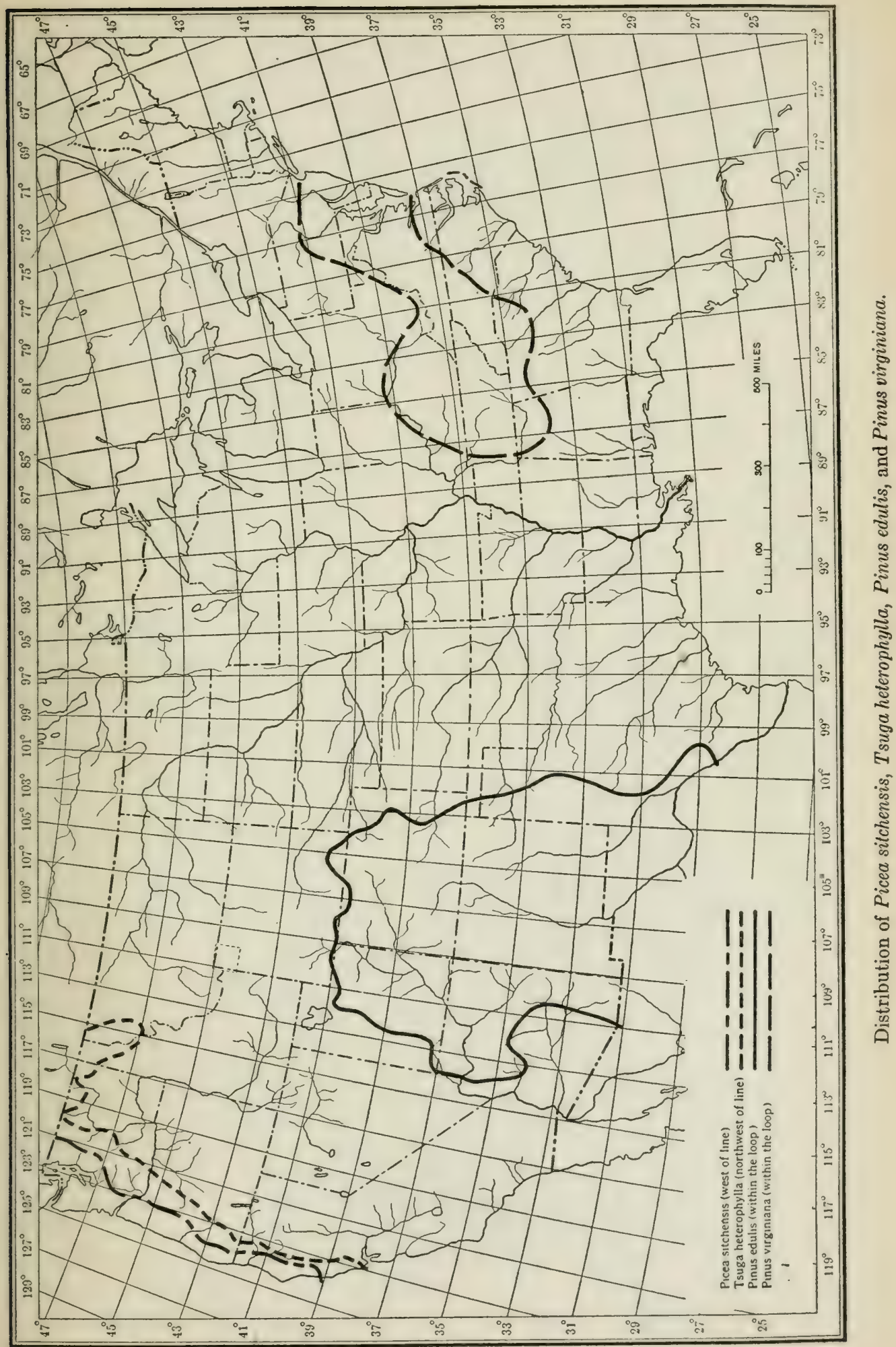




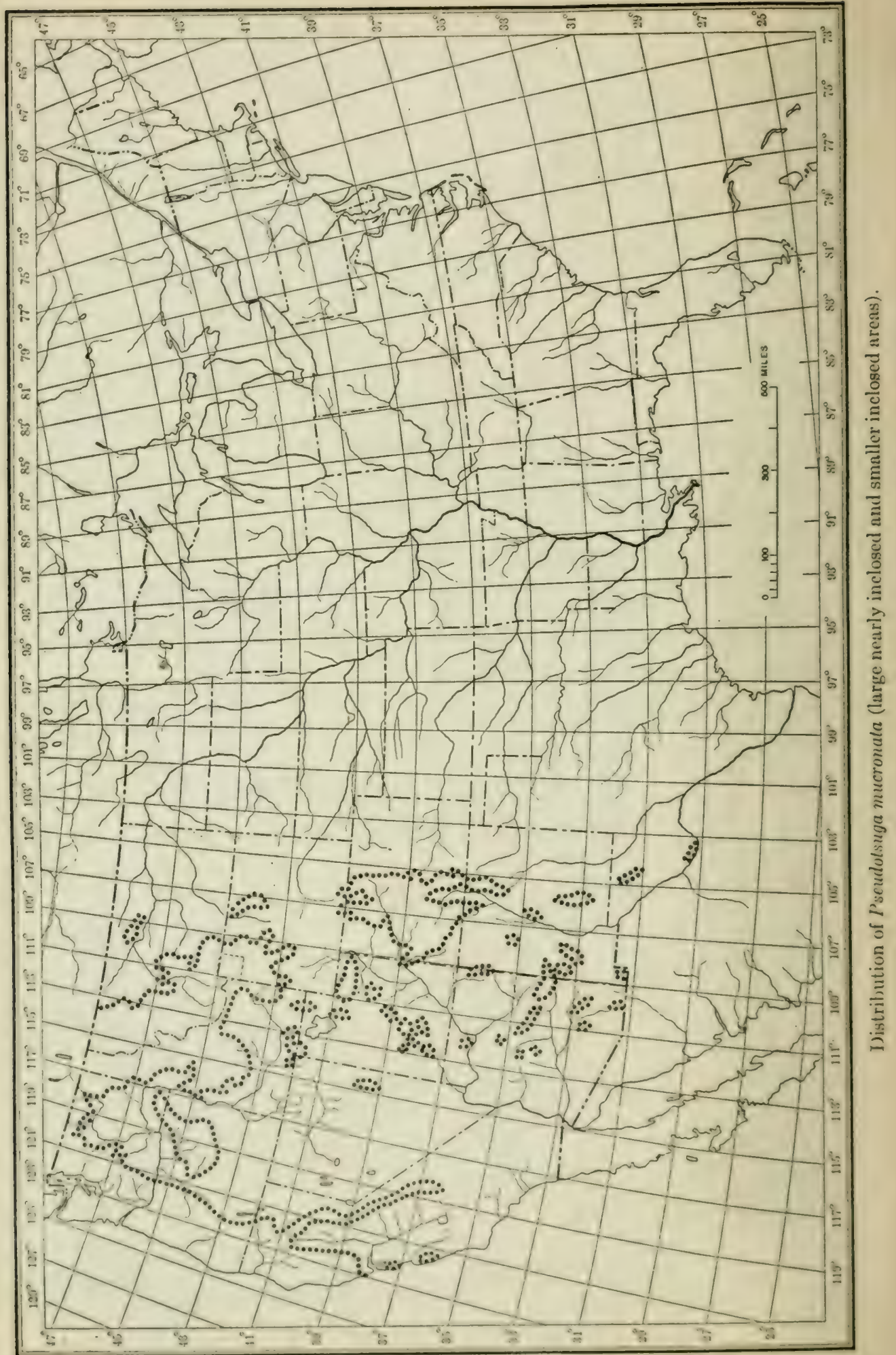




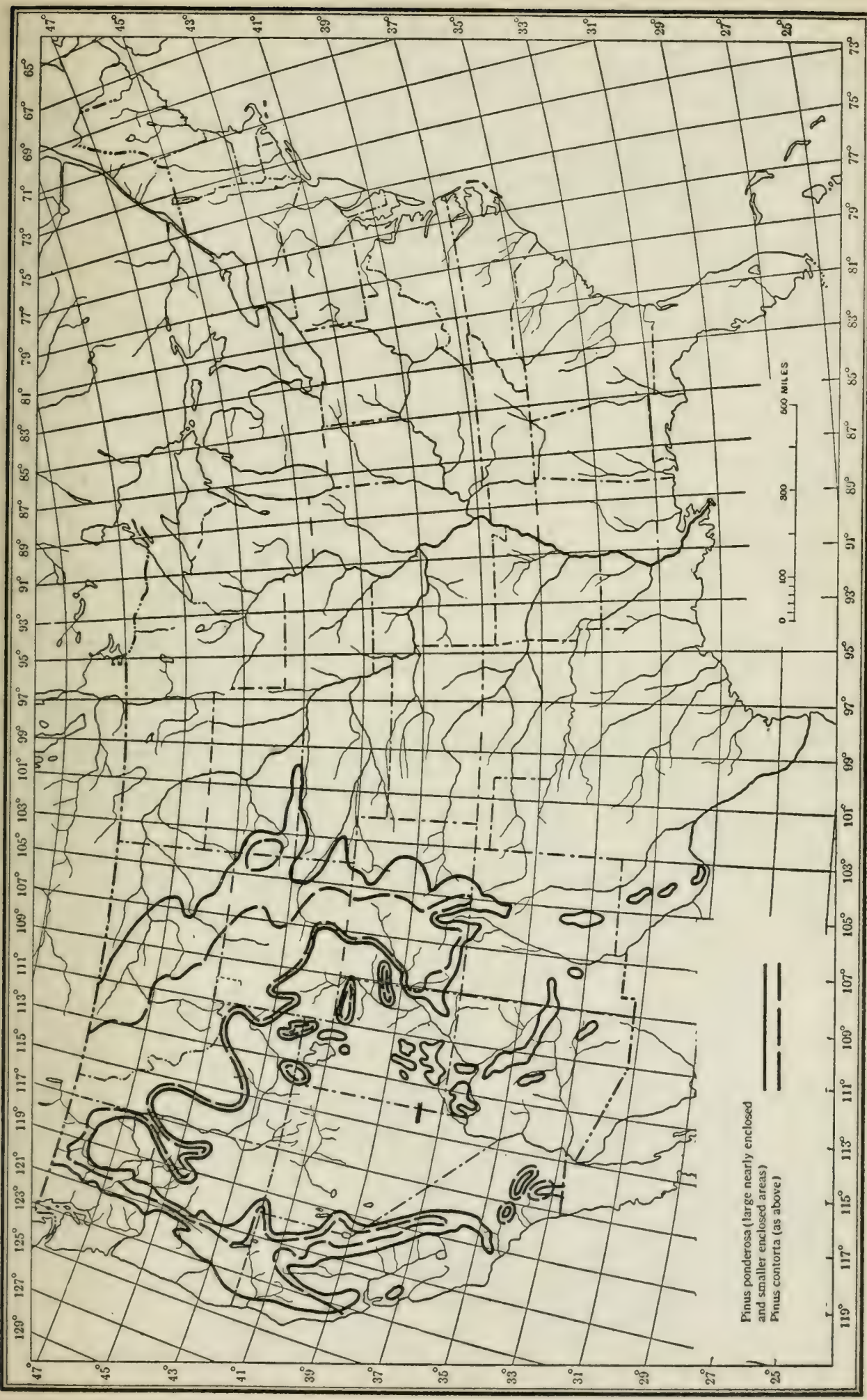




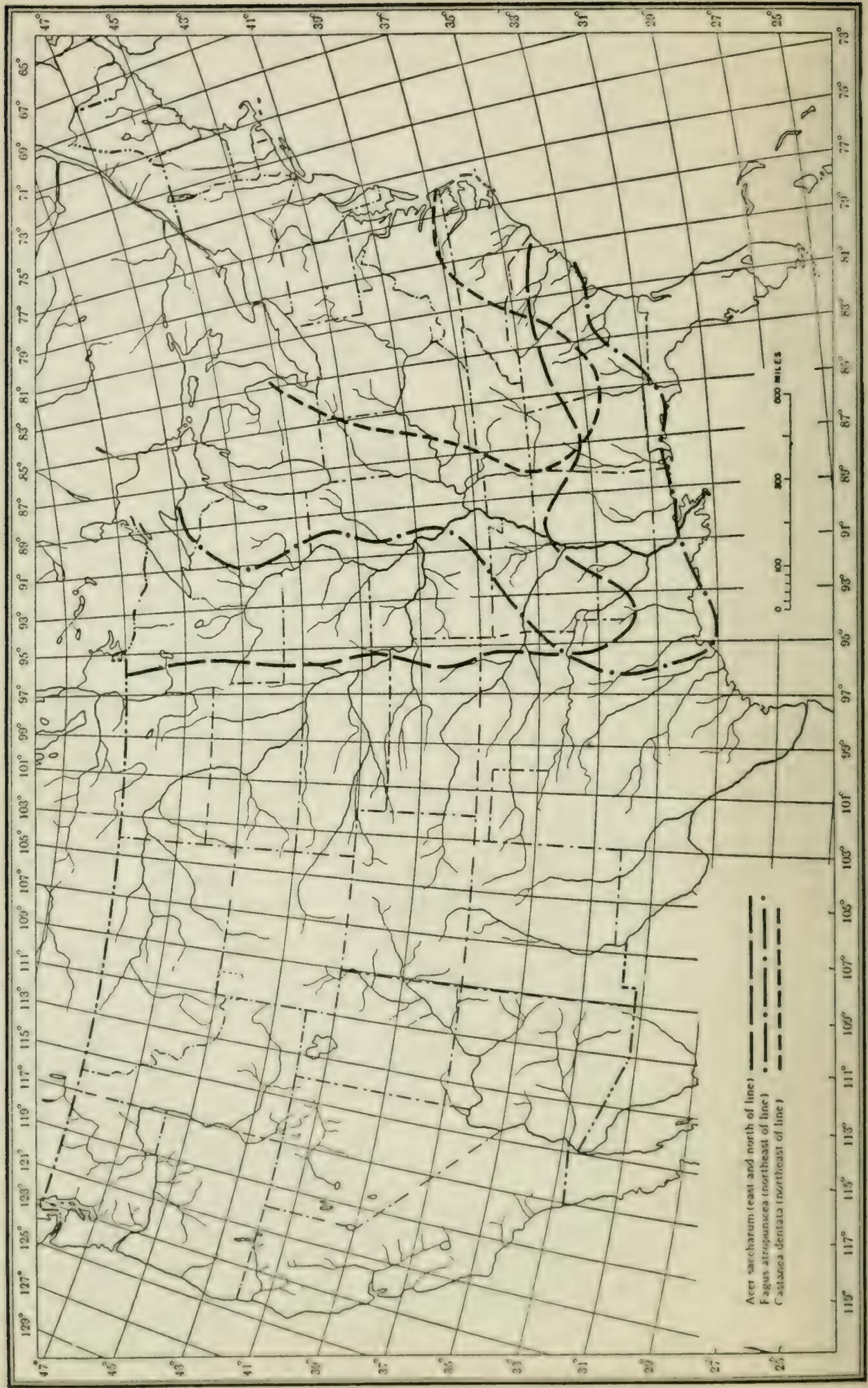


through the forested portions of the western United States from the Canadian boundary to Mexico. Pinus contorta is very nearly coincident in its range with the northern portion of the area occupied by Pinus ponderosa. Pinus edulis is used as an example of the type of evergreen needle-leaved trees which is dominant in the Western Xerophytic Evergreen Forest. It has not been possible from any data which are at hand to represent the range of this tree in as great detail as we used for the other members of this group.

\section{Southeastern Evergreen Needle-Leaved Trees. (Plate 6.)}

Pinus palustris Mill.

Pinus tæda L.

Pinus echinata Mill. (=P. mitis Michx.).

Pinus caribæa Morelet ( $=$ P. heterophylla (Ell.) Sudw.).

The range of the members of this group of characteristic evergreen needle-leaved trees of the Southeastern Mesophytic Forest has already been discussed on a previous page.

4. Northeastern Evergreen Needle-Leaved Trees. (Plates 7 and 13.)

Pinus strobus L.

Tsuga canadensis (L.) Carr.

Pinus virginiana Mill. (=P. inops Ait.).

Pinus divaricata (Ait.) DuMont. (=P. banksiana Lamb.).

Abies balsamea (L.) Mill.

The distribution of four of these species has also been discussed in showing the relation of their ranges to the range of the eastern portion of the Northern Mesophytic Evergreen Forest. Pinus virginiana has been used as an example of a type of distribution which is somewhat unusual among the evergreen needle-leaved trees, occupying an area between the northern and southern areas of evergreen needle-leaved forest and lying almost wholly in the deciduous region.

\section{Eastern Deciduous Trees. (Plates 16 and 17.)}

\section{Quercus alba L.}

Fagus atropunicea Ehrh. (=F. americana Sweet =F. ferruginea Ait.).

Castanea dentata (Marsh.) Borkh. (=C. sativa var. americana Sarg.).

Acer saccharum Marsh. (=A. saccharinum Wang.).

This group comprises four of the commonest trees of the deciduous forest, all of which were used in the map of cumulative occurrence of trees of this type given in plate 6. Quercus alba is found practically throughout the eastern half of the United States, with the exception of peninsular Florida and northern Michigan and Minnesota. Fagus atropunicea is also found throughout the greater part of the eastern United States, although it is somewhat less restricted in range than Quercus alba. Castanea dentata is more strictly confined in its occurrence to the Alleghenian region and its adjacent areas. Acer saccharum is similar in its range to Quercus alba, but extends farther to the north and not quite so far to the south. 


\section{Southeastern Deciduous Trees. (Plates 17 and 18.)}

Quercus falcata Michx. (=Q. digitata Sudw.).

Sapindus marginatus Willd.

These trees have been used as typical of the southeastern distributions, the former being very nearly coincident with the Atlantic Coastal Plain in its range, the latter occupying its largest area in Texas and Oklahoma, and extending eastward along the Gulf coast to Florida.

\section{Northern Deciduous Trees. (Plates 17 and 18.)}

Populus balsamifera L. (including P. hastata Dode). Quercus macrocarpa Michx.

These trees have been selected because of their wide northern range, which is limited in the former to the eastern portion of the Northern Evergreen Needle-leaved Forest and to a small area in the northern Rocky Mountains, while the latter tree extends south to the inner edge of the Coastal Plain and is remarkable for its extreme western extension into the grassland region.

8. Southeastern Evergreen Broad-Leaved Trees. (Plate 19.)

Ilex opaca Ait.

Magnolia grandiflora L. (=M. fœtida (L.) Sarg.).

Ilex has been used as an example of an evergreen broad-leaved tree which is nearly coincident in its range with the extent of the Atlantic Coastal Plain, and Magnolia has been used as another tree of the same growth-form which is confined to a more southern section of the Coastal Plain.

\section{Palms. (Plate 20.)}

Sabal palmetto (Walt.) R. and S.

Serenoa serrulata (Michx.) Hook.

Washingtonia filamentosa Wendl. (=Neowashingtonia filamentosa (Wendl.) Sudw.).

The three palms which are most widely distributed in the United States have been used as the sole representatives of this type of plant and as groups which are confined to the warmest portions of the United States. Sabal and Screnoa are both confined to western Florida and the adjacent coasts, while Washingtonia is found only in the interior deserts of southern California.

\section{Palustrine Simubs. (Plate 22.)}

Cephalanthus occidentalis L.

Adelia acuminata Michx. (= Forestiera acuminata Poir.)

Decodon verticillatus (L.) Lil.

Itea virginica $L$. 


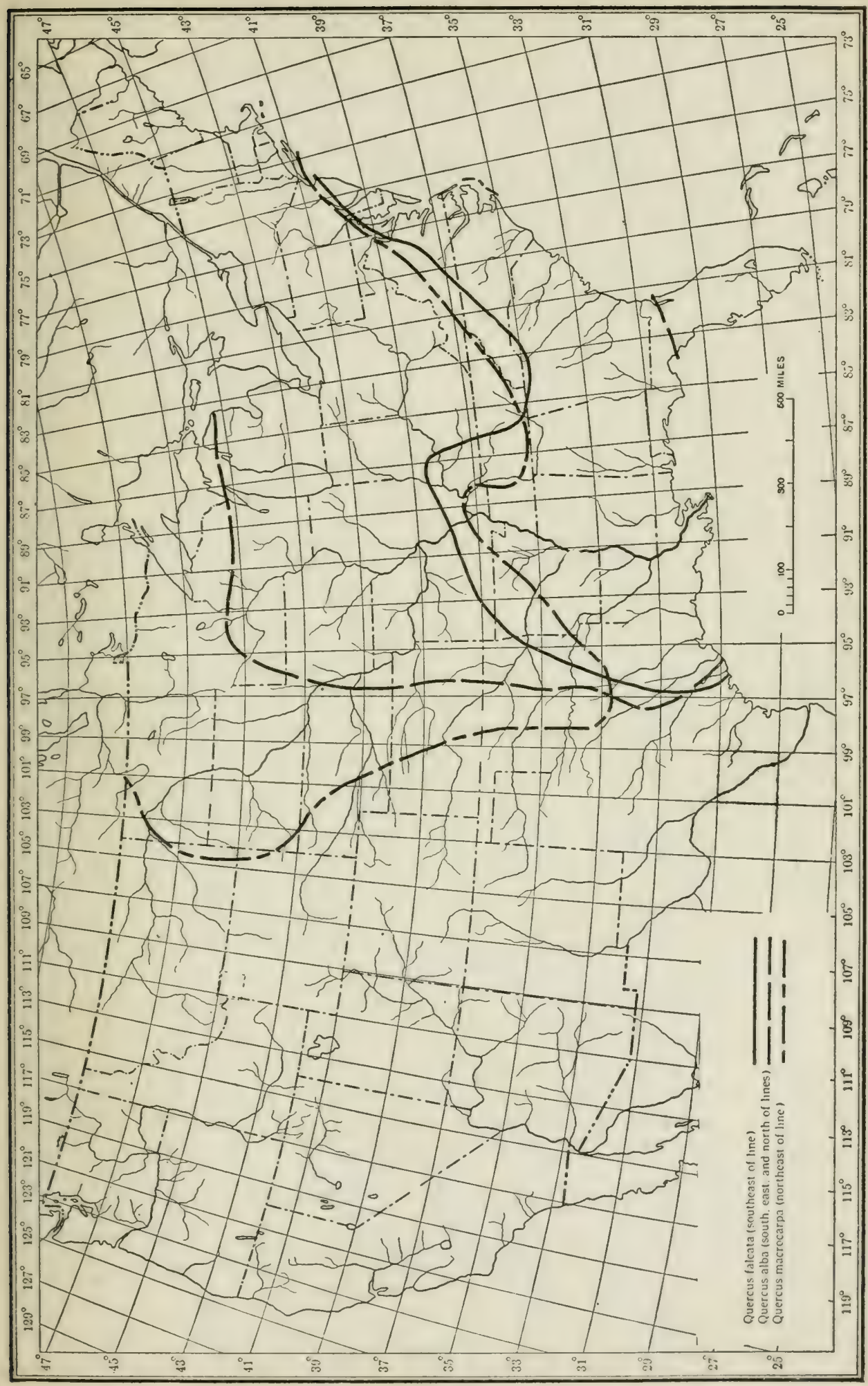




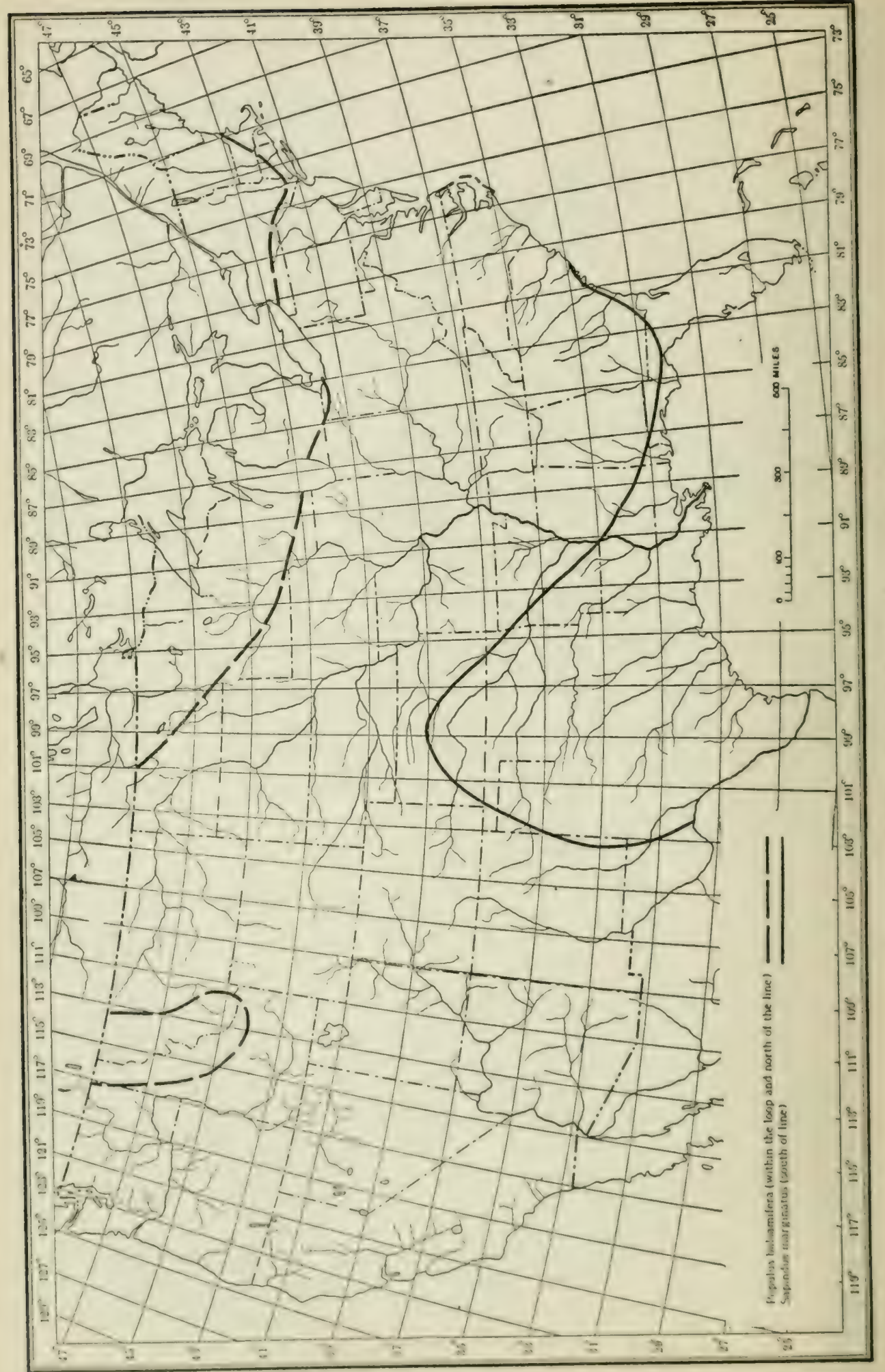

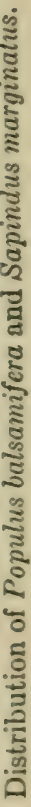




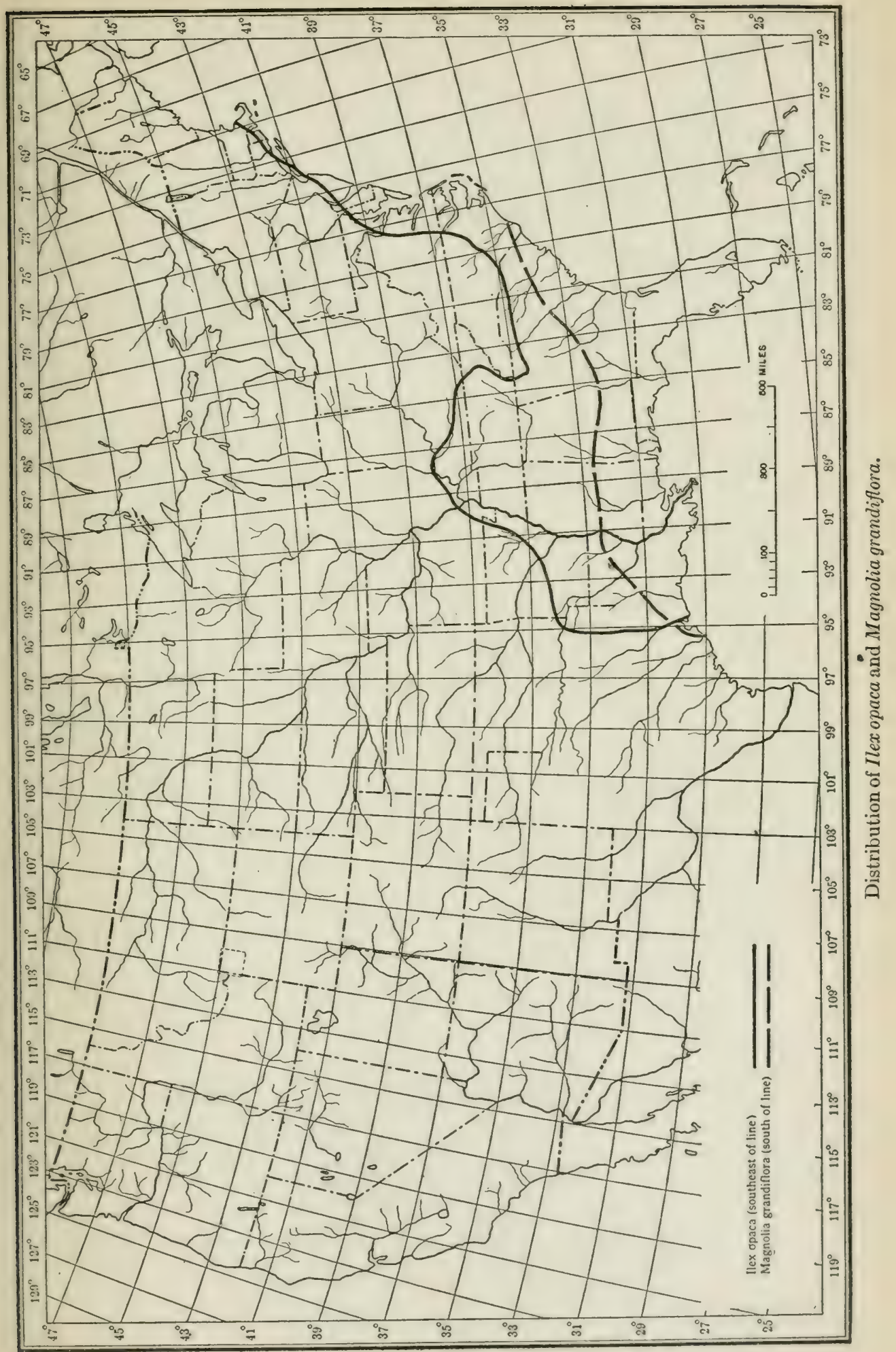




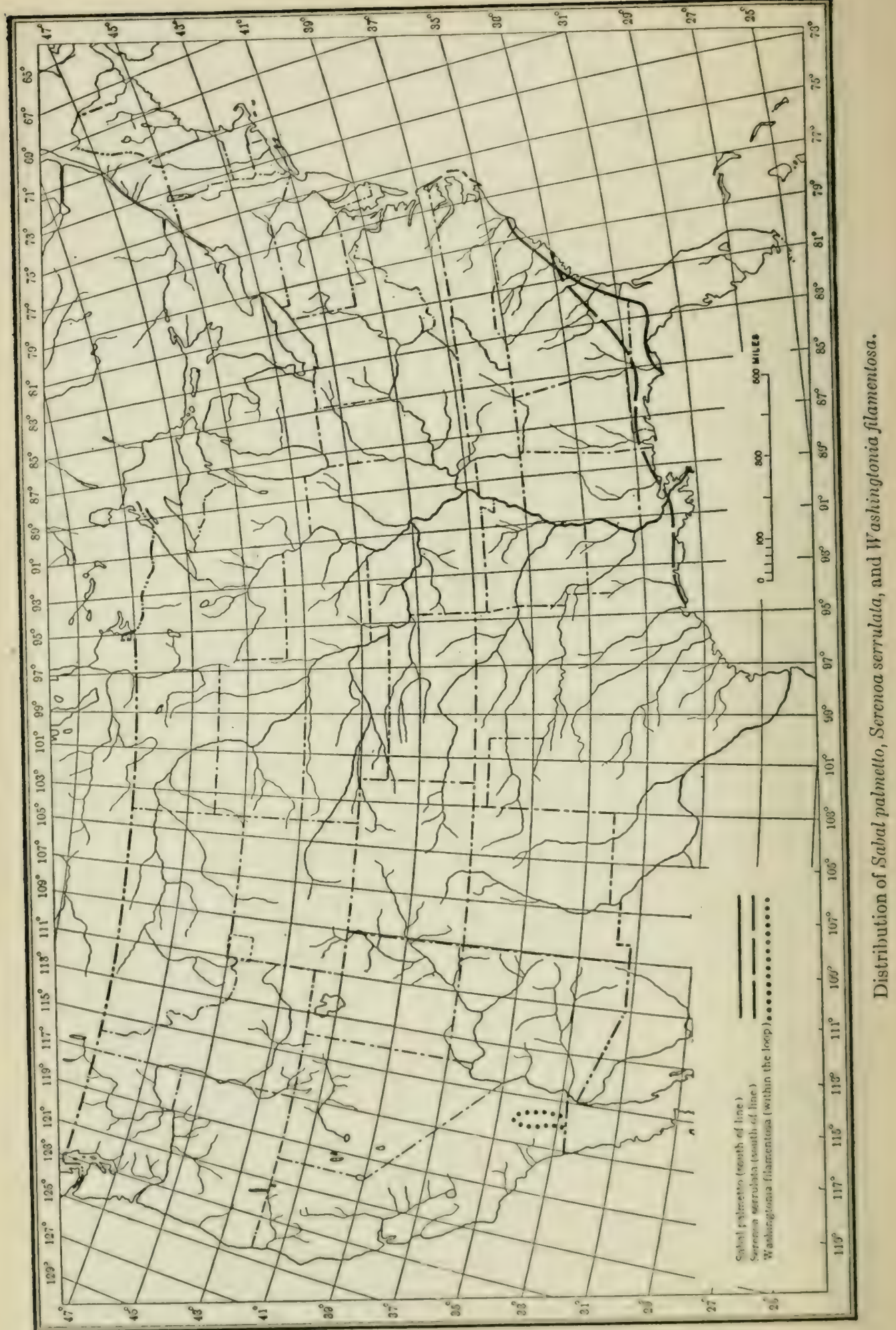




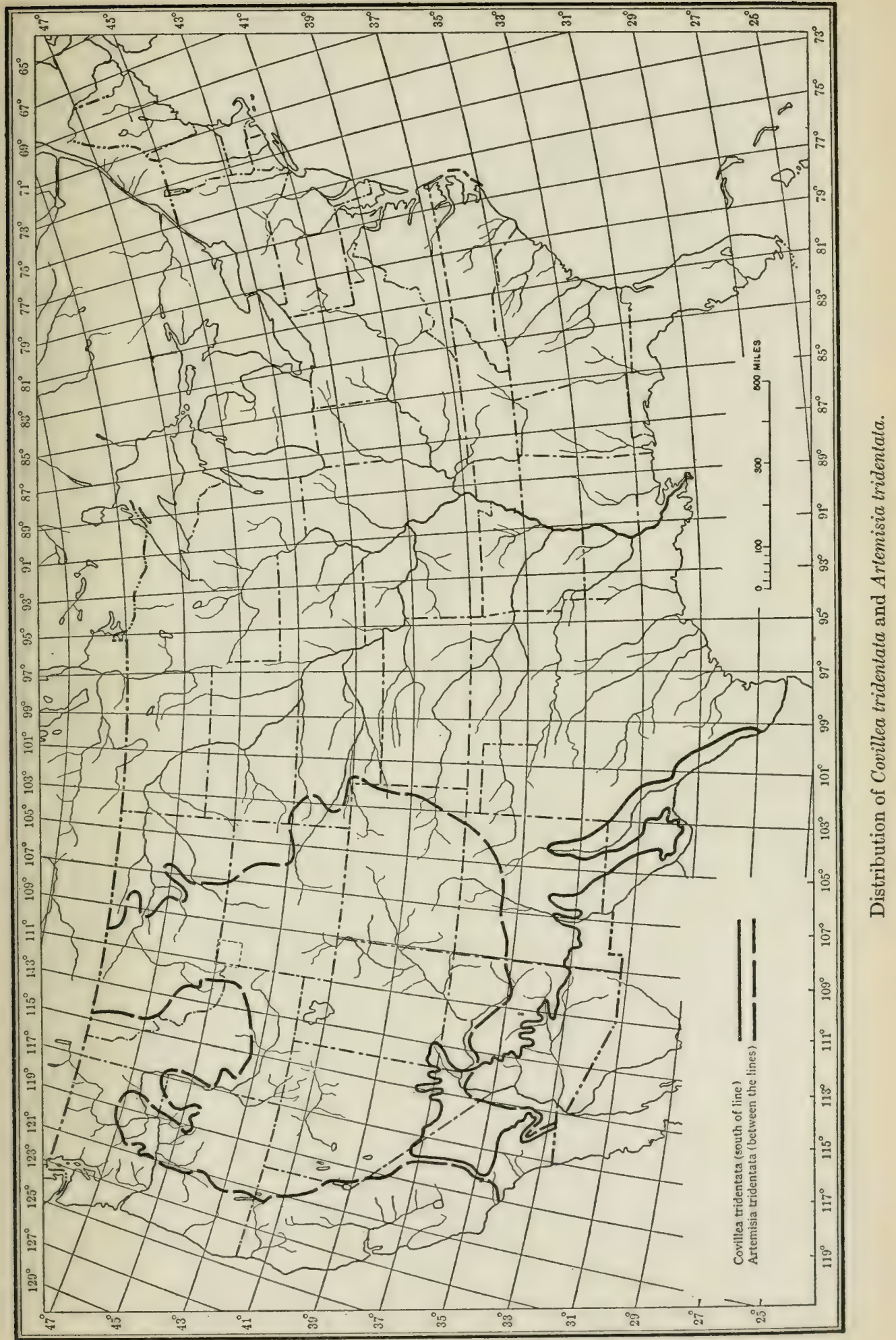




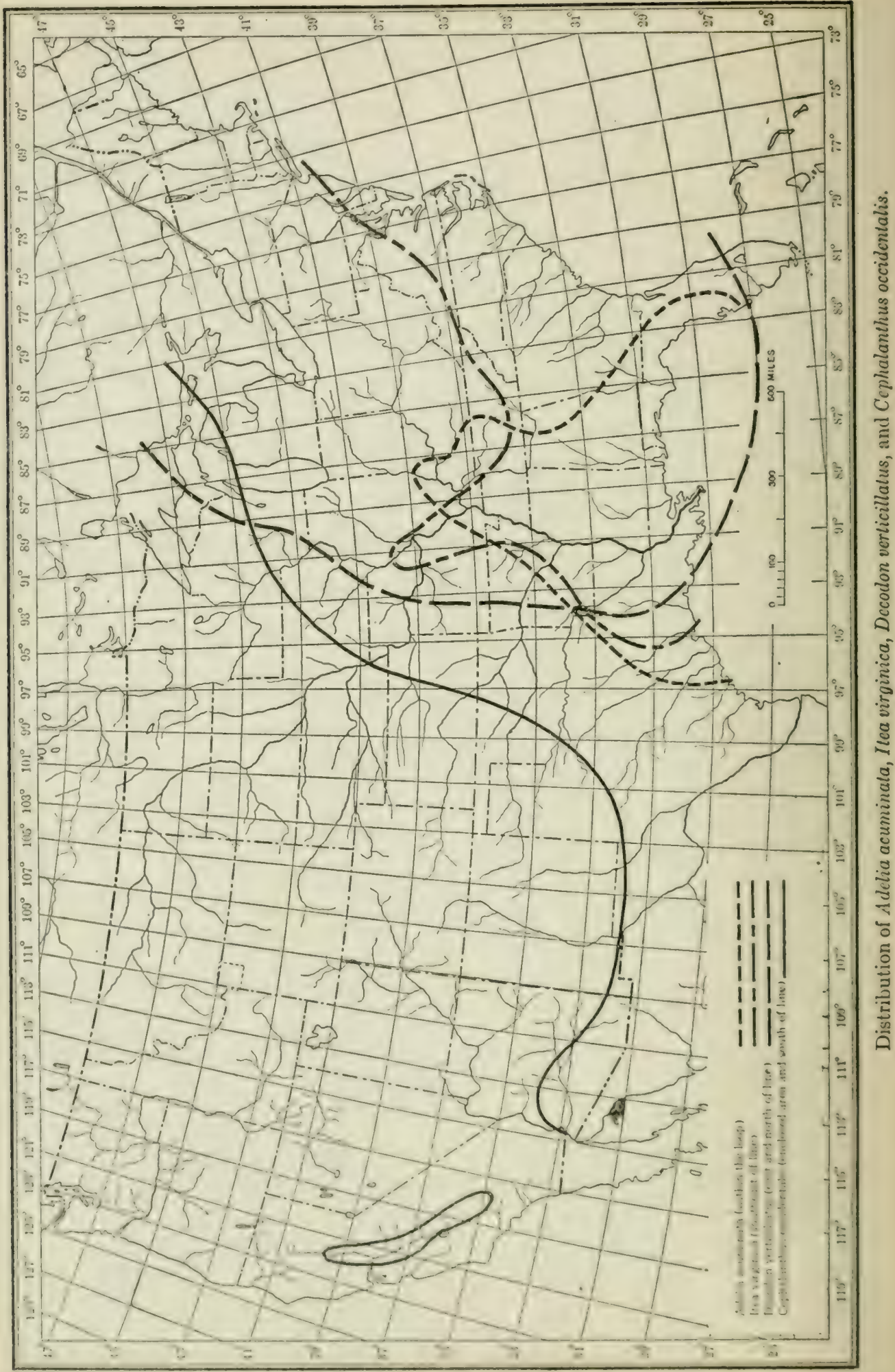




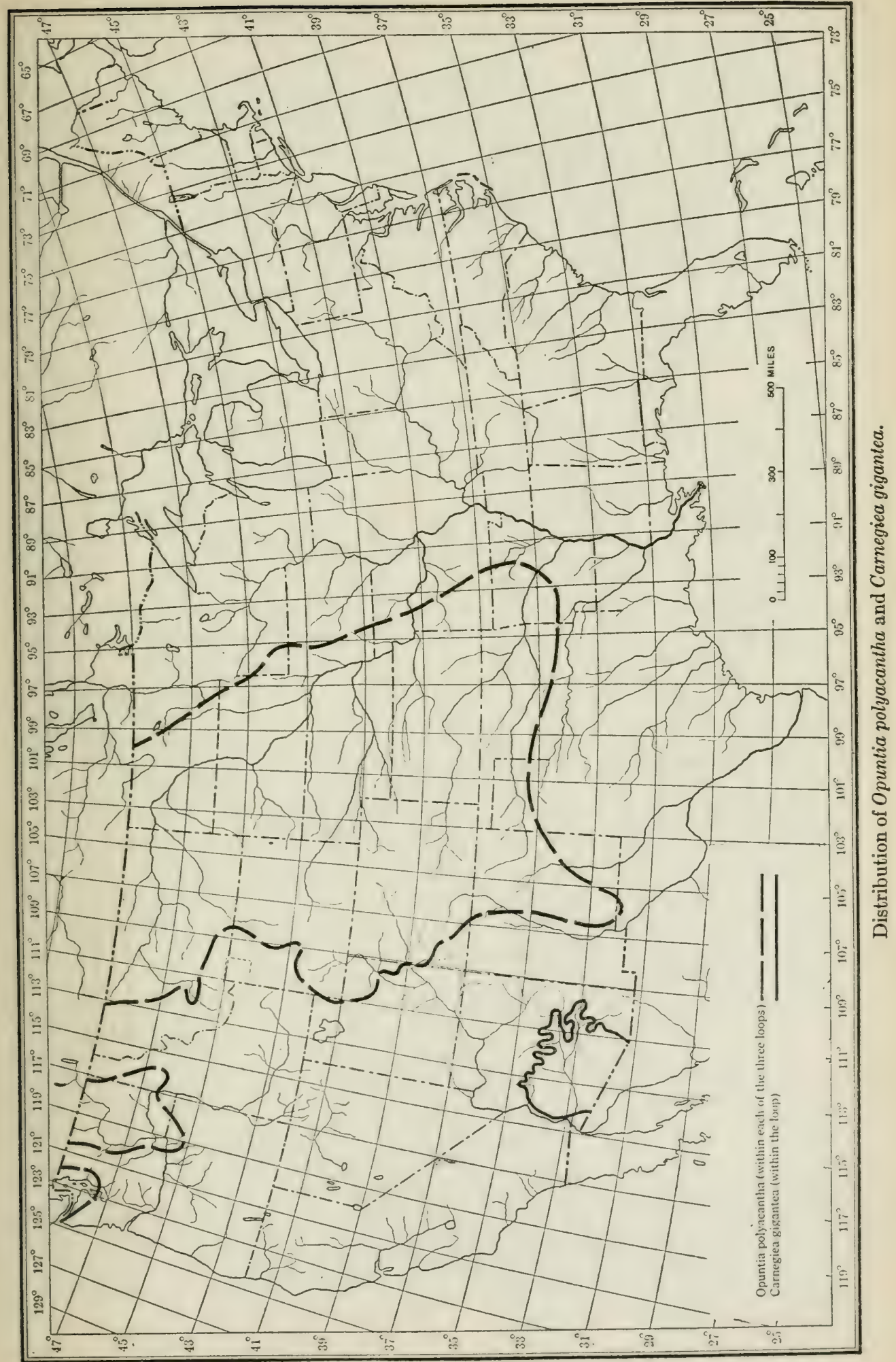




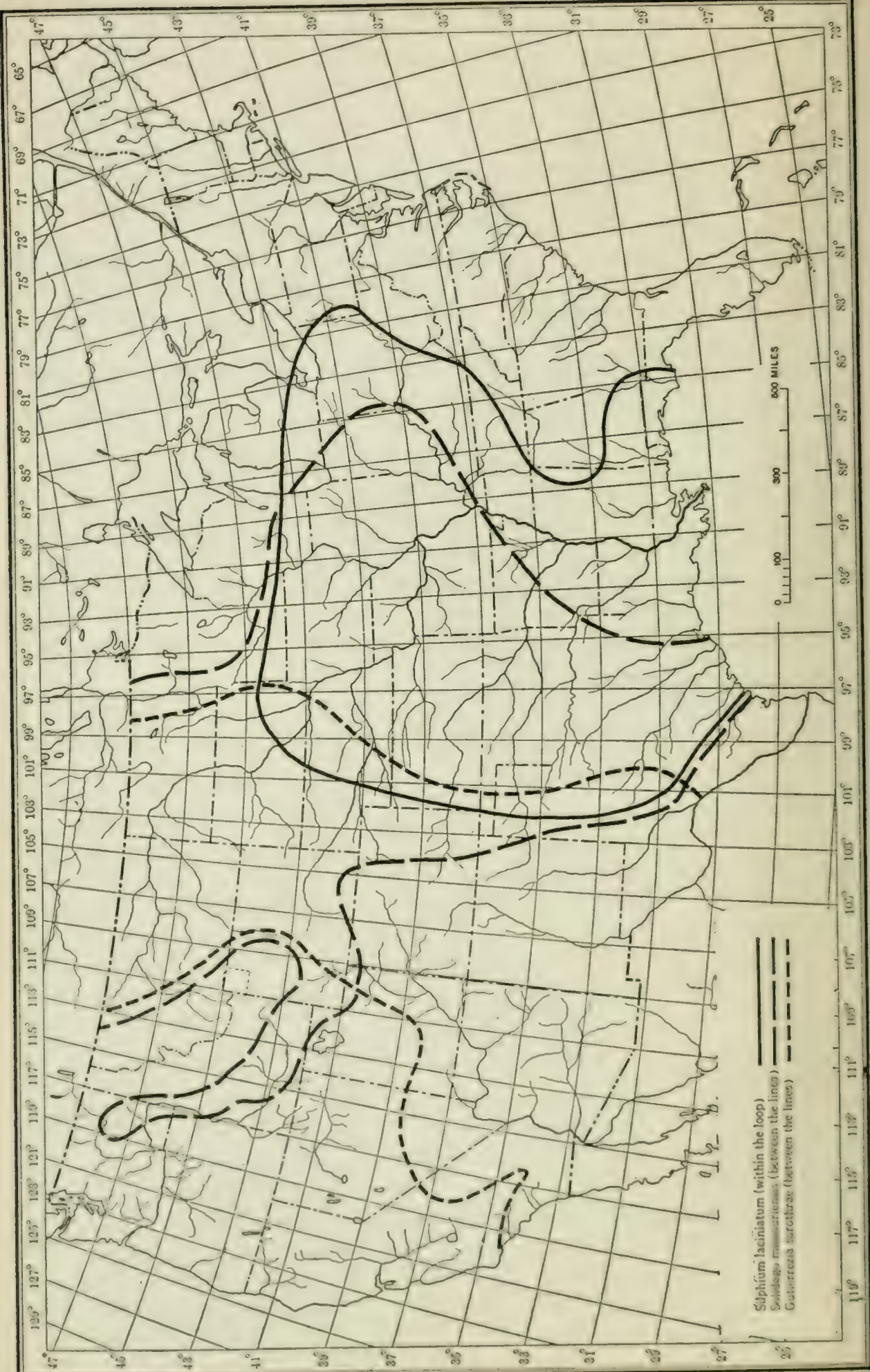


The members of this group are all shrubs of swamp and palustrine habitat and have been selected for use in our work with a view to bringing out the features of climatic control for plants which exist under a practically uniform set of soil-moisture conditions. Adelia is confined to the central portion of the Atlantic Coastal Plain. Itea occupies a similar area, but extends throughout the Coastal Plain as far north as New Jersey. Decodon is found in palustrine situations throughout the eastern United States, and Cephalanthus occupies a similar area with a remarkable westward prolongation of its range into southern Arizona and the central valley of California.

\section{Microphyllous Desert Shrubs. (Plate 21.)}

Artemisia tridentata Nutt.

Covillea tridentata (DC.) Vail (including C. glutinosa (Engelm.) Rydb.).

These shrubs have been used in view of the fact that they are the most abundant plants in two of the most important of the desert areas. Artemisia is found throughout the Great Basin Microphyll Desert and extends beyond its limits to the east and northeast into the higher portions of the Grassland area. Covillea is the leading plant throughout the California Microphyll Desert and the Arizona and Texas Succulent Deserts, extending eastward into the Texas Semidesert.

\section{Cacti. (Plate 23.)}

Opuntia polyacantha Haw. (including closely related varieties).

Carnegiea gigantea (Engelm.) Britton and Rose (=Cereus giganteus Engelm.)

The species of cacti which have been used were taken as examples of this great group of stem-succulent plants, the former representing one of the most widely distributed of the species found in the United States, and the latter one of the most restricted of the species which is in any place an important element of the vegetation. Opuntia polyacantha is found throughout the Grassland region, extending from southern New Mexico to the Canadian boundary and occurring in small areas in Washington. Carnegiea is limited to southwestern Arizona at elevations below 4,000 feet.

\section{Characteristic Composites of the Great Plains. (Plate 24.)}

Silphium laciniatum L.

Solidago missouriensis Nutt.

Gutierrezia sarothræ (Pursh) B. and R.

These suffrutescent perennials are found throughout the Grassland and Grassland-Deciduous areas and, in their range and relations to climate, may be taken as typical of a large number of similar plants. Silphium occupies the most easterly range of this group of plants, 
extending as far as Pennsylvania and Florida, with the suspicion of its having been introduced along its easternmost limit. Solidago is closely confined to the Grassland and Grassland-Deciduous Transition area, with a northwestward extension in regions of this type through southern Idaho and eastern Washington. Gutierrezia is found in the western portion of the Grassland and in the higher portions of all areas in the extreme southwestern portion of the country. In addition to its natural habitats, this plant is an extremely common one in all areas in which the original cover of grasses has been disturbed by grazing or other unnatural conditions.

14. Characteristic Grasses of the Great Plains and the Prairies. (Plates 11 and 25.)

Bouteloua oligostachya (Nutt.) Torr.

Bulbilis dactyloides (Nutt.) Raf. (=Buchlöe dactyloides Nutt.).

Koleria cristata (L.) Pers.

Agropyron spicatum (Pursh) Rydb. (=A. divergens . Nees, =Festuca spicata Pursh. Not A. spicatum Scribn, and Smith).

Hilaria jamesii (Torr.) Benth.

Andropogon virginicus $L$.

Bouteloua hirsuta Lag.

Four of the members of this group of grasses characteristic of the Grassland and Grassland-Deciduous Forest Transition regions have already been used in constructing the map of cumulative occurrence of grasses (plate 12). The ranges of three other species are given in plate 25. Agropyron and its allies have been selected because of their importance in the formation of the Grassland of the northwestern States, Hilaria because of its importance in the southernmost extension of the Grassland and the Desert-Grassland Transition, and Andropogon because of its importance in the easternmost portion of the prairies of the Grassland-Deciduous Forest Transition.

\section{Palustrine Herbaceous Plants. (Plates 26 and 27.)}

Sparganium americanum Nutt. (including S. americanum var. androcladum (Engelm.) Fern, and Eames).

Dianthera americana $\mathrm{L}$.

Sium cicutrefolium Gmel.

These palustrine herbaceous plants are of interest for the same reason that has been mentioned in connection with palustrine shrubs. Sparganium is extensively distributed in the Eastern States, but its precise range is not well known. Dianthera is found in the eastern half of the United States south of Wisconsin and outside the eastern half of the Coastal l'lain. Sium is found practically throughout the United States, with the exception of the continental desert areas, its occurrence throughout a large part of this region being extremely infrequent 


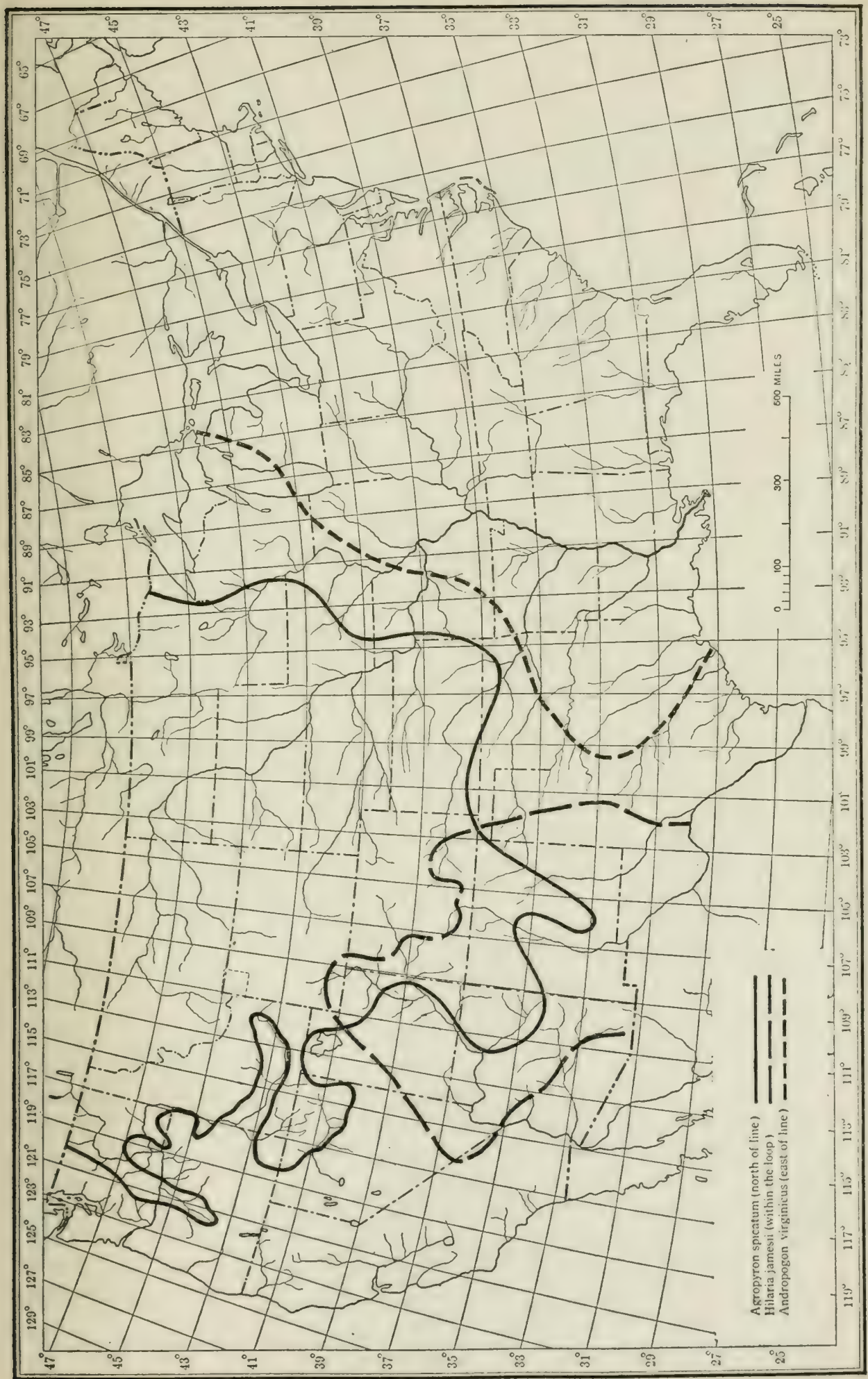




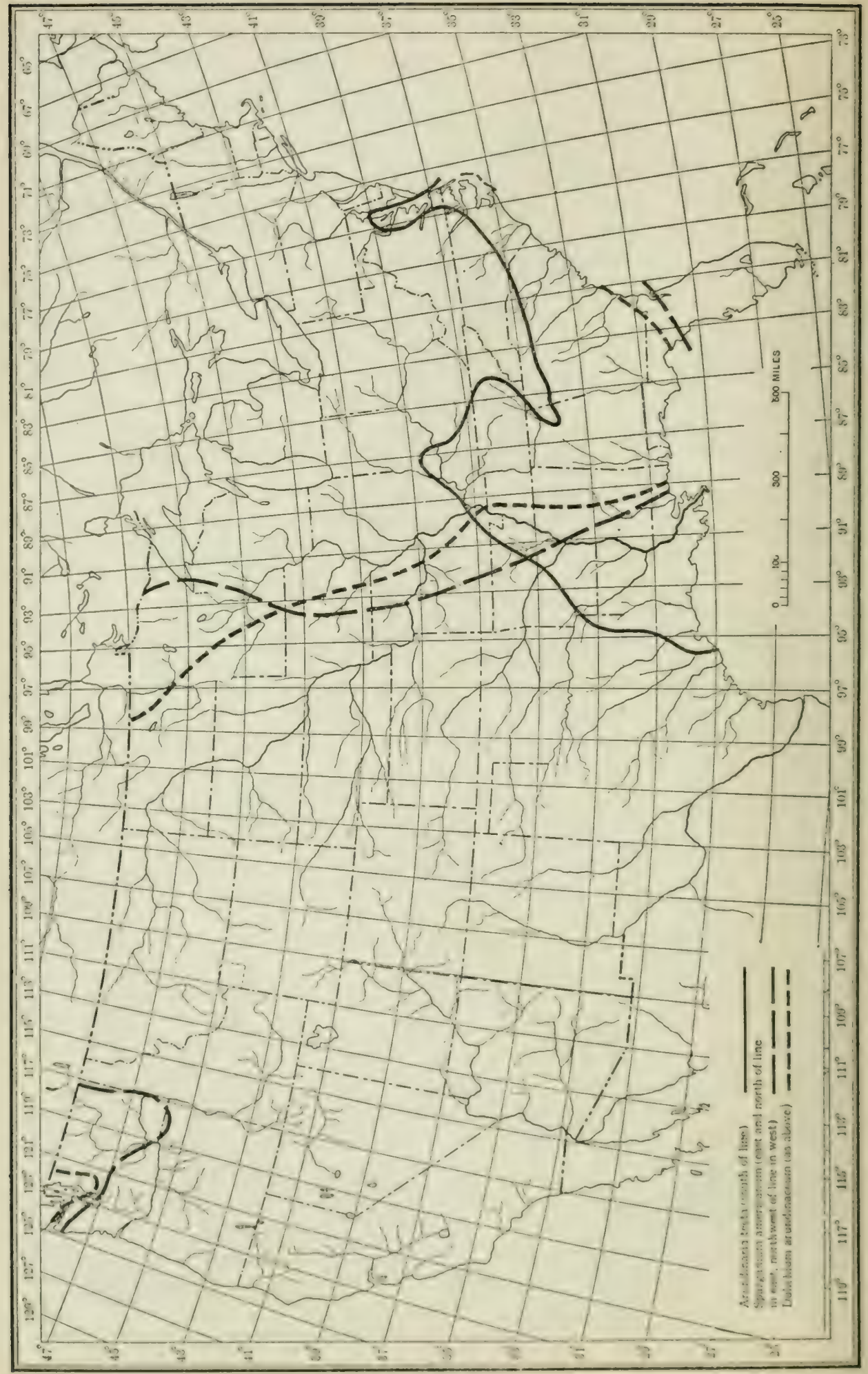

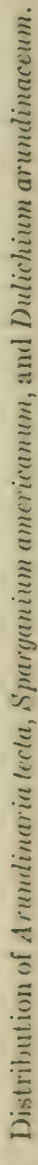




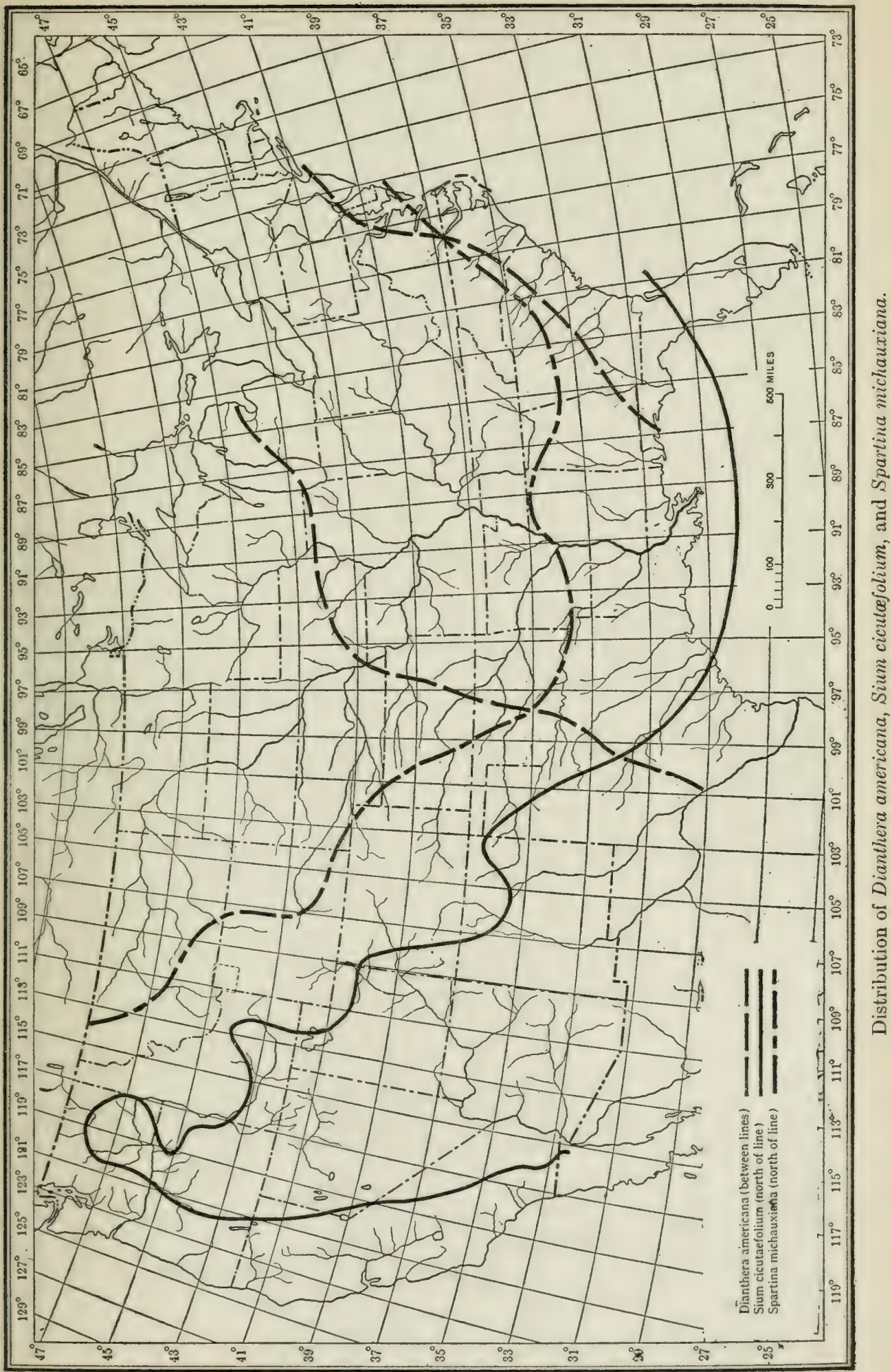




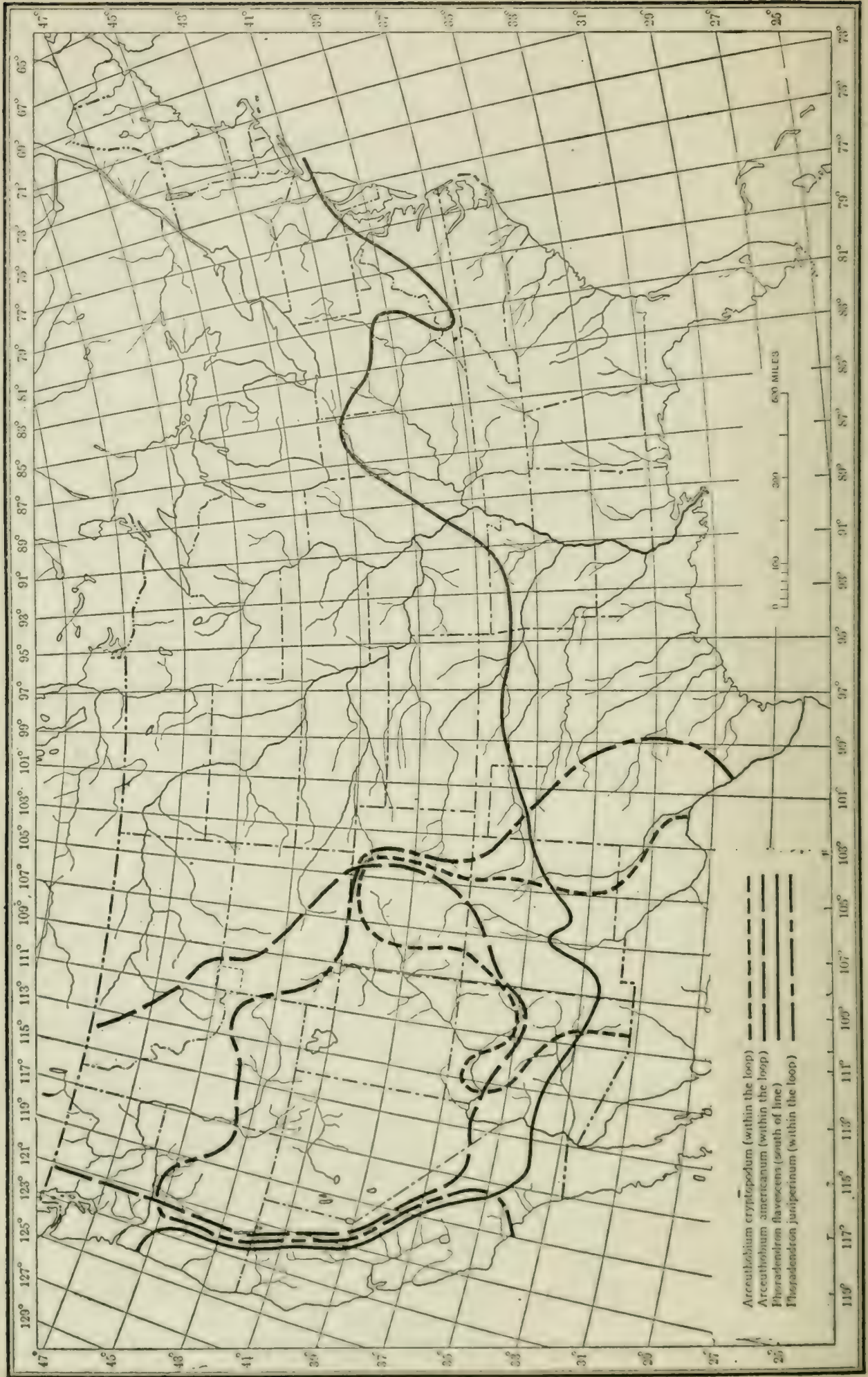

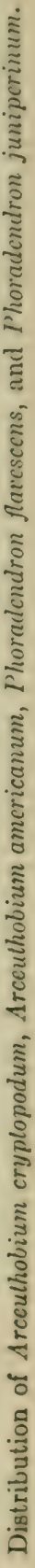


16. Palustrine Grasses and Sedges. (Plates 26 and 27.)

Arundinaria tecta (Walt.) Muhl.

Dulichium arundinaceum (L.) Britton.

Spartina michauxiana Hitchk. (=S. cynosuroides (L.) Willd.).

While not strictly a plant of palustrine habitat, Arundinaria is found in moist alluvial soil and is of interest as one of the largest grasses found in the United States. Dutichium is distributed over an area very similar to that occupied by Sparganium, while Spartina occurs in palustrine situations throughout the northeastern portion of the country from Georgia, Oklahoma, and Wyoming to Canada.

\section{Mistletoes. (Plate 28.)}

Arceuthobium cryptopodum Engelm. (=Razoumofskya cryptopoda (Engelm.) Coville.

Arceuthobium americanum Nutt. (=Razoumofskya americana (Nutt.) Kze.).

Phoradendron flavescens (Pursh) Nutt. (including varieties).

Phoradendron juniperinum Engelm.

In view of the fact that the mistletoes are independent of the conditions which the ordinary terrestrial plant encounters in its relation to the substratum, a series of four of these plants has been selected for our correlational work. The moisture conditions of the substratum in which these plants grow are doubtless determined in large measure by the moisture conditions that exist for the hosts themselves. Phoradendron flavescens, together with its varieties, possesses an extremely wide range from the Atlantic to the Pacific throughout the southern half of the United States. The other species that have been used are western in their range, and we have been under the unfortunate necessity of representing their areas of distribution by smooth lines which surround the territory in which they are of scattered occurrence, chiefly in the forested mountains.

18. Plants of Northern Transcontinental Range. (Plate 29.)

Arenaria lateriflora L. (=Mœhringia lateriflora (L.) Fenzl.

Parietaria pennsylvanica Muhl.

Cornus canadensis L.

The two herbaceous plants and the single shrub which form this group extend entirely across the North American continent and range southward to different distances, the southernmost range being that of Parietaria. Cornus is the least southerly in its range in the Eastern States, but is the most southerly in California. Transcontinental ranges of this character are extremely abundant among plants of still more northerly distribution than these, a number of trees and shrubs being found almost continuously from Labrador to Alaska. We are 
here concerned solely with the southern limits of distribution of these plants, all of which range northward into the forested belt of Canada.

19. Plants of Southern Transcontinental Range. (Plate 30.)

Spermolepis echinata (Nutt.) Hell.

Daucus pusillus Michx.

Parietaria debilis Forst. (=P. floridana Nutt.)

Similar ranges extending from the Atlantic to the Pacific are found in the case of a few herbaceous plants which grow and mature during the different portions of the year in different parts of their transcontinental ranges. Daucus extends from North Carolina through Louisiana, Texas, and California, and up the Pacific coast to Washington, although it is relatively infrequent at the extremes of this range. All of these plants extend beyond the limits of the United States and we are able to investigate only the northern limits of their distributions.

\section{Herbaceous Plants of Southwestern Range. (Plate 31.)}

Kallstrœmia grandiflora Torr. (=Tribulus grandiflorus Wats.).

Cladothrix lanuginosa Nutt.

Pectis papposa Harv, and Gray.

Euphorbia serpyllifolia Pers. (=Chamæsyce serpyllifolia (Pers.) Small.

A small group of plants has here been selected as representing types of distribution applying to a very large number of plants in the southwestern arid regions. Cladothrix, Kallstromia, and Pectis are all confined to the extremely warm regions of Texas, New Mexico, and Arizona. Euphorbia is found throughout the western half of the United States in varying abundance.

\section{Herbaceous Plants of Central Distribution (Nyctaginacem).} (Plate 32.)

Bœrhàavia erecta L.

Oxybaphus nyctagineus (Michx.) Sweet (=Allionia nyctaginea Michx.).

Oxybaphus angustifolius (Nutt.) Sweet (=Allionia linearis Pursh).

Oxybaphus floribundus Chois. (=Allionia floribunda (Chois.) Kze.).

In view of the fact that we have been concerned in so many cases with only one of the two edges of distribution of plants, we have here selected a group of central occurrence so as to make it possible to investigate the conditions of their eastern and western limits. Members of the same family have been chosen in this case because of the desirability of working out the behavior of a group of plants which are closely related in growth-form as well as in taxonomic relationship. Boerhania is found in the extreme south from (ieorgia to Arizona, while the other species of this group have their main regions of occurrence in the Grassland and Grassland-Deciduous Forest Transition. 


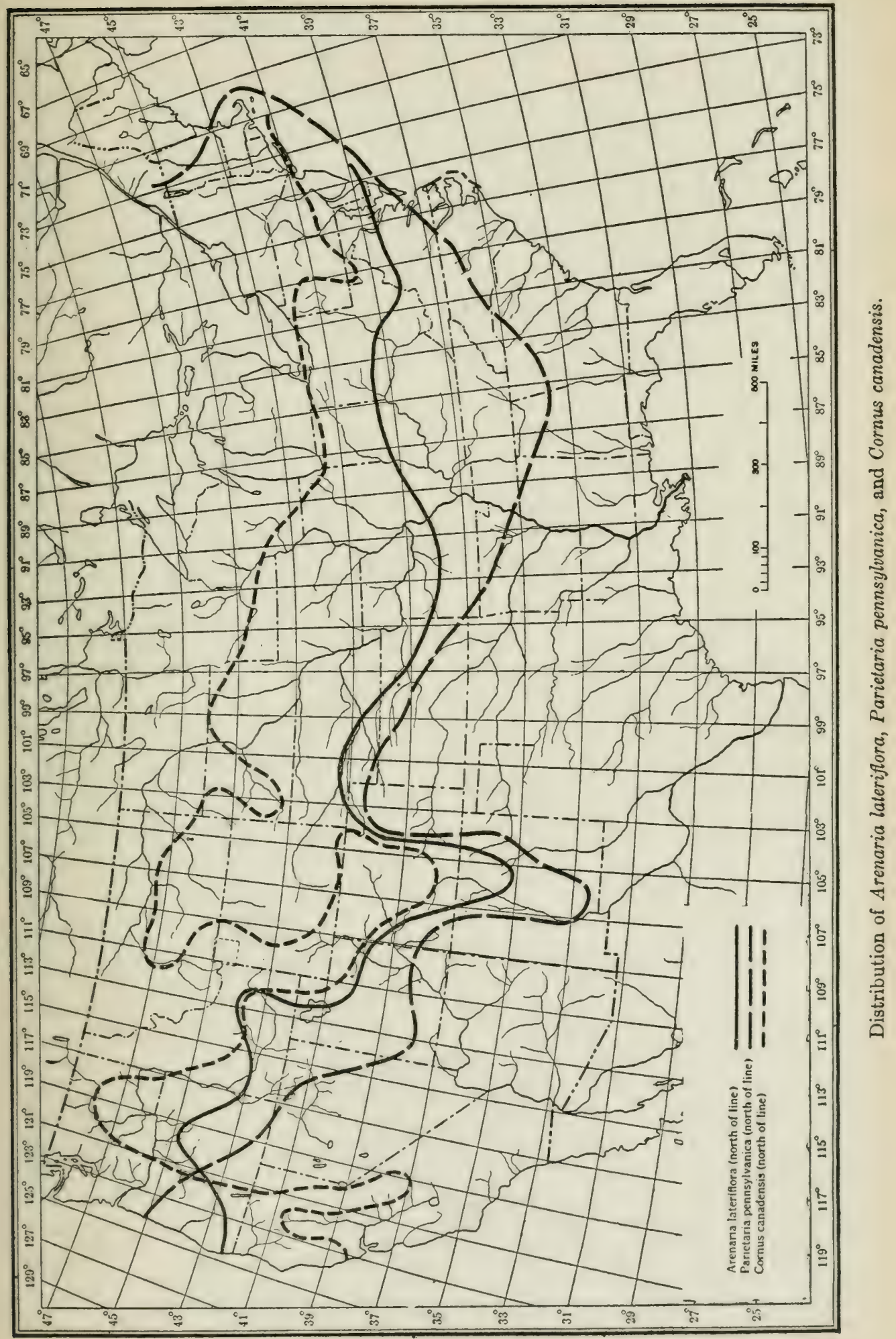




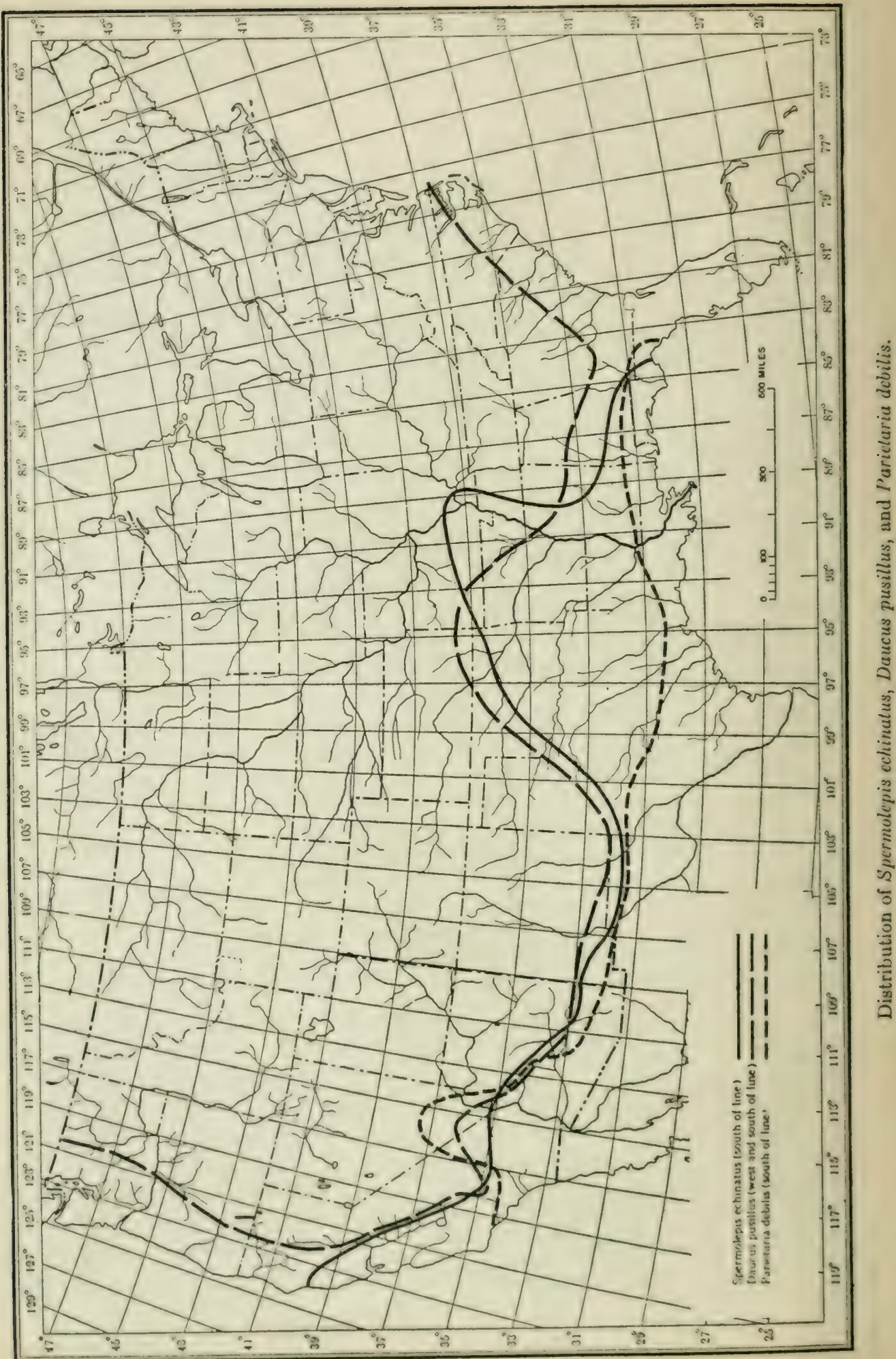




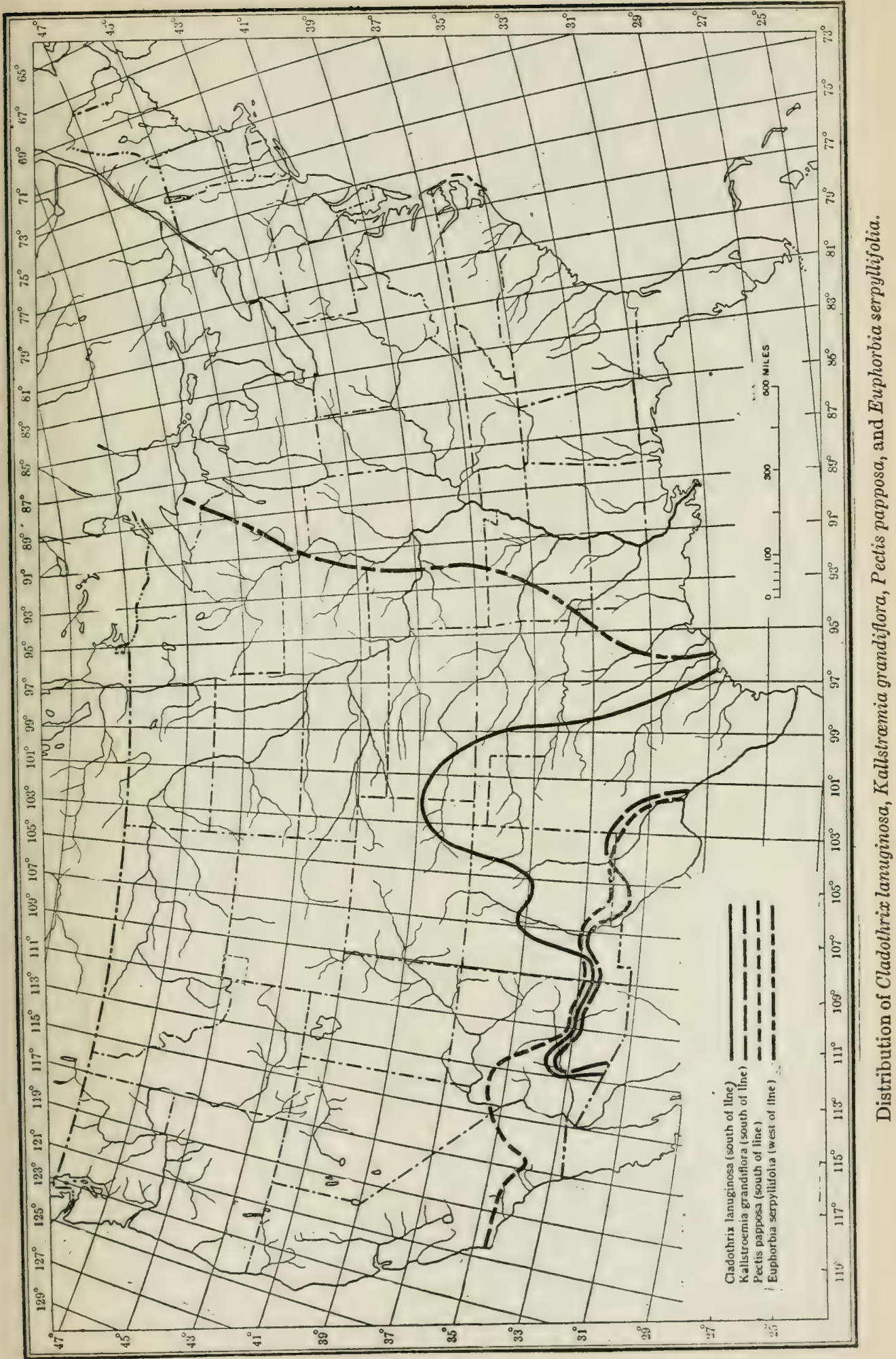




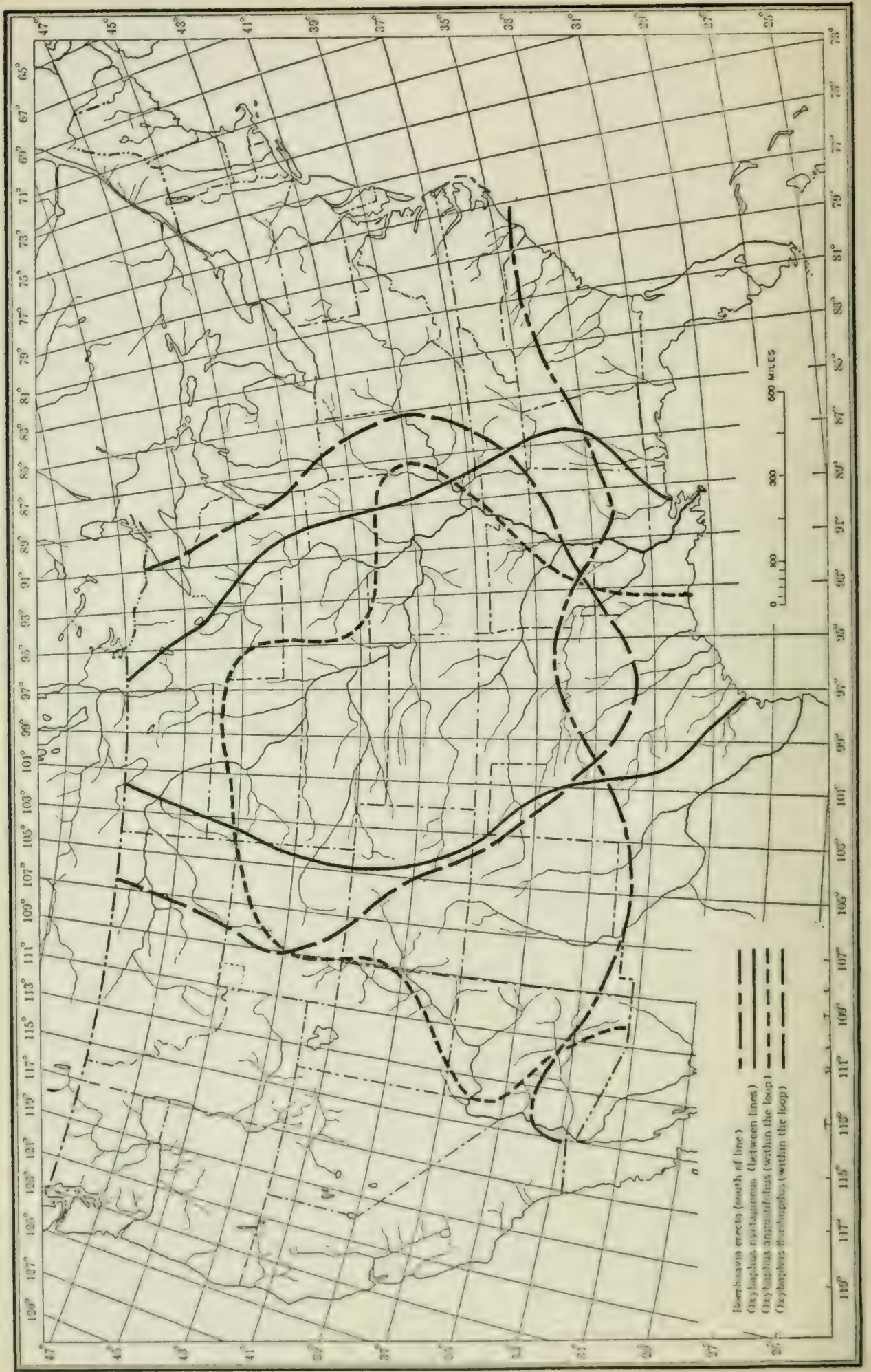

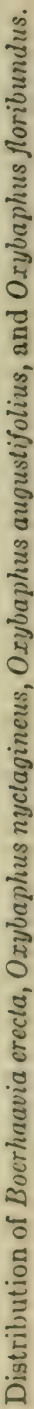


22. Paired Species of Eastern and Western Ranges, Respectively. (Plate 33.)

Flœrkea occidentalis Rydb.

Flœrkea proserpinacoides Willd.

Trautvetteria grandis Nutt.

Trautvetteria carolinensis (Walt.) Vail (=T. palmata Fisch. and Mey.).

Cebatha diversifolia (DC.) Kze. (=Cocculus diversifolius DC.).

Cebatha carolina (L.) Britton (=Cocculus carolinus (L.) DC.).

There are a few cases in the flora of the United States in which a genus has two species, one of which is eastern and the other western in range. We have selected the paired species of each of the three genera, Floerkea, Trautvetteria, and Cebatha, with a view to investigating the climatic conditions characteristic of the present separated ranges of these pairs of closely related plants. There is some doubt in the case of the two species of Fløerkia as to their specific distinctness. The two species of Cebatha apparently overlap in central Texas, whereas the other two species are widely separated. 


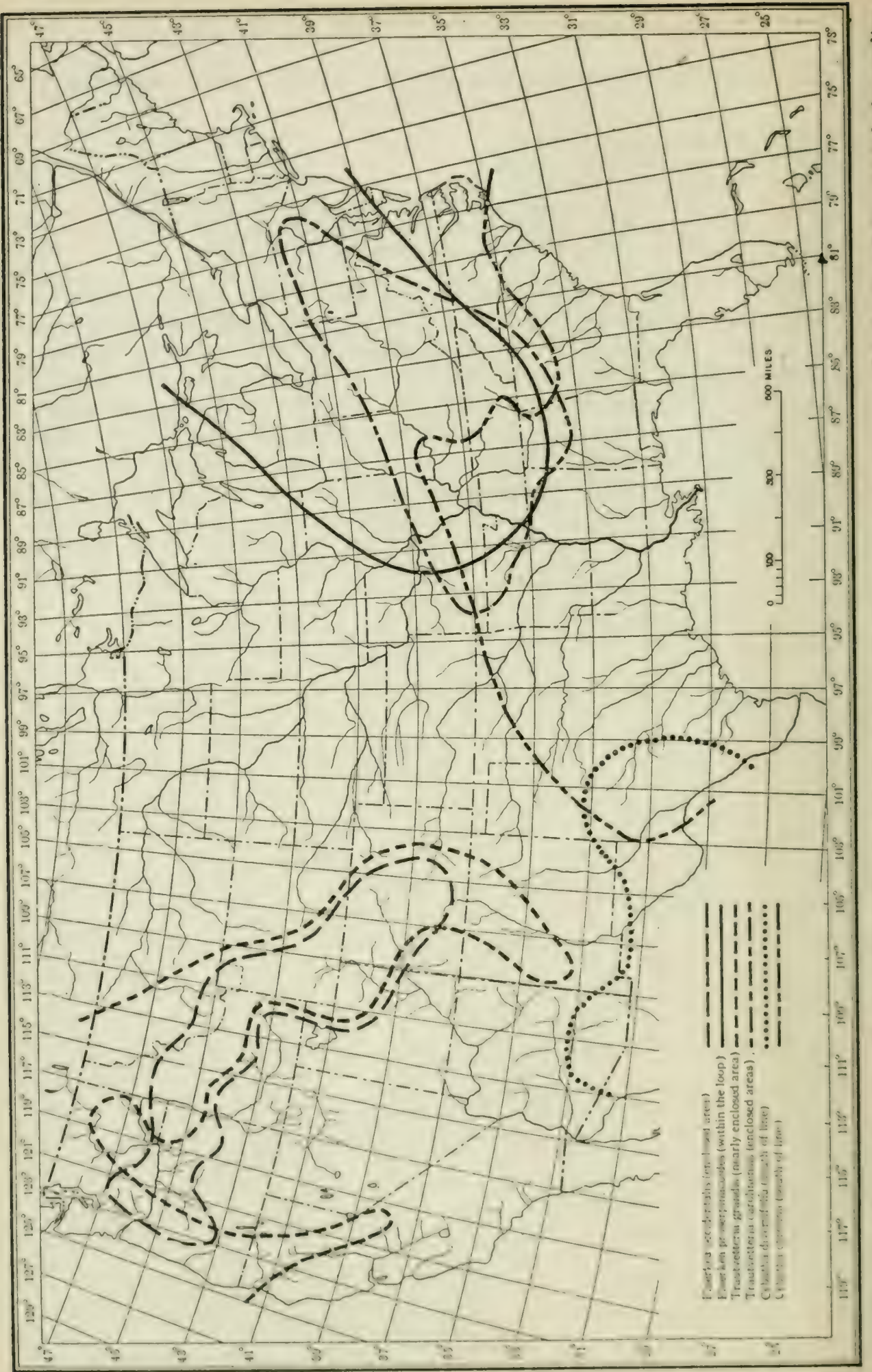


PART II.

\section{ENVIRONMENTAL CONDITIONS.}





\section{INTRODUCTION.}

The physiological point of view has been constantly held before us, as has been said, in planning and carrying out the complicated comparisons and correlations with which the present publication deals. Part I shows how the numerous vegetation features employed by us were derived, emphasis being placed on the physiological characteristics of the plants considered. Part II deals with the principles and methods by which the climatic features that we have used were selected, and shows how the requisite numerical computations were made and how the maps were prepared. This selection had to be based, as has already been indicated, upon two different kinds of circumstances: the physiological importance of the climatic features (as these are known to influence plant activity in general) and the availability of climatic data suited to our purposes. These matters will be set forth under three general headings: (I) general influence of the environment on plant life; (II) chief environmental conditions and the general nature of their effects upon plants; and (III) climatic conditions of the United States. The first two of these sections are mainly physiological-ecological in nature and are general in their scope; the last is mainly climatological-ecological and deals with the actual climatic data employed in our researches. 



\section{GENERAL INFLUENCE OF THE ENVIRONMENT ON PLANT LIFE.}

\section{EXTERNAL AND INTERNAL CONDITIONS AND PLANT ACTIVITY.}

The behavior of any plant is said to be controlled by the surrounding conditions, variations in these being the stimuli, or causes, which produce in the internal, physiological complex of the organism various responses or effects. Such responses are, however, quite as dependent upon the nature of the responding organism as upon the nature of the stimuli. With the same set of environmental conditions different plants behave differently, merely because their internal conditions differ, and with unlike environmental complexes plants of the same form exhibit quite different behaviors. The behavior of plants in general thus depends upon two interacting sets of conditions, the one set being external and the other internal. The latter set of conditions makes up, of course, the nature of the plant and serves to define it physiologically. These internal factors determine the ability of the organism to respond to exposure to any given constant external condition, or to any given change in any condition, and they also determine the extent of such responses. A plant might be rigorously defined by means of these powers or capabilities to respond to stimuli, and it is some of these powers that are, indeed, unconsciously used by taxonomists in their descriptions; but, although certain groups of the bacteria are now described by conscious reference to their physiological properties, the physiological nature of the taxonomic description in botany may be said hardly to be generally recognized as yet.

As a plant develops, its internal conditions pass through a series of more or less profound alterations, and the different developmental phases of the same plant often show greater divergency in response to the same environmental complex than do the corresponding phases of distinct plant-forms. Thus, environmental conditions that are favorable to seed germination or to regetative growth may be markedly unfavorable to the production of flowers or fruit. It follows that for the best growth and reproduction of many forms the external conditions must vary from phase to phase as growth proceeds. This is one of a number of considerations that make for great difficulty in the inception of any satisfactory quantitative study of the relation of external conditions to the characters of individual plants and of vegetation in general.

A second consideration that enormously complicates our problem is this, that the response, or effect of the external system upon the organism, is definitely dependent upon the duration of the component 
conditions that make up the environment. Considerable time is required for most responses, and a momentary alteration in the environment may often pass without apparent effect upon the plant. Thus, outside of the time factor, the necessary and sufficient conditions for the production of those changes in manner of growth that are termed etiolation are present every night, but these conditions are not effective over a long enough period of time to result in risible responses. In the study of any external factor or complex of factors it is logically necessary and inevitable that the time element enter seriously into consideration.

Physiologists have found it advantageous to analyze the environment into its component conditions or factors. While some factors have so far received but a minimum of attention, a large amount of reliable information is already at hand bearing upon the effects produced by the action, over various time intervals, of different intensities of heat, light, oxygen-supply, etc. The method of such determinations has been to hold all factors but one as nearly constant as possible, and to cause rarious selected intensities of this one factor to register their effects upon the plant, in the form of alterations in growth or other activity.

But this study of the simple component factors of the environment is only the learning of the alphabet, and the task of really reading the book of plant phenomena in the light of cause and effect still rests with the future. We are already well aware, in a general way, that the responses brought about in the organism by a certain quality, intensity, and duration of any external factor are totally dependent upon the nature of the other concomitant factors which are comprised in the environmental system. For example, a given increase in the rate of water-supply may fail to produce any marked acceleration of growth in certain forms existing under excessive drought conditions, but if the increase in soil-moisture be accompanied by a decrease in the evaporating power of the air, growth response may be immediate and definite enough. ${ }^{1}$ Igain, the agriculturist is well aware that with many soils an increase of the nitrate content is without full effect unles: other salts are simultaneously added. In such eases the result of these several increases together is not generally a simple summation of the results obtained by the single additions separately.

While a large amount of laboratory experimentation of the most refined physieal sort will be reculured before we shall even approach an aderiuate knowledge of the influence of single eonditions upon plants, the far more diffeult study of the complex envirommental system of which these single conditions are always components has already begun to attract attention. It seems safe to predict that the line of work thus started will rapidly gain in prominenee, and it is

'Livingston, B. E., Evaporation and Plant Development, Plant World, 10:269-276, 1907. 
conceivable that plant physiology may eventually work out the principles whereby the behavior of plants may be one day explained, predicted, and controlled. This is, of course, the hope of ecology and of the developing science of plant-culture. It is our aim in the present chapter to present merely a tentative outline of the general and more superficial relations that apparently obtain between the plant and its environmental conditions. No attempt is here made to make our consideration logically complete, either from the standpoint of physical causation or from that of the plant kingdom in general; attention is mainly turned upon some of the most obvious physiological considerations and upon the behavior of ordinary vascular land-plants.

\section{THEORY OF PHYSIOLOGICAL LIMITS.}

In an etiological study of plant distribution, either natural or artificial, the conception of physiological limits must hold a very prominent place. We understand by this term the extremes of intensity, etc., in a given factor which a certain plant can withstand. Starting from the optimum intensity of heat for any plant, for example, we may reduce the temperature till death ensues, thus attaining the minimum temperature limit for life. If the temperature be increased sufficiently above the optimum, another death-point is reached, the temperature maximum. The plant is thus able to retain life only under temperature conditions that fall within these physiological limits. With some other factors a similar pair of limits can be determined; with many, however, only a single limit exists. For example, a submerged aquatic possesses a definite minimum rate of watersupply, but it is impossible to produce death or injury by increasing this rate even to its physical limit, as by surrounding the entire plant with water. It is obvious that in such a case there exists no maximum limit for life. Cases where there is a well-marked maximum but no minimum are also frequent, most toxic substances furnishing examples of this. Plants live normally in utter absence of these substances; they also live normally in their presence, so long as the amount supplied does not surpass a fixed maximum limit.

Life is able to proceed, then, in any particular plant, only so long as the external conditions do not surpass the physiological limits for the life processes of the form considered. In different plants and in different developmental phases or stages of the same plant these limits may be very different, so that an environmental complex that inhibits life in one phase or form may allow healthy activity in another. It is mainly in accord with this generalization that distinct climatic areas are characterized by corresponding types of vegetation, and the principle is therefore probably of primary importance in the study of plant distribution. 
The generalization just mentioned is very greatly complicated, as has been indicated, by the variations and fluctuations in the internal physiological conditions as development proceeds. The limits for life are often very different in the various developmental phases of the same form. Thus, mature seeds of many temperate and boreal annuals show temperature minima far below the freezing-point of water, while the vegetative phases of the same forms may succumb to the first frost. Winter buds of northern deciduous trees possess high powers of resistance to low temperatures, while summer buds of the same plants may not bear temperatures as low as the centigrade zero.

Besides the variations in limits of growth and life in different phases of the same form, it must be remembered that, in any phase or at any time in the life of the organism, there are a number of different processes going on, such as photosynthesis, respiration, digestion, secretion, and the like, each of which has its physiological limits, and the limits for one process are frequently not at all the same as for another. In general, the pair of limits that characterize these simpler processes, which together make up the vegetative or reproductive activities of a plant, are much less widely divergent than those for the mere retention of vitality itself. By retention of vitality we probably mean the occurrence of the life processes at their lowest intensity, an intensity that is just sufficient to maintain life, though this expression may be taken in a general way to denote simply the power of initiating the various more vigorous and obvious processes when conditions become right. Seeds retain their power to start the germination processes for long periods of time under conditions that preclude germination itself. Again, with increasing scarcity of water or lowering of temperature, the growth processes of all plants are sooner or later brought to a standstill, long before death ensues. Moisture conditions that are optimal for vegetative growth frequently prevent the production of fruit, so that gardeners make it a practice, with the coming of the flowering-time in many plants, to diminish the water-supply.

That the factor of time enters into the determination of physiological limits is obvious. Many plants are able to survive a short period with the soil about their roots in a saturated eondition, but succumb to a longer period of exposure to a saturated soil. Numerous forms retain their vitality through long periods of drought, when the soil is nearly air-dry, but if the dry period is sufficiently prolonged death is the inevitable result. As has been mentioned, general growth is not noticeably affected by the regularly recurring nocturnal period without illumination, but etiolation becomes marked, and various other pathological conditions are induced when ordinary plants are kept in continuous darkness for but a few days. In physical terms this means merely that the effects of any set of external conditions upon the plant are always cumulative and are exaggerated, in one way or another, with the lapse of time. 
A combination of the time factor and that of intensity is, frequently if not always, the effective condition which determines the success or failure of plants in nature. With a somewhat rapid alternation of favorable and unfavorable conditions, where the unfavorable factors for any short period do not in themselves at first produce death, the organism may generally lose its power of resistance and finally go to the ground. In regions where such rapid fluctuations in the intensity of external conditions occur, the natural vegetation must necessarily comprise only those forms which can bear this sort of fluctuation. Alpine plants are reputed to be especially resistant to the great daily ranges of temperature that occur in their habitats, and many plants, such as lichens and certain liverworts and club-mosses, exhibit a high power of resistance to repeated wetting and drying-out.

The limits for life, growth, or reproductive activity (the latter a sort of growth) define the resisting power of an organism in respect to the particular condition considered, and, by maintaining the quality and intensity of the other environmental factors and causing the one in question to vary, the range between the limits for that one may be more or less approximately determined. But, with two or more factors varying at the same time, the problem of physiological limits becomes much more difficult. The study of the behavior of plants when several factors are simultaneously in a state of change has, as we have pointed out, only just begun. We may be sure, however, that the resisting power of a plant to any single condition will prove to be markedly influenced by other concomitant conditions. The antagonistic action of certain salts, such as those of calcium and magnesium, in the work of Loew, Osterhout, and others, is a case in point, as is also the well-known fact that, by a degree of desiccation that does not surpass the death-limit, the power of many organisms to withstand both high and low temperatures is markedly increased. The common experiment comparing the effect of high and low temperatures upon dry and moist seeds is a clear illustration of the latter case; within limits, the less water a seed contains, the more freezing or heating it can bear without losing its vitality .

\section{RELATION OF PLANT DISTRIBUTION TO THE PHYSIOLOGICAL LIMITS OF THE VARIOUS DEVELOPMENTAL PHASES.}

Each plant-form, each developmental phase, and each physiological process exhibits a minimum or a maximum, or both, for each of the environmental factors, these limits depending, of course, on the other conditions that prevail within and without the plant. Whenever an environmental factor falls below the minimum for life, in either quality, intensity, or duration, the annihilation of the organism of course ensues. A like result follows any increase above the life maximum. It is only with each of the environmental conditions falling between 
its respective life-limits, under the given set of relations between the other conditions, that a plant can exist at all. ${ }^{1}$ But the mere existence of a given plant-form, its mere retention of vitality, is not sufficient to give it a permanent place in the vegetation of a given region; each plant must pass through various developmental stages, must come to maturity, and must reproduce. Since the intensity limits for the retention of life do not approach each other so closely as do those for growth and reproduction, it is easy to understand that the duration of the different intensities or qualities of certain factors must determine whether or not a given form may come periodically to maturity in any region. Though the lower temperature limit for life in seeds, bulbs, rhizomes, and the like, and in resistant perennials, is not attained in the temperate and boreal winter, yet the temperature conditions for growth and the production of fruit obtain only in the summer season. Similarly, the moisture conditions in a desert fall, for the greater portion of the year, below the minimum for the growth of many desert shrubs, these producing new leaves and flowers only in and immediately following the rainy seasons. The same is true of root and bulb peremnials and of those annuals which succeed in the desert. It is thus seen that, in regions characterized by an alternation of seasons of plant activity and of dormancy, the lengths of the seasons of activity must determine whether or not the plant reproduces adequately; and since adequate reproduction is essential to the maintenance of the form in the given region, this length of season must determine whether that form succeeds or fails.

While a mature plant, or a portion thereof, may exist in a relatively inactive condition for a long period of time, in an environment whose factors lie without the limits for most forms of activity (but within those for the retention of life), the resisting power thus evidenced is usually of but a low order when compared with that exhibited by ripe seeds or spores. From a physiological point of view such bodies represent merely a certain phase in the development of the plant, a phase in which the life processes are even more in abeyance than during the dormant periods of the mature form. An annual may play a very important rôle in the vegetation of a region, although during the greater

'It is of course to be borne in mind, in this connection, that an alteration in one environmental condition may result in death simply by eausing one or more of the vital processes to be so greatly aceelerated or retarded that the other given external conditions, although they have not been changed, become fatal. Thus, while a given rate of watcr-supply may be sufficient for life and growth under a low evaporating power of the air, an increase in the evaporation-rate, unaccompanied by a corresponding increase in the rate of supply, may result in death. Had the rate of water-supply been aderuately increased as the transpiration-rate rose, such a plant might have survived. It is frequently said that in such $₫$ case death is due to a condition which was not altered. 'This simply means that internal conditions have been changed, so that an environmental factor heretofore favornble to life becomes unfavorable, without itself changing. It is in this connection that the "law of the minimum" of agriculturists has been developed. (See: for example, L. J. Russell, Soil conditions and plant growth. Third ed., London, 1917, chap. II. Also see: F. F. Blackman, Optimn and limiting fuctors, Ann. Bot. 19: 251-295. 1905.) 
portion of the year the environmental conditions surpass the limits for both growth and life in the plant itself; the conditions in their adverse period do not surpass the limits for the retention of life in the seed. During a comparatively short period the environment may allow germination, growth, and the production of more seeds, and this short growing-season, together with the bridging of the adverse period by dormant seeds, constitute the conditions that are necessary and essential in order that such a plant may maintain a permanent place in the vegetation of its region.

External conditions frequently surpass even the life-limits of many seeds; in such cases it must, of course, follow that the plant can not become a permanent part of the natural vegetation. This is probably true of the majority of cultivated plants that do not volunteer in the second season. A tropical plant, such as the castor-bean, may make a luxuriant growth in the temperate summer, but its seeds must be protected through the winter and sown each spring.

In general, the natural plants of the temperate and frigid regions must necessarily experience a longer or shorter period, usually each year, with proper conditions for growth and reproduction, and they exist through the adverse periods in some dormant phase. Not only must the conditions of the active period lie within the limits for growth and reproduction, but the period itself must be of adequate length, otherwise the necessary amount of growth could not take place, and fruit would not be matured. The principle that a plant, to become a part of the permanent vegetation of a locality, must have an adequate growing-season and must not meet its death during the remainder of the year, must be regarded as fundamental to the study of all problems dealing with the study of plant behavior under natural conditions. This principle is commonly accepted, though perhaps seldom formulated.

With regard to any geographical area or region, we may conceive that all plants may succeed therein, for which the physiological limits for life and those for growth and reproduction do not approach each other more closely than do the extremes of the physical conditions in the respective seasons for the given region. This view has led to a form of analogy which may be termed the sieve conception of environments. According to this, we may regard the physical conditions of the surroundings as resembling a sieve or screen, with meshes of a certain magnitude, through which, as we may imagine, will pass only those successful forms which withstand the most adverse conditions of the environment. The analogy is but roughly applicable; to make it more so we may suppose that the size of the meshes in our screen is continually changing throughout the year, while the size of the imaginary particles. which are to be screened are also undergoing continuous change with the advance of the organism from phase to phase of its development. With the progress of the season the con- 
ditions change and the powers of resistance also vary. Thus modified, the sieve analogy becomes unwieldy and does not aid us very much in our thinking.

Since the behavior of a plant is nothing but the summation of the behaviors of its active parts, and since all higher plants exhibit, at any given phase in their growth, various gradations in the activities of their different tissues, it follows that any adequate consideration of the physiological limits of plant activity as a whole must be exceedingly difficult. It is therefore impossible, in the present state of our knowledge, to treat the question of complex limits quantitatively. The best that may be done in the discussions which follow is to attempt to bring togrether a series of confessedly incomplete and exceedingly inadequate treatments of the main environmental factors and their general mode of action upon ordinary autotrophic land-plants.

\section{GENETIC CONTINUITY OF PROTOPLASM AND ITS CYCLIC ACTIVI- TIES, IN CONNECTION WITH PROBLEMS OF DISTRIBUTION.}

In the preceding sections of this chapter the general terms of the problem of plant distribution have been presented in the words of the present-day physiology of plant organs. There can be little doubt that the day of this organic physiology is about to pass. It has been, of necessity, mainly descriptive and has not concerned itself primarily with the details of the actual causes of plant response and their mode of action, but there is already a strong tendency to turn attention from the description of plant organs and their responses to the physical causation of these, a development of physiology which bids fair to place this branch of science on the same quantitative etiologieal basis as that upon which physics and chemistry are now working. From the current literature of plant distribution and of ecology in general it is suggested that many workers in this field have so far failed to realize the present status of the physiology which lies at the base of all ecological facts. Ecology, which was at first regarded as a purely descriptive study, a mere cataloging of relatively superficial descriptions of phenomena and a classification of these, was an outgrowth of taxonomy. But it has advanced with more rapidity, perhaps, than any other branch of science, and it has already aceumulated enough descriptions so that a beginning at least in the study of cause and effect has been made. Such a study must, by the very nature of its subjectmatter, take aceount of all the contributions so far made by physiology towards an etiology of plant phenomena in their broader aspeets. For a long time the phyciology of organs must be the basis of ecological considerations, and it is with this in mind that we have taken the principles of organie physiology as the basis of our discussions. We have consciously aroided such ideas as that of purposeful adaptation and other teleologieal coneeptions, still too common in botanieal writing-with what suecess the reader will best be able to judge-and have 
aimed to place our treatment of plant distribution on a basis as free from anthropomorphic conceptions as is that of physiography, a science with which plant geography must always be closely related.

But the modern trend of physiology, with its application of the methods and findings of physico-chemistry and its tendency to seek explanations of all phenomena in the properties of matter and energy, offers to the study of distribution at least one conception which goes far to simplify the logic of physiological limits and their modes of variation. The present section will deal with this conception.

From the standpoint of general physiology, the reproductive activities are to be regarded as a special form of growth. The protoplasm of any species is a continuously existing thing, to be likened, perhaps, to a river, in which the material particles are ever changing and of which the form, course, activities, etc., show a continuous variation. The channel of our river moves from place to place within the limits of its valley; the river accomplishes much work of excavation and the like at certain seasons of the year; at other seasons it is inactive and, in the arid regions, often disappears from sight completely, flowing only underground. In the latter condition we may perhaps speak of it as dormant. Following this analogy, the living substance of any species may be thought of as continually existent, but varying widely in situation, amount, activity, etc., with the ever-fluctuating physical conditions within and without its mass. Thus the conditions for the success or failure of any species in any region or habitat are that its protoplasm be indefinitely maintained in that area, and that the various cyclic phases of physiological activity follow one another in a certain order. By such a conception we are enabled to generalize without the complication incident to the special consideration of the reproductive activities.

Scrutinized in this manner, the individuals of a given plant-form are seen in somewhat the same light as are the buds of an indefinitely growing perennial, such as a tree. These buds are continuously dying and being formed, but the system of growing-points which make up the tree possesses form, size, etc., and maintains itself throughout years. Growth here consists partly in the approximate replacement of parts which have been destroyed by the action of adverse conditions, and partly in an actual increase in the number of growing-points comprised within the entire system.

By this sort of generalization we may bring all plant-forms into the same broad category, as far as the general influence of external conditions is concerned. The protoplasm of any species, in any region, passes through variously active and dormant phases with the march of the seasons, the success of a species requiring that there be, at least, no progressive and permanent decrease in the number of growingpoints or individual groups of active cells. But there may occur, and usually do occur-outside the tropics at any rate-great periodic fluctuations in the number and activity of these biotic units. 
Plant protoplasm may be said to pass through alternating phases or stages of diastole and of systole. In the condition of diastole it is very active, increasing rapidly in amount and extending and altering its configuration to a remarkable degree. These changes are due directly to numerous physico-chemical processes and energy transformations which, during the period of this phase, are quite violently active. In the condition of systole, on the other hand, the various characteristic life processes are at a low ebb, some of them being apparently altogether abated. The mass and extent of the protoplasm falls off more or less markedly, in the death of many parts which were previously the seats of vigorous activity, and in many cases the whole organism practically fails to exhibit any form of life at all. This rhythmic pulsation is of course immediately due to internal conditions, but the latter are, in turn, to be causally related either to changes in the environment or to effects of a constant environment summed or integrated by the organism.

While the point of view just suggested is not at all new, and has been of great service to some students of heredity, we are not aware that it has been resorted to in studies of the influence of external conditions upon the maintenance of plant population in a distributional sense. It seems to promise such utility as a logical tool that we venture, in the following paragraphs, to outline the behavior of some of the main plant types in terms of this conception.

Attention may first be directed to the case of a perennial which propagates vegetatively, omitting for the present any question as to whether seeds are produced. An excellent example of this is seen in several forms of much-branched cylindrical opuntias, as the various "cholla" cactus-forms of the North American Southwest. By the action of various agencies, such as wind and animals, short branches are easily broken off from these plants, and are widely distributed by the operation of the same agencies and by that of flood-water. Under farorable conditions these framents possess the property of taking root and forming new plants. Such may be regarded as the simplest form of species maintenance, and it is of course the rule among the lowest forms of plants. We have here to do with the mature plant at. all times, the only complication in vegetative phases lying in the fact that roots are produced and proceed with their characteristie activit ies under the peeculiar conditions offered by the dispersal of the joints. With the coming of each farorable season, mainly defined by conditions of moisture and temperature, growth in size oceurs in numerous branches, some of the latter being portions of larger plants, while others lie singly upon the ground. By the action of adverse conditions many branches are destroyed, but enough survive apparently to maintain the status of the species in the regetation of its area. During the rear great fluctuations oceur in the quality and intensity of the environmental factors; periods of extreme drought and heat, periods of drought and cold, periods of abundant moisture and either high or low tem- 
peratures, follow one another in unending succession. In certain periods foliage is produced and growth proceeds with rapidity; in others the leaves disappear and growth is retarded or checked; in still others nearly all activities cease and the branches remain dormant till the return of conditions favorable to growth.

In a manner similar to the above, we may consider all perennial forms that maintain themselves or spread by vegetative propagation, as in the case of those with underground branches, rhizomes, stolons, dividing-bulbs, and the like. In a form like Solomon's seal (Polygonatum), vegetative propagation is very important; a single rhizome may give rise to numerous new growing-points (by means of branching), which eventually become separated from the parent rhizome with the decay of the connecting portions, so that, if time suffices, a considerable area may be occupied without other activity than this purely vegetative one.

Of course bulbs, tubers, and the like are to be regarded as plant phases which are characterized by dormancy and highly resistant properties, for which the physiological limits are widely separated. There occur all gradations in stem and bud vitality between the bulb or tuber and the great deciduous or evergreen tree, all agreeing in the essential point that with the coming of adverse conditions they undergo a check in their activities. The deciduous forms lose a large proportion of all of their vegetative organs. All these forms remain alive but dormant till the return of the growing-season.

A second example may be taken from those tropical forms which reproduce by seeds, but in which vivipary vaults the resting-period usually so characteristic of the seed. A history of the activities in the mangrove, for instance, might run somewhat as follows: In the mature phase of this plant there are manifested cell activities which result in the dormancy of many cells. Some of the dormant cells, the eggs, are capable of resuming growth in size under certain conditions, the main condition being the entrance of protoplasmic material from another cell; that is, the occurrence of fertilization. If fertilization takes place, and this is to be looked upon as merely one of the environmental changes which act upon the plant in its cycle of developmental phases, then the ovum develops into an embryo and continues growth without any marked pause or resting-stage, forming ultimately a new plant. External conditions must furnish stimuli by changing in quality or intensity at various times in this development, one of the most special of which is the falling of the germinated seed into the mud below. We see in such forms all the usual phenomena of production by seed, but the pronounced dormant phase of the majority of seeds is omitted. However, unless the embryo root reaches the mud of the substratum (which signifies pronounced environmental change) the cycle of growth is checked.

A third example may be chosen from among the plants which reproduce through seeds, but in which parthenogenesis bridges the interruption which usually precedes the formation of the embryo 
from the mature egg. Here no external change is apparently required to induce the ovum to proceed to the embryonic phase, but the embryo finally reaches a resting-stage, where growth activities are checked. Other alterations in the internal conditions of the dormant phase which we term a seed often require a prolongation of the restingperiod, but, in any event, before active growth in size is again manifest definite changes in the external complex are required; the seed must absorb water to a certain degree, the temperature and rate of oxygensupply must fall within the limits for germination, and various other conditions must be fulfilled in order that the embryo may emerge from its dormant state.

The dormant phase of the mature egg is omitted or very much reduced in parthenogenesis, that of the seed in vivipary. The periods of rest, or of internal conditions adverse to growth, often coincide with periods of adverse external conditions, and the dormant tissue usually possesses high powers of resistance to the latter. This is a consideration the importance of which, in general climatic behavior and distribution of plants, can hardly be overestimated.

Our fourth and last example is taken from the great majority of plants, where fertilization is necessary and a more or less prolonged period of dormancy intervenes between the maturation of the seed and germination. The annual plant perhaps illustrates this sort of rhythmic activity in its simplest form. Germination occurs in the spring, when temperature, moisture-supply, etc., are favorable for this kind of growth. Later, the various developmental phases follow each other with more or less pronounced alterations in the external conditions, and when seeds have been matured the parent plant dies. This final death may occur because of the action of internal conditions, perhaps connected with the ripening of the seed, or because of the action of external factors, such as drought or frost. But the dormant phase represented by the seeds is highly resistant, and these bodies carry the living protoplasm forward through the winter of adverse conditions to the beginning of a new cycle of activity.

The general conception outlined above may be expressed briefly to the effect that each particular sort of plant protoplasm (form, species) is indefinitely perennial, ever passing through repeated cycles, ever changing in internal nature from one developmental phase to another, growing, fragmenting (as in reproduction of all sorts), resting in a dormant condition, and always again taking up the endlessly repeating series. Of course our conception of the repetition here involved must be broad enough to include such alterations from cycle to rycle (variation, mutation, ete.) as the study of evolution demands for the origin of new forms from old. This mode of contemplating plant activities should be as valuahle in physiology and ecology as has been the conception of the alternation of generations in the descriptions of the consecutive steps in plant phylogeny. 


\section{CHIEF ENVIRONMENTAL CONDITIONS AND THE GENERAL NATURE OF THEIR EFFECTS UPON PLANTS.}

\section{GENERAL CLASSIFICATION OF ENVIRONMENTAL FACTORS.}

The environmental conditions that are commonly most potent in the determination of plant development, and that therefore appear most important in distribution, may be classified under the following headings: (1) Moisture conditions; (2) temperature conditions; (3) light conditions; (4) chemical conditions; (5) mechanical conditions. The present section will be devoted to a brief summary of the nature and effects of these factors as they vary in quality, intensity, and duration. No attempt is made to denote more by the order of items in the above list than a very general estimate of the relative importance of the various factors as they are usually operative in limiting plant distribution. There is, of course, nothing to be gained in a discussion of the relative importance of a number of factors, all of which are necessary in order that a given phenomenon may occur. Such consideration were as bootless as a discussion of the relative importance of the hub, spokes, felloes, and tire of a wheel. The reader is therefore requested to make nothing of our order of arrangement.

Students of ecological plant distribution have usually classified these sets of conditions according to their origin or source, rather than according to their mode of physically affecting the plant. Thus, the literature contains many references to climatic and edaphic conditions, physical and biotic ones, and the like. Such groupings seem, however, not to have led much farther than to the mere description and arbitrary classification of distribution conditions, and, since by their very nature they point to the causes of the factors immediately involved rather than to the real nature of these factors or their effects upon the plant, they promise little for our present purpose. Thus, shade produced by a natural rock arch or overhanging cliff may be impossible of physical or physiological differentiation from that produced by a tree; yet the former is said to be a physical factor and the latter a biotic one. Again, the mechanical relation of the physical separation of plant and soil, together with the accompanying ruptures and lesions of the plant tissues, might arise equally well from the action of animals (a biotic factor) and from that of wind or torrential water (undoubtedly physical factors). In a study of the ultimate causes that bring the proximate, effective factors into being, such classification has its value, but in such studies we have assuredly left our field of plant distribution for the adjoining one of climatology, physiography, and the rest. In the beginning it appears more promis- 
ing, because simpler and logically more direct, to attend strictly to the various factors as they actually affect the plant, leaving the analysis of the sources of these factors to other studies, perhaps at a later day. We shall here consider only the proximate determining conditions in plant behavior and distribution, merely mentioning a few points bearing on the more remote determination of these controlling conditions, which are available from researches in climatology and other fields.

Since every effect upon the plant must be supposed to be directly traceable to conditions that previously prevailed within and without the organism, and since we make no attempt here to analyze the various physiological processes that constitute the varied plant responses, it has been deemed best in these considerations to regard as an external condition or factor any status of the processes of the external world that directly influences changes within the plant. Many logical difficulties arise here, as is usual when any attempt is made to subdivide a continuous series into regions, and the only satisfactory method of procedure is to subdivide the series into arbitrary portions, being sure to define the limits chosen. Thus, for ecological purposes it seems quite as undesirable at the present time to enter into the exceedingly complex physiological considerations involved in a study of the details of plant activities as these are controlled by conditions as it is to take up in detail the more remote causes which bring the various effective conditions into existence. This logical difficulty arises, of course, from the fact that the plant is not an independent system, but is perfectly continuous with the universe about it; the classification, into external and internal, of the conditions determining chemical and physical changes within the plant, is at best but a subjective affair with the mind that classifies. Whether a given condition is to be taken as external or internal will always depend largely upon the previous experience and point of view of the observer, upon his internal conditions. For the present needs it seems desirable to base our arbitrary definition of the controlling factors upon the spatial limits of the plant-body as ordinarily considered. Thus we define as cffective external conditions all phases of universal progress outside the plant-body which directly affect the latter in such a - manner as to produce alterations in the chemieal, physieal, and physiological processes that occur within. An example may illustrate this. The passing of a given region of the earth's surface from shadow into sunlight at dawn is not a condition immediately effective upon plant processes, nor is the influx of radiant energy to the surfaces of objects in the vicinity of the organism. The plant-body is first affected in this case when there oecurs an increased rate of transmission of light or heat energy through the periphery (or a decreased loss of heat, which amounts to the same thing as an increased ineome), so that some substance actually a part of the plant-body becomes lighted or warmed. 
It is only by a logical short-cut, not always tenable, that we may say that the rising of the sun produces such responses as the opening of stomata, the eastward bending of flower-heads, the assumption of the day position by nyctitropic leaves, and the like. From the logical standpoint, attention is here to be confined to changes in the rate of inward or outward transfer of the various forms of matter and energy between the surroundings and the plant-body itself.

It must be confessed that, while most existing types of vegetation have been rather carefully, and in some cases perhaps even somewhat quantitatively, described, yet there appears so far in the literature scarcely anything of a fundamental nature upon the external factors and their modes of action in determining plant distribution. ${ }^{1}$ It is thus largely upon quantitative studies of the intensity, duration, etc., of the external conditions and upon the true physiological interpretation of these that the future conquests of this branch of ecology must depend. It therefore seems desirable, even at the risk of appearing to "carry coals to Newcastle," to venture the following physiological discussion before taking up such meager contributions as can be brought together in regard to the geographical distribution of the different environmental complexes of the United States, as these may be related to the distribution of the various vegetational types.

The different categories of environmental conditions will be considered in the order of the preceding list, and attention wil be turned briefly to the various modes in which these are effective to bring the different plant responses about. In the interest of clear presentation, it will now and again be advantageous to overstep our logical limits in the other direction, and to touch upon some of the relations of the more remote conditions that, in their turn, influence or control the proximate and immediately effective environmental conditions.

\section{MOISTURE.}

\section{WATER REQUIREMENT WITHIN THE PLANT.}

The very complex moisture-relations to which plants are subjected are to be considered as of the utmost importance in the great majority of distributional problems. These may be physiologically best studied from two standpoints - that of the requirement for water and that of the water-supply - since these two factors determine by their interaction the moisture conditions of the plant. They may be taken up in order.

Since every active cell is filled with water, it follows that there must occur constantly, or with but brief interruptions, a movement of water

\footnotetext{
${ }^{1}$ Serious beginnings in the direction of physiologically experimental investigation of the relations holding between plant-growth in the open and the controlling conditions of the environment have been made by a very few workers. In this connection, see McLean's studies of the control of seedling soy-beans by the conditions of the Maryland summer climate (McLean, F. T., A preliminary study of climatic conditions in Maryland, as related to the growth of soybean seedlings, Physiol. Res. 2: 129-208, 1917). References to earlier literature are there given. See also: Hildebrandt, F. M., A physiological study of the climatic conditions of Maryland, as related to plant growth. Physiol. Res, 2:341-405, 1921.
} 
into all enlarging portions of the plant-body. This demand for water, due to growth, is usually quite insignificant in degree, but it is nevertheless real, and the absence or inadequacy of the water-supply to growing cells must always act as a check upon enlargement.

Also, since water is destroyed in the process of photosynthesis in green plants, there must be a continuous influx of moisture into all photosynthetizing cells. If this supply is cut off, the formation of carbohydrates must cease in a short time. With insufficient watersupply, growth ceases before photosynthesis, but both processes must soon be brought to a standstill. The water demand occasioned by photosynthesis is perhaps usually more pronounced than that for enlargement, yet the rate of influx thus brought about is far too small in amount to permit of measurement by simple methods. Also, water disappears when hydrolytic decompositions occur, so that such processes as the digestion of starch are essentially drying processes. On the whole, these water requirements may be safely assumed to be only of relatively slight importance in comparison with that of transpiration, which is next considered.

The fact that all plant tissues contain water, and that no cuticular or other covering is absolutely impervious to this liquid-many leafcuticles, etc., being rather freely permeable to water-makes it logically follow that there must ever be a more or less pronounced evaporation of water from all plant surfaces that are exposed to the outer air. This superficial evaporation through externally exposed membranes makes up the so-called cuticular transpiration, which varies in amount in different forms, depending upon structure, and is often of great importance in determining the need of the plant for water. The water lost by cuticular transpiration is replaced from more deeply-lying tissues, according to the principles of diffusion and of imbibition, and sooner or later there must occur an inward movement of water from some region without the plant-body, or else death must ensue.

But, while cuticular transpiration is a rery real and almost constant source of water requirement, it is of relatively little account in comparison with stomatal transpiration. The presence of membranes of high moisture-content within the leaves-the walls of the mesophyll, etc.-which are in direct comnection with the external air through the stomata, makes continuous evaporation from these internal tissues an incritable condition, unless, indeed, the foliar surface be eovered with a film of water or of a solution of higher vaportension than that which occurs within the tissues. The internal atmosphere is maintatined more or less nearly in moisture equilibrium with the wet membranes that bound it, and ordinary diffusion through the stomatal pores eonstantly removes water-vapor to the outer air. 
This water-loss, constituting stomatal transpiration, usually occurs at a rate much higher than that evidenced by cuticular transpiration. The ubiquity of this form of water requirement and its generally great magnitude render it the dominating physiological moisture condition for most plant-forms growing in the open air.

However it may be removed, the water-content of an active tissue that is being depleted is always replenished at a greater or less rate from other tissues in the vicinity or from without the body. If such renewal of the water-content fails for a considerable time, partial or complete loss of activity must result. In plants without storageorgans the usual transpiration-rate can continue but a short time without entrance of water from the outside. Where storage-tissues are present the external supply may, of course, be cut off for a longer time.

Since transpiration is the most important factor in determining the need for an external water-supply to the plant, it will be necessary to consider here some of the conditions that influence this process. Its rate is dependent upon three conditions: (1) the structure and condition of the leaves or other transpiring parts; (2) the evaporating power of the air; (3) the intensity and quality of illumination. As is well known, there occur in different plants, under identical external conditions, great differences in the rate of transpiration per unit of surface. Certain structures, such as waxy and hairy coverings, palisade tissue, and other features, make cuticular transpiration markedly less pronounced than it might otherwise be, and many stomatal character's similarly influence the rate of stomatal water-loss. Many anatomical characters are known to become permanently altered by age, of course with reference to the nature of the environmental complex, as where cuticular thickening may increase or fail to do so, according to the age and surroundings of the plant. Such physiological responses are apparently dependent upon the rate of movement of transpirationwater (Pfeffer-Ewart, Plant Physiology, 2: 121). In regions where the evaporating power of the air rises rapidly during the growingseason, plants with a highly developed cuticular response of the form just mentioned may be expected to survive longer than others with a less-marked response of this kind. Stomatal movements, of opening and closing, may also be of considerable importance in certain cases, though this whole question is sadly in need of a more thoroughgoing physical investigation than it has yet received. ${ }^{1}$

\footnotetext{
${ }^{1}$ Concerning the quantitative aspect of differences in the power of plant leaves to retain water and thus retard water-loss, see the following papers: Jivingston, B. E., The relation of desert plants to soil-moisture and to evaporation, Carnegie Inst. Wash. Pub. 50, 1906.Idem, The resistance offered by leaves to transpirational water-loss, Plant World 16: 1-35, 1913.-Livingston, B. E., and A. H. Estabrook, Observations on the degree of stomatal movement in certain plants, Bull. Torr. Bot. Club 39: 15-22, 1912.-Bakke, A. L., Studies on the transpiring power of plants as indicated by the method of standardized hygrometric paper. Jour. Ecol. 2: 145-173, 1914.
} 
The amount of water in the transpiring organs appears also to be important in determining the rate of water-loss. Thus, transpiration is frequently checked in the daytime, with no apparent wilting and no closure of stomata, but with a marked fall in foliar water-content, while the evaporating power of the air is maintained or even increases in magnitude. ${ }^{1}$

Second only to the nature and condition of the leaves, etc., the evaporating power of the air exerts an enormous controlling influence upon the rate of water-loss from plants. For any particular plantform it appears to be by far the most potent of all the climatic factors affective above the soil-surface. The evaporating power of the air is a compound factor, dependent itself upon three other factors, as commonly considered-temperature, humidity, and wind velocity. Its resultant effects are the summation of the partial effects brought about through the influence of these three factors upon the vaporization of water and the removal of the water-vapor from the evaporating surface. Evaporation, as a climatic factor, will be more thoroughly considered in another place.

The intensity of the sunlight and its quality are also potent factors in the determination of transpiration from plants. While a certain small proportion of the energy of the solar rays is made potential and entrapped in the plant by the photosynthetic process, by far the greater portion of that which is neither reflected nor transmitted becomes potential in the water-vapor that escapes from the plant by transpiration. Thus, intense sunlight with a good proportion of the longer waves is markedly effective to increase the transpiration-rate of plant organs whereon it falls. The color and structure of plant organs have also to do with this, through the influence these exert on reflection and transmission. With a smaller proportion of the greater wave-lengths or with less intensity the effect is not so marked. ${ }^{2}$

Ecologists have classified plants, as to their ability to withstand different degrees of light intensity, into those which thrive best in shade, in bright sunshine, etc., and have given to these groups Greek names, but inasmuch as there are all possible gradations in this power of withstanding sunlight, and since there is as yet so little information of a quantitative nature bearing upon these matters, it seems advisable here merely to emphasize the fact that one of the most important influences of sunlight on plants is upon the rate of water-loss, and, therefore, upon the water-requirement.

Another form of water-loss from certain plants is the active exeretion of liquid moisture from nectaries and other superficial glands. such as water-pores, ete. The process by which this is brought about

\footnotetext{
${ }^{1}$ Livingston, B. E., and W. H. Brown, Relation of the daily march of transpiration to variations in the water-content of folinge leaves, 13ot. Gnz., 53: 311-330, 1912. - Shreve, Edith 13., The daily march of transpiration in a desert perennial, Carnegie Inst. Wash. Pub. No. 194, 1914.

2 Livingston, B. E., Light intensity and transpiration, Bot. Gaz., 52: $-115-135,1911$.
} 
is not at all understood, but we may be sure that the water-loss thus occasioned is of no great general importance in the distribution of plant-forms, especially since the greatest excretion of water, as in guttation, usually occurs at times when the transpiration-rate is low and the supply of water within the tissues is relatively great. Indeed, one of the common teleological conceptions bearing upon the so-called regulation of water-loss by plants is that these organisms actively and purposefully force water out of their tissues whenever they have been compelled by external eircumstances to absorb more of the liquid than they want.

To summarize the preceding paragraphs, the entrance of water into the ordinary active plant is essential to its activity: (1) because it is necessary for the enlargement of water-saturated cells; (2) because it is destroyed in photosynthesis, etc.; (3) because it is continually being lost by cuticular and stomatal transpiration and by excretion.

\section{SUPPLY OF WATER TO THE PLANT.}

As has been emphasized above, the transpiring tissues of a plant must receive water from elsewhere, otherwise they would soon become wilted and collapse, and other active tissues must likewise receive water, though frequently in much smaller amounts. There are several sources from which this water may come. Water-storage tissues and dying cells, or cells passing into a dormant stage, may furnish more or less water to other tissues, according to the form of plant considered and its phase of development. A relatively very small amount of water, fixed previously by photosynthesis, must be set free in the tissues by the activity of respiration, and this may become available for growing cells or may be again transformed by photosynthesis. Also, the reverse of hydrolytic decomposition (such as the synthesis of starch from glucose) results in the chemical formation of some water. For the transpiration of ordinary plants the latter sources are surely inadequate. ${ }^{1}$

During rains, and when the temperature of the foliage falls below the dew-point of the surrounding air, the external surfaces of the plant become wet, and a considerable amount of moisture may enter the plant-body through the cuticle and even through stomatal openings. This source of water is especially important only for certain forms, such as mosses, liverworts, and plants of similar water-relations. With heavy cuticle, trichome coverings, etc., very little water

\footnotetext{
${ }^{1}$ Fitting suggests that respiration water may be important in this connection in certain desert tubers, etc. (See Fitting, Hans, Die Wasserversorgung und die osmostischen Druckverhältnisse der Wüstenpflanzen, Zeitschr. Bot. 3: 209-275, 1911.-Livingston, B. E., The relation of the osmotic pressure of the cell-sap in plants to arid habitats, Plant World 14: 153-164, 1911.) The latter is in part a review of Fitting's paper. An excellent discussion and the most valuable experimental treatment yet available of the amount and importance of respiration water in plants and animals is the following: Babcock, S. M., Metabolic water: its production and rôle in vital phenomena, Wisconsin Agric. Exp. Sta. Bull. 22, 1912.
} 
can find its way into the tissues by these channels, and it follows that those plants that transpire the least must exhibit the smallest amount of leaf absorption. The main source of water-supply to the majority of plants is of course the soil or other substratum in which the plant is rooted. We may consider in greater detail the first and the last of the sources just mentioned.

In relatively large plants the diffusion of water from non-active to active parts is often of great importance. Thus, as a tissue dies its contained moisture may pass into other portions and there support growth and other activities. A familiar example of this is exhibited by bulbs, rhizomes, etc., that produce leaves, shoots, and even flowers and fruits without the influx of any water from without. Water from the bulb moves gradually into the more active portion and supplies the moisture for growth and transpiration; here the so-called storagetissue plays the same rôle of water source as does the moist substratum in the case of ordinary rooted and absorbing plants. In many instances water-bearing tissues may lose much of their water during the growth period of the plant and may still retain vitality and the power to absorb, so that at another season, when the external watersupply is greater, such tissues may receive water in larger amounts from the substratum and so return to their original turgid condition. In many cacti, fleshy euphorbias, and the like, all of the water for transpiration-a relatively small but nevertheless important amountand even for reproduction may be derived from the quiescent stem parenchyma for long periods of time. Many water-storage plants of the desert can retain vitality and maintain their reduced transpiration for several years after they have been removed from the soil and are thus able to absorb no water from without. ${ }^{1}$

Such isolated plants often effect new growth and ripen fruits with the return of the proper season, the conditions that bring about renewed activity in such cases being probably mainly those of temperature. Of course, these forms must eventually succumb to lack of water, as must any other form when deprived of a water-supply, but the interesting point here is simply that they may withstand the absolute lack of a water-supply from without for exceedingly long periods of time.

To most plants, a root system or its analog is essential throughout the actively transpiring phases of its development. Is has just been implied, such a system must likewise be present a part of the time, at long intervals perhaps, even in the most extreme water-storage forms. Through these water-absorbing organs the moisture of the substratum finds its way to the tissues of the plant. This water often traverses long distances of stem, etc., and it is thus seen that the rate at.

\footnotetext{
'MacDougal, D. T., E. R. Long, and J. G. Brown, End results of desiccation and respiration in succulent plants, Physiol. Res., 1: 259-325, 1915.
} 
which the transpiring portions of a non-storage plant may receive water depends upon several conditions: the rate at which water may move in through to the absorbing surfaces, the nature and condition of the roots, and the nature and condition of the water-conducting tissues.

In most cases where growth ceases or wilting occurs, the inadequacy in the water-supply seems to arise, not from the attainment of the physiological maximum of absorption and transmission, but from a greater or less drying-out of the soil, whereby it fails to transfer water to the absorbing roots at a rate adequate to supply the demand of absorption. Quantitative information bearing upon the relations between soil-moisture and plant absorption and transpiration is not yet available, and our consideration of this exceedingly important subject must be very brief and very tentative. ${ }^{1}$

It is certain that, with diminished supply of soil-moisture and with other conditions remaining unchanged, transpiration in any plant must be decreased in amount, also that this diminution in the transpiration-rate does not progress parallel to the continuous drying of the soil, so that it ultimately comes about that the supply fails to equal the demand, transpiration becomes greater than absorption, the nonstorage plant ceases to grow, and wilting or even partial or total death ensues.

Such a decrease in the rate of movement of water to the roots does not necessarily mean any considerable fall in the average percentage of moisture present in the soil, but gives evidence merely of the fact that the movement of water through the soil-films and into the roots has become less rapid. Under such conditions the soil immediately surrounding the absorbing portions of a root-system becomes drier than that at a greater distance, and the movement of water into the drier layer is too slow to keep the surface of the root adequately moist. This matter of the possible rate of water transfer from soil to root, fundamental as it is, is greatly in need of thorough investigation. The subject has been opened by Livingston and Hawkins and by Livingston and Pulling in the papers cited above. This should prove a wonderfully productive field, both for scientific ecology and for agriculture when serious attention is at length turned to it.

Great differences in the water-relations of plants in different habitats are secondarily occasioned by the nature and exposure of the soil in which they are rooted. Surface drainage often conducts the water of precipitation away before it can penetrate the soil to an adequate degree, and underground drainage frequently depletes the moisturesupply of porous soils almost as fast as water enters from a shower. Evaporation removes water rapidly from some soils and but slowly from

${ }^{1}$ In this connection see Livingston, B. E., and Lon A. Hawking, The water-relation between plant and soil, Carnegie Inst. Wash. Pub. No. 204: 3-48, 1915.-Pulling, H. E., and B. E. Livingston, The water-supplying power of the soil as indicated by atmometers, Ibid. 204: 49-84, 1915. Also see: Livingston, B. E., and Riichiro Koketsu, The water-supplying power of the soil as related to the wilting of plants, Soil Science, 9: 469-485, 1920. 
others, and the supply remaining to a plant after a period of dry weather depends very largely upon this factor. Fine-grained soils resist both evaporation and subdrainage, but they also resist waterabsorption by plants, while coarser ones give up their water more readily ${ }^{1}$ so that in a region frequently visited by drought the upland vegetation is, in general, most highly developed on the heavier soils.

As to the internal conditions that influence the rate of water-supply to the transpiring parts of the plant, there appears to be, for any plant at any time, a maximum rate at which water can enter the roots and pass through the vessels. This rate seems to depend upon the extent of the root-system and upon the condition of the absorbing portions of the roots as well as upon that of the conducting tissues in general. It is probably never for very long periods that this maximum rate is attained in moist weather; most plants at such times do not appear to transpire at a rate that exceeds their maximum rate of absorption and conduction. Evidence has been obtained in the arid regions, however, and this probably holds also for dry periods in humid areas, that this maximum rate may frequently be reached when the rate of transpiration is greatly increased through high evaporating power of the air. ${ }^{2}$

In such cases growth ceases even with an ample supply of soilmoisture and the plant remains quiescent, without other sign of injury, till a lower evaporation-rate allows absorption and transmission again to surpass the rate of water-loss. Thus, in the spring dry season at Tueson, Arizona, morning-glory plants attained a few leares and then rested without growth until the higher humidity of the summer scason arrived, although the soil in which they were rooted was kept continuously at or somewhat above its optimum water-content by irrigation. When the evaporation-rate had fallen markedly, with the coming of the cloudy and more humid summer rainy season, these plants resumed their growth in the usual manner. ${ }^{3}$

The ability of a plant to absorb and conduct water is, of course, an internal condition, which depends upon many things. Naturally, the more extensive is the absorbing surface of the roots the more

\footnotetext{
1 Of several soils with approximately the same chemical composition, but differing in the size of their particles, that with the finest particles gives ordinarily the most luxurious vegetation. The physical and physiological reasons for these phenomena have apparently not been taken up in detail. In this connection seo Livingston, B. E., and G. H. Jensen, An experiment on the relation of soil physies to plant growth, Bot. Gaz., 38:67-71, 190t. On the capillary movement of water in natural soils, seo: Pulling $\mathrm{H}$. E., The rate of water movement in aerated soils, Soil Science, 4:239-268, 1917.

${ }^{2}$ Livingston and Brown (1912): Brown, W. H., The relation of evaporation to the watercontent of the eoil at the time of wilting, Plant World, 15:121-134, 1912.-Briggs, L. J., and H. L. Shantz, Tho wilting coefficient for different plants and its indirect determination, U. S. Dept. Agric., Bur. Plant Ind. Bull. 230, 1912. Other citations of this work are given in the two following papers: Caldwell, J. S., The relation of environmental conditions to the phenomenon of permanent wilting in plants, Physiol. Res., 1: 1-56.1913.-Shive, J. W., and 13. E. Livingston, The relation of atmospheric cvaporating power to soil-moisture content at permanent wilting in plants, Plant World, 17:81-121, 1914.

${ }^{3}$ Livingston, 1907. a
} 
rapidly may moisture enter, providing, of course, that the maximum rate of supply of soil-moisture to this surface is not surpassed. Also, the rate of absorption must be markedly affected by the condition of the absorbing membranes and the cells adjacent to them. If these tissues are pathologically modified, as by the presence of poisons, even a large extent of root surface may fail to allow as much water entrance as might occur through a smaller root-system in a healthy condition. ${ }^{1}$

The condition of the vessels in the stem, etc., whether well or poorly developed, whether the lumina are large or small, and whether crosswalls are frequent or not, is an important factor in determining the maximum rate of water conduction with a given pressure gradient. It will be remembered in this connection that the primary deleterious effect of certain fungus growths within the vessels is due to a simple stopping of these passages. In such cases the plant might suffer from lack of water, although its roots possessed an adequate power of absorption and were in a soil of adequate water-supplying power.

Of course, the causes of the internal conditions above mentioned are to be sought in previously effective external and internal conditions, as the effects of which any present status of affairs must be considered; but this phase of environmental influence lies far beyond the matters with which we are here concerned.

\section{RELATIONS BETWEEN WATER-REQUIREMENT AND WATER-SUPPLY.}

From the above consideration of the water-requirement and watersupply of plants it is clear that growth and other activities are not dependent upon either of these factors alone, but depend upon the relation that holds between them. It is this relation which gives the clue to all physiological and ecological problems concerning moisture. So long as water moves into any tissue as rapidly as it is removed, that tissue may maintain itself in a quiescent state; so long as the possible rate of influx surpasses the actual rate of loss, the tissue may increase in size and carry on any processes requiring the fixation or destruction of water; and whenever the supply falls below the demand (i.e., whenever the demand exceeds the supply), growth and many other activities must cease. If the latter condition continues long, partial or total death must follow, or at least the more or less complete entrance of the organism into a state of dormancy. The effect upon the plant is the same, whether the physiological lack of water be brought about through an increase in the demand, through a decrease in the supply, or through both of these acting together.

${ }^{1}$ Livingston, B. E., Note on the relation between the growth of roots and of tops in wheat, Bot. Gaz., 41: 139-143, 1906.-Livingston, B. E., J. C. Britton, and F. E. Reid, Studies on the properties of an unproductive soil, U. S. Dept. Agric., Bur. Soils Bull. 28, 1905.-Livingston, B. E., Further studies on the properties of unproductive soils, U. S. Dept. Agric., Bur. Soils Bull. 36, 1907. 
Higher evaporation-rate or increased solar intensity may raise the rate of transpiration in any plant until it surpasses the possible rate of supply to the transpiring parts. On the other hand, the dryingout of the soil or a pathological condition of the absorbing or conducting system of the plant may reduce the rate of entrance or transmission of moisture until the transpiring tissues suffer from dryness. The effect of this physiological drought, however caused, is a gradual loss of water and hence of turgor, which results finally in plasmolysis and wilting. Under such conditions tissue enlargement must cease before plasmolysis is accomplished, and can not begin again until a certain amount of turgidity has been regained.

As long as the ratio between the rate of possible water-supply and the rate of water-demand in any tissue or organ is greater than unity, growth may occur. When this water ratio falls to unity growth must soon cease, though the organ may retain its form and vitality. When the water ratio becomes less than unity, incipient drying occurs and plasmolysis must soon follow if the ratio continues less than unity. Whether plasmolysis and wilting result in the death of the tissue inrolved depends upon the extent to which the ratio falls below unity and upon the length of the period during which this condition obtains. ${ }^{1}$ Of course, it must be remembered that the matter here brought forward is very much complicated by the free interchange of water by various parts of the plant itself; the wilting of a certain tissue may not denote anything out of the ordinary in the plant as a whole, for the normal process of development often includes many reversals in growth. Thus, a tuber grows for a long time and then loses its water and other contents, while the entire plant of which such tuber is a part may be said to be continually advancing through its development phases.

With the continuation of a drought period most plants die only by degrees; the lower and older leaves are apt to succumb first, and it is only after a somewhat protracted dry period that total death of an individual occurs. Even in such cases the existence of seeds usually carries the vital substance forward to the next favorable season. The withering and falling away of a few of the older leaves often acts as an automatic removal of the drought conditions, for such a decrease in the transpiring surface may so diminish the transpiration-rate as to prevent further wilting. The same result is frequently brought about by a temporary lowering of the evaporating power of the air or of the light intensity. The tendeney to wilt, which is manifest in most plants on dry, sunny afternoons, though no actual wilting may occur, is regularly checked by the eoming on of night with its consequent lowering of the evaporation-rate, and also, sometimes at least. through closure of the stomata. The water ratio of transpiring organs

\footnotetext{
1913.

'Livingston, B. E., Incipient drying in plants, Science, n.s., 35: 391-395, 1912.-Caldwell
} 
thus falls during bright days and rises again at night. In the arid regions it appears that this night period of recovery is of very great importance. Many plants that are normally very resistant to drought conditions as they occur would probably succumb completely on the second day if the night period of recovery of the water ratio were omitted. A shower of rain affects both terms of the water ratio; it increases soil-moisture and decreases evaporation, while incipient drying or partial plasmolysis may sometimes be almost immediately corrected through actual absorption of moisture through leaf surfaces wetted by rain.

In the majority of ordinary plants the water of transpiration passes with comparative directness from the absorbing surfaces of the rootsystem to the transpiring surfaces of the foliage. Stored water is here of little general importance. Outside of the arid regions such plants appear to absorb and transmit moisture from a moist or wet soil with sufficiently great rapidity to prevent any serious wilting, even with the highest transpiration-rates; that is, the maximum possible rate of absorption is seldom inadequate. But, with a soil that is becoming dry, there comes a time when the actual rate of absorption fails to keep the water ratio above unity, and in such cases wilting soon occurs. If a plant wilts for this cause it may be made to revive by mere addition of water to the soil about its roots. However, if the maximum possible rate of conduction is at fault (which depends, as has been seen, upon the structure and condition of the roots, vessels, etc.), such treatment will fail to produce a complete return to the usual condition. (Caldwell 1913.)

Before the problem of the quantitative aspects of wilting and of general plant behavior with regard to moisture may be seriously approached, the study of soil physics and of water absorption, conduction, and transpiration, must furnish us with means of determining with fair accuracy the terms of the water ratio. The study of soil transmission and plant absorption have been strangely neglected by students of plant physiology. That of transpiration and the conditions controlling it has progressed somewhat further, but much remains to be determined. No field of plant physiology promises greater conquests than this one of the water-relations, either from the standpoint of pure science or from that of a rational plant-culture. (Livingston and Hawkins 1915; Pulling and Livingston 1915, also Pulling 1917.

When a plant wilts from lack of soil-moisture it is well known that the soil about its roots is not dry, but always contains a considerable amount of water. This residual water, left after the roots have ceased to absorb, has been called "non-available." Under a given set of conditions this moisture-content appears to be constant for any plant and for any soil, but the conditions upon which the magnitude of the residual soil-moisture content depend are much more complex than has usually been thought (Shive and Livingston 1914). 
As the soil adjoining the absorbing membranes becomes drier, the surface tension of the capillary films about its particles increases until it finally equals or surpasses the imbibition attraction for moisture exerted by the exposed walls of the absorbing-cells. These capillary phenomena are the main factor in the attraction of the soil for water, and it is this capillary force against which the forces that produce water-entrance into plant roots must operate. It therefore appears that, at the time when absorption ceases, we may expect to find the vapor-tension of the exposed root-membranes just balanced by that of the soil solution.

The amount of water remaining in different soils, with different plants, has been determined by various workers, and it has been taken to vary with the nature of the plant and with that of the soil. It is lower in sandy soils than in heavier ones, depending thus upon the specific attraction of the soil for water, a variable which depends largely upon the size of the soil particles. Non-available soil-moisture has often been treated briefly and summarily in texts and monographs as a soil constant. ${ }^{1} \quad$ This it assuredly is not, ${ }^{2}$ for with a given plant and a given soil, this factor may be made to vary within wide limits, according to the status of the other conditions. It depends, indeed, for any soil and any plant, upon the transpiration-rate for the period during which wilting occurs. The higher the rate of water-loss from a plant the more water will there be in the soil about its roots when permanent wilting occurs. Plants in a moist room remove more water from the soil in which they are potted before permanent wilting occurs than do other similar ones in a dry room or in the open. This state of affairs might have been inferred from the principles already brought out, that the drier the soil becomes the less rapidly will it conduct moisture to the roots of a plant, and that when the transpiration-rate surpasses that of intake, there must be a tendency toward wilting. The residual moisture content of a soil, with reference to a certain plant when wilting occurs, is simply the amount of water which that soil contains when the rate of water absorption and conduction to the foliage have been, for an adequate period, less than the rate of loss from the leaves. The length of the period of lag which elapses between the time when the rate of foliar water-supply first falls below that of transpiration and the time when permanent wilting ensues

\footnotetext{
1 The best general treatise on soils and their relation to plants is, so far as we are aware, Mitscherlich, E. A., Bodenkunde für Land und Fortswirte, Berlin, 1913. The work can not be too highly commended as, in general, a logically and physically sound treatise on this, one of the most difficult of biological subjects. On the general phenomena of capillarity and the complex principles upon which these depend the reader may be referred to Freundlich, H. Kapillarchemie, Leipzig. 1909.

${ }^{2}$ Livingston, B. E., Present problems of soil physics as related to plant activities, Amer. Nat., 46: 291-301, 1912.-Briggs and Shantz 1912, Brown 1912, Caldwell 1913. - Shive and Livingston 1914.
} 
must be a function of the internal water-conditions of the plant-body and of the difference between the rate of supply to the roots and the rate of transpiration. It will be seen at once that a statement of the residual water-content for any soil and plant is meaningless without a statement of the transpiration-rate at the time the determination was made, or, at least, the statement of some measure of the condi tions that determine transpiration. It is apparently quite possible to define these conditions with some precision by means of measurements of the evaporating power of the air.

Aside from actual dryness of the soil, another condition produces the same effect upon the plant. This is included in what has been termed by Schimper physiological dryness. This condition exists where a plant apparently suffers from drought and yet is rooted in a moist or even wet soil. The optimally moist soil of the experiment with morning-glory described on page 119, might be said to be physiologically dry for that plant under those conditions of transpiration. The term more commonly connotes those cases where the plant suffers from lack of water, due either to some pathological condition of the roots or conducting organs or to a too high physical concentration (osmotic pressure) of the soil solution. In either case the symptoms are those produced by a dry soil, but the actual amount of moisture present in the soil may still be relatively very high, or the soil may even be completely saturated.

The best known cases of adverse osmotic conditions in the soil solution are those of the so-called "alkali"' soils, where the salt-content is usually high, although the component salts are not highly toxic. In such soils ordinary plants suffer from lack of water, apparently not because water movement into the roots is checked through increased external capillary resistance, as in a soil that is actually nearly dry, nor because of toxic effects, but merely because of the high osmotic pressure of the soil solution itself, which may result in plasmolysis of the superficial root-cells and the consequent derangement of the absorbing mechanism. Plants that are characterized by an unusually high osmotic pressure in their absorbing organs seem to succeed in such soils. ${ }^{1}$

Those instances of physiological dryness which are produced by injury or by a pathological condition of the absorbing or conducting system need not here be treated in detail; it needs only to be remarked that any condition leading to an inadequate power of absorption or conduction may bring about a correspondingly inadequate water-supply and, in general, may result in the same symptoms as those produced by soils of low moisture-content or of low power of water delivery.

\footnotetext{
${ }^{1}$ See in this regard Fitting, 1911, and the remarks on this paper by Livingston, 1911.
} 
The factor of duration is exceedingly important in the moisturerelations of plants. While cessation of enlargement must immediately ensue with incipient plasmolysis of a growing tissue, a partially plasmolyzed tissue may retain vitality for a long time and may immediately recover with the return of an adequate water-supply, provided that the process of desiccation has not progresssed too far. Thus, many plants complete their growth in seasons and in regions where wilting occurs for several hours daily, this being corrected and positive growth being accomplished during the cooler or more moist hours of the day. For such forms a change in the rhythm of fluctuation of the water ratio might prevent maturation or reproduction, although the fraction of the total period of growth represented by the total period of wilting might remain unchanged. Also, the after-effect of adverse conditions being, as it seems, generally more pronounced than that of favorable ones, rapid fluctuations between adequate and inadequate water ratios frequently result in much less growth than would have occurred in a continuous period of favorable conditions, although the latter were of no greater duration than the total of all the short favorable periods really experienced by the plant in the first case. This factor of fluctuation or variation in the environment is especially difficult to consider at the present time; it is mentioned here only to throw emphasis on an important phase of the duration factor which will need careful investigation in the future.

With regard to the quantitative aspect of the moisture limits which plants are able to withstand, very little information is available. Since the activities of the plant as a whole are the summation of the activities of its various parts, we must regard the primary moisture condition that is effective in the control of plant activity as simply the water ratio obtaining in the active tissues. But practically nothing has so far been done with this dynamic aspect of the waterrelation. The determinations that are available bear simply upon the amount of desiccation which various forms or organs may bear. Many of the scattered observations on this point are presented in Ewart's translation of Pfeffer's Plant Physiology under the heading "Desiccation." To obtain further information bearing upon the point in which we are at present interested, the whole riewpoint needs to be somewhat different from that heretofore employed. The moisture-contents will need to be uniformly calculated to comparable terms, such as to the hasis of dry weight or natural volume, and the different regions of the bodies of higher plants will have to be separately considered.

In connection with the foregoing discussion of the relation between the rates of entrance of water into the plant and those of its exit. Woodward's ${ }^{1}$ eneeption of the water requirement of plants should

\footnotetext{
IWoodward, J., Some thoughts and experiments concerning vegetation, Phil. Trans. Roy. Soc. London, 21: 193-227, 1699.
} 
receive some attention. The water requirement, in this special sense, denotes the ratio of the total water-loss by transpiration to the yield of plant material for any given period of time, usually for the entire growing-season. This conception has recently received renewed attention and very thorough study at the hands of Briggs and Shantz, ${ }^{1}$ who have employed it as a physiological criterion by which to compare the relative drought resistance of agricultural plants.

If several different plant-forms be grown under the same set of climatic conditions, it is found that the different forms differ in their water requirements; the amount of water required to produce unit weight of crop is greater in one case than in another. In such a case the plant with the lower water requirement is the one giving the larger crop with the smaller amount of water, and it is obvious that this criterion must be very valuable in the study of agricultural conditions in arid and semiarid regions. It is also clear that such comparisons between plant-forms are always made with reference to some given set of climatic conditions; if one form has a lower water requirement than another for one climate it does not follow that the same relation must hold for the same two forms in another climate. Thus, relative water requirements need always to be stated with reference to a particular environmental complex, which must be defined as precisely as is possible. Of course the water requirement of a given plant-form may also be employed as a criterion by which different sets of environmental conditions may be compared, the physiological properties of this plant-form being the standard of measurement in such a case. This whole matter promises much for both agricultural science and the ecology of uncultivated plants, and it may be predicted that water requirement will assume greater importance in discussions of plant water-relations, as this field becomes more thoroughly investigated.

The foregoing general and incomplete treatment of the subject of the influence of water conditions upon plants may suffice for the present. It is to be hoped that the future may furnish well-collected and well-related data on these questions and that some of the experimentation to be carried out in the future may be more adequate to the purposes of ecology and agriculture than is much of the haphazard experimentation so far predominating in the literature.

\footnotetext{
${ }^{1}$ Briggs, L. J., and H. L. Shantz, The water requirement of plants, II, A review of the literature, U. S. Dept. Agric., Bur. Plant Ind. Bull. 285, 1913. This is a very complete and valuable annotated bibliography of the subject and includes discussion of and references to the writers' own work. In this connection see also Shive, J. W., A study of physiological balance in nutrient media, Physiol. Res., 1: 327-397, 1915. P. 379.
} 


\section{TEMPERATURE.}

\section{TEMPERATURE REQUIREMENT WITHIN THE PLANT.}

One of the fundamental conditions that have to be fulfilled in order that life processes may go forward is that the body of the organism must possess a temperature lying between certain limits; the temperature of the living cells must be neither too high nor too low. If the temperature rises beyond the maximum temperature limit for life, or if it falls below the corresponding minimum, death must follow.

In this consideration, which is one of the most clearly established principles of physiological science, it is to be borne in mind that the numerous processes, or material and energy transformations, that make up life are partly chemical in their nature and partly physical. All processes that result in an alteration in the kind of matter within the plant are chemical. Here belong photosynthesis in green plants, all the various kinds of chemosynthesis, and all processes of oxidation and reduction, of hydration and dehydration in the chemical sense, of polymerization and hydrolysis, etc. On the other hand, all processes that result merely in a change of state of the matter within the plant-body are physical. These latter do not usually receive so much attention at the hands of physiologists as do the others, and they are probably not as well known, but they are certainly no less important. As examples of such physical changes may be mentioned such processes as coagulation or precipitation of substances out of solution or suspension, the various possible alterations in the viscosity of liquids, and even the transformations that may occur between the solid, liquid, and gaseous states of matter. It is frequently true of physiological phenomena that the chemical and physical processes are so closely related that it is impossible to relegate a material change to either category alone. In this connection it may be recalled how modern researches along the border-line between physies and chemistry are tending more and more to erase this line and to prove it to be quite an arbitrary demarcation.

All of the innumerable processes, physical and chemical, that occur in the living plant must be thought of as having their temperature limits, just as has the grand summation of these processes. In so far as physiological studies have gone in this connection, it appears that each component process possesses temperature limits more or less different from those of others, and also different from those of the grand summation. Thus, with falling temperature growth in size is checked when a certain minimum temperature is reached, at lower minima cell-division and photosynthesis are also checked, and at a still lower minhimum respiration ceases and death ensues. It follows from this that general plant activity can not proced at any tempera- 
ture that might prevent the occurrence of any of the simpler physical and chemical changes essential to the make-up of this general activity.

According to the kinetic theory of matter an alteration in the temperature of any body is to be considered as a change in the rate of motion of its component particles and, consequently, as a gain or loss of kinetic energy by the latter. The degree to which this energy of motion is possessed by the molecules, etc., of a mixture- that is, its temperature - is to be regarded as the proximate condition determining the nature of the transformations that occur therein. Therefore every temperature change must be regarded as affecting a more or less marked alteration in the velocity of each of the many physical and chemical processes that make up the life activity of a plant.

It is possible, therefore, to proceed a step farther than we have gone in our generalization above. Not only is it true that the prime essential temperature condition for general vitality is that a temperature must obtain under which all the necessary component processes may occur, but the temperature must be such that these component processes may go on with adequate velocities. They must not proceed with too high nor with too low rates; otherwise death must occur. Thus photosynthesis, for example, might occur in a plant at a certain temperature, but the rapidity of the formation of carbohydrates might at the same time not be great enough to make up for the loss entailed by respiration, growth, etc., at that temperature.

As has been mentioned in another place, however, it is quite possible for an organism to survive a brief period of exposure to a condition to which it would succumb with a more prolonged exposure. It is clear, then, that the temperature limits usually given for plants are not definite and quantitative measures of the limiting conditions for vital activity unless they are taken in connection with the length of time during which the organism is subjected to these temperatures. The question of the duration of temperature conditions in connection with the establishment of physiological limits has received attention from Blackman, ${ }^{1}$ from Miss Matthaei, ${ }^{2}$ and from Lehenbauer, ${ }^{3}$ and is worthy of still further study.

From the foregoing paragraph we may formulate the following statement of a general and fundamental principle regarding the relation between temperature and vital activity. The temperature of the living plant-body must not remain for more than a maximum time period at any temperature which, if longer continued, would cause any essential physical or chemical process of the general life activity to surpass the minimum or maximum limit of its velocity.

\footnotetext{
1 Blackman, (1905)-Idem, The metabolism of the plant considered as a catalytic reaction, Science, n.s., 28: 628-636, 1908.

2 Matthaei, Gabrielle L. C., Experimental researches on vegetable assimilation and respiration. III. On the effect of temperature on carbon dioxide assimilation Phil. Trans. Roy. Soc. London, B. 197: 47-105, 1904.

${ }^{3}$ Lehenbauer, P. A., Growth of maize seedlings in relation to temperature, Physiol. Res., 1: 247-288, 1914.
} 
The temperature limits for vigorous activity, as these are usually given, are very different for different plants. The lowest minima are somewhat below $0^{\circ} \mathrm{C}$., while the highest maxima are above $60^{\circ} \mathrm{C}$. For the retention of life in dormant phases the range is, of course, much greater than for vigorous activity. Dry seeds can endure, for long periods, temperatures far below the freezing-point of water and far above the boiling-point. It needs to be emphasized that the vatercontent of a tissue is highly important in determining what may be its minimum and maximum temperature and the duration of such temperatures that it may survive.

\section{RELATION OF TEMPERATURE WITHIN THE PLANT TO CONDITIONS OF THE ENVIRONMENT.}

Material changes, whether physical or chemical, generally result in the warming or cooling of the medium; some heat is generally produced or else disappears with each material alteration. Wherever material processes are going forward in the plant, heat must be continuously supplied, or else it must be as continuously removed; otherwise the temperature of the body must fall or rise and a corresponding alteration in the processes themselves must ensue.

In the case of a living plant the many physical and chemical processes of its life must, of course, influence one another, in velocity and direction in various ways, among which the mutual effect of heat absorption and liberation must be important. Thus the heat set free by respiration may be of primary importance in maintaining a possible temperature for cell growth, and the absorption of heat by transpiration is probably often the prime condition that prevents a too great rise in tissue temperature.

If there were no outward or inward passage of heat through the periphery of the plant, it is obvious that life could be possible only so long as this complicated interplay of the heat effects of the various physiologieal processes were automatically so limited that no essential process might be too greatly altered by temperature change. But, just as the moisture conditions of the plant-body are usually much more influenced by water changes between it and its surroundings than by the generally insignificant destruction and formation of water within the tissues, so also the internal temperature conditions usually depend mainly upon heat exchanges with the exterior, and the internal absorption and liberation of heat. which has just been considered are of prime importance only in relatively few cases, if they ever are at all in nature.

The temperature of the plant tends closely to follow that of its environment; roots ean seldom possess a temperature markedly different from that of the surrounding soil, and stems and leaves are never very much warmer or cooler than the air that bathes them. If the vital processes result, at any time, in the liberation of heat, a 
marked rise in temperature is prevented by outward conduction and radiation, and the body-temperature remains automatically at about the same point as that of the material surroundings. On the other hand, if the summed result of physiological changes be a disappearance of heat within the cells, then any considerable fall in tissue-temperature is automatically adjusted at an incipient stage by intake of heat from without. The only exceptions to this rule that are worthy of mention here are the cooling effect of transpiration, whereby the temperature of foliage is sometimes as much as a few centigrade degrees below that of the air, and the heating effect of sunshine, whereby leaves are sometimes a few degrees warmer than their surroundings.

From thermodynamics we may be sure that the outward and inward conduction of heat are automatically self-controlling, heat not being conducted from a cooler to a warmer body. Thus an atmosphere at a given temperature will never, by heat conduction, render a plant either warmer or cooler than the air itself. But it is possible for a plant, or other body, to receive heat by radiation from an immediate environment the temperature of which is much lower than its own. Conversely, for a time at least, it may radiate heat into an immediate environment having a higher temperature. The first case is very important in many instances, as in the absorption of sunlight by green leaves, which will be considered under the topic "Light." The second is of much less frequent occurrence, but may sometimes be important in the cooling of leaves on clear nights when radiation is rapid.

As in the case of the entrance and exit of water, the nature and condition of the plant surfaces exert a considerable influence upon the possible rate of entrance or exit of heat, either by radiation or conduction. The effect of a more or less thorough insulation of the plantbody would, of course, be the introduction of a correspondingly pronounced lag in the temperature changes of the plant, as far as these are due to radiation or to conduction to or from the exterior. Thus, after a temperature change in the surroundings, some time may elapse before uniform temperatures within and without again prevail. In the case of roots this feature is probably of but little importance, although the results of secondary growth often produce on old organs of this sort a layer of cork and other modified cells of sufficient thickness, so that a considerable retardation of heat conduction no doubt ensues. Heat radiation appears to be of little or no importance in subterranean organs.

The aerial portions of the plant exhibit more considerable effects of the retardation of heat transmission. This is especially notable in many buds, in the stems of the larger plants, such as trees, and in densely hairy leaves. The bark of the cork oak is familiar to every- 
one as a substance with a low power of heat conduction, and the hairy buds and younger leaves of such forms as mullein (Verbascum thapsus) are known to assume the temperature of their surroundings, after a rapid temperature change in the latter, with a distinctly sensible lag. One of the main internal conditions affecting the rate of outward and inward heat conduction is that of moisture-content, which, as we have seen, is also of great importance in the determination of the rates of intake and outgo of water.

The rate of absorption or elimination of radiant heat is affected also by the nature of the plant periphery; rough surfaces radiate and absorb a greater proportion of energy rays than do smooth ones; plaited and folded surfaces radiate and absorb much less than plain ones of equal area; the color of the tissue is highly important in this connection, and the exposure of the surfaces considered, with reference to the earth's surface and to the sky, is also of primary importance. Most of the heat radiation and absorption by plants occurs in the direction toward and from the sky, and by far the greater portion of the radiant energy absorbed is from direct sunshine. This latter feature has been emphasized in our discussion of transpiration and will be touched upon again in connection with the treatment of light. Of course, it is to be remembered that the effect of heat radiation in producing a body-temperature higher or lower than that of the surroundings is soon limited by the increased rates of both radiation and conduction in the opposite direction, so that very great differences between outside and inside temperature are not to be expected. The heating effect of direct sunshine upon green, transpiring leaves is limited, not only by outward conduction and radiation, but also by the cooling effect of transpiration. Even in intense sunshine the temperature of turgid, rapidly transpiring leaves is frequently or usually below that of the surrounding air. ${ }^{3}$

The main generalization in connection with the temperature relation is simply that the temperature of the plant is never very different from that of its immediate surroundings. The important effect of the different rates of heat exchange between plant and enviromment is practically confined to the determination of the temperatures of leaves and other similarly exposed parts when under the direet rays of the sun, and to the production of a more or less pronounced lag in the tissue temperature changes brought about by great and rapid alteration in the environmental temperature. 


\section{THE DURATION ASPECT OF THE TEMPERATURE RELATION.}

The velocities of the many physical and chemical changes that compose vital activity as a whole, depend, as has been seen, upon the temperature. It follows that the outcome of each separate process, and that of the entire complex, is largely determined by the degree of temperature and by the length of the time period during which any temperature has obtained. The accomplishment of the entire plant, as measured by the amount of its growth, for example, might be the summation of the various accomplishments of the component processes during the given time period, which latter are simply the integrations of the various process velocities with respect to time. Since we are very far from being able satisfactorily to separate the component processes that go to make up the activity of any plant, it is necessary here only to call attention to the above proposition as representing a general principle the details of which must occupy many minds in the future. For the present the physiological ecologist can do nothing but consider the varying velocities of certain broad, complex processes such as growth, crop production, and the like. But our generalization is of use at least in this, that it enables us to lay out the field for future study, with a probability of satisfactory results that might otherwise be absent.

We have seen that physical and chemical processes are intimately commingled in the intricate complex which we call life. To this conception may be appended the additional one that many of the physical properties, probably all of them in the final reckoning, depend upon chemical changes which have previously occurred. It is quite impossible for a physical change to occur in the substance of a plant unless the various materials involved be present, and these may be assumed, in general, to have resulted from chemical changes. Since we know already that chemical action is exceedingly important in most plant phenomena, the last statement allows us tentatively to state that this action may probably be found to have the controlling influence in general life activities. ${ }^{1} \quad$ If this be true, the general activities of the plant should follow more or less accurately the principles of chemical action. The relation of chemical processes to temperature have been much worked upon and the law of Van't Hoff and Arrhenius has been developed in this connection. It states that for each rise in temperature over a range amounting to 10 centigrade degrees, there is a doubling or tripling of the reaction velocity. Often the coefficient is 2 or a little more or less $\left(\mathrm{PH}_{3}=\mathrm{P}+\mathrm{H}_{3}, 1.2 ; \mathrm{C}_{2} \mathrm{H}_{3} \mathrm{O}_{2} \cdot \mathrm{C}_{2} \mathrm{H}_{5}+\mathrm{NaOH}\right.$, 1.89 $)^{2}$. Usually it is between 2 and $3\left(\mathrm{C}_{2} \mathrm{H}_{3} \mathrm{ONH}_{2} \mathrm{Ag}, 2.12 ; \mathrm{C}_{2} \mathrm{H}_{3} \mathrm{C}_{2}\right.$.

${ }^{1}$ The whole matter here brought forward has been given somewhat more thorough consideration than is needed here, in the following paper: Livingston, B. E., and G. J. Livingston, Temperature Coefficients in Plant Geography and Climatology, Bot. Gaz., 56: 349-375, 1913.

${ }^{2}$ Van't Hoff, J. H., Lectures on theoretical and physical chemistry, translated by R. A. Lehfeldt. London, no date (author's preface date 1898), part 1, p. 228. 
$\mathrm{Ag}, 2.55)$, sometimes it is over $3\left(\mathrm{NaOC}_{2} \mathrm{H}_{5}+\mathrm{CH}_{3} \mathrm{I}, 3.34\right)^{20}$, and it may be still higher.

Since the temperature of the plant follows so closely that of the surroundings, it will be safe to consider these two temperatures as identical for our present purpose. Of course, we shall expect to find that the principle of Van't Hoff and Arrhenius may be applied to plant phenomena only between certain limits of temperature. It is perfectly clear that the generalization can hold only so long as all or nearly all of the component or partial processes are progressing according to this principle. In purely chemical processes there are always a minimum and a maximum, beyond which this principle of Van't Hoff and Arrhenius no longer expresses the relation of temperature to velocity.

A somewhat extensive literature already exists regarding the application of this principle to physiological phenomena. We may mention the main results with plants. Clausen ${ }^{1}$ determined the velocity of carbon-dioxide excretion from seedlings and buds at different temperatures. He found that the rate somewhat more than doubled for each temperature rise of $10^{\circ} \mathrm{C}$., to an upper limit about $40^{\circ} \mathrm{C}$. Miss Matthaei ${ }^{2}$ studied the effect of temperature on the evolution of the same gas from leaves in darkness, and also on its fixation by leaves in light, showing that the Van't Hoff-Arrhenius principle holds here in a very satisfactory manner. Blackman ${ }^{3}$ has presented a very good statement of this entire problem, especially in regard to plants, and his concluding sentences are worthy of quotation here. He writes:

"To me it seems impossible to avoid regarding the fundamental processes of anabolism, katabolism, and growth as slow chemical reactions catalytically accelerated by protoplasm and inevitably accelerated by temperature. This soon follows if we once admit that the atoms and molecules concerned possess the same essential properties during their brief sojourn in the living nexus as they do before and after."

On the whole, it seems allowable to conclude that the majority of the elementary chemical processes of living things proceed according to the general principle of Van't Hoff and Arrhenius, and that such processes exhibit temperature coefficients, within the ordinary limits of environmental temperature of from 2.0 to 2.5. When, however, these elementary or component processes are combined into such a complex resultant as growth, it does not necessarily follow that the temperature coefficient of the complex process must be the same as that of its components. Russell' states that "the effect of temperature on the rate of grouth of a plant is in no wise like its effect in accelerating chemical change," eiting Bialoblocki ${ }^{5}$ to support this view.

\footnotetext{
${ }^{1}$ Clausen, H., Beitrïge zur Kenntnis der Athmung der Gewächse und des pflanzlichen Stoffwechsels, Landw. Jahrb., 19: 893-930, 1890.

${ }^{2}$ Matthaei, 1904.

3 Blackman, 1908.

4 Russell, E. J., Soil conditions and plant growth, London, 1917.

- Bialoblocki, J., Ueber den Einfluss der Bodenwirme auf dio Entwichlung ciniger Culturpflanzen, Landw. Versuchsstat., 13: 424-472, 1870.
} 
The last-named author studied the influence of temperature upon the rate of growth of barley, and his results seem to show (see Russell's graph, page 21 ) that the temperature coefficient here varies markedly with the temperature itself. Since later workers have failed to record the same conclusion, it seems that Russell may perhaps give to Bialoblocki's results too conclusive a weight. It is clear that in some cases, at least, the operation of the law of the minimum ${ }^{1}$ may interfere in such experimentation, thus precluding the full acceleration affect of a given rise in temperature. As Livingston and Livingston (1913) have pointed out, "it seems highly probable that complex vital processes such as growth may frequently fail, under natural conditions, to exhibit the chemical temperature coefficient. In some of these cases proper alterations in other environmental factors might disclose the otherwise masked coefficient, in other cases the limitations might be internal, as in the nature of the protoplasmic mixture, and the obscuring of the coefficient might persist in spite of any attempt at external adjustment."

In favor of the supposition that growth-rates of plants do show a temperature coefficient of 2.0 or above, may be mentioned the experimental studies of Price, ${ }^{2}$ who determined the temperature coefficients for the opening of flower-buds on cut twigs of the plum, peach, apple, and other fruits. The time period required for resting buds to produce flowers was shown to be reduced about one-half for each rise in temperature of $10^{\circ} \mathrm{C}$. Lehenbauer's ${ }^{3}$ extensive study of the relation of growth rate, in shoots of maize seedlings, to maintained temperature shows much more clearly than had ever been done before how important the duration factor is in determining the effect of temperature on growth. As regards the temperature coefficient, he found that this has a value of from 2.40 to 1.88 for a range of temperature from $20^{\circ}$ to $32^{\circ} \mathrm{C}$., the seedlings being exposed to the given temperature for 12 hours. For temperatures below $20^{\circ}$ the coefficient has higher values (for the decade from $12^{\circ}$ to $22^{\circ}$ its value is 6.56 ) and for temperatures above $32^{\circ}$ the coefficient is much lower (for the decade from $33^{\circ}$ to $43^{\circ}$ it is 0.06 ).

Our present knowledge of this whole matter leads to the idea, as Livingston and Livingston have stated, "that there are many cases in which growth-rates and other complex processes in plants and animals exhibit temperature coefficients of about 2.0, and that in other cases this same coefficient is probably operative but is obscured by the limiting effect of some other environmental condition." These authors also point out that temperature coefficients of other orders of magnitude than that given may be expected, both for elementary and

\footnotetext{
1 Blackman, F. F. (1908), Idem, (1905),-Mitscherlich, E. A., Das Gesetz des Minimums und das Gesetz des abnehmenden Bodenertrages, Landw. Jahrb. 38: 537-552, 1909.-Idem, Ueber das Gesetz des Minimums und die sich aus diesem ergebenden Schlussfolgerungen, Landw. Versuchsstat, 75: 231-263, 1911.

${ }^{2}$ Price, H. L., The application of meteorological data in the study of physiological constants. Ann. Rept. Virginia Agric. Exp. Sta., 1909-10: 206-212, 1911.

${ }^{3}$ Lehenbauer, 1914.
} 
for complex life processes. The processes upon which growth immediately depends are wholly or mainly physical, as has been stated above, and these in turn depend upon chemical phenomena. Thus, the formation of cell walls is surely a physical process (precipitation, coagulation, ete.), but it is conditioned by such chemical processes as the formation of cellulose from water-soluble carbohydrates. Under such conditions it is reasonable to expect such physical processes to exhibit chemical temperature coefficients, this being, again, an instance of the operation of the law of the minimum. The true physical temperature coefficient of cell-wall formation may never be evidenced, since the rate of chemical formation of wall constituents at the periphery of the protoplasmic mass may never be sufficient to allow their solidification at the maximum rate for any given temperature. Nevertheless, some physical phenomena that have to do with vital processes show, independently of chemical phenomena, temperature coefficients that rather closely approach a value of 2.0. The authors last mentioned call attention to the fact that such is the case with the vapor-tension of water between $4^{\circ}$ and $34^{\circ} \mathrm{C}$.

It appears that we have here a general principle that seems to hold with rather satisfactory approximation for a number of different physiological processes in different organisms, for a considerable range of temperatures such as is frequently met with in nature. This problem of the temperature coefficient for physiological processes is by far the most important temperature question now awaiting investigation. Its solution for a large number of plant-forms and for a large number of developmental phases should do much for climatic plant geography and for agriculture. ${ }^{1}$ We shall return to this matter in another place.

Aside from the simple matter of amount and duration of the temperature of the environment, it is rather widely held that alterations in temperature, if these are of great magnitude and if they occur rapidly and frequently, are in themselves a potent cause for a change, often a retardation, in the rate of plant growth. Frequent changes of temperature see m, per sc, to act as a stimulus upon some plants and to bring about a different form of development from that which might occur under more stationary conditions of temperature.

Nevertheless, Price (1911) has tested this last proposition in the case of the flower-buds of peach and plum and finds that "a sudden drop of temperature to some point below $50^{\circ} \mathrm{F}$. results in the cessation of all development, but that normal development is resumed immediately when favorable temperature conditions are restored, i.e., that the retardation of development by eold is altogethex temporary and direetly proportional to the time during which the low temperature prevails." It is obvious that this matter is in need of a throughgoing investigation.

\footnotetext{
${ }^{2}$ Certain general aspects of the temperature relations of organisms are well brought out in the following: Fawcett, II. S., The temperature relations of growth in certain parasitic fungi. Univ. Calif. Puh. Arric. Sci. 4: 18:3-232, 1921.
} 


\section{LIGHT.}

\section{GENERAL NATURE OF LIGHT.}

The relations of water and temperature to plants, which have already been considered, involve variations only in intensity, there being no qualitative differences involved; the amount of moisture and the degree of temperature in the plant-body are all that need to be specified in order that these conditions be defined. Light, however, may vary not only in amount, that is, in intensity, but also in quality, and herein lies a most serious complication. Furthermore, making matters still more difficult, it is impossible, excepting on purely arbitrary grounds, to distinguish the radiant energy that we term light from radiant heat on the one hand and from the ultra-violet rays on the other. It thus appears that the term "light" itself is nothing more than an arbitrary term, denoting a range of different sorts of radiant energy, the range being characterized by certain wave-lengths. This range is extended on either side, beyond the arbitrarily limited region, by still other wave-lengths which are not included under the term "light."

Light is usually understood to mean radiant energy that is capable of affecting the human eye, having a range, then, of wave-lengths from about 400 to about 750 millionths of a millimeter. The sun's spectrum, however, extends to wave-lengths of about $293 \mu \mu$, where the opacity of the earth's atmosphere to these ultra-violet rays brings its range to a rather abrupt limit. ${ }^{1}$ Radiant energy with wave-lengths greater than about $750 \mu \mu$, and extending beyond 2,400 $\mu \mu$ (Nutting, 1912, page 202) are termed heat.

The study of the characteristics of radiant energy has been facilitated in certain aspects, and perhaps retarded in others, by the fact that all these various radiations may be mostly transformed, on being allowed to fall upon a blackened surface, into the molecular vibrations of matter. It has thus come about that the intensity of all these forms of radiant energy is uşually measured by converting them into molecular heat and by determining the temperature acquired by the heated body. It is thus that the intensity of light, or other radiant form of energy transfer, is commonly measured and described in terms of calories received per square centimeter of the cross-section of the impinging beam per unit of time.

The quality of light is defined by its range of wave-length and by the relative intensities for the different portions of the range. The range of wave-length may be determined through the use of a properly constructed spectroscope. The plant never receives light of just a single wave-length; it always receives a mixture of wave-lengths with a more or less broad range. Since light is most easily perceived by us

\footnotetext{
${ }^{1}$ Nutting, P. G., Outlines of applied optics, Philadelphia, 1912, p. 2.
} 
through its physiological effect upon our eyes, the range of any given light mixture is usually thought of in terms of the spectral eolors, which are simply names of certain physiological responses of the human organism to light of various wave-lengths and intensities. Thus, we may state that a certain light mixture ranges from red to green, for example, is particularly intense in the green, etc. It is highly desirable, however, that biological measurements of light quality be made in terms of wave-lengths, for such definition does not depend upon the eye. ${ }^{1}$

The only practical way to describe light conditions that is so far available is arbitrarily to divide the spectral range of wave-lengths into smaller ranges, and to state the intensities of these smaller ranges in terms of their respective equivalent heat intensities. Certain of the well-known Fraunhofer lines of the sun's spectrum may conveniently be used in this arbitrary subdivision.

\section{EFFECT OF LIGHT UPON PLANTS.}

We have already seen that radiant heat is effective upon plants in controlling their temperature. Light also has the same effect, in so far as it is absorbed by the plant-body and converted into molecular vibrations of thermal nature. Where this effect is alone to be considered it is not directly necessary to analyze the impinging waves into their spectral groups or component ranges of wave-lengths; it is only requisite to determine the total heating effect produced by the absorbed portion of the total impinging radiation. Since, however, any given surface, as of a plant, absorbs the different wave-lengths in different amounts, the study of light qualities may become essential even in this connection. The heating effect of light upon plants is only incidental in our present discussion; absorbed radiation is seldom if ever of primary importance in determining the temperature of plant parts.

$\Lambda$ second lind of effect produced by light upon plants is a morphogenic one and does not seem to depend upon the heating of the tissues. This is, in all probability, a photochemical effect, but the very difficult question thus raised still awaits investigation. Here we need to consider, perhaps, the retardation of growth in length, apparently due to the action of light upon most cylindrical plant-parts and the corresponding acceleration of enlargement in most dorsirentral organs. It is usually supposed that ordinarily plants would not assume their usual form without this action of light; the characteristic, much elongated stems and greatly dwarfed leaves of etiolated plants are an example of the effect of lack of light, though the moisture relation

1 Watson and Yerkes's valuable monograph on light measurement for biological purposes should be referred to in this connection: Watson, J. B., and R. M. Yerkes, Methods of studying vision in animals, Behavior Monographs, Serial No. 2, 1910. Sce also: Pulling, H. E., Sunlight and its measurement. Plant World 22: 151-171, 187-209, 1919. 
surely plays an important rôle here. Likewise, the development of certain tissues, as of leaf-palisade, are apparently often dependent upon the quality, intensity, and direction of luminous rays, and the relative positions assumed by most ordinary leaves are largely due to the asymmetrical effects of light as such. The "leaf mosaics" of plant ecology are considered as due to the operation of this condition. Also, the positions assumed by many stems and other parts are primarily due to light conditions. Such morphogenic activities are directed, not by light in general, but only by radiant energy of certain ranges of wave-length. Certain intensities within these ranges are necessary for the usual development of ordinary plants.

A third effect of light, and the one that is most fundamentally important for all terrestrial life, is the photochemical process called photosynthesis. It is only by the action of a certain range of wavelengths of radiant energy, within certain limits of intensity, that the production of carbohydrates from water and carbon dioxid may occur in chlorophyll-bearing cells. Fundamental as is the photosynthetic process, the conditions determining its velocity have hardly begun to be studied quantitatively, and this statement is especially true with regard to the light relation. In order to begin a study of the relation of plants to light it will be necessary first to possess some suitable method by which the light conditions may be measured. It is essential that both quality and intensity of the impinging light be determined for the different hours of the day and for the different days of the growing-season. The present apparent difficulty of obtaining such measurements of the environmental conditions is surpassed only by its fundamental importance to plant physiology and by its practical bearing upon the problems of ecology and agriculture.

\section{DURATION ASPECT OF LIGHT RELATION OF ORDINARY PLANTS.}

It is obvious that the result, the amount of material change, produced by any of the physiological processes that are dependent upon light must be determined by the duration of the process as well as by the nature of the light conditions determining its velocity. It seems highly probable, also, that mere fluctuation of the light conditions, as between daylight and darkness, may have a more or less definite effect upon the development of plants. We are certain that many physiological rhythms depend primarily upon this sort of fluctuations. As we have seen, however, the feature of duration is usually the last one to be carefully considered in the case of any environmental condition, and we need not be surprised to note that very little indeed has been accomplished in this direction regarding light influence upon plants. Until we are able to measure and control light conditions it must be quite hopeless to attempt any but the most superficial consideration of this aspect of the general problem of plant control. 


\section{CHEMICAL CONDITIONS.}

\section{REQUIREMENT OF MATERIAL WITHIN THE PLANT.}

At the beginning of this treatment of the main categories of environmental conditions we discussed the water-relation of plants. Water was given a place by itself in our series because its importance in the organism, as has been seen, appears to depend more upon its solvent powers and power to be imbibed in the plant colloids than upon its chemical influence. But it has been pointed out that water also acts chemically in the plant, being one of the two substances chemically transformed in photosynthesis and likewise one of the two products of the process of respiration. It is probably chemically important in other ways, certainly playing an essential part in many processes of hydration polymerization, hydrolysis, etc.

Of course there are innumerable other substances, besides water, that take part chemically in plant activities. We are apt to think first of the three great groups of compounds that have been called foods - the carbohydrates, fats, and proteins. These are apparently all essential to vital activity and even to the mere retention of life in the most dormant phases. Besides these there are a large number of substances of a more or less complex nature that are sometimes considered as foods and sometimes not. Here may be mentioned glucosides, alkaloids, various lipoids like the cholesterins, phytostearins, etc. Also, substances of importance in certain of the simpler component processes of vital activity, like chlorophyll; the various enzymes - still of questionable nature - ete., may be classified here. Finally, in order that life may occur, there must be in the tissues a number of inorganic salts and their ionized products. These are not classified as foods by physiologists, although conservative agriculturists are still prone to speak of them as "plant-foods." They might better be termed auxiliary substances until some such time as the word "foods" may be dropped from physiology. The conditions in different protoplasms, in different plants, in different developmental phases of the same plant and different parts of the same individual, are, however, so extremely varied that there seems little ultimate value in attempting to classify the various materials that are essential to plant activity. It is certainly far simpler, and probably as satisfactory in every way, to consider merely the material conditions of life, classifying the different substances on purely chemieal grounds. We need here merely emphasize the well-known point that one of the prime conditions for organie life is the presence in the organism of innumerable kinds of chemical compounds.

Since all vital activity must be regarded as material change of some sort, it is clear that the quantitative and qualitative relations between these many substances must be continually changing; the substances 
of the cell are always tending toward chemical and physical equilibrium. Such equilibrium is, of course, never really attained, even in the case of the dormant phases of plants, like seeds and spores. Thus, as long as life exists there is always in progress a more or less pronounced interchange of materials between the organism and its surroundings. Oxygen, for example, disappears in the process of normal respiration and carbon dioxid is produced. If no material exchange were possible, if the system of the plant were not continuous with that of the universe about it, then this process must shortly come to a standstill; an equilibrium between the internal diffusion tensions of oxygen and carbon dioxid must be reached, and no further oxidation might occur. Under existing conditions, however, a fall in the diffusion tension of oxygen within the plant-body immediately creates a diffusion gradient between the interior and exterior, and the gas finds its way in from the outside. Conversely, the mere occurrence of the process of respiration sets up an outward diffusion of carbon dioxid.

It appears probable that, other conditions remaining constant, every substance might prove to have its maximum concentration, or its maximum and minimum, below or between which life is possible. We must expect, however, that the concentrations of other substances, as well as light and temperature conditions, will be found to alter these limits for any given substance. It hardly needs to be mentioned here that variations in the concentrations of non-aqueous materials accompany alterations in water-content.

\section{MATERIAL EXCHANGES BETWEEN THE PLANT AND ITS SURROUNDINGS.}

From the preceding paragraph it is to be inferred that the importance of the chemical environment, in determining the nature of plant growth, etc., and in limiting the kinds of plants that can exist in any given habitat, is definitely dependent upon the generalization that diffusion tends always to bring the plant-body and the surrounding media into concentration equilibrium. Two groups of conditions militate more or less against the attainment of this equilibrium: (1) The degree of permeability of the plant periphery to the diffusing materials, and (2) the rate of their transformation within the plant or of their removal from or supply to the immediate environment. Thus, if the air about a plant should contain ether-vapor, for example, the diffusion gradient would insure an inward diffusion of the ether until the vapor-pressure of the ether solution within the plant-body just equaled its partial pressure in the surroundings. If the plant epidermis were readily permeable to the poison it is clear that death must soon ensue, and a lower permeability could only postpone, but could not prevent, this result. It is probable that no substance exists to which the plant periphery is absolutely impermeable, though there are many that penetrate only very slowly. 
If the inwardly diffusing substance be altered chemically upon reaching the interior of the plant, and if this process of alteration be capable of removing it as rapidly as it enters, it is clear that equilibrium between interior and exterior, or even any considerable solution concentration within the plant, can not be reached. Similarly, if some poison be produced within the tissues, as organic acids in the case of certain roots growing under low oxygen pressure, ${ }^{1}$ and if the epidermal tissues be adequately permeable to this substance, then the concentration that is obtained within the cells must be determined by the possible rate of removal of the poison from the immediate surroundings. A substance diffusing from roots may diffuse away through soilmoisture films, it may be absorbed by the solid-liquid surfaces of the soil, or it may be oxidized or otherwise transformed; but it is obviously essential that the poison be removed from the soil solution in the immediate neighborhood of the excreting roots; otherwise the rate of outward passage must be lowered, and the consequent rise of the internal concentration of this particular substance (supposing the process of its formation to continue at the original rate) might soon bring about a general upsetting of all the physiological processes so that death might finally ensue.

If plant activity depends upon the absolute and relative concentrations of various substances within the body, then it is clear that variations in the concentrations of these substances in the environment must be accompanied by more or less profound alterations in the physiological processes. We thus arrive at the well-known proposition that the concentration or diffusion tension of the various substances in the environment is of prime importance in determining how any plant may develop and, indeed, whether it may exist at all in a given habitat. It is immaterial whether the environmental concentration of a substance at any time be the result of causes acting wholly without the plant or of internal processes; the end-result must be the same in either case. Thus, the lactic-acid organism of souring milk is checked or killed by the accumulation of its own excretions just as truly as though the acid content of the medium had arisen solely from external causes.

It thus emerges that the chemical relation, unlike those of water and temperature but like that of light, must always be considered not only with reference to intensity but also in regard to quality. Just as there are many different wave-lengths of light that influence the plant differently, so there are innumerable chemical compounds, all differing qualitatively in their effect upon plants. Moreorer, the effect of cach one of these compounds varies not only with its own

\footnotetext{
1 Stoklasa, J., and A. Ernest, Dio chemische Charakter der Wurzelausscheidung verschicdenartiger Iíulturpflaazen, Jahrb. wiss. Bot., 46:52-102, 1908.
} 
concentration (intensity), but also with that of many others. The general problem of the chemical relation of plants is, therefore, an exceedingly complex one, so complex, indeed, that the problem of the water or temperature relation becomes, by comparison, a very simple matter.

Upon the chemical relation of plants depends, in large measure, our agricultural practice, and it is instructive to bear in mind the $a$ priori considerations of the above paragraphs when perusing the current writings upon such questions as that regarding the use of fertilizers, for example. It is to be hoped that the succeeding developments of plant ecology and of agricultural theory may be characterized by greater catholicity of perception than has prevailed in the past, and that the forthcoming literature may be burdened with less of that familiar type of argument by which a single one out of many interrelated conditions is enthusiastically proclaimed as the real and only cause of some particular physiological phenomenon.

There is much promise for the future in the study of chemical relations, however. The qualities of the chemical environment of plants can already be quite readily determined; the identification of chemical compounds is no longer a general source of serious difficulty, and we are beginning to see some light in the darkness of our prolonged endeavors to determine the intensities (concentrations, diffusion tensions) of the various substances with which we have to deal. The interdependence of the influences exerted by the various chemical compounds occurring in the environment has recently attracted much attention, and this bids fair to be an important way by which agricultural theory may at length become physiological. Salt antagonisms - the influence of the presence of a certain concentration of one salt upon the effect produced upon the plant by a certain concentration of another-were first brought into prominence by Loew ${ }^{1}$, and are attracting much attention at the present time. ${ }^{2}$

\section{CHEMICAL ENVIRONMENT IN NATURE.}

In a discussion of environmental conditions, Livingston ${ }^{3}$ has suggested that perhaps the simplest and most obvious classification of these promises most at the present time, and he divides these conditions as a whole into those that are effective above the soil surface and

${ }^{1}$ Loew, O., Die Bedeutung der Kalk-Magnesiazalze in der Landwirtzchaft, Landw. Versuchsstat, 41: 467-475, 1892.-Loew, O., and D. W. May, The relation of lime and magnesia to plant growth, U. S. Dept. Agric., Bur. Plant Ind. Bull. 1, 1901.

2 Osterhout, W. J. V., On the importance of physiologically balanced solutions for plants, [I, fresh water and terrestrial plants, Bot. Gaz, 44: 259-292, 1907.-Tottingham, W. E., A quantitative chemical and physiological study of nutrient solutions for plant cultures, Physiol. Res. 1: 133-245, 1914 (this paper contains many literature references).-Shive, $1905 b$.

${ }^{3}$ Livingston, B. E., Present problems of physiological plant ecology., Am. Nat., 43: 369378,1909 . The same paper, with some omissions and modifications, appeared under the same title in Plant World 12: 41-46, 1909. 
those that are effective below. All of our categories of external conditions are effective both above and below the soil surface, excepting light alone (as far as we now know), but it seems especially profitable to consider this classification with reference to chemical conditions.

The chemical conditions above the soil surface are characterized by a striking and almost complete uniformity and symmetry. The air of different regions and of different habitats comprises practically always the same gases, and these, with the exception of water-vapor, occur with but little variation in their partial pressures. Of the substances influencing plants, other than water, carbon dioxid exhibits the greatest variation, but even this variation appears to be, comparatively speaking, of but little account. We may safely conclude that few plants in nature are ever appreciably influenced by variations or differences in the quality or intensity of the chemical environment above the soil surface. ${ }^{1}$ Small supplies of ammonia and inorganic salts may reach the plant from its aerial environment, but with these generally insignificant phenomena of absorption we need not deal here.

When we turn our attention to the soil, we find a very different state of affairs. Every soil differs chemically from every other soil, the soil solution varying between wide limits in the nature and amount of solutes present. ${ }^{2}$ At one extreme of the series are thoroughly washed sands, in which are almost no dissolved material; at the other extreme are alkali soils, which are highly impregnated with soluble inorganic salts. In the middle region between these extremes, in most ordinary soils, it appears that the quality and concentration of the soil solution are without very great differences as far as inorganic compounds are concerned, but that these ordinary soils show very great differences in the kinds and amounts of organic matter present. ${ }^{3}$

In spite of the great amount of work that has been devoted to the problems of the soil, the whole question remains as one that has hardly been really touched in a way to be of any present aid in problems of plint distribution. Of course, our general knowledge of the paucity of soluble matter in a few sands and of the superabundance of certain compounds in alkali soils is of definite value in this regard: but even here the strietly quantitative aspect of our problems remains wholly for the future to develop.

\footnotetext{
${ }^{3}$ If atmospheric ionization should prove an important chemical feature influencing plants in nature, and if this varies from place to place and from season to season, then this statement may require modification in this regard. See report of Spochr's work in MacDougal, D. T., Annual Report of the Director of the Department of Botanical Research, Carnegie Inst. Wash. Year Book No. 13, 87-88, 1915.

${ }^{2}$ Cameron, F. K., The soil solution, the nutrient medium for plant growth, Easton, Pennsylvania, 1911.

${ }^{3}$ Livingston, Britton, and Reid, 1905.-Livingston, 1907b, Schrciner, O.. Organic compounds and fertilizer action, U. S. Dept. Agric., Bur. Soils Bull. 77, 1911. - Schreiner, O., and E. C. Lathrop, Dihydroxysteario acid in good and poor soils, Jour. Amer. Chem. Soc., 33: 1412-1417, 1911. Also numerous other papers from the Bureau of Soils, U. S. Department of Agriculture, deal with this matter.
} 


\section{DURATION ASPECT OF CHEMICAL CONDITIONS.}

Since the chemical environment of the plant is effective through controlling the internal chemical conditions, and since such control is manifested by outward and inward diffusion of material, it follows that any given change in environment may produce the response of changed activity in the plant only after the lapse of an adequate time period. Diffusion of material through water requires considerable time in every instance. Also, the physiological processes of the plant can produce material transformations only in proportion to the length of time during which they are operative at their different velocities, these velocities being in part controlled by internal chemical conditions.

The study of this feature of the chemical relation has just begun, and it surely demands much attention. What may be the effect upon the final result of a plant's activity, of frequent fluctuations in the chemical nature and intensity of the surroundings, we are unable as yet to surmise.

\section{MECHANICAL CONDITIONS.}

\section{GENERAL CONSIDERATIONS.}

All environmental conditions that are effective to influence plant activity through pressure of material en masse are to be classified as mechanical. From this point of view it matters not how the pressure may have originated or whether actual molar motion be protuced. We should thus consider the flattened root which is confined within a rock-cleft, the one-sided development of trees growing in a windswept mountain pass where the direction of air-movement is predominantly the same, the deformed branches, etc., produced by a heavy fall of snow, and the fantastic forms often exhibited by shrubs such as the hawthorns when continually browsed by animals, as all due to mechanical conditions. A mass pressure applied from without may merely hinder expansion of tissues, it may tend to compress certain parts or organs, or it may actually bring about a tearing or cutting of the tissues.

One special form of mechanical pressure, which is of basic importance in plant growth, is definitely due to external conditions, but is first developed within the plant-body. We refer to the asymmetrical pressures produced in tissues and cells by the action of gravitation. Here an influence, still practically unknown excepting in its most general aspects, not a simple pressure of body upon body nor a diffusion of material, nor yet any form of energy transfer that is apparently at all immediately related to light, heat, and electricity, reaches from the external world through the periphery of the plant and largely controls certain forms of cell activity. In this we may be fairly certain that the material condition within the organism, 
which is proximately or immediately responsible for the peculiar influence of gravitation, is pressure asymmetrically developed. That this asymmetrical internal pressure that results when the position of a plant is altered with references to the earth's center of inertia first produces a molar movement of certain portions of the cell-contents, and that this pressure and movement, with the new configuration of protoplasmic particles when gravitational equilibrium is again established, is the cause of the altered cell activities known to be produced by such change in the position of a plant, is the logical supposition which has been developed into what of theory we as yet possess in this general connection. Since the variations in the intensity of the gravitational influence that occur over the surface of the earth are quite negligible when considered with reference to the effect of this factor on plant development, it is obvious that gravitation does not require a thorough consideration at the hands of the ecologist or agriculturist. Here is one external factor, at least, which is practically identical in all habitats, as far as plant control is concerned.

\section{DESTRUCTIVE INFLUENCES OF MECHANICAL CONDITIONS.}

It seems probable that the pressures developed when roots grow against or between rocks and other objects that they can not penetrate may sometimes be a considerable factor in determining the success or failure of individual plants; but it is not at all likely that this consideration is important in the distribution of plants among different habitats. The only forms of mechanical influence from without that appear to be generally important to plant distribution and to agriculture are those due to (1) wind, (2) water, and (3) animals. In relatively few cases soil-movements, such as landslides, the caving of bluffs, etc., need to be brought directly into account in explaining the vegetation of habitats of limited extent. Ice-morements, as at. the lower ends of some glaciers and at the margins of streams and lakes, are often the source of plant destruction in such places. With the mechanically destructive action of animals may be mentioned a somewhat similar action of other plants or plant parts, but here relations other than mechanical are also frequently to be considered.

The action of wind in differentiating the regetation of different plant habitats has often been dwelt upon in ecological literature. It must be remembered, however, that this action is at least twofold; air-movement not only exerts a deforming or breaking pressure upon the plantbody, but it also profoundly affects the water-relation through increasing the evaporating power of the air, as this is effective both upon plant and soil. In connection with wind influenee may be mentioned the eutting action of blown sand, really a factor that should be considered with that of soil-movement. which includes the influenee of rolling stones), and with that of ice, as above mentioned. 
The direct mechanical effect of flowing water is familiar to everyone, and it must be accounted of great importance in determining the nature of the vegetation of many stream margins, as well as of streams themselves, of intermittently flooded stream-channels in the arid regions, and of sea and lake beaches. Other factors undoubtedly are coeffective in such cases, however.

The influence of animals upon vegetation has not been much emphasized in plant ecology, but it is undoubtedly of considerable importance in many cases, as when seeds are thus mechanically destroyed in such large numbers that the establishment or spread of a species is rendered practically impossible. This factor in distribution is usually operative only on certain developmental phases of the plant; often the seedling stage is preeminently in danger of destruction by animals. In agriculture and horticulture-practical ecology under more or less artificial conditions - the influence of animals is of prime importance and has perforce received much attention. Fences, traps, scarecrows, insecticides, and even trespass warnings are material evidences of the importance ascribed to the direct mechanical influence of animals upon the plant population. For the most part, methods have been readily devised for more or less thoroughly removing this source of danger to cultivated plants. Among the different groups of animals, insects have probably been the least easily combated, and much attention is still being devoted to this important destructive factor. It will be an interesting and important chapter of agricultural ecology when the geographical distribution of various forms of animals is correlated with that of the regions where the various crops may be successfully grown. Livingston ${ }^{1}$ has mentioned the apparent importance of animals in determining the very existence of irrigated seedlings in the dry season at Tucson, and we have often made observations in that same region that suggest a rather important relation between certain plant-forms and animal activity. It seems probable that the destructive action of animals is relatively more important in arid regions than in most others.

\section{FAVORABLE INFLUENCES OF MECHANICAL CONDITIONS.}

Wind, water, animals, etc., as is well known, frequently accelerate the spread of plants throughout large areas. In the majority of these cases it is a dormant phase, as of seeds, that is moved about. It is also true, however, that fragments of the plant-body other than seeds may be torn or broken away, being removed to another locality and there continuing development. Such is often the case with willow twigs, which float downstream and find lodgment and conditions for growth in a muddy bank. The movement of cactus branches in the arid regions of the American Southwest has been mentioned above. 
Books on ecology may be consulted for many instances of mechanical influences that increase the geographic range of the activities of plants, these being usually and curiously described as adaptations by which plants "have come to be" fitted to growth in certain habitats, rather than as environmental adaptations by which the habitats have become fitted to support certain kinds of plants!

Practically none of these considerations, however, pertain logically to a study of the proximate or immediate conditions controlling plant activities; these mechanical agencies of transport are of only secondary interest; they may be said to be only causes of causes. Thus the immediate external conditions usually considered as causing the germination of a seed must be the entrance of water, of oxygen, and of heat, and the reason for the occurrence of these immediate conditions is to be sought in the preceding mechanical transport of the seed. Of course such secondary causes are of great importance, and it is often practically impossible to approach nearer than these to the real seat of the external control of plant processes. While superficial and merely qualitative studies upon such influences have been frequent, the deeper-going quantitative and comparative work upon them remains almost entirely for the future.

\section{INTERRELATIONS OF THE ENVIRONMENTAL CONDITIONS.}

The present section is appended here merely to emphasize a feature of the discussion of environments that has already received some attention at several points in the foregoing pages, namely, that external influences are seldom or never singly effective upon plants. Three considerations in this connection require a short treatment:

(1) The more remote conditions of the external world, in bringing about the occurrence of any given influence upon plants, usually inaugurate other influences at the same time. Thus, with an increase in the amount of soil-moisture, the permeability of the soil to oxygen, the concentration of the soil solution in salts, ete., and the power of the soil to retain or give up heat, are more or less profoundly altered. With an increase in the intensity of impinging light comes also an increased income of heat to the foliage, and consequent alterations in aerial convection currents about the plant.

(2) The same external condition usually influences the velocity of more than one of the elementary component processes of the organism. Thus, phosynthesis, respiration, digestion, exeretion, secretion, growth, ete, are all greatly influeneed by such fundamental environmental relations as those of water, temperature, light, etc.

(3) Since every elementary physiologieal process is thoroughly bound up with many other concomitant processes, it follows that an external change that alter's only one process directly may indirectly be the cause of alteration in many others. If the secretion process, 
for example, by which cell-walls are thickened or modified, be increased in velocity, this internal change must directly or indirectly alter the concentration and chemical nature of the solutions of the affected tissues. The formation of cork, cuticle, etc., profoundly alters the transpiring power of aerial plant surfaces, and similar modifications in roots must produce a changed absorbing power for solutes as well as for water.

It is thus emphasized how difficult and arbitrary must be any attempt sharply to distinguish external from internal conditions, and how practically impossible it is at the present time logically to analyze the latter so as to begin to attain quantitative information concerning the various relations that have been roughly and crudely outlined in this chapter. Our reason for submitting this unsatisfactory treatment of the general subject of plant-relations is that the fundamental importance of these is not only theoretically but practically very great, and it seems time that a systematic beginning were made in some of the directions suggested by the foregoing incomplete analysis. If our treatment stimulates quantitative and comparative studies of plant environments, so that the present publication may soon be looked upon as useless and quite out of date, our aim will have been realized. The difficulty involved in really scientific studies of plant-relations ought not to be a legitimate reason for their omission and for the continuation of the pioneer sort of qualitative descriptions and teleological interpretations, which appear to belong rather in the realm of "nature-study" and natural mythology than in that of true science. There are, however, already many ecological and agricultural studies on record, wherein the more logical point of view of the more advanced physical sciences is given prominence, and the future of this aspect of biology seems to be assured.

\section{EXPERIMENTAL DETERMINATION OF RELATIONS BETWEEN PLANT ACTIVITY AND ENVIRONMENTAL CONDITIONS.}

It is perhaps not out of place here to devote some space to a consideration of the general character of the methods which must be employed in the more accurate determination of the relations with which the present chapter has had superficially to deal. As in all such cases, the only possible method of procedure is the experimental, and the experiments must be carried out with all the foresight and logical planning that characterize the work of the modern physical or chemical laboratory. The importance of this line of inquiry can not be overestimated; it is to be regarded as quite indispensable to the scientific advancement not only of ecological knowledge but of that most essential of all human activities, agricultural practice, and its pursuit is surely well worth the time, energy, and money that it would require. 
The experimentation needed is exceedingly complicated and expensive, at least from the present standpoint of biological science, but would probably not prove particularly difficult in competent hands. A special laboratory is of course required-not a series of office rooms, nor the mere contents of an architectural exterior, but a carefully planned and elaborately and logically equipped building or series of buildings, with the requisite greenhouses, cellars, constant-temperature rooms and the like. The main requirements of the work here contemplated are a variety of controlled conditional complexes, under which plants may be grown. Many of the methods of such control have still to be devised, but enough has been accomplished so that ultimate success may be regarded as assured. The moisture conditions of soil and air can be controlled with comparatively little trouble, as can also those of temperature. The control of chemical conditions offers a field for the exercise of ingenuity, and that of light and electric conditions will require still more attention.

Many investigators in plant physiology have been able to control, in more or less satisfactory ways, one or two, rarely three or four, of the influential conditions, but no plant has ever yet been studied with even approximate control of all the influential conditions of its surroundings. Since the influence of any condition is determined by the others, it is clear that, for any true appreciation of the relations between plant and enviroment, all of the influential conditions must be quantitatively known.

The suggestion here put forward, that thoroughgoing quantitative studies on the relations between environmental conditions and plant development are to be regarded as the only logical basis for a truly scientific ecology and agriculture, and that such studies are not possible without the elaborate facilities of a specially constructed laboratory, was largely included in a plea for a climatic laboratory made by $A$. P. de Candolle as early as 1855 in his Geographie Botanique Raisonée. Apparently the idea has never borne fruit. In $1891 . \mathrm{Abbe} \mathrm{b}^{1}$ repeated and indorsed the suggestion of de Candolle. The utter lack of appreciation with which the arduous work of Abbe was received, in bringing together what he could in a limited time, of the literature bearing upon the relation of agricultural crops to climatic conditions, is to be estimated from the mere fact that his summary lay unpublished for 14 years and was at length brought out, in apparently perfunctory form, only in 1905 !

\footnotetext{
${ }^{3}$ Abbe, Cleveland. A first report on the relations between climates and crops, U. S. Dept. Arric., Weather Bur. Bull. 36, 1905. See especially p. 23 ct scq.
} 


\section{THE CLIMATIC CONDITIONS OF THE UNITED STATES.}

\section{INTRODUCTORY.}

From the last paragraph of the preceding chapter it is clear that no adequate description of the environmental conditions that obtain in any area is even to be attempted for a long time. In the present chapter will be brought together merely the results of certain studies which we have been able to carry out upon a very few conditions, and upon large areas. Some of the conditions studied do not directly affect plant life at all, it being usually impossible as yet to obtain quantitative information upon the subjects most pertinent to our general line of inquiry. Only in a single case have we attempted actually to obtain measurements of an environmental factor de novo; for the rest we have simply made use of data already collected. As is clear from the preceding analyses, to obtain the kinds of information most needed for such a study as the present methods, will have to be employed which are as yet quite unknown; adequate procedures remain to be devised. Nevertheless, so great is the inertia of routine that there is little hope that the trend of observational work will alter very profoundly in the near future, and, as has been stated, we have deemed it advisable to make what use is now possible of the information at hand, with the hope that the very inadequacy of our whole presentation may itself be a potent stimulus toward the acquirement, in the future, of the kind of climatic observations upon which alone anything like a scientific ecology or agriculture may eventually be founded.

Of the five main groups of external conditions which influence plant activities, discussed in outline in Chapter II, we shall consider here, and very inadequately, only the first three-moisture, temperature, and light. For the subjects of chemical and mechanical conditions no information that is at present available can be brought to bear upon the problem of plant distribution in a broad way. It seems probable, indeed, that the distribution of vegetation types is only rarely determined by any of the last-named conditions, though the detailed distribution of many species in any relatively small area is probably often related to chemical and mechanical influences.

The information so far accumulated upon environmental conditions has not been obtained primarily with reference to plant activities; it has been brought together mainly in the interest of meteorology, climatology, and weather prediction. Therefore it is impossible at present generally to select for study those conditions that directly affect the plant. We have been forced, in the main, to study conditions or factors that are more or less remote causes of the immediate conditions 
influencing plants. This is not always the case, however, for the air temperature of the climatologist and meteorologist is the temperature condition of the aerial environment of organisms, and the evaporating power of the air is a factor that directly affects the rate of water-loss from the aerial parts of plants and animals. Both of these immediate, and thus truly environmental, conditions we have been able to consider to some extent. On the other hand, such climatic factors as rainfall, humidity, vapor-tension of water, wind velocity, and duration of sunshine are all recognized climatic factors, concerning the distribution of the various intensities of which many data have been accumulated, but which have no direct influence upon plant activities. These climatic factors are very important, however, and often exert a controlling influence upon the more directly effective environmental conditions. Thus, the partial pressure of water-vapor in the air and the rate of air-movement influence the evaporating power of the surroundings. Rainfall greatly influences soil-moisture, and hence the ability of the soil to supply water to root surfaces, but it does not detcrmine this environmental condition, for other factors, such as the physical nature of the soil, its exposure, subterranean water-flow, etc., must be taken into consideration in this connection. It therefore became necessary not to restrict our studies to immediately effective conditions, but to consider in most cases the more remote climatic factors which meteorology and climatology have placed at our disposal.

The subterranean environment of plants has not, as yet, been studied in any way at all adequate to the present purpose, and our knowledge of the relation of this to plant distribution is still in the first stages of the purely observational phase. It has therefore been impossible for us to devote serious attention to this exceedingly important category of environmental conditions. Nevertheless, on account of a general similarity of the prevailing soils of most of the broad vegetational areas of the United States, our studies of the relation between the available measurements of the aerial conditions in connection with vegetational distribution are not as unsatisfactory as they might otherwise be. In the majority of the great regetational areas with which we have to deal, the prevailing soil is a clay or a clay loam, with usually a rather deep-lying subterranean watertable, and with a more or less pronounced admixture of organic matter, and the prevailing vegetational types are, in the majority of cases, found upon this character of soil. Exceptions to this generalization are swamps and marshes on the one hand and sandy regions on the other. The general vegetational type of broad marshlands appears to be about the same for a great range of acrial conditions; thus, these bear the same physiological types of plants under the climatic conditions of Baja California as under the very different ones of the southeastern coast. Also, the physiologieal characters of many plants 
of the sand dunes of the Atlantic coast, of the Great Lake region, and of the Southwest are very similar. Furthermore, the pine forest of the Southeast is characteristic only of sandy soils and has the same physiological character as have the sandy pine plains of New Jersey, Michigan, Wisconsin, etc. The heavier soils of these regions all bear a very different type of vegetation.

It has long been the practice of ecological and agricultural writers, in discussing any given region, to present rather elaborate tabulations of meteorological data as a sort of description of the region considered, and then to turn to the discussion of the vegetational phenomena in hand, usually without any attempt to correlate the two sets of descriptive data. There seems to have been no doubt that there is some sort of relation between vegetation and the usual sets of climatological observations, but each author has contented himself with presenting the results of such observations, apparently with a faith that someone in the future might be able to interpret them. It has appeared to us high time that some serious attempt were set on foot to develop promising methods for such interpretations.

It has therefore been necessary, throughout our studies of environment, to choose and devise methods for handling the climatic data that are at hand, so as to derive from them as much information as possible about their probable influence upon plants. In many cases this choice of method has been based mainly upon general physiological theory rather than upon actual knowledge, since, as has been pointed out, adequate results of actual tests of the influence of climatic conditions upon plants are not to be looked for until very special facilities for this work have become available. One of the ideal aims that we have held in view during the years which these studies have occupied is the ultimate attainment of what may be termed environmental formulas, which might express the minimum, optimum, and maximum for each of the effective environmental conditions for any given plant-form or vegetation type. It is clear that the time is not yet ripe for the establishment of more than tentative and general suggestions in this direction, but several such suggestions will appear in following pages. It is here to be emphasized, however, that such formulas are the legitimate end of such investigations as these. ${ }^{1}$

In the following sections we shall bring out the results that we have been able to obtain by various methods of treatment of the available climatological data. Most of these data have been obtained by the U. S. Weather Bureau for other purposes, commercial and political, as well as meteorological, as it appears. Our sources will be given in their proper places.

\footnotetext{
1 The idea of such formulas is not new; it has received attention, but from a different standpoint, from some of the phenologists. Simple formulas by which two factors are combined to give a single climatic index have been brought forward by Transeau, by Shreve, and by Livingston. These will be considered in another place.
} 
The present treatment is a purely geographical and climatological study, wherein it is sought to determine the approximate distribution of different climatic types in the United States. Since the aim of Part III of this publication will be to study the comparative distribution of vegetation types and climatic areas, it has seemed desirable to prepare many of our climatic charts upon a base-map showing by different patterns the distribution of the great general vegetational areas of the country. The discussion of the climatic ranges of the several vegetational types will be reserved for Part III.

The climatological charts themselves have been prepared, as far as possible, directly from the particular set of data involved, in the same manner as is the common practice in climatological work in general. The numerical data were first placed upon a copy of the Relief Map of the United States, of the U. S. Geological Survey, (17 by $27 \frac{3}{4}$ inches, hypsometrically colored and also furnished with contours for altitude), the numbers being written near the positions of the respective stations. Then the isoclimatic lines were sketched in in pencil and the map laid away for a time. At a later date it was worked over a second time and changes made that seemed to bring the lines nearer toward expressing the probable truth.

In this revision the topography of the country was constantly scrutinized and the contour-lines of the base-map were allowed to influence the course of the isoclimatic lines in many instances, especially where the stations for which data were at hand were too far apart to show the true directions of these lines. The preparation of charts of this sort is at best largely a matter of guesswork; information is not available for the plotting of climatic details. For meteorological purposes it is usually quite undesirable to have charts showing such details, but for our purposes they were quite essential. It thus became evident early in the present studies that the method commonly used in the drawing of meteorological charts-of carrying isoclimatic lines from plain to plain directly over high mountain ranges, with little or no attention to altitude - should not be resorted to here; at least it should not be used on so grand a scale as is common in meteorological work. Furthermore, the degree of approximation to the actual truth is surely often greatly increased by a due regard to topography, rather than by an almost blind following of inadequate climatic data modified only by a desire for lines as smooth as possible. If we are aiming at as true a picture of natural conditions as is attainable, it is obviously more undesirable to pass a given line over a locality where we are absolutely certain it should not pass than to draw it through some other area where it surely does pass and wherein only its proper placing is questionable. Thus, if there be given two stations with the same climatic datum, on either side of a range of mountains, it may be taken as certain that the conventional joining 
of these two points on the chart, by means of a line passing directly over the mountains, is absolutely certain to be wrong. On the other hand, whether to bend the connecting-line to right or left in passing around the range may not be apparent at all from the data of other stations, so that it might be drawn either way. Whicherer way the truth might require the line to be turned, it seems that either way is a better approximation than the method of directly connecting the two datum-points, which we may be sure is incorrect. Usually other climatic factors, known to be related to the one receiving attention, may be used to throw the weight of probability in one direction or the other.

In our charts we have followed this method of rational guessing and have tried not to pass isoclimatic lines through points where we are practically certain they do not belong. We have also attempted to interpret the various climatic features by as nearly similar criteria as are possible in the nature of the different cases, and have tried to correlate our guesses in regard to related climatic features. The future may be expected to show egregious errors in many instances, but the discovery of such errors may lead to progress.

After the revision of the charts, by means of the topographic contours and hypsometric coloring of the base-map, they were again laid aside and later were carefully scrutinized and corrected where apparently necessary, with reference to one another and to a large reliefmap of the United States. ${ }^{1}$ Only after these repeated studies and revisions had been accomplished were the climatological charts traced upon the generalized vegetation maps, or whatever base maps were requisite, and the lines inked in.

The charts resulting from the above-described methods of procedure are characterized by very irregular lines in the western part. While we are convinced that many of these western isoclimatic lines are quite probably wrongly drawn (the data at hand are so unsatisfactory and the available stations are of such inadequate number and distribution), yet we think that an attempt to interpret, as far as possible, the scanty information that is available should advance our knowledge of this important subject of climatological zonation more than would be possible from smoother lines drawn mainly without reference to topography, or from the complete omission of any attempt at a chart of these complex western regions.

As has been pointed out above, the drawing of isoclimatic lines is frequently a matter of selecting the most probable of several directions and positions, all of which are possible from the standpoint of the limited climatic data. Even with an excellent series of data, no two workers would place a given line in exactly the same position through-

\footnotetext{
1The "Relief map of the United States," Atlas School Supply Co., Chicago, on which altitude is actually expressed by magnified relief.
} 
out its course. This possible difference in the interpretations of two students is the more emphasized, the more complex are the climatic characters of the regions dealt with, and the less adequate are the data available. We have therefore become convinced that, for the sort of subject that is here involved, it is quite wide of the mark for a writer to transform his series of data into charted lines and to publish merely the chart. It is quite essential that the data themselves, on which the chart is based, be placed in the hands of those who are interested. In the climatic studies which follow, we have been careful to point out just how each series of data have been derived and have uniformly presented these data by means of tables. The positions of the stations for which data were employed are generally shown upon the charts by small circles.

In the following discussions, the different conditions or climatic features will be treated serially, under the three sectional headings. "Temperature," Moisture," and "Light," each one of which will be subdivided.

\section{TEMPERATURE CONDITIONS.}

\section{DURATION OF TEMPERATURE CONDITIONS.}

(A) PRELIMINARY CONSIDERATIONS.

Probably the most important environmental condition in the determination of plant distribution is the length of season or seasons, in each year, during which growth may occur. These are seasons, or time periods, during which every one of the factors of the surroundings exists in an intensity or quality such that growth activities can go forward. If a single factor were effective beyond the limits for growth-in its quality or intensity - then this must suffice to throw the plant into a dormant phase, in spite of the fact that other factors might still be favorable to growth activities.

Directly or indirectly, the ebb and flow of the environmental conditions affecting organic life are dependent upon astronomical causes, and the annual rhythm so commonly manifest in plant activities, as far as this is due to alterations in the surroundings, may be traced finally to the movement of the obliquely placed earth along its orbit and to the resulting procession of the equinoxes. This rhythm is always, then, either itself a temperature rhythm or else it is more or less directly connected with a temperature rhythm.

This fact that the seasonal temperature fluctuations stand in a casual relation to the fluctuations in the other environmental conditions has given us logical reason for basing many of our elinuatic studies upon the duration aspect of the temperature factor. To this must be added the practical reason that temperature fluctuations, in the United states as well as elsewhere, have been much more thoroughly 
studied than have the seasonal changes occurring in any other climatic factor. Still another consideration to be mentioned in this connection is this, that the most advanced modern civilizations of the world have developed in humid temperate regions, where temperature changes seem to be actually the immediate causes of the annual rhythm in plant activities, and hence the temperature-relation in its general aspect is more familiar and more useful to most of us than might be any other. Practically only in arid regions do moisture conditions play an important and direct rôle in determining the march of the natural vegetational seasons, and the light-relation as such is perhaps never important in this connection with outdoor vegetation. By greenhouse culture in temperate regions the summer temperature season is prolonged throughout the winter, and here alone is it noticeable that plants often suffer, during the early winter months, apparently for lack of light. In the open, however, whenever temperatures favorable to growth prevail, the light intensity is much greater than it is in winter in our greenhouses.

Since natural fluctuations in temperature are usually characterized by being gradual and continuous, it is quite impossible, even for a single year and for a single plant, to determine sharply what are the time limits of the growing-season. It is much more difficult to fix seasonal limits in general; the only method at all possible here is that of averages. Thanks to the elaborate routine observations continued through many years by the United States Signal Service and its successor, the United States Weather Bureau, a vast accumulation of temperature data are at hand for a large number of stations in the United States. From these it is possible to determine averages and means, of various types and by various procedures, which may be taken as fairly representative of the average conditions of the country throughout a series of years. It is these temperature data, and the results of various mathematical treatments of these, upon which we base our temperature considerations.

Data bearing upon plant activities, sufficiently detailed to be at all comparable to the temperature data just mentioned, are totally lacking, and must remain so long after the much-needed laboratories for the study of environmental relations shall have become available. While the discouraging character of this state of affairs is pronounced enough, nevertheless it need not cause us to refrain altogether from attempts to relate growth activities of plants to temperature conditions. Considerable preliminary information, and perhaps some that will later prove to be of a deeper-going sort, may be obtained if we merely approximate the temperature limits of general plant growth by a simple inspection of the knowledge which is available at present. Everyone who has dealt with plants at all, from the standpoint of the temperature relation, is convinced that the occurrence of a "killing 
frost." (in the agricultural sense) practically marks the end of the active season for the vast majority of plants. The average dates of occurrence of the last killing frost in spring and of the first in autumn should furnish us with a valuable index to the approximate length of the temperature season of general plant activity at any given station. It is of course well known that the growth activities of many plantforms are checked and that death of all but dormant phases frequently ensucs with temperatures far above those requisite for the recurrence of a killing frost. It is just as elear, however, that many forms make a considerable growth before and after the frostless season, which we consider as limited by the average dates of occurrence of the last and first killing frosts respectively.

It seems therefore safe, in default of any better means for improving our knowledge, to resort to the average length of the frostless season as the basis for the duration aspect of the temperature conditions in the United States. ${ }^{2}$ A somewhat detailed consideration of the frostless season in the United States will comprise the next following subsection.

It will have been remarked that no attention has here been directed toward an approximation of the upper temperature limit for plant growth. This matter will receive some consideration in the sequel, but it may be remarked here that it is not nearly so easy to attain to an approximation of this general maximum as of the general minimum for plant growth. This is partly due to the fact that the effect of freezing is quickly manifested upon many living plants, while injurious effects of high temperature are generally but slowly exhibited and therefore less readily observed. Furthermore, the response to frost is almost always a direct and unequivocal effect of the surrounding temperature upon the organism, while with high temperatures, alterations in the transpiration-rate, and in the rate of possible watersupply to plant roots - alterations in the water-relation, in shortbecome quite hopelessly confused with the temperature effects. It seems probable that relatively few plants will be found that are directly prevented by high temperature from thriving anywhere in the United States. Wherever this appears to be the case, a more thorough examination of the facts has usually resulted in the conclusion that the high temperature is at least primarily effective only as the more remote climatic cause of an alteration in the moisture-relation.

\footnotetext{
${ }^{2}$ On the employment of the average length of the frostless season in this sort of studies, see the following: Livingston, B. E. Climatic areas of the United States as related to plant growth; invitation paper read before the American Philosophical Society, Philadelphia, April 1913, Proc. Amer. Phil. Soc. 52: 257-275, 1913.-Livingston and Livingston, 1913.-Fassig, O. L., The period of safo plant growth in Maryland and Delaware, Monthy Weather Rev.. 42: 152-158, 1914. Fassig calls attention to the fact that the occurrence of the last and first minimum of $32^{\circ} \mathrm{F}$. furnishes as good a criterion for the determination of the average length of the growing season, and perhaps a better one, than does the occurrence of actual killing frost.
} 
But the occurrence or non-occurrence of a given plant-form in a given region depends upon other features than length of the season of active growth. As was mentioned earlier, it frequently occurs that the limiting condition preventing the occurrence of a certain plant in any area is to be sought in the nature of the surroundings during a dormant period. The length of the season or seasons during which growth can not occur is perhaps frequently as important in plant distribution as is the duration of the growing-period itself. Also, if environmental conditions are adverse enough they may result in the destruction of plant protoplasm even in its dormant phases, and it thus becomes necessary to study the duration of extremely low temperature, a thing which it seems quite possible to do and to which we shall devote some space in the present section.

We turn our attention now to the variations in the length of the average frostless season throughout the United States.

\section{(B) THE LENGTH OF THE PERIOD OF THE AVERAGE FROSTLESS}

SEASON. (TABLE 2, PLATE 34.)

Although data for the determination of this exceedingly and very obviously fundamental condition of plant growth, whether it be agriculturally or ecologically considered, ${ }^{1}$ have been in existence and have been increasing in volume and in value for a long time, it is apparently not until very recently that the subject has received even cursory mention in the literature. Abbe (1909), in his excellent review of the literature upon the relation of climates to crops, already cited, makes no reference to the length of the frostless season as a climatological feature. As has been mentioned, this work should be connected with the date of its preface (1891) rather than with that of its long-delayed publication. It is thus highly improbable that this feature of climate had entered very seriously into the considerations of workers in climatology up to about the year 1891 .

The publication in 1906 of Henry's elaborate presentation of the climatological data available for the United States ${ }^{2}$ put the information upon spring and autumn frosts, that had been collected up to that time in this country, into a form so that it could be made use of. In the early stages of the studies reported in the present publication, in the winter of 1909-10, Mrs. Grace J. Livingston undertook to derive an approximation of the average length of frostless season for each station for which Henry gives the requisite data. This was done by

1 "Probably no factor in the study of climate from the standpoint of the agriculturalist should be given more consideration than the average length of the growing-season. This is the key to an actual knowledge as to the possibilities of success or failure in the production of crops, since in practically all portions of the United States agricultural products are menaced by frost at some period of their growth." (Day, F. C., Frost data of the United States, and length of the crop growing season, as determined from the average of the latest and earliest dates of killing frost, U. S. Dept. Agric., Weather Bu. Bull. V, 1911.)

'Henry, A. J., Climatology of the United States, U. S. Dept. Agric., Weather Bu. Bull. Q. 1906. 
determining the number of days intervening between the average dates of the last killing frost in spring and the first in autumn. - It was realized that these average dates for many of the stations were not based upon adequate observations, but it was thought desirable to make what use was possible of the data at hand as being the best that were available, and our studies progressed satisfactorily, using the lengths of the frostless season thus derived.

About $1910^{1}$ a series of frost data for the United States, much more complete than that presented by Henry, became available through the publication by the United States Weather Bureau of the 106 summaries by sections. In the following year appeared Day's "Frost Data of the United States" (loc. cit.,) which comprises several forms of frost charts, including one (Chart V) of the average length of the crop growing season, days. This bulletin contains no presentation of the data from which the charts were prepared and no reference to any publication wherein the student may find these, ${ }^{2}$ but, through the kindness of Professor Day, we have been informed that most of the data for the preparation of the charts were taken from the summary above mentioned.

Under the heading "Source of Data," the following statements are made in Bulletin V:

To secure data that would show more nearly the actual conditions that prevail in the fields, orchards, and gardens, the most extensive compilation of frost data yet undertaken by the Weather Bureau has been accomplished and the results have been spread upon the accompanying charts.

The data from approximately 1,000 of our cooperative stations having the longest records, usually from 10 to 30 years, except in the most newly settled localitics of the West, where records for shorter periods only are available, have been summarized, and the local conditions due to physical environments brought out in much greater detail than has heretofore been attempted.

These charts being based upon the results of observations made in the open country and therefore not subject to the artificial conditions prevailing in the large eities where the regular stations of the Bureau are mainly located, differ from any that have appeared in the past in that areas having peculiar climatic features not heretofore shown on sucb charts are now clearly set forth.

Chart V of Bulletin V shows isoclimatic lines, for the country east of the one hundred and third meridian, "the average length of the crop growing season, days, being the number of days between the arerage date of the last killing frost in spring and the arerage date of the first killing frost in autumn." The distance between any two adjacent lines

\footnotetext{
1 Summary of the elimatological data for the United States, by Sections, U. S. Dept. Acric., Weather Bur. This elabosate presentation of "all the available data as they stand" (reprint of section 1, page 1, introductory remarks) comprises 106 separate pamplalets, all of them without any date of publication. They include, for tho most part, data for periods extending through 1908 or 1909.$)$

${ }^{2}$ On page 1 of Bulletin VIl we find the somewhat unsatisfactory bit of information which follows: "The chnrt showing the average length in days of the crop growing season was prepared from a somewhat different list of stations than was used for the charts of average dates of frosts, hence an actual determination of the length of the senson from charts I and it might differ a few days from the date shown on chart $v . "$ But the data are not given for any of the charts.
} 
represents a variation of 10 days in the average length of the frostless season. West of the one-hundred-and-third meridian the stations are so few, so poorly located, and the periods through which observations have been accumulated are in many cases so short, that it was not deemed advisable to continue the chart farther west. Here, however, the data themselves are placed upon the map.

The chart just described is the first one of the length of the frostless season to be published for the United States, and its appearance is to be considered as marking a very great step in advance in the climatology of the country. It should be of great value to agriculturists and ecologists, and it is to be hoped that the future may witness more uniformity and completeness in the keeping of the records from which a more perfect chart of this feature, not unaccompanied by an adequate tabulation of the source data, may evenually be obtained.

Since our own studies have been so largely based ur on the average length of the frostless season as the duration factor for many climatic conditions, the bare chart (as presented by Day) has been of comparatively little value in our work. After the publication of the Summary by Sections, Mrs. Livingston made a recalculation of most of the great mass of derived data that had already been prepared, using the new average lengths of the frostless season obtained from the average dates of the summary. The result exhibited some considerable modifications in our derived data. Since the information of the summary is later and much more complete than that given by Henry, we have adopted the data from the latter source as the basis of much of our work, using those from Henry's Bulletin Q only for a few stations for which the summary fails to give frost data and for which these are furnished by the other source.

In table 2 are presented all the frost data that have been used in our studies. In the first column are given the names of the stations, alphabetically arranged under each State, the States being also alphabetically arranged. An $n$ after the station name denotes that the observations were made in the vicinity of the place marked. In the second column are given the altitudes of the stations (in feet) so far as these have been available. ${ }^{1}$ The third and fourth columns, respectively, contain the average data of the last killing frost in spring and of the first killing frost in autumn. These data are quoted directly from the Summary by Sections (the number of the section in which the station occurs being given in parentheses directly after the station name) excepting in relatively few cases (indicated by an $\mathrm{H}$ after the station name in the first column), where the average dates have been obtained from Henry's Climatology of the United States. In a few

${ }^{3}$ These altitudes have been obtained from several sources and we have been unable to verify all of them. If there are errors in some cases they are probably but slight ones. 


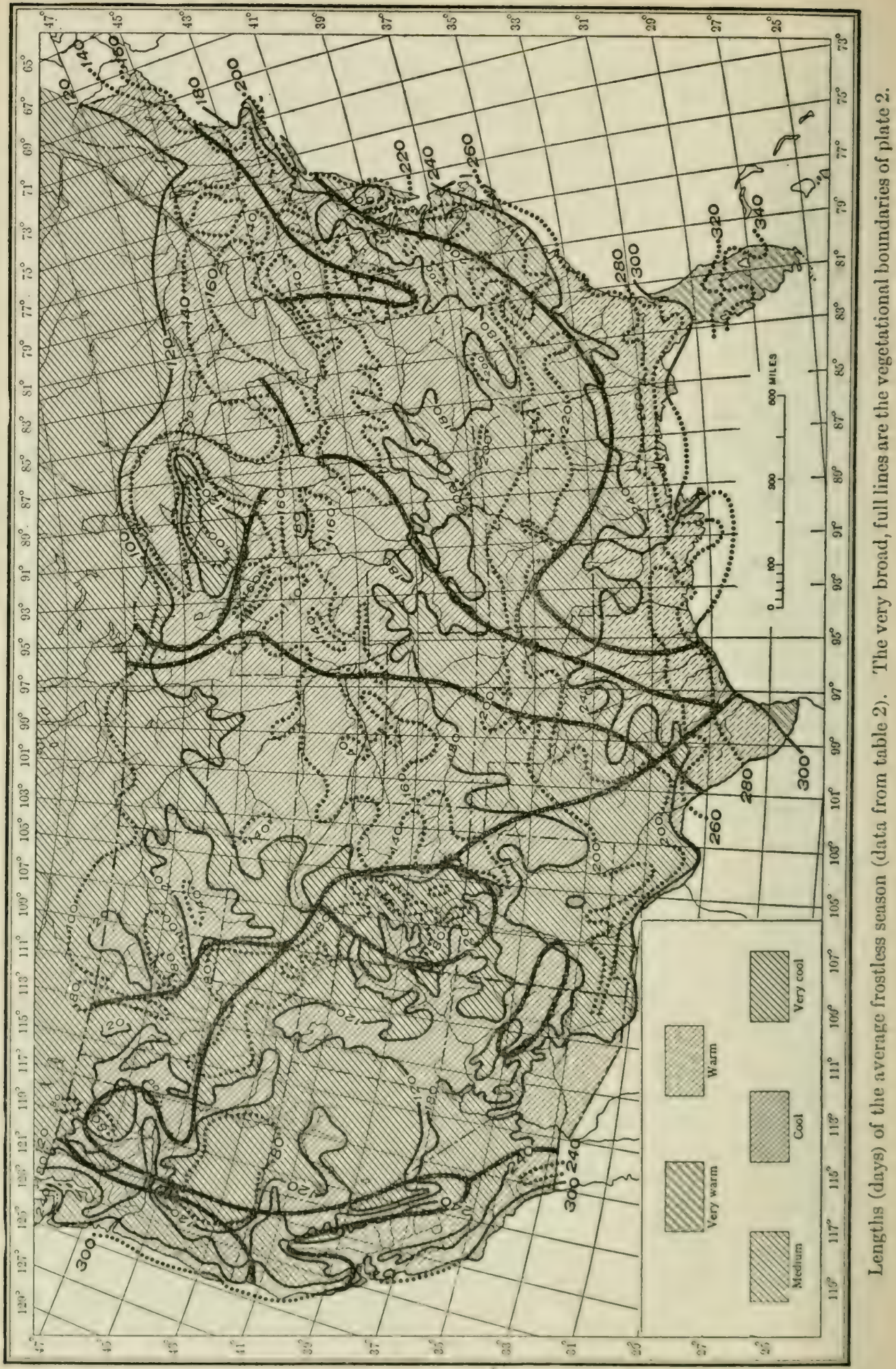


cases frosts are so infrequent that no average dates can yet be obtained. In such cases the words "very infrequent" are entered in these columns. The frostless season should be considered as approximately the entire year in these cases. Similarly, for a few stations the words "possible throughout year" indicate that the data at hand do not indicate any reliable average dates of last and first killing frosts and that the average frostless season is either very short or absent. For such stations the duration factor which we are considering should be regarded as practically nil. The fifth column of table 2 gives the average length of the frostless season (in days) for each station, this being derived from the dates of the third and fourth columns, not employing the first date, but including the last.

TABLE 2.-Frost data and length of average frostless season for 1803 stations in the United States. (Plate 34.)

\begin{tabular}{|c|c|c|c|c|c|}
\hline \multirow{2}{*}{ Station. } & \multirow{2}{*}{ Altitude. } & \multirow{2}{*}{$\begin{array}{l}\text { No. of } \\
\text { years of } \\
\text { record. }\end{array}$} & \multicolumn{2}{|c|}{ Average date of - } & \multirow{2}{*}{$\begin{array}{l}\text { Length of } \\
\text { average } \\
\text { frostless } \\
\text { season. }\end{array}$} \\
\hline & & & $\begin{array}{l}\text { Last frost } \\
\text { in spring. }\end{array}$ & $\begin{array}{l}\text { First frost } \\
\text { in autumn. }\end{array}$ & \\
\hline Alabama: & feet. & & & & days. \\
\hline Anniston (82)a........ & 741 & 18 & Apr. 2 & Oct. 20 & 201 \\
\hline Asheville (82) . . . . . . . . & 685 & 16 & Mar. 31 & Oct. 27 & 210 \\
\hline Birmingham (81)....... & 700 & 21 & Mar. 19 & Nov. 5 & 231 \\
\hline Camp Hill (82)......... & 738 & 8 & Mar. 19 & Nov. 2 & 228 \\
\hline Clanton (82).......... & 590 & 16 & Mar. 24 & Nov. 6 & 227 \\
\hline Decatur $(81) \ldots \ldots \ldots \ldots$ & 573 & 27 & Apr. 5 & Oct. 15 & 193 \\
\hline Eufaula $(82) \ldots \ldots \ldots \ldots$ & 200 & 25 & Mar. 14 & Nov. 9 & 240 \\
\hline Evergreen (82)......... & 285 & 25 & Mar. 13 & Nov. 12 & 244 \\
\hline Flomaton $(82) \ldots \ldots \ldots$ & 91 & 18 & Mar. 5 & Nov. 20 & 260 \\
\hline Florence $(81) \ldots \ldots \ldots \ldots$ & 563 & 25 & Apr. 1 & Oct. 30 & 212 \\
\hline Gadsden $(82) \ldots \ldots \ldots \ldots$ & 621 & 23 & Mar. 29 & Oct. 29 & 214 \\
\hline Goodwater (82)......... & 826 & 14 & Mar. 20 & Nov. 9 & 234 \\
\hline Greensboro (81)......... & 220 & 27 & Mar. 20 & Nov. 8 & 233 \\
\hline Healing Springs (81).... . & $\ldots \ldots \ldots \ldots$ & 10 & Mar. 6 & Nov. 17 & 256 \\
\hline Highland Home (82)..... & $\ldots \ldots \ldots \ldots$ & 17 & Mar. 15 & Nov. 21 & 251 \\
\hline Livingston (81) .......... & 160 & 25 & Mar. 17 & Nov. 3 & 231 \\
\hline Maple Grove (82)........ & $\cdots$ & 16 & Apr. 6 & Oct. 23 & 200 \\
\hline Mobile (82).............. & 57 & 38 & Feb. 24 & Nov. 30 & 279 \\
\hline Montgomery (82)........ & 240 & 37 & Mar. 10 & Nov. 8 & 243 \\
\hline Oneonta $(81) \ldots \ldots \ldots \ldots$ & 857 & 15 & Apr. 10 & Oct. 15 & 188 \\
\hline Opelika (82)............ & 817 & 30 & Mar. 17 & Nov. 9 & 237 \\
\hline Ozark (82) ............. & 400 & 7 & Mar. 10 & Nov. 13 & 248 \\
\hline Pine Apple (82) ....... & $\ldots \ldots \ldots$ & 20 & Mar. 14 & Nov. 10 & 241 \\
\hline Pushmataha $(\mathrm{H}) \ldots$. & $\cdots \cdots$ & ... & Mar. 21 & Nov. 12 & 236 \\
\hline Rock Mills (82) ... . . . & $\cdots \cdots$ & 8 & Mar. 24 & Nov. 6 & 227 \\
\hline Scottsboro (81)...... & 652 & 25 & Apr. 10 & Oct. 28 & 201 \\
\hline Selma $(82) \ldots \ldots \ldots \ldots$ & 147 & 34 & Mar. 13 & Nov. 8 & 240 \\
\hline Thomasville $(81) \ldots \ldots \ldots$ & 385 & $\begin{array}{l}20 \\
18\end{array}$ & $\begin{array}{l}\text { Mar. } 26 \\
\text { Mar. } 15\end{array}$ & $\begin{array}{ll}\text { Uet. } & 30 \\
\text { Noy } & 8\end{array}$ & $\begin{array}{l}218 \\
238\end{array}$ \\
\hline Tuscaloosa $(81) \ldots \ldots \ldots$. & 230 & 28 & Mar. 23 & Nov. & 228 \\
\hline Union Springs $(82) \ldots \ldots \ldots$ & 216 & 22 & Mar. 9 & Nov. 18 & 254 \\
\hline Uniontown (82)......... & 273 & 23 & Mar. 15 & Nov. 8 & 238 \\
\hline Valley Head (82) ........ & 1,031 & 24 & Apr. 5 & Oct. 20 & 198 \\
\hline
\end{tabular}

aWere the frost dates have been obtained from the Summary by Sections the number of the section in which any station occurs is placed in parentheses directly after the station name. Where the dates have been obtained from Henry's Climatology of the United States this fact is shown by $\mathrm{H}$ in parentheses after the station name. The letter $n$ in parentheses after a station name means that the data were obtained from a location in the immediate vicinity of the station named. 
TABle 2.-Frost data and length of average frostless season for 1803 stations in the United States. (Plate 34.)-Continued.

\begin{tabular}{|c|c|c|c|c|c|}
\hline \multirow{2}{*}{ Station. } & \multirow{2}{*}{ Altitude. } & \multirow{2}{*}{$\begin{array}{l}\text { No. of } \\
\text { years of } \\
\text { record. }\end{array}$} & \multicolumn{2}{|c|}{ Average date of - } & \multirow{2}{*}{$\begin{array}{l}\text { Length of } \\
\text { average } \\
\text { frostless } \\
\text { season. }\end{array}$} \\
\hline & & & $\begin{array}{l}\text { Last frost } \\
\text { in spring. }\end{array}$ & $\begin{array}{l}\text { First frost } \\
\text { in autumn. }\end{array}$ & \\
\hline Arizona: & feet. & & & & days. \\
\hline Arizona Canal Co. Dam (3) & 1,372 & 10 & Feb. 15 & Dec. 18 & 296 \\
\hline Benson (3) ............ & 3,523 & 7 & Mar. 20 & Nov. 10 & 235 \\
\hline Casa Grande (3)......... & 1,396 & 5 & Mar. 5 & Nov. 22 & 262 \\
\hline Columbia (4)........... & 1,900 & 8 & Mar. 20 & Nov. 22 & 247 \\
\hline Congress (4)........... & 3,688 & 8 & Feb. 20 & Nov. 30 & 283 \\
\hline Dudleyville (3).......... & 2,360 & 13 & Mar. 30 & Nov. 13 & 228 \\
\hline Flagstaff (4)........... & 6,907 & 11 & June 7 & Sept. 20 & 105 \\
\hline Ft. Apache (3) ......... & 5,200 & 14 & May 10 & Oct. 13 & 156 \\
\hline Fort Defiance $(\mathbf{H}) \ldots \ldots$. & 6,850 & $\ldots$ & June 2 & Sept. 23 & 113 \\
\hline Fort Grant (3) ......... & 4,916 & 14 & Apr. 1 & Nov. 26 & 239 \\
\hline Fort Hauchuca (3)....... & 5,100 & 13 & Apr. 5 & Nov. 28 & 237 \\
\hline Fort Mohave (4)........ & 604 & 8 & Feb. 16 & Nov. 21 & 278 \\
\hline Gila Bend (3) .......... & 737 & 4 & Jan. 27 & Nov. 28 & 305 \\
\hline Grand Canon (4)....... & 6,866 & 8 & June 16 & Sept. 25 & 101 \\
\hline Holbrook (4)........... & 5,069 & 8 & May 19 & Oct. $\quad 5$ & 139 \\
\hline Jerome (4) . . . . . . . . . . . & 4,743 & 8 & Apr. 8 & Nov. 6 & 212 \\
\hline Keams Canyon (4)....... & 3,326 & 3 & June 10 & Sept. 24 & 106 \\
\hline Kingman (4) ........... & 3,326 & 8 & Apr. 21 & Nov. 8 & 201 \\
\hline Maricopa (3)........... & 1,173 & 8 & Mar. 3 & Nov. 23 & 265 \\
\hline Oracle $(3) \ldots \ldots \ldots \ldots . . . .$. & 4,500 & 13 & Mar. 29 & Dec. 4 & 250 \\
\hline Parker $(4) \ldots . . . \ldots \ldots$ & 345 & 8 & Mar. 1 & Nov. 22 & 266 \\
\hline Phoenix (3)........... & 1,108 & 8 & Feb. 23 & Dec. 3 & 283 \\
\hline Prescott (4)............ & 5,320 & 8 & May 21 & Sept. 29 & 131 \\
\hline St. Johns (4) ......... . . & 5,650 & 8 & May 15 & Sept. 29 & 137 \\
\hline St. Michaels (4) ........ & 6,950 & 8 & June 7 & Sept. 17 & 102 \\
\hline San Simon (3).......... & 3,609 & 5 & Apr. 3 & Oct. 23 & 203 \\
\hline Seligman (4)........... & 5,219 & 4 & May 29 & Sept. 26 & 120 \\
\hline Showlow (4) .......... & 6,300 & 4 & Apr. 28 & Oct. $\quad 9$ & 164 \\
\hline Signal (4) ............. & 1,652 & 5 & Mar. 27 & Nov. 13 & 231 \\
\hline Supai (4)............. & 3,200 & 3 & Feb. 25 & Nov, 18 & 266 \\
\hline 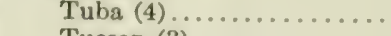 & 4,500 & 8 & May 1 & Oct. 3 & 155 \\
\hline Tucson (3)............. & 2,390 & 14 & Mar. 26 & Nov. 22 & 241 \\
\hline Willeox (3),........... & 4,164 & 8 & Apr. 11 & Nov. 5 & 208 \\
\hline Williams (4) ........... & 6,750 & 6 & June 4 & Sept. 22 & 110 \\
\hline Young (4) ........... & 4,400 & 5 & May 14 & Oct. $\quad 2$ & 141 \\
\hline Arkansas: & & & & & \\
\hline Amity (47). & 250 & 12 & Apr. 13 & Oct. 28 & $19 S$ \\
\hline Arkadelphia (47) ..... & 250 & 10 & Mar. 26 & Nov. & 227 \\
\hline Batesville (48) ....... & 271 & 10 & Apr. 9 & Nov. & 207 \\
\hline Bee Branch (48) ..... & ... & 12 & Apr. & Oct. 31 & 206 \\
\hline Brinkley (48) ......... & 226 & 12 & Mar. 27 & Oct. $2 S$ & 215 \\
\hline Camden $(47) \ldots \ldots \ldots \ldots$ & 158 & 12 & Mar. 17 & Nov. & 237 \\
\hline Conway $(48) \ldots \ldots \ldots \ldots$ & 309 & 12 & Mar. 27 & Nov. & 222 \\
\hline Corning (48) ........... & 293 & 12 & Mar. 28 & Oct. 20 & 207 \\
\hline Dallas $(\mathbf{H}) \ldots \ldots \ldots$ & 1,100 & ... & Apr. & Nov. & 214 \\
\hline Dodd City (48).......... & 1,175 & 12 & Apr. 20 & Oct. 19 & 182 \\
\hline Fayettovillo (48)..... & 1,451 & 12 & Apr. 14 & Oct. 27 & 196 \\
\hline Forrest City (48).... & 286 & 11 & Mar. $2 S$ & Nov. 2 & 219 \\
\hline Fort Smith (47).... & 481 & 27 & Mar. 21 & Nov. & 230 \\
\hline Holens $(48) \ldots . . . .$. & 182 & 12 & Mar. 22 & Nov. 8 & 231 \\
\hline Hope $(47) \ldots . . . .$. & 377 & 5 & Mar. 7 & Oct. 27 & 235 \\
\hline Hot Springs (17) ........ & 600 & 5 & Apr. & Oct. 26 & 205 \\
\hline Jonestoro (18) ........... & 345 & 12 & Mnr. 30 & Oet. 30 & 214 \\
\hline LaCrosse (48) ....... & ... & 12 & Apr. 9 & Oct. 29 & 203 \\
\hline Little Rock $(47) \ldots . .$. & 357 & 30 & Mar, 19 & Nov. 11 & 237 \\
\hline
\end{tabular}


TABLE 2.-Frost data and length of average frostless season for 1803 stations in the United States. (Plate 34.)-Continued.

\begin{tabular}{|c|c|c|c|c|c|}
\hline \multirow{2}{*}{ Station. } & \multirow{2}{*}{ Altitude. } & \multirow{2}{*}{$\begin{array}{l}\text { No. of } \\
\text { years of } \\
\text { record. }\end{array}$} & \multicolumn{2}{|c|}{ Average date of- } & \multirow{2}{*}{$\begin{array}{c}\text { Length of } \\
\text { average } \\
\text { frostless } \\
\text { season. }\end{array}$} \\
\hline & & & $\begin{array}{l}\text { Last frost } \\
\text { in spring. }\end{array}$ & $\begin{array}{l}\text { First frost } \\
\text { in autumn. }\end{array}$ & \\
\hline Arkansas-Continued: & feet. & & & & days. \\
\hline Malvern (47) ........... & 277 & 12 & Mar. 28 & Nov. 1 & 218 \\
\hline Marvell $(48) \ldots \ldots \ldots \ldots$ & 200 & 12 & Mar. 22 & Oct. 30 & 222 \\
\hline Mena (47) ............. & 1,100 & 4 & Mar. 14 & Oct. 26 & 226 \\
\hline Mossville (48) ............ & & 12 & Apr. 9 & Oct. 30 & 204 \\
\hline Mount Nebo (47)......... & 1,750 & 11 & Mar. 30 & Nov. 7 & 222 \\
\hline Newport $(48) \ldots \ldots \ldots \ldots$ & 231 & 12 & Mar. 27 & Oct. 31 & 218 \\
\hline 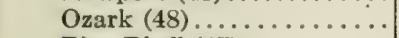 & 377 & 12 & Mar. 21 & Nov. 5 & 229 \\
\hline Pine Bluff (47) . . . . . . & 215 & 12 & Mar. 26 & Nov. 2 & 221 \\
\hline Pocahontas $(48) \ldots \ldots \ldots$. & .... & 12 & Apr. 3 & Oct. 28 & 208 \\
\hline Prescott $(47) \ldots \ldots \ldots \ldots$ & 327 & 12 & Mar. 20 & Nov. 4 & 229 \\
\hline Rogers $(48) \ldots \ldots \ldots \ldots$ & 1,385 & 12 & Apr. 13 & Oct. 20 & 190 \\
\hline Russellville (48) ......... & 348 & 12 & Apr. 5 & Oct. 28 & 206 \\
\hline Spielerville (47) ........ & 1,050 & 12 & Mar. 23 & Nov. 2 & 224 \\
\hline Stuttgart $(48) \ldots \ldots \ldots \ldots$ & 495 & 12 & Mar. 26 & Oct. 29 & 217 \\
\hline Texarkana $(47) \ldots \ldots \ldots$ & 332 & 12 & Mar. 20 & Nov. 9 & 234 \\
\hline Warren $(47) \ldots \ldots \ldots \ldots$ & 304 & 12 & Mar. 27 & Nov. & 219 \\
\hline Wiggs $(47) \ldots \ldots \ldots \ldots \ldots$ & & 12 & Apr. 6 & Oct. 24 & 201 \\
\hline California: & & & से & & \\
\hline Antioch (14) & 46 & .. & Feb. 22 & Dec. & 289 \\
\hline Aptos (14)... & 102 & $\ldots \ldots \ldots$ & Mar. 25 & Nov. 17 & 237 \\
\hline Auburn (15). & 1,360 & $\ldots \ldots \ldots$ & Jan. 21 & Dec. 25 & 338 \\
\hline Ben Lomond (14)........ & 300 & $\ldots \ldots \ldots$ & Mar. 15 & Nov. 7 & 237 \\
\hline Berkeley (14)........... & 320 & $\ldots \ldots \ldots$ & Jan. 28 & Dec. 15 & 321 \\
\hline Blocksburg (16) ......... & - & $\ldots \ldots \ldots$ & Apr. 7 & Nov. 2 & 209 \\
\hline Boulder Creek (14)....... & 470 & $\ldots \ldots \ldots$ & Mar. & Oct. 29 & 236 \\
\hline Bowman's Dam (14)..... & 5,500 & $\ldots \ldots \ldots$ & Apr. 26 & Oct. 25 & 182 \\
\hline Branscomb (16).......... & 2,000 & $\ldots \ldots \ldots$ & Apr. 9 & Nov. 9 & 214 \\
\hline Byron (14)............. & 33 & $\ldots \ldots \ldots$ & Feb. & Dec. & 300 \\
\hline Campbell (14)........... & 217 & $\ldots \ldots \ldots$ & Mar. 23 & Nov. 22 & 244 \\
\hline Cedarville $(15) \ldots \ldots \ldots \ldots$ & 4,675 & $\ldots \ldots \ldots$ & Mar. 15 & Oct. 6 & 205 \\
\hline 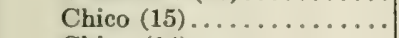 & 189 & $\ldots \ldots \ldots$ & Mar. 27 & Dec. & 254 \\
\hline Chino (14) ............ & 714 & $\ldots \ldots \ldots$ & Feb. 28 & Dec. & 277 \\
\hline Claremont (14)......... & 1,200 & $\ldots \ldots \ldots$ & Mar. 17 & Dec. & 263 \\
\hline Cloverdale $(14,16) \ldots \ldots \ldots$ & 340 & $\ldots \ldots \ldots$ & Mar. 4 & Dec. & 277 \\
\hline 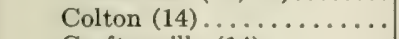 & 965 & $\ldots \ldots \ldots$ & Jan. 22 & Dec. & 316 \\
\hline Craftonville (14) .......... & 1,759 & $\ldots \ldots \ldots$ & Feb. 7 & Dec. 26 & 322 \\
\hline Crescent City (16)....... & 50 & $\ldots \ldots \ldots$ & May 10 & Nov. 27 & 201 \\
\hline Davisville $(\mathrm{H}) \ldots . . . .$. & 51 & $\ldots \ldots \ldots$ & Feb. 26 & Dec. 7 & 284 \\
\hline 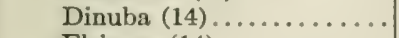 & 335 & $\ldots \ldots \ldots$ & May 2 & Nov. 27 & 209 \\
\hline Elsinore (14) ............ & 1,234 & $\ldots \ldots \ldots$ & Mar. 23 & Nov. 26 & 248 \\
\hline Eureka (16)............. & 64 & $\ldots \ldots \ldots$ & Mar. 29 & Nov. 29 & 245 \\
\hline Fallbrook (14)........... & 700 & $\ldots \ldots$ & Feb. 20 & Dec. 7 & 290 \\
\hline Fort Bragg (16) ......... & & $\ldots \ldots$ & Feb. 4 & Dec. & 307 \\
\hline Fort Ross (16).......... & 100 & $\ldots$ & Feb. 14 & Dec. 29 & 318 \\
\hline Fresno $(14) \ldots \ldots \ldots \ldots$ & 293 & $\ldots \ldots$ & Mar. & Nov. 14 & 258 \\
\hline Georgetown (15)......... & 2,650 & $\ldots \ldots$ & Mar. & Nov. 29 & 270 \\
\hline Gilroy (14)............. & 193 & $\ldots \ldots$ & Feb. 25 & Nov. 5 & 253 \\
\hline Guinda (14)......... & 350 & $\ldots \ldots$ & Mar. 14 & Nov. 30 & 261 \\
\hline Hanford (14)....... & 249 & $\ldots \ldots \ldots$ & Mar. 16 & Nov. 9 & 237 \\
\hline Healdsburg (16)... & 52 & $\ldots \ldots \ldots$ & Apr. & Nov. 16 & 222 \\
\hline Hollister $(\mathbf{H}) \ldots . .$. & 284 & $\ldots \ldots \ldots$ & Mar. 16 & Nov. 23 & 252 \\
\hline Hullville (16). & & $\ldots \ldots \ldots$ & June 10 & Sept. 8 & 90 \\
\hline Independence $(\mathrm{H}) \ldots$ & 3,884 & $\ldots \ldots \ldots$ & Mar. 17 & Oct. 25 & 222 \\
\hline Kernville (14)..... & 2,600 & $\ldots$ & May 10 & Sept. 27 & 140 \\
\hline Kingsburg (14)......... & 301 & $\ldots \ldots \ldots$ & Feb. 28 & Dec. 27 & 302 \\
\hline
\end{tabular}


TABLE 2.-Frost data and length of average frostless season for 1803 stations in the United States. (Plate 34.)-Continued.

\begin{tabular}{|c|c|c|c|c|c|}
\hline \multirow{2}{*}{ Station. } & \multirow{2}{*}{ Altitude. } & \multirow{2}{*}{$\begin{array}{l}\text { No. of } \\
\text { years of } \\
\text { record. }\end{array}$} & \multicolumn{2}{|c|}{ Average date of- } & \multirow{2}{*}{$\begin{array}{c}\text { Length of } \\
\text { average } \\
\text { frostless } \\
\text { season. }\end{array}$} \\
\hline & & & $\begin{array}{l}\text { Last frost } \\
\text { in spring. }\end{array}$ & $\begin{array}{l}\text { First frost } \\
\text { in autumn. }\end{array}$ & \\
\hline California-Continued: & feet. & & & & days. \\
\hline LaPorte $(15) \ldots . . . . .$. & 5,000 & $\ldots \ldots \ldots$ & May 30 & Oct. 9 & 132 \\
\hline Lemon Grove (14) ........ & 600 & $\ldots \ldots \ldots$ & Feb. 22 & Dec. 10 & 291 \\
\hline Lick Observatory (14).... & 4,209 & $\ldots \ldots \ldots$ & Mar. 28 & Oct. 11 & 197 \\
\hline Livermore $(\mathrm{H}) \ldots . . . \ldots$. & 485 & $\ldots \ldots \ldots$ & Feb. 23 & Dec. 2 & 282 \\
\hline Lodi (14)................ & 35 & $\ldots \ldots \ldots$ & Mar. 12 & Nov. 16 & 249 \\
\hline Los Angeles (14)......... & 293 & $\ldots \ldots \ldots$ & Jan. 27 & Dec. ${ }^{\circ} 27$ & 334 \\
\hline Los Gatos (14).......... & 600 & $\ldots \ldots \ldots$ & Jan. 22 & Dec. 14 & 326 \\
\hline Manzana (14)............. & 2,870 & $\ldots \ldots \ldots$ & Mar. 25 & Oct. 16 & 205 \\
\hline Menlo Park (14)......... & 64 & $\ldots \ldots \ldots$ & Jan. 22 & Dec. 24 & 336 \\
\hline Merced (14)............ & 173 & $\ldots \ldots \ldots$ & Apr. & Dec. & 240 \\
\hline Mills College (14)........ & 200 & $\ldots \ldots \ldots$ & Jan. 30 & Dec. 4 & 308 \\
\hline Milo $(14) \ldots \ldots \ldots \ldots \ldots$ & 1,600 & $\ldots \ldots \ldots$ & Feb. 16 & Nov. 29 & 286 \\
\hline Milton (n) (14).......... & 660 & $\ldots \ldots \ldots$ & Feb. & Nov. 29 & 294 \\
\hline Mokelumne (14).......... & 1,550 & $\ldots \ldots \ldots$ & Mar. 21 & Dec. 15 & 269 \\
\hline Monumental (16).......... & $\cdots \cdots$ & $\ldots \ldots \ldots$ & May 30 & Oct. 16 & 139 \\
\hline Mt. Tamalpais (16)....... & 2,375 & $\ldots \ldots \ldots$ & Mar. 3 & Dec. 13 & 285 \\
\hline 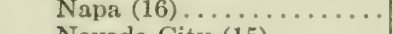 & 20 & $\ldots \ldots . .$. & Mar. 20 & Nov. 15 & 240 \\
\hline Nevada City (15)......... & 2,580 & $\ldots \ldots \ldots$ & May 8 & Oct. 19 & 164 \\
\hline Niles $(14) \ldots \ldots \ldots \ldots \ldots$ & 87 & $\ldots \ldots \ldots$ & Feb. 11 & Nov. 30 & 292 \\
\hline North Ontario (14)....... & 1,750 & $\ldots \ldots \ldots$ & Mar. 14 & Dec. 14 & 275 \\
\hline Oakland (14) ............ & 36 & $\ldots \ldots \ldots$ & Jan. & Dec. 20 & 347 \\
\hline Ontario (n) (14) .......... & 860 & ........... & Mar. & Dec. 16 & 284 \\
\hline Orleans (16) ............ & $\because{ }^{\prime}$ & .......... & Jan. 29 & Mar. 14 & 289 \\
\hline & 70 & $\ldots \ldots \ldots$ & Feb. 19 & Dec. 25 & 309 \\
\hline $\begin{array}{l}\text { Paso Robles (14)......... } \\
\text { Peachland (16) }\end{array}$ & 800 & (n......... & Apr. & Nov. 5 & 218 \\
\hline Placerville $(14) \ldots \ldots \ldots$ & 190 & $\cdots \cdots \cdots$ & Apr. & Nov, 21 & 221 \\
\hline Porterville $(14) \ldots \ldots \ldots$ & $\begin{array}{r}1,020 \\
461\end{array}$ & $\cdots \cdots \cdots$ & Mar. 15 & $\begin{array}{ll}\text { Dec. } & 31 \\
\text { Dec. } & 10\end{array}$ & $\begin{array}{l}291 \\
306\end{array}$ \\
\hline Poway $(14) \ldots \ldots \ldots \ldots$ & 460 & $\cdots \ldots$ & Jan. 26 & Dec. 3 & 311 \\
\hline Red Bluff (15)........... & 307 & ... & Mar. 27 & Dec. 16 & 264 \\
\hline Redding (15)........... & 552 & $\ldots \ldots \ldots \ldots$ & Feb. 20 & Nov. 27 & 280 \\
\hline Redlands $(\mathrm{H}) \ldots \ldots \ldots \ldots$ & 1,352 & $\ldots \ldots \ldots$ & Feb. 19 & Dec. 12 & 296 \\
\hline Sacramento (15)......... & 71 & $\ldots \ldots \ldots$ & Feb. 16 & Nov. 15 & 272 \\
\hline Salinas (14).............. & 40 & $\ldots \ldots \ldots$ & Feb. 18 & Dec. & 287 \\
\hline San Ardo (14) ............ & 236 & $\ldots \ldots \ldots$ & Mar. & Dec. 20 & 292 \\
\hline San Francisco (14)........ & 207 & $\ldots \ldots \ldots$ & Jan. 25 & Dec. 10 & 319 \\
\hline San Jacinto (14).......... & 1,550 & $\ldots \ldots \ldots$ & Mar. 27 & Nov. 20 & 235 \\
\hline San Jose $(14) \ldots \ldots \ldots . .$. & 95 & $\ldots \ldots \ldots$ & Feb. & Nov. 27 & 294 \\
\hline San Leandro (14)......... & 50 & $\ldots \ldots \ldots$ & Mar. & Dec. & 274 \\
\hline San Luis Obispo (14) ..... & 201 & $\ldots \ldots \ldots$ & Mar. & Nov. 18 & 260 \\
\hline San Mipuel (14) ......... & 616 & $\ldots \ldots \ldots$ & Feb. 22 & Nov. 13 & 264 \\
\hline Santa Barbara (14)...... & 130 & $\ldots \ldots \ldots$ & Jan. & Dec. 13 & 328 \\
\hline Santa Clara (14)......... & 90 & $\ldots \ldots \ldots$ & Feb. 27 & Nov. 25 & 271 \\
\hline Santa Cruz (14)........ & 20 & $\ldots \ldots \ldots$ & Mar. 10 & Dec. & 274 \\
\hline Santa Margarita (14).... & 996 & $\ldots \ldots$ & Feb. 15 & Nov. 25 & 283 \\
\hline Santa Paula (14)......... & 350 & $\ldots \ldots \ldots$ & Jan. 23 & Dec. 18 & 329 \\
\hline Santa Rosa (16)......... & 181 & $\ldots \ldots \ldots$ & Apr. 24 & Dec. 10 & 230 \\
\hline Sisson $(15) \ldots \ldots \ldots \ldots \ldots$ & 3,555 & $\ldots \ldots$ & Mar. 19 & Oct. $\quad 12$ & 207 \\
\hline Soledad $(14) \ldots . . . \ldots$ & 188 & $\ldots \ldots$ & Fcb. & Dec. & 301 \\
\hline 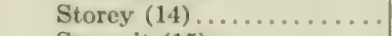 & 296 & & Feb. 23 & Dec. & 283 \\
\hline Summit (15).. & 7,017 & & Feb. 13 & Oct. $\quad 13$ & 242 \\
\hline Susanville (15).......... & 4,195 & & May 10 & Sept. 22 & 135 \\
\hline Tehachapi (14) .......... & 3,964 & $\ldots \ldots$ & Mar. 17 & Nov. $2 S$ & 256 \\
\hline Tracy $(14) \ldots \ldots \ldots \ldots$ & 64 & $\ldots \ldots \ldots$ & Feb. 17 & Nov. 18 & 274 \\
\hline Tulare $(14) \ldots . . . \ldots$ & 274 & $\ldots \ldots \ldots$ & Mar. & Nov. 15 & 253 \\
\hline
\end{tabular}


TABLE 2.-Frost data and length of average frostless season for 1803 stations in the United States. (Plate 34.)-Continued.

\begin{tabular}{|c|c|c|c|c|c|}
\hline \multirow{2}{*}{ Station. } & \multirow{2}{*}{ Altitude. } & \multirow{2}{*}{$\begin{array}{l}\text { No. of } \\
\text { years of } \\
\text { record. }\end{array}$} & \multicolumn{2}{|c|}{ Average date of - } & \multirow{2}{*}{$\begin{array}{l}\text { Length of } \\
\text { average } \\
\text { frostless } \\
\text { season. }\end{array}$} \\
\hline & & & $\begin{array}{l}\text { Last frost } \\
\text { in spring. }\end{array}$ & $\begin{array}{l}\text { First frost } \\
\text { in autumn. }\end{array}$ & \\
\hline California-Continued: & feet. & & & & days. \\
\hline 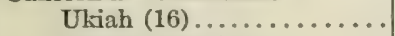 & 620 & $\ldots \ldots \ldots$ & Apr. 14 & Nov. & 201 \\
\hline Upper Lake (16).......... & 1,350 & & Mar. 30 & Nov. 13 & 228 \\
\hline Upper Mattole (16)....... & 244 & $\ldots \ldots$ & Mar. 7 & Nov. 18 & 256 \\
\hline Valley Springs (14)....... & 673 & $\ldots \ldots$ & Apr. 12 & Dec. 17 & 249 \\
\hline 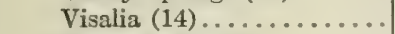 & 334 & & Mar. 13 & Nov. 27 & 259 \\
\hline Watsonville (14)......... & 23 & $\ldots$. & Mar. 8 & Nov. 3 & 240 \\
\hline Westpoint (14)......... & 2,326 & & Apr. 16 & Nov. & 206 \\
\hline Willows (15) ... & 136 & $\ldots \ldots$ & Jan. 22 & Dec. 11 & 323 \\
\hline Colorado: & & & & & \\
\hline Blaine (7) & 3,935 & & Apr. 29 & Oct. 11 & 165 \\
\hline Boulder (8). & 5,347 & 12 & Apr. 27 & Oct. 9 & 165 \\
\hline Canon City (7).. & 5,329 & ... & Apr. 29 & Oct. & 159 \\
\hline Castle Rock (8)......... & 6,220 & 15 & May 28 & Sept. 19 & 114 \\
\hline Cedar Edge (9).......... & 6,175 & 11 & May 20 & Sept. 23 & 126 \\
\hline Cheyenne Wells (38)...... & 4,279 & 15 & May 10 & Sept. 22 & 135 \\
\hline Collbran (9).............. & 6,000 & 8 & May 26 & Sept. 24 & 121 \\
\hline Colorado Springs (7)..... & 6,098 & & May 3 & Oct. 3 & 153 \\
\hline Cope $(8) \ldots \ldots \ldots \ldots \ldots$ & 4,400 & 11 & May & Sept. 30 & 145 \\
\hline Delta $(9) \ldots . . .$. & 4,965 & 13 & May 16 & Sept. 25 & 132 \\
\hline Denver $(8) \ldots \ldots \ldots \ldots$ & 5,272 & 38 & May 6 & Oct. 6 & 153 \\
\hline Durango (9) ............. & 6,534 & 12 & May 28 & Sept. 26 & 121 \\
\hline Fort Collins (8) .......... & 4,985 & 18 & May 9 & Sept. 24 & 138 \\
\hline Fort Morgan (8)......... & 4,338 & 13 & May & Sept. 28 & 143 \\
\hline Glen Eyrie (7) ......... & 6,500 & & May 12 & Sept. 27 & 138 \\
\hline Grand Junction (9) ...... & 4,608 & 16 & Apr. 18 & Oct. 18 & 183 \\
\hline Grand Valley (9)........ & 5,089 & 14 & May 10 & Sept. 29 & 142 \\
\hline Greeley (8)............. & 4,639 & 16 & May 6 & Oet. 1 & 148 \\
\hline Hamps (7).............. & 5,400 & $\ldots \ldots$ & May 17 & Sept. 24 & 130 \\
\hline Hoehne (7)............. & 5,721 & $\ldots \ldots \ldots$ & May 20 & Oct. 2 & 135 \\
\hline Holly (7) ............. & 3,380 & $\ldots \ldots \ldots$ & May 1 & Oct. & 153 \\
\hline Holyoke (8)............ & 3,745 & 9 & May & Sept. 22 & 136 \\
\hline Husted (7) ............ & 6,596 & .. & May 15 & Sept. 25 & 133 \\
\hline 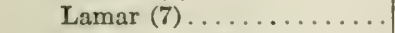 & 3,592 & ... & Apr. 27 & Oct. 3 & 159 \\
\hline Las Animas (7).......... & 3,899 & .. & May 2 & Oct. & 153 \\
\hline Lay $(9) \ldots . . . \ldots \ldots$ & 6,190 & 9 & June 16 & Sept. & 82 \\
\hline Le Roy $(8) \ldots \ldots \ldots \ldots$ & 4,380 & 16 & May 2 & Sept. 29 & 150 \\
\hline Long's Peak (n) (8)....... & 8,700 & 9 & June 26 & Sept. 2 & 68 \\
\hline Mancos (9)............. & 6,960 & 10 & June 9 & Sept. 17 & 100 \\
\hline Meeker (9) .............. & 6,183 & 14 & June 12 & Sept. 12 & 92 \\
\hline Montrose (9)............ & 5,811 & 10 & May 16 & Oct. 2 & 142 \\
\hline Moraine (8)............ & 7,750 & 12 & June 15 & Sept. 13 & 90 \\
\hline Pagoda (9).............. & 6,500 & 13 & June 12 & Sept. 3 & 83 \\
\hline Paonia (9)............. & 5,694 & 8 & May & Oct. & 151 \\
\hline Pueblo (7) .............. & 4,734 & .. & Apr. 27 & Oct. & 163 \\
\hline Rocky Ford (7)......... & 4,177 & T & May & Oct. & 154 \\
\hline Saguache $(H) \ldots \ldots \ldots$. & 7,740 & & May 24 & Sept. 17 & 116 \\
\hline Salida (7) ............. & 7,050 & $\ldots . .$. & May 31 & Sept. 20 & 112 \\
\hline - San Luis (H).......... & 7,794 & . & June & Sept. 11 & 94 \\
\hline Santa Clara (7).......... & 8,250 & 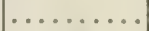 & June & Sept. 24 & 113 \\
\hline Silt (9).............. & 5,441 & 12 & May 21 & Sept. 27 & 129 \\
\hline Sugar Loaf $(8) \ldots . . . . .$. & 9,300 & 16 & June & Sept. 13 & 102 \\
\hline Trinidad (7) ............. & 5,994 & $\cdots$ & May & Oct. 7 & 159 \\
\hline T. S. Ranch (9) .......... & 5,200 & 8 & Apr. 27 & Oct. 10 & 166 \\
\hline Westcliffe (7) ........... & 7,864 & . & June 12 & Sept. 18 & 98 \\
\hline Wray $(8) \ldots . . . \ldots \ldots$ & 3,512 & 14 & May 8 & Sept. 27 & 142 \\
\hline
\end{tabular}


TABLE 2.-Frost data and length of average frostless season for 1803 stations in the United States. (Plate 34.)-Continued.

\begin{tabular}{|c|c|c|c|c|c|c|c|}
\hline \multirow{2}{*}{ Station. } & \multirow{2}{*}{ Altitude. } & \multirow{2}{*}{$\begin{array}{l}\text { No. of } \\
\text { years of } \\
\text { record. }\end{array}$} & \multicolumn{4}{|c|}{ Average date of- } & \multirow{2}{*}{$\begin{array}{l}\text { Length of } \\
\text { average } \\
\text { frostless } \\
\text { season. }\end{array}$} \\
\hline & & & $\begin{array}{l}\text { Last fros } \\
\text { in spring }\end{array}$ & & $\begin{array}{l}\text { First frc } \\
\text { in autur }\end{array}$ & $\begin{array}{l}\text { rost } \\
\text { mn. }\end{array}$ & \\
\hline Connecticut: & feet. & & & & & & days. \\
\hline Colchester (150) . & $370(\mathrm{H})$ & 22 & May & & Sept. & & 147 \\
\hline Cream Hill (105). & 1,300 & 11 & May & 1 & Sept. : & & 148 \\
\hline Hartford (105)... & 159 & 23 & Apr. 2 & & Oct. & 10 & 165 \\
\hline Middletown (105). & 125 & 17 & Apr. 2 & & Oct. & & 158 \\
\hline New Haven (105). & 107 & 36 & Apr. 2 & & Oct. & 17 & 180 \\
\hline New London $(\mathrm{H}) \ldots \ldots \ldots$ & 47 & & Apr. 1 & & Oct. & 18 & 186 \\
\hline North Grosvenor Dale (105) & 400 & 18 & Apr. 2 & & Oet. & 4 & 161 \\
\hline Norwalk $(\mathrm{H}) \ldots \ldots \ldots \ldots$ & 116 & .... & May & 1 & Oct. & 10 & 162 \\
\hline Southington $(\mathrm{H}) \ldots \ldots \ldots$ & 140 & & May 1 & & Oct. & 8 & 151 \\
\hline Storrs $(\mathrm{H}) \ldots \ldots \ldots$ & 640 & & May & 8 & Oct. & 10 & 155 \\
\hline Voluntown $(\mathrm{H})$. & 260 & & May 1 & & Sept. & 20 & 131 \\
\hline Waterbury $\mathrm{H}) \ldots$... & 400 & & May & & Oct. & 9 & 160 \\
\hline \multicolumn{8}{|l|}{ Delaware: } \\
\hline Milford (95) .. & 20 & 8 & Apr. 2 & & Oct. & 17 & 180 \\
\hline Millsboro (95). . & 20 & 14 & Apr. 2 & & Oct. : & 20 & 180 \\
\hline Newark (95)... & 136 & 12 & Apr. 1 & & Oct. & 17 & 183 \\
\hline Seaford (95)... & 40 & 14 & Apr. 2 & & Oct. & 21 & 183 \\
\hline \multicolumn{8}{|l|}{ Florida: } \\
\hline Apalachicola (82). & 24 & 5 & Jan. 3 & & Dec. & 6 & 310 \\
\hline Arcadia $(84) \ldots \ldots \ldots \ldots$ & 61 & 9 & Feb. 1 & & Dec. & 29 & 319 \\
\hline $\operatorname{Archer}(\mathrm{H}) \ldots$. & 92 & & Mar. & & Nov. & 28 & $26-1$ \\
\hline Avon Park (84). & 150 & 8 & Jan. & & Dec. & 22 & 348 \\
\hline Bartow (84)...... & 115 & 14 & Feb. 1 & & Dec. & 22 & 315 \\
\hline Brooksville (84).... . & 126 & 17 & Feb. 1 & & Dec. & 21 & 311 \\
\hline Carrabelle (83).. & 10 & 8 & Feb. 1 & 9 & Dec. & 18 & 302 \\
\hline Cedar Keys (83)..... & 6 & 11 & \multicolumn{5}{|c|}{ Very infre quent. } \\
\hline De Funiak Springs (82).. & 193 & 12 & Feb. 2 & 281 & Nov. & 17 & 262 \\
\hline Eustis $(\mathrm{H}) \ldots \ldots \ldots \ldots \ldots$ & 180 & & Feb. 1 & 18 & Dec. & $2 S$ & 313 \\
\hline Flamingo (84). & 4 & 7 & \multicolumn{5}{|c|}{ Very infre quent. } \\
\hline Ft. Myers (84).. & 12 & 17 & Jan. & 5 & Dec. & 14 & 343 \\
\hline Ft. Pierce (84).. & 6 & 8 & Jan. 1 & & Dec. & 31 & 347 \\
\hline Gainesville (83). & 176 & 12 & Feb. 2 & 20 & Dec. & 11 & 294 \\
\hline Huntington (83). & 51 & 11 & Feb. 1 & 18 & Dec. & 22 & 307 \\
\hline Jacksonville (83)..... & 8 & 53 & Feb. 1 & 14 & Dec. & 4 & 293 \\
\hline Jupiter ( $(x)$. & 28 & 21 & Feb. 1 & & Dec. & 29 & $31 \mathrm{~s}$ \\
\hline Key West (84). & 14 & 38 & No frost & & & & 365 \\
\hline Lake City (83).. & 210 & 16 & Mar. & 4 & Dec. & 2 & 273 \\
\hline Macclenny (83). & 125 & 12 & Mar. & 2 & Nov. & 25 & 268 \\
\hline Madison (83).... & 200 & 8 & Mar. & 1 & Nov. & 29 & 273 \\
\hline Malabar (84).. & 24 & 7 & \multicolumn{5}{|c|}{ Very infre quent. } \\
\hline Manatee (84)... & 8 & 17 & Jan. 2 & 26 & & 3 & 342 \\
\hline Marco $(84) \therefore$ & 5 & 6 & \multicolumn{4}{|c|}{ Very infre quent. } & \\
\hline Marianna (82). & 80 & 8 & Mar. & & & 21 & 265 \\
\hline Merritt's Island (84). & 20 & 17 & \multirow{2}{*}{\multicolumn{4}{|c|}{$\begin{array}{l}\text { Very infre quent. } \\
\text { Very infre quent. }\end{array}$}} & \\
\hline Miami (si). & 5 & 13 & & & & & \\
\hline Monticello (83).... & 207 & 5 & \multicolumn{4}{|c|}{ Very infre quent. } & \\
\hline New Smyrna (83)... . & 9 & 16 & Feb. 1 & 15 & Dec. & 23 & 311 \\
\hline Ocala (s3). & 83 & 18 & Feb. 1 & 17 & Dee. & 8 & 294 \\
\hline Orlando (84)......... & 111 & 17 & Feb. & 4 & Dec. & 20 & 319 \\
\hline Pensacola (83). & 12 & 31 & Feb. 2 & 23 & Dee. & 5 & 255 \\
\hline St. Augustine (83). & 10 & 14 & Feb. 1 & 15 & Dees: & 30) & 315 \\
\hline Stephensville (83). & 10 & 8 & Feh. 2 & 22 & Nor: & 30 & $2 \leqslant 1$ \\
\hline Tallahassee (83). . & 192 & 1s & Mar. & 3 & Dee. & 5 & 277 \\
\hline 'Tampa $(8.1) \ldots \ldots \ldots \ldots$ & 20 & 19 & Feb. & 8 & Jan. & 9 & 335 \\
\hline
\end{tabular}


TABLE 2.-Frost data and length of average frostless season for 1803 stations in the United States. (Plate 34.)-Continued.

\begin{tabular}{|c|c|c|c|c|c|}
\hline \multirow{2}{*}{ Station. } & \multirow{2}{*}{ Altitude. } & \multirow{2}{*}{$\begin{array}{l}\text { No. of } \\
\text { years of } \\
\text { record. }\end{array}$} & \multicolumn{2}{|c|}{ Average date of -} & \multirow{2}{*}{$\begin{array}{c}\text { Length of } \\
\text { average } \\
\text { frostless } \\
\text { season. }\end{array}$} \\
\hline & & & $\begin{array}{l}\text { Last frost } \\
\text { in spring. }\end{array}$ & $\begin{array}{l}\text { First frost } \\
\text { in autumn. }\end{array}$ & \\
\hline Georgia: & feet. & & & & days. \\
\hline Adairsville (85) ......... & 772 & 17 & Apr. & Oct. 27 & 205 \\
\hline Albany $(85) \ldots . . . . .$. & 230 & 10 & Mar. 6 & Nov. 11 & 250 \\
\hline Allapaha $(85) \ldots \ldots \ldots$. & 293 & 10 & Mar. 2 & Nov. 17 & 260 \\
\hline Americus (85) ........... & 362 & 14 & Mar. 8 & Nov. 13 & 250 \\
\hline Athens $(86) \ldots \ldots \ldots$. & 694 & 12 & Mar. 27 & Nov. 8 & 226 \\
\hline Atlanta (85)... & 1,218 & 18 & Mar. 23 & Nov. & 225 \\
\hline Augusta $(86) \ldots \ldots \ldots \ldots$ & 180 & 18 & Mar. 24 & Nov. 7 & 228 \\
\hline Bainbridge (85) .......... & 119 & 6 & Mar. 15 & Nov. 11 & 241 \\
\hline Blakely $(85) . . . . . . . .$. & 300 & 15 & Mar. 15 & Nov. 14 & 244 \\
\hline Camak (86)... & 613 & 10 & Mar. 25 & Nov. 3 & 223 \\
\hline Carrolton (85).. & ....... & 4 & Apr. 4 & Oct. 29 & 208 \\
\hline Clayton (86).... & 2,100 & 16 & Apr. 15 & Oct. 19 & 187 \\
\hline Columbus (85).. & 262 & 12 & Mar. 6 & Nov. 17 & 256 \\
\hline Covington (86) .......... & 800 & 14 & Mar. 31 & Nov. 8 & 222 \\
\hline Dahlonega $(86) \ldots \ldots \ldots$. & 1,519 & 18 & Apr. 7 & Oct. 31 & 207 \\
\hline Dudley $(86) \ldots . . . . .$. & ........ & 12 & Mar. 22 & Nov. 6 & 229 \\
\hline Eastman $(86) \ldots \ldots \ldots \ldots$ & 361 & 8 & Feb. 26 & Nov. 11 & 258 \\
\hline Eatonton $(86) \ldots \ldots \ldots$ & ..... & 6 & Mar. 19 & Nov. 4 & 230 \\
\hline Elberton (86)............ & 710 & 18 & Mar. 26 & Nov. 8 & 227 \\
\hline Forsyth (86) ........... & 735 & 9 & Mar. 24 & Oct. 31 & 221 \\
\hline Fort Gaines (85) ......... & 166 & 13 & Mar. 13 & Nov. 9 & 241 \\
\hline Gainesville (86) ......... & 1,254 & 14 & Mar. 31 & Nov. & 217 \\
\hline Gillsville $(86) \ldots \ldots \ldots$. . & 1,052 & 17 & Mar. 31 & Nov. 3 & 217 \\
\hline Greenbush (85)......... & $\cdots \ldots$ & 10 & Apr. 2 & Oct. 30 & 211 \\
\hline Greensboro (86)......... & 598 & 7 & Apr. 1 & Nov. 1 & 214 \\
\hline Griffin (85) ............ & 975 & 9 & Mar. 20 & Nov, 4 & 229 \\
\hline Harrison (86) ........... & 245 & 10 & Mar. 23 & Nov. 10 & 232 \\
\hline Hawkinsville (86)........ & 235 & 14 & Mar. 17 & Nov. 12 & 240 \\
\hline Jesup $(H) \ldots \ldots \ldots \ldots$ & 100 & .. & Mar. 17 & Nov. 20 & 248 \\
\hline Lost Mountain (85)....... & .... & 9 & Apr. 3 & Nov. 4 & 215 \\
\hline Louisville (86).......... & 259 & 17 & Mar. 15 & Nov. 10 & 240 \\
\hline Lumpkin (85) ............ & 650 & 15 & Mar. 17 & Nov. 11 & 239 \\
\hline Macon $(86) \ldots \ldots \ldots \ldots$ & 370 & 12 & Mar. 20 & Nov. 13 & 238 \\
\hline Marshallville (85) ......... & 500 & 17 & Mar. 19 & Nov. 6 & 232 \\
\hline Milledgeville (86) ........ & 276 & 13 & Mar. 22 & Nov. & 228 \\
\hline Monticello (86)........... & 800 & 11 & Mar. 17 & Nov. 13 & 241 \\
\hline Morgan (85)........... & 337 & 16 & Mar. 16 & Nov. 6 & 235 \\
\hline Newnan (85)... & 959 & 13 & Mar. 25 & Nov. & 228 \\
\hline Point Peter $(86) \ldots . . .$. & 1,000 & 18 & Apr. 1 & Nov. 1 & 214 \\
\hline Poulan $(85) \ldots \ldots \ldots \ldots$ & 365 & 17 & Mar. 16 & Nov. 9 & 238 \\
\hline Quitman (85).......... & 173 & 14 & Mar. 11 & Nov. 13 & 247 \\
\hline Ramsey (85) ........... & 1,363 & 14 & Apr. 14 & Oct. 18 & 187 \\
\hline Rome $(85) \ldots . . . \ldots \ldots$ & 576 & 17 & Mar. 31 & Nov. 1 & 215 \\
\hline St. Marys (86) . . . . . . . & 20 & 7 & Mar. 6 & Nov. 24 & 263 \\
\hline Savannah $(86) \ldots \ldots \ldots$. & 65 & 18 & Mar. 9 & Nov. 27 & 263 \\
\hline Statesboro $(86) \ldots \ldots \ldots \ldots$ & 253 & 9 & Mar. 9 & Nov. 13 & 249 \\
\hline Talbotton (85).......... & 750 & 16 & Mar. 25 & Nov. 9 & 229 \\
\hline Tallapoosa (85).......... & 1,150 & 10 & Apr. 7 & Oct. 30 & 206 \\
\hline Thomasville (85)........ & 273 & 12 & Mar. & Nov. 18 & 257 \\
\hline Valona $(86) \ldots \ldots \ldots \ldots$ & 12 & 9 & Mar. 11 & Nov. 17 & 251 \\
\hline Waycross (86).......... & 131 & 17 & Mar. 11 & Nov. 16 & 250 \\
\hline West Point (85)......... & 620 & 10 & Mar. 20 & Nov. 2 & 227 \\
\hline Idaho: & & & & & \\
\hline American Falls (22). & 4,341 & 15 & May 27 & Sept. 8 & 104 \\
\hline Blackfoot (H). & 4,503 & & May 29 & Sept. 12 & 106 \\
\hline
\end{tabular}


TABLE 2.-Frost data and length of average frostless season for 1803 stations in the United Slates. (Plate 34.)-Continued.

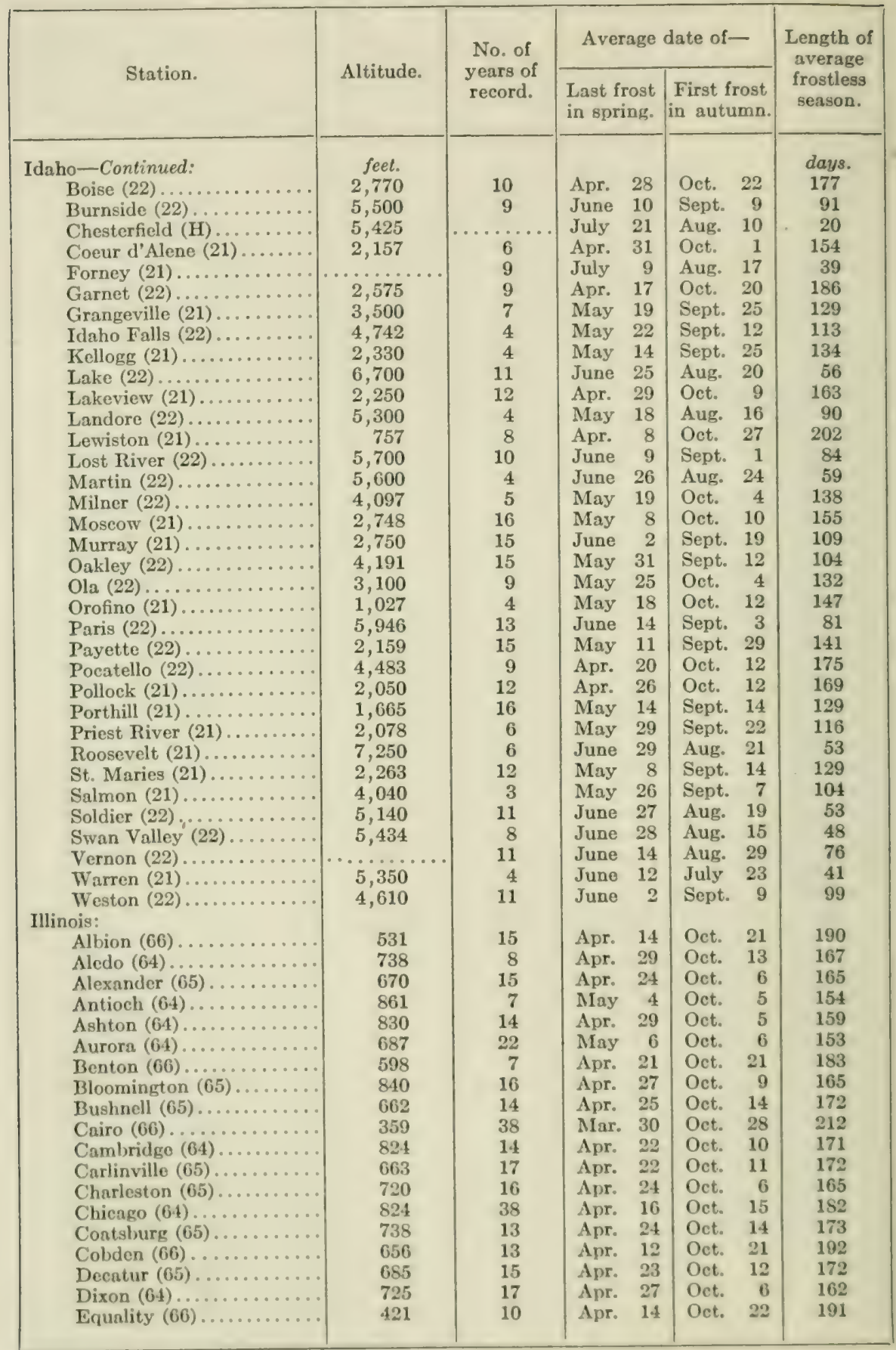


TABLE 2.-Frost data and length of average frostless season for 1803 stations in the United States. (Plate 34.)-Continued.

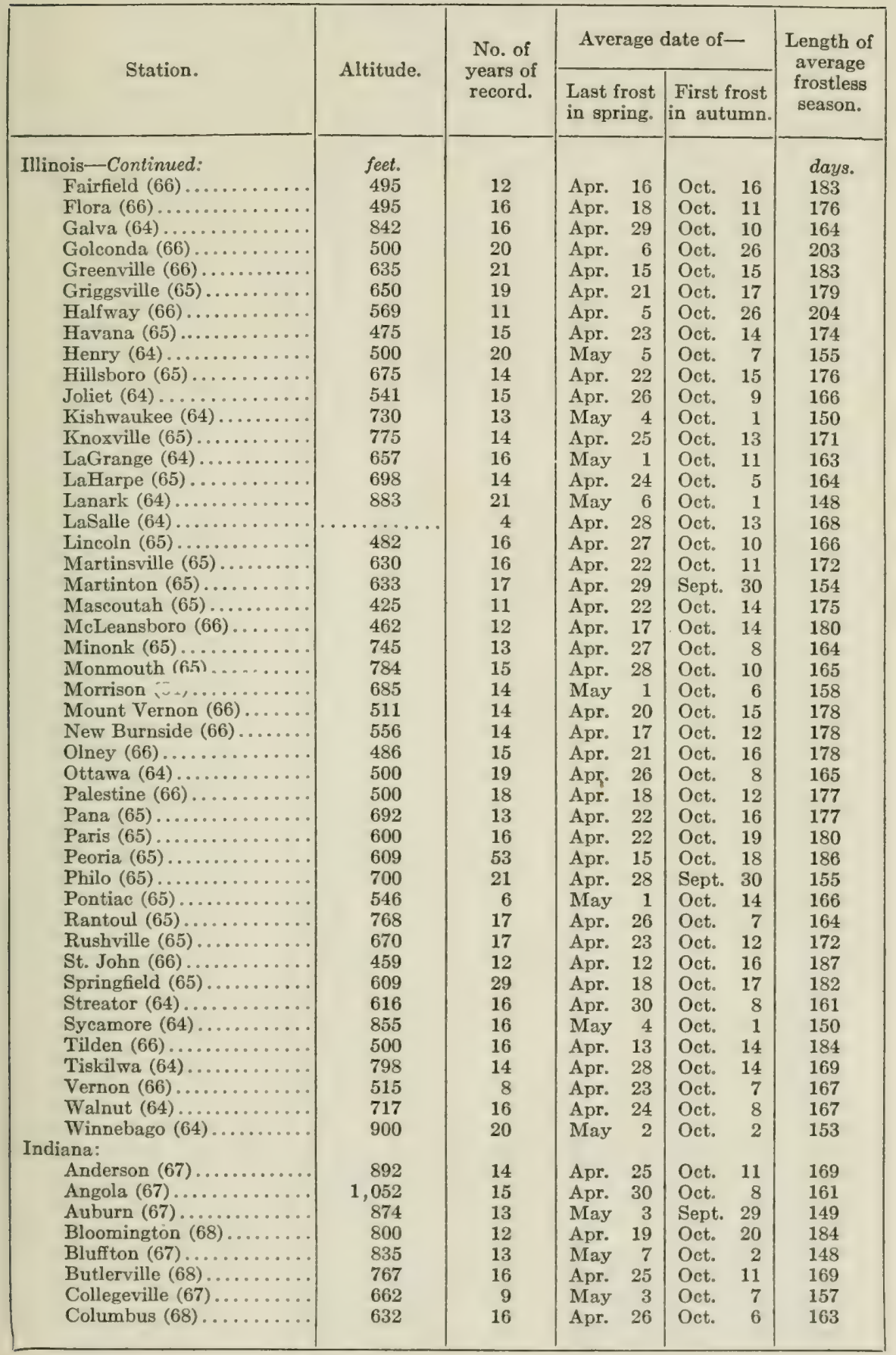


TABLE 2.-Frost data and length of average frostless season for 1803 stations in the United States. (Plate 34.)-Continued.

\begin{tabular}{|c|c|c|c|c|c|}
\hline \multirow{2}{*}{ Station. } & \multirow{2}{*}{ Altitude. } & \multirow{2}{*}{$\begin{array}{l}\text { No. of } \\
\text { years of } \\
\text { record. }\end{array}$} & \multicolumn{2}{|c|}{ Average date of- } & \multirow{2}{*}{$\begin{array}{l}\text { Length of } \\
\text { average } \\
\text { frostless } \\
\text { season. }\end{array}$} \\
\hline & & & $\begin{array}{l}\text { Last frost } \\
\text { in spring. }\end{array}$ & $\begin{array}{l}\text { First frost } \\
\text { in autumn. }\end{array}$ & \\
\hline $\begin{array}{l}\text { Indiana-Continued: } \\
\text { Connersville }(\mathrm{H}) \ldots \ldots \ldots .\end{array}$ & $\begin{array}{l}\text { feet. } \\
844\end{array}$ & & Apr. 27 & Oct. & $\begin{array}{c}\text { days. } \\
159\end{array}$ \\
\hline Delphi $(67) \ldots . . . \ldots$. & 668 & 16 & May 3 & Sept. 30 & 150 \\
\hline Evansville $(68) \ldots \ldots \ldots \ldots$ & 386 & 12 & Apr. & Oct. 27 & 203 \\
\hline Farmersburg (68) .......... & $\ldots \ldots \ldots$ & 6 & Apr. 22 & Oct. 18 & 179 \\
\hline Farmland $(67) \ldots . . . \ldots$ & 1,101 & 16 & Apr. 25 & Oct. 10 & 168 \\
\hline Fort Wayne (67)......... & 775 & 11 & May & Oct. & 160 \\
\hline Greenfield $(68) \ldots \ldots \ldots \ldots$ & 905 & 6 & Apr. 21 & Oct. 16 & 178 \\
\hline Greensburg (68) ......... & 954 & 12 & Apr. 22 & Oct. 16 & 177 \\
\hline Hammond (67)........... & 598 & 16 & Apr. 27 & Oct. 15 & 171 \\
\hline Huntington (67)......... & 741 & 15 & May & Oct. & 156 \\
\hline Indianapolis $(68) \ldots \ldots \ldots$ & 822 & 30 & Apr. 16 & Oct. 19 & 186 \\
\hline Jeffersonville (68) ......... & 455 & 18 & Apr. 16 & Oct. 18 & 185 \\
\hline Kokomo (67) ............. & 840 & 16 & Apr. 26 & Oct. & 158 \\
\hline Lafayette $(67) \ldots \ldots \ldots \ldots$ & 617 & 18 & Apr. 26 & Oct. & 162 \\
\hline Laporte $(67) . . . . . . . . .$. & 810 & 12 & May & Oct. & 157 \\
\hline Logansport (67) ......... & 620 & 16 & Apr. 24 & Oct. & 166 \\
\hline Marengo (68)............ & 363 & 16 & Apr. 19 & Oct. & 175 \\
\hline Marion (67)............. & 814 & 18 & May & Oct. & 148 \\
\hline Mauzy $(68) \ldots \ldots \ldots \ldots$ & 980 & 18 & May & Oct. & 155 \\
\hline Moore's Hill (68) ......... & 918 & 7 & May & Oct. & 159 \\
\hline Mount Vernon (68)....... & 410 & 16 & Apr. 18 & Oct. & 185 \\
\hline Northfield (67).......... & 900 & 13 & May & Oct. & 152 \\
\hline Paoli $(68) \ldots . . . \ldots \ldots \ldots$ & 611 & 11 & Apr. 18 & Oct. & 150 \\
\hline Princeton $(68) \ldots \ldots \ldots \ldots$ & 481 & 16 & Apr. 17 & Oct. 17 & 183 \\
\hline Richmond $(68) \ldots \ldots \ldots \ldots$ & 972 & 12 & May & Oct. & 149 \\
\hline Rockville (68)............ & 722 & 16 & Apr. 27 & Oct. & 163 \\
\hline Rome $(68) \ldots \ldots \ldots \ldots$ & 370 & 6 & Apr. 21 & Oct. & 175 \\
\hline Scottsburg $(68) \ldots \ldots \ldots$ & 570 & 15 & Apr. 16 & Oct. & 183 \\
\hline Seymour $(68) \ldots \ldots \ldots \ldots$ & 610 & 15 & Apr. 19 & Oct. & 177 \\
\hline South Bend $(67) \ldots \ldots \ldots$ & 726 & 15 & May & Oct. & 150 \\
\hline Terre Haute $(68) \ldots . . .$. & 498 & 16 & Apr. 19 & Oct. & 186 \\
\hline Veedersburg (67) ........ & 612 & 10 & May & Oct. & 157 \\
\hline Vevay $(68) \ldots \ldots \ldots \ldots$ & 525 & 17 & Apr. 19 & Oet. & 158 \\
\hline Vincennes $(68) \ldots \ldots \ldots$. & 431 & 15 & Apr. 14 & Oet. & 159 \\
\hline Washington (68)......... & 484 & 12 & Apr. 16 & Oct. & 186 \\
\hline Worthington (68)........ & 526 & 15 & Apr. 22 & Oet. & 185 \\
\hline va: & & & & & \\
\hline Algona (54)... & 1,500 & 16 & Apr. 26 & Oct. & 161 \\
\hline Alta $(5-1) \ldots \ldots \ldots \ldots \ldots$ & 1,440 & 16 & May & Sept. 2s & 142 \\
\hline $\operatorname{Amana}(H) \ldots \ldots \ldots$. & 763 & ... & Apr. 23 & Oct. & 165 \\
\hline Atlantic $(\mathrm{H}) \ldots \ldots \ldots \ldots$ & 1,164 & .... & May 11 & Sept. 19 & 131 \\
\hline Belle Plaine $(\mathrm{H}) \ldots . . . .$. & 864 & .... & May & Oct. & 156 \\
\hline Bonaparte $(\mathbf{H}) \ldots \ldots \ldots$. & 700 & ... & Apr. 20 & Oct. $\quad 7$ & 170 \\
\hline Carroll $(\mathrm{H}) \ldots \ldots \ldots \ldots$ & 1,272 & $\cdots$ & May & Sept. 22 & 1.40 \\
\hline Cedar Rapids (54) ....... & 733 & 16 & Apr. 24 & Oet. 6 & 165 \\
\hline Charles City (54)........ & 1,015 & 17 & May 16 & Sent. 26 & 133 \\
\hline Clarinda $(54) \ldots \ldots \ldots \ldots$ & $1,064(\mathrm{H})$ & 16 & Apr. $: 26$ & Ort. $\quad 5$ & 162 \\
\hline Clinton $(\mathbf{H}) \ldots \ldots \ldots \ldots$ & 591 & ... & Apr. 2s & Oct. & 157 \\
\hline Corning $(\mathrm{H}) \ldots \ldots \ldots \ldots$ & 1,127 & ... & Apr. 26 & Oet. & 150 \\
\hline Corydon (54)............. & $1,100(\mathrm{H})$ & 15 & May & Sept. 30 & 152 \\
\hline Davenport (54).......... & 580 & 37 & Apr. 22 & Oct. 13 & 174 \\
\hline Decornh $(54) \ldots \ldots$. & 975 & 15 & May 10 & Sept. 26 & 139 \\
\hline Des Moines $(54) \ldots . . \ldots$. & 861 & 31 & Apr. 22 & Oct. 10 & 171 \\
\hline Dubuque $(5.4) \ldots \ldots \ldots$. & 639 & 36 & Apr. 20 & Oet. 13 & 170 \\
\hline Elkader $(\mathbf{H}) \ldots \ldots \ldots \ldots$ & 727 & .. & May & Seprt. $2 \%$ & 141 \\
\hline
\end{tabular}


TABLE 2.-Frost data and length of average frostless season for 1803 stations in the United States. (Plate 34.)-Continued.

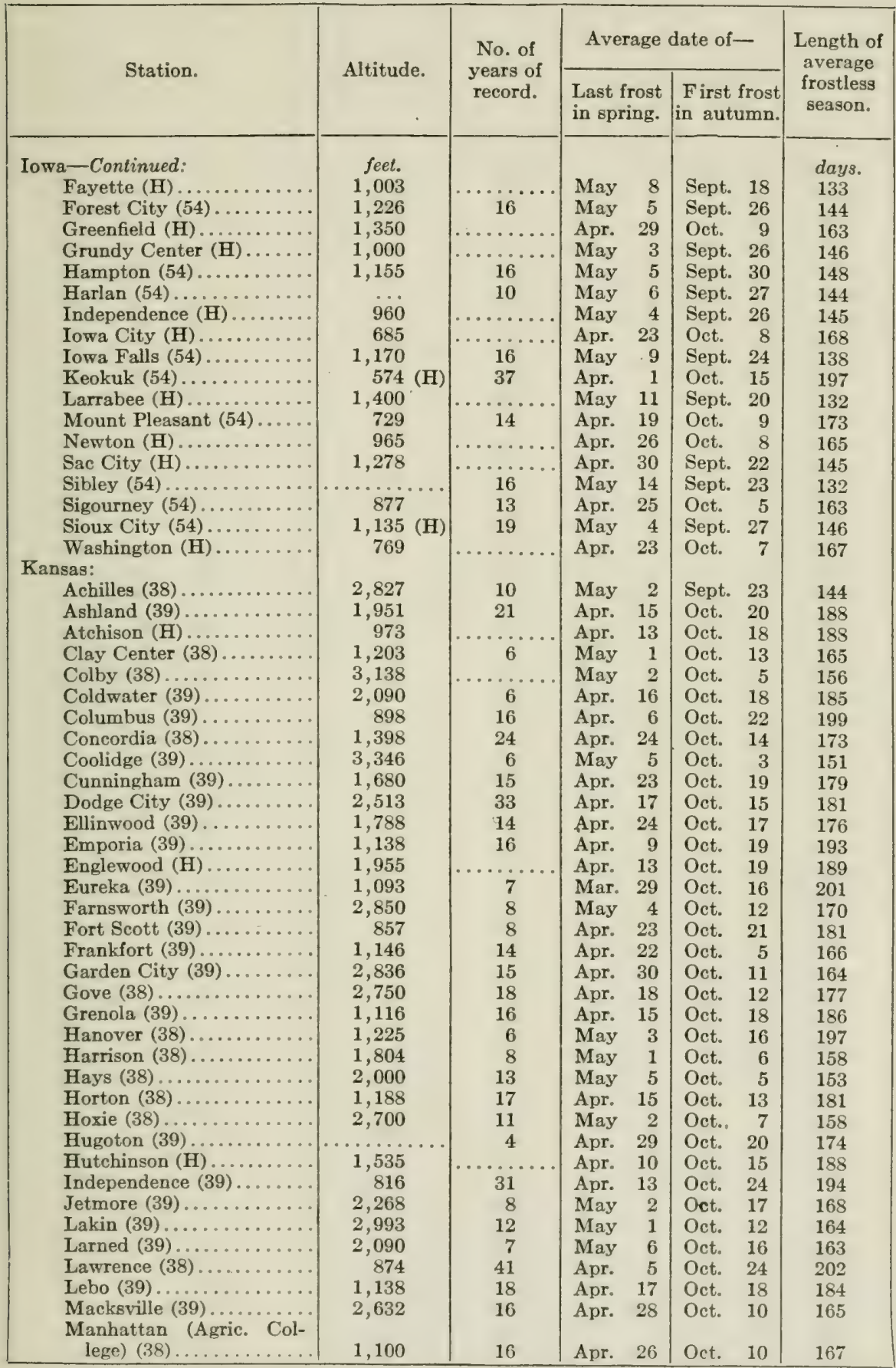


TABLE 2.-Frost data and length of average frostless season for 1803 stations in the United States. (Plate 34.)-Continued.

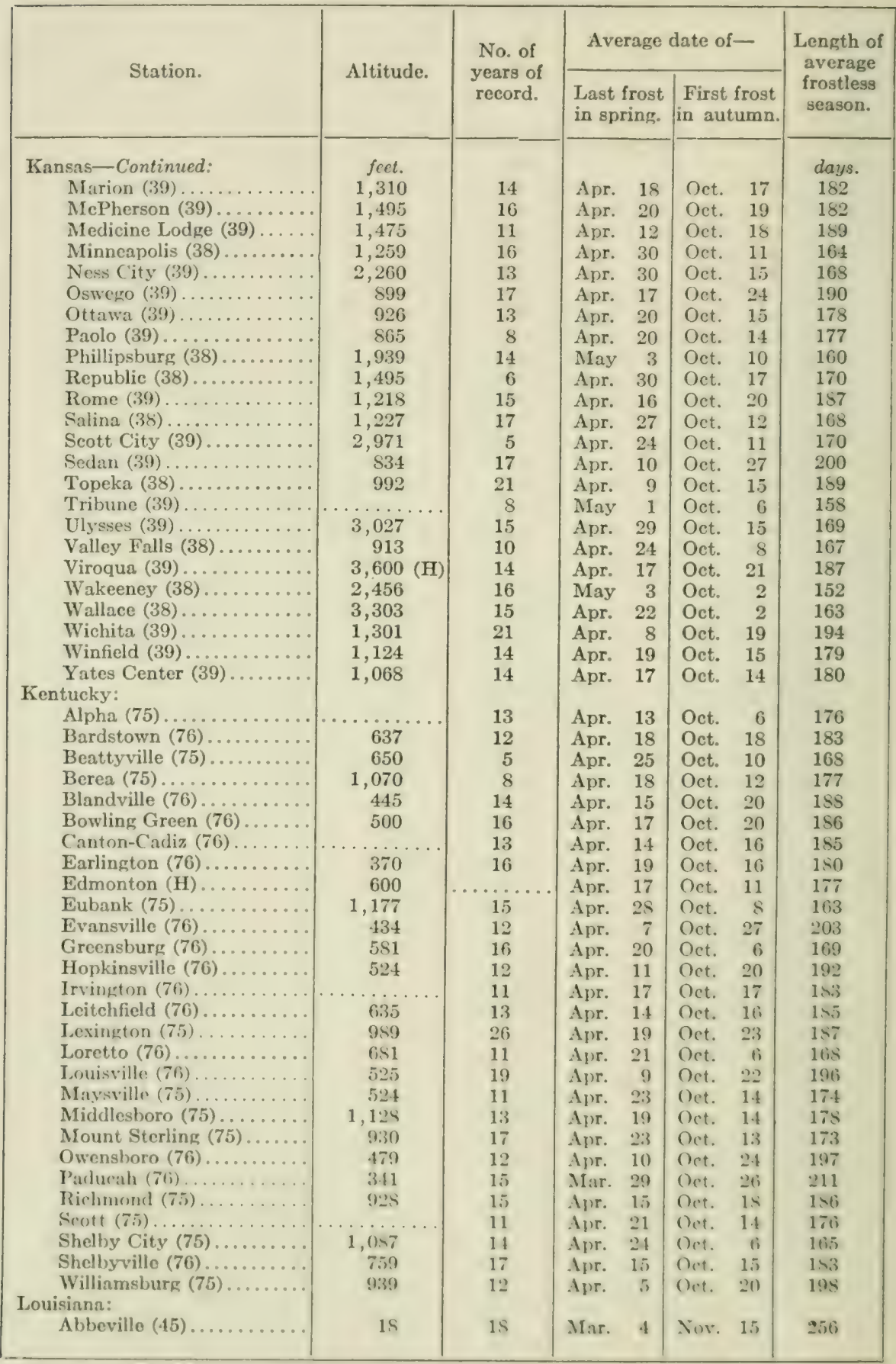


TABLE 2.-Frost data and length of average frostless season for 1803 stations in the United States. (Plate 34.)-Continued.

\begin{tabular}{|c|c|c|c|c|c|}
\hline \multirow{2}{*}{ Station. } & \multirow{2}{*}{ Altitude. } & \multirow{2}{*}{$\begin{array}{l}\text { No. of } \\
\text { years of } \\
\text { record. }\end{array}$} & \multicolumn{2}{|c|}{ Average date of- } & \multirow{2}{*}{$\begin{array}{l}\text { Length of } \\
\text { average } \\
\text { frostless } \\
\text { season. }\end{array}$} \\
\hline & & & $\begin{array}{l}\text { Last frost } \\
\text { in spring. }\end{array}$ & $\begin{array}{l}\text { First frost } \\
\text { in autumn. }\end{array}$ & \\
\hline Louisiana-Continued: & feet. & & & & days \\
\hline Alexandria (46) ........... & 77 & 19 & Mar. & Nov. 10 & 247 \\
\hline Amite $(45), \ldots \ldots \ldots \ldots$ & 130 & 18 & Mar. 13 & Nov. 10 & 242 \\
\hline Baton Rouge (45)........ & 35 & 15 & Mar. & Nov. 19 & 263 \\
\hline Burrwood (45),.......... & 1 & 14 & Feb. & Dec. & 300 \\
\hline Calhoun (46) ........... & 180 & 15 & Mar. 15 & Nov. & 239 \\
\hline Cameron (45) .............. & 6 & 15 & Feb. 22 & Nov. 26 & 277 \\
\hline Cheneyville (45) ......... & 67 & 18 & Mar. & Nov. & 252 \\
\hline Clinton (45) ................ & 113 & 17 & Mar. 12 & Nov. & 242 \\
\hline Collinston (46) .......... & 65 & 8 & Mar. & Nov. 10 & 253 \\
\hline Covington (45) $\ldots \ldots \ldots \ldots$ & 39 & 17 & Mar. & Nov. 18 & 260 \\
\hline Donaldsonville (45)....... & 33 & 17 & Feb. 25 & Nov. 21 & 269 \\
\hline Farmerville (46) . . . . . . & 177 & 16 & Mar. 19 & Nov. & 231 \\
\hline Franklin (45) .............. & 10 & 18 & Feb. 20 & Nov. 25 & 278 \\
\hline Grand Cane (46).......... & 302 & 13 & Mar. 11 & Nov. 10 & 244 \\
\hline Hammond (45)........... & 44 & 15 & Mar. & Nov. 17 & 255 \\
\hline Houma (45)............. & $\cdots$ & 18 & Feb. 28 & Nov. 20 & 265 \\
\hline Jennings $(45) \ldots \ldots \ldots \ldots$ & 30 & 13 & Feb. 22 & Nov. 21 & 272 \\
\hline Lafayette $(45) \ldots \ldots \ldots \ldots$ & 36 & 20 & Mar. & Nov. 13 & 255 \\
\hline Lake Charles (45) ......... & 22 & 18 & Feb. 24 & Nov. 25 & 274 \\
\hline Lake Providence $(\mathrm{H}) \ldots .$. & 107 & 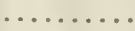 & Mar. 14 & Nov. & 239 \\
\hline Lakeside $(45) \ldots \ldots \ldots \ldots$. . . & $\cdots$ & 8 & Feb. 13 & Dec. & 295 \\
\hline Lawrence (45) ............ & 6 & 17 & Feb. 13 & Dec. 11 & 301 \\
\hline Liberty Hill (46) .......... & $\cdots$ & 19 & Mar. 22 & Nov. & 227 \\
\hline Melville (45)............ & 45 & 18 & Mar. & Nov. & 244 \\
\hline Minden (46) ............ & 194 & 20 & Mar. 14 & Nov. 13 & 244 \\
\hline Monroe (46) ............. & 82 & 16 & Mar. 14 & Nov, 12 & 243 \\
\hline New Iberia (45) .......... & 15 & 17 & Feb. 20 & Nov. 29 & 282 \\
\hline New Orleans (45)........ & 8 & 37 & Feb. & Dec. 10 & 310 \\
\hline Opelousas $(45) \ldots \ldots \ldots \ldots$ & 83 & 17 & Mar. & Nov. 17 & 257 \\
\hline 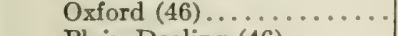 & $\ldots$ & 13 & Mar. 28 & Nov. & 221 \\
\hline Plain Dealing (46) ....... & 268 & 16 & Mar. 27 & Nov. & 227 \\
\hline Port Eads $(\mathrm{H}) \ldots \ldots \ldots \ldots$ & 3 & .. & Jan. 26 & Dec. 20 & 328 \\
\hline 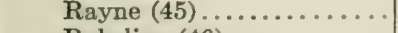 & 44 & 18 & Feb. 25 & Nov. 20 & 268 \\
\hline Robeline (46)........... & 147 & 13 & Mar. 21 & Nov. & 228 \\
\hline Ruston (46) ............ & 312 & 14 & Mar. 23 & Nov. & 225 \\
\hline Schriever (45) ... . . . . . & 17 & 17 & Feb. 28 & Nov. 16 & 261 \\
\hline Shreveport (46).......... & 249 & 39 & Mar. & Nov. 11 & 252 \\
\hline Sugartown (45) ......... & ... & 16 & Feb. 26 & Nov. 22 & 269 \\
\hline Maine: & & & & & \\
\hline Bar Harbor (106)........ & 20 & 20 & May 18 & Oct. 12 & 147 \\
\hline Cornish (106) ............ & 784 & 15 & May 23 & Sept. 12 & 112 \\
\hline Eastport (106)........... & 53 & 35 & Apr. 28 & Oct. 12 & 167 \\
\hline Fairfield (H) ............ & 90 & $\cdots$ & May 13 & Sept. 24 & 134 \\
\hline Farmington (106)........ & ... & 16 & May 16 & Sept. 15 & 122 \\
\hline Flagstaff $(106) \ldots \ldots \ldots \ldots$ & . & 5 & May 22 & Sept. 15 & 116 \\
\hline Gardiner (106) .......... & 163 & 16 & May & Oct. & 148 \\
\hline Lewiston (106) .......... & 185 & 24 & May & Oct. & 150 \\
\hline Mayfield (106).......... & 1,000 & 18 & May 17 & Sept. 22 & 128 \\
\hline Millinocket (106)......... & 386 & 6 & May 16 & Sept. 20 & 127 \\
\hline North Bridgton (106)..... & 450 & 15 & May 15 & Sept. 15 & 123 \\
\hline Orono $(106) \ldots \ldots \ldots \ldots$ & 129 & 23 & May 11 & Sept. 24 & 136 \\
\hline Portland (106)............ & 99 & 35 & May 14 & Oct. 18 & 157 \\
\hline Rumford Falls (106)...... & 505 & 15 & May 15 & Sept. 20 & 128 \\
\hline Winslow (106)........... & 90 & 11 & May 10 & Sept. 22 & 135 \\
\hline
\end{tabular}


TABLE 2.-Frost data and length of average frostless season for 1803 stations in the United States. (Plate 34.)-Continued.

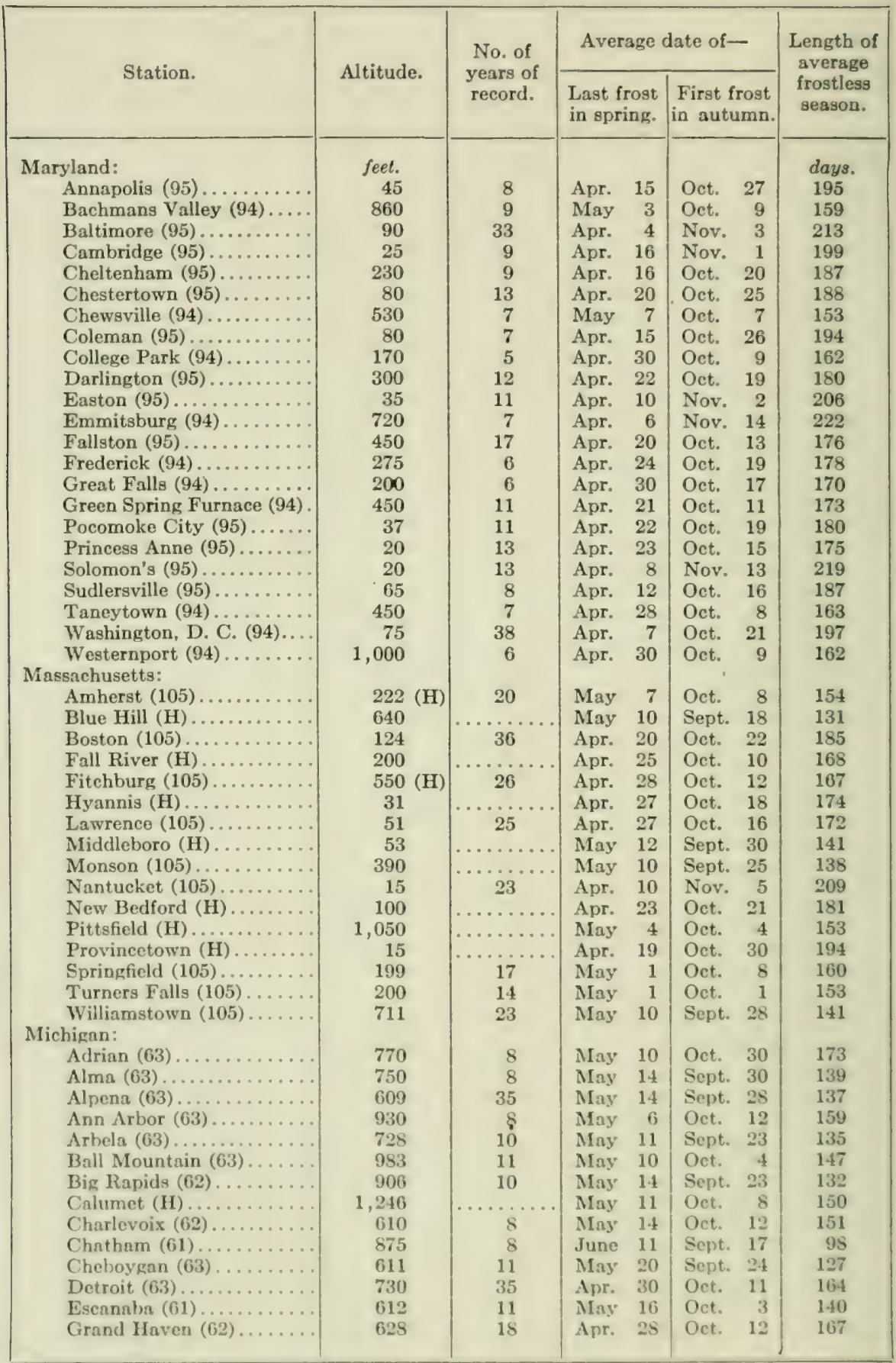


TABLE 2.-Frost data and length of average frostless season for 1803 stations in the United States. (Plate 34.)-Continued.

\begin{tabular}{|c|c|c|c|c|c|c|}
\hline \multirow{2}{*}{ Station. } & \multirow{2}{*}{ Altitude. } & \multirow{2}{*}{$\begin{array}{l}\text { No. of } \\
\text { years of } \\
\text { record. }\end{array}$} & \multicolumn{3}{|c|}{ Average date of - } & \multirow{2}{*}{$\begin{array}{l}\text { Length of } \\
\text { average } \\
\text { frostless } \\
\text { season. }\end{array}$} \\
\hline & & & $\begin{array}{l}\text { Last } \mathrm{f} \\
\text { in spr }\end{array}$ & $\begin{array}{l}\text { rost } \\
\text { ing. }\end{array}$ & $\begin{array}{l}\text { First frost } \\
\text { in autumn. }\end{array}$ & \\
\hline Michigan-Continued: & feet. & & & & & days. \\
\hline $\begin{array}{l}\text { Grand Rapids }(62) \ldots \ldots \ldots \\
\text { Grayling }(63) \ldots \ldots \ldots\end{array}$ & $\begin{array}{r}707 \\
1,147\end{array}$ & $\begin{array}{r}15 \\
9\end{array}$ & May & 1 & Oct. 12 & 164 \\
\hline Harbor Beach $(63) \ldots \ldots$. . . & $\begin{array}{r}1,147 \\
635\end{array}$ & 9 & $\begin{array}{l}\text { May } \\
\text { May }\end{array}$ & 18 & Sept. 18 & 123 \\
\hline Harrison (63) ........... & 1,159 & 8 & May & $\begin{array}{l}11 \\
17\end{array}$ & $\begin{array}{lr}\text { Oct. } & 5 \\
\text { Sent. } & 27\end{array}$ & $\begin{array}{l}147 \\
133\end{array}$ \\
\hline Harrisville $(62) \ldots \ldots \ldots \ldots$ & 616 & 8 & May & 16 & $\begin{array}{l}\text { Sept. } \\
\text { Oct. } 47\end{array}$ & 141 \\
\hline Hart $(61) \ldots \ldots \ldots \ldots \ldots$ & 698 & 4 & May & 13 & Oct. 1 & 141 \\
\hline Hastings $(\mathrm{H}) \ldots \ldots \ldots \ldots$. & 770 & & May & 10 & Sept. 15 & 128 \\
\hline Houghton $(61) \ldots \ldots \ldots \ldots$ & 668 & 10 & May & 8 & Oct. 7 & 152 \\
\hline Humbolt (61).......... & 1,536 & 11 & June & 7 & Sept. 5 & 92 \\
\hline Iron River $(61) \ldots \ldots \ldots$ & 1,504 & 10 & June & 3 & Sept. 15 & 104 \\
\hline Ivan $(\mathrm{H}) \ldots \ldots \ldots \ldots$ & ...... & $\cdots$ & May & 23 & Sept. 14 & 114 \\
\hline Kalamazoo (62)........ & 955 & 9 & May & 4 & Oct. 7 & 156 \\
\hline Lansing (63) .......... & 820 & 11 & May & 4 & Oct. 9 & 158 \\
\hline Mancelona $(62) \ldots \ldots \ldots$. & 1,121 & 8 & May & 24 & Sept. 28 & 127 \\
\hline Manistee (62)......... & 600 & 6 & May & 13 & Oct. 3 & 143 \\
\hline Marquette $(61) \ldots . . . .$. & 734 & 21 & May & 15 & Oct. & 140 \\
\hline Menominee $(61) \ldots \ldots \ldots$ & 581 & 9 & May & 16 & Oct. 6 & 143 \\
\hline Muskegon (62)......... & 587 & 9 & May & 5 & Sept. 29 & 147 \\
\hline Newberry $(61) \ldots \ldots \ldots \ldots$ & 773 & 5 & May & 29 & Sept. 11 & 105 \\
\hline Olivet $(62) \ldots \ldots \ldots \ldots$ & 934 & 9 & May & 7 & Oct. 5 & 151 \\
\hline Omer $(63) \ldots \ldots \ldots \ldots$ & 616 & 8 & May & 28 & Sept. 13 & 108 \\
\hline Port Huron (63) ......... & 639 & 35 & May & 7 & Oct. $\quad 9$ & 155 \\
\hline Saginaw, WV. S. (63) ...... & 601 & 9 & May & 9 & Oct. 1 & 145 \\
\hline St. Ignace $(61) \ldots \ldots \ldots$ & 593 & 7 & May & 11 & Sept. 28 & 140 \\
\hline St. Joseph (62) . . . . . . & 593 & 6 & Apr. & 17 & Oct. 12 & 178 \\
\hline Saranac $(62) \ldots$ & 639 & 7 & May & 13 & Oct. 2 & 142 \\
\hline Sault Ste. Marie (61)..... & 614 & 22 & May & 14 & Sept. 29 & 138 \\
\hline Thomaston (61)........ & 1,347 & 10 & June & 8 & Sept. 1 & 85 \\
\hline Traverse City (62)....... & 588 & 8 & May & 17 & Oct. 5 & 141 \\
\hline Wasepi $(62) \ldots \ldots \ldots \ldots$ & 842 & 10 & May & 4 & Oct. 11 & 160 \\
\hline Whitefish Point (61).... & 610 & 9 & May & 17 & Oct. 10 & 146 \\
\hline Minnesota: & $P$ & & & & & \\
\hline Bird Island (55) ......... & 1,039 & 16 & May & 9 & Sept. 25 & 139 \\
\hline Collegeville (56)......... & 1,282 & 16 & May & 7 & Sept. 28 & 144 \\
\hline Crookston (57) ......... & 863 & 16 & May & 15 & Sept. 22 & 130 \\
\hline Duluth $(58) \ldots \ldots \ldots \ldots$ & 1,133 & 39 & May & 4 & Oct. 3 & 152 \\
\hline Fairmont (n) (55) ........ & 753 & 16 & May & 6 & Oct. & 150 \\
\hline Fergus Falls (55)........ & 1,210 & 17 & May & 11 & Sept. 22 & 134 \\
\hline Grand Meadow (56)...... & 1,338 & 16 & May & 16 & Sept. 25 & 132 \\
\hline Lake Winnibigoshish (57). . & 1,300 & 12 & May & 22 & Sept. 24 & 125 \\
\hline Long Prairie (55) ......... & 1,299 & 16 & May & 18 & Sept. 23 & 128 \\
\hline Luverne $(\mathrm{H}) \ldots \ldots \ldots \ldots$ & 1,480 & & May & 11 & Sept. 19 & 131 \\
\hline Lynd $(55) \ldots \ldots \ldots \ldots$ & 1,175 & 15 & May & 12 & Sept. 25 & 136 \\
\hline Milan $(55) \ldots \ldots \ldots \ldots$ & 995 & 16 & May & 14 & Sept. 21 & 130 \\
\hline Minneapolis $(56) \ldots \ldots \ldots$ & 918 & 1.5 & Apr. & 29 & Oct. 7 & 161 \\
\hline Moorhead (57) .......... & 903 & $2 s$ & May & 13 & Sept. 22 & 132 \\
\hline Mnrris (55) ........... & 1,170 & 17 & May & 12 & Sept. 25 & 136 \\
\hline Mt. Iron (57) ......... & 1,510 & 15 & June & 3 & Sept. 9 & 98 \\
\hline New London (55)....... . & 1,215 & 15 & May & 9 & Sept. 30 & 144 \\
\hline New Ulm (55) .......... & 791 & 16 & May & 6 & Sept. 27 & 144 \\
\hline Park Rapids (57) ....... & 1,426 & 16 & May & 20 & Sept. 19 & 122 \\
\hline Pine River Dam (56).... & 1,251 & 15 & May & 18 & Sept. 20 & 125 \\
\hline Red Wing (56) ......... & $70 \mathrm{~s}$ & 9 & May & 4 & Oct. 11 & 160 \\
\hline Rolling Green $(\mathrm{H}) \ldots . .$. & 1,240 & & May & 4 & Oct. 3 & 152 \\
\hline St. Charles $(56) \ldots \ldots \ldots$. & 850 & 16 & May & 16 & Sept. 27 & 134 \\
\hline
\end{tabular}


TABLE 2.-Frost data and length of average frostless season for 1803 stations in the United States. (Plate 34.)-Continued.

\begin{tabular}{|c|c|c|c|c|c|}
\hline \multirow{2}{*}{ Station. } & \multirow{2}{*}{ Altitude. } & \multirow{2}{*}{$\begin{array}{l}\text { No. of } \\
\text { years of } \\
\text { record. }\end{array}$} & \multicolumn{2}{|c|}{ Average date of -} & \multirow{2}{*}{$\begin{array}{l}\text { Length of } \\
\text { average } \\
\text { frostless } \\
\text { season. }\end{array}$} \\
\hline & & & $\begin{array}{l}\text { Last frost } \\
\text { in spring. }\end{array}$ & $\begin{array}{l}\text { First frost } \\
\text { in autumn. }\end{array}$ & \\
\hline $\begin{array}{l}\text { Minnesota-Continued: } \\
\text { St. Paul (56).................... }\end{array}$ & $\begin{array}{l}\text { feet. } \\
848\end{array}$ & 36 & Apr. 27 & Oct. & $\begin{array}{l}\text { days. } \\
159\end{array}$ \\
\hline St. Peter $(56) \ldots \ldots \ldots \ldots$ & 840 & 14 & May 17 & Sept. 27 & 133 \\
\hline St. Vincent Pembine (57).. & 789 & 14 & June 3 & Sept. 14 & 103 \\
\hline Sandy Lake Dam (57).... & 1,234 & 17 & May 21 & Sept. 23 & 125 \\
\hline Shakopee $(56) \ldots \ldots \ldots \ldots$ & 750 & 14 & May 12 & Sept. 25 & 136 \\
\hline Tower $(57) \ldots \ldots \ldots \ldots$ & 1,350 & 7 & June 5 & Sept. 13 & 100 \\
\hline Wabasha $(58) \ldots \ldots \ldots \ldots$ & 681 & 14 & May & Oct. 5 & 157 \\
\hline Winnebago $(56) \ldots \ldots \ldots$. & 1,100 & 11 & May & Sept. 28 & 145 \\
\hline Worthington $(55) \ldots \ldots \ldots$ & 979 & 15 & May & Sept. 23 & 139 \\
\hline Mississippi : & & & & Oct. 29 & \\
\hline $\begin{array}{l}\text { Austin }(79) \ldots \ldots \ldots \ldots \\
\text { Batesville }(\mathrm{H}) \ldots \ldots\end{array}$ & $\begin{array}{l}200 \\
230\end{array}$ & 15 & $\begin{array}{ll}\text { Mar. } & 30 \\
\text { Mar. } & 24\end{array}$ & $\begin{array}{ll}\text { Uct. } & 29 \\
\text { Oct. } & 24\end{array}$ & $\begin{array}{l}213 \\
214\end{array}$ \\
\hline $\begin{array}{l}\text { Batesville }(\mathrm{H}) \ldots \ldots \ldots \ldots \\
\text { Biloxi }(80) \ldots \ldots \ldots \ldots\end{array}$ & $\begin{array}{r}230 \\
24\end{array}$ & $\cdots \cdots$ & Feb. 25 & Nov. 28 & $\begin{array}{l}214 \\
276\end{array}$ \\
\hline & 504 & 15 & Mar. 29 & Nov. 1 & 217 \\
\hline Brookhaven $(80) \ldots \ldots \ldots$. & 500 & 16 & Mar. 18 & Nov. & 231 \\
\hline Canton $(80) \ldots \ldots \ldots \ldots$ & 228 & 16 & Mar. 19 & Nov. 4 & 230 \\
\hline Columbus $(79) \ldots \ldots \ldots$. & 250 & 14 & Mar. 27 & Oct. 31 & 218 \\
\hline Crystal Springs (80)...... & 468 & 16 & Mar. 24 & Oct. 31 & 221 \\
\hline Greenville $(79) \ldots \ldots \ldots$ & 126 & 18 & Mar. 18 & Oct. 31 & 227 \\
\hline Greenwood (79) ......... & 140 & 14 & Mar. 19 & Oct. 27 & 222 \\
\hline Hattiesburg $(80) \ldots . . .$. & 154 & 16 & Mar. 11 & Nov. 9 & 243 \\
\hline Holly Springs (79) ....... & 600 & 14 & Mar. 28 & Nov. & 219 \\
\hline Leakesville $(80) \ldots \ldots \ldots \ldots$ & $\ldots \ldots \ldots$ & 16 & Mar. 5 & Nov. 10 & 250 \\
\hline Louisville (79) ........... & 561 & 16 & Mar. 26 & Oct. 30 & 218 \\
\hline Magnolia $(80) \ldots . . . .$. & 415 & 15 & Mar. 15 & Nov. 9 & 239 \\
\hline Meridian $(80) \ldots \ldots \ldots \ldots$ & 375 & 20 & Mar. 17 & Nov. 2 & 230 \\
\hline Natchez $(80) \ldots . . . . .$. & 206 & 16 & Mar. 9 & Nov. 12 & 248 \\
\hline Palo Alto $(\mathbf{H}) \ldots \ldots \ldots \ldots$ & 300 & $\ldots$ & Mar. 27 & Nov. 4 & 222 \\
\hline Pearlington $(80) \ldots . . .$. & 10 & 16 & Mar. 1 & Nov. 24 & 268 \\
\hline Pontotoc $(79) \ldots \ldots \ldots \ldots$ & 475 & 18 & Mar. 28 & Oct. 28 & 214 \\
\hline Vicksburg $(80) \ldots \ldots \ldots$. & 247 & 38 & Mar. 6 & Nov. 13 & 252 \\
\hline Water Valley (79) ........ & 300 & 17 & Mar. 27 & Oct. 31 & 218 \\
\hline Waynesboro $(80) \ldots . . .$. & 191 & 17 & Mar. 20 & Nov. 4 & 229 \\
\hline Woodville $(80) \ldots \ldots \ldots$. & 560 & 16 & Mar. 12 & Nov. 14 & 247 \\
\hline Yazoo City $(80) \ldots \ldots \ldots$ & 116 & 16 & Mar. 28 & Nov. 2 & 219 \\
\hline $\begin{array}{l}\text { Missouri: } \\
\text { Appleton (49) .......... }\end{array}$ & & 18 & & Oct. 19 & 182 \\
\hline $\begin{array}{l}\text { Appleton }(49) \ldots \ldots \ldots \ldots \\
\text { Avalon }(51) \ldots \ldots \ldots \ldots\end{array}$ & $\cdots \cdots$ & 12 & $\begin{array}{l}\text { Apr. } 20 \\
\text { Apr. } 22\end{array}$ & Oct. 12 & 173 \\
\hline $\begin{array}{l}\text { Avalon }(51) \ldots \ldots \ldots \ldots \ldots \\
\text { Belle }(50) \ldots \ldots \ldots \ldots \ldots\end{array}$ & 1,000 & 8 & Apr. 17 & Oct. 15 & 181 \\
\hline Bethany (51)............ & 881 & 16 & Apr. 26 & Oct. & 165 \\
\hline Birchtree $(50) \ldots \ldots \ldots \ldots$ & 1,200 & 16 & Apr. 17 & Oct. $\quad 16$ & 182 \\
\hline Brunswick (51) .......... & 652 & 20 & Apr. 17 & Oct. 19 & 185 \\
\hline Caruthersville $(50) \ldots \ldots$. & 860 & 13 & Apr. & Oct. 21 & 199 \\
\hline Columbia (51).......... & 784 & 20 & Apr. 18 & Oet. 14 & 179 \\
\hline Darksville (51)........ & 826 & 17 & Apr. 23 & Oct. 14 & 174 \\
\hline Dean $(49) \ldots \ldots \ldots \ldots$ & $\ldots \ldots$ & 10 & Apr. 16 & Oct. $\quad 13$ & 180 \\
\hline DeSoto $(50) \ldots \ldots \ldots$ & 498 & 7 & Apr. 21 & Oct. 12 & 174 \\
\hline Fairport $(51) \ldots \ldots \ldots$ & 535 & 12 & May & Oct. & 160 \\
\hline Fayette (51)... & 725 & 24 & Apr. 21 & Oct. 14 & 176 \\
\hline Fulton $(51) \ldots$ & 818 & 14 & Apr. 21 & Oct. & 170 \\
\hline Gallatin (51)... & 803 & 17 & Apr. 17 & Oct. $\quad 19$ & 155 \\
\hline Gano $(50) \ldots \ldots$ & $\ldots \ldots$ & 6 & Apr. 23 & Oct. 15 & 175 \\
\hline Gorin $(51) \ldots .$. & 700 & 15 & Apr. 29 & Oet. & 164 \\
\hline Grant City (51). & 1,130 & 12 & Apr. 26 & Oct. 13 & 170 \\
\hline Greenville $(50) \ldots$ & .... & 12 & Apr. 16 & Oct. & 176 \\
\hline
\end{tabular}


TABLE 2.-Frost data and length of average frostless season for 1803 stations in the United States. (Plate 34.)-Continued.

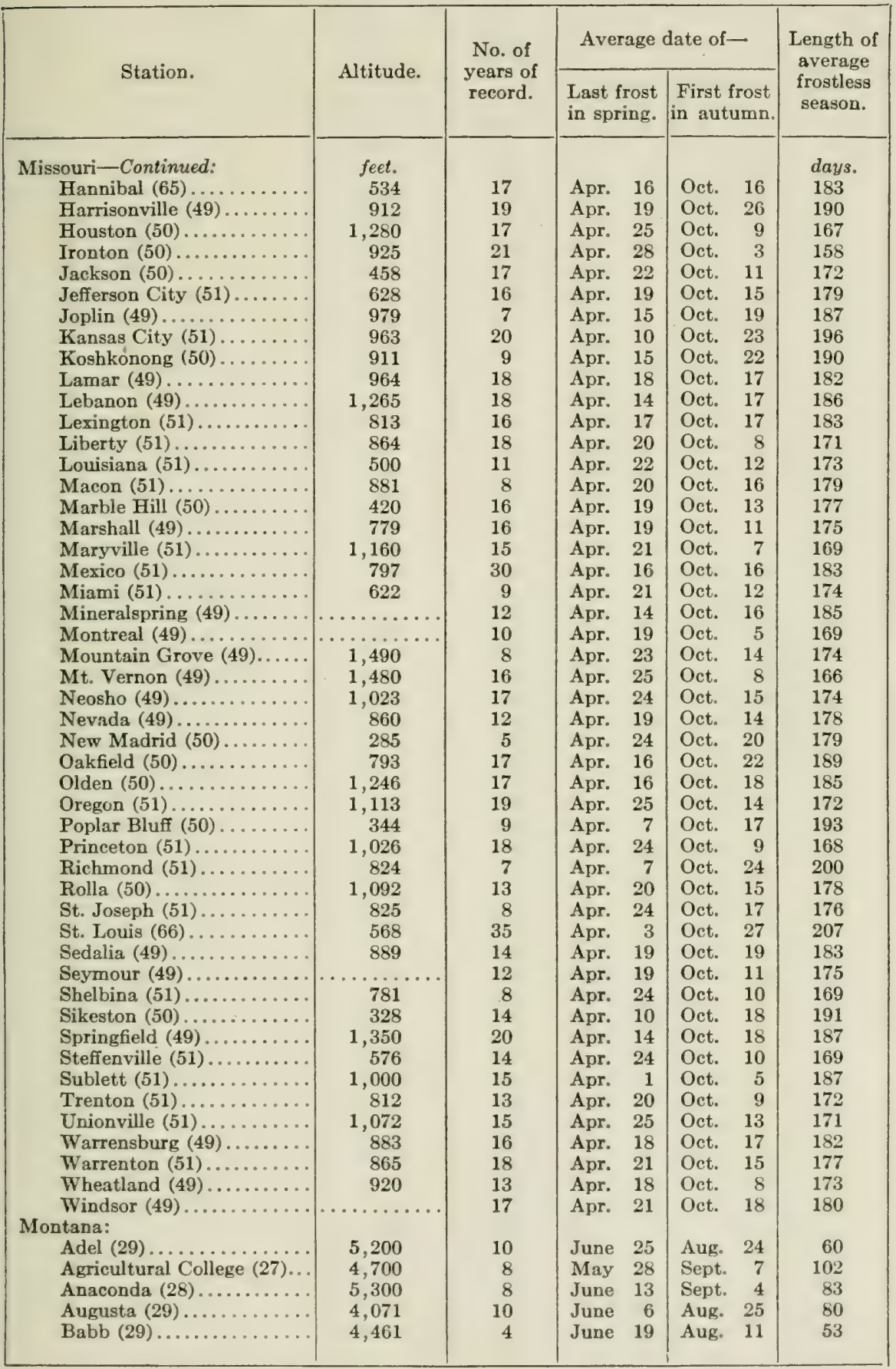


TABLE 2.-Frost data and length of average frostless season for 1803 stations in the United States. (Plate 34.)-Continued.

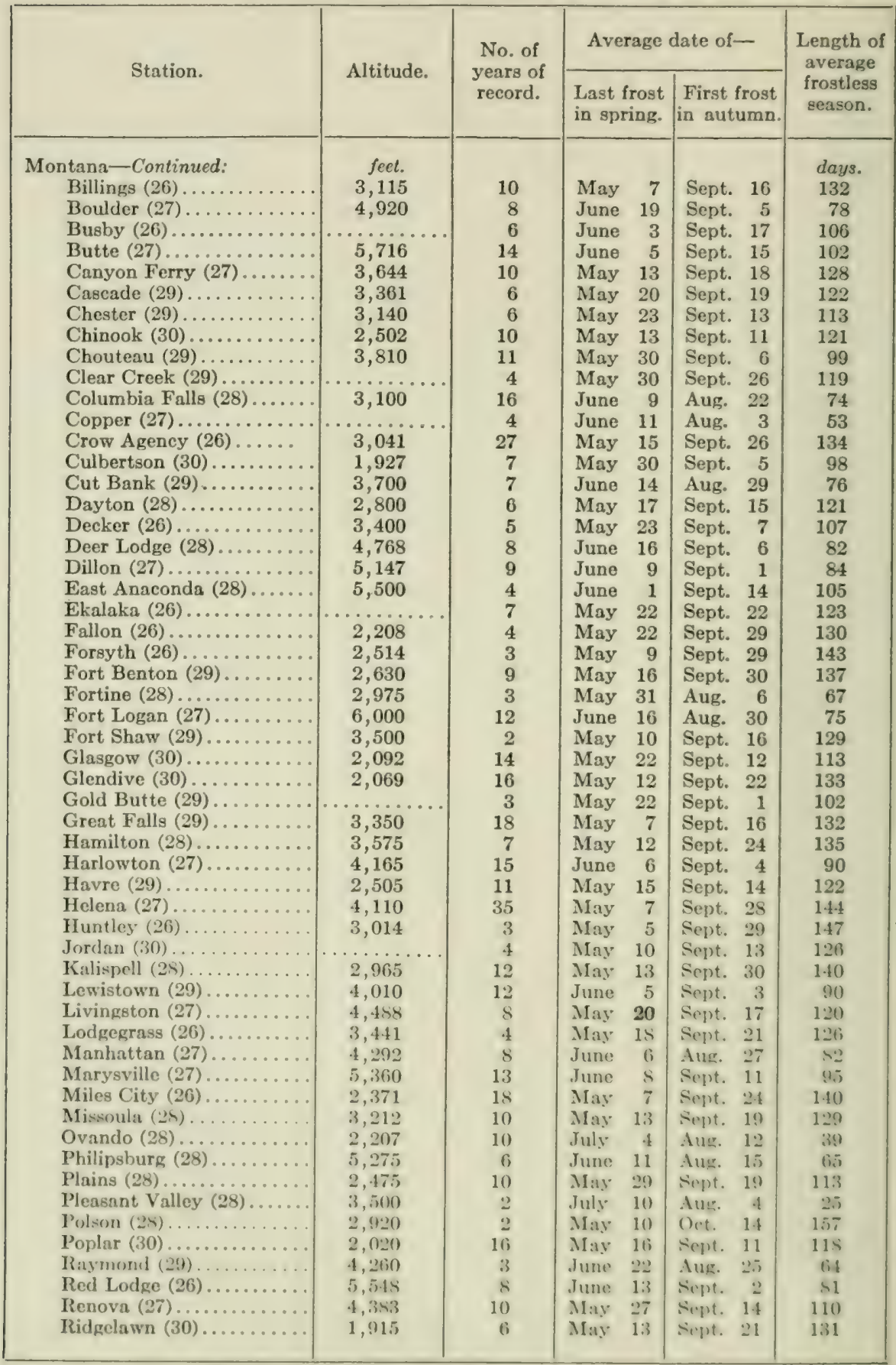


TABLE 2,-Frost data and length of average frostless season for 1803 stations in the United States. (Plate 34.)-Continued.

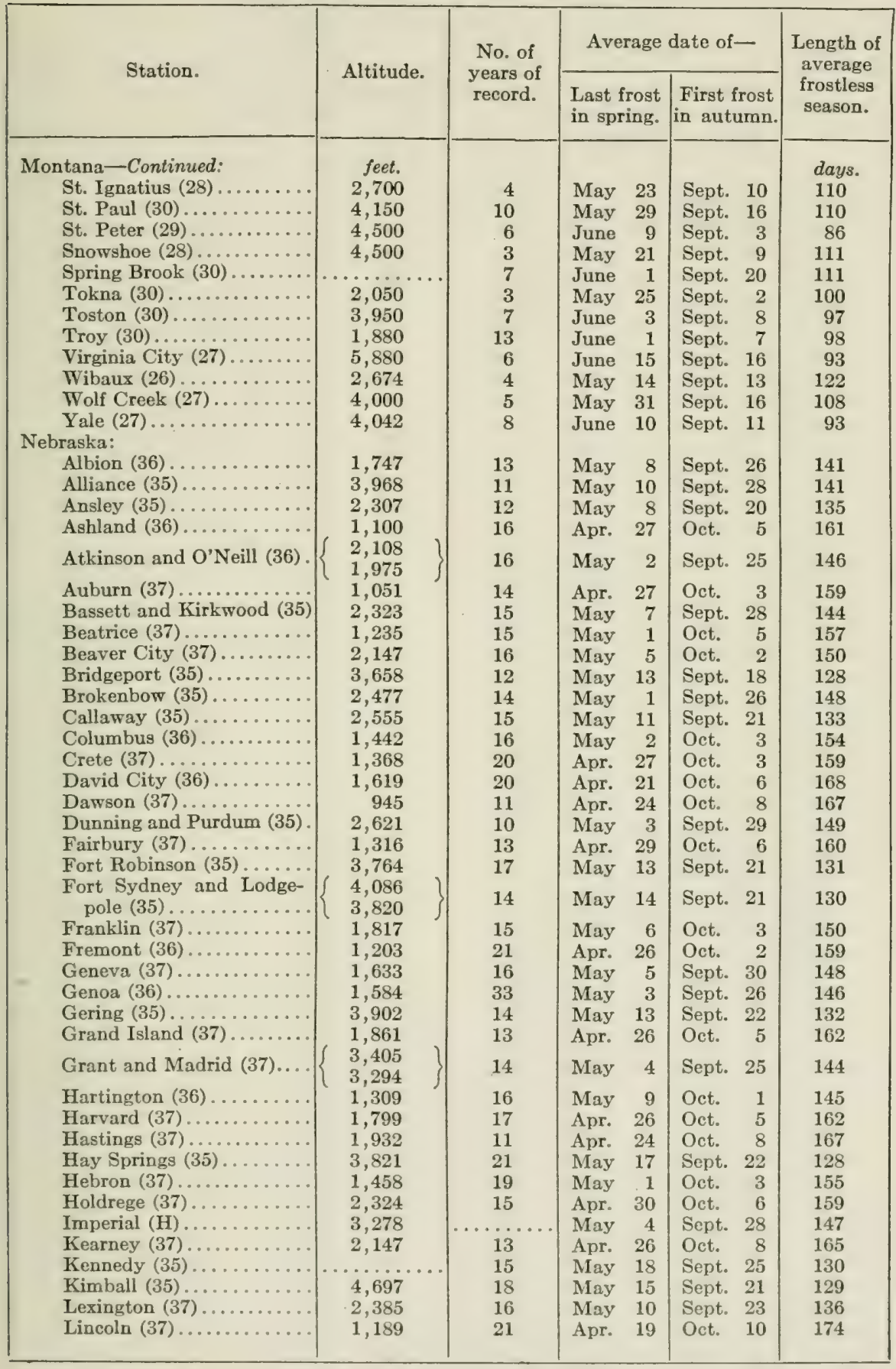


TABLE 2.-Frost data and length of average frostless season for 1803 stations in the United States. (Plate 34.)-Continued.

\begin{tabular}{|c|c|c|c|c|c|c|}
\hline \multirow{2}{*}{ Station. } & \multirow{2}{*}{ Altitude. } & \multirow{2}{*}{$\begin{array}{l}\text { No. of } \\
\text { years of } \\
\text { record. }\end{array}$} & \multicolumn{3}{|c|}{ Average date of- } & \multirow{2}{*}{$\begin{array}{l}\text { Length of } \\
\text { average } \\
\text { frostless } \\
\text { season. }\end{array}$} \\
\hline & & & $\begin{array}{l}\text { Last fr } \\
\text { in sprix }\end{array}$ & ost & $\begin{array}{l}\text { First frost } \\
\text { in autumn. }\end{array}$ & \\
\hline Nebraska-Continued: & feet. & & & & & days. \\
\hline Lynch $(36) \ldots \ldots \ldots \ldots$ & $1,965(\mathrm{H})$ & 16 & May & 7 & Sept. 24 & 140 \\
\hline Madison (36) ............. & 1,585 & 14 & May & 2 & Sept. 30 & 151 \\
\hline Nesbit (35)............. & $\ldots \ldots \ldots$ & 9 & May & 12 & Sept. 21 & 132 \\
\hline Norfolk $(36) \ldots \ldots \ldots \ldots$ & 1,532 & 16 & May & 7 & Sept. 30 & 146 \\
\hline North Loup (36) .......... & 1,961 & 16 & May & 7 & Sept. 28 & 144 \\
\hline North Platte (35)....... & 2,826 & 34 & May & 1 & Sept. 29 & 151 \\
\hline Oakdale $(36) \ldots \ldots \ldots \ldots$ & 1,722 & 20 & May & 3 & Sept. 26 & 146 \\
\hline Omaha $(36) \ldots \ldots \ldots \ldots$ & 1,103 & 37 & Apr. & 26 & Oct. 13 & 170 \\
\hline 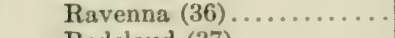 & 2,028 & 24 & May & 10 & Sept. 30 & 143 \\
\hline Redcloud (37).......... & 1,687 & 12 & Apr. & 24 & Oct. 4 & 163 \\
\hline St. Paul (36) ........... & 1,796 & 12 & May & 8 & Oct. 3 & 148 \\
\hline Santee (35) ............ & $\ldots \ldots \ldots \ldots$ & 16 & May & 6 & Sept. 30 & 147 \\
\hline Springview (36) .......... & $\cdots \ldots \ldots . .$. & 16 & May & 10 & Sept. 30 & 143 \\
\hline 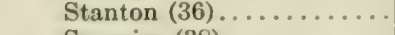 & 1,472 & 16 & May & 2 & Oct. 1 & 152 \\
\hline Superior (38) ........... & 1,574 & 8 & Apr. & 28 & Oct. & 156 \\
\hline Tecumseh (37) ......... & 1,114 & 20 & May & 2 & Oct. & 157 \\
\hline Tekamah (36) ............ & 1,060 & 18 & Apr. & 27 & Oct. & 157 \\
\hline $\begin{array}{l}\text { Turlington and Syracuse } \\
(37) \ldots \ldots \ldots \ldots \ldots \ldots\end{array}$ & $\begin{array}{l}1,224 \\
1,059\end{array}$ & 23 & Apr. & 21 & Oct. & 169 \\
\hline Valentine (35) ............ & 2,859 & 20 & May & 9 & Sept. 18 & 132 \\
\hline Wakefield (36) ............ & 1,387 & 13 & May & 8 & Sept. 28 & 143 \\
\hline Weeping Water (37)...... & 1,080 & 23 & May & 8 & Oct. & 146 \\
\hline West Point (36) . . . . . . . & 1,313 & 14 & May & 3 & Oct. & 154 \\
\hline $\begin{array}{l}\text { York (37) } \ldots \ldots \ldots \ldots \ldots \ldots \\
\text { Nevada: }\end{array}$ & 1,633 & 16 & Apr. & 21 & Oct. & 165 \\
\hline $\begin{array}{l}\text { Nevada: } \\
\text { Austin (12). }\end{array}$ & 6,594 & & May & 21 & Sept. 21 & 123 \\
\hline Beowawe (12). & 4,695 & $\ldots \ldots$ & May & 15 & Oct. 1 & 139 \\
\hline Carson City $(\mathrm{H}) \ldots \ldots \ldots$ & 4,674 & $\ldots \ldots \ldots$ & May & 20 & Sept. 20 & 123 \\
\hline Ely $(12) \ldots \ldots \ldots \ldots \ldots$ & 6,421 & $\ldots \ldots \ldots$ & June & 1 & Sept. 18 & 109 \\
\hline Eurcka (12)............ & 6,500 & $\ldots \ldots \ldots$ & June & 8 & Sept. 20 & 104 \\
\hline 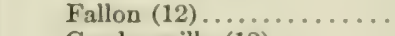 & 3,965 & $\ldots \ldots \ldots$ & May & 25 & Oct. 4 & 132 \\
\hline Gardnerville (12). & 4,830 & $\ldots \ldots \ldots$ & June & 15 & Sept. 23 & 100 \\
\hline Geyser (12)........... & ..... & $\ldots \ldots \ldots$ & June & 23 & Sept. 3 & 72 \\
\hline Lewer's Ranch (12)...... . & 6,282 & $\ldots \ldots \ldots$ & May & 26 & Sept. 28 & 125 \\
\hline 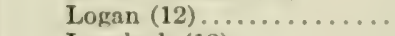 & 1,700 & $\ldots \ldots \ldots$ & Apr. & 14 & Nov. 6 & 206 \\
\hline Lovelock (12).......... & 3,977 & $\ldots \ldots \ldots$ & May & 22 & Sept. 22 & 123 \\
\hline Palmetto (12).......... & 6,500 & $\ldots \ldots \ldots$ & May & 30 & Sept. 21 & 114 \\
\hline Potts $(12) \ldots \ldots \ldots \ldots$. & 6,990 & $\ldots \ldots \ldots$ & June & 16 & Oct. & 105 \\
\hline Quinn Riv. Ranch (12).... & 4,850 & $\ldots \ldots \ldots$ & June & 19 & Seput. 6 & 79 \\
\hline Reno (12)............. & 4,532 & $\ldots \ldots \ldots$ & May & 16 & Sept. 31 & 138 \\
\hline Tecoma (12).......... & 4,812 & $\ldots \ldots \ldots$ & May & $2 S$ & Sept. 14 & 109 \\
\hline Winnemucer (12)........ & 4,344 & $\ldots \quad \ldots \ldots$ & May & 15 & Sept. 23 & 131 \\
\hline \multicolumn{7}{|l|}{ New Hampshire: } \\
\hline Bethlehem (105).. & 1,470 & 18 & May & 22 & Sept. 19 & 120 \\
\hline Concord $(105) \ldots \ldots \ldots \ldots$ & 350 & 38 & May & 7 & Sept. 30 & 146 \\
\hline Durham $(\mathbf{H}) \ldots \ldots \ldots \ldots$ & 93 & 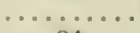 & May & 8 & Oct. 3 & 148 \\
\hline Hanover (105) ........... & 603 & 24 & May & 18 & Sept. 25 & 130 \\
\hline Keene (105) ............ & 506 & 16 & May & 16 & Sept. 20 & 127 \\
\hline Nashua (105) ....... & 125 & 24 & May & 5 & Sept. 10 & 125 \\
\hline Plymouth (105)........... & 500 & 21 & May & 17 & Sept. 26 & 132 \\
\hline Stratford (105)..... & 1,000 & 9 & May & 23 & Sept. 20 & 120 \\
\hline \multicolumn{7}{|l|}{ New Jersey: } \\
\hline Asbury Park $(\mathrm{H})$.. & 30 & $9=$ & Apr. & 19 & Oct. 21 & 185 \\
\hline Atlantic City (99) ......... & 16 & 35 & Apr. & 11 & Nov. 4 & 207 \\
\hline Bayonne $(100) \ldots . . . . .$. & 50 & 16 & Apr. & 16 & Oct. 19 & 186 \\
\hline
\end{tabular}


TABLE 2.-Frost data and length of average frostless season for 1893 stations in the United States. (Plate 34.)-Continued.

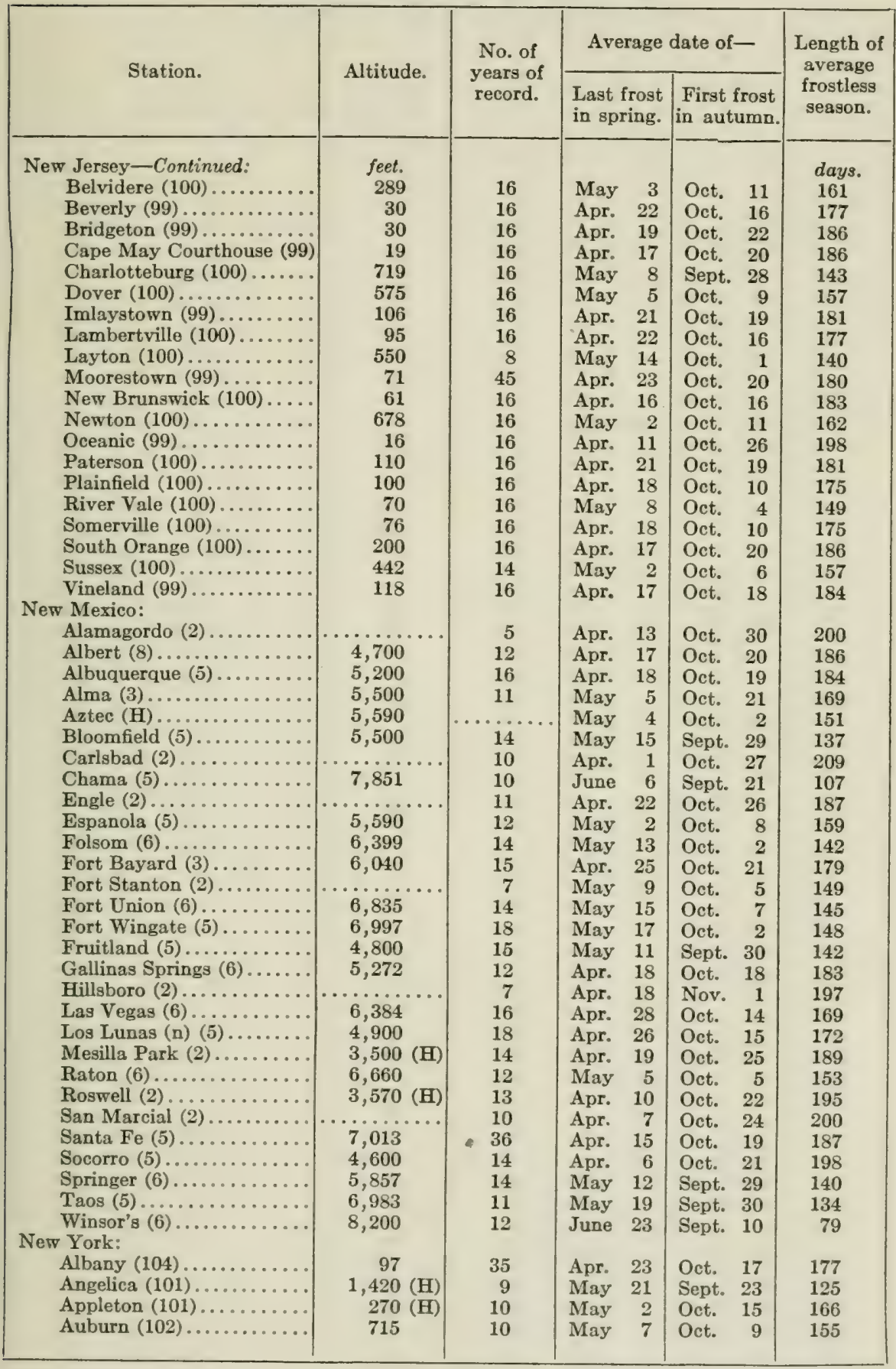


TABLE 2.-Frost data and length of average frostless season for 1803 stations in the United States. (Plate 34.)-Continued.

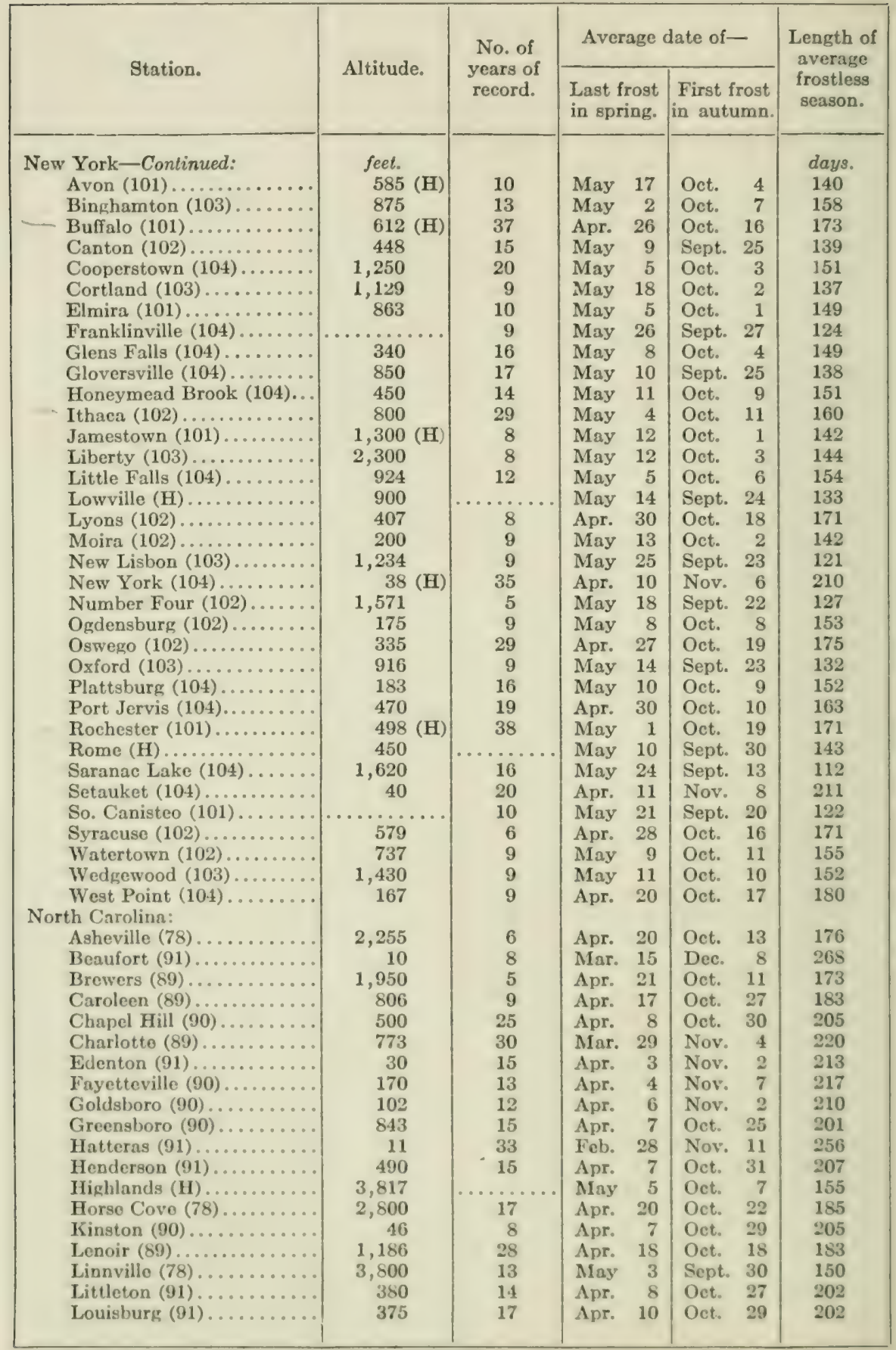


TABLE 2.-Frost data and length of average frostless season for 1803 stations in the United States. (Plate 34.)-Continued.

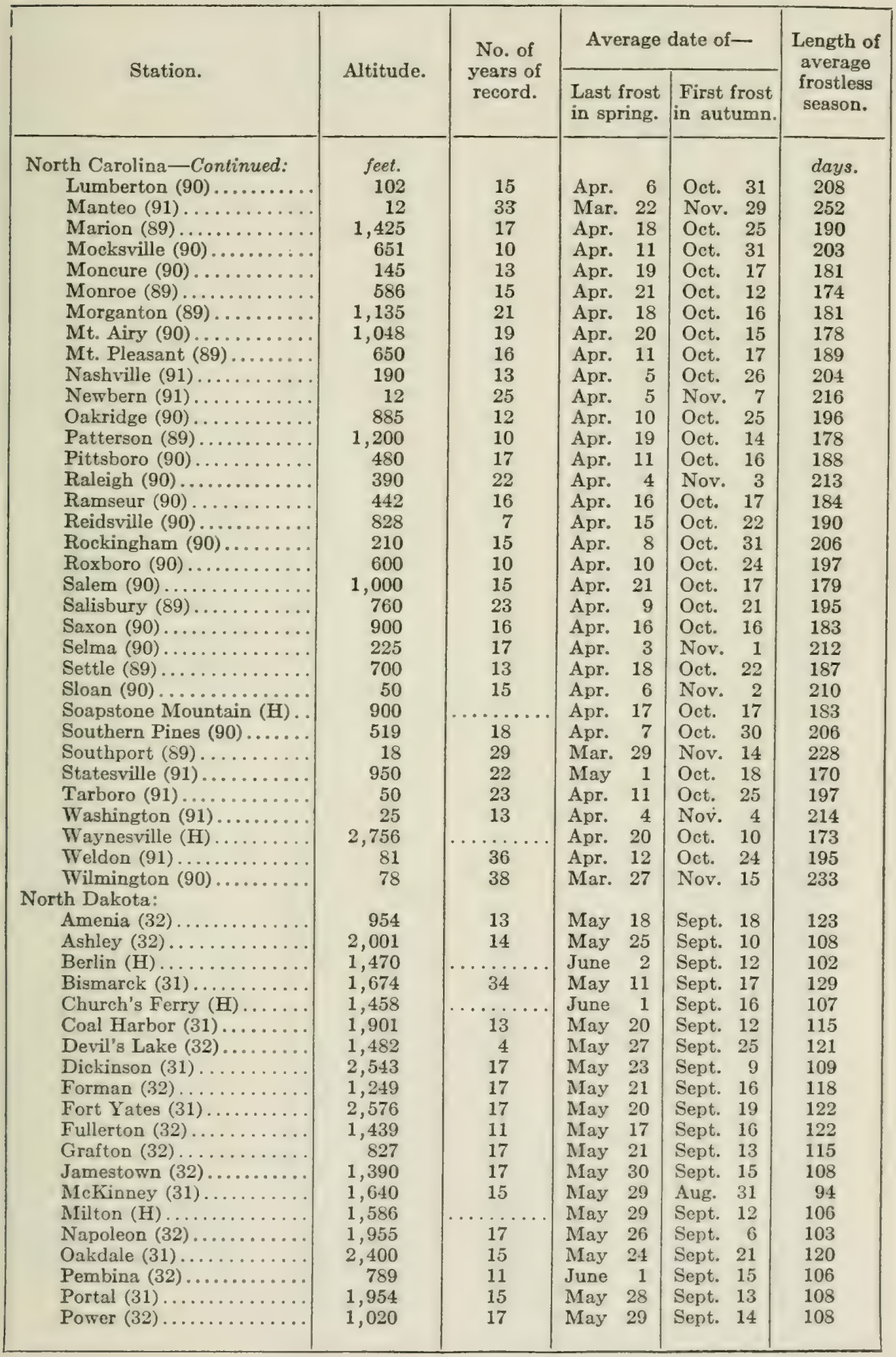


TABLE 2.-Frost data and length of average frostless season for 1803 stations in the United States. (Plate 34.)-Continued.

\begin{tabular}{|c|c|c|c|c|c|c|}
\hline \multirow{2}{*}{ Station. } & \multirow{2}{*}{ Altitude. } & \multirow{2}{*}{$\begin{array}{l}\text { No. of } \\
\text { years of } \\
\text { record. }\end{array}$} & \multicolumn{3}{|c|}{ Average date of- } & \multirow{2}{*}{$\begin{array}{l}\text { Length of } \\
\text { average } \\
\text { frostless } \\
\text { season. }\end{array}$} \\
\hline & & & $\begin{array}{l}\text { Last fr } \\
\text { in spri }\end{array}$ & $\begin{array}{l}\text { rost } \\
\text { ng. }\end{array}$ & $\begin{array}{l}\text { First frost } \\
\text { in autumn. }\end{array}$ & \\
\hline North Dakota-Continued: & feet. & & & & & days. \\
\hline $\begin{array}{l}\text { University }(32) \ldots \ldots \ldots \ldots \\
\text { Wahpeton }(\mathrm{H}) \ldots \ldots \ldots \ldots\end{array}$ & 830 & 17 & May & 20 & Sept. 14 & 117 \\
\hline Wahpeton $(\mathrm{H}) \ldots \ldots \ldots \ldots$ & 962 & ....... & May & 8 & Sept. 15 & 130 \\
\hline Williston (31)........... & 1,872 & 30 & May & 18 & Sept. 14 & 119 \\
\hline Willow City (31).......... & 1,471 & 17 & May & 30 & Sept. 11 & 104 \\
\hline \multicolumn{7}{|l|}{ Ohio: } \\
\hline $\begin{array}{l}\text { Akron }(69) \\
\text { Bangorville }(72) \ldots \ldots \ldots\end{array}$ & $\begin{array}{l}1,081 \\
1,380\end{array}$ & $\begin{array}{l}18 \\
22\end{array}$ & $\begin{array}{l}\text { Apr. } \\
\text { May }\end{array}$ & 27 & $\begin{array}{l}\text { Oct. } \\
\text { Oct. }\end{array}$ & $\begin{array}{l}165 \\
150\end{array}$ \\
\hline $\begin{array}{l}\text { Bangorville }(72) \ldots \ldots \ldots \ldots \\
\text { Bellefontaine }(70) \ldots \ldots \ldots\end{array}$ & 1,276 & $\begin{array}{l}22 \\
15\end{array}$ & $\begin{array}{l}\text { May } \\
\text { Apr. }\end{array}$ & $\begin{array}{r}6 \\
28\end{array}$ & $\begin{array}{l}\text { Oct. } \\
\text { Oct. }\end{array}$ & $\begin{array}{l}150 \\
162\end{array}$ \\
\hline Bowling Green (69)....... & 670 & 17 & May & 10 & Oct. & 145 \\
\hline Bucyrus $(69) \ldots \ldots \ldots \ldots$ & 1,000 & 15 & May & 9 & Oct. & 147 \\
\hline Cambridge (72) .......... & 803 & 16 & May & 5 & Sept. 28 & 146 \\
\hline Camp Dennison (70)..... & 570 & 16 & Apr. & 25 & Oct. 11 & 169 \\
\hline Canal Dover (72)........ & 884 & 15 & May & 8 & Oct. & 147 \\
\hline Canton $(69) \ldots \ldots \ldots \ldots$ & 1,065 & 18 & Apr. & 27 & Oct. & 161 \\
\hline Cincinnati $(70) \ldots \ldots \ldots$ & 628 & 37 & Apr. & 14 & Oct. 25 & 194 \\
\hline Circleville (71) .......... & 694 & 14 & Apr. & 28 & Oct. & 160 \\
\hline Clarksville (70)........... & 1,010 & 16 & Apr. & 25 & Oct. & 165 \\
\hline Cleveland $(69) \ldots \ldots \ldots$ & 762 & 38. & Apr. & 16 & Oct. 31 & 198 \\
\hline Coalton (71)............. & 7.18 & 14 & May & 3 & Oct. & 154 \\
\hline Columbus (71) .......... & 918 & 31 & Apr. & 16 & Oct. 17 & 184 \\
\hline Dayton $(70) \ldots . . . . . .$. & 790 & 15 & Apr. & 27 & Oct. 10 & 166 \\
\hline Defiance $(69) \ldots \ldots \ldots \ldots$ & 712 & 15 & May & 7 & Sept. 29 & 145 \\
\hline Delaware $(71) \ldots . . . .$. & 927 & 13 & May & 3 & Oct. & 153 \\
\hline $\operatorname{Demos}(72) \ldots . . . \ldots \ldots$ & 1,325 & 18 & May & 3 & Oct. 10 & 160 \\
\hline Findlay $(69) \ldots \ldots \ldots \ldots$ & 776 & 18 & May & 3 & Oct. & 154 \\
\hline Garrettsville (69) ......... & 1,005 & 22 & May & 18 & Sept. 29 & 134 \\
\hline Granville (72) ......... & 960 & 20 & Apr. & 30 & Oct. 5 & 158 \\
\hline Gratiot (72)............ & 1,000 & 18 & May & 5 & Oct. & 153 \\
\hline Green $(70) \ldots \ldots \ldots \ldots$ & 500 & 15 & Apr. & 21 & Oct. 14 & 176 \\
\hline Greenfield $(71) \ldots \ldots \ldots$ & $\ldots \ldots$ & 12 & Apr. & 19 & Oct. 12 & 176 \\
\hline Green Hill $(69)$.......... & 1,135 & 17 & May & 16 & Sept. 28 & 131 \\
\hline Greenville (70).......... & 1,060 & 16 & Apr. & 30 & Oct. 10 & 161 \\
\hline Hedges $(69) \ldots . . . . . . .$. & 725 & 15 & May & 13 & Sept. 30 & 140 \\
\hline Hillhouse $(69), \ldots \ldots \ldots$. & 997 & 17 & May & 18 & Oct. & 138 \\
\hline $\operatorname{Hiram}(\mathbf{H}) \ldots \ldots \ldots \ldots$. & 1,260 & & Apr. & 28 & Oct. $\quad 14$ & 169 \\
\hline Hudson $(69) \ldots \ldots \ldots \ldots$ & 1,153 & 15 & May & 8 & Oct. & 147 \\
\hline Ironton $(71) \ldots \ldots \ldots \ldots$ & 575 & 17 & Apr. & 21 & Oct. 11 & 173 \\
\hline Jacksonburg (70)........ & 975 & 18 & May & 2 & Oct. 13 & 164 \\
\hline Kenton (71)............. & 1,015 & 16 & May & 9 & Oct. & 149 \\
\hline Killbuck (72) ........... & 1,087 & 16 & May & 2 & Oct. & 152 \\
\hline Lancaster (71)........... & 898 & 14 & Apr. & 24 & Oct. & 162 \\
\hline Marietta (72)........... & 627 & 25 & Apr. & 18 & Oct. 20 & 185 \\
\hline 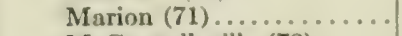 & 980. & 17 & May & 7 & Oct. & 147 \\
\hline MeConnellsville (72)...... & 710 & 24 & Apr. & 29 & Oct. & 162 \\
\hline Medina $(69) \ldots \ldots \ldots \ldots$ & 944 & 15 & May & 9 & Oct. 6 & 150 \\
\hline Milfordton (72) ........... & 1,200 & 16 & May & 6 & Sept. 30 & 147 \\
\hline Milligan (72)........... & 875 & 15 & May & 9 & Sept. $2 S$ & 142 \\
\hline Millport (72) ........... & 1,145 & 16 & May & 9 & Oct. & 147 \\
\hline Montpelier (69) ........... & 880 & 17 & May & 2 & Sept. 30 & 151 \\
\hline Napoleon $(69) \ldots . . . . .$. & 680 & 21 & May & 4 & Oct. & 154 \\
\hline Now Alexandria (72)...... & 1,050 & 24 & May & 2 & Oct. & 158 \\
\hline New Waterford (69)....... & 1,053 & 14 & May & 9 & Oct. & 145 \\
\hline North Lewisburg (71)..... & 1,095 & 21 & May & 4 & Oct. & 157 \\
\hline North Royalton (69)...... & 1,000 & 17 & May & 2 & Oct. 11 & 162 \\
\hline Norwalk (69).......... & 719 & 16 & May & 10 & Oet. & 148 \\
\hline
\end{tabular}


TABLE 2.-Frost data and length of average frostless season for 1803 stations in the United States. (Plate 34.)-Continued.

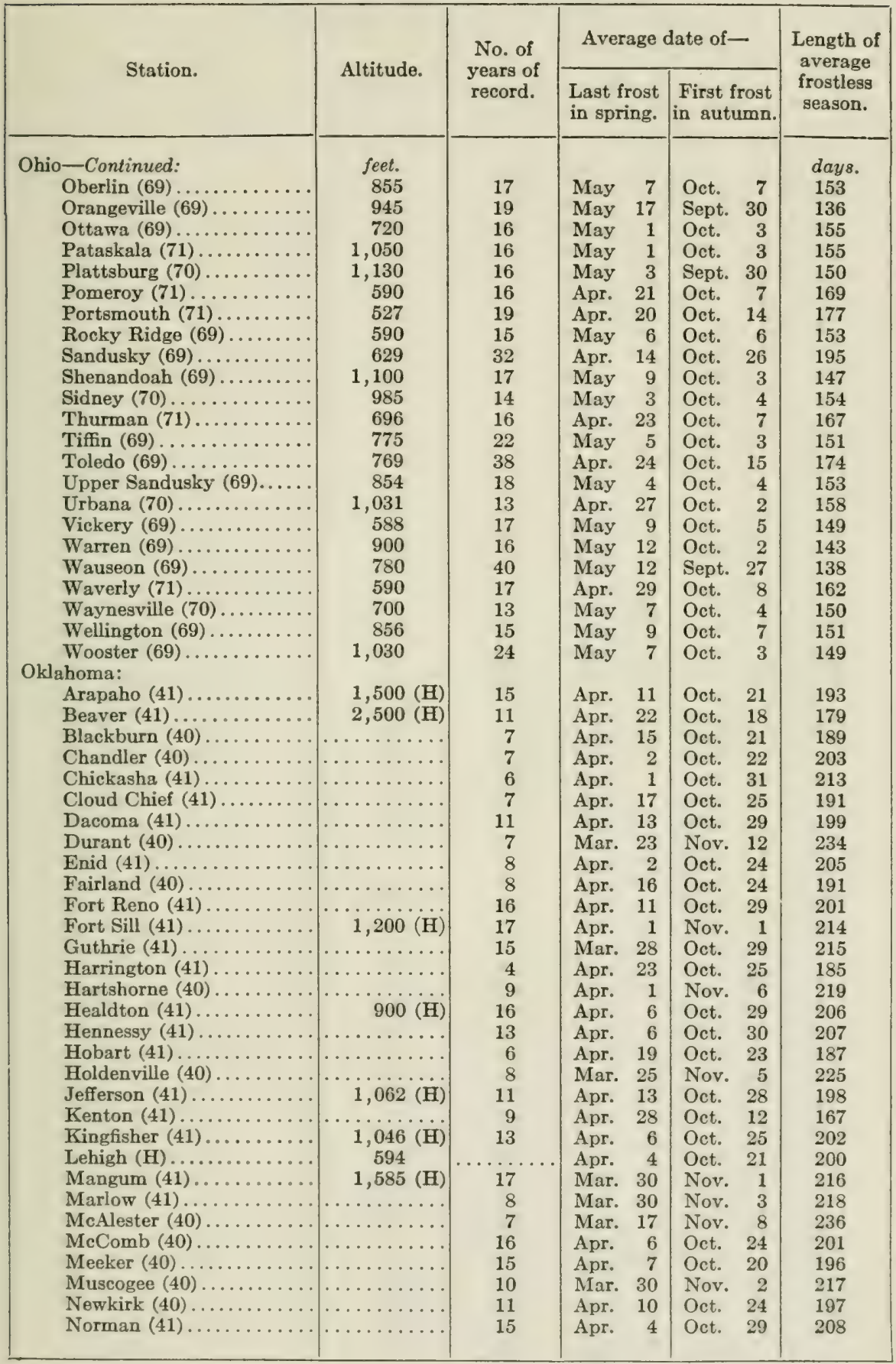


TABLE 2.-Frost data and length of average frostless season for 1803 stations in the United States. (Plate 34.)-Continued.

\begin{tabular}{|c|c|c|c|c|c|}
\hline \multirow{2}{*}{ Station. } & \multirow{2}{*}{ Altitude. } & \multirow{2}{*}{$\begin{array}{l}\text { No. of } \\
\text { years of } \\
\text { record. }\end{array}$} & \multicolumn{2}{|c|}{ Average date of- } & \multirow{2}{*}{$\begin{array}{l}\text { Length of } \\
\text { average } \\
\text { frostless } \\
\text { season. }\end{array}$} \\
\hline & & & $\begin{array}{l}\text { Last frost } \\
\text { in spring. }\end{array}$ & $\begin{array}{l}\text { First frost } \\
\text { in autumn. }\end{array}$ & \\
\hline Oklahoma-Continued: & fect. & & & & days. \\
\hline Oklahoma (41).... & $1,196(\mathrm{H})$ & 18 & Apr. & Nov. & 214 \\
\hline Paul's Valley (41)........ & $\ldots \ldots \ldots \ldots$ & 8 & Apr. 11 & Oct. 20 & 192 \\
\hline Pawhuska (40)......... & $\ldots \ldots \ldots \ldots$ & 10 & Apr. 13 & Oct. 26 & 196 \\
\hline Perry $(41) \ldots \ldots \ldots \ldots \ldots$ & $\ldots \ldots \ldots$ & 10 & Apr. 9 & Oct. 30 & 204 \\
\hline Ravia $(40) \ldots \ldots \ldots \ldots \ldots$ & $\ldots \ldots \ldots \ldots$ & 6 & Apr. & Nov. 8 & 218 \\
\hline Sac and Fox Agency (40).. & $\ldots \ldots \ldots$ & 13 & Apr. & Oct. 21 & 200 \\
\hline Shawnee $(40) \ldots \ldots \ldots \ldots$ & $\ldots \ldots \ldots$ & 7 & Apr. 2 & Oct. 29 & 210 \\
\hline Stillwater $(40) \ldots \ldots \ldots \ldots$ & $880(\mathrm{H})$ & 15 & Apr. 10 & Oct. 23 & 196 \\
\hline Temple (41)........... & $\ldots \ldots \ldots \ldots$ & 6 & Apr. 11 & Oct. 30 & 202 \\
\hline Wagoner $(40) \ldots \ldots \ldots \ldots$ & $\ldots \ldots \ldots \ldots$ & 12 & Apr. 10 & Oct. 30 & 203 \\
\hline Waukomis (41)... & . & 12 & Apr. 8 & Oct. 30 & 205 \\
\hline Weatherford (41)....... & & 8 & Apr. 13 & Oct. 30 & 200 \\
\hline Webber's Falls $(40) \ldots . .$. & & 8 & Apr. 8 & Oct. 28 & 203 \\
\hline \multicolumn{6}{|l|}{ Oregon: } \\
\hline Albany $(\mathrm{H})$ & 224 & & Mar. 27 & Nov. 4 & 222 \\
\hline Ashland (17). & 1,940 & 20 & Apr. 20 & Oct. 14 & 177 \\
\hline Astoria (17) .......... & 11 & 22 & Mar. 6 & Dec. 2 & 271 \\
\hline Baker City (18)......... & 3,471 & 20 & May 24 & Sept. 28 & 127 \\
\hline Bandon $(\mathrm{H}) \ldots \ldots$ & 55 & & Mar. 10 & Nov. 25 & 260 \\
\hline Bend (17) .... & 4,000 & & \multicolumn{3}{|c|}{ Possible throughout year. } \\
\hline Beulah (18)........... & 3,269 & 16 & June 27 & Aug. 29 & 63 \\
\hline Blalock (18) ............ & 237 & 10 & Mar. 23 & Nov. 2 & 224 \\
\hline Buckhorn Farm (17)..... & 1,300 & 11 & May 1 & Oct. 16 & 168 \\
\hline Burns (18) ............ & 4,157 & 16 & June 25 & Aug. 26 & 62 \\
\hline Canyon City (18)........ & 3,000 & 5 & May 19 & Oct. 6 & 140 \\
\hline Cascade Locks (17)...... & 100 & 18 & Apr. 1 & Nov. 13 & 226 \\
\hline Conrton (18) ........... & 2,891 & 5 & June & Sept. 14 & 97 \\
\hline Dayville $(18) \ldots \ldots \ldots \ldots$ & 2,500 & 14 & May 18 & Sept. 20 & 125 \\
\hline Detroit (17) ........... & 1,500 & 5 & May 4 & Sept. 21 & 140 \\
\hline Doraville (17) .......... & 600 & 7 & Apr. 13 & Nov. 4 & 205 \\
\hline Drain $(17) \ldots \ldots \ldots \ldots$ & 300 & 6 & Apr. 27 & Sept. 26 & 152 \\
\hline Fort Klamath (17) ....... & 4,200 & & Possible & throughout & year. \\
\hline Gardiner (17) .......... & 72 & 19 & Mar. 27 & Dec. 8 & 256 \\
\hline Glenora (17) . . . . . . . & 575 & 17 & May 10 & Oct. 11 & 154 \\
\hline Grant's Pass (17) ........ & 956 & 20 & May 6 & Oct. 12 | & 159 \\
\hline Happy Valley (18)....... & 4,200 & 10 & \multicolumn{3}{|c|}{ Possible throughout year. } \\
\hline Heppner (18) .......... & 1,950 & 10 & May 6 & Sept. 29 & 146 \\
\hline Joseph (18)............. & 4,400 & 19 & June 15 & Sept. 71 & 84 \\
\hline Klamath Falls (17)....... & 4,250 & & \multicolumn{3}{|c|}{ Possible throughout year. } \\
\hline LaGrande (18) ........... & 2,784 & 19 & May 20 ] & Sept. $22 \mid$ & $1: 5$ \\
\hline Lakeview (18)......... & 4,825 & 25 & \multicolumn{3}{|c|}{ Possible throughout year. } \\
\hline Lono Rock $(\mathrm{H}) \ldots \ldots \ldots \ldots$ & 3,114 & & June 23 & Sept. 16 & 85 \\
\hline McKenzio Bridge (17) . ... & 1,400 & 7 & June 1 & Sept. 12 & 103 \\
\hline Monroe (17) .......... & 350 & 12 & Apr. 19 & Nov. 1 & 196 \\
\hline Newport (17) .......... & 69 & 18 & Mar. 19 & Dec. 22 & 278 \\
\hline Paisley (18) ............ & 4,500 & 5 & May 25 & Sept. 24 & 122 \\
\hline Pendleton (18) ........... & 1,272 & 18 & May & Oct. & 150 \\
\hline Pompeii (17).......... & 3,879 & 14 & \multicolumn{3}{|c|}{ All month 8 . } \\
\hline Portland (17) ........... & 57 & 37 & Mnr. 16 & Nov. 16 & 245 \\
\hline Port Orford (17) ........ & 300 & 4 & \multicolumn{3}{|c|}{ Very infre quent. } \\
\hline Prineville (18) .......... & 2,860 & 12 & Juno 8 & Aug. 21 & 74 \\
\hline Riverside (18) .......... & 3,000 & 12 & June 26 & Aug. 30 & 6.5 \\
\hline Roseburg (17) .... . . . . & 510 & is & Apr. $\quad 15$ & Oct. (it) & 105 \\
\hline Salem (17) ............ & 120 & 16 & Apr. 10 & Nov. 2 & 205 \\
\hline Silver Lake (17) . . . . . . . & 4,700 & . & \multicolumn{3}{|c|}{ All month s. } \\
\hline
\end{tabular}


TABLE 2.-Frost data and length of average frostless season for 1803 stations in the United States. (Plate 34.)-Continued.

\begin{tabular}{|c|c|c|c|c|c|c|}
\hline \multirow{2}{*}{ Station. } & \multirow{2}{*}{ Altitude. } & \multirow{2}{*}{$\begin{array}{l}\text { No. of } \\
\text { years of } \\
\text { record. }\end{array}$} & \multicolumn{3}{|c|}{ Average date of- } & \multirow{2}{*}{$\begin{array}{l}\text { Length of } \\
\text { average } \\
\text { frostless } \\
\text { season. }\end{array}$} \\
\hline & & & $\begin{array}{l}\text { Last fr } \\
\text { in sp ri }\end{array}$ & $\begin{array}{l}\text { ost } \\
\text { ing }\end{array}$ & $\begin{array}{l}\text { First frost } \\
\text { in autumn. }\end{array}$ & \\
\hline Oregon-Continued: & feet. & & & & & days. \\
\hline Sparta (18) . . & 4,150 & 13 & May & 13 & Sept. 28 & 138 \\
\hline The Dalles (17).......... & 112 & 19 & Apr. & 10 & Nov. 5 & 209 \\
\hline Umatilla (18)........... & 340 & 7 & Apr. & 14 & Oct. 22 & 191 \\
\hline Vale $(18) \ldots$. & 3,047 & 17 & May & 30 & Sept. $\quad 9$ & 102 \\
\hline Warmspring (17) ........ & 1,600 & 7 & May & 24 & Sept. 25 & 124 \\
\hline Weston (18) ............. & 1,800 & 19 & May & 13 & Sept. 24 & 134 \\
\hline \multicolumn{7}{|l|}{ Pennsylvania: } \\
\hline Emporium $(97) \ldots \ldots \ldots \ldots$ & 1,050 & . & May & 11 & Oct. & $\begin{array}{l}151 \\
149\end{array}$ \\
\hline Erie $(96) \ldots \ldots \ldots \ldots \ldots$ & 713 & $\ldots$ & Apr. & 20 & Oct. 31 & 194 \\
\hline Everett $(97) \ldots \ldots \ldots \ldots$ & 1,080 & $\cdots$ & May & 5 & Oct. & 156 \\
\hline Franklin (Venango Co.) (96) & .... & $\cdots$ & May & 17 & Oct. 11 & 147 \\
\hline Harrisburg (97) .......... & 361 & $\ldots$ & Apr. & 10 & Oct. 23 & 196 \\
\hline Huntingdon $(\mathrm{H}) \ldots \ldots \ldots$ & 650 & . & May & 1 & Oct. $\quad 15$ & 167 \\
\hline Lebanon $(\mathrm{H}) \ldots \ldots \ldots \ldots$ & 458 & .. & Apr. & 25 & Oct. 25 & 183 \\
\hline LeRoy $(97) \ldots$ & $1,400(\mathrm{H})$ & $\ldots$ & May & 6 & Oct. & 154 \\
\hline Mauch Chunk (98)... . . . & 634 & $\cdots$ & May & 3 & Oct. 11 & 161 \\
\hline Philadelphia (98) ........ & 117 & $\cdots$ & Apr. & 8 & Oct. 31 & 206 \\
\hline Pittsburg (96). & 842 & . & Apr. & 22 & Oct. 18 & 179 \\
\hline Quakertown (H) ......... & 536 & . & Apr. & 20 & Oct. & 183 \\
\hline Saegerstown (H) ......... & 1,116 & $\cdots$ & May & 14 & Sept. 24 & 133 \\
\hline Scranton (97). & $\ldots$ & $\cdots$ & Apr. & 20 & Oct. 13 & 176 \\
\hline Selinsgrove $(\mathrm{H})$. & 455 & . & May & 8 & Oct. & 146 \\
\hline South Eaton $(\mathrm{H})$. & 660 & . & Apr. & 22 & Sept. 28 & 159 \\
\hline State College $(97) \ldots \ldots \ldots$ & 1,191 & • & May & 9 & Oct. & 146 \\
\hline Westchester $(\mathrm{H}) \ldots \ldots \ldots \ldots$ & 460 & . & Apr. & 23 & Oct. & 186 \\
\hline York $(\mathrm{H}) \ldots$. & 385 & $\cdots$ & Apr. & 25 & Oct. & 159 \\
\hline \multicolumn{7}{|l|}{ Rhode Island: } \\
\hline Block Island (105). & 26 & 21 & Apr. & 12 & Nov. 16 & 218 \\
\hline Kingston (H). & 250 & $\cdots$ & Apr. & 26 & Oct. 17 & 174 \\
\hline Narragansett $(H) \ldots \ldots \ldots$ & 33 & * & Apr. & 20 & Nov. 11 & 205 \\
\hline Providence (105) ......... & 182 & 24 & Apr. & 15 & Oct. 22 & 190 \\
\hline \multicolumn{7}{|l|}{ South Carolina: } \\
\hline Aiken (87). & 565 & 18 & Mar. & 11 & Nov. 18 & 252 \\
\hline Allendale $(86) \ldots \ldots \ldots \ldots$ & 186 & 15 & Mar. & 24 & Nov. 20 & 241 \\
\hline Batesburg (87) . . . . . . . & 656 & 13 & Mar. & 24 & Nov. & 223 \\
\hline Beaufort $(88) \ldots \ldots \ldots \ldots$ & 20 & 20 & Mar. & 9 & Nov. 23 & 259 \\
\hline Blackville (88) .......... & 296 & 18 & Mar. & 19 & Nov. 16 & 242 \\
\hline Charleston (88) ....... & 48 & 38 & Mar. & 1 & Dec. 2 & 276 \\
\hline Cheraw (88)........ & 144 & 20 & Apr. & 5 & Nov. & 210 \\
\hline Clemson College (87) ..... & 850 & 13 & Apr. & 5 & Oct. 31 & 209 \\
\hline Columbia (87) .......... & 351 & 22 & Mar. & 22 & Nov. & 231 \\
\hline Conway (88). & 25 & 10 & Mar. & 26 & Nov. 13 & 232 \\
\hline Florence (88). & 136 & 18 & Mar. & 31 & Nov. & 221 \\
\hline Georgetown (88). & 12 & 14 & Mar. & 19 & Nov. 13 & 239 \\
\hline Greenville (87)... & 989 & 14 & Apr. & 8 & Nov. & 213 \\
\hline Greenwood (86)... & 671 & 20 & Mar. & 22 & Nov. & 227 \\
\hline Newberry (87) .... & 502 & 13 & Apr. & 11 & Oct. 31 & 203 \\
\hline Santuc (87)... & 512 & 15 & Apr. & 3 & Oct. 26 & 206 \\
\hline Society Hill $(\mathrm{H}) \ldots$ & 192 & $\cdots$ & Mar. & 18 & Nov. 15 & 242 \\
\hline Spartanburg (87).... & 875 & 14 & Mar. & 31 & Nov. 6 & 220 \\
\hline Stateburg (88) ........ & 500 & 27 & Apr. & 2 & Nov. & 221 \\
\hline Summerville (88) ........ & 75 & 10 & Mar. & 20 & Nov. 17 & 242 \\
\hline Trenton (87) ........... & 620 & 15 & Mar. & 24 & Nov. 12 & 233 \\
\hline Trial $(88) \ldots \ldots \ldots$ & 85 & 20 & Apr. & & Nov. 4 & 215 \\
\hline
\end{tabular}


TABLE 2.-Frost data and length of average frostless season for 1803 stations in the United States. (Plate 34.)-Continued.

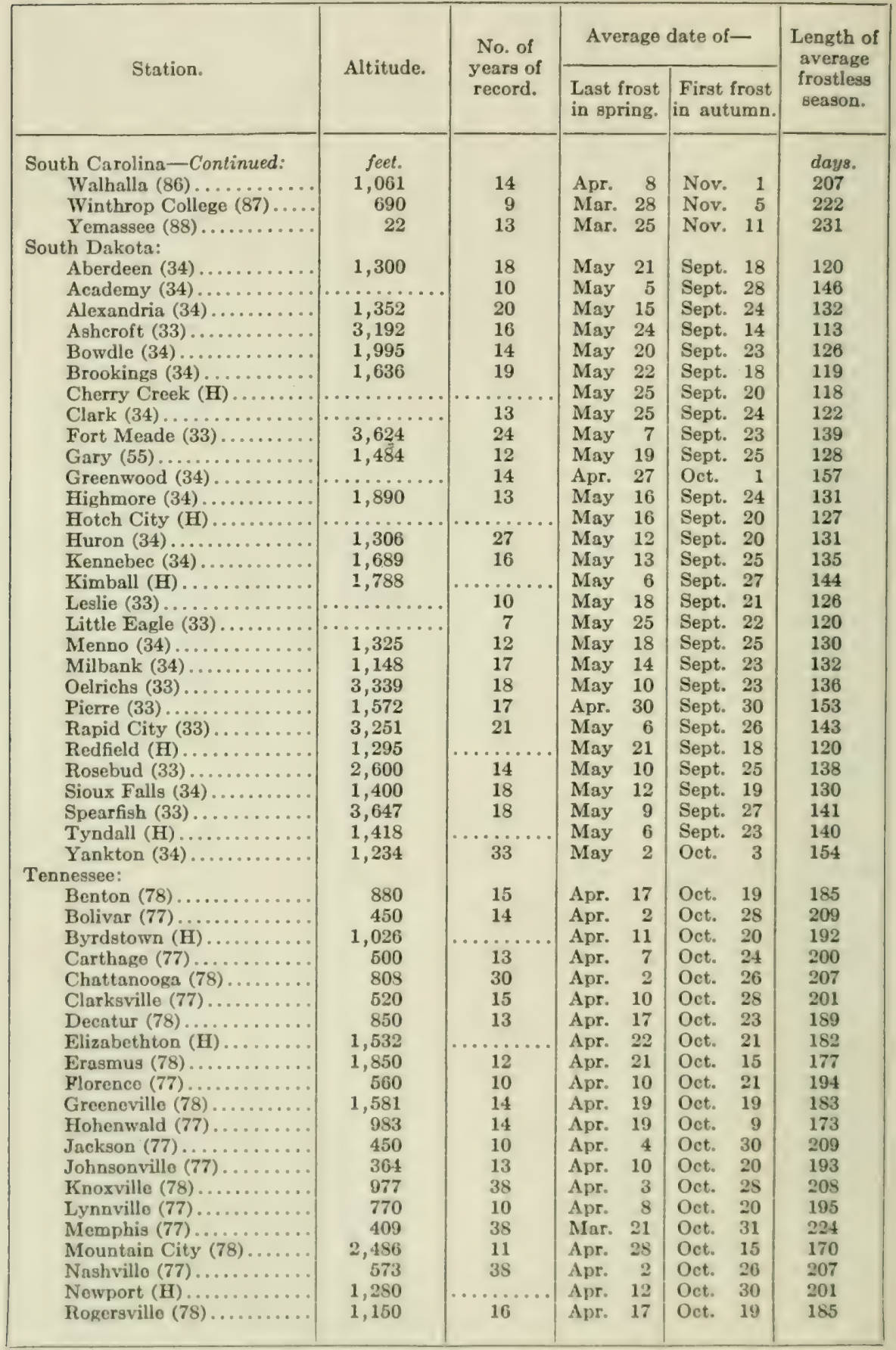


TABLE 2.-Frost data and length of average frostless season for 1803 stations in the United States. (Plate 34.)-Continued.

\begin{tabular}{|c|c|c|c|c|c|}
\hline \multirow{2}{*}{ Station. } & \multirow{2}{*}{ Altitude. } & \multirow{2}{*}{$\begin{array}{l}\text { No. of } \\
\text { years of } \\
\text { record. }\end{array}$} & \multicolumn{2}{|c|}{ Average date of - } & \multirow{2}{*}{$\begin{array}{c}\text { Length of } \\
\text { average } \\
\text { frostless } \\
\text { season. }\end{array}$} \\
\hline & & & $\begin{array}{l}\text { Last frost } \\
\text { in spring. }\end{array}$ & $\begin{array}{l}\text { First frost } \\
\text { in autumn. }\end{array}$ & \\
\hline Tennessee-Continued: & feet. & & & & days. \\
\hline Rugby (78) ...... & 1,410 & 16 & Apr. 22 & Oct. 13 & 174 \\
\hline Savannah (77)........... & 442 & 12 & Apr. 7 & Oct. 23 & 199 \\
\hline Springdale $(78) \ldots \ldots \ldots$ & 1,058 & 16 & Apr. 21 & Oct. 15 & 177 \\
\hline Springville (77) ......... & 377 & 6 & Apr. 16 & Oct. 13 & 180 \\
\hline Trenton (77) ........... & 345 & 15 & Apr. 5 & Oct. 18 & 196 \\
\hline Tullahoma (77)......... & 1,075 & 13 & Apr. 17 & Oct. $\quad 18$ & 184 \\
\hline Texas: & & & & & \\
\hline Abilene (42) ... & 1,738 & 23 & Mar. 15 & Nov. 15 & 245 \\
\hline Albany (43) ............. & 1,429 & 13 & Mar. 25 & Nov. 9 & 229 \\
\hline Amarillo (42) ............. & 3,676 & 18 & Apr. 16 & Nov. 1 & 199 \\
\hline 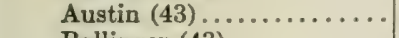 & 593 & 15 & Mar. 12 & Nov. 18 & 251 \\
\hline Ballinger (43) ............ & 1,637 & 5 & Mar. 23 & Nov. 9 & 231 \\
\hline Beaumont (1).......... & 29 & 12 & Feb. 24 & Dec. 2 & 281 \\
\hline Beeville (1) ............ & 225 & 15 & Feb. 14 & Nov. 30 & 289 \\
\hline Big Spring (2) ......... & $\cdots \cdots \cdots \cdots$ & 6 & Apr. 6 & Nov. 7 & 215 \\
\hline Blanco $(43) \ldots . . . . . .$. & 1,350 & 4 & Mar. 22 & Nov. 7 & 230 \\
\hline Boerne (1).............. & 1,412 & 16 & Mar. 1 & Nov. 24 & 268 \\
\hline 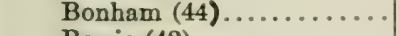 & 566 & 7 & Apr. 6 & Nov. 13 & 221 \\
\hline Bowie (42)............. & 1,113 & 9 & Mar. 16 & Nov. 10 & 239 \\
\hline Brady (43) .............. & 1,500 & 6 & Mar. 29 & Nov. 7 & 223 \\
\hline Brenham (44) .......... & 350 & 19 & Feb. 23 & Nov. 25 & 275 \\
\hline Brighton (1) ............ & 12 & 16 & Feb. 10 & Dec. 9 & 302 \\
\hline Brownsville (1)........... & 38 & 18 & Feb. & Dec. 21 & 318 \\
\hline Brownwood (43)......... & 1,342 & 13 & Mar. 27 & Nov. 10 & 228 \\
\hline Channing (42)........... & $\ldots \ldots \ldots$ & 5 & Apr. 25 & Oct. 18 & 176 \\
\hline Childress (42) .......... & 1,869 & 9 & Apr. & Nov. & 211 \\
\hline Claude $(42) \ldots \ldots \ldots \ldots$ & 3,397 & 4 & Apr. 21 & Oct. 29 & 191 \\
\hline Claytonville (43) ......... & 2,100 & 11 & Mar. 25 & Nov. & 225 \\
\hline Coleman (43) ............ & 1,710 & 8 & Mar. 18 & Nov. 17 & 244 \\
\hline College Station (44) ....... & 308 & 15 & Mar. 4 & Nov. 21 & 262 \\
\hline Colorado (43)............ & 2,066 & 8 & Apr. 11 & Nov. & 212 \\
\hline Columbia (1)........... & 34 & 16 & Feb. 24 & Nov. 28 & 277 \\
\hline Corpus Christi (1)....... & 20 & 24 & Feb. 21 & Dec. 16 & 298 \\
\hline Corsicana (44).......... & 445 & 19 & Mar. 15 & Nov. 14 & 244 \\
\hline Cuero $(1) \ldots \ldots \ldots \ldots \ldots$ & 177 & 17 & Feb. 25 & Nov. 23 & 271 \\
\hline Dallas $(44) \ldots . . . . . . .$. & 466 & 19 & Mar. 19 & Nov. 13 & 239 \\
\hline Danevang (1)........... & 145 & 14 & Feb. 24 & Nov. 21 & 270 \\
\hline Del Rio (1)............. & 952 & 5 & Feb. 23 & Nov. 21 & 271 \\
\hline Dublin (43)........... & 1,466 & 12 & Mar. 17 & Nov. 14 & 242 \\
\hline Eagle Pass (1)........... & 800 & 17 & Feb. 27 & Nov. 21 & 267 \\
\hline El Paso (2).............. & $3,702(\mathrm{H})$ & 29 & Mar. 20 & Nov. 11 & 236 \\
\hline Fairland $(43) \ldots \ldots \ldots \ldots$ & 1,000 & 14 & Mar. 16 & Nov. 20 & 249 \\
\hline Fort Clark (1).......... & 1,050 & $18-$ & Feb. 24 & Nov. 23 & 272 \\
\hline Fort McIntosh (1)....... & 460 & 18 & Feb. 18 & Nov. 28 & 283 \\
\hline Fort Ringgold (1)........ & 230 & 14 & Feb. 12 & Dec. 11 & 302 \\
\hline Fort Worth (43)........ & 670 & 17 & Mar. 8 & Nov. 24 & 261 \\
\hline Fredericksburg (43) ....... & 1,742 & 18 & Mar. 13 & Nov. 14 & 246 \\
\hline Gainesville (44) ......... & 738 & 77 & Mar. 31 & Nov. 7 & 221 \\
\hline Galveston (1)..... & 69 & 39 & Jan. 27 & Dec. 24 & 331 \\
\hline Graham (42)............ & 1,040 & 7 & Apr. & Nov. 10 & 222 \\
\hline Greenville (44)......... & 550 & 8 & Mar. 10 & Nov. 18 & 253 \\
\hline Hale Center (42) ......... & ....... & 11 & Apr. & Oct. 30 & 211 \\
\hline Hallettsville (1).......... & 235 & 17 & Mar. & Nov. 21 & 263 \\
\hline Haskell (42).......... & 1,553 & 12 & Apr. & Nov. 12 & 222 \\
\hline Henrietta $(42) \ldots \ldots \ldots \ldots$ & 915 & 12 & Mar. 25 & Nov. 8 & 228 \\
\hline
\end{tabular}


TABLE 2.-Frost data and length of average frostless season for 1803 stations in the United States, (Plate 34.)-Continued.

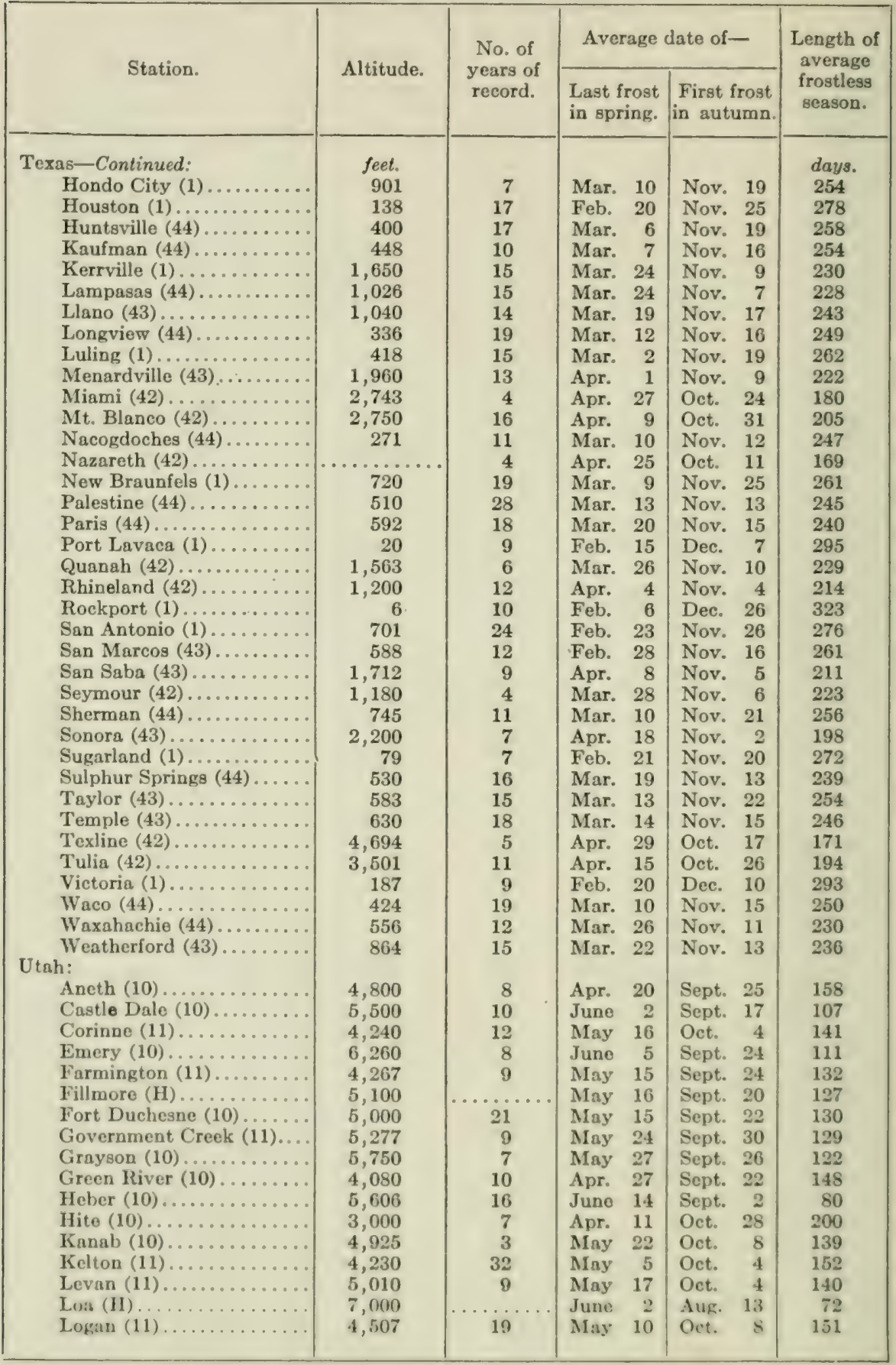


TABLE 2.-Frost data and length of average frostless season for 1803 stations in the United States. (Plate 34.)-Continued.

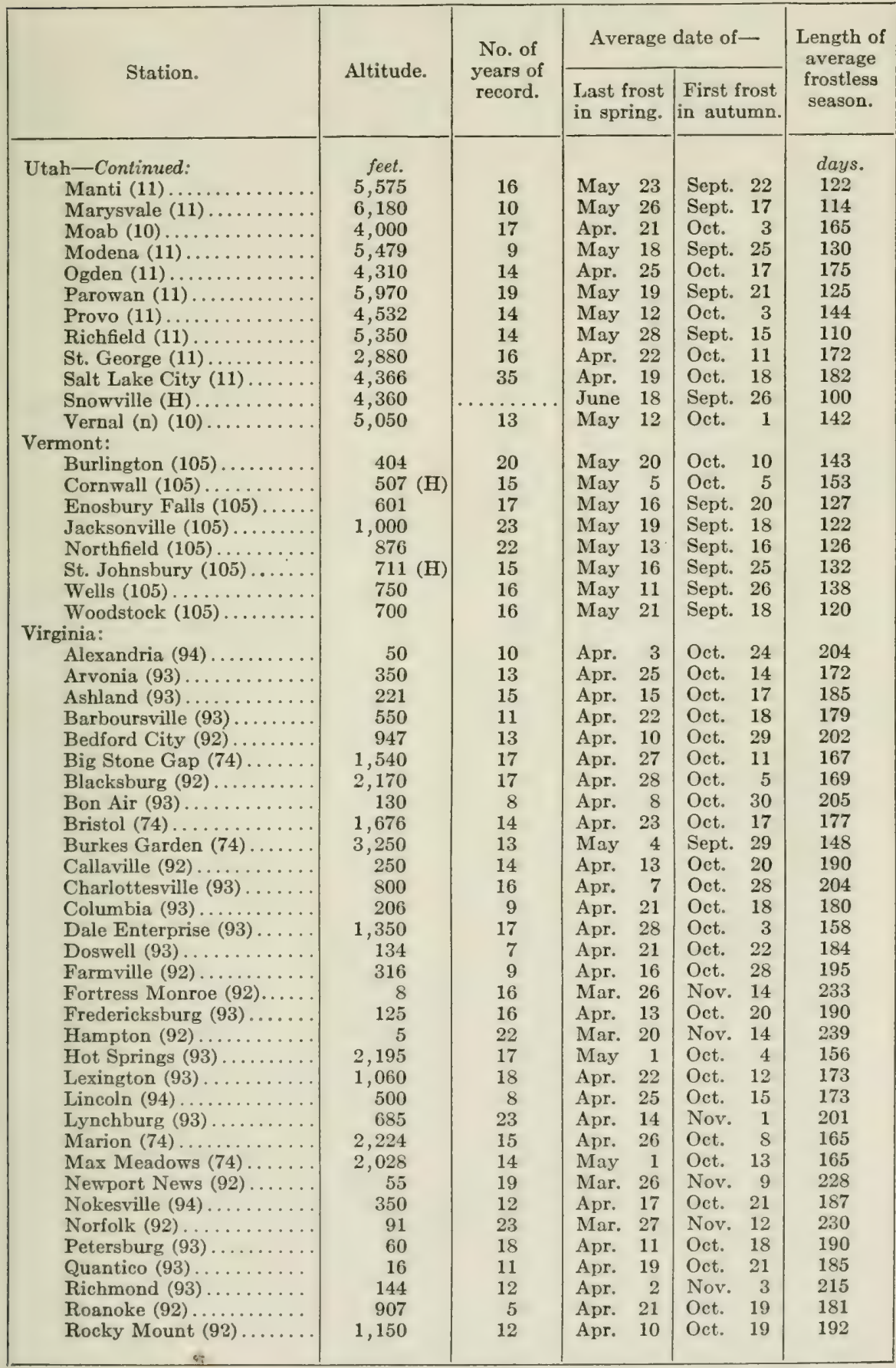


TABLE 2.-Frost data and length of average frostless season for 1803 stations in the Uniled States. (Plate 34.)-Continued.

\begin{tabular}{|c|c|c|c|c|c|c|c|}
\hline \multirow{2}{*}{ Station. } & \multirow{2}{*}{ Altitude. } & \multirow{2}{*}{$\begin{array}{l}\text { No. of } \\
\text { years of } \\
\text { record. }\end{array}$} & \multicolumn{4}{|c|}{ Average date of- } & \multirow{2}{*}{$\begin{array}{c}\text { Length o } \\
\text { average } \\
\text { frostless } \\
\text { season. }\end{array}$} \\
\hline & & & $\begin{array}{l}\text { Last fro } \\
\text { in sprin }\end{array}$ & $\begin{array}{l}\text { rost } \\
\text { ing. }\end{array}$ & $\begin{array}{l}\text { First fr } \\
\text { in autu }\end{array}$ & rost & \\
\hline $\begin{array}{l}\text { Virginia-Continusd: } \\
\text { Salem }(92) \ldots . . .\end{array}$ & $\begin{array}{r}\text { feet. } \\
1,000\end{array}$ & 9 & Apr. 1 & & Oct. & 17 & $\begin{array}{l}\text { days. } \\
190\end{array}$ \\
\hline Saxe $(92) \ldots$. & 350 & 8 & Apr. 1 & & Oct. & 21 & 190 \\
\hline Shenandoah (93) & 937 & 3 & Apr. 2 & & Oct. & 9 & 163 \\
\hline Spottsville (92).. & 15 & 17 & Apr. 1 & 17 & Oct. & 16 & 182 \\
\hline Stanardsville (93) & 670 & 12 & Apr. 1 & 12 & Oct. & 25 & 196 \\
\hline Staunton (93).... & 1,380 & 17 & Apr. 2 & 25 & Oct. & 12 & 170 \\
\hline Stephens City (94). & 710 & 14 & Apr. 2 & 21 & Oct. & 14 & 176 \\
\hline Sunbeam $(92) \ldots \ldots$ & 60 & 6 & Apr. 1 & 11 & Oct. & 31 & 203 \\
\hline Warsaw (93). & 200 & 16 & Apr. 1 & 15 & Oct. & 22 & 190 \\
\hline West Brook (93). & & 4 & Apr. 1 & 15 & Oct. & 20 & 188 \\
\hline Williamsburg (93) & 74 & 8 & Apr. & 9 & Oct. & 26 & 200 \\
\hline Woodstock $(94) \ldots$ & 927 & 11 & Apr. 2 & 22 & Oct. & 7 & 168 \\
\hline Wytheville $(74) \ldots \ldots$ & 2,293 & 15 & Apr. 1 & 18 & Oct. & 10 & 175 \\
\hline \multicolumn{8}{|l|}{ Washington: } \\
\hline Aberdeen (19) & $162(\mathrm{H})$ & 10 & Apr. 2 & 27 & Oct. & 22 & 178 \\
\hline Bellingham (19). & & 10 & Apr. 2 & 22 & Oct. & 21 & 182 \\
\hline Centralia (19). & $212(\mathrm{H})$ & 9 & May & 6 & Oct. & 14 & 161 \\
\hline Clearwater (19) & & 9 & Apr. 2 & 27 & Nov. & 7 & 194 \\
\hline Cle Elum (20).. & 1,930 & 9 & June & $\begin{array}{ccc}9 & & \\
\end{array}$ & Sept. & 7 & 90 \\
\hline Colfax $(20) \ldots$ & 2,300 & 13 & May 2 & 25 & Sept. & 10 & 108 \\
\hline Colville $(20)$. & 1,635 & 9 & June & 5 & Sept. & 7 & 94 \\
\hline Conconully (20). & 2,300 & 9 & May 1 & 18 & Sept. & 21 & 126 \\
\hline Coupeville (19).. & & 9 & Apr. & $\begin{array}{l}9 \\
\end{array}$ & Nov. & 8 & 213 \\
\hline Crescent (20)... & 2,250 & 9 & May 2 & 23 & Sept. & 22 & 122 \\
\hline Ellensburg (20) & 1,571 & 15 & May 2 & 23 & Sept. & 21 & 121 \\
\hline Kennewick (20). & 367 & 7 & Apr. 2 & 28 & Oct. & 15 & 170 \\
\hline La Center (19). & & 10 & Apr. 2 & 20 & Oct. & 25 & 188 \\
\hline Lakeside (20)... & 1,116 & 16 & Apr. 1 & 10 & Oct. & 19 & 192 \\
\hline Lester (19).... & & 5 & May 1 & 18 & Sept. & 13 & 118 \\
\hline Lyle (20) & 600 & 16 & Apr. 2 & 23 & Oct. & 18 & 178 \\
\hline Moxee (20). & 1,000 & 16 & May 2 & 23 & Sept. & 21 & 121 \\
\hline North Head (19). & & 6 & Feb. & 9 & Dec. & 22 & 316 \\
\hline Olga $(19) \ldots \ldots$. & $50(\mathrm{H})$ & 10 & Mar. 2 & 27 & Nov. & 21 & 239 \\
\hline Olympia (19)..... & $17(\mathrm{H})$ & 10 & Apr. 2 & 28 & Nov. & 2 & 188 \\
\hline Pomeroy $(\mathrm{H})$. & 1,500 & & Apr. 2 & 26 & Sept. & 28 & 155 \\
\hline Republic (20). & 2,628 & 8 & June 1 & 15 & Sept. & 3 & 80 \\
\hline Rosalia (20). & 2,425 & 9 & June & 1 & Sept. & 14 & 105 \\
\hline Seattle (19). & $46(\mathrm{H})$ & 18 & May 2 & 21 & Nov. & 22 & 246 \\
\hline Snohomish $(H)$. & 50 & & Apr. 2 & 21 & Oct. & 21 & 183 \\
\hline Snoqualmie Falls (19) & & 8 & May & 9 & Oct. & 24 & 168 \\
\hline Spokane $(20) \ldots \ldots \ldots$ & 1,943 & 28 & Mar. 2 & 26 & Oct. & 14 & 202 \\
\hline Sunnyside (20). & 740 & 9 & May & 7 & Oct. & 8 & 154 \\
\hline Tatoosh (H). & 86 & & Mar. 1 & 13 & Dec. & 9 & 271 \\
\hline Union City (19). & & 8 & Apr. 2 & 27 & Oct. & 27 & 183 \\
\hline Walla Walla $(20)$. & 1,000 & 23 & $\Lambda$ pr. & 1 & Nov. & 3 & 216 \\
\hline Waterville (20). & 2,624 & 16 & May 3 & 31 & Sept. & 20 & 112 \\
\hline Wenatcheo $(\mathrm{n})(20)$. & 1,169 & & Apr. 3 & 30 & Oct. & 21 & 174 \\
\hline Wilbur $(20) \ldots \ldots$. & 2,203 & 9 & June 2 & 23 & Sept. & 6 & 75 \\
\hline Zindel $(20) \ldots \ldots \ldots$ & 715 & 7 & Apr. 1 & 14 & Oet. & 29 & 198 \\
\hline \multicolumn{8}{|l|}{ West Virginia: } \\
\hline Ben's Run (73) & 622 & 8 & Apr. 2 & 24 & Oet. & 16 & 175 \\
\hline Buckhannon (73) & 1,472 & 16 & Apr. 2 & 27 & Oct. & 6 & 162 \\
\hline Burlington (94).. & 875 & & May & 7 & Oct. & 2 & 148 \\
\hline Central Station (73). & 953 & 11 & May 1 & 10 & Oct. & 7 & 150 \\
\hline Charleston (74). & 598 & 6 & Apr. 2 & 24 & Oct. & 19 & 178 \\
\hline
\end{tabular}


TABLE 2.-Frost data and length of average frostless season for 1803 stations in the United States. (Plate 34.)-Continued.

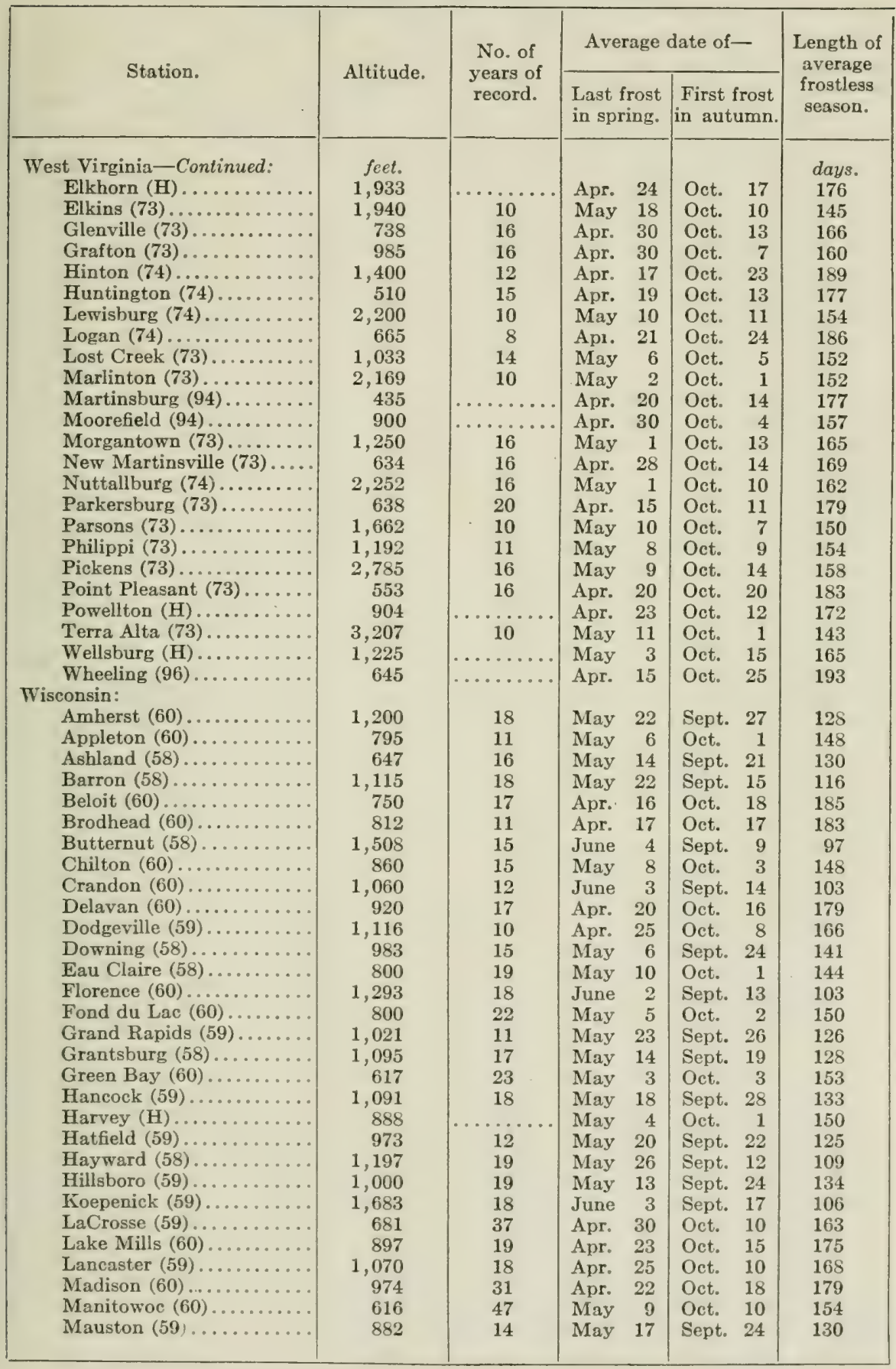


TABLE 2.-Frost data and length of average frostless season for 1803 stations in the United States. (Plate 34.)-Continued.

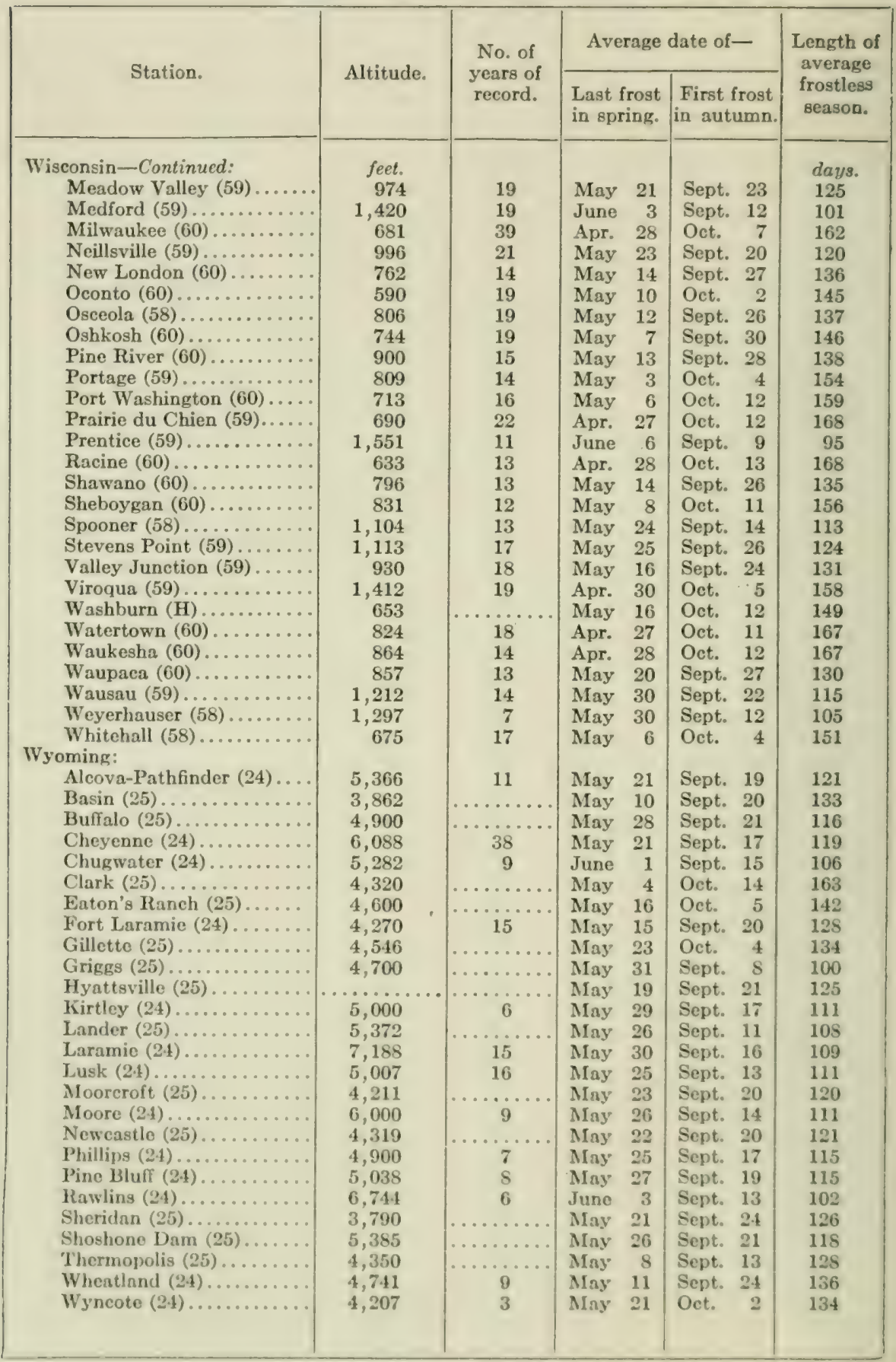


Most of the data of table 2 have been plotted on a map of the United States, to represent them in a graphic way. After the numerous stations had been located and the average length of their respective frostless seasons had been placed beside the points representing them on the map, lines were traced as accurately as possible through points having a common length of frostless season, a line for each increment of 20 days, beginning with 80 . By this means the map was subdivided into 15 sorts of seasonal areas. The first sort has, according to our data, an average frostless season of less than 80 days, the second has a season of 80 to 100 days, and so on by steps of 20 days until the average frostless season for the fourteenth sort of areas is 320 to 340 days, and for the fifteenth, over 340 days. The only one of our stations that is unquestionably without frost, thus having an average frostless season of 365 days, is Key West, Florida.

In tracing the equiseasonal lines we have followed the data of table 2 as accurately as possible, making no attempt to smooth the lines. Topography has been allowed to exert a deciding influence in many cases where observational stations are too far apart satisfactorily to determine the positions of the lines. This is especially the case for that portion of the chart which lies west of the one hundred and fifth meridian of west longitude. In a very few cases the data of single stations have been ignored, where the length of season given in the table is obviously a marked exception for its region, thus suggesting the possibility of error or inadequacy in the data themselves. Wherever a small local area is indicated by the data from two or more stations, however, the area has been shown on the chart.

It was found at once that the approximate equiseasonal lines for 20 -day increments were altogether too crowded in the mountainous region of the West, when drawn upon a chart of any convenient size, and for this region all lines have been discarded in this region, excepting those for $80,120,180,240$, and 300 days. This method probably presents the details for this part of the country as accurately as is to be expected from the data now at hand. The omitted lines, all drawn for the East, have been abruptly terminated wherever they would enter the more generalized portion of our chart. Plate 34 is a representation of the chart just described. For the sake of clearness, the chart has been shaded so as to fall primarily into the five classes of areas. These areas denote regions with average lengths, in days, of frostless season of (1) less than 120, (2) 120 to 180, (3) 180 to 240, (4) 240 to 300 , and (5) over 300 . The other lines, representing greater detail, are shown in dotted form.

A comparison of plate 34 with Day's plate V (loc. cit.) shows, at first glance, a remarkable series of differences. Closer scrutiny brings it out, however, that the apparent discrepancies are mainly due to the fact that the lines of Day's chart have been obviously subjected to a 
very strenuous smoothing process. Since a smoothed chart is thus already in existence, we have thought it best to let plate 34 represent as nearly as possible the present status of our climatic and physiographic information, and, as has been pointed out, we have made no attempt to smooth our equiseasonal lines. Professor Day informs us that some scattered data other than those available for our use were included in his study of the frostless season, and it seems probable that a few discrepancies between his chart and ours may be related to this fact. At any rate, for all practical purposes, and until such time as much more complete and reliable observational data may have been obtained, we may say that these two charts are in very good agreement. So far as we are aware, no attempt, other than Day's and our own, to prepare a chart of the average length of frostless period for the United States has yet been made.

The main generalization to be derived from plate 34 is that the areas of equal frostless seasons traverse the country, roughly, in a west-east direction, being displaced, however, to the northward in the vicinity of the Pacific and Atlantic coasts and to the southward in the regions of the western and eastern mountain systems. The coastal displacement is especially pronounced on the Pacific, where the 300-day season reaches as far north as Washington. Here the season of 180 days seems to extend even into British Columbia. On the eastern coast the last-named season extends as far northward as Massachusetts. The northward extension of the same season is seen to be limited in the central portion of the country, approximately by the southern boundary of Iowa, while the western mountains displace its northern limit, in southern New Mexico, to about latitude $33^{\circ}$ north, and the Appalachians displace it, in northern Georgia, to about latitude $35^{\circ}$ north. Again, the 120-day season is not represented at all on either coast, but extends as far southward as latitude $35^{\circ}$ north, in Arizona and New Mexico.

The Great Lakes exhibit a tendency, as far as our data extend, to lengthen the average frostless season in their vicinity. The chart shows also a frequent tendency toward an upstream extension of any given length of average frostless season in the ricinity of the larger rivers, even where this has no obvious relation to altitude.

As has already been stated, the data for the average length of the frostless season have been made the basis for many of our studies of other climatie features, on the assumption that this time period may be taken as a rough approximation of the length of the season of active growth for a large number of plant-forms. It seems probable that it is proportional to the average growing season for most plants, at any rate. 
(C) LENGTH OF PERIOD OF AVERAGE FROST SEASON.

By the frost season is here indicated the period of the year during which frost is apt to occur. In this season such plant-forms as are killed or thrown into the dormant state by the occurrence of freezing temperatures should not be active. While actual growth of such plants often occurs within this season of any year, in frostless periods of a few days, yet this growth is soon checked, and foliage, etc., thereby produced is usually destroyed by the recurrence of frost, so that the result of such short growing-periods is seldom to be considered as advancing the organism very much toward maturity or reproduction. It may thus be generally assumed that the average frost season for any region represents the average period of dormancy for a large number of plant forms.

It is obvious that the average length of the frost season is the complement of the average length of the frostless period." Thus, from table 2, the mean length of the frost season may be obtained for any station by subtracting the number of days given for the frostless season from 365 , the total number of days in the year. It is also obvious that the chart of the mean duration of the frostless season is simultaneously a chart of the mean length of the frost season. Thus, on plate 34 , the area represented as having a mean length of frostless season of less than 120 days is characterized by an average period of general plant dormancy of over 245 days, etc.

It seems highly probable, though there is at hand no direct information in this connection, that many plant-forms are excluded from certain areas in the United States, not by the lack of an adequately long growing-season nor by killing temperatures, but by too great a duration of the dormant period. It may thus be possible that, for a given plant, a certain locality might possess a growing-season quite adequate in every way for maturation and reproduction, and yet the length of the enforced period of dormancy might be so great that death from autolysis, respiration, and the like might ensue before the return of the conditions requisite for full activity. The question thus raised can not be answered until after the accumulation of a much more thorough knowledge of the limiting conditions of plant-life than is now available. Indeed, the first prerequisite for an attack upon such questions is some such laboratory for the study of environmental relations as has attracted our attention earlier in the present publication.

(D) LENGTH OF PERIOD OF HIGH NORMAL DAILY MEAN TEMPERATURES. (TABLE 3, PLATE 35.)

On the supposition that high temperature may, directly or indirectly, prevent the appearance of certain plants in certain areas, or that this may be the critical requisite for the complete development of certain 
forms, it follows that the duration of relatively high temperature may, in some cases, be a limiting vegetational condition. It has therefore seemed worth while to attempt a cartographical study of this feature.

The temperature observations that have been carried out by the Signal Service and by the United States Weather Bureau have already resulted in an enormous mass of data. From our present point of view these data are very unsatisfactory in many respects. The distribution of the stations of observation is, as has been remarked previously, exceedingly unequal, and seems to have been the result of political rather than of scientific interests. Furthermore, the exposure of the thermometers at the various stations follows no general rule; sometimes the instruments are placed on the tops of high buildings, sometimes near the ground; they are seldom in the open country, and are almost always subjected to whatever peculiar conditions prevail in or over cities. Nevertheless, in spite of the many quite obvious fundamental errors which a more rational guidance might have been able to avoid, the faithful labors of the observers and interpreters of the Bureau have resulted in a very valuable mass of statistical information upon temperature conditions, and it is from this alone that information such as we require may be obtained. Especially valuable to us is the Herculean work of Professor Frank H. Bigelow, who has done what was possible to bring order and meaning out of the chaos of existing observations.

In the present instance, as also in the next following, we have drawn our fundamental statistics directly from Bulletin $\mathrm{R}$ of the United States Weather Bureau. ${ }^{1}$ In this bulletin Bigelow has presented the normal daily mean temperature for every day in the year for 177 stations in the United States. Although the introductory statements of this work are none too clear as to the sources of the temperature data used in the computations, it is implied that these normal daily means are the direct outcome of a graphically mathematical treatment of the normal monthly mean temperatures as given in Bulletin $\mathrm{S}$ of the United States Weather Bureau." Since, however, the list of the linstmentioned bulletin comprises but 123 stations, it is obvious that other data than these have been employed in the preparation of the daily normal means. It thus appears that the fundamental homogeneous reductions for at least 54 stations have never been jublished and that these have nevertheless been made use of in the preparation of the list. of 177 stations which we are to employ. While there ean be no rational doubt of the approximate reliability of all the data given in the firstmentioned of these two publieations, it is to be regretted, where there

\footnotetext{
1 Bigelow, Frank Iagar, The daily normal temperature and the daily normal precipitation of tho United States, U.S. Dept. Agric., Weather Bur. 13ull. R, 190 s.

2Bigelow, Frank H., Report on the temperatures and vapor tensions of the United States, reduced to a homogeneous system of 24 hourly observations for the 33-year interval 15731905, U. S. Dept. Agric., Wenther Bur. Bull. S, 1909.
} 
are so many mathematical steps between the actual observations and the finally resulting daily normal means, that so many of the fundamental properties and characteristics of the data upon which the latter have been based still remain practically unattainable to the student of these important statistics.

The enormous amount of work represented by the portion of Bulletin $\mathrm{S}$ that deals with the normal monthly mean temperatures resulted in the elimination, as far as this was possible, of the error-producing effects of variations and alterations in the hours of observation at the different stations throughout the long period of observations, as well as in the reduction of the variously derived daily means to a homogeneous system. For an account of the ingenious methods employed in this work the reader is referred to Chapter I of Bulletin S; for not nearly all of the stations are observations for the full 33 years available, and the possible approximate reductions of the means of short-record stations to a 33-year basis were not carried out in Bulletin S, though a method for this sort of reduction is given on page 32 . Whether these reductions have been carried out for the temperature data of Bulletin $R$ we are not informed, but it may safely be supposed that the statement of the normal daily means of temperature for the 177 stations dealt with in Bulletin $\mathrm{R}$, approach the truth as nearly as was possible, all circumstances being considered, at the time of the preparation of the bulletin.

It is interesting and worthy of remark here that the letter of transmittal accompanying Bulletin S, signed by Willis L. Moore, Chief, sounds a truly prophetic note in this sentence:

These data, and the normals that have been deduced from them, will form the fundamental basis for future studies on climatology and for the investigation of the relations between plant life and the thermal and hygrometric conditions that prevail in nature.

We have chosen $68^{\circ} \mathrm{F}$. $\left(20^{\circ} \mathrm{C}\right.$.) as our critical normal daily mean temperature in the present connection, and have determined from the tables of Bulletin $R$ the number of days in the year to which are ascribed normal daily means of $68^{\circ} \mathrm{F}$. or above. These days are consecutive in every case, owing to the smoothing process by which the normals have been derived. There are comparatively few stations in the United States without any such days, and for one station, Key West, Florida, this period of what most dwellers in temperate regions would probably term hot days is extended throughout the year. Scarcity of information has no doubt led us into more considerable errors in the western mountainous area than elsewhere. 
TABLE 3.-Length of period with normal daily mean temperatures of $68^{\circ} \mathrm{F}$. or above, and of period with similar means of $32^{\circ} F$. or below, within the year. (Plates 35 and 36.)

\begin{tabular}{|c|c|c|c|c|c|}
\hline Station. & $\begin{array}{c}\text { Mean of } \\
68^{\circ} \mathrm{F} . \mathrm{cr} \\
\text { above. }\end{array}$ & $\begin{array}{l}\text { Mean of } \\
32^{\circ} \mathrm{F} \text { or } \\
\text { below. }\end{array}$ & Station. & $\begin{array}{c}\text { Mean of } \\
68^{\circ} \mathrm{F} \text {. or } \\
\text { above. }\end{array}$ & $\begin{array}{l}\text { Mean of } \\
32^{\circ} \mathrm{F} \text { or } \\
\text { below. }\end{array}$ \\
\hline Alabama: & days. & days. & Iowa-Continued: & days. & days. \\
\hline Anniston............ & 141 & 0 & Des Moines........... & 97 & 105 \\
\hline Birmingham........ & 160 & 0 & Dubuque........... & 89 & 112 \\
\hline Mobile............. & 176 & 0 & Ireokuk............ & 107 & 89 \\
\hline Montgomery......... & 171 & 0 & Sioux City.......... & 91 & 119 \\
\hline Arizona: & & & Kansas: & & \\
\hline Flagstaff............ & 0 & 92 & Concordia.......... & 112 & 82 \\
\hline Phoenix............ & 186 & 0 & Dodge.............. & 113 & 70 \\
\hline Yuma.............. & 211 & 0 & Topeka............. & 118 & 74 \\
\hline Arkansas: & & & Wichita............ & 125 & 54 \\
\hline Fort Smith.......... & 149 & 0 & Kentucky: & & \\
\hline Little Rock......... & 153 & 0 & Lexington.......... & 115 & 0 \\
\hline California: & & & Louisville............ & 128 & 0 \\
\hline Eureka............. & 0 & 0 & Louisiana: & & \\
\hline Fresno............. & 150 & 0 & New Orleans......... & 192 & 0 \\
\hline Independence........ & 118 & 0 & Shreveport.......... & 172 & 0 \\
\hline Los Angeles.......... & 54 & 0 & Maine: & & \\
\hline Red Bluff........... & 140 & 0 & Eastport............ & 0 & 121 \\
\hline Sacramento.......... & 110 & 0 & Portland.............. & 31 & 110 \\
\hline San Diego........... & 50 & 0 & Maryland: & & \\
\hline San Francisco........ & 0 & 0 & Baltimore............ & 118 & 0 \\
\hline San Jose.............. & 0 & 0 & TVashington, D. C.... & 116 & 0 \\
\hline San Luis Obispo...... & 0 & 0 & Massachusetts: & & \\
\hline Colorado: & & & 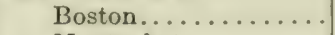 & 63 & 86 \\
\hline 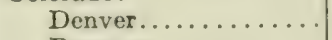 & 74 & 73 & Nantucket............ & 38 & 43 \\
\hline 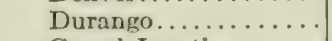 & 38 & 88 & Michigan: & & \\
\hline Grand Junction...... & 105 & 80 & 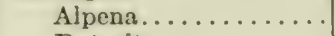 & 0 & 135 \\
\hline Pueblo............. & 89 & 70 & 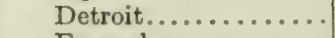 & 78 & 102 \\
\hline Connecticut: & & & Escanaba............ & 0 & 144 \\
\hline Fartford . . . . . . . . & 69 & 94 & Grand Haven....... & 53 & 107 \\
\hline New Haven.......... & 77 & 84 & Grand Rapids........ & 77 & 103 \\
\hline Florida: & & & Houghton.......... & 0 & 145 \\
\hline Jacksonville.......... & 191 & 0 & Marquette........... & 0 & 143 \\
\hline Jupiter............. & 285 & 0 & Port Huron. . . . . . . & 46 & 117 \\
\hline Key West......... & 365 & 0 & Sault Ste. Marie..... & 0 & 149 \\
\hline Pensacola........... & 189 & 0 & Minnesota: & & \\
\hline Tampa............ & 226 & 0 & Duluth........... & 0 & 146 \\
\hline Georgia: & & & Moorhead.......... & 39 & 150 \\
\hline 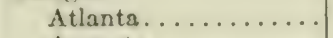 & 148 & 0 & St. Paul.......... & 71 & 132 \\
\hline Augrusta........... & 160 & 0 & Mississippi: & & \\
\hline Macon............. & 157 & 0 & Meridian............ & 157 & 0 \\
\hline Savannah.......... & 169 & 0 & Vicksburg. . . . . . . . & 168 & 0 \\
\hline Thomasville........ & 183 & 0 & Missouri: & & \\
\hline Idaho: & & & Columbia........... & 114 & 05 \\
\hline Boise............. & 72 & 56 & Hannibal........... & 116 & 77 \\
\hline Lewiston.......... & SS & 0 & Kansas City ....... & 113 & 74 \\
\hline Pocatello.......... & 6.1 & 96 & St. Louis. . . . . . . . . & 127 & 42 \\
\hline Illinois: & & & Springfield........... & 114 & 43 \\
\hline 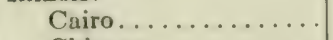 & 132 & 0 & Montana: & & \\
\hline Chicngo........... & 79 & 97 & Havre............. & 37 & 136 \\
\hline La Sallo............ & 90 & 95 & IIelena............ & 18 & 125 \\
\hline Peoria.............. & 95 & 99 & Kalispel........... & 0 & 121 \\
\hline Springfield.......... & 109 & 78 & Miles City........... & 71 & 132 \\
\hline Indians: & & & Nebraska: & & \\
\hline Evansville.......... & 129 & 22 & Lincoln ..... . & 102 & 101 \\
\hline Indianapolis........ & 108 & 68 & North Platte......... & 85 & 107 \\
\hline Iown: & & & 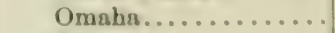 & 105 & 102 \\
\hline Charles City........ & 81 & 126 & Valentine........... & is & 119 \\
\hline Davenport.......... & 96 & 103 & & & \\
\hline
\end{tabular}


TABLE 3.-Length of period with normal daily mean temperatures of $68^{\circ} \mathrm{F}$. or above, and of period with similar means of $32^{\circ} \mathrm{F}$. or below, within the year. (Plates 35 and 36.)

\begin{tabular}{|c|c|c|c|c|c|}
\hline Station. & $\begin{array}{c}\text { Mean of } \\
68^{\circ} \mathrm{F} \text {. or } \\
\text { above. }\end{array}$ & $\begin{array}{c}\text { Mean of } \\
32^{\circ} \mathrm{F} \text {. or } \\
\text { below. }\end{array}$ & Station. & $\begin{array}{c}\text { Mean of } \\
68^{\circ} \mathrm{F} \text {. or } \\
\text { above. }\end{array}$ & $\begin{array}{c}\text { Mean of } \\
32^{\circ} \mathrm{F} \text {. or } \\
\text { below. }\end{array}$ \\
\hline Nevada: & days. & days. & South Dakota: & days. & days. \\
\hline Reno................ & 32 & 26 & 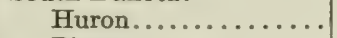 & 67 & 140 \\
\hline Winnemucca......... & 61 & 68 & Pierre.............. & 85 & 129 \\
\hline New Hampshire: & & & Rapid City......... & 55 & 121 \\
\hline 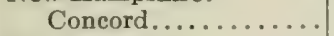 & 44 & 113 & Yankton........... & 89 & 122 \\
\hline New Jersey: & & & Tennessee: & & \\
\hline Atlantic City.......... & 91 & 27 & Chattanooga............ & 141 & 0 \\
\hline Cape May........... & 101 & 0 & Knoxville........... & 128 & 0 \\
\hline New Mexico: & & & Memphis............ & 153 & 0 \\
\hline Roswell. . & 140 & 0 & Nashville........... & 141 & 0 \\
\hline Santa Fe........... & 57 & 76 & Texas: & & \\
\hline New York: & & & Abilene... & 163 & 0 \\
\hline 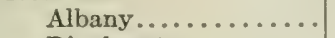 & 76 & 107 & Amarillo............. & 114 & 0 \\
\hline Binghamton......... & 58 & 109 & Corpus Christi....... & 218 & 0 \\
\hline Buffalo.............. & 64 & 104 & El Paso............. & 156 & 0 \\
\hline 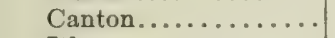 & 47 & 129 & Fort Worth.......... & 173 & 0 \\
\hline Ithaca............. & 62 & 108 & Galveston.......... & 215 & 0 \\
\hline New York........... & 94 & 64 & Palestine........... & 172 & 0 \\
\hline Oswego............... & 57 & 107 & San Antonio......... & 197 & 0 \\
\hline Rochester........... & 63 & 109 & Taylor............. & 187 & 0 \\
\hline Syracuse.............. & 67 & 108 & Utah: & & \\
\hline North Carolina: & & & Modena............. & 55 & 65 \\
\hline Asheville........... & 90 & 0 & Salt Lake City...... & 87 & 63 \\
\hline Charlotte.......... & 138 & 0 & Vermont: & & \\
\hline Hatteras............. & 148 & 0 & Burlington.......... & 32 & 129 \\
\hline Raleigh............ & 137 & 0 & Northfield.......... & 0 & 137 \\
\hline Wilmington.......... & 147 & 0 & Virginia: & & \\
\hline North Dakota: & & & Cape Henry........ & 128 & 0 \\
\hline . Bismarck.......... & 4 & 148 & Lynchburg.......... & 121 & 0 \\
\hline Devils Lake.......... & 30 & 158 & Norfolk............ & 132 & 0 \\
\hline Williston............ & 51 & 152 & Richmond.......... & 133 & 0 \\
\hline Ohio: & & & Wytheville........... & 85 & 0 \\
\hline Cincinnati... & 121 & 25 & Washington: & & \\
\hline Cleveland............ & 83 & 92 & North Head. . . . . . . . . & 0 & 0 \\
\hline Columbus.......... & 102 & 68 & Port Crescent........ & 0 & 0 \\
\hline Sandusky........... & 90 & 89 & Seattle............. & 0 & 0 \\
\hline Toledo.............. & 88 & 92 & Spokane............ & 45 & 78 \\
\hline Oklahoma: & & & Tacoma............. & 0 & 0 \\
\hline Oklahoma........... & 141 & 0 & Tatoosh Island....... & 0 & 0 \\
\hline Oregon: & & & Walla Walla......... & 89 & 0 \\
\hline Baker City........... & 0 & 102 & West Virginia: & & \\
\hline Portland............ & 0 & 0 & Elkins............. & 63 & 66 \\
\hline Roseburg........... & 0 & 00 & Parkersburg. . . . . . . . & 104 & 43 \\
\hline Pennsylvania: & & & Wisconsin: & & \\
\hline Erie............... & 75 & 93 & Green Bay............ & 50 & 135 \\
\hline Harrisburg. . . . . . . . . . & 95 & 75 & La Crosse........... & 77 & 122 \\
\hline Philadelphia........ & 107 & 44 & Madison............. & 71 & 123 \\
\hline Pittsburgh.......... & 102 & 56 & Milwaukee........... & 55 & 116 \\
\hline Scranton.............. & 71 & 95 & Wyoming: & & \\
\hline Rhode Island: & & & Cheyenne...... & 28 & 110 \\
\hline Block Island.......... & 47 & 55 & Lander............. & 32 & 134 \\
\hline Providence........... & 82 & 83 & Yellowstone........... & 0 & 149 \\
\hline \multicolumn{6}{|l|}{ South Carolina: } \\
\hline Charleston............. & 168 & 0 & & & \\
\hline Columbia...... & 159 & 0 & & & \\
\hline
\end{tabular}




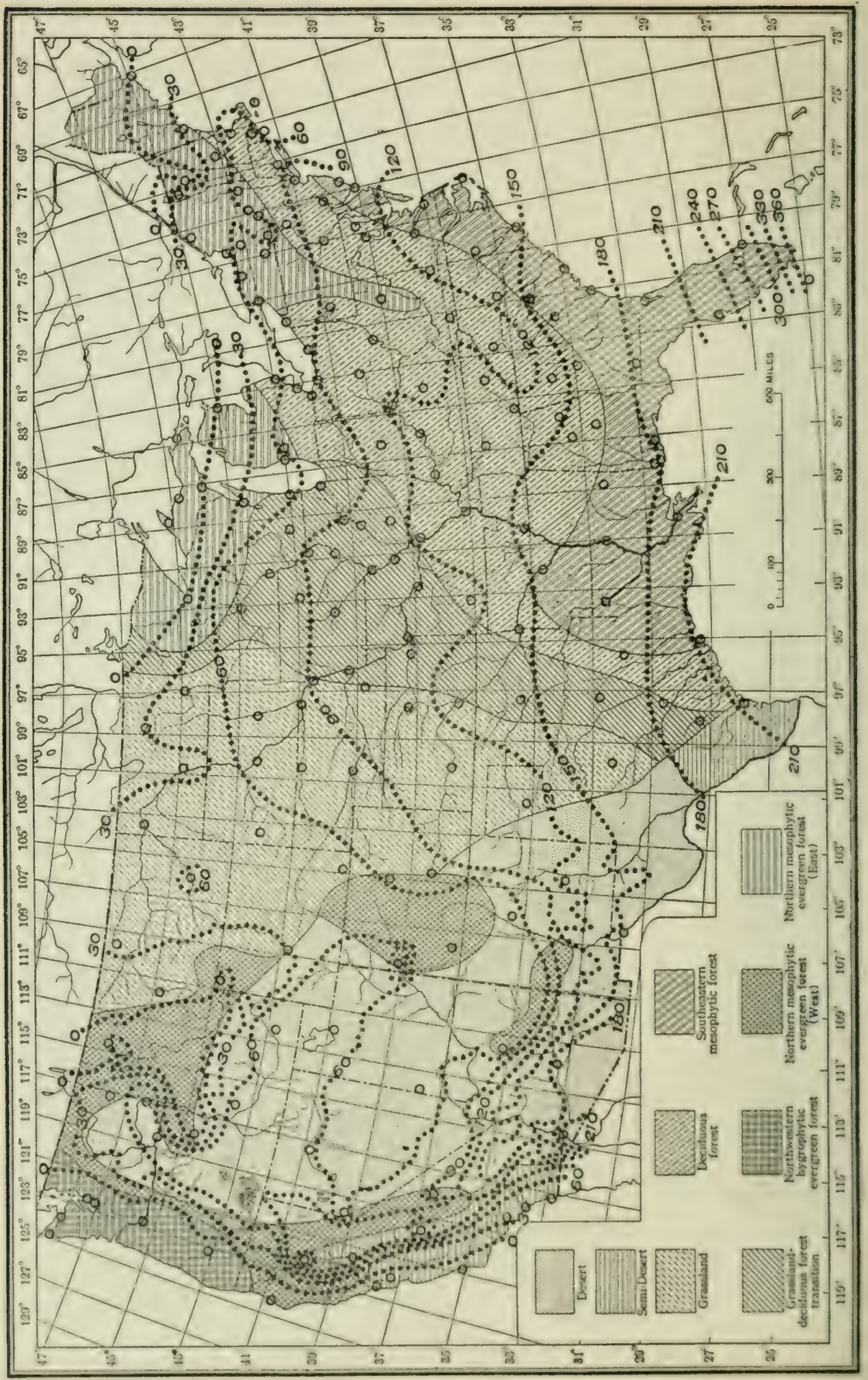

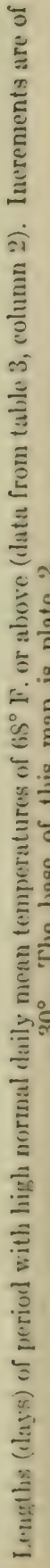




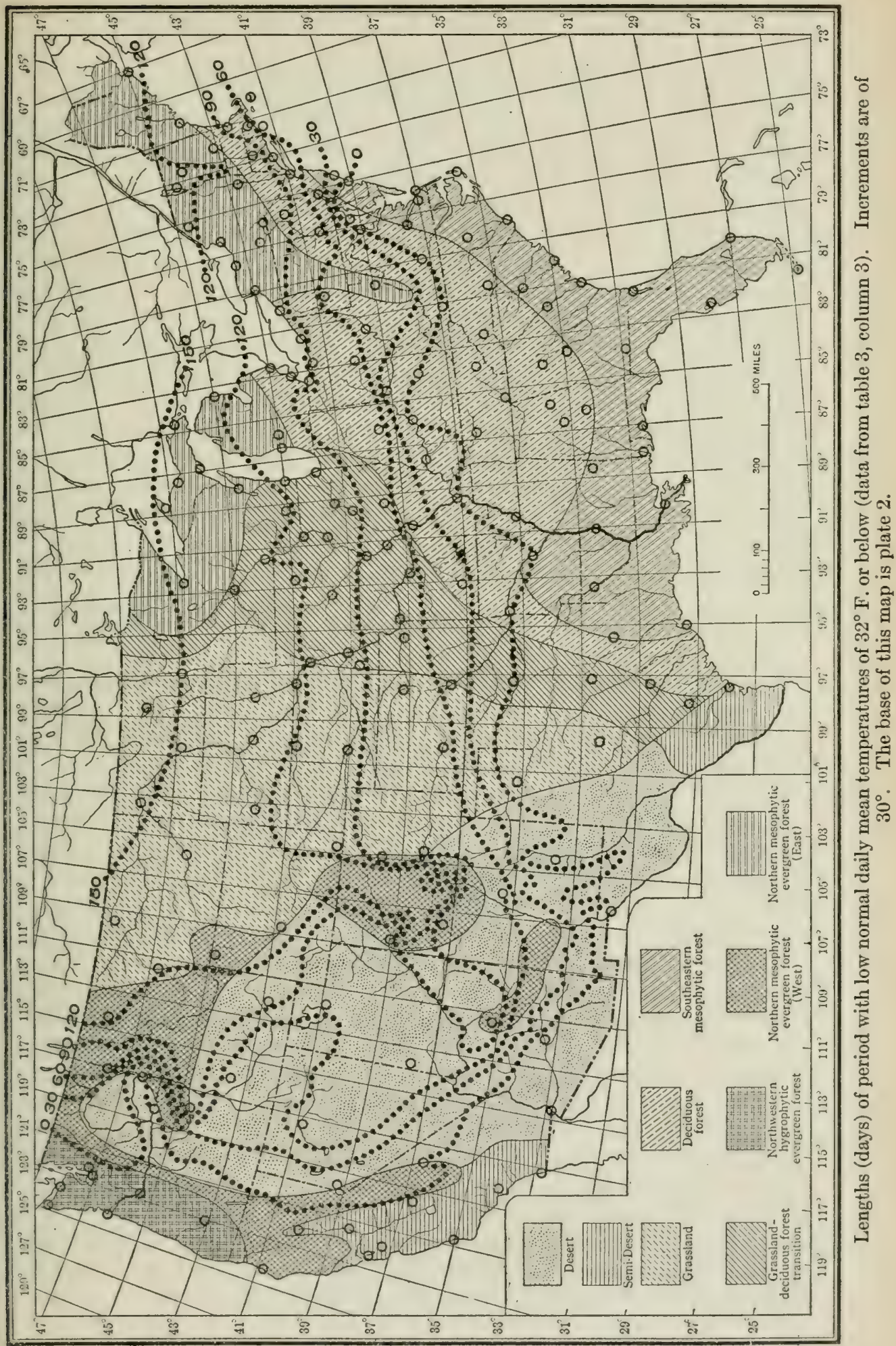


The numbers obtained by our study are given in table 3 , and the chart formed with these is reproduced as plate 35 , the locations of the stations used being denoted by small circles. The equiseasonal lines exhibit the data in a graphic manner. Increments of 30 days in the length of this hot season are represented.

It is noticeable on plate 35 that our lines assume a generally eastand-west direction to the eastward of the one hundredth meridian of west longitude, and a north-and-south direction in the vicinity of the Pacific ocean. Both mountain systems exhibit a tendency to displace the various climatic belts to the southward, a tendency shown still more markedly by the Pacific Ocean, and the same tendency is exhibited to a relatively slight degree by the Great Lakes. The region thus normally without the hot period here considered embraces almost the entire Pacific coast, from the vicinity of San Luis Obispo to the Canadian boundary; also the northwestern part of Minnesota, the whole of the northern peninsula of Michigan, a small adjacent part of Wisconsin, the northern extremity of the southern peninsula of Michigan, and the extremely northern parts of New England. This entire region is generally in high repute for summer resorts.

(E) LENGTH OF PERIOD OF LOW NORMAL DAILY MEAN TEMPERATLRES. (TABLE 3, PLATE 36.)

Just as the length of the hottest period may be supposed to influence the appearance or non-appearance of certain plants, so the mean length of the coldest period of the year may have its effect upon the distribution of the sarne or other forms. It seems improbable, however, that a normally long period of very cold weather may be essential to the full development of any organism. This coldest season always finds the plant in a dormant condition, and it is hardly possible, on physiological grounds, that extreme cold should be directly advantageous to its survival, but it is to be realized that nothing is yet quantitatively known in this connection. On the other hand, it is quite clear, in general at least, that cold weather tends to exclude many plantforms from the regetation of any region where such weather may occur. Numerous plants that readily survive a single frost are completely annihilated by the oceurrence of several days of freezing weather. The question here raised refers mainly to the power of dormant plants to retain life under more or less persistent conditions of temperature below that of frost.

As a critical normal daily temperature mean we here take $32^{\circ} \mathrm{F}$. $\left(0^{\circ} \mathrm{C}\right.$. $)$ For each station included in the temperature tahles of Bulletin $R$ of the U. S. Weather Bureau, it has been determined how many days in the year possess a normal daily mean of $32^{\circ} \mathrm{F}$. or below. Owing to the averaging and smoothing process by which the daily normals have been derived, these data exhibit a regular annual march, and all 
dates accredited with normals of $32^{\circ}$ or below occur consecutively. Thus the period so characterized may be termed the normal season of cold weather.

Our method of procedure in the present case brings out the fact that nearly one-half of the United States is without such normal periods of cold weather as are here considered. The longest period encountered is of 158 days. The numbers obtained from this study are shown in table 3 , and the chart of plate 36 exhibits the equiseasonal lines derived therefrom, this chart thus exhibiting graphically the extent of the areas having different lengths of cold season as here characterized. The station locations are again indicated by small circles on the chart. Increments of 30 days have again been employed, as in the charting of the periods of hot days (plate 35 ).

From plate 36 it is seen that the area indicated as without cold season occupies the entire country south of a line passing, approximately, from Cape May, New Jersey, to Tucson, Arizona, and is extended westward and northward to embrace approximately the southern third of California, the western two-thirds of the remainder of that State, and the western half of Oregon and of Washington. It is in this area that the most highly reputed winter resorts occur.

The area characterized by 150 or more days of normal daily means of $32^{\circ} \mathrm{F}$. or below occupies only the northern third of Minnesota, the northern half of North Dakota, and a little of northeastern Montana.

The eastern mountains and the Great Lakes appear to have little tendency to extend the areas of cold winter weather southward, but such an effect is noticeable in the case of the western mountains. The influence of the Pacific Ocean is conspicuous, crowding the area of cold season far back from the coast, even north of the Canadian boundary.

\section{INTENSITY OF TEMPERATURE CONDITIONS.}

(A) PRELIMINARY CONSIDERATIONS.

The physical conception of life phenomena allows us to regard the organism as a spatial system, in which a complex series of chemical and physical changes are ever in progress; during life, material and energy are always entering the system and are as unceasingly leaving it. The accomplishment of growth, maturation, reproduction, etc., of a plant is thus to be regarded, at any moment in its history, as the summation of the effects produced by the innumerable physical and chemical changes which have thus far occurred. Since all energy and material transformations are influenced to a greater or less extent by temperature, and since this influence is usually very important, it follows that, if other conditions were but constant, or if they always varied in the same manner, the state of a plant at any moment might be treated almost as a direct function of the various aerial temperature conditions to which it has been subjected in the past. Of course the 
other conditions do not always vary in the same way and they are never constant, not even as related to criteria without the plant-body. Even though they were constant for any plant, progressive alteration within the organism would assuredly produce great variations in the relations of the external world to internal conditions as criteria. It is therefore quite hopeless to contemplate an accurate causal interpretation of plant states merely on the basis of integrations of temperature effects. Nevertheless, the same incubus of hopelessness overshadows similar attempts along lines of approach based upon other external factors, and since we are sure that no single criterion alone will lead very definitely toward the solution of our problem, progress may be sought only by treating the different factors separately and studying the results, after which artificial and natural combinations of factors may be attacked. Furthermore, the study of the temperature conditions of plant environments appears promising in this particular, namely, that temperature not only directly influences plant activities, but also influences all of the other effective environmental conditions to a greater or less degree.

There appear to be, in general, two possible criteria for comparing the temperature intensities of several different localities. By one of these the comparison is made of the extremes, merely, of the yearly maximal and minimal temperatures for the several stations. Thus the duration factor is completely left out of account. It is, however, possible to consider and compare maxima and minima, not for the entire year, but for shorter seasons, and these seasons may be of different length at the different stations. The difference between the maximum and minimum temperatures may then be obtained, giving the range of normal temperature for each particular season and place to be considered. The last-named function of the temperature conditions does take some account of the duration factor (since the season is described in each case), and may, in certain instances, have an important relation to plant activities. By the second general criterion the intensity conditions are summed or integrated, in some manner, through a given length of time, and the duration factor is thus seen to play a direct and important part in this procedure.

Mean temperatures for long periods of time are not apt to be of value in studies of the relations between plant activities and the environment; seasonal or yearly means, which comprise such an important part of the usual meteorological reports, seem never to have given any real promise in this direction. Such means do not take aceount of the duration factor; the summed daily means are divided by the number of days considered, so that either this number of days must be the same for all localities compared, or else the numbers obtained can bear no relation to the corresponding extent of plant growth and other activities. Thus, a long growing-season with a given mean tempera- 
ture is not at all the same, as far as vegetation is concerned, as a short season with the same mean.

In studies regarding the relations between temperature intensity and plant development it is necessary to measure and compare the relative effectiveness of the temperature conditions at one station with that of the conditions at another; it is not the temperature of the atmosphere or of the plant that primarily interests us, but it is the possible effect which the various degrees of temperature may have in controlling plant activity. To make the following subsection clear we shall digress at this point to explain the various concepts and the ter minology which will here be employed, thus presenting a tentative discussion of the various sorts of temperature indices that may be used in vegetational or other dynamically applied climatology.

The word "temperature" is used with a variety of different meanings, some of which are very vague. Temperature and heat-content are frequently confused, but the heat-content of a body is only one of the conditions that determine its temperature; the heat capacity or specific heat of the matter in question and the amount (mass) of matter considered being also influential in determining the temperature. The definition of temperature involves difficulties unless based on the kinetic theory of matter, in which case the temperature of a body is considered as a measure of the mean kinetic energy of its particles. Temperature is often said to be the relative measure of the sensible heat of the body in question, of its hotness or coldness, this conception being based on the heat-sense of human beings. The latter definition must assume that the matter considered does not change its state (as solid, liquid, or gas) when the human sense-organ is applied to it as a measuring instrument, but the heat-conductivity of the material is important in this connection. Thus, a block of steel and a similar one of wood seem to have different temperatures by the criterion of sense, although they may be of quite the same temperature as determined by a thermometer. The commonest way of measuring temperature is to state it in terms of the relative volume assumed by a given mass of some standard substance (such as air, mercury, alcohol, ete.) when that mass of substance is in heat equilibrium with the body whose temperature is to be determined, this equilibrium being attained when heat does not migrate in either direction between the standard mass and the body under consideration. Thus air-temperature is measured in terms of the relative volume assumed by the liquid in a thermometer when this liquid neither gives heat to the air nor receives heat from the air. In short, the thermometer liquid is allowed to come to the same temperature as the air and then this temperature is stated in terms of the volume occupied by this liquid under these conditions. Since the mass of the thermometer liquid is constant for any instrument, the different volumes assumed at various temperatures may be 
indicated as a temperature scale on the thermometer-tube, and these volumetric graduations may be of any convenient magnitude. Thus, centigrade, Fahrenheit, etc., degrees are the respective increments in the volume of the thermometer liquid corresponding to equal increments in temperature rise.

The term "temperature" often means temperature reading (on some thermometer scale), and it is also used in a self-evident adjectival sense, meaning pertaining to temperature. In order to apply constructive reasoning to temperature conditions it is necessary to be somewhat more explicit than is usually the case, so that the terms we shall use require definition.

Temperature rcadings or measurements are simply numbers that represent comparative temperatures, and they may therefore be considered as conventional indices of temperature. The numbers of the Fahrenheit and centigrade scales are merely these indices expressed in two different kinds of units. Thus, the normal daily means of Bulletin $R$ of the United States Weather Bureau are to be regarded as averages of a number of means for the given date, these means themselves being averages of a series of temperature indices representing the different temperatures encountered in the air throughout the day. According to this terminology, all climatological studies of temperature conditions thus start with temperature indices. These indices tell nothing about the possible effects of the temperatures represented, as these may accelerate or retard any process; they have reference only to the state of molecular motion of the particular body whose temperature is considered.

For the problems before us, as has been mentioned, the various degrees of temperature effectiveness upon plant activities must be measured and compared, rather than temperatures themselves, and it thus follows that we require indices of temperature efficiency. These are to be derived from the indices of temperature, with due regard to the nature of the process to be studied, and rarious attempts have been made to obtain from temperature indices these other indiees that are to be measures of the plant-producing power, ete, of given atmospherie temperatures. We shall deal below with the various methods that have been tried. The relative applieability of these methods is to be determined only empirically, but certain a priori considerations need to enter into the critieal discussion of this relatively new and rery important subject. According to the way in which indiees of temprerature efficiency are derived from temperature indices, we may have four distinet classes of the former, which will now be taken up separately.

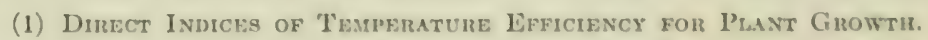

Direct effieieney indiees are ohtained direetly from the eorresponding temperature indiees, the numerieal values being the same in both eases 
This method of derivation assumes that the rate of plant growth varies proportionally to the environmental temperature. Any thermometer scale may be used and a set of efficiency indices thus obtained may be transferred from one scale to another by arithmetical treatment. The assumption here is exemplified as follows. If a plant grows 2 units per time period at $2^{\circ} \mathrm{C}$. it should grow 10 units per time period at $10^{\circ} \mathrm{C}$., 25 units at $25^{\circ} \mathrm{C}$., etc. While direct indices of temperature efficiency (on the absolute thermometer scale) are of great value in studying simple physical processes, such as the expansion of gases, they do not promise much in connection with the study of physiological processes, and need not be seriously considered in our practical applications.

(2) Remainder Indices of Temperature Efficiency for Plant Grow jh.

The derivation of what we shall here term remainder indices of temperature efficiency is but little more complicated than that of direct indices. A constant difference between the temperature indices and the corresponding efficiency indices is assumed (or derived from experiment), and this difference is subtracted from every temperature index, thus giving the required efficiency indices. It will be seen that this method virtually does nothing but alter the position of the zero of the thermometer scale, after which alteration it employs direct efficiency indices as these have been defined above. Thus, the rate of plant growth at $40^{\circ} \mathrm{F}$. may be considered as unity and it may be assumed that this rate becomes 2 at $41^{\circ}, 10$ at $49^{\circ}, 50$ at $89^{\circ}$, etc., the constant difference above mentioned being here 39 . In the phenological studies that have employed this sort of efficiency indices it has been assumed that if the plant does not attain the particular growth-rate that is taken as unity, it does not grow at all; that is, with a temperature of $39.5^{\circ}$, for example, no growth is supposed to occur, when unit rate of growth would be obtained with a temperature of $40^{\circ}$. It is clear that the method of direct indices of efficiency is a special case of that of remainder indices, the constant difference being reduced to zero in the case of direct derivation.

The integration of temperature data by these remainder indices has received the attention of workers in phenology for many years and $\mathrm{a}$ large amount of literature bears upon this subject. For a review and citations of the earlier phenological studies, the reader is referred to Abbe's Relations between Climates and Crops, already mentioned. Because of their close relation to our special field of study, Merriam's researches upon the zonation of temperature conditions in the Lnited States must be considered here. The conclusions arrived at by this author have been largely adopted by plant and animal geographers in this country, and Merriam's zonal terminology has come into very general use, despite the exceedingly tentative nature of the data on which this is based. 


\section{Merriam's work ${ }^{1}$ and that of his colleagues of the Bureau of Biologi-} cal Survey of the U. S. Department of Agriculture constitute by far the most thorough study that has yet been brought forth of the relations of plant and animal distribution to temperature conditions in the United States, and Merriam's temperature integrations have furnished, for two decades, practically the only available information in this regard. In his most complete account of this arduous work of integration, Merriam writes:

Several years ago I endeavored to show that the distribution of terrestrial animals and plants is governed by the temperature of the period of growth and reproductive activity, not by the temperature of the whole year; but how to measure the temperature concerned was not then worked out. $* * *$ At one time I believed that the mean temperature of the actual period of reproductive activity in each locality was the factor needer, but such means are almost impossible to obtain, and subsequent study has convinced me that the real temperature control may be better expressed by other data. *** If it is true that the same stage of vecretation is attained in different years when the sum of the mean daily temperature reaches the same value, it is obvious that the physiological constant of a species must be the total quantity of heat or sum of positive temperatures required by thal species to complete its cycle of development and reproduction. *** I am not aware that an attempt has been made to correlate the facts thus obtained with the boundaries of the life zone. *** If the computation can be transferred from the species to the zone it inhabits-if a zone constant can be substituted for a species constant - the problem will be well nigh solved. This I have attempted to do. In conformity with the usage of botanists, a minimum temperature of $6^{\circ} \mathrm{C}$. $\left(43^{\circ} \mathrm{F} \text {. [ } 42.8^{\circ} \mathrm{F} \text {, see footnote, p. 231] }\right)^{2}$ has been assumed as marking the inception of the period of physiological activity in plants and of reproductive activity in animals. The effective temperature or degrees of normal mean daily heat in excess of this minimum has been added together for each station, beginning when the normal mean daily temperature rises higher than $6^{\circ} \mathrm{C}$. in spring and continuing until it falls to the same point at the end of the season. The sums thus obtained have been platted on a large scale map of the United States, and isotherms have been run which are found to conform in a most gratifying manner to the northern boundaries of the several life zones. ${ }^{*} * *$ While the available data are not so numerous as might be desired, the stations in many

1Merriam (1894.) A very much abbreviated statement of the results embodied in the above paper was published as Part III of the same author's Life Zones and Crop Zones of the United States, U.S. Dept. Agric., Div. Biol. Survey. Bull. 10, 1898. This latter involves but two pages $(54,55)$ and does not include the climatic map. Nothing approaching an adequate presentation of the data upon which these important studies are based has, as far as we are aware, ever appeared.

It can not be too strongly emphasized that work of this sort is deprived of by far the greater part of its possible usefulness in building up our knowledge whenever a derived chart is published without the station data upon which it is based. It appears that most writers who have dealt with climatic charts have considered these as an end rather than as a means. Such charts are simply broad and necessarily very general presentations of the facts or observations upon which they are constructed, and can accomplish little more for the student of plant distribution or of agriculture than to inform him where in the given region to look for stations with certain climatic characteristics. As soon, however, as his interest is thus aroused he requires the station data, and if these are not at hand, further quantitative studies therewith are effectually precluded. We will not suppose that this common suppression of basic data is to be related at all to any desire on the part of writers to veil the exact methods of their procedure in the preparation of charts; we suppose rather that the suppression here in view has usually arisen from lack of facilities for publication or from lack of time and energy requisite for the preparation of tables or for the placing of the numerical data upon the published charts. It is hardly conceivable that a writer who has derived important generalization from a mass of figures should not appreciate the probability that the same figures may be utilized, in the same or in different ways, by other students of the subject.

Had Merrian's publications included $\mathrm{n}$ list of stations each with its numerical climatic indices, the latter might have been put to many other uses than the mere preparation of the simple charts.

${ }^{2} \mathrm{As}$ the chart was published, however, the minimum here referred to was $0^{\circ} \mathrm{C}$. (32. $\mathrm{F}^{\circ}$ ). See Merriam's note, Seience, n.s., 9: 116, 1 S99. 
instances being too far apart, still enough are at hand to justify the belief that animals and plants are restricted in northward distribution by the total quantity of heat during the season of growth and reproduction.

Merriam's chart (1894, plate 12) of the summations just described is here reproduced in its essentials, as our plate 37 , for purposes of comparison, and because of its pioneer nature and present scarcity. From this map it is seen that the warmest zone of the United States, as here indicated, is characterized by a summation temperature of 26,000 on the Fahrenheit scale $(14,500$ on the centigrade scale), and that this zone is restricted to the lower Colorado Valley, the extreme southern portion of Texas, and the southern half of the Florida peninsula. The zone characterized by temperature summations below 10,000, F. $(5,500$ C. $)$ occupies, in general, the highest portion of the Cascade and Sierra Nevada Mountains, the Rocky Mountains, northern Minnesota, a little of northern Wisconsin, the northern half of Michigan, and the northern half of Maine. The isoclimatic lines for $11,500 \mathrm{~F} .(6,400 \mathrm{C}$.), and $18,000 \mathrm{~F} .(10,000 \mathrm{C}$.) are seen to have a west-east trend, but are more or less markedly displaced southward by the western and eastern mountains and northward by the Pacific and Atlantic oceans.

Our own work with remainder indices will be presented farther on.

(3) Exponential Indices of Temperature Efficiency for Plant Growth.

As has been stated in our earlier discussions, it appears that some possibility of advance lies in studying climatic temperature conditions with reference to the chemical principle of Van't Hoff and Arrhenius, and a first attempt in this direction has been made by Livingston and Livingston in the paper already cited. This principle states that chemical reaction velocity usually about doubles (or somerwhat more than doubles) for each rise in temperature of $10^{\circ} \mathrm{C}$., or of $18^{\circ} \mathrm{F}$. It is to be understood that the principle of Van't Hoff and Arrhenius is applicable, even in purely chemical problems, only within certain temperature limits, and it is sufficiently clear that the same general sort of limitation must influence its applicability in physiology and ecology. We are primarily interested here in growth processes and their rates, and, obviously, there may occur natural temperatures either above the maximum or below the minimum for growth, so that here are unquestionable limits for the application of the principle just stated. Furthermore, the principle itself supposes that the process considered increases its velocity with each rise of temperature between the limits of applicability, and we are well aware that increasing temperature is not accompanied by increased growth-rate throughout the range from the minimum to the maximum for growth in plants. An optimum temperature can always be found above which the previously increasing growth-rate begins to decrease. Thus the applicability of the Van't Hoff-Arrhenius principle to organic growth phe- 
nomena as a whole can not be maintained beyond the limits set by the minimum and optimum temperatures for growth. However, these physiological constants are not the same for different plant-forms, and they may also be assumed to vary with other conditions within and without the plant. It therefore seemed desirable, in an attempt to apply this law of temperature coefficients to the climatological delimitation of geographical areas, to choose such limiting temperatures as should give promise of merely approximating the physiological limits for a large number of plant-forms. In this we follow Livingston and Livingston, who give a more complete discussion of this whole question than is required here.

The authors just mentioned calculated their indices of temperature efficiency on the basis of the supposition that general plant activity occurs at unity rate when the daily mean temperature is $40^{\circ} \mathrm{F}$., and that this rate is doubled with each rise of $18^{\circ} \mathrm{F}$. in the daily mean. Thus, with a daily mean temperature of $58^{\circ} \mathrm{F}$. the rate becomes 2.0, with a mean of $76^{\circ} \mathrm{F}$. it becomes 4.0 , etc. It thus becomes clear that the relation here assumed between any index of temperature efficiency and the corresponding index of temperature itself is an exponential one, expressed by the equation $I=2^{\frac{l-40}{18}}$, where $I$ is the index of efficiency and $t$ is the corresponding index of temperature. From this equation, by substituting the various values of $t$, Livingston and Livingston prepared a table of the efficiency index values corresponding to the temperature indices from $40^{\circ}$ to $99^{\circ} \mathrm{F}$. This table is reproduced as our table 4. Of course, constants other than 2, 18, and 40 might be employed, thus giving other values to the indices of efficiency; these indices, as has been stated, are based on the supposition that plant

TABLe 4.-Exponential indices of temperature efficiency for plant grouth, based on a coefficient of 2.0 for each rise in temperature of $18^{\circ}$ above $40^{\circ} \mathrm{F}$., for each temperature from $41^{\circ}$ to $100^{\circ} \mathrm{F}$.

\begin{tabular}{|c|c|c|c|c|c|c|c|c|c|}
\hline 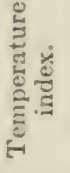 & 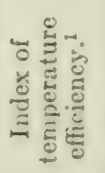 & 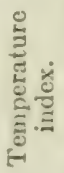 & 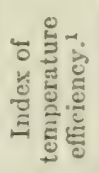 & 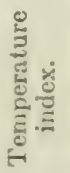 & 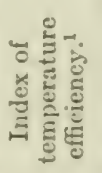 & 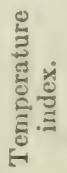 & 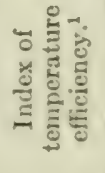 & 递 & 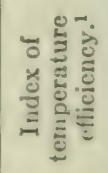 \\
\hline${ }^{\circ} \mathrm{F}$. & & ${ }^{\circ} \mathrm{F}$. & & ${ }^{\circ} \mathrm{F}$. & & ${ }^{\circ} \mathrm{F}$. & & ${ }^{\circ} \mathrm{F}$. & \\
\hline 41 & 1.0393 & 53 & 1.6493 & 65 & 2.6192 & 77 & 4.1572 & 89 & 6.5972 \\
\hline 42 & 1.0802 & 54 & 1.7412 & 66 & 2.7212 & 78 & 4.3206 & 90 & 6.8566 \\
\hline 43 & 1.1226 & 5.5 & 1.7815 & 67 & 2.8284 & 79 & 4.4902 & 91 & 7.1258 \\
\hline 44 & 1.1666 & 56 & 1.8512 & 68 & 2.9391 & 80 & 4.6662 & 92 & $7.40-48$ \\
\hline 45 & 1.2123 & 57 & 1.9240 & 69 & 3.05 .45 & 81 & 4.8490 & 93 & 7.6960 \\
\hline 46 & 1.2599 & 58 & 2.0000 & 70 & 3.1748 & 82 & $5.03966^{\circ}$ & 9.4 & 8.0000 \\
\hline 47 & 1.3096 & 59 & 2.0786 & 71 & 3.2956 & 83 & 5. 2354 & 95 & 8.3144 \\
\hline 48 & 1.3603 & 60 & 2.1603 & 72 & 3.42 & 84 & 5.44 & 96 & 8.6412 \\
\hline 49 & 1.4142 & 61 & 2.2451 & 73 & 3.5629 & 85 & 5.6568 & 97 & 8.9804 \\
\hline 50 & 1.4696 & 62 & 2.33331 & 7.4 & 3.7024 & 86 & 5.8782 & $9 \mathrm{~s}$ & 9.3329 \\
\hline 51 & 1.5273 & 6.3 & 2.4215 & 75 & 3.8450 & 87 & 6.1090 & 99 & 9.6950 \\
\hline 52 & 1.5574 & 64 & 2.5198 & 76 & 4.0000 & ss & 6.3496 & 100 & 10.0792 \\
\hline
\end{tabular}

${ }^{2}$ The temperature efficiency index is here assumed to be doubled for each rise of $10^{\circ} \mathrm{C}$. (15 $\mathrm{F}^{\circ}$ ) above $40^{\circ} \mathrm{F}$, at which temperature the index of efficiency is taken to be unity. 
activity in general doubles with each rise in temperature above $40^{\circ} \mathrm{F}$., at which temperature the rate of development is considered as unity.

(4) Physiological Indices of Temperature Efficiency for Plant Growth.

All three methods so far considered for deriving temperature efficiency indices from temperature indices assume that the rates of plant activity increase continuously as the temperature rises. If these various series of efficiency index values be plotted as ordinates on graphs whose abscissas are the temperature indices, then the graphs for direct and remainder indices both take the form of straight lines with an upward slope of $45^{\circ}$. The graph for exponential indices, on the other hand, has the form of a curved line slightly concave upward, which much more nearly approaches being horizontal in the region of climatic temperatures than do the other graphs. In other words, within the range of temperature indices encountered in climatology, the calculated efficiency index increases much more rapidly with rise in temperature for the direct and remainder methods of calculation than it does for the exponential method, as adopted by Livingston and Livingston. None of these graphs, however, shows a maximum, and we know that the true graph of temperature efficiency for plant growth must possess two points where the ordinate is zero and must have a maximum somewhere between these points. This maximum of the graph has a relatively large ordinate and its abscissa is the index of the optimum temperature for growth. It thus follows that none of the methods so far discussed can possibly furnish true indices of temperature efficiency for plant activities, excepting, as has been emphasized, within certain limits of temperature range. If a perfectly satisfactory method of calculating efficiency indices from temperature indices is to be devised, it must be of such nature that both low and high temperature values will give efficiency indices of zero, and intermediate temperature values must give indices whose graph shows a well-defined maximum.

Livingston ${ }^{1}$ has attempted to obtain efficiency indices from the variations in plant growth-rate experimentally determined for different temperatures. As has been said, the best study of the actual relations between temperature and growth is that of Lehenbauer, and Livingston has employed the results of that writer in this connection. $\mathrm{He}$ considered the average hourly growth-rates of shoots of maize seedlings exposed to the same temperature for a 12-hour period, the temperatures included in Lehenbauer's study ranging, by increments of one degree, from $12^{\circ}$ to $43^{\circ} \mathrm{C}$. Lehenbauer's curve, plotted with growth-rates as ordinates and temperature indices as abscissas, was first smoothed by the use of a flexible spline, so as to give a generalized

${ }^{1}$ Livingston, B. E., Physiological temperature indices for the study of plant growth in relation to climatic conditions, Physiol. Res. 1: 399-420, 1916. 
curve, which was continued at either end to cut the axis of abscissas at $2^{\circ}$ and $48^{\circ} \mathrm{C}$. The ordinates corresponding to the various abscissal temperature values were then actually measured. Finally, all the new ordinates thus derived were expressed in terms of the ordinates for $40^{\circ} \mathrm{F}$. considered as unity. The resulting series of values are Livingston's physiological indices of temperature efficiency. He presents a table showing these values for each single degree of temperature from $36^{\circ}$ to $118^{\circ} \mathrm{F}$., and that table is here reproduced as our table 5 .

TABLE 5.-Physiological indices of temperature efficiency for grouth, based on Lehenbauer's 12-hour exposures with maize seedlings.

\begin{tabular}{|c|c|c|c|c|c|}
\hline \multicolumn{2}{|c|}{ Centigrade scale. } & \multicolumn{2}{|c|}{ Fahrenheit scale. } & \multicolumn{2}{|c|}{ Fahrenheit scale. } \\
\hline Degrees. & Index. & Degrees. & Index. & Degrees. & Index. \\
\hline 3 & 0.333 & 36 & 0.111 & 82 & 106.889 \\
\hline 4 & 0.667 & 37 & 0.222 & 83 & 110.778 \\
\hline 4.5 & 1.000 & 38 & 0.342 & 84 & 115.000 \\
\hline 5 & 1.333 & 39 & 0.667 & 85 & 118.111 \\
\hline 6 & 1. 889 & 40 & 1.000 & 86 & 120.000 \\
\hline 7 & 2.778 & 41 & 1.333 & 87 & 121.222 \\
\hline 8 & 3.667 & 42 & 1.667 & 88 & 122.000 \\
\hline 9 & 4.889 & 43 & 2.000 & 89 & 122.333 \\
\hline 10 & 6.333 & 44 & 2.344 & 90 & 121.667 \\
\hline 11 & 8.000 & 45 & 3.000 & 91 & 117.667 \\
\hline 12 & 9.889 & 46 & 3.500 & 92 & 113.444 \\
\hline 13 & 12.111 & 47 & 4.000 & 93 & 108.333 \\
\hline 14 & 14.778 & 48 & 4.778 & 94 & 103.333 \\
\hline 15 & 17.778 & 49 & 5.500 & 95 & 96.000 \\
\hline 10 & 21.556 & 50 & 6.333 & 96 & 91.444 \\
\hline 17 & 26.000 & 51 & 7.111 & 97 & 85.000 \\
\hline 18 & 31.333 & 52 & 8.167 & 98 & 79.444 \\
\hline 19 & 38.000 & 53 & 9.222 & 99 & 73.111 \\
\hline 20 & 46.000 & 54 & 10.333 & 100 & 66.667 \\
\hline 21 & 54.778 & 55 & 11.667 & 101 & 60.000 \\
\hline 22 & 63.444 & 56 & 12.778 & 102 & 52.667 \\
\hline 23 & 71.111 & 57 & 14.444 & 103 & 44.444 \\
\hline 24 & 79.111 & 58 & 16.111 & 104 & 36.000 \\
\hline 25 & 86.556 & 59 & 17.778 & 105 & 25.667 \\
\hline 26 & 94.000 & 60 & 19.883 & 106 & 21.859 \\
\hline 27 & 101.222 & 61 & 22.000 & 107 & 16.778 \\
\hline 28 & 108.444 & 62 & 24.333 & 108 & 12.556 \\
\hline 29 & 115.778 & 63 & 27.111 & 109 & 9.444 \\
\hline 30 & 120.000 & 64 & 30.000 & 110 & 7.000 \\
\hline 31 & 121.889 & 65 & 33.333 & 111 & 5.222 \\
\hline 32 & 122.333 & 66 & 37.222 & 112 & 3.785 \\
\hline 33 & 116.111 & 67 & 41.333 & 113 & 2.778 \\
\hline 34 & 107.333 & 68 & 46.000 & 114 & 2.000 \\
\hline 35 & 96.000 & 69 & 50.833 & 115 & 1.414 \\
\hline 36 & 86.556 & 50 & 56.000 & 116 & 1.000 \\
\hline 37 & 75.667 & 71 & 60.333 & 117 & 0.500 \\
\hline 38 & $64.333: 3$ & 72 & 65.3333 & 118 & 0.111 \\
\hline 39 & 50.6667 & 73 & 69.000 & & \\
\hline 40 & 36.000 & 74 & 73.667 & & \\
\hline 41 & 23.3333 & 75 & 7R. 111 & & \\
\hline 42 & $14 .(1)(0)$ & 76 & 82.333 & & \\
\hline 43 & 8.3333 & 77 & Siti.5ist & & \\
\hline 44 & 4..$\leqslant \leqslant !)$ & is & (4). fitit & & \\
\hline 45 & 2.778 & 79 & 9.5 .000 & & \\
\hline 46 & 1.66i7 & 80 & $\begin{array}{r}0.6967 \\
103000\end{array}$ & & \\
\hline 41 & $0.66 i 6$ & $s 1$ & 10.3000 & & \\
\hline
\end{tabular}


This physiological method of deriving temperature efficiency indices is of course empirical, but it is not more so than either the direct or the remainder method, and it has what seems to be a better experimental foundation than has either of these. Furthermore, Livingston's physiological indices appear to be much more in accord with what is actually observed in the case of growing plants than are the exponential indices of Livingston and Livingston, and it may be said that we have at last obtained a method for estimating temperature efficiency that is applicable throughout the entire range of possible temperatures, the graph of these indices showing approximately the same form as Lehenbauer's graph of actual growth-rates. Of course it is desirable that this set of relations be eventually broadened so as to include other plants than maize and other developmental phases than that of the seedling, and when this is accomplished the efficiency index values for general use will probably need to be altered; it would be surprising if indices for seedling maize plants should prove to be representative for plants in general. Enough data are now at hand, however, for a somewhat rational beginning, and we regard these physiological indices of Livingston as the most promising of all the different kinds of indices of temperature efficiency that have been proposed.

We turn now to the employment of these four different kinds of temperature efficiency indices in our study of the climatic conditions of the United States. In the application of temperature efficiency indices to climatological study we have followed phenological workers in summing these indices throughout a certain period, the resulting summation being supposed to represent, in the form of a single number, the efficiency value of that period, and for the station in question, as far as temperature is concerned. We have used Bigelow's normal daily mean temperatures (Bulletin $\mathrm{R}$ of the United States Weather Bureau) as our daily temperature indices, with which all our computations begin. For the time-period we have here again used the period of the average frostless season. The procedure has been (1) to find the efficiency index corresponding to the temperature index for each day of the average frostless season, at each station, and (2) to add these daily efficiency indices all together to give the seasonal efficiency index for the station in question. This method allows the length of the average frostless season, as well as the values of the daily efficiency indices throughout that season, to take part in the control of the final value which represents the seasonal temperature efficiency for growth. Thus, a long season with relatively low temperatures may give a higher summation index than does a shorter season with higher daily mean temperatures. The use of Bigelow's normal daily means of temperature as original data should give to these studies an exceedingly broad and general character, and may be supposed to produce some approximation to the average conditions of temperature for many years. 
(B) SUMMATIONS OF DIRECT INDICES OF TEMPERATLRE EFFICIENCY FOR PERIOD OF AVERAGE FROSTLESS SEASON.

As has been said, direct indices of temperature efficiency offer little promise for our present purpose. We therefore present here only the numerical values (in the third column of table 6 , summations above $0^{\circ} \mathrm{F}$.) and do not reproduce the climatic chart based upon these values. This chart shows practically the same climatic zonation as is shown by Merriam's chart (our plate 37) and by our charts based on the remainder indices, the numerical values given to the various isoclimatic lines being of course different in each case.

(C) SUMMATIONS OF REMAINDER INDICES OF TEMPERATLRE EFFICIENCY FOR PERIOD OF AVERAGE FROSTLESS SEASON. (TABLE 6, PLATE 38.)

Remainder indices of temperature efficiency have been derived in the present study by the use of three different values for the constant difference, 32,39 and 50 . It will be remembered that the efficiency index is here taken to be equal to the corresponding temperature index minus the constant difference. Thus we have subtracted 32,39 , or 50 , as the case might be, from each of the daily normal means given in Bulletin $R$, for the period of the average frostless season, and have then summed the daily normal mean efficiency indices thus obtained for each station considered. Columns 4,5 , and 6 of table 6 present the results of these three kinds of summations, above $32^{\circ}$, above $39^{\circ}$, and above $50^{\circ} \mathrm{F}$. The method here followed is the same as that employed by Livingston and Livingston, in their summations above $39^{\circ} \mathrm{F}$, and our final results for that series of summations are the same as those presented on their chart of "direct summations," figure 1 in the paper already cited. Our summations above $32^{\circ} \mathrm{F}$. are derived by the method actually employed by Merriam, but his failure to publish the data used in making his chart (our plate 37) make detailed comparison impossible. 
TABLE 6.-Summations of normal daily mean remainder indices of temperature efficiency for plant growth, for the period of the average frostless season. (Plate 38.)

[The daily indices are derived by subtracting $0,32,39$, or 50 from the values of the normal daily mean temperature on the Fahrenheit scale.]

\begin{tabular}{|c|c|c|c|c|c|}
\hline \multirow{2}{*}{ Station. } & \multirow{2}{*}{$\begin{array}{l}\text { Length of } \\
\text { frostless } \\
\text { season. }\end{array}$} & \multicolumn{4}{|c|}{$\begin{array}{c}\text { Direct summation of normal daily mean } \\
\text { temperatures for period of average } \\
\text { frostless season. }\end{array}$} \\
\hline & & $\begin{array}{c}\text { Above } \\
0^{\circ} \mathrm{F} \text {. }\end{array}$ & $\begin{array}{l}\text { Above } \\
32^{\circ} \mathrm{F} \text {. }\end{array}$ & $\begin{array}{l}\text { Above } \\
39^{\circ} \mathrm{F} \text {. }\end{array}$ & $\begin{array}{l}\text { Above } \\
50^{\circ} \mathrm{F} \text {. }\end{array}$ \\
\hline Alabama: & days. & & & & \\
\hline 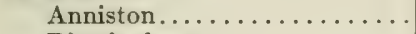 & 201 & 14,882 & 8,450 & 7,043 & 5,033 \\
\hline Birmingham............. & 231 & 16,660 & 9,268 & 7,651 & 5,341 \\
\hline Mobile.................. & 279 & 19,756 & 10,828 & 8,875 & 6,085 \\
\hline Montgomery.............. & 243 & 17,618 & 9,812 & 8,141 & 5,711 \\
\hline \multicolumn{6}{|l|}{ Arizona: } \\
\hline Flagstaff............... & 105 & 6,492 & 3,132 & 2,397 & 1,347 \\
\hline Phoenix................ & 283 & 21,108 & 12,052 & 10,071 & 7,241 \\
\hline \multicolumn{6}{|l|}{ Arkansas: } \\
\hline Fort Smith............ & 230 & 16,254 & 8,894 & 7,284 & 4,984 \\
\hline Little Rock............... & 237 & 16,749 & 9,165 & 7,506 & 5,136 \\
\hline \multicolumn{6}{|l|}{ California: } \\
\hline Eureka................. & 245 & 13,056 & 5,216 & 3,501 & 1,051 \\
\hline 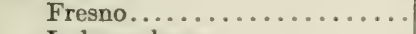 & 258 & 17,974 & 9,718 & 7,912 & 5,332 \\
\hline Independence............. & 222 & 15,003 & 7,899 & 6,345 & 4,125 \\
\hline Los Angeles............... & 334 & 20,381 & 9,693 & 7,355 & 4,015 \\
\hline Red Bluff. ............... & 264 & 17,944 & 9,496 & 7,648 & 5,008 \\
\hline 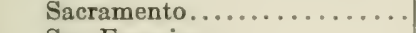 & 272 & 17,368 & 8,664 & 6,760 & 4,040 \\
\hline 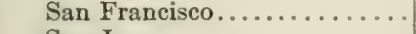 & 319 & 17,819 & 7,611 & 5,378 & 2,188 \\
\hline San Jose. . . . . . . . . . . . & 294 & 17,745 & 8,337 & 6,279 & 3,339 \\
\hline San Luis Obispo. . . . . . . . . & 260 & 15,367 & 7,047 & 5,227 & 2,627 \\
\hline \multicolumn{6}{|l|}{ Colorado: } \\
\hline 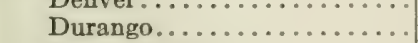 & 121 & 7,775 & 3,903 & 3,056 & $\begin{array}{l}2,563 \\
1,846\end{array}$ \\
\hline Grand Junction............ & 183 & 12,554 & 6,698 & 5,417 & 3,587 \\
\hline Pueblo................. & 163 & 10,950 & 5,734 & 4,593 & 2,963 \\
\hline \multicolumn{6}{|l|}{ Connecticut: } \\
\hline Hartford................ & 165 & 10,660 & 5,380 & 4,225 & 2,575 \\
\hline 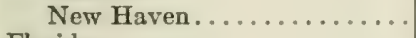 & 180 & 11,560 & 5,800 & 4,540 & 2,740 \\
\hline \multicolumn{6}{|l|}{ Florida: } \\
\hline Jacksonville.............. & 293 & 20,765 & 11,389 & 9,338 & 6,408 \\
\hline Jupiter.................. & 318 & 23,721 & 13,545 & 11,319 & 8,139 \\
\hline 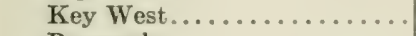 & 365 & 28,757 & 17,077 & 14,522 & 10,872 \\
\hline 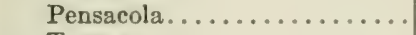 & 285 & 20,519 & 11,399 & 9,404 & 6,554 \\
\hline 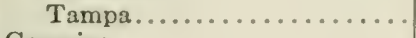 & 335 & 23,909 & 13,189 & 10,844 & 7,494 \\
\hline \multicolumn{6}{|l|}{ Georgia: } \\
\hline Atlanta..... & 225 & 15,731 & 8,531 & 6,956 & 4,706 \\
\hline 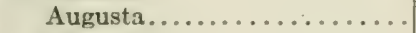 & 228 & 16,436 & 9,140 & 7,544 & 5,264 \\
\hline 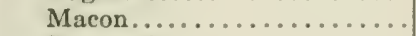 & 238 & 16,831 & 9,215 & 7,549 & 5,169 \\
\hline 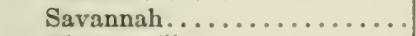 & 263 & 19,288 & 10,258 & 8,417 & 5,787 \\
\hline Thomasville.............. & 257 & 18,748 & 10,524 & 8,725 & 6,155 \\
\hline \multicolumn{6}{|l|}{ Idaho: } \\
\hline Boise..... & 177 & 11,457 & 5,793 & 4,554 & 2,781 \\
\hline Lewiston.... & 202 & 13,030 & 6,566 & 5,152 & 3,132 \\
\hline Pocatello.................. & 175 & 10,980 & 5,380 & 4,155 & 2,405 \\
\hline \multicolumn{6}{|l|}{ Illinois: } \\
\hline Cairo........ & 212 & 14,750 & 7,966 & 6,482 & 4,362 \\
\hline 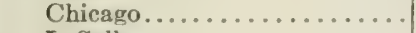 & 182 & 11,671 & 5,847 & 4,573 & 2,753 \\
\hline 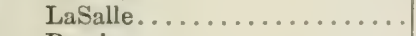 & 168 & 11,309 & 5,933 & 4,757 & 3,077 \\
\hline 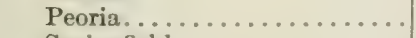 & 186 & 12,356 & 6,404 & 5,102 & 3,242 \\
\hline Springfield... & 182 & 12,442 & 6,618 & 5,344 & 3,524 \\
\hline \multicolumn{6}{|l|}{ Indiana: } \\
\hline Evansville.... & 203 & 14,196 & 7,700 & 6,279 & 4,249 \\
\hline Indianapolis.............. & 186 & 12,595 & 6,643 & 5,341 & 3,481 \\
\hline
\end{tabular}


TABLE 6. - Summations of normal daily mean remainder indices of temperature efficiency for plant growth, for the period of the average frostless season. (Plate 38.)-Continued.

\begin{tabular}{|c|c|c|c|c|c|}
\hline \multirow{2}{*}{ Station. } & \multirow{2}{*}{$\begin{array}{l}\text { Length of } \\
\text { irostless } \\
\text { season. }\end{array}$} & \multicolumn{4}{|c|}{$\begin{array}{c}\text { Direct summation of normal daily mean } \\
\text { temperatures for period of average } \\
\text { frostless season. }\end{array}$} \\
\hline & & $\begin{array}{c}\text { Above } \\
0^{\circ} \mathrm{F}\end{array}$ & $\begin{array}{l}\text { Above } \\
32^{\circ} \mathrm{F} \text {. }\end{array}$ & $\begin{array}{l}\text { Above } \\
39^{\circ} \mathrm{F} \text {. }\end{array}$ & $\begin{array}{l}\text { Above } \\
50^{\circ} \mathrm{F} \text {. }\end{array}$ \\
\hline Iowa: & days. & & & & \\
\hline Charles City................ & 133 & 9,095 & 4,839 & 3,908 & 2,578 \\
\hline 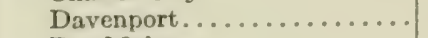 & 174 & 11,755 & 6,187 & 4,969 & 3,229 \\
\hline Des Moines............... & 171 & 11,594 & 6,122 & 4,925 & 3,215 \\
\hline 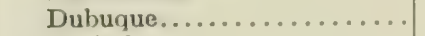 & 176 & 11,688 & 6,056 & 4,824 & 3,064 \\
\hline Keokuk................. & 197 & 13,215 & 6,911 & 5,532 & 3,562 \\
\hline Sioux City............. & 146 & 10,041 & 5,396 & 4,347 & 2,887 \\
\hline \multicolumn{6}{|l|}{ Kansas: } \\
\hline 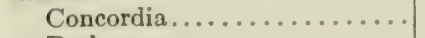 & 173 & 12,171 & 6,635 & 5,424 & 3,694 \\
\hline 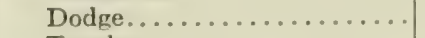 & 181 & 12,612 & 6,820 & 5,553 & 3,743 \\
\hline Topeka................. & 189 & 13,086 & 7,038 & 5,715 & 3,825 \\
\hline 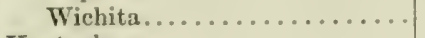 & 194 & 13,660 & 7,452 & 6,094 & 4,154 \\
\hline \multicolumn{6}{|l|}{ Kentucky: } \\
\hline Lexington. . & 187 & 12,880 & 6,896 & 5,587 & 3,717 \\
\hline \multirow{2}{*}{\multicolumn{6}{|c|}{ Louisiana: }} \\
\hline & & & & & \\
\hline New Orleans... & 310 & 21,971 & 1,251 & 9,881 & - 6,781 \\
\hline Shreveport.... & 252 & 18,228 & 10,164 & 8,400 & 5,880 \\
\hline \multicolumn{6}{|l|}{ Maine: } \\
\hline Eastport... & 167 & 2,119 & 3,775 & 2,606 & 936 \\
\hline Portland... & 157 & 2,699 & 4,675 & 3,576 & 2,006 \\
\hline \multicolumn{6}{|l|}{ Maryland: } \\
\hline 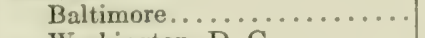 & 213 & 14,281 & 6,816 & 5,974 & 3,844 \\
\hline Washington, D. C ......... & 197 & 13,385 & 7,081 & 5,702 & 3,732 \\
\hline \multicolumn{6}{|l|}{ Massachusetts: } \\
\hline $\begin{array}{l}\text { Boston................... } \\
\text { Nantucket. . . . . . . . . . }\end{array}$ & 209 & 12,411 & 5,723 & $\begin{array}{l}4,410 \\
4,260\end{array}$ & 2,565 \\
\hline $\begin{array}{l}\text { Nantucket.............. } \\
\text { Michigan: }\end{array}$ & \multicolumn{5}{|c|}{ Michigan: } \\
\hline $\begin{array}{l}\text { Michigan: } \\
\text { Alpena..... }\end{array}$ & 137 & 8,330 & 3,946 & 2,987 & 1,617 \\
\hline$\ldots \ldots \ldots \ldots$ & 164 & 10,738 & 5,490 & 4,342 & 2,702 \\
\hline 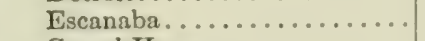 & 140 & 8,536 & 4,056 & 3,076 & 1,676 \\
\hline 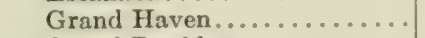 & 167 & 10,481 & 5,137 & 3,968 & 2,298 \\
\hline Grand Rapids............ & 164 & 10,745 & 5,497 & 4,349 & 2,709 \\
\hline 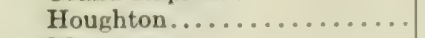 & 152 & 8,979 & 4,115 & 3,051 & 1,531 \\
\hline 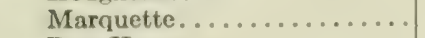 & 140 & 8,367 & 3,887 & 2,907 & 1,507 \\
\hline Port Huron............... & 155 & 9,777 & 4,817 & 3,732 & 2,182 \\
\hline Sault Ste. Marie........... & 138 & 7,959 & 3,543 & 2,577 & 1,197 \\
\hline \multicolumn{6}{|l|}{ Minnesota: } \\
\hline 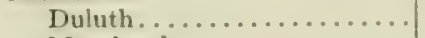 & 152 & 8,973 & 4,109 & 3,045 & 1,525 \\
\hline Moorhead................ & 132 & 8,499 & 4,275 & 3,351 & 2,031 \\
\hline St. Paul................. & 159 & 10,342 & 5,254 & 4,141 & 2,551 \\
\hline \multicolumn{6}{|l|}{ Mississippi: } \\
\hline 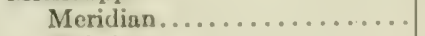 & 230 & 16,424 & 9,064 & 7,454 & 5,154 \\
\hline 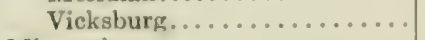 & 252 & 18,032 & 9,968 & 8,204 & 5,684 \\
\hline \multicolumn{6}{|l|}{ Missouri: } \\
\hline Columbia............ & 179 & 12,487 & 6,759 & 5,506 & 3,716 \\
\hline Hannibal................ & 183 & 12,701 & 6,845 & 5,564 & 3,734 \\
\hline Kansas City............... & 196 & 13,362 & 6,090 & $5,71 \mathrm{~s}$ & 3,755 \\
\hline St. Louis................. & 207 & 14,395 & 7,771 & 6,322 & 4,252 \\
\hline 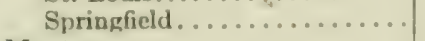 & 187 & 12,907 & 6,923 & 5,614 & 3,744 \\
\hline \multicolumn{6}{|l|}{ Montans: } \\
\hline Havro..... & 122 & 7,811 & 3,907 & 3,053 & 1,833 \\
\hline Helenn................. & 144 & 8,785 & $4,177$. & 3,169 & - 1,729 \\
\hline Kalispell................ & 140 & 8,283 & 3,503 & 2,823 & 1,423 \\
\hline Miles City............... & 140 & 9,337 & 4,857 & 3,877 & 2,477 \\
\hline
\end{tabular}


TABLE 6.-Summations of normal daily mean remainder indices of temperature efficiency for plant growth, for the period of the average frostless season. (Plate 38.)-Continued.

\begin{tabular}{|c|c|c|c|c|c|}
\hline \multirow{2}{*}{ Station. } & \multirow{2}{*}{$\begin{array}{l}\text { Length of } \\
\text { frostless } \\
\text { season. }\end{array}$} & \multicolumn{4}{|c|}{$\begin{array}{c}\text { Direct summation of normal daily mean } \\
\text { temperatures for period of average } \\
\text { frostless season. }\end{array}$} \\
\hline & & $\begin{array}{c}\text { Above } \\
0^{\circ} \mathrm{F} \text {. }\end{array}$ & $\begin{array}{l}\text { Above } \\
32^{\circ} \mathrm{F} \text {. }\end{array}$ & $\begin{array}{l}\text { A bove } \\
39^{\circ} \mathbf{F} \text {. }\end{array}$ & $\begin{array}{l}\text { A bove } \\
50^{\circ} \mathrm{F} \text {. }\end{array}$ \\
\hline Nebraska: & days. & & & & \\
\hline 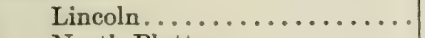 & 174 & 11,912 & 6,344 & 5,126 & 3,386 \\
\hline North Platte.............. & 151 & 10,195 & 5,363 & 4,306 & 2,796 \\
\hline 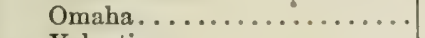 & 170 & 11,723 & 6,283 & 5,093 & 3,393 \\
\hline Valentine................ & 132 & 8,971 & 4,747 & 3,823 & 2,503 \\
\hline \multicolumn{6}{|l|}{ Nevada: } \\
\hline Reno. & 138 & 8,649 & 4,233 & 3,267 & 1,887 \\
\hline Winnemucca.............. & 131 & 8,641 & 4,449 & 3,532 & 2,222 \\
\hline \multicolumn{6}{|l|}{ New Hampshire: } \\
\hline Concord ...... & 146 & 9,280 & 4,608 & 3,586 & 2,126 \\
\hline \multicolumn{6}{|l|}{ New Jersey: } \\
\hline Atlantic City.... & 207 & 13,348 & 6,724 & 5,275 & 3,205 \\
\hline Cape May. . . . . . . . . . . & 186 & 12,362 & 6,410 & 5,108 & 3,248 \\
\hline \multicolumn{6}{|l|}{ New Mexico: } \\
\hline Roswell ... & 195 & 14,064 & 7,824 & 6,459 & 4,509 \\
\hline Santa Fe... & 187 & 11,510 & 5,526 & 4,217 & 2,347 \\
\hline \multicolumn{6}{|l|}{ New York: } \\
\hline Albany... & 177 & 11,382 & 5,918 & 4,479 & 2,709 \\
\hline 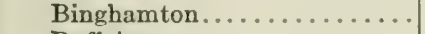 & 158 & 10,088 & 5,032 & 3,926 & 2,346 \\
\hline 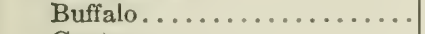 & 173 & 10,907 & 5,371 & 4,160 & 2,430 \\
\hline 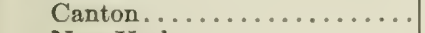 & 139 & 8,933 & 4,485 & 3,512 & 2,122 \\
\hline 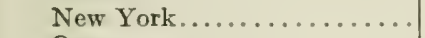 & 210 & 13,422 & 6,702 & 5,232 & 3,132 \\
\hline 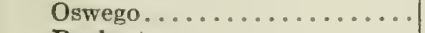 & 175 & 10,950 & 5,350 & 4,125 & 2,375 \\
\hline Rochester.............. & 171 & 10,864 & 5,392 & 4,195 & 2,485 \\
\hline Syracuse.................... & 171 & 10,918 & 5,446 & 4,249 & 2,539 \\
\hline \multicolumn{6}{|l|}{ North Carolina: } \\
\hline Asheville................ & 176 & 11,693 & 6,061 & 4,829 & 3,069 \\
\hline Charlctte............... & 220 & 15,316 & 8,276 & 6,736 & 4,536 \\
\hline Hatteras.................. & 256 & 17,650 & 9,460 & 7,668 & 5,108 \\
\hline 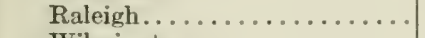 & 213 & 14,485 & 8,069 & 6,578 & 4,448 \\
\hline Wilmington $\ldots \ldots \ldots \ldots \ldots$ & 233 & 16,359 & 8,903 & 7,272 & 4,942 \\
\hline \multicolumn{6}{|l|}{ North Dakota: } \\
\hline Bismarck.............. & 129 & 7,932 & 3,804 & 2,901 & 1,611 \\
\hline Devils Lake. . . . . . . . . . . . & 121 & 7,658 & 3,786 & 2,939 & 1,729 \\
\hline Williston...$\ldots \ldots \ldots \ldots \ldots$ & 119 & 7,785 & 3,977 & 3,144 & 1,954 \\
\hline \multicolumn{6}{|l|}{ Ohio: } \\
\hline Cincinnati............. & 194 & 13,416 & 7,208 & 5,850 & 3,910 \\
\hline Cleveland ................ & 198 & 12,546 & 6,210 & 4,824 & 2,844 \\
\hline Columbus.............. & 184 & 12,373 & 6,485 & 5,197 & 3,357 \\
\hline Sandusky ............... & 195 & 12,491 & 6,251 & 4,886 & 2,936 \\
\hline Toledo..................... & 174 & 11,494 & 5,926 & 4,708 & 2,968 \\
\hline \multicolumn{6}{|l|}{ Oklahoma: } \\
\hline \multicolumn{6}{|l|}{ Oregon: } \\
\hline 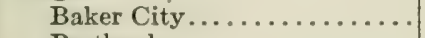 & 127 & 7,767 & 3,703 & 2,814 & 1,544 \\
\hline Portland................. & 245 & 14,172 & 6,332 & 4,617 & 2,167 \\
\hline Roseburg................ & 198 & 11,848 & 5,512 & 4,126 & 2,146 \\
\hline \multicolumn{6}{|l|}{ Pennsylvania: } \\
\hline Erie ........... & 194 & 12,232 & 6,024 & 4,666 & 2,726 \\
\hline Harrisburg. .............. & 196 & 12,882 & 6,610 & 5,238 & 3,278 \\
\hline Philadelphia............ & 206 & 13,629 & 7,037 & 5,595 & 3,535 \\
\hline Pittsburgh............... & 179 & 12,102 & 6,374 & 5,121 & 3,331 \\
\hline 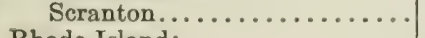 & 176 & 11,304 & 5,672 & 4,440 & 2,680 \\
\hline \multicolumn{6}{|l|}{ Rhode Island: } \\
\hline Block Island.............. & 218 & 12,946 & 5,970 & 4,444 & 2,264 \\
\hline Providence............... & 190 & 12,164 & 6,084 & 4,754 & 2,854 \\
\hline
\end{tabular}


TABLE 6.-Summations of normal daily mean remainder indices of lemperature efficiency for plant growth, for the period of the average frostless season. (Plate 38.)-Continued.

\begin{tabular}{|c|c|c|c|c|c|}
\hline \multirow{2}{*}{ Station. } & \multirow{2}{*}{$\begin{array}{l}\text { Length of } \\
\text { frostless } \\
\text { season. }\end{array}$} & \multicolumn{4}{|c|}{$\begin{array}{c}\text { Direct summation of normal daily mean } \\
\text { temperatures for period of average } \\
\text { frostless season. }\end{array}$} \\
\hline & & $\begin{array}{c}\text { Above } \\
0^{\circ} \mathrm{F}\end{array}$ & $\begin{array}{l}\text { Above } \\
32^{\circ} \mathrm{F} \text {. }\end{array}$ & $\begin{array}{l}\text { Above } \\
39^{\circ} \mathrm{F} \text {. }\end{array}$ & $\begin{array}{l}\text { Above } \\
50^{\circ} \mathrm{F} \text {. }\end{array}$ \\
\hline South Carolina: & days. & & & & \\
\hline Charleston................ & 276 & $\begin{array}{l}19,470 \\
16.593\end{array}$ & $\begin{array}{r}10,638 \\
9,201\end{array}$ & $\begin{array}{l}8,706 \\
7,584\end{array}$ & 5,946 \\
\hline \multicolumn{6}{|l|}{ South Dakota: } \\
\hline Huron....... & 131 & 8,788 & 4,596 & 3,631 & 2,321 \\
\hline Pierre................. & 153 & 10,387 & 5,491 & 4,420 & 2,890 \\
\hline Rapid City................. & 143 & 9,149 & 4,573 & 3,572 & 2,142 \\
\hline 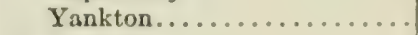 & 154 & 10,470 & 5,542 & 4,464 & 2,924 \\
\hline \multicolumn{6}{|l|}{ Tennessee: } \\
\hline 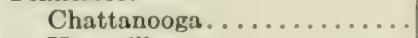 & 207 & 14,602 & 7,978 & 6,529 & 4,459 \\
\hline 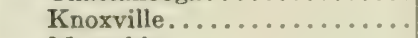 & 208 & 14,229 & 7,573 & 6,117 & 4,037 \\
\hline 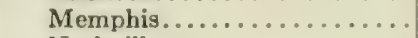 & 224 & 15,712 & 8,544 & 6,976 & 4,736 \\
\hline Nashville................. & 207 & 14,700 & 8,076 & 6,627 & 4,557 \\
\hline \multicolumn{6}{|l|}{ Texas: } \\
\hline$\ldots \ldots \ldots \ldots$ & 245 & 17,536 & 9,696 & 7,981 & 5,531 \\
\hline 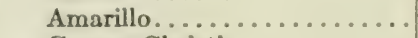 & 199 & 13,542 & 7,174 & 5,781 & 3,791 \\
\hline Corpus Christi........... & 298 & 21,898 & 12,362 & 10,276 & 7,296 \\
\hline 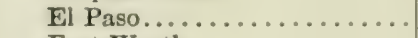 & 236 & 16,819 & 9,267 & 7,615 & 5,255 \\
\hline 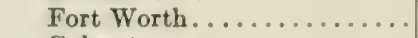 & 261 & 18,816 & 10,464 & 8,637 & 6,027 \\
\hline 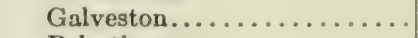 & 331 & 23,538 & 12,946 & 10,629 & 7,319 \\
\hline 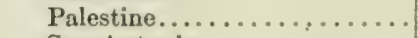 & 245 & 17,690 & 9,850 & 8,135 & 5,685 \\
\hline 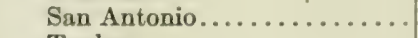 & 276 & 20,119 & 11,287 & 9,355 & 6,595 \\
\hline 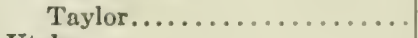 & 254 & 18,656 & 10,528 & 8,750 & B, 210 \\
\hline \multicolumn{6}{|l|}{ Utah: } \\
\hline 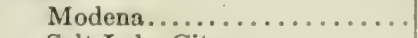 & 130 & 8,451 & 4,291 & 3,391 & 2,091 \\
\hline Salt Lake City............ . & 182 & 12,078 & 6,254 & 4,980 & 3,160 \\
\hline \multicolumn{6}{|l|}{ Vermont: } \\
\hline Burlington............. & 143 & 9,000 & 4,424 & 3,423 & 1,993 \\
\hline Northfield.............. & 126 & 7,812 & 3,780 & 2,898 & 1,638 \\
\hline \multicolumn{6}{|l|}{ Virginia: } \\
\hline Lynchburg.... . & 201 & 13,759 & 7,327 & 5,920 & 3,910 \\
\hline Norfolk.................. & 230 & 15,691 & 8,331 & 6,721 & 4,421 \\
\hline 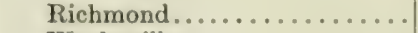 & 215 & 14,942 & 8,062 & 6,557 & 4,407 \\
\hline 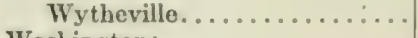 & 175 & 11,544 & 5,944 & 4,719 & 2,969 \\
\hline \multicolumn{6}{|l|}{ Washington: } \\
\hline North Head............... & 316 & 16,180 & 6,068 & 3,856 & 696 \\
\hline 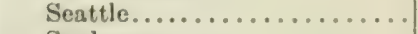 & 246 & 13,770 & 5,898 & 4,176 & 1,716 \\
\hline 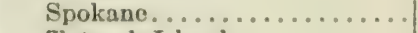 & 202 & 11,992 & 5,528 & 4,114 & 2,094 \\
\hline Tatoosh Island............. & 271 & 13,623 & 4,951 & 3,054 & 344 \\
\hline Walla Walla............... & 216 & 13,819 & 6,907 & 5,395 & 3,235 \\
\hline \multicolumn{6}{|l|}{ West Virginia: } \\
\hline Elkins................ & 145 & 9,521 & 4,881 & 3,866 & 2,416 \\
\hline $\begin{array}{l}\text { Parkersburg............... } \\
\text { Wisconsin: }\end{array}$ & 179 & 12,195 & 6,467 & 5,214 & 3,424 \\
\hline \multicolumn{6}{|l|}{ Wisconsin: } \\
\hline 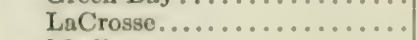 & 163 & 10,707 & 5,491 & 4,350 & 2,720 \\
\hline 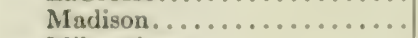 & 179 & 11,370 & 5,642 & 4,389 & 2,599 \\
\hline Milwaukeo................ & 162 & 10,191 & 5,007 & 3,573 & 2,253 \\
\hline \multicolumn{6}{|l|}{ Wyoming: } \\
\hline Cheyenne............... & 119 & 7,528 & 3,720 & $2, \mathrm{SS7}$ & 1,697 \\
\hline Lander................... & 108 & 6,911 & 3,455 & 2,699 & 1,619 \\
\hline
\end{tabular}




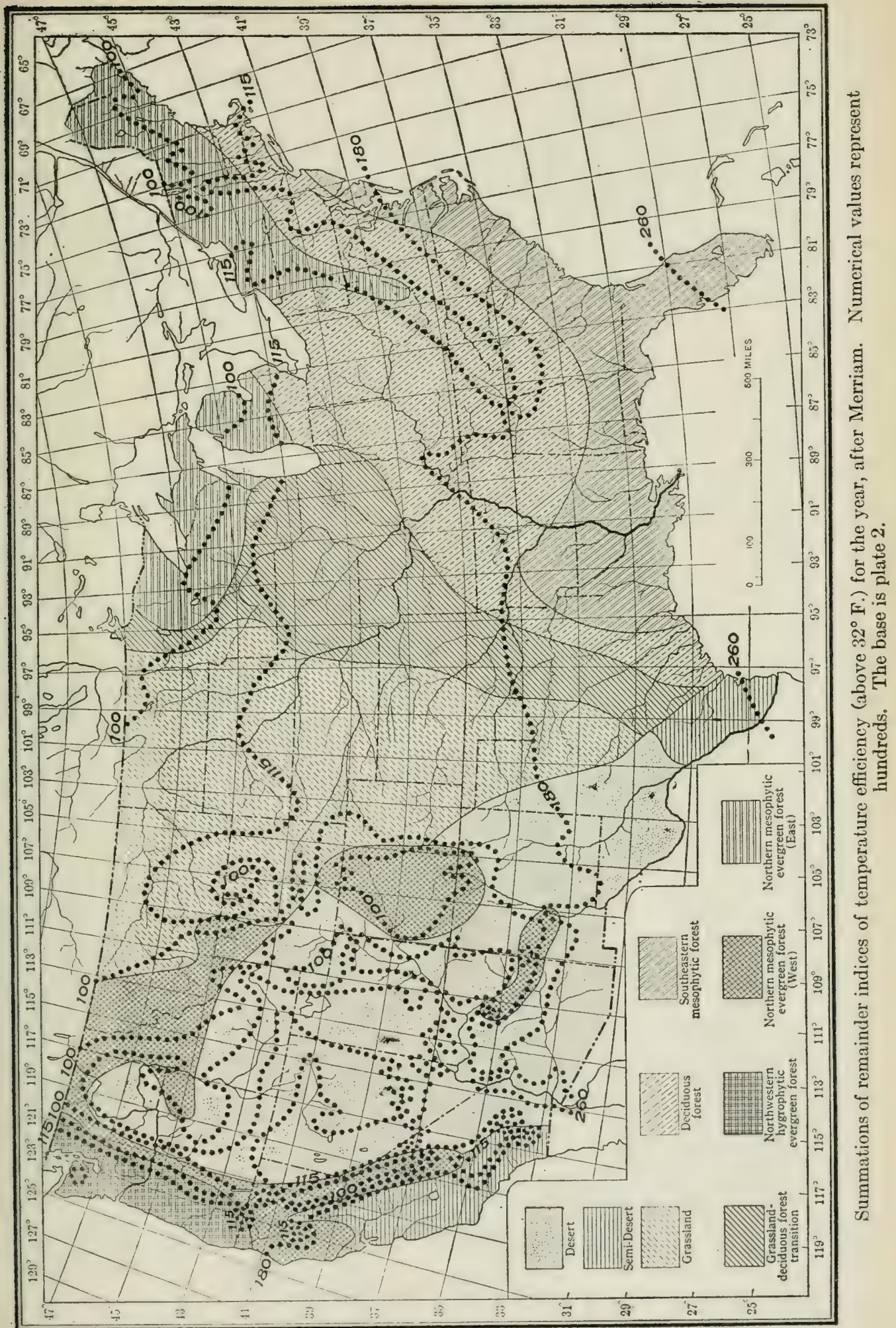




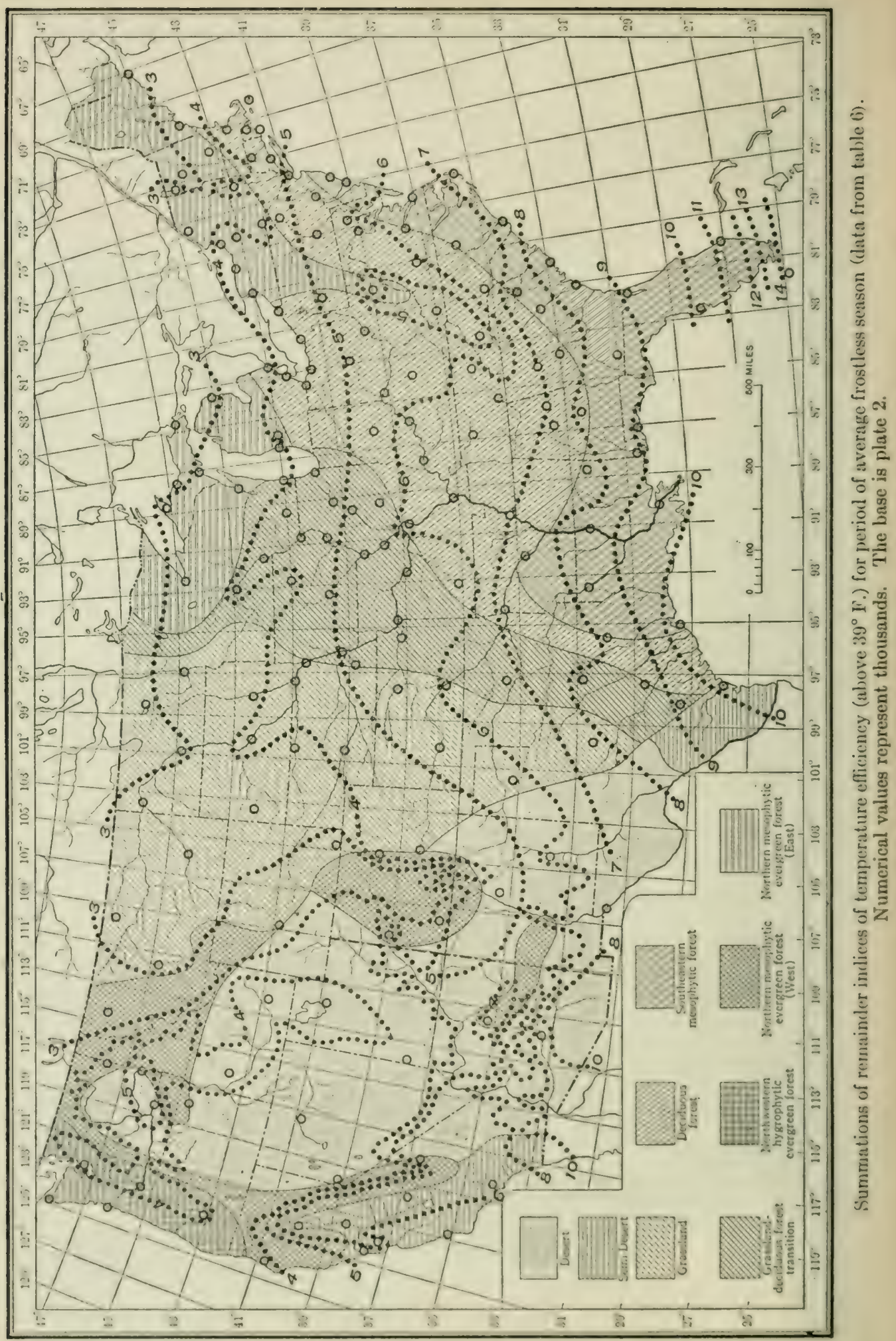




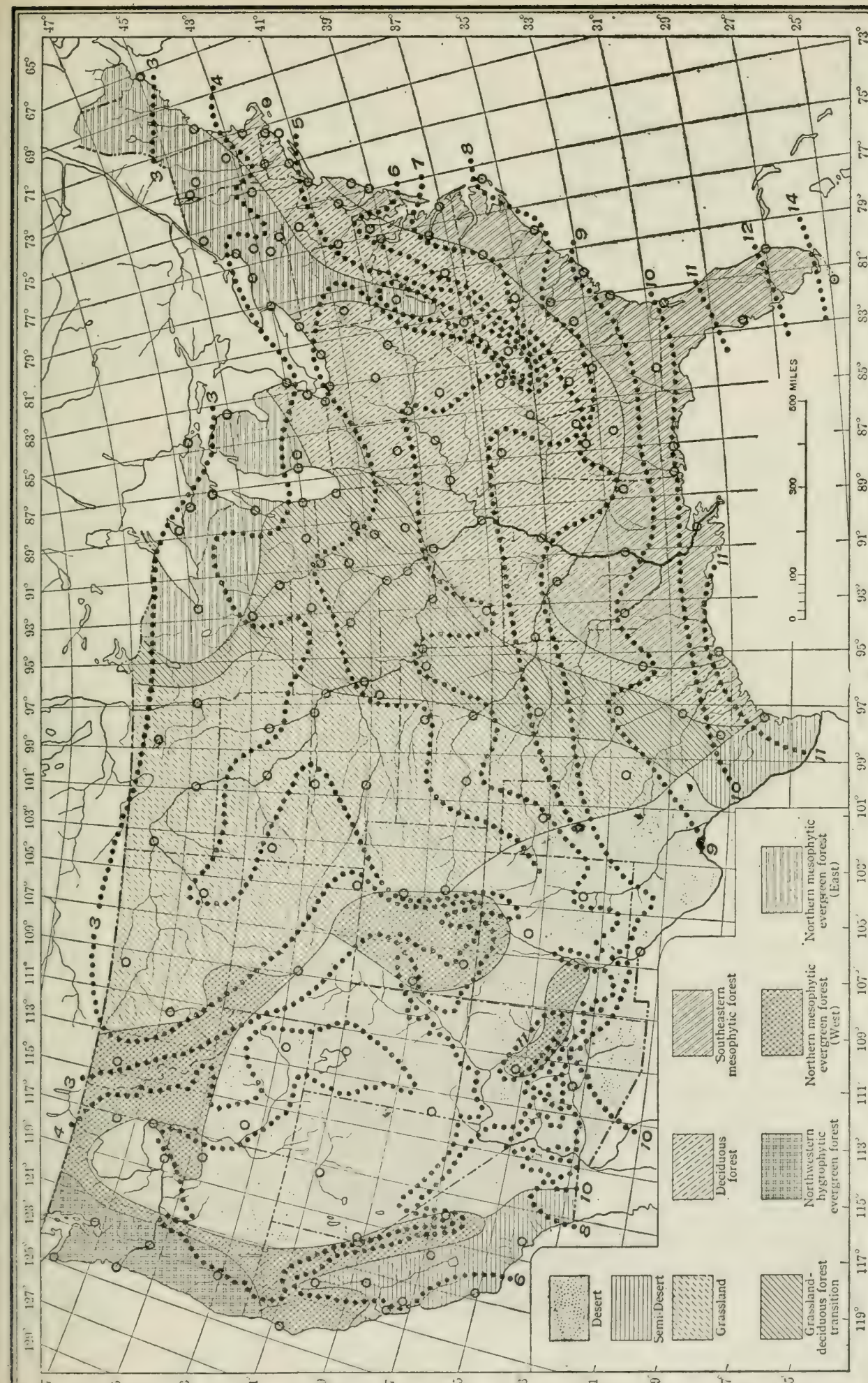

눈

胥

这

눙.

등

马

당

일

逑

6.

.

氠

(2)

공

동

ले

G

욜

ह

잉

迅

능

至N

总

ज

पू

콜

矛

봉 


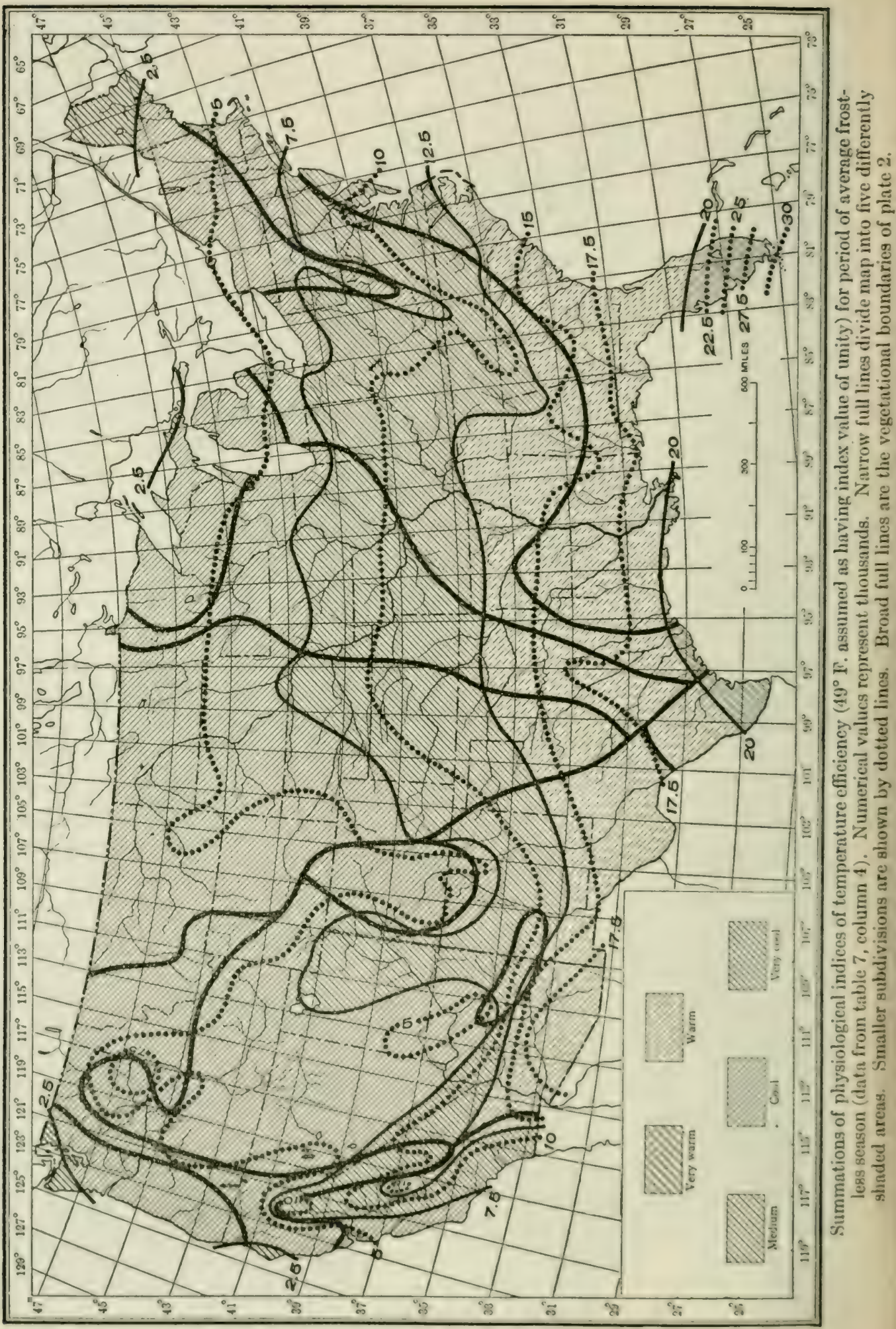


Charts were made for each of these four series of seasonal efficiency indices, but they all agree in the general delineation of the climatic zones and only the one for summations above $39^{\circ} \mathrm{F}$. is here presented, in plate $38^{1}$. This chart differs from the corresponding one of Livingston in a few details, but the two are practically identical.

The increments of seasonal temperature efficiency indices shown on the chart of plate 38 are each 1,000 in the East, and the numbers placed upon the isoclimatic lines denote thousands. These values may be reduced to the corresponding ones based on the centigrade thermometer scale by the use of the familiar factor $5 / 9$, the starting-point for our summations being $39^{\circ} \mathrm{F}$., or $3.9^{\circ} \mathrm{C}$.

The lines of this chart are seen to have a generally west-east direction east of the Rocky Mountains, several of them being southwardly displaced by the Appalachians. The western mountains produce a very great southward displacement, and another considerable displacement of some lines, in the same direction, appears due to the immediate vicinity of the Pacific Ocean. It is interesting to note that the area having an index of 7,000 or less extends southward on the California coast nearly to the parallel of latitude $33^{\circ}$ north, while the same area on the Atlantic coast extends southward only to about $35^{\circ}$ north latitude.

Most of the country appears to be characterized by these seasonal indices of temperature efficiency having values between 3,000 and 10,000. The region where these indices are less than 3,000 seems to occupy northern New England, northeastern Michigan, northern Minnesota and North Dakota, western Montana, central Wyoming, and the Rocky Mountain system. The region having indices above 10,000 appears to occupy the valleys of the Gila and lower Colorado Rivers, a narrow strip of the Gulf coast of Texas, and the southern half of the peninsula of Florida. A closed area with indices between 4,000 and 6,000 is shown on this chart as occupying a region extending from the Columbia River to Great Salt Lake.

(D) SUMMATION OF EXPONENTIAL INDICES OF TEMPERATURE EFFICIENCY FOR PERIOD OF AVERAGE FROSTLESS SEASON. (TABLE 7, PLATE 39.)

In applying the exponential method of deriving temperature efficiency indices from normal daily mean temperature indices as given in Bulletin R, we have followed Livingst on and Livingston. The efficiency index corresponding to each normal daily mean within the period of the average frostless season, for each station considered, was first obtained from table 5, and then all these indices were summed to give the seasonal index in each case. The results of these summations, which are the data given on the chart of Livingston and Livingston's figure 2, are presented in the second column of table 7.

\footnotetext{
1 The chart derived from these summations of normal daily means above $32^{\circ} \mathrm{F}$. has been presented by Livingston. See Livingston, $1913 a$.
} 
TABLE 7.-Summation of normal daily indicrs of temperature efficiency for plant grouth, for the period of the average frosiless season. (Plates 39 and 40.)

[The mean daily efficiency indices are derived from the corresponding temperature indices, (1) by the exponential equation of chemical reaction velocities and (2) by the empirical growthrate coefficients for maize seedlings as found by Lehenbauer for a 12-hour exposure to maintained temperature. The temperature efficiency for $40^{\circ} \mathrm{F}$. is taken as unity in both cases.]

\begin{tabular}{|c|c|c|c|c|c|}
\hline Station. & 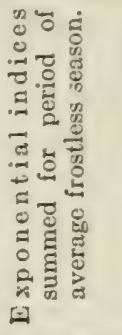 & 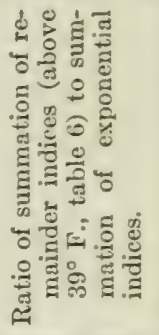 & 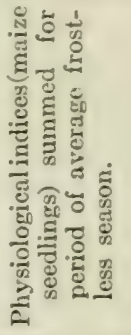 & 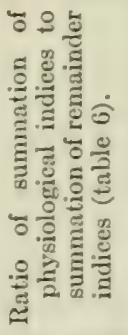 & 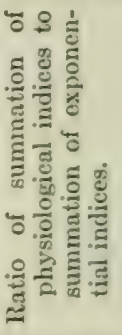 \\
\hline \multicolumn{6}{|l|}{ Alabama: } \\
\hline Anniston........... & 681.8 & 10.33 & 12,326 & 1.75 & 18.1 \\
\hline Birmingham..... & 816.7 & 9.37 & 15,025 & 1.96 & 18.4 \\
\hline Mobile.......... & 963.7 & 9.21 & 17,340 & 1.95 & 18.0 \\
\hline Montgomery..... & 886.0 & 9.19 & 16,511 & 2.03 & 18.6 \\
\hline \multicolumn{6}{|l|}{ Arizona: } \\
\hline Flagstaff........ & 245.2 & 9.78 & 2,652 & 1.11 & 10.8 \\
\hline Phoenix......... & $1,183.6$ & 8.51 & 20,640 & 2.05 & 17.4 \\
\hline \multicolumn{6}{|l|}{ Arkansas: } \\
\hline Fort Smith..... & 789.5 & 9.23 & 14,168 & 1.95 & 18.0 \\
\hline Little Rock....... & 811.7 & 9.25 & 14,567 & 1.94 & 17.9 \\
\hline \multicolumn{6}{|l|}{ California: } \\
\hline Eureka......... & 410.1 & 8.54 & 2,388 & 0.68 & 5.8 \\
\hline Fresno.......... & 862.9 & 9.17 & 15,007 & 1.90 & 17.5 \\
\hline Independence.... & 677.6 & 9.36 & 11,228 & 1.77 & 16.6 \\
\hline Los Angeles...... & 764.8 & 9.62 & 8,451 & 1.15 & 11.0 \\
\hline Red Bluff....... & 844.9 & 9.05 & 14,339 & 1.88 & 17.0 \\
\hline Sacramento...... & 706.1 & 9.57 & 9,884 & 1.46 & 14.0 \\
\hline San Francisco.... & 586.4 & 9.17 & 4,122 & 0.77 & 7.0 \\
\hline San Jose......... . & 657.9 & 9.54 & 7,000 & 1.12 & 10.6 \\
\hline San Luis Obispo.. & 500,5 & 10.44 & 4,963 & 0.95 & 9.9 \\
\hline \multicolumn{6}{|l|}{ Colorado: } \\
\hline Denver.......... & 422.0 & 9.70 & 6,271 & 1.53 & 14.9 \\
\hline Durango........ & 311.5 & 9.81 & 4,077 & 1.33 & 13.1 \\
\hline Grand Junction... & 581.9 & 9.31 & 9,921 & 1.83 & 17.1 \\
\hline Pueblo........... & 477.1 & 9.63 & 7,604 & 1.66 & 15.9 \\
\hline \multicolumn{6}{|l|}{ Connecticut: } \\
\hline Hartford........ & 436.8 & 9.67 & 6,181 & 1.46 & 14.2 \\
\hline New Haven...... & 473.3 & 9.59 & 6,703 & 1.48 & 14.2 \\
\hline \multicolumn{6}{|l|}{ Florida: } \\
\hline Jacksonville...... & $1,033.1$ & 9.04 & 18,791 & 2.01 & 18.2 \\
\hline Jupiter.......... & $1,260.0$ & 8.98 & 24,872 & 2.20 & 19.7 \\
\hline Key West........ & $1,541.8$ & 9.42 & 31,063 & 2.14 & 20.1 \\
\hline Pensacola....... & $1,028.4$ & 9.14 & 18,914 & 2.01 & 18.4 \\
\hline Tampa........... & $1,175.4$ & 9.23 & 21,420 & $1.9 \mathrm{~s}$ & 18.2 \\
\hline \multicolumn{6}{|l|}{ Georgia: } \\
\hline Atlanta......... & 737.3 & 9.43 & 13,019 & 1.57 & 11.7 \\
\hline Augusta......... & 816.8 & 9.24 & 15,134 & 2.01 & 15.6 \\
\hline Macon........... & 810.3 & 9.32 & $1+1,564$ & 1.93 & 18.0 \\
\hline Savannah........ & 909.8 & 9.25 & 16,407 & 1.95 & 18.0 \\
\hline Thomasville...... & 953.8 & 9.15 & 17,858 & 2.05 & 18.7 \\
\hline \multicolumn{6}{|l|}{ Idaho: } \\
\hline Boise........... & 467.7 & 9.74 & 6,716 & 1.47 & 14.3 \\
\hline Lewiston......... & 544.5 & 9.46 & 8,065 & 1.57 & 14.5 \\
\hline Pocatello......... & 437.4 & 9.50 & 5,893 & 1.42 & 13.4 \\
\hline
\end{tabular}


TABLE 7.-Summation of normal daily indices of temperature efficiency for plant growth, for the period of the average frostless season. (Plates 39 and 40.)-Continued.

\begin{tabular}{|c|c|c|c|c|c|}
\hline Station. & 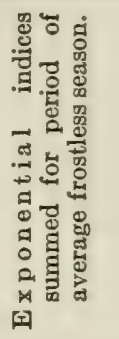 & 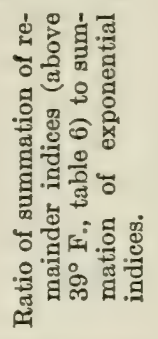 & 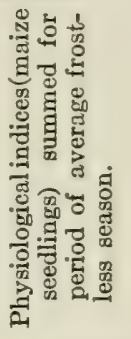 & 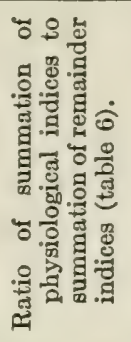 & 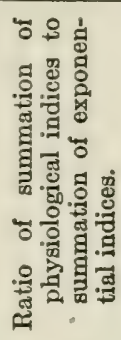 \\
\hline \multicolumn{6}{|l|}{ Illinois: } \\
\hline Cairo............... & 691.8 & 9.37 & 12,122 & 1.82 & 17.5 \\
\hline Chicago.......... & 479.6 & 9.54 & 6,892 & 1.51 & 14.4 \\
\hline La Salle......... & 496.3 & 9.58 & 7,975 & 1.68 & 16.1 \\
\hline Peoria............ & 535.8 & 9.52 & 8,466 & 1.66 & 16.1 \\
\hline Springfield....... & 562.7 & 9.50 & 9,464 & 1.77 & 16.8 \\
\hline \multicolumn{6}{|l|}{ Indiana: } \\
\hline Evansville........ & 672.6 & 9.34 & 11,879 & 1.89 & 17.7 \\
\hline Indianapolis...... & 567.3 & 9.41 & 9,441 & 1.77 & 16.6 \\
\hline \multicolumn{6}{|l|}{ Iowa: } \\
\hline Charles City...... & 403.1 & 9.69 & 6,630 & 1.70 & 16.5 \\
\hline Davenport....... & 519.6 & 9.56 & 8,464 & 1.70 & 16.3 \\
\hline Des Moines....... & 514.3 & 9.58 & 8,417 & 1.71 & 16.4 \\
\hline Dubuque........ & 503.6 & 9.58 & 7,865 & 1.63 & 15.6 \\
\hline Keokuk.......... & 588.8 & 9.40 & 9,681 & 1.75 & 16.4 \\
\hline Sioux City....... & 450.5 & 9.65 & 7,548 & 1.74 & 16.8 \\
\hline \multicolumn{6}{|l|}{ Kansas: } \\
\hline Concordia......... & 575.8 & 9.42 & 10,206 & 1.88 & 17.7 \\
\hline Dodge........... & 589.6 & 9.42 & 10,308 & 1.86 & 17.5 \\
\hline Topeka.......... & 607.5 & 9.41 & 10,546 & 1.85 & 17.4 \\
\hline Wichita......... & 650.8 & 9.36 & 11,563 & 1.90 & 17.8 \\
\hline \multicolumn{6}{|l|}{ Kentucky: } \\
\hline Lexington........ & 588.2 & 9.50 & 10,051 & 1.80 & 17.1 \\
\hline Louisville........ & 651.8 & 9.43 & 11,581 & 1.89 & 17.8 \\
\hline \multicolumn{6}{|l|}{ Louisiana: } \\
\hline New Orleans...... & $1,077.3$ & 9.17 & 19,323 & 1.96 & 18.0 \\
\hline Shreveport...... & 921.0 & 9.12 & 17,023 & 2.03 & 18.5 \\
\hline \multicolumn{6}{|l|}{ Maine: } \\
\hline Eastport......... & 299.3 & 8.71 & 2,102 & 0.80 & 7.0 \\
\hline Portland......... & 371.5 & 9.63 & 4,362 & 1.22 & 11.7 \\
\hline \multicolumn{6}{|l|}{ Maryland: } \\
\hline Baltimore......... & 635.9 & 9.39 & 10,457 & 1.75 & 16.5 \\
\hline $\begin{array}{l}\text { Washington, D. C. } \\
\text { Massachusetts: }\end{array}$ & 602.6 & 9.46 & 10,087 & 1.77 & 16.8 \\
\hline Boston.............. & 462.5 & 9.55 & 6,157 & 1.40 & 13.3 \\
\hline Nantucket....... & 459.0 & 9.28 & 5,032 & 1.18 & 11.0 \\
\hline \multicolumn{6}{|l|}{ Michigan: } \\
\hline Alpena.......... & 310.1 & 9.63 & 3,277 & 1.10 & 10.6 \\
\hline Detroit.......... & 448.5 & 9.68 & 6,622 & 1.53 & 14.8 \\
\hline Escanaba........ & 319.7 & 9.62 & 3,472 & 1.13 & 10.9 \\
\hline Grand Haven.... & 412.3 & 9.62 & 5,283 & 1.33 & 12.8 \\
\hline Grand Rapids.... & 450.4 & 9.66 & 6,687 & 1.54 & 14.8 \\
\hline Houghton....... & 323.8 & 9.42 & 3,147 & 1.03 & 9.7 \\
\hline Marquette....... & 304.7 & 9.54 & 3,019 & 1.04 & 9.9 \\
\hline Port Huron ....... & 385.0 & 9.69 & 4,884 & 1.31 & 12.6 \\
\hline Sault Ste. Marie.. & 276.1 & 9.33 & 2,322 & 0.90 & 8.4 \\
\hline \multicolumn{6}{|l|}{ Minnesota: } \\
\hline Duluth...... & 325.8 & 9.35 & 3,299 & 1.08 & 10.1 \\
\hline Moorhead......... & 333.9 & 9.84 & 4,283 & 1.28 & 12.8 \\
\hline St. Paul ......... & 428.7 & 9.66 & 6,230 & 1.50 & 14.5 \\
\hline
\end{tabular}


TABLE 7.-Summation of normal daily indices of temperature efficiency for plant growth, for the period of the average frosiless season. (Plates 39 and 40.)-Continued.

\begin{tabular}{|c|c|c|c|c|c|}
\hline Station. & 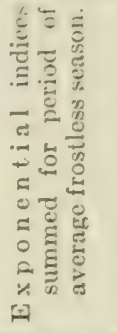 & 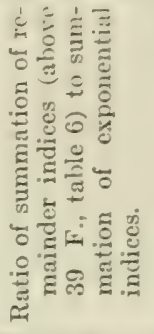 & 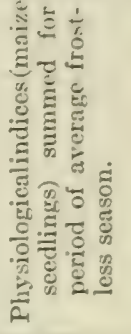 & 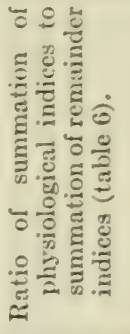 & 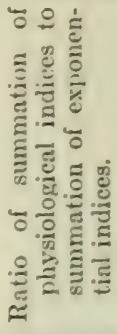 \\
\hline \multicolumn{6}{|l|}{ Mississippi: } \\
\hline Meridian........ & 800.6 & 9.31 & 14,565 & 1.95 & 18.2 \\
\hline Vicksburg........ & 892.6 & 9.19 & 16,194 & 1.97 & 18.1 \\
\hline \multicolumn{6}{|l|}{ Missouri: } \\
\hline Columbia........ & 583.8 & 9.43 & 10,241 & 1.86 & 17.6 \\
\hline Hannibal......... & 585.4 & 9.50 & 10,189 & 1.83 & 17.4 \\
\hline Kansas City..... . & 612.8 & 9.33 & 10,368 & 1.81 & 16.9 \\
\hline St. Louis........ . & 677.6 & 9.33 & 11,868 & 1.88 & 17.5 \\
\hline Springfield. . . . . . & 588.8 & 9.53 & 10,031 & 1.79 & 17.1 \\
\hline \multicolumn{6}{|l|}{ Montana: } \\
\hline Helena........... & 331.0 & 9.57 & 3,710 & 1.17 & 11.2 \\
\hline Kalispell......... & 297.2 & 9.50 & 2,827 & 1.00 & 9.5 \\
\hline Miles City...... & 402.2 & 9.64 & 6,253 & 1.61 & 15.6 \\
\hline \multicolumn{6}{|l|}{ Nebraska: } \\
\hline Lincoln......... & 538.9 & 9.51 & 9,062 & 1.77 & 16.8 \\
\hline North Platte.... & 446.7 & 9.64 & 7,192 & 1.67 & 16.1 \\
\hline Omaha.......... & 534.7 & 9.52 & 9,087 & 1.78 & 17.0 \\
\hline Valentine....... & 394.5 & 9.69 & 6,393 & 1.67 & 16.2 \\
\hline \multicolumn{6}{|l|}{ Nevada: } \\
\hline Reno........... . & 338.5 & 9.65 & 4,134 & 1.27 & 12.2 \\
\hline Winnemucca..... & 364.7 & 9.68 & 5,463 & 1.55 & 15.1 \\
\hline \multicolumn{6}{|l|}{ New Hampshire: } \\
\hline Concord........ & 368.0 & 9.74 & 4,724 & 1.32 & 12.8 \\
\hline \multicolumn{6}{|l|}{ New Jersey: } \\
\hline Atlantic City..... & 544.2 & 9.69 & 7,878 & 1.50 & 14.5 \\
\hline Cape May....... & 533.5 & 9.57 & 8,417 & 1.65 & 15.8 \\
\hline \multicolumn{6}{|l|}{ New Mexico: } \\
\hline Roswell......... & 678.4 & 9.52 & 12,448 & 1.93 & 18.4 \\
\hline Santa Fe....... & 442.5 & 9.53 & 5,350 & 1.30 & 12.0 \\
\hline \multicolumn{6}{|l|}{ New York: } \\
\hline Albany ......... & 466.3 & 9.61 & 6,633 & 1.49 & 14.3 \\
\hline Binghamton...... & $40 \cdot 4.4$ & 9.71 & 5,399 & 1.38 & 13.3 \\
\hline Buffalo......... & 433.8 & 9.59 & 5,761 & 1.39 & 13.3 \\
\hline Canton.......... & 358.5 & 9.80 & 4,713 & 1.31 & 13.1 \\
\hline New York........ & 554.4 & 9.44 & 8,104 & 1.55 & 14.6 \\
\hline Oswego.......... & 430.3 & 9.59 & 5,524 & 1.34 & 12.8 \\
\hline Rochester...... & $4: 34.6$ & 9.65 & 5.807 & 1.38 & 13.3 \\
\hline Syracuse... . . . . & 440.4 & 9.65 & 6,022 & 1.43 & 13.7 \\
\hline \multicolumn{6}{|l|}{ North Carolina: } \\
\hline Asheville....... & 495.4 & 9.75 & 7,504 & 1.55 & 15.1 \\
\hline Charlotte....... & 717.5 & 9.39 & 12,554 & 1.86 & 17.5 \\
\hline Hatteras........ & 80.4 .9 & 9.52 & 13,771 & 1.80 & 17.1 \\
\hline Raleigh......... & 700.3 & 9.39 & 12,329 & 1.88 & 17.6 \\
\hline Wilmington..... & 769.0 & 9.46 & 13,561 & 1.87 & 17.0 \\
\hline \multicolumn{6}{|l|}{ North Dnkota: } \\
\hline Bismarck.... & 342.4 & 8.47 & 4,792 & 1.65 & 14.0 \\
\hline Devils Lake.... . . & 301.2 & 9.76 & 3,754 & 1.28 & 12.5 \\
\hline Williston......... & 320.5 & 9.81 & 4,508 & 1.46 & 14.1 \\
\hline
\end{tabular}


TABLE 7.-Summation of normal daily indices of temperature efficiency for plant growth, for the period of the average frostless season. (Plates 39 and 40.)-Continued.

\begin{tabular}{|c|c|c|c|c|c|}
\hline Station. & 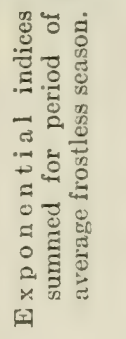 & 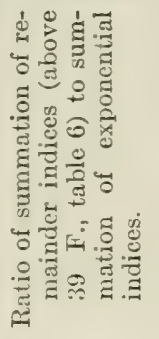 & 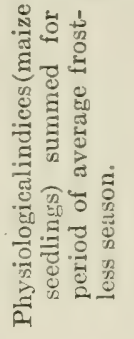 & 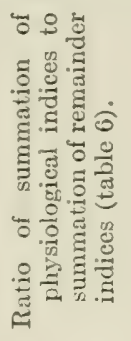 & 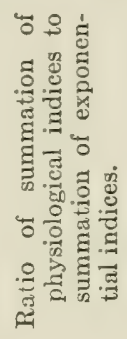 \\
\hline \multicolumn{6}{|l|}{ Ohio: } \\
\hline Cincinnati........ & 620.2 & 9.43 & 10,725 & 1.83 & 17.3 \\
\hline Cleveland........ & 508.9 & 9.48 & 7,151 & 1.48 & 14.1 \\
\hline Columbus........ & 543.9 & 9.56 & 8,799 & 1.70 & 16.2 \\
\hline Sandusky........ & 524.8 & 9.31 & 7,766 & 1.59 & 14.8 \\
\hline Toledo.......... & 489.5 & 9.62 & 7,508 & 1.59 & 15.3 \\
\hline $\begin{array}{l}\text { Oklahoma: } \\
\text { Oklahoma........ } \\
\text { Oregon: }\end{array}$ & 729.9 & 9.29 & 13,098 & 1.93 & 17.9 \\
\hline Baker City....... & 290.7 & 9.68 & 3,116 & 1.11 & 10.7 \\
\hline Portland......... & 502.9 & 9.18 & 4,780 & 1.04 & 9.4 \\
\hline \multirow{2}{*}{\multicolumn{6}{|c|}{ Pennsylvania: }} \\
\hline & & & & & \\
\hline Erie............ & 491.2 & 9.50 & 6,741 & 1.45 & 13.7 \\
\hline Harrisburg...... & 546.4 & 9.59 & 8,367 & 1.60 & 15.3 \\
\hline Philadelphia..... & 591.7 & 9.46 & 9,397 & 1.68 & 15.9 \\
\hline Pittsburg....... & 532.9 & 9.61 & 8,659 & 1. 69 & 16.3 \\
\hline Scranton.......... & 460.8 & 9.64 & 6,463 & 1.46 & 14.0 \\
\hline \multicolumn{6}{|l|}{ Rhode Island: } \\
\hline Block Island..... & 481.7 & 9.22 & 5,447 & 1.23 & 11.3 \\
\hline Providence........ & 500.9 & 9.49 & 7,241 & 1.52 & 14.5 \\
\hline \multicolumn{6}{|l|}{ South Carolina: } \\
\hline Charleston....... & 946.4 & 9.20 & 16,874 & 1.80 & 17.8 \\
\hline Columbia........ & 823.3 & 9.21 & 15,140 & 2.00 & 18.4 \\
\hline \multicolumn{6}{|l|}{ South Dakota: } \\
\hline Huron........... & 369.8 & 9.82 & 5,604 & 1.54 & 15.2 \\
\hline Pierre.......... & 461.8 & 9.57 & 7,566 & 1.72 & 16.4 \\
\hline Rapid City...... & 370.1 & 9.65 & 5,059 & 1.42 & 13.6 \\
\hline Yankton......... & 464.4 & 9.61 & 7,616 & 1.71 & 16.4 \\
\hline \multicolumn{6}{|l|}{ Tennessee: } \\
\hline Chattanooga.... & 692.5 & 9.43 & 12,395 & 1.90 & 17.9 \\
\hline Knoxville....... & 643.6 & 9.50 & 10,886 & 1.78 & 16.9 \\
\hline Memphis........ & 788.0 & 8.85 & 14,392 & 2.06 & 18.5 \\
\hline Nashville....... & 710.9 & 9.32 & 12,886 & 1.95 & 18.1 \\
\hline \multicolumn{6}{|l|}{ Texas: } \\
\hline Abilene.......... & 873.6 & 9.14 & 15,937 & 2.00 & 18.2 \\
\hline Amarillo......... & 598.9 & 9.65 & 10,668 & 1.85 & 17.8 \\
\hline Corpus Christi.... & $1,131.9$ & 9.08 & 21,392 & 2.08 & 18.8 \\
\hline El Paso.......... & 826.8 & 9.21 & 15,043 & 1.98 & 18.2 \\
\hline Fort Worth....... & 961.0 & 8.99 & 17,652 & 1.88 & 18.4 \\
\hline Galveston........ & $1,175.2$ & 9.04 & 21,163 & 1.99 & 18.0 \\
\hline Palestine........ & 888.8 & 9.15 & 16,468 & 2.02 & 18.5 \\
\hline San Antonio...... & $1,027.8$ & 9.10 & 19,202 & 2.08 & 18.7 \\
\hline Taylor............ & 963.5 & 9.08 & 18,200 & 2.08 & 19.0 \\
\hline \multicolumn{6}{|l|}{ Utah: } \\
\hline Modena.......... & 345.9 & 9.80 & 4,826 & 1.42 & 13.9 \\
\hline $\begin{array}{l}\text { Salt Lake City.... } \\
\text { Vermont: }\end{array}$ & 529.3 & 9.41 & 8,416 & 1.69 & 15.9 \\
\hline 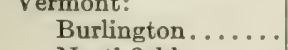 & 352.1 & 9.72 & 4,341 & 1.27 & 12.3 \\
\hline Northfield....... . & 297.6 & 9.74 & 3,348 & 1.16 & 11.3 \\
\hline
\end{tabular}


TABLE 7.-Summation of normal daily indices of temperaluere efficiency for plant growth, for the period of the average frostless season. (Plates 39 and 40.)-Continued.

\begin{tabular}{|c|c|c|c|c|c|}
\hline Station. & 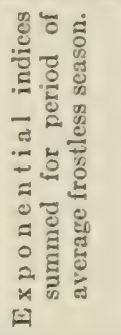 & 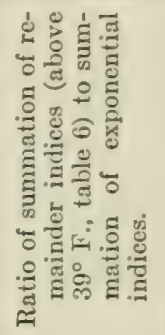 & 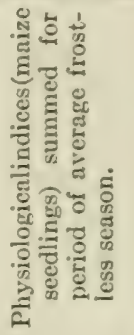 & 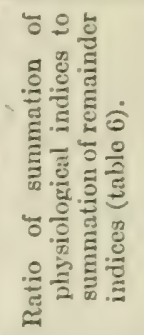 & 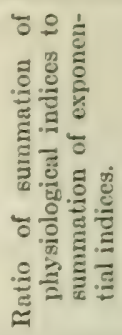 \\
\hline \multicolumn{6}{|l|}{ Virginia: } \\
\hline Lynchburg. & 626.2 & 9.45 & 10,631 & 1.80 & 17.0 \\
\hline Norfolk... & 718.0 & 9.36 & 12,194 & 1.81 & 17.0 \\
\hline Richmond.. & 702.8 & 9.33 & 12,305 & 1.88 & 17.5 \\
\hline Wytheville. & 487.0 & 9.69 & 7,313 & 1.55 & 15.0 \\
\hline \multicolumn{6}{|l|}{ Washington: } \\
\hline North Head. & 496.3 & 7.77 & 2,693 & 0.70 & 5.4 \\
\hline Seattle....... & 462.2 & 9.04 & 3,692 & 0.88 & 8.0 \\
\hline Spokane. & 446.3 & 9.22 & 5,059 & 1.23 & 11.3 \\
\hline Tatoosh Island. & 407.8 & 7.49 & 1,947 & 0.65 & 4.8 \\
\hline Walla Walla.... & 573.3 & 9.41 & 8,378 & 1.55 & 14.6 \\
\hline \multicolumn{6}{|l|}{ West Virginia: } \\
\hline Elkins.... & 395.2 & 9.78 & 5,685 & 1.47 & 14.3 \\
\hline Parkersburg.. & 543.9 & 9.59 & 9,009 & 1.73 & 16.6 \\
\hline \multicolumn{6}{|l|}{ Wisconsin: } \\
\hline Green Bay. . & 382.2 & 9.67 & 4,942 & 1.34 & 12.9 \\
\hline LaCrosse.... & 449.9 & 9.67 & 6,705 & 1.54 & 14.9 \\
\hline Madison..... & 460.9 & 9.52 & 6,434 & 1.47 & 14.0 \\
\hline Milwaukee.... & 403.2 & 9.61 & 5,261 & 1.36 & 13.1 \\
\hline \multicolumn{6}{|l|}{ Wyoming: } \\
\hline Cheyenne... & 295.3 & 9.78 & 3,640 & 1.26 & 12.3 \\
\hline Lander.... & 275.5 & 9.80 & 3,548 & 1.31 & 12.9 \\
\hline
\end{tabular}

It is seen at once that the values given in the first column of table 7 are much smaller than are the corresponding ones of table 6 . Livingston and Livingston made a study of the ratios obtained by dividing each exponential seasonal index by the corresponding remainder index, derived by using the constant difference of 39 , and the resulting ratio values are here reproduced in the third column of table 7 .

The chart of our plate 39 presents the climatic zonation exhibited by the summations of efficiency indices derived by the application of the exponential method (Van't Hoff-Arrhenius principle of chemical reaction velocity), and it is essentially the same as the second chart (fig. 2) of Livingston and Livingston.

In a general way, the two charts of plates 38 and 39 agree in the positions and directions of the isoclimatic lines, but they difier in a number of details. I somewhat thorough comparison of these two charts has been made hy Livingston and Livingston, using the ratio of the value of one index to that of the other (column 3 of our table 7 ). and they present a chart of these ratios as their third figure, which we do not here reproduce. As has been pointed out, these authors con- 
clude from their study of these two methods of estimating temperature effectiveness that the method that derives efficiency indices by subtracting 39 from each daily mean temperature index gives, "in a broadly general way, and for most of the area of the United States, nearly the same climatic zones" (loc. cit., p. 375) as those given by summations of temperature efficiencies based on the chemical coefficient of 2.0. Nevertheless, these authors point out that "the similarity between the results derived by these two methods of temperature integration is, however, only superficial and roughly approximate. The ratios of direct summation (above $39^{\circ} \mathrm{F}$.) to chemical efficiency summation, range in magnitude, for the mean frostless season in the United States, from a minimum of 7.49 to a maximum of 10.44." Their chart (fig. 3) shows clearly that these ratio values (column 3 of our table 7) are to be considered as some sort of climatic measure. The marginal regions of the United States are frequently characterized by low ratio values and the two main mountain systems seem to have high ratio values. For most of the area of the country the ratio of the summation index derived by the method of subtraction, to the index derived from the chemical coefficient, has a value of about 9.5 , and the assumption of this as a constant ratio between the two indices does not introduce very large errors for most of the area with which we are dealing.

The feature of these chemical efficiency indices that should attract our attention, however, is their relative values; according to the fundamental assumptions upon which these efficiency indices are based, these values should be proportional to the amounts of plant accomplishment within the frostless season, at the corresponding localities. Thus, referring to plate 40, if plant production in the region of Eastport, Maine, has a value of 300 for the average frostless season at that station, that in the vicinity of Jacksonville, Florida, should have a value of 1,000 for the frostless season there. The extreme range of this seasonal temperature efficiency, as shown by the chart of plate 39 and by table 7, column 2, is from 276 (Sault Ste. Marie, Michigan) to 1,538 (Key West, Florida), or from unity to about 5.6. By the remainder indices (plate 38 , table 6), the corresponding range is from 3,543 to 17,077 , or from unity to about 4.8 . It is thus brought out that, while one method of deriving efficiency indices would lead us to expect only 4.8 times as much plant activity at Key West as at Sault Ste. Marie, the other would lead us to expect this ratio to have the value 5.6. Since the physiological indices of temperature efficiency promise to be much more valuable in climatological study than either of the kinds of indices so far applied in our study, we need not here enter further into this comparison. 
(E) SUMMATIONS OF PHYSIOLOGICAL INDICES OF TEMPERATURE EFFICIENCY FOR PERIOD OF AVERAGE FROSTLESS SEASON. (TABLE 7. PLATE 40.)

The physiological indices here employed, as indeed the summations themselves, are reproduced from Livingston's paper $(1916,1)$ already cited. For each normal daily mean within the period of the average frostless season the corresponding physiological index was obtained from Livingston's tabulation (our table 5, Fahrenheit scale), and all the indices thus obtained were summed for each station considered. The seasonal physiological indices of temperature efficiency thus obtained are reproduced in column 4 of our table 7 . In the same table are also given the ratios of the physiological seasonal index to the corresponding remainder index (above $39^{\circ} \mathrm{F}$.; column 5) and to the exponential seasonal index (column 6).

The geographical distribution of the seasonal indices of temperature efficiency, physiologically derived, are shown on the chart of our plate 40 , the lines of which are reproduced from Livingston's paper $(1916,1)$. As that writer states, the general delineation of climatic zones is here much the same as in the case of the other two kinds of summations. The lines again show a general west-east trend, and are again displaced southward in the vicinity of the occans (especially on the west) and of the mountain systems. A cursory glance at these three charts of temperature efficiency summations for the period of the arerage frostless scason (plates 38, 39, and 40) shows them to be so generally similar that one might almost serve for either of the other two, as far as the forms of the various elimatic zones is concerned. Which method of derivation of the efficiency indices is used seems not to be of great importance in the general seasonal result. As far as present knowledge goes, then, one method appears to be as satisfactory as either of the others in this respect.

The authors of these methods have discussed some of the main features wherein these three charts differ in detail, and we do not need to enter deeply into this matter here; but the following points may receive brief mention. The actual values are much lower in the ense of the exponential indices (plate 39) than in either of the other cases (plates 40 and 41). Furthermore, the values obtained by the remainder method are generally, but not always, somewhat smaller than those derived from the Lehenbauer measurements for maize growth. It is not possible, however, to reduce the values of one of these three series to those of another, by employing any constant ratio, as is shown by the variations in each of the three sets of ratio values giren in table 7 . For convenience, we may represent the summation by the remainder or difference method (above $39^{\circ} \mathrm{F}$.) by $D$, that by the exponential or

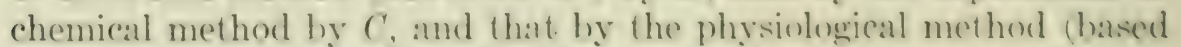
on growth of maize seedlings) by ( $\mathrm{f}$. We find (table 7 ) that the arer- 
age value of $D / C$ is 9.44 and that this value ranges from 7.49 to 10.44 . Similarly, the average value of $G / D$ is 1.60 , the range being from 0.65 to 2.20 , and the average value of $G / C$ is 15.0 , the range being from 4.8 to 20.0 . While the first ratio value $D / C$ shows a geographic variation that is not apparently related to temperature conditions (Livingston and Livingston, 1913), both of the other ratio values, $G / D$ and $G / C$, exhibit a variation that is obviously related to temperature, and the charts of these values (not here reproduced) appear very much alike and also very similar to the charts of the summations themselves, as far as the direction of zonation is concerned.

The main differences between these three summation charts, that require attention at the present time, have to do with the relative magnitudes of the summation or seasonal efficiencies indicated for the various stations. By the remainder method (table 6, plate 38) the seasonal temperature efficiency of southern Florida is about four times as great as that of middle New England. By the exponential method this ratio appears to be about 3.7 (table 7 , plate 39 ), and by the physiological method it is 6.1 (table 7 , plate 40 ). Which of these three ratios most nearly expresses the actual relation between the seasonal temperature efficiency for middle New England and that for southern Florida can not be determined without much more knowledge than is at present available. The ratios just given show clearly that the physiological method indicates a much greater range of seasonal temperature efficiency throughout the country than is indicated by either of the other methods, but whether this greater range is also represented by corresponding differences in plant growth must be left an open question for the present. As has been stated, we follow Livingston in deeming it highly probable, for various theoretical reasons, some of which have been expressed above, that the physiological method of obtaining efficiency summations for temperature will prove of more service than either of the others.

Attention should finally be called to the fact that the chart of our plate 40 brings out five zones or provinces of temperature efficiency for plant growth. These zones are somewhat similar to those shown on plate 34 , but the present chart is of course much less detailed.

(F) ABSOLUTE TEMPERATURE MAXIMA.

The absolute maximum temperatures as given in the Summary by Sections were placed upon a chart and isotherms were drawn for $100^{\circ}$ and $110^{\circ} \mathrm{F}$. Most of the area of the United States was thus shown to lie between these two lines. It is a remarkable fact, and one that emphasizes the importance of the duration factor in climatology, that there is, on the whole, so little variation between the highest temperatures on record throughout the country. The following list of stations and their highest observed temperatures (according to the Summary) are given here merely as an illustration of the fact just mentioned: 


\begin{tabular}{|c|c|}
\hline${ }^{n}$, Mich........ ${ }^{\circ}{ }_{103}$ & Vicksburg, Miss. \\
\hline g, Mich......... 98 & Natchez, Miss... \\
\hline ba, Mich. . & New Orleans, La. . \\
\hline Mont.... & Galveston, Tex. . \\
\hline Mont. . & Silver City, N. Mex... \\
\hline Chicago, Ill............ 103 & Tampa, Fla........... 10 \\
\hline Cairo, Ill.. & Miami, Fla. \\
\hline
\end{tabular}

The area characterized by maxima of less than $100^{\circ} \mathrm{F}$. comprises northwestern Washington and coastal points on the Pacific north of about the fortieth parallel of north latitude, central Idaho, western Montana and Wyoming, northern Minnesota, northwestern Wisconsin, and the entire northern peninsula of Michigan, some of the western and not so much of the eastern margin of the southern peninsula of the last-named State, eastern Ohio, the northern half of Pennsylvania, the Appalachian area south of about the thirty-eighth parallel, all of New York, the western half of New England, Atlantic coastal stations from southern Maine to North Carolina, the southern third of Florida, and a few coastal stations on the Gulf of Mexico. Besides the area thus described, there are several restricted areas of maxima below $100^{\circ}$, the main ones of which occupy the regions of the Sierra Nevada and of the Rocky Mountains. It is thus clear that only a very small portion of the United States has been characterized, during the periods of record, by maxima below $100^{\circ} \mathrm{F}$.

The area characterized by maxima of $110^{\circ}$ or above comprises localized sections, the main ones of which are as follows: (1) the southern third of California, the great Sacramento-San Joaquin Valley, and Arizona south of the great plateau; (2) western Texas and the Rio Grande region; (3) southwestern North Dakota, northwestern and southeastern South Dakota; (4) western Kansas, south central Nebraska and northwestern Oklahoma; (5) northeastern Arkansas, southeastern Missouri, southwestern Illinois, and a little of northeastern Missouri, and southeastern Iowa.

It seems clear that the variation in absolute maxima throughout the country is so slight that this criterion can be of no practical use for our present purpose. Of course, it is patent that the lengths of the periods of observation are very unequal for the different stations, and it seems probable that, with much longer periods, the absolute maxima will approach about $\mathbf{6} 10^{\circ} \mathrm{F}$. for approximately the whole country.

\section{(G) ABSOLUTE TEMPERATURE MINIMA. (PLATES 41 AND 42.)}

1 chart of the absolute minima of temperature (as these are given in the Summary by Feetions) was prepared in the same manner as was that of the absolute maxima. This chart brings out some rather definite climatie relations, and appears to be valuable for our purpose: it is therefore here reprodued as plate 41. The Fahrenheit intervals have been so chosen in the making of this chart that they correspond 


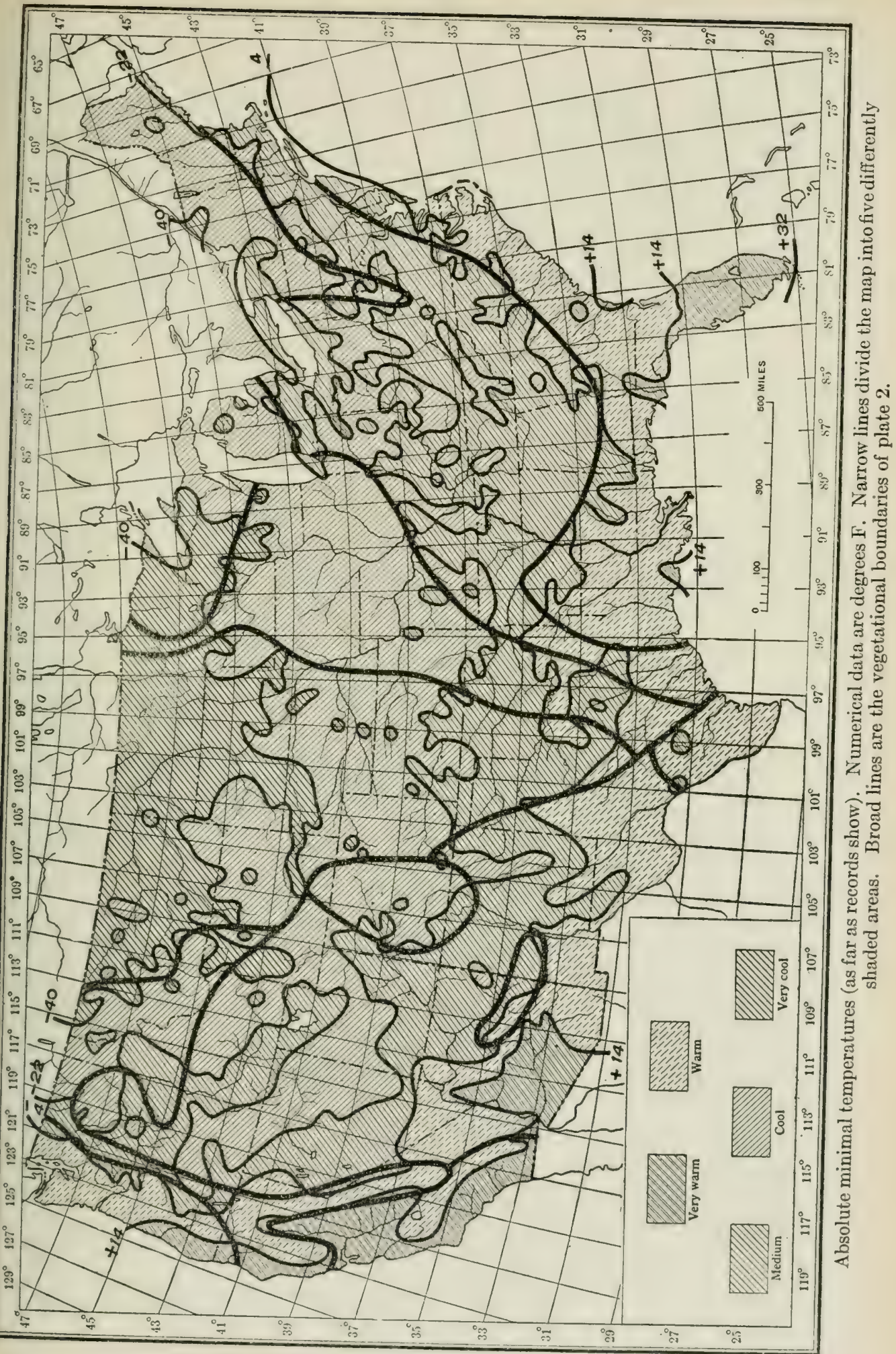




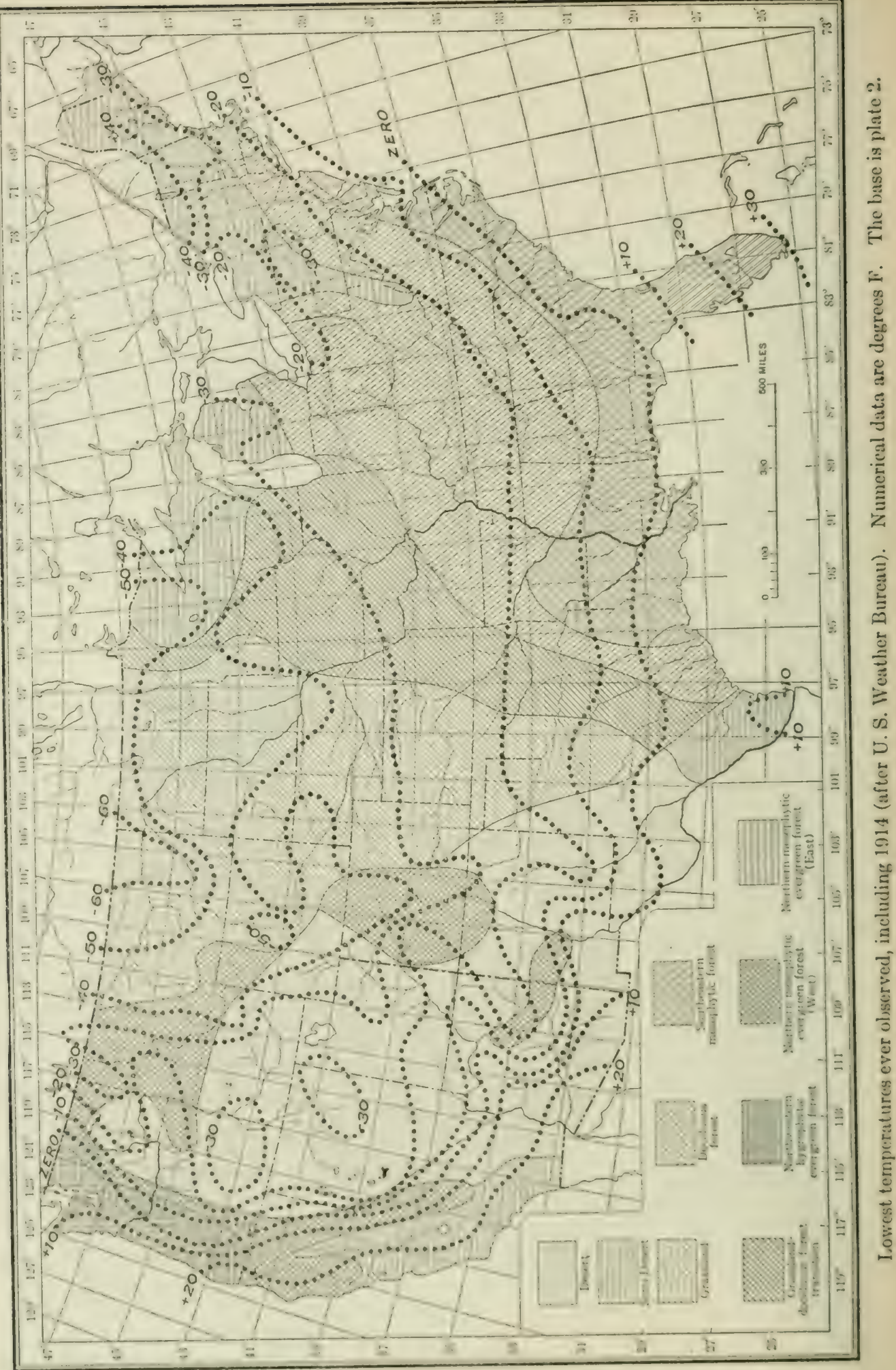


to centigrade intervals of $10^{\circ}$, between the extremes of $0^{\circ}$ and $-40^{\circ}$. Thus, there appear 5 lines, representing $32^{\circ} \mathrm{F} .\left(0^{\circ} \mathrm{C}.\right), 14^{\circ} \mathrm{F} .\left(-10^{\circ}\right.$ C. $), 4^{\circ}$ F. $\left(-20^{\circ}\right.$ C. $),-22^{\circ}$ F. $\left(-30^{\circ}\right.$ C. $)$ and $-40^{\circ}$ F. $\left(-40^{\circ}\right.$ C. $)$. No attempt has been made to smooth the lines; they represent, as nearly as possible, the actual data given in the Summary by Sections, the topography being also taken into account, as usual, in the placing of the lines. The five different zones are shown by different patterns in plate 41 . There is only one station with a value of over $32^{\circ} \mathrm{F}$. (Key West, Florida), so that no temperature province for values above $32^{\circ}$ is shown.

Plate 41 shows that the isoclimatic lines based on this criterion have generally the usual east-and-west trend of temperature lines. Here they are markedly displaced to the northward in the vicinity of either ocean. Southward displacement by the mountains is also more or less pronounced. The regions with absolute minima above $14^{\circ} \mathrm{F}$. contain most of the popular winter resorts.

The United States Weather Bureau chart showing the lowest temperature ever observed ${ }^{1}$ is here reproduced as plate 42 , for comparison with our plate 41 . The increments represented by the lines are each $10^{\circ} \mathrm{F}$. It is seen that this chart agrees with ours in its main points, but that its lines have been subjected to an effective smoothing, so that they are much more regular than those of plate 41 .

(H) AVERAGE DAILY NORMIAL TEMPERATURE FOR COLDEST 14 DAYS OF YEAR. (TABLE 8, PLATE 43.)

Since the absolute minima of temperature do not furnish an indication of the intensity of cold usually encountered at the various stations, it seems desirable to employ some normal temperature mean that may represent this. We have chosen for this purpose the average of the normal daily means for the 14 days having the lowest normal daily means, as given in Bulletin $\mathrm{R}$ of the United States Weather Bureau. It is to be noted that this 14-day period does not include the same days for the different stations, so that this climatic feature may be expected to be somewhat different from the mean temperature of some uniform period, such as the first two weeks of January, etc. The nature of the normal daily means of Bulletin $\mathrm{R}$ is such that it is impossible always to select 14 days as representing the lowest values. Thus, for Anniston, Alabama, the normal daily mean is $42^{\circ} \mathrm{F}$. for all days from December 27 to January 24 , and the average of any 14 of these 29 days remains $42^{\circ}$. In table 8 , which gives these averages, the first and last dates of the period considered are given for each station. Wherever the period includes more than 14 days the normal daily mean is constant (and the same as the average given) for the entire period. When a 14-day period includes several values of the normal daily mean, the day repre-

\footnotetext{
${ }^{1}$ U. S. Weather Bureau, Chart of lowest temperatures ever observed. (To and including 1914. - Lelter from Professor C. F. Marvin).
} 
senting (as nearly as possible) the middle of the shorter period having the minimum value is taken as the seventh or eighth day of the 14day period, by which method the position of this period in the calendar is determined closely enough.

The data given in the last column of table 8 are represented by the chart of plate 43 , on which the isotherms are shown for increments of $5^{\circ} \mathrm{F}$, from $0^{\circ}$ to $60^{\circ}$. The climatic zonation is here seen to be generally similar to that of the other temperature charts, but there are differences of detail.

TABLE 8.-Average normal daily temperalures for the coldest 14 days of the year. (Plate 43.) [The period is frequently more than $\mathbf{1 4}$ days in length. Where this is true the daily normal is constant throughout the period given.]

\begin{tabular}{|c|c|c|c|c|c|}
\hline Station. & Period. & Temp. & Station. & Period. & Temp. \\
\hline Alabama: & & ${ }^{\circ} \mathrm{F}$. & Illinois: & & ${ }^{\circ} \mathrm{F}$. \\
\hline Anniston & Dec. 27 to Jan. 24 & 42 & Cairo. & Dec. 30 to Feb. 3 & 35 \\
\hline Birmingham. & Jan. 1 to Jan. 22 & 45 & Chicago & Jan. 17 to Feb. 4 & 23 \\
\hline Mobile......... & Jan. 2 to Jan. 15 & 49 & La Salle. & Jan. 14 to Jan. 27 & 21 \\
\hline Montgomery.... & Dec. 30 to Jan. 14 & 47 & Peoria. & Jan, 3 to Feb. 2 & 23 \\
\hline Arizona: & & & Springfield. & Jan. 9 to Jan. 29 & 26 \\
\hline Flagstaff. & Dec. 28 to Jan. 14 & 26 & Indiana: & & \\
\hline Phoenix... & Dec. 31 to Jan. 13 & 50 & Evansville & Jan. 3 to Jan. 24 & 32 \\
\hline Yuma.... & Dec. 27 to Jan. 16 & 54 & Indianapolis & Jan. 4 to Jan. 28 & 28 \\
\hline Arkansas: & & & Iowa: & & \\
\hline Fort Smith. & Jan. 3 to Jan. 25 & 38 & Charles Cits & Jan. 7 to Jan. 26 & 11 \\
\hline Little Rock. . & Jan. 4 to Jan. 17 & 40 & Davenpo & Jan. 13 to Jan. 26 & 20 \\
\hline California: & & & Des Moines & Jan. 8 to Jan. 26 & 20 \\
\hline Eureka.. & Jan. 26 to Feb. 8 & 46 & Dubuqu & Jan. 5 to Jan. 26 & 18 \\
\hline Fresno.. & Dec. 24 to Jan. 20 & 45 & $\mathrm{Kec}$ & Jan. 10 to Jan. 23 & 23 \\
\hline Independen & Dec. 24 to Jan. 19 & 40 & Sioux City. & Jan. 11 to Jan. 24 & 15 \\
\hline Los Ängeles. & Jan. 4 to Feb. 5 & 53 & Kansas: & & \\
\hline $\operatorname{Red} \mathrm{B}$ & Dec. 31 to Jan. 13 & 45 & Concordia & Jan. 6 to Jan. 25 & 24 \\
\hline Sacrame & Dec. 21 to Jan. 17 & 45 & Dor & Jan. 2 to & 27 \\
\hline San I & Dec. 29 to Feb. 12 & 54 & To & Jan. 9 to Jan. 21 & 25 \\
\hline San Fra & Dec. 30 to Jan. 16 & 49 & Wi & Jan. 10 to Jan. 23 & 29 \\
\hline San Jo: & Dec. 28 to $J_{\text {an. }} 22$ & 48 & Kentucky: & & \\
\hline San Luis Obispo. & Dec. 29 to Jan. 31 & 51 & Lexington. & Dec. 31 to Jan. 31 & 33 \\
\hline Colorado: & & & ille. & Jan. 7 to Feb. 2 & 34 \\
\hline Denver. & Jan. 8 to Feb. 3 & 29 & Louisiana: & & \\
\hline Durango . & Jan. 1 to J & 24 & New Orlear & Dec. 23 to Jan. 27 & 53 \\
\hline unctic & Jan. 2 to Jan. 19 & 24 & Shreveport.. & Dec. 28 to Jan. 25 & 46 \\
\hline Pueblo.. & Jan. 3 to Jan. 31 & 29 & Maine: & & \\
\hline Connecticut: & & & Eastport. & b. 9 & 20 \\
\hline Hartford. . & Jan. 13 to $J_{\mathrm{an}} .29$ & 25 & Por & Jan. 18 to Jan. 31 & 22 \\
\hline New Haver & Jan. 10 to Feb. 7 & 27 & Maryland: & & \\
\hline Florida: & & & Baltimore. & Jan. 13 to Feb. 6 & 33 \\
\hline Jacksonville. & Dec. 19 to Jan. 28 & 54 & Washington, D. C. & Jan. 13 to Jan. 26 & 33 \\
\hline Jupi & Dec. $31 \mathrm{t}$ & 64 & Massachusetts: & & \\
\hline Key Wes & Dec. 22 to $\mathrm{J} t$ & 69 & Bos & Jan. 19 & 26 \\
\hline Pensa & Dec. 28 to & 52 & $\mathrm{Nn}$ & Jan. 4 to Feb. 15 & 32 \\
\hline Tamp & Jan. 1 to Jan. 20 & 57 & Michigan: & & \\
\hline Georgia: & & & Alpe & Jan. 27 & 17 \\
\hline Att & Jan. 1 to $J$ & 42 & Det & Jan. 23 & 23 \\
\hline Augt & Dec. 17 to & 46 & & Jnn. 12 & 14 \\
\hline $\mathbf{M r}$ & Dec. 29 & 45 & & Jan. 23 & 23 \\
\hline Sava & Dec. $21 t$ & 50 & Gri & Jan. 13 to J & 23 \\
\hline Thomasville. & Dec. 30 to Jan. 12 & 50 & Io & $J_{\mathrm{nn}}, 13$ to Feb. 3 & 14 \\
\hline Idaho: & & & $M_{\Omega}$ & Jan. 17 to Feb. 14 & 15 \\
\hline Boise. & Dec. $30 \mathrm{t}$ & 29 & Pot & Jan. 17 to Feb. 14 & 21 \\
\hline Lewiston & Jan. 11 to $J$ & 34 & Snult Ste. Maric & Jan. 23 to Feb. 7 & 12 \\
\hline \multirow[t]{2}{*}{ Pocatello......... } & Jan. 3 to Jan. 29 & 25 & Minnesn & & \\
\hline & & & & Jan. 10 to Feb. 1 & 10 \\
\hline
\end{tabular}


TABLE 8.-Average normal daily temperatures for the coldest 14 days of the year. (Plate 43.) -Cont'd.

\begin{tabular}{|c|c|c|c|c|c|}
\hline Station. & Period. & Temp. & Station. & Period. & Temp. \\
\hline Minnesota-Cont'd: & & ${ }^{\circ} \mathrm{F}$. & Pennsylvania: & & ${ }^{\circ} \mathrm{F}$. \\
\hline Moorhead.... & Jan. 13 to Jan. 26 & 2 & Erie. & Jan. 29 to Feb. 11 & 25 \\
\hline & Jan. 10 to Jan. 23 & 11 & Harrisburg. & Jan. 16 to Feb. 4 & 28 \\
\hline Mississippi: & & & Philadelphia. & Jan. 15 to Jan. 28 & 31 \\
\hline Meridian. & Jan. 3 to Jan. 16 & 45 & Pittsburgh... & Jan. 20 to Feb. 4 & 30 \\
\hline Vicksburg. & Jan. 5 to Jan. 18 & 47 & Scranton.. & Jan. 15 to Feb. 4 & 25 \\
\hline Missouri: & & & Rhode Island: & & \\
\hline Columbia.. & Jan. 8 to Feb. 1 & 27 & Block Island. & Jan. 26 to Feb. 8 & 30 \\
\hline Hannibal... & Jan. 13 to Jan. 26 & 26 & Providence.. & Jan. 6 to Feb. 3 & 27 \\
\hline Kansas City. & Jan. 6 to Jan. 29 & 26 & S. Carolina: & & \\
\hline St. Louis.... & Jan. 10 to Jan. 23 & 31 & Charleston.. & Jan. 7 to Jan. 27 & 49 \\
\hline Springfield.. & Jan. 8 to Jan. 29 & 31 & Columbia.... & Dec. 30 to Jan. 27 & 45 \\
\hline Montana: & & & South Dakota: & & \\
\hline Havre.. & Jan. 12 to Feb. 7 & 13 & Huron...... & Jan. 11 to Jan. 26 & 9 \\
\hline Helena.. & Jan. 5 to Feb. 5 & 20 & Pierre... & Jan. 4 to Feb. 3 & 14 \\
\hline Kalispell. & Jan. 6 to Jan. 19 & 19 & Rapid City.. & Jan. 12 to Jan. 27 & 21 \\
\hline Miles City. & Jan. 14 to Feb. 1 & 14 & Yankton .... & Jan. 16 to Jan. 29 & 14 \\
\hline Nebraska: & & & Tennessee: & & \\
\hline Lincoln... & Jan. 5 to Jan. 28 & 21 & Chattanooga. & Jan. 1 to Jan. 14 & 40 \\
\hline North Platte.. & Jan. 15 to Jan. 28 & 21 & Knoxville.. & Jan. 2 to Jan. 19 & 37 \\
\hline Omaha........ & Jan. 9 to Jan. 25 & 20 & Memphis... & Jan. 4 to Jan. 25 & 40 \\
\hline Valentine. . & Jan. 6 to Feb. 1 & 18 & Nashville... . & Jan. 8 to Jan. 21 & 38 \\
\hline Nevada: & & & Texas: & & \\
\hline Reno.. & Dec. 24 to Jan. 18 & 32 & Abilene. & Jan. 6 to Jan. 20 & 42 \\
\hline Winnemucca. & Jan. 1 to Jan. 14 & 28 & Amarillo.. & Jan. 7 to Jan. 20 & 34 \\
\hline N. Hampshire: & & & Corpus Christ & Jan. 3 to Jan. 19 & 53 \\
\hline Concord... & Jan. 7 to Jan. 31 & 21 & El Paso..... & Dec. 29 to Jan. 12 & 43 \\
\hline New Jersey: & & & Fort Worth. & Jan. 2 to Jan. 24 & 44 \\
\hline Atlantic City & Jan. 15 to Feb. 10 & 32 & Galveston... & Jan. 10 to Jan. 23 & 52 \\
\hline Cape May... & Jan. 28 to Feb. 11 & 33 & Palestine. & Dec. 31 to Jan. 20 & 46 \\
\hline New Mexico: & & & San Antonio. & Dec. 28 to Jan. 27 & 51 \\
\hline Roswell... & Dec. 30 to Jan. 26 & 39 & Taylor...... & Jan. 7 to Jan. 21 & 47 \\
\hline Santa Fe. & Dec. 29 to Jan. 19 & 28 & Utah: & & \\
\hline New York: & & & Modena. & Jan. 3 to Jan. 20 & 27 \\
\hline Albany... & Jan. 13 to Feb. 9 & 22 & Salt Lake Cit & Jan. 4 to Jan. 17 & 28 \\
\hline Binghamton. & Jan. 3 to Feb. 6 & 23 & Vermont: & & \\
\hline Buffalo..... & Jan. 29 to Feb. 12 & 23 & Burlington. . & Jan. 9 to Feb. 6 & 16 \\
\hline Canton.... & Jan. 8 to Feb. 7 & 16 & Northfield.. & Jan. 7 to Feb. 4 & 15 \\
\hline Ithaca.. & Jan. 3 to Feb. 10 & 24 & Virginia: & & \\
\hline New York. & Jan. 6 to Feb. 15 & 30 & Cape Henry. & Jan. 8 to Feb. 9 & 40 \\
\hline Oswego..... & Jan. 22 to Feb. 14 & 23 & Lynchburg... & Jan. 10 to Jan. 23 & 36 \\
\hline Rochester.. & Jan. 24 to Feb. 12 & 23 & Norfolk. & Jan. 13 to Feb. 2 & 40 \\
\hline Syracuse. . & Jan. 22 to Feb. 4 & 22 & Richmond. & Jan. 1 to Feb. 3 & 38 \\
\hline N. Carolina: & & & Wytheville. & Dec. 29 to Feb 3 & 33 \\
\hline Asheville.. & Jan. 3 to Jan. 20 & 35 & Washington: & & \\
\hline Charlotte. & Dec. 29 to Jan. 21 & 40 & North Head. & Jan. 1 to Feb. 24 & 42 \\
\hline Hatteras. . & Jan. 14 to Jan. 27 & 46 & Port Crescent & Dec. 31 to Feb. 5 & 36 \\
\hline Raleigh.. & Dec. 30 to Jan. 19 & 40 & Seattle. & Jan. 5 to Jan. 25 & 39 \\
\hline Wilmington.. & Jan. 7 to Jan. 20 & 45 & Spokane. & Jan. 10 to Jan. 23 & 26 \\
\hline North Dakota: & & & Tacoma & Jan. 1 to Jan. 28 & 38 \\
\hline Bismarck... & Jan. 20 to Feb. 2 & 6 & Tatoosh Islar & Jan. 11 to Feb. 27 & 41 \\
\hline Devils Lake. & Jan. 7 to Jan. 27 & 0 & Walla Walla. & Jan. 1 to Jan. 25 & 33 \\
\hline Williston. & Jan. 11 to Feb. 10 & 6 & West Virginia: & & \\
\hline Ohio: & & & Elkins.. & Jan. 2 to Jan. 31 & 29 \\
\hline Cincinnati. & Jan. 10 to Feb. 3 & 32 & Parkersburg. & Jan. 7 to Jan. 27 & 31 \\
\hline Cleveland & Jan. 21 to Feb. 3 & 26 & Wisconsin: & & \\
\hline Columbus. & Jan. 11 to Jan. 24 & 28 & Green Bay. & Jan. 13 to Jan. 26 & 14 \\
\hline Sandusky & Jan. 10 to Feb. 13 & 26 & La Crosse. & Jan. 7 to Feb. 1 & 15 \\
\hline Toledo. . & Jan. 15 to Feb. 6 & 25 & Madison & Jan. 9 to Jan. 26 & 16 \\
\hline Oklahoma: & & & Milwaukee. & Jan. 13 to Jan. 26 & 19 \\
\hline Oklahoma & Jan. 8 to Jan. 21 & 34 & Wyoming: & & \\
\hline Oregon: & & & Cheyenne. & Jan. 14 to Feb. 9 & 25 \\
\hline Baker City & Jan. 4 to Jan. 17 & 24 & Lander.... & Dec. 26 to Jan. 20 & 17 \\
\hline Portland. & Jan. 2 to Jan. 29 & 39 & & & \\
\hline Roseburg.. & Dec. 29 to Jan. 11 & 41 & & & \\
\hline
\end{tabular}




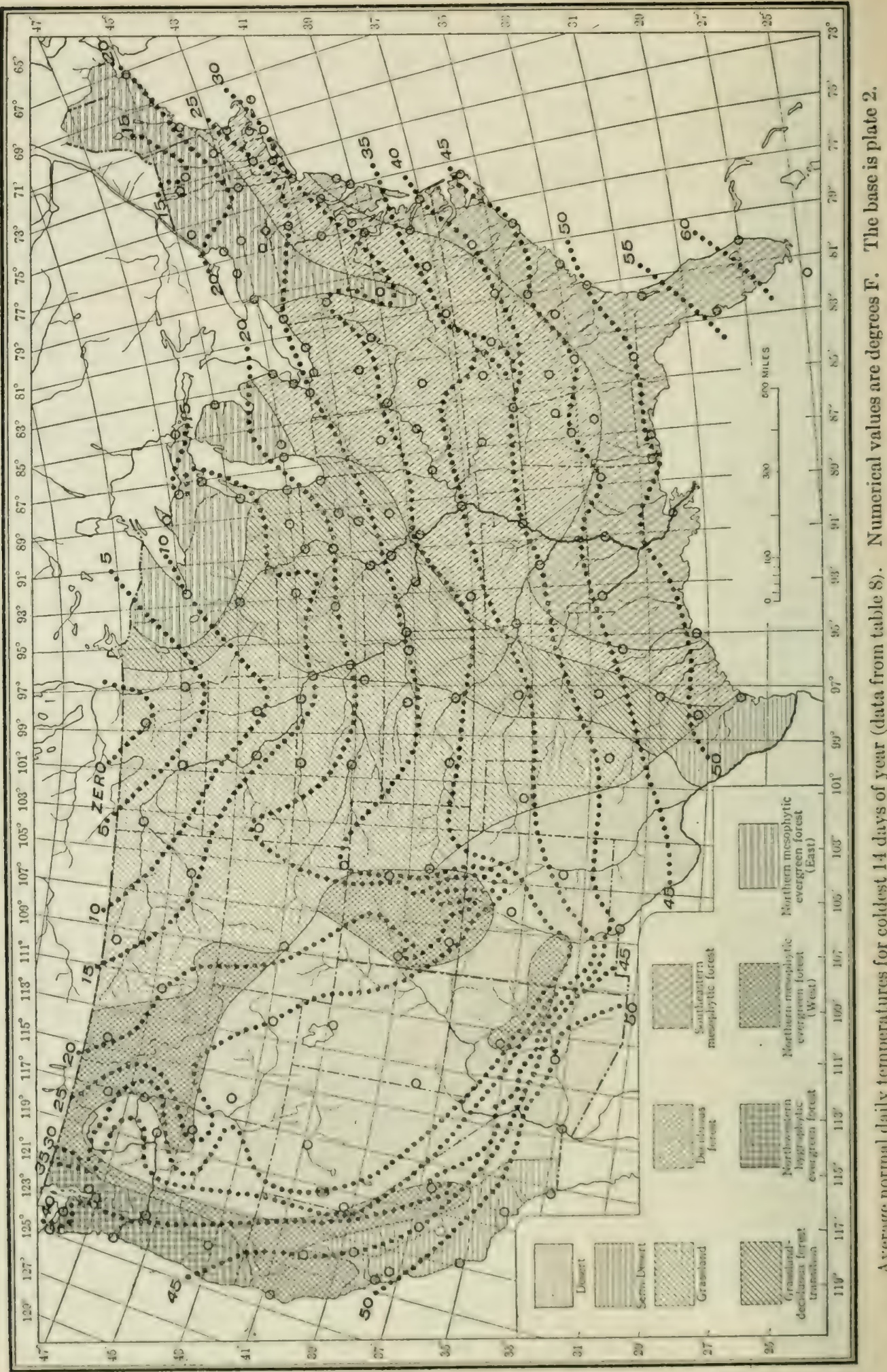




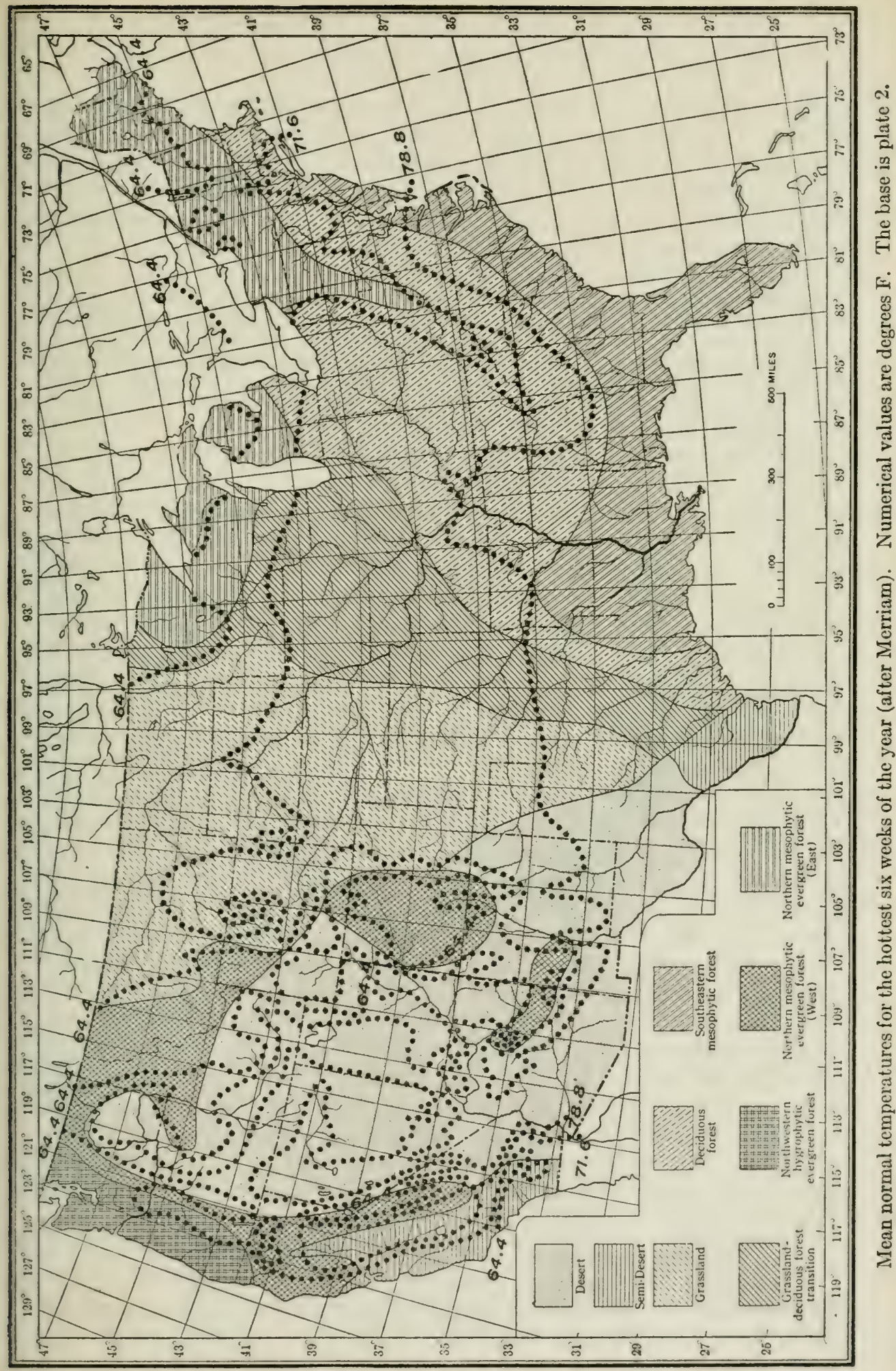


(I) MERRIAM'S MEAN NORMAL TEMPERATLRE FOR HOTTEST SIX WEEKS OF YEAR. (PLATE 44.)

In the same paper (1894) from which we have already made extracts, Merriam calls attention to the fact that, while his summation indices (our plate 37) appear to furnish satisfactory criteria for relating temperature conditions to the northward limits of species distribution, yet these do not seem at all satisfactory in connection with the southward extension of northern forms. This author writes $(1894$, p. 233):

It is evident $* * *$ that the southward range of Boreal species $* * *$ is regulated by some cause other than the total quantity of heat [i.e., his summation indices]. This cause was believed to be the mean temperature of the hottest part of the year, for it is reasonable to suppose that Boreal species in ranging southward will encounter, sooner or later, a degree of heat they are unable to endure. ${ }^{* * *}$ For experimental purposes, and without attempting unnecessary refinement, the mean normal temperature of the 6 hottest consecutive weeks of summer was arbitrarily chosen and platted on a large contour map of the United States, as in the case of the total quantity of heat.

We here reproduce in its essentials, as our plate 44 , the chart thus obtained-Merriam's (1894) plate 13- because of its scarcity and of its interest in connection with our own studies. The marked differences between this chart and that of our plate 37 (also reproduced from Merriam) are practically confined to the Pacific Slope. East of the Sierra Nevada, Cascade, and San Bernardino Ranges the zone with a normal for the hottest 6 weeks of above $79^{\circ} \mathrm{F}$. $\left(26^{\circ} \mathrm{C}\right.$.) corresponds well with that of the Merriam summation above 18,000 (F.) or 10,000 (C.); the zone characterized by a 6 -weeks normal of from $72^{\circ} \mathrm{F} .\left(22^{\circ}\right.$ C.) to $79^{\circ} \mathrm{F} .\left(26^{\circ} \mathrm{C}\right.$.) corresponds with that having a summation of

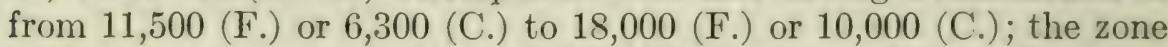
with a 6 -weeks normal of from $64^{\circ} \mathrm{F}$. $\left(18^{\circ} \mathrm{C}\right.$.) to $72^{\circ} \mathrm{F}$. $\left(22^{\circ} \mathrm{C}\right.$.) corresponds to that with a summation from 10,000 (F.) or 5,500 (C.), to 11,500 (F.) or 6,400 (C.) ; and a similar correspondence is noted between the zone having a 6 -weeks normal below $64^{\circ} \mathrm{F}$. $\left(18^{\circ} \mathrm{C}\right.$.) and that with a summation of less than 10,000 (F.) or 5,500 (C.). On the Pacific Slope, however, no such series of comparisons can be instituted. While the coldest zone of the summation chart does not appear at all on the Pacific Slope of the United States, the zone of the 6-weeks normals, which corresponds to this elsewhere, occupies the whole coast as far south as Los Angeles. Furthermore, the next to the coldest zone of normals extends much farther westward and southward in the region under discussion than does the corresponding zone of summations; the former occupies the coastal area west of the san Bernardino and San Jacinto Mountains, south of Los Angeles. Merriam has drawn important conclusions from these differences, bearing upon the delimitation of his life-zones, a matter which will receive some attention in Part III of the present publieation.

(J) NORMAL MEAN ANNUAL TEMPERATURE. (PLATE 45.)

The normal mean ammal temperature is eommonly employed by climatologists for comparing climatie temperature intensities, and it 


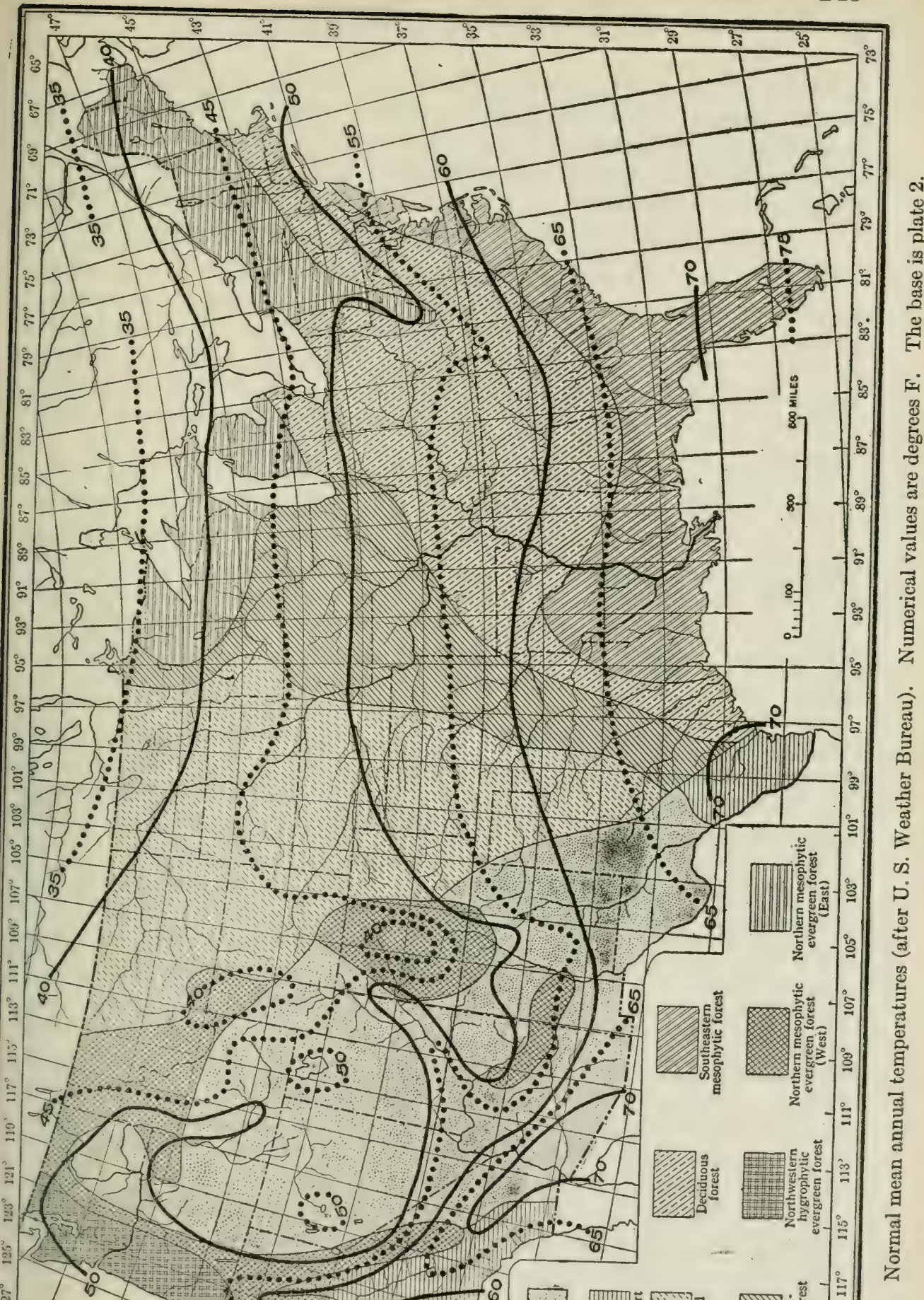


is added to our list of temperature features for this reason more than for any other. Our chart for this (plate 45) is reproduced from that of the United States Weather Bureau. ${ }^{1}$ It requires no special comment, excepting that we have represented four of the lines as full, so as to bring out the general temperature zonation according to this criterion.

3. CONCLUSIONS FROM THE STUDY OF TEMPERATURE CONDITIONS.

The most obvious generalization to be drawn from our temperature studies, as represented by plates 34 to 45 , is patent to everyone, namely, that the temperature zonation of the United States has a predominantly west-east direction. Latitude is of course the controlling geographical feature that brings this about, and the values of the various forms of temperature indices increase toward the south and decrease toward the north.

Modifying features are the mountain systems and the oceans. The isoclimatic lines bend northward near the Atlantic and Pacific coasts. Also, they generally bend southward on either side of each of the three main mountain systems.

On plate 34 the different patterns represent the country as divided into 5 climatic zones or provinces, accolding to temperature conditions, and Merriam's chart for summation indices above $32^{\circ} \mathrm{F}$. (our plate 37 ) shows a similar convention. Of course, any number of zones might be considered, but it is perhaps most useful to follow Merriam in this matter if a few definite zones are required. These 5 temperature provinces do not, however, need to be given names of the sort used by the author just mentioned, and if special names were requisite they should be climatically descriptive; they should of course not be named after geographic areas. We therefore suggest, in this connection, that a 5-zonal arrangement for temperature conditions will probably prove satisfactory, these being subdivisions of the larger temperate zone of geographers, and that these 5 temperature provinces of the United States may be termed simply and directly: very warm, warm, medium, cool, and very cool. It might be as well for scientific purposes to number these provinces serially, but such a procedure would not be satisfactory in non-technical discussions. The simple, descriptive terminology here suggested is clearly understood by everyone, while such terms as upper and lower Austral (Nerriam) apparently fail to be understood by many who actually employ them. It should also be noted that the area of the United States does not include all of the north temperate zone, and our suggested terms leave opportunity for other subdivisions lying north and south of our group of 5. Thus, south of the very warm temperate temperature province may be one called hot and still another called very hot, while north of the very cool temperate province may be two more temperate subdivisions, the cold and the very cold.

\footnotetext{
${ }^{1}$ U. S. Weather Bureau, Chart of normal annual temperature. (To and including 1914.
} Leller from Professor C. $F^{\prime}$. MAarvin.) 
Probably the most generally useful of our charts of the temperature conditions is the one for the length of the period of the average frostless season, and the five temperature provinces just mentioned are indicated on that chart (plate 34). In terms of that particular temperature index, the relation between our simple names and the index values is shown in table 9 . For other temperature indices the values would

TABLE 9.

\begin{tabular}{|c|c|}
\hline $\begin{array}{l}\text { Temperature prov- } \\
\text { inces of temperate } \\
\text { zone in United } \\
\text { States. }\end{array}$ & $\begin{array}{l}\text { Length of } \\
\text { period of } \\
\text { average } \\
\text { frostless } \\
\text { season. }\end{array}$ \\
\hline 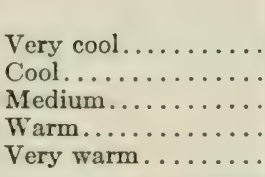 & $\begin{array}{l}\text { days. } \\
\text { Below } 120 \\
120 \text { to } 180 \\
180 \text { to } 240 \\
240 \text { to } 300 \\
\text { Above } 300\end{array}$ \\
\hline
\end{tabular}

of course be entirely different, but the general zonation for these other indices may be generally comparable to that of plate 34 , if proper limiting values are chosen. In the case of the temperature summation indices obtained by the physiological method (plate 40 and fig. 1), for example, the zones noted in table 10 , roughly comparable to those

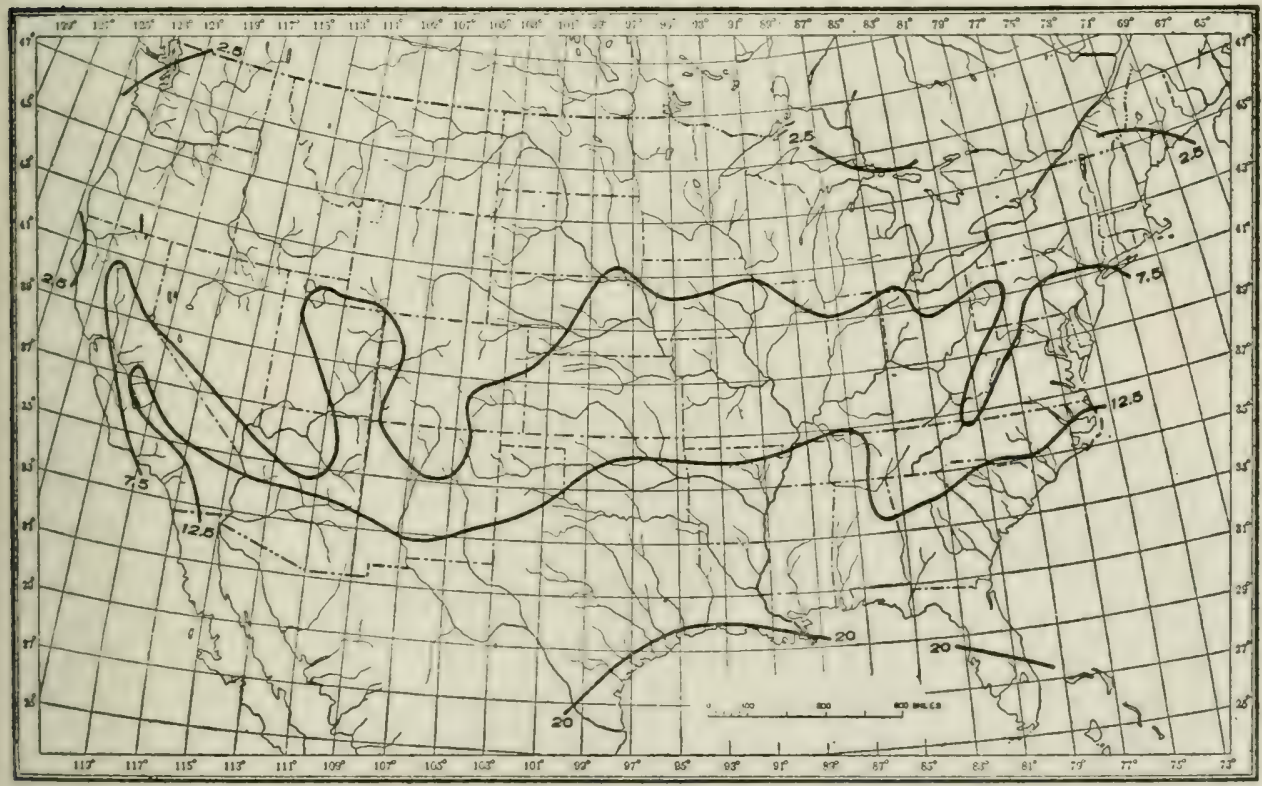

F1G. 1.-Temperature zonation, according to physiological summations for period of average frostless season. Temperature efficiency provinces: very warm, more than 20 ; warm, 12.5 to 20 ; medium, 7.5 to 12.5 ; cool, 2.5 to 7.5 ; uery cool, less than 2.5 . Numerical values represent thousands. (See also plate 40.$)$ 
shown on plate 34 , may be distinguished. These temperature provinces are shown on figure 1 , reproduced from plate 40 , for ready reference here.

TABle 10.

\begin{tabular}{|c|c|}
\hline $\begin{array}{l}\text { Temperature prov- } \\
\text { inces of temperate } \\
\text { zone in United } \\
\text { States. }\end{array}$ & $\begin{array}{l}\text { Physiological } \\
\text { summation in- } \\
\text { dices of tem- } \\
\text { perature effi- } \\
\text { ciency for plant } \\
\text { growth. }\end{array}$ \\
\hline 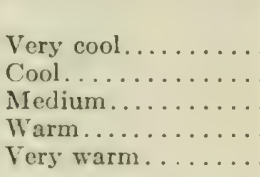 & $\begin{array}{l}\text { thousands. } \\
\text { Below } 2.5 \\
2.5 \text { to } 7.5 \\
7.5 \text { to } 12.5 \\
12.5 \text { to } 20 \\
\text { Above } 20\end{array}$ \\
\hline
\end{tabular}

Of course, it is not expected that any two charts, based upon different forms of climatic indices, will agree as to details. Thus, the Pacific coastal region is seen to be mostly included in the cool province on plate 40 , or figure 1, while it lies mostly within the medium prorince on plate 34. What particular form of temperature index, or what combination of such indices, will be found most valuable in distinguishing the climatic zones for studies of dynamic plant geography remains to be determined, but it may be safely predicted that no single form of index will be sufficient for the purpose of ecological and agricultural climatology. It is unfortunate that Merriam's zones (our plate 37) and the unsatisfactory terminology that goes with them should have been allowed to become stereotyped; climatic temperature conditions have many dimensions and the useful comparison of climates requires the employment of many more than a single one of these.

\section{MOISTURE CONDITIONS.}

\section{INTRODUCTORY.}

As has been emphasized carlier in the present part (page 120), the moisture condition immediately effective to control plant activity is the water-content of the particular cells and tissues involved, and if it were possible to study the duration and intensity aspects of this condition such a study ought to be fundamental for the ecological relations with which we have to deal. Is in the case of the temperature relation, howerer, it is impossible to make any progress at present by attacking the problem in this idcally logical mamer; here also it is necessary to consider less inmediate conditions and to pass to what is considered as the external enviromment, without even attempting at present to inqt ire, in more than a very siperficial way, concerning the nature of the internal water-relations which directly determine plant phenomena. Our analysis of the mattor before is proceeds somewhat as follows: 
Vital activity is influenced by internal moisture-conditions that mainly remain to be studied by physiological science. We are sure that these internal conditions are largely dependent upon external water-relations, and our task is to find ways of measuring and defining the latter as they exist in nature, in such a way as to render our description of the moisture conditions of the environment valuable to those interested in geographical distribution. It has been already noted that such a procedure is rather simple in the case of the temperature-relation, for the immediate and internal temperature conditions effective in the control of plant activity are closely paralleled at all times by the more remote conditions of the environmental temperature; there is usually no great lag between the march of the external, or ecological, and that of the internal, or physiological, temperature conditions. This is, of course, simple because heat migrates with comparative readiness either into or away from the plant and hence equilibrium in this regard, between plants and their surroundings, is seldom very far from being attained. Similarly, the environmental moisture conditions are also effective to control the immediate, internal moisture conditions, through the relative rates of the entrance and exit of water. In this case we have to deal with material instead of energy, but the general relations are the same. Therefore, it is with those conditions of the environment that may influence the rates of entrance and exit of water that the present section has to deal. A plant may suffer from lack of water, (1) because of too slow a rate of entrance of this substance into its body during some previous time period, (2) because of too rapid a rate of exit, or (3) because these two conditions have been simultaneously effective. The environmental conditions to be here considered for the area of the United States will be presented under the two captions, the supply of water to the plant, and the removal of water from the plant.

\section{SUPPLY OF WATER TO PLANT.}

(A) PRELIMINARY CONSIDERATIONS.

A discussion of the power of the surroundings to supply water to plants should begin (for ordinary plants) with the power of the soil to supply moisture to roots; it makes no difference, for this primary inquiry, what conditions may determine this power, for the only thing directly affecting the plant in this connection is this power itself. ${ }^{1}$ Since, however, ways and means for comparing the water-supplying power of the soil at various times and places are still to be perfected,

\footnotetext{
${ }^{1}$ Livingston has emphasized the crying need for methods of measuring and comparing the powers of soil to supply moisture to unit absorbing surface, and he and his co-workers have suggested three methods for the quantitative measurement of this power, all of which appear promising in this direction, but little has yet been done of a positive character. See, in this connection, the following papers: Livingston, 1906, b.-Idem, 1909.-Idem, 1912, b.-Livingston and Hawkins, 1915.-Pulling and Livingston, 1915.
} 
it is obviously impossible to deal here with this fundamental factor. We turn, therefore, to the conditions next in order of remoteness from the plant itself, and recognize at once that the water-supplying power of the soil is determined by its water-content and its physical make-up. Charts can not yet be made, however, to represent the mean watercontent of the soil throughout any considerable area, and the charts now in existence, ${ }^{1}$ of physical soil properties, can be of no quantitative value in such discussions as the present, until the corresponding moisture-contents (with their seasonal fluctuations) may be similarly represented. Our inquiry is thus forced back once more to a consideration of the factors determining the soil-moisture content. These factors are (1) precipitation, (2) superficial supply by overflow, superficial drainage, and subterranean supply and run-off, and (3) removal of water from the soil by plant-absorption and by direct evaporation.

In the first of these tertiary conditions influencing the supply of moisture to vegetation we have, finally, a well-recognized climatic factor that has been measured and recorded, in a way, for many years throughout the area of the United States. It is impossible at the present time, however, to make any quantitatively comparative use of what little information is at hand regarding the second set of factors just mentioned $;^{2}$ this information is still far too general and qualitative to be of service in an inquiry such as the present. With the third set of conditions above mentioned (plant absorption and direct evaporation) we shall have to deal in the following subsection, for the same environmental conditions that control the removal of water from the plant are effective to determine plant absorption-in a great measure, at least - and loss of soil-moisture by evaporation into the air.

While precipitation is thus clearly seen to be in no sense a direct or immediate condition influencing water-supply to plants, it is very frequently a condition that may be roughly related to plant activity, as is well recognized by everyone; the mean annual rainfall of a given area has long been regarded as of great value in estimating the possibility of plant growth in such an area. ${ }^{3}$

As in the case of temperature, precipitation and evaporation should be considered as they affect plants, rather than as they affect any given

\footnotetext{
${ }^{1}$ See, in this connection, the numerous soil surveys of the Bureau of Soils of the U. S. Department of Agriculture.

2 The reader interested in underground waters will find numerous bits of still unrelated information in the series of Water Supply and Irrigation Papers published by the U. S. Geological Survey. Especially interesting is also the following paper: McGee, W J, Wells and subsoil water, U. S. Dept. Arric., Bur. Soils Bull. 92, 1913.

${ }^{3}$ Of course it is obvious enough that this proposition holds only with certnin restrictions, as, for example, where the subterranean water-table is considerably below the soil surface. 'Thus, the cat-tail ( $T y p h a)$ or tule swamps in the vicinity of springs in the Salton 13asin of California have the same ecological aspect as have similar marshes near the Atlantic seaboard, though a comparison of the precipitation data for these two regions utterly" fails to show any reasons for expecting such similarity. The sand-dunes of the Salton Basin and those of the Lake Michigan shores, on the other hand, show differences in vegetational aspect which may clearly be related to differences in rainfall between these portions of the continent.
} 
rain-gage or atmometer tank, but these conditions have just begun to attract attention in connection with the quantitatively dynamic aspect of the study of plant activities, and we are not able to go nearly so far with their treatment as is possible with temperature. Work such as that of Köppen and of Lehenbauer for temperature influence upon plants is greatly needed for the corresponding influence of the moisture conditions, but this sort of work has not yet been attempted, even if it may have occurred to anyone. When such work is accomplished (which will be possible only with good equipment for the general control of environmental conditions), then it will be time to consider efficiency indices of the moisture conditions in somewhat the same way as we have attempted to deal with the suggested indices of temperature efficiency.

(B) PRECIPITATION.

(1) INTroductory.

Since rainfall is so remote from being the immediate environmental condition controlling the water-supply to plants in nature, the measurement of this climatic condition must not be expected to show very definite relations to plant activity or distribution. As has been indicated, we employ rainfall data not because they are desirable, but because they are the nearest approach to what is desirable that the present state of our knowledge affords. In this case, as in that of temperature, we usually employ the length of the average frostless season as our duration factor. Since the effect of precipitation is markedly cumulative, we have also tentatively established a second annual period, which may prove to be more satisfactory for this condition than is the length of the average frostless season. This period is obtained by adding to the average frostless season, at its beginning, a period of 30 days. By this scheme the rain falling during the last 30 days of the frost season is considered as pertaining to the following frostless season. Thus the snow and rain of March is frequently very influential in determining the kind of plant growth that can occur in the following month, especially if the latter is comparatively without precipitation. The length of this added period is taken as 30 days quite gratuitously; perhaps it should be longer or shorter and it probably should have different lengths, according to other climatic conditions, for different localities. At any rate, it has seemed desirable to make test of this modification. In the discussions that follow we shall let $P$ represent the normal total precipitation for the period of the average frostless season, while $\pi$ will represent the corresponding normal total precipitation for the longer period just described.

It is obviously not to the point at all to employ the summed precipitation for a portion of the year as a measure of the water-supplying power of the environment available for plant growth during that period. Rather is it requisite to study the average water-supplying 
power of the surroundings throughout the period in question. A usable index of this is obtained by dividing the quantity $P$ or $\pi$ by the number of days in the average frostless season. This procedure is logically no better here than the average temperature for the frostless season would be in the case of temperature relations, but, as has been said, lack of knowledge prevents as logical a treatment of the moisturerelation as is now possible in this aspect of the other case.

Rainfall is universally measured in terms of depth units, which denote volume or weight units per unit of horizontal surface. The position of the horizontal surface of reference is assumed to be at the level of the soil surface. Raising this surface a few meters above the soil has no considerable influence upon the readings in most regions, though it would be undesirable to place the rain-gage funnels at any very great distance above the ground in an arid country. In such a country a considerable amount of rain might frequently be recorded on a gage supported a few hundred meters above the ground, while a gage directly beneath, at the ground-level, might remain quite dry; the rain-drops often evaporate as they descend. Of course, the opposite is sometimes true in a very moist region, where the drops may increase in size as they fall. Our precipitation data are in terms of inches of depth, since inches are still employed in the tables of the United States Weather Bureau publications, from which we derive our original values. All such data may of course be readily converted into metric values, where more universally comparable numbers are desired.

As a basis for our computations we have again had recourse to Bigelow's tables of normal daily values (Bulletin $\mathrm{R}$ of the $\mathrm{U}$. S. Weather Bureau). These tables present the results of an elaborate treatment of the observation data in the United States, resulting in a precipitation value for each day in the year for each station considered. Thus the normal precipitation is given for each day of the year and for each station in the list. If a "normal" year, in this sense, erer occurred, then the actual precipitation for each day in the year, for any station, would be the value given in Bigelow's table. We have treated these normal daily precipitation values in somewhat the same manner as was followed in handling the normal daily means of temperature given by Bigelow in the same publication. By the use of these tables it is possible to study the comparative lengths of what may be called normal drought periods and normal rainy periods, as will be brought out below. All of our computations involve both duration and intensity factors, as will also appear in the discussions that follow.

(2) Nonmal Mean Darly Prechptation for Period of Average Frostless

$$
\text { Season }\left(\frac{P}{S}\right) \text {. (Table 11, Plate 46, and Fig. 2.) }
$$

The mean daily rainfall for the period of the frostless season should be a general measure of aridity in a certain sense, and we have obtained 


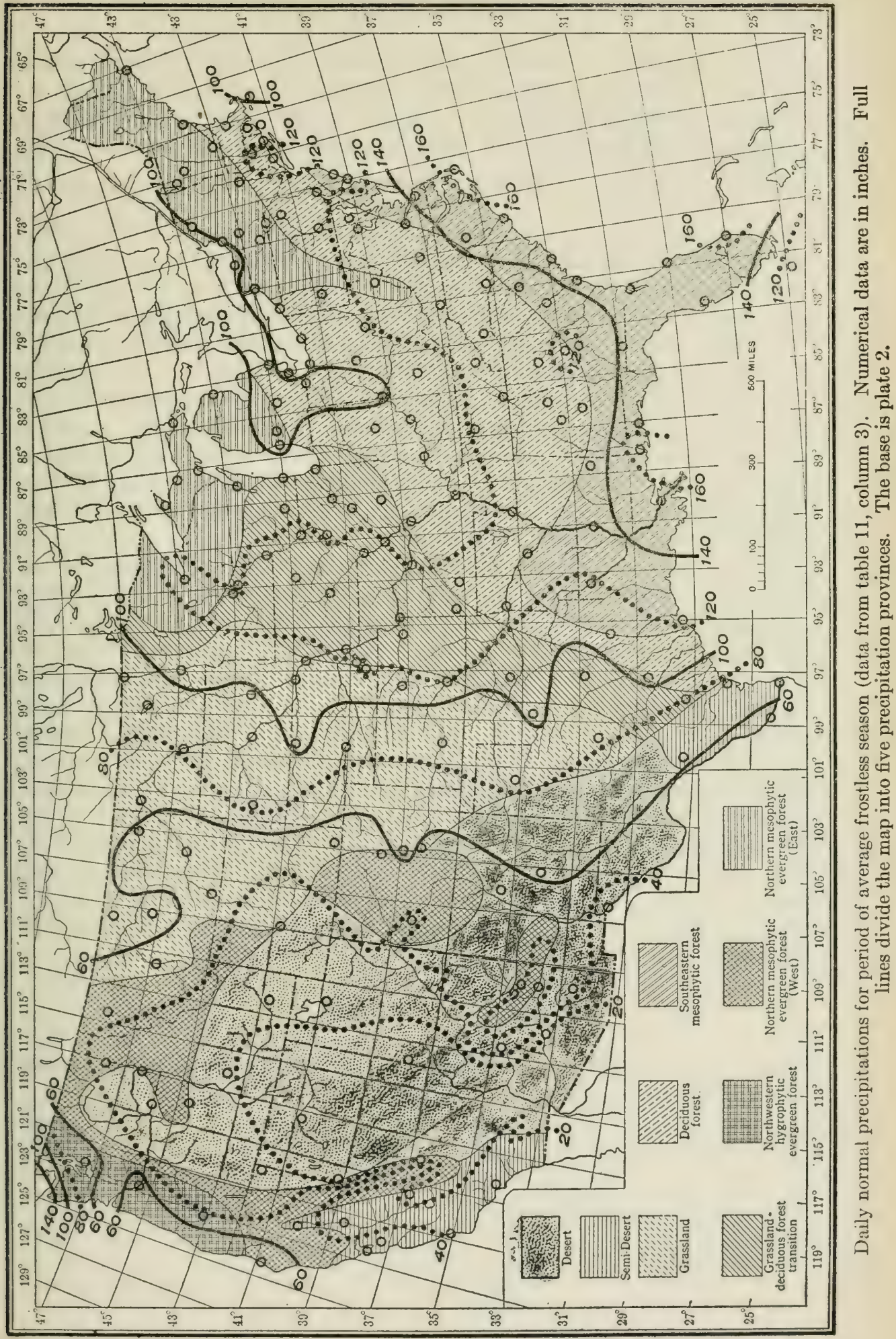


indices for this by summing the normal daily precipitation values within the period of the average frostless season (Bulletin R, U.S. Weather Bureau) and then dividing the result by the number of days represented in the period. These averages may be represented by the symbol $\frac{P}{S}$, where $P$ is the total rainfall for the average frostless period and $S$ is the number of days in that period. The values of this ratio, for the stations considered, are given in the third column of table 11, the values of $P$ being placed in the second column. The precipitation data for a few of the stations listed are not from Bulletin R: they are either from Bulletin Q (in which case the station name is followed by an $\mathrm{H}$ in parentheses), or they are from the Summary by Sections (in which case the name is followed by an $\mathrm{S}$ and the number of the section of the Summary in which it is listed, in parentheses). Table 11 also includes evaporation data, and where two stations are given for the same data, the first (not in parentheses) is the one to which the precipitation data refer. The second one (in parentheses) is the one referred to by the evaporation data.

Where the precipitation data are not derived from Bulletin $\mathrm{R}$ (those marked $\mathrm{H}$ or $\mathrm{S}$ ), the total precipitation for the period of the average frostless season was approximated by calculation from the normal monthly precipitation data as given by Henry (Bulletin Q) or in the Summary by Sections. To accomplish this approximation the monthly normals for all whole months included in the period of the average frostless season were added together, and to this sum were added a fractional part of the next preceding and of the next following monthly normal, these fractional parts being, in each case, that part of the value for the whole month that is represented by the number of days of that month included in the frostless season. Thus, if the average frostless season extends from June 5 to September 6 and if the monthly normal precipitation values for the months involved are $a, b, c$, and $d$, then the approximate total precipitation for the period of the frostless season is

$$
\frac{25}{30} a+b+c+\frac{6}{30} d=P
$$

Here $S$, the number of days in the average frostless season is $25+31+$ $31+6=93$, and

$$
\frac{P}{S}=\frac{5 / 6 a+b+c+1 / 5 d}{93}
$$


TABLE 11.-Precipitation and evaporation data for the period of the average frostless season. (Plates 46, 57, and 58.)

\begin{tabular}{|c|c|c|c|c|c|c|c|c|}
\hline \multirow[b]{2}{*}{ Station. } & \multirow{2}{*}{ 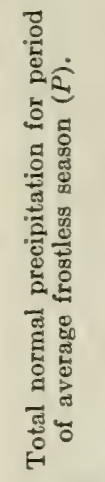 } & \multirow{2}{*}{ 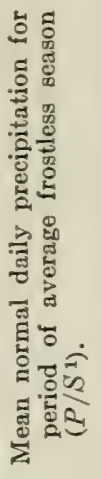 } & \multirow{2}{*}{ 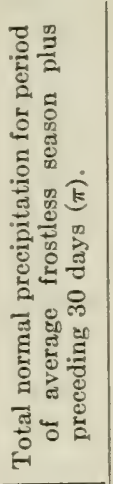 } & \multirow[b]{2}{*}{$\frac{\pi^{1}}{S}$} & \multirow{2}{*}{ 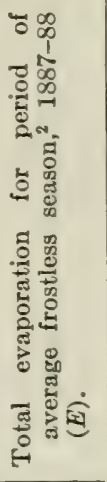 } & \multirow{2}{*}{ 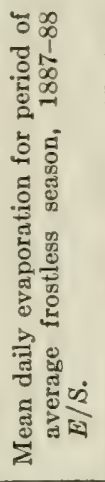 } & \multicolumn{2}{|c|}{$\begin{array}{l}\text { Moisture ratios } \\
\text { for period of } \\
\text { average frostless } \\
\text { season, } P / E \text {. }\end{array}$} \\
\hline & & & & & & & $P / E$ & $\pi / E$ \\
\hline $\begin{array}{l}\text { Alabama: } \\
\text { Anniston. }\end{array}$ & $\begin{array}{l}\text { inches. } \\
24.43\end{array}$ & $\begin{array}{l}\text { inches. } \\
0.122\end{array}$ & $\begin{array}{l}\text { inches. } \\
30.11\end{array}$ & $\begin{array}{l}\text { inch. } \\
0.150\end{array}$ & $\begin{array}{l}\text { inches. } \\
\text {........ }\end{array}$ & $\begin{array}{l}\text { inches. } \\
\text {......... }\end{array}$ & & \\
\hline Birmingham & 28.41 & 0.123 & 33.46 & 0.145 & & & & \\
\hline $\begin{array}{l}\text { Mobile......... } \\
\text { Montgomery.. }\end{array}$ & 47.97 & 0.172 & 53.51 & 0.192 & 35.16 & 0.130 & 1.36 & 1.52 \\
\hline $\begin{array}{l}\text { Montgomery........ } \\
\text { Arizona: }\end{array}$ & & & 37.72 & 0.155 & 41.90 & 0.172 & 0.75 & 0.90 \\
\hline Fort Apache (S3) & 9.82 & 0.063 & 10.57 & \multicolumn{2}{|c|}{ Arizona: } & 0.236 & 0.27 & 0.29 \\
\hline Fort Grant (S3).... & 9.98 & 0.042 & 10.85 & 0.045 & 78.91 & 0.330 & 0.13 & 0.14 \\
\hline $\begin{array}{l}\text { Phoenix....... } \\
\text { Prescott }(\mathrm{S} 4) . .\end{array}$ & 5.57 & 0.020 & 6.35 & 0.022 & & & & \\
\hline $\begin{array}{l}\text { Prescott (S4)..... } \\
\text { Arkansas: }\end{array}$ & 7.48 & 0.057 & 8.10 & 0.062 & 29.72 & 0.227 & 0.25 & 0.27 \\
\hline Fort Smith. & \multicolumn{8}{|c|}{ Arkansas: } \\
\hline Little Rock & 30.60 & 0.129 & 35.25 & 0.149 & 39.63 & 0.167 & 0.77 & $\begin{array}{l}0.85 \\
0.89\end{array}$ \\
\hline \multicolumn{9}{|l|}{$\begin{array}{l}\text { California: } \\
\quad \text { Cedarville (S15)... }\end{array}$} \\
\hline $\begin{array}{l}\text { Cedarville (S15).... } \\
\text { Fort Bidwell. . . . }\end{array}$ & $\begin{array}{l}5.12 \\
\ldots \ldots\end{array}$ & 0.025 & 7.07 & 0.034 & 37.52 & 0.183 & 0.14 & 0.19 \\
\hline Eureka.... & 17.25 & $\begin{array}{l}0.070 \\
0.017\end{array}$ & $\begin{array}{r}24.10 \\
5.94\end{array}$ & $\begin{array}{l}0.098 \\
0.023\end{array}$ & & & & 0.10 \\
\hline $\begin{array}{l}\text { Fresno......... } \\
\text { Independence. }\end{array}$ & 4.48 & 0.017 & $\begin{array}{l}5.94 \\
4.17\end{array}$ & $\begin{array}{l}0.023 \\
0.091\end{array}$ & 56.87 & $\begin{array}{l}0.220 \\
0.340\end{array}$ & 0.08 & 0.06 \\
\hline (Keeler)....... & 2.79 & 0.014 & 4.17 & 0.021 & 69.55 & 0.349 & 0.04 & 0.06 \\
\hline Los Angeles.. & 12.88 & 0.039 & 15.52 & $\begin{array}{l}0.046 \\
0.059\end{array}$ & $\begin{array}{l}34.81 \\
70.75\end{array}$ & $\begin{array}{l}0.104 \\
0.268\end{array}$ & $\begin{array}{l}0.37 \\
0.17\end{array}$ & 0.45 \\
\hline $\begin{array}{l}\text { Red Bluff.. } \\
\text { Sacramento }\end{array}$ & $\begin{array}{r}11.88 \\
9.62\end{array}$ & $\begin{array}{l}0.045 \\
0.035\end{array}$ & $\begin{array}{l}15.55 \\
13.41\end{array}$ & $\begin{array}{l}0.059 \\
0.049\end{array}$ & $\begin{array}{l}70.75 \\
46.38\end{array}$ & $\begin{array}{l}0.268 \\
0.171\end{array}$ & 0.21 & $\begin{array}{l}0.22 \\
0.29\end{array}$ \\
\hline $\begin{array}{l}\text { Sacramento........ } \\
\text { San Francisco.... }\end{array}$ & 15.70 & 0.048 & $\begin{array}{l}13.41 \\
19.84\end{array}$ & 0.062 & 32.49 & 0.102 & 0.48 & 0.61 \\
\hline San Jose... & 12.63 & 0.043 & 16.86 & 0.057 & & & $\ldots \ldots$. & ....... \\
\hline San Luis Obispo... & 8.63 & 0.033 & 12.43 & 0.048 & & & & .... \\
\hline \multicolumn{9}{|l|}{ Colorado: } \\
\hline $\begin{array}{l}\text { Colorado Sps. (S7) } \\
\text { Denver............. }\end{array}$ & $\begin{array}{r}10.12 \\
7.49\end{array}$ & $\begin{array}{l}0.066 \\
0.049\end{array}$ & $\begin{array}{r}11.76 \\
9.82\end{array}$ & $\begin{array}{l}0.077 \\
0.064\end{array}$ & $\begin{array}{l}30.48 \\
38.92\end{array}$ & 0.254 & 0.19 & $\begin{array}{l}0.39 \\
0.25\end{array}$ \\
\hline $\begin{array}{l}\text { Denver............... } \\
\text { Montrose (S9) ..... }\end{array}$ & 3.90 & 0.028 & 4.71 & 0.034 & 37.17 & 0.262 & 0.10 & 0.13 \\
\hline Pueblo..................... & 7.65 & 0.047 & 9.09 & 0.056 & & 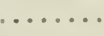 & & .... \\
\hline \multicolumn{9}{|l|}{ Connecticut: } \\
\hline $\begin{array}{l}\text { Hartford.......... } \\
\text { Nerw Haven . }\end{array}$ & 20.28 & $\begin{array}{l}0.123 \\
0.131\end{array}$ & 23.88 & 0.145 & & 0.111 & & \\
\hline $\begin{array}{l}\text { New Haven....... } \\
\text { New London }(\mathrm{H}) \text {.. }\end{array}$ & $\begin{array}{l}23.55 \\
21.02\end{array}$ & $\begin{array}{l}0.131 \\
0.113\end{array}$ & $\begin{array}{l}27.57 \\
24.75\end{array}$ & $\begin{array}{l}0.153 \\
0.133\end{array}$ & $\begin{array}{l}20.05 \\
20.40\end{array}$ & 0.108 & 1.03 & 1.21 \\
\hline \multicolumn{9}{|l|}{ Florida: } \\
\hline Jacksonville. & 45.97 & 0.157 & 49.61 & 0.169 & 39.67 & 0.135 & 1.16 & 1.25 \\
\hline Jupiter. & $\begin{array}{l}55.02 \\
38.66\end{array}$ & $\begin{array}{l}0.173 \\
0.106\end{array}$ & $\begin{array}{l}58.39 \\
38.66\end{array}$ & $\begin{array}{l}0.184 \\
0.106\end{array}$ & 51.60 & 0.141 & 0.75 & 0.75 \\
\hline acey the & 38.66 & 0.100 & & & & & & \\
\hline
\end{tabular}

1 The values of $S$ are given in tables 1 and 6 , number of days in the period of the average frostless season.

${ }^{2}$ Approximated from Russell's data for 1887-88.

${ }^{3}$ Numbers with $\mathrm{S}$ in parentheses after the station name refer to the section number, in the Summary by Sections, under which the given station appears. 
TABLE 11.-Precipitation and evaporation data for the period of the average frostless scason. (Plates 46, 57, and 58.)-Continued.

\begin{tabular}{|c|c|c|c|c|c|c|c|c|}
\hline \multirow{2}{*}{ Station. } & \multirow{2}{*}{ 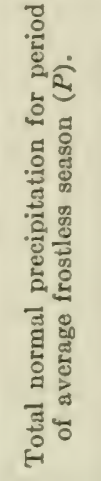 } & \multirow{2}{*}{ 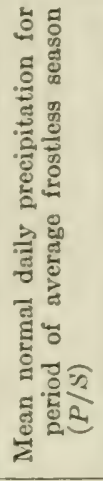 } & \multirow{2}{*}{ 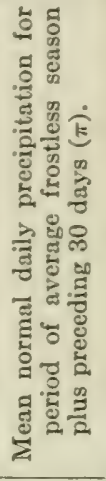 } & \multirow{2}{*}{$\frac{\pi^{2}}{S}$} & \multirow{2}{*}{ 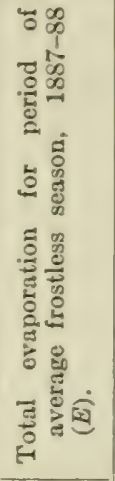 } & \multirow{2}{*}{ 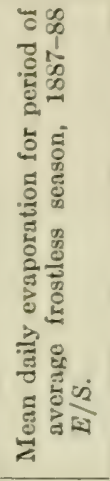 } & \multicolumn{2}{|c|}{$\begin{array}{l}\text { Moisture ratios } \\
\text { for period of } \\
\text { average frostless } \\
\text { season, } P / E \text {. }\end{array}$} \\
\hline & & & & & & & $P / E$ & $\pi / E$ \\
\hline Florida-Continued: & inches. & inches. & inches. & inches. & inches. & inches. & & \\
\hline (Titusville)............ & 45.34 & 0.146 & 48.52 & 0.156 & 38.51 & 0.124 & 1.18 & 1.26 \\
\hline Pensacola............. & 45.01 & 0.158 & 49.51 & 0.174 & 41.59 & 0.146 & 1.08 & 1.19 \\
\hline 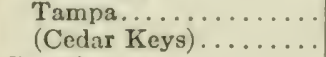 & 50.13 & 0.150 & 53.13 & 0.159 & 46.35 & 0.138 & 1.03 & 1.15 \\
\hline \multicolumn{9}{|l|}{ Georgia: } \\
\hline 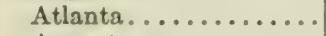 & 27.48 & 0.122 & 32.67 & 0.145 & 36.95 & 0.164 & 0.74 & 0.88 \\
\hline Augusta.............. & 29.97 & 0 . & 34. & 0.151 & 35.21 & 0.154 & 0.85 & 0.98 \\
\hline 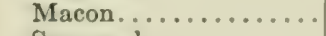 & 27.44 & 0 . & 32.27 & 0.136 & 8 & $\cdots$ & & \\
\hline Savannah............. & 39.63 & 51 & 42.98 & 0.163 & 35.53 & 0.135 & 1.12 & 1.21 \\
\hline Thomasville......... & 36.22 & 0.141 & 41.00 & 0.160 & & & & \\
\hline \multicolumn{9}{|l|}{ Idaho: } \\
\hline$\ldots \ldots \ldots$ & 3.90 & 0.022 & 5.14 & 0.029 & 43.80 & 0.247 & 0.09 & 0.12 \\
\hline Lewiston............. & 5.91 & 29 & 7.20 & .036 & $\ldots \ldots$ & $\ldots \ldots$ & $\cdots$ & $\cdots \cdots$ \\
\hline $\begin{array}{l}\text { Pocatello........... } \\
\text { Illinois: }\end{array}$ & 6.49 & 0.037 & 8.17 & 0.047 & & & & $\ldots$ \\
\hline Cairo.... & 23.07 & 9 & 98 & 0.127 & 5 & 0 . & 0.64 & 0.75 \\
\hline Chicago.............. & 19.18 & & 96 & 0.121 & 25.94 & 0.143 & 0.74 & 0.85 \\
\hline La Salle............ & 18.63 & 0.111 & 21.70 & 0.129 & $\ldots \ldots$ & $\ldots \ldots \ldots$ & $\ldots \ldots \ldots$ & $\ldots \ldots$ \\
\hline Peoria................ & 21.09 & & 24.19 & 0.130 & … & -. . & $\therefore$ & ....... \\
\hline Springfield........... & 20.99 & 0.115 & 24.21 & 0.133 & 28.26 & 0.155 & 0.74 & 0.86 \\
\hline \multicolumn{9}{|l|}{ Indiana: } \\
\hline $\begin{array}{l}\text { Evansville............. } \\
\text { Indianapolis......... }\end{array}$ & 22.09 & 19 & 25.94 & 0.139 & 34.95 & $0.188^{\circ}$ & 0.63 & 0.74 \\
\hline \multicolumn{9}{|l|}{ Iowa: } \\
\hline Charles City......... & 17.04 & 0.128 & 20.95 & 0.158 & & & & \\
\hline Davenport........... & 20.52 & 0.118 & 23.38 & 0.134 & 27.77 & 0.160 & 0.74 & 0.81 \\
\hline Des Moines............ & 21.93 & 0.128 & 24.51 & 0.143 & 24.75 & 0.145 & 0.89 & 0.99 \\
\hline Dubuque............ & 22.15 & 0.126 & 24.80 & 0.141 & 23.31 & 0.132 & 0.95 & 1.06 \\
\hline Keokuk............. & 24.44 & 0.124 & 26.76 & 0.136 & 32.70 & 0.166 & 0.75 & 0.82 \\
\hline Sioux City ........... & 16.60 & 0.114 & 19.64 & 0.135 & & & & \\
\hline \multicolumn{9}{|l|}{ Kansas: } \\
\hline Concordia........... & 20.07 & 0.116 & 22.27 & 0.129 & 28.79 & 0.166 & 0.70 & 0.77 \\
\hline Dodge City........... & 15.93 & 0.088 & 17.36 & 0.096 & 36.69 & 0.203 & 0.43 & 0.47 \\
\hline Topeka.............. & 25.60 & 0.135 & 28.09 & 0.149 & 25.45 & 0.135 & 1.01 & 1.10 \\
\hline Wichita.......... & 23.02 & 0.119 & 25.39 & 0.131 & & . & 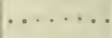 & $\cdots$ \\
\hline \multicolumn{9}{|l|}{ Kentucky: } \\
\hline Lexington... & 20.93 & 0.112 & 24.92 & 0.133 & & & & \\
\hline Louisville.... & 22.31 & 0.114 & 26.59 & 0.136 & 39.19 & 0.200 & 0.57 & 0.65 \\
\hline \multicolumn{9}{|l|}{ Louisiann: } \\
\hline New Orlenns..... & 49.15 & 0.159 & 53.57 & 0.173 & 40.61 & 0.131 & 1. 21 & 1. 32 \\
\hline Shreveport....... & 30.11 & 0.119 & 33.93 & 0.135 & 36.90 & 0.147 & 0.32 & 0.92 \\
\hline
\end{tabular}


TABLE 11.-Precipitation and eva poration data for the period of the average frostless season. (Plates 46, 57, and 58.)-Continued.

\begin{tabular}{|c|c|c|c|c|c|c|c|c|}
\hline \multirow[b]{2}{*}{ Station. } & \multirow{2}{*}{ 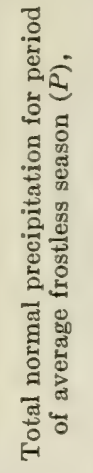 } & \multirow{2}{*}{ 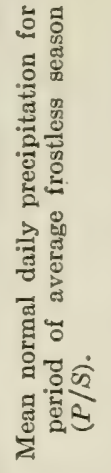 } & \multirow{2}{*}{ 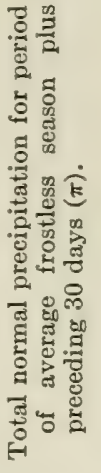 } & \multirow[b]{2}{*}{$\frac{\pi^{1}}{S}$} & \multirow{2}{*}{ 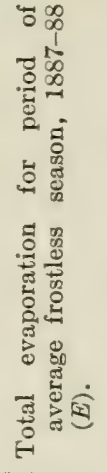 } & \multirow{2}{*}{ 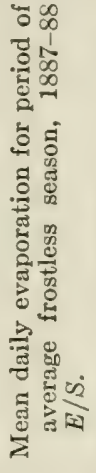 } & \multicolumn{2}{|c|}{$\begin{array}{l}\text { Moisture ratios } \\
\text { for period of } \\
\text { average frostles } \\
\text { season, } P / E \text {. }\end{array}$} \\
\hline & & & & & & & $P / E$ & $\pi / E$ \\
\hline $\begin{array}{l}\text { Maine: } \\
\quad \text { Eastport. . . }\end{array}$ & $\begin{array}{r}\text { inches. } \\
18.26\end{array}$ & $\begin{array}{l}\text { inches. } \\
0.109\end{array}$ & $\begin{array}{l}\text { inches. } \\
21.23\end{array}$ & $\begin{array}{l}\text { inch. } \\
0.127\end{array}$ & $\begin{array}{r}\text { inches. } \\
13.97\end{array}$ & $\begin{array}{r}\text { inches. } \\
0.084\end{array}$ & 1.31 & 1.52 \\
\hline $\begin{array}{l}\text { Portland...... } \\
\text { Maryland: }\end{array}$ & 17.79 & 0.113 & 20.95 & 0.133 & 17.13 & 0.109 & 1.04 & 1.22 \\
\hline Baltimore. & 26.42 & 0.124 & 30.16 & 0.142 & 34.78 & 0.163 & 0.76 & 0.87 \\
\hline $\begin{array}{l}\text { Washington, D. C. . } \\
\text { Massachusetts: }\end{array}$ & 24.96 & 0.127 & 28.71 & 0.146 & 30.26 & 0.154 & 0.83 & 0.95 \\
\hline Boston.......... & 21.12 & 0.114 & 24.84 & 0.134 & 22.75 & & & \\
\hline Nantucket. & 18.99 & 0.091 & 22.74 & 0.109 & 18.40 & $\begin{array}{l}0.123 \\
0.088\end{array}$ & $\begin{array}{l}0.93 \\
1.03\end{array}$ & $\begin{array}{l}1.09 \\
1.24\end{array}$ \\
\hline Michigan: & & & & & & & & \\
\hline $\begin{array}{l}\text { Alpena..... } \\
\text { Detroit.... }\end{array}$ & $\begin{array}{l}14.97 \\
16.82\end{array}$ & $\begin{array}{l}0.109 \\
0.103\end{array}$ & $\begin{array}{l}17.77 \\
19.15\end{array}$ & $\begin{array}{l}0.128 \\
0.117\end{array}$ & 14.85 & 0.108 & 1.01 & 1.20 \\
\hline Escanaba... & 16.15 & 0.115 & $\begin{array}{l}19.15 \\
18.93\end{array}$ & 0.135 & 24.39 & 0.149 & 0.69 & 0.82 \\
\hline Grand Haven. & 15.28 & 0.091 & 17.73 & 0.106 & 19.28 & 0.115 & 0.79 & 0.92 \\
\hline Grand Rapids..... & 15.19 & 0.093 & 17.64 & 0.108 & & & ...... & .... \\
\hline Houghton. & 16.25 & 0.107 & 18.57 & 0.122 & & & & \\
\hline Lansing $(\mathrm{H}) \ldots \ldots$ & 14.02 & 0.089 & 16.51 & 0.104 & 17.59 & 0.111 & 0.80 & 0.94 \\
\hline Marquette... & 14.94 & 0.107 & 17.59 & 0.126 & 14.48 & 0.103 & 1.03 & 1.21 \\
\hline Port Huron: & 14.73 & 0.095 & 16.98 & 0.110 & 18.85 & 0.122 & 0.78 & 0.90 \\
\hline Sault Ste. Marie... & 14.07 & 0.102 & 16.48 & 0.119 & & & . & 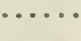 \\
\hline $\begin{array}{l}\text { Minnesota: } \\
\text { Duluth... }\end{array}$ & 18.66 & 0.123 & 20.97 & 0.138 & 15.13 & 0.100 & 1.23 & 1.39 \\
\hline Minneapolis. & 20.00 & 0.124 & 22.37 & 0.139 & & & & \\
\hline Moorhead............ & 14.59 & 0.111 & 17.03 & 0.129 & 15.46 & 0.117 & 0.94 & 1.10 \\
\hline St. Paul. & 18.91 & 0.119 & 21.09 & 0.133 & 18.33 & 0.115 & 1.03 & 1.15 \\
\hline $\begin{array}{l}\text { St. Vincent. . } \\
\text { Mississippi: }\end{array}$ & 9.54 & 0.093 & 12.05 & 0.117 & 10.39 & 0.101 & 0.92 & 1.16 \\
\hline Meridian. . & 30.63 & 0.133 & 35.73 & 0.155 & & & & \\
\hline 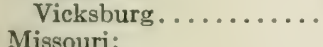 & 34.87 & 0.138 & 39.83 & 0.158 & 37.34 & 0.148 & 0.93 & 1.07 \\
\hline Missouri: & & & & & & & & \\
\hline 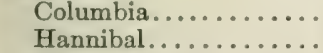 & $\begin{array}{l}21.57 \\
22.02\end{array}$ & $\begin{array}{l}0.120 \\
0.120\end{array}$ & $\begin{array}{l}24.80 \\
24.88\end{array}$ & $\begin{array}{l}0.139 \\
0.135\end{array}$ & & & $\cdots$ & $\cdots$ \\
\hline $\begin{array}{l}\text { Kansas City }, \ldots . . . . \\
\text { (Leavenworth, Kans). }\end{array}$ & 25.89 & 0.132 & 28.94 & 0.148 & 30.26 & 0.154 & 0.86 & $\ddot{0} 9$ \\
\hline Lamar (S49) ......... & 26.58 & 0.146 & 30.20 & 0.166 & 25.83 & 0.142 & 1.03 & 1.17 \\
\hline St. Louis......... & 22.59 & 0.112 & 25.83 & 0.129 & 39.29 & 0.195 & 0.58 & 0.66 \\
\hline $\begin{array}{l}\text { Springfield............ } \\
\text { Montana: }\end{array}$ & 27.54 & 0.147 & 31.12 & 0.166 & 24.96 & 0.133 & 1.00 & 1.25 \\
\hline $\begin{array}{l}\text { Montana: } \\
\text { Crow Agency (S26) }\end{array}$ & & & & & & & & 29 \\
\hline $\begin{array}{l}\text { (Fort Custer) . . . . } \\
\text { Havre........... }\end{array}$ & 7.08 & 0.053 & 8. & 0.067 & 31.30 & $t$ & 0.23 & 29 \\
\hline (Fort Assiniboine). & 7.78 & 0.064 & 9.45 & 0.077 & 20.86 & 0.171 & 0.37 & 0.45 \\
\hline Helena... & 6.48 & 0.045 & 7.82 & 0.054 & 29.70 & 0.206 & 0.22 & 0.62 \\
\hline
\end{tabular}


TABLE 11.-Precipitation and evaporation data for the period of the average frostless season. (Plates 46, 57, and 58.)-Continued.

\begin{tabular}{|c|c|c|c|c|c|c|c|c|}
\hline \multirow[b]{2}{*}{ Station. } & \multirow{2}{*}{ 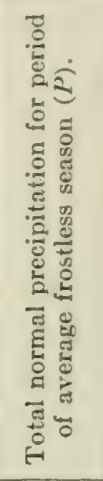 } & \multirow{2}{*}{ 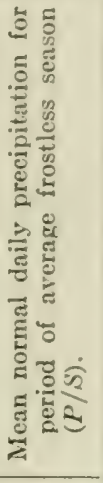 } & \multirow{2}{*}{ 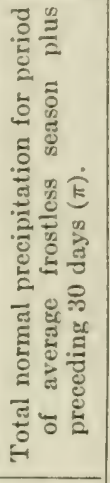 } & \multirow[b]{2}{*}{$\frac{\pi^{1}}{5}$} & \multirow{2}{*}{ 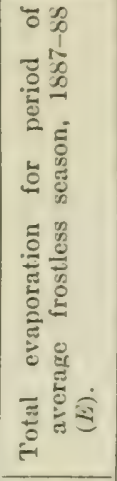 } & \multirow{2}{*}{ 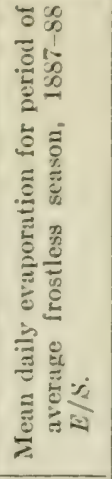 } & \multicolumn{2}{|c|}{$\begin{array}{l}\text { Moisture ratios } \\
\text { for period of } \\
\text { average frostless } \\
\text { season, } P / E \text {. }\end{array}$} \\
\hline & & & & & & & $P / E$ & $\pi / E$ \\
\hline $\begin{array}{l}\text { Montana-Continucd: } \\
\text { Kalispell............. }\end{array}$ & $\begin{array}{l}\text { inches. } \\
6.07\end{array}$ & $\begin{array}{l}\text { inches. } \\
0.043\end{array}$ & $\begin{array}{l}\text { inches. } \\
7.47\end{array}$ & $\begin{array}{l}\text { inch. } \\
0.053\end{array}$ & inches. & inches. & & \\
\hline $\begin{array}{l}\text { Lewistown (S29)...... } \\
\text { (Fort Maginnis)....... }\end{array}$ & 7.06 & 0.078 & 9.61 & 0.107 & 15.61 & 0.173 & 0.45 & 0.62 \\
\hline Miles City............ & 7.60 & 0.054 & 8.98 & 0.064 & .. & $\ldots$ & $\cdots \cdots$ & \\
\hline $\begin{array}{l}\text { Poplar }(\text { S } 30) \ldots \ldots \ldots \\
\text { (Poplar River) ............ }\end{array}$ & 6.89 & 0.058 & 8.34 & 0.071 & 20.48 & 0.174 & 0.34 & 0.41 \\
\hline Nebraska: & & & & & & & & \\
\hline Crete... & 20.24 & 0.127 & 22.81 & 0.143 & 22.60 & 0.142 & 0.90 & 1.01 \\
\hline Lincoln. . . . . . . . . & 20.65 & 0.119 & 22.72 & 0.131 & $\because \cdots \cdots$ & $\cdots \cdots$ & $\cdots \cdots$ & $\ldots \ldots$ \\
\hline North Platte......... & 12.83 & 0.055 & 15.03 & 0.100 & 25.05 & 0.166 & 0.51 & 0.60 \\
\hline Omaha.............. & 22.08 & 0.130 & 24.83 & 0.146 & 27.09 & 0.159 & 0.82 & 0.92 \\
\hline Valentine............ & 13.64 & 0.103 & 16.39 & 0.124 & 22.59 & 0.171 & 0.60 & 0.73 \\
\hline $\begin{array}{l}\text { Nevada: } \\
\quad \text { Reno............... }\end{array}$ & 1.22 & 0.009 & 2.01 & 0.015 & & & & \\
\hline Winnemucea ......... & 1.67 & 0.013 & 2.64 & 0.020 & 45.99 & 0.351 & 0.01 & $0.05 j$ \\
\hline New Hampshire: & & & & & & & & \\
\hline Concord............ & 16.68 & 0.114 & 19.37 & 0.133 & 17.84 & 0.122 & 0.94 & 1.09 \\
\hline New Jersey: & & & & & & & & \\
\hline Atlantic City .......... & 22.74 & 0.110 & $\begin{array}{l}26.21 \\
23.64\end{array}$ & $\begin{array}{l}0.127 \\
0.127\end{array}$ & 17.48 & 0.084 & 1.30 & 1.50 \\
\hline $\begin{array}{l}\text { Cape May............ } \\
\text { New Mexico: }\end{array}$ & 20.39 & 0.110 & 23.64 & 127 & & & & $\cdots \cdots$ \\
\hline Fort Stanton.......... & $10.8 \mathrm{~S}$ & 0.073 & 11.50 & 0.077 & 43.19 & 0.290 & 0.23 & 0.27 \\
\hline $\begin{array}{l}\text { Santa Fe.......... } \\
\text { New York: }\end{array}$ & 10.01 & 0.054 & 10.82 & 0.058 & 54.79 & 0.293 & 0.18 & 0.20 \\
\hline 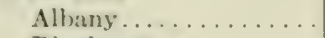 & 20.00 & 0.113 & 22.39 & 0.126 & 23.71 & 0.134 & 0.84 & 0.94 \\
\hline Binghamton . . . . . . & 16.91 & 0.107 & 19.14 & 0.121 & & $\ldots \ldots$ & & \\
\hline 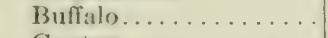 & 17.95 & 0.104 & 20.45 & 0.11 s & 22.94 & 0.133 & 0.78 & 0.83 \\
\hline Canton............ & 13.50 & 0.099 & 10.01 & 0.115 & $\ldots \ldots$ & $\ldots \ldots$ & $\cdots \cdots$ & $\ldots \ldots$ \\
\hline 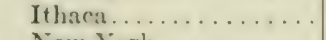 & 17.49 & 0.112 & 20.21 & 0.126 & & ........ & $\cdots \cdots$ & $\cdots \cdots$ \\
\hline New York........... & 25.52 & 0.121 & 29.47 & 0.110 & 29.43 & 0.140 & 0.57 & 1.00 \\
\hline Oswergo $\ldots \ldots \ldots \ldots$ & 17.29 & 0.099 & 19.61 & 0.112 & 19.97 & 0.114 & 0.87 & 0.98 \\
\hline Rochester. . . . . . . & 16.01 & $0 .(19 \cdot 4$ & 18.43 & $0.10 \mathrm{~s}$ & 22.67 & 0.133 & 0.71 & 0.51 \\
\hline Syracuse .......... & 18.91 & 0.111 & 21.20 & 0.124 & $\ldots \ldots$ & $\ldots \ldots$ & $\ldots \ldots$ & $\ldots \ldots$ \\
\hline North Carolina: & & & & & & & & \\
\hline $\begin{array}{l}\text { Asheville... . . . . } \\
\text { Charbtte.... . . . }\end{array}$ & $\begin{array}{l}23.09 \\
23 \\
300\end{array}$ & $\begin{array}{l}0.131 \\
0.136 i\end{array}$ & 27.6 .3 & $\begin{array}{l}0.1 .17 \\
0.1 .2 i\end{array}$ & 34.06 & 0.155 & 0.88 & 1.01 \\
\hline 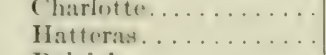 & $\begin{array}{l}29.90 \\
43.46\end{array}$ & $\begin{array}{l}0.136 i \\
0.170\end{array}$ & $\begin{array}{l}34.30 \\
14.24\end{array}$ & 0.1 is & 24.6 .5 & 0.096 & 1.76 & 1.96 \\
\hline 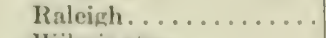 & 31.75 & 0.149 & 3.5 .90 & 0.1169 & 20.13 & 0.123 & 1.22 & 1.37 \\
\hline Wilmington ......... & 36.75 & 0.1 .54 & 40.10 & 0.172 & 27.35 & 0.117 & 1.35 & 1.47 \\
\hline $\begin{array}{l}\text { North Dakota: } \\
\text { Bismarck......... }\end{array}$ & 10.35 & $0.11-1$ & $1: .41$ & $0.09+i$ & 15.94 & 0.147 & 0.55 & $0.66 i$ \\
\hline
\end{tabular}


TABLE 11.-Precipitation and evaporation data for the period of the average frostless season. (Plates 46, 57, and 58.) - Continued.

\begin{tabular}{|c|c|c|c|c|c|c|c|c|}
\hline \multirow[b]{2}{*}{ Station. } & \multirow{2}{*}{ 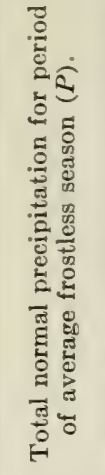 } & \multirow{2}{*}{ 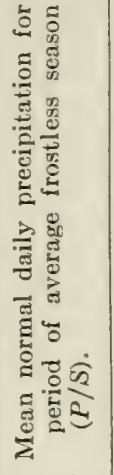 } & \multirow{2}{*}{ 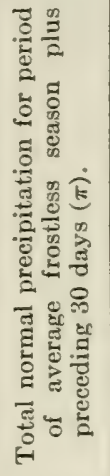 } & \multirow[b]{2}{*}{$\frac{\pi^{1}}{S}$} & \multirow{2}{*}{ 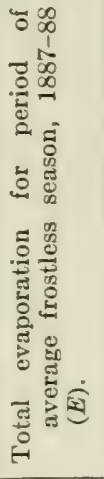 } & \multirow{2}{*}{ 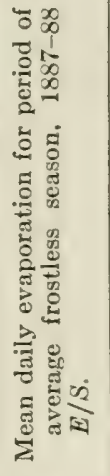 } & \multicolumn{2}{|c|}{$\begin{array}{l}\text { Moisture ratios } \\
\text { for period of } \\
\text { average frostless } \\
\text { season, } P / E \text {. }\end{array}$} \\
\hline & & & & & & & $P / E$ & $\pi / E$ \\
\hline $\begin{array}{l}\text { North Dakota-Cont'd: } \\
\text { Devils Lake........ } \\
\text { (Fort Totten) }\end{array}$ & $\begin{array}{l}\text { inches. } \\
11.57\end{array}$ & $\begin{array}{l}\text { inches. } \\
0.096\end{array}$ & $\begin{array}{r}\text { inches. } \\
13.67\end{array}$ & $\begin{array}{l}\text { inch. } \\
0.113\end{array}$ & $\begin{array}{r}\text { inches. } \\
15.38\end{array}$ & $\begin{array}{l}\text { inches. } \\
0.127\end{array}$ & 0.75 & 0.89 \\
\hline $\begin{array}{l}\text { Williston (S31)... } \\
\text { (Fort Buford)... }\end{array}$ & 8.22 & 0.069 & 9.96 & 0.084 & 20.31 & 0.171 & 0.40 & 0.49 \\
\hline Ohio: & & & & & & & & \\
\hline $\begin{array}{l}\text { Cincinnati. } \\
\text { Cleveland. }\end{array}$ & 19.13 & 0.099 & 22.38 & 0.115 & 37.20 & 0.192 & 0.51 & 0.60 \\
\hline $\begin{array}{l}\text { Cleveland. } \\
\text { Columbus. }\end{array}$ & 20.66 & 0.104 & 23.22 & 0.117 & 26.35 & 0.133 & 0.78 & 0.88 \\
\hline bus. & 19.34 & 0.105 & 22.36 & 0.122 & 33.29 & 0.181 & 0.58 & 0.67 \\
\hline $\begin{array}{l}\text { Sandusky. } \\
\text { Toledo... }\end{array}$ & $\begin{array}{l}20.35 \\
16.60\end{array}$ & $\begin{array}{l}0.104 \\
0.095\end{array}$ & $\begin{array}{l}22.99 \\
18.93\end{array}$ & $\begin{array}{l}0.118 \\
0.109\end{array}$ & $\begin{array}{l}27.36 \\
26.84\end{array}$ & $\begin{array}{l}0.140 \\
0.154\end{array}$ & $\begin{array}{l}0.74 \\
0.62\end{array}$ & $\begin{array}{l}0.84 \\
0.71\end{array}$ \\
\hline Oklahoma: & & & & & & & & \\
\hline $\begin{array}{l}\text { Fort Siil (S41). } \\
\text { Oklahoma..... }\end{array}$ & 23.49 & 0.110 & 25.04 & 0.117 & 33.81 & 0.158 & 0.70 & 0.74 \\
\hline $\begin{array}{l}\text { Oklahoma... } \\
\text { Oregon: }\end{array}$ & 23.03 & 0.108 & 25.37 & 0.119 & & & & \\
\hline $\begin{array}{l}\text { Astoria...... } \\
\text { Baker City }\end{array}$ & 41.25 & 0.152 & 51.09 & 0.189 & 21.77 & 0.080 & 1.90 & 2.35 \\
\hline $\begin{array}{l}\text { Baker City.. } \\
\text { Portland.... }\end{array}$ & $\begin{array}{r}3.23 \\
19.43\end{array}$ & $\begin{array}{l}0.025 \\
0.079\end{array}$ & 4.73 & 0.037 & & 0.120 & 0.66 & \\
\hline Roseburg. & $\begin{array}{r}19.43 \\
8.33\end{array}$ & $\begin{array}{l}0.079 \\
0.042\end{array}$ & $\begin{array}{l}25.08 \\
11.59\end{array}$ & $\begin{array}{l}0.102 \\
0.059\end{array}$ & $\begin{array}{l}29.42 \\
28.35\end{array}$ & $\begin{array}{l}0.120 \\
0.143\end{array}$ & $\begin{array}{l}0.00 \\
0.29\end{array}$ & $\begin{array}{l}0.85 \\
0.41\end{array}$ \\
\hline Pennsylvania: & & & & & & & & \\
\hline Erie...... . & 22.52 & 0.116 & 25.07 & 0.129 & 25.10 & 0.129 & 0.90 & 1.00 \\
\hline $\begin{array}{l}\text { Harrisburg... } \\
\text { Philadelphia. }\end{array}$ & 21.91 & 0.112 & 24.92 & 0.127 & & & & \\
\hline $\begin{array}{l}\text { Philadelphia. } \\
\text { Pittsburgh... }\end{array}$ & 23.97 & 0.116 & $\begin{array}{l}27.35 \\
22 \\
25\end{array}$ & $\begin{array}{l}0.133 \\
0.124\end{array}$ & 32.13 & $\begin{array}{l}0.156 \\
0.166\end{array}$ & $\begin{array}{l}0.75 \\
0.66\end{array}$ & 0.85 \\
\hline $\begin{array}{l}\text { Pittsburgh... } \\
\text { Scranton.... }\end{array}$ & $\begin{array}{l}19.45 \\
19.88\end{array}$ & $\begin{array}{l}0.109 \\
0.113\end{array}$ & $\begin{array}{l}22.25 \\
22.81\end{array}$ & $\begin{array}{l}0.124 \\
0.130\end{array}$ & 29.69 & 0.166 & 0.66 & $\begin{array}{c}0.75 \\
\ldots \ldots\end{array}$ \\
\hline Rhode Island: & & & & & & & & \\
\hline $\begin{array}{l}\text { Block Island.. } \\
\text { Providence... }\end{array}$ & 24.37 & 0.112 & 28.66 & 0.131 & 17.56 & 0.081 & 1.39 & 1.63 \\
\hline $\begin{array}{l}\text { Providence... } \\
\text { South Carolina: }\end{array}$ & 21.63 & 0.114 & 25.56 & 0.135 & & & & . \\
\hline Charleston... & 42.15 & 0.153 & 45.78 & 0.166 & 36.25 & 0.131 & 1.16 & 1.26 \\
\hline $\begin{array}{l}\text { Columbia...... } \\
\text { South Dakota: }\end{array}$ & 31.16 & 0.135 & 34.74 & 0.150 & 31.81 & 0.138 & 0.98 & 1.09 \\
\hline $\begin{array}{c}\text { South Dakota: } \\
\text { Huron...... }\end{array}$ & 12.49 & 0.095 & 15.37 & 0.117 & 19.00 & 0.145 & 0.66 & 0.81 \\
\hline $\begin{array}{l}\text { Pierre...... } \\
\text { (Fort Sully). }\end{array}$ & 10.68 & 0.070 & 11.98 & 0.078 & 27.60 & 0.180 & 0.39 & 0.43 \\
\hline Rapid City. . & 11.70 & 0.082 & 14.30 & 0.100 & & & & \\
\hline $\begin{array}{l}\text { Yankton... } \\
\text { Tennessee: }\end{array}$ & 17.17 & 0.111 & 20.11 & 0.131 & 18.79 & 0.122 & 0.91 & 1.07 \\
\hline Chattanooga. & 25.13 & 0.121 & 31.22 & 0.151 & 31.00 & 0.150 & 0.81 & 1.01 \\
\hline Knoxville... & 25.28 & 0.122 & 30.71 & 0.148 & 30.70 & 0.148 & 0.82 & $\begin{array}{l}1.01 \\
1.00\end{array}$ \\
\hline Memphis.... & 27.87 & 0.124 & 32.91 & 0.147 & 37.00 & 0.165 & 0.75 & 0.89 \\
\hline Nashville..... & 25.41 & 0.123 & 30.69 & 0.148 & 36.66 & 0.177 & 0.69 & 0.84 \\
\hline
\end{tabular}


TABLE 11.-Precipitation and craporation data for the period of the average frostless scason. (Plates 46, 57, and 58.)-Continued.

\begin{tabular}{|c|c|c|c|c|c|c|c|c|}
\hline \multirow[b]{2}{*}{ Station. } & \multirow{2}{*}{ 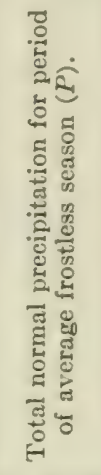 } & \multirow{2}{*}{ 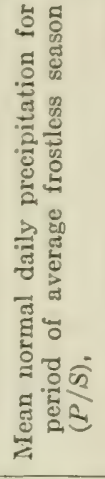 } & \multirow{2}{*}{ 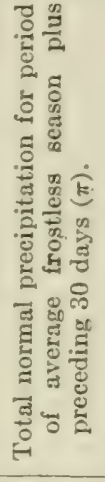 } & \multirow[b]{2}{*}{$\frac{\pi^{1}}{S}$} & \multirow{2}{*}{ 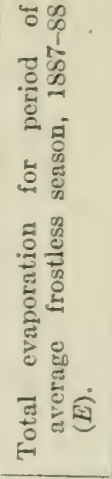 } & \multirow{2}{*}{ 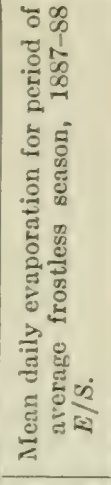 } & \multicolumn{2}{|c|}{$\begin{array}{l}\text { Moisture ratios } \\
\text { for period of } \\
\text { average frostless } \\
\text { season, } P / E \text {. }\end{array}$} \\
\hline & & & & & & & $P / E$ & $\pi / E$ \\
\hline $\begin{array}{l}\text { Texas: } \\
\text { Abilene... }\end{array}$ & $\begin{array}{l}\text { inches. } \\
20.30\end{array}$ & $\begin{array}{r}\text { inches. } \\
0.083\end{array}$ & $\begin{array}{l}\text { inches. } \\
21.65\end{array}$ & $\begin{array}{l}\text { inch. } \\
0.088\end{array}$ & $\begin{array}{r}\text { inches. } \\
46.00\end{array}$ & $\begin{array}{l}\text { inches. } \\
0.188\end{array}$ & 0.44 & 0.47 \\
\hline $\begin{array}{l}\text { Amarillo..... } \\
\text { (Fort Eliot). }\end{array}$ & 17.52 & 0.088 & 18.76 & 0.094 & 40.02 & 0.201 & 0.44 & 0.47 \\
\hline $\begin{array}{l}\text { Fort Brown (H) } \\
\text { (Brownsville)... }\end{array}$ & 24.79 & 0.078 & 26.12 & 0.082 & 32.41 & 0.102 & 0.77 & 0.81 \\
\hline Corpus Christi. & 22.83 & 0.077 & 25.22 & 0.085 & 35.09 & 0.118 & 0.65 & 0.72 \\
\hline $\begin{array}{l}\text { El Paso........ } \\
\text { Fort Clark.... }\end{array}$ & $\begin{array}{r}7.71 \\
19.52\end{array}$ & $\begin{array}{l}0.033 \\
0.072\end{array}$ & $\begin{array}{r}8.09 \\
20.31\end{array}$ & $\begin{array}{l}0.034 \\
0.075\end{array}$ & 64.41 & 0.273 & 0.12 & 0.13 \\
\hline Fort Ringgold (S1) & 15.82 & 0.0 .52 & & & 46.90 & & & $\cdots \cdots$ \\
\hline $\begin{array}{l}\text { (Rio Grande City) } \\
\text { Fort Worth. }\end{array}$ & $\begin{array}{l}10.82 \\
22.75\end{array}$ & 0.087 & $\begin{array}{l}16.62 \\
24.28\end{array}$ & $\begin{array}{l}0.00 i) \\
0.093\end{array}$ & $\$ 0.90$ & 0.155 & $0.3 \%$ & 0.35 \\
\hline Gart Weston... & $\begin{array}{l}22.75 \\
42.82\end{array}$ & 0.129 & $\begin{array}{l}24.28 \\
46.50\end{array}$ & 0.140 & 44.06 & 0.133 & 0.97 & 1.05 \\
\hline Palestine. : & 33.55 & 0.117 & 32.18 & 0.131 & 36.25 & 0.148 & 0.79 & 0.89 \\
\hline $\begin{array}{l}\text { San Antonio... } \\
\text { Taylor. }\end{array}$ & 22.08 & 0.080 & 23.70 & 0.086 & 43.63 & 0.158 & 0.51 & 0.54 \\
\hline $\begin{array}{l}\text { Taylor............ } \\
\text { Utah: }\end{array}$ & 25.66 & 0.101 & 28.48 & 0.112 & 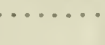 & & & \\
\hline $\begin{array}{l}\text { Modena........ } \\
\text { Salt Lake City }\end{array}$ & 1.99 & 0.015 & 3.57 & 0.027 & & & & \\
\hline $\begin{array}{l}\text { Salt Lake City. } \\
\text { Vermont: }\end{array}$ & 6.49 & 0.036 & 8.70 & 0.048 & 51.71 & 0.284 & 0.13 & 0.17 \\
\hline $\begin{array}{l}\text { Vermont: } \\
\text { Burlington. }\end{array}$ & 16.55 & 0.116 & 18.83 & 0.132 & & & & \\
\hline Northfield. . & 14.26 & 0.113 & 16.16 & 0.128 & 12.28 & 0.097 & 1.16 & 1.32 \\
\hline Virminia: & & & & & & & & \\
\hline Lynchburg. & 24.88 & 0.124 & 28.50 & 0.142 & 28.68 & 0.143 & 0.57 & 0.99 \\
\hline $\begin{array}{l}\text { Norfolk...... } \\
\text { Richmond... }\end{array}$ & 33.69 & 0.146 & 37.79 & 0.164 & 27.26 & 0.119 & 1.24 & 1.39 \\
\hline $\begin{array}{l}\text { Richmond... } \\
\text { Wytheville.. }\end{array}$ & 26.41 & 0.123 & 30.09 & 0.140 & & & 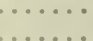 & \\
\hline $\begin{array}{l}\text { Wytheville.. } \\
\text { Washington: }\end{array}$ & 22.67 & 0.130 & 26.75 & 0.153 & & & & \\
\hline $\begin{array}{l}\text { North Head. } \\
\text { (Fort Canby). }\end{array}$ & 34.94 & 0.111 & 41.17 & 0.130 & 20.18 & 0.064 & 1.73 & 2.04 \\
\hline $\begin{array}{l}\text { Olympia (S19). } \\
\text { Senttle. }\end{array}$ & 13.81 & 0.073 & 17.69 & 0.094 & 19.85 & 0.106 & 0.70 & 0.89 \\
\hline $\begin{array}{l}\text { Seattle........ } \\
\text { Spokane..... }\end{array}$ & $\begin{array}{r}18.29 \\
7.69\end{array}$ & $\begin{array}{l}0.074 \\
0.038\end{array}$ & $\begin{array}{r}21.71 \\
9.14\end{array}$ & $\begin{array}{l}0.088 \\
0.045\end{array}$ & & & & \\
\hline Tatoosh Island. & 53.92 & 0.199 & 62.88 & 0.232 & $\begin{array}{l}34.00 \\
14.05\end{array}$ & 0.052 & 3.84 & 4.45 \\
\hline Walla Walla. & 8.08 & 0.037 & 9.95 & 0.046 & 45.87 & 0.212 & 0.18 & 0.22 \\
\hline $\begin{array}{l}\text { West Virginia: } \\
\text { Elkins....... }\end{array}$ & & & & & & & & \\
\hline $\begin{array}{l}\text { Elkins.......... } \\
\text { Parkersburg. }\end{array}$ & $\begin{array}{l}19.01 \\
21.32\end{array}$ & $\begin{array}{l}0.131 \\
0.119\end{array}$ & $\begin{array}{l}22.26 \\
24.70\end{array}$ & $\begin{array}{l}0.154 \\
0.135\end{array}$ & & & & \\
\hline Wisconsin: & & & & 0.130 & & & & \\
\hline Green Bay.... & 16.75 & 0.109 & 19.43 & 0.126 & 19.39 & 0.127 & 0.86 & 1.00 \\
\hline $\begin{array}{l}\text { La Crosse... } \\
\text { Madison. . . }\end{array}$ & 20.62 & 0.126 & 22.91 & 0.141 & 21.97 & 0.135 & 0.94 & 1.04 \\
\hline $\begin{array}{l}\text { Madison..... } \\
\text { Milwaukce. }\end{array}$ & 20.37 & 0.114 & 22.63 & 0.126 & & & & \\
\hline $\begin{array}{l}\text { Milwaukee. . } \\
\text { Wyoming: }\end{array}$ & 16.63 & 0.103 & 19.28 & 0.119 & 19.11 & 0.118 & 0.87 & 1.01 \\
\hline Cheyenno. & 6.33 & 0.054 & 8.57 & 0.073 & 32.48 & 0.275 & 0.20 & 0.26 \\
\hline Lander... & 3.37 & 0.031 & 6.27 & $0.05 s$ & & & & \\
\hline
\end{tabular}


The daily normals of precipitation for the period of the average frostless season are shown graphically by the chart of plate 46 , where the isoclimatic lines represent increments of 20 thousandths of an inch. The values are seen to be high for the southeast (the maximum being 170, for Cape Hatteras, North Carolina). The lowest values occur in the arid region as this term is commonly understood (the minimum being 9 , for Reno, Nevada). On this chart the lines for index values 60,100 , and 140 are represented as broader than the others, since these lines are useful in delimiting the main precipitation zones or provinces of the country. The line for value 60 extends northward from about Cape Mendocino, roughly parallelling the Pacific coast and passing into Canada near the western margin of the Rocky Mountains system. This same line reenters the United States near the eastern margin of the same mountain system, passes southward and somewhat eastward, so as to lie just east of the Rocky Mountains proper, and enters Mexico near the mouth of the Rio Grande. This line includes, within the loop thus formed, all of the region commonly called arid, and somewhat more. Northwest from the area thus demarked as arid the values increase, and the lines for values 100 and 140 lie just within the United States, in the neighborhood of Tatoosh Island, Washington (which station has the value 199).

East of the arid region as above defined the values of these daily normals increase slowly, and the line for value 100 passes from north to south through the eastern plains or western prairies, approximating a line drawn from Corpus Christi, Texas, to Winnipeg, Manitoba. At its northern end, however, this line bends eastward, apparently passing somewhere north of the upper Great Lakes, and then bends southward so as to reenter the United States north of Port Huron, Michigan. It crosses Michigan from east to west, bends southwestward to Grand Haven, Michigan, and reaches Cincinnati, Ohio. From this point it passes northward to Detroit and then follows the valley of the lower Great Lakes and St. Lawrence River, to pass again into Canada near the northern end of Lake Champlain. It apparently again bends southward and touches the Atlantic coast once more near Nantucket, Massachusetts. Between the arid region and the northsouth portion of this line, and north of the portion about the Great Lakes, lies a region that may be called semiarid, the values lying between 60 and 100 .

The line for value 140 delimits what may be called the southeastern rainy region, which here includes southeastern Louisiana, southern Mississippi, Alabama, and Georgia, all of Florida, and the Atlantic coastal region north as far as the entrance to Chesapeake Bay. Key West, Florida, lies outside of this rainy zone.

The region lying between the line for value 100 and that for value 140 , including most of the eastern half of the country, may be con- 
sidered here as a semirainy region. There are thus four precipitation zones or provinces roughly marked out on this chart, which may be defined as in table 12 .

TABLE 12.

\begin{tabular}{|c|c|}
\hline Province. & $\begin{array}{l}\text { Normal daily pre- } \\
\text { cipitation for the } \\
\text { period of average } \\
\text { frostless season. }\end{array}$ \\
\hline 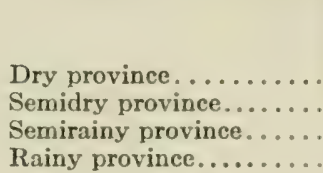 & $\begin{array}{l}\text { thousandths } \\
\text { inch. } \\
\text { Below } 60 \\
60 \text { to } 100 \\
100 \text { to } 140 \\
\text { Above } 140\end{array}$ \\
\hline
\end{tabular}

The four provinces thus indicated will be repeatedly referred to in our further discussion of moisture conditions.

(3) Total Normal Precipitation for Period of Average Frostlegs Season Plus Preceding 30 Days, Divided by Number of Days in Average Frostless Season $(\pi / S)$. (Table 11.$)$

This rather artificial index of precipitation intensity is based, as has been mentioned, upon the consideration that some of the precipitation occurring just before the opening of the frostless season is still effective in the early part of that season. In many places the first few weeks of the average frostless season are normally more or less dry, and yet plants may be able to begin their activities with the advent of frostless weather, on account of soil-moisture left over from the latter part of the preceding frost season. The length of the additional period of 30 days was chosen quite arbitrarily, in an attempt to bring these considerations into the index, which we term $\pi$. Bigelow's precipitation normals (Bulletin $\mathrm{R}$ ) were again used. The values obtained are given in column 4 of table 11, and these totals divided, in each case, by the corresponding number of days in the period of the average frostless season $(\pi / S)$ are given in column 5 of the same table.

The chart obtained from these averages $(\pi / S)$ shows no pronounced differences from that presented in plate 46 , and it is not reproduced here. This chart is mentioned, since the method by which it was obtained is new and may be of value in the future, for special studies of certain regions. The values of $\pi$ will be otherwise employed below.

(4) Number of Normaley Rainy Days in Period of Average Frostless Season.

(Table 13, Plate 47.)

This kind of index of precipitation intensity is frequently employed by climatologists, though for other duration factors than the one here 


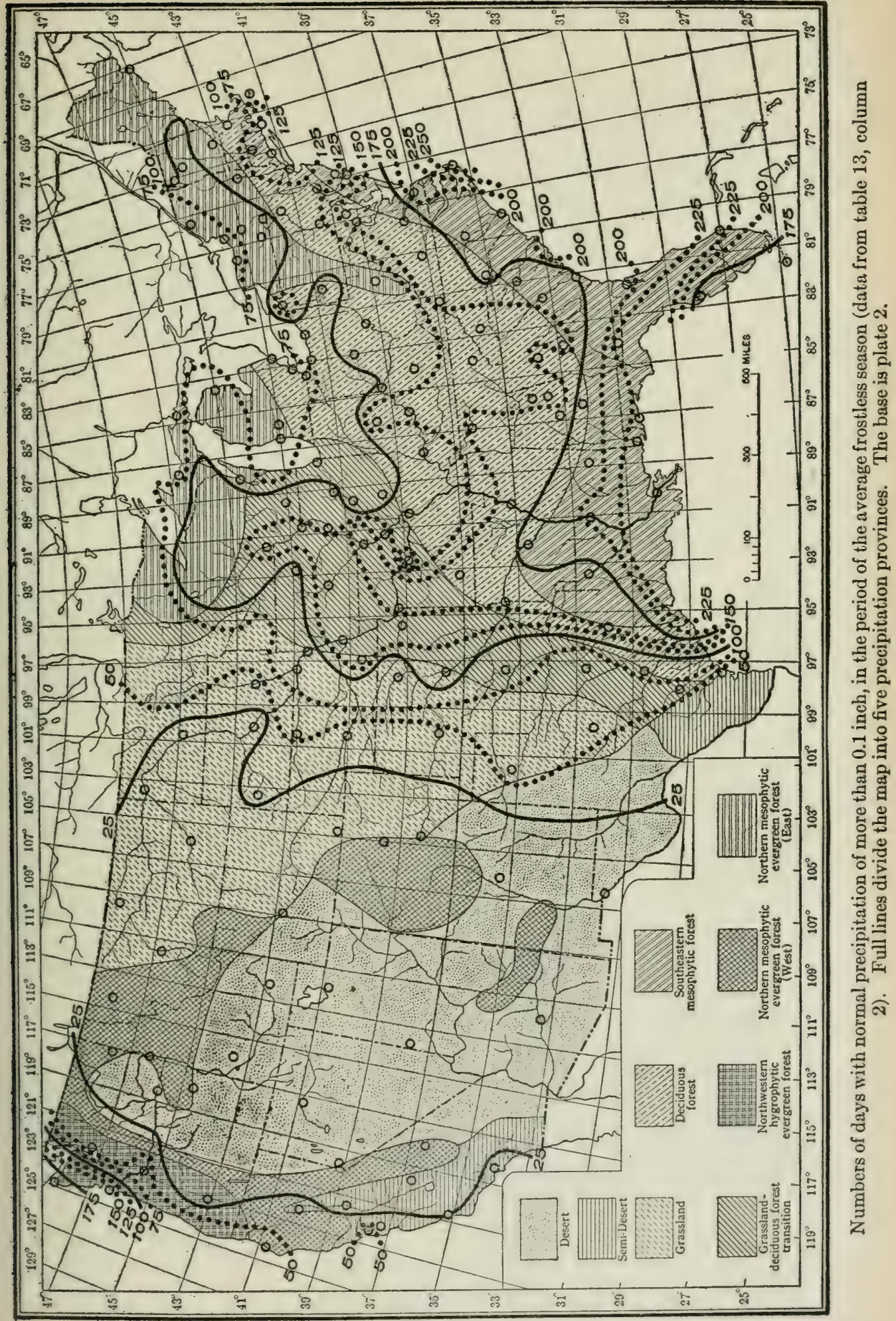




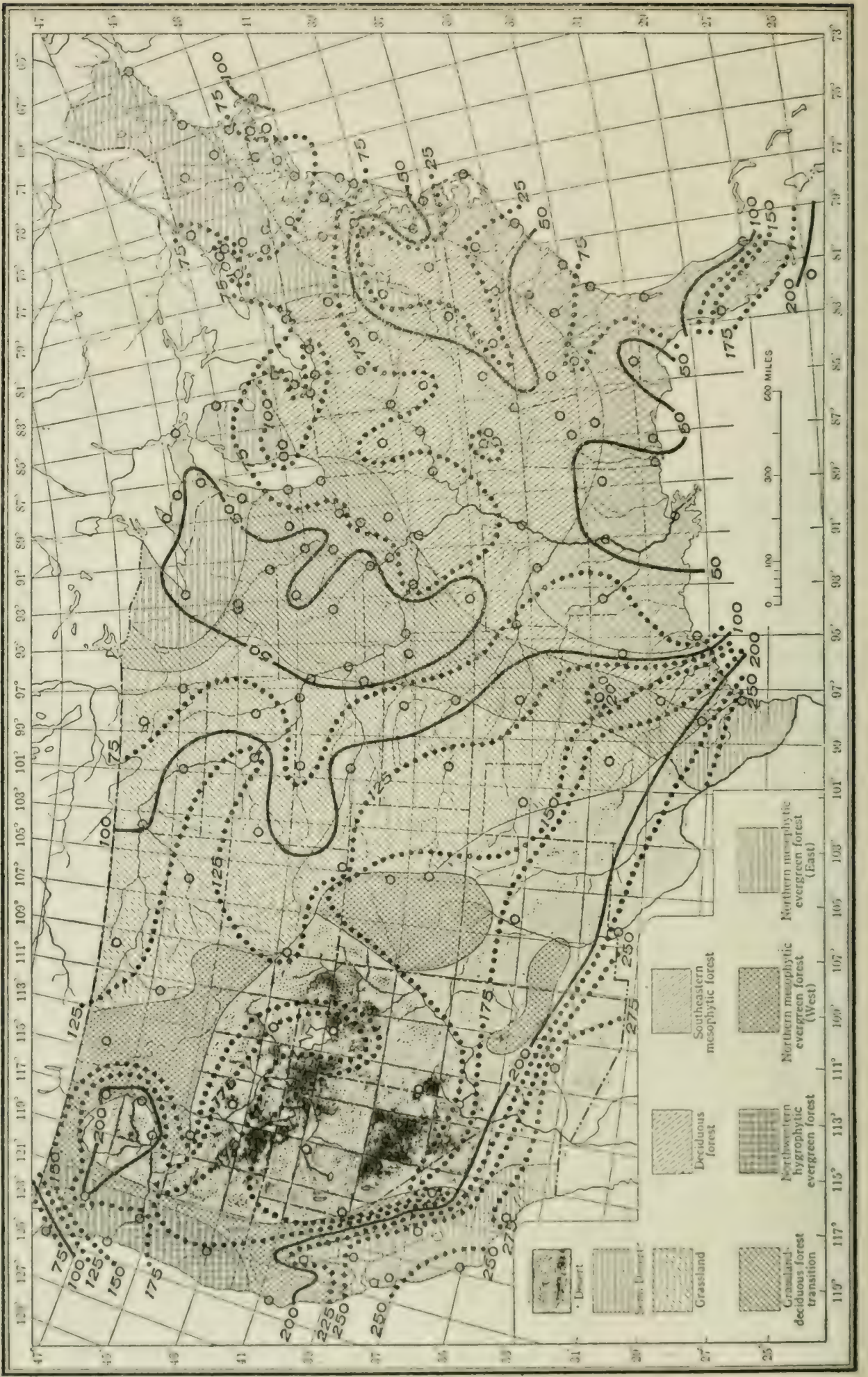

क्. 


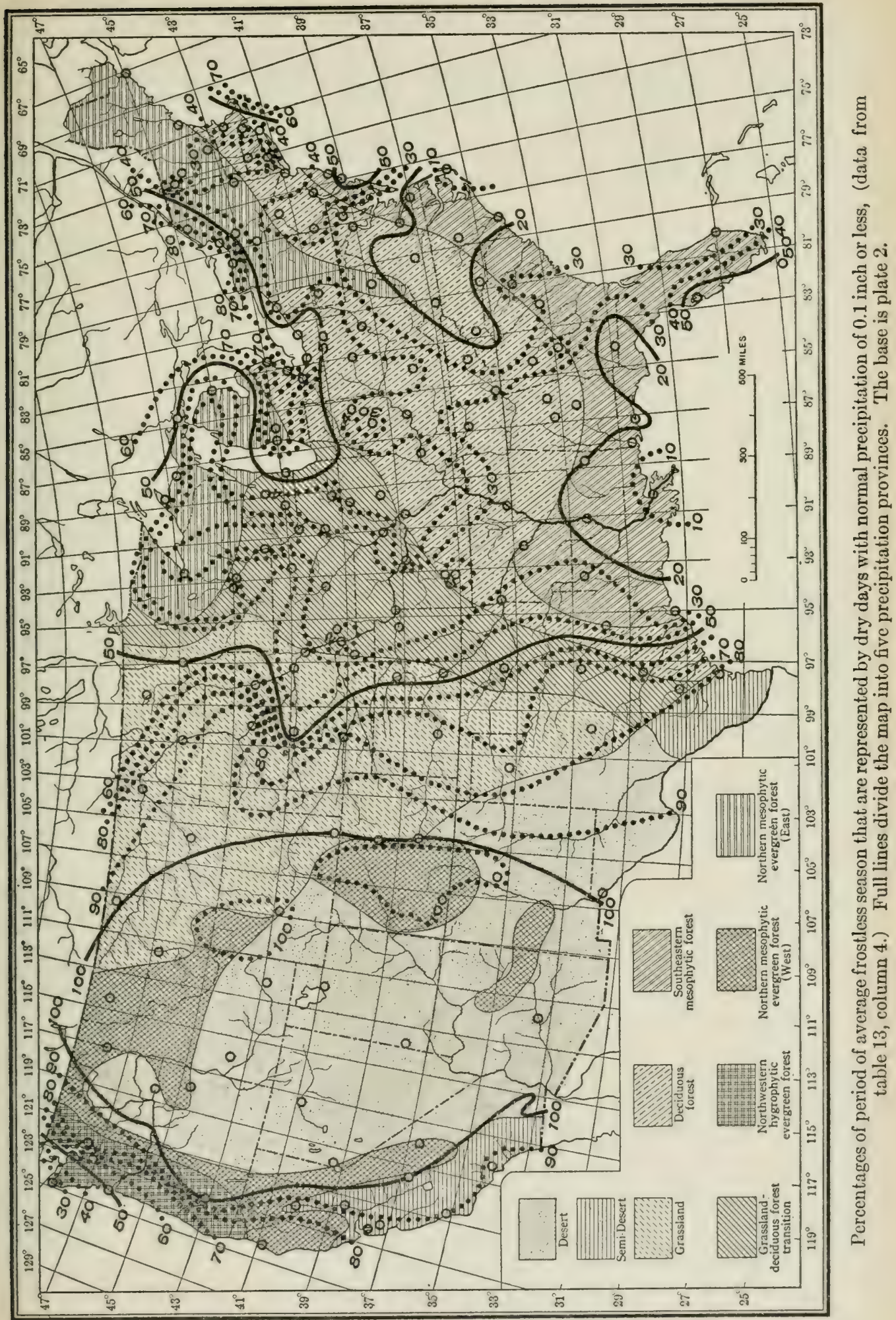


used. Normally rainy days are here considered (arbitrarily) as those with normal daily means (Bigelow's table, Bull. R) of over 0.10 inch. The days are counted without reference to when they occur in the period, so that we do not touch here upon the question of rainy periods. These data are given in column 2 of table 13.

TABLE 13.-Number of days in the period of the average frostless season with normal precipilation of more than 0.10 inch and with normal precipitation of 0.10 inch or less, the latter also expressed as percentage of the number of days in the average frostless season. (Plates 47,48 , and 49.)

\begin{tabular}{|c|c|c|c|c|c|c|c|}
\hline Station. & 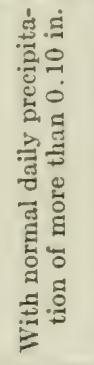 & 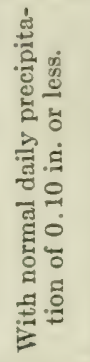 & 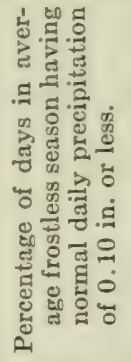 & Station. & 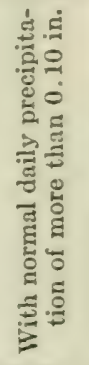 & 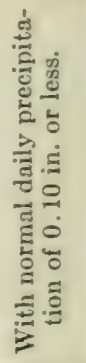 & 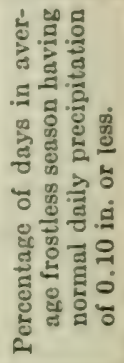 \\
\hline Alabama: & days. & days. & p.ct. & Idaho: & days. & days. & p. $c t$. \\
\hline Anniston . . . . . . . & 145 & 56 & 28 & Boise. . . . . . . . & 00 & 177 & 100 \\
\hline Birmingham...... & 166 & 65 & 28 & Lewiston......... & 00 & 202 & 100 \\
\hline Mobile........... & 248 & 31 & 11 & Pocatello.......... & 00 & 175 & 100 \\
\hline Montgomery....... & 177 & 66 & 27 & Illinois: & & & \\
\hline Arizona: & & & & Cairo............ & 122 & 90 & 42 \\
\hline Phoenix.......... & 00 & 283 & 100 & Chicago......... & 89 & 93 & 51 \\
\hline Arkansas: & & & & La Salle.......... & 103 & 65 & 39 \\
\hline Fort Smith....... & 159 & 71 & 30 & Peoria............ & 97 & s9 & 48 \\
\hline Little Rock........ & 182 & 55 & 23 & Springfield........ & 95 & 87 & 43 \\
\hline California: & & & & Indiana: & & & \\
\hline Eureka........... & 55 & 190 & 78 & Evansville........ & 120 & 83 & 41 \\
\hline Fresno........... & 00 & 258 & 100 & Indianapolis....... & 133 & 53 & 29 \\
\hline Independence..... & 00 & 199 & 100 & Iowa: & & & \\
\hline Los Angeles........ & 40 & 294 & sis & Charles City....... & So & 53 & 40 \\
\hline Red Bluff......... & 33 & $2: 31$ & 88 & Davenport......... & 117 & 57 & 33 \\
\hline Sacramento....... & 13 & 259 & 95 & Des Moines........ & 134 & 37 & 22 \\
\hline San Francisco...... & 62 & 257 & 81 & Dubuque......... & 141 & 35 & 20 \\
\hline San Jose.......... & 43 & 251 & 85 & Kieokuk......... & 152 & 45 & 23 \\
\hline San Luis Obispo.... & 25 & 235 & 90 & Sioux City....... & 97 & 49 & 34 \\
\hline Colorado: & & & & Kansas: & & & \\
\hline Denver............ & 00 & $15: 3$ & 100 & Concordia... & $\$ 9$ & $\therefore 4$ & 49 \\
\hline Pueblo............. & 00 & 163 & 100 & Dodge City....... & 48 & $13: 3$ & 74 \\
\hline Connecticut: & & & & Topeka.......... & 141 & 45 & 25 \\
\hline Hartford........... & 128 & 37 & 22 & Wichita........ & 111 & $\therefore 3$ & 43 \\
\hline New Haven........ & 148 & 32 & is & Kentucky: & & & \\
\hline Florida: & & & & Lexington . . . . . . & $10: 3$ & 84 & 45 \\
\hline Jacksonville........ & 198 & $9 \bar{i}$ & 32 & Louisville........ & 125 & (is) & 35 \\
\hline Jupiter........... & 234 & 84 & 26 & Louisiana: & & & \\
\hline Key West........ & 161 & 201 & 56 & New Orleans....... & $2 \times 4$ & 26 & 8 \\
\hline Pensacola......... & 226 & 59 & 21 & Shreveport........ & 166 & $s i$ & $3 t$ \\
\hline Tampa............ & 165 & 170 & 51 & Mnine: & & & \\
\hline Georgin: & & & & Eustport... & $10: 3$ & 6.4 & 38 \\
\hline Atlanta........... & 155 & 70 & 31 & Portland ......... & 100 & 57 & 36 \\
\hline Augusta......... & 160 & 6is & 30 & Maryland: & & & \\
\hline Macon.......... & 144 & 9.1 & 40 & Baltimore. . . . . . & $16 i 3$ & 50 & 21 \\
\hline Savannah........ & 175 & 85 & 34 & Washington, D. C. . & 151 & $4: 3$ & 22 \\
\hline Thomnsville...... & 229 & 28 & 11 & & & & \\
\hline
\end{tabular}


TABLE 13.-Number of days in the period of the average frostless season with normal precipitation of more than $0.10 \mathrm{inch}$ and with normal precipitation of 0.10 inch or less, the latter also expressed as percentage of the number of days in the average frostless season. (Plates 47, 48, and 49.)-Continued.

\begin{tabular}{|c|c|c|c|c|c|c|c|}
\hline Station. & 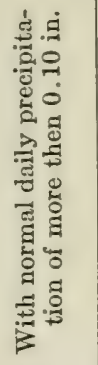 & 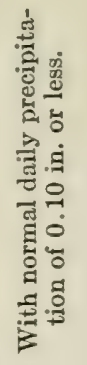 & 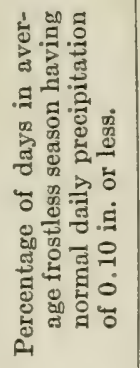 & Station. & 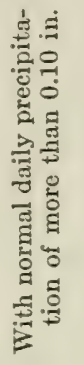 & 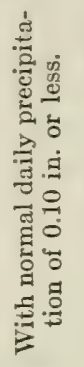 & 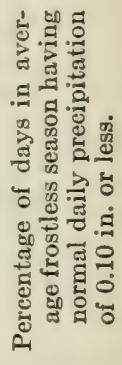 \\
\hline Massachusetts: & days. & days. & p.ct. & New York: & days. & days. & p. ct. \\
\hline 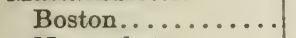 & 110 & 75 & 41 & 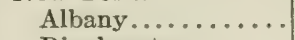 & 110 & 67 & 38 \\
\hline Nantucket........ & 55 & 154 & 74 & Binghamton....... & 95 & 63 & 40 \\
\hline Michigan: & & & & Buffalo............ & 80 & 93 & 54 \\
\hline Alpena... & 92 & 45 & 33 & Canton............ & 43 & 96 & 69 \\
\hline Detroit.... & 66 & 98 & 60 & Ithaca........... & 97 & 63 & 39 \\
\hline Escanaba.... & 105 & 35 & 25 & New York......... & 143 & 67 & 32 \\
\hline Grand Haven..... & 51 & 116 & 70 & Oswego............ & 55 & 120 & 69 \\
\hline Grand Rapids...... & 46 & 118 & 72 & Rochester......... & 35 & 136 & 80 \\
\hline Houghton........ & 84 & 68 & 45 & Syracuse............ & 98 & 73 & 43 \\
\hline Marquette......... & 71 & 69 & 49 & North Carolina: & & & \\
\hline Port Huron........ & $26^{\circ}$ & 129 & 83 & Asheville......... & 142 & 34 & 19 \\
\hline Sault Ste. Marie.... & 64 & 74 & 54 & Charlotte......... & 175 & 45 & 21 \\
\hline Minnesota: & & & & Hatteras......... & 256 & 00 & 00 \\
\hline Duluth........... & 124 & 28 & 18 & Raleigh.......... & 180 & 33 & 16 \\
\hline Minneapolis....... & 120 & 41 & 26 & Wilmington........ & 189 & 44 & 19 \\
\hline Moorhead........ & 67 & 65 & 49 & North Dakota: & & & \\
\hline St. Paul.......... & 106 & 53 & 33 & Bismarck.......... & 21 & 108 & 84 \\
\hline Mississippi: & & & & Devils Lake. . . . . . . & 53 & 68 & 56 \\
\hline Meridian........... & 185 & 45 & 20 & Williston......... & 21 & 98 & 82 \\
\hline Vicksburg......... & 207 & 45 & 18 & Ohio: & & & \\
\hline Missouri: & & & & Cincinnati......... & 101 & 93 & 48 \\
\hline Columbia.......... & 98 & 81 & 45 & Cleveland........ & 95 & 103 & 52 \\
\hline Hannibal......... & 127 & 57 & 31 & Columbus......... & 106 & 78 & 42 \\
\hline Kansas City....... & 151 & 45 & 23 & Sandusky......... & 89 & 106 & 54 \\
\hline St. Louis......... & 115 & 86 & 43 & Toledo............ & 49 & 125 & 72 \\
\hline Springfield......... & 161 & 26 & 14 & Oklahoma: & & & \\
\hline Montana: & & & & Oklahoma. & 90 & 124 & 58 \\
\hline Havre............ & 11 & 111 & 91 & Oregon: & & & \\
\hline Helena............ & 00 & 144 & 100 & Baker City........ & 00 & 127 & 100 \\
\hline Kalispell......... & 00 & 140 & 100 & Portland......... & 73 & 172 & 70 \\
\hline Miles City......... & 3 & 137 & 98 & Roseburg......... & 1 & 197 & 100 \\
\hline Nebraska: & & & & Pennsylvania: & & & \\
\hline Lincoln.... & 131 & 43 & 25 & Erie............ & 130 . & 64 & 33 \\
\hline North Platte...... & 29 & 122 & 81 & Harrisburg. . . . . . . & 113 & 83 & 42 \\
\hline Omaha............ & 138 & 32 & 19 & Philadelphia...... & 114 & 92 & 45 \\
\hline Valentine......... & 72 & 60 & 46 & Pittsburgh........ & 91 & 88. & 49 \\
\hline Nevada: & & & & Scranton.......... & 102 & 74 & 42 \\
\hline Reno........ & 00 & 138 & 100 & Rhode Island: & & & \\
\hline Winnemucca....... & 00 & 131 & 100 & Block Island....... & 142 & 76 & 35 \\
\hline New Hampshire: & & & & Providence........ & 111 & 79 & 42 \\
\hline Concord........... & 104 & 42 & 28 & South Carolina: & & & \\
\hline New Jersey: & & & & Charleston........... & 206 & 70 & 25 \\
\hline Atlantic City...... & 108 & 99 & 48 & Columbia........... & 154 & 77 & 33 \\
\hline Cape May.... . & 91 & 95 & 51 & South Dakota: & & & \\
\hline New Mexico: & & & & 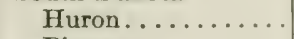 & 47 & 84 & 64 \\
\hline Santa Fe... & 8 & 179 & 96 & Pierre............ & 13 & 140 & 92 \\
\hline
\end{tabular}


TABLE 13.-Number of days in the perion of the average frostless srason with normal precipitation of more than 0.10 inch and with normal precipitation of 0.10 inch or less, the latter also expressed as percentage of the number of days in the average frostless season. (Plates 47, 48, and 49.)-Continued.

\begin{tabular}{|c|c|c|c|c|c|c|c|}
\hline Station. & 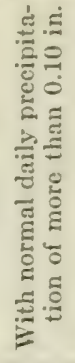 & 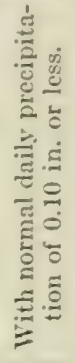 & 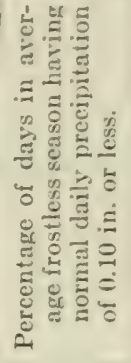 & Station. & 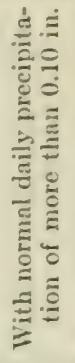 & 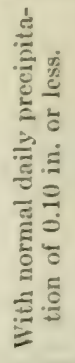 & 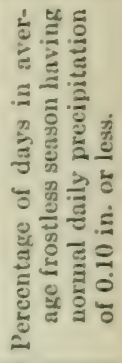 \\
\hline S. Dakota-Cont'd: & days. & days. & p. $\mathrm{cl}$. & Virginia: & days. & days. & p. ct. \\
\hline Rapid City........ & 28 & 115 & 81 & Lynchburg. . . . . . . & 166 & 35 & 17 \\
\hline Yankton......... & 99 & 55 & 36 & Norfolk........... & 202 & 28 & 12 \\
\hline Tennessee: & & & & Richmond......... & 160 & 55 & 26 \\
\hline Chattanooga....... & 143 & 64 & 31 & Wytheville....... & 156 & 19 & 11 \\
\hline Knoxville........ & 147 & 61 & 29 & Washington: & & & \\
\hline Memphis......... & 161 & 63 & 28 & North Head..... & 150 & 166 & 53 \\
\hline Nashville......... & 158 & 49 & 24 & Seattle........... & 46 & 200 & 81 \\
\hline Texas: & - & & & Spokane.......... & 00 & 202 & 100 \\
\hline Abilene........... & 53 & 192 & 78 & Tatoosh Island..... & 199 & 72 & 27 \\
\hline Amarillo.......... & 57 & 142 & 71 & Walla Walla....... & 00 & 216 & 100 \\
\hline Corpus Christi.... & 39 & 259 & 87 & West Virginia: & & & \\
\hline El Paso.......... & 2 & 234 & 99 & Elkins........... & 113 & 32 & 22 \\
\hline Fort Worth....... & 58 & 203 & 79 & Parkersburg....... & 120 & 59 & 33 \\
\hline Galveston........ & 257 & 74 & 22 & Wisconsin: & & & \\
\hline Palestine......... & 153 & 92 & 38 & - Green Bay......... & 85 & 68 & 44 \\
\hline San Antonio...... & 65 & 211 & 77 & La Crosse........ & 135 & 28 & 17 \\
\hline Taylor........... & 85 & 169 & 67 & Madison.......... & 105 & 71 & 40 \\
\hline Utah: & & & & Milwaukce........ & 72 & 90 & 55 \\
\hline Modena.......... & 00 & 130 & 100 & Wyoming: & & & \\
\hline Salt Lake City..... & 00 & 182 & 100 & Cheyenne........ & 00 & 118 & 100 \\
\hline Vermont: & & & & Lander............ & 4 & 104 & 95 \\
\hline Burlington......... & 108 & 35 & 25 & & & & \\
\hline Northfield......... & 86 & 40 & 32 & & & & \\
\hline
\end{tabular}

The values representing the number of normally rainy days in the period of the average frostless season were plotted in the regular way and the resulting chart is given as plate 47. This chart shows isoclimatic lines at intervals of 25 days; the total range for the country is from 284 days (New Orleans, Louisiana) to none at all (various stations in the arid region). The general zonation is seen to be very similar to that shown in plate 46 . The heavy lines show the four provinces above described.

(5) Number of Nomalut Dry Days in Period of Average Frostless Season.

('Table 13, Plate 4S.)

This index is the complement of the preceding one, and is derived by subtracting that from the number of days in the period, in each case. A dry day is thus one that has a normal daily mean precipitation (Bigelow, Bull. R) of 0.10 inch or less. If the former be considered as 
an index of raininess, this may be taken as an index of dryness or aridity. The values are given in column 3 of table 13 and are shown graphically on plate 48 . This chart shows a total range for the country of from 294 days (Los Angeles, California) to no days (Cape Hatteras, North Carolina). The isoclimatic lines here again represent increments of 25 days each, full lines being shown for the values 50, 100, and 200. In a general way, the climatic zonation of the country is similar to that of plates 46 and 47 , but this chart is markedly different from the others in certain details, and of course the actual values are different. Some of these differences will be considered below.

(6) Percentage of Days in Peiriod of Average frostless Season that are Dry Days (with Normal Precipitation of 0.10 Inch or Less). (Table 13, Plate 49.)

This index of precipitation intensity is obtained simply by expressing as percentage each value of the third column of table 13 , in terms of the corresponding length of the period of the average frostless season. These percentages are given in column 4 of table 13 . They express the relative frequency of dry days in the period.

These values are shown graphically by the chart of plate 49 . The total range for the country is from nil (Cape Hatteras, North Carolina) to 100 per cent (various stations in the arid region). The lines of the chart are drawn at intervals of 10 per cent, those for 20,50 , and 100 being full lines, and the zonation is once more similar to that of the other precipitation charts already mentioned.

(7) Lengti of Longest Normally Rainy Period in Period of Average Frostless Season. (Table 14, Plate 50.)

In many regions the duration factor for the favorable range of moisture conditions is not as great as that for the corresponding range of temperature conditions, and the former thus becomes the main duration factor influencing plant activities. In such cases only a portion of the period of the average frostless season is suitable for active plant growth. In southern Arizona, for example, the normal frostless season is very long (241 days at Tucson, from March 26 to November 22), but all of this period is practically without rain, excepting only a portion of the summer. The summer rainy period at Tucson extends from about July 1 to about September 15, but there is also a spring period of general plant activity, extending from the cessation of frost to about May 1. The latter period is nearly rainless, but the soil-moisture content is high, due to the residual effects of the winter precipitation. Thus there are here two periods of general plant activity within the period of the average frostless season, one from about March 26 to about May 1 (at which time the winter moisture is about dried out of the soil) and the other from about July 1 to about October 15 (when the summer moisture has largely disappeared). It 
is thus possible to consider two different periods of plant growth in this region, both of which lie within the limits set by the average frostless season, but neither of which is as long as that season. In some other regions there is but one period of general plant growth, but this is not as long as that of the average frostless season. When moisture conditions have been more thoroughly studied it may become possible to consider both the frostless season and that with moist soil, in deriving the duration factor for plant growth in general, but we have not found it expedient to attempt this at the present time; the relations encountered are too complicated and information is too meager.

Nevertheless, we have been able to derive two duration factors for precipitation, which may be superimposed upon the temperature duration factor here generally employed. These two new factors are the lengths of the longest rainy period and of the longest dry period within the period of the average frostless season. The first of these is considered here and the other will receive attention under the nextfollowing heading.

In attempting to derive an index of the normal duration of moist conditions, we have again begun our computations with the data of normal daily precipitation given by Bigelow (Bulletin $R$ ). Our procedure has been quite arbitrary. In the case of each station the series of daily normals given by Bigelow has been considered as separated into a series of overlapping groups of 5 days each. Numbering the days of the period of the average frostless season consecutively, days 1 to 5 constitute the first group, days 2 to 6 constitute the second group, days 3 to 7 constitute the third, etc. The 5 daily normals for each group are averaged to give the mean daily normal precipitation for that group, and these means are set down to form a new series. Beginning with the first (in the period of the average frostless season) of these new group means, the groups are marked as rainy or dry, accordingly as the value of their means are or are not greater than 0.10 inch. Thus, a 5-day group is called rainy if its group-mean is over 0.10 inch, dry if this mean is 0.10 inch or less. If we designate the successive 5-day groups throughout the normal frostless season by the alphabet letters, and if we follow each letter by an R or D, to denote "rainy" or "dry," as the case may be, we obtain a series more or less similar to the following: IR, BR, CR, DR, ED, FD, GR, HD, ID, JR, KR, I.D, MR, NR, etc. In this example the first four groups (A to D) are seen to be "rainy." Then follow two "dry" groups (E, F), after which is a single "rainy" group (G), which in turn is followed by two "dry" groups (H, I), etc. Now, the last day of each "rainy" group is eonsidered as occurring in a normally "rainy" period, and the last day of each "dry" group is considered as within a normally "dry" period, and it this becomes possible to determine the extents of the various "rainy" and "dry" periods thus established. If, for example, group 
A includes April 1 to 5, group B April 2 to 6, group C April 3 to 7, etc., it follows that the period April 1 to 8 (inclusive) is a "rainy" one; the period April 9 to 10 is "dry"; that of April 11 (a single day) is "rainy"; that of April 12 to 13 is "dry," etc.

The dates of beginning and ending of each normally "rainy" and normally "dry" period of the normal frostless season having been thus obtained for each station, the length of each period is noted, in days, and the lengths of the longest normally rainy period and of the longest normally dry period become the two indices desired for the station in question.

The beginning and ending and the length of each normally dry period in the period of the average frostless season are given in the second column of table 14, and the corresponding dates and length of the longest normally rainy period are given in the third column. Roman numerals refer to months, the arabic numerals not in parentheses represent the days of the month, and these data are followed, in each case, by the length of the period, in parentheses. Thus, the longest normally rainy period in the period of the average frostless season for Anniston, Alabama, extends from May 18 to September 8 and includes 114 days.

As has been remarked, this method of treatment is quite arbitrary, but it seems to furnish indices of normal raininess and droughtiness that may be valuable. At least, these indices are worthy of a test, and may be employed till more satisfactory ones may be devised. It should be noted that the smoothing process applied to the precipitation data by Bigelow (in deriving the daily normals) is here overlaid by another very efficient smoothing process of our own (the use of 5-day averages), so that the natural irregularity of precipitation is very largely obliterated, which is to be desired when normals are requisite. It may also be mentioned that the indices here set forth might have a still greater value for such work as this if the constant 0.10 inch were made somewhat smaller. Such an alteration would of course render the normally rainy periods longer and the normally dry periods shorter. The testing of such modifications may, however, be left to a later time, and to other workers, if they will take up this important phase of the climatology of precipitation. 


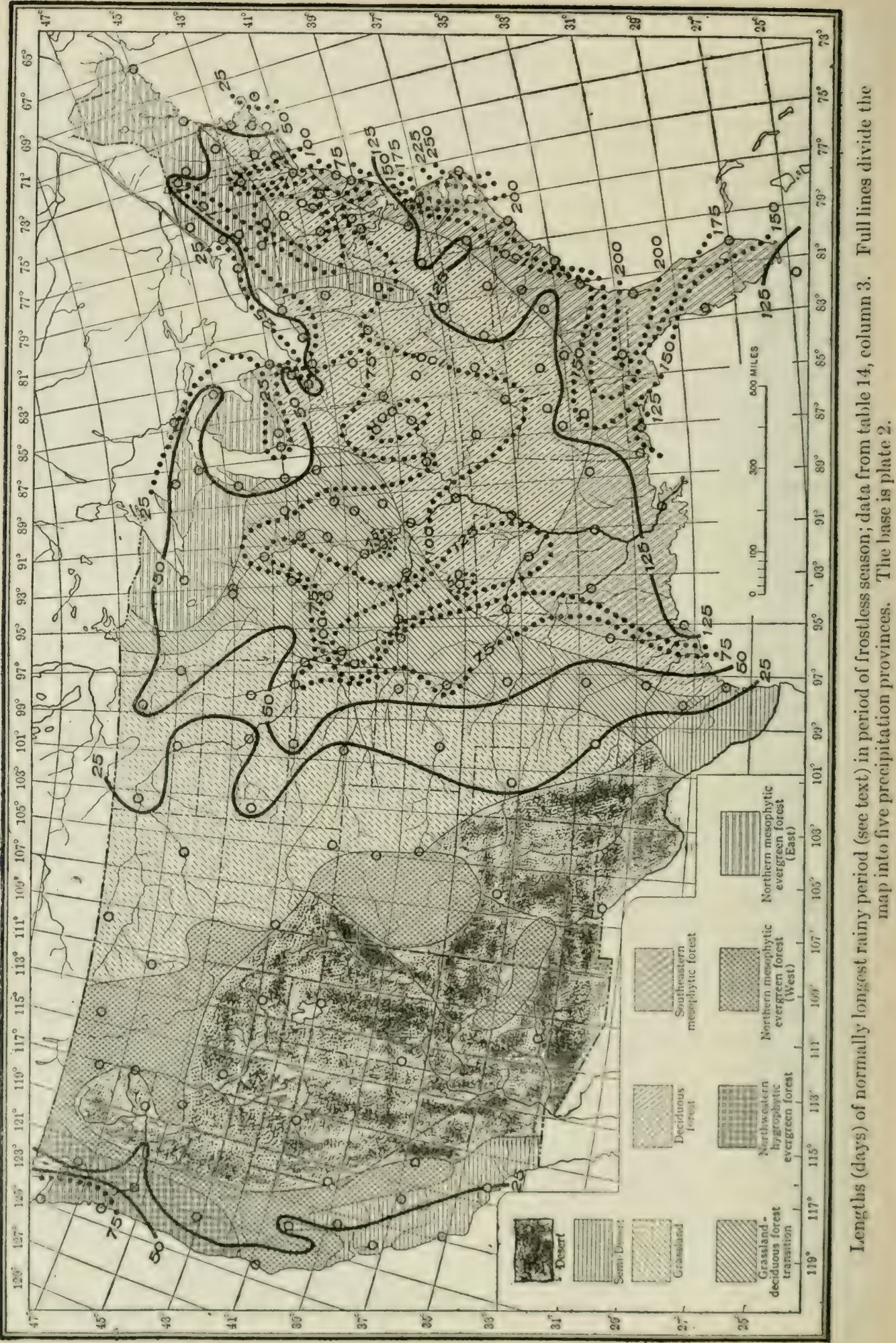




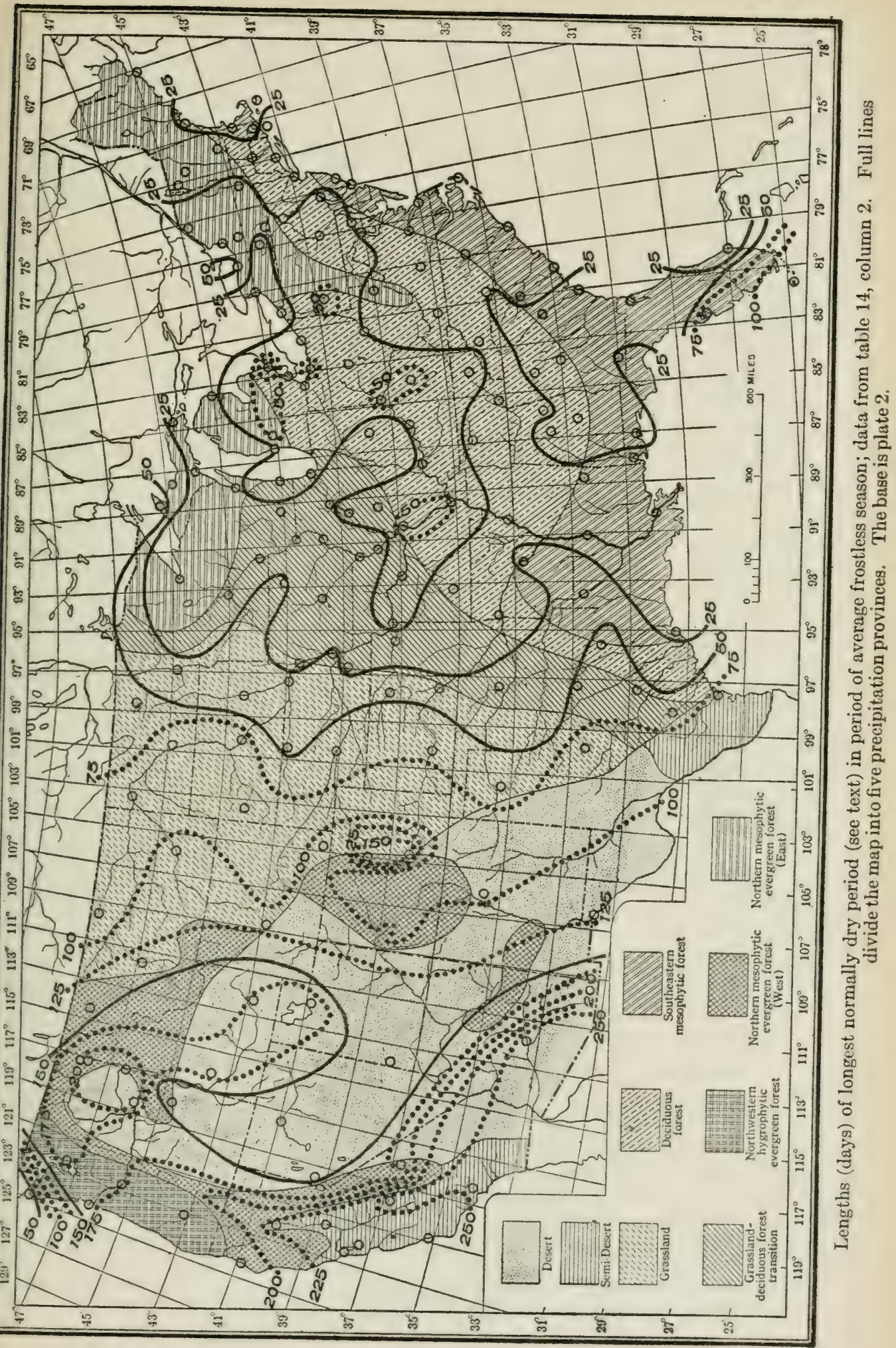


TABLE 14.-Beginning, ending, and duration of each normally dry period and of longest normally rainy period within the period of the average frostless season. (Plates 50 and 51.)

A normally "dry period" begins on the last day of the first 5-day period whose average normal daily precipitation is not over 0.10 inch; a normally "rainy period" begins on the last day of the first 5-day period whose average normal daily precipitation is greater than 0.10 inch. Months are represented by Roman and days of the month by Arabic numerals; numbers in parentheses are the duration of the respective periods, in days, these being in full-face type for the longest dry period in each case.

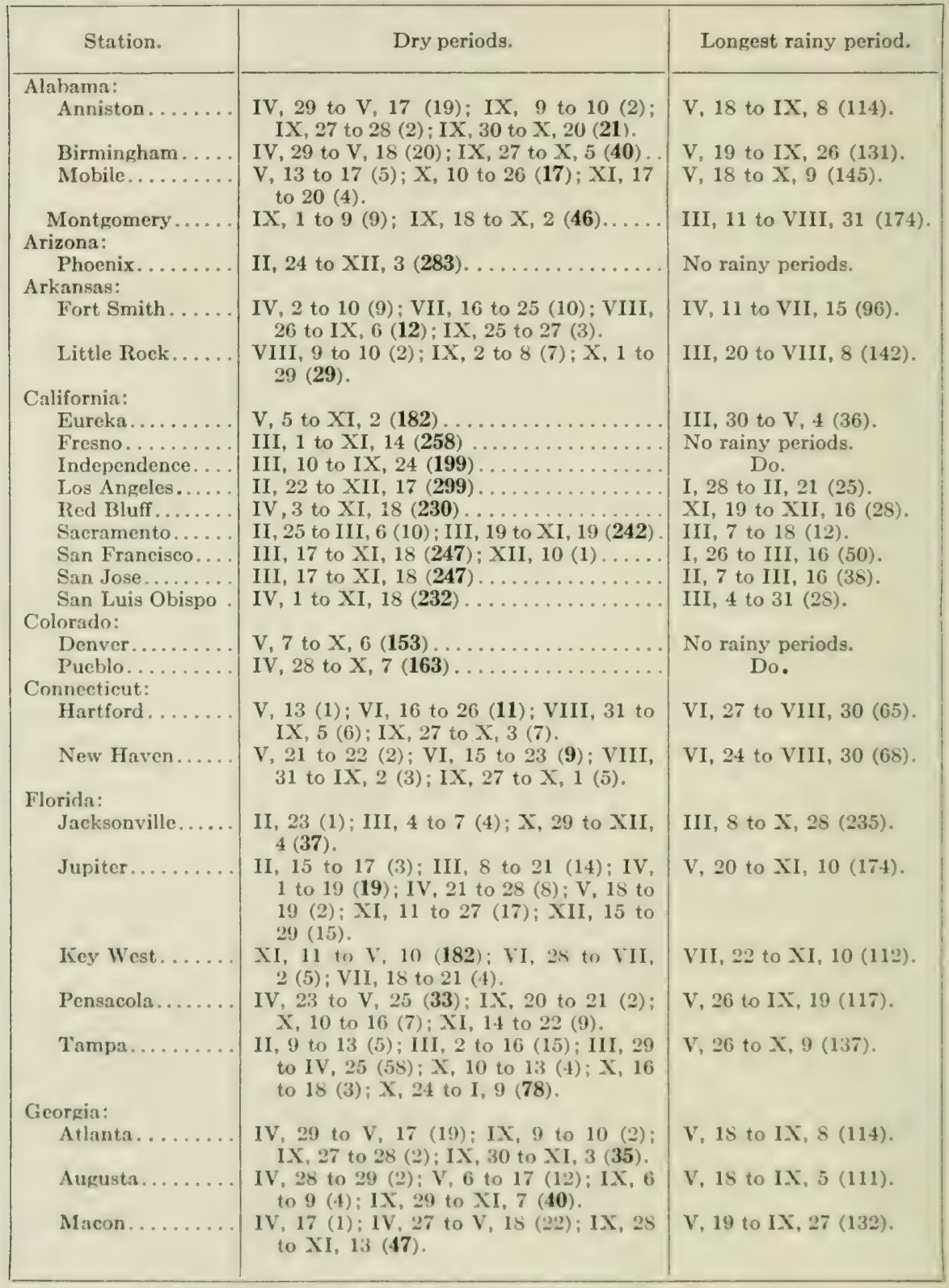


TABLE 14.-Beginning, ending, and duration of each normally dry period and of longest normally rainy period within the period of the average frostless season. (Plates 50 and 51.)-Continued.

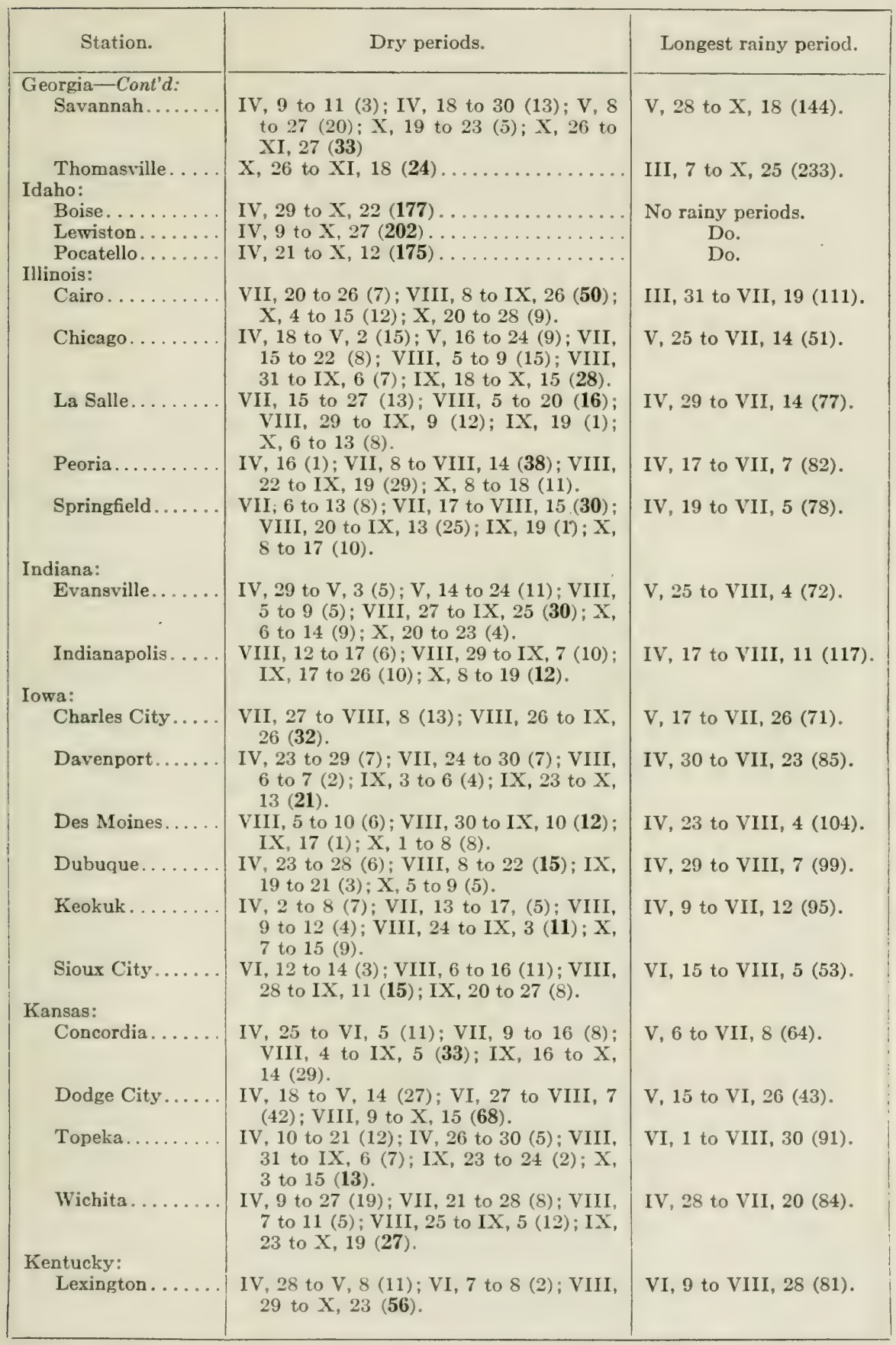


TABLE 14.-Beginning, ending, and duration of ench normally dry period and of longest normally rainy period within the period of the average frostless season, (Plates 50 and 51.)-Continued.

\begin{tabular}{|c|c|c|}
\hline Station. & Dry periods. & Longest rainy period. \\
\hline $\begin{array}{l}\text { Kentucky-Cont'd: } \\
\text { Jouisville....... }\end{array}$ & $\begin{array}{l}\text { V, } 13 \text { to } 18(6) ; \text { VIII, } 30 \text { to } \mathrm{IX}, 26(28) \\
\quad \mathrm{X}, 4 \text { to } 22(19) \text {. }\end{array}$ & V, 19 to VIII, 29 (103). \\
\hline $\begin{array}{l}\text { Louisiana: } \\
\quad \text { New Orleans.... }\end{array}$ & & V. 18 to IX. 7 (143) \\
\hline $\begin{array}{l}\text { New Orleans..... } \\
\text { Shreveport...... }\end{array}$ & $\begin{array}{l}\text { V, } 10 \text { to } 17(8) ; I X, 8 \text { to } 25(18) ; X I, 1(1) \\
\text { VI, } 19 \text { to } 22(4) ; \text { VII, } 10 \text { to } 17(8) ; \text { VIII, } \\
3 \text { to IX, } 5(34) ; \text { IX, } 20 \text { to } 23(4) ; I X \text {, } \\
28 \text { to X, } 17(20) \text {. }\end{array}$ & III, 5 to VI, 18 (106). \\
\hline $\begin{array}{l}\text { Maine: } \\
\text { Eastport.. }\end{array}$ & $\mathrm{V}, 9$ to $12(4) ; \mathrm{VI}, 10$ to $19(10) ; \mathrm{VII}$, & VI, 20 to VII, 30 (41). \\
\hline Portland. . & $\begin{array}{l}\text { (18); IX, } 26 \text { to X, } 5 \text { (10). } \\
\text { VI, } 26 \text { to VII, } 21(26) \text {; VIII, } 30 \text { to IX, } \\
12(14) ; X, 1 \text { to } 3(3) \text {. }\end{array}$ & $\mathrm{V}, 15$ to $\mathrm{VI}, 25(42)$ \\
\hline $\begin{array}{l}\text { Maryland: } \\
\text { Baltimore. }\end{array}$ & $\begin{array}{l}\text { IV, } 16 \text { to } 23(8) ; \mathrm{VI}, 1(1) ; \mathrm{IX}, 27 \text { to } \mathrm{X} \\
21(25) ; \mathrm{XI}, 1 \text { to } 3(3) .\end{array}$ & VI, 2 to IX, 26 (117). \\
\hline Washington, D. C. & $\begin{array}{l}\text { IV, } 18 \text { to } 23(6) ; I X, 3 \text { to } 8(6) ; \mathrm{IX} \text {, } \\
26 \text { to } \mathrm{X}, 19(\mathbf{2 4}) \text {. }\end{array}$ & IV, 24 to IX, 2 (132). \\
\hline $\begin{array}{l}\text { Massachusetts: } \\
\text { Boston...... }\end{array}$ & $\begin{array}{l}\text { IV, } 21 \text { to } 22(2) ; \mathrm{V}, 13(1) ; \mathrm{V}, 31(1) \\
\text { VI, } 15 \text { to VII, } 19(35) ; \text { VIII, } 31 \text { to }\end{array}$ & VII, 20 to VIII, 30 (41). \\
\hline Nantucket.. & $\begin{array}{l}\text { IX, } 10(11) \\
\text { IV, } 17 \text { to V, } 23(37) ; \mathrm{VI}, 4 \text { to } 20(17) ; \\
\text { VI, } 22 \text { to VII, } 4(44) ; \text { VIII, } 22 \text { to } \\
\text { IX, } 13(23) ; \mathrm{IX}, 20 \text { to } \mathrm{X}, 1(12) ; \mathrm{X} \\
19 \text { to } 24(6) ; \mathrm{XI}, 2 \text { to } 5(4) \text {. }\end{array}$ & VIII, 5 to $21(17)$. \\
\hline $\begin{array}{l}\text { Michigan: } \\
\text { Alpena... }\end{array}$ & $\begin{array}{l}\mathrm{V}, 25 \text { to } 28(4) \text {; VII, } 9 \text { to } 16(8) \text {; VII, } \\
22 \text { to VIII, } 6(\mathbf{1 6}) \text {. }\end{array}$ & VIII, 7 to IX, 28 (53). \\
\hline Detroit. & $\begin{array}{l}\mathrm{V}, 1 \text { to } 6(6) ; \mathrm{V}, 11 \text { to } 21(11) ; \mathrm{VIII}, 2 \\
\text { to } 18(17) ; \mathrm{VIII}, 25 \text { to } \mathrm{X}, 11(48)\end{array}$ & V, 22 to VIII, 1 (72). \\
\hline $\begin{array}{l}\text { Escanalia....... } \\
\text { Grand Haven. }\end{array}$ & VII, 10 to 19 (10); VIII, 20 to 31 (12), . & $\begin{array}{l}\text { V, } 17 \text { to VII, } 9(54) \text {. } \\
\text { V, } 7 \text { to } 28(22) \text {. }\end{array}$ \\
\hline Grand Haven... & $\begin{array}{l}\text { VI, } 9 \text { to VII, } 2(24) ; \mathrm{VII}, 4 \text { to } 28(25) ; \\
\text { VIII, } 7 \text { to } 21 \text { (15); VIII, } 26 \text { to IX, } 8 \\
\text { (14); } \mathrm{X}, 1 \text { to } 12 \text { (12). }\end{array}$ & \\
\hline Grand Rapids... & $\begin{array}{l}\mathrm{V}, 2 \text { to } 7(6) ; \mathrm{VI}, 1 \text { to } \mathrm{VII}, 28(58) ; \mathrm{VIII}, \\
7 \text { to IX, } 8(33) ; \mathrm{X}, 1 \text { to } 12(12) \text {. }\end{array}$ & $\mathrm{V}, 8$ to $31(24)$ \\
\hline Houghton. . & $\begin{array}{l}\text { V, } 25 \text { (1); VII, } 8 \text { to VIII, } 31 \text { (55).... } \\
\text { VII } 8 \text { to } 19 \text { (12); VII } 29 \text { to VIII, } 31 \text { (34). }\end{array}$ & $\begin{array}{l}\text { V, } 26 \text { to VII, } 7 \text { (43). } \\
\text { V, } 16 \text { to VII, } 7 \text { (53). }\end{array}$ \\
\hline Port Huron..... & $\begin{array}{l}\mathrm{V}, 19 \text { to } 23(5) ; \mathrm{VI}, 14 \text { to } \mathrm{IX}, 12(\mathbf{9 1}) \\
\text { IX, } 19 \text { to } \mathrm{X}, 9(2 \mathrm{I}) \text {. }\end{array}$ & $\begin{array}{l}\text { V, } 16 \text { to VII, } 7(53) \text {. } \\
\text { V, } 24 \text { to VI, } 13(21) \text {. }\end{array}$ \\
\hline Sault Ste. Marie. & $\begin{array}{l}\text { V, } 15 \text { (1); VI, } 9 \text { to } 26(18) ; \text { VII, } 8 \text { to } 30 \\
(23) ; \text { VIII, } 12 \text { to } 27 \text { (16); IX, } 21 \text { to } \\
29 \text { (9). }\end{array}$ & $\mathrm{V}, 16$ to VI, S (24). \\
\hline $\begin{array}{l}\text { Minnesota: } \\
\text { Iuluth.. }\end{array}$ & $\begin{array}{l}\mathrm{V}, 5 \text { (1); VII, } 17 \text { to } 27 \text { (11); VIII, } 28 \text { to } \\
31 \text { (4), }\end{array}$ & V, 6 to VII, $16(72)$ \\
\hline Minneapolis. & $\begin{array}{l}\text { VII, } 12 \text { to } 24(13) ; \text { VIII, } 15 \text { to } 18 \text { (4); } \\
\text { IX, } 26 \text { to } \mathrm{X}, 1(6) \text {. }\end{array}$ & IV, 30 to VII, 11 (73). \\
\hline Moorhead. & $\begin{array}{l}\text { V, } 14 \text { to } 16(3) ; \mathrm{V}, 25 \text { to } 31 \text { (7); VII, } \\
23 \text { to VIII, } 13(22) \text {; VIII, } 28 \text { to IX, } \\
22(26) \text {. }\end{array}$ & VI, 1 to VII, 22 (52). \\
\hline St. Paul. . & $\begin{array}{l}\mathrm{IV}_{1} 28 \text { to } \mathrm{V}, 8(11) ; \mathrm{VII}, 12 \text { to } 25(\mathbf{1 4}) \\
\text { VIII, } 18 \text { to } 26(9) ; \mathrm{IX}, 23 \text { to } \mathrm{X}, 3(11) .\end{array}$ & V, 9 to VII, 11 (64). \\
\hline $\begin{array}{l}\text { Mississipni: } \\
\text { Meridian. }\end{array}$ & $\begin{array}{l}\mathrm{V}, 18 \text { to } 19(2) ; \mathrm{VHI}, 20 \text { to } 28(9) ; \mathrm{X} \text {, } \\
\text { 4 to } 27(24) \text {. }\end{array}$ & V. 20 to VIII, 19 (92). \\
\hline
\end{tabular}


TABLE 14.-Beginning, ending, and duration of each normally dry period and of longest normally rainy period within the period of the average frostless season. (Plates 50 and 51.)-Continued.

\begin{tabular}{|c|c|c|}
\hline Station. & Dry periods. & Longest rainy period. \\
\hline $\begin{array}{l}\text { Mississippi-Cont'd: } \\
\text { Vicksburg........ }\end{array}$ & $\begin{array}{l}\text { V, } 14 \text { to } 16(3) ; \text { VIII, } 20 \text { to } 27(8) ; I X \text {, } \\
6 \text { to } 12(7) ; X, 3 \text { to } 21(\mathbf{1 9}) \text {. }\end{array}$ & V, 17 to VIII, 19 (95). \\
\hline $\begin{array}{l}\text { Missouri: } \\
\quad \text { Columbia... }\end{array}$ & $\begin{array}{l}\text { VII, } 18 \text { to } 21(4) ; \mathrm{VII}, 28 \text { to VIII, } 17 \\
\text { (21); VIII, } 28 \text { to } \mathrm{X}, 14(48) \text {. }\end{array}$ & IV, 19 to VII, $17(90)$ \\
\hline Hannibal... & $\begin{array}{l}\text { IV, } 15 \text { to } 20(6) ; \mathrm{VI}, 9 \text { to } 18(10) \text {; VII, } \\
12 \text { to } 15(4) ; \text { VIII, } 24 \text { to IX, } 5(13) \\
\text { IX, } 30 \text { to X, } 15(16) \text {. }\end{array}$ & IV, 21 to VI, 8 (49). \\
\hline Kansas City..... & $\begin{array}{l}\text { IV, } 14(1) ; \mathrm{IV}, 16 \text { to } 17(2) ; \mathrm{IX}, 1 \text { to } 3 \\
(2) ; \mathrm{IX}, 27 \text { to } \mathrm{X}, 23 \text { (27). }\end{array}$ & IV, 18 to VIII, 31 (136). \\
\hline St. Louis........ & $\begin{array}{l}\text { VII, } 13 \text { to } 19(7) ; \text { VIII, } 5 \text { to } \text { IX, } 24 \\
(51) ; X, 4 \text { to } 18(15) ; X, 20 \text { to } 24(5) .\end{array}$ & IV, 7 to VII, 12 (97). \\
\hline Springfield....... & $\mathrm{X}, 4$ to $18(\mathbf{1 5}) \ldots \ldots \ldots \ldots \ldots \ldots \ldots$ & IV, 15 to $\mathrm{X}, 3$ (172). \\
\hline Montana: & V 16 to VI 6 (92) : VT 20 to IV 14 (87) & \\
\hline $\begin{array}{l}\text { Hawre........... } \\
\text { Helena.......... }\end{array}$ & $\begin{array}{l}V, 16 \text { to } V 1,6(22) ; V 1,20 \text { to } \mathrm{IX}, 14(87) . \\
V, 8 \text { to } \mathrm{IX}, 28(\mathbf{1 4 4}) \ldots \ldots \ldots \ldots \ldots\end{array}$ & $\begin{array}{l}\text { VI, } 7 \text { to } 19(13) \text {. } \\
\text { No rainy periods. }\end{array}$ \\
\hline Kalispell......... & $\mathrm{V}, 14$ to $\mathrm{IX}, 30(\mathbf{1 4 0}) \ldots \ldots \ldots$ & Do. \\
\hline Miles City....... & V, 8 to VI, 6 (29); VI, 13 to IX, 24 (104). & $\mathrm{VI}, 7$ to $12(6)$ \\
\hline $\begin{array}{l}\text { Nebraska: } \\
\text { Lincoln. }\end{array}$ & $\begin{array}{l}\text { IV, } 20 \text { to } 22(3) \text {; VIII, } 26 \text { to } \mathrm{IX}, 10(16) \text {; } \\
\text { IX, } 20 \text { to } \mathrm{X}, 10(21) \text {. }\end{array}$ & IV, 23 to VIII, 25 (125). \\
\hline North Platte. & $\begin{array}{l}\mathrm{V}, 2 \text { to } 19(18) ; \mathrm{VI}, 9 \text { to } 10(2) ; \mathrm{VI}, 25 \\
\text { to VII, } 23 \text { (29); VII, } 29 \text { to IX, } 29(63) \text {. }\end{array}$ & V, 20 to VI, $8(20)$ \\
\hline Oms & IX, 1 to $7(7)$;X, 19 to $X, 13(25) \ldots \ldots$ & IV, 27 to VIII, 31 (127). \\
\hline Vale & $\begin{array}{l}\text { V, } 10 \text { to } 15(6) ; \text { VII, } 14 \text { to } 17(4) \text {; VII, } \\
20(1) ; \text { VIII, } 12 \text { to IX, } 18(38) \text {. }\end{array}$ & V, 16 to VIX, 13 (59). \\
\hline $\begin{array}{l}\text { Nevada: } \\
\text { Reno. }\end{array}$ & V, 17 to IX, 31 (138). & No rainy periods, \\
\hline Winnemucca..... & $\mathrm{V}, 16$ to $\mathrm{IX}, 23(\mathbf{1 3 1}) \ldots \ldots \ldots \ldots$ & Do. \\
\hline $\begin{array}{l}\text { New Hampshire: } \\
\text { Concord...... }\end{array}$ & $\begin{array}{l}\text { V, } 8 \text { to } 14(7) ; \text { VI, } 28(1) ; \text { VIII, } 31 \text { to } \\
\text { IX, } 13(14) ; \mathrm{IX}, 28 \text { to } 30 \text { (3). }\end{array}$ & VI, 29 to VIII, 30 (63). \\
\hline $\begin{array}{l}\text { New Jersey: } \\
\text { Atlantic City. }\end{array}$ & $\begin{array}{l}\text { IV, } 13 \text { to } 24(12) ; V, 3 \text { to } 13(11) ; \mathrm{V}, 23 \\
\quad \text { to VI, } 8(17) ; \mathrm{VI}, 19 \text { to } 28(10) .\end{array}$ & VII, 14 to VIII, 29 (47). \\
\hline Cape May & $\begin{array}{l}\text { IV, } 18 \text { to } 25(8) ; \mathrm{V}, 3 \text { to } 14(12) ; \mathrm{V}, 24 \\
\text { to VI, } 8(\mathbf{1 6}) ; \mathrm{VI}, 19 \text { to } 28(10) ; \mathrm{VII}, \\
9 \text { to } 12(4) ; \mathrm{VIII}, 30 \text { to } \mathrm{IX}, 8(10) ; \mathrm{IX} \text {, } \\
23 \text { to } \mathrm{X}, 6(14) ; \mathrm{X}, 15 \text { to } 20(6) .\end{array}$ & VII, 13 to VIII, 29 (48). \\
\hline $\begin{array}{l}\text { New Mexico: } \\
\text { Santa Fe... }\end{array}$ & $\begin{array}{l}\text { IV, } 16 \text { to VII, } 15 \text { (91); VII, } 26 \text { to } \mathrm{IX} \text {, } \\
19 \text { (86). }\end{array}$ & VII, 16 to $25(10)$ \\
\hline $\begin{array}{l}\text { New York: } \\
\text { Albany. }\end{array}$ & $\begin{array}{l}\text { IV, } 24 \text { to } V, 21(28) ; I X, 5 \text { to } 9(5) ; I X, \\
24 \text { to } X, 7(14) ; X, 13 \text { to } 17(5) \text {. }\end{array}$ & V, 22 to IX, 4 (106). \\
\hline Binghamton..... & $\begin{array}{l}\mathrm{V}, 3 \text { to } 15(13) ; \mathrm{VII}, 10 \text { to } 14(5) \text {; VIII, } \\
12 \text { to } 18(7) ; \mathrm{IX}, 5 \text { to } \mathrm{X}, 6(32) .\end{array}$ & V, 16 to VII, 9 (55). \\
\hline Buffalo.. & $\begin{array}{l}\text { IV, } 27 \text { to } V, 17(21) ; V I, 5 \text { to } 18(14) \\
\text { VIII, } 31 \text { to IX, } 11(12) \text {. }\end{array}$ & VI, 19 to VIII, 30 (73). \\
\hline Canton... & $\begin{array}{l}\text { V, } 10 \text { to } 25(16) ; \mathrm{V}, 28 \text { to } 30(3) ; \mathrm{VII}, \\
12 \text { to } 23(12) ; \text { VII, } 30 \text { to VIII, } 27(29) \\
\text { IX, } 3 \text { to } 14(12) ; \mathrm{IX}, 19 \text { to } 25(7) \text {. }\end{array}$ & V, 31 to VII, 11 (42). \\
\hline Ithaca... & $\begin{array}{l}\mathrm{V}, 5 \text { to } 13(9) ; \mathrm{VIII}, 11 \text { to } 18(8) ; \mathrm{VIII} \text {, } \\
31 \text { to } \mathrm{IX}, 1(2) ; \mathrm{IX}, 3 \text { to } 13(11) ; \mathrm{IX} \text {, } \\
16 \text { to } \mathrm{X}, 7(22) \text {. }\end{array}$ & V, 14 to VIII, $10(89)$ \\
\hline New York... & $\begin{array}{l}\text { IV, } 16 \text { to } 23(8) ; V, 16 \text { to } 23(8) ; \mathrm{IX} \text {, } \\
1 \text { to } 8(8) ; \mathrm{IX}, 26 \text { to } \mathrm{X}, 4(9)\end{array}$ & V, 24 to VIII, $31(100)$ \\
\hline
\end{tabular}


TABLE 14.-Beginning, ending, and duration of each normally dry period and of longest normally rainy period within the period of the average frostless season. (Plates 50 and 51.)-Continued.

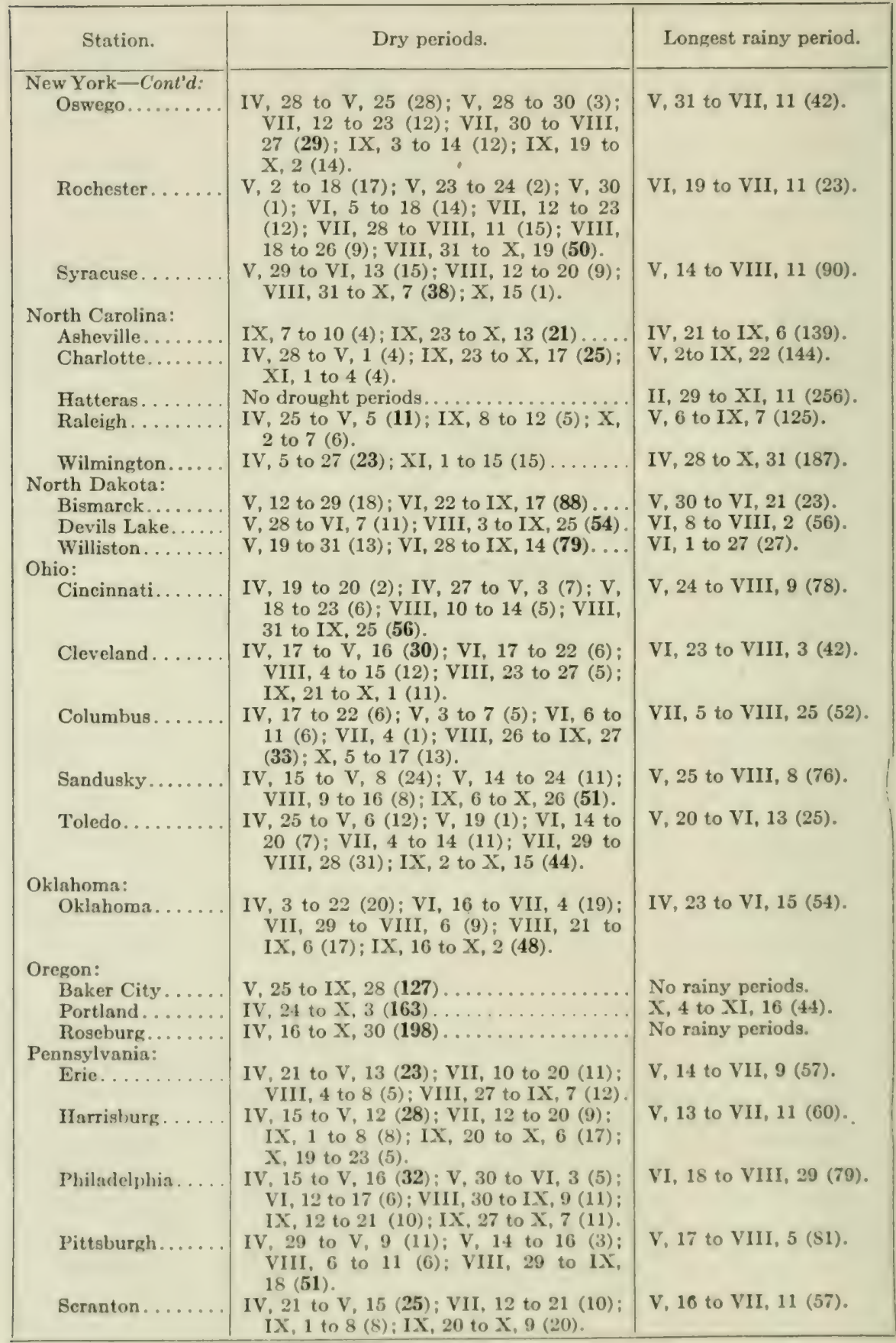


TABLE 14.-Beginning, ending, and duration of each normally dry period and of longest normally rainy period within the period of the average frostless season. (Plates 50 and 51.)-Continued.

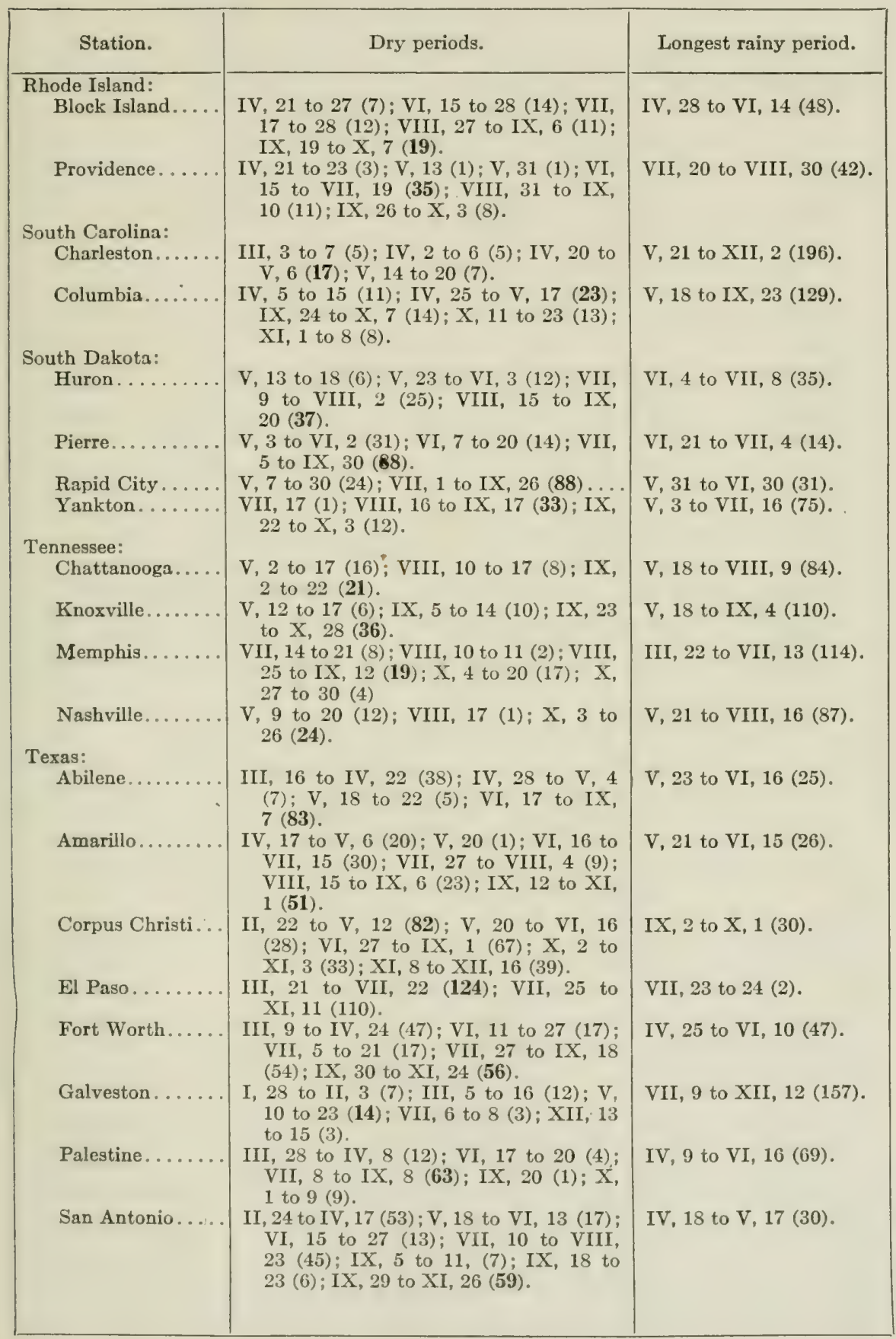


TABLE 14.-Beginning, ending, and duration of each normally dry period and of longest normally rainy period within the period of the average frostless season. (Plates 50 and 51.)-Continued.

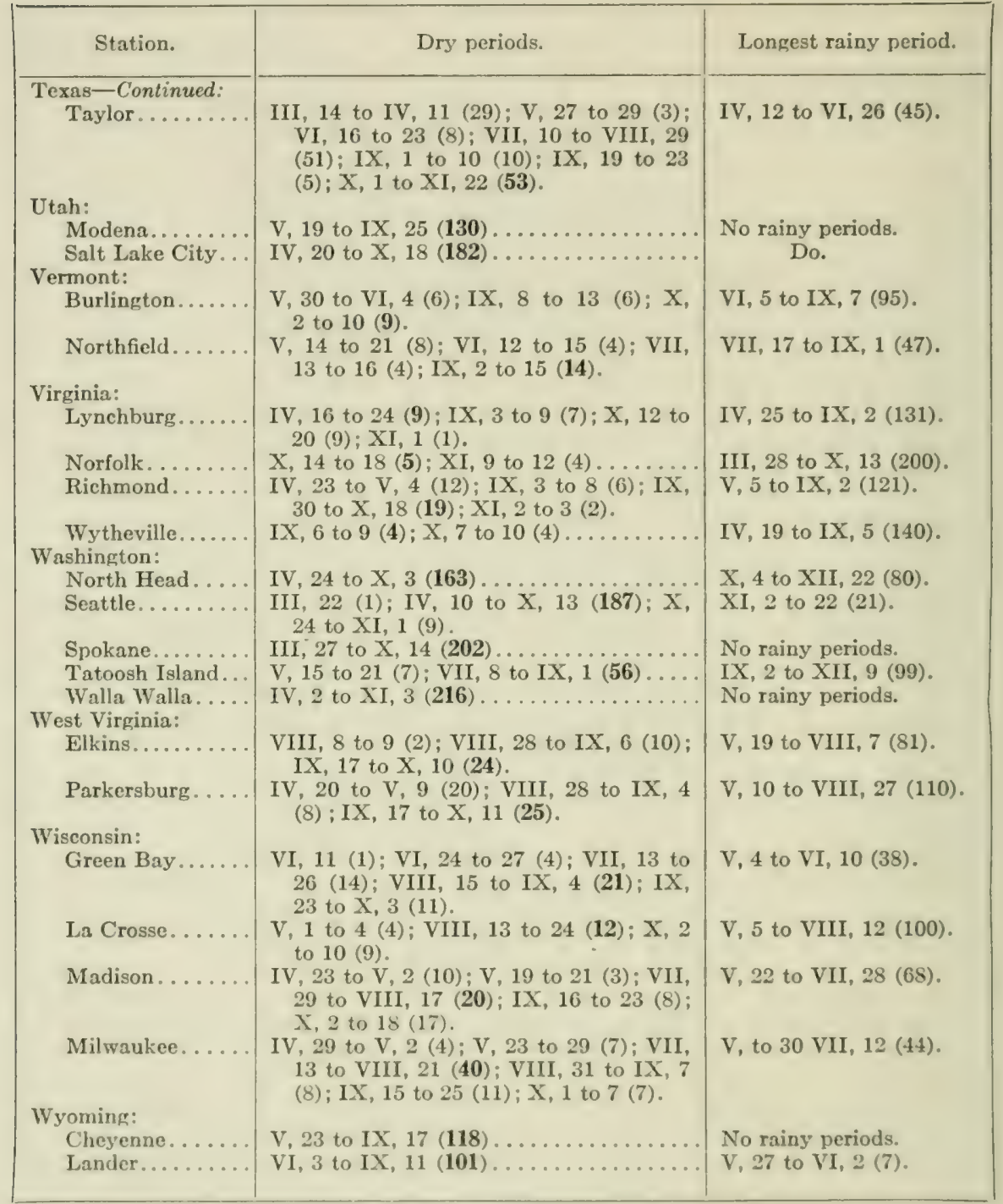

The lengths of the longest normally rainy periods in the period of the average frostless season, as given in the third column of table 14 , are shown graphically on the chart of plate 50, in which the isoclimatic lines represent increments of 25 days. The total range for the eountry is from nil to 256 (Cape Hatteras, North Carolina). All of the region west of about the one-hundred-and-first meridian has exceedingly low values, most of them being zero, excepting the strip of country border- 
ing the Pacific. The droughtiness of the intermountain area is here relatively exaggerated, on account of the choice of 0.10 inch as our constant in deriving these indices; the stations here characterized as having no normally rainy periods are not to be considered as really all alike in this particular. For the detailed study of the arid regions there seems to be no doubt that a lower value than 0.10 inch will be required. The precipitation provinces of the country, as indicated by the full lines of plate 51, are similar to those shown on plates 46 , 47 , and 49 .

(8) Length of Longest Normally Dry Period in Period of Average Frostless Season. (Table 14, Plate 51.)

These indices for droughtiness, corresponding to those for raininess just discussed, are given in the third column of table 14, and the method by which they were obtained has already been described. Plate 51 shows the chart constructed from these values. The isoclimatic lines are shown for intervals of 25 , with full lines for the values 150 , 50 , and 25. The total range of this index, for the entire country, is from zero (Cape Hatteras, North Carolina) to 299 (Los Angeles, California). The meridian of $101^{\circ}$ west longitude again forms an important demarcation, separating the more humid east from the more arid west, and some other features are like those of the other precipitation charts. In the details of the Southwest this chart has peculiarities somewhat like those of plate 48 , and will be referred to below.

(9) Normal Annual Precipitation. (Plate 52.)

Since total annual precipitation is surely of considerable value in a general estimate of the aridity or humidity of a region, we include in our series a reproduction of the chart of this feature published by Gannett. ${ }^{1}$ This chart has been drawn, as its author states, with considerable reference to topography as well as to the records of about 4,000 stations, and it appears to us to be the most useful annual rainfall chart of the United States thus far published. Data for normal annual precipitation for 167 stations are given in the second column of table 15 , for use in other connections, but these are not the data from which this chart was constructed. The data given in that table are taken from Bulletin R of the U. S. Weather Bureau. Table 15 also includes other data, which will be considered below.

In Gannett's chart, as here reproduced (plate 52), the isohyetal lines for 10, 30, and 50 inches are dotted and the others are full. All are drawn for increments of 10 inches. By the emphasized line for a precipitation of 30 inches the country is divided into 3 main

\footnotetext{
${ }^{3}$ Gannett, Henry, Distribution of rainfall, U. S. Geol. Survey, Water Supply Paper No. 234, reprinted from report of National Conservation Commission, 1909, Washington, 1909. The chart referred to is Plate $I$, and is published in color. The isohyetal lines of our chart (plate 52 ) have been copied from Gannett's plate I by means of a pantograph.
} 


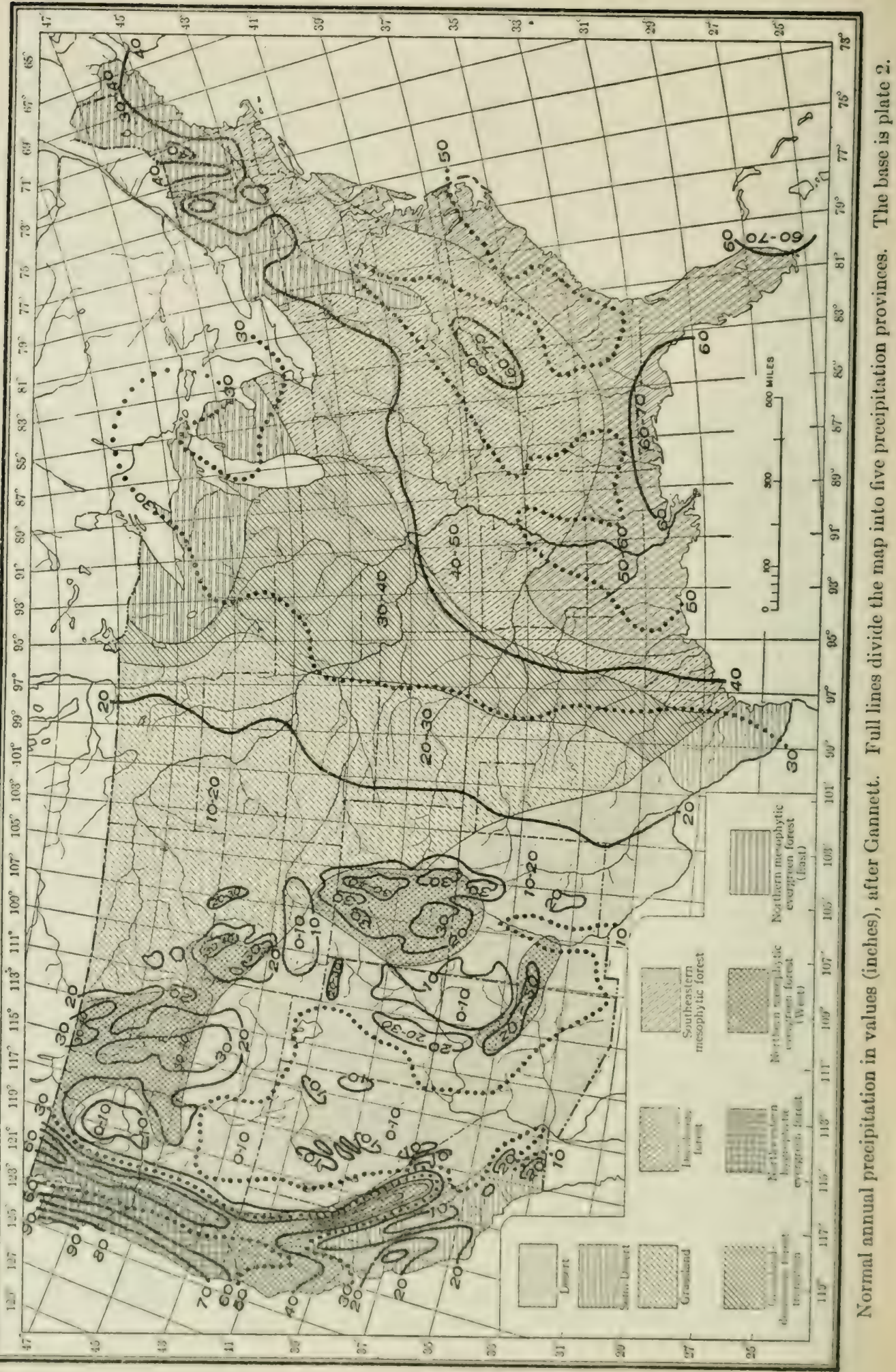


regions. The humid northwest occupies the western half of Washington and of Oregon, practically the full width of northern California, and the whole of the Sierra Nevada Mountains. The arid and semiarid central region occupies the country south and east of the zone just described, and extends eastward as far as a line drawn from the western end of Lake Superior to the Rio Grande at a point about 100 miles above its mouth. In this general description we of course neglect the restricted mountain areas of Idaho, Wyoming, Colorado, etc. The humid east occupies the region lying east of the line just mentioned. It should be added that the northern half of the southern peninsula of Michigan is also to be classed as semiarid on this basis, since its normal annual precipitation is less than 30 inches; this is a restricted area.

The arid precipitation zone is here shown as having values below 10 , and it occupies the Great Basin and extends southward into Mexico from Arizona and southern California. The humid region, as above defined, is here considered as divided into two provinces by the line for value 50, thus indicating a humid and a semihumid, or a rainy and semirainy province. These four precipitation provinces do not require special discussion; their general characteristics are very similar to those pointed out for plate 46 .

The general north and south trend of the isohyetal zones is here seen to be modified by the Gulf of Mexico and by the southern Atlantic so that the lines of the eastern portion of the humid region trend northeastward, or even eastward, instead of southward. The mountainous regions, of course, have higher precipitation indices than lowland regions of the same latitude. In general, the zones tend toward an arrangement parallel to the two coast-lines, which is readily explainable on meteorological grounds and which is the reason for the northsouth trend noted in all charts representing moisture relations.

(10) Conclusions from Study of Precipitation Conditions.

The charts of precipitation conditions (plates 46 to 52) exhibit features that are markedly unlike those of the temperature charts (plates 34 to 45), as was of course to be expected. The precipitation zones have a strong general tendency to extend in a north-south direction, while those of temperature generally extend from west to east. We have found it convenient to consider the following four precipitation provinces in the United States: (1) the humid (or rainy) rainprovince, occupying a small area of the extreme Northwest and a larger area of the southeastern Gulf and Atlantic coasts; (2) the semihumid (or semirainy) rain-province, occupying a rather narrow strip of country southeast of the northwestern humid area and nearly all of the country east of a line drawn from Corpus Christi, Texas, to Winnipeg, Manitoba; (3) the semiarid (or semidry) rain-province, occupying a narrow strip 
east and south of the northwestern semihumid area, the plains region, roughly defined as between the mountains and the Winnipeg-Corpus Christi line, and portions of the States bordering the Great Lakes; (4) the arid (or dry) rain-province, occupying, roughly, the region west of the Rocky and Big Horn Mountains that is not included in the western and northwestern portions of the other provinces. The general forms of these rain-provinces are set forth in figure 2 , which is taken from plate 46 .

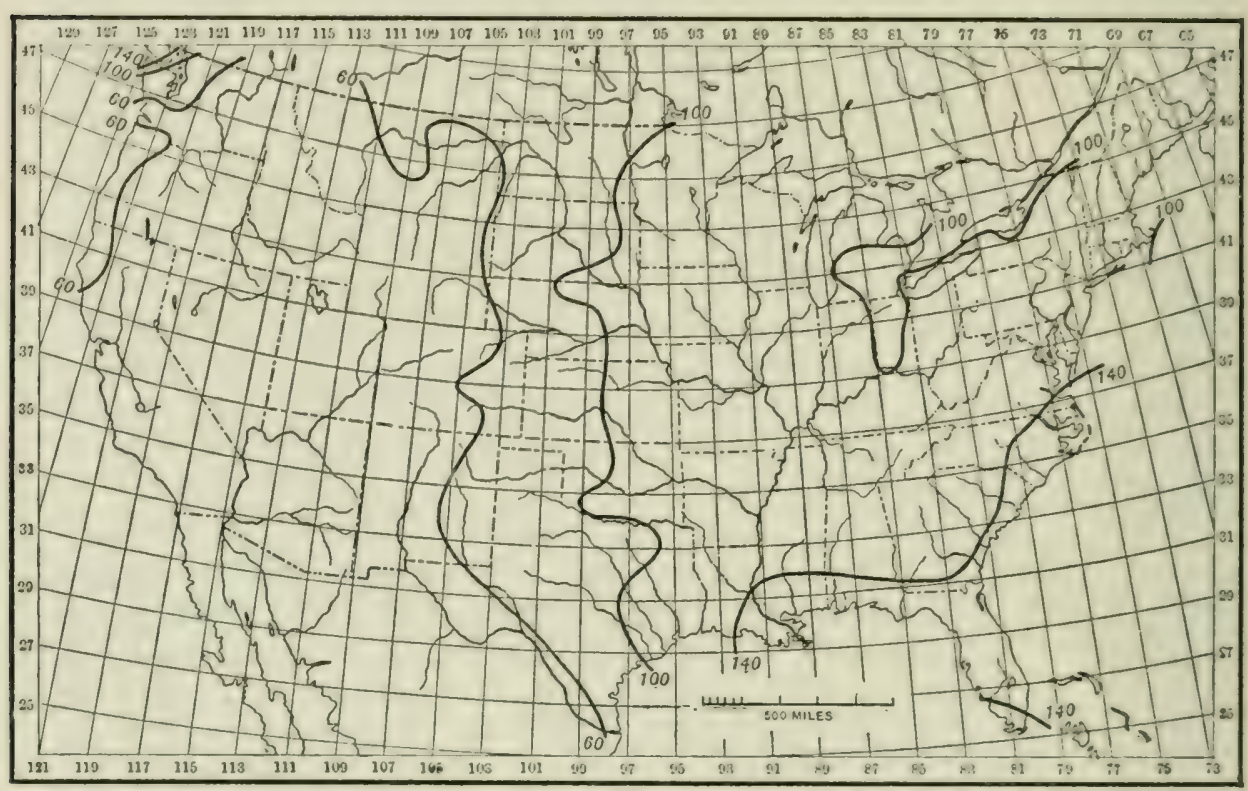

Fig. 2. Moisture zonation, according to precipitation indices for period of arerage frostless season. Precipitation provinces: Humid, more than 140; semihumid, 100 to 140 ; semiarid, 60 to 100 ; arid, less than 60. Numerical values are in the thousandth of an inch. (See also plate 46.)

Plates 48 and 51 show somewhat marked departures from this generalization, consisting mainly in the westward or southwestward displacement of the arid province, so that the latter comes to include the southern half or more of the Pacific coast and little or none of the Great Basin.

The other features of these two plates are somewhat different from those of the other precipitation charts, but still agree with them in a general way. The southeastern humid province appears on plate 49 as three localized areas, two of which are where they would be expected (from a study of plates $46,47,49,50$, and 51). The third area, however, is differently placed, occupying portions of Minnesota, Iowa, Missouri, Wisconsin, and Illinois. On plate 51 this southeastern humid. or rainy, region is much more extensive northward and northwestward 
(than in the case of plate 46, for example), and southern Georgia and Alabama, and most of Florida are here shown as in the semiarid province.

\section{REMOVAL OF WATER FROM THE PLANT.}

\section{A. INTRODUCTORY.}

(1) General Control of Water-Loss.

The external conditions ${ }^{1}$ that are effective in the control of waterloss from ordinary land-plants are generally confined to the aerial environment, for water is probably seldom lost through the subterranean periphery of the plant-body. The water-extracting conditions of the aerial environment are more directly related to climate than are the water-supplying conditions of the subterranean surroundings. Some of these conditions have been studied by meteorologists and climatologists, and the published records of the U. S. Weather Bureau will once more be drawn upon for climatological information wherever possible.

There are just two features of the aerial environment of plants that are directly effective in controlling their rates of water-loss-the evaporating power of the air and the intensity of absorbed radiant energy. These two conditions should not be confused; one is dependent upon air-temperature, air-humidity, and velocity of air movement, and the other depends upon the quality, intensity, and duration of sunshine, which is not generally a function of the air conditions immediately about the plant. Also, these two conditions should not be confused with evaporation, which is almost always done in common parlance. The rate of evaporation from a given water-surface is determined by various internal conditions (resident in or back of the surface) and by these two external conditions. We shall, however, still frequently employ the term "evaporation" as practically synonymous

\footnotetext{
${ }^{1}$ On the internal conditions that are effective in this regard, see Livingston $(1906,2)$. - Idem $(1913,2)$. Bakke 1914. - Shreve, F., The vegetation of a desert mountain range as conditioned by climatic factors, Carnegie Inst. Wash. Pub. No. 217, 1915, and the citations given in these papers. The xerophytism, mesophytism, etc., of plant forms, as these have been roughly considered by observational and classifying ecologists, are mainly based upon the appearance or structure of the aerially exposed parts and, until the recent development of the concept of transpiring power, or resistance to transpirational water-loss (which is quite distinct from the transpiration-rate itself), but little progress has been made toward the quantitative definition of plants in this regard. If the transpiring power of plants might be as well known as the shapes of their leaves and the arrangements of their floral parts, a great impetus should be given to the more permanent aspect of ecological study. With a knowledge of this power for the various plant forms should of course go a similar knowledge of water-absorbing power and water-conducting power (see especially Livingston and Hawkins (1915), in this general connection), for the xerophytism, etc., of a plant may depend on one of these latter rather than upon transpiring power alone. The measurement of these more recondite internal conditions has not as yet been seriously attempted, and methods therefor are still to be devised. Ecological plant geography will eventually need to define its plants physiologically as well as taxonomically, and the geographical distribution of species (itself as yet attempted only in a crude way) will become of little interest without some real knowledge of the physiological qualities by which these species resist or favor the various influences of the environment. In the present part of our study we confine attention to environmental conditions.
} 
with atmospheric evaporating power, since this will be readily understood and since an attempt to avoid such practice seems somewhat pedantic at the present time. When the evaporating power of the air begins to enter seriously into climatogical studies this meaning of the word "evaporation" may be dropped. In short, it seems desirable to avoid clouding the main issue for the present, and we shall frequently employ the word "evaporation" to correspond with the word "precipitation" as here used. The expression "evaporating power of the air," or "atmospheric evaporating power," will also be used, however.

(2) Atmospheric Evaporating Power.

This term is here used in Livingston' $\mathrm{s}^{1}$ sense, meaning the tendency of the air about the plant to accelerate transpiration. The expression has been seriously opposed as an "inaccurate and misleading expression," by certain members of the staff of the United States Weather Bureau. Some of the discussion that has been raised in this connection is indicated in one of Livingston's papers $(1915,2)$ and in footnotes, editorial and otherwise, incorporated therein.

The objection to the term, as so far brought out in the literature, seems to reside mainly in the consideration that the non-aqueous gases of the air actually hinder vaporization of water (which they do to a comparatively slight degree), so that a decrease in the amount of these gases present must increase the evaporating power of the air. It does not appear, however, that this is really an objection to the term in question, especially since the meaning is understood immediately by everyone, and since no better term seems available as yet. Doubtless some of the misunderstanding brought out in this discussion hinges on what may be meant by air. The air is a mixture of varying proportions of various gases; it always contains (in nature) nitrogen, oxygen, and a little argon, but it also contains carbon dioxide and water-vapor, and frequently numerous other gases. We see no reason for not considering these last-named gases as a part of the mixture, and it is in the sense of air as the gas mixture bathing the evaporating surface in question that the word has been employed by Livingston and is here employed. Now, such a gas-mixture as the air may vary in the nature and proportions of its constituents, and it may also vary in its density, or pressure. Is the pressure decreases the air becomes less dense, and this purely physical change makes it possible for evapora-

\footnotetext{
${ }^{1}$ In this connection, see the following papers: Livingston (1906, 2). - Idem, Evaporation as a climatic factor influencing vegetation, Hort. Soc. New York, Mem. 2: 43-54, 1910.-Idem, A schematic representation of the water-relations of plants, a pedagogical suggestion, Plant World 15: 214-218, 1912.-Atmometry and the porous cup atmometer, Plant World 18: 21-30, 51-74, 95-111, 143-149, 1915.-Idcm. Atmospheric influence upon evaporation and its direct measurement, Monthly Weather Rev. 43: 126-131, 1915.-Idem, A modification of the Bellani porous plate atmometer, Science, n. s., 41: $872-574,1915 .-I d c m$, A single climatic index to represent both moisture and temperature conditions as related to plants, Physiol. Res. 1: 421-1.10, 1916.-Idem, Atmospheric units, Johns Hopkins Univ. Circ., 160-170, Mar., 1917.
} 
tion to proceed at a higher rate than is obtained with greater pressure. It is likewise true that an alteration in the water-content of the air changes the rate at which evaporation may occur, ceteris paribus. Furthermore, a change in the temperature or velocity of movement of the air (wind) over the evaporating surface also alters the possible rate of evaporation. It is thus seen that both physical and chemical changes in the air exert an influence on the rate of evaporation from exposed liquid or solid water. That a decrease in the amount of gas present per given volume should accelerate evaporation is surely not a matter to cause misunderstanding, for we regard the mixture as air, at whatever density it may occur. It thus appears that the evaporating power of the air increases as the density of the air decreases, and this consideration appears to clear up the whole difficulty above mentioned. The usual popular quibble over the conception of limits arises here, as elsewhere in physical science, when we consider the result of decreasing the air-pressure to zero. In such a case the air approaches, and finally should become, an absolutely empty space, without temperature and without chemical nature. Such an absolute vacuum would have the highest possible evaporating power, in Livingston's sense, and after such a condition had been reached (if it could be maintained) the rate of evaporation from exposed liquid or solid water should be controlled only by conditions resident in the liquid or solid itself. This condition is impossible of attainment, of course, so that the evaporating power of the air never becomes infinite, but this consideration is valuable in that it shows clearly how this power becomes greatest when there is the least gas present in the air-space. The quibble arises over the popular interpretation of the apparently paradoxical statement that the evaporating power of the air is greatest when all the air has been removed. Of course, when the limit is reached and the air-pressure is actually zero, we have to broaden our definition of air so as to let the term mean the space abutting against the evaporating surface into which water vapor may diffuse. That this is necessary at the limit of reduced pressure (which is never really attained) seems to be no reason for changing our term, though if it seems desirable we are free to admit that the term in question really denotes the evaporating power of the circumambient space in which air usually occurs.

Another objection to the term "evaporating power of the air" is parallel to the one always raised against the word suction. The water vaporizes because of conditions resident in its solid or liquid phase, and the energy thus transformed does not come (directly) from the air-space. Just as the term "suction," or sucking power, has to be regarded as referring to the removal or decrease of a resistance, rather than to the application of a driving force, so the evaporating power of the air is to be regarded as proportional to the reciprocal of the measure of the resistance offered by the air to evaporation. The resistance thus 
offered may be expressed in terms of the water-condensing power of the air, but, as Livingston has remarked, air without actual tendency to precipitate or deposit liquid or solid water still offers resistance to evaporation:

\footnotetext{
"Air without water-vapor offers resistance to evaporation but has no condensing power; it can not deposit water upon a surface, no matter what its pressure may be. The resistance offered by such dry air can be expressed in terms of an equivalent condensing power, however."
}

In answering this second objection to the term "evaporating power of the air," a third possible objection is also partly answered. This objection arises from the various senses in which the word power is used. If we are interested only in the statical phase of the matter before us, then the evaporating power of the air is proportional to the reciprocal of the measure of the capacity of the air-space to retard the vaporization of water, from a liquid or solid water surface exposed to that space. The dynamic phase of the problem of evaporation, however, allows the use of the word "power" in its ordinary physical sense, as denoting the time-rate of doing work. The conditions resident in the air-space are thus thought of as somewhat like a brake on a wheel, and we consider a time-rate of the reciprocal of resistance to evaporation. Thus, our use of the word "power" is not with the meaning of spatial capacity, but we employ the word in its true physical sense, as though the air were a machine acting to retard evaporation. As in other cases of power measurement, it is necessary to measure the power in question in terms of the amount of work capable of being performed in a given time period. Internal conditions, resident in the solid or liquid phase of the water, determine what would be the rate of evaporation if the air-space offered absolutely no resistance, and if these internal conditions remain constant the amount of evaporation occurring per time period is proportional to the reciprocal of the power of the air to behave as though it were condensing water-vapor upon the exposed surface. The reciprocal of the rate at which water would be condensed if all of the tendency of the air-space to retard evaporation were effective toward actual condensation is thus proportional to the tendency of the air conditions to allow evaporation to proceed, and this may be relatively measured as a power by determining the amount of water actually vaporized per time period. Of course, the conditions resident within the solid or liquid surface are never even sensibly constant for long, and the actual rate of evaporation depends not only upon the evaporating power of the air, as above defined, but also upon the internal conditions. The evaporating power of the air is thus relatively measured as the time rate of the reciprocal of the resistance offered by the air to evaporation, this resistance being measured in terms of equivalent condensation. But condensation is merely negative evaporation, so that when the air conditions are such as to make the external resistance just equal to the internal tendency 
(within the solid or liquid phase) to vaporize water, then the apparent rate of evaporation becomes zero. Going further, the external conditions frequently become such that the resistance offered by the air to vaporization of water is greater than the tendency of the internal conditions to cause vaporization, this resistance being due to a tendency of the air to deposit water on the surface, and actual condensation ensues; $i$. $e$., the evaporating power of the air becomes negative and the evaporating surface gains water instead of losing it.

There seems never to have been any attempt to define air according to its chemical content; it would still be air if it were largely carbon dioxide or hydrogen, etc., and it seems unadvisable to attempt a restriction of the terms "air" and "atmosphere" at this late day. For the rest, the expression "evaporating power of the air" has been in use among students of this power at least since 1906 (when Livingston used it). It will probably appeal to most students of this power as quite unobjectionable and it need not be dropped. Livingston $(1917,1)$ has suggested atmometric index as still another term, to avoid the difficulty just mentioned and to avoid the necessity of employing evaporation to mean both the process and one of the conditions controlling its rate. We shall not employ this new expression, however, preferring to allow others to decide the question thus raised.

The evaporating power of the air is of the utmost physiological importance to vegetation, and it can be rather readily and directly measured, in relative terms. Nevertheless it has not been seriously studied in the United States, and most of the information so far obtained in regard to it is only indirect. To appreciate what ones of the climatic conditions usually measured may be valuable here, it is necessary to consider the secondary conditions, upon which depends the atmospheric evaporating power.

The vapor-tension deficit.- Without air-movement, and supposing the air and water temperatures to be the same, the evaporation-rate should be nearly proportional to the vapor-tension deficit; that is, to the difference between the maximum vapor-tension for the given airtemperature and the tension of water-vapor actually present in the air. The actual vapor-tension in the air is a closely approximate measure of the tendency toward condensation and the maximum vaportension for the given temperature and pressure is a measure of the whole tendency toward evaporation; the former tendency overcomes a portion of the latter and what remains is very nearly the actual tendency toward evaporation. The maximum vapor-tension of water is, of course, a constant for any temperature and barometric pressure, and its value may be obtained from physical tables. The actual vapor-tension is seldom as great as the maximum; it is so only in the case of water-saturated air. If we allow $E$ and $E^{\prime}$ to represent evaporation-rates from the same surface at different times, $P$ and $P^{\prime}$ the 
maximum vapor-pressures corresponding to the respective air-temperatures, and $p$ and $p^{\prime}$ the corresponding vapor-pressures of water actually in the air at those times, then

$$
\frac{E}{E^{\prime}}=\frac{P-p}{P^{\prime}-p^{\prime}}
$$

Under such conditions $P-p$ and $P^{\prime}-p^{\prime}$ are measures of the respective forces tending to drive water-vapor off from the surface into the air. To determine the values of $p$ and $p^{\prime}$, we may measure the absolute humidity by chemical methods, we may resort to the sling psychrometer or any form of wet and dry bulb thermometer with constant and rapid air-movement, or we may employ the Regnault dew-point apparatus. It is clear that the values of $p$ and $p^{\prime}$ depend upon the absolute humidity and upon the air-temperature and barometric pressure, while the values $P$ and $P^{\prime}$ depend only upon the temperature and barometric pressure. Since the influence of barometric pressure is relatively small under natural conditions, it need not be seriously considered here. The vapor-tension deficit is seen to include the airtemperature influence.

Relative humidity.-The vapor-pressure deficit is not one of the climatic features usually determined by climatologists, who have rather uniformly followed earlier workers in the employment of the concept of relative humidity in its stead. Relative humidity is the vapor-pressure of the water-vapor actually present in the air expressed as percentage of the maximum vapor pressure for the given temperature and pressure; it is simply the percentage of water-saturation of the air. This bears no quantitative relation to atmospheric evaporating power, even with wind and barometric effects left out of considera. tion, for it is obvious that air with a given relative humidity must be more effective in promoting evaporation at a higher temperature than at a lower. It is not the percentage of the maximum vapor-pressure actually present, but the difference between the maximum pressure and the actual, which measures this influence upon eraporation-rate. Since the maximum increases with temperature (though not proportionally), a given percentage of deficit must represent a larger actual deficit as the temperature rises.

If $H$ and $H^{\prime}$ represent the relative humidity of the air at different times, the remaining symbols being the same as above, then

$$
\frac{I I}{H^{\prime}}=\frac{p / I^{\prime}}{p^{\prime} / P^{\prime}}
$$

From this it is clear that, if the air-temperature is known in cach case, thus furnishing the values of $P$ and $l^{\prime}$, the vapor-pressure deficits may be found; from this equation and the one for $E, E^{\prime}$, given abore, it follows that

$$
\frac{E}{E^{\prime}}=\frac{P(1-H)}{P^{\prime}\left(1-H^{\prime}\right)}
$$


In other terms, the rate of evaporation is, under the assumed conditions, proportional to the product of the maximum vapor-pressure of water, for the given air-temperature, and the complement of the relative humidity.

Relative humidity is commonly measured and discussed in climatological studies, and its complement is sometimes employed as a measure of atmospheric dryness. They are both easily seen to have no definite relation to the evaporating power of the air. There is here, however, a general and merely qualitative relation; high relative humidity usually corresponds to low atmospheric evaporating power, and the reverse. We shall have to deal with relative humidity in our discussion of the climatic conditions influencing evaporation, but this concept is to be clearly appreciated as without logical foundation; it is simply a mathematical abstraction and its value to agriculture or ecology will have to be determined by direct empiricism. It may be here suggested that vapor-tension deficit is the climatic dimension that should be measured by ecological workers, if the analysis needs to be carried so far. ${ }^{1}$ Fortunately, the evaporating power of the air can be directly measured, and much more readily and usefully than can this deficit, and it seems not at all necessary at present, for ecological purposes, to analyze this power into its components.

Wind.-Besides the vapor-tension deficit, atmospheric evaporating power is greatly influenced by air-movement; with increasing wind, ceteris paribus, the evaporation-rate is accelerated. Here again, however, the relation between wind velocity and evaporation-rate is not a linear one; with low velocities the effect of alteration in wind velocity is great; with high velocities this effect practically vanishes, and the relation of the two features for any given range of velocity depends upon the kind and upon the exposure of the evaporating surface. As has been mentioned, an enormous amount of effort has been expended in attempts to find empirically a formula by which evaporation might be calculated from measurements of other climatic conditions, and the argument over the wind factor has been greatly prolonged. Such attempts have failed, as they always must until the problem is first solved by controlled physical methods, which solution has not yet been seriously attempted. When a solution is reached, however, it will obviously hold only for some particular kind, size, etc., of evaporating-pan or other atmometer.

As a climatic feature that must surely influence water-loss from plants, but the exact nature of whose influence is still quite beyond our reach, wind velocity will be but briefly touched upon in our study. On theoretical grounds this is not a promising criterion for ecological

${ }^{1}$ Livingston, B. E., The vapor tension deficit as an index of the moisture conditions of the air, Johns Hopkins Univ. Circ., Mar. 1917, pp. 170-175.-Johnston, Earl S., The seasonal march of climatic conditions as related to plant growth, Maryland Agric. Exp. Sta., in press. 
climatology, and it is practically unsatisfactory on account of the inadequacy of the information in this regard which is now available.

\section{(3) Absorbed Radiation.}

Reverting again to the conditions controlling water-loss from plants, we have said that there are, generally, two of these - the evaporating power of the air and the intensity of absorbed radiant energy. The first of these has been discussed in sufficient detail for present purposes and the second remains to be considered. By far the greater portion of the radiant energy intercepted by plant surfaces comes directly from the sun; other sources of radiant energy appear to be practically negligible under most natural conditions. It is therefore absorbed sunshine (light and heat) that needs attention at this point.

The intensity of absorbed solar radiation is determined by three conditions - the intensity of the impinging rays, the angle at which they meet the exposed surfaces, and the absorbing power of the surfaces. The last is an internal condition, effective within the plant, with like transpiring power, water-absorbing power, etc. With this, as other internal conditions, practically nothing of a quantitative nature has yet been attempted. ${ }^{1}$

The angle at which the impinging rays meet plant surfaces varies with the time of day, with the season, and with the shape and position of the plant; but since ordinary plants offer absorbing surfaces to solar radiation at all possible angles, it is only in special studies of special species (as of "compass plants," for example) that this matter may require attention. We may ignore the angle of incidence in our present discussion. ${ }^{2}$

The intensity of the impinging radiation is obriously the feature dealt with in climatology as sunshine intensity. For the measurement of this, various methods have been devised from time to time (such as the black-bulb thermometer, the bolometer, the pyrheliometer, the Hicks solar radio-integrater and several forms of photographic actinometers), but no data are available for a quantitative climatological study of this condition. It appears probable that the radio-atmometer ${ }^{3}$ may furnish adequate information for ecological purposes, when its value in this connection has become appreciated.

A very distant approach toward the measurement of sunshine intensity, and the only systematic attempt in this direction thus far

\footnotetext{
${ }^{1}$ See, in this regard, Livingston $1911, a$.

2 Briggs and Shantz have argued that only the vertical component of solar radiation is to be considered as effective upon plants. It seems to us that this question requires experimental investigation before its detailed discussion may be attempted. We may add here the remark that the surfaces of most plant leaves occupy almost all conceivable angles with the vertical, so that the exposure of the plant as a whole must approach being equivalent to that of a sphere or of a vertical cylinder with spherical top. For the opposite argument, see L. J. Briggs and H. C. Shantz, Hourly transpiration rate on clear days as determined by cyclic environmental factors, U. S. Dept. Agric., Jour. Agric. Res. 5: 583-649, 1916.

${ }^{2}$ Livingston, B. E., A radio-atmometer for measuring light intensity, Plant World 14: 96-99. 1911.-Idem 1911, b; Idem 1915, a; Idem 1916, b.
} 
carried out by the United States Weather Bureau, consists in the determination of the number of hours of sunshine occurring each day at the various stations. It is to be emphasized that the sunshine recorders now generally in use give but little information as to the intensity of the sunshine itself; they record the duration aspect of that range of intensities which is called direct sunshine, but the limits of this range have never attracted attention and are not established, so that the whole mass of data so derived are any thing but precise. Nevertheless, some of the sunshine data of the United States Weather Bureau will be considered below, since they furnish the only available measurements having any bearing at all upon the matter before us.

\section{B. ATMOSPHERIC EVAPORATING POWER IN THE UNITED STATES.}

(1) Very Limited Nature of Available Data.

To obtain data bearing on atmospheric evaporating power it is only necessary to operate a number of atmometers of the same form in the various climatic regions dealt with, being sure that all have similar local exposures. The importance of this condition to plant and animal life and the relative ease with which it may be measured makes it appear surprising that practically no organized study of evaporation throughout the United States has yet been undertaken. Had evaporation been recorded as thoroughly as precipitation has been, we should now be able to construct relatively satisfactory atmometric charts, but the almost utter lack of data makes this practically impossible at present. To render our position in this connection still less satisfying, it is to be remembered that observations of any climatic condition, extending through a single year, are of but little value; if evaporation measurements were to be systematically begun in the present and were to be systematically continued, it would require many years of records to render these measurements as valuable climatologically as are those of temperature and precipitation at the present time. It seems now, however, that students of climate will hardly be able to persist much longer in their too common attitude of ignoring the evaporating power of the air. As we have emphasized, this climatic feature is probably as important from the standpoint of agriculture and etiological plant geography as either temperature or precipitation, and its investigation seems likely to be carried forward first by agriculturists and ecologists.

While it is possible to collect from the literature numerous instances in which evaporation has been measured at a single station for a longer or shorter period of years, such measurements can not usually be correlated with those for other stations, either because the same years are not involved or because different kinds or sizes of atmometers have been employed. Aside from such cases, ${ }^{1}$ which are all valuable-at

\footnotetext{
${ }^{1}$ Most of these cases are mentioned in: Livingston, Grace J., An annotated bibliography o evaporation, Monthly Weather Rev. 36 : 181-186,301-306, 375-381, 1908; 37 : 68-72, 103-109 157-160, 193-199, 248-253, 1909.
} 
least in showing the importance of this climatic condition-there are available just two logically planned series of atmometric measurements in the United States. One of these series was carried out by Russell for a single year, beginning in the summer of 1887. The other was conducted by the present writers during the summer of 1908 . Approximately 20 years elapsed between these two series of observations, and no thorough study of this feature has been completed since the last-named year, although the U.S. Weather Bureau is giving increasing (but always secondary) attention to evaporation. It should be noted that the United States Signal Service, the precursor of the United States Weather Bureau, carried out the earlier of these series, the second series being under the auspices of the Department of Botanical Research of the Carnegie Institution of Washington. These two series of atmometric observations, and the results derived from them, will now be considered in order.

(2) Russell's Data of Evaporation in the United States.

Evaporation intensities for period of average frostless season. (Table 11, plate 53 and fig. 14.) - Russell's ${ }^{1}$ study of evaporation extended from July 1887 to June 1888 inclusive, and this author prepared an evaporation chart of the country, but the data thus used were calculated. For the period from June to September 1888, Piche atmometers were exposed in louvred instrument shelters at 19 stations. An experiment in a closed room, employing two Piche instruments and two open pans of water, gave data from which Russell calculated that the Piche instrument lost 1.33 times as much water as did his free water surface. ${ }^{2}$ By use of this ratio the readings of the Piche instruments in the louvred shelters were converted into losses from the free water surface of the particular kind of pan used in the laboratory test, and these, as tri-daily readings, were compared with the corresponding dew-points and wet-bulb temperatures within the shelters. From this comparison Russell derived a formula by which he afterwards calculated the evaporational loss from free water surfaces in the shelter, for 140 stations in the United States. In his paper he presents a table of the monthly calculated rates (July 1887 to June 1888), and also the annual totals.

In order to make use of these data in connection with the length of the average frostless season, as we have employed the latter, we have proceeded as follows for each station involved. The evaporation data for all whole months included in the average frostless season have been

\footnotetext{
'Russell, T., Depth of evaporation in the United States, Mo. Wenther Rev. 16: 235-239. 1888. See also Kimball, H. H., Evaporation observations in the United States, Monthly Weather Rev., 32: 556-559, 1905.

${ }^{2}$ Of course this relation must vary more or less markedly with temperature and humidity conditions, even where the wind influence is out of account; but Russell seems to have ignored this consideration entirely, along with the other important consideration that the amount of evaporation is dependent on the sort of pan used.
} 


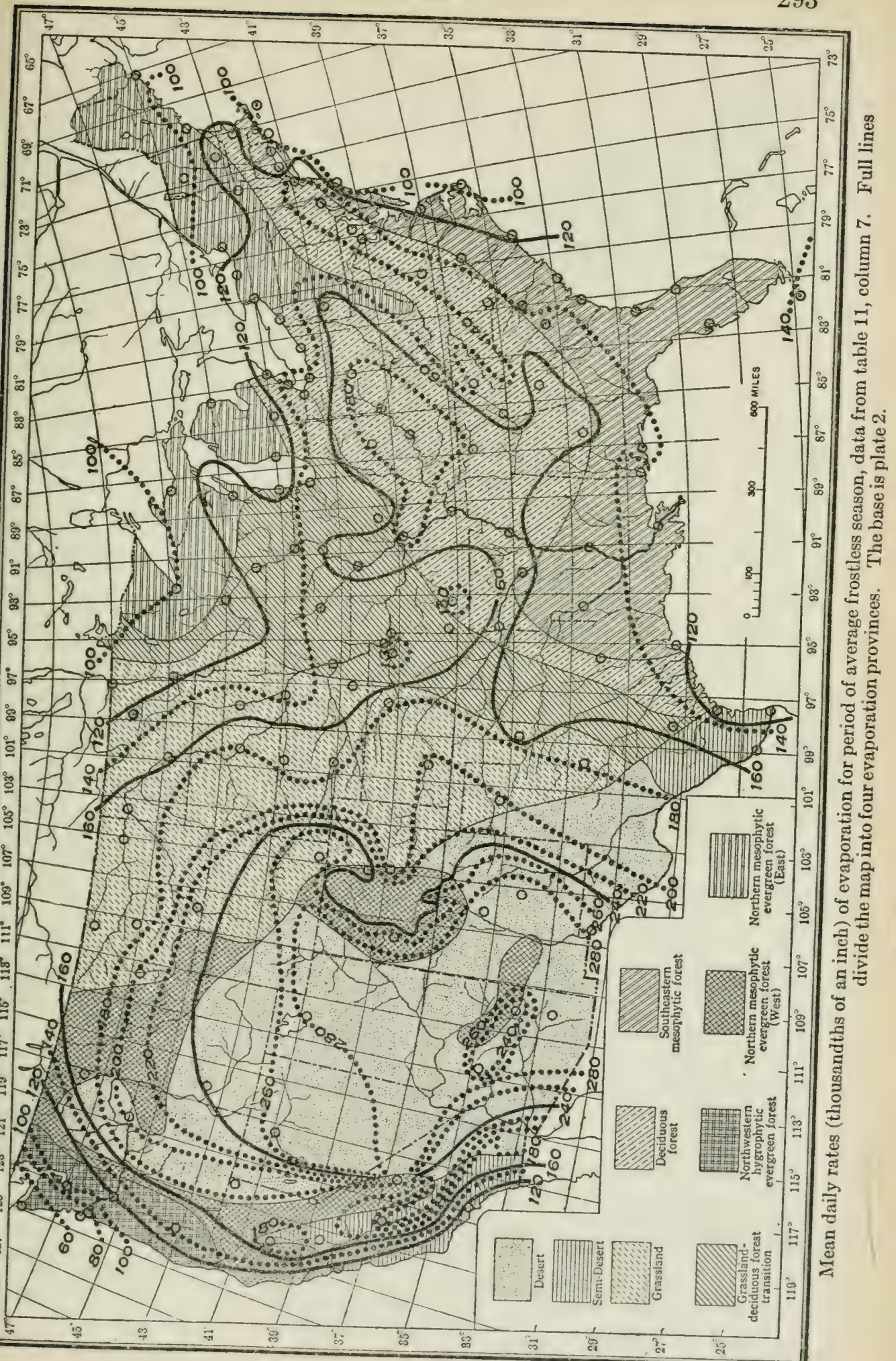




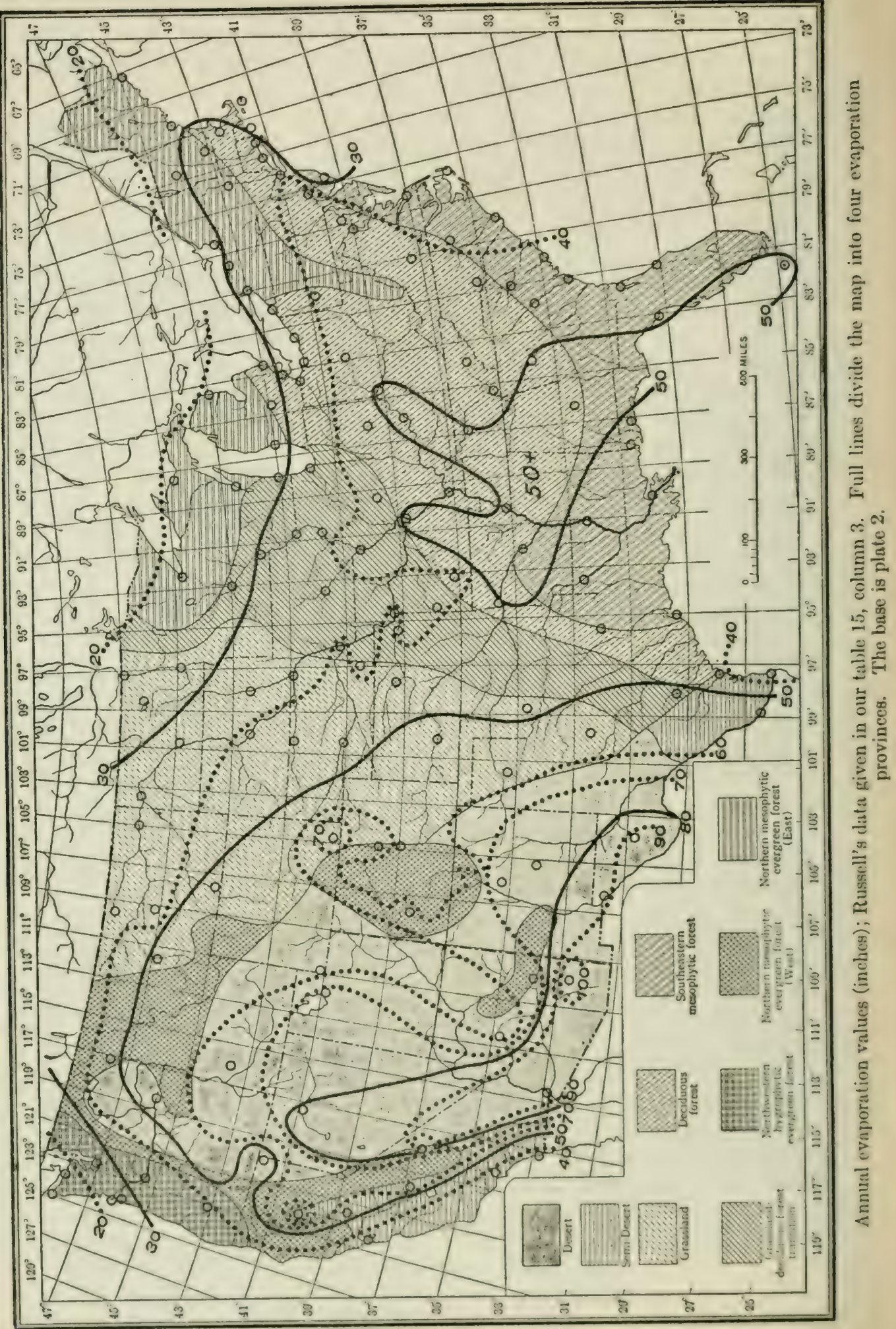


summed, and to this sum have been added two quantities, representing the evaporation for the two fractional parts of a month at the beginning and at the end of the frostless season, respectively. These added amounts have been derived, in each case, by dividing Russell's evaporation-rate for the month in question by the number of days in that month, and then multiplying the resulting quotient by the number of days of the same month comprised in the period of the average frostless season.

The total evaporation for the season thus obtained is next divided by the number of days in the period of the average frostless season. Thus, for example, if the season extends from April 24 to October 3, we sum Russell's monthly rates for May to September, inclusive, and add to this six-thirtieths of Russell's rate for April and three thirtyfirsts of his rate for October. We then divide this total by 162, the number of days from April 24 to October 3. The seasonal totals and the approximate average daily evaporation-rates (1887-88) for the period of the average frostless season, obtained as just described, for 133 stations, are given in columns 6 and 7 of table 11, and the latter data are spread on the chart of plate 53 , where the positions of the stations employed are again shown by small circles. It should be emphasized that Russell's data for June, and earlier, refer to 1888, while those for July, and later, refer to 1887 . We have merely made the best possible use of the available data.

The isoatmic lines of this chart are drawn at intervals of 20 thousandths of an inch of average daily depth of loss from some hypothetical pan of water, Russell's measurements being in such terms. The total range of values is from 52 (Tatoosh Island, Washington) to 349 (Independence, California).

On this chart of the approximate average daily intensities of atmospheric evaporating power for the period of the average frostless season, from data of 1887 and 1888, it appears that the isoatmic lines in the vicinity of the oceans have a very pronounced north-south trend. They also have a north-south direction in the plains region. Little relation to latitude, or to temperature, is here discoverable; during the frostless season temperature is not usually a prime condition in the determination of differences between different stations in the evaporating power of the air.

The lines for values 120,160 , and 240 are shown on plate 53 as distinct from the others, and these may be taken as dividing the country into four evaporation provinces. Following our usage in the case of the precipitation indices, these provinces will be termed arid, semiarid, humid, and semihumid. They are shown also in figure 14, which is derived from plate 53. This zonation is different from that shown for precipitation in several important respects. Here the humid province (values above 120) appears again as a western and an 
eastern portion. The western portion is much larger in this case, however, occupying the Pacific Slope for practically the full length of the western coastline and widening at the north to include about the western half of Washington. The eastern humid region has an entirely different form from that shown on the precipitation charts. Here it does not occupy the southeastern part of the country, but embraces the northern margin from about the one-hundredth meridian eastward. It also occupies portions of the Atlantic coast as far south as Cape Fear. It appears that the line for value 120 passes into Canada from Washington and reenters the United States in North Dakota, so that these two portions of the humid province are probably to be regarded as a single one. It should be noted, furthermore, that the Atlantic coastal portion from Massachusetts, or New Jersey, southward appears to be separated from the northeastern portion, and that a small area of humid conditions is shown about Corpus Christi and Brownsville, Texas. These features will appear more prominently on the charts of precipitation-evaporation ratios and on those of relative humidity, to be considered below.

The arid province (values above 240) occupies much the same region as in the case of the precipitation charts, but it does not here extend west of the Sierra Nevada Mountains. Of course, the western mountains are largely humid, but our charts do not generally present such details. The semiarid province (values between 160 and 240) occupies a belt outside of the area of the arid province, and this belt is extended eastward in the middle of the country nearly to the Appalachian Mountains. This eastern lobe of the semiarid province will also be pronounced on the charts of moisture ratios and relative humidities. The line separating the semiarid from the eastern semihumid area (value 160) does not here bend eastward at its northern end as it does on the precipitation charts; on the contrary, it here bends westward and apparently joins the corresponding line which enters Canada from western Montana.

Annual eraporation intensities. (Table 15, plate 54.)-Russell's table gives the yearly totals for his series of stations, in inches of depth from some hypothetical pan of water, and he also presents a chart to represent these annual data. The data are reproduced in the third column of table 15 and they are shown graphically by the chart of plate 54, which is not exactly the same as Russell's chart, a number of obvious errors in the latter having been corrected here. The total range for the country is from 18.1 (Tatoosh Island, Washington) to 101.2 (Fort (irant, Arizona), and the isoatmie lines are placed at intervals of 10 inches, with full lines for the values 30,50 , and 80 .

This chart has a pronounced general resemblance to the one representing evaporation for the period of the average frostless season (plate 5.3), but it differs quantitatively therefrom in several important 


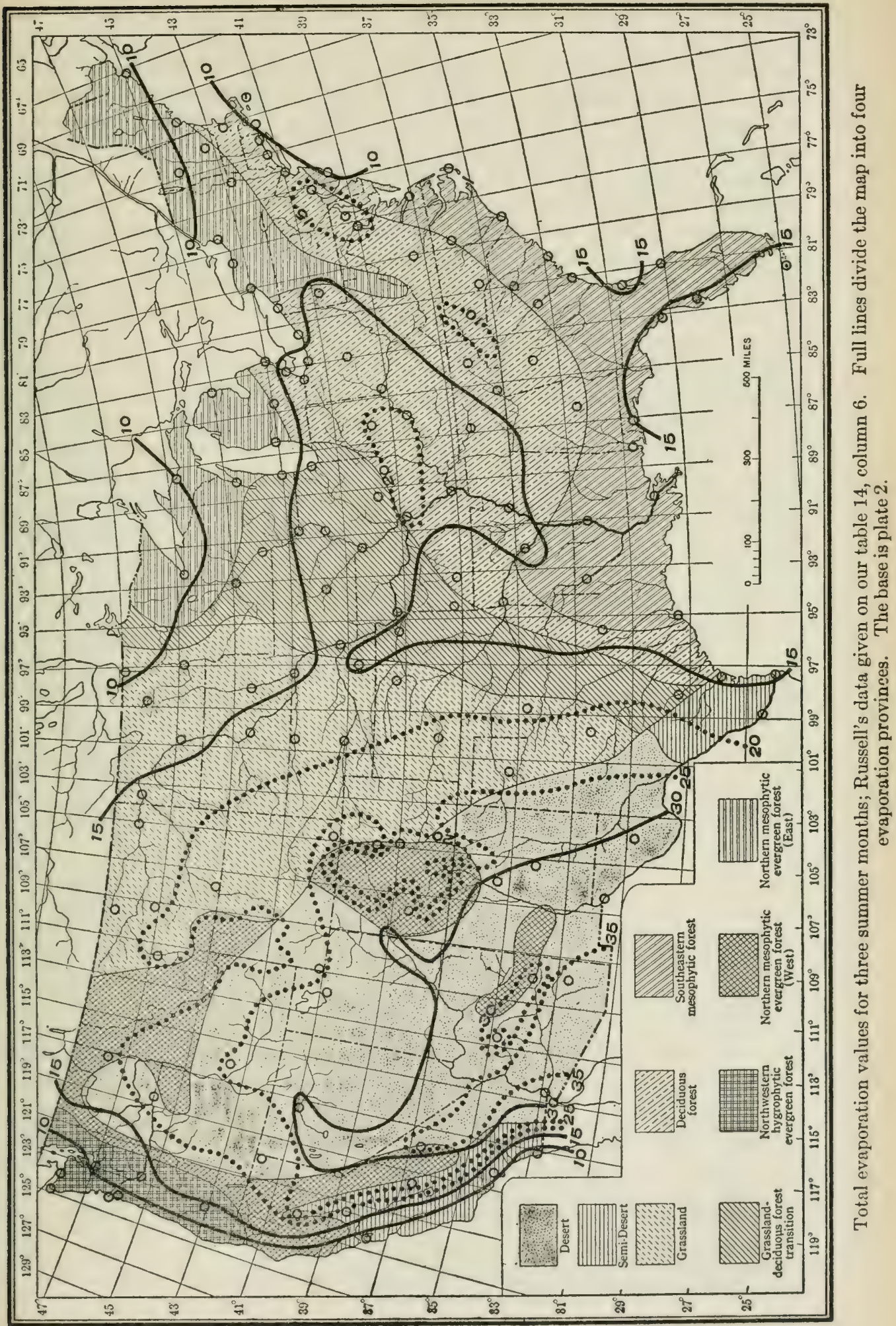


features. The northwestern portion of the humid province (values below 30) is less extensive, and the Pacific Slope is here depicted as in the semihumid province. The eastern portion of the humid province appears here about as in the case of plate 53 , but it does not extend southward farther than Rhode Island, on the Atlantic coast. The arid province (values above 80 ) is here shown as smaller than in the preceding case. The great eastern lobe of the semiarid province (values between 50 and 80 ), reaching nearly to the eastern mountains on plate 53 , is not present on plate 54 , but a large area of values above 80 is shown as extending from St. Louis, Missouri, and Louisville, Kentucky, to Key West, Florida.

Evaporation intensities for the three summer months. (Table 15, plate 55.) - Because we shall wish to compare the Russell evaporation data with those obtained by ourselves (to be considered below), and since it is impossible to employ the length of the mean frostless season as duration factor in connection with the latter, it is expedient here to study Russell's data for the period of the three summer months, June, July, and August. For a period approximately comparable to this our own data may be studied.

TABLE 15.-Precipitation and evaporation data for the year and for the three summer months June to August.

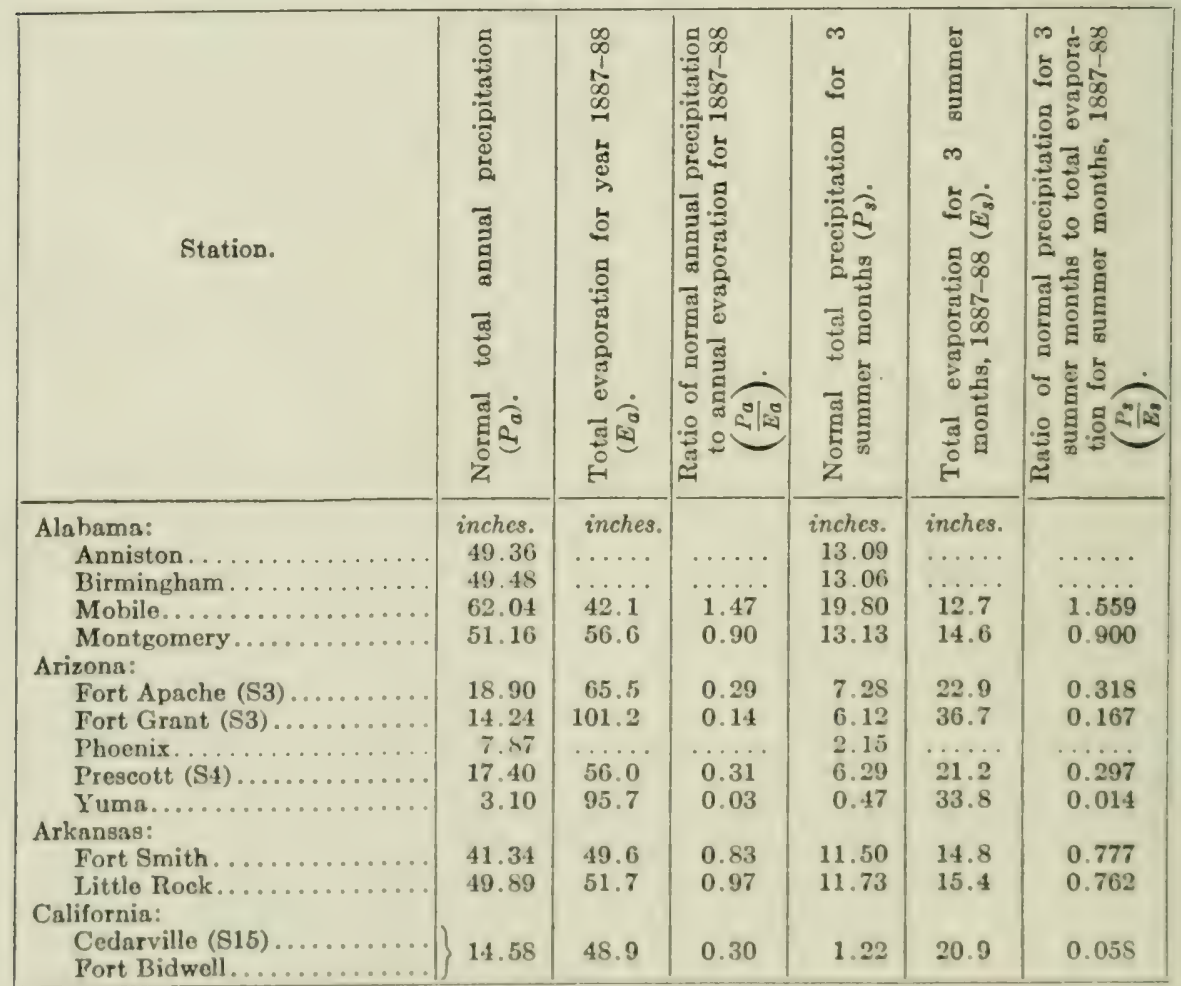


TABLE 15.-Precipitation and evaporation data for the year and for the three summer months June to August.-Continued.

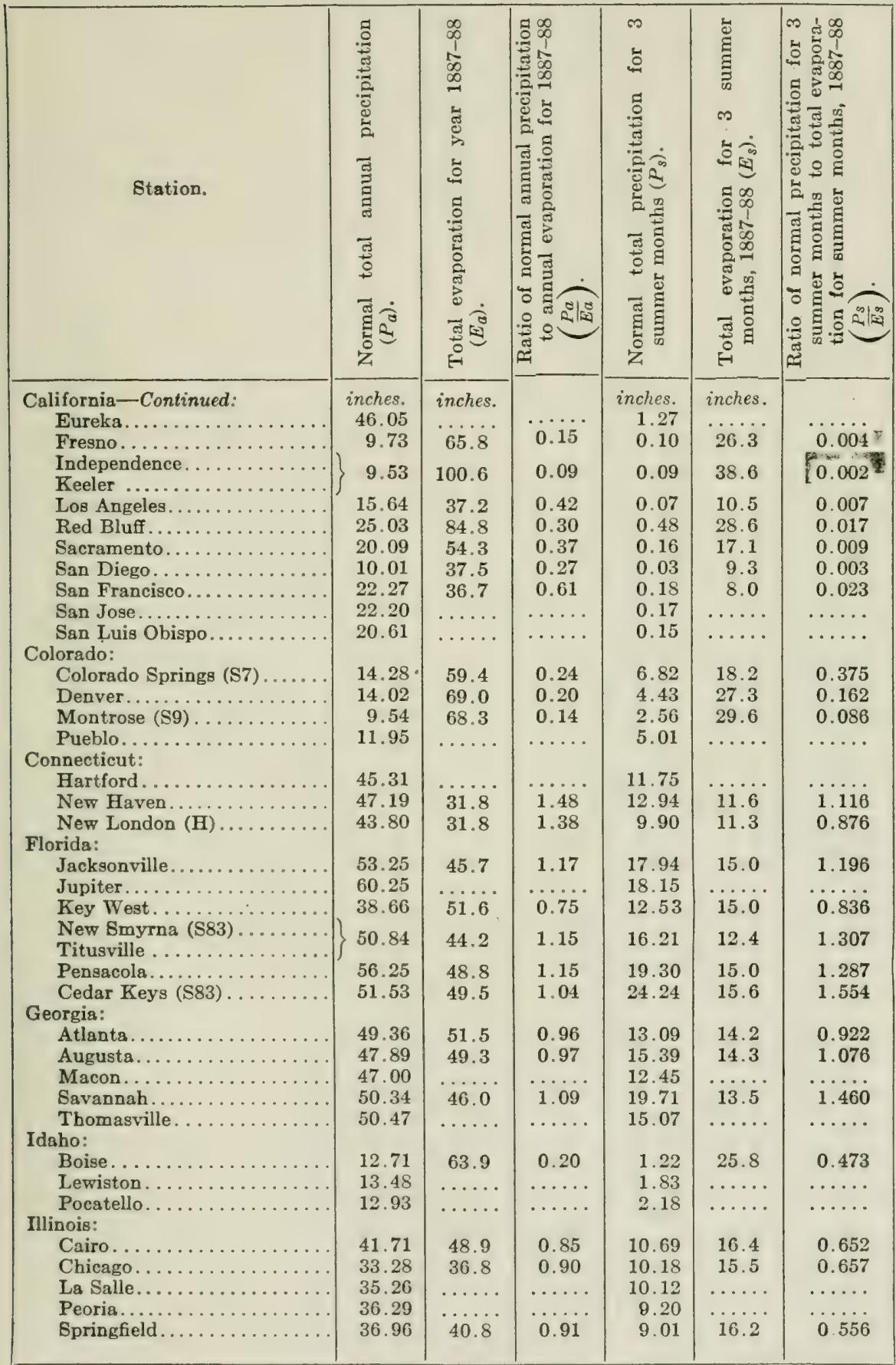


TABLE 15.-Precipitation and evaporation data for the ycar and for the three summer months June to August. - Continued.

\begin{tabular}{|c|c|c|c|c|c|c|}
\hline Station. & 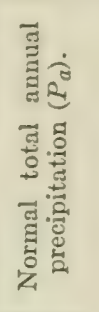 & 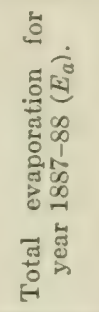 & 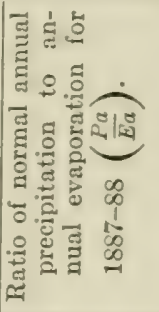 & 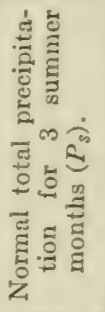 & 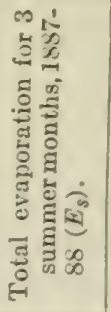 & 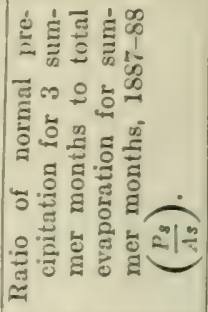 \\
\hline $\begin{array}{l}\text { Indiana: } \\
\quad \text { Evansville.. }\end{array}$ & $\begin{array}{l}\text { inches. } \\
43.16\end{array}$ & $\begin{array}{l}\text { inches. } \\
\ldots \ldots\end{array}$ & & $\begin{array}{l}\text { inches. } \\
11.22\end{array}$ & inches. & \\
\hline Indianapolis: & 41.48 & 48.6 & 0.85 & 11.77 & 20.3 & 0.580 \\
\hline \multicolumn{7}{|l|}{$\begin{array}{l}\text { Iowa: } \\
\text { Charles City }\end{array}$} \\
\hline $\begin{array}{l}\text { Charles City } \\
\text { Davenport.. }\end{array}$ & $\begin{array}{l}31.23 \\
32.69\end{array}$ & 39.0 & 0.84 & $\begin{array}{l}12.10 \\
11.30\end{array}$ & 17.7 & \\
\hline $\begin{array}{l}\text { Davenport..... } \\
\text { Des Moines.... }\end{array}$ & 32.45 & 36.0 & 0.90 & 12.43 & 15.5 & $\begin{array}{l}0.639 \\
0.802\end{array}$ \\
\hline Dubuque..... & 34.01 & 33.2 & 1.02 & 11.89 & 15.2 & 0.783 \\
\hline Keokuk.. & 35.07 & 42.9 & 0.82 & 11.62 & 18.1 & 0.642 \\
\hline \multirow{2}{*}{\multicolumn{6}{|c|}{ Kansas: }} & \\
\hline & & & & & 18.2 & 0.626 \\
\hline Dodge City.. & 20.84 & 54.6 & 0.38 & 9.29 & 22.3 & 0.417 \\
\hline Topeka... & 33.76 & 36.1 & 0.94 & 13.95 & 13.9 & 1.004 \\
\hline Wichita. . & 30.61 & ‥ & $\ldots \ldots$ & 11.48 & & $\cdots \cdots$ \\
\hline \multicolumn{7}{|l|}{ Kentucky: } \\
\hline Louisville... & 44.33 & 54.8 & 0.81 & 11.58 & 20.0 & 0.579 \\
\hline \multicolumn{7}{|l|}{ Louisiana: } \\
\hline New Orleans... & 57.42 & 45.4 & 1.27 & 18.24 & 12.5 & 1.459 \\
\hline $\begin{array}{l}\text { Shreveport......... } \\
\text { Mnine: }\end{array}$ & 45.68 & 45.6 & 1.00 & 9.54 & 14.3 & 0.667 \\
\hline $\begin{array}{l}\text { Maine: } \\
\text { Eastport. }\end{array}$ & 43.27 & 25.2 & \multicolumn{4}{|c|}{ Maine: } \\
\hline Portland... & 42.51 & 29.7 & 1.43 & 10.18 & 11.0 & 0.926 \\
\hline \multicolumn{7}{|l|}{ Maryland: } \\
\hline Baltimore.. & 43.18 & 48.1 & 0.90 & 12.87 & 16.6 & 0.775 \\
\hline Washington, D. C. . & 43.50 & 45.6 & 0.95 & 13.23 & 16.3 & 0.812 \\
\hline \multicolumn{7}{|l|}{ Massachusetts: } \\
\hline $\begin{array}{l}\text { Boston....... } \\
\text { Nantucket.. }\end{array}$ & $\begin{array}{l}43.38 \\
37.00\end{array}$ & $\begin{array}{l}34.4 \\
25.6\end{array}$ & 1.26 & $\begin{array}{r}10.42 \\
8.14\end{array}$ & $\begin{array}{r}13.1 \\
9.2\end{array}$ & 0.796 \\
\hline \multicolumn{7}{|l|}{ Michigan: } \\
\hline Alpena.. & 33.20 & 24.3 & 1.37 & 9.95 & 11.1 & 0.896 \\
\hline Detroit..... & 32.16 & 36.0 & 0.89 & 10.14 & 15.9 & 0.638 \\
\hline Escanaba... & 31.51 & & 1 & 10.55 & & 0 \\
\hline Grand Haven... & 31.37 & 28.6 & 1.10 & 7.67 & 12.3 & 0.624 \\
\hline Grand Rapids.. & $\begin{array}{l}31.47 \\
32.62\end{array}$ & $\cdots \cdots$ & $\cdots \cdots$ & $\begin{array}{l}7.74 \\
9.46\end{array}$ & $\cdots \cdots$ & $\cdots \cdots$ \\
\hline $\begin{array}{l}\text { Houghton..... } \\
\text { Lansing (S63). }\end{array}$ & $\begin{array}{l}32.62 \\
30.99\end{array}$ & 27.6 & 1.12 & $\begin{array}{r}9.40 \\
10.10\end{array}$ & 12.2 & 0.828 \\
\hline $\begin{array}{l}\text { Lansing (\$63)........ } \\
\text { Marquette....... }\end{array}$ & $\begin{array}{l}30.99 \\
32.63\end{array}$ & 24.5 & 1.33 & 9.47 & 10.0 & 0.947 \\
\hline Port Huron. & 30.65 & 29.3 & 1.05 & 8.61 & 12.6 & 0.683 \\
\hline Sault Ste. Mario.. & 31.38 & $\therefore \ldots$. & $\ldots \ldots$ & 8.66 & ….. & $\cdots \cdots$ \\
\hline \multicolumn{7}{|l|}{ Minnesota: } \\
\hline $\begin{array}{l}\text { Duluth....... } \\
\text { Minneapolis. }\end{array}$ & 29.31 & & & 11.51 & 3.0 & 1.190 \\
\hline Moorhead. & 24.92 & 26.3 & 0.95 & 10.97 & 10.8 & 1.015 \\
\hline St. Paul........ & 28.68 & 28.1 & 1.02 & 11.27 & 12.8 & 0.853 \\
\hline $\begin{array}{l}\text { Pembina (S57). } \\
\text { (St. Vincent)... }\end{array}$ & 20.31 & 22.1 & 0.92 & 9.04 & 9.6 & 0.942 \\
\hline \multicolumn{7}{|l|}{ Mississippi: } \\
\hline $\begin{array}{l}\text { Meridlian..... } \\
\text { Virkshurg. . . }\end{array}$ & $\begin{array}{l}5.3 .20 \\
53.74\end{array}$ & 17.1 & 1.14 & $\begin{array}{l}12.67 \\
12.44\end{array}$ & 13.8 & 0.902 \\
\hline
\end{tabular}


TABLE 15.-Precipitation and evaporation data for the year and for the three summer months, June to August.-Continued.

\begin{tabular}{|c|c|c|c|c|c|c|}
\hline Station. & 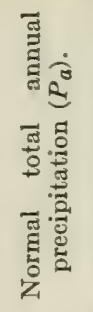 & 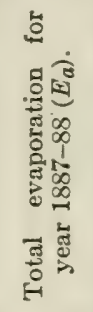 & 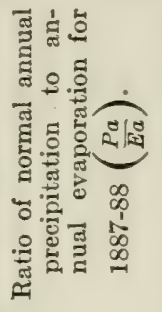 & 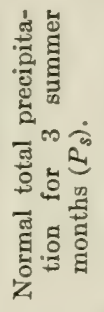 & 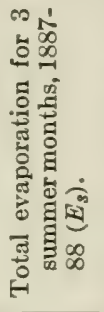 & 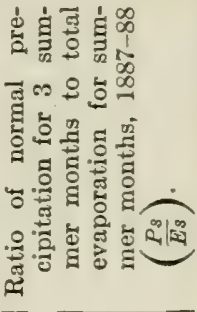 \\
\hline Missouri- & inches. & inches. & & inches. & inches. & \\
\hline $\begin{array}{l}\text { Missouri: } \\
\text { Columbia. }\end{array}$ & 36.61 & & $\cdots$ & 11.07 & & \\
\hline Hannibal.: & 34.26 & $\ldots \ldots$ & $\ldots \ldots$ & 10.65 & $\ldots \ldots$ & ..... \\
\hline $\begin{array}{l}\text { Kansas City....... } \\
\text { (Leavenworth,Kan.) }\end{array}$ & 37.28 & 41.6 & 0.90 & 14.25 & 15.8 & 0.902 \\
\hline Lamar $(\mathrm{S} 49) \ldots \ldots$. & 41.24 & 39.6 & 1.04 & 13.51 & 14.6 & 0.925 \\
\hline St. Louis. . & 37.20 & 52.2 & 0.71 & 10.56 & 20.5 & 0.515 \\
\hline Springfield. & 44.57 & 38.3 & 1.16 & 14.29 & 12.4 & 1.152 \\
\hline Montana: & & & & & & \\
\hline $\begin{array}{l}\text { Crow Agency. . } \\
\text { (Fort Custer). }\end{array}$ & 14.56 & 52.0 & 0.28 & 5.04 & 22.5 & 0.224 \\
\hline $\begin{array}{l}\text { Havre .............. } \\
\text { (Fort Assiniboine) }\end{array}$ & 13.67 & 39.5 & 0.35 & 6.00 & 16.5 & 0.364 \\
\hline Helena............ & 12.77 & 53.4 & 0.24 & 3.87 & 20.4 & 0.190 \\
\hline Kalispell ......... & 16.94 & & $0-4$ & 3.47 & 16.0 & \\
\hline $\begin{array}{l}\text { Fort Maginnis (S30) } \\
\text { Miles City }\end{array}$ & 16.52 & 35.8 & 0.46 & $\begin{array}{l}5.20 \\
5.17\end{array}$ & $\begin{array}{l}16.0 \\
\ldots \ldots\end{array}$ & $\begin{array}{l}0.320 \\
\therefore \ldots\end{array}$ \\
\hline Poplar (S30)... & $\begin{array}{l}13.17 \\
13.59\end{array}$ & 35.4 & 0.38 & 5.66 & 16.5 & 0.343 \\
\hline $\begin{array}{l}\text { (Poplar } R \\
\text { Nebraska: }\end{array}$ & & & & & & \\
\hline Crete (S37). & 29.06 & 35.5 & 0.82 & 13.02 & 14.8 & 0.880 \\
\hline Lincoln... & 27.51 & & & 11.86 & & \\
\hline $\begin{array}{l}\text { North Platte...... } \\
\text { Omaha............ }\end{array}$ & $\begin{array}{l}18.86 \\
30.66\end{array}$ & $\begin{array}{l}41.3 \\
41.7\end{array}$ & $\begin{array}{l}0.46 \\
0.74\end{array}$ & $\begin{array}{r}8.39 \\
13.00\end{array}$ & $\begin{array}{l}17.7 \\
16.6\end{array}$ & $\begin{array}{l}0.474 \\
0.783\end{array}$ \\
\hline Valentine.. & 22.46 & 43.8 & 0.51 & 10.03 & 17.2 & 0.584 \\
\hline Nevada: & & & & & & \\
\hline Reno.. & 10.43 & & & 0.63 & & \\
\hline Winnemucca.. & 8.40 & 83.9 & 0.10 & 0.98 & 33.6 & 0.029 \\
\hline $\begin{array}{l}\text { New Hampshire: } \\
\text { Concord...... } \\
\text { (Manchester) }\end{array}$ & 40.11 & 33.3 & 1.20 & 10.87 & 12.4 & 0.877 \\
\hline New Jersey: & & & & & & \\
\hline Atlantic City. & 40.82 & 25.2 & 1.62 & 11.11 & 9.8 & 1.133 \\
\hline $\begin{array}{l}\text { Cape May... } \\
\text { New Mexico: }\end{array}$ & 40.75 & & & 10.98 & & ...... \\
\hline Fort Stanton (S2) & 16.70 & 76.0 & 0.22 & 8.07 & 31.9 & 0.253 \\
\hline $\begin{array}{l}\text { Sante Fe. } \\
\text { Sew }\end{array}$ & 14.49 & 79.8 & 0.18 & 6.11 & 31.9 & 0.192 \\
\hline New York: & & & & & & \\
\hline $\begin{array}{l}\text { Albany....... } \\
\text { Binghamton. }\end{array}$ & $\begin{array}{l}36.38 \\
32.94\end{array}$ & 34.8 & 1.05 & $\begin{array}{l}11.62 \\
10.48\end{array}$ & 14.2 & 0.818 \\
\hline Buffalo...... & 37.28 & 32.9 & 1.13 & 9.53 & 14.0 & 0.683 \\
\hline Canton. & 36.18 & $\ldots \ldots$ & $\ldots \ldots$ & 9.35 & ..... & $\ldots \ldots$ \\
\hline Ithaca. . & 34.23 & $\ldots \ldots$ & & 10.87 & & \\
\hline New York.. & 44.63 & 40.6 & 1.10 & 12.33 & 14.8 & 0.834 \\
\hline Oswego... & 36.18 & 28.9 & 1.25 & 9.35 & 11.7 & 0.799 \\
\hline Rochester.. & 34.27 & 32.4 & 1.06 & 9.18 & 13.6 & 0.675 \\
\hline Syracuse. & 34.30 & & $\ldots \ldots$ & 10.90 & …... & \\
\hline
\end{tabular}


TABLE 15.-Precipitation and evaporation data for the year and for the three summer month8, June to August.-Continued.

\begin{tabular}{|c|c|c|c|c|c|c|}
\hline Station. & 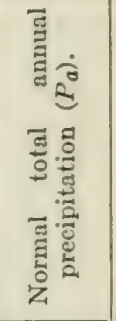 & 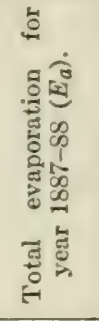 & 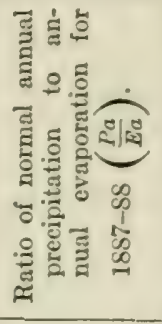 & 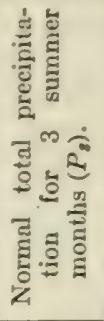 & 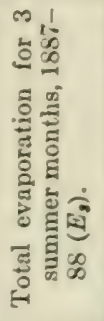 & 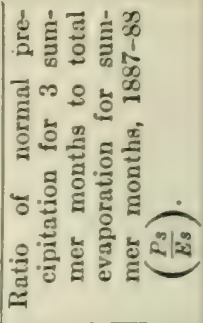 \\
\hline $\begin{array}{l}\text { North Carolina: } \\
\text { Asheville.... }\end{array}$ & $\begin{array}{c}\text { inches. } \\
49.56\end{array}$ & inches. & & $\begin{array}{l}\text { inches. } \\
14.00\end{array}$ & inches. & \\
\hline Charlotte... & 49.20 & 49.0 & 1.00 & 15.50 & 13.8 & 1.123 \\
\hline Hatteras. . & 60.85 & 31.3 & 1.94 & 16.30 & 10.4 & 1.567 \\
\hline Raleigh. . & 49.60 & 37.0 & 1.34 & 16.73 & 12.8 & 1.307 \\
\hline Wilmington.... & 51.05 & 38.4 & 1.33 & 19.10 & 11.7 & 1.632 \\
\hline $\begin{array}{l}\text { North Dakota: } \\
\text { Bismarck... }\end{array}$ & 17.64 & 31.0 & 0.57 & 7.66 & 13.9 & 0.550 \\
\hline $\begin{array}{l}\text { Devils Lake... } \\
\text { (Fort Totten). }\end{array}$ & 20.16 & 27.2 & 0.74 & 10.07 & 11.7 & 0.861 \\
\hline $\begin{array}{l}\text { Williston....... } \\
\text { (Fort Buford).. }\end{array}$ & 15.07 & 35.5 & 0.42 & 6.91 & 16.1 & $0.42 \theta$ \\
\hline Ohio: & & & & & & \\
\hline Cincinna & 37.33 & 52.0 & 0.72 & 10.85 & 19.5 & 0.556 \\
\hline Cleveland. & 35.04 & 35.7 & 0.98 & 10.38 & 14.5 & 0.716 \\
\hline Columbus. & 36.92 & 47.8 & 0.77 & 10.36 & 19.1 & 0.543 \\
\hline Sandusky . & 34.02 & 36.6 & 0.93 & 10.98 & 15.4 & 0.713 \\
\hline Toledo.: & 30.62 & 38.6 & 0.79 & 9.32 & 17.0 & 0.548 \\
\hline $\begin{array}{l}\text { Oklahoma: } \\
\text { Fort Sill (S41). }\end{array}$ & 30.85 & 46.1 & 0.67 & 9.83 & 16.7 & 0.589 \\
\hline $\begin{array}{l}\text { Fort Sill (S41). } \\
\text { Oklahoma..... }\end{array}$ & 31.69 & & $\ldots .$. & 9.89 & $10.8:$ & 0.009 \\
\hline Oregon: & & & & & & \\
\hline Astoria (S17). & 75.35 & 25.3 & 2.98 & 5.62 & 8.6 & 0.653 \\
\hline Baker City... & 13.20 & 347 & 1.30 & $\begin{array}{l}2.03 \\
2.97\end{array}$ & 128 & 0.232 \\
\hline $\begin{array}{l}\text { Portland........ } \\
\text { Roseburg.... }\end{array}$ & $\begin{array}{l}45.13 \\
34.43\end{array}$ & $\begin{array}{l}34.7 \\
39.2\end{array}$ & 0.88 & $\begin{array}{l}2.97 \\
1.72\end{array}$ & 13.6 & 0.127 \\
\hline Pennsylvania: & & & & & & \\
\hline Erie. : & 38.55 & 33.8 & 1.14 & 10.22 & 14.9 & 0.686 \\
\hline Harrisburg. & 37.27 & & & 11.67 & & \\
\hline Philadelphia. & 41.17 & 45.0 & 0.91 & 12.24 & 16.6 & 0.738 \\
\hline Pittsburgh.. & 36.35 & 44.5 & 0.82 & 11.49 & 17.6 & 0.653 \\
\hline $\begin{array}{l}\text { Scranton. : } \\
\text { Rhode Island: }\end{array}$ & 37.05 & & $\cdots$ & 11.65 & …... & $\cdots \cdots$ \\
\hline $\begin{array}{l}\text { Rhode Island: } \\
\text { Block Island. }\end{array}$ & 44.36 & 24.0 & 1.85 & 9.66 & 8.2 & 1.178 \\
\hline Providence. . & 43.38 & & ....... & 10.42 & & ...... \\
\hline South Carolina: & & & & & & \\
\hline Charleston.... & 52.07 & 43.7 & 1.19 & 19.52 & 13.7 & 1.425 \\
\hline Columbia... & 46.08 & 43.2 & 1.07 & 17.02 & 13.4 & 1.268 \\
\hline $\begin{array}{l}\text { South Dakota: } \\
\text { Huron.... }\end{array}$ & 21.10 & 33.0 & 0.64 & 9.36 & 14.0 & 0.868 \\
\hline $\begin{array}{l}\text { Huron....... } \\
\text { Pierre...... }\end{array}$ & 16.63 & 41.9 & 0.40 & 7.44 & 17.8 & 0.418 \\
\hline $\begin{array}{l}\text { (Furt Sully) } \\
\text { Rapid City. }\end{array}$ & 18.69 & 32.0 & & 8.25 & & \\
\hline Yankton..... & 25.43 & 31.0 & 0.82 & 10.91 & 12.7 & 0.859 \\
\hline Tennessee: & & & & & & \\
\hline Chattanooga. & 50.68 & 46.4 & 1.09 & 11.93 & 13.6 & 0.877 \\
\hline Knoxville... & 49.35 & 45.9 & 1.08 & 12.38 & 14.1 & 0.879 \\
\hline Memphis.. & 50.34 & 50.0 & 1.01 & 11.08 & 15.1 & 0.734 \\
\hline Nashville. & 48.49 & 50.1 & 0.97 & 12.19 & 16.9 & 0.722 \\
\hline
\end{tabular}


TABLE 15.-Precipitation and evaporation data for the year and for the three summer months, June to August.-Continued.

\begin{tabular}{|c|c|c|c|c|c|c|}
\hline Station. & 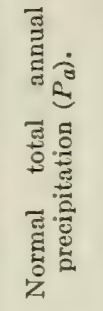 & 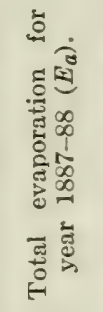 & 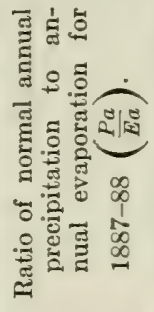 & 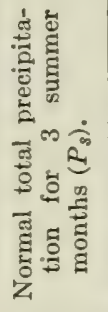 & 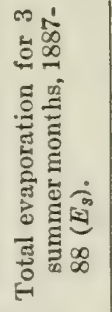 & 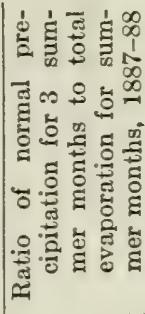 \\
\hline $\begin{array}{l}\text { Texas: } \\
\text { Abilene. . }\end{array}$ & $\begin{array}{l}\text { inches. } \\
24.74\end{array}$ & $\begin{array}{c}\text { inches. } \\
54.4\end{array}$ & 0.46 & $\begin{array}{r}\text { inches. } \\
7.54\end{array}$ & $\begin{array}{c}\text { inches. } \\
22.8\end{array}$ & 0.331 \\
\hline Amarillo.......... & & 55.4 & 0.41 & 8.97 & 22.0 & 0.408 \\
\hline (Fort Eliot) ...... & 22.00 & $\begin{array}{ll}30.4 \\
38.8\end{array}$ & 0.41 & 6.88 & 120 & 0.530 \\
\hline Corpus Christi.... & 27.18 & 38.8 & 0.70 & 6.68 & 12.6 & $\begin{array}{l}0.530 \\
0.143\end{array}$ \\
\hline El Paso..... & 9.84 & 82.0 & 0.12 & 4.40 & 30.7 & $\begin{array}{l}0.143 \\
0.580\end{array}$ \\
\hline $\begin{array}{l}\text { Brownsville (S1)... } \\
\text { Fort Davis (S2) ... }\end{array}$ & $\begin{array}{l}26.89 \\
17.46\end{array}$ & $\begin{array}{l}37.0 \\
96.4\end{array}$ & $\begin{array}{l}0.73 \\
0.18\end{array}$ & $\begin{array}{l}6.96 \\
9.01\end{array}$ & $\begin{array}{l}12.0 \\
32.4\end{array}$ & $\begin{array}{l}0.580 \\
0.278\end{array}$ \\
\hline $\begin{array}{l}\text { Fort Ringgold (S1) } \\
\text { (Rio Grande City). }\end{array}$ & 17.46 & 53.1 & 0.33 & 5.21 & 18.5 & 0.282 \\
\hline Fort Worth....... & 26.89 & & & 7.88 & & \\
\hline Galveston........ & 47.06 & 46.0 & 1.02 & 13.74 & 14.7 & 0.934 \\
\hline Palestine......... & 43.02 & 47.1 & 0.91 & 9.22 & 14.9 & 0.618 \\
\hline San Antonio....... & 26.83 & 52.4 & 0.51 & 8.02 & 16.9 & 0.474 \\
\hline Taylor........... & 35.47 & $\cdots \cdots$ & $\ldots \ldots$ & 8.61 & ...... & $\cdots \cdots$ \\
\hline Utah: & & & & & & \\
\hline Modena.. & 10.15 & & & 1.14 & $\cdots \cdots$ & ..... \\
\hline Salt Lake City..... & 16.03 & 74.4 & 0.22 & 2.09 & 28.8 & 0.073 \\
\hline Vermont: & & & & & & \\
\hline Burlington... & 31.56 & $\because \cdots \cdots$ & & 11.05 & $\ldots \ldots$ & $\because \cdots$ \\
\hline Northfield........ & 33.84 & 33.9 & 1.00 & 10.86 & 9.6 & 1.131 \\
\hline Virginia: & & & & & & \\
\hline Lynchburg........ & 43.42 & 45.5 & 0.95 & 12.17 & 14.6 & 0.834 \\
\hline Norfolk. ......... & 49.54 & 35.6 & 1.39 & 16.10 & 12.5 & 1. 288 \\
\hline Richmond........ & 41.63 & $\ldots \ldots$ & $\ldots \ldots$ & 12.35 & $\ldots \ldots$ & $\cdots \cdots$ \\
\hline Wytheville........ & 46.71 & $\ldots \ldots$ & $\ldots \ldots$ & 13.09 & $\cdots \cdots$ & $\cdots \cdots$ \\
\hline Washington: & & & & & & \\
\hline $\begin{array}{l}\text { North Head....... } \\
\text { (Fort Canby) ... }\end{array}$ & 45.77 & 21.1 & 2.17 & 2.87 & 7.07 & 0.410 \\
\hline Olympia (S19).... & 55.23 & 26.8 & 2.06 & 3.12 & 11.6 & $0.26 \theta$ \\
\hline Port Angeles...... & $\ldots \ldots$ & 19.1 & $\ldots \ldots$ & $\ldots \ldots$ & 6.0 & $\cdots \cdots$ \\
\hline Seattle.......... & 36.59 & $\ldots \ldots$ & $\cdots \cdots$ & 2.90 & $\because \cdots$ & $\ldots \ldots$ \\
\hline Spokane......... & 18.85 & 42.8 & 0.44 & 2.76 & 18.5 & 0.149 \\
\hline Tacoma ........... & 45.41 & $\cdots \cdots$ & $\cdots \cdots$ & 3.49 & $\cdots \cdots$ & $\because \because 7$ \\
\hline Tatoosh Island.... & 88.78 & 18.1 & 4.90 & 8.07 & 4.6 & 1.754 \\
\hline Walla Walla...... & 17.67 & 57.7 & 0.31 & 2.03 & 23.5 & 0.086 \\
\hline West Virginia: & & & & & & \\
\hline Elkins.......... & 42.75 & $\ldots$ & $\ldots \ldots$ & 13.33 & $\cdots \cdots$ & $\cdots \cdots$ \\
\hline Parkersburg. ... & 40.22 & $\cdots \cdots$ & $\cdots \cdots$ & 12.84 & $\cdots \cdots$ & $\cdots \cdots$ \\
\hline $\begin{array}{l}\text { Wisconsin: } \\
\text { Green Ba. }\end{array}$ & 31.12 & 28.2 & 1.10 & 10.16 & 13.9 & 0.731 \\
\hline La Crosse...... & 31.17 & 32.9 & 0.95 & 11.91 & 14.5 & 0.822 \\
\hline Madison........ & 31.71 & $\cdots \cdots$ & $\cdots \cdots$ & 11.30 & …․ & \\
\hline Milwaukee....... & 31.40 & 29.0 & 1.08 & 9.50 & 12.3 & 0.774 \\
\hline Wyoming: & & & & & & \\
\hline Cheyenne..... & 13.60 & 76.5 & 0.18 & 5.03 & 26.1 & 0.193 \\
\hline $\begin{array}{l}\text { Evanston }(\mathrm{H}) \ldots \\
\text { (Fort Bridger).... }\end{array}$ & 13.1 & 56.1 & 0.23 & 1.90 & 21.0 & 0.090 \\
\hline Lander......... & 13.92 & $\cdots \cdots$ & $\ldots \ldots$ & 2.51 & ... & $\cdots \cdots$ \\
\hline
\end{tabular}


Column 6 of table 15 presents the sums of Russell's three monthly losses, for each station considered, for June 1888 and July and August 1887, these numbers representing inches of depth. To obtain the average monthly rate, each number is to be divided by 3 ; to obtain the weekly rate each is to be divided by 13 , etc.; but since any such alteration in the time increment considered would result in applying the same coefficient to the entire series of indices, and since relative indices for the different stations are all that are here requisite, we employ the totals simply. These are charted in plate 55, where isoatmic lines are represented for increments of 5 inches, those for the values 10,15 , and 30 being distinct. This chart exhibits about the same features as does that based upon the length of the mean frostless season as the duration factor.

(3) Evaponation Studies of 1908.

Presentation of data.-As has been stated, a series of evaporation observations were carried out from the Desert Laboratory in the summer of 1908, the cylindrical porous-cup atmometer being employed. This is the first and only fairly representative series of direct measurements of this climatic feature that has been carried out for the United States, and we shall here enter into considerable detail in the discussion of our results.

As has been mentioned, certain aspects of this evaporation study formed the subject of a paper by Livingston. ${ }^{1}$ The following presentation will proceed without attempting to distinguish between what is now published for the first time and what is here repeated from Livingston's paper. Since that paper was published the data have been thoroughly revised, which accounts for some numerical discrepancies between our tables and those of Livingston.

Two standardized porous cups were sent to each of 38 stations in the United States and Canada, in the spring of 1908, and were there operated side by side in the open till midsummer, when one of them was returned to the Desert Laboratory and restandardized. The restandarized cups were then sent back to their respective stations and again installed, and the two then continued to operate till the work was discontinued. In the fall both cups were returned and restandardized. Thus coefficients of correction were determined for the beginning, middle, and end of the season's operation, and the amount of variation in these coefficients was determined without interrupting the series of observations. All standardizations were made with reference to standard cups, the same standard being employed as is still in use for cylindrical cups supplied by the Plant World. Thus the corrected readings here given are directly comparable to corrected

'Livingston, B. E., A study of the relation between summer evaporation intensity and centers of plant distribution in the United States, Plant World 14: 205-222, 1911. 
readings from all cups related to the Livingston cylindrical standard, wherever and whenever they may have been operated.

The cups were freely exposed to sunshine and wind in every case. The center of the cup was $15 \mathrm{~cm}$. above the soil surface, so that our records may be considered as proportional to the evaporating powers of the air (as this feature would affect the transpirational water-loss from a plant of about the above-named height, growing in the open) at the respective stations and for the respective time periods. The mountings were of the simple absorbing type ${ }^{1}$ and some rain absorption is undoubtedly involved in the records. At that time the nonabsorbing form had not yet been devised. Weekly readings were made.

When the coefficient of correction of any cup altered, as shown by the mid-summer or final calibrations, interpolations were made to give probable coefficients for each of the weeks intervening between calibrations, it being borne in mind that, where two cups operate side by side, their weekly readings constitute a continuous series of measurements of the relative fluctuations of their coefficients. After the derivation of the coefficients for each week of the entire series of observations, all readings were corrected (by multiplying the actual reading by the corresponding coefficient), and the resulting pair of records (when two cups had operated simultaneously at the same place - that is, excepting for the period of mid-summer calibration, when but one cup was exposed) were averaged, to give the corrected rate for each week and station.

To the observers at the 38 stations involved in this study our sincere thanks are due; without their kind and sustained help the study could not have been carried out at all. The names of the observers who so generously cooperated in this work have been given by Livingston (Plant World, 1911).

Owing to various considerations, observations were not begun at the same time at all stations, and were not discontinued simultaneously. Also, accidents and interruptions of various sorts occurred, so that the final records are of unequal completeness in several respects. Such as they are, they are presented in table 16.

The observations will be considered in five 5-week periods and a final 3-week period, and each of these periods constitutes a subdivision of table 16 , which gives the weekly evaporation data and also the corresponding weekly records of precipitation whenever the latter are available. The units employed for evaporation are cubic centimeters (of water lost by the standard cylindrical porous-cup atmometer), and precipitation is given in inches of depth in the customary way.

\footnotetext{
1 The mounting as actually used is described and figured in the following papers: Livingston, 1907, a.-Idem, A simple atmometer, Science, n. s., 28: 319-330, 1908. The non-absorbing mounting was first described by Livingston (Livingston, B. E., A rain-correcting atmometer for ecological instrumentation, Plant World 13: 79-82, 1910). It was improved by Shive (Shive, J. W., An improved non-absorbing porous cup atmometer, Plant World, 18: 7-10, 1915). See also Livingston, 1915, $a$.
} 
TABLE 16.-Weekly precipitation $(P)$ and weekly rates of evaporation $(E)$, the latter from cylindrical porous-cup atmometers, summer of 1908.

First 5-Week Period (Apr. 21 to May 25).

\begin{tabular}{|c|c|c|c|c|c|c|c|c|c|c|c|c|}
\hline \multirow{2}{*}{ Station. } & \multicolumn{2}{|c|}{$\begin{array}{l}\text { Apr. } 21 \\
\text { to } 27 \text {. }\end{array}$} & \multicolumn{2}{|c|}{$\begin{array}{l}\text { Apr. } 28 \\
\text { to May } 4 .\end{array}$} & \multicolumn{2}{|c|}{$\begin{array}{l}\text { May } 5 \\
\text { to } 11 .\end{array}$} & \multicolumn{2}{|c|}{$\begin{array}{l}\text { May } 12 \\
\text { to } 18 .\end{array}$} & \multicolumn{2}{|c|}{$\begin{array}{l}\text { May } 19 \\
\text { to } 25 .\end{array}$} & \multicolumn{2}{|c|}{ Average. } \\
\hline & $P$ & $E$ & $P$ & $E$ & $P$ & $E$ & $P$ & $E$ & $P$ & $E$ & $P$ & $E$ \\
\hline Arizona: Tueson. & in. & c.c. & in. & $\begin{array}{l}\text { c.c. } \\
\text {. }\end{array}$ & in. & c.c. & in. & $\begin{array}{l}\text { c.c. } \\
\text {. }\end{array}$ & $\begin{array}{l}i n . \\
0.11\end{array}$ & $\begin{array}{l}\text { c.c. } \\
390\end{array}$ & $\begin{array}{l}\text { in. } \\
0.11\end{array}$ & $\begin{array}{l}\text { c.c. } \\
390 \text { (1) }\end{array}$ \\
\hline $\begin{array}{l}\text { Florida: } \\
\text { Gainesville... }\end{array}$ & & & & & 0.70 & 251 & 0.57 & 269 & 0.17 & 254 & 0.48 & $268(3)$ \\
\hline 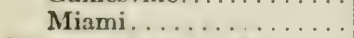 & 0.35 & 184 & ${ }^{1} 0.82$ & 133 & ${ }^{1} 0.82$ & 133 & 0.21 & 232 & 0.62 & 222 & 0.56 & 181 \\
\hline Illinois: Charleston ${ }^{2}$. & $\ldots \ldots$ & $\ldots$ & & & & & & & $0 . \leqslant 1$ & 149 & 0.81 & 149 (1) \\
\hline Louisiana: Cameron². & & & & & & & & & 0.28 & 245 & $0.2 \varepsilon$ & 245 (1) \\
\hline Maryland: Easton ${ }^{2}$. & & & 1.46 & 166 & 1.44 & 61 & 0.13 & $15 \mathrm{~S}$ & 0.36 & 8.3 & 0.85 & $117(4)$ \\
\hline Michigan: Grand Rapids... & & & & & & & & & 0.39 & 156 & 0.39 & $156(1)$ \\
\hline $\begin{array}{l}\text { Missouri: St. Louis. . . . . . . } \\
\text { Nebraska: }\end{array}$ & 1.75 & 104 & 0.54 & 91 & 3.50 & 71 & 0.95 & 134 & 1.55 & 131 & 1. 66 & 108 \\
\hline Lincoln . . . . . . . . . . . . & & & & & & & & & 2.11 & 45 & 2.11 & $48(1)$ \\
\hline North Platte. & & & & & & & & & 0.61 & 245 & 0.64 & $245(1)$ \\
\hline $\begin{array}{l}\text { NewBrunswick:Fredericton } \\
\text { North Carolina: }\end{array}$ & & & & & & & 0.37 & 137 & 0.0 & 160 & 0.19 & $149(2)$ \\
\hline West Raleigh. . . . . . . . . & 0.50 & 189 & 0.73 & 215 & 0.21 & $20 s$ & 0.19 & 242 & 1.40 & 156 & 0.61 & 202 \\
\hline North Dakota: Dickinson ${ }^{2}$. & ... & $\ldots$. & & $\ldots$ & & 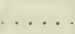 & 1.46 & 107 & 1.98 & 93 & 1.72 & $100(2)$ \\
\hline Ohio: Oxford ${ }^{3} \ldots \ldots \ldots \ldots$ & & & & & & & ${ }^{4} T$ & 133 & 1. 28 & $7 S$ & 0.61 & $106(2)$ \\
\hline Oklahoma: Stillwater..... & & & 1.51 & 168 & 0.12 & 168 & 1.86 & 95 & 8.31 & 94 & 2.95 & $131(4)$ \\
\hline Oregon: Eugene.......... & . . & & & $\ldots$ & $\ldots$ & & 0.38 & 42 & 0.80 & 63 & 0.59 & $53(2)$ \\
\hline Tennesree: Knoxville... & & & & & & & 0.02 & 132 & 1. 29 & 72 & 0.66 & $102(2)$ \\
\hline Texas: Dalhart........ & & & & & & & $\ldots \ldots$ & $\ldots$. & 0.52 & 522 & 0.52 & $522(1)$ \\
\hline
\end{tabular}

1 Calculated from 2-week total.

2 Precipitation data from monthly weather reports.
${ }^{3}$ Precipitation data are for Jacksonburg.

4 means trace, less than 0.01 inch. 
TABLE 16.-Weekly precipitation $(P)$ and weekly rates of evaporation $(E)$, the latter from cylindrical porous-cup atmometers, summer of 1908.-Continued.

\begin{tabular}{|c|c|c|c|c|c|c|c|c|c|c|c|c|}
\hline \multirow{2}{*}{ Station. } & \multicolumn{2}{|c|}{$\begin{array}{c}\text { May } 26 \\
\text { to June } 1 .\end{array}$} & \multicolumn{2}{|c|}{$\begin{array}{c}\text { June } 2 \\
\text { to } 8 .\end{array}$} & \multicolumn{2}{|c|}{$\begin{array}{l}\text { J une } 9 \\
\text { to } 15 .\end{array}$} & \multicolumn{2}{|c|}{$\begin{array}{l}\text { June } 16 \\
\text { to } 22 .\end{array}$} & \multicolumn{2}{|c|}{$\begin{array}{l}\text { June } 23 \\
\text { to } 29 \text {. }\end{array}$} & \multicolumn{2}{|c|}{ Average. } \\
\hline & $P$ & $E$ & $P$ & $E$ & $P$ & $E$ & $P$ & E & $P$ & $E$ & $P$ & $E$ \\
\hline & in. & c.c. & in. & c.c. & $i n$. & c.c. & in. & c.c. & in. & c.c. & in. & c.c. \\
\hline Arizona: Tueson... & 0.00 & 407 & 0.00 & 427 & 0.00 & 541 & 0.00 & 543 & $1 T$ & 635 & 0.00 & 511 \\
\hline California: San Diegc & & & & & & & 0.00 & 194 & 0.00 & 201 & 0.00 & $198(2)$ \\
\hline Colorado: Boulder... & 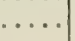 & 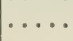 & & .... & 0.25 & 141 & 0.00 & 281 & 0.02 & 252 & 0.09 & $225(3)$ \\
\hline Florida: & & & & & & & & & & & & \\
\hline Gainesville ${ }^{2}$ & ${ }^{4} T$ & 277 & 2.14 & 237 & 1.50 & & 2.95 & 120 & 2.36 & 170 & 1.86 & $201(4)$ \\
\hline Miami.... & 4.44 & 165 & 3.30 & 144 & 1.21 & 137 & 4.02 & 109 & 3.18 & 124 & 3.23 & 136 \\
\hline Illinois: & & & & & & & & & & & & \\
\hline Charleston ${ }^{2}$ & 0.07 & 176 & 1.41 & 201 & 0.37 & 153 & 0.62 & 208 & 1.02 & 205 & 0.70 & 189 \\
\hline Urbana... & & & 0.09 & 145 & 0.46 & 114 & 1.52 & 88 & ${ }^{4} T$ & 104 & 0.52 & $113(4)$ \\
\hline Louisiana: Camero & 0.00 & 283 & 0.19 & 265 & 4.46 & 195 & 0.26 & 256 & 0.25 & 170 & 1.03 & 234 \\
\hline Maine: Orono ${ }^{2}$. & 2.30 & 48 & 0.70 & 139 & ${ }^{4} T$ & 128 & 0.53 & 165 & 0.12 & & 0.88 & $120(4)$ \\
\hline Maryland: Easton ${ }^{2}$ & 1.35 & 133 & 0.33 & 121 & 0.53 & 178 & 0.59 & 156 & 0.12 & 203 & 0.58 & \\
\hline Michigan: & & & & & & & & & & & & \\
\hline Grand Rapids. & 0.84 & 104 & 0.00 & 227 & 0.61 & 121 & 0.08 & 144 & 0.19 & 168 & 0.34 & 153 \\
\hline Houghton... & & $\ldots$. & & & & & 1.51 & 198 & 0.31 & 146 & 0.91 & $172(2)$ \\
\hline Minnesota: Minneapolis & & & 0.86 & 72 & 1.92 & 98 & 1.35 & 81 & 2.19 & 122 & 1.58 & $93(4)$ \\
\hline Missouri: St. Loui & 1.29 & 119 & 1.02 & 99 & 0.68 & 148 & 0.63 & 192 & 0.15 & 201 & 0.75 & 152 \\
\hline Montana: Bozema & 4.45 & 16 & 2.71 & 14 & 0.15 & 64 & 1.49 & 10 & 0.06 & 93 & 1.77 & 39 \\
\hline Nebraska: & & & & & & & & & & & & \\
\hline Lincoln... & 1.38 & 50 & 6.09 & 36 & 2.92 & 51 & 1.43 & 105 & 0.79 & 125 & 2.25 & 73 \\
\hline North Pla & 0.22 & 148 & 0.30 & 153 & 2.27 & 86 & 2.32 & 95 & 0.09 & 108 & 1.04 & 118 \\
\hline Nevada: Reno & & & 0.17 & & 0.07 & & ${ }^{4} T$ & & 0.00 & & & \\
\hline New Brunswick: Fredericton & 2.37 & 21 & 0.70 & 106 & 0.14 & 121 & 1.43 & 91 & 0.14 & 132 & 0.96 & 94 \\
\hline New York: & & & & & & & & & & & & \\
\hline New York. & & & 0.00 & 167 & 0.00 & 136 & 0.80 & 93 & 0.20 & 132 & 0.25 & $132(4)$ \\
\hline Syracu & & & & & & & & & 0.67 & 320 & 0.67 & $320(1)$ \\
\hline North Carolina: & & & & & & & & & & & & \\
\hline Pisgah $\mathrm{F}$ & & & 1.23 & 40 & 1.25 & 52 & 0.39 & 76 & 0.63 & 99 & 0.88 & $67(4)$ \\
\hline West Raleigh. & 0.23 & 242 & 0.76 & 192 & 1.45 & 190 & 2.30 & 175 & 1.69 & 183 & 1.29 & 196 \\
\hline North Dakota: Dickinson? & 0.74 & 179 & 1.88 & 187 & 0.10 & 305 & 0.58 & 243 & 0.95 & 427 & 0.85 & 268 \\
\hline Ohio: Oxfor & 0.04 & 116 & 0.04 & 134 & 0.47 & 123 & 1.22 & 155 & 0.82 & 216 & 0.52 & 149 \\
\hline Oklahoma: Stillwater. & 0.07 & 72 & 3.55 & 48 & 2.07 & 53 & 0.29 & 205 & 0.59 & 167 & 1.32 & 109 \\
\hline Oregon: Eugene....... & 0.08 & 112 & 0.12 & 96 & 0.00 & 77 & 1.40 & 77 & 0.46 & 114 & 0.41 & 95 \\
\hline Quebec: & & & & & & & & & & & & \\
\hline $\begin{array}{l}\text { Ste. Anne de } \mathrm{Be} \\
\end{array}$ & 0.84 & & 0.06 & & 0.00 & & 0.58 & 161 & 0.14 & $\begin{array}{l}181 \\
203\end{array}$ & 0.36 & $\begin{array}{l}171(2) \\
155(2)\end{array}$ \\
\hline $\begin{array}{l}\text { Saskatchewan: Regina. } \\
\text { Tennessee: Knoxville.. }\end{array}$ & 0.03 & 117 & 1.24 & $\ddot{7 i}$ & 0.62 & 92 & 0.20 & 107 & 0.01 & 203 & 0.63 & $\begin{array}{r}155(2) \\
93(3)\end{array}$ \\
\hline Texas: Dalhart. & 0.00 & 550 & 0.51 & 483 & 0.00 & 581 & 2.06 & 656 & 0.00 & 556 & 0.51 & 565 \\
\hline Utah: Salt Lake City. & 2.53 & & 0.74 & 74 & 0.08 & 89 & 0.87 & 55 & 0.10 & 70 & 0.45 & $72(4)$ \\
\hline Vermont: Burlington. & 0.77 & 129 & 0.08 & 198 & 1.09 & 150 & 1.12 & 147 & 0.07 & 160 & 0.63 & 157 \\
\hline Washington: Seattle. & & & & & & & 0.02 & 134 & 0.01 & 177 & 0.02 & $156(2)$ \\
\hline
\end{tabular}

${ }^{1}$ Calculated from 2-week total.

2 Precipitation data from monthly weather reports.

3 Precipitation data are for Jacksonburg.

T $T$ means trace, less than 0.01 inch.

- Precipitation data are for Sapphire. 
TABLE 16.-Weekly precipitation $(P)$ and wcelily rates of evaporation $(E)$, the latler from cylindrical porous-cyp atmometers, summer of 1908.-Continued.

\begin{tabular}{|c|c|c|c|c|c|c|c|c|c|c|c|c|}
\hline \multirow{2}{*}{ Station. } & \multicolumn{2}{|c|}{$\begin{array}{c}\text { June } 30 \\
\text { to July } 6 .\end{array}$} & \multicolumn{2}{|c|}{$\begin{array}{l}\text { July } 7 \\
\text { to } 13 .\end{array}$} & \multicolumn{2}{|c|}{$\begin{array}{c}\text { July } 14 \\
\text { to } 20 .\end{array}$} & \multicolumn{2}{|c|}{$\begin{array}{c}\text { July } 21 \\
\text { to } 27 \text {. }\end{array}$} & \multicolumn{2}{|c|}{$\begin{array}{l}\text { July } 28 \\
\text { to Aug. } 3 \text {. }\end{array}$} & \multicolumn{2}{|c|}{ Average. } \\
\hline & $P$ & $E$ & $P$ & $E$ & $P$ & $E$ & $P$ & $E$ & $P$ & $E$ & $P$ & $E$ \\
\hline Alabama: Florence & in. & $c$. & & & & c.c. & in. & $c$ & & c.c. & in.. & \\
\hline $\begin{array}{l}\text { Alabama: Florence } \\
\text { Arizona: Tucson. }\end{array}$ & $\begin{array}{l}1.24 \\
4\end{array}$ & 100 & 0.13 & $\begin{array}{l}142 \\
460\end{array}$ & 1.29 & 144 & 1.05 & 116 & 0.40 & 110 & 0.82 & 122 \\
\hline California: San Diego & 0.00 & $\begin{array}{l}554 \\
207\end{array}$ & $\begin{array}{l}0.04 \\
0.00\end{array}$ & $\begin{array}{l}460 \\
264\end{array}$ & $\begin{array}{l}4.41 \\
0.00\end{array}$ & $\begin{array}{l}206 \\
249\end{array}$ & 2.88 & 271 & $\begin{array}{r}0.42 \\
0.13\end{array}$ & 298 & 1.55 & 358 \\
\hline Colorado: Boulder. & 0.59 & 180 & 0.04 & 225 & 0.16 & $\begin{array}{l}249 \\
148\end{array}$ & $\begin{array}{l}0.00 \\
4 T\end{array}$ & 195 & $\begin{array}{l}0.13 \\
1.28\end{array}$ & 153 & 0.03 & 221 \\
\hline Florida: & & & & & & & & & & & 0.41 & 180 \\
\hline Gainesville². & 0.67 & 163 & 1.78 & 161 & 2.76 & 100 & 0.04 . & & & & 1.74 & $141(3)$ \\
\hline $\begin{array}{l}\text { Miami..... } \\
\text { Illinois: }\end{array}$ & 1.86 & 171 & 0.62 & 132 & 0.26 & 184 & 1.23 & 125 & 0.63 & 109 & 0.92 & 144 \\
\hline $\begin{array}{l}\text { Illinois: } \\
\text { Charleston }\end{array}$ & & & & & & & & & & & & \\
\hline & 0.66 & 125 & 0.08 & 162 & 2.20 & 153 & 0.00 & 153 & 0.00 & 196 & 0.59 & 158 \\
\hline & 1.27 & 89 & 0.42 & 122 & 1.04 & 124 & 0.59 & 72 & 0.00 & 143 & 0.66 & 110 \\
\hline Iowa: Iowa City ${ }^{2} \ldots$. & & & & & 3.51 & 118 & 0.28 & 155 & 0.00 & 222 & 1.26 & $165(3)$ \\
\hline Louisiana: Cameron ${ }^{2}$ & 3.52 & 123 & 3.80 & 99 & 0.67 & 278 & 0.41 . & & 4.62 . & & 2.66 & $167(3)$ \\
\hline Maine: Orono2. & 0.70 & 121 & 0.00 & 178 & 0.90 & 117 & 0.86 & 91 & 0.39 & 122 & 0.57 & 126 \\
\hline Manitoba: Winnipeg & & 180 & & 209 & & 84 & & 126 & & 180 & & 156 \\
\hline $\begin{array}{l}\text { Maryland: Easton }{ }^{2} . \\
\text { Michigan: }\end{array}$ & 0.04 & 281 & 0.12 & 301 & 0.34 & 308 & 4.27 & 122 & 0.00 & 135 & 0.95 & 229 \\
\hline Grand Rapids. & 0.75 & 103 & 0.13 & 243 & 0.58 & 136 & 0.00 & 140 & 1.71 & 182 & 0.63 & 161 \\
\hline Houghton. & 0.92 & 92 & 0.06 & 188 & 1.00 & 86 & 0.02 & 147 & 1.73 & 120 & 0.75 & 127 \\
\hline St. Helen ${ }^{6}$. & 1.22 & 107 & 0.52 & & 1.45 & & 0.00 & & 0.00 & 296 & 0.61 & $202(2)$ \\
\hline Minnesota: Minneapol & 0.91 & 96 & 0.13 & 119 & 1.77 & 149 & ${ }^{4} T$ & 151 & 0.35 & 204 & 0.63 & 144 \\
\hline Missouri: St. Louis & 1.70 & 146 & 1.31 & 167 & 0.41 & 125 & 1.36 & 126 & 0.00 & 206 & 0.96 & 154 \\
\hline Montana: Bozeman². & 0.12 & 84 & 0.04 & 148 & 0.02 & 144 & 0.25 & 199 & 0.00 & 234 & 0.09 & 162 \\
\hline $\begin{array}{l}\text { Nebraska: } \\
\text { Lincoln. }\end{array}$ & 3.81 & 29 & 3.64 & & 0.07 & & 0.15 & & 0.42 & & 3.81 & $29(1)$ \\
\hline North Platte. & 1.30 & 64 & 0.69 & 125 & 0.22 & 116 & 0.96 & 119 & 0.00 & 212 & 0.63 & 127 \\
\hline Nevada: Reno ${ }^{7}$. & & & 0.04 & 536 & $4 T$ & 499 & 0.01 & 484 & 0.08 & 379 & 0.03 & $475(4)$ \\
\hline $\begin{array}{l}\text { New Brunswick: Fredericton } \\
\text { New York: }\end{array}$ & 0.20 & 129 & 0.19 & 161 & 1.27 & 98 & 0.70 & 76 & 0.06 & 128 & 0.48 & 118 \\
\hline New York. & 0.05 & 105 & $T$ & 161 & 0.85 & 178 & 2.39 & & 0.00 & & 0.30 & $148(3)$ \\
\hline $\begin{array}{l}\text { Syracuse. } \\
\text { North }\end{array}$ & 0.31 & 340 & 1.10 & & 1.41 & 270 & 1.08 & 225 & ${ }^{4} T$ & 383 & 0.70 & $305(4)$ \\
\hline $\begin{array}{l}\text { North Carolina: } \\
\text { Pisgah Forest. }\end{array}$ & & & & & & & & & & & & \\
\hline $\begin{array}{l}\text { Pisgah Forest.. } \\
\text { West Raleigh.. }\end{array}$ & $\begin{array}{l}4.50 \\
0.70\end{array}$ & $\begin{array}{r}32 \\
140\end{array}$ & 0.43 & $\begin{array}{r}68 \\
198\end{array}$ & 0. & $\begin{array}{l}104 \\
216\end{array}$ & $\begin{array}{l}0.78 \\
0.74\end{array}$ & $\begin{array}{r}68 \\
190\end{array}$ & $\begin{array}{l}0.27 \\
3.52\end{array}$ & $\begin{array}{l}81 \\
74\end{array}$ & $\begin{array}{l}1.42 \\
1.23\end{array}$ & $\begin{array}{r}70 \\
164\end{array}$ \\
\hline North Dakota: Dickinson. & 0.11 & 289 & 0.39 & 393 & & 320 & 0.33 & 309 & 0.00 & 285 & 0.30 & 319 \\
\hline Ohio: Oxford .. & 1.86 & 132 & 0.00 & 207 & 0.87 & 201 & 0.72 & 153 & 0.00 & 249 & 0.69 & 188 \\
\hline Oklahoma: Stillwater. & 1.52 & 167 & 1.22 & 189 & 0.04 & 256 & 0.30 & 140 & 0.01 & 201 & 0.62 & 191 \\
\hline $\begin{array}{l}\text { Orogon: Eugene........ } \\
\text { Quehec: }\end{array}$ & 0.00 & 62 & 0.00 & 234 & 0.00 & 262 & 0.00 & 236 & 0.00 & 300 & 0.00 & 219 \\
\hline Ste. Anne de Bellevue & 0.22 & 147 & 0.00 & 150 & 0.88 & 82 & 0.26 & 51 & 0.30 & 67 & 0.33 & 99 \\
\hline Saskatche & & 178 & & 234 & & 109 & & 118 & & 180 & & \\
\hline Tennessee: Knoxvill & 1.73 & & 0.20 & & 0.67 & & 0.81 & 126 & 0.46 & 130 & 0.65 & $128(2)$ \\
\hline Tex: & 1.6.4 & 390 & 0.00 & 470 & 1.17 & 329 & 0.03 & 427 & 1.05 & & 0.71 & $404(4)$ \\
\hline Utah: Salt I & ${ }^{4} T$ & 117 & 0.00 & 143 & 0.02 & 189 & 0.01 & 226 & 0.22 & 175 & 0.05 & 170 \\
\hline Ver & 0.11 & 169 & 0.14 & 349 & 1.58 & 156 & 0.72 & 122 & 0.21 & 184 & 0.55 & 196 \\
\hline Washington: Seattle.. & 0.09 & 190 & ${ }^{4} \mathrm{~T}$ & 200 & 0.10 & 128 & 0.05 & 174 & 0.00 & 142 & 0.05 & 167 \\
\hline
\end{tabular}

${ }^{2}$ Precipitation data from monthly weather reports.

- $T$ means trace, less than 0.01 inch.

- Precipitation data are for Boscommon.

7 Much interpolation on account of infrequent readings. 
TABLE 16.-Weekly precipitation $(P)$ and weekly rates of evaporation $(E)$, the latter from cylindrical porous-cup atmometers, summer of 1908. - Continued.

\begin{tabular}{|c|c|c|c|c|c|c|c|c|c|c|c|c|}
\hline \multirow{3}{*}{ Station. } & \multicolumn{12}{|c|}{ Fourth 5-week Period (Aug. 4 to Sept. 7). } \\
\hline & \multicolumn{2}{|c|}{$\begin{array}{l}\text { Aug. } 4 \\
\text { to } 10 .\end{array}$} & \multicolumn{2}{|c|}{$\begin{array}{l}\text { Aug. } 11 \\
\text { to } 17 .\end{array}$} & \multicolumn{2}{|c|}{$\begin{array}{l}\text { Aug. } 18 \\
\text { to } 24 .\end{array}$} & \multicolumn{2}{|c|}{$\begin{array}{c}\text { Aug. } 25 \\
\text { to } 31 .\end{array}$} & \multicolumn{2}{|c|}{$\begin{array}{l}\text { Sept. } 1 \\
\text { to } 7 .\end{array}$} & \multicolumn{2}{|c|}{ Average. } \\
\hline & $P$ & $E$ & $P$ & $E$ & $P$ & $E$ & $P$ & $E$ & $P$ & $E$ & $P$ & $E$ \\
\hline Alabama: Florence ${ }^{2}$ & $\begin{array}{l}\text { in. } \\
0.85\end{array}$ & $\begin{array}{r}\text { c.c. } \\
34\end{array}$ & ${ }_{0.11}^{i n}$ & $\begin{array}{l}\text { c.c. } \\
132\end{array}$ & $\begin{array}{l}i n . \\
0.59\end{array}$ & $\begin{array}{c}c . c . \\
75\end{array}$ & in. & $\begin{array}{ll}\text { c.c. } \\
139\end{array}$ & in. & c.c. & in. & c.c. \\
\hline Arizona: Tueson... & $\begin{array}{l}0.80 \\
0.08\end{array}$ & $\begin{array}{r}34 \\
252\end{array}$ & 1.76 & $\begin{array}{l}132 \\
230\end{array}$ & 1.67 & 232 & $\begin{array}{l}0.00 \\
1.67\end{array}$ & 229 & 0.41 & $\begin{array}{l}184 \\
302\end{array}$ & $\begin{array}{l}0.07 \\
1.12\end{array}$ & 249 \\
\hline California: San Diego & 0.55 & 179 & 0.00 & 204 & 0.00 & 230 & 0.00 & 235 & 0.07 & 157 & 0.12 & 201 \\
\hline Colorado: Boulder ${ }^{2}$. & 0.43 & 165 & 0.85 & 94 & 2.02 & 104 & 0.00 & 205 & 0.00 & 235 & 0.66 & 161 \\
\hline Florida: Miami. . & 2.60 & 96 & 1.81 & 83 & 0.43 & 73 & 1.81 & 72 & 0.04 & 134 & 1.34 & 92 \\
\hline Illinois: & & & & & & & & & & & & \\
\hline $\begin{array}{l}\text { Charleston }{ }^{2} . \\
\text { Urbana...... }\end{array}$ & $\begin{array}{l}0.19 \\
T\end{array}$ & $\begin{array}{l}230 \\
148\end{array}$ & $\begin{array}{l}0.59 \\
1.95\end{array}$ & 227 & ${ }^{4} T$ & & $\ddot{0.10}$ & & 0.48 & & $\begin{array}{r}0.39 \\
0.00\end{array}$ & $\begin{array}{l}229(2) \\
148(1)\end{array}$ \\
\hline Iowa: Iowa City². & 0.03 & 178 & 5.09 & $6 i$ & 0.02 & 153 & 2.33 & 133 & 0.28 & 170 & 1.55 & 139 \\
\hline Louisiana: Cameron ${ }^{2}$. & 1.05 & & 2.26 & 137 & 1.38 & 75 & ${ }^{4} T$ & 123 & 1.30 & 130 & 1.49 & $116^{\circ}(4)$ \\
\hline Maine: Orono ${ }^{2} . \ldots \ldots$ & 2.12 & 61 & 2.39 & 83 & 0.18 & 122 & 0.00 & 97 & 0.04 & 73 & 0.95 & 87 \\
\hline Manitoba: Winnipeg. & & $\begin{array}{r}130 \\
05\end{array}$ & & & & & & & & & & $130(1)$ \\
\hline $\begin{array}{l}\text { Maryland: Easton }{ }^{2} . \\
\text { Michigan: }\end{array}$ & 2.69 & 95 & 0.62 & 157 & 1.36 & 113 & 2.93 & 59 & 1.60 & 112 & 1.84 & 107 \\
\hline Grand Rapids. & 0.13 & 136 & 3.62 & 68 & 0.09 & 120 & 0.14 & 154 & 0.00 & 160 & 0.80 & 128 \\
\hline Houghton.... & 0.16 & 164 & 0.12 & 99 & 0.34 & 212 & 0.01 & 128 & 0.37 & 212 & 0.20 & 163 \\
\hline St. Helen... & 0.88 & 249 & 1.13 & 180 & 0.00 & 314 & $0.00 \mid$ & & 0.05 & 191 & 0.52 & $234(4)$ \\
\hline Minnesota: Minneapolis & 0.53 & 127 & 0.24 & 100 & 0.01 & 159 & 0.14 & 177 & 0.25 & 231 & 0.23 & 159 \\
\hline Missouri: St. Louis.. & 1.01 & 125 & 0.15 & 176 & 0.36 & 177 & 0.03 & 191 & 0.00 & 212 & 0.31 & 176 \\
\hline Montana: Bozeman. & $T$ & 190 & 0.49 & 105 & 0.24 & 144 & 0.36 & 124 & $T$ & 188 & 0.22 & 150 \\
\hline $\begin{array}{l}\text { Nebraska: } \\
\text { Lincoln. }\end{array}$ & 0.29. & & 1.01 & 108 & 0.52 & 95 & 0.98 & 138 & 0.24 & 193 & & $134(4)$ \\
\hline North Platte. & 0.56 & 192 & 0.27 & 130 & 0.46 & 98 & 0.28 & 140 & 0.00 & 295 & 0.31 & 171 \\
\hline Nevada: $\operatorname{Reno}^{7}$. & 0.00 & 379 & 0.12 & 367 & ${ }^{4} T$ & 367 & 0.00 . & & 0.10 . & & 0.04 & $371(3)$ \\
\hline New Brunswick: Fredericton & 1.50 & 69 & 1.35 & 50 & 0.76 & 66 & 0.00 & 73 & 0.40 & 37 & 0.80 & 59 \\
\hline $\begin{array}{l}\text { New York: } \\
\text { New York. }\end{array}$ & 0.63 & 62 & 0.35 & 85 & 2.28 & & 3.63 & & & 62 & & 71 \\
\hline Syracuse.. & $\begin{array}{l}0.03 \\
0.32\end{array}$ & 346 & $\begin{array}{l}0.35 \\
0.23\end{array}$ & $\begin{array}{r}80 \\
299\end{array}$ & 0.11 & $\begin{array}{l}100 \\
371\end{array}$ & $\begin{array}{l}3.03 \\
0.00\end{array}$ & $\begin{array}{r}41 \\
319\end{array}$ & $\begin{array}{l}0.00 \\
0.13\end{array}$ & $\begin{array}{r}02 \\
406\end{array}$ & $\begin{array}{l}1.59 \\
0.16\end{array}$ & 348 \\
\hline North Carolina: & & & & & & & & & & & & \\
\hline Pisgah Forest. & 3.11 & 42 & 3.05 & 79 & 7.15 & 14 & 1.24 & 38 & 2.20 . & & 3.64 & $43(4)$ \\
\hline West Raleigh. . & 1.16 & 109 & 0.21 & 120 & 3.09 & 50 & 9.17 & 14 & 3.07 & 76 & 3.34 & \\
\hline North Dakota: Dickinson². & 0.00 & & 0.53 & 181 & 0.54 & 297 & 0.34 & 243 & 0.00 & 312 & 0.35 & $258(4)$ \\
\hline Ohio: Oxford. & 0.62 & 167 & 0.47 & 190 & 0.00 & 238 & 0.00 & 248 & ${ }^{4} T$ & 233 & 0.22 & 215 \\
\hline Oklahoma: Stillwater. & 0.24 & 221 & 1.07 & 224 & 1.27 & 115 & 0.14 & 168 & 3.91 & 85 & 1.33 & 163 \\
\hline Oregon: Eugene... & 0.00 & 305 & 0.00 & 170 & 0.00 & 174 & 0.00 & 110 & 0.00 & 134 & 0.00 & 179 \\
\hline $\begin{array}{l}\text { Quebec: } \\
\text { Ste. Anne de Bellevue. }\end{array}$ & 0.82 & 43 & 0.10 & 60 & 0.77 & & & & & 96 & 0.34 & 65 \\
\hline Saskatchewan: Regina.... & 0.82 & $\begin{array}{r}45 \\
128\end{array}$ & 0.10 & 56 & 0.88 & 75 & 0.02 & 28 & 0.00 & $\begin{array}{l}96 \\
95\end{array}$ & 0.34 & $\begin{array}{l}65 \\
76\end{array}$ \\
\hline Tennessee: Knoxville. & 1.45 & 55 & 0.01 & 129 & 2.38 & & 0.11 . & & 1.54 . & & 0.73 & $92(2)$ \\
\hline Texas: Dalhart.... & 0.00 & 504 & 0.28 & 461 & 0.70 & 353 & 0.10 & 435 & 0.15 & 447 & 0.25 & 440 \\
\hline Utah: Salt Lake Cit & 0.72 & 178 & 0.62 & 168 & 0.01 & 181 & 0.04 & 195 & 0.00 & 170 & 0.28 & 178 \\
\hline Vermont: Burlington & 0.38 & 114 & 0.79 & 120 & 0.53 & 179 & 0.00 & 171 & 0.50 & 214 & 0.44 & 160 \\
\hline Washington: Seattle....... & 0.00 & 160 & 0.03 & 112 & 0.03 & 92 & 0.76 & 51 & 0.01 & 67 & 0.17 & 96 \\
\hline
\end{tabular}

2 Precipitation data from monthly weather reports.

- $T$ means trace, less than 001 inch.

- Precipitation data are for Roscommon.

Much interpolation on account of infrequent readings. 
TABLE 16.-Weekly precipitation $(P)$ and weekly rates of evaporation $(E)$, the latter from cylindrical porous-cup almometers, summer of 1908. - Continued.

\begin{tabular}{|c|c|c|c|c|c|c|c|c|c|c|c|c|}
\hline \multicolumn{13}{|c|}{ Fifth 5-week Period (Sept. 8 to Oct. 12). } \\
\hline \multirow[t]{2}{*}{ Station. } & \multicolumn{2}{|c|}{$\begin{array}{l}\text { Sept. } 8 \\
\text { to } 14 .\end{array}$} & \multicolumn{2}{|c|}{$\begin{array}{l}\text { Sept. } 15 \\
\text { to } 21 .\end{array}$} & \multicolumn{2}{|c|}{$\begin{array}{c}\text { Sept. } 22 \\
\text { to } 28 \text {. }\end{array}$} & \multicolumn{2}{|c|}{$\begin{array}{r}\text { Sept. } 29 \\
\text { to Oct. } 5 \text {. }\end{array}$} & \multicolumn{2}{|c|}{$\begin{array}{l}\text { Oct. } 6 \\
\text { to } 12 .\end{array}$} & \multicolumn{2}{|c|}{ Average. } \\
\hline & $P$ & $E$ & $P$ & $E$ & $P$ & E & $P$ & $E$ & $P$ & E & $P$ & E \\
\hline & & & & & in. & c.c. & in. & c.c. & in. & c.c. & & \\
\hline Arizona: Tues & 0.19 & 276 & & & & & & & & & 0.19 & $276(1)$ \\
\hline California: San & 0.00 & 214 & 0.00 & 164 & 0.22 & 167 & 0.00 & 252 & 0.00 & 218 & 0.04 & 203 \\
\hline Colorado: Boulder ${ }^{2}$. & 0.02 & 247 & 0.00 & 300 & 1.53 & 258 & 0.14 & 109 & 0.00 & 165 & 0.34 & 216 \\
\hline Florida: Mian & $|0.10|$ & 140 & 6.68 & 82 & 3.36 & 104 & 9.00 & 35 & 10.52 & 61 & 93 & 84 \\
\hline Illinois: Urhana. & 0.00 & & 0.00 & 132 & 1.33 & 116 & 0.14 & 114 & ${ }^{4} T$ & 109 & 0.37 & $118(4)$ \\
\hline Iowa & $0.00 \mid$ & 149 & 0.00 & 145 & 1.53 & 92 & & & & & 0.51 & $129(3)$ \\
\hline Lou & 1.76 & 78 & 17.75 & & 0.47 & 83 & 0.00 & 155 & & & 0.74 & $105(3)$ \\
\hline Mai & ${ }^{4} T$ & 147 & ${ }^{4} T$ & 113 & ${ }^{4} T$ & 113 & & & & & 0.00 & $124(3)$ \\
\hline Man & & 171 & & 139 & & 62 & & & & & & $124(3)$ \\
\hline Mary! & 0.02 & 98 & 0.05 & 106 & 0.08 & 63 & 0.32 & 120 & ${ }^{4} T$ & 102 & 0.09 & 98 \\
\hline Michi & & & & & & & & & & & & \\
\hline $\mathrm{G}$ & 0.00 & 154 & 0.03 & 126 & 0.72 & 76 & & & & & $0.25 \mid$ & $119(3)$ \\
\hline $\mathrm{H}$ & 0.04 & 172 & 0. & 85 & 1 & 76 & 2.34 & & & & 0.69 & 11 \\
\hline St & 0.00 & 297 & 0.00 & 237 & 0.47 & 240 & $|0.00|$ & 170 & & & 0.12 & $236(4)$ \\
\hline Minnes & 3.23 & 174 & & & & & & & & & 3.23 & $174(1)$ \\
\hline Mis & 0.00 & 171 & 0.06 & 149 & 1.18 & & & & & & 0.03 & $160(2)$ \\
\hline Mont & 0.05 & 147 & 0.38 & 131 & & & & & & & 0.22 & $139(2)$ \\
\hline Nebraska: & & & & & & & & & & & & \\
\hline & 0.00 & 185 & ${ }^{4} T$ & 189 & 0.51 & 120 & & & & & 0.17 & $165(3)$ \\
\hline $\mathrm{N}$ & ${ }^{4} T$ & 206 & 0.00 & 209 & & & & & & & $0.00 \mid$ & $208(2)$ \\
\hline Neva & 0.05 & & 0.28 & & 0.06 & & & & & & 0.13 & \\
\hline New $B$ & 0.13 & 63 & 0.00 & 84 & 0.12 & 44 & & & & & $\mid 0.08$ & $64(3)$ \\
\hline New & & & & & & & & & & & & \\
\hline & 0.00 & 80 & 0.00 & 101 & 0 & 33 & & & & & .00 & $71(3)$ \\
\hline & 0.00 & 311 & 4 & 294 & ${ }^{4} T$ & 275 & 1.00 & & 0.87 & & $0.00 \mid$ & $293(3)$ \\
\hline North C & & & & & & & & & & & & \\
\hline & & 124 & & & & & & & & & & 124 \\
\hline Wes & 0.00 & 7 & 0 . & 108 & 0 & 83 & 0.48 & 97 & & & .15 & \\
\hline North 1 & 0.02 & 273 & & 269 & & 204 & & & & & .54 & 249 (3) \\
\hline $\mathrm{Oh}$ & 0.00 & 233 & & 185 & & 199 & & & & & 12 & $206(3)$ \\
\hline $\mathrm{OH}$ & 0.00 & 95 & & 74 & 1 & 52 & 0.00 & 90 & 0.60 & 90 & 0.54 & 80 \\
\hline ugene.. & 0.00 & 138 & 0.00 & 112 & 0.00 & 128 & 0.23 & 102 & & & 0.06 & $120(4)$ \\
\hline Quel & & & & & & & & & & & & \\
\hline & 0.00 & 108 & 0.02 & & 0.40 & & & & & & $0.00 \mid$ & $108(1)$ \\
\hline Sas & & 101 & & 49 & & 76 & & & & & & \\
\hline Te & 0.00 & 9 & 0 . & 9 & 0 & 106 & 0.24 & 141 & 1.32 & 81 & 0 & \\
\hline & 0.00 & 538 & & 426 & & 478 & & & & & $0.0 \mathrm{~s}$ & $481(3)$ \\
\hline & 0.87 & 107 & 0. & 141 & 1. & 63 & 0.75 & & & & 0.91 & $104(3)$ \\
\hline & 0.00 & 186 & 0 . & 172 & 0.0 & 155 & & & & & $0.00 \mid$ & $171(3)$ \\
\hline Washington: Sen & $|0.18|$ & 89 & $4 T$ & 55 & 0.04 & 55 & 0.50 & 42 & 0.31 & 15 & $|0.21|$ & 51 \\
\hline
\end{tabular}

LAst 3 WeEks (OCt. 13 to Nov. 2).

\begin{tabular}{|c|c|c|c|c|c|c|c|c|}
\hline \multirow{2}{*}{ Station. } & \multicolumn{2}{|c|}{ Oct. 13 to 19.} & \multicolumn{2}{|c|}{ Oct. 20 to 26.} & \multicolumn{2}{|c|}{$\begin{array}{l}\text { Oct. } 27 \text { to } \\
\text { Nov. } 2 .\end{array}$} & \multicolumn{2}{|c|}{ Avernge. } \\
\hline & $P$ & $E$ & $P$ & E & $P$ & $E$ & l & E \\
\hline California: San Diem & in. & c.c. & in. & c.c. & in. & $c . c$. & in. & c.c. \\
\hline Florida: Mi:mi..... & $\begin{array}{l}4.28 \\
4.25\end{array}$ & 103 & 0.40 & 110 & & & 2.34 & $107(2)$ \\
\hline Illinois: Urbann. . & $\because T$ & 111 & 0.09 & so & & & 0.05 & $96(2)$ \\
\hline Maryland: Fatston. & 0.00 & 122 & 0.00 & 125 & 3.92 & 89 & 1.31 & 102 \\
\hline Tenuesser: Kunxville. & 0.00 & 108 & 0.89 & 52 & 0.36 & is & 0.42 & 73 \\
\hline Washington: Seattle & 0496 & 1.5 & & & & & $0 \div 3$ & $15(1)$ \\
\hline
\end{tabular}

- Precipitation data from monthly wenther reports. "Precipitation data aro for Roscommon.

- $T$ means trace, less than 0.01 inch. TIuch interpolation on account of infrequent readings. 
Summer march of evaporation at selected stations. - The variation in the intensity of atmospheric evaporating power as the season advances at any given station is surely of very great importance in determining the kind and extent of the corresponding variations of developmental changes occurring in plants. These variations are therefore of considerable interest in other ways than because of the general novelty of atmometric data. We therefore give, in the following paragraphs, a short discussion of this feature for each of the 10 stations represented by the graphs of figures 3 to 12 . These graphs are of gradatory form and each one is double. The upper graph of each pair represents the weekly evaporation rates (in cubic centimeters) from the standard cylindrical porous-cup atmometer, and the lower one represents the weekly precipitation. The dates given below, in each case, denote the endings of the consecutive weeks of the series, and the numbers on the graphs themselves are the ordinate values, representing cubic centimeters of evaporation and inches of rainfall. The features brought out by these figures will now be considered.

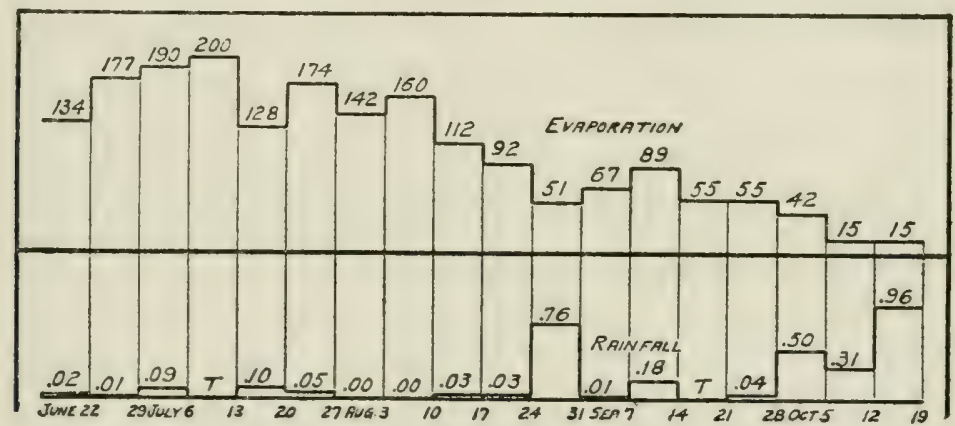

Fra. 3.-Weekly precipitation and evaporation indices, summer of 1908, Seattle, Washington.

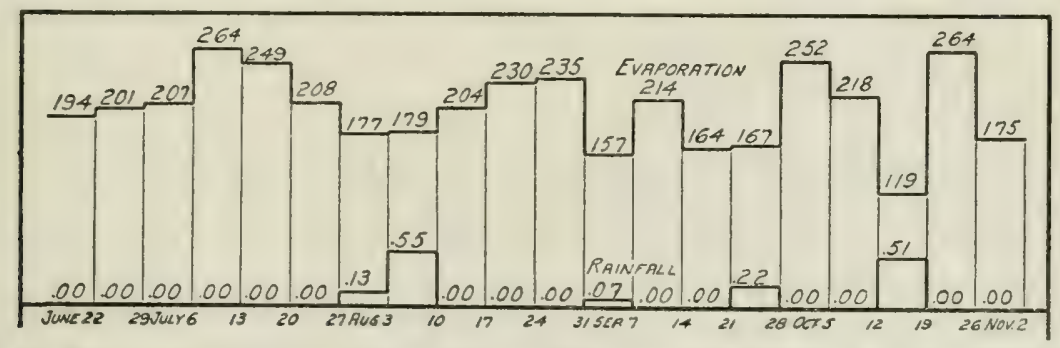

Fra. 4.-Weekly precipitation and evaporation indices, summer of 1908, San Diego, California.

Seattle, Washington (fig. 3): In this graph the intensity of atmospheric evaporating power attained an early maximum of 200 c.c. (week ending July 13) and then fell generally to the end of our series. The weeks ending October 12 and 19 showed an average rate of 15 c.c. per week, which is the minimum rate encountered. In the latter 
half of our period considerable precipitation occurred. It is to be noted, as is generally the case, that weeks with much precipitation are characterized by low evaporation intensity.

San Diego, California (fig. 4): For this station the weekly rates of evaporation show a striking uniformity for the period, with a minimum of 119 c.c. and a maximum of 264 c.c. The period was nearly rainless.

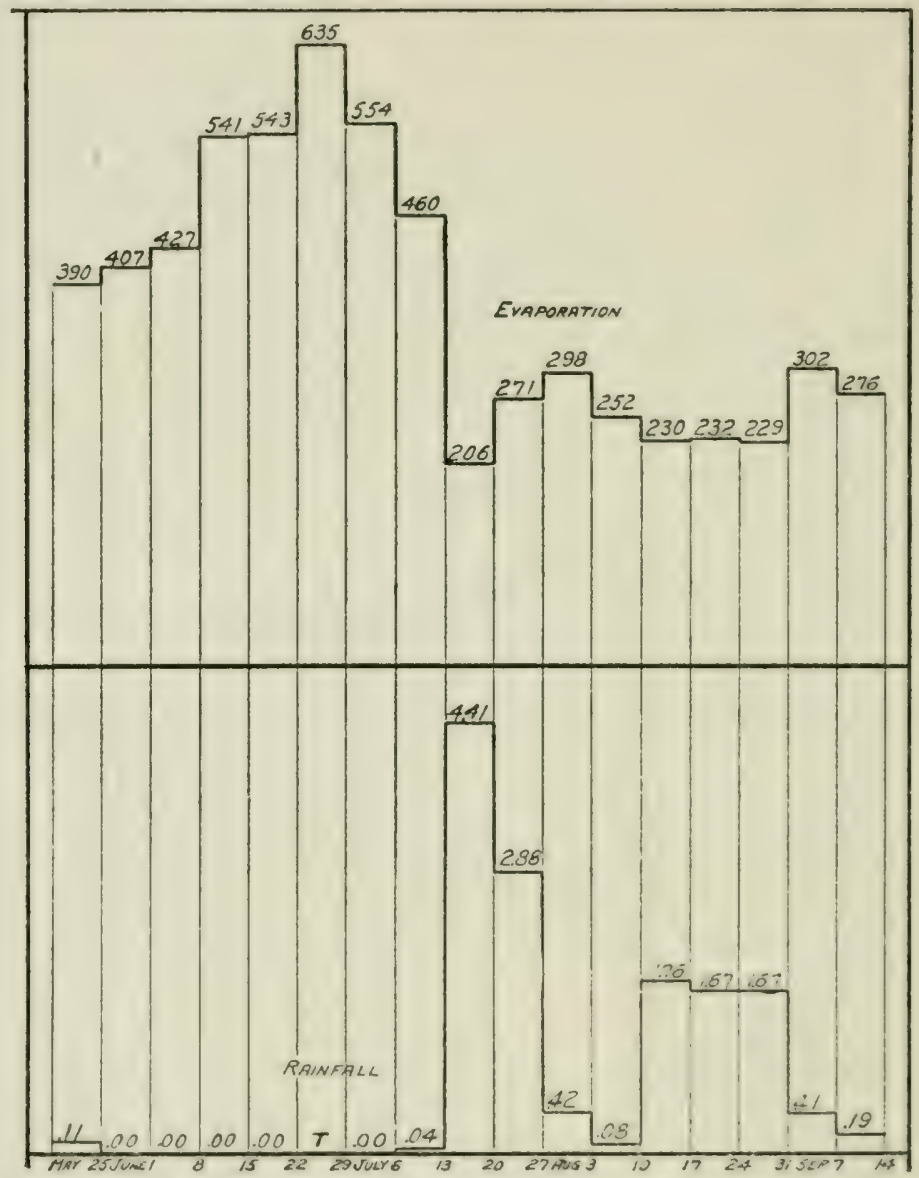

Fı. 5.-Weekly precipitation and evaporation indices, summmer of 190S, Tucson, Arizona.

Tucson, Arizona (fig. 5): From the beginning of the series to June 29 the intensity of evaporation rose rapidly to a maximum of 635 c.c. for the week ending on the latter date. With the advent of the summer rainy season the rate then fell in 3 weeks to a magnitude of 206, less than one-third of the maximum. During the remainder of the series (ending September 14) the rates raried between 229 and $3(12$. This latter half of the summer is a season of relatively great precipitation at this station, and the low evaporation intensity of the rainy 
period is here well shown. ${ }^{1}$ This is the time when summer annuals are active.

Dalhart, Texas (fig. 6): This station is characterized by exceedingly high rates for our series of observations - higher rates than were obtained at any other place - in spite of the fact that there was here considerable precipitation throughout the summer. No observation was obtained for the week ending August 3. The maximum weekly rate was 656 c.c. (week ending June 22) and the minimum was 329 c.c. (week ending July 20). The march of the index of evaporation intensity suggests a periodicity with a period of several weeks.

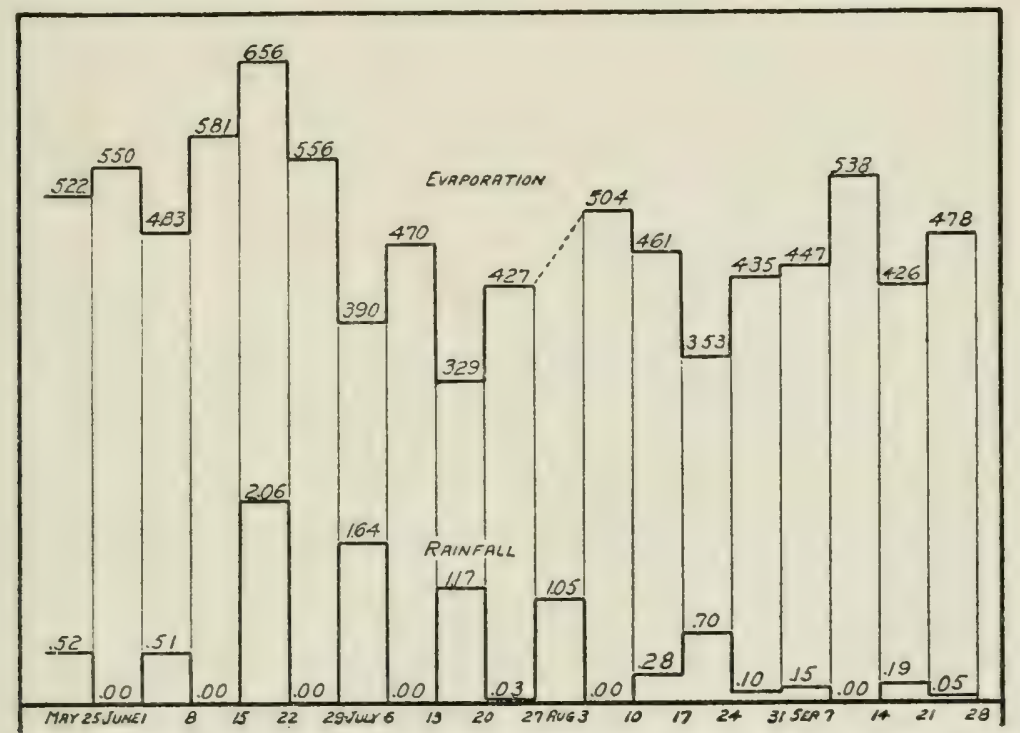

FIG. 6.-Weekly precipitation and evaporation indices, summer of 1908 , Dalhart, Texas.

St. Louis, Missouri (fig. 7): The maximum weekly rate (212 c.c.) was not attained here until the week ending September 7 , and the minimum (71 c.c.) occurred early in the season, for the week ending May 11. The highest rate at this station was but little higher than the lowest encountered at Tucson. There appears to have been a general upward trend of the rate values throughout the whole series, to about September 7. There is here also a suggestion of a periodicity. Rains were frequent and plentiful until the latter part of the series of observations.

Oxford, Ohio (fig. 8): Here there was a general increase in evaporation intensity throughout the season, until about August 31. The rates vary irregularly, from a minimum of 78 c.c. (week ending May 25 ) to a maximum of 249 c.c. (week ending August 3), the highest ones being nearly as great as those for San Diego. 
Ste. Anne de Bellevue, Quebec (Macdonald College, fig. 9): The highest weekly rate for this station was 181 c.c. (week ending June 29) and the lowest was 43 c.c. (week ending August 10). The last 3 weeks of July and the first 3 of August were characterized by low evaporation intensity. Here the highest intensity recorded is much lower than the lowest experienced at Tucson. Rainfall at Ste. Anne de Bellevue was not great, but the rainy weeks were somewhat regularly spaced.

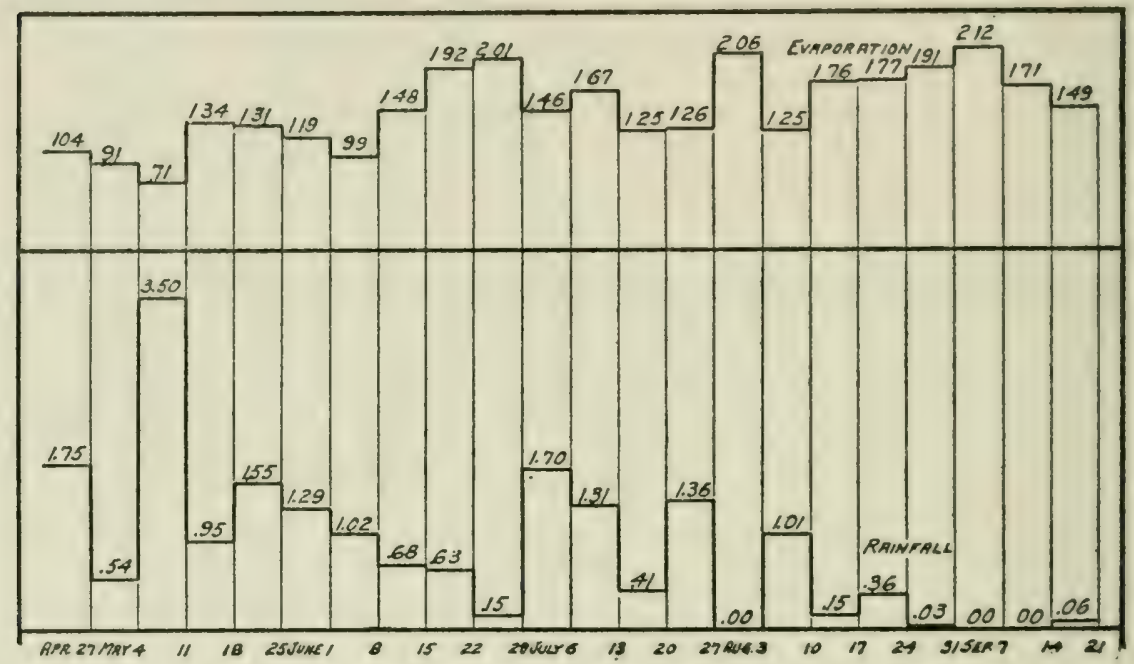

Fra. 7.-Weekly precipitation and evaporation indices, summer of 1908, St. Louis, Missouri.

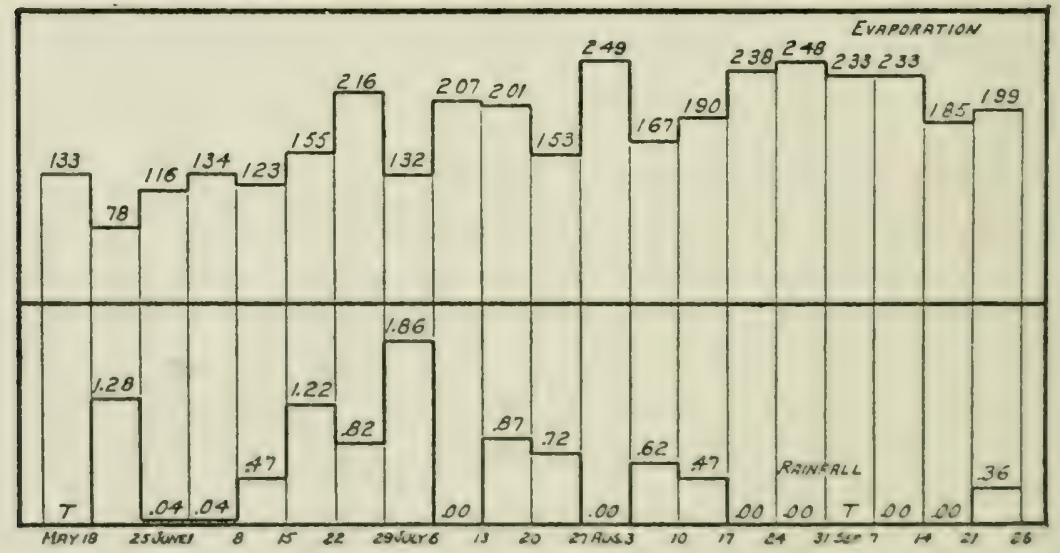

Fra. 8.-Weekly precipitation and evaporation indices, summer of 190S, Oxford, Ohio.

Orono, Maine (fig. 10): The minimum rate for Orono is 48 c.c. (week ending June 1) and the maximum is 178 c.c. (week ending July 13). The week ending June 29 is without a record for evaporation. 
The evaporation-rates are here shown to have been rather uniform, on the whole, with a suggestion of a 3 -week periodicity.

Easton, Maryland (fig. 11): Here the weekly rates of evaporation varied from a minimum value of 59 c.c. (weeks ending August 31 and November 2) to a maximum of 308 c.c. (week ending June 20). There occurred a general rise in these rates from the beginning of the series to about July 20, after which the evaporation intensity was low and strikingly uniform. It is interesting to note that the highest rate for Easton is higher than any rate encountered in the summer rainy season

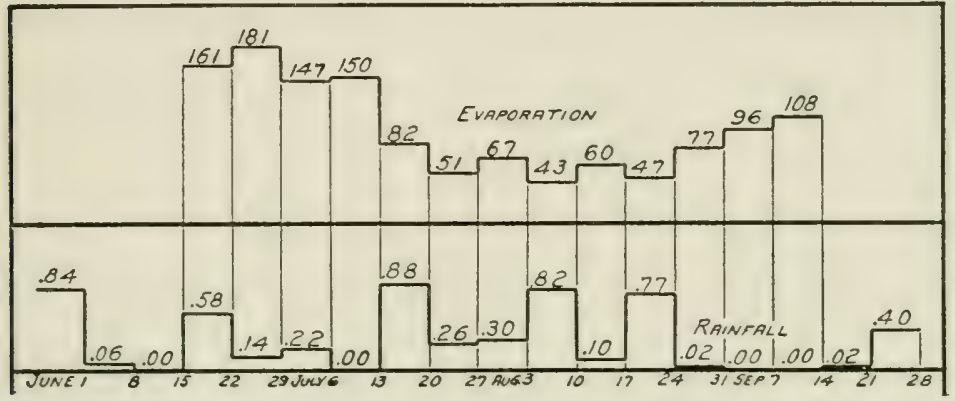

FIG. 9.-Weekly precipitation and evaporation indices, summer of 1908, MacDonald College, Ste. Anne de Bellevue, Quebec.

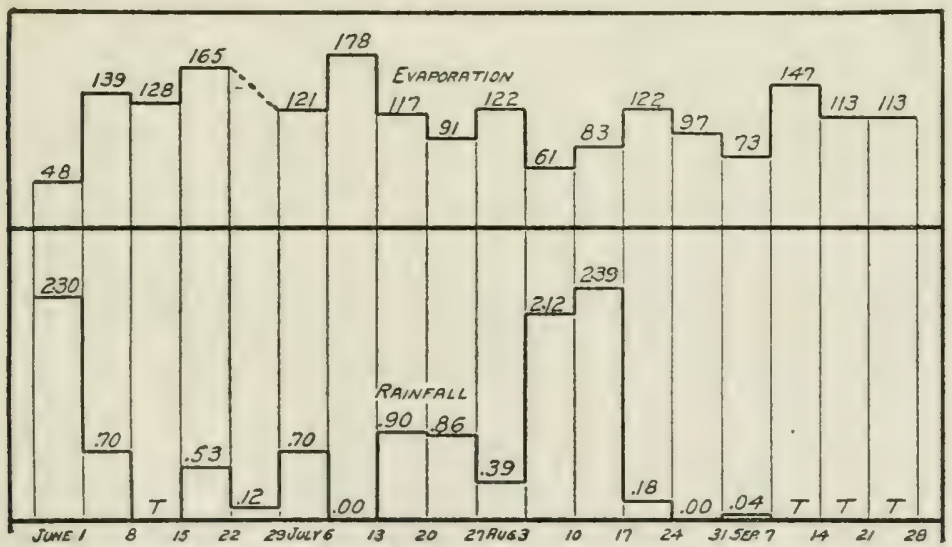

FIG 10.-Weekly precipitation and evaporation indices, summer of 1908 , Orono, Maine.

at Tucson, and the three highest for Easton are higher than any rate experienced at San Diego during the whole series of observations at that station. Curiously enough, the two graphs for Easton are similar in their general form to those for Tucson. In both cases a fore-summer of low rainfall and of generally increasing evaporation intensity is finally broken by a heavy rain, which ushers in a period of greater 
rainfall and of relatively low evaporation values. This droughtbreaking rain occurred in the week ending July 20 at Tucson, in the next following week at Easton.

Miami, Florida (fig. 12): The exceptionally long record for Miami shows a maximum evaporation rate of 232 c.c. early in the season (week ending May 18), and a minimum of 35 c.c. near the end of the series (week ending October 5). While the general trend of the graph of atmospheric evaporating power is downward as the season advances, there are rather pronounced variations, which have some tendency to occur periodically, with an interval of about 8 weeks. The intensity of precipitation appears to increase throughout the season, and heavy rains have relatively but little relation to the evaporation values.

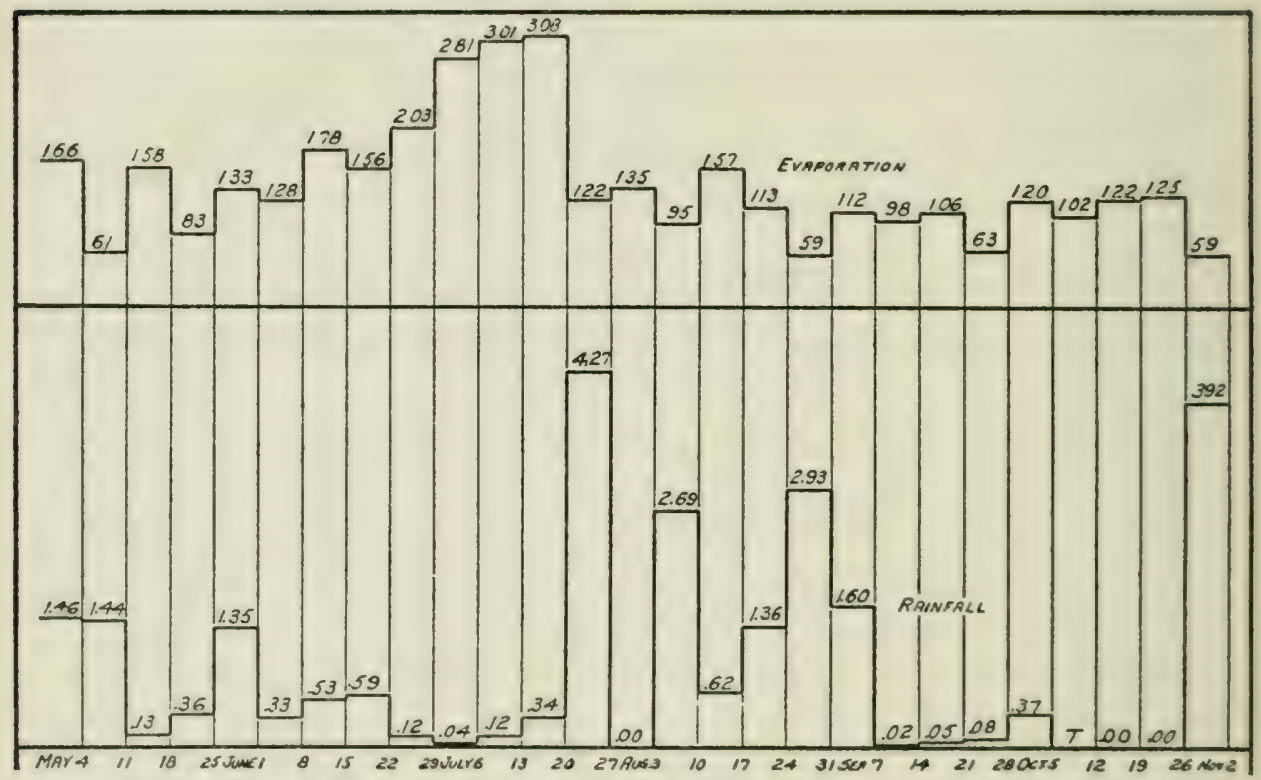

FIG. 11.-Weekly precipitation and evaporation indices, summer of 1908, Easton, Maryland.

Mean evaporation values for 5-week periods and for 15-week season (Table 17, plate 56.) - As has been indicated, the average weekly rates of loss from the porous-cup atmometer, at the several stations, have been calculated for the 5-week periods, April 20 to May 25, May 26 to June 29, June 20 to August 3, August 4 to September 7, and September 8 to October 12. For a few stations the average rates for the last 3 weeks of the series (from October 12 to November 2) have also been calculated. These averages are given in the final columns of table 16 and they are all brought together in table 17 . Where data are not available for all the 5 (or 3 ) weeks of a period, the average has been made from the smaller number of weekly records at hand, and the 
number of records so used is denoted by the number following the average, in parentheses. It will be seen at once that many of our 5 -week averages are rendered unsatisfactory in this way, because full records are lacking.

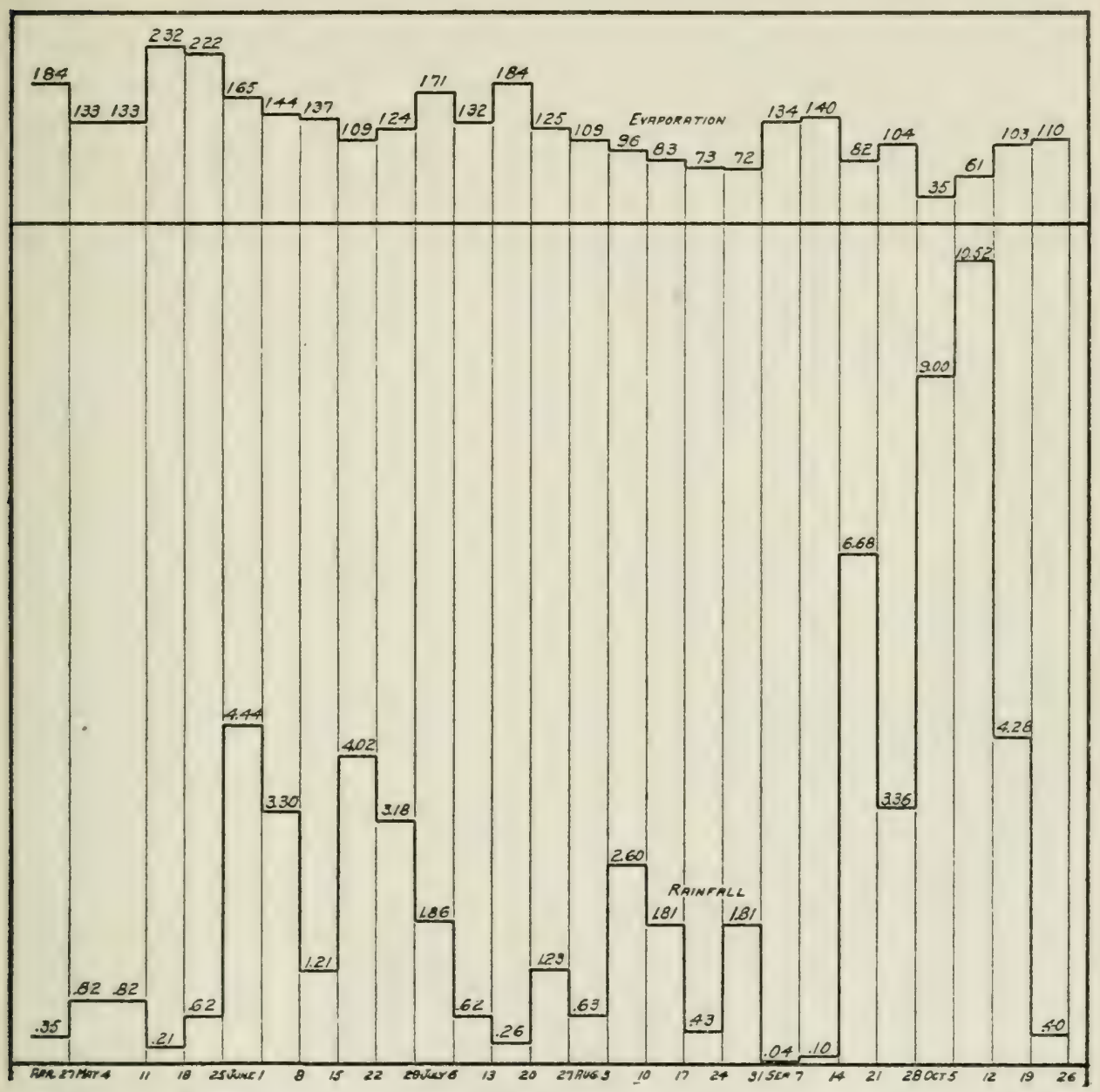

Fra. 12.-Weekly precipitation and evaporation indices, summer of 1908, Miami, Florida.

For the three 5-week periods (May 26 to June 29, June 30 to August 3 , and August 4 to September 7 ) the records are generally more nearly complete than for the earlier and later periods, and these three averages form the basis of our chart of summer evaporation intensities. 
TABLE 17.-Summary of data of precipitation $(P)$ and cvaporalion (E) for three 5-week periods for the summer of 1908 (data from table 16), together with averages and precipitation-evaporation ratios $(P / E)$ for the 15-week period May 26 to September 7.

\begin{tabular}{|c|c|c|c|c|c|c|c|c|c|}
\hline \multirow{3}{*}{$\begin{array}{c}\cdot \\
\text { Station. }\end{array}$} & \multirow{2}{*}{\multicolumn{2}{|c|}{ First period. }} & \multirow{2}{*}{\multicolumn{2}{|c|}{ Second period. }} & \multirow{2}{*}{\multicolumn{2}{|c|}{ Third period. }} & \multicolumn{3}{|c|}{ 15-week period. } \\
\hline & & & & & & & \multicolumn{2}{|c|}{ Average. } & \multirow{2}{*}{$\frac{P_{8}, 1908}{E_{8}, 1908}$} \\
\hline & $P$ & $E$ & $P$ & $E$ & $P$ & E & $P$ & $E$ & \\
\hline & $i n$. & c.c. & in. & c.c. & in. & c.c. & in. & c.c. & \\
\hline Alabama: Florence. & & & 0.82 & 122 & 0.57 & 113 & 0.70 & $118(2)$ & $0.0059(2)$ \\
\hline Arizona: Tueson... & 0.00 & 511 & 1.55 & 358 & 1.12 & 249 & 0.89 & 373 & 0.0024 \\
\hline California: San Diego & 0.00 & $198(2)$ & 0.03 & 221 & 0.12 & 201 & 0.05 & & 0.002 \\
\hline Colorado: Boulder.. & 0.09 & $225(3)$ & 0.41 & 180 & 0.66 & 161 & 0.39 & 189 & 0.0021 \\
\hline $\begin{array}{l}\text { Florida: } \\
\text { Gainesville. }\end{array}$ & 1.86 & $201 \%$ & 1.74 & $141(3)$ & & & 1.80 & & \\
\hline Miami..... & $\begin{array}{l}1.00 \\
3.23\end{array}$ & 136 & 0.92 & 144 & 1.34 & 92 & $\begin{array}{l}1.80 \\
1.83\end{array}$ & 124 & $\begin{array}{l}0.0105(2) \\
0.0148\end{array}$ \\
\hline Illinois: & & & & & & & & & \\
\hline Charleston. & 0.70 & 189 & 0.59 & 158 & 0.39 & $229(2)$ & 0.56 & 192 & 0.0029 \\
\hline Urbana... & 0.52 & $113(4)$ & 0.66 & 110 & 0.00 & & 0.59 & $112(2)$ & 0.0053 \\
\hline Iowa: Iowa City... & & & 1.26 & $165(3)$ & 1.55 & 139 & 1.41 & $152(2)$ & $0.0093(2)$ \\
\hline Louisiana: Cameron. & 1.03 & 234 & 2.66 & $167(3)$ & 1.49 & $116(4)$ & 1.73 & 172 & 0.0101 \\
\hline $\begin{array}{l}\text { Maine: Orono........ } \\
\text { Manitoba: Winnipeg. }\end{array}$ & 0.88 & $120(4)$ & 0.57 & $\begin{array}{l}126 \\
156\end{array}$ & 0.95 & 87 & 0.80 & 111 & 0.0072 \\
\hline Maryland: Easton... & 0.58 & 158 & 0.95 & 229 & 1.84 & 107 & 1.12 & 165 & 0.0068 \\
\hline Michigan: & & & & & & & & & \\
\hline Grand Rapids. . & 0.34 & 153 & 0.63 & 161 & 0.80 & 128 & 0.59 & 147 & 0.0040 \\
\hline Houghton....... & 0.91 & $172(2)$ & 0.75 & 127 & 0.20 & 163 & 0.62 & 154 & 0.0040 \\
\hline St. Helen. . & & & 0.61 & $202(2)$ & 0.52 & $234(4)$ & 0.57 & $218(2)$ & $0.0026(2)$ \\
\hline Minnesota: Minneapolis. & 1.58 & $93(4)$ & 0.63 & 144 & 0.23 & 159 & 0.81 & 132 & 0.0061 \\
\hline Missouri: St. Louis... & 0.75 & 152 & 0.96 & 154 & 0.31 & 176 & 0.67 & 161 & 0.0042 \\
\hline Montana: Bozeman.. & 1.77 & 39 & 0.09 & 162 & 0.22 & 150 & 0.69 & 117 & 0.0059 \\
\hline Nebraska: & & & & & & & & & \\
\hline Lincoln.... & 2.52 & 73 & 3.81 & & 0.69 & $134(4)$ & 1.61 & 104 & 0.0155 \\
\hline North Platte... & 1.04 & 118 & 0.63 & 127 & 0.31 & 171 & 0.66 & 139 & 0.0047 \\
\hline Nevada: Reno.... & & & 0.03 & $475(4)$ & 0.04 & $371(3)$ & 0.04 & $423(2)$ & 0.0001 \\
\hline $\begin{array}{l}\text { New Brunswick: Fredericton } \\
\text { New York: }\end{array}$ & 0.96 & 94 & 0.48 & 118 & 0.80 & 59 & 0.75 & 90 & 0.0083 \\
\hline New York. & 0.25 & $132(4)$ & 0.30 & $148(3)$ & 1.39 & 71 & 0.65 & & \\
\hline Syracuse... & 0.67 & & 0.70 & $305(4)$ & 0.16 & 348 & $\begin{array}{l}0.05 \\
0.43\end{array}$ & 327 & $\begin{array}{l}0.0056 \\
0.0013\end{array}$ \\
\hline North Carolina: & & & & & & & & & \\
\hline Pisgah Forest... & 0.88 & $67(4)$ & 1.42 & 70 & 3.64 & $43(4)$ & 1.98 & 60 & 0.0330 \\
\hline West Raleigh... & 1.29 & 196 & 1.23 & 164 & 3.34 & 74 & 1.95 & 145 & 0.0134 \\
\hline North Dakota: Dickinson.. & 0.85 & 268 & 0.30 & 319 & 0.35 & $258(4)$ & 0.50 & 282 & 0.0018 \\
\hline Ohio: Oxford... & 0.52 & 149 & 0.69 & 188 & 0.22 & 215 & 0.48 & 184 & 0.0026 \\
\hline Oklahoma: Stillwater. & 1.31 & 109 & 0.62 & 191 & 1.33 & 163 & 1.09 & 154 & 0.0071 \\
\hline Oregon: Eugene..... & 0.41 & 95 & 0.00 & 219 & 0.00 & 179 & 0.14 & 164 & 0.0009 \\
\hline Queber: St. Anne de Bellevue & 0.36 & $171(2)$ & 0.33 & 99 & 0.34 & 65 & 0.34 & 112 & 0.0030 \\
\hline Saskatchewan: Regina....... & & $155(2)$ & & 164 & & 76 & & 132 & \\
\hline Tennessee: Knoxville..... & 0.63 & $93(3)$ & 0.65 & $128(2)$ & 0.73 & $92(2)$ & 0.67 & 104 & 0.0064 \\
\hline Texas: Dalhart... & 0.51 & 565 & 0.71 & $404(4)$ & 0.25 & 440 & 0.49 & 470 & 0.0010 \\
\hline Utah: Salt Lake City & 0.45 & $72(4)$ & 0.05 & 170 & 0.28 & 178 & 0.26 & 140 & 0.0019 \\
\hline Vermont: Burlington. & 0.63 & 157 & 0.55 & 196 & 0.44 & 160 & 0.54 & 171 & 0.0032 \\
\hline Washington: Seattle. & 0.02 & $156(2)$ & 0.05 & 167 & 0.17 & 96 & 0.08 & 140 & 0.0006 \\
\hline
\end{tabular}

To obtain a single number to represent each station for this 15 -week period (May 26 to September 7 ), the three 5-week means have been averaged, to give a mean intensity of atmospheric evaporating power for approximately the three summer months. The three 5-week averages and the resulting 15 -week means are shown in table 17 , together 
with the corresponding precipitation means and the precipitationevaporation ratios for the 15 -week period, the latter to be considered below. Where the 15 -week mean had been obtained from only two averages this is indicated by the figure 2 in parentheses.

Scrutiny of the means for this summer season shows them to vary, with the geographic position of the stations, in an apparently rational manner, and there is no reason to doubt that they furnish an approximately correct picture of the distribution of intensities of evaporation over the United States for the period in question. There are two marked exceptions to the last statement-the means for Bozeman, Montana, and Salt Lake City, Utah, appear to be far too low, though no explanation is at hand to account for this. The data for these two stations have been ignored in the preparation of our chart.

Plate 56 is a chart of evaporation intensities constructed from the means just considered. The relatively few stations for which data are available makes it unadvisable to attempt any detailed study of climatic zones in this case, and isoatmic lines are shown for only 150 and 300 c.c. $^{1}$

The most significant features of the chart of plate 56 are the following:

(1) The Canadian region of low summer evaporation (less than 150 c.c. per week) extends, as a great lobe, southwestwar d from Lake Superior, as far as the valley of the Arkansas River. Another southern extension of the northern area of low summer evaporation intensities reaches south-southwestward from southern New England, occupying the whole of the Appalachian Mountain system south of Massachusetts. This area broadens toward the south and embraces most of South Carolina, Georgia, Alabama, Mississippi, and eastern Kentucky and Tennessee. Miami, Florida, northern and western Washington, and northwestern Oregon lie in the region of low intensity, as must also the high altitudes of the Rocky and Sierra Nevada Mountains though our numerical data do not show this feature.

(2) The main region of high evaporation intensity (over 300 c.c. per week) extends northward from Mexico and occupies western Texas, New Mexico, and Arizona south of the plateau, and the lower altitudes of Nevada and of southeastern California. This is obviously the so-called desert or arid region of the United States, and corresponds to the arid evaporation province as shown on plates 53 to 55 , and in

\footnotetext{
I I is to be remembered that the numerical data are in terms of cubic centimeters of weekly loss from the Livingston standard cylindrical porous-cup atmometer, exposed as were our instruments. They are to be regarded merely as comparable indices of atmospheric evaporating power with reference to this instrument, just as are numerical data in terms of depth of water-loss from some specified water-surface exposed in a specified manner. Prevalent ideas in this connection require repeated emphasis upon the fact that rates of water-loss from one form of atmometer can not be mathematically deduced from those obtained with another form of instrument, excepting in a very general way. "If this can be generally appreciated it will aid much toward atmometric progress.
} 
-figure 2. There is also clearly indicated a limited region of high summer evaporation in the vicinity of Syracuse, New York.

(3) The region of intermediate evaporation intensities (from 150 to 300 c.c. per week), lying between the other two, of course extends eastward from the California coast to about the one-hundredth meridian of west longitude, where it is nearly replaced by the great embayment of the northern region and by the northeastern termination of the arid region. It then broadens southward to include the Gulf coast and also extends northeastward. It occupies the Atlantic coastal region as far north as southern New England (excepting southeastern Florida). Between the two great southern extensions of the zone of low intensities this zone of medium intensities extends northeastward as far as Burlington, Vermont, broadening to include most of Michigan.

Comparison between plates 55 and 56.-Plate 55, as has been stated, presents a chart of summer evaporation intensities based upon Russell's data, and is here published in order to allow a comparison of our own summer data. (1908) with those of Russell (1887-88). As might be expected, the details of these two charts are not at all in agreement, but a study of the two brings out certain features which may be worthy of brief attention.

(1) It is seen at once that the desert region is clearly shown on both charts. On one scale this zone has an evaporation intensity of over 300 c.c. per week; on the other scale, of over 30 inches for the three months, and the geographic areas represented by such intensities are satisfactorily similar in the two cases.

(2) The region of low intensities of evaporation, characterized by indices below 150 c.c. on the 1908 chart, may be taken to correspond to the region of intensities below 15 inches on the other chart. Such a convention shows, not an agreement between the two, but such differences as might be expected to occur between charts for different summers, even though these were derived by the same methods. On the chart from Russell's data we find the zone of low evaporation (below 15 inches) to extend southward along the Pacific so as to include the whole coastal region, while in the 1908 chart the corresponding zone apparently can not be extended nearly so far southward. On the 1888 chart, instead of the southward-projecting lobe of the zone of low intensities, west of the Great Lakes (shown on the other), this northern zone merely widens to include all of the Great Lakes and the country west of them to the middle of the Dakotas, and continues eastward to the Atlantic coast. On approaching the coast its southern boundary (plate 55) still bends southward and southwestward and reaches the continental margin only at the mouth of the Rio Grande, thus producing the counterpart of the eastern or Atlantic lobe of this zone (below 150 c.c.) on the 1908 chart. Thence the isoatmic line of 15 inches (plate 55) apparently bends agnin to the 
east (in the Gulf of Mexico) and reenters the continent sufficiently to demark the Gulf coasts of Florida and also a little of the Atlantic coast near Jacksonville, as pertaining to the zone of medium evaporation intensities. Thus the northern area of low intensities is broadened southeastward from New England, to include nearly all of the Atlantic and Gulf coast region, which suggests no very great alteration from the condition of affairs depicted by the 1908 chart. This way of regarding the zonation thus considers that the Minnesota-Kansas lobe of the northern zone of low intensities is represented only on the 1908 chart, that the northern zone is otherwise much widened southward throughout its eastern half on the 1908 chart, and that the MaineMississippi lobe of this same zone is represented on both charts, being, however, more extensive on that for 1888 .

(3) One of the main differences between the two charts lies in the portion of the great eastern lobe of the zone of medium evaporation intensities. While this lobe extends northeastward from Oklahoma and Texas in the 1908 chart, it extends eastward from Nebraska in the 1888 chart. In the latter it does not attain as great a northerly extension as in the former, and it practically reaches farther east in the region of Chesapeake Bay (on account of the localized area of that region) in the 1888 chart. As has been noted above, the zone of intermediate atmospheric evaporating power occupies practically all of the Gulf coast and all of the Atlantic coast as far north as New Jersey, on the 1908 chart, but this zone is, as it were, nearly crowded off from the continent on the 1888 chart.

From the above considerations it appears that the two charts are not in nearly so great disagreement as a first view might suggest. They agree very well in depicting the desert region. They agree in showing a southward extension of the northern zone of low evaporation, on each ocean border, and in showing the eastern of these extensions as embracing the Appalachian Mountains south of Pennsylvania. They agree in depicting a zone of intermediate evaporating power, including, roughly, the western half of the country (excepting the most arid part), the region of the main great mountain-mass, and the high plains east of this. Finally, they agree in showing that a great lobe of this intermediate zone extends eastward or northeastward from the main area, and that this lobe embraces the region immediately west of the Appalachian Mountains.

Whether all these generalizations from imperfect observations, for two periods 20 years apart, may prove generally true throughout a long period of years will hardly be known by the present generationnor even by the next following one, unless atmometry begins to attract serious attention in the very near future. They are of little value at present, excepting as very rough approximations and as they indicate how important among climatic features is the evaporating 
power of the air, and how readily it might have been and may be studied.

Summer evaporation, 1908, as shoun by geographic profiles.-To obtain another kind of picture of the geographical variation in summer evaporating power of the air, as brought out by the 15 -week means, attention may be directed to the 6 profiles shown in figure 13. These profiles aim to present graphically the changes in summer evaporation intensity to be encountered in traversing the country from west to east and from north to south. Two west-east profiles are shown, one

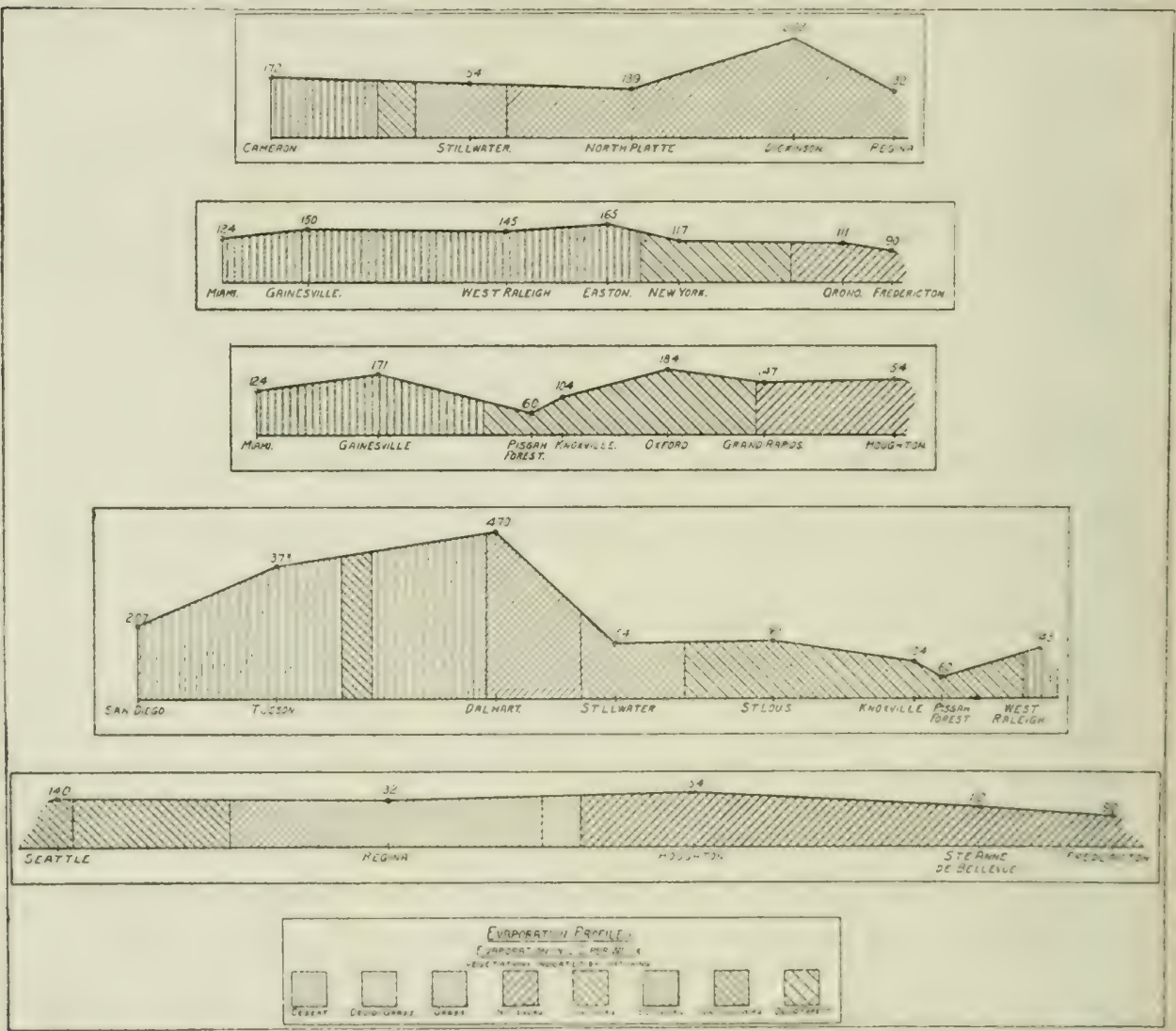

Fio. 13.-Evaporation profiles, weekly rates, summer of 1908 , with vegetation indicated. The lines along which these profiles are constructed are indicsted on Plate 56.

passing from Seattle, Washington, to Fredericton, New Brunswick; the other passing from sian Diego, California, to West Raleigh, North ('arolina. Three north-south profiles are presented, the first passing from Regina, Faskatchewan, to Cameron, Louisiana; the second from Houghton, Michigan, to Miami, Florida; and the third from Fredericton, New Brunswick, to Miami, Florida. Those of our stations through 
which these profiles pass are indicated at proper distances along the horizontal axis. As ordinates, extending upward at the points so marked, are represented the mean weekly losses from the porous-cup atmometer, as given in table 17, these numerical data being placed upon the profiles. Below each profile are conventionally indicated the vegetational types traversed and their approximate boundary lines, these boundaries having been obtained from the generalized vegetation chart (plate 2). We need not digress here to discuss the relations brought out between vegetation and summer evaporation intensity as here indicated; these matters will receive attention in their own place.

The northern west-east profile (lowest in figure 13) shows little variation in the intensity of evaporation throughout its extent. The southern west-east profile, on the other hand, shows a very great variation in evaporation values, the maximum being at Dalhart and the minimum at Pisgah Forest. The north-south profiles bring out the relatively high summer evaporation values obtained at Dickinson, at Easton, and at Gainesville and Oxford.

(4) Conclusions from the Study of Evaporation Conditions.

All the evaporation charts agree in their main features, especially the charts derived from Russell's observations. The four evaporation provinces are represented in figure 14, based on plate 53, and may be

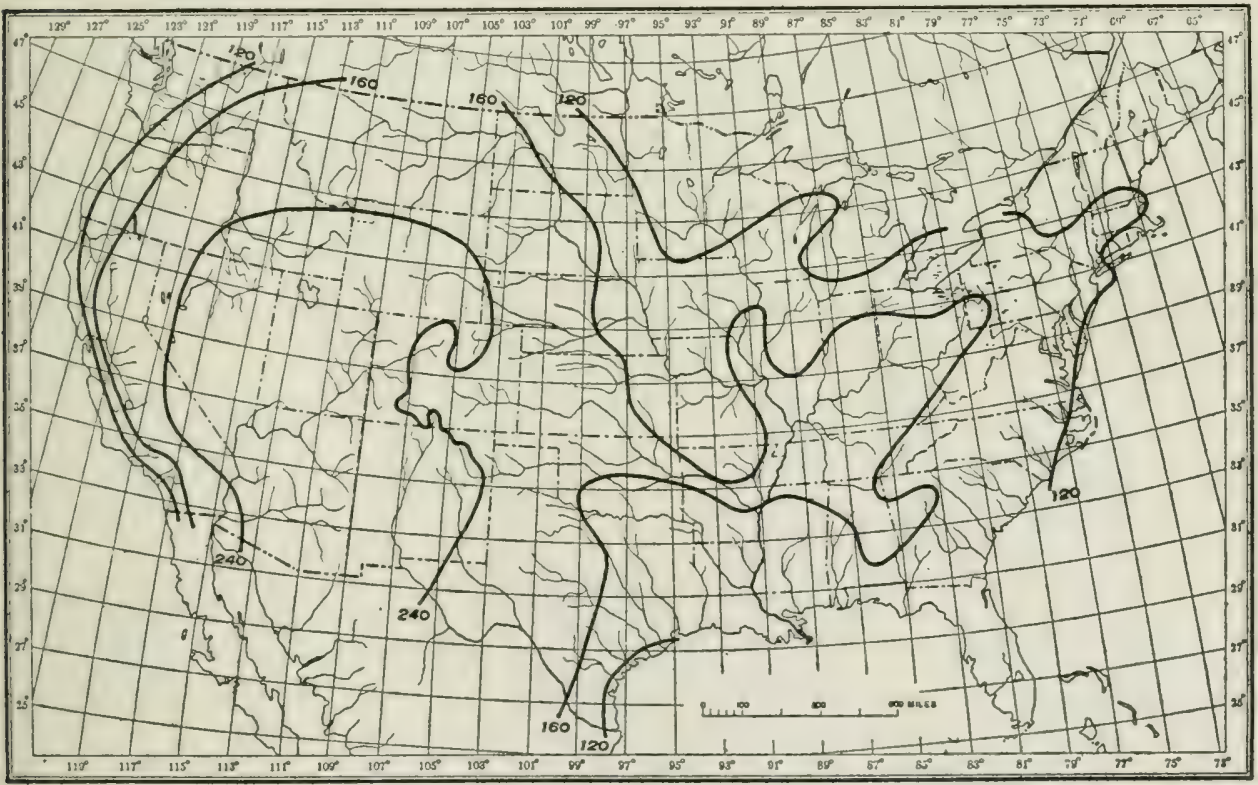

Fra. 14.-Moisture zonation, according to evaporation indices (1887-88) for period of average frostless season. Evaporation provinces: Humid, less than 120; semihumid, 120 to 160; semiarid, 160 to 240 ; arid, more than 240 . Numerical values are in thousandths of an inch. (See also Plate 53.) 


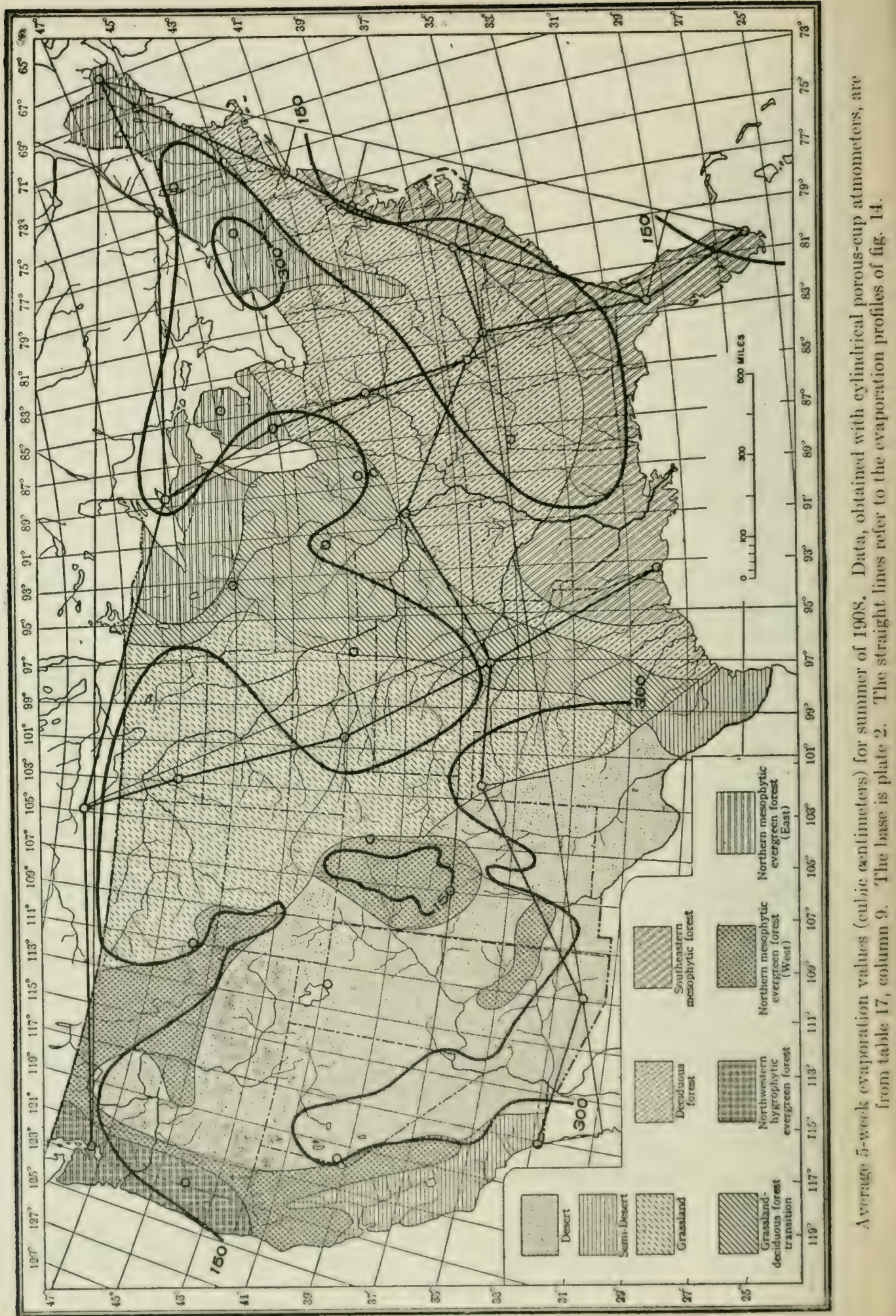




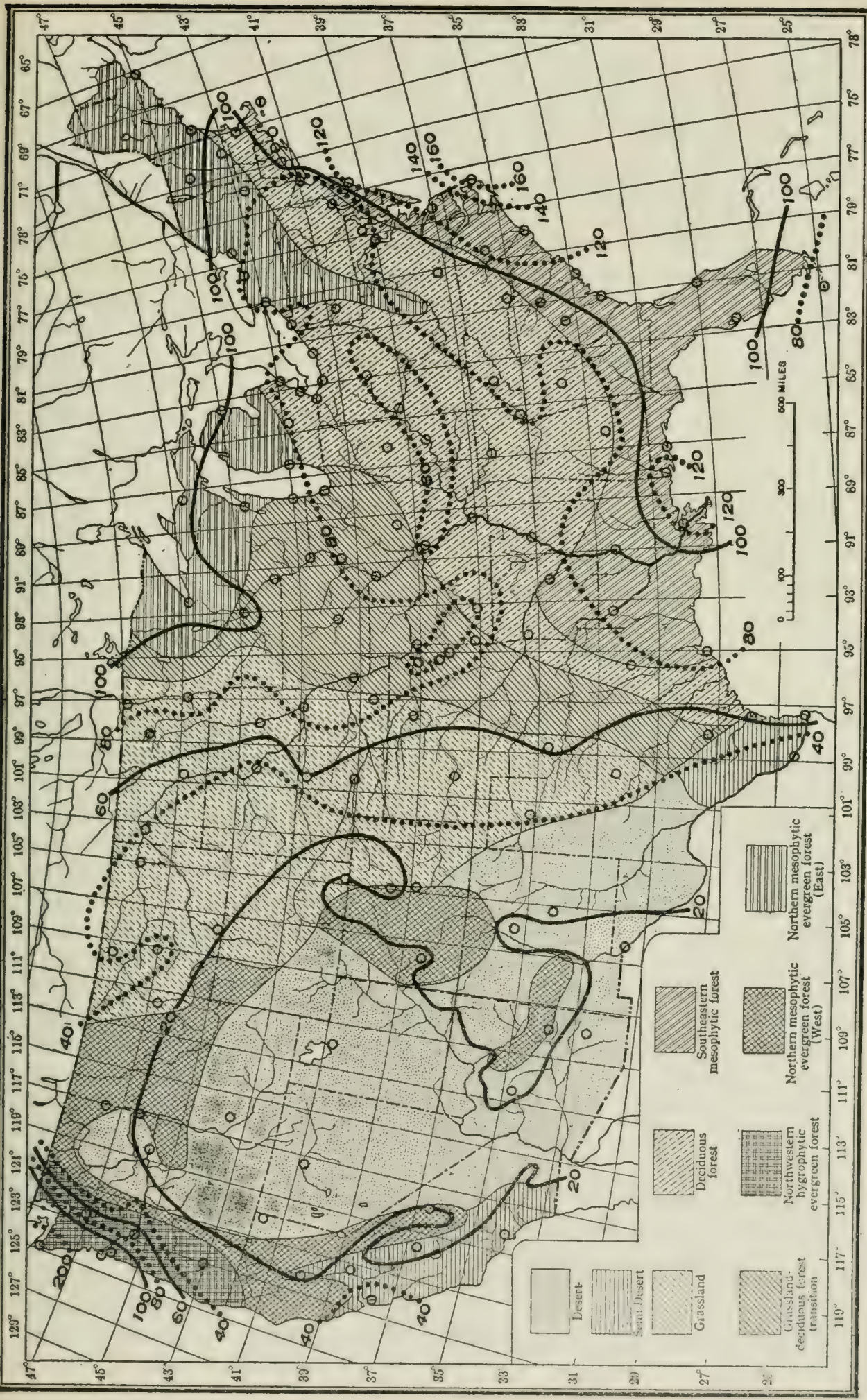

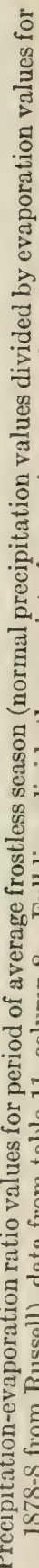


characterized as follows: The arid province extends northward from Mexico and occupies the Great Basin. The semiarid province is shown as a belt lying outside of the arid area, this belt not being wide enough to reach the Pacific at any point, but extending into Canada at the north and also extending eastward, from Nebraska and Oklahoma, in the form of a large lobe that reaches nearly to the Appalachian Mountains. The semihumid province occupies a narrow belt outside of the semiarid one, but not reaching to the Pacific coast (on plates 53 and 55, at least). This belt broadens at the north, extends into Canada, and lies just outside of the great eastern lobe of the semiarid province. It includes much of the Gulf and southern Atlantic region, a feature that is quite unlike the corresponding state of affairs on the precipitation charts. The humid province occupies a small portion of the northwest (reaches southward along the Pacific coast on plate 54, fig. 14), extends into Canada at the north, reenters the United States west of the Great Lakes, and occupies most of Minnesota, Wisconsin, Michigan, New York, and the three northern States of New England. Another portion of the humid province lies along the Atlantic coast, from Massachusetts (or southern New Jersey, see fig. 14) to North Carolina, and a narrow strip appears on the eastern coast of Texas.

\section{RATIOS OF PRECIPITATION TO EVAPORATION.}

(1) Preliminary Considerations.

Following the lead of Transeau (1905), we have employed the ratio of precipitation to evaporation as the nearest approach that is as yet possible toward an ideal index of the external moisture-relation of plants. Transeau's introduction of this ratio marked a very definite and important forward step in climatology, which will, no doubt, become more thoroughly appreciated as data of evaporation become available. Of course this ratio, like other intensity factors, may be employed in connection with any duration factor or factors that may seem desirable, and we have thus employed it in six different ways. As has been said, however, it is not yet possible to obtain evaporation normals, and all that can be done is to use Russell's data for the single year, July 1887 to June 1888. Transeau obtained his ratio by dividing the normal annual precipitation, for each station considered, by the corresponding total evaporation for Russell's year of evaporation data. Our various applications of the Transeau ratio will receive attention below.

(2) Ratios of Normal 'Total Precipitation, for Period of Average frostless Season, to Total Evaporation as omtained by Rusgell for the game Period, for the Year July 1887 to June 1888. P/E. (Table 11, Plate 57, Fig. 16.)

The two terms of this ratio, for each of the stations included in our list, are given in the second and sixth columns, respectively, of table 11 , and the ratiovalues themselves $(P / E)$ are given in the eighth column 
of the same table. These ratios are shown graphically on plate 57, where the numbers on the lines represent hundredths.

Inspection of table 11 and plate 57 shows that the moisture-ratio values range from 0.04 (Independence, California, and Winnemucca, Nevada) to 1.76 (Cape Hatteras, North Carolina) and to 3.84 (Tatoosh Island, Washington). The highest values are in western Washington and Oregon and the lowest are in the region of the Great Basin and in the arid Southwest. The isoclimatic lines show that the country may be divided into three main zones or regions: (1) an arid zone (ratio values below 0.20 ), (2) a humid zone (ratio values above 1.00 ), and (3) an intermediate zone (ratio values between 0.20 and 1.00). The intermediate zone is conveniently subdivided into a semiarid zone (ratio values between 0.20 and 0.60 ) and a semihumid zone (ratio values between 0.60 and 1.00). There are thus four climatic zones or provinces to be considered, as in the case of our precipitation and evaporation charts. Their limits are denoted by full lines on plate 57 .

The arid province extends, roughly, from the Rocky and Big Horn Mountains westward to the Cascades, the Sierra Nevadas, and the Coast ranges. It includes a little of southeastern Washington.

The semiarid province forms a belt lying west, east, and north of the arid region, extending westward nearly to the coast of Washington and northern Oregon, and to the coast of southern Oregon and California, northward into Canada, and eastward to about the ninetyninth meridian of west longitude.

The semihumid province is shown northwest and east of the semiarid area. It includes a narrow strip of western Washington and northwestern Oregon and extends eastward from the semiarid region to about the ninety-third meridian at north and south, but is broadened to include most of the country in its middle portion. This zone also includes southern peninsular Florida. The western portion of the humid region occupies western Washington and a little of northwestern Oregon, while the eastern portion includes the Gulf coast east of the Mississippi River (excepting extreme southern Florida) and the Atlantic coast north of southern Florida (excepting the northern part of the New Jersey coast and the coast about Boston). It also occupies the country north of middle New England and extends southward from Canada to include northern Michigan, northern Wisconsin, and eastern Minnesota.

The most interesting special feature of this chart is the enormous eastward enlargement of the semihumid region, which corresponds to a similar eastern lobe evident on the evaporation chart for the period of the average frostless season (plate 53). It should be remarked that a localized portion of the semiarid region here appears to be located in the middle of this enlargement, occupying southern Illinois, Indiana, and Ohio, and northern Kentucky. A similar localized arid area is shown 
on plate 53. There is also apparent here a small localized area with ratio values of 1.00 or 1.03 , including Topeka, Kansas, and Lamar and Springfield, Missouri, but this deserves no special attention. It is especially interesting to note, as will need to be done also in connection with a number of the following charts, that the line for value 1.00 apparently leaves the mainland in the middle of the New Jersey coast, returns at the western edge of Connecticut, leaves it again south of Boston, and finally reenters at the southwestern extremity of Maine. This brings it about that the eastern portion of the humid zone is divided into two parts, a northern one extending from Minnesota to northern New England and, an eastern and southern one extending from about Boston or New York to the Rio Grande and beyond. This feature appears to be an important one, and it will receive more attention later.

(3) Ratiog of Total Precipitation for Period of Average Frostless Season, for the Year July 1887 to June 1888, to Total Evaporation as obtained by Russell for the Same Period and Year (P/E). (Table 18, Plate 58.)

The method of deriving the moisture ratios that has just been described involves the use of normal precipitation data along with evaporation data for the single year of Russell's study. It was thought that this would give to the derived ratios somewhat more of the character of normals than would be the case if both precipitation and evaporation data had been taken for the single year in question, but the novelty and great promise of this climatic ratio render it worth while to present the values obtained by the latter method. These are given in table 18. To make comparison easier, the ratio values from table 11 are here repeated, in the last column. The second column of table 18 gives the total precipitation for the period of the average frostless season, derived for the actual months of Russell's observations. His 12 months have been treated as though they all pertained to the same calendar year, and our usual method of approximating average frostless season data from monthly data has been employed, the original monthly data of precipitation for the year July 1887 to June 1888 being taken from the Summary by Sections. We term this precipitation value $p$, to distinguish it from $P$. The third column gives the corresponding evaporation values $(E)$ for the period of the average frostless season, being taken from the sixth column of our table 11. The fourth column gives the new ratio values $(p / E)$. When the name of one station is followed by that of another (the latter in parentheses), the evaporation data are for the latter station and the precipitation data are for the former. 
TABLE 18.-Data of precipitation and evaporation for the period of the average frostless season, for the year of Russell's observation (July 1887 to June 1888), and corresponding ratios of precipitation to evaporation, together with similar ratios derived by employing normal data of precipitation instead of those for this single year.

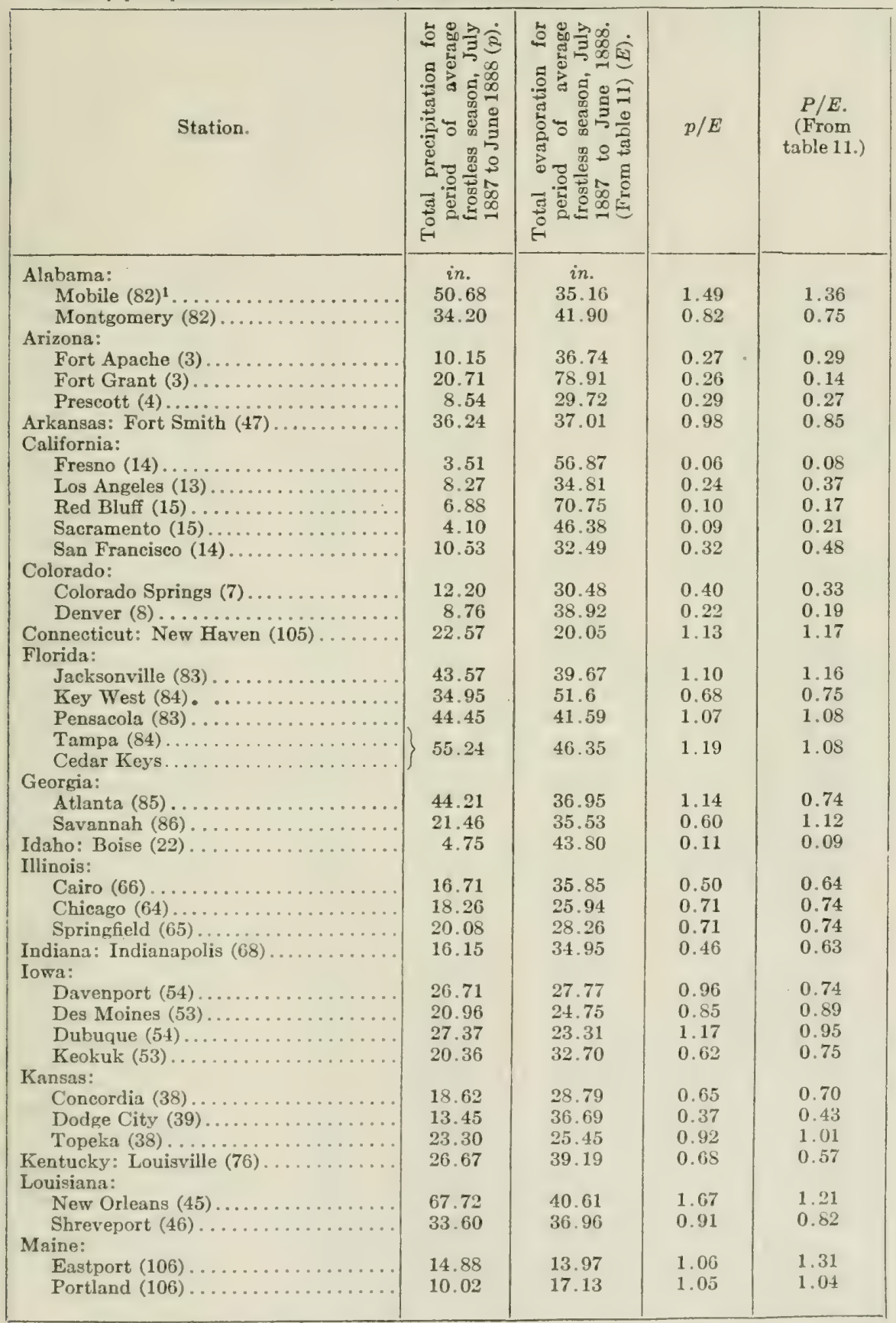

1 Numbers in parentheses after station names denote the section number, in the Summary by Sections, under which this station is given. 
TABLE 18.- Data of precipilation and evaporation for the period of the average frostless season, for the year of Russell's observalion (July 1887 to June 1888), and corresponding ratios of precipitation to evaporation, together with similar ratios derived by employing normal data of precipitation instead of those for this single year.-Continued.

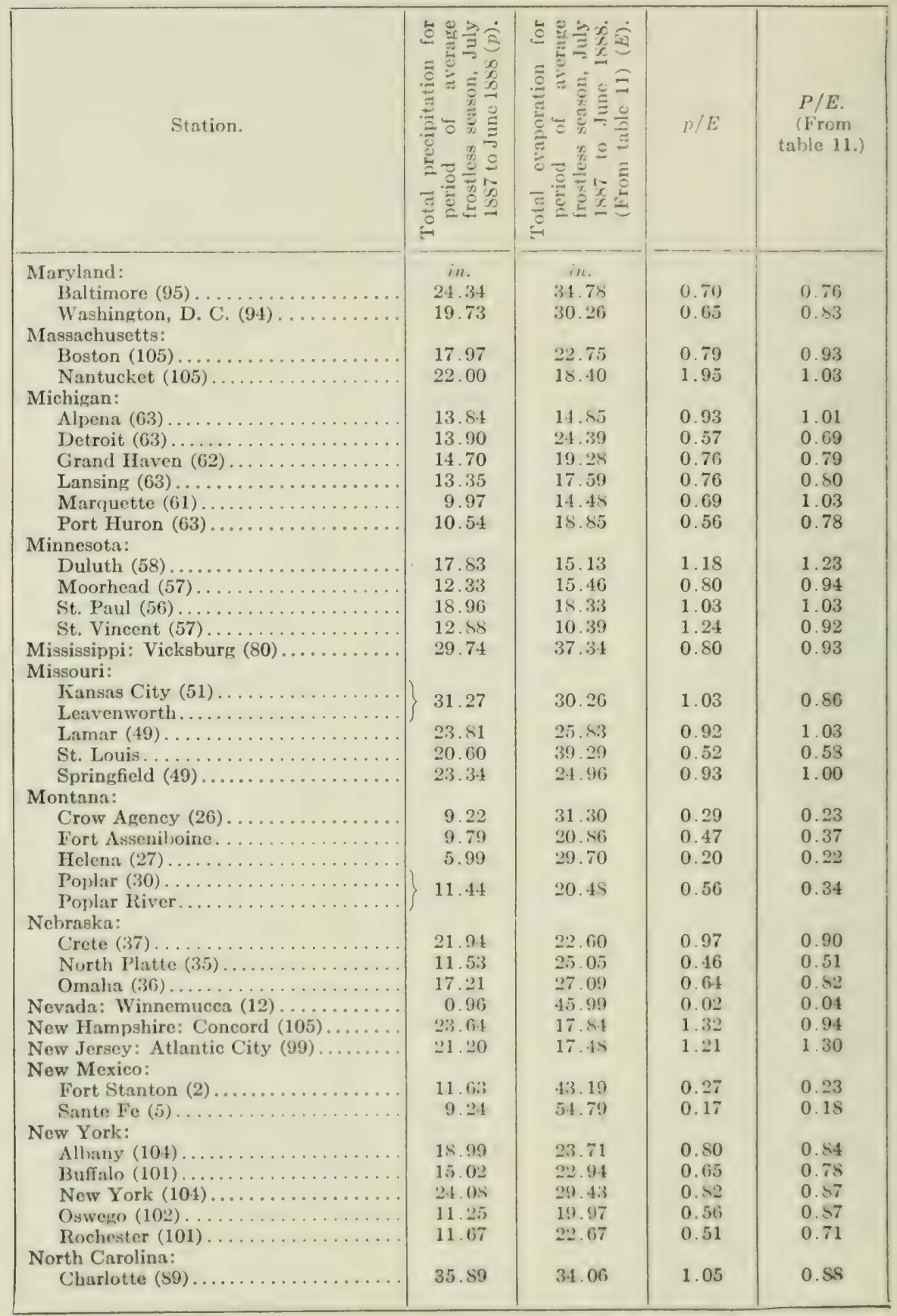


TABLE 18.-Data of precipitation and vaporation for the period of the average frostless season, for the year of Russell's observation (July 1887 to June 1888), and corresponding ratios of precipitation to evaporation, together with similar ratios derived by employing normal data of precipitation instead of those for this single year.-Continued.

\begin{tabular}{|c|c|c|c|c|}
\hline Station. & 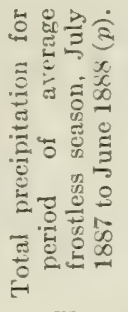 & 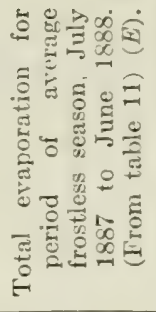 & $p / E$ & $\begin{array}{c}P / E . \\
\text { (From } \\
\text { table 11.) }\end{array}$ \\
\hline $\begin{array}{l}\text { North Carolina-Continued: } \\
\quad \text { Hatteras }(91) \ldots \ldots \ldots \ldots \ldots\end{array}$ & in. & 2465 & & \\
\hline $\begin{array}{l}\text { Hatteras }(91) \ldots \ldots \ldots \ldots \ldots \ldots \\
\text { Raleigh }(90) \ldots \ldots\end{array}$ & $\begin{array}{l}44.00 \\
41.03\end{array}$ & $\begin{array}{l}24.65 \\
26.13\end{array}$ & $\begin{array}{l}1.81 \\
1.57\end{array}$ & $\begin{array}{l}1.76 \\
1.22\end{array}$ \\
\hline Wilmington $(90) \ldots \ldots$ & 33.84 & 27.35 & 1.24 & 1.35 \\
\hline North Dakota: & & & & \\
\hline Bismark (31)... & 13.09 & 18.94 & 0.66 & 0.55 \\
\hline $\begin{array}{l}\text { Williston }(31) \ldots \\
\text { Fort Buford...... }\end{array}$ & 13.56 & 20.31 & 0.67 & 0.40 \\
\hline Devils Lake (32). & 14.96 & 15.38 & 0.97 & 0.75 \\
\hline Ohio: & & & & \\
\hline Cincinnati (70) & 11.55 & 37.20 & 0.31 & 0.51 \\
\hline Cleveland (69). & 21.31 & 26.35 & 0.81 & 0.78 \\
\hline Columbus (71). & 11.39 & 33.29 & 0.34 & 0.58 \\
\hline Sandusky (69).. & 12.33 & 27.36 & 0.45 & 0.74 \\
\hline Toledo $(69) \ldots \ldots \ldots \ldots$ & 13.48 & 26.84 & 0.50 & 0.62 \\
\hline Oklahoma: Fort Sill (41)... & 31.80 & 33.81 & 0.94 & 0.70 \\
\hline Oregon: & & & & \\
\hline Astoria (17).. & 36.29 & 21.77 & 1.67 & 1.90 \\
\hline Portland (17). & 16.35 & 29.42 & 0.56 & 0.66 \\
\hline Roseburg (17) ..... & 9.19 & 28.33 & 0.32 & 0.29 \\
\hline Pennsylvania: & & & & \\
\hline Erie $(96) \ldots \ldots \ldots$ & 25.10 & 17.48 & 1.52 & 0.90 \\
\hline Philadelphia (98)........ & 22.14 & 32.13 & 0.69 & 0.75 \\
\hline Pittsburgh....... & 20.55 & 29.69 & 0.69 & 0.66 \\
\hline Rhode Island: Block Island (105) ..... & 20.80 & 17.56 & 1.18 & 1.39 \\
\hline $\begin{array}{l}\text { South Carolina: } \\
\quad \text { Charleston }(88) \ldots \ldots \ldots \ldots \ldots \ldots\end{array}$ & 31.85 & 36.25 & 0.88 & \\
\hline $\begin{array}{l}\text { Charleston }(88) \ldots \ldots \ldots \ldots \ldots \ldots \ldots \ldots \\
\text { Columbia }(87) \ldots \ldots \ldots \ldots \ldots \ldots\end{array}$ & 30.94 & 31.81 & 0.97 & $\begin{array}{l}1.10 \\
0.98\end{array}$ \\
\hline South Dakota: & & & & \\
\hline Huron (34).... & 15.43 & 19.00 & 0.81 & 0.66 \\
\hline Pierre $(33) \ldots .$. & 12.87 & 27.60 & 0.47 & 0.39 \\
\hline Yankton $(34) . .$. & 25.27 & 18.79 & 1.34 & 0.91 \\
\hline Tennessee: & & & & \\
\hline Chattanooga (78).... & 27.25 & 31.00 & 0.88 & 0.81 \\
\hline Knoxville (78)...... & 25.56 & 30.70 & 0.83 & 0.82 \\
\hline Memphis (77)....... & 18.46 & 37.00 & 0.50 & 0.75 \\
\hline Nashville (77) .................. & 26.67 & 36.66 & 0.73 & 0.69 \\
\hline Texas: & & & & \\
\hline Abilene (42) .... & 23.83 & 46.00 & 0.52 & 0.44 \\
\hline $\begin{array}{l}\text { Brownsville (1)..... } \\
\text { Fort Brown....... }\end{array}$ & 49.39 & 32.41 & 1.52 & 0.77 \\
\hline $\begin{array}{l}\text { Corpus Christi }(1) \ldots \ldots \\
\text { El Paso }(2) \ldots \ldots \ldots\end{array}$ & $\begin{array}{r}38.12 \\
6.06\end{array}$ & $\begin{array}{l}35.09 \\
64.41\end{array}$ & $\begin{array}{l}1.08 \\
0.09\end{array}$ & $\begin{array}{l}0.65 \\
0.12\end{array}$ \\
\hline Fort Ringgold (1)... & 21.60 & 46.90 & 0.46 & 0.34 \\
\hline $\begin{array}{l}\text { Rio Grande City } \ldots \ldots \ldots \ldots \ldots \ldots \\
\text { Galveston }(1) \ldots \ldots \ldots\end{array}$ & 51.84 & 44.06 & 1.17 & 0.97 \\
\hline Palestine $(44) \ldots \ldots \ldots \ldots \ldots \ldots \ldots$ & 32.97 & 36.25 & 0.91 & 0.79 \\
\hline San Antonio $(1) \ldots \ldots \ldots \ldots \ldots$ & 29.00 & 43.63 & 0.66 & 0.51 \\
\hline
\end{tabular}


TABLE 18.-Data of precipilation and evaporation for the periort of the average frostless season, for the year of Russell's observation (July 1887 to June 1888), and corresponding ratios of precipitation to evaporation, together with similar ratios derived by employing normal data of precipitation instead of those for this single year.-Continued.

\begin{tabular}{|c|c|c|c|c|}
\hline Station. & 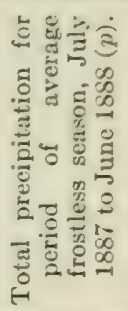 & 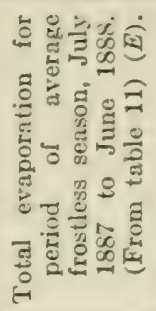 & $p / E$ & $\begin{array}{c}P / E, \\
\text { (From } \\
\text { table 11.) }\end{array}$ \\
\hline Utah: Salt Lake City (11).... & $\begin{array}{l}\text { in. } \\
4.32\end{array}$ & $\begin{array}{l}\text { in. } \\
51.71\end{array}$ & 0.08 & 0.13 \\
\hline Vermont: Northfield (105).... & 19.02 & 12.28 & 1.55 & 1.16 \\
\hline Virginia: & 2405 & & & \\
\hline $\begin{array}{l}\text { Lynchburg (93). } \\
\text { Norfolk }(92) . . .\end{array}$ & $\begin{array}{l}24.95 \\
39.82\end{array}$ & $\begin{array}{l}28.68 \\
27.26\end{array}$ & $\begin{array}{l}0.87 \\
1.46\end{array}$ & $\begin{array}{l}0.87 \\
1.24\end{array}$ \\
\hline Washington: & & & & \\
\hline $\begin{array}{l}\text { North Head (19).. } \\
\text { Fort Canby.... }\end{array}$ & 43.68 & 20.18 & 2.16 & 1.73 \\
\hline Olympia $(19) \ldots . . .$. & 11.22 & 19.85 & 0.56 & 0.70 \\
\hline Spokane $(20) \ldots$ & 11.28 & 33.80 & 0.33 & 0.23 \\
\hline Walla Walla $(20) \ldots \ldots \ldots \ldots$. & 7.37 & 45.87 & 0.16 & 0.18 \\
\hline Wisconsin: & & & & \\
\hline Green Bay $(60)$ & 14.57 & 19.39 & 0.75 & 0.86 \\
\hline La Crosse $(59) \ldots \ldots \ldots \ldots \ldots \ldots$ & 18.67 & 21.97 & 0.85 & 0.94 \\
\hline Milwaukee $(60) \ldots$ & 17.63 & 19.11 & 0.92 & 0.87 \\
\hline Wyoming: Cheyenne $(24) \ldots \ldots \ldots$. & 7.09 & 32.48 & 0.22 & 0.20 \\
\hline
\end{tabular}

The chart of plate 58 was prepared from these ratio values $(p / E)$, the isoclimatic lines representing increments of 0.20 . This chart agrees in its essentials with that of plate 57 ; the great eastern lobe of the semihumid region (values between 0.60 and 1.00 ) is here again apparent and the localized area of semiarid conditions, within this lobe, is more pronounced than in the former case. The arid region (values below 0.20 ) is here indicated much as in plate 57 , but the line for the value 0.60 , in the middle of the country, here swings farther westward at its northern end than in the other case. In general, it appears to make no serious difference which of these two charts is studied, since all their essential features are so nearly alike.

Whenever agricultural climatology begins to receive attention in this country, and when evaporation observations are made for the period without frost, it will be desirable to prepare a chart similar to that of plate 58 for every year. Finally, a normal ratio value for each station may be actually obtained, after which the value for any particular station and season may be stated by comparison with the normal for that station, just as is now done in the case of temperature and rainfall. 
(4) Ratios of Normal Total Precipitation for Period of Average Frostlesg Season Plus preceding 30 Days, to Total Evaporation for the Same Period, July 1887 to June $1888(\pi / E)$. (Table 11, Plate 59.)

This form of the moisture ratio is based on the idea, already mentioned, that some of the precipitation occurring before the beginning of the frostless season is effective to supply water for plant activities in the early part of that season. The numerator $(P)$ of the first form of ratio has thus been increased, in each case, by adding to it the normal total precipitation for the 30 days just preceding the beginning of the average frostless season. This increased precipitation value has been termed $\pi$, to distinguish it from $P$ and $p$, so that the form of ratio here considered becomes $\pi / E$. The values of $\pi$ and $E$, and those of $\pi / E$, are given, for our list of stations, in table 11 , the ratio values occupying the last column.

The chart based on these ratios is shown as plate 59. The essentials of this chart are so nearly like those shown by plates 57 and 58 that no special comment is here needed. It may be noted, however, that the full line separating the humid from the semihumid zone is here taken as haring the value 1.10 instead of 1.00 , as in plates 57 and 58 .

(5) Ratios of Normal Total Annual Precipitation to Total Annual Evaporation, July 1887 to June $1888\left(P_{a} / E_{a}\right)$. (Table 15 , Plate 60.)

These annual ratios are derived by the method employed by Transeau. We have termed the normal total annual precipitation $P_{a}$, and

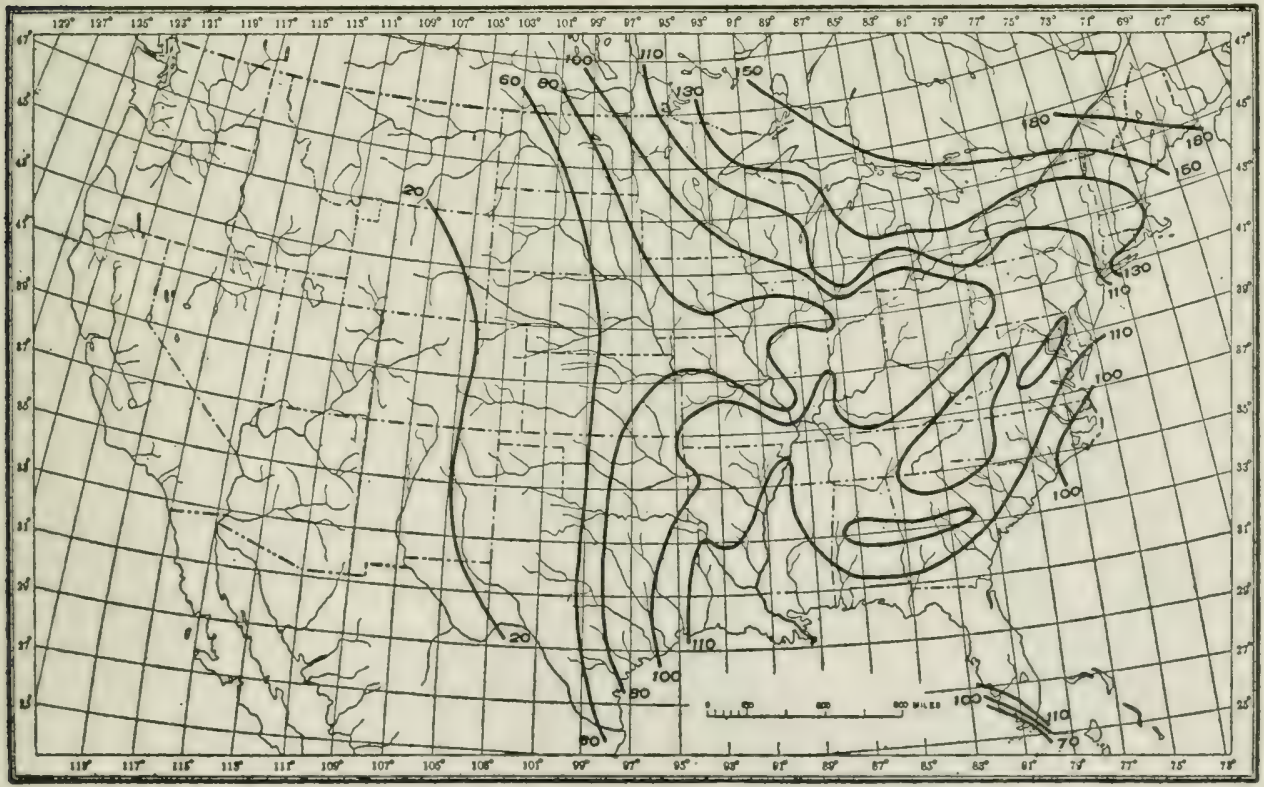

Frg. 15.-Moisture zonation of the eastern United States, according to precipitation-evaporation ratio (annual), after Transeau. 


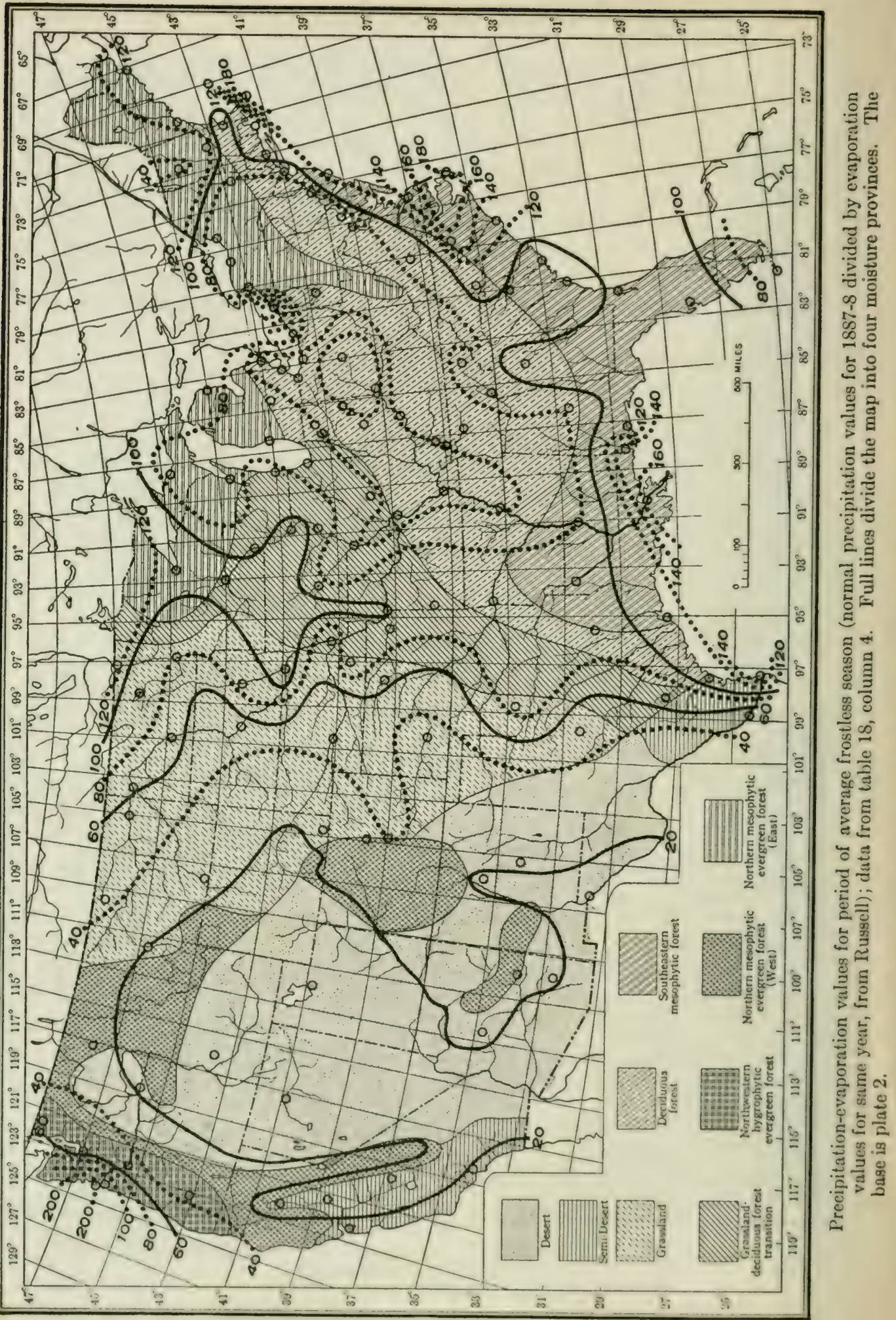




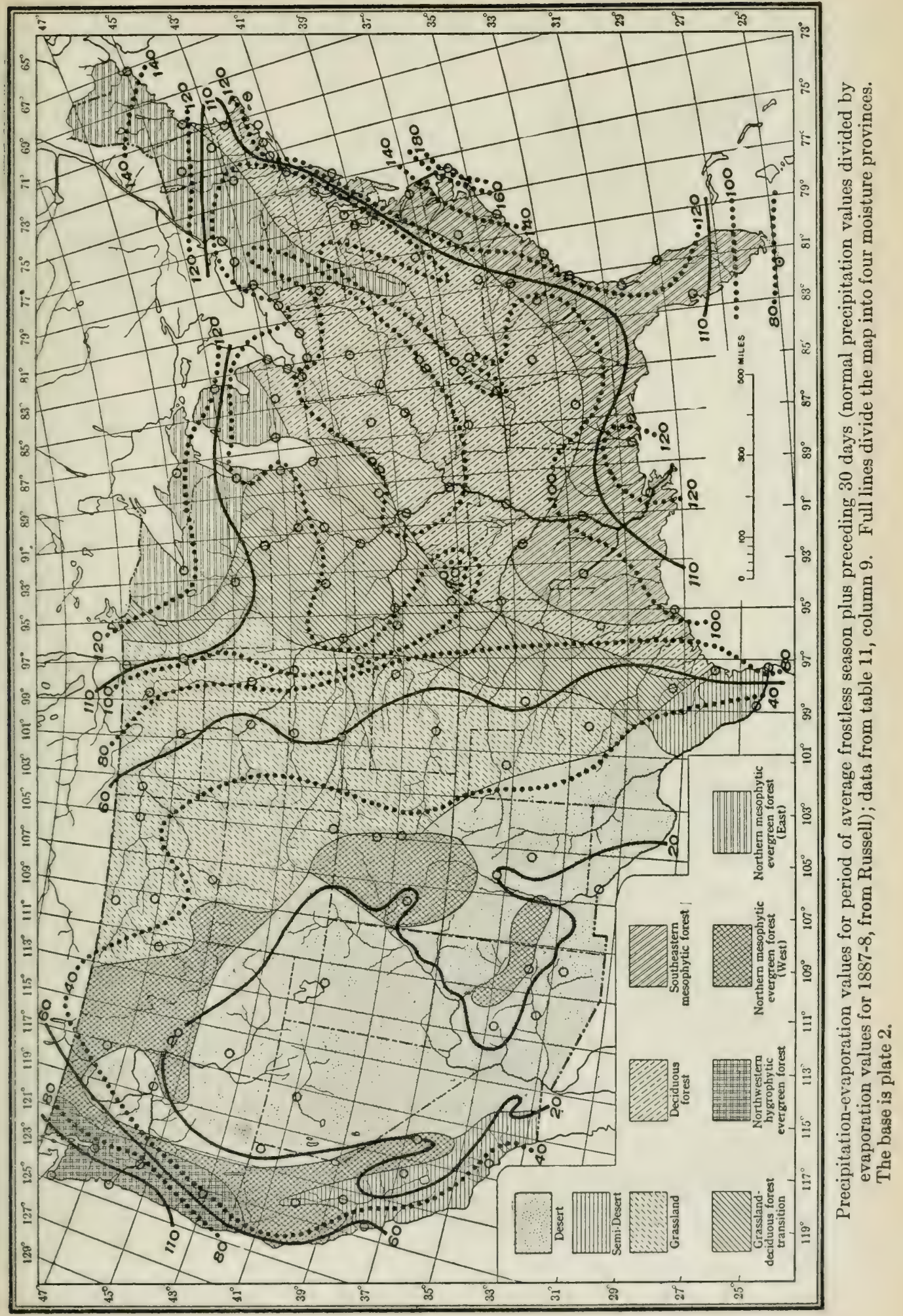




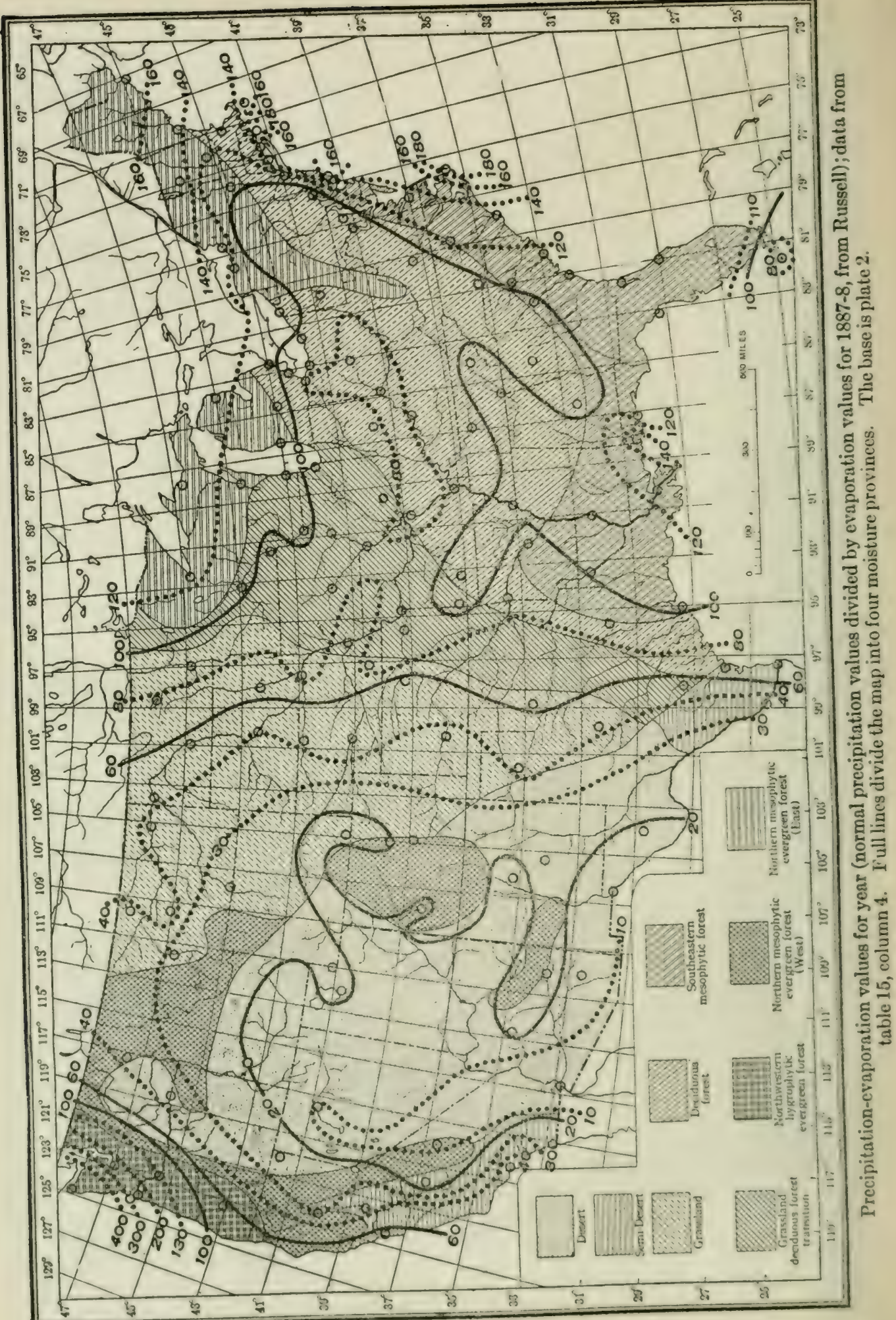




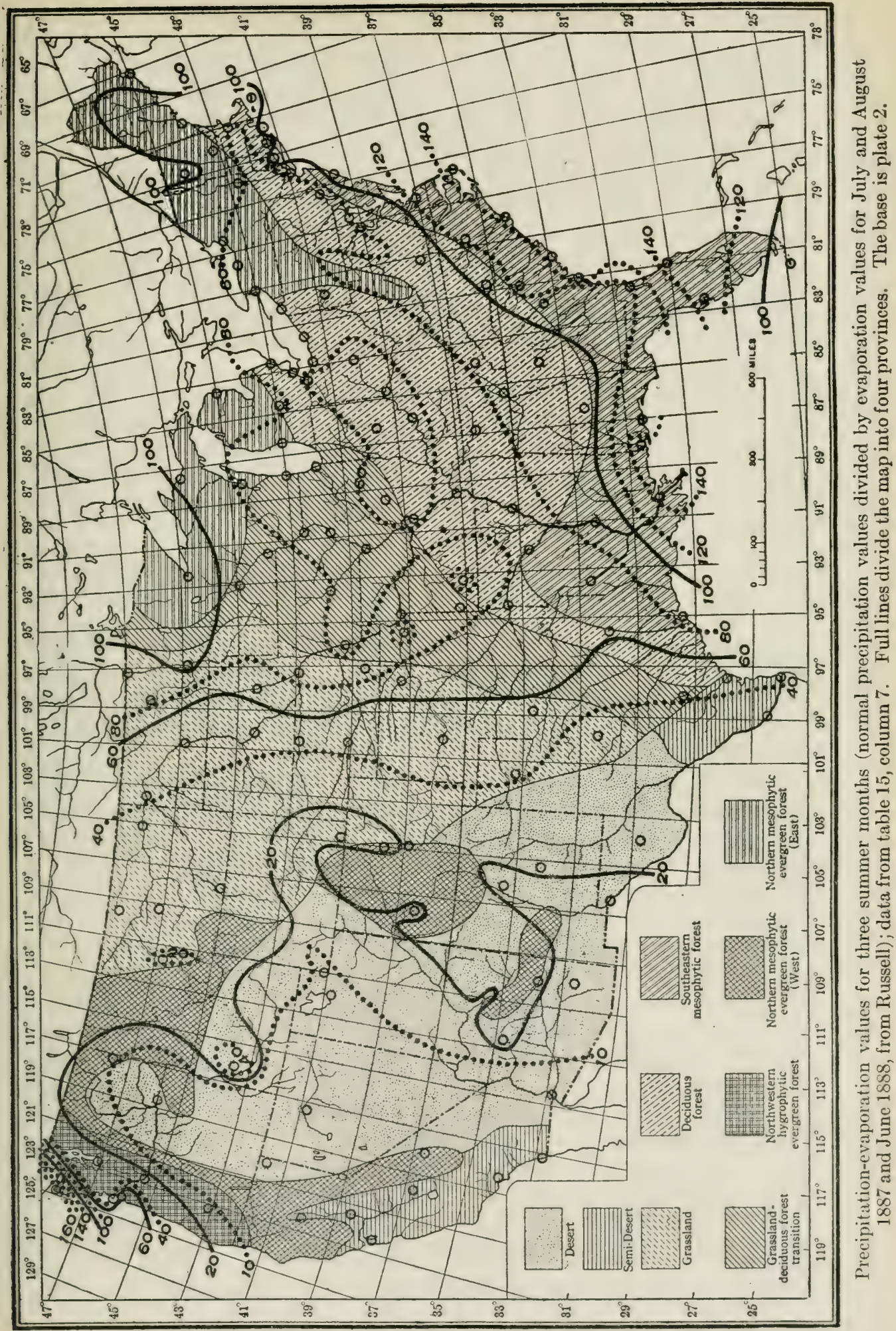


the total annual evaporation for Russell's year, $E_{a}$, so that this form of ratio becomes $P_{a} / E_{a}$. The values for both terms and for the ratios themselves are given in table 15, and the chart derived from the ratio values is here presented as plate 60 .

This chart has the same essential characteristics as those of plates 57,58 , and 59, and it agrees, in general, with Transeau's similar chart, which is here reproduced for comparison, as figure 15. It includes only the eastern half of the country. Since he did not publish the station data on which it is based, it is impossible to determine just wherein lie the discrepancies between Transeau's calculations and our own. The agreement between the two charts is close enough, however, for present purposes.

If the chart of plate 60 is compared with those of plates 57,58 , and 59 , the main difference is seen to lie in the fact that the line separating the humid from the semihumid zone, in the East, bends northward in plate 60 , to include in the humid zone all of Mississippi and Alabama and parts of Tennessee and Missouri, which is not true for any other moisture-ratio chart of our series:

(6) Ratios of Normal Total Precipitation for the Three Summer Montis, June to August, to Total Evaporation for July and August 1887 and June $1888, P_{s} / E_{s}$. (Table 15, Plate 61.)

As also in the case of the annual ratios just discussed, the duration factor employed for these summer ratios is the same for all stations; all represent the period of the months June, July, and August. The two terms and the ratio are shown, for each station considered, in table 15 , the ratios occupying the last column.

Plate 61 shows the chart based on these summer ratios. Here the zonation is different from that of the preceding moisture-ratio charts in several particulars. In the first place, the arid region (values below 0.20 ) is here extended west to the Pacific and includes nearly all of Washington and Oregon and all of California, this difference from the preceding charts of this feature being probably related to the characteristic summer drought of California. The semiarid and semihumid regions indicated in the northwest are very restricted. Looking at this chart from any point of view, it is clear that the whole Pacific coast and the Pacific Northwest are characterized as far more arid for the summer months than for the period of the arerage frostless season or for the year.

In the East, the most pronouneed difference between this chart and the preceding ones lies in the fact that the great semihumid lobe projecting eastward from the Plains is here shown as extending farther to the north than on the preceding charts. This suggests that New lingland and the states bordering on the Great Lakes are more arid for the period of the three summer months than for the other periods we have considered. 
(7) Ratios of Total Precipitamion for 15 Weeks in the Summer of 1908 to Total Evaporation for the Same Period and Year, the Evaporation Data obtained wite the Cxlindrical Porous-cup Atmometer $\left(\frac{P}{S} 1908 / \frac{E}{S} 1908\right)$. (Table 17, Plate 62.)

These ratios were obtained to accompany the evaporation and rainfall data of 1908. The numerators and denominators and the ratio values are presented in table 17 . The chart of plate 62 represents these ratio values, as well as the small number of stations will permit.

It is to be noted at once that the values obtained by dividing inches of precipitation by cubic centimeters of evaporation have an entirely different order of magnitude from those heretofore considered, being very much smaller. In order to render these numbers more readily ('omparable with those used on the preceding ratio charts, the values from the last column of table 17 have all been multiplied by 10,000 before employing them for the chart of plate 62 . On this chart the humid zone is considered as having ratio values above 75 , the semihumid zone is characterized by values between 25 and 75 , the semiarid zone has values between 10 and 25 , and the values representing the arid zone are all below 10. By this convention the chart before us appears to agree in a rather satisfactory manner with the other moisture-ratio charts. Like the chart for the three summer months (plate 61 ), the arid zone is here shown as including the Great Basin, the Pacific coast, and most of the Colorado Desert. In the present case, however, the arid zone is extended northward, so that no semiarid, semihumid, or humid conditions are encountered in the extreme western part of the United States. The eastern margin of this zone lies farther west (except at the south, where it is farther east) than the corresponding margin on plate 61 . In short, the arid zone of plate 62 may be approximately obtained from plate 61 if we conceive that this zone on the earlier chart is simply extended northward, curtailed along most of its eastern margin, and extended eastward at its southern end. The line for the value 25 here corresponds with that for 60 on plate 61 , indicating that the eastern margin of the semiarid zone here lies much farther west than in the other case. The semihumid zone, as shown on plate 62 , appears as nearly cut into two portions by the great lobe of the humid zone that extends northward from eastern Texas, but its eastern projection is still as clear as on the other ratio charts, only this is displaced northward. This great eastern lobe of the semihumid zone here occupies the whole Atlantic region from southern Maryland nearly to the Canadian boundary, being thus also more extensive eastward than in the case of plate 61 . The localized area of semiarid conditions is once more apparent in the region of the lower Great Lakes, being displaced northeastward from its position on the other ratio charts. Lack of stations in Canada brings it about that no humid zone can here be depicted north of the Great Lakes region; it appears simply to be displaced northward from its position 


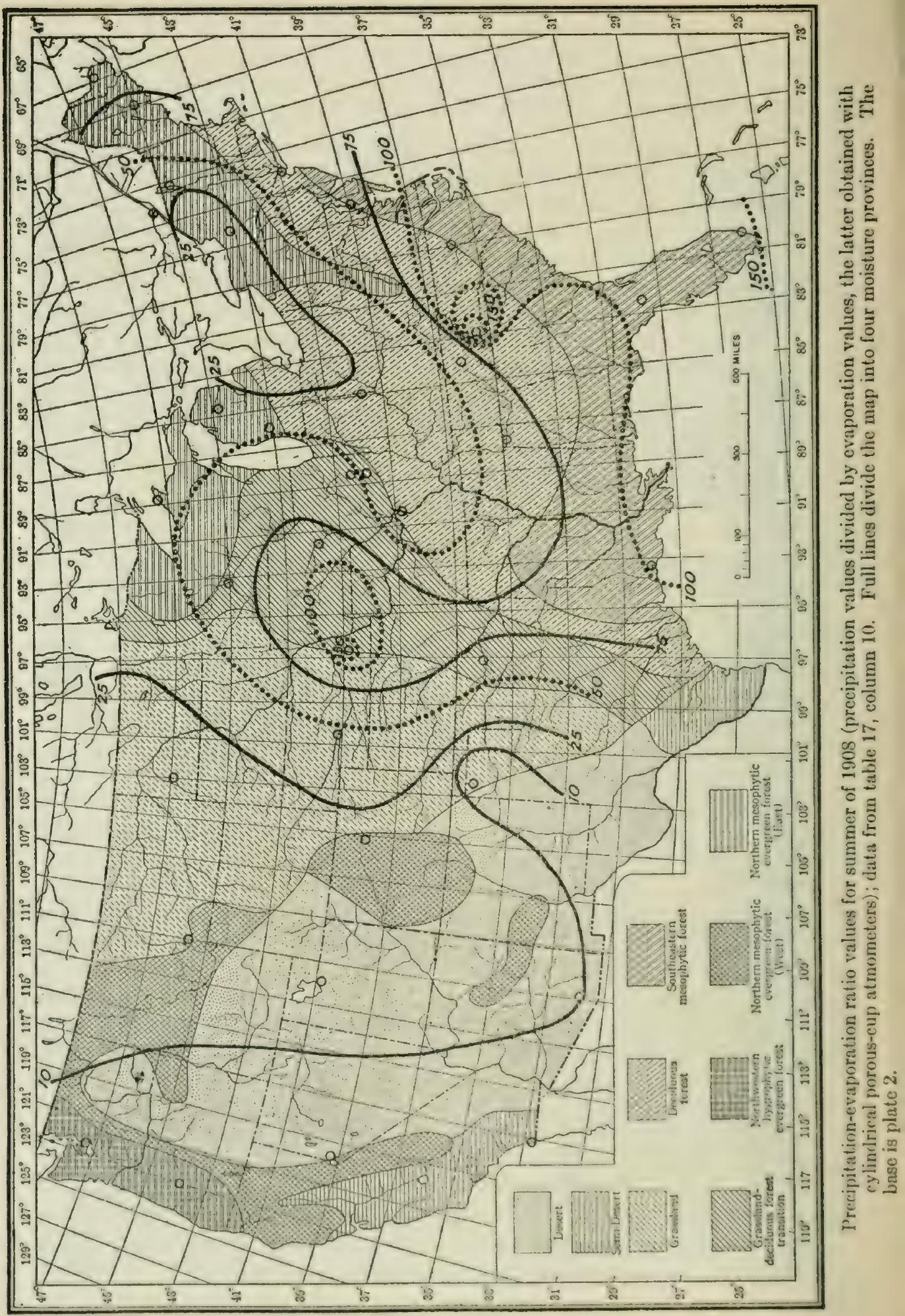




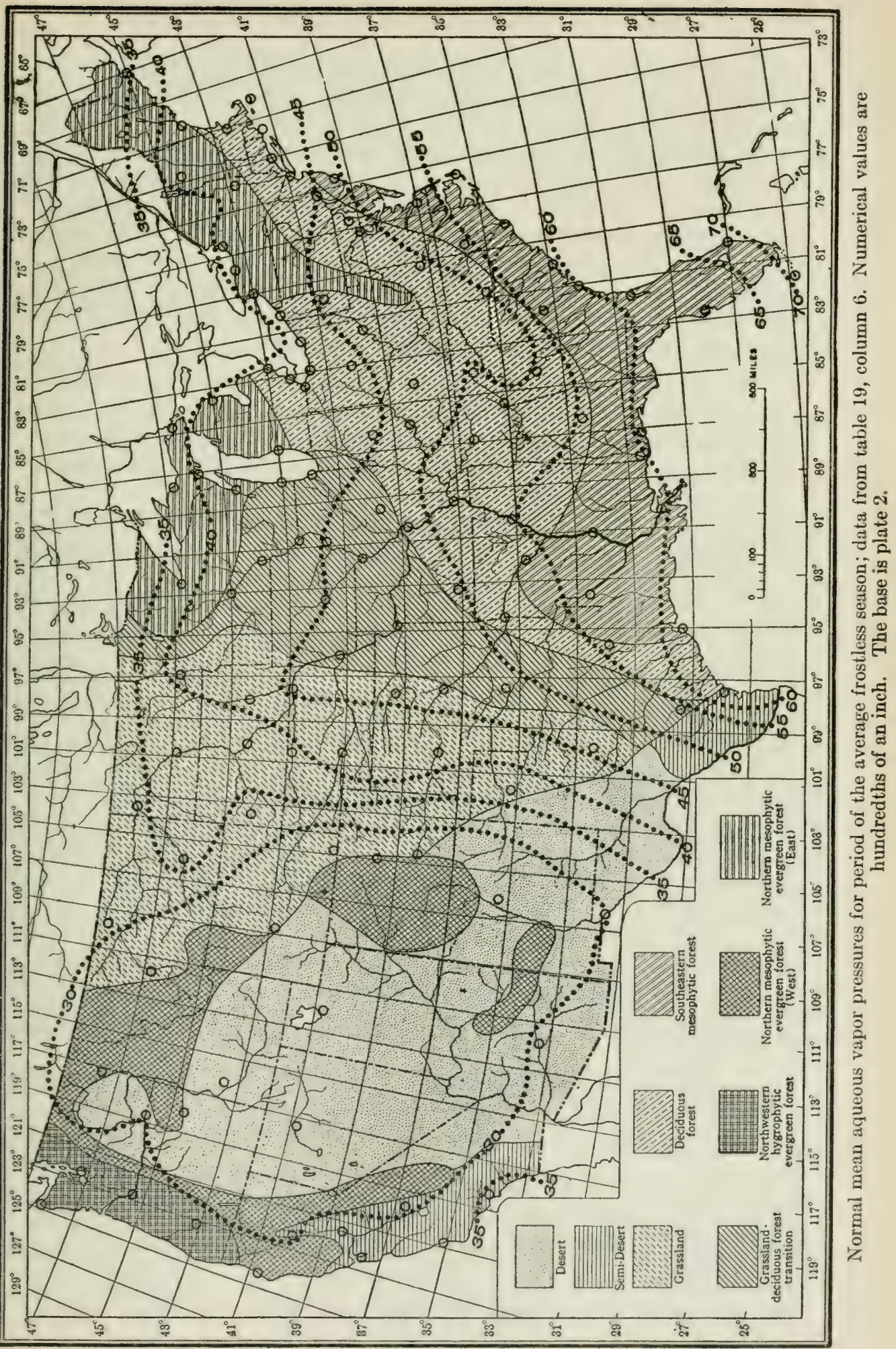


on the other plates. The separation of this eastern humid region into a northern and a southern portion, indicated on all the ratio charts, is here much more pronounced than in any other case. The northern portion is here indicated as occupying eastern Maine, while the southern portion extends from eastern Texas to southern Maryland, reaching farther inland than in any of the other cases, especially at its southeastern extremity, where its great northern lobe reaches southern Minnesota. This lobe appears here like an exaggeration of the similar but smaller lobe shown on the chart of plate 60 . It is thus seen that plate 61 differs from the other ratio charts only in relatively minor details, the main essentials being about the same.

(8) Conclusions from the Study of the Precipitation-Evaporation Ratios.

The results of our study of the various forms of Transeau ratios above described lead clearly to the conclusion, which is in full agreement with that reached by Transeau from his first use of this ratio,

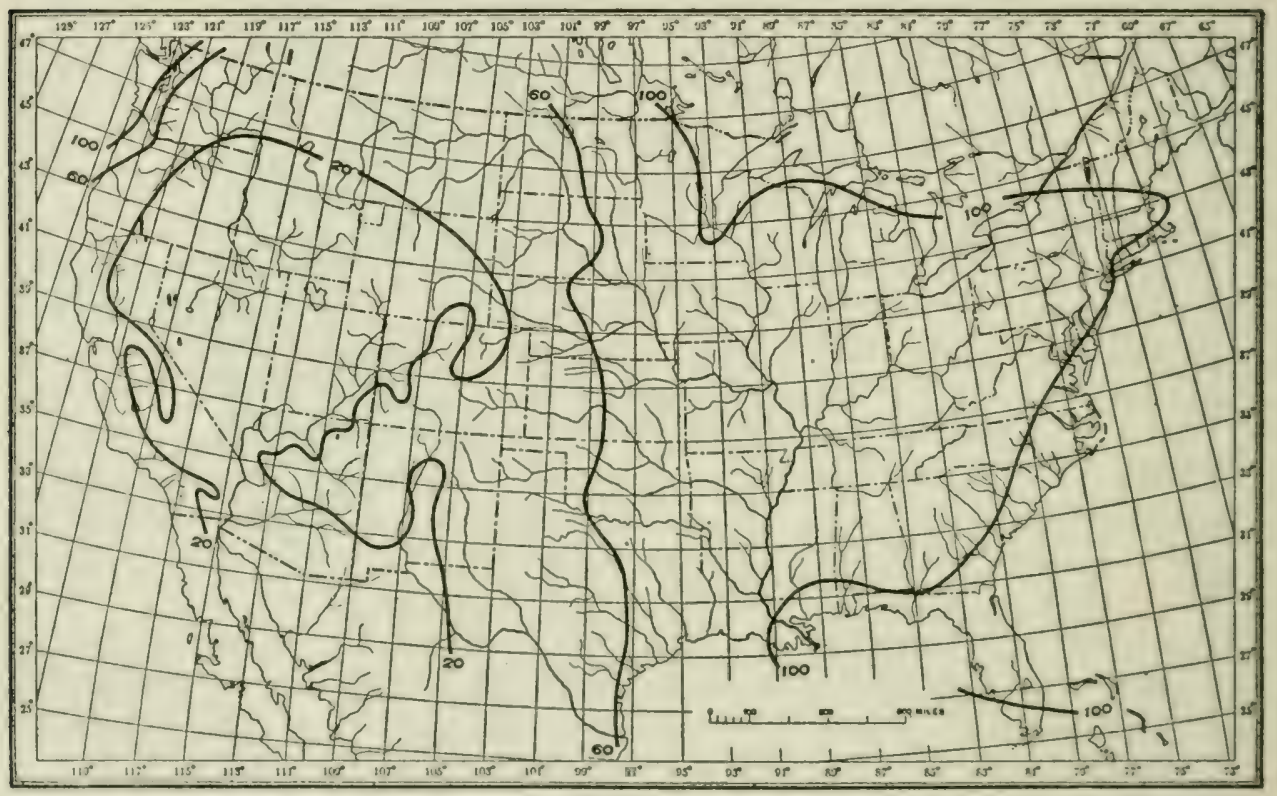

FIG. 16.-Moisture zonation, accordin frostless season). Moisture provinees: II umid, more than 100; semihumid, 60 to 100 ; semiarid, 20 to 60 ; arid, less than 20 . (See also Plate 57.)

that we have here a climatic index by means of which the zonation of the country with regard to moisture conditions maly he clearly shown. As will receive emphasis later, the climatic zomition thus brought out is elosely paralleled by certain prominent features of the zonation of vegetation types, and there is little room to douth that this division

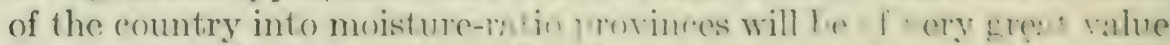
in the study of climatolory with weferenes to ayri, we and forestry. 
Our charts represent the country as divided into climatic provinces, which may be conveniently considered once more as (1) humid, (2) semihumid, (3) semiarid, and (4) arid. The following general description of these areas will serve to summarize the descriptions of the separate charts. Reference may be made to figure 16 , which is derived from plate 57.

Three humid areas are apparent, one in the Pacific Northwest, a second in the region of the Great Lakes and northern New England, and a third in the Southeast along the Gulf of Mexico and the Atlantic as far north as northern New Jersey or southern New England. The last two humid provinces are nearly continuous, and may be represented as such by charts drawn from certain forms of moisture ratio or from data of certain years. Our plate 60 shows them as continuous by a narrow belt that embraces about the southern half of Delaware and the eastern half of New Jersey.

The two semihumid areas are irregular in shape. The eastern one occupies all of the country not included in the humid area and east of about the one-hundredth meridian of west longitude. The western semihumid area occupies a rather narrow strip of country east of the western humid province and extending southward along the Pacific, south of that province, to about the middle of the California coast. These two areas are almost surely joined at the north, in Canada, though this point is not actually demonstrated by any of our data.

The semiarid area appears to be made up of an eastern and a western portion, joined together at the north. The eastern portion extends, approximately, from the one-hundredth meridian westward to the Rocky Mountains and to the San Francisco Mountains of the Arizona Plateau. Its eastern boundary appears to cross the Rio Grande somewhat southeast of El Paso. In its northern part this eastern semiarid region apparently broadens westward and extends through the lower passes of the mountains to Washington, where it joins the western portion of the same region. This western portion lies east of the western semihumid area as above described and extends southward to the Mexican boundary, along the coast of southern California. From Washington to Lower California it is a rather narrow belt.

The arid area occupies the intermontane region of the West, extending from the Rocky Mountains to the Sierras and from southern California and Arizona to Idaho and southwestern Washington. It is thus surrounded, on three sides within the United States, by the belt of the semiarid area.

Of course, it is understood that all the higher mountain ranges are to be considered as belonging to the humid area, at least in their upper portions, and that such high mountain masses are surely each bordered by a zone of semihumid conditions when they lie in an arid area. No attempt is here made to consider the innumerable small areas of 
humid, semihumid, and semiarid conditions included in the arid, semiarid, etc., areas as above described. A detailed moisture-ratio chart of a single county in Arizona or Nevada would doubtless be vastly more complicated than is any of our general charts for the entire country.

\section{AQUEOUS-VAPOR PRESSURE.}

(1) Preliminary Considerations.

The pressure of aqueous vapor in the air should be an index of the relative influence of the air (aside from its rate of movement) toward the retardation of evaporation from wet surfaces. Roughly, this index should be somewhat nearly inversely proportional to the evaporating power of the air, disregarding the wind factor. This is, therefore, a climatic dimension the measurement of which should be valuable in studies of the relations between plant activities and environmental conditions.

The vapor-pressure data for the United States, for the years 1873 to 1905 , have been reduced to a system of homogeneous monthly and annual means by Bigelow (Bull. S., 1909), and these means have furnished the basis for our work in this connection. We have employed (1) the normal mean vapor-pressure for the period of the average frostless season, and (2) the normal mean annual vapor pressure.

(2) Normal Mean Aqueous-Vapor Pressures for Period of Average Frostless Season (Table 19, Plate 63.)

The indices here employed were derived from the monthly means of Bigelow, in the way already described for such cases. Our results are given in the last column of table 19 , and plate 63 represents them graphically.

TABLE 19. - Normal mean relative humidities, for the year and for the period of the average frostless scason, mean relative humidities for the three summer months, 1908, and normal mean vapor-pressures for the year and for the period of the average froslless season.

\begin{tabular}{|c|c|c|c|c|c|}
\hline \multirow[b]{2}{*}{ Station. } & \multicolumn{3}{|c|}{ Mean relative humidity. } & \multicolumn{2}{|c|}{$\begin{array}{c}\text { Mean vapor-pressure } \\
\text { of water. }\end{array}$} \\
\hline & Annual. & $\begin{array}{c}\text { For period } \\
\text { of average } \\
\text { frostless } \\
\text { season. }\end{array}$ & $\begin{array}{l}\text { For June, } \\
\text { July, and } \\
\text { August } \\
190 \text { s. }\end{array}$ & Annual. & $\begin{array}{l}\text { For period } \\
\text { of average } \\
\text { frostless } \\
\text { season. }\end{array}$ \\
\hline Alabama: & p. ct. & $p . c t$. & p. $c l$. & inch. & inch. \\
\hline Birmingham............. & $\cdots .$. & ............. & 76 & $\cdots \cdots \cdots$ & $\ldots \ldots \ldots$ \\
\hline Mobile................. & 80.9 & 80.6 & 78 & 0.530 & 0.599 \\
\hline Montgomery.............. & 72.4 & 71.3 & 71 & .457 & .553 \\
\hline $\begin{array}{l}\text { Arizona: } \\
\quad \text { Flarstaff..... }\end{array}$ & & & 54 & & \\
\hline Phoenix...... & 38.7 & 36.3 & 35 & .278 & .305 \\
\hline Yuma...... & 41.6 & $\ldots \ldots \ldots$ & 45 & .334 & \\
\hline Arkansas: & & & & & \\
\hline Fort Smith... & 70.6 & 70.8 & 75 & .399 & .522 \\
\hline Little Rock................ & 72.8 & 72.4 & 74 & .424 & .539 \\
\hline
\end{tabular}


TABLE 19.-Normal mean relative humidities, for the year and for the period of the average frostless season, mean relative humidities for the three summer months, 1918, and normal mean vapor-pressures for the year and for the period of the average frostless season.Continued.

\begin{tabular}{|c|c|c|c|c|c|}
\hline \multirow[b]{2}{*}{ Station. } & \multicolumn{3}{|c|}{ Mean relative humidity. } & \multicolumn{2}{|c|}{$\begin{array}{l}\text { Mean vapor-pressure } \\
\text { of water. }\end{array}$} \\
\hline & Annual. & $\begin{array}{c}\text { For period } \\
\text { of average } \\
\text { frostless } \\
\text { season. }\end{array}$ & $\begin{array}{c}\text { For June, } \\
\text { July, and } \\
\text { August } \\
1908 .\end{array}$ & Annual. & $\begin{array}{c}\text { For period } \\
\text { of average } \\
\text { frostless } \\
\text { season. }\end{array}$ \\
\hline California: & p. ct. & p. ct. & p. ct. & inch. & inch. \\
\hline$\ldots \ldots \ldots \ldots \ldots$ & 86.8 & 87.5 & 89 & .325 & .348 \\
\hline 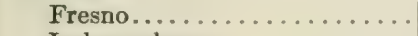 & 57.2 & 48.9 & 30 & .271 & .279 \\
\hline Independence............ & 29.7 & 22.6 & 30 & $\ldots \ldots$ & $\ldots \ldots$ \\
\hline Los Angeles.............. & 71.7 & 72.2 & 74 & .367 & .378 \\
\hline Red Bluff . . . . . . . . . . . & 48.1 & 49.9 & 36 & .281 & .296 \\
\hline 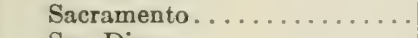 & 66.7 & 62.8 & 55 & .324 & .347 \\
\hline San Diego............... & 74.8 & & 73 & .399 & \\
\hline 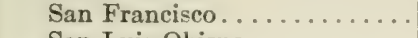 & 79.9 & 80.0 & 83 & .340 & .348 \\
\hline San Luis Obispo........... & 69.0 & 69.9 & 77 & .314 & .335 \\
\hline \multicolumn{6}{|l|}{ Colorado: } \\
\hline Denver................. & 48.7 & 46.7 & 54 & .185 & .285 \\
\hline 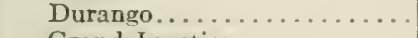 & $\cdots \cdots$ & $\cdots \cdots$ & 53 & … & $\ldots \ldots$ \\
\hline Grand Junction. . . . . . . . . & $\ldots$ & & 36 & & $\cdots \cdots$ \\
\hline Pueblo........................ & 48.1 & 45.8 & 47 & .177 & .274 \\
\hline \multirow{2}{*}{\multicolumn{6}{|c|}{$\begin{array}{l}\text { Connecticut: } \\
\quad \text { Hartford } \ldots \ldots \ldots \ldots \ldots \ldots\end{array}$}} \\
\hline & 75.9 & & & & 447 \\
\hline \multicolumn{6}{|l|}{ Florida: } \\
\hline Jacksonville. . . . . . . . . . . & 79.5 & 76.8 & 85 & .551 & .604 \\
\hline Jupiter. . . . . . . . . . . . . & 80.5 & 80.5 & 84 & .664 & .692 \\
\hline Key West................ & 77.1 & 77.1 & 77 & .707 & .707 \\
\hline Pensacola.............. & 78.3 & 77.6 & ... & .549 & .610 \\
\hline 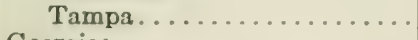 & 80.5 & 80.4 & 80 & .592 & .612 \\
\hline \multicolumn{6}{|l|}{ Georgia: } \\
\hline 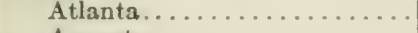 & 72.1 & 71.0 & 76 & .396 & .503 \\
\hline 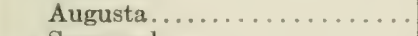 & 73.4 & 73.8 & 79 & .450 & .565 \\
\hline Savannah............... & 78.5 & 79.0 & 81 & .514 & .600 \\
\hline Thomasville ............. & $\cdots \cdots$ & $\cdots \cdots$ & 82 & $\cdots$ & $\cdots \cdots$ \\
\hline \multicolumn{6}{|l|}{ Idaho: } \\
\hline Boise. ................. & 56.7 & 45.6 & 42 & .209 & .262 \\
\hline Pocatello............... & . & $\cdots$ & 47 & $\cdots \cdots$ & $\cdots \cdots$ \\
\hline \multicolumn{6}{|l|}{ Illinois: } \\
\hline Cairo................... & 74.2 & 73.5 & 74 & .390 & .534 \\
\hline Chicago ................. . . & 74.8 & 71.1 & 71 & .286 & .423 \\
\hline 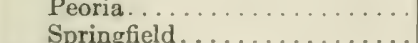 & & $\ldots \ldots$ & 72 & $\ldots \ldots$ & $\cdots \ldots$ \\
\hline $\begin{array}{l}\text { Springheld. . . . } \ldots \ldots \ldots \ldots \\
\text { Indiana: Indianapolis. . . . . . . }\end{array}$ & 71.4 & 69.0 & 65 & .311 & .464 \\
\hline \multirow{2}{*}{\multicolumn{6}{|c|}{$\begin{array}{l}\text { Iowa: } \\
\quad \text { Charles City } \ldots \ldots \ldots \ldots \ldots\end{array}$}} \\
\hline & & & & & \\
\hline 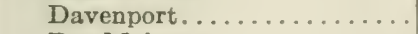 & 72.6 & 68.5 & 71 & .290 & .452 \\
\hline Des Moines............... & 71.1 & 68.5 & 71 & .285 & .450 \\
\hline 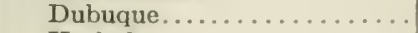 & 75.0 & 70.7 & 70 & .275 & .432 \\
\hline Keokuk.................. & 72.0 & 64.5 & 72 & .313 & .458 \\
\hline Sioux City . . . . . . . . . . & 69.0 & 65.3 & & & $\ldots \ldots$ \\
\hline \multicolumn{6}{|l|}{ Kansas: } \\
\hline Concordia............... & 68.6 & 66.3 & 74 & .298 & .463 \\
\hline Dodge City.............. & 66.9 & 62.9 & 69 & .284 & .431 \\
\hline 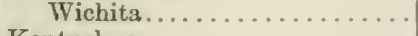 & 67.7 & 66.0 & 71 & .321 & .466 \\
\hline \multicolumn{6}{|l|}{ Kentucky: } \\
\hline Lexington...... & 70.1 & 69.0 & & .328 & .466 \\
\hline Louisville. ............... & 67.5 & 65.6 & 64 & .340 & .476 \\
\hline \multicolumn{6}{|l|}{ Louisiana: } \\
\hline New Orleans....... & 77.3 & 75.9 & so & .545 & .585 \\
\hline
\end{tabular}


TABLE 19.-Normal mean relative humidities, for the year and for the period of the average frostless season, mean rclative humiditics for the three summer months, 1908, and normal mean vapor-pressures for the year and for the period of the average frostless season.Continued.

\begin{tabular}{|c|c|c|c|c|c|}
\hline \multirow[b]{2}{*}{ Station. } & \multicolumn{3}{|c|}{ Mean relative humidity. } & \multicolumn{2}{|c|}{$\begin{array}{c}\text { Mean vapor-pressure } \\
\text { of water. }\end{array}$} \\
\hline & Annual. & $\begin{array}{c}\text { For period } \\
\text { of average } \\
\text { frostless } \\
\text { season. }\end{array}$ & $\begin{array}{c}\text { For June, } \\
\text { July, and } \\
\text { August } \\
1908 .\end{array}$ & Annual. & $\begin{array}{c}\text { For period } \\
\text { of average } \\
\text { frostless } \\
\text { season. }\end{array}$ \\
\hline $\begin{array}{l}\text { Louisiana-Continued: } \\
\text { Shreveport........ }\end{array}$ & $\begin{array}{l}\text { p.ct. } \\
72.9\end{array}$ & $\begin{array}{l}\text { p. ct. } \\
72.9\end{array}$ & $\begin{array}{l}\text { p. } c t . \\
76\end{array}$ & $\begin{array}{l}\text { inch. } \\
.470\end{array}$ & $\begin{array}{l}\text { inch. } \\
.567\end{array}$ \\
\hline \multicolumn{6}{|l|}{ Maine: } \\
\hline Eastport.. & 77.9 & 81.8 & 79 & .229 & .345 \\
\hline Portland.. & 75.1 & 78.2 & 72 & .259 & .410 \\
\hline \multicolumn{6}{|l|}{ Maryland: } \\
\hline Baltimore.. & 69.5 & 68.6 & 68 & .336 & .460 \\
\hline Washington, D. C.. & 72.3 & 73.5 & 74 & .343 & .488 \\
\hline \multicolumn{6}{|l|}{ Massachusetts: } \\
\hline Boston...... & 72.1 & 73.0 & 69 & .280 & .411 \\
\hline Nantucket. & 82.1 & 83.9 & 87 & .316 & .425 \\
\hline \multicolumn{6}{|l|}{ Michigan: } \\
\hline Alpena... & 80.2 & 77.4 & 76 & .240 & .403 \\
\hline Detroit. . & 74.4 & 70.2 & 74 & .282 & .437 \\
\hline Escanaba... & & & 71 & .232 & .401 \\
\hline Grand Haven... & 77.5 & 73.1 & 74 & .273 & .417 \\
\hline Grand Rapids. . & & & 64 & & \\
\hline Marquette..... & 78.6 & 74.8 & 67 & .222 & .371 \\
\hline Port Huron..... & 76.7 & 74.2 & 71 & .266 & .423 \\
\hline Sault Ste. Marie... & 79.9 & 78.8 & 77 & .221 & .374 \\
\hline \multicolumn{6}{|l|}{ Minnesota: } \\
\hline Duluth. & 75.1 & 72.3 & 81 & .212 & .355 \\
\hline Moorhead. & 74.1 & 71.5 & 75 & $.221^{\circ}$ & .405 \\
\hline St. Paul.... & 71.9 & 67.3 & 68 & .247 & .416 \\
\hline \multicolumn{6}{|l|}{ Mississippi: } \\
\hline Meridian.. & 78.3 & 77.6 & 79 & & \\
\hline Vicksburg........ & 73.5 & 73.9 & 78 & .474 & .569 \\
\hline \multicolumn{6}{|l|}{ Missouri: } \\
\hline Hannibal. & 70.7 & 69.0 & & & \\
\hline Kansas City . & 70.3 & 68.4 & 72 & .322 & .466 \\
\hline St. Louis...... & 69.5 & 67.3 & 69 & .342 & .485 \\
\hline Springfield. & 72.6 & 71.9 & 75 & .343 & .495 \\
\hline \multicolumn{6}{|l|}{ Montana: } \\
\hline Havre.. & 66.4 & 56.7 & 62 & .186 & .305 \\
\hline Helena.. & 56.1 & 48.0 & 56 & .165 & .249 \\
\hline Kalispell. & & & 61 & & \\
\hline Miles City. & 69.7 & 57.8 & 59 & .223 & .367 \\
\hline \multicolumn{6}{|l|}{ Nebraska: } \\
\hline Lincoln. & 70.0 & 66.9 & 73 & & \\
\hline North Platte. & 66.3 & 65.3 & 69 & .250 & .421 \\
\hline Omaha......... & 69.2 & 66.4 & 71 & .289 & .463 \\
\hline Valentine....... & 66.5 & 63.8 & 67 & .222 & .386 \\
\hline \multicolumn{6}{|l|}{ Nevada: } \\
\hline Carson City. . & 50.2 & 39.6 & ${ }^{1} 40$ & & \\
\hline Tonapah ..... & & & 28 & & \\
\hline Winnemucca. . & 46.5 & 30.4 & 45 & .148 & .183 \\
\hline New Jersey: & & & & & \\
\hline Atlantic City. & 81.4 & 82.3 & 78 & .356 & .491 \\
\hline \multicolumn{6}{|l|}{ Now Mexico: } \\
\hline $\begin{array}{l}\text { Roswell........... } \\
\text { Santa Fo........ }\end{array}$ & 45.4 & 41.5 & $\begin{array}{l}63 \\
50\end{array}$ & .169 & .233 \\
\hline
\end{tabular}

1 Reno instend of Caraon. 
TABLE 19.-Normal mean relative humidities, for the year and for the period of the average frostless season, mean relative humidities for the three summer months, 1908, and normal mean vapor-pressures for the year and for the period of the average froslless season.Continued.

\begin{tabular}{|c|c|c|c|c|c|}
\hline \multirow[b]{2}{*}{ Station. } & \multicolumn{3}{|c|}{ Mean relative humidity. } & \multicolumn{2}{|c|}{$\begin{array}{c}\text { Mean vapor-pressure } \\
\text { of water. }\end{array}$} \\
\hline & Annual. & $\begin{array}{c}\text { For period } \\
\text { of average } \\
\text { frostless } \\
\text { season. }\end{array}$ & $\begin{array}{l}\text { For June, } \\
\text { July, and } \\
\text { August } \\
1908 .\end{array}$ & Annual. & $\begin{array}{c}\text { For period } \\
\text { of average } \\
\text { frostless } \\
\text { season. }\end{array}$ \\
\hline New York: & p. ct. & p. ct. & p. ct. & inch. & inch. \\
\hline 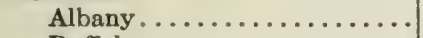 & 76.2 & 74.0 & 68 & .284 & .405 \\
\hline Buffalo................ & 73.4 & 71.6 & 72 & .271 & .414 \\
\hline New York................ & 73.2 & 73.3 & 69 & .314 & .433 \\
\hline 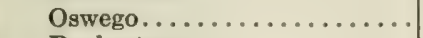 & 75.8 & 73.4 & 72 & .270 & .408 \\
\hline Rochester................ & 73.4 & 70.4 & 67 & .267 & .410 \\
\hline \multirow{2}{*}{\multicolumn{6}{|c|}{$\begin{array}{l}\text { North Carolina: } \\
\text { Asheville................ }\end{array}$}} \\
\hline & & & & & \\
\hline Charlotte................ & 71.1 & 71.3 & 78 & .382 & .500 \\
\hline 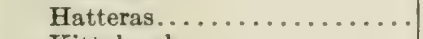 & 82.9 & 82.7 & 86 & .484 & .573 \\
\hline Kittyhawk. . . . . . . . . . & 80.7 & & & & \\
\hline 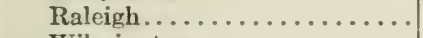 & 73.8 & 74.6 & 78 & .394 & .527 \\
\hline Wilmington............. & 80.1 & 80.9 & 82 & .471 & .588 \\
\hline \multicolumn{6}{|l|}{ North Dakota: } \\
\hline Bismarck... & 69.9 & 65.8 & 64 & .206 & .377 \\
\hline Devils Lake. . . . . . . . . . . . & & & 65 & & \\
\hline 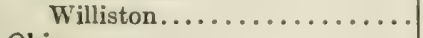 & 68.8 & 59.7 & 60 & .184 & .334 \\
\hline \multicolumn{6}{|l|}{ Ohio: } \\
\hline Cincinnati. . & 69.0 & 65.8 & 60 & .325 & .459 \\
\hline Cleveland............... & 72.9 & 70.9 & 68 & .290 & .414 \\
\hline Columbus............... & 72.6 & 68.9 & 66 & .305 & .442 \\
\hline Sandusky............... & 74.4 & 69.7 & . . & .294 & .423 \\
\hline Toledo.................. & 73.9 & 69.9 & 67 & .290 & .440 \\
\hline \multicolumn{6}{|l|}{ Oklahoma: } \\
\hline Oklahoma. . & 69.8 & 68.7 & 75 & .361 & .494 \\
\hline \multicolumn{6}{|l|}{ Oregon: } \\
\hline Baker City................ & 60.7 & 49.3 & 54 & .173 & .236 \\
\hline 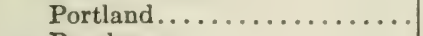 & 75.5 & 71.4 & 67 & .293 & .329 \\
\hline 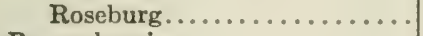 & 74.6 & .... & 63 & .282 & .323 \\
\hline \multicolumn{6}{|l|}{ Pennsylvania: } \\
\hline Erie...$\ldots \ldots \ldots \ldots \ldots$ & 75.5 & 72.6 & 66 & .286 & .416 \\
\hline Harrisburg............... & 73.5 & 72.0 & 69 & … & $\cdots \cdots$ \\
\hline Philadelphia.............. & 70.3 & 69.8 & 67 & .322 & .450 \\
\hline Pittsburgh............. & 72.2 & 69.3 & 65 & .312 & .455 \\
\hline 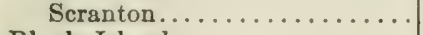 & $\ldots \ldots$ & $\ldots \ldots \ldots$ & 66 & $\ldots \ldots$ & $\ldots \ldots$ \\
\hline \multicolumn{6}{|l|}{ Rhode Island: } \\
\hline Block Island. . . . . . . . . . . & 81.2 & 84.0 & 85 & .320 & .428 \\
\hline Providence.............. & $\ldots \ldots$ & $\ldots \ldots \ldots$ & 76 & $\cdots \cdots$ & $\ldots \ldots \ldots$ \\
\hline \multicolumn{6}{|l|}{ South Carolina: } \\
\hline 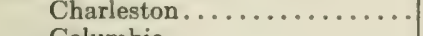 & 78.0 & 78.2 & 80 & .511 & .586 \\
\hline \multicolumn{2}{|l|}{ South Dakota: } & $\ldots \ldots \ldots$ & 76 & $\cdots \cdots$ & $\cdots \cdots \cdots$ \\
\hline Huron............. & 70.2 & 65.2 & 75 & .229 & .415 \\
\hline Pierre...... & 64.7 & 57.6 & 60 & .219 & .370 \\
\hline Rapid City..... & 59.8 & 53.2 & 61 & .197 & .322 \\
\hline 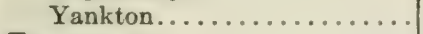 & 70.4 & 67.8 & ... & .262 & .454 \\
\hline \multicolumn{6}{|l|}{ Tennessee: } \\
\hline Chattanooga. & 72.7 & 72.8 & 72 & .391 & .520 \\
\hline Knoxville.... & 73.7 & 73.7 & 75 & .374 & .505 \\
\hline 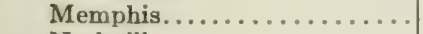 & 71.8 & 71.3 & 76 & .417 & .545 \\
\hline 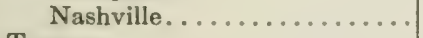 & 70.7 & 69.1 & 74 & .379 & .513 \\
\hline \multicolumn{6}{|l|}{ Texas: } \\
\hline Abilene. . & 63.9 & 62.6 & 65 & .369 & .456 \\
\hline
\end{tabular}


TABLE 19.-Normal mean relative humidities, for the year and for the period of the average frostless season, mean rclative humidities for the three summer months, 1908, and normal mean vapor-pressures for the year and for the period of the average frostless season.Continued.

\begin{tabular}{|c|c|c|c|c|c|}
\hline \multirow[b]{2}{*}{ Station. } & \multicolumn{3}{|c|}{ Mean relative humidity. } & \multicolumn{2}{|c|}{$\begin{array}{c}\text { Mean vapor-pressure } \\
\text { of water. }\end{array}$} \\
\hline & Annual. & $\left|\begin{array}{c}\text { For period } \\
\text { of average } \\
\text { frostless } \\
\text { season. }\end{array}\right|$ & $\begin{array}{c}\text { For June, } \\
\text { July, and } \\
\text { August } \\
\text { 1908. }\end{array}$ & Annual. & $\begin{array}{l}\text { For period } \\
\text { of average } \\
\text { frostless } \\
\text { season. }\end{array}$ \\
\hline Texas-Continued: & p. ct. & p. ct. & p. ct. & inch. & inch. \\
\hline 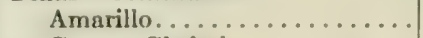 & 59.3 & 59.4 & 68 & .262 & .373 \\
\hline Corpus Christi............ & 82.1 & 81.9 & 80 & .618 & .675 \\
\hline El Paso................. & 38.8 & 37.0 & 43 & .243 & .300 \\
\hline Galveston................. & 85.2 & 80.2 & 74 & .595 & .622 \\
\hline Palestine................. & 73.8 & 73.7 & 78 & .467 & .569 \\
\hline 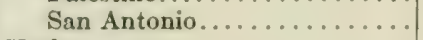 & 66.6 & 66.7 & 73 & .472 & .540 \\
\hline Utah: & & & & & \\
\hline Salt Lake City............. & 52.8 & 40.9 & 45 & .193 & .250 \\
\hline Vermont: & & & & & \\
\hline Northfield. & 77.6 & 78.1 & 86 & .239 & .423 \\
\hline Virginia: & & & & & \\
\hline Lynchburg. . . . . . . . . . . . . . & 71.1 & 72.8 & 77 & .356 & .498 \\
\hline Norfolk................. & 78.6 & 79.1 & 79 & .410 & .525 \\
\hline Wy theville............... & 列 & $\ldots \ldots \ldots$ & 90 & $\ldots \ldots$ & $\ldots \ldots$ \\
\hline Washington: & & & & & \\
\hline 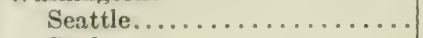 & 76.2 & 73.6 & 75 & .286 & .318 \\
\hline Spokane............... & 64.0 & 52.9 & 46 & .209 & .253 \\
\hline 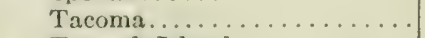 & $\ldots \ldots \ldots$ & $\ldots \ldots \ldots$ & 73 & $\ldots$. & \\
\hline Tatoosh Island. . . . . . . . . . . & $\ldots \ldots \ldots$ & $\ldots \ldots \ldots$ & 89 & .304 & .328 \\
\hline Walla Walla............ & 64.8 & 54.6 & 46 & .250 & .297 \\
\hline West Virginia: & & & & & \\
\hline Elkins............... & & & 83 & & \\
\hline Parkersburg. . . . . . . . . . . & 75.7 & 73.2 & 73 & 331 & .482 \\
\hline Wisconsin: & & & & & \\
\hline Green Bay............... & 74.0 & 70.6 & 69 & .245 & .407 \\
\hline La Crosse................. & 72.2 & 69.5 & & .266 & .438 \\
\hline Madison................ & $\cdots \cdots$ & $\ldots \ldots \ldots$ & 71 & & $\ldots \ldots \ldots$ \\
\hline Milwaukee.............. & 74.9 & 72.6 & 71 & .265 & .420 \\
\hline Wyoming: & & & & & \\
\hline 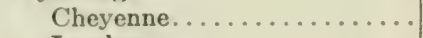 & 53.7 & 52.9 & 61 & .163 & .274 \\
\hline 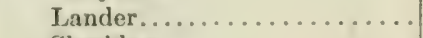 & 57.8 & 47.9 & 58 & .153 & .253 \\
\hline Sheridan................ & $\ldots \ldots \ldots$ & $\ldots \ldots \ldots$ & 62 & $\ldots \ldots \ldots$ & $\ldots \ldots \ldots$ \\
\hline Yellowstone............. & $\ldots \ldots \ldots$ & $\ldots \ldots$ & 61 & $\ldots \ldots$ & $\ldots \ldots \ldots$ \\
\hline
\end{tabular}

The chart of plate 63 is markedly different from any of the moisture charts heretofore considered, excepting that it somewhat resembles some of those for precipitation. It is not very similar to any of the temperature charts with which we have been concerned, but it appears to partake of the general characteristics of both temperature and moisture charts. In the East the zonation here shown is markedly similar to that for temperature, the isobars having generally a westeast direction. Each of these lines, however, is seen to bend rather sharply southward in the middle of the country, so that the region west of about the ninety-eighth meridian of west longitude is generally characterized by isobars that have a north-south trend. The western 
mountainous region, west to about the middle of Washington, Oregon, and California, is shown as an area of low vapor-pressures (below 0.300 inch), this area apparently extending a little into Canada at the north, but not reaching the Mexican boundary at the south. The whole Pacific coast region is shown as having very low values (mainly between 0.300 and 0.350 inch), about like those of the region of the one hundred and third meridian, in the plains area. Southern Florida has the highest values, that for Key West being 0.707 inch.

(3) Normal Mean Aqueous Vapor-pressure for the Year. (Table 19, Plate 64.)

The means here used are taken directly from Bigelow's tables. They are reproduced in the fifth column of table 19 and are shown graphically by the chart of plate 64 .

The zonation shown by this chart is so similar to that of plate 63 that no special discussion is here needed. Neither is it necessary to derive any generalization from these two vapor-pressure charts, since the discussion given for plate 63 brings out all the points that might be mentioned in a generalized statement.

\title{
E. RELATIVE AIR HUMIDITY.
}

(1) Preliminary Considerations.

Data of relative air humidity, obtained by means of stationary or whirled wet and dry bulb thermometers, have been accumulated for many years at the various stations of the United States Signal Service and of the United States Weather Bureau. These were brought together by Stockman, ${ }^{1}$ as monthly and annual means for the period 1888-1901, for 130 stations. Stockman's means are stated to have been "derived from observations made at 8 a. m. and $8 \mathrm{p}$. m., seventyfifth meridian time." They form the original source of our studies of humidity in the United States.

The theoretical inadequacy of data of relative air humidity in characterizing climate is tacitly suggested by a brief but well-chosen statement with which Stockman prefaces the table above referred to. $\mathrm{He}$ says:

\begin{abstract}
"The relative humidity furnishes no direct indication of the absolute amount of moisture in the air. For purposes of comparison consideration should be given to the fact that as the temperature is lowered the eapacity of the air to retain moisture is decreased. Of two stations having the same average percentage of relative humidity, but different mean temperatures, that station which has the higher mean temperature will have the greater amount of moisture."
\end{abstract}

We have already emphasized the fact that vapor-tension deficit appears to be the climatic feature which should be recorded in connection with air humidity, this being the difference (in pressure units, as bars or as millimeters of a mercury column) between the maximum

\footnotetext{
1 Stockman, W. B., Temperature and relative humidity data, U. S. Dept. Agric., Weather Bur. Bull. O: 25-29. 1905.
} 
vapor-pressure of water for the given air-temperature and the actual vapor-pressure of water in the air. As has been pointed out, this deficit should be an index of the relation of air humidity to evaporation; it should measure that portion of the atmospheric evaporating power for any given time, which is not related to wind-movement. The arguments of air-temperature and air moisture-content are thus combined in a single function, which becomes the more significant when it is pointed out that different parts of the country do not generally differ very marliedly in relation to wind. The terms from which the vapor-pressure deficit might have been obtained were at hand when the relative humidity observations were recorded, but it is not possible to deduce them from the recorded percentages, especially since Stockman's published data have been reduced to normal monthly means. It is readily seen, however, that the higher the temperature (i.e., the farther south is the location of a given station), the more a given percentage of relative humidity is to be discounted, as it were. Thus, if a northern and a southern station (as Duluth, Minnesota, and Little Rock, Arkansas, for example) agree in having the same normal mean relative humidity for the period of the average frostless season-say 72 per cent-we are perfectly safe in concluding that the normal mean evaporating power of the air at the southern station is greater than at the northern, supposing the air-movement to be alike in the two cases. But it is not possible in such a case to weight the humidity records in a quantitative way.

Before proceeding to our results in this connection it is necessary to mention one other very important point, which requires $\mathrm{s}$ attention from climatologists who hope to relate climate to plant activities in a detailed way. As has been stated, the humidity data of Stockman's table were derived from observations "made at 8 a. m. and $8 \mathrm{p} . \mathrm{m}$., seventy-fifth meridian time." This is the regular practice of the United States Weather Bureau in dealing with humidity, and it will be seen that the longitude of a station determines the times in its day when observations are to be obtained. Thus, the observations for any day at North Head, Washington, come to us as though they were made at about $4^{\mathrm{h}} 40^{\mathrm{m}}$ a. m. and p. m., while those for a day at Eastport, Maine, are to be similarly considered as made at about $8^{\mathrm{h}} 30^{\mathrm{m}}$ a. $\mathrm{m}$. and p. $\mathrm{m}$. Now, humidity alters very rapidly, in most cases, during an hour or two about sunrise and about sunset, and the morning observation on the Pacific coast is usually made well before sumrise, while that in the northeast is made well after sunrise. A similar eonsideration applies to the evening observations. It is clear that neither the morning observations nor the evening ones, throughout the country, represent the true humidity conditions for the given date, and it remains to be determined what the mean of these two percentages may denote. The question is, simply: Can the humidity con- 
ditions for any day be as well derived from observations made, say, at 5 a. m. and 5 p. m. as from those made at, say, 8 a. m. and 8 p. m.? When vapor-pressure deficit attracts the attention it deserves the same problem will arise in connection with it, and then the local times of observation will have to be seriously considered before the data may be regarded as quite suitable for studies involving plant transpiration and other ecological or agricultural features. ${ }^{1}$

On the whole, it needs to be borne in mind simply that the moisture conditions of the air deserve as much attention as do its temperature conditions, if agriculture and ecology are to employ climatological results. Our aim in the above paragraphs has been, not to point out what might or might not have been done in the past, which can not now be changed, but rather to emphasize what seem to us to be the needs of ecological and agricultural climatology for the future.

We shall deal here with three kinds of indices of relative humidity: (1) the normals for the period of the average frostless season, (2) for the year, and (3) the means for the three summer months of 1908.

(2) Percentages Representing Normal Mean Relative Air Humidity for the Period of the Average Frostless Season. (Table 19, Plate 65, and Fig. 17.)

The data here employed were derived from the monthly values given by Stockman (1905), by the same general method as we have heretofore resorted to in obtaining indices for the period of the average frostless season from monthly normals. Our values are given in the third column of table 19, and they are shown graphically on the chart of plate 65 .

The chart of plate 65 shows that the relative humidity values are high (above 75 per cent) for the Pacific coast region and also for the Northeast, East, and Southeast. The lowest values (below 40 per cent) are found in the arid southwest. As a whole, this chart resembles the charts of precipitation-evaporation ratios (plates 57 to 62), and certain lines are here shown as broader than the others, to bring out the division of the country into climatic provinces, as was done on those charts. The arid region may be characterized as having relativehumidity percentage values below 50 , the semiarid region shows values between 50 and 65 , the semihumid regions show values between 65 and 75 , and values of over 75 characterize the humid regions. In this case the northwestern humid area is extended southward, along the Pacific coast, to middle California, and the adjoining semihumid area extends to the Mexican boundary. The semiarid region extends eastward to about the hundredth meridian of longitude, somewhat farther at the south and not as far at the north, thus agreeing, in general, with

1 The hours of temperature observation in the United States have been very thoroughly studied, in relation to the daily means derived therefrom, and several important considerations bearing on the readings of the dry and wet bulb thermometers have been taken up. In these connections see Bigelow, 1909. Also see O. L. Fassig, Report on the climate and weather of Baltimore, Maryland Weather Service 2: 29-312, 1907; especially pp. 152-158. 


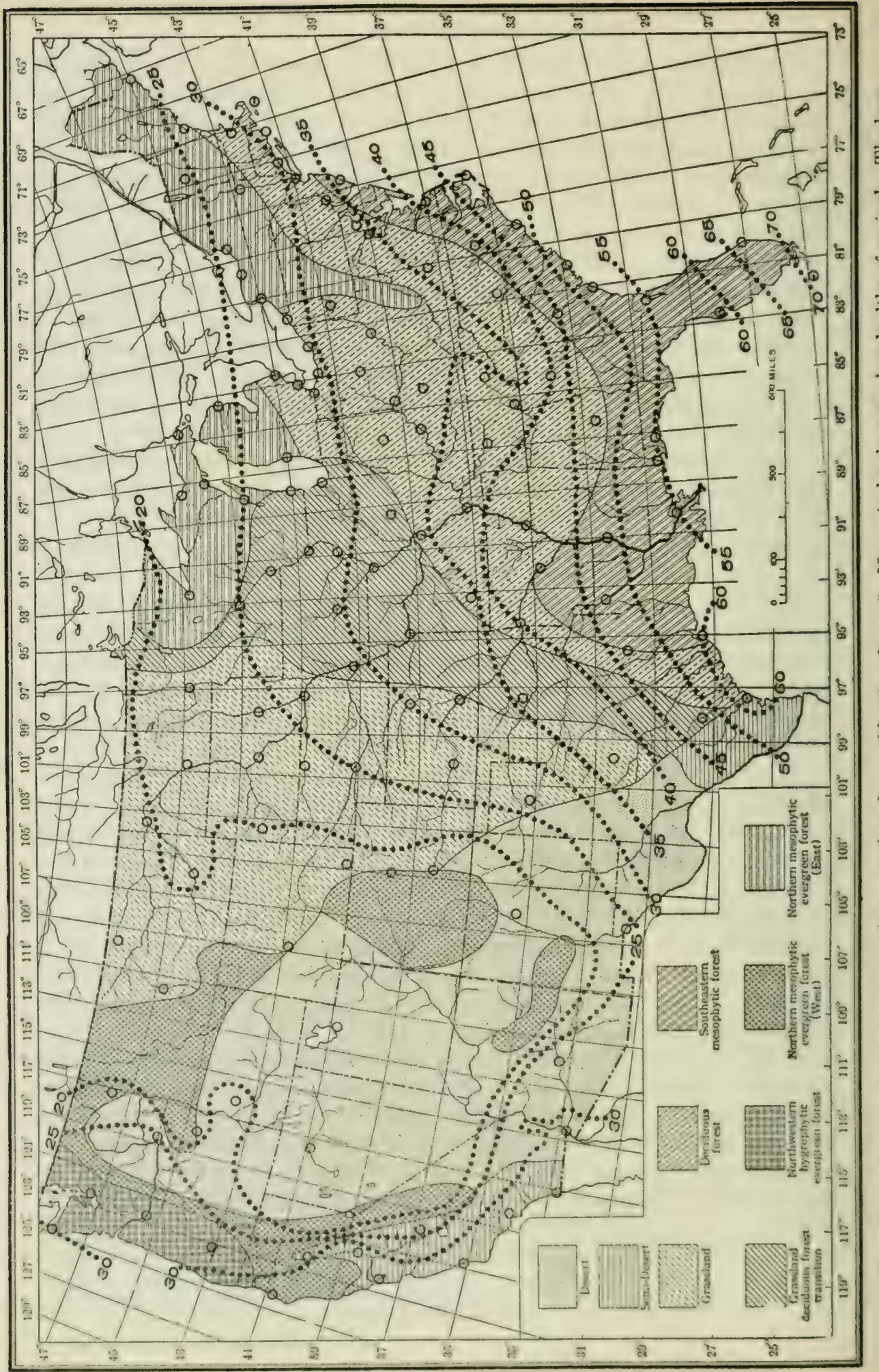




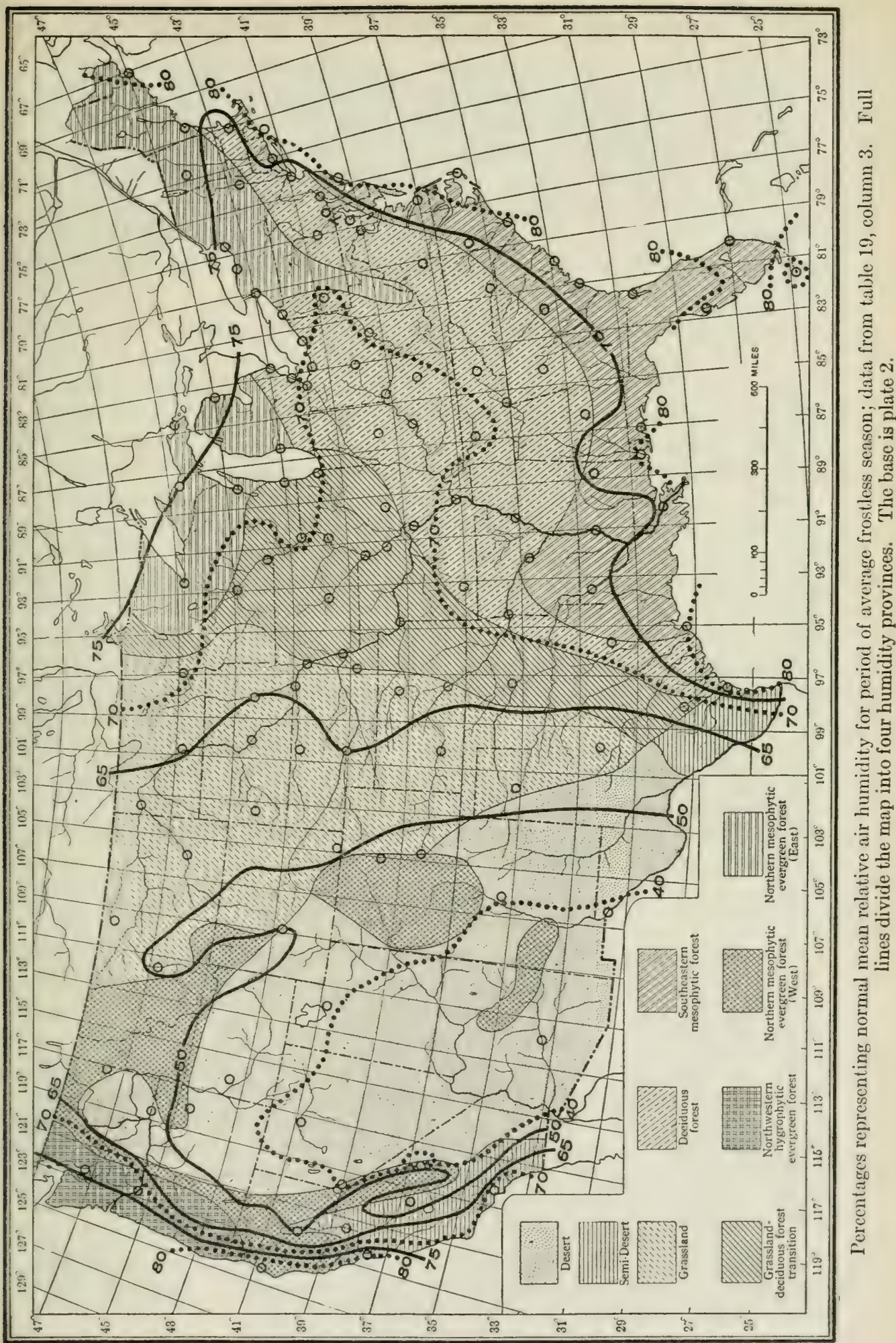


plates 57 to 62 . This relative-humidity chart also agrees, in general, with the moisture-ratio charts just mentioned, by showing an eastward projection of the semihumid area from Nebraska to New England. The eastern humid area occupies the Gulf coast from the Rio Grande eastward, practically all of the Atlantic coast (excepting northern New Jersey and southeastern New York), northern New England, northern New York, and northern Michigan. In its northeastern portion this chart resembles that of plate 61 more than any of the other moisture-ratio charts.

(3) Percentages Representing Normal Mean Relative Air Humidity for the Year. (Table 19, Plate 66.)

Our data for this index are taken directly from Stockman's table. They are reproduced in the second column of table 19, and are represented by the chart of plate 66 .

This chart also shows a pronounced general agreement with our charts for the Transeau moisture ratios. The general zonation of the country is again shown by broad lines, but the lines for the value 65 on plate 65 are here represented by those for the value 70 . The semiarid region is here thus characterized by values between 50 and 70 , and the values for the semihumid region lie between 70 and 75 . The line for the value 72 has been added in the central part of the country, to emphasize the eastern lobe of the semihumid area (shown also on plates 57 to 61 and on plate 65). An area of values a little below 70 is shown about St. Louis, Louisville, and Indianapolis, thus differing from plate 65 , but agreeing, in general, with plates 57 to 61 . The eastern humid region is here shown as much like that of plate 65 , but the line for 75 here lies considerably farther south in the regions of the Great Lakes and of New England. In this respect plate 66 resembles plates 57 to 60 more than plates 61 and 65 .

(4) Pencentages Representing Mean Relative Air Humidity for June, July, and August 1908. (Table 19, Plate 67.)

The data for these indices were obtained from the Monthly Weather Review for 1908 , the means for the three separate months being simply averaged in each case. The results are given in the fourth column of table 19 and are charted in the usual way in plate 67.

This chart has the lines for the index values 50,70 , and 75 represented as full lines, to exhibit the general zonation of the country; just as in the case of plate 66 . The northwestern humid area (values above 75) here extends southward, along the Pacific coast, nearly to Ios Angeles, California, and the corresponding semihumid area reaches to the Mexican boundary. The arid area is depicted in much the same way as on the other two charts of relative humidity, but is not quite as extensive here as in the other eases, this being due, no doubt, to the effect of the characteristic summer rains in western Texas and Vew 
Mexico. Instead of completely surrounding the Rocky mountains, as in the other two charts, this zone extends eastward only to these mountains, but there is here a small local area of values of 50 or below, indicated by the data of Santa Fe, New Mexico, and Pueblo, Colorado. The eastern limit of the semiarid region (70) is here placed about the 99th meridian and this line has nearly a north-south direction. The region here called semihumid, in the east, is somewhat restricted in this case, but shows a very large eastern lobe, reaching from Nebraska to Maine. The localized area of semiarid conditions within this lobe is here very large, extending from Minnesota and Illinois to Maine. It will be remembered that the area in question is not shown at all on the humidity chart for the period of the average frostless season (plate 65) and is represented as much smaller on that for the year (plate 66). The eastern humid region is here exceptionally wide in the southeast and is narrowed at the north, somewhat as on the chart for the moisture ratio for the year (plate 60 ).

This chart of relative-humidity values for the three summer months of 1908 was prepared for comparison with the chart of evaporation for a similar period of the same year (plate 56). The stations for the 1908 series were not numerous enough to give a satisfactory chart, but these two charts agree, in a general way, in showing the great' eastern extension of the region of semihumid and semiarid summer conditions, from Oklahoma to northern Michigan and New England. For details, the two charts can not be compared.

(5) Generalizations from the Three Charts of Relative Humidity Values.

(Plates 65 to 67, and Fig. 17.)

The main points brought out by our study of relative humidity values may be briefly summarized as follows, reference being made to figure 17, which is derived from plate 65 . The country may be divided, on the basis of relative humidity - as on that of other moisture features-into four humidity provinces, which may be termed arid, semiarid, semihumid, and humid.

The humid relative humidity province occupies: (1) western Washington and Oregon and a variable portion of the California coast region (depending on the form of index employed); (2) the whole of the Gulf coast region and that of the Atlantic coast as far north as Long Island or Massachusetts; (3) northern New England and New York and a variable portion of Michigan, Wisconsin, and Minnesota. It appears probable that portions (1) and (3), as just defined, are continuous through Canada, so as to form a single northern humid region. The southeastern portion (2) appears to be quite cut off from the northern one, but only by a narrow neck. The semihumid province lies just interior to the humid one, being very narrow in the Northwest and West. The eastern and western portions of this zone, as shown on 


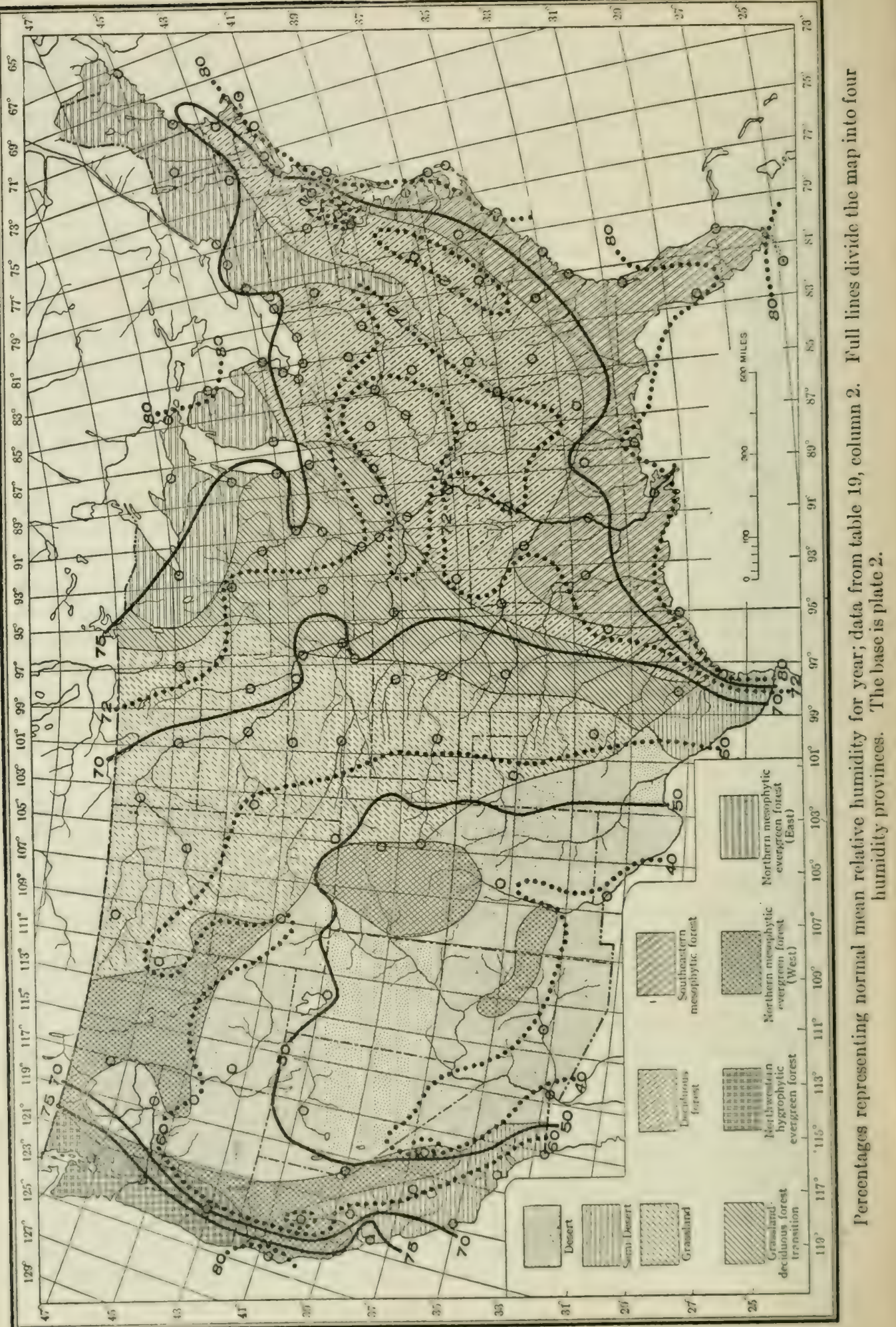




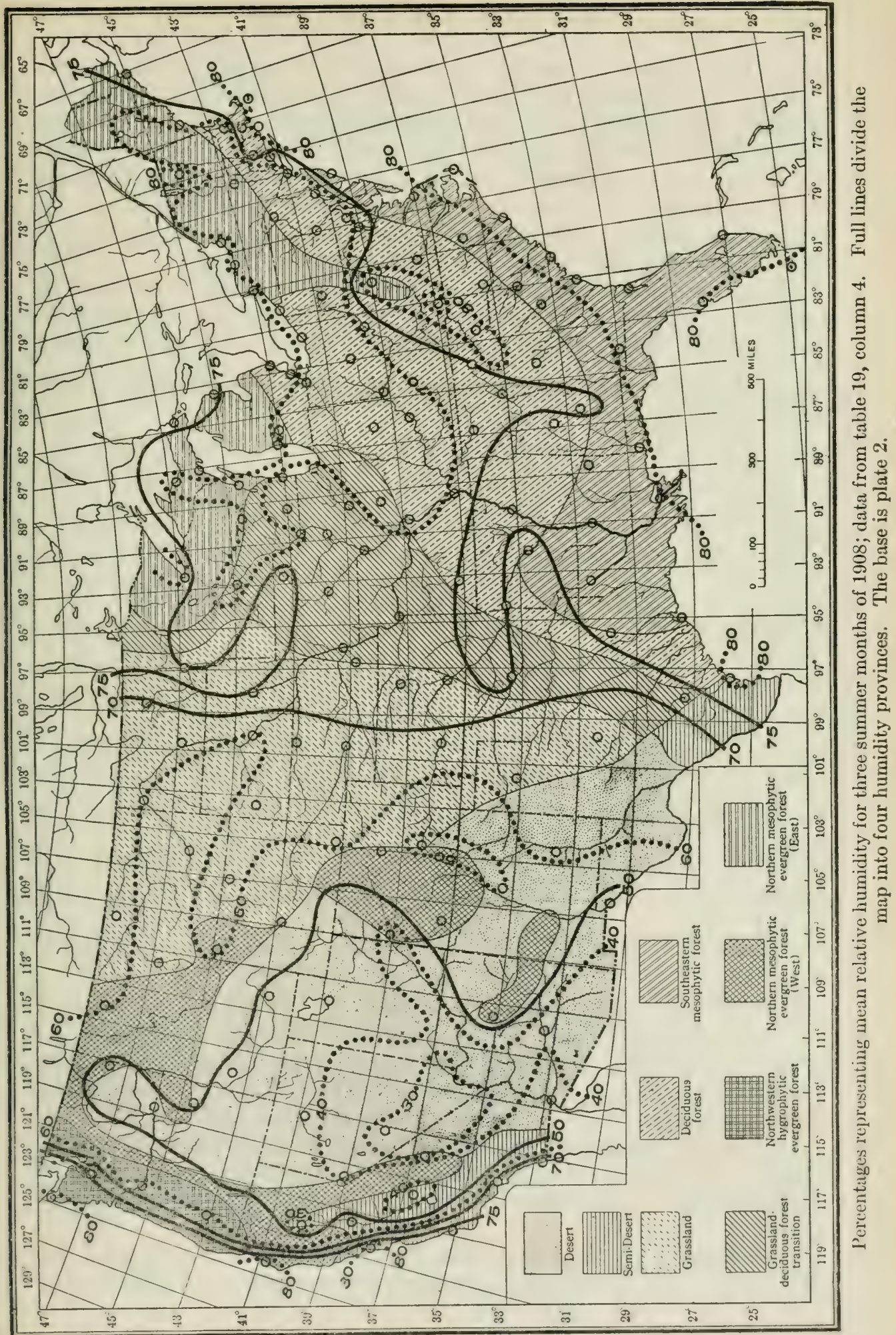


these three charts, are probably continuous in Canada, to form a single zone. In the eastern half of the country this zone occupies all of the interior, as far west as about the hundredth meridian of west longitude. The semiarid province embraces the Great Plains and a belt lying east of the western semihumid zone. Finally, the arid province occupies the region generally known as arid, extending approximately from Arizona to eastern Washington. This zonation is in general agreement with that shown by the precipitation-evaporation ratios.

From these considerations it appears quite clear that the data of relative humidity, as here employed, furnish an exceedingly valuable and rational climatic zonation of the United States. This may be taken

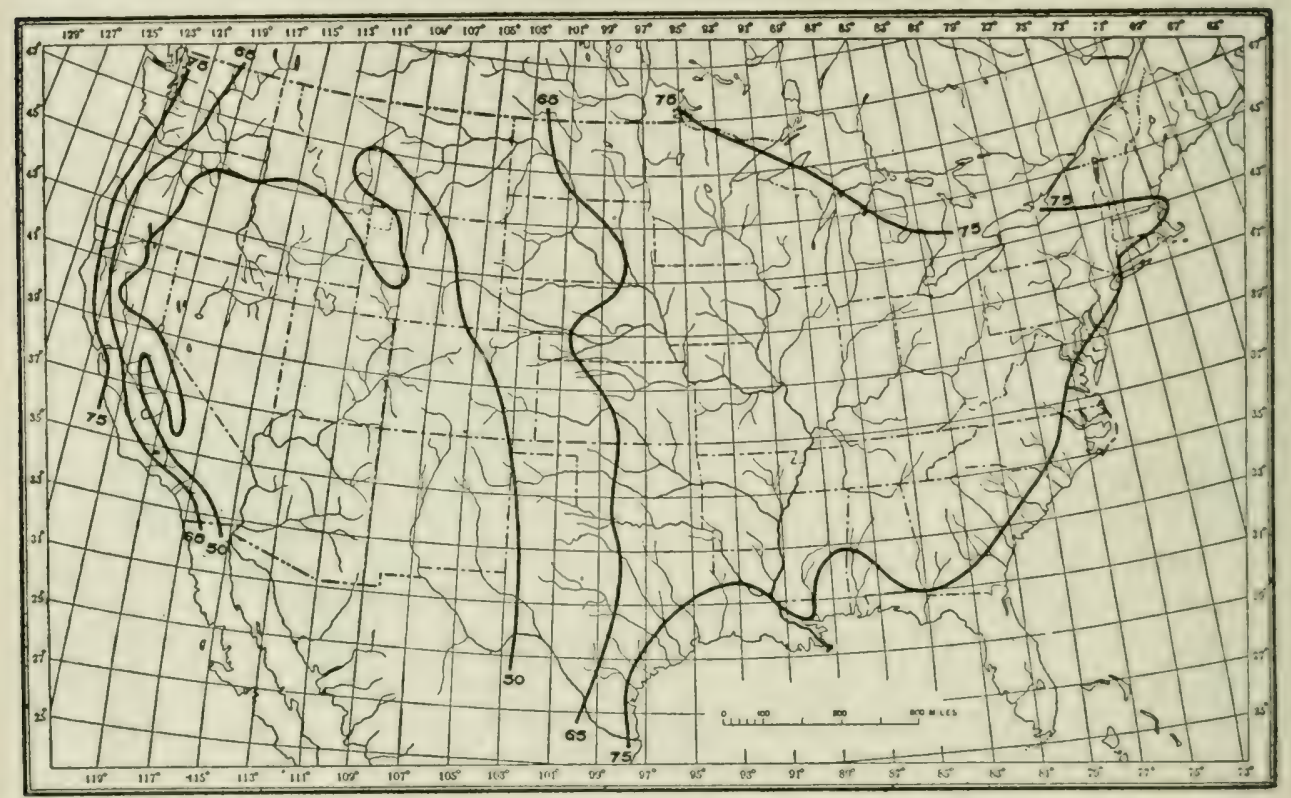

FIG. 17.-Moisture zonation, according to indices of relative humidity for period of average frostless season. Humidity provinces: Humid, more than 75; semihumid, 65 to 75 ; semiarid, 50 to 65 ; arid, less than 50 . Numerical values represent percentages. (See also Plate 65.)

as satisfactory evidence that the theoretical objections to the concept of relative humidity and to the usual manner of its employment are not serious enough to prevent this method giving a clear zonation that generally agrees with that obtained from the precipitation-evaporation ratios as we have used the latter. It seems hardly probable, howerer, that detailed studies of the annual march of climatic conditions as related to plants may be as well served by these data as by those of vapor-tension deficit, evaporation, and the precipitation-evaporation ratio.

Considering the fact that data from 14 years of observations are here employed, and that many more years are now available, so that 
much more satisfactory charts might probably now be made, it appears that these charts of relative humidity must be regarded as perhaps the most valuable of all air-moisture charts with which we have been able to deal in a practical way.

\section{F. WIND. (TABLE 20, PLATE 68.)}

Wind influence upon plant growth occurs in several ways, the most generally important of which is probably effective through increased transpiration. As has been emphasized, air-movement is very influential in determining the evaporating power of the air, which, in turn, is the main external condition governing the transpiration-rate as well as the rate of water-loss directly from the soil-surface. This influence depends upon the velocity of air-movement. Strong winds tend to break plants as well as to dry them, but this feature of wind influence appears to be of importance to vegetation only in relatively restricted areas. If wind were to be studied in this regard, it is very high windpressure and its duration that would require attention. The direction of the wind, so important in weather predictions, is of no importance to plants in general. If the ecology of individual plants or small groups is to be studied, then wind direction may sometimes become important.

Wind as a climatic condition influencing vegetation may be considered: (1) in terms of its average velocity-perhaps the most useful wind-dimension as far as the evaporating power of the air is concerned; (2) in terms of its maximum velocity and the duration of very high rates of air-movement; and (3) in terms of the maximum pressure developed and the duration of very high pressures. The United States Weather Bureau has accumulated anemométric data for many stations in the United States, and we have employed some of these data to prepare a chart of average wind-velocities for the period of the average frostless season. The data employed are as yet unpublished, but have been very kindly furnished us by Professor P. C. Day, of the United States Weather Bureau. They consist in a table of average wind-velocities (in miles per hour) for each month of the year and for 151 stations, based generally upon the 20-year period 18911910. For each of these stations the average wind-velocity has been calculated for the period of the average frostless season, as in the other cases where monthly averages were used in deriving means for the frostless season. The results of our computations, together with the annual average velocity in each case (obtained from the records of the United States Weather Bureau) and the height above the ground of the anemometer are given in table 20 . 
TABLE 20.-Average wind velocities for the year and for the period of the average frostless season, usually from records for the period 1891-1910.

\begin{tabular}{|c|c|c|c|c|c|c|c|}
\hline Station. & 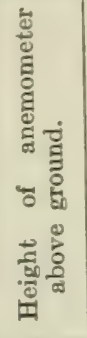 & 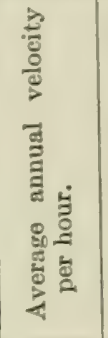 & 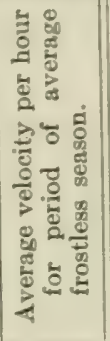 & Stzion. & 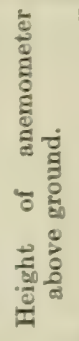 & 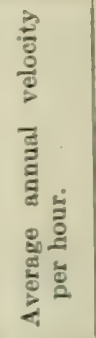 & 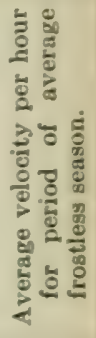 \\
\hline Alabama: & feet. & miles. & miles. & Iowa: & feet. & miles. & miles. \\
\hline Birmingham.... & 48 & 7.4 & 6.7 & Charles City.. & 8 & 7.4 & 6.2 \\
\hline Mobile.......... & 106 & 7.4 & 7.2 & Davenport.... & 79 & 8.2 & 7.3 \\
\hline Montgomery....... & 112 & 6.1 & 5.8 & Des Moines.. & 98 & 8.3 & 7.7 \\
\hline Arizona: & & & & Dubuque... & 115 & 6.6 & 6.0 \\
\hline Flagstaff. & 57 & 7.4 & 6.7 & Keokuk. & 79 & 7.5 & 6.8 \\
\hline Phoenix. . & 56 & 4.3 & 4.5 & Sioux City. & 164 & 12.2 & 11.4 \\
\hline Yuma... & 58 & 6.2 & & Kansas: & & & \\
\hline Arkansas: & & & & Concordia.. & 50 & 7.6 & $\begin{array}{r}7.4 \\
11.5\end{array}$ \\
\hline Fort Smith.... & 94 & 7.6 & 7.0 & Dodge...... & 51 & $\begin{array}{r}11.1 \\
9.3\end{array}$ & $\begin{array}{r}11.5 \\
8.8\end{array}$ \\
\hline $\begin{array}{l}\text { Little Rock... } \\
\text { California: }\end{array}$ & 147 & 7.3 & 6.7 & $\begin{array}{l}\text { Wichita...... } \\
\text { Kentucky: }\end{array}$ & 121 & 9.3 & \\
\hline $\begin{array}{l}\text { California: } \\
\text { Eureka. }\end{array}$ & 88 & 6.6 & 6.6 & Lexington... & 102 & 10.8 & 9.0 \\
\hline Fresno..... & 70 & 5.6 & 6.1 & Louisville..... & 132 & 7.9 & 6.9 \\
\hline Los Angeles......... & 191 & 4.5 & 4.5 & Louisiana: & & & \\
\hline Point Reyes Light. . & 18 & 19.7 & & New Orleans........ & 121 & 8.5 & 8.3 \\
\hline Red Bluff........... & 56 & 5.8 & 5.5 & Shreveport........ & 74 & 6.8 & 6.4 \\
\hline Sacramento.. & 117 & 8.2 & 8.4 & Maine: & & & \\
\hline San Diego.......... & 102 & 5.5 & & Eastport. & 85 & 11.0 & 8.7 \\
\hline San Francisco........ & 204 & 9.7 & 10.1 & Portland. . & 117 & 8.5 & 7.8 \\
\hline San Luis Obispo.... & 54 & 5.2 & 5.2 & Maryland: & & & \\
\hline S. E. Farrallon... & 48 & 15.9 & & Baltimore... & 113 & 6.8 & 6.5 \\
\hline Colorado: & & & & Washington, D. C. . & 85 & 6.7 & 6.0 \\
\hline Denver.... & 172 & 7.8 & 7.4 & Massachusetts: & & & \\
\hline Durango.. . & 56 & 5.7 & 5.8 & Boston...... & 183 & 10.8 & 9.8 \\
\hline Grand Junction. & 51 & 5.0 & 5.6 & Nantucket...... & 90 & 13.8 & 12.5 \\
\hline Pueblo............ & 86 & 7.3 & 7.2 & Michigan: & & & \\
\hline Connecticut: & & & & Alpena... & 92 & 10.1 & 8.8 \\
\hline New Haven. & 155 & 9.1 & 8.2 & Escanaba. & 60 & 9.1 & 8.4 \\
\hline Florida: & & & & Detroit. & 258 & 11.3 & 9.9 \\
\hline Jacksonville. & 129 & 8.2 & 8.2 & Grand Haven. . & 92 & 11.0 & 9.3 \\
\hline Key West... & 53 & 9.7 & 9.7 & Grand Rapids. . & 162 & 10.8 & 9.5 \\
\hline Pensacola.. & 183 & 9.9 & 9.7 & Houghton...... & & 7.2 & 6.8 \\
\hline Tampa.... & 96 & 6.7 & 6.7 & Marquette... & 116 & 10.4 & 9.3 \\
\hline Georgia: & & & & Port Huron. & 120 & 11.3 & 9.7 \\
\hline Atlanta. . & 216 & 10.4 & 9.3 & Sault Ste. Marie... & 61 & 8.7 & 7.6 \\
\hline Augusta.... & 97 & 5.9 & 5.6 & Minnesota: & & & \\
\hline Macon. & 87 & 5.8 & 5.5 & Duluth. & 47 & 13.5 & 12.4 \\
\hline Savannah... & 194 & 8.2 & 8.0 & Moorhead. & 57 & 10.6 & 9.9 \\
\hline Thomasville....... & 57 & 5.3 & 5.1 & St. Paul... & 212 & 8.6 & 7.8 \\
\hline Idaho: & & & & Mississippi: & & & \\
\hline Boise.... & 86 & 5.0 & 4.9 & Vicksburg & 74 & 6.8 & 6.3 \\
\hline Lewiston. . & 51 & 5.6 & 5.7 & Missouri: & & & \\
\hline Pocatello. & 54 & 9.0 & 8.5 & Columbia. . & 84 & 8.8 & 6.8 \\
\hline Illinois: & & & & Hannibal.. & 109 & 9.1 & 8.2 \\
\hline Cairo. & 93 & 8.5 & 7.4 & Kansas City. & 181 & 9.1 & 8.5 \\
\hline Chicago..... & 310 & 16.3 & 14.9 & St. Louig. . & 217 & 10.4 & 9.4 \\
\hline Springfield... & 91 & 9.2 & 8.0 & Springfield. & 104 & 10.2 & 9.0 \\
\hline Indiana: & & & & Montana: & & & \\
\hline Evansville.. & 82 & 7.3 & 6.5 & Havre... & 44 & 9.5 & 8.8 \\
\hline Indianapolis. . & 164 & 9.0 & 7.9 & Helena.......... & 56 & 7.3 & 7.4 \\
\hline
\end{tabular}


TABLE 20.-Average wind velocities for the year and for the period of the average frostless season, usually from records for the period 1891-1910.-Continued.

\begin{tabular}{|c|c|c|c|c|c|c|c|}
\hline Station. & 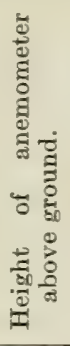 & 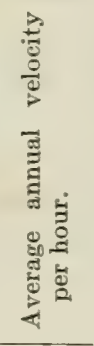 & 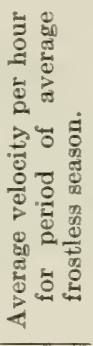 & Station. & 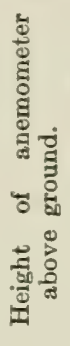 & 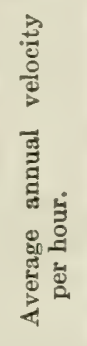 & 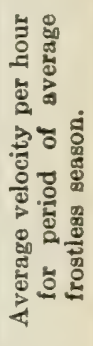 \\
\hline $\begin{array}{l}\text { Indiana-Continued: } \\
\text { Kalispell......... }\end{array}$ & $\begin{array}{r}\text { feet. } \\
34\end{array}$ & $\begin{array}{r}\text { miles. } \\
\mathbf{5} .0\end{array}$ & $\begin{array}{r}\text { miles. } \\
\mathbf{5 . 4}\end{array}$ & $\begin{array}{l}\text { Rhode Island: } \\
\text { Block Island. . }\end{array}$ & $\begin{array}{r}\text { feet. } \\
46\end{array}$ & $\begin{array}{l}\text { miles. } \\
16.8\end{array}$ & $\begin{array}{r}\text { miles. } \\
14.9\end{array}$ \\
\hline Nebraska: & & & & South Carolina: & & & \\
\hline North Platte. & 51 & 9.0 & 8.8 & Charleston... & 10 & 10.0 & 9.9 \\
\hline Omaha........ & 121 & 8.6 & 7.8 & Columbia... & 57 & 7.0 & 6.5 \\
\hline Valentine.. & 54 & 10.7 & 10.6 & South Dakota: & & & \\
\hline Nevada: & & & & Huron..... & 67 & 12.3 & 11.8 \\
\hline Carson City. . & 92 & 6.8 & 6.7 & Pierre....... & 75 & 9.2 & 9.5 \\
\hline Winnemucea.. & 56 & 9.0 & 8.6 & Rapid City. . & 50 & 8.0 & 7.8 \\
\hline New Hampshire: & & & & Yankton. & 57 & 8.3 & 7.5 \\
\hline Concord........ & 79 & 5.5 & 4.7 & Tennessee: & & & \\
\hline New York: & & & & Chattanooga. & 213 & 6.0 & 5. \\
\hline Albany.. & 115 & 7.7 & 7.1 & Knoxville.. & 100 & 6.1 & \\
\hline Binghamton. & 88 & 6.1 & 5.1 & Memphis.. & 97 & 8.7 & 7.9 \\
\hline Buffalo...... & 206 & 13.7 & 11.5 & Nashville.. & 191 & 6.6 & 5.9 \\
\hline Canton. & 61 & 11.1 & 9.7 & Texas: & & & \\
\hline New York.. & 314 & 12.7 & 11.2 & Abilene.... & 52 & 9.8 & 9. \\
\hline Oswegc & 91 & 11.0 & 8.9 & rillo... & 49 & 14.4 & 14. \\
\hline Rochester. & 102 & 8.5 & 7.3 & Corpus Christi. & 77 & 12.0 & 12. \\
\hline Syracuse. . & 113 & 11.8 & 10.0 & El Paso... & 133 & 10.3 & 10. \\
\hline New Jersey: & & & & Fort Worth. & 114 & 10.8 & 10. \\
\hline Atlantic City. & 48 & 10.3 & 9.6 & Galveston. . & 112 & 11.1 & 11.0 \\
\hline New Mexico: & & & & Pales & 79 & 7.1 & 6. \\
\hline Roswell. & 57 & 6.1 & 5.8 & San Antonio & 91 & 7.6 & 7. \\
\hline Santa Fe. . & 56 & 6.8 & 6.8 & Tay & 63 & 9.0 & 8.7 \\
\hline North Carolina: & & & & Utah: & & & \\
\hline Asheville.. & 84 & 7.6 & 6.0 & Moder & 43 & 10.5 & 11. \\
\hline Char & 76 & 6.8 & 6.2 & Salt Lake Cit & 189 & 6.0 & 6. \\
\hline Hattera & 47 & 14.3 & 13.5 & Vermont: & & & \\
\hline Ralei & 110 & 6.4 & 5.9 & Northfie & 60 & 8.4 & \\
\hline Wilmington. . & 91 & 8.3 & 8.0 & Virginia: & & & \\
\hline North Dakota: & & & & $\mathrm{Ly}$ & 88 & 4.2 & 3.6 \\
\hline Bism & 57 & 10.2 & 10.0 & & 111 & 9.1 & 8. \\
\hline Devil & 44 & 12.1 & 10.9 & & 52 & 6.8 & 6. \\
\hline Willis & 47 & 9.4 & 9.3 & lle... & 47 & 5.4 & 4.2 \\
\hline Ohio: & & & & Washing & & & \\
\hline Cincinn & 160 & 7.2 & 6.3 & $\mathrm{~N}$ & 56 & 16.8 & 16. \\
\hline Cler & 201 & 14.7 & 12.2 & & 224 & 6.6 & 6 . \\
\hline $\mathrm{C}$ & 222 & 9.3 & 8.9 & Sp & 110 & 5.9 & 6 . \\
\hline & 70 & 8.6 & & & 121 & 5.8 & \\
\hline Toledo & 246 & 11.0 & 9.7 & Island. & 57 & 14.9 & \\
\hline Oklahoma: & & & & West Virginia: & & & \\
\hline Oklahoma.. & 47 & 11.5 & 11.0 & & 50 & 4.2 & 3. \\
\hline Orego & & & & Parkersburg & 84 & 5.6 & 47 \\
\hline Po & 106 & 7.7 & 7. & Wiscon & & & \\
\hline Roseby & 57 & 3.4 & 3.5 & Gr & 144 & 9.3 & \\
\hline Pennsy & & & & & 49 & 6.9 & \\
\hline Erie. & 102 & 11.2 & 9.7 & Milwaukee. & 139 & 10.7 & 9.2 \\
\hline & 104 & 7.2 & 6.3 & Wyoming: & & & \\
\hline Phila & 184 & 10.3 & 9.6 & Cheyenne. & 64 & 10.7 & 8. \\
\hline Pittshur & 410 & 7.5 & 6.5 & Lander. & 36 & 4.1 & \\
\hline Scranton. & 119 & 7.2 & 6.4 & Yellowstone Par & 48 & 7.7 & \\
\hline
\end{tabular}


The average wind-velocities for the period of the average frostless season, given in table 20, are presented in the form of a chart in plate 68. Perhaps the most striking features of this chart are the prevalence of high average wind-velocities near the coasts and near the Great Lakes and the apparently low average velocities encountered in the mountain regions. In connection with the latter feature it is to be noted that stations in mountainous regions are generally in valleys, and are therefore protected from the general air-drift of the region. This chart appears to be of little or no value in defining rational climatic zones; either the average wind-velocity per hour during the period of the average frostless season is not a suitable feature by which to measure climate as related to plants, or the data upon which our calculations have been based are too inadequate to bring out any relations that may exist between this feature and plant distribution. We are persuaded that both of these alternatives express the truth. The average wind-velocity during the growing-season for plants is so little different in different parts of the country, and the evaporating power of the air is so greatly influenced by other conditions than wind, that it seems hardly to be expected that average wind-velocity may prove of great value in vegetational climatology. At the same time, if the distribution of climatological stations and the methods of wind observation employed by the United States Weather Bureau should ever be reorganized according to the needs of this sort of study, it is possible that average wind-velocities might assume more importance than is here apparent. A glance at the various heights of the anemometers above the ground, as given in table 20, makes it clear that these instruments have been placed rather for convenience than for the obtaining of useful results, as far as climatology is concerned. As Livingston has remarked (1913), the population of the cities of the United States may be estimated from decade to decade by the average height of the Weather Bureau instruments above the ground; the instruments seem to have risen higher in the air as the population has increased. This, of course, is due to the pernicious habit of locating the recording-stations generally in eities and towns instead of in the open country, where the first principles of climatological study demand that such stations should be placed. As towns have become cities and cities have enlarged, the anemometers have been elevated from time to time, so that the backwardness of a town-as far as large buildings are concerned-may be inferred from the low elevation of its Weather Bureau station. Table 20 shows that the anemometers are generally about 50 feet above the ground in small towns. In New York City, the anemometer is 314 feet above the ground, and for Pittsburgh (the highest elevation given on our list) its height is 410 feet.

When better wind data become available other lines of attack may be begun, as, for instance, a study of the relation holding between 
vegetation and maximum wind-velocity, but such attempts would be well-nigh useless at present.

G. SUNLIGHT AS A CONDITION INFLUENCING WATER-LOSS FROM PLANTS.

(TABLE 21, PLATE 69.)

As has been remarked, the influence exerted by light conditions upon plant growth is very complex, comprising several kinds of influences that are themselves quite different. With regard to general plant growth, which mainly interests us in the present studies, it is obvious that the most important light-relations are those of transpiration and photosynthesis.

The second of these relations is quite out of reach at present, for before the light conditions of different climatic regions may be compared with reference to the possibility of plant photosynthesis, the light-supply must be measured especially in terms of those ranges of wave-lengths that are known to influence the photosynthetic process; it is clear enough that the total light-supply is not in itself the condition to be studied in this connection, and spectrophotometry has not yet progressed far enough to furnish the instruments and methods here required. When it becomes possible to measure and record, at each climatological station, the daily supply of solar radiant energy of certain relatively small ranges of wave-lengths, then, and then only, will the photosynthetic powers of different regions become capable of being satisfactorily compared, but this seems unlikely to occur in the very near future.

The light-relation of transpiration is much more easily approachable than is that of photosynthesis, but the climatological records so far available are not suitable for even the relatively simple comparisons required in this connection. Here the climatological condition to be studied is the power of the surroundings to accelerate the rate of water-loss from plants through the action of impinging solar radiant energy. Since no two plants are to be regarded as exactly alike, and since the internal conditions of the light-absorbing surface are as influential in determining the light effect upon evaporation as are the external light conditions, it becomes necessary here (as in the case of the evaporating power of the air) to adopt some standard lightabsorbing surface and to measure the light-effect upon that surface. The measurement of sunshine has usually been accomplished by methods that depend upon the increase in temperature occasioned by the exposure of a certain blackened surface for a specified time period. Thus, the black-bulb thermometer has been employed by various authors in the comparison of the total intensity of sunshine energy received at different stations or for different days, etc., at the same station. The instruments so far described have not been generally satisfactory, being difficult of adequate operation and of calibration. 


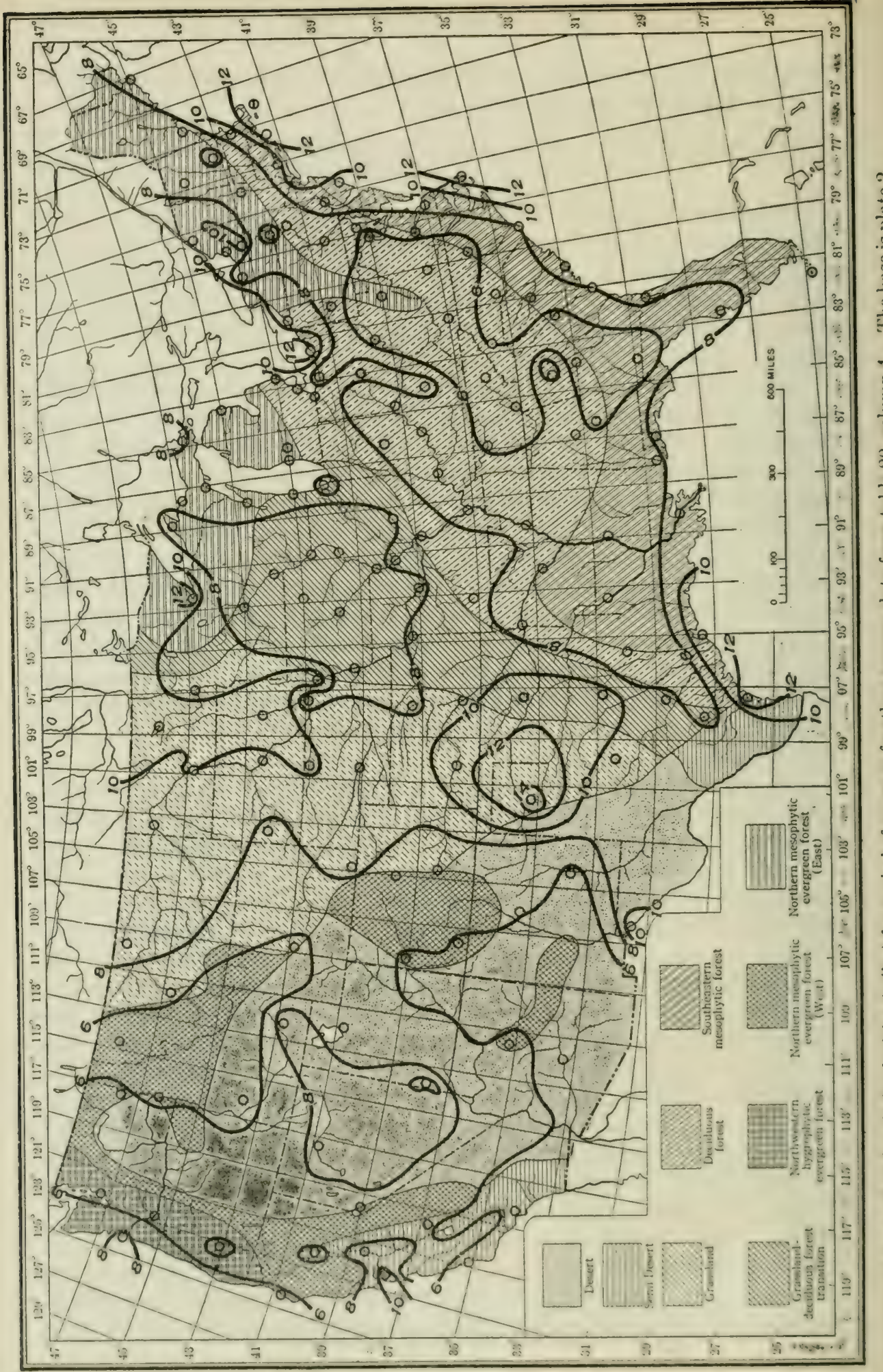




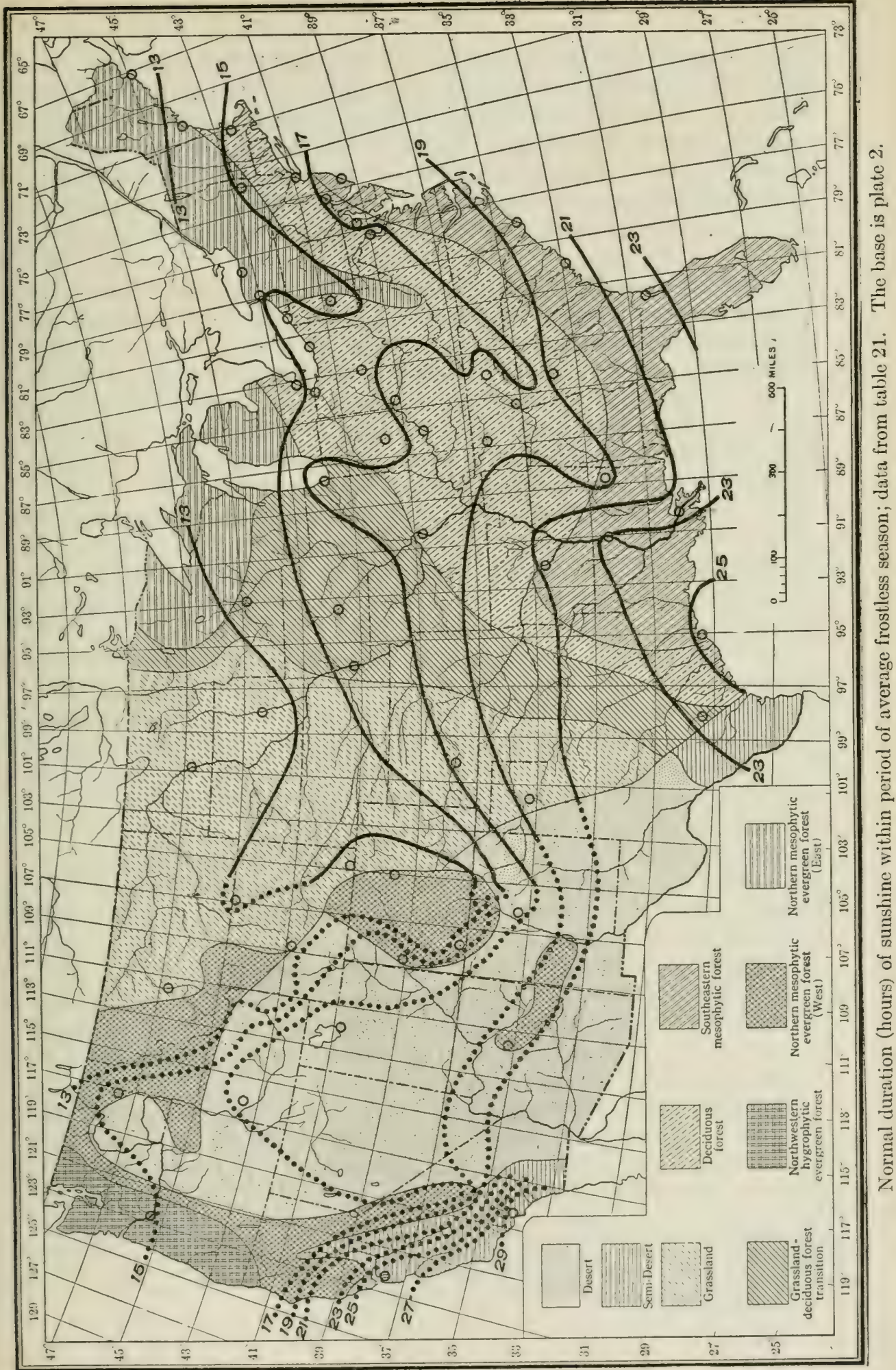


The bolometer and the pyrheliometer seem not yet to have been employed in climatological comparisons and the initial cost of these instruments, as well as the labor required in obtaining readings therefrom, make it probable that they will not, in their present forms at least, be of much climatological value. It must be remembered that, for such studies as we are considering, the operation of the instruments must be simple, the results must be satisfactory, and the cost must be low.

Livingston ${ }^{1}$ has discussed and compared various other simple forms of instruments intended for measuring light intensity, including the Hicks solar radio-integrator, two forms of actinometer employing photographic paper, and his own radio-atmometer. Reference should be made to his paper for what little has been done by way of comparing the readings obtained from these instruments with the corresponding degrees of light influence upon plant transpiration. This extremely important subject is deserving of much further study.

The Hicks instrument is faulty in its theoretical conception, in several ways; its readings are as much determined by air-temperature as by the intensity of the light which it aims to measure, and they are also greatly influenced by the changing amounts of liquid in the exposed bulb, in the shaded reservoir, etc.

The various forms of photographic-paper actinometers, such as the Wynne photographic exposure meter, the Clements "photometer,"' and the instrument employed by Wiesner ${ }^{3}$ are all valuable in measuring and comparing sunlight intensities with reference to their possible photochemical effect upon the particular paper or film employed, but they show nothing in regard to the corresponding possible photosynthetic or evaporational influence upon plants, since the photosynthetic process in plants has nothing essentially in common with the photochemical alteration of silver salts, excepting that both are photochemical, and transpiration has nothing at all in common with the photographic process.

Livingston's radio-atmometer has been greatly improved since its first description, ${ }^{4}$ and it seems probable that this instrument may prove to be of very great value in climatology as well as in plant physiology, ecology, agriculture, etc., but its general employment in such lines of study is yet to be accomplished.

The most improved form of the radio-atmometer consists of two spherical porous-cup atmometers, one of the spheres being black and the other white, but the two being otherwise alike. These areseparately mounted and are operated side by side in the location where the light

${ }^{1}$ Livingston, $(1911 a)$. See also, for the best sunshine records yet available, Briggs and Shantz, 1916.

${ }^{2}$ Clements, F. E., Research Methods in Ecology, Lincoln, Nebraska, 1905.

${ }^{3}$ Wiesner, J., Der Lichtgenuss dor Pllanzen, Leipzig, 1907.

- Livingston, 1915, 6. 
intensity is to be measured. The white surface absorbs but little radiant energy, while the black one absorbs a large proportion of the sunlight that reaches it. Both instruments are similarly affected by alterations in the evaporating power of the air, due to whatever cause, and the difference between their losses for any given time period is the amount of water vaporized on account of the energy absorbed by the black surface, over and above what is absorbed by the white one. This difference is thus an approximate measure of the radiation intensity for the given period, as this might accelerate evaporation from moist exposed surfaces of the kind here employed. The instrument is calculated to integrate the effects of sunshine throughout the time period that occurs between the readings, and it is exceedingly sensitive to relatively weak light intensities, so that it can give a wide range of rates. The period of exposure may of course be made of any convenient length. Attention should be called to the fact that the readings are to be interpreted as the time-rates of work done in vaporizing water from the standard surface, so that it thus becomes possible to consider sunshine, from the climatological point of view, as to its power to do the work of accelerating evaporation from the instrument. For a simple term to denote this power we may use "the evaporating power of the sunshine." No doubt this expression can be shown to be faulty in certain respects, if interest seems to lie in such a direction, but until the very important climatological factor of light intensity begins to attract serious attention it makes little difference in what terms we emphasize its present neglect and its great importance.

The sunshine records of the United States Weather Bureau consist of observations on the number of hours of sunshine occurring each day at each of the stations provided with the Marvin sunshine recorder. This instrument is virtually a differential thermometer, having two bulbs, the surface of one being a very good reflector and that of the other being blackened. The automatic recording device records the time periods when the blackened bulb has a temperature higher than that of the other bulb by more than a certain small amount. During periods of sunshine these two temperatures differ in this way. It is thus seen that the instrument is not calculated to give any information regarding comparative intensities of the impinging or absorbed radiant energy. It simply records for each day the amount of time when the sunshine was intense enough to produce the stated difference between the temperatures of the two bulbs. While this recorder leaves much to be desired, its records are probably more valuable than are periodic ocular observations of the amount of cloudiness during daylight hours. ${ }^{1}$

\footnotetext{
${ }^{1}$ In this connection see F. T. McLean, A preliminary study of climatic conditions in Maryland as related to the growth of soy-bean seedlings, Physiol. Res. 2: 129-208, 1917. See also: F. Merrill Hildebrandt, A method for approximating sunshine intensity from ocular observations of cloudiness, Johns Hopkins Univ. Circ., March, 1917, pp. 205-208.-Idem, 1921.
} 
From the records of the Marvin sunshine recorder, the United States Weather Bureau has derived data of the normal number of hours of sunshine, for each day of the year, for each of the stations included in the sunshine study. These numbers are expressed as percentages of the possible daily hours of sunshine in each case, the possible number of hours being, for each day and station, the number of hours between sunrise and sunset. Through the kindness of Professor P. C. Day, of the United States Weather Bureau, we have been able to obtain these data of the percentage of possible hours of sunshine for each month and for 57 stations in the United States, and these have been employed as basis for our sunshine computations.

From the present point of view the percentage of possible hours of sunshine is quite without interest; what affects plant activities is, of course, simply the amount of sunshine, and, if we consider this in terms of hours of light intensity above the threshold of the sunshine recorder, it is the actual number of hours of sunshine which should attract our attention. Our first step was, then, to calculate the normal number of hours of sunshine for each month, in each case. This was done by finding the number of possible hours of sunshine for the latitude of each station and for each month included in the period of the average frostless season, from Marvin's Sunshine Tables, ${ }^{1}$ and then multiplying this number by the corresponding percentage of the possible, considered as hundredths. The next step was to sum the numbers thus obtained for all whole months occurring in the average frostless season for the station in question, and to add to this sum quantities calculated to represent the fractions of a month with which the average frostless season generally begins and ends. The final sum represents the normal number of hours of sunshine occurring in the period of the average frostless season, for the particular station in question. The sums thus obtained are given in table 21 , the summations being plotted on a chart, with isoclimatic lines drawn in the usual way. The chart is given as plate 69. It is obvious, from the small number of stations for which data are available, that this chart is very crude and superficial. Nevertheless, a rational and self-consistent arrangement of climatic zones is here brought out, and this zonation is very similar to that based on temperature conditions. The stations receiving the most sunshine (measured in terms of hours by the Marvin recorder) are in the extreme Southwest, while those receiving the least lie near the northern boundary of the country or in the mountain regions. The lines of the western portion of this chart are shown as distinet from the rest, to suggest the greater uncertainty with which they have been placed.

\footnotetext{
1 Marvin, C. F., Sunshine tables, Edition of 1905, giving the times of sunrise and sunset in mean solar time and the total duration of sunshine for every day in the year, latitudes $20^{\circ}$ to $50^{\circ}$ North, U. S. Dept. Agric., Weather Bur., 1905 (numbered "W. B. No. 320").
} 
TABLE 21.-Normal total number of hours of sunshine within the period of the average frostless season.

\begin{tabular}{|c|c|c|c|}
\hline Station. & $\begin{array}{l}\text { Normal } \\
\text { total } \\
\text { duration } \\
\text { of } \\
\text { sunshine. }\end{array}$ & Station. & $\begin{array}{l}\text { Normal } \\
\text { total } \\
\text { duration } \\
\text { of } \\
\text { sunshine. }\end{array}$ \\
\hline Arizona: Flagstaff. & $\begin{array}{c}\text { hours. } \\
1,134\end{array}$ & New Mexico: Santa Fe... & $\begin{array}{c}\text { hours. } \\
1,892\end{array}$ \\
\hline Arkansas: Little Rock.......... & 2,166 & New York: & \\
\hline California: & & Albany... & 1,504 \\
\hline Los Angeles. & 2,995 & Buffalo.................. & 1,479 \\
\hline San Francisco.. & 2,615 & New York................ & 1,626 \\
\hline Colorado: & & 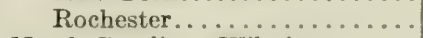 & 1,418 \\
\hline Denver... & 1,261 & North Carolina: Wilmington.... & 1,942 \\
\hline Durango...... & 1,367 & North Dakota: Bismarck....... & 1,227 \\
\hline Grand Junction. . . . . . . . . . & 1,865 & Ohio: & \\
\hline Florida: Jacksonville. ......... & 2,297 & 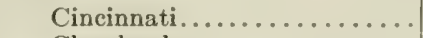 & 1,775 \\
\hline 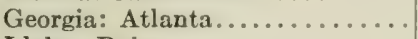 & 1,946 & 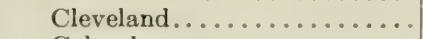 & 1,567 \\
\hline Idaho: Boise................ & 1,870 & Columbus................ & 1,651 \\
\hline Illinois: Chicago.............. & 1,774 & 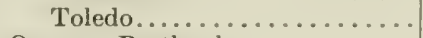 & 1,512 \\
\hline Indiana: Indianapolis.......... & 1,571 & 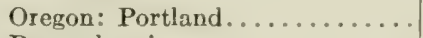 & 1,578 \\
\hline Iowa: Des Moines............. & 1,544 & Pennsylvania: & \\
\hline Kansas: Dodge............... & 1,784 & Erie........ & 1,577 \\
\hline Kentucky: Louisville........... & 1,743 & Philadelphia............. & 1,732 \\
\hline Louisiana: New Orleans........ & 2,123 & Pittsburgh............. & 1,403 \\
\hline Maine: & & South Carolina: Charleston..... & 2,026 \\
\hline Eastport... & 1,225 & South Dakota: Huron.......... & 1,267 \\
\hline Portland.... & 1,365 & Tennessee: & \\
\hline Maryland: & & Chattanooga... & 1,836 \\
\hline Baltimore.... & 1,736 & Knoxville............... & 1,772 \\
\hline Washington, D. C......... & 1,646 & Nashville................. & 1,878 \\
\hline Massachusetts: Boston........ & 1,499 & Texas: & \\
\hline Michigan: Detroit............ & 1,468 & Amarillo.... & 2,057 \\
\hline Minnesota: St. Paul........... & 1,367 & Galveston............... & 2,650 \\
\hline Mississippi: & & San Antonio.............. & 2,343 \\
\hline Meridian................. & 1,895 & Utah: Salt Lake City........... & 1,927 \\
\hline Vicksburg............... & 2,301 & Washington: Spokane......... & 1,927 \\
\hline Missouri: St. Louis............. & 1,832 & Wyoming: & \\
\hline Montana: Helena............. & 1,286 & Cheyenne.............. & 1,127 \\
\hline Nebraska: Omaha............. & 1,548 & 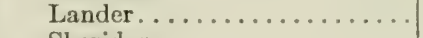 & 1,167 \\
\hline New Jersey: Atlantic City...... & 1,827 & Sheridan............... & 1,307 \\
\hline
\end{tabular}




\section{MOISTURE-TEMPERATURE INDICES.}

A. INTRODUCTORY.

An attempt on the part of Livingston ${ }^{1}$ to obtain a single climatic index for moisture and temperature efficiencies combined resulted in a climatic chart of the United States that has certain interesting characteristics. These indices are based on the tentative suppositions: (1) that the temperature efficiency of a climate, to produce plant growth, is proportional to the temperature summation-index of that climate (obtained by whatever method may prove most satisfactory), and (2) that the moisture efficiency is proportional to the Transeau ratio of precipitation to evaporation. This last supposition considers that if two stations differ only in rainfall and in the intensity of the evaporating power of the air, then plant growth at these two stations should be directly proportional to the rainfall and inversely proportional to the atmospheric evaporating power, as far as climatic conditions are concerned. In short, the moisture-temperature index of a climate (for any given duration factor) is taken as the product of the temperature summation-index and the moisture ratio. To employ Livingston's terminology, if $I_{m t}$ represents the moisture-temperature index, if $I_{t}$ represents the temperature summation-index, if $I_{p}$ represents the summed precipitation for the period considered, and if $I_{e}$ represents the total evaporation from some standard atmometer for the same period, then

$$
I_{m \ell}=I_{\ell} I_{p} / I_{e} \text {. }
$$

Inspection of this formula shows that the value of this moisturetemperature index is increased by higher temperature (supposing that the optimum temperature for plant growth is not surpassed) and also by lengthening of the time period taken into account. The higher are the daily temperature-index values and the more of them are summed, the greater must be the resulting sum $\left(I_{t}\right)$, and the product index is of course increased by increasing its first factor. Also, this product index is increased by higher values of the Transeau moisture-ratio $\left(I_{p} / I_{c}\right)$. This ratio value, in turn, is increased by more rainfall and by lower atmospheric evaporating power. The efficiency, for plant growth, of the moisture-temperature complex is thus greatest with a long growing-season, with high temperatures (not surpassing the optimum), with great rainfall, and with low evaporating power.

Livingston's product indiees were based upon the duration factor of the length of the period of the average frostless season and upon the physiological summation-index of temperature efliciency. We have calculated these values also for temperature indies derived by the remainder method and for those derived by the exponential method. The results obtained for these two forms of moisture- 
temperature index are presented below, and these are followed by those obtained by Livingston.

It is perhaps not out of place here to remark that these moisturetemperature indices represent no more than a first rough approximation toward an environmental index, which might state the efficiency of the environment as a whole to produce plant growth. It is quite obvious that such an environmental index will not really be attainable for a very long time; it must embrace many other terms besides those representing climatic conditions, and also terms for all of the influential climatic ones, and, as has been emphasized, methods for the measurement and weighting of most of the environmental conditions are yet to be devised. Nevertheless, progress can best be favored by employing the two climatic indices that seem most promising, with the hope that the shortcomings of the resulting interpretations may suggest closer approximations to the form of index required.

It may also be remarked that but little real progress can be hoped for in this direction until laboratory facilities become available, by which the relations between plant growth and environmental conditions may be experimentally studied. As has been emphasized, this sort of experimentation will require well-planned physical equipment for the control of environmental conditions. It will also require a group of workers who can bend their energies toward gaining a common end, for a single individual, no matter how well equipped with apparatus, can not hope to find it in his power to enter very deeply into these complex relations. Nevertheless, expensive and difficult as the project may seem at present, there can be no doubt that it will be eventually undertaken, nor can it be doubted that the benefits to be derived from properly planned and conducted experimental studies on plant environmental relations will prove fully as great and as valuable to the human race as have been those derived from experimental physics and astronomy. It is in the laboratories and observatories of these sciences that the nearest approach to the sort of work here contemplated is now being carried on. On the practical, breadwinning side, it needs only to be suggested that the greatest and most important of all human industries, agriculture, rests entirely upon what little knowledge we already happen to possess in regard to the relations between plant growth and environmental conditions. Whenever a workable environmental index for plant growth may be approached, it is certain that the arts of agriculture and forestry will be markedly improved.

B. MOISTURE-TEMPERATURE INDICEA BASED ON TEMPERATURE SUMMATIONINDICES OBTAINED BY THE REMAINDER METHOD (ABOVE $39^{\circ}$ F.), FOR THE PERIOD OF THE AVERAGE FROSTLESS SEASON. (TABLE 22, PLATE 70.)

These values were derived by multiplying the summation index for the period of the average frostless season, for each station (table 


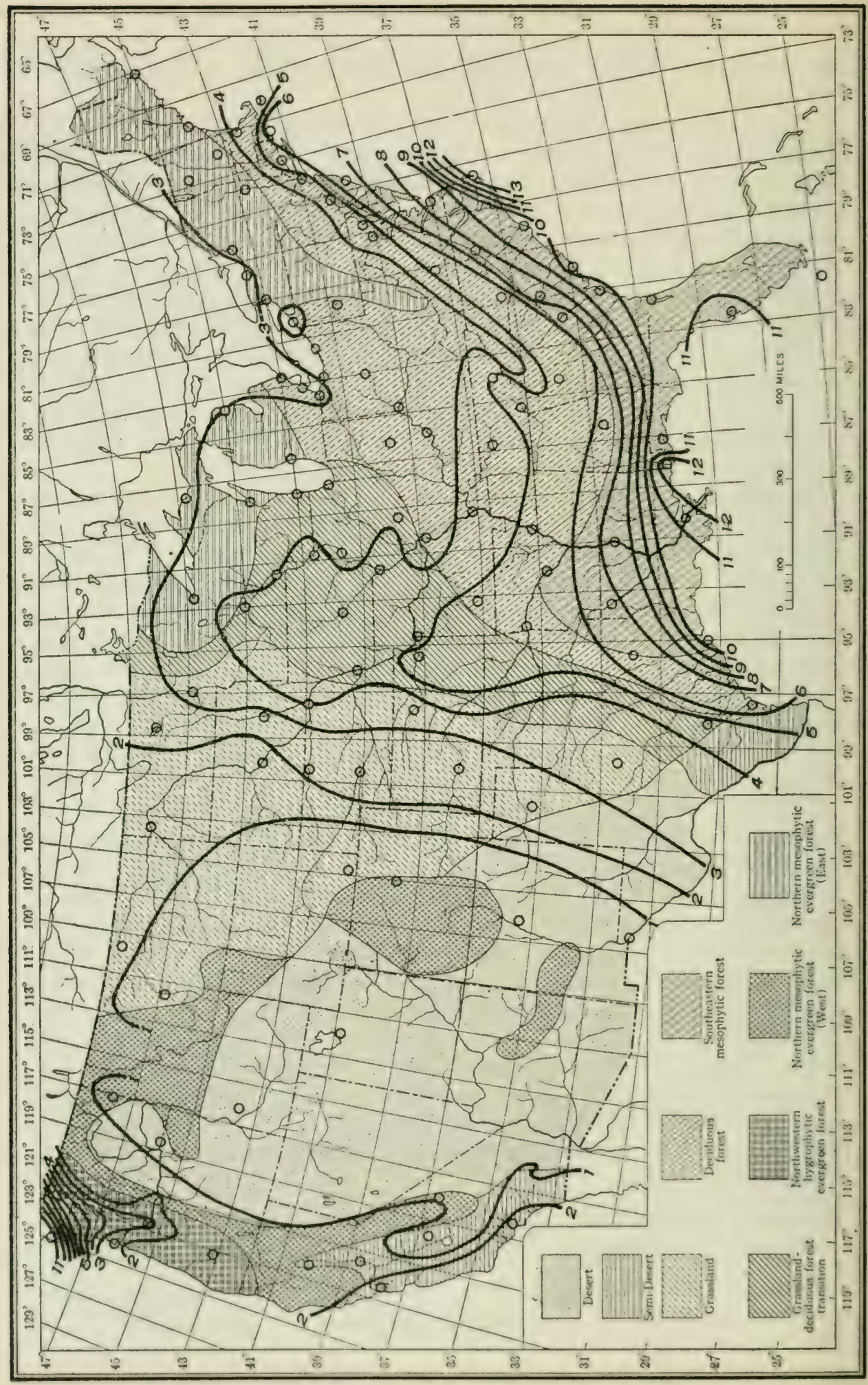

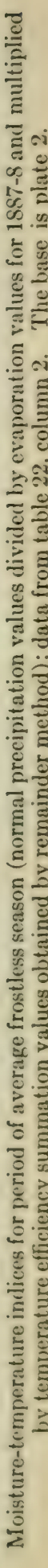


6 , column 5), by the corresponding Transeau ratio (table 11 , column 8). For simplicity, we may represent the first of these factors by the letter $T$ and retain the expression already used $(P / E)$ for the second, whence we may term the resultant index $P / E \times T$. The results obtained are set forth in the second column of table 22.

The chart for this series of values is shown as plate 70. Its discussion may best be combined with those of the two following charts.

TABLE 22.-Moisture-temperature indices $(P / E \times T)$ for the period of the average frostless season, by remainder (above $89^{\circ} F$.), exponential, and physiological methods.

The moisture ratios $(P / E)$ from table 12 , and the summation indices $(T)$ from tables 9 and 10.

\begin{tabular}{|c|c|c|c|c|c|c|c|}
\hline \multirow[b]{2}{*}{ Station. } & \multicolumn{3}{|c|}{$\begin{array}{c}\text { Temperature summation } \\
\text { obtained by- }\end{array}$} & \multirow[b]{2}{*}{ Station. } & \multicolumn{3}{|c|}{$\begin{array}{c}\text { Temperature summation } \\
\text { obtained by - }\end{array}$} \\
\hline & $\begin{array}{c}\text { Remain- } \\
\text { der } \\
\text { method } \\
\text { (above } \\
39^{\circ} \text { F.). }\end{array}$ & $\begin{array}{l}\text { Expo- } \\
\text { nential } \\
\text { method. }\end{array}$ & $\begin{array}{c}\text { Physio- } \\
\text { logical } \\
\text { method.2 }\end{array}$ & & $\begin{array}{c}\text { Remain- } \\
\text { der } \\
\text { method } \\
\text { (above } \\
39^{\circ} \text { F.). }\end{array}$ & $\begin{array}{c}\text { Expo- } \\
\text { nential } \\
\text { method. }\end{array}$ & $\begin{array}{c}\text { Physio- } \\
\text { logical } \\
\text { method.2 }\end{array}$ \\
\hline Alabama: & & & & Indiana: & & & \\
\hline $\begin{array}{l}\text { Mobile............... } \\
\text { Montgomery }\end{array}$ & $\begin{array}{r}12,106 \\
6,114\end{array}$ & $\begin{array}{r}1,314 \\
665\end{array}$ & $\begin{array}{l}23,652 \\
12,400\end{array}$ & Indianapolis......... & 3,376 & 359 & 5,967 \\
\hline Arkansas: & 0,114 & 665 & 12,400 & $\begin{array}{l}\text { 10wa: } \\
\text { Davenport........... }\end{array}$ & 3,672 & 384 & 6,255 \\
\hline Fort Smith......... & 5,543 & 601 & 10,782 & Des Moines........... & 4,364 & 456 & 7,457 \\
\hline Little Rock... . . . . . & 5,795 & 627 & 11,246 & Dubuque.............. & 4,583 & 478 & 7,472 \\
\hline California: & & & & Keokuk........... & 4,138 & 440 & 7,241 \\
\hline Fresno............. & 625 & 68 & 1,186 & Kansas: & & & \\
\hline Independence....... & 254 & 27 & 449 & Concordia.......... & 3,781 & 401 & 7,114 \\
\hline Los Angeles......... & 2,721 & 283 & 3,127 & Dodge............ & 2,410 & 256 & 4,474 \\
\hline Red Bluff........... & 1,285 & 142 & 2,409 & Topeka............ & 5,744 & 611 & 10,599 \\
\hline Sacramento......... & 1,399 & 146 & 2,046 & Kentucky: & & & \\
\hline San Francisco....... & 2,598 & 283 & 1,991 & Louisville.......... & 3,498 & 371 & 6,590 \\
\hline $\begin{array}{l}\text { Colorado: } \\
\text { Denver }\end{array}$ & & & & Louisiana: & & & \\
\hline $\begin{array}{l}\text { Denver..... } \\
\text { Connecticut: }\end{array}$ & 786 & 81 & 1,204 & New Orleans........ & 11,956 & 1,304 & 23,381 \\
\hline $\begin{array}{l}\text { Connecticut: } \\
\text { New Haven. }\end{array}$ & 5,330 & 556 & 7,869 & $\begin{array}{l}\text { Shreveport........ } \\
\text { Maine: }\end{array}$ & 6,846 & 751 & 13,874 \\
\hline Florida: & & & & Eastport. . . . . . . . . & 3,406 & 391 & 2,747 \\
\hline Jacksonville........ & 10,813 & 1,196 & 21,760 & Portland............ & 3,712 & 386 & 4,528 \\
\hline Key West.......... & 10,877 & 1,155 & 23,266 & Maryland: & & & \\
\hline Pensacola........... & 10,175 & 1,113 & 20,465 & Baltimore......... & 4,540 & 483 & 7,947 \\
\hline $\begin{array}{l}\text { Tampa......... } \\
\text { (Cedar Keys) }\end{array}$ & $\{11,722$ & 1,271 & 23,155 & $\begin{array}{l}\text { Washington, D. C. . } \\
\text { Massachusetts: }\end{array}$ & 4,704 & 497 & 8,322 \\
\hline Georgia: & & & & Boston..... & 4,097 & 429 & 5,714 \\
\hline 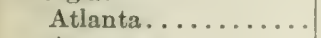 & 5,175 & 549 & 9,686 & Nantucket... & 4,396 & 474 & 5,193 \\
\hline Augusta............ & 6,420 & 695 & 12,879 & Michigan: & & & \\
\hline Savannah............ & 9,385 & 1,014 & 18,294 & Alpena. & 3,008 & 312 & 3,300 \\
\hline Idaho: & & & & Detroit........ & 2,996 & 309 & 4,569 \\
\hline Boise... & 405 & 42 & 598 & Grand Haven...... & 3,147 & 327 & 4,189 \\
\hline Illinois: & & & & Marquette........ & 2,997 & 314 & 3,113 \\
\hline Cairo... & 4,174 & 446 & 7,807 & Port Huron......... & 2,918 & 301 & 3,819 \\
\hline Chicago..... & 3,384 & 355 & 5,100 & Minnesota: & & & \\
\hline Springfield... & 3,971 & 418 & 7,032 & Duluth.. & 3,751 & 401 & 4,064 \\
\hline
\end{tabular}

1 Where a second station is named in parentheses, the evaporation value is for this station.

${ }^{2}$ The values in this column have appeared in Livingston's paper $(1916,2)$. 
TABLE 22-Moisture-temperature indices $(P / E \times T)$ for the period of the atrage frostless season, by remainder (above $39^{\circ} \mathrm{F}$.), exponcntial, and physiological methods. - Continued.

The moisture ratios $(P / E)$ from table 12 , and the summation indices $(T)$ from tables 9 and 10.

\begin{tabular}{|c|c|c|c|c|c|c|c|}
\hline \multirow[b]{2}{*}{ Station, } & \multicolumn{3}{|c|}{$\begin{array}{c}\text { Temperature summation } \\
\text { obtained by- }\end{array}$} & \multirow[b]{2}{*}{ Station. } & \multicolumn{3}{|c|}{$\begin{array}{l}\text { Temperature summation } \\
\text { obtained by- }\end{array}$} \\
\hline & 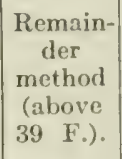 & $\begin{array}{c}\text { Expo- } \\
\text { nential } \\
\text { method. }\end{array}$ & $\begin{array}{c}\text { Physio- } \\
\text { logical } \\
\text { method. }^{2}\end{array}$ & & $\begin{array}{l}\text { Remain- } \\
\text { der } \\
\text { method } \\
\text { (above } \\
39 \text { F.). }\end{array}$ & $\begin{array}{c}\text { Expo- } \\
\text { nential } \\
\text { method. }\end{array}$ & $\begin{array}{l}\text { Physio- } \\
\text { logical } \\
\text { method.2 }\end{array}$ \\
\hline $\begin{array}{l}\text { Minnesota-Continued: } \\
\text { Moorhead........... }\end{array}$ & 3,163 & 315 & 4,043 & $\begin{array}{l}\text { Oregon: } \\
\quad \text { Portland. }\end{array}$ & 3,052 & 332 & 3,160 \\
\hline St. Paul............. & 4,269 & 442 & 6,423 & Roseburg.. & 1,213 & 126 & 1,313 \\
\hline $\begin{array}{l}\text { Mississippi: } \\
\text { Vicksburg. }\end{array}$ & & & & Pennsylvania: & & & \\
\hline $\begin{array}{l}\text { Vicksburg. } \\
\text { Missouri: }\end{array}$ & 7,663 & 834 & 15,125 & Erie......... & $\begin{array}{l}4,185 \\
4,174\end{array}$ & $\begin{array}{l}441 \\
441\end{array}$ & $\begin{array}{l}6,047 \\
7,010\end{array}$ \\
\hline $\begin{array}{l}\text { Missouri: } \\
\text { Kansas City,......... } \\
\text { (Leavenworth,Kans.) }\end{array}$ & 4,895 & 525 & 8,875 & Pittsburgh... & 3,354 & 349 & $\begin{array}{l}7,010 \\
5,672\end{array}$ \\
\hline $\begin{array}{l}(\text { Leavenworth,Kans.) })^{1} \\
\text { St. Louis............. }\end{array}$ & 3,635 & 390 & 6,824 & $\begin{array}{l}\text { Rhode Island: } \\
\text { Block Island. }\end{array}$ & 6,164 & 668 & 7,555 \\
\hline Springfield......... & 5,631 & 591 & 10,061 & South Carolina: & & & \\
\hline Montana: & & & & Charleston.. & 10,116 & 1,100 & 19,608 \\
\hline $\begin{array}{l}\text { Havre ............ } \\
\text { (Fort Assiniboine) }\end{array}$ & 1,139 & 116 & 1,505 & $\begin{array}{l}\text { Columbia... } \\
\text { South Dakota: }\end{array}$ & 7,432 & 807 & 14,837 \\
\hline Helena.......... & 691 & 72 & 809 & Huron.... & 2,389 & 243 & 3,657 \\
\hline Nebraska: & 2,205 & 229 & 3,682 & $\begin{array}{l}\text { Pierre........ } \\
\text { (Fort Sully) }^{i} \text {. }\end{array}$ & 1,711 & 179 & 2,928 \\
\hline $\begin{array}{l}\text { North Platte.. } \\
\text { Omaha....... }\end{array}$ & $\begin{array}{l}2,205 \\
4,151\end{array}$ & $\begin{array}{l}229 \\
436\end{array}$ & 7,406 & Yankton.... & 4,080 & 424 & 6,961 \\
\hline Valentine..... & 2,309 & 238 & 3,861 & Tennessee: & & & \\
\hline Nevada: & & & & Chattanooga. & 5,295 & 562 & 10,052 \\
\hline Winnemucca. . & 127 & 13 & 197 & Knoxville.... & 5,040 & 530 & 8,970 \\
\hline New Hampshire: & & & & Memphis.. & 5,253 & 593 & 10,837 \\
\hline $\begin{array}{l}\text { Concord............. } \\
\text { (Manchester) }^{2} \text {. }\end{array}$ & 3,356 & 344 & 4,422 & $\begin{array}{l}\text { Nashville... } \\
\text { Texas: }\end{array}$ & 4,593 & 493 & 8,930 \\
\hline New Jersey: & & & & Abilene... & 3,520 & 385 & 7,028 \\
\hline Atlantic City . & 6,858 & 707 & 10,241 & $\begin{array}{l}\text { Amarillo...... } \\
\text { (Fort Eliot) }\end{array}$ & 2,532 & 262 & 4,673 \\
\hline $\begin{array}{l}\text { New Mexico: } \\
\text { Santa Fe..... }\end{array}$ & 772 & 81 & 979 & Corpus Christi. & 6,690 & 737 & 13,926 \\
\hline New York: & & & & El Paso...... & 906 & 98 & 1,790 \\
\hline Albany... & 3,780 & 39.4 & 5,598 & Galveston.. & 10,331 & 1,142 & 20,570 \\
\hline Buffalo.. & 3,257 & 340 & 4,511 & Palestine.... & 6,410 & 700 & 12,977 \\
\hline New York.... & 4,541 & 481 & 7,034 & San Antonio... & 4,734 & 520 & 9,716 \\
\hline Oswego....... & 3,572 & 373 & 4,784 & Utah: & & & \\
\hline Rochester.. & 2,962 & 307 & 4,100 & Salt Lake City.. & 623 & 66 & 1,052 \\
\hline North Carolina: & & & & Vermont: & & & \\
\hline Charlotte... & 5,914 & 630 & 11,022 & Northfield & 3,362 & 345 & 3, SS4 \\
\hline $\begin{array}{l}\text { Hatteras..... } \\
\text { Raleigh.... }\end{array}$ & $\begin{array}{r}13,511 \\
7,992\end{array}$ & $\begin{array}{r}1,418 \\
851\end{array}$ & $\begin{array}{l}24,265 \\
14,980\end{array}$ & $\begin{array}{l}\text { Virginia: } \\
\text { Lynchburg... }\end{array}$ & 5,139 & 544 & 9,225 \\
\hline Wilmington. & 9,781 & 1,034 & 18,240 & Norfolk..... & 8,300 & 887 & 15,060 \\
\hline North Dakota: & & & & Washington: & & & \\
\hline Bismarck... & 1,590 & 188 & 2,626 & $\begin{array}{l}\text { North Head. . } \\
\text { (Fort Canby) }\end{array}$ & 2,684 & 345 & 1,874 \\
\hline $\begin{array}{l}\text { Devils Lake... } \\
\text { (Fort Totten) }\end{array}$ & 2,210 & 227 & 2,823 & $\begin{array}{l}\text { (Fort Canby) }^{1} \\
\text { Spokane....... }\end{array}$ & 934 & 101 & 1,148 \\
\hline $\begin{array}{l}\text { Williston.... } \\
\text { (Fort Buford) }\end{array}$ & 1,327 & 135 & 1,902 & Tatoosh Island & 11,724 & 1,566 & 7,475 \\
\hline $\begin{array}{l}\text { (Fort Bufo } \\
\text { Ohio: }\end{array}$ & & & & $\begin{array}{l}\text { Walla Walla. } \\
\text { Wisconsin: }\end{array}$ & 950 & 101 & 1,475 \\
\hline Cincinnati... & 3,007 & 319 & 5,513 & Green Bay.... & $\cdot 3,193$ & 330 & 4,270 \\
\hline Cleveland. . & 3,782 & 399 & 5,606 & La Crosse... & 4,085 & 422 & 6,296 \\
\hline Columbus... & 3,019 & 316 & 5,112 & Milwaukee. . & 3,373 & 351 & 4,582 \\
\hline Sandusky... & 3,635 & 390 & 5,778 & Wyoming: & & & \\
\hline Toledo.... & 2,914 & 303 & 4,617 & Cheyenne. . & 563 & $5 \mathrm{~s}$ & 710 \\
\hline
\end{tabular}

1 Where a second station is named in parentheses, the evaporation value is for this station.

${ }^{2}$ The values in this column have appeared in Livingston's paper $(1916,2)$. 
C. MOISTURE-TEMPERATURE INDICES $(P / E \times T)$ BASED ON TEMPERATURE SUMMATION-INDICES OBTAINED BY THE EXPONENTIAL METHOD (TEMPERATURE COEFFICIENT OF 2.0), FOR THE PERIOD OF THE AVERAGE FROSTLESS SEASON. (TABLE 22, PLATE 71.)

The same values for $P / E$ are used here as in the preceding case, but the temperature indices are taken from table 7 , column 2 . The products are presented in the third column of table 22. They are shown graphically on the chart of plate 71 , the discussion of which will be postponed until the next following chart has been presented.

D. MOISTURE-TEMPERATURE INDICES BASED ON TEMPERATURE SUMMATION-INDICES OBTAINED BY THE PHYSIOLOGICAL METHOD (LIVINGSTON'S, 1916) INDICES FROM LEBENBAUER'S 1915 MEASUREMENTS FOR YOUNG MAIZE SHOOTS, FOR THE PERIOD OF THE AVERAGE FROSTLESS SEASON. (TABLE 22, PLATE 72, AND FIG. 18.)

The values for $P / E$ are the same here as in the two preceding cases, but the temperature indices are taken from table 7 , column 4 . The products are given in the fourth column of table 22 , and the chart therefor is shown as plate 72. The discussion for plates 70, 71, and 72 will now be given.

\section{E. CONCLUSIONS FROM THE STUDY OF THE THREE FORMS OF MOISTURE-} TEMPERATURE PRODUCTS. (FIG. 18.)

The direction of zonation on all three of these moisture-temperature charts is at once seen to be essentially the same. A glance at the data

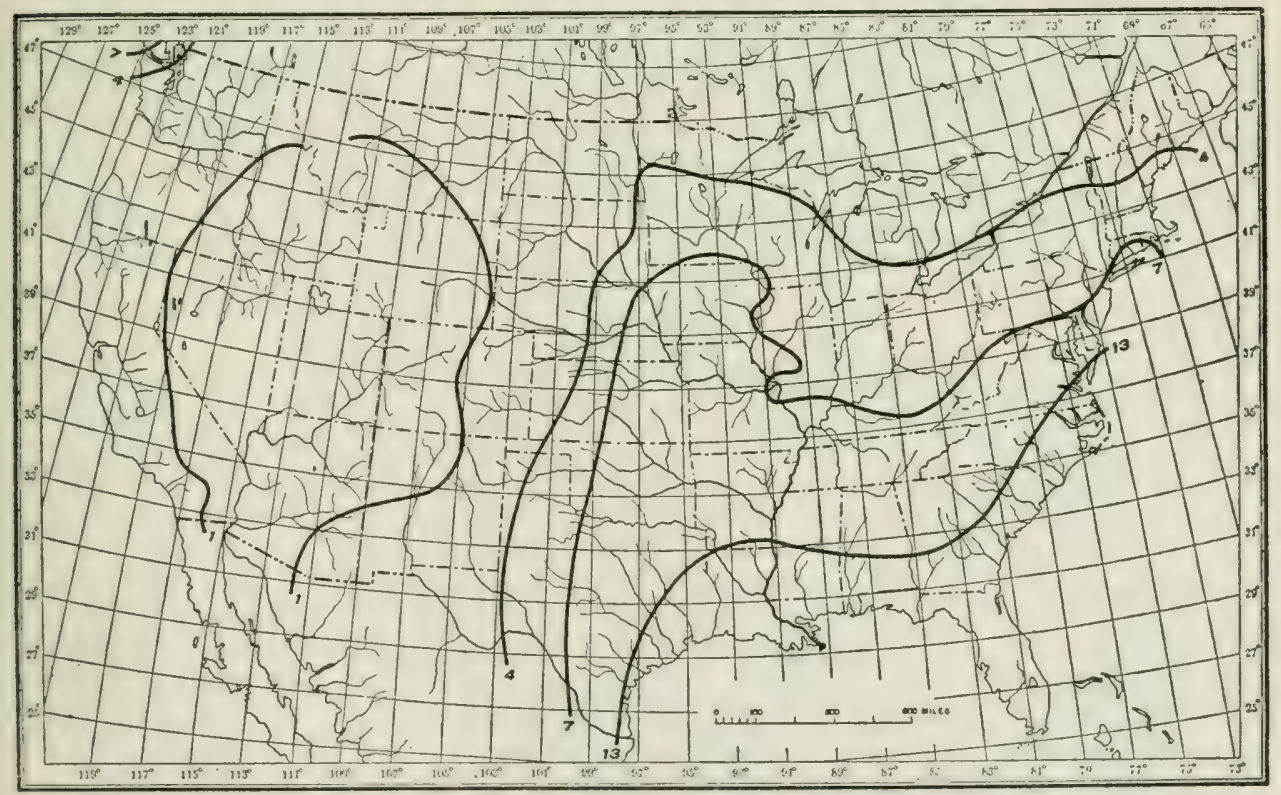

FIG. 18.-Moisture-temperature zonation, according to moisture-temperature products (physiological method) for period of average frostless season. Moisture-temperature provinces: Very high, more than 13 ; high, 7 to 13 ; medium, 4 to 7 ; low, 1 to 4 ; very low, less than 1 . Numerical values represent thousands. (See also Plate 72.) 


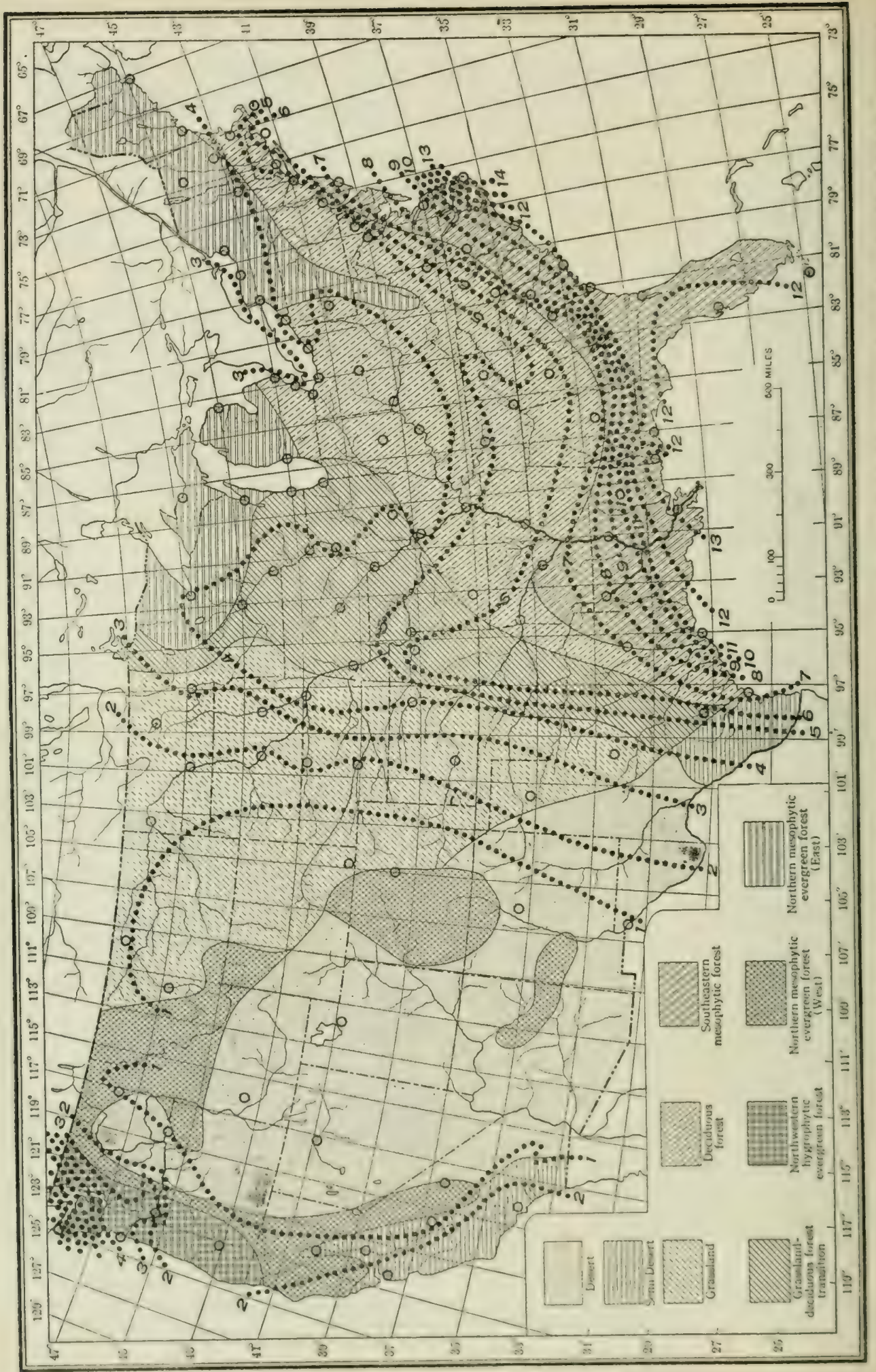




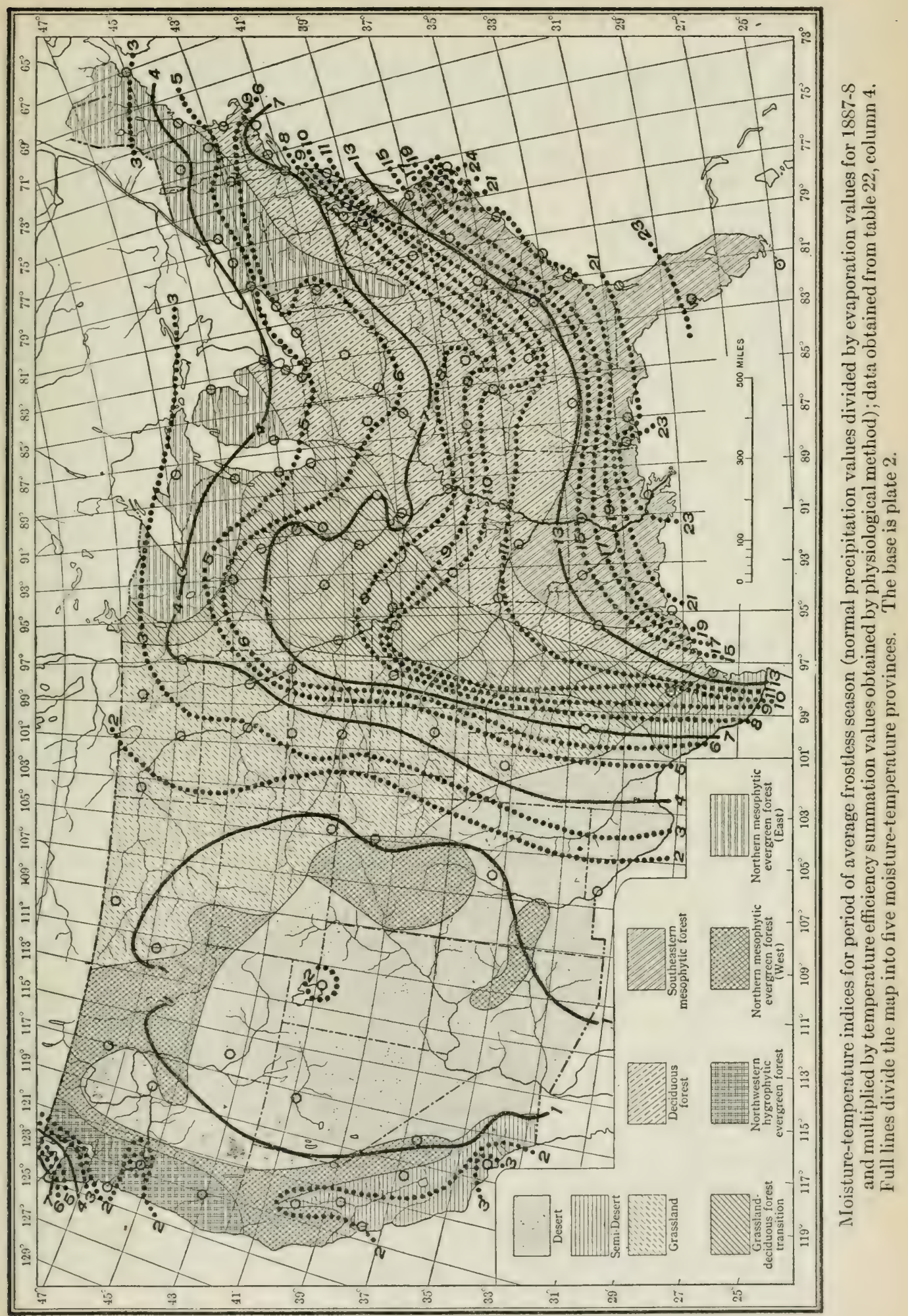


of table 22 shows that the indices themselves are markedly different in the three cases, however. The values indicated for the lines of plates 70 and 72 are thousands, while those for the lines of plate 71 are hundreds, and the table shows that the indices obtained from the exponential summations are always the smallest of the three. Those obtained from the remainder summations are roughly 10 times as great as those from the exponential ones, and those from the physiological summations are about twice as great as those from the remainder summations, or 20 times as great as those from the exponential ones. These statements are only roughly approximate, however, so that the values indicated on the three charts are: from 1 to 13 thousands, from 1 to 15 hundreds, and from 1 to 24 thousands, respectively. It is thus seen that the actual values given for any given station are quite different on these three charts, but, as has been said, the general zonation is nearly the same for all.

Peninsular Florida, a small area about Cape Hatteras, and the very humid Northwest show the highest values. (See fig. 18, derived from plate 72.) The Pacific coast region south of the humid Northwest has relatively low values, which are similar to those of the plains just east of the Rocky Mountains. The Great Basin region and the arid deserts of Arizona and southern California belong in the area of lowest indexvalues. From the plains belt eastward the values increase to about the one-hundredth meridian of west longitude. East of this meridian the lines of the charts roughly parallel the gulf and Atlantic coasts.

It is at once clear that these moisture-temperature charts combine the features of moisture charts with those of temperature charts. The index values here brought forward are predominantly controlled by moisture conditions in the arid regions and similarly controlled by temperature conditions in the cold regions, while for regions of intermediate moisture and temperature conditions, neither the one nor the other group is dominant in this control.

As Livingston has pointed out, it is not to be expected that this climatic zonation will bre found to correspond generally with the distributional zonation of plants; these charts indicate, for example, that Portland, Maine; Milwaukee, Wisconsin; Dodge, Kansas; and Amarillo, Texas, all belong in the same climatic zone. This system of indices was planned, however, to represent, not plant distribution, but the climatic possibility of plant grouth, in general. The most valuable use of these charts will doubtless come in studies of annual plant production, without particular regard to the forms of plants involved. They will probably be most useful in connection with agricultural and forestal studies. In this eonnection Livingst on has remarked:

"If it were possible to improve the temperature conditions (length and temperature efficiency of the season of plant growth) for Portland until they were as good as those for Tampa, Florida, then the potential annual plant product per acre (aside from soil influences) for the northern station should about equal that for the southern. But temperature con- 
ditions are not as easily controlled by artificial means as are moisture conditions; it is much easier to make a desert moist than to make winter into summer. So it comes about that large areas of the arid Southwest are annually producing nearly as much as their temperature conditions allow, while only exceedingly small areas in the Portland region are producing as much per year as their moisture conditions might allow. These latter areas are of course under glass; greenhouses are the only localities where the natural winter is transformed into an artificial summer."

And even greenhouse conditions fail to give summer light during the winter months. Quoting further from the author just mentioned,

"It appears that the most efficient climate for plant growth, in the United States, is that of peninsular Florida, as far as moisture and temperature conditions are concerned. But this climate is not the most comfortable for buman beings; its moisture ratio is too high in the season of active plant growth. The reason why the climate of southern California is so generally regarded as better than that of Florida [the temperature conditions being similar for the two regions] is to be found in the facts, (1) that the moisture ratio here is very low (making it a very poor climate for plant growth but a very pleasant one for human beings, and (2) that the moisture ratio is here artificially raised for plants (by irrigation), but not thus generally raised for human beings. The southern California climate for cultivated plants is an artificial one, in as true a sense as is that of a greenhouse in winter in Maine. In the latter case the value of the temperature index is artificially increased."

It should be remarked, in conclusion, that the main essentials of these moisture-temperature charts are also shown by the charts of vapor-pressure of water in the air (plates 63 and 64), and that the same is true in a more general way (but not so true in detail) of the four precipitation charts, plates 46 (fig. 2), 47, 49, and 50. The chart for normal annual precipitation (plate 52) also indicates some of these features. None of the charts showing temperature conditions and none of the other moisture charts (aside from those for vapor-pressure just mentioned) exhibit fundamental similarity to the moisture-temperature charts here considered.

\section{CARTOGRAPHICAL COMBINATION OF TEMPERATURE AND MOISTURE INDICES.}

While the arithmetical combination of temperature and moisture indices (giving such moisture-temperature indices as those just considered) does not give a chart that promises to be of great general value in the study of the geographical distribution of species or vegetation types, a chart formed by separately plotting the isoclimatic lines for moisture and for temperature values upon the same map appears to be much more promising for the present purposes. Such a combination chart was first presented, in a roughly generalized form. by Merriman's system of life-zones, in which each temperature province was divided into two portions according to moisture conditions. Livingston ${ }^{1}$ published three charts representing combinations of moisture and temperature data, and emphasized this general method of studying climatic data.

${ }^{1}$ Livingston, (1913, 1). 
We have prepared another chart of this kind by superimposing the chart of the physiological summation indices of temperature for the average frostless season (plate 40 and fig. 1) upon that of the precipitation-evaporation ratio for the average frostless season (plate 57 and fig. 16), and the result is shown in figure 19, where the broken lines represent temperature conditions and the full ones represent conditions of moisture. On this chart each of the five temperature provinces is subdivided into four moisture provinces and the country is thus represented as a mosaic of small areas of various shapes and sizes, each area being characterized by a certain range and amplitude of the temperature index and also of the moisture index. Following our previous usage, climatically descriptive adjectives may be employed

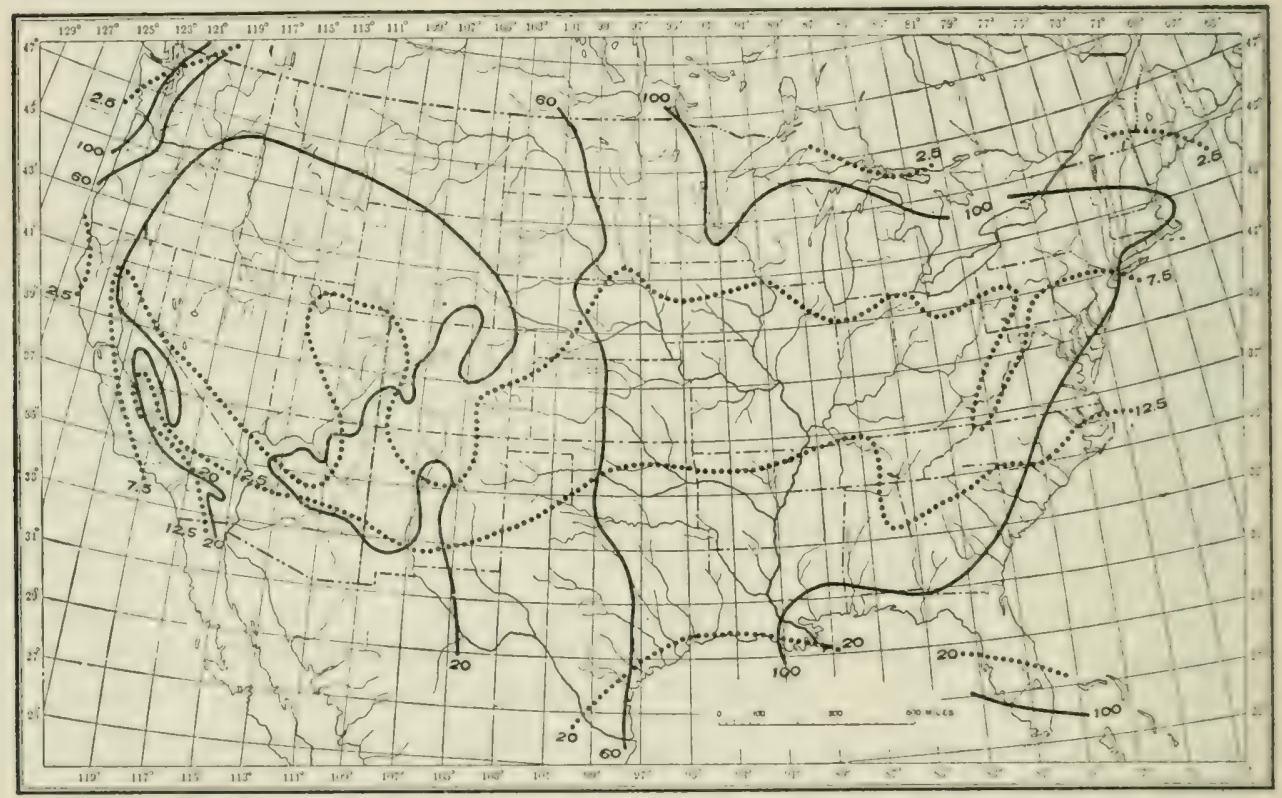

Fig. 19. - Two dimensional moisture-temperature provinees, being a combination of figures 1 and 16. Broken lines limit temperature efficiency provinees (fig. 1), full lines limit precipitationevaporation provinces. (fig.16). (See also Plates 40 and 57.)

in designating these ranges of index values, and two such adjectives suffice to describe any one of the irregular areas shown on the chart. Thus, we may refer to the warm semiarid province, the medium humid province, the cool scmihumid province, etc., each of these provinces including the coincident or overlapping portions of the corresponding temperature and moisture provinces.

An examination of figure 19 shows, howerer, that several different geographical areas may be characterized by the same pair of adjectives, and these may be designated by geographically deseriptive terms, 
employing either political designations (as the names of States), physiographical ones (such as rivers and mountain ranges), or the geographical ones of latitude and longitude. It is not to be implied that the different and separated portions of any climatic province, as these provinces are here characterized, have the same climatic conditions in general. The chart under consideration has been derived from moisture and temperature indices of certain specific kinds, obtained in certain specified ways, and the separate portions of the same province are to be considered as alike only with reference to the ranges of the indices employed. If other climatic indices were used these areas might not appear alike. It is obvious, for example, that other climatic conditions than those actually represented by the chart of figure 19 render the northwestern portion of the very cool humid province decidedly different climatically from the northeastern portion of the same province.

\section{GENERAL CONCLUSIONS FROM STUDY OF CLIMATIC CONDITIONS IN THE UNITED STATES.}

The climatic conditions considered in the preceding sections are mainly those of temperature and moisture. These are surely the most generally important climatic conditions met with in the natural control of plant distribution, and they are also the ones for which the best data are available, although the data for temperature conditions are much more satisfactory than those for moisture conditions. It is also true that methods for interpreting temperature observations are considerably farther advanced than are those for interpreting observations on the moisture conditions; methods by which temperature values may be weighted and integrated are available (though it must not be forgotten that these methods are susceptible of great improvement), but no general system for weighting moisture conditions has yet been suggested. The studies here repoted all lead unmistakably to the conviction that climatological methods and climatological interpretation, as so far developed, are wofully inadequate for the solution of problems dealing with the control of plant distribution. From the standpoint of ecology and agriculture no great progress is to be expected until much more attention is given to the devising of new methods for obtaining the climatic records and new methods for interpreting these records, and until a new point of view is reached, different in many respects from that hitherto held by workers in climatology. It can not be too strongly emphasized that the whole field of ecological climatology first requires original research in these fundamental and primary lines, research of an originality pronounced enough to be able to set aside many of the now stereotyped methods of observation and interpretation employed in climatology and meteorology, and it is to be hoped that those entering this new field of scientific endeavor will 
not be too strongly impressed by the dicta of meteorological and climatological science. They should be encouraged to leave the beaten paths and to approach the climatological problems from new angles, angles determined by the principles of physiology rather than merely by past custom or by the principles of meteorology. It may readily happen that some of the most satisfactory methods for ecological climatology will be strenuously opposed by students of climatology as this special science has been hitherto developed, but such students may remember that the main reason why greater progress concerning the relations of climate to organisms has not been made lies in the fact that those interested in climate have seldom been seriously interested in physiology, while most writers in physiology have had little active interest in climate. What ecological climatology requires is fundamental study of the climatic conditions as these are effective to alter physiological processes. Here there should he comparatively little interest in the meteorological causes of climatic conditions; attention is rather to be directed to the physiological effects of these conditions, without confusing the main issue by considerations as to how the conditions themselves have been brought into existence.

The most general conclusion derived from our investigations is, therefore, that very little real advance in this field is to be looked for until many new methods have been devised and tested. This proposition may appear disappointing to some ecological students, and our failure to place great reliance upon our own methods and results may be regarded by some as a confession that the work itself has been without valuable outcome. On the contrary, as has been repeatedly stated and implied, we have been convinced, throughout this prolonged study, that the only present way to make progress in ecological climatology is to utilize the available climatic records as far as possible and to test every method for their interpretation that appears at all plausible or promising. If some or all of the methods of integration, etc., here employed shall finally prove to be without permanent value. the present studies may have been useful in showing this to be true, and they may stimulate subsequent workers to devise other and better methods. Whether a method for handling climatic observations may or may not be useful in the study of plant distribution can not be known, of course, until it is subjected to rather thorough test. Our climatic charts have been prepared to test, in this regard, some of the most plausible methods that have been suggested.

Turning to the results of the work itself, the following paragraphs of the present section are to be read with the mental reservation that the conclusions stated, as far as they are general, are but tentative; they are known to be true only within the limits imposed by the nature of the climatic data used and by the methods of interpretation 
employed. At best, they are perhaps indications as to the nature of better-grounded generalizations that are to be developed in the future, from more satisfactory data, and by means of more adequate methods than are now available. The conditions will be considered in their order of presentation in the preceding sections.

\section{A. TEMPERATURE CONDITIONS.}

All of our temperature charts, whether for duration or intensity of the temperature conditions, represent the area of the United States as divided into temperature zones or provinces, these having a generally west-east direction, but being distorted more or less by mountain systems and proximity of the ocean. These zones result from arbitrary divisions, depending on selected ranges or amplitudes of the index values concerned, but, since the climatic characters are geographically continuous from province to province this is the only method by which they may be profitably studied. For general purposes it has seemed desirable to recognize five temperate temperature provinces in the United States, which we have termed: very warm, warm, medium, cool, and very cool. This terminology may be applied to all our temperature charts, but it is necessary to name the temperature index employed in each case and also the index amplitude representing each province. Thus, the warm province based on length of the average frostless season is not exactly coextensive with the province of the same name based on the physiological summation index, etc.

Special emphasis should be placed on the length of the average frostless season as an index of temperature duration. It has proved to be of great value, not only as a temperature index per se, but also as a duration factor for intensity indices of both temperature and moisture conditions. This promises to be one of the most useful temperature indices for use in ecological climatology, although it has not yet attracted the attention that it deserves.

Other duration indices of temperature that may prove valuable are (1) the length of the period of high daily normals and (2) the length of the period of low daily normals.

The most promising intensity index of temperature conditions appears to be that of the physiological summation for the duration of the average frostless season, as devised by Livingston, but much more physiological study will be required before this index can be regarded as established. As here employed, however, this index has proved to be very satisfactory in many ways. Absolute temperature minima have also proved to be valuable as intensity indices. We have also employed the average daily normal for the coldest 14 days of the year, as well as Merriam's chart of the mean normal for the hottest 6 weeks. The normal mean annual temperature is the most promising of the rarious temperature indices for which values are directly available in 
the publications of the United States Weather Bureau, but it appears to be of comparatively little use in interpreting climate in connection with the physiological activities of plants and animals.

\section{B. MOISTURE CONDITIONS.}

The moisture conditions with which we have dealt are those of (1) precipitation, (2) evaporation, (3) aqueous-vapor pressure, (4) relative humidity, (5) wind, and (6) sunlight. As far as the environment above the soil-surface is concerned, precipitation is the condition that determines the supply of water to plants, but indices of precipitation are also indirect indications of the evaporating power of the air, atmospheric humidity, and sunlight intensity; for abundant precipitation is generally accompanied by high air-humidity and much cloudiness. But no methods are as yet available for obtaining an index of the power of the soil to supply water to plants, which is the subterranean moisture condition that corresponds to atmospheric evaporating power, and we have employed several precipitation indices as representing either the moisture conditions in general or else the moisture-supplying power of the climatic complex.

We have employed eight different precipitation charts, seven of them original and the remaining one (normal annual precipitation, plate 52) after Gannett. ${ }^{1}$ Probably the most valuable single criterion for the water-supplying power of the climatic complex is the normal mean daily precipitation for the period of the average frostless season (plate 46). But the precipitation of the United States can not be adequately interpreted by any single criterion; using the observational data that are at hand several different indices are required. Only when data for soil-moisture conditions shall have become available can a true study of precipitation as an influence on plant growth be undertaken.

For the various precipitation indices the country appears to be made up of a series of more or less parallel precipitation zones, which may be represented by four precipitation provinces. These we have termed humid, semithumid, semiarid, and arid. The same terms are applied to the corresponding four provinces based on the desiccation features of the climate, evaporation, aqueous-rapor pressure, relative humidity, etc. The exact extent of each province of course depends upon the nature of the climatic index employed, but there is generally shown an eastern and northwestern portion of the humid province, while the arid province lies in the region of the southwestern desert and semi-desert.

Our evaporation charts represent three different climatic indices derived from Russell's data for a single your (1S57-SS), and also

${ }^{1}$ A more satisfactory chart of this fenture, by Iincer, has recently apneared, too late to be included in our study. See Kincer, J. 13., Average annual preeipitation in inches [for the United States], Based on Records of Alout 1,600 Stations for the 20-year Period 1895-1914, and 2,000 Additional records, from 5 to 19 years in length, uniformly adjusted to the same period, advance slseet 1, Atlas of Amer. Apric., U. S. Dept. Apric., Weather Bur., Jan. 1917. 
another index based on atmometric measurements carried out from the Desert Laboratory in the summer of 1908. From our work with these and other atmometric indices, as well as on a priori grounds, it appears that evaporation is one of the most important climatic features, as far as the climatic control of vegetation is concerned. It deserves more attention than does precipitation, and as much as does temperature, but observational data on the evaporating power of the air are as yet exceedingly meager, and serious interest has only recently been directed toward atmometry.

Transeau's ratio, the normal annual precipitation divided by the annual evaporation for the single year tested (Russell), is a very valuable index of climatic moisture conditions, and we have used this ratio in our series. The method employed by Transeau has been modified in several ways, to give other moisture indices. Of these modifications two appear to be particularly worthy of special mention here: (1) the ratio calculated for the period of the average frostless season, and (2) a ratio derived by dividing the total evaporation for the period of the average frostless season (Russell) by the total normal precipitation for the period of the average frostless season plus the preceding 30 days. The use of the frostless season as duration factor needs no comment in this place, but it may be emphasized that the second index of the two just mentioned (or some similar one) should prove worthy of much more study than is now possible. It is based on the idea that the precipitation of the early spring, before the beginning of the frostless season, is effective to offset a portion of the evaporation of the earlier part of the frostless season itself.

Aqueous-vapor pressure and relative air humidity furnish several moisture indices. Special attention should be directed to the normal mean percentage of relative humidity for the period of the average frostless season, an index that is probably the most valuable of all the desiccation indices we have used. The reason why this index is here accounted as more valuable than those of evaporation or aqueousvapor pressure apparently lies in the fact that relative humidity data have been accumulated for a long period of time, while the other data are unsatisfactory in this respect. It should be emphasized that the saturation deficit is what ought to be recorded, instead of relative humidity, however, and that the simultaneous observation of moisture conditions throughout an area embracing as many degrees of longitude as does the United States is at least misleading and apt to introduce artifacts in the charts. For use in climatological ecology a much better method of making the records needs to be devised.

From the available data on wind velocity and sunlight intensity we have derived climatic indices that may be regarded as pertaining to the moisture aspect of the climatic complex, but the information at hand, and especially the very unsatisfactory method now used for sunshine records by the United States Weather Bureau, are not adequate for a general consideration of these conditions for the area studied. 


\section{COMBINATIONS OF TEMPERATURE AND MOISTURE CONDITIONS.}

It has been pointed out that temperature indices show a zonation of the country, the zones extending generally west and east. A similar generalization may be stated for the moisture indices that we have studied, only the direction of the isoclimatic lines is generally north and south in this case. Of course these statements are true only in a general way; what is most worthy of emphasis is that the two kinds of zones tend to cross each other, so that if our area had been large enough we should have had 4 moisture provinces within each temperature province and 5 temperature provinces within each moisture province. This observation has suggested the cartographical combination of moisture and temperature indices, by which the country becomes a mosaic of irregularly shaped areas representing 34 temperate moisturetemperature provinces. Since such combination charts bid fair to be very largely used in climatological ecology, the descriptive names of these various provinces, as we have suggested them, may be tabulated here for convenience.

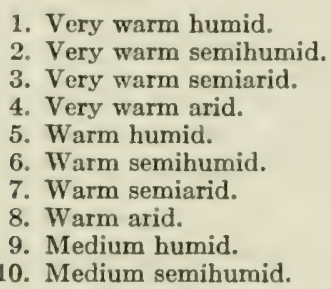

11. Medium semiarid.
12. Medium arid.
13. Cool humid.
14. Cool semihumid.
15. Cool semiarid.
16. Cool arid.
17. Very cool humid.
18. Very cool semihumid.
19. Very cool semiarid.
20. Very cool arid.

Livingston's moisture-temperature products receive attention, but, while they promise to be of value in some ways, as, for example, in the comparative evaluation of land in different parts of the country, their very nature precludes their being of very great general service in the etiological study of plant distribution. They refer to the efficiency of the climatic complex for plant growth in general rather than to its efficiency for any particular species or kind of vegetation. 
PART III.

THE CORRELATION OF DISTRIBUTIONAL FEATURES WITH CLIMATIC CONDITIONS. 



\section{INTRODUCTION.}

After presenting in the preceding pages the data which have been selected to exhibit the principal features of plant distribution in the United States, together with climatological data that have been elaborated for use, we are now in a position to proceed to the final stage of our investigation and to discuss the correlation of features of plant distribution with various intensities of the climatic conditions. All that has gone before has been, in a large measure, preparatory to this phase of our work.

We have sought primarily to discover the amplitude or extreme range of the climatic differences that may be found within the distributional area of each vegetation, each species, or each group of species. Such amplitudes, as shown by the climatological figures that we have used, are those of the average year, or the average frostless season. Even where we have used such data as the number of cold days, the number of days in the longest rainy period, or the number of days in the longest dry period, we have been concerned with the average or normal operation of these extreme factors. Our work disregards, in other words, the actual absolute extremes by which every region is visited with respect to every element of the climate, except in the single instance in which we have employed data as to the absolute minimum temperature. The individual climatic extremes of a given year may be of great importance to plants, and may change distributional limits or influence migratory movements. For perennial plants, however, it is probably the average conditions of decades and groups of decades that determine the mean distributional limits, and it is the average conditions that should be taken into account in a preliminary and generål investigation of this character.

In addition to ascertaining the amplitude of each climatic index for each plant area, we have endeavored, in as many cases as possible, to discover evidence that would show which of the various climatic conditions appears to be most influential in controlling the distribution of a given vegetation or plant, and what particular intensities of such main controls appear to be the critical points. It requires little experience with such problems to come to a realization that the various parts of the boundary of a plant or vegetational area are not controlled by the same factor or group of factors. In the case of plants which have a distribution of east-and-west extension it is obvious that their northern and southern boundaries are determined by different intensities of some condition, or possibly by entirely distinct sets of conditions. In the case of plants which have extensive and irregular distributional boundaries it may be possible to state what the limiting conditions are for certain portions of this boundary without being able to discover how far the given controls extend their domination. 
In the United States there are many plants which appear to have three sets of controlling factors, one limiting them toward the north, one toward the south, and one toward the west.

The investigation of distributional limits is still further complicated by the fact that the potency of a given condition in limiting the geographic range of a plant may vary with the intensity of some other condition or conditions. This is very well shown in the case of the westward extension of Quercus macrocarpa (see plate 18), which reaches eastern Montana in the north and extends only to central Oklahoma in the south. The fact that this tree reaches its western limit in flood-plains points to the influence of water-relations in limiting its western extension, as is doubtless true of all trees of the Deciduous Forest region. All climatic lines which have to do with the water relations, however, have a nearly north-and-south direction in this part of the United States. It is obvious that a given set of moisture conditions may permit the growth of Quercus macrocarpa in South Dakota, while the same moisture conditions, under much more exacting conditions of temperature, and consequently of evaporation, would inhibit the occurrence of the tree in the Panhandle region of Texas. Similar considerations are doubtless involved in the western limit of the Grassland area.

Another case of this extremely common state of affairs is to be found in connection with the northward distributional limit of Opuntia missouriensis. This plant, like many of its congeners, appears to have its northward distribution determined by some phase of the wintertemperature conditions. The cacti appear to be able to withstand low temperatures much better when their water-content is low than when it is high. In the dry late summer of the Grassland region Opuntia missouriensis becomes desiccated to such an extent that it is able to withstand the winter temperatures up to a latitude of $53^{\circ}$. In the regions to the east and west of its northernmost area the winter conditions are scarcely more severe, but the soil-moisture conditions of the late summer are such as would bring the cactus to the winter season in a state of turgidity that would prove fatal. This is a case, in brief, in which the annual march of soil-moisture conditions appears to cooperate with the winter temperature conditions in determining the limitation of a species.

There are very many cases in which it is possible to demonstrate that a particular climatic condition at a particular intensity is responsible for the position of a distributional limit. But in no case does the operation of this factor fail to be influenced by the intensities of other factors. The most that we ean do is to analyze the environmental complex, to discover which of the various environmental conditions is of the greatest relative importance in determining a given distribution.

The coincidence of a charted distributional linit and an isoclimatic line ean not be taken as an absolute and logieally complete proof that 
the particular climatic intensity in question causes the limitation of the plant or vegetation in question. The probabilities to be inferred from such a correspondence are extremely strong, but we may place full confidence in such a deduction only when we are sure that no other factor, intensity, or combination of factors, also follows the given vegetational limit.

It frequently happens, particularly in the United States, that a rapid geographic change in a given climatic condition is accompanied by changes in other conditions, and that the isoclimatic lines for one feature of the climate are parallel to those for another. There is also a frequent reciprocal relation between factors, one approaching lower values as the other approaches higher. All of these circumstances make more difficult the task of correlating vegetation and climate. All isoclimatic lines which refer to temperature conditions run, in general, in a west-east direction, and in the United States those referring to moisture conditions run mainly north and south. This makes it easy to distinguish at least the temperature controls from moisture controls, however difficult it may remain to discover which of several temperature conditions, or of several moisture conditions, may be the most critical in a given case of distribution.

Wherever the method of correlation breaks down or is inconclusive in its evidence, there is an opportunity opened for more intensive study of correlations with reference to critical localities. It might be possible to secure the most conclusive and logically precise knowledge of the critical factors for plants by using the experimental methods of a physiological laboratory with equipment for the control of conditions. It is, however, much easier to determine the optimal points for a plant by laboratory methods than it is to determine the limiting points in the scale of conditions. It is relatively easy, too, to subject a shortlived plant to experimentally controlled conditions, although it is difficult to give it conditions - particularly of soil and light-similar to these occurring anywhere in nature, so that the results will have a definite ecological bearing. Perennial plants may be experimentally investigated with respect to their early life-history, or with respect to individual phases of activity, but there are many problems in connection with their physiology which demand field experimentation, or the measurement of the uncontrolled conditions of the natural habitat, and all problems in connection with their ecology demand it. The study of the optimal and limiting conditions for vegetation, as contrasted with individual species, appears to be quite beyond the pale of experimental methods and must be carried on by means of instrumentation and correlation.

The rather crude cartographic method of correlation that we have used is adapted only to general studies covering large areas. The same method might well be used on a more refined scale for a study of correlations over any topographically simple area for which there was 
an abundant and well-distributed set of climatic data. The State of Texas is particularly well suited to such an investigation, both by reason of its sharp gradients of vegetational and climatic conditions and by reason of the absence of mountains and their complicating effects.

While many distributional limits are sharp, the great majority of plants pass gradually from abundance to rarity, sometimes over a wide belt of territory. It is often possible by purely observational methods to discover that the outermost localities for plants, on the edges of their ranges, present conditions which are rare in that region but are common or universal in the region in which this plant is abundant. All of the deciduous trees of the Mississippi Valley find their western limits along the watereourses of the Great Plains, and all of the cacti of the Arizona Succulent Desert find their northern limits on arid slopes of southern exposure along the southern edge of the Colorado Plateau. These cases make it difficult to correlate vegetation and climate on a coarse scale, and they invite the use of more refined methods with reference to small areas.

The study of distributional limits and the climatic extremes by which they appear to be controlled is only one phase of the correlation of climate and vegetation. An equally important field is that in which attention is given to the ecological centers of the distribution of plants or the development of vegetations, and to the optimal conditions which appear to determine the location of these centers. Faunal naturalists have long been interested in the subject of ecological centers, and numerous writers have proposed criteria by which they may be recognized. Adams ${ }^{1}$ has brought these together, the criteria which are applicable to plants being as follows: (a) location of greatest differentiation, (b) location of greatest individual abundance, (c) location of closely allied forms, $(d)$ location of maximum size of individuals, $(e)$ ocation of greatest reproductive activity, $(f)$ location of convergence of lines of dispersal, $(g)$ location of greatest catholicity of habitat, $(h)$ location of convergence of lines of individual variation. To these might be added, for plants, the location of most rapid rate of growth and the location in which a form is accompanied by the largest number of individuals which are specifically distinct but of the same growth-form.

It is obvious that the locating of the ecological center for a plant. or a vegetation is a matter which requires the assembling of a considerable body of data. There are calses in which one or two of these criteria have been very carefully determined, but we know of no ease in which all of them have been determined for any plant. If it had been possible to secure such data in the published literature, it would have been an easy and fruitful task to have applied our climatological figures to such eenters for the sake of learning the optimum climatic

iAdams, C. C. Southeastern United States as a center of geographical distribution of flora and fauna, Biol. 13ull. 3: 115-131, 1902. 
conditions of the plant in question, in addition to its limiting ones. We publish our climatic data in detail partly with the hope that it may be possible for future workers in this field to do more than we have been able to do with this other and equally important half of the field of distributional etiology, and that our assemblage of figures may be of use in that connection.

It is in default of full data as to the location of the ecological centers of distribution that we have used the maps showing the cumulative occurrence of trees and grasses and the maps showing the relative abundance of Pinus toxda, Liriodendron tulipifera, and Bulbilis dactyloides, in the different portions of their areas. These maps probably approach the appearance that might characterize maps drawn by combining all of the criteria mentioned by Adams if such charts were possible.

With respect to both the climatological and the vegetational data that we have been able to secure, the limitations of our material have at all times been more serious than the limitations of our methods, and the methods might readily have been much improved in many respects if the available observational data had seemed to warrant this.

\section{PRESENTATION OF THE CORRELATION DATA.}

\section{METHODS OF CORRELATION.}

The method here adopted for determining the extreme climatic values for each botanical area is a simple cartographic one. After assembling all of the values for a given element or condition of the climate, these were placed on a base-map of the United States (U. S. Geological Survey sheets, 17 by 28 inches), with a heavy dot locating each station used. From each of the 39 climatic maps (see Part II) it was then possible to learn at a glance the climatic index value for any given station and to follow the regions of similar readings by aid of the isoclimatic lines. The data on plant and vegetational distribution were placed on maps of the same size, and from them overlays were made, on thin tracing-paper, for each of the 33 botanical maps. It was then possible to take each distributional tracing in turn and to lay it successively on each of the climatological maps, reading the figures for the stations which showed the highest and lowest values within each of the distributional areas. In this manner 126 botanical areas were compared with 31 of the climatological maps and 3,906 readings of climatic extremes were secured. These readings are presented in tables 23 to 151 .

Our method of determining optimum conditions, in the few cases in which we obtained centers of distribution by the method of cumulative occurrences, was to secure the readings of highest and lowest climatic 
values for the center, and also for each of the zones, of diminishing abundance.

The discovery of close relationship between the position of plant boundaries and isoclimatic lines has been made by a mere comparison of the vegetational and climatic maps, checked by the use of the vegetational overlays on the climatic maps.

It is true of both sets of the maps which embody our basic data that some are constructed from much fuller figures or information than others. Over 1,600 stations have been used for the map of absolute minimum temperature and over 1,200 stations for the map of average length of frostless season, whereas, on the other hand, the map showing the annual total evaporation is based on only 139 stations, and that showing the normal mean annual precipitation is based on Gannett's chart, showing no readings for individual stations and smoothed to exhibit the means by 10 -inch increments. The most poorly determined plant ranges are those of Florkea occidentalis, based on four published occurrences, and of Trautvetteria grandis, based on sixteen occurrences, nine of which are in western Washington. The most satisfactory plant ranges are those of the southeastern species of pines, worked out by Mohr, and those of Pseudotsuga mucronata, Pinus ponderosa, P. contorta, and other western and eastern trees, worked out by the United States Forest Service.

A large percentage of our botanical areas extend beyond the geographical limits of the United States, and this circumstance has proved particularly unfortunate in connection with our efforts to express the climatic extremes characterizing their limits. Nearly all of the climatic factors also reach higher or lower values in Canada or Mexico than are shown for the United States. In the table showing the extreme values of the climatic data for the United States (table 152) the bold-faced figures indicate the cases in which the highest or lowest possible values are found in this country. These cases are only 11 out of a total of 62 extremes, for the 31 climatic features.

In a number of cases the extreme values of the climatic indices are found to occur on capes or coastal islands. The smallest number of dry days is found at Cape Hatteras, as well as the highest value of the physiological moisture-temperature index; the highest normal daily mean precipitation and the highest values of the moisture ratios are found at Cape Flattery, and the highest temperature summations at Key West. Nineteen out of the 62 extremes have been derived from stations of this character. The fact that all of these coastal stations are situated where vegetation is extremely sparse and rery different from that of the adjacent mainland, has led us to attribute to some of our botanical areas extreme values which may be slightly too high or too low. We have considered, however, that the elimatic conditions of these coastal stations are very nearly like those that are endured by the nearest bodies of regetation growing on the mainland, and that they probably represent the extremes for this regetation 
much more closely than do the climatic values for the nearest mainland stations, which are often far distant.

It has been particularly difficult to determine the southern limits of southeastern plants in peninsular Florida, and our data as to the climatic extremes for plants reaching southern Florida should be regarded as only tentative in those cases in which the values for Key West or Miami have been used.

It is impossible, in general, to determine the optimum value of a given climatic condition by any method which involves taking into account the number of stations with readings of a given value, owing to the irregular distribution of the climatic stations. As an example of what might be done by this method, however, we have constructed the diagram shown in figure 20 , in which is shown graphically the

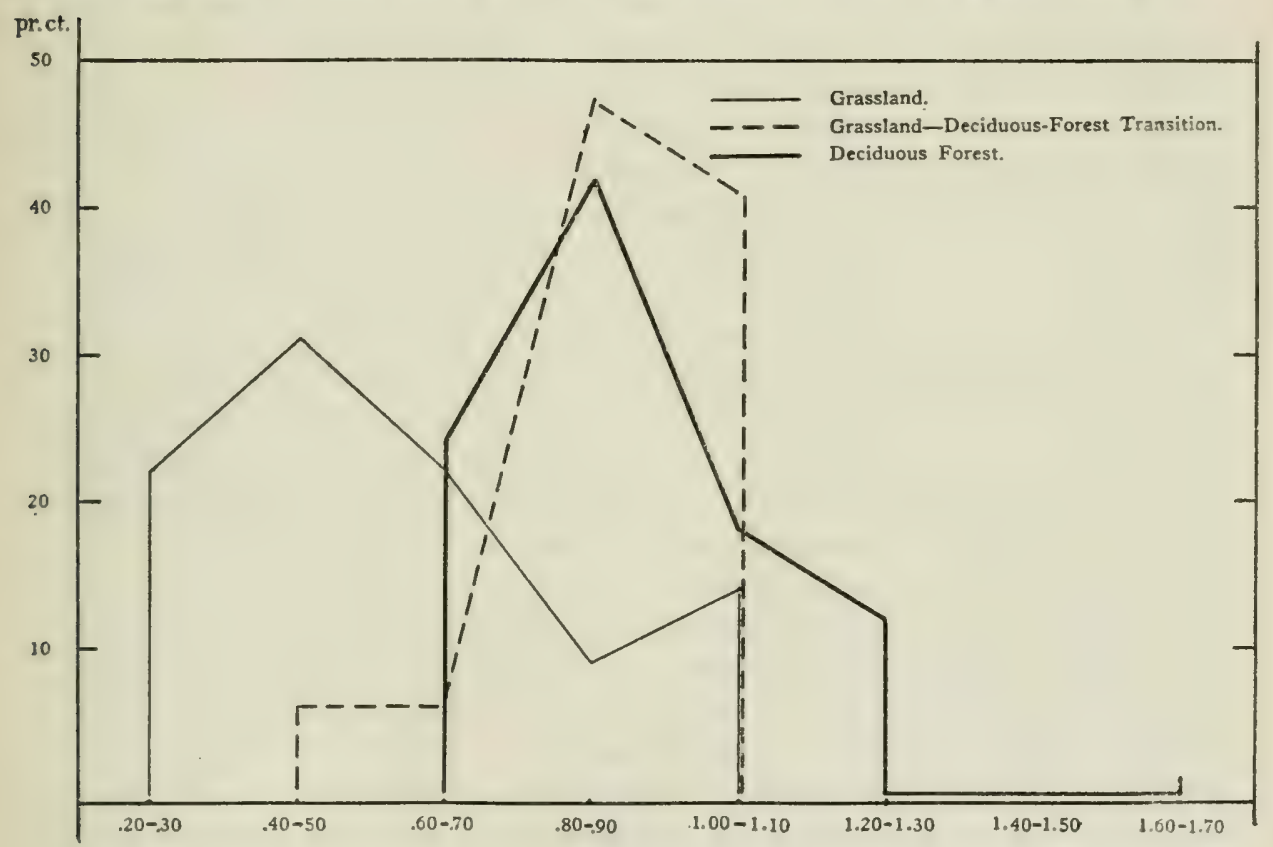

Fig. 20.-Graphs showing the frequency in each of three different vegetations, of the grouped stations to which the progressive values of the moisture ratio $\pi / \mathbf{E}$ correspond.

Ordinates represent frequencies of occurrence of the ratio-value ranges shown as abscissas.

number of stations in the Grassland, the Grassland-Deciduous Forest Transition, and the Deciduous Forest, at which have been determined the several progressive values of the moisture ratio $\pi / E$ that are indicated. As there is a relative uniformity in the distribution of the climatic stations in these three vegetations, and as results have been expressed in percentages of the total number of stations in each vegetation, we may regard this graph as a rough means of showing which of the intensities of this condition is found over the largest area in each of the given vegetations. No ratio values below 0.20 are found in the Grassland, and the greatest number of stations have values 
between 0.40 and 0.50. There are, however, Grassland stations which have readings as high as those of the minimum values for the Transition region. The maxima and minima of the Transition lie within those of the Grassland and the Deciduous Forest, as is shown by the simpler form of graph which we have generally used (figs. 21 to 74). The maximum number of stations for both the Transition and the Deciduous Forest is in the group having values of 1.00 to 1.10 for the moisture ratio. There are only a few stations in the Deciduous Forest for which the readings are higher than in the Transition region. It is impossible to draw definite conclusions from the graph presented, but it may be seen that this method of expressing results would be valuable for cases in which data had been secured for evenly spaced localities.

The climatic extremes given in the accompanying tables have been derived from all of the climatic maps sufficiently detailed for this use. In view of the large number of values involved it seemed desirable to attempt a means of giving a graphic presentation of a portion of these data. This has been done by constructing the graphs shown in figures 21 to 74 . The manner in which these were made is such as to show the relation between the extremes for each plant or vegetation and the extremes for the United States as a whole. The graphs were made by constructing a special scale for each one of the climatic charts. All scales were of the same length and each bore at its left end the minimum value. The scale was then marked so as to read in convenient units the successive values that might be expected at different localities in the United States. To give a concrete example: The lowest and highest values of the normal daily mean precipitation (plate 46) are 0.009 and 0.199 inch respectively. These values were placed at the left and right ends, respectively, of the scale for this climatic feature. The length of the scale in millimeters was then divided by the difference between the extremes, $0.190 \mathrm{inch}$, and the length of scale was calculated that would express 0.010 inch. With this length as a unit the scale was then subdivided by 19 lines, with calculated allowance for the fact that the scale neither started nor ended with the even tens. Out of the 31 sets of climatic extremes given in the tables, 17 were selected as most important, and scales were made for each of them by the above method. The graphs were then constructed by using the appropriate scale to mark off the distances in each block that would express the extremes shown in the table.

Two kinds of graphs have been made, the first of which (figs. 21 to 26) show the limits of the same climatic feature for each of the generalized vegetational areas of the United states. In these graphs each block has been marked off by the same scale, and the maximum and minimum values are therefore given at the top of the graph. In the remaining graphs (figs. 27 to 74 ) are shown the extreme values of 
each of the 17 leading climatic features as read from the distributional areas of various vegetations and species, as described above. In these a different scale has been used in measuring the length of the range of climate in each of the blocks, and therefore it has been impossible to give on them the figures for the extreme values. The same scale was, of course, used in laying out the range of the same climatic feature on all of the graphs.

The reading of these graphs or diagrams may be illustrated by a case of each kind. In figure 21 are shown the extremes of the number of days in the normal frostless season for the 9 types of generalized vegetation of the United States, the extreme range, or amplitude, of this feature being from 25 to 365 days. Reference to the tables will show that the values for the Desert region show a minimum of 25 (or 0 in some anomalous stations in the Klamath Lake region) and a maximum of 305 . The first block of the diagram shows, therefore, the portion of the whole amplitude of this factor in the United States that is to be encountered in the Desert region. It has no reference to the variations in this factor from year to year in the Desert, and gives no indication of the relative proportions of the Desert that are visited by short or long frostless seasons. The second block of this graph shows the minimum, 197, and the maximum, 319, for the Semidesert region. A comparison of this block with the former one gives a means of contrasting the amplitudes of the length of frostless season for these two vegetations. In the seventh block, showing the amplitude for the Southeastern Mesophytic Evergreen Forest, we have a minimum of 131 and a maximum of 365 , this being the vegetational area in which is found the highest value for this climatic feature.

As a rule, the maximum and minimum values are each found only in a single vegetation. In the graph showing the amplitude in the number of hot days (mean daily temperature of $68^{\circ}$ or over), however, it will be seen that the minimum (no hot days) is found in 4 vegetations, the maximum in only 1, while the Hygrophytic Evergreen Forest shows no hot days at all in any part of its area. The position of the shaded portion of each block is determined entirely by the values for the absolute extremes in the United States. These graphs merely depict, in other words, the comparative amplitudes of climatic conditions in the 9 leading vegetational areas of the United States.

The graphs shown in figures 27 to 74 are constructed in the same manner as those just considered. Each figure gives a picture of the range of each of the 17 leading climatic conditions for the area in question. A slight familiarity with the extreme values of these conditions, as given in table 152 , will enable the reader to interpret the graphs, and it will be found much easier to compare the graphs for two species or areas than it is to compare the numerical values on which they are based. 
In a few cases the comparison of graphs has been facilitated by using double blocks. This has been done to show the comparative conditions of the Deciduous Forest region and the Evergreen Needleleaved areas considered collectively (fig. 36), and to show the comparison between the climatic extremes of plants of very different range, in the cases of Sapindus marginatus and Populus balsamifera, and of Cornus canadensis and Spermolepis echinatus.

It is difficult to devise any means of graphically representing the conditions that accompany the zones of diminishing abundance in the case of the charts for the cumulative occurrence of plants of the same growth-form. Three diagrams have been prepared to show the amplitudes of conditions in these cases. One is for the 13 species of commonest eastern deciduous trees, showing the conditions for the areas that have 8 to 12 and 1 to 7 species, respectively. This graph has triple blocks, each of which was constructed by use of the scales that have been described, and the significance of the shading is explained by the key. The other diagrams are for the zones of abundance of Bulbilis and Liriodendron, and are also explained by keys.

All of the graphs showing the range of climatic conditions would have a much greater value if they could be drawn in such a way as to show what part of the total range of a climatic index is characteristic of the largest, best-developed, or most typical part of a given vegetational or distributional area. Several test graphs were prepared showing by vertical lines in each block the values for each of the stations in the distributional area selected. These graphs, however, proved to be not so much an exhibit of the conditions prevailing through most of the area as they were of the irregularity in the location of available meteorological stations, a matter over which we of course had no control. For this reason it has seemed unwise to present these graphs, or to enter into a discussion of this aspect of the correlation problem.

\section{CLIMATIC EXTREMES FOR EACH OF THE VARIOUS VEGETATIONAL FEATURES.}

In the following tables are presented the climatic extremes for all of the botanical areas investigated, as obtained by the methods just described. These cover the general vegetational areas, the life-zones, the areas of relative abundance of growth-forms, the areas of relative abundance of individual species (their ecological distribution), and the distributional areas of individual species. In each table are given the highest and lowest values for each of about 31 climatic conditions for the botanical area in question. The numbers given in the first column of each table refer to the plates on which the climatic data are given in map form. The same numbers are also used in the figures shown on subsequent pages (figs. 21 to 74 ). Temperatures are given in 
degrees Fahrenheit, the length of the period of the average frostless season is expressed by $f s$. Other abbreviations are self-explanatory.

In the majority of cases the highest and lowest climatic values for each area have been read from maps bearing the detailed readings of all stations, but in several cases we have used generalized maps based on the work of others, for which the detailed data have not been published. This is true of the normal daily mean temperature and of the mean annual precipitation (lines 46 and 53 of the tables). In these cases we have derived the extremes directly from the isoclimatic lines, often being compelled to use + and - signs to indicate that the extremities of an area lie well beyond or well short of a given isoclimatic line. These signs have also been used in connection with other conditions whenever a scarcity of stations made it appear more accurate to do so. The use of an isoclimatic line rather than a station for the derivation of a reading is indicated by a superscript $a$ in each case.

In the last table of this series (table 152) are to be found the lowest and highest readings recorded in the United States for each of the 31 climatic conditions, together with the names of the stations to which these extreme values correspond. This table affords a means of comparing the climatic extremes of a given botanical area with the extremes of the entire country. In many cases this comparison has been facilitated by the preparation of graphs, in which blocks representing the entire range of each condition for the United States have been shaded throughout the portion of the range encountered by a given plant or vegetation. Of the 62 national extremes, 11 are the lowest minima or the highest maxima possible in any region, as the minimum of 0 hot days or the maximum of 100 per cent of dry days in the frostless season. These absolute extremes are printed in bold-faced type in table 152. Several other conditions approach their logically absolute values, as the minimum readings of 0.03 and 0.04 for two derivations of the moisture ratio will exemplify. In the majority of the cases, however, the extremes for the United States are exceeded either in Mexico or in Canada as well as elsewhere, as has been said. It is unfortunate that the incompleteness of the basic data on both climate and vegetation have made it impossible to extend the present investigation so as to include the whole land area from the Isthmus of Tehuantepec to the Arctic Ocean. 
TABLE 23.-Climatic extremes for the desert.

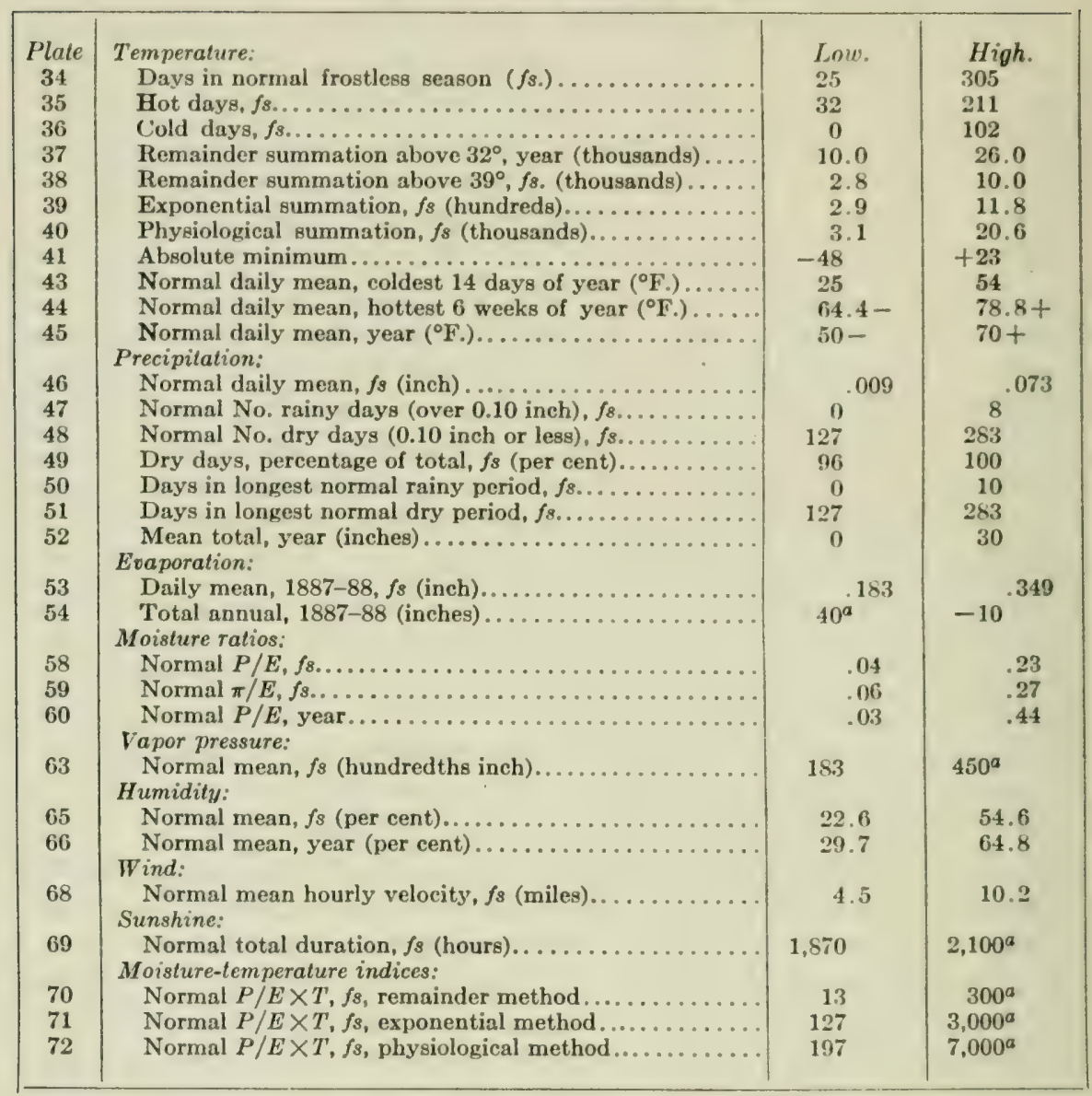

TABLE: 24.-Climatic extremes for the semidesert.

\begin{tabular}{|c|c|c|c|}
\hline Plate & Temperature: & Love. & High. \\
\hline 34 & Days in normal frostless season $f_{s} \ldots . .$. & 197 & 319 \\
\hline 35 & Hot days, $f s_{2} \ldots \ldots \ldots \ldots \ldots \ldots$ & 0 & 218 \\
\hline 36 & Cold days, $f s$. . & 0 & 0 \\
\hline 37 & Remainder summation above $32^{\circ}$, year (thousands) & 11.5 & 26.0 \\
\hline 38 & Remainder summation above $39^{\circ}, f_{s}$ (thousands)... & 5.2 & 10.3 \\
\hline 39 & Exponential summation, $f s$ (hundreds)............. & 5.0 & 11.3 \\
\hline 40 & Physiological summation, $f s$ (thousands)... & 4.1 & 21.4 \\
\hline 41 & 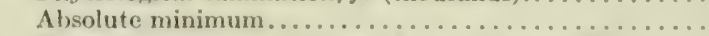 & +5 & +32 \\
\hline 43 & Normal daily mean, coldest 14 days of year $\left({ }^{\circ} \mathrm{F}\right.$. $)$. & $45-$ & 54 \\
\hline 44 & Normal daily mean, hottest 6 weeks of year $\left({ }^{\circ} \mathrm{F}.\right)$. & $6.4 .4-$ & $7 \times .8+$ \\
\hline 45 & Normal daily mean, year $\left({ }^{\circ} F_{0}\right) \ldots \ldots \ldots \ldots \ldots$ & 6i) - & $70+$ \\
\hline 46 & $\begin{array}{l}\text { Precipitation: } \\
\text { Normat daily mean, } f_{3} \text { (inch). }\end{array}$ & $01-$ & $07 \mathrm{~s}$ \\
\hline 47 & Normal No. rainy days (over $0.10 \mathrm{inch}$ ), fs.. & 0 & $62^{0.0}$ \\
\hline 48 & Normal No. dry days (0.10 inch or less), $f s$.. & 231 & 29.4 \\
\hline 49 & Dry days, percentage of total, $f_{s}$ (per cent)... & \&l & 100 \\
\hline 50 & Day's in longest normal rainy period, $f_{s}$. & 0 & 50 \\
\hline 51 & Days in longest normal dry period, $f_{s} .$. & s: & 299 \\
\hline 52 & Mean total, year (inches).............. & 10 & $30+$ \\
\hline
\end{tabular}


TABLE 24.-Climatic extremes for the semidesert-Continued.

\begin{tabular}{|c|c|c|c|}
\hline Plate & Eraporation: & Low. & High. \\
\hline 53 & Daily mean, $1887-88, f_{s}$ (inch) $\ldots \ldots \ldots \ldots \ldots \ldots \ldots$ & .102 & .268 \\
\hline 54 & 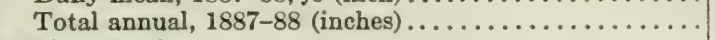 & 32.5 & 65.8 \\
\hline & Moisture ratios: & & \\
\hline 58 & Normal $P / E, f s .$. & .08 & .77 \\
\hline 59 & 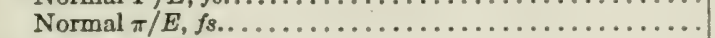 & .10 & .81 \\
\hline 60 & 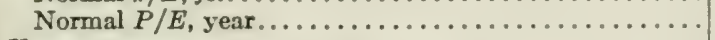 & .15 & .76 \\
\hline 63 & $\begin{array}{l}\text { Vapor pressure: } \\
\text { Normal mean, fs (hundredths inch)................ } \\
\text { Humidity; }\end{array}$ & 296 & 675 \\
\hline 65 & 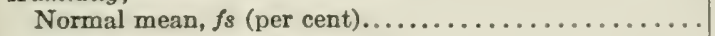 & 48.9 & 81.9 \\
\hline 66 & $\begin{array}{l}\text { Normal mean, year (per cent) } \ldots \ldots \ldots \ldots \ldots \ldots \ldots \ldots \\
\text { Wind: }\end{array}$ & 48.1 & 82.1 \\
\hline 68 & $\begin{array}{l}\text { Normal mean hourly velocity, fs (miles)............. } \\
\text { Sunshine: }\end{array}$ & 4.5 & 12.3 \\
\hline 69 & $\begin{array}{l}\text { Normal total duration, } f_{s} \text { (hours) } \ldots \ldots \ldots \ldots \ldots \ldots \\
\text { Moisture-temperature indices: }\end{array}$ & 2,615 & 2,995 \\
\hline 70 & Normal $P / E \times T, f s$, remainder method... & 68 & 737 \\
\hline 71 & Normal $P / E \times T, f_{s}$, exponential method........... & 625 & 6,690 \\
\hline 72 & 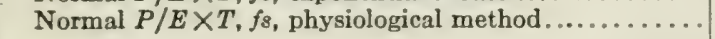 & 1,186 & 13,926 \\
\hline
\end{tabular}

TABLE 25.-Climatic extremes for the grassland.

\begin{tabular}{|c|c|c|c|}
\hline Plate & Temperature: & Low. & High. \\
\hline 34 & Days in normal frostless season $(f s) \ldots \ldots \ldots \ldots$ & 36 & 245 \\
\hline 35 & 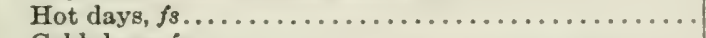 & 18 & 163 \\
\hline 36 & Cold days, $f_{s} \ldots \ldots \ldots \ldots \ldots \ldots \ldots \ldots \ldots \ldots$ & 0 & 158 \\
\hline 37 & Remainder summation above $32^{\circ}$, year (thousands).... & 10.0 & 18.0 \\
\hline 38 & Remainder summation above $39^{\circ}, f_{s}$ (thousands)...... & 2.9 & 7.9 \\
\hline 39 & Exponential summation, $f_{s}$ (hundreds) ............. & 3.0 & 8.7 \\
\hline 40 & Physiological summation, $f_{s}$ (thousands) ............ & 3.7 & 15.9 \\
\hline 41 & 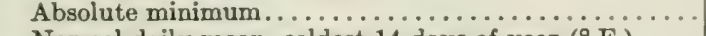 & -65 & +4 \\
\hline 43 & Normal daily mean, coldest 14 days of year $\left({ }^{\circ} \mathrm{F}_{2}\right) \ldots .$. & 0 & 42 \\
\hline 44 & Normal daily mean, hottest 6 weeks of year $\left({ }^{\circ}\right.$ F.)... & $64.4-$ & $78.8+$ \\
\hline 45 & $\begin{array}{l}\text { Normal daily mean, year }\left({ }^{\circ} \mathrm{F} .\right) \ldots \ldots \ldots \ldots \ldots \ldots \ldots \\
\text { Precipitation: }\end{array}$ & 35 & $65+$ \\
\hline 46 & Normal daily mean, $f_{s}$ (inch) $\ldots \ldots \ldots \ldots \ldots \ldots$ & .045 & .116 \\
\hline 47 & Normal No. rainy days (over $0.10 \mathrm{inch}$ ), $f s \ldots . \ldots \ldots$ & 0 & 99 \\
\hline 48 & Normal No. dry days (0.10 inch or less), fs......... & 55 & 192 \\
\hline 49 & Dry days, percentage of total, $f s$ (per cent)......... & 36 & 100 \\
\hline 50 & Days in longest normal rainy period, $f_{8} \ldots \ldots \ldots \ldots$ & 0 & 75 \\
\hline 51 & Days in longest normal dry period, $f s \ldots . . . \ldots \ldots$ & 26 & 153 \\
\hline 52 & $\begin{array}{l}\text { Mean total, year (inches) } \ldots \ldots \ldots \ldots \ldots \ldots \ldots \ldots \\
\text { Evaporation: }\end{array}$ & 20 & 30 \\
\hline 53 & Daily mean, $1887-88, f s$ (inch) $\ldots \ldots \ldots \ldots \ldots \ldots$ & .117 & .275 \\
\hline 54 & $\begin{array}{l}\text { Total annual, 1887-88 (inches) } \ldots \ldots \ldots \ldots \ldots \ldots \ldots \\
\text { Moisture Ratios: }\end{array}$ & 22.1 & 54.4 \\
\hline 58 & Normal $P / E, f_{3} \ldots \ldots \ldots \ldots \ldots \ldots \ldots \ldots$ & .19 & .94 \\
\hline 59 & Normal $\pi / E, f_{s} \ldots \ldots \ldots \ldots \ldots \ldots \ldots \ldots$ & .25 & 1.10 \\
\hline 60 & 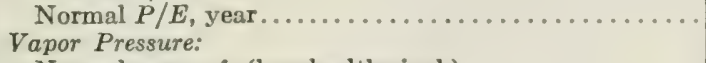 & .20 & 1.74 \\
\hline 63 & $\begin{array}{l}\text { Normal mean, } f_{s} \text { (hundredths inch)....... } \\
\text { Humidity: }\end{array}$ & 253 & 456 \\
\hline 65 & 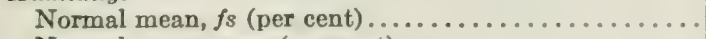 & 45.8 & 71.5 \\
\hline 66 & $\begin{array}{l}\text { Normal mean, year (per cent) } \ldots \ldots \ldots \ldots \ldots \ldots \ldots \\
\text { Wind: }\end{array}$ & 48.1 & 74.1 \\
\hline 68 & $\begin{array}{l}\text { Normal mean hourly velocity, fs (miles) ................ } \\
\text { Sunshine: }\end{array}$ & 7.4 & 14.2 \\
\hline 69 & $\begin{array}{l}\text { Normal total duration, } f s \text { (hours)..... } \\
\text { Moisture-temperature indices: }\end{array}$ & 1,127 & $2,100+$ \\
\hline 70 & Normal $P / E \times T, f_{s}$ remainder method.... & 116 & 385 \\
\hline 71 & Normal $P / E \times T, f_{8}$, exponential method......... & 563 & 3,520 \\
\hline 72 & Normal $P / E \times T, f s$, physiological method.......... & 710 & 7,028 \\
\hline
\end{tabular}


TABLE 26.-Climatic extremes for the grassland-deciduous-forest transition.

\begin{tabular}{|c|c|c|c|}
\hline Plate & Temperalure: & Lovo. & High. \\
\hline 34 & Days in normal frostless season $(f s)$ & 125 & 276 \\
\hline 35 & Hot days, $f_{s} \ldots \ldots \ldots \ldots \ldots \ldots$. & $0^{\alpha}$ & 173 \\
\hline 36 & Cold days, $f_{8} \ldots \ldots \ldots$. & 0 & $150+a$ \\
\hline 37 & Remainder summation above $32^{\circ}$, year (thousands) & 10.0 & 18.0 \\
\hline 38 & Remainder summation above $39^{\circ}, f_{s}$ (thousands). & 3. $0^{2}$ & 9.3 \\
\hline 39 & Exponential summation, $f_{s}$ (hundreds).......... & $3.0-a$ & 10.3 \\
\hline 40 & Physiological summation, $f_{s}$ (thousands) & $5.0^{3}$ & 19.2 \\
\hline 41 & Absolute minimum............... & -48 & +4 \\
\hline 43 & Normal daily mean, coldest 14 days of year $\left({ }^{\circ} \mathrm{F}.\right)$. & $5-a$ & 51 \\
\hline 44 & Normal daily mean, hottest 6 weeks of year $\left({ }^{\circ} \mathrm{F}\right.$.). & 64.4 & $78.8+$ \\
\hline 45 & Normal daily mean, year $\left({ }^{\circ} \mathrm{F}.\right) \ldots \ldots \ldots \ldots \ldots \ldots$ & 35 & \\
\hline 46 & $\begin{array}{l}\text { Precipilation: } \\
\quad \text { Normal daily mean, } f_{s} \text { (inch) }\end{array}$ & .080 & .135 \\
\hline 47 & Normal No. rainy days (over 0.10 inch), $f s$. & 58 & 151.00 \\
\hline 48 & Normal No. dry days (0.10 inch or less), $f_{s}$. & 25 & 211 \\
\hline 49 & Dry days, percentage of total, $f_{s}$ (per cent). & 19 & 78 \\
\hline 50 & Days in longest normal rainy period, $f s$. & 18 & 136 \\
\hline 51 & Days in longest normal dry period, $f s \ldots$ & 12 & 59 \\
\hline 52 & $\begin{array}{l}\text { Mean total, year (inches) .............. } \\
\text { Evaporation. }\end{array}$ & $30-$ & 40 \\
\hline & $\begin{array}{l}\text { Evaporation: } \\
\text { Daily mean, } 1887-88, f s \text { (inch). }\end{array}$ & & \\
\hline $\begin{array}{l}53 \\
54\end{array}$ & Total annual, 1887-88 (inches)... & $20^{3}$ & 52.4 \\
\hline & Moisture ratios: & & \\
\hline 58 & Normal $P / E, f_{s}$. . & .51 & 1.03 \\
\hline 59 & Normal $\pi / E, f_{s}$. & .64 & 1.14 \\
\hline 60 & Normal $P / E$, year.................... & .65 & 1.02 \\
\hline 63 & $\begin{array}{l}\text { Vapor pressure: } \\
\text { Normal mean, } f_{s} \text { (hundredths inch). } \\
\text { Humidity: }\end{array}$ & $350-a$ & 540 \\
\hline 65 & Normal mean, fs (per cent). & 64.5 & 70.7 \\
\hline 66 & $\begin{array}{l}\text { Normal mean, year (per cent).......... } \\
\text { Wind: }\end{array}$ & 66.6 & 75.0 \\
\hline 68 & $\begin{array}{l}\text { Normal mean hourly velocity, } f_{s} \text { (miles). } \\
\text { Sunshine: }\end{array}$ & 6.0 & 12.3 \\
\hline 69 & $\begin{array}{l}\text { Normal total duration, } f_{s} \text { (hours). } \\
\text { Moisture-temperature indices: }\end{array}$ & $1,300-$ & 2,343 \\
\hline 70 & Normal $P / E \times T, f s$, remainder method. & $300^{a}$ & 611 \\
\hline 71 & Normal $P / E \times T, f s$, exponential method. & 3,373 & 4,734 \\
\hline 72 & Normal $P / E \times T, f s$, physiological method. & $3,000-$ & 9,716 \\
\hline
\end{tabular}

TABLE 27.-Climatic extremes for the deciduous-forest.

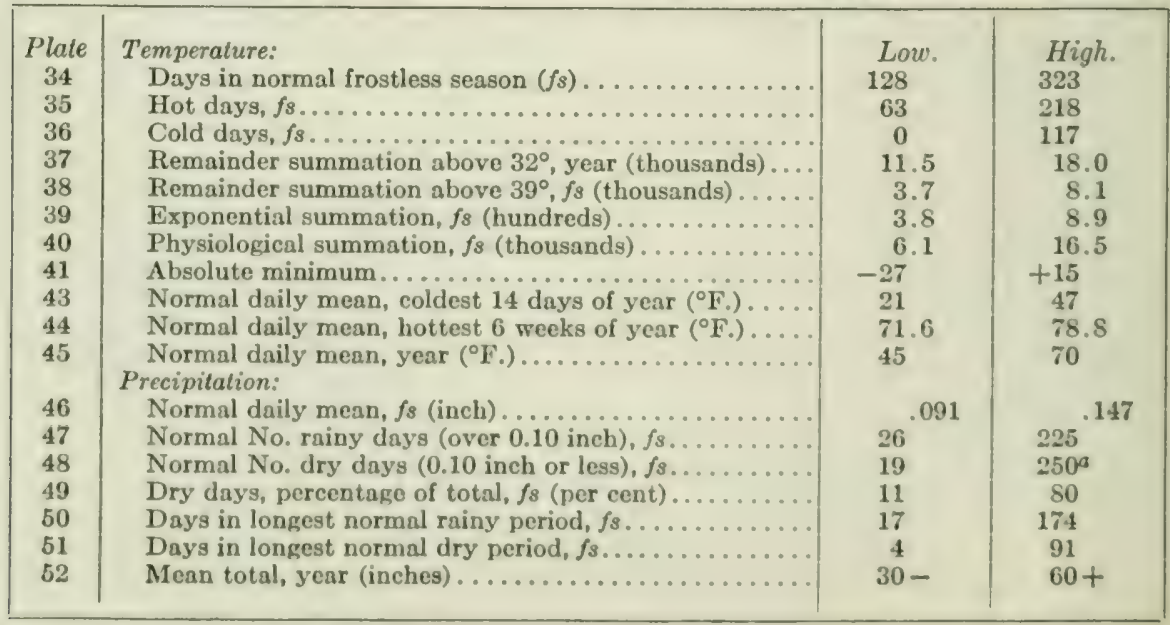


TABLE 27.-Climatic extremes for the deciduous-forest-Continued.

\begin{tabular}{|c|c|c|c|}
\hline Plate & Evaporation: & Low. & High. \\
\hline 53 & Daily mean, $1887-88, f_{s}($ inch $) \ldots \ldots \ldots \ldots \ldots \ldots$ & .081 & .200 \\
\hline 54 & Total annual, 1887-88 (inches) $\ldots \ldots \ldots \ldots \ldots \ldots \ldots$ & 20.3 & 54.8 \\
\hline 58 & $\begin{array}{l}\text { Monsture ratios: } \\
\text { Normal } P / E, f s . .\end{array}$ & .51 & 1.39 \\
\hline 59 & Normal $\pi / E, f 8 \ldots \ldots \ldots \ldots \ldots \ldots \ldots \ldots$ & .66 & 1.63 \\
\hline 60 & $\begin{array}{l}\text { Normal } P / E, \text { year. } \ldots \ldots \ldots \ldots \ldots \ldots \ldots \ldots \ldots \ldots \\
\text { Vapor pressure: }\end{array}$ & .51 & 1.85 \\
\hline 63 & $\begin{array}{l}\text { Normal mean, fs (hundredthe inch) } \ldots \ldots \ldots \ldots \ldots \ldots \\
\text { Humidity: }\end{array}$ & 411 & $600+$ \\
\hline 65 & Normal mean, $f_{s}$ (per cent) ............ & 65.6 & 83.9 \\
\hline 66 & $\begin{array}{l}\text { Normal mean, year (per cent) } \ldots \ldots \ldots \ldots \ldots \ldots \ldots \\
\text { Wind: }\end{array}$ & 67.5 & 82.1 \\
\hline 68 & $\begin{array}{l}\text { Normal mean hourly velocity, } f s \text { (miles) ........... } \\
\text { Sunshine: }\end{array}$ & 3.6 & 12.5 \\
\hline 69 & $\begin{array}{l}\text { Normal total duration, } f s \text { (hours) } \ldots \ldots \ldots \ldots \ldots \ldots \\
\text { Moisture-temperaiture indices: }\end{array}$ & 1,468 & $2,300+$ \\
\hline 70 & Normal $P / E \times T, f s$, remainder method... & 301 & $1,100^{a}$ \\
\hline 71 & Normal $P / E \times T, f_{s}$, exponential method.......... & 2,914 & $10,000^{a}$ \\
\hline 72 & Normal $P / E \times T, f s$, physiological method......... & 3,819 & $20,000^{a}$ \\
\hline
\end{tabular}

TABLE 28.-Climatic extremes for the northwestern hygrophytic evergreen forest.

\begin{tabular}{|c|c|c|c|}
\hline Plate & Temperature: & Low. & High. \\
\hline 34 & Days in normal frostless season $(f s) \ldots \ldots \ldots \ldots \ldots$ & 103 & 316 \\
\hline 35 & 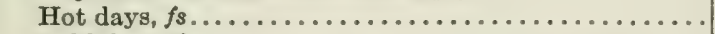 & 0 & 0 \\
\hline 36 & Cold days, $f s \ldots \ldots \ldots \ldots \ldots \ldots \ldots \ldots \ldots \ldots \ldots \ldots$ & $\mathbf{0}$ & 0 \\
\hline 37 & Remainder summation above $32^{\circ}$, year (thousands).... & $11.5 \pm$ & $\ldots$ \\
\hline 38 & Remainder summation above $39^{\circ}, f s$ (thousands)...... & 3.8 & 4.6 \\
\hline 39 & 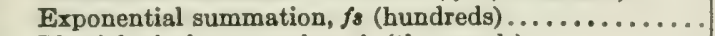 & 4.1 & 5.0 \\
\hline 40 & 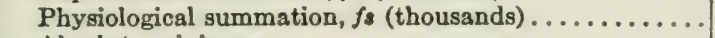 & 1.9 & 4.8 \\
\hline 41 & 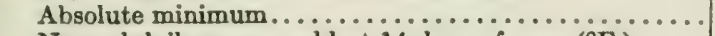 & -6 & +30 \\
\hline 43 & Normal daily mean, coldest 14 days of year $\left({ }^{\circ} \mathrm{F}.\right) . .$. & $35^{a}$ & $45 \mathrm{~s}$ \\
\hline 44 & Normal daily mean, hottest 6 weeks of year $\left({ }^{\circ} \mathrm{F}.\right) \ldots$. & $64.4 \pm$ & $\because \cdots$ \\
\hline 45 & $\begin{array}{l}\text { Normal daily mean, year }\left({ }^{\circ} \mathrm{F} .\right) \ldots \ldots \ldots \ldots \ldots \ldots \ldots \\
\text { Precipitation: }\end{array}$ & $50-$ & $55+$ \\
\hline 46 & 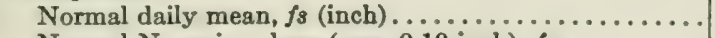 & .042 & .199 \\
\hline 47 & Normal No. rainy days (over 0.10 inch), $f_{3} \ldots \ldots \ldots \ldots$ & 1 & 199 \\
\hline 48 & Normal No. dry days $(0.10$ inch or less $), f s \ldots . . .$. & 72 & 200 \\
\hline 49 & Dry days, percentage of total, $f s$ (per cent).......... & 27 & 100 \\
\hline 50 & Days in longest normal rainy period, $f_{s} \ldots \ldots \ldots \ldots$ & 0 & 99 \\
\hline 51 & Days in longest normal dry period, $f_{s} \ldots \ldots \ldots \ldots \ldots$ & 56 & 198 \\
\hline 52 & $\begin{array}{l}\text { Mean total, year (inches) } \ldots \ldots \ldots \ldots \ldots \ldots \ldots \ldots \ldots \ldots \\
\text { Evaporation: }\end{array}$ & 50 & 90 \\
\hline 53 & Daily mean, $1887-88, f_{8}$ (inch) $\ldots \ldots \ldots \ldots \ldots \ldots$ & .052 & .143 \\
\hline 54 & $\begin{array}{l}\text { Total annual, } 1887-88 \text { (inches) } \ldots \ldots \ldots \ldots \ldots \ldots \ldots \ldots \\
\text { Moisture ratios: }\end{array}$ & 18.1 & 39.2 \\
\hline 58 & Normal $P / E, f s \ldots \ldots \ldots \ldots \ldots \ldots \ldots \ldots \ldots \ldots \ldots \ldots \ldots \ldots \ldots$ & .29 & 3.84 \\
\hline 59 & Normal $\pi / E, f_{s} \ldots \ldots \ldots \ldots \ldots \ldots \ldots \ldots \ldots \ldots$ & .41 & 4.48 \\
\hline 60 & 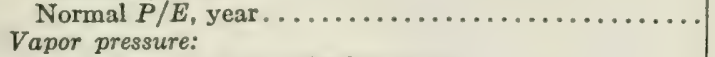 & .88 & 4.90 \\
\hline 63 & $\begin{array}{l}\text { Normal mean, } f_{s} \text { (hundredths inch) } \ldots \ldots \ldots \ldots \ldots \ldots \\
\text { Humidity: }\end{array}$ & 318 & 328 \\
\hline 65 & 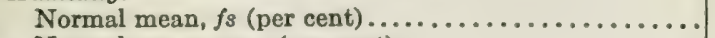 & 71.4 & $80+a$ \\
\hline 66 & $\begin{array}{l}\text { Normal mean, year (per cent) } \ldots \ldots \ldots \ldots \ldots \ldots \ldots \ldots \\
\text { Wind: }\end{array}$ & 74.6 & $76.2+$ \\
\hline 68 & $\begin{array}{l}\text { Normal mean hourly velocity, fs (miles)............ } \\
\text { Sunshine: }\end{array}$ & 3.5 & 16.4 \\
\hline 69 & $\begin{array}{l}\text { Normal total duration, } f s \text { (hours) } \ldots \ldots \ldots \ldots \ldots \ldots \\
\text { Moisture-temperature indices: }\end{array}$ & 1,500 & $1,500+$ \\
\hline 70 & Normal $P / E \times T, f_{s}$, remainder method... & 126 & 1,566 \\
\hline 71 & Normal $P / E \times T, f s$, exponential method........... & 1,213 & 11,724 \\
\hline 72 & Normal $P / E \times T, f s$, physiological method........... & 1,313 & 7,475 \\
\hline
\end{tabular}


TABLE 29.-Climatic extremes for the southeastern mesophytic evergreen forest.

\begin{tabular}{|c|c|c|c|}
\hline Plate & Temperature: & Low. & High. \\
\hline 34 & Days in normal frostless season $\left(f_{s}\right)$. & 181 & 365 \\
\hline 35 & Hot days, $f_{s} \ldots \ldots \ldots \ldots \ldots \ldots \ldots$. & 91 & 365 \\
\hline 36 & Cold days, $f_{8} \ldots \ldots \ldots \ldots \ldots \ldots \ldots$ & 0 & 27 \\
\hline 37 & Remainder summation above $32^{\circ}$, year (thousands) & 11.5 & 26.0 \\
\hline 38 & Remainder summation above $39^{\circ}, f_{8}$ (thousands) .. & 5.1 & 14.5 \\
\hline 39 & Exponential summation, $f_{s}$ (hundreds)... & 5.3 & 15.4 \\
\hline 40 & Physiological summation, $f_{8}$ (thousands). & 7.8 & 31.1 \\
\hline 41 & Absolute minimum............... & -15 & +41 \\
\hline 43 & Normal daily mean, coldest 14 days of year $\left({ }^{\circ} \mathrm{F}\right.$. & 32 & 69 \\
\hline 44 & Normal daily mean, hottest 6 weeks of year $\left({ }^{\circ} \mathrm{F}\right.$. $)$ & 78.8 & $78.8 \pm$ \\
\hline 45 & $\begin{array}{l}\text { Normal daily mean, year }\left({ }^{\circ} \mathrm{F} .\right) \ldots \ldots \ldots \ldots \ldots \\
\text { Precipitation: }\end{array}$ & $55-$ & $75+$ \\
\hline 46 & Normal daily mean, $f s$ (inch). . & 106 & .172 \\
\hline 47 & Normal No. rainy days (over $0.10 \mathrm{inch}$ ), $f_{s}$. & 91 & 284 \\
\hline 48 & Normal No. dry days ( 0.10 inch or less), $f_{s}$. & 0 & 204 \\
\hline 49 & Dry days, percentage of total, $f_{8}$ (per cent). & 0 & 56 \\
\hline 50 & Days in longest normal rainy period, $f_{3} \ldots$ & 47 & 256 \\
\hline 51 & Days in longest normal dry period, $f 8 \ldots$ & 0 & 182 \\
\hline 52 & $\begin{array}{l}\text { Mean total, year (inches)............ } \\
\text { Evaporation: }\end{array}$ & $50-$ & $60+$ \\
\hline 53 & Daily mean, $1887-88, f_{s}$ (inch). & .084 & .148 \\
\hline 54 & $\begin{array}{l}\text { Total annual, 1887-88 (inches).... } \\
\text { Moisture ratios: }\end{array}$ & 25.2 & 51.6 \\
\hline 58 & Normal $P / E, f_{s}$. & .75 & 1.76 \\
\hline 59 & Normal $\pi / E, f_{3} \ldots$ & .89 & 1.96 \\
\hline 60 & $\begin{array}{l}\text { Normal } P / E \text {, year. } \\
\text { Vapor pressure: }\end{array}$ & .91 & 1.94 \\
\hline 63 & $\begin{array}{l}\text { Normal mean, } f_{s} \text { (hundredths inch). } \\
\text { Humidity: }\end{array}$ & 491 & 707 \\
\hline 65 & Normal mean, $f_{s}$ (per cent).... & 72.9 & 82.7 \\
\hline 66 & $\begin{array}{l}\text { Normal mean, year (per cent)... } \\
\text { Wind: }\end{array}$ & 72.9 & 85.2 \\
\hline 68 & $\begin{array}{l}\text { Normal mean hourly velocity, } f_{s} \text { (miles). . . } \\
\text { Sunshine: }\end{array}$ & 6.3 & 13.5 \\
\hline 69 & Normal total duration, $f_{s}$ (hours) ......... & $1,700^{a}$ & 2,650 \\
\hline 70 & Normal $P / E \times T, f_{8}$, remainder method. & 707 & 1,314 \\
\hline 71 & Normal $P / E \times T, f_{s}$, exponential method. & $6,000-$ & 13,511 \\
\hline 72 & Normal $P / E \times T, f s$, physiological method. & $8,000^{a}$ & 24,265 \\
\hline
\end{tabular}

TABLE 30.-Climatic extremes for the northern mesophytic evergreen forest (West).

\begin{tabular}{|c|c|c|c|}
\hline Plate & Temperalure: & Low. & High. \\
\hline 34 & Days in normal frostless season $(f s)$.. & & \\
\hline & Hot days, $f s \ldots \ldots \ldots \ldots \ldots \ldots \ldots$ & & 105 \\
\hline 36 & Cold days, $f_{s} \ldots \ldots \ldots \ldots \ldots \ldots$ & 78 & $149+$ \\
\hline 37 & Remainder summation above $32^{\circ}$, year (thousands). & 10.0 & 11.5 \\
\hline 38 & Remainder summation above $39^{\circ}, f_{s}$ (thousands).... & 2.4 & 5.2 \\
\hline 39 & Exponential summation, $f_{s}$ (hundreds) ............. & 2.4 & 5.4 \\
\hline 40 & Physiological summation, $f_{s}$ (thousands) ......... & 2.6 & 9.9 \\
\hline 41 & Absolute minimum.......... & -49 & -7 \\
\hline 43 & Normal daily mean, coldest 14 days of year $\left({ }^{\circ} \mathrm{F}.\right)$. & 19 & 41 \\
\hline 44 & Normal daily mean, hottest 6 weeks of year $\left({ }^{\circ} \mathrm{F}\right.$.). & 64.4 & 71.6 \\
\hline 45 & Normal daily mean, year $\left({ }^{\circ} \mathrm{F}.\right) \ldots \ldots \ldots \ldots \ldots$ & $40-$ & $60+$ \\
\hline 46 & $\begin{array}{l}\text { Precipitalion: } \\
\text { Normal daily mean, } f_{s} \text { (inch).. }\end{array}$ & .025 & .070 \\
\hline 47 & Normal No. rainy days (over $0.10 \mathrm{inch}$ ), $f_{3}$. & 0 & 55 \\
\hline 48 & Normal No. dry days (0.10 inch or less), fs. & 140 & 202 \\
\hline 49 & Dry days, percentage of total, $f_{8}$ (per cent). & 78 & 100 \\
\hline 50 & Days in longest normal rainy period, $f_{s} \ldots \ldots \ldots$ & 0 & 36 \\
\hline 51 & Days in longest normal dry period, $f_{s} .$. & 140 & 202 \\
\hline 52 & Mean total, year (inches) ............... & 20 & $50+$ \\
\hline
\end{tabular}


TABLE 30.-Climatic extremes for the northern mesophytic evergreen forest (West)-Continued.

\begin{tabular}{|c|c|c|c|}
\hline Plate & Evaporation: & Low. & High. \\
\hline 53 & Daily mean, $1887-88, f_{s}$ (inch) $\ldots \ldots \ldots \ldots \ldots \ldots$ & $.120-a$ & .262 \\
\hline 54 & $\begin{array}{l}\text { Total annual, } 1887-88 \text { (inches) } \ldots \ldots \ldots \ldots \ldots \ldots \ldots \ldots \\
\text { Moisture ratios: }\end{array}$ & $30-$ & 68.3 \\
\hline 58 & 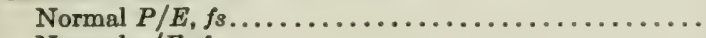 & .10 & .23 \\
\hline 59 & Normal $\pi / E, f_{s} \ldots \ldots \ldots \ldots \ldots \ldots \ldots \ldots \ldots$ & .12 & $.60-a$ \\
\hline 60 & $\begin{array}{l}\text { Normal } P / E \text {, year. . . . . } \ldots \ldots \ldots \ldots \ldots \ldots \ldots \\
\text { Vapor pressure: }\end{array}$ & .14 & $1.30-0$ \\
\hline 63 & $\begin{array}{l}\text { Normal mean, } f_{s} \text { (hundredths inch) } \ldots \ldots \ldots \ldots \ldots \ldots \\
\text { Humidity: }\end{array}$ & 253 & 348 \\
\hline 65 & Normal mean, $f_{s}$ (per cent. . . . . . . . . . . . . & 52.9 & 87.5 \\
\hline 66 & $\begin{array}{l}\text { Normal mean, year (per cent) } \ldots \ldots \ldots \ldots \ldots \ldots \ldots \\
\text { Wind: }\end{array}$ & 60.7 & 86.8 \\
\hline 68 & $\begin{array}{l}\text { Normal mean hourly velocity, } f_{s} \text { (miles) ............ } \\
\text { Sunshine: }\end{array}$ & 5.4 & 6.6 \\
\hline 69 & $\begin{array}{l}\text { Normal total duration, } f s \text { (hours) . . . . . . . . . . . . } \\
\text { Moisture-temperature indices: }\end{array}$ & 1,134 & $2,300^{a}$ \\
\hline 70 & Normal $P / E \times T, f s$, remainder method. & 101 & $200^{a}$ \\
\hline 71 & Normal $P / E \times T, f s$ exponential method........ & 934 & $1,000+a$ \\
\hline 72 & Normal $P / E \times T, f s$, physiological method..... & $1,000^{\alpha}$ & $2,000^{a}$ \\
\hline
\end{tabular}

TABLE 31.-Climatic extremes for the northern mesophytic evergreen forest (East).

\begin{tabular}{|c|c|c|c|}
\hline Plate & Temperature: & Low. & High. \\
\hline 34 & Days in normal frostless season $(f s) \ldots \ldots \ldots \ldots \ldots$ & 85 & $167^{\circ}$ \\
\hline 35 & Hot days, $f_{s} \ldots \ldots \ldots \ldots \ldots \ldots \ldots \ldots \ldots \ldots$ & 0 & 77 \\
\hline 36 & Cold days, $f s . . . \ldots \ldots \ldots \ldots .$. & 66 & $149+$ \\
\hline 37 & Remainder summation above $32^{\circ}$, year (thousands).... & 10.0 & 11.5 \\
\hline 38 & Remainder summation above $39^{\circ}, f_{s}$ (thousands)...... & 2.6 & 4.5 \\
\hline 39 & 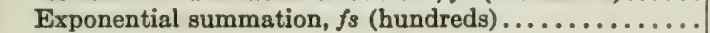 & 2.8 & 4.7 \\
\hline 40 & Physiological summation, $f_{s}$ (thousands)........... & 2.1 & 6.7 \\
\hline 41 & 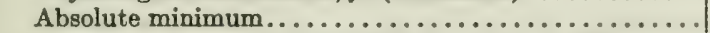 & -48 & -7 \\
\hline 43 & Normal daily mean, coldest 14 days of year ( $\left.{ }^{\circ} \mathrm{F}.\right) . . .$. & $5-a$ & $30+a$ \\
\hline 44 & Normal daily mean, hottest 6 weeks of year ( $\left.{ }^{\circ} \mathrm{F}.\right) . .$. . & 64.4 & 71.6 \\
\hline 45 & $\begin{array}{l}\text { Normal daily mean, year }\left({ }^{\circ} \mathrm{F} .\right) \ldots \ldots \ldots \ldots \ldots \ldots \ldots \\
\text { Precipilation: }\end{array}$ & 35 & 50 \\
\hline 46 & Normal daily mean, $f_{s}($ inch $) \ldots \ldots \ldots \ldots \ldots \ldots$ & .091 & .131 \\
\hline 47 & Normal No. rainy days (over 0.10 inch), $f_{s} . . . \ldots . .$. & 35 & 124 \\
\hline 48 & Normal No. dry days $(0.10$ inch or less $), f s \ldots . . . .$. & 28 & 136 \\
\hline 49 & Dry days, percentage of total, $f s$ (per cent)........... & 18 & 80 \\
\hline 50 & Days in longest normal rainy period, $f s \ldots \ldots \ldots \ldots$ & 22 & 106 \\
\hline 51 & Days in longest normal dry period, $f s . . . \ldots \ldots \ldots$ & 9 & 58 \\
\hline 52 & $\begin{array}{l}\text { Mean total, year (inches) } \ldots \ldots \ldots \ldots \ldots \ldots \ldots \ldots \ldots \\
\text { Evaporation: }\end{array}$ & $30-$ & $50+$ \\
\hline 53 & Daily mean, $1887-88, f s$ (inch) $\ldots \ldots \ldots \ldots \ldots \ldots$ & .084 & .149 \\
\hline 54 & $\begin{array}{l}\text { Total annual, } 1887-88 \text { (inches) } \ldots \ldots \ldots \ldots \ldots \ldots \ldots \ldots \\
\text { Moisture ratios: }\end{array}$ & 21.3 & $30+$ \\
\hline 58 & 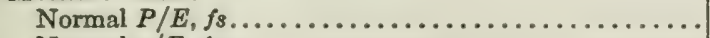 & .71 & 1.23 \\
\hline 59 & Normal $\pi / E, f_{s} \ldots \ldots \ldots \ldots \ldots \ldots \ldots \ldots$ & .81 & 1.52 \\
\hline 60 & 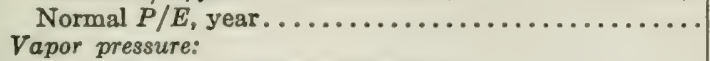 & .82 & 1.72 \\
\hline 63 & $\begin{array}{l}\text { Normal mean, } f_{s} \text { (hundredths inch)................ } \\
\text { Humidity: }\end{array}$ & 345 & $450-a$ \\
\hline 65 & Normal mean, $f s$ (per cent)..... & 70.4 & 81.8 \\
\hline 66 & $\begin{array}{l}\text { Normal mean, year (per cent) } \ldots \ldots \ldots \ldots \ldots \ldots \ldots \\
\text { Wind: }\end{array}$ & 73.4 & 80.2 \\
\hline 68 & $\begin{array}{l}\text { Normal mean hourly velocity, } f_{s} \text { (miles) } \ldots \ldots \ldots \ldots \ldots \\
\text { Sunshine: }\end{array}$ & 4.7 & 12.9 \\
\hline 69 & $\begin{array}{l}\text { Normal total duration, } f_{s} \text { (hours) . . . . . . . . } \\
\text { Moisture-temperature indices: }\end{array}$ & 1,225 & $1,500+a$ \\
\hline 70 & Normal $P / E \times T, f_{s}$, remainder method.... & 301 & 401 \\
\hline 71 & Normal $P / E \times T, f_{s}$, exponential method........... & 2,997 & 3,780 \\
\hline 72 & Normal $P / E \times T, f s$, physiological method........... & 2,747 & $7,000+a$ \\
\hline
\end{tabular}


TABLE 32.-Climatic extremes for the Boreal Region.

\begin{tabular}{|c|c|c|c|}
\hline Plate & Temperature: & Low. & High. \\
\hline 34 & Days in normal frostless season $(f s)$.. & 25 & 183 \\
\hline 35 & Hot days, $f 8 \ldots \ldots \ldots \ldots \ldots \ldots \ldots \ldots \ldots$ & 0 & 105 \\
\hline 36 & Cold days, $f_{8} \ldots$ & 0 & 149 \\
\hline 37 & Remainder summation above $32^{\circ}$, year (thousands). & $10-a$ & $18^{a}$ \\
\hline 38 & Remainder summation above $39^{\circ}, f_{8}$ (thousands) ... & 2.4 & 5.4 \\
\hline 39 & Exponential summation, $f_{8}$ (hundreds) ........... & 245 & 582 \\
\hline 40 & Physiological summation, $f s$ (thousands) ..... & 1,947 & 9,921 \\
\hline 41 & Absolute minimum....... & -54 & +30 \\
\hline 43 & Normal daily mean, coldest 14 days of year $\left({ }^{\circ} \mathrm{F}.\right)$. & 0 & \\
\hline 44 & Normal daily mean, hottest 6 weeks of year $\left({ }^{\circ} \mathrm{F}\right.$. ). & $64.4-^{a}$ & $71.6+6$ \\
\hline 45 & Normal daily mean, year $\left({ }^{\circ} \mathbf{F}.\right) \ldots \ldots \ldots \ldots$ & $35^{a}$ & $55+a$ \\
\hline & Precipitation: & & \\
\hline 46 & Normal daily mean, $f_{s}$ (inch). & .025 & 199 \\
\hline 47 & Normal No. rainy days (over $0.10 \mathrm{inch}$ ), $f s$. & $25-$ & 175 \\
\hline 48 & Normal No. dry days ( 0.10 inch or less), $f_{s}$. & 75 & 200 \\
\hline 49 & Dry days, percentage of total, $f_{s}$ (per cent). & 78 & 100 \\
\hline 50 & Days in longest normal rainy period, $f_{s} . .$. & $25-a$ & $75+a$ \\
\hline 51 & Days in longest normal dry period, $f_{3} \ldots$ & $25-a$ & $200^{a}$ \\
\hline 52 & Mean total, year (inches) ............. & $20-a$ & $90+a$ \\
\hline & Evaporation: & & \\
\hline 53 & Daily mean, $1887-88, f_{8}$ (inch) & .052 & 262 \\
\hline 54 & Total annual, 1887-88 (iuches). & $20-a$ & $80+a$ \\
\hline & Moisture ratios: & & \\
\hline 58 & Normal $P / E, f_{8}$. . & .10 & 3.84 \\
\hline 59 & Normal $\pi / E, f_{3} .$. & .12 & 4.48 \\
\hline 60 & Normal $P / E$, year. & $.20^{a}$ & $4.00^{a}$ \\
\hline & Vapor pressure: & & \\
\hline 63 & $\begin{array}{l}\text { Normal mean, fs (hundredths inch). } \\
\text { Humidity: }\end{array}$ & 249 & 328 \\
\hline 65 & Normal mean, $f_{s}$ (per cent).. & $40-a$ & $80+a$ \\
\hline 66 & $\begin{array}{l}\text { Normal mean, year (per cent).......... } \\
\text { Wind: }\end{array}$ & $40^{a}$ & $80^{\circ}$ \\
\hline 68 & $\begin{array}{l}\text { Normal mean hourly velocity, } f_{s} \text { (miles). } \\
\text { Sunshine: }\end{array}$ & $6-a$ & $10+a$ \\
\hline 69 & $\begin{array}{l}\text { Normal total duration, } f_{s} \text { (hours). } \\
\text { Moisture-temperature indices: }\end{array}$ & $1,300^{a}$ & $1,700^{a}$ \\
\hline 70 & $\begin{array}{l}\text { Moisture-temperature indices: } \\
\text { Normal } P / E \times T, f_{s} \text {, remainder method. }\end{array}$ & $1-a$ & $4^{a}$ \\
\hline 71 & Normal $P / E \times T, f_{s}$ exponential method. & $1-a$ & $15+a$ \\
\hline 72 & Normal $P / E \times T, f_{s}$, physiological method.. & $1-a$ & $7^{a}$ \\
\hline
\end{tabular}

TABLE 33.-Climatic extremes for the transition zone.

\begin{tabular}{|c|c|c|c|}
\hline Plate & Temperature: & Lou. & Hioh. \\
\hline 34 & Days in normal frostless season $(f s) \ldots \ldots \ldots \ldots$ & 25 & 316 \\
\hline 35 & Hot days, $f_{s} \ldots \ldots \ldots \ldots \ldots \ldots \ldots \ldots \ldots$ & 0 & 105 \\
\hline 36 & Cold days, fs.... & 0 & 152 \\
\hline 37 & Remainder summation above $32^{\circ}$, year (thousands). & $10-a$ & $18+a$ \\
\hline 38 & Remainder summation above $39^{\circ}, f_{8}$ (thousands)... & 3,053 & 4,617 \\
\hline 39 & Exponential summation, $f_{8}$ (hundreds)............ & 297 & 545 \\
\hline 40 & Physiological summation, $f_{8}$ (thousands) $\ldots \ldots \ldots \ldots$. & 2,693 & 6,271 \\
\hline 41 & 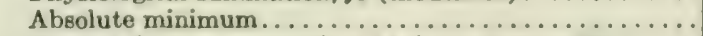 & -59 & +22 \\
\hline 43 & Normal daily mean, coldest 14 days of year $\left({ }^{\circ} \mathrm{F}\right.$ ) . & 6 & 51 \\
\hline 44 & Normal daily mean, hottest 6 weeks of year $\left({ }^{\circ} \mathrm{F}.\right)$.. & $64.4-6$ & $78.8^{a}$ \\
\hline 45 & Normal daily mean, year $\left({ }^{\circ} \mathrm{F}.\right) \ldots$ & $40-a$ & $60+a$ \\
\hline & Precipitation: & & \\
\hline 46 & Normal daily mean, $f_{8}$ (inch). & .009 & 152 \\
\hline 47 & Normal No. rainy days (over $0.10 \mathrm{inch}$ ), $f_{8} \ldots$ & 25 & 175 \\
\hline 48 & Normal No. dry days (0.10 inch or less), fs... & 75 & 250 \\
\hline 49 & Dry days, percentage of total, $f_{8}$ (per cent).. & 53 & 100 \\
\hline 50 & Days in longest normal rainy period, $f_{s} . . . \ldots \ldots$ & $25-0$ & $75+0$ \\
\hline 51 & Days in longest normal dry period, $f_{s} \ldots \ldots \ldots \ldots$. & $50^{a}$ & $250^{\circ}$ \\
\hline 52 & Mean total, year (inches) . . . . . . . . . . . . . . . & $10^{a}$ & $80^{\circ}$ \\
\hline
\end{tabular}


TABLE 33.-Climatic extremes for the transition zone-Continued.

\begin{tabular}{|c|c|c|c|}
\hline Plate & Evaporation: & Low. & High. \\
\hline 53 & Daily mean, $1887-88, f s$ (inch). & .064 & 293 \\
\hline & Total annual, 1887-88 (inches). & & $90^{\alpha}$ \\
\hline & Moisture ratios: & & \\
\hline 58 & Normal $P / E, f_{s}$. & 18 & 1.90 \\
\hline 59 & Normal $\pi / E, f_{s} .$. & 25 & 2.35 \\
\hline 60 & Normal $P / E$, year. & $20-a$ & $4.00^{\circ}$ \\
\hline & Vapor pressure: & & \\
\hline 63 & $\begin{array}{l}\text { Normal mean, fs (hundredths inch)....... } \\
\text { Humidity: }\end{array}$ & 183 & 377 \\
\hline 65 & Normal mean, fs (per cent..... & $40-a$ & $80+a$ \\
\hline 66 & $\begin{array}{l}\text { Normal mean, year (per cent)........ } \\
\text { Wind: }\end{array}$ & $40^{a}$ & $80^{a}$ \\
\hline 68 & $\begin{array}{l}\text { Normal mean hourly velocity, } f_{s} \text { (miles). } \\
\text { Sunshine: }\end{array}$ & $6^{a}$ & $16^{a}$ \\
\hline 69 & $\begin{array}{l}\text { Normal total duration, } f s \text { (hours). . } \\
\text { Moisture-temperature indices: }\end{array}$ & 1,127 & 2,995 \\
\hline 70 & Normal $P / E \times T, f s$, remainder method. & $1-a$ & $3+a$ \\
\hline 71 & Normal $P / E \times T, f_{s}$ exponential method.. & $1-a$ & $15+c$ \\
\hline 72 & Normal $P / E \times T, f s$, physiological method.... & $1-a$ & $3^{\alpha}$ \\
\hline
\end{tabular}

TABLE 34.-Climatic extremes for the Alleghanian zone.

\begin{tabular}{|c|c|c|c|}
\hline Plate & Temperature: & Low. & High. \\
\hline 34 & Days in normal frostless season $(f s) \ldots$ & 106 & 211 \\
\hline 35 & Hot days, $f s \ldots \ldots \ldots \ldots \ldots \ldots \ldots \ldots$ & 0 & 128 \\
\hline 36 & Cold days, $8 . . . . .$. & 0 & 150 \\
\hline 37 & Remainder summation above $32^{\circ}$, year (thousands). & $10-a$ & $11.5+a$ \\
\hline 38 & Remainder summation above $39^{\circ}, f_{s}$ (thousands).. & 2,606 & 5,238 \\
\hline 39 & Exponential summation, $f_{s}$ (hundreds) $\ldots \ldots \ldots \ldots$ & 299 & 546 . \\
\hline 40 & Physiological summation, fs (thousands). . & 2,102 & 10,886 \\
\hline 41 & Absolute minimum .................. & -59 & -4 \\
\hline 43 & Normal daily mean, coldest 14 days of year ( ${ }^{\circ} \mathrm{F}$.). & 0 & 40 \\
\hline 44 & Normal daily mean, hottest 6 weeks of year $\left({ }^{\circ} \mathrm{F}.\right)$. & $64.4-a$ & $71.6+a$ \\
\hline 45 & $\begin{array}{l}\text { Normal daily mean, year }\left({ }^{\circ} \mathrm{F} .\right) \ldots \ldots \ldots \ldots \ldots \ldots \\
\text { Precipitation: }\end{array}$ & $40-a$ & $55+^{a}$ \\
\hline 46 & Normal daily mean, fs (inch)...... & 093 & 1.31 \\
\hline 47 & Normal No. rainy days (over $0.10 \mathrm{inch}$ ), $f s$. & 25 & 150 \\
\hline 48 & Normal No. dry days ( 0.10 inch or less), $f_{8}$. . & 50 & 100 \\
\hline 49 & Dry days, percentage of total, fs (per cent). & 17 & 83 \\
\hline 50 & Days in longest normal rainy period, $f_{s} .$. & $25^{a}$ & $125^{a}$ \\
\hline 51 & Days in longest normal dry period, $f_{3} .$. & $25-a$ & $75^{a}$ \\
\hline 62 & Mean total, year (inches). & $20^{a}$ & $60+a$ \\
\hline & Evaporation: & & \\
\hline 53 & Daily mean, $1887-88$, fs (inch).. & .084 & 1.66 \\
\hline 54 & $\begin{array}{l}\text { Total annual, } 1887-88 \text { (inches).. } \\
\text { Moisture ratios: }\end{array}$ & $20^{a}$ & $50^{a}$ \\
\hline 58 & Normal $P / E, f_{s} \ldots$ & 66 & 1.31 \\
\hline 59 & Normal $\pi / E, f_{8} \ldots \ldots$ & 82 & 1.63. \\
\hline 60 & $\begin{array}{l}\text { Normal } P / E \text {, year...................... } \\
\text { Vapor pressure: }\end{array}$ & $60^{a}$ & $1.80^{\circ}$ \\
\hline 63 & $\begin{array}{l}\text { Normal mean, } f_{s} \text { (hundredths inch).... } \\
\text { Humidity: }\end{array}$ & 405 & 428 \\
\hline 65 & Normal mean, $f_{8}$ (per cent). & $60^{a}$ & $80^{a}$ \\
\hline 66 & $\begin{array}{l}\text { Normal mean, year (per cent)......... } \\
\text { Wind: }\end{array}$ & $70^{a}$ & $80^{\circ}$ \\
\hline 68 & $\begin{array}{l}\text { Normal mean hourly velocity, } f_{8} \text { (miles)... } \\
\text { Sunshine: }\end{array}$ & $6^{a}$ & $12^{a}$ \\
\hline 69 & $\begin{array}{l}\text { Normal total duration, } f s \text { (hours). } \\
\text { Moisture-temperature indices: }\end{array}$ & 1,225 & 1,836 \\
\hline 70 & Normal $P / E \times T, f_{8}$, remainder method. . & $2^{a}$ & $6^{a}$ \\
\hline 71 & Normal $P / E \times T, f_{s}$, exponential method.... & $2^{a}$ & $6+a$ \\
\hline 72 & Normal $P / E \times T, f_{s}$, physiological method.... & $3^{a}$ & $10^{a}$ \\
\hline
\end{tabular}


TABLE 35.-Climatic extremes for the upper Sonoran zone.

\begin{tabular}{|c|c|c|c|}
\hline Plate & Temperature: & Low. & Hioh. \\
\hline 34 & Days in normal frostless season $(f s)$. & 25 & \\
\hline 35 & Hot days, $f s \ldots \ldots \ldots \ldots \ldots \ldots \ldots$ & 0 & 140 \\
\hline 36 & Cold days, $f_{s} \ldots \ldots \ldots$. . & 0 & 134 \\
\hline 37 & Remainder summation above $32^{\circ}$, year (thousands). & $11.5-a$ & $18+a$ \\
\hline 38 & Remainder summation above $39^{\circ}, f_{8}$ (thousands).. & 2,699 & 6,459 \\
\hline 39 & Exponential summation, $f_{s}$ (hundreds) ......... & 402 & 678 \\
\hline 40 & Physiological summation, $f_{8}$ (thousands)....... & 3,640 & 12,448 \\
\hline 41 & Absolute minimum .................. & -65 & +20 \\
\hline 43 & Normal daily mean, coldest 14 days of year $\left({ }^{\circ} \mathrm{F}.\right)$. & $10-$ & $45+$ \\
\hline 44 & Normal daily mean, hottest 6 weeks of year $\left({ }^{\circ} \mathrm{F}.\right)$. & $71.6-^{a}$ & $78.8+a$ \\
\hline 45 & Normal daily mean, year $\left({ }^{\circ} F_{0}\right) \ldots \ldots \ldots \ldots \ldots$ & $40^{a}$ & $65^{a}$ \\
\hline & Precipitation: & & \\
\hline 46 & Normal daily mean, $f_{3}$ (inch). & .022 & 1.03 \\
\hline 47 & Normal No. rainy days (over $0.10 \mathrm{inch}$ ), $f_{s} .$. & $25-$ & $50+$ \\
\hline 48 & Normal No. dry days $(0.10$ inch or less), fs... & 75 & $275+$ \\
\hline 49 & Dry days, percentage of total, $f_{8}$ (per cent).. & 46 & 100 \\
\hline 50 & Days in longest normal rainy period, $f_{s}$. & $25-a$ & $50^{3}$ \\
\hline 51 & Days in longest normal dry period, $f_{3} \ldots$ & $50-^{a}$ & $250+a$ \\
\hline 52 & Mean total, year (inches) ........... & $10-a$ & $30+a$ \\
\hline 5.3 & $\begin{array}{l}\text { Evaporation: } \\
\text { Daily mean, } 1887-88, f_{s} \text { (inch). }\end{array}$ & .166 & .330 \\
\hline 54 & Total annual, 1887-88 (inches)... & $40^{x}$ & $100^{\circ}$ \\
\hline 58 & $\begin{array}{l}\text { Moisture ratios: } \\
\text { Normal } P / E \text { fs. }\end{array}$ & 12 & 60 \\
\hline 59 & Normal $\pi / E, f_{8} .$. & 15 & 73 \\
\hline 60 & 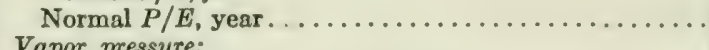 & $10^{a}$ & $40+a$ \\
\hline 63 & $\begin{array}{l}\text { apor pressure: } \\
\text { Normal mean, fs (hundredths inch)... } \\
\text { Humidity: }\end{array}$ & 253 & 386 \\
\hline 65 & Normal mean, $f_{8}$ (per cent). & $40-a$ & $80^{a}$ \\
\hline 66 & $\begin{array}{l}\text { Normal mean, year (per cent) } \ldots \ldots \ldots \ldots \ldots \ldots \\
\text { Wind: }\end{array}$ & $40-a$ & $70+a$ \\
\hline 68 & $\begin{array}{l}\text { Normal mean hourly velocity, } f_{s} \text { (miles)... } \\
\text { Sunshine: }\end{array}$ & $6^{a}$ & $14^{a}$ \\
\hline 69 & $\begin{array}{l}\text { Normal total duration, } f 8 \text { (hours). } \\
\text { Moisture-temperature indices: }\end{array}$ & 1,307 & 2,615 \\
\hline 70 & Normal $P / E \times T, f_{s}$, remainder method. & $1-a$ & $3^{a}$ \\
\hline 71 & Normal $P / E \times T, f s$, exponential method. & $1-a$ & $3^{a}$ \\
\hline 72 & Normal $P / E \times T, f s$, physiological method..... & $1-a$ & $6^{a}$ \\
\hline
\end{tabular}

TABLE 36.-Climatic extremes for the Carolinian zone.

\begin{tabular}{|c|c|c|c|}
\hline Plate & Temperalure: & Low. & High. \\
\hline 34 & Days in normal frostless season $(f s)$. & 127 & 241 \\
\hline 35 & Hot days, $f_{3} \ldots \ldots \ldots \ldots \ldots \ldots \ldots$ & 53 & 148 \\
\hline 36 & Cold days, $f 8, \ldots$. & 0 & 140 \\
\hline 37 & Remainder summation above $32^{\circ}$, year (thousands). & $11.5-a$ & $15+a$ \\
\hline 38 & Remainder summation above $39^{\circ}, f_{s}$ (thousands)... & 3,631 & 7,043 \\
\hline 39 & 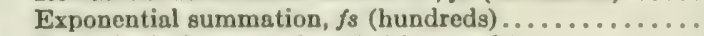 & 370 & 737 \\
\hline 40 & Physiological summation, fs (thousands) ........... & 5,604 & 14,564 \\
\hline 41 & Absolute minimum.. & -43 & +10 \\
\hline 43 & Normal daily mean, coldest 14 days of year $\left({ }^{\circ} \mathrm{F}.\right)$. & $10-$ & 45 \\
\hline 44 & Normal daily mean, hottest 6 weeks of year $\left({ }^{\circ} \mathrm{F}\right.$.). & $71.6-a$ & $78.8+6$ \\
\hline 45 & Normal daily mean, year $\left({ }^{\circ} \mathrm{F}.\right) \ldots \ldots \ldots \ldots \ldots \ldots$ & $45-$ & 65 \\
\hline & Precipitation: & & \\
\hline 46 & Normal daily mean, $f_{s}$ (inch). & 095 & .147 \\
\hline 47 & Normal No. rainy days (over $0.10 \mathrm{inch}$ ), $f_{s} .$. & 25 & 175 \\
\hline 48 & Normal No. dry days (0.10 inch or less), $f s . .$. & 25 & 125 \\
\hline 49 & Dry days, percentage of total, $f_{s}$ (per cent).... & 17 & $5 s$ \\
\hline 50 & Dnys in longest normal rainy period, $f_{s} \ldots \ldots \ldots \ldots$ & $25-a$ & $12.5+9$ \\
\hline 51 & Days in longest normal dry period, $f_{s} \ldots . . . \ldots$. & $25-a$ & $7.5^{a}$ \\
\hline 52 & Mean total, year (inches) $\ldots \ldots \ldots \ldots \ldots \ldots \ldots \ldots$ & $20-0$ & $60^{\circ}$ \\
\hline
\end{tabular}


TABLE 36.-Climatic extremes for the Carolinian zone-Continued.

\begin{tabular}{|c|c|c|c|}
\hline Plate & Evaporation: & Low. & High. \\
\hline 53 & Daily mean, $1887-88, f_{s}$ (inch) $\ldots \ldots \ldots \ldots \ldots \ldots \ldots$ & .81 & .195 \\
\hline 54 & $\begin{array}{l}\text { Total annual, } 1887-88 \text { (inches) } \ldots \ldots \ldots \ldots \ldots \ldots \ldots \ldots \\
\text { Moisture ratios: }\end{array}$ & $30-a$ & $50^{\circ}$ \\
\hline 58 & 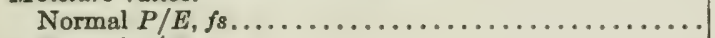 & 43 & 139 \\
\hline 59 & Normal $\pi / E, f_{3} \ldots \ldots \ldots \ldots \ldots \ldots \ldots \ldots \ldots$ & 47 & 163 \\
\hline 60 & $\begin{array}{l}\text { Normal } P / E \text {, year. } \ldots \ldots \ldots \ldots \ldots \ldots \ldots \ldots \ldots \ldots \\
\text { Vapor pressure: }\end{array}$ & $40^{a}$ & $180^{a}$ \\
\hline 63 & $\begin{array}{l}\text { Normal mean, } f_{s} \text { (hundredths inch) .................. } \\
\text { Humidity: }\end{array}$ & 415 & 513 \\
\hline 65 & 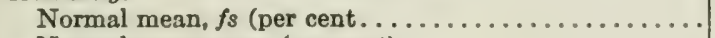 & $60^{\alpha}$ & $80^{\circ}$ \\
\hline 66 & $\begin{array}{l}\text { Normal mean, year (per cent) } \ldots \ldots \ldots \ldots \ldots \ldots \ldots \ldots \\
\text { Wind: }\end{array}$ & $70-a$ & $80+a$ \\
\hline 68 & $\begin{array}{l}\text { Normal mean hourly velocity, } f s \text { (miles)............ } \\
\text { Sunshine: }\end{array}$ & $8^{a}$ & $12^{a}$ \\
\hline 69 & $\begin{array}{l}\text { Normal total duration, } f_{s} \text { (hours) } \ldots \ldots \ldots \ldots \ldots \ldots \\
\text { Moisture-temperature indices: }\end{array}$ & 1,267 & 1,946 \\
\hline 70 & 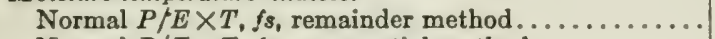 & $-2^{a}$ & $7^{a}$ \\
\hline 71 & Normal $P / E \times T, f_{s}$, exponential method.......... & $2^{a}$ & $7^{a}$ \\
\hline 72 & Normal $P / E \times T, f s$, physiological method........... & $4-a$ & $12^{a}$ \\
\hline
\end{tabular}

TABLE 37.-Climatic extremes for the lower Sonoran zone.

\begin{tabular}{|c|c|c|c|}
\hline Plate & Temperature: & Low. & High. \\
\hline 34 & Days in normal frostless season $(f s)$. . & 106 & 331 \\
\hline 35 & 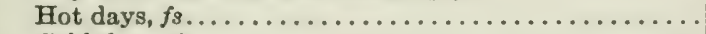 & 50 & 197 \\
\hline 36 & Cold days, $f_{s} \ldots \ldots \ldots \ldots \ldots \ldots \ldots \ldots \ldots \ldots \ldots$ & 0 & 0 \\
\hline 37 & Remainder summation above $32^{\circ}$, year (thousands).... & $11.5-a$ & $18+a$ \\
\hline 38 & Remainder summation above $39^{\circ}, f_{s}$ (thousands)..... & 6,760 & 10,071 \\
\hline 39 & Exponential summation, $f_{s}$ (hundreds) $\ldots \ldots \ldots \ldots \ldots$ & 599 & 1,184 \\
\hline 40 & Physiological summation, $f s$ (thousands) . . . . . . . . & 9,884 & 20,640 \\
\hline 41 & Absolute minimum $\ldots \ldots \ldots \ldots \ldots \ldots \ldots \ldots$ & -14 & +32 \\
\hline 43 & Normal daily mean, coldest 14 days of year $\left({ }^{\circ} \mathrm{F}.\right) \ldots$ & 42 & 54 \\
\hline 44 & Normal daily mean, hottest 6 weeks of year $\left({ }^{\circ} \mathrm{F}\right.$.).... & $78.8-a$ & $78.8+a$ \\
\hline 45 & $\begin{array}{l}\text { Normal daily mean, year }\left({ }^{\circ} \mathrm{F} .\right) \ldots \ldots \ldots \ldots \ldots \ldots \ldots \\
\text { Precipitation: }\end{array}$ & $50-a$ & $70+a$ \\
\hline 46 & Normal daily mean, $f_{s}$ (inch). & .017 & .083 \\
\hline 47 & Normal No. rainy days (over $0.10 \mathrm{inch}$ ), $f_{s} \ldots \ldots \ldots$ & 0 & $50+$ \\
\hline 48 & Normal No. dry days $(0.10$ inch or less $), f s \ldots \ldots \ldots$ & $150-$ & $250+$ \\
\hline 49 & Dry days, percentage of total, $f s$ (per cent) .......... & 77 & 100 \\
\hline 50 & Days in longest normal rainy period, $f s \ldots \ldots \ldots \ldots$ & $25-a$ & $25+a$ \\
\hline 51 & Days in longest normal dry period, $f s \ldots \ldots \ldots \ldots \ldots$ & $50^{a}$ & $250+a$ \\
\hline 52 & $\begin{array}{l}\text { Mean total, year (inches) } \ldots \ldots \ldots \ldots \ldots \ldots \ldots \ldots \\
\text { Evaporation: }\end{array}$ & $10-a$ & $30+a$ \\
\hline 53 & Daily mean, $1887-88, f_{s}$ (inch) $\ldots \ldots \ldots \ldots \ldots \ldots$ & 104 & 273 \\
\hline 54 & $\begin{array}{l}\text { Total annual, } 1887-88 \text { (inches) } \ldots \ldots \ldots \ldots \ldots \ldots \ldots \ldots \\
\text { Moisture ratios: }\end{array}$ & $40-a$ & $100^{a}$ \\
\hline 58 & Normal $P / E, f_{s} \ldots \ldots \ldots \ldots \ldots \ldots \ldots \ldots$ & 08 & 51 \\
\hline 59 & 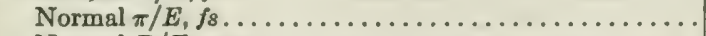 & 13 & 54 \\
\hline 60 & $\begin{array}{l}\text { Normal } P / E, \text { year. } \ldots \ldots \ldots \ldots \ldots \ldots \ldots \ldots \ldots \ldots \ldots \\
\text { Vapor pressure: }\end{array}$ & $10-a$ & $60+^{a}$ \\
\hline 63 & $\begin{array}{l}\text { Normal mean, } f_{s} \text { (hundredths inch) } \ldots \ldots \ldots \ldots \ldots \ldots \\
\text { Humidity: }\end{array}$ & $300^{a}$ & $600^{a}$ \\
\hline 65 & Normal mean, $f s$ (per cent).... & $40-a$ & $70+a$ \\
\hline 66 & $\begin{array}{l}\text { Normal mean, year (per cent)... } \\
\text { Wind: }\end{array}$ & $40-a$ & $72+a$ \\
\hline 68 & $\begin{array}{l}\text { Normal mean hourly velocity, } f_{s} \text { (miles)............ } \\
\text { Sunshine: }\end{array}$ & $6-^{a}$ & $12+a$ \\
\hline 69 & $\begin{array}{l}\text { Normal total duration, } f s \text { (hours) } \ldots \ldots \ldots \ldots \\
\text { Moisture-temperature indices: }\end{array}$ & $2,100-$ & $2,900+$ \\
\hline 70 & 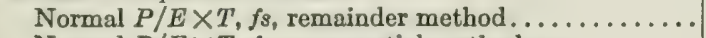 & $1-a$ & $6+a$ \\
\hline 71 & Normal $P / E \times T, f_{8}$, exponential method.......... & $1-a$ & $7^{a}$ \\
\hline 72 & Normal $P / E \times T, f s$, physiological method.......... & $1-a$ & $13^{a}$ \\
\hline
\end{tabular}


TABLE 38.-Climatic extremes for the Austroriparian zone.

\begin{tabular}{|c|c|c|c|}
\hline Plate & Temperature: & Low. & High. \\
\hline 34 & Days in normal frostless season $(f s) \ldots \ldots \ldots$. & 187 & 289 \\
\hline 35 & Hot days, $f 8 \ldots \ldots \ldots \ldots \ldots \ldots \ldots \ldots \ldots \ldots$ & 128 & 197 \\
\hline 36 & Cold days, $f_{8}, \ldots \ldots \ldots \ldots \ldots \ldots \ldots$ & 0 & 22 \\
\hline 37 & Remainder summation above $32^{\circ}$, year (thousands). & $18-a$ & $18+a$ \\
\hline 38 & Remainder summation above $39^{\circ}, f_{8}$ (thousands). & 6,279 & 9,355 \\
\hline 39 & Exponential summation, $f_{s}$ (hundreds). & 673 & 1,028 \\
\hline 40 & Physiological summation, fs (thousands). & 11,879 & 19,202 \\
\hline 41 & Absolute minimum.......... & -31 & +16 \\
\hline 43 & Normal daily mean, coldest 14 days of year $\left({ }^{\circ} \mathrm{F}.\right)$. & 32 & 51 \\
\hline 44 & Normal daily mean, hottest 6 weeks of year $\left({ }^{\circ} \mathrm{F}\right.$.). & $78.8-a$ & $78.8+a$ \\
\hline 45 & Normal daily mean, year $\left({ }^{\circ} \mathrm{F}.\right) \ldots \ldots \ldots \ldots \ldots$ & $60-a$ & $70+a$ \\
\hline 46 & $\begin{array}{l}\text { Precipitation: } \\
\quad \text { Normal daily mean, } f_{s} \text { (inch)... }\end{array}$ & 050 & 170 \\
\hline 47 & Normal No. rainy days (over $0.10 \mathrm{inch}), f_{8}$. & 50 & 250 \\
\hline 48 & Normal No. dry days ( 0.10 inch or less), $f s$. & $25-$ & $250^{a}$ \\
\hline 49 & Dry days, percentage of total, $f_{s}$ (per cent). & 0 & 78 \\
\hline 50 & Days in longest normal rainy period, $f_{s}$.. & $25^{a}$ & $250^{a}$ \\
\hline 51 & Days in longest normal dry period, $f_{s} \ldots .$. & $25^{a}$ & $75^{a}$ \\
\hline 52 & Mean total, year (inches). & $30-a$ & $50+{ }^{\circ}$ \\
\hline & Evaporation: & & \\
\hline 53 & Daily mean, $1887-88, f_{8}$ (inch).. & 96 & \\
\hline 54 & $\begin{array}{l}\text { Total annual, } 1887-88 \text { (inches) ................... } \\
\text { Moisture ratios: }\end{array}$ & $40-a$ & $50+a$ \\
\hline 58 & Normal $P / E, f_{s} \ldots \ldots \ldots \ldots \ldots$ & 51 & 176 \\
\hline 59 & Normal $\pi / E, f_{s} \ldots \ldots \ldots \ldots \ldots \ldots$ & 54 & 196 \\
\hline 60 & Normal $P / E$, year.............. & $60-a$ & $180^{a}$ \\
\hline 63 & $\begin{array}{l}\text { Vapor pressure: } \\
\text { Normal mean, } f_{s} \text { (hundredths inch).. }\end{array}$ & $450^{a}$ & 600 \\
\hline & Humidity: & & \\
\hline 65 & Normal mean, $f_{s}$ (per cent). & $70-a$ & $80+a$ \\
\hline 66 & $\begin{array}{l}\text { Normal mean, year (per cent)............ } \\
\text { Wind: }\end{array}$ & $70-a$ & $80++^{a}$ \\
\hline 68 & $\begin{array}{l}\text { Normal mean hourly velocity, } f_{s} \text { (miles).... } \\
\text { Sunshine: }\end{array}$ & $6-a$ & $12^{a}$ \\
\hline 69 & $\begin{array}{l}\text { Normal total duration, } f_{s} \text { (hours) ............. } \\
\text { Moisture-temperature indices: }\end{array}$ & 1,895 & 2,343 \\
\hline 70 & Normal $P / E \times T, f s$, remainder method. & $4-a$ & $13+a$ \\
\hline 71 & Normal $P / E \times T, f s$, exponential method.. & $4^{a}$ & $14^{a}$ \\
\hline 72 & Normal $P / E \times T, f s$, physiological method. & $8^{a}$ & $20^{a}$ \\
\hline
\end{tabular}

TABLE 39.-Climatic extremes for gulf strip of lower Austral zone.

\begin{tabular}{|c|c|c|c|}
\hline Plate & Temperalure: & Low. & High. \\
\hline 34 & Days in normal frostless sesson $(f s)$. & 247 & \\
\hline 35 & Hot days, $f 8 \ldots \ldots \ldots \ldots \ldots \ldots \ldots$ & 168 & \\
\hline 36 & Cold days, $f_{8} \ldots \ldots \ldots \ldots \ldots \ldots \ldots$ & 0 & \\
\hline 37 & Remainder summation above $32^{\circ}$, year (thousands). & $18+a$ & $26+0$ \\
\hline 38 & Remainder summation above $39^{\circ}$, ss (thousands)... & 8,725 & 10,844 \\
\hline 39 & Exponential summation, fs (hundreds)... & 893 & 1,115 \\
\hline 40 & Physiological summation, fs (thousands) & 16,194 & 21,420 \\
\hline 41 & Absolute minimum $\ldots \ldots \ldots \ldots \ldots \ldots \ldots \ldots \ldots$ & -5 & +23 \\
\hline 43 & Normal daily mean, coldest 14 days of year $\left({ }^{\circ} F_{0}\right)$. & 47 & -60 \\
\hline 44 & Normal daily mean, hottest 6 weeks of year $\left({ }^{\circ} \mathrm{F}.\right)$. & $78.8+a$ & \\
\hline 45 & $\begin{array}{l}\text { Normal daily mean, year }\left({ }^{\circ} \mathrm{F} .\right) . . \\
\text { Precipilation. }\end{array}$ & $65+a$ & $70+8$ \\
\hline 46 & $\begin{array}{l}\text { Preciptation: } \\
\text { Normal daily mean, fs (inch). }\end{array}$ & .077 & .172 \\
\hline 47 & Normal No. rainy days (over $0.10 \mathrm{inch}$ ), $f_{3}$. & $50-$ & $225+$ \\
\hline 48 & Normal No. dry days (0.10 inch or less), fs. . & $50-$ & $250+$ \\
\hline 49 & Dry days, percentage of total, $f_{s}$ (per cont). . & 8 & 87 \\
\hline 50 & Days in longest normal rainy period, $f_{8} \ldots \ldots$ & $50^{-a}$ & $200+a$ \\
\hline 51 & Days in longest normal dry period, $f s \ldots \ldots \ldots$. & $25-a$ & $75+a$ \\
\hline 52 & Mean total, year (inches)..... & $40^{-a}$ & $60+a$ \\
\hline
\end{tabular}


TABLE 39.-Climatic extremes for gulf strip of lower Austral zone-Continued.

\begin{tabular}{|c|c|c|c|}
\hline Plate & Evaporation: & Low. & High. \\
\hline 53 & Daily mean, $1887-88, f_{s}($ inch) $\ldots \ldots \ldots \ldots \ldots \ldots \ldots$ & .118 & .148 \\
\hline 54 & $\begin{array}{l}\text { Total annual, } 1887-88 \text { (inches) } \ldots \ldots \ldots \ldots \ldots \ldots \ldots \\
\text { Moisture ratios: }\end{array}$ & $40^{a}$ & $50+a$ \\
\hline 58 & Normal $P / E, f_{s}, \ldots \ldots \ldots \ldots \ldots \ldots \ldots$ & 6 & 136 \\
\hline 59 & Normal $\pi / E, f_{s} \ldots . .$. & 72 & 152 \\
\hline 60 & $\begin{array}{l}\text { Normal } P / E \text {, year. . . . } \ldots \ldots \ldots \ldots \ldots \ldots \ldots \ldots \ldots \\
\text { Vapor pressure: }\end{array}$ & $80-a$ & $140+a$ \\
\hline 63 & $\begin{array}{l}\text { Normal mean, } f s \text { (hundredths inch) } \ldots \ldots \ldots \ldots \ldots \ldots \\
\text { Humidity: }\end{array}$ & 569 & 675 \\
\hline 65 & Normal mean, $f_{8}$ (per cent). & $80-a$ & $80++^{\circ}$ \\
\hline 66 & $\begin{array}{l}\text { Normal mean, year (per cent) } \ldots \ldots \ldots \ldots \ldots \ldots \ldots \\
\text { Wind: }\end{array}$ & $80-a$ & $80+a$ \\
\hline 68 & $\begin{array}{l}\text { Normal mean hourly velocity, } f s \text { (miles) ............. } \\
\text { Sunshine: }\end{array}$ & $6-4$ & $12+a$ \\
\hline 69 & $\begin{array}{l}\text { Normal total duration, } f s \text { (hours) ................. } \\
\text { Moisture-temperature indices: }\end{array}$ & 2,123 & 2,650 \\
\hline 70 & Normal $P / E \times T, f_{s}$, remainder method.... & $7-a$ & $12+a$ \\
\hline 71 & Normal $P / E \times T, f s$, exponential method........ & $8-a$ & $13+a$ \\
\hline 72 & Normal $P / E \times T, f s$, physiological method.......... & $14-a$ & $23+a$ \\
\hline
\end{tabular}

TABLE 40.-Climatic extremes for the tropical region.

\begin{tabular}{|c|c|c|c|}
\hline Plate & Temperature: & Low. & High. \\
\hline 34 & Days in normal frostless season $(f s) \ldots \ldots \ldots \ldots \ldots$ & 266 & 365 \\
\hline 35 & 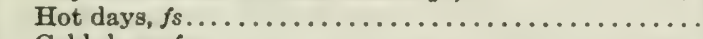 & 211 & 365 \\
\hline 36 & Cold days, $f 8 \ldots \ldots \ldots \ldots \ldots \ldots \ldots \ldots \ldots \ldots \ldots$ & 0 & 0 \\
\hline 37 & Remainder summation above $32^{\circ}$, year (thousands)... & $26^{a}$ & $26+a$ \\
\hline 38 & Remainder summation above $39^{\circ}, f_{8}$ (thousands).... & $10,000^{a}$ & 14,522 \\
\hline 39 & 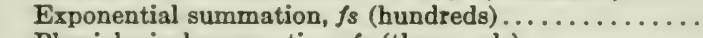 & 1,260 & 1,540 \\
\hline 40 & Physiological summation, $f s$ (thousands).......... & $15,000+a$ & 31,063 \\
\hline 41 & Absolute minimum $\ldots \ldots \ldots \ldots \ldots \ldots \ldots \ldots \ldots \ldots$ & +10 & +41 \\
\hline 43 & Normal daily mean, coldest 14 days of year $\left({ }^{\circ} \mathrm{F}.\right) \ldots$ & 54 & 69 \\
\hline 44 & Normal daily mean, hottest 6 weeks of year $\left({ }^{\circ} \mathrm{F}.\right) \ldots$ & $71.6^{a}$ & $78.8+a$ \\
\hline 45 & $\begin{array}{l}\text { Normal daily mean, year }\left({ }^{\circ} \mathrm{F} .\right) \ldots \ldots \ldots \ldots \ldots \ldots \ldots \\
\text { Precipitation: }\end{array}$ & $70+a$ & $75+a$ \\
\hline 46 & Normal daily mean, $f_{s}$ (inch). & .078 & 173 \\
\hline 47 & Normal No. rainy days (over 0.10 inch), $f 8 \ldots \ldots \ldots$ & $25-$ & $225+$ \\
\hline 48 & Normal No. dry days (0.10 inch or less), $f_{s} \ldots \ldots \ldots$ & $100-$ & $275+$ \\
\hline 49 & Dry days, percentage of total, $f s$ (per cent)......... & 26 & 56 \\
\hline 50 & Days in longest normal rainy period, $f_{s} \ldots \ldots \ldots \ldots$ & $25-a$ & $175^{a}$ \\
\hline 51 & Days in longest normal dry period, $f_{s} \ldots \ldots \ldots \ldots \ldots$ & $25-a$ & $250+a$ \\
\hline 52 & $\begin{array}{l}\text { Mean total, year (inches) } \ldots \ldots \ldots \ldots \ldots \ldots \ldots \ldots \\
\text { Evaporation: }\end{array}$ & $10-a$ & $60+a$ \\
\hline 53 & Daily mean, $1887-88, f_{s}$ (inch).. & 102 & $260^{a}$ \\
\hline 54 & $\begin{array}{l}\text { Total annual, } 1887-88 \text { (inches) } \ldots \ldots \ldots \ldots \ldots \ldots \ldots \ldots \\
\text { Moisture ratios: }\end{array}$ & $40-a$ & $90+a$ \\
\hline 58 & Normal $P / E, f_{s} \ldots$. & $20-a$ & $100+a$ \\
\hline 59 & Normal $\pi / E, f_{8} \ldots \ldots \ldots \ldots \ldots \ldots \ldots \ldots \ldots \ldots \ldots \ldots \ldots \ldots$ & $20-a$ & $120^{\circ}$ \\
\hline 60 & $\begin{array}{l}\text { Normal } P / E \text {, year. } \ldots \ldots \ldots \ldots \ldots \ldots \ldots \ldots \ldots \\
\text { Vapor pressure: }\end{array}$ & $.10^{a}$ & $1.20^{\circ}$ \\
\hline 63 & $\begin{array}{l}\text { Normal mean, } f s \text { (hundredths inch).... } \\
\text { Humidity: }\end{array}$ & $200^{\circ}$ & 707 \\
\hline 65 & Normal mean, $f s$ (per cent)...... & $40^{a}$ & $80+a$ \\
\hline 66 & $\begin{array}{l}\text { Normal mean, year (per cent) } \ldots \ldots \ldots \ldots \ldots \ldots \ldots \ldots \\
\text { Wind: }\end{array}$ & $40^{a}$ & $80+a$ \\
\hline 68 & $\begin{array}{l}\text { Normal mean hourly velocity, fs (miles).. } \\
\text { Sunshine: }\end{array}$ & $6-a$ & $12+a$ \\
\hline 69 & $\begin{array}{l}\text { Normal total duration, } f_{s} \text { (hours) } \ldots \ldots \ldots \ldots \ldots \ldots \ldots \\
\text { Moisture-temperature indices: }\end{array}$ & $2,300+$ & \\
\hline 70 & Normal $P / E \times T, f s$, remainder method.......... & $1-a$ & $11+a$ \\
\hline 71 & Normal $P / E \times T, f s$, exponential method $\ldots \ldots \ldots \ldots$ & $1-a$ & $12+a$ \\
\hline 72 & Normal $P / E \times T, f s$, physiological method.......... & $1-a$ & $23+a$ \\
\hline
\end{tabular}


TABLE 41.-Climatic extremes for region with no species of evergreen broad-leaved trees.

\begin{tabular}{|c|c|c|c|}
\hline Plate & Temperature: & Low. & High. \\
\hline 34 & Days in normal frostless season $\left(f_{8}\right) \ldots \ldots \ldots \ldots$ & 25 & 305 \\
\hline 35 & 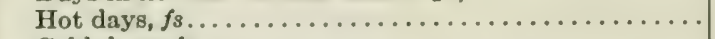 & 0 & 187 \\
\hline 36 & Cold days, $f_{s} \ldots \ldots \ldots \ldots \ldots \ldots \ldots \ldots \ldots \ldots$ & 0 & 158 \\
\hline 37 & Remainder summation above $32^{\circ}$, year (thousands).... & 10.0 & $18.0+$ \\
\hline 38 & Remainder summation above $39^{\circ}, f s$ (thousands)...... & 2.4 & 10.1 \\
\hline 39 & 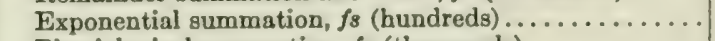 & 2.4 & 11.8 \\
\hline 40 & Physiological summation, $f_{s}$ (thousands)........... & 2.1 & 18.2 \\
\hline 41 & 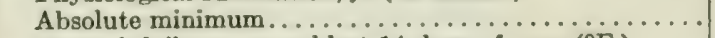 & -65 & +4 \\
\hline 43 & Normal daily mean, coldest 14 days of year $\left({ }^{\circ} \mathrm{F}.\right) \ldots$ & 0 & 54 \\
\hline 44 & Normal daily mean, hottest 6 weeks of year $\left({ }^{\circ} \mathrm{F}.\right) . .$. & $64.4-$ & $78.8+$ \\
\hline 45 & Normal daily mean, year $\left({ }^{\circ} \mathrm{F}.\right) \ldots \ldots \ldots \ldots \ldots \ldots$ & & $70+$ \\
\hline 46 & $\begin{array}{l}\text { Precipitation: } \\
\quad \text { Normal daily mean, } f_{8} \text { (inch). }\end{array}$ & .009 & .147 \\
\hline 47 & Normal No. rainy days (over 0.10 inch), $f_{8} \ldots \ldots \ldots$ & 0 & 159 \\
\hline 48 & Normal No. dry days $(0.10$ inch or less), $f s . \ldots \ldots \ldots$ & 19 & 283 \\
\hline 49 & Dry days, percentage of total, $f_{s}$ (per cent)......... & 14 & 100 \\
\hline 50 & Days in longest normal rainy period, $f_{s} \ldots \ldots \ldots \ldots$ & 0 & 172 \\
\hline 51 & Days in longest normal dry period, $f s \ldots \ldots \ldots \ldots \ldots$ & 4 & 283 \\
\hline 52 & $\begin{array}{l}\text { Mean total, year (inches) } \ldots \ldots \ldots \ldots \ldots \ldots \ldots \ldots \\
\text { Evaporation: }\end{array}$ & $10-$ & $60+$ \\
\hline 53 & Daily mean, $1887-88, f_{s}$ (inch) $\ldots \ldots \ldots \ldots \ldots \ldots \ldots$ & .084 & .351 \\
\hline 54 & $\begin{array}{l}\text { Total annual, } 1887-88 \text { (inches) } \ldots \ldots \ldots \ldots \ldots \ldots \ldots \\
\text { Moisture ratios: }\end{array}$ & 22.1 & 100.6 \\
\hline 58 & Normal $P / E, f s \ldots \ldots \ldots \ldots$ & .04 & 1.31 \\
\hline 59 & 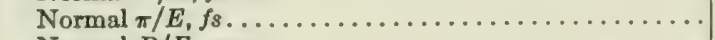 & .06 & 4.48 \\
\hline 60 & 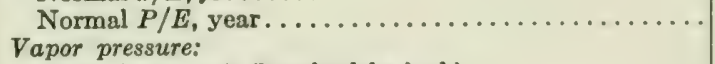 & .03 & 1.72 \\
\hline 63 & $\begin{array}{l}\text { Normal mean, } f_{s} \text { (hundredths inch).......... } \\
\text { Humidity: }\end{array}$ & 183 & 569 \\
\hline 65 & 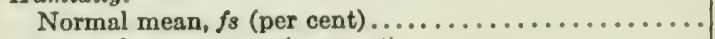 & 22.6 & 81.8 \\
\hline 66 & $\begin{array}{l}\text { Normal mean, year (per cent) } \ldots \ldots \ldots \ldots \ldots \ldots \ldots \ldots \\
\text { Wind: }\end{array}$ & 29.7 & 80.2 \\
\hline 68 & $\begin{array}{l}\text { Normal mean hourly velocity, } f_{s} \text { (miles)............ } \\
\text { Sunshine: }\end{array}$ & 3.1 & 14.9 \\
\hline 69 & $\begin{array}{l}\text { Normal total duration, } f s \text { (hours) } \ldots \ldots \ldots \ldots \ldots \ldots \ldots \\
\text { Moisture-temperature indices: }\end{array}$ & 1,127 & $2,100^{a}$ \\
\hline 70 & Normal $P / E \times T, f s$, remainder method... & 13 & 851 \\
\hline 71 & 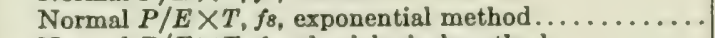 & 127 & 7,992 \\
\hline 72 & Normal $P / E \times T, f s$, physiological method................... & 197 & 14,950 \\
\hline
\end{tabular}

TABLE 42.-Climatic extremes for region with 1 to 4 species of evergreen broad-leaved trees (East).

\begin{tabular}{|c|c|c|c|}
\hline Plate & Temperature: & Lov. & High. \\
\hline 34 & Days in normal frostless season $(f s) \ldots \ldots \ldots \ldots$ & 140 & 318 \\
\hline 35 & 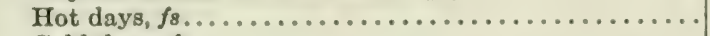 & 38 & 218 \\
\hline 36 & 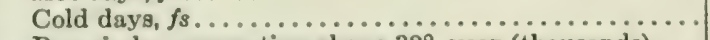 & 0 & 86 \\
\hline 37 & Remainder summation above $32^{\circ}$, year (thousands).... & $10.0+$ & $18.0+$ \\
\hline 38 & Remainder summation above $39^{\circ}, f_{8}$ (thousands)...... & 4.3 & 10.3 \\
\hline 39 & 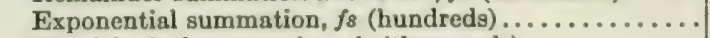 & 4.4 & 11.3 \\
\hline 40 & Physiological summation, fs (thousands)............ & 5.0 & 21.4 \\
\hline 41 & 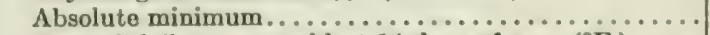 & -23 & +15 \\
\hline 43 & Normal daily mean, coldest 14 days of year $\left({ }^{\circ} \mathrm{F}.\right) . .$. . & 26 & 53 \\
\hline 44 & Normal daily mean, hottest 6 weeks of year $\left({ }^{\circ} \mathrm{F}.\right) . .$. & $71.6-$ & $78.8+$ \\
\hline 45 & $\begin{array}{l}\left.\text { Normal daily mean, year ( }{ }^{\circ} \mathrm{F} .\right) \ldots \ldots \ldots \ldots \ldots \ldots \ldots \ldots \\
\text { Precipitation: }\end{array}$ & $50-$ & $70+$ \\
\hline 46 & Normal daily mean, $f_{s}$ (inch) ............ & .072 & .146 \\
\hline 47 & Normal No. rainy days (over 0.10 inch), $f_{3} \ldots \ldots \ldots$ & 2 & 202 \\
\hline 48 & Normal No. dry days $(0.10$ inch or less), fs......... & 32 & 259 \\
\hline 49 & Dry days, percentage of total, $f_{s}$ (per cent).......... & 24 & 99 \\
\hline 50 & Days in longest normal rainy period, $f_{s} \ldots \ldots \ldots \ldots$ & 17 & 200 \\
\hline 51 & Days in longest normal dry period, $f_{s} \ldots \ldots \ldots . . .$. & 9 & $100^{a}$ \\
\hline 52 & 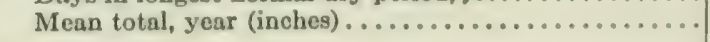 & $10+$ & 60 \\
\hline
\end{tabular}


TABLE 42.-Climalic extremes for region with 1 to 4 species of evergreen broad-leaved trees (East)-Continued.

\begin{tabular}{|c|c|c|c|}
\hline Plate & Evaporation: & Low. & High. \\
\hline 53 & Daily mean, $1887-88, f_{s}$ (inch) $\ldots \ldots \ldots \ldots \ldots \ldots$ & .081 & $.180^{a}$ \\
\hline 54 & $\begin{array}{l}\text { Total annual, } 1887-88 \text { (inches) } \ldots \ldots \ldots \ldots \ldots \ldots \ldots \\
\text { Moisture ratios: }\end{array}$ & 20.3 & 96.4 \\
\hline 58 & 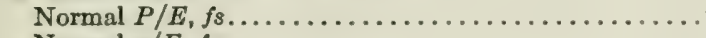 & .34 & 1.39 \\
\hline 59 & Normal $\pi / E, f_{8} \ldots \ldots \ldots \ldots \ldots \ldots \ldots \ldots \ldots$ & .13 & 1.63 \\
\hline 60 & $\begin{array}{l}\text { Normal } P / E \text {, year. } \ldots \ldots \ldots \ldots \ldots \ldots \ldots \ldots \ldots \\
\text { Vapor pressure: }\end{array}$ & .24 & 1.85 \\
\hline 63 & $\begin{array}{l}\text { Normal mean, } f s \text { (hundredths inch) } \ldots \ldots \ldots \ldots \ldots \ldots \\
\text { Humidity: }\end{array}$ & 300 & 675 \\
\hline 65 & 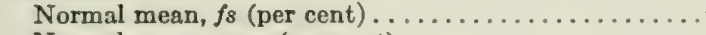 & 37.0 & 84.0 \\
\hline 66 & $\begin{array}{l}\text { Normal mean, year (per cent) ................. } \\
\text { Wind: }\end{array}$ & 38.8 & 82.1 \\
\hline 68 & $\begin{array}{l}\text { Normal mean hourly velocity, } f s \text { (miles)............ } \\
\text { Sunshine: }\end{array}$ & 5.0 & 14.9 \\
\hline 69 & $\begin{array}{l}\text { Normal total duration, } f s \text { (hours) } \ldots \ldots \ldots \ldots \ldots \ldots \\
\text { Moisture-temperature indices: }\end{array}$ & 1,626 & 2,343 \\
\hline 70 & Normal $P / E \times T, f 8$, remainder method. & 98 & 887 \\
\hline 71 & Normal $P / E \times T, f s$, exponential method. & 906 & 10,331 \\
\hline 72 & Normal $P / E \times T, f s$, physiological method. & 1,790 & 20,570 \\
\hline
\end{tabular}

TABLE 43.-Climatic extremes for region with 1 to 4 species of evergreen broad-leaved trees (West).

\begin{tabular}{|c|c|c|c|}
\hline Plate & Temperature: & Low. & High. \\
\hline 34 & Days in normal frostless season $(f s) \ldots \ldots \ldots \ldots \ldots$ & 108 & 316 \\
\hline 35 & Hot days, $f s \ldots \ldots \ldots \ldots \ldots \ldots \ldots \ldots \ldots \ldots \ldots$ & 0 & $120-a$ \\
\hline 36 & Cold days, fs . . . . . . . . . . . . . & 0 & 0 \\
\hline 37 & Remainder summation above $32^{\circ}$, year (thousands).... & $10.0-$ & $18.0+$ \\
\hline 38 & Remainder summation above $39^{\circ}, f s$ (thousands)...... & 3.8 & $8.0+a$ \\
\hline 39 & Exponential summation, $f_{s}$ (hundreds).............. & 4.3 & $8.0+$ \\
\hline 40 & Physiological summation, $f s$ (thousands)........... & 1.9 & $12.5+$ \\
\hline 41 & 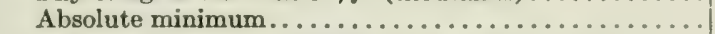 & -6 & $+14+$ \\
\hline 43 & Normal daily mean, coldest 14 days of year $\left({ }^{\circ} \mathrm{F}.\right) \ldots$ & 39 & $50+a$ \\
\hline 44 & Normal daily mean, hottest 6 weeks of year $\left({ }^{\circ} \mathrm{F}.\right) \ldots$ & $64.4-$ & $78.8+$ \\
\hline 45 & $\begin{array}{l}\text { Normal daily mean, year }\left({ }^{\circ} \mathrm{F} .\right) \ldots \ldots \ldots \ldots \ldots \ldots \ldots \\
\text { Precipitation: }\end{array}$ & $50-$ & $65+$ \\
\hline 46 & Normal daily mean, $f_{s}($ inch $) \ldots \ldots \ldots \ldots \ldots \ldots$ & .042 & .199 \\
\hline 47 & Normal No. rainy days (over $0.10 \mathrm{inch}$ ), $f_{s} . . . \ldots .$. & 1 & 199 \\
\hline 48 & Normal No. dry days $(0.10$ inch or less $), f s \ldots \ldots \ldots$ & 72 & 197 \\
\hline 49 & Dry days, percentage of total, $f s$ (per cent)........... & 27 & 100 \\
\hline 50 & Days in longest normal rainy period, $f_{s} \ldots \ldots \ldots \ldots \ldots$ & 0 & 99 \\
\hline 51 & Days in longest normal dry period, $f_{s} \ldots \ldots \ldots \ldots \ldots$ & 56 & 198 \\
\hline 52 & $\begin{array}{l}\text { Mean total, year (inches) } \ldots \ldots \ldots \ldots \ldots \ldots \ldots \ldots \ldots \\
\text { Evaporation: }\end{array}$ & $20+$ & 90 \\
\hline 53 & Daily mean, $1887-88, f_{s}$ (inch)... & .052 & .143 \\
\hline 54 & $\begin{array}{l}\text { Total annual, } 1887-88 \text { (inches) } \ldots \ldots \ldots \ldots \ldots \ldots \ldots \ldots \\
\text { Moisture ratios: }\end{array}$ & 18.1 & $60+a$ \\
\hline 58 & Normal $P / E, f s . .$. & .29 & 3.84 \\
\hline 59 & Normal $\pi / E, f_{s} \ldots \ldots \ldots \ldots \ldots \ldots \ldots \ldots \ldots$ & .41 & 4.48 \\
\hline 60 & 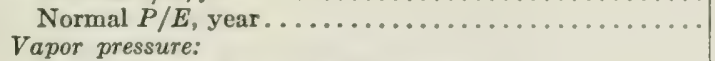 & .88 & 4.90 \\
\hline 63 & $\begin{array}{l}\text { Normal mean, } f s \text { (hundredths inch)......................... } \\
\text { Humidity: }\end{array}$ & 323 & 329 \\
\hline 65 & 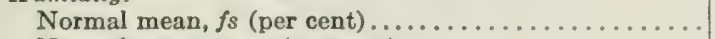 & $60.0-a$ & 73.6 \\
\hline 66 & $\begin{array}{l}\text { Normal mean, year (per cent) ......................... } \\
\text { Wind: }\end{array}$ & 74.6 & 76.2 \\
\hline 68 & $\begin{array}{l}\text { Normal mean hourly velocity, } f_{s} \text { (miles) } \ldots \ldots \ldots \ldots \ldots \\
\text { Sunshine: }\end{array}$ & 3.5 & 16.4 \\
\hline 69 & $\begin{array}{l}\text { Normal total duration, } f_{s} \text { (hours) ........ } \\
\text { Moisture-temperature indices: }\end{array}$ & $1,500-a$ & $2,700 \pm a$ \\
\hline 70 & Normal $P / E \times T, f s$, remainder method... & 126 & 1,566 \\
\hline 71 & Normal $P / E \times T, f_{s}$, exponential method.......... & $1,000-a$ & 11,724 \\
\hline 72 & Normal $P / E \times T, f s$, physiological method.......... & 1,313 & 7,475 \\
\hline
\end{tabular}


TABLE 44.-Climatic extremes for region with 5 to 9 species of evergreen broad-leaved trees (East).

\begin{tabular}{|c|c|c|c|}
\hline Plate & Temperature: & Low. & High. \\
\hline 34 & Days in normal frostless season $\left(f_{8}\right)$. & 205 & \\
\hline 35 & Hot days, $f_{s} \ldots \ldots \ldots \ldots \ldots \ldots \ldots \ldots \ldots \ldots \ldots \ldots$ & 128 & 168 \\
\hline 36 & Cold days, $f_{s}, \ldots \ldots \ldots \ldots \ldots \ldots \ldots \ldots \ldots$ & 0 & 0 \\
\hline 37 & Remainder summation above $32^{\circ}$, year (thousands). & $18.0+$ & $18.0+$ \\
\hline 38 & Remainder summation above $39^{\circ}$, fs (thousanda)... & 6.7 & 8.2 \\
\hline 39 & Exponential summation, fs (hundreds)............ & 7.9 & 8.9 \\
\hline 40 & Physiological summation, fs (thousanda) ......... & 12.2 & 18.2 \\
\hline 41 & Absolute minimum.............. & -3 & +12 \\
\hline 43 & Normal daily mean, coldest 14 days of year $\left({ }^{\circ} F_{0}\right)$. & 40 & 52 \\
\hline 44 & Normal daily mean, hottest 6 weeks of year $\left({ }^{\circ} \mathrm{F}\right.$.). & $78.8+$ & $78.8+$ \\
\hline 15 & $\begin{array}{l}\text { Normal daily mean, year }\left({ }^{\circ} \mathrm{F} .\right) \ldots \ldots \ldots \ldots \ldots \ldots \ldots \\
\text { Precipitation: }\end{array}$ & & $65+$ \\
\hline 46 & Normal daily mean, fs (inch). & 129 & 170 \\
\hline 47 & Normal No. rainy days (over $0.10 \mathrm{inch}$ ), $f_{8}$. & 185 & 256 \\
\hline 48 & Normal No. dry days ( 0.10 inch or less), fs. & 0 & $75^{a}$ \\
\hline 49 & Dry days, percentage of total, $f_{s}$ (per cent).. & 0 & 27 \\
\hline 50 & Days in longest normal rainy period, $f_{8} .$. & 92 & 256 \\
\hline 51 & Days in longest normal dry period, $f_{3} \ldots .$. & 0 & 24 \\
\hline 52 & $\begin{array}{l}\text { Mesn total, year (inches) } . . . \ldots \ldots \ldots \ldots \\
\text { Exaporation: }\end{array}$ & $50-$ & $60+$ \\
\hline 53 & Daily mean, $1887-88, f_{8}$ (inch). & .096 & .148 \\
\hline 54 & $\begin{array}{l}\text { Total annual, } 1887-88 \text { (inches) ........ } \\
\text { Moisture ratios: }\end{array}$ & 31.3 & 47.1 \\
\hline 58 & Normal $P / E$, fs.... & .93 & 1.76 \\
\hline 59 & Normal $\pi / E, f_{s} \ldots$ & 1.07 & 1.96 \\
\hline 60 & $\begin{array}{l}\text { Normal } P / E \text {, year. . . . . . . . . } \ldots \ldots \ldots \ldots \ldots \ldots \\
\text { Vapor pressure: }\end{array}$ & 1.02 & 1.94 \\
\hline 63 & $\begin{array}{l}\text { Normal mean, } f_{8} \text { (hundredths inch) ... } \\
\text { Humidity: }\end{array}$ & 568 & 622 \\
\hline 65 & Normal mean, fs (per cent)...... & 73.9 & 82.7 \\
\hline 66 & $\begin{array}{l}\text { Normal mean, year (per cent) ................ } \\
\text { Wind: }\end{array}$ & 73.5 & 82.9 \\
\hline 68 & $\begin{array}{l}\text { Normal mean hourly velocity, fs (miles). } \\
\text { Sunshine: }\end{array}$ & 6.3 & 13.5 \\
\hline 69 & $\begin{array}{l}\text { Normal total duration, } f_{s} \text { (hours).. } \\
\text { Moisture-temperature indices: }\end{array}$ & 1,895 & 2,650 \\
\hline 70 & Normal $P / E \times T, f s$, remainder method.. & 665 & 1,418 \\
\hline 71 & Normal $P / E \times T, f_{s}$, exponential method. & 7,663 & 13,511 \\
\hline 72 & Normal $P / E \times T, f_{s}$, physiological method. & 12,400 & 24,265 \\
\hline
\end{tabular}

TABle 45.-Climatic extremes for region with 5 to 9 species of cvergreen broad-leaved trees (West).

\begin{tabular}{|c|c|c|c|}
\hline Plate & Temperature: & Low. & High. \\
\hline 34 & Days in normal frostless season $\left(f_{s}\right) \ldots \ldots \ldots \ldots \ldots$ & 139 & 311 \\
\hline 35 & 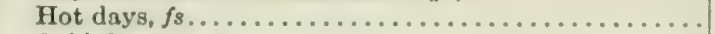 & 0 & $60+$ \\
\hline 36 & Cold days, $f_{s} \ldots \ldots \ldots \ldots \ldots \ldots \ldots \ldots \ldots \ldots$ & 0 & 0 \\
\hline 37 & Remainder summation above $32^{\circ}$, year (thousands).... & $11.5-$ & $18.0+$ \\
\hline 38 & Remainder summation above $39^{\circ}, f_{s}$ (thousands)...... & 3.5 & $8.0+a$ \\
\hline 39 & Exponential summation, $f s$ (hundreds) .............. & 4.1 & 8. $0+$ \\
\hline 40 & Physiological summation, fs (thousands)............ & 2.4 & 15.0 \\
\hline 41 & 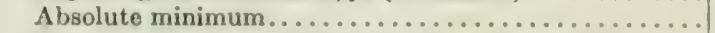 & +12 & +30 \\
\hline 43 & Normal daily mean, coldest 14 days of year $\left({ }^{\circ} \mathrm{F}.\right) \ldots$. & $45-$ & $50+a$ \\
\hline 44 & Normal daily mean, hottest 6 weoks of year $\left({ }^{\circ} \mathrm{F}.\right) \ldots$. & $64.4-$ & $78.8+$ \\
\hline 45 & $\begin{array}{l}\text { Normal daily mean, year }\left({ }^{\circ} \mathrm{F} .\right) \ldots \ldots \ldots \ldots \ldots \ldots \\
\text { Precipitation: }\end{array}$ & $55-$ & $65+$ \\
\hline 46 & Normal daily mean, $f_{8}$ (inch). & .017 & .070 \\
\hline 47 & Normal No. rainy days (over 0.10 inch), $f 8 \ldots . . . .$. & 0 & 55 \\
\hline 48 & Normal No. dry days $(0.10$ inch or less $), f s \ldots . . .$. & 190 & 259 \\
\hline 49 & Dry days, percentage of total, $f s$ (per cent).......... & 78 & 100 \\
\hline 50 & Days in longest normal rainy period, $f_{s} \ldots \ldots \ldots \ldots \ldots$ & 0 & 36 \\
\hline 51 & Days in longest normal dry period, $f_{s} \ldots \ldots \ldots \ldots \ldots$ & 182 & 258 \\
\hline 52 & 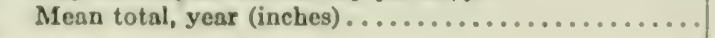 & $20+$ & 70 \\
\hline
\end{tabular}


TABLE 45.-Climatic extremes for region with 5 to 9 species of evergreen broad-leaved trees (West)-Continued.

\begin{tabular}{|c|c|c|c|}
\hline Plate & Evaporation: & Low. & High. \\
\hline 53 & Daily mean, $1887-88, f_{s}$ (inch) $\ldots \ldots \ldots \ldots \ldots \ldots$ & .171 & .220 \\
\hline 54 & $\begin{array}{l}\text { Total annual, } 1887-88 \text { (inches) } \ldots \ldots \ldots \ldots \ldots \ldots \ldots \ldots \\
\text { Moisture ratios: }\end{array}$ & $40-a$ & 84.8 \\
\hline 58 & 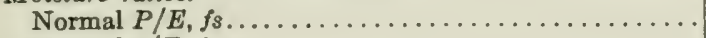 & .08 & .21 \\
\hline 59 & 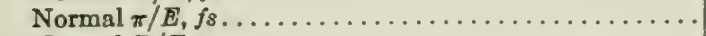 & .10 & .29 \\
\hline 60 & 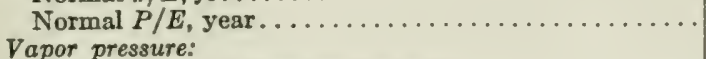 & .15 & .37 \\
\hline 63 & $\begin{array}{l}\text { Normal mean, } f_{8} \text { (hundredths inch) } \ldots \ldots \ldots \ldots \ldots \ldots . \\
\text { Humidity: }\end{array}$ & 279 & 348 \\
\hline 65 & 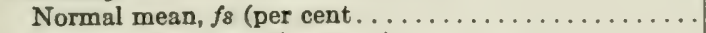 & 48.9 & 87.5 \\
\hline 66 & $\begin{array}{l}\text { Normal mean, year (per cent) } \ldots \ldots \ldots \ldots \ldots \ldots \ldots \ldots \\
\text { Wind: }\end{array}$ & 48.1 & 86.8 \\
\hline 68 & $\begin{array}{l}\text { Normal mean hourly velocity, } f_{8} \text { (miles) } \ldots \ldots \ldots \ldots \ldots \\
\text { Sunshine: }\end{array}$ & 5.5 & 8.4 \\
\hline 69 & $\begin{array}{l}\text { Normal total duration, } f_{s} \text { (hours) } \ldots \ldots \ldots \ldots \ldots \ldots \ldots \\
\text { Moisture-tomperature indices: }\end{array}$ & $1,700-a$ & $2,700 \pm a$ \\
\hline 70 & Normal $P / E \times T, f s$, remainder method........ & 68 & 146 \\
\hline 71 & 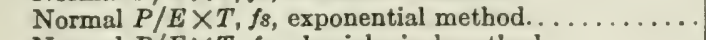 & 625 & 1,399 \\
\hline 72 & Normal $P / E \times T, f_{s}$, physiological method...... & 1,186 & 2,409 \\
\hline
\end{tabular}

TABLE 46.-Climatic extremes for region with 10 to 14 species of evergreen broad-leaved trees (East).

\begin{tabular}{|c|c|c|c|}
\hline Plate & Temperature: & Low. & High. \\
\hline 34 & Days in normal frostless season $(f s) \ldots \ldots \ldots \ldots$ & 228 & 328 \\
\hline 35 & Hot days, $f_{3} \ldots \ldots \ldots \ldots \ldots \ldots$ & 147 & 192 \\
\hline 36 & Cold days, $f s \ldots \ldots \ldots \ldots \ldots \ldots$ & 0 & 0 \\
\hline 37 & Remainder summation above $32^{\circ}$, year (thousands)... & $18.0+$ & $26.0+$ \\
\hline 38 & Remainder summation above $39^{\circ}, f_{s}$ (thousands)...... & 7.3 & 9.9 \\
\hline 39 & Exponential summation, $f_{s}$ (hundreds) ............. & 7.7 & 11.1 \\
\hline 40 & 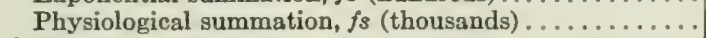 & 13.5 & 19.3 \\
\hline 41 & 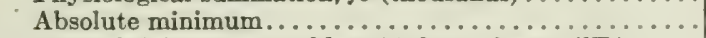 & -1 & +22 \\
\hline 43 & Normal daily mean, coldest 14 days of year $\left({ }^{\circ} \mathrm{F}.\right) \ldots$ & 45 & 57 \\
\hline 44 & Normal daily mean, hottest 6 weeks of year $\left({ }^{\circ} \mathrm{F}.\right) . .$. & $78.8+$ & $78.8+$ \\
\hline 45 & Normal daily mean, year $\left({ }^{\circ} \mathrm{F}.\right) \ldots \ldots \ldots \ldots \ldots \ldots . . . . .$. & $65-$ & $70+$ \\
\hline 46 & $\begin{array}{l}\text { Precipitation: } \\
\text { Normal daily mean, } f s \text { (inch)..... }\end{array}$ & .141 & .172 \\
\hline 47 & Normal No. rainy days (over 0.10 inch), fs......... & 175 & 284 \\
\hline 48 & Normal No. dry days $(0.10$ inch or less $), f s \ldots \ldots \ldots$ & 26 & 95 \\
\hline 49 & Dry days, percentage of total, $f_{s}$ (per cent).......... & 8 & 34 \\
\hline 50 & Days in longest normal rainy period, $f s \ldots \ldots \ldots \ldots$ & 143 & 235 \\
\hline 51 & Days in longest normal dry period, $f s \ldots \ldots \ldots \ldots$. & 18 & 37 \\
\hline 52 & Mean total, year (inches)...$\ldots \ldots \ldots \ldots \ldots \ldots \ldots \ldots$ & $50-$ & $60+$ \\
\hline & Evaporation: & & \\
\hline 53 & Daily mean, $1887-88, f_{s}$ (inch). & .130 & .146 \\
\hline 54 & $\begin{array}{l}\text { Total annual, } 1887-88 \text { (inches).. } \\
\text { Moisture ratios: }\end{array}$ & 38.4 & 49.6 \\
\hline 58 & Normal $P / E, f s \ldots$ & 1.08 & 1.36 \\
\hline 59 & Normal $\pi / E, f s . . . \ldots \ldots .$. & 1.12 & 1.52 \\
\hline 60 & $\begin{array}{l}\text { Normal } P / E \text {, year............ } \\
\text { Vapor pressure: }\end{array}$ & 1.09 & 1.47 \\
\hline 63 & $\begin{array}{l}\text { Normal mean, } f_{s} \text { (hundredths inch)... } \\
\text { Humidity: }\end{array}$ & 585 & 610 \\
\hline 65 & Normal mean, $f_{s}$ (per cent). & 73.2 & 80.6 \\
\hline 66 & $\begin{array}{l}\text { Normal mean, year (per cent) .................... } \\
\text { Wind: }\end{array}$ & 77.3 & 80.9 \\
\hline 68 & $\begin{array}{l}\text { Normal mean hourly velocity, } f_{s} \text { (miles) . . . . . . . . } \\
\text { Sunshine: }\end{array}$ & 5.1 & 9.9 \\
\hline 69 & $\begin{array}{l}\text { Normal total duration, } f_{s} \text { (hours) } \ldots \ldots \ldots \\
\text { Moisture-temperature indices: }\end{array}$ & 1,942 & 2,297 \\
\hline 70 & 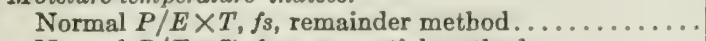 & 1,014 & 1,314 \\
\hline 71 & 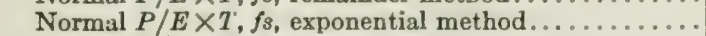 & 9,385 & 12,106 \\
\hline 72 & Normal $P / E \times T, f_{B}$, physiological method........... & 18,294 & 23,652 \\
\hline
\end{tabular}


TABLE 47.-Climatic extremes for region with 10 to 14 species of evergreen broad-leaved trees (West).

\begin{tabular}{|c|c|c|c|}
\hline Plate & Temperature: & Low. & High. \\
\hline 34 & Days in normal frostless season $\left(f_{s}\right)$. & 237 & \\
\hline 35 & Hot days, $f_{s} \ldots \ldots \ldots \ldots \ldots \ldots \ldots$ & 0 & 54 \\
\hline 36 & Cold days, $f s \ldots \ldots \ldots \ldots \ldots \ldots \ldots$ & 0 & 0 \\
\hline 37 & Remainder summation above $32^{\circ}$, year (thousands). & $18.0+$ & $18.0+$ \\
\hline 38 & Remainder summation above $39^{\circ}, f_{8}$ (thousands)... & 5.2 & $8.0+a$ \\
\hline 39 & Exponential summation, $f_{8}$ (hundreds). & 5.01 & $8.0+$ \\
\hline 40 & Physiological summation, $f_{s}$ (thousands). & 4.1 & 8.4 \\
\hline 41 & Absolute minimum.................. & +13 & +32 \\
\hline 43 & Normal daily mean, coldest 14 days of year $\left({ }^{\circ} \mathrm{F}.\right)$. & 49 & 54 \\
\hline 44 & Normal daily mean, hottest 6 weeks of year $\left({ }^{\circ} \mathrm{F}.\right)$. & $64.4-$ & 64.4 \\
\hline 45 & Normal daily mean, year $\left({ }^{\circ} \mathrm{F}.\right) \ldots \ldots \ldots \ldots \ldots$ & $60-$ & $60+$ \\
\hline & Precipitation: & & \\
\hline 46 & Normal daily mean, $f s$ (inch) . ............. & $25^{.033}$ & $62^{.048}$ \\
\hline $\begin{array}{l}47 \\
48\end{array}$ & $\begin{array}{l}\text { Normal No. rainy days (over } 0.10 \mathrm{inch}), f_{B} \\
\text { Normal No. dry days }\left(0.10 \mathrm{inch} \text { or less), } f_{s} .\right.\end{array}$ & 235 & $\begin{array}{l}02 \\
294\end{array}$ \\
\hline 49 & Dry days, percentage of total, $f s$ (per cent).. & 81 & 90 \\
\hline 50 & Days in longest normal rainy period, $f_{3} \ldots .$. & 22 & 50 \\
\hline 51 & Days in longest normal dry period, $f_{3} \ldots \ldots \ldots$ & 232 & 299 \\
\hline 52 & $\begin{array}{c}\text { Mean total, year (inches) ........................ } \\
\text { Evaporation: }\end{array}$ & $20-$ & $30+$ \\
\hline 53 & Daily mean, $1887-88, f s$ (inch) .... & .102 & .104 \\
\hline 54 & $\begin{array}{l}\text { Total annual, } 1887-88 \text { (inches)......... } \\
\text { Moisture ratios: }\end{array}$ & 32.5 & 36.7 \\
\hline 58 & Normal $P / E, f_{s}$. & .37 & .48 \\
\hline 59 & Normal $\pi / E, f_{8} \ldots$ & .45 & .61 \\
\hline 60 & $\begin{array}{l}\text { Normal } P / E \text {, year....... } \\
\text { Vapor pressure: }\end{array}$ & .27 & .60 \\
\hline 63 & $\begin{array}{l}\text { Normal mean, } f_{s} \text { (hundredths inch)... } \\
\text { Humidity: }\end{array}$ & 335 & 378 \\
\hline 65 & Normal mean, $f_{s}$ (per cent).. & 69.9 & 80.1 \\
\hline 66 & $\begin{array}{l}\text { Normal mean, year (per cent) ............ } \\
\text { Wind: }\end{array}$ & 69.0 & 79.9 \\
\hline 68 & $\begin{array}{l}\text { Normal mean hourly velocity, } f s \text { (miles). } \\
\text { Sunshine: }\end{array}$ & 4.5 & 17.2 \\
\hline 69 & $\begin{array}{l}\text { Normal total duration, } f s \text { (hours) } \ldots \ldots \ldots \ldots \\
\text { Moisture-temperature indices: }\end{array}$ & 2,615 & 2,995 \\
\hline 70 & Normal $P / E \times T, f s$, remainder method... & 283 & 283 \\
\hline 71 & Normal $P / E \times T, f_{s}$, exponential method... & 2,598 & 2,721 \\
\hline 72 & Normal $P / E \times T, f_{s}$, physiological method.... & 1,191 & 3,127 \\
\hline
\end{tabular}

TABLE 48.-Climatic extremes for region with 15 to 63 species of evergreen broad-leared trees (East).

\begin{tabular}{|c|c|c|c|}
\hline Plate & Temperature: & Low: & High. \\
\hline 34 & Days in normal frostless season $(f s) \ldots \ldots \ldots \ldots \ldots$ & 311 & 335 \\
\hline 35 & Hot days, $f_{s} \ldots \ldots \ldots \ldots \ldots \ldots \ldots \ldots \ldots \ldots$ & $210-a$ & 226 \\
\hline 36 & Cold days, $f_{s}, \ldots \ldots \ldots \ldots \ldots \ldots \ldots \ldots . . . . . . .$. & 0 & 0 \\
\hline 37 & Remainder summation above $32^{\circ}$, year (thousands).... & $18.0+$ & $26.0+$ \\
\hline 38 & Remainder summation above $39^{\circ}, f_{s}$ (thousands)..... & $9.0+a$ & $10.0+a$ \\
\hline 39 & Exponential summation, $f_{8}$ (hundreds) ............ & $10.0+$ & $11.0+$ \\
\hline 40 & Physiological summation, $f_{s}$ (thousands) ............ & $17.5+$ & 22.5 \\
\hline 41 & 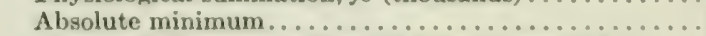 & +16 & +22 \\
\hline 43 & Normal daily mean, coldest 14 days of year $\left({ }^{\circ} \mathrm{F}.\right)$. & $55^{\circ}$ & tio-a \\
\hline 44 & Normal daily mean, hottest 6 weeks of year $\left({ }^{\circ} \mathrm{F}\right.$. $) . .$. & $78.8+$ & $7 s .8+$ \\
\hline 45 & $\begin{array}{l}\text { Normal daily mean, year }\left({ }^{\circ} \mathrm{F}_{0}\right) \ldots \ldots \ldots \ldots \ldots \ldots \ldots \\
\text { Precipitation: }\end{array}$ & $70+$ & $75-$ \\
\hline 46 & Normal daily mean, $f s$ (inch). & .147 & .150 \\
\hline 47 & Normal No. rainy days (over 0.10 inch), $f s$. & $165^{\circ}$ & $2.25+3$ \\
\hline 48 & Normal No. dry days $(0.10$ inch or less $), f s . . . . .$. & $100+a$ & 170 \\
\hline 49 & Dry days, percentage of total, $f s$ (per cent).......... & $30-a$ & 51 \\
\hline 50 & Days in longest normal rainy period, $f_{s} \ldots \ldots \ldots \ldots \ldots$ & 137 & $2(1))^{a}$ \\
\hline 51 & Days in longest normal dry period, $f_{s} \ldots \ldots \ldots \ldots \ldots$ & $25^{a}$ & $7 x$ \\
\hline 52 & 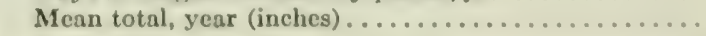 & $50+$ & $50+$ \\
\hline
\end{tabular}


TABLE 48.-Climatic extremes for region with 15 to 63 species of evergreen broad-leaved trees (East)-Continued.

\begin{tabular}{|c|c|c|c|}
\hline Plate & Evaporation: & Low. & High. \\
\hline 53 & Daily mean, $1887-88$, $f s$ (inch). & .124 & .138 \\
\hline 54 & $\begin{array}{l}\text { Total annual, } 1887-88 \text { (inches) ..... } \\
\text { Moisture ratios: }\end{array}$ & 44.2 & $50+^{a}$ \\
\hline 58 & Normal $P / E, f_{8}$. & 1.08 & 1.19 \\
\hline 59 & Normal $\pi / E$, fs.............. & 1.15 & 1.28 \\
\hline 60 & $\begin{array}{l}\text { Normal } P / E \text {, year.......................... } \\
\text { Vapor pressure: }\end{array}$ & 1.07 & 1.36 \\
\hline 63 & $\begin{array}{l}\text { Normal mean, } f_{s} \text { (hundredths inch).... } \\
\text { Humidity: }\end{array}$ & 612 & $650^{a}$ \\
\hline 65 & Normal mean, fs (per cent) & $80.0-a$ & 80.4 \\
\hline 66 & $\begin{array}{l}\text { Normal mean, year (per cent) ............ } \\
\text { Wind: }\end{array}$ & $80.0-a$ & 80.5 \\
\hline 68 & $\begin{array}{l}\text { Normal mean hourly velocity, } f_{s} \text { (miles)...... } \\
\text { Sunshine: }\end{array}$ & 6.7 & $8.0+a$ \\
\hline 69 & $\begin{array}{l}\text { Normal total duration, } f_{s} \text { (hours) ...... } \\
\text { Moisture-temperature indices: }\end{array}$ & $2,300+a$ & $2,300+a$ \\
\hline 70 & Normal $P / E \times T, f s$, remainder method. & $1,200-a$ & 1,271 \\
\hline 71 & Normal $P / E \times T, f s$, exponential method. . & $11,000-a$ & 11,722 \\
\hline 72 & Normal $P / E \times T, f s$, physiological method. & $22,000^{a}$ & 23,155 \\
\hline
\end{tabular}

TABLE 49.-Climatic extremes for region with 64 to 87 species of evergreen broad-leaved trees (East).

\begin{tabular}{|c|c|c|c|}
\hline Plate & Temperature: & Low. & High. \\
\hline 34 & Days in normal frostless season $(f s) \ldots \ldots \ldots \ldots$ & 312 & 348 \\
\hline 35 & 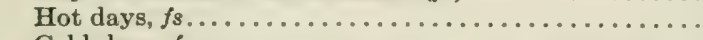 & $240^{\alpha}$ & $330+a$ \\
\hline 36 & Cold days, $f_{s} \ldots \ldots \ldots \ldots \ldots \ldots \ldots \ldots \ldots \ldots \ldots \ldots \ldots \ldots \ldots \ldots \ldots$ & 0 & 0 \\
\hline 37 & Remainder summation above $32^{\circ}$, year (thousands)... & $26.0+$ & $26.0+$ \\
\hline 38 & Remainder summation above $39^{\circ}, f_{s}$ (thousands) ...... & 10.8 & $12.0+a$ \\
\hline 39 & 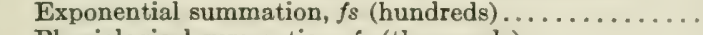 & $11.0+$ & $12.0+$ \\
\hline 40 & Physiological summation, $f_{s}$ (thousands)........... & 20.0 & $27.5+$ \\
\hline 41 & 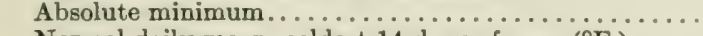 & +19 & +30 \\
\hline 43 & Normal daily mean, coldest 14 days of year $\left({ }^{\circ} \mathrm{F}.\right) \ldots$ & $60-$ & 64 \\
\hline 44 & Normal daily mean, hottest 6 weeks of year $\left({ }^{\circ} \mathrm{F}.\right) \ldots$ & $78.8+$ & $78.8+$ \\
\hline 45 & $\begin{array}{l}\text { Normal daily mean, year }\left({ }^{\circ} \mathrm{F} .\right) \ldots \ldots \ldots \ldots \ldots \ldots \ldots \\
\text { Precipitation: }\end{array}$ & $75-$ & $75+$ \\
\hline 46 & Normal daily mean, $f s$ (inch). & $.140^{-a}$ & .173 \\
\hline 47 & Normal No. rainy days (over 0.10 inch), $f 8 \ldots \ldots \ldots$ & $175-a$ & 234 \\
\hline 48 & Normal No. dry days $(0.10$ inch or less), $f s \ldots \ldots \ldots$ & 84 & $175^{a}$ \\
\hline 49 & Dry days, percentage of total, $f s$ (per cent)........... & 26 & $50+a$ \\
\hline 50 & Days in longest normal rainy period, $f s . \ldots \ldots \ldots \ldots$ & $125^{a}$ & 174 \\
\hline 51 & Days in longest normal dry period, $f s \ldots \ldots \ldots \ldots \ldots$ & 19 & $100^{a}$ \\
\hline 52 & $\begin{array}{l}\text { Mean total, year (inches) } \ldots \ldots \ldots \ldots \ldots \ldots \ldots \ldots \\
\text { Evaporation: }\end{array}$ & $50-$ & $60+$ \\
\hline 53 & Daily mean, $1887-88, f_{s}$ (inch). & $.140^{-a}$ & \\
\hline 54 & $\begin{array}{l}\text { Total annual, } 1887-88 \text { (inches) } \ldots \ldots \ldots \ldots \ldots \ldots \ldots \\
\text { Moisture ratios: }\end{array}$ & $50-a$ & $50+a$ \\
\hline 58 & Normal $P / E, f s \ldots$. & $.80+a$ & $1.00+a$ \\
\hline 59 & Normal $\pi / E, f_{s} \ldots \ldots \ldots \ldots \ldots$ & $1.00-a$ & 1.20 \\
\hline 60 & $\begin{array}{l}\text { Normal } P / E \text {, year. . . . . . . } \ldots \ldots \ldots \ldots \ldots \ldots \ldots \\
\text { Vapor pressure: }\end{array}$ & $1.00-a$ & 1.36 \\
\hline 63 & $\begin{array}{l}\text { Normal mean, } f_{s} \text { (hundredths inch). } \\
\text { Humidity: }\end{array}$ & $650-a$ & 692 \\
\hline 65 & Normal mean, $f_{s}$ (per cent).... & $80.0-a$ & 80.5 \\
\hline 66 & $\begin{array}{l}\text { Normal mean, year (per cent).. } \\
\text { Wind: }\end{array}$ & $80.0-a$ & 80.5 \\
\hline 68 & $\begin{array}{l}\text { Normal mean hourly velocity, fs (miles)............ } \\
\text { Sunshine: }\end{array}$ & $8.0-a$ & $8.0+a$ \\
\hline 69 & $\begin{array}{l}\text { Normal total duration, } f_{s} \text { (hours) } \\
\text { Moisture-temperature indices: }\end{array}$ & $2,300+a$ & $2,300+a$ \\
\hline 70 & 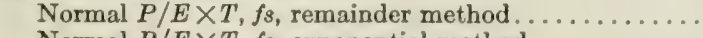 & $1,200-a$ & $1,200+a$ \\
\hline 71 & 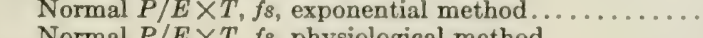 & $11,000-a$ & $11,000+a$ \\
\hline 72 & Normal $P / E \times T, f s$, physiological method.......... & $23,000++^{a}$ & $23,000+^{a}$ \\
\hline
\end{tabular}


TABle 50.-Climatic extremes for region with 88 or more species of evergreen broad-leaved trees (East).

\begin{tabular}{|c|c|c|c|}
\hline Plate & Temperalure: & Low. & High. \\
\hline 34 & Days in normal frostless season $\left(f_{8}\right)$.. & $340^{\alpha}$ & 365 \\
\hline 35 & Hot days, $f_{8} \ldots \ldots \ldots \ldots \ldots \ldots \ldots$ & $300+$ & 365 \\
\hline 36 & Cold days, $f_{8}, \ldots \ldots \ldots \ldots \ldots \ldots \ldots$ & 0 & 0 \\
\hline 37 & Remainder summation above $32^{\circ}$, year (thousands). & $26.0+$ & $26.0+$ \\
\hline 38 & Remainder summation above $39^{\circ}, f_{8}$ (thousands).. & $12.0^{a}$ & 14.5 \\
\hline 39 & Exponential summation, $f_{8}$ (hundreds)... & $12.0+$ & 15.4 \\
\hline 40 & Physiological summation, $f_{8}$ (thousands). & $25.0+$ & 31.1 \\
\hline 41 & Absolute minimum. . . . . . . . . . . . . . . & +29 & +41 \\
\hline 43 & Normal daily mean, coldest 14 days of year $\left({ }^{\circ} \mathrm{F}.\right)$ & $60+$ & 69 \\
\hline 44 & Normal daily mean, hottest 6 weeks of year $\left({ }^{\circ} \mathbf{F}\right.$.). & 78.8 & $78.8+$ \\
\hline 45 & $\begin{array}{l}\text { Normal daily mean, year }\left({ }^{\circ} \mathrm{F} .\right) \ldots \ldots \ldots \ldots \\
\text { Precipitation: }\end{array}$ & $75+$ & $75+$ \\
\hline 46 & Normal daily mean, $f_{s}$ (inch). & .106 & $160^{a}$ \\
\hline 47 & Normal No. rainy days (over $0.10 \mathrm{inch}$ ), $f s$. & 161 & $200^{a}$ \\
\hline 48 & Normal No. dry days (0.10 inch or less), fs. & $150^{a}$ & 204 \\
\hline 49 & Dry days, percentage of total, $f_{8}$ (per cent). & $30-a$ & \\
\hline 50 & Days in longest normal rainy period, $f_{8} \ldots \ldots$ & 112 & $150+a$ \\
\hline 51 & Days in longest normal dry period, $f 8 \ldots$ & $75-a$ & \\
\hline 52 & $\begin{array}{l}\text { Mean total, year (inches).............. } \\
\text { Evaporation: }\end{array}$ & $50+$ & $60+$ \\
\hline 53 & Daily mean, $1887-88, f_{s}$ (inch). & $.140-a$ & 141 \\
\hline 54 & $\begin{array}{l}\text { Total annual, } 1887-88 \text { (inches).. } \\
\text { Moisture ratios: }\end{array}$ & $50-$ & 51.6 \\
\hline 58 & Normal $P / E, f_{s}$. & .75 & $.80+6$ \\
\hline 59 & Normal $\pi / E, f s .$. & .75 & $.80+a$ \\
\hline 60 & $\begin{array}{l}\text { Normal } P / E \text {, year...... } \\
\text { Vapor pressure }\end{array}$ & .75 & $1.00+a$ \\
\hline 63 & $\begin{array}{l}\text { Vapor pressure: } \\
\text { Normal mean, fs (hundredths inch).... } \\
\text { Humidity: }\end{array}$ & $700-a$ & 707 \\
\hline 65 & 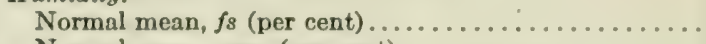 & 77.1 & $80.0+a$ \\
\hline 66 & $\begin{array}{l}\text { Normal mean, year (per cent)............ } \\
\text { Wind: }\end{array}$ & 77.1 & $80.0+a$ \\
\hline 68 & $\begin{array}{l}\text { Normal mean hourly velocity, fs (miles)... } \\
\text { Sunshine: }\end{array}$ & $8.0+a$ & 9.7 \\
\hline 69 & $\begin{array}{l}\text { Normal total duration, } f s \text { (hours) ....... } \\
\text { Moisture-temperature indices: }\end{array}$ & $2,300+a$ & $2,300+a$ \\
\hline 70 & Normal $P / E \times T, f_{s}$, remainder method.. & 1,155 & $1,200-a$ \\
\hline 71 & Normal $P / E \times T, f s$, exponential method.. & 10,877 & $11,000-a$ \\
\hline 72 & Normal $P / E \times T, f s$, physiological method. & $23,000+a$ & 23,266 \\
\hline
\end{tabular}

TABLE 51.-Climatic extremes for region with no species of Microphyllous trees.

\begin{tabular}{|c|c|c|c|}
\hline Plate & Temperature: & Low. & High. \\
\hline 34 & Days in normal frostless season $(f s)$. & 25 & \\
\hline 35 & Hot days, $f_{s} \ldots \ldots \ldots \ldots \ldots \ldots \ldots$ & 0 & 365 \\
\hline 36 & Cold days, $f s . . . .$. & 0 & 158 \\
\hline 37 & Remainder summation above $32^{\circ}$, year (thousands) & $10.0-$ & $26.0+$ \\
\hline 38 & Remainder summation above $39^{\circ}, f_{s}$ (thousands).. & 2.4 & 14.5 \\
\hline 39 & Exponential summation, $f_{s}$ (hundreds) .......... & 2.4 & 15.4 \\
\hline 40 & Physiological summation, $f_{s}$ (thousands). & 21.0 & 31.1 \\
\hline 41 & Absolute minimum... & -65 & +41 \\
\hline 43 & Normal daily mean, coldest 14 days of year $\left({ }^{\circ} \mathrm{F}\right.$. & 0 & 69 \\
\hline 44 & Normal daily mean, hottest 6 weeks of year $\left({ }^{\circ} \mathrm{F}_{0}\right)$. & $64.4-$ & $78.5+$ \\
\hline 45 & $\begin{array}{l}\text { Normal daily mean, year }\left({ }^{\circ} \mathrm{F}_{\mathrm{f}}\right) \ldots \\
\text { Precipitation: }\end{array}$ & 35 & $75+$ \\
\hline 46 & Normal daily mean, $f_{s}$ (inch). & .009 & 199 \\
\hline 47 & Normal No. rainy days (over 0.10 inch), fs.. & 0 & 284 \\
\hline 48 & Normal No. dry days ( 0.10 inch or less), $f_{s} \ldots$ & 0 & 257 \\
\hline 49 & Dry days, percentage of total, $f_{s}$ (per cent) ..... & 0 & 100 \\
\hline 50 & Days in longest normal rainy period, $f s \ldots \ldots$ & 0 & 256 \\
\hline 51 & Days in longest normal dry period, $f_{s} \ldots \ldots \ldots$ & 0 & 258 \\
\hline 52 & Mean total, year (inches). & $10-$ & $60+$ \\
\hline
\end{tabular}


TABLE 51.-Climatic extremes for region with no species of Microphyllous trees-Continued.

\begin{tabular}{|c|c|c|c|}
\hline Plate & Evaporation: & Low. & High. \\
\hline $\begin{array}{l}53 \\
54\end{array}$ & $\begin{array}{l}\text { Daily mean, } 1887-88, f s \text { (inch) } \ldots \ldots \ldots \ldots \\
\text { Total annual, } 1887-88 \text { (inches) } \ldots \ldots \ldots \ldots\end{array}$ & 18.052 & 100.6 \\
\hline & Moisture ratios: & & \\
\hline 58 & Normal $P / E, f s$. & .04 & 3.84 \\
\hline 59 & Normal $\pi / E, f s$. & .06 & 4.48 \\
\hline 60 & Normal $P / E$, year.. & .09 & 4.90 \\
\hline 63 & $\begin{array}{l}\text { Vapor pressure: } \\
\text { Normal mean, fs (hundredths inch).. }\end{array}$ & 183 & 707 \\
\hline 65 & Normal mean, $f_{s}$ (per cent........... & 22.6 & 87.5 \\
\hline 66 & $\begin{array}{l}\text { Normal mean, year (per cent).......... } \\
\text { Wind: }\end{array}$ & 29.7 & 86.8 \\
\hline 68 & $\begin{array}{l}\text { Normal mean hourly velocity, fs (miles). } \\
\text { Sunshine: }\end{array}$ & 3.1 & 16.4 \\
\hline 69 & $\begin{array}{l}\text { Normal total duration, } f_{s} \text { (hours) ...... } \\
\text { Moisture-temperature indices: }\end{array}$ & 1,127 & 2,615 \\
\hline 70 & Normal $P / E \times T, f s$, remainder method. & 13 & 1,566 \\
\hline 71 & Normal $P / E \times T, f s$, exponential method. . & 127 & 13,511 \\
\hline 72 & Normal $P / E \times T, f s$, physiological method. & 197 & 24,265 \\
\hline
\end{tabular}

TABLE 52.-Climatic extremes for region with 1 to 4 species of Microphyllous trees.

\begin{tabular}{|c|c|c|c|}
\hline Plate & Temperature: & Low. & High. \\
\hline 34 & Days in normal frostless season $(f s) \ldots \ldots \ldots \ldots \ldots$ & 172 & 334 \\
\hline 35 & 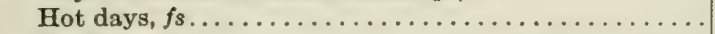 & 50 & 215 \\
\hline 36 & Cold days, $f s \ldots \ldots \ldots \ldots \ldots \ldots \ldots \ldots \ldots$ & 0 & 0 \\
\hline 37 & Remainder summation above $32^{\circ}$, year (thousands).... & 11.5 & $18.0+$ \\
\hline 38 & Remainder summation above $39^{\circ}, f_{s}$ (thousands)...... & 3.4 & 10.6 \\
\hline 39 & Exponential summation, $f_{s}$ (hundreds) . . . . . . . . . & 3.5 & 11.7 \\
\hline 40 & Physiological summation, $f_{s}$ (thousands) . . . . . . . . & 8.4 & 21.2 \\
\hline 41 & 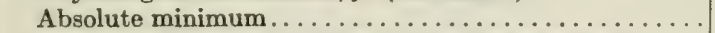 & 32 & +15 \\
\hline 43 & Normal daily mean, coldest 14 days of year $\left({ }^{\circ} \mathrm{F}.\right) . .$. & $30^{a}$ & 54 \\
\hline 44 & Normal daily mean, hottest 6 weeks of year $\left({ }^{\circ} \mathrm{F}.\right)$.... & $64.4-$ & $78.8+$ \\
\hline 45 & $\begin{array}{l}\text { Normal daily mean, year }\left({ }^{\circ} \mathrm{F} .\right) \ldots \ldots \ldots \ldots \ldots \ldots \ldots \\
\text { Precipitation: }\end{array}$ & 55 & $70+$ \\
\hline 46 & Normal daily mean, $f_{s}$ (inch) ... & .039 & 129 \\
\hline 47 & Normal No. rainy days (over 0.10 inch),$f_{s} \ldots \ldots \ldots \ldots$ & 0 & 257 \\
\hline 48 & Normal No. dry days $(0.10$ inch or less), $f 3 \ldots \ldots \ldots \ldots$ & 74 & 294 \\
\hline 49 & Dry days, percentage of total, $f_{s}$ (per cent) $\ldots \ldots \ldots \ldots$ & 22 & 100 \\
\hline 50 & Days in longest normal rainy period, $f s \ldots \ldots \ldots \ldots$ & 0 & 157 \\
\hline 51 & Days in longest normal dry period, $f s \ldots \ldots \ldots \ldots \ldots$ & 14 & 299 \\
\hline 52 & $\begin{array}{l}\text { Mean total, year (inches) } \ldots \ldots \ldots \ldots \ldots \ldots \ldots \ldots \ldots \\
\text { Evaporation: }\end{array}$ & $10-$ & 50 \\
\hline 53 & Daily mean, $1887-88, f_{8}$ (inch) $\ldots \ldots \ldots \ldots \ldots \ldots$ & .104 & .330 \\
\hline 54 & $\begin{array}{l}\text { Total annual, } 1887-88 \text { (inches) } \ldots \ldots \ldots \ldots \ldots \ldots \ldots \ldots \\
\text { Moisture ratios: }\end{array}$ & 32.5 & 101.2 \\
\hline 58 & 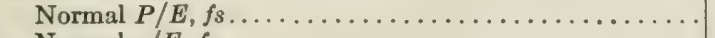 & .12 & .97 \\
\hline 59 & 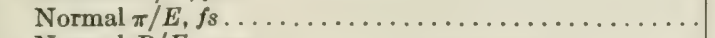 & .15 & 1.05 \\
\hline 60 & $\begin{array}{l}\text { Normal } P / E \text {, year. } \ldots \ldots \ldots \ldots \ldots \ldots \ldots \ldots \ldots \\
\text { Vapor pressure: }\end{array}$ & .21 & 1.02 \\
\hline 63 & $\begin{array}{l}\text { Normal mean, } f_{s} \text { (hundredths inch) } \ldots \ldots \ldots \ldots \ldots \ldots \\
\text { Humidity: }\end{array}$ & $300-a$ & 622 \\
\hline 65 & Normal mean, $f_{s}$ (per cent) $\ldots \ldots \ldots \ldots \ldots \ldots$ & $40.0-a$ & 80.2 \\
\hline 66 & $\begin{array}{l}\text { Normal mean, year (per cent) } \ldots \ldots \ldots \ldots \ldots \ldots \ldots \\
\text { Wind: }\end{array}$ & $40.0-a$ & 85.2 \\
\hline 68 & $\begin{array}{l}\text { Normal mean hourly velocity, } f_{s} \text { (miles) } \ldots \ldots \ldots \ldots \ldots \\
\text { Sunshine: }\end{array}$ & 4.5 & 11.0 \\
\hline 69 & $\begin{array}{l}\text { Normal total duration, } f s \text { (hours) . . . . . . } \\
\text { Moisture-temperature indices: }\end{array}$ & .. & 2,995 \\
\hline 70 & Normal $P / E \times T, f_{s}$, remainder method... & 283 & 1,142 \\
\hline 71 & Normal $P / E \times T, f_{s}$, exponential method..... & $1,000-a$ & 10,331 \\
\hline 72 & 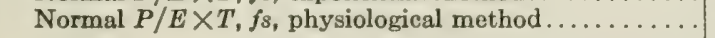 & $1,000-a$ & 20,570 \\
\hline
\end{tabular}


TABLE 53.-Climatic extremes for region with 5 to 9 species of Microphyllous trees.

\begin{tabular}{|c|c|c|c|}
\hline Plate & Temperature: & Low. & High. \\
\hline 34 & Days in normal frostless season $\left(f_{s}\right) \ldots \ldots \ldots . .$. & 226 & 323 \\
\hline 35 & Hot days, $f_{8} \ldots \ldots \ldots \ldots \ldots \ldots \ldots \ldots \ldots \ldots$ & 156 & 218 \\
\hline 36 & Cold days, $f_{s} \ldots \ldots \ldots \ldots \ldots \ldots \ldots \ldots \ldots$ & 0 & 0 \\
\hline 37 & Remainder summation above $32^{\circ}$, year (thousands).. & $18.0-$ & $26.0+$ \\
\hline 38 & Remainder summation above $39^{\circ}, f s$ (thousands).... & 7.6 & 10.3 \\
\hline 39 & Exponential summation, $f_{8}$ (hundreds) ........... & 8.3 & 11.8 \\
\hline 40 & 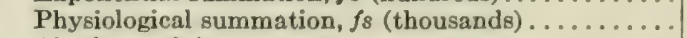 & 15.0 & 21.4 \\
\hline 41 & 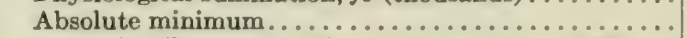 & -5 & +22 \\
\hline 43 & Normal daily mean, coldest 14 days of year $\left({ }^{\circ} \mathrm{F}.\right) \ldots$ & 43 & 54 \\
\hline 44 & Normal daily mean, hottest 6 weeks of year $\left({ }^{\circ} \mathrm{F}.\right)$. & $71.6-$ & $78.8+$ \\
\hline 45 & $\begin{array}{l}\text { Normal daily mean, year }\left({ }^{\circ} \mathrm{F} .\right) \ldots \ldots \ldots \ldots \ldots \ldots \ldots \\
\text { Precipitation: }\end{array}$ & $65-$ & $70+$ \\
\hline 46 & Normal daily mean, $f_{8}$ (inch) $\ldots \ldots \ldots \ldots \ldots \ldots$ & .020 & .080 \\
\hline 47 & Normal No. rainy days (over 0.10 inch), $f_{8} \ldots \ldots \ldots$ & 2 & $100^{a}$ \\
\hline 48 & Normal No. dry days $(0.10$ inch or less $), f_{s} \ldots \ldots .$. & 211 & 259 \\
\hline 49 & Dry days, percentage of total, $f_{s}$ (per cent)......... & 77 & 99 \\
\hline 50 & Days in longest normal rainy period, $f s \ldots \ldots \ldots$. & 2 & 30 \\
\hline 51 & Days in longest normal dry period, $f_{8} \ldots \ldots \ldots \ldots$ & $75-a$ & 124 \\
\hline 52 & $\begin{array}{l}\text { Mean total, year (inches) } \ldots \ldots \ldots \ldots \ldots \ldots \ldots \ldots \\
\text { Evaporation: }\end{array}$ & $10-$ & 40 \\
\hline 53 & Daily mean, $1887-88, f_{8}$ (inch) $\ldots \ldots \ldots \ldots \ldots$ & .118 & $.260+a$ \\
\hline 54 & $\begin{array}{l}\text { Total annual, } 1887-88 \text { (inches) ................. } \\
\text { Moisture ratios: }\end{array}$ & 38.0 & 95.7 \\
\hline 58 & Normal $P / E, f_{s} \ldots$. & .12 & .65 \\
\hline 59 & Normal $\pi / E, f_{s} \ldots \ldots \ldots . .$. & .13 & .72 \\
\hline 60 & 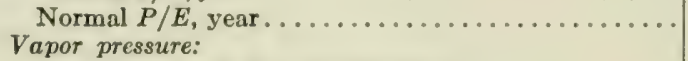 & .03 & .70 \\
\hline 63 & $\begin{array}{l}\text { Normal mean, } f s \text { (hundredths inch)... } \\
\text { Humidity: }\end{array}$ & 300 & 675 \\
\hline 65 & Normal mean, $f_{3}$ (per cent).. & 37.0 & 81.9 \\
\hline 66 & $\begin{array}{l}\text { Normal mean, year (per cent)... } \\
\text { Wind: }\end{array}$ & 38.7 & 82.1 \\
\hline 68 & $\begin{array}{l}\text { Normal mean hourly velocity, } f_{s} \text { (miles).... } \\
\text { Sunshine: }\end{array}$ & 4.5 & 12.3 \\
\hline 69 & $\begin{array}{l}\text { Normal total duration, } f s \text { (hours.......... } \\
\text { Moisture-temperature indices: }\end{array}$ & $2,300+$ & $2,300+$ \\
\hline 70 & Normal $P / E \times T, f 3$, remainder method... & 98 & 737 \\
\hline 71 & Normal $P / E \times T, f_{s}$, exponential method........ & 906 & 6,690 \\
\hline 72 & Normal $P / E \times T, f s$, physiological method........ & 1,790 & 13,926 \\
\hline
\end{tabular}

TABLE 54.-Climatic extremes for region with 10 or more species of Microphyllous trees.

\begin{tabular}{|c|c|c|c|}
\hline Plate & Temperature: & Low. & High. \\
\hline 34 & Days in normal frostless season $(f s) \ldots \ldots \ldots \ldots \ldots$ & $280+a$ & $318^{\circ}$ \\
\hline 35 & Hot days, $f_{s} \ldots \ldots \ldots \ldots \ldots \ldots \ldots \ldots \ldots \ldots$ & $180 \div$ & $210+$ \\
\hline 36 & Cold days, $f_{s} \ldots \ldots \ldots$. & 0 & 0 \\
\hline 37 & Remainder summation above $32^{\circ}$, year (thousands)... & 1. $0+$ & $26.0+$ \\
\hline 38 & Remainder summation above $39^{\circ}, f_{8}$ (thousands).... & $9.0+$ & $10.0+$ \\
\hline 39 & Exponential summation, $f_{s}$ (hundreds). & $11.0+$ & \\
\hline 40 & Physiological summation, $f_{s}$ (thousands) & 20.0 & $21.0+$ \\
\hline 41 & 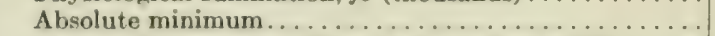 & +6 & +12 \\
\hline 43 & Normal daily mean, coldest 14 days of year $\left({ }^{\circ} \mathrm{F}_{0}\right) \ldots$ & $50+$ & $50+$ \\
\hline 44 & Normal daily mean, hottest 6 wecks of year ( $\left.{ }^{\circ} \mathrm{F}.\right) \ldots$ & $7 x .4+$ & $78.5+$ \\
\hline 45 & $\begin{array}{l}\text { Normal daily mean, year }\left({ }^{\circ} \mathrm{F} .\right) \ldots \ldots \ldots \ldots \ldots \ldots \ldots \\
\text { Precipitation: }\end{array}$ & $70+$ & $70+$ \\
\hline 46 & Normal dnily mean, $f s$ (inch) $\ldots \ldots \ldots \ldots$ & 0,12 & $.07 \mathrm{~s}$ \\
\hline 47 & Normal No. rainy days (over $0.10 \mathrm{inch}$ ), $f s \ldots$ & $39-$ & $39-$ \\
\hline 48 & Normal No. dry days $(0.10$ inch or less $), f_{s} \ldots \ldots$. & $259+$ & $259+$ \\
\hline 49 & Dry days, percentage of total, $f_{s}$ (per cent)..... & $s+$ & $87+$ \\
\hline 50 & Days in longest normal rainy period, $f_{s} \ldots \ldots \ldots \ldots \ldots$ & $25-$ & $25-$ \\
\hline 51 & Days in longest normal dry period, $f_{3} \ldots \ldots \ldots \ldots \ldots$ & $75 \div$ & $75+$ \\
\hline 62 & 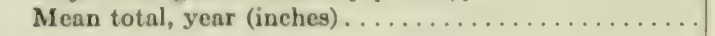 & $30-$ & $30+$ \\
\hline
\end{tabular}


TABLE 54.-Climatic extremes for region with 10 or more species of Microphyllous trees-Continued.

\begin{tabular}{|c|c|c|c|}
\hline Plate & Evaporation: & Low. & Hiah. \\
\hline 53 & Daily mean, $1887-88, f_{s}$ (inch) $\ldots \ldots \ldots \ldots \ldots \ldots$ & .102 & $.160^{\alpha}$ \\
\hline 54 & $\begin{array}{l}\text { Total annual, } 1887-88 \text { (inches) } \ldots \ldots \ldots \ldots \ldots \ldots \ldots \ldots \\
\text { Moisture ratios: }\end{array}$ & 37.0 & 53.1 \\
\hline 58 & Normal $P / E, f_{s}, \ldots \ldots \ldots \ldots \ldots \ldots \ldots \ldots$ & .34 & .77 \\
\hline 59 & Normal $\pi / E, f_{s} \ldots \ldots \ldots \ldots \ldots \ldots \ldots \ldots \ldots$ & .35 & .81 \\
\hline 60 & 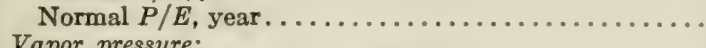 & $.60-a$ & .76 \\
\hline 63 & 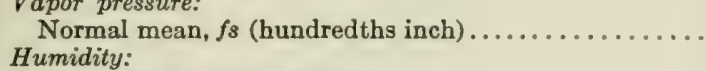 & $550-a$ & $600+a$ \\
\hline 65 & Normal mean, $f_{S}$ (per cent). & $70.0-a$ & $80.0+a$ \\
\hline 66 & $\begin{array}{l}\text { Normal mean, year (per cent) } \ldots \ldots \ldots \ldots \ldots \ldots \ldots \\
\text { Wind: }\end{array}$ & $70.0-a$ & $80.0+a$ \\
\hline 68 & $\begin{array}{l}\text { Normal mean hourly velocity, } f_{s} \text { (miles) ......... } \\
\text { Sunshine: }\end{array}$ & $10-a$ & $10+a$ \\
\hline 69 & $\begin{array}{l}\text { Normal total duration, } f_{s} \text { (hours) . . . . . . . . . . } \\
\text { Moisture-temperature indices: }\end{array}$ & $2,300+$ & $2,300+$ \\
\hline 70 & Normal $P / E \times T, f s$, remainder method... & $500-a$ & $700+a$ \\
\hline 71 & Normal $P / E \times T, f_{s}$, exponential method......... & $5,000-a$ & $6,000+a$ \\
\hline 72 & Normal $P / E \times T, f s$, physiological method.......... & $8,000^{a}$ & $13,000+a$ \\
\hline
\end{tabular}

TABLE 55.-Climatic extremes for region with no species of a selected group of 15 deciduous trees of the southeastern states (South).

\begin{tabular}{|c|c|c|c|}
\hline Plate & Temperature: & Low. & High. \\
\hline 34 & Days in normal frostless season $(f s) \ldots \ldots \ldots \ldots \ldots$ & 315 & 365 \\
\hline 35 & Hot days, $f_{s} \ldots \ldots \ldots \ldots \ldots \ldots \ldots \ldots \ldots$ & 226 & 365 \\
\hline 36 & 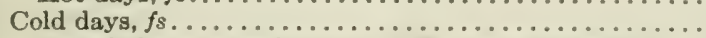 & 0 & 0 \\
\hline 37 & Remainder summation above $32^{\circ}$, year (thousands)... & $26.0+$ & \\
\hline 38 & Remainder summation above $39^{\circ}, f_{s}$ (thousands)..... & 10.8 & 14.5 \\
\hline 39 & Exponential summation, $f_{8}$ (hundreds) ............ & 11.1 & 15.4 \\
\hline 40 & Physiological summation, $f_{s}$ (thousands) ............ & 21.4 & 31.1 \\
\hline 41 & 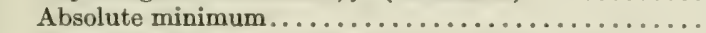 & +19 & +41 \\
\hline 43 & Normal daily mean, coldest 14 days of year $\left({ }^{\circ} \mathrm{F}.\right) \ldots$ & 57 & 69 \\
\hline 44 & Normal daily mean, hottest 6 weeks of year $\left({ }^{\circ} \mathrm{F}.\right) \ldots$ & $78.8+$ & \\
\hline 45 & $\begin{array}{l}\text { Normal daily mean, year }\left({ }^{\circ} \mathrm{F} .\right) \ldots \ldots \ldots \ldots \ldots \ldots \\
\text { Precipitation: }\end{array}$ & $75-$ & $75+$ \\
\hline 46 & Normal daily mean, $f_{s}$ (inch). & .106 & $: 173$ \\
\hline 47 & Normal No. rainy days (over 0.10 inch), $f_{s} \ldots \ldots \ldots$ & 161 & 234 \\
\hline 48 & Normal No. dry days $(0.10$ inch or less $), f_{s} \ldots \ldots \ldots \ldots$ & 84 & 204 \\
\hline 49 & Dry days, percentage of total, $f s$ (per cent) .......... & 26 & 56 \\
\hline 50 & Days in longest normal rainy period, $f s \ldots \ldots \ldots \ldots$ & 112 & 174 \\
\hline 51 & Days in longest normal dry period, $f s . \ldots \ldots \ldots \ldots$ & 19 & 182 \\
\hline 52 & $\begin{array}{l}\text { Mean total, year (inches) } \ldots \ldots \ldots \ldots \ldots \ldots \ldots \ldots \\
\text { Evaporation: }\end{array}$ & 60 & $60+$ \\
\hline 53 & Daily mean, $1887-88, f_{s}$ (inch)... & $.140-a$ & .141 \\
\hline 54 & $\begin{array}{l}\text { Total annual, } 1887-88 \text { (inches) } \ldots \ldots \ldots \ldots \ldots \ldots \ldots \\
\text { Moisture ratios: }\end{array}$ & $50-a$ & 51.6 \\
\hline 58 & Normal $P / E, f s . . . \ldots \ldots$ & .75 & $1.00+a$ \\
\hline 59 & Normal $\pi / E, f_{s} \ldots \ldots \ldots \ldots \ldots \ldots$ & .75 & $1.20^{a}$ \\
\hline 60 & 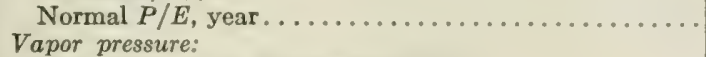 & .75 & 1.36 \\
\hline 63 & $\begin{array}{l}\text { Normal mean, } f s \text { (hundredths inch) ........................ } \\
\text { Humidity: }\end{array}$ & 692 & 707 \\
\hline 65 & Normal mean, $f_{s}$ (per cent)......... & 77.1 & 80.5 \\
\hline 66 & $\begin{array}{l}\text { Normal mean, year (per cent) ..................... } \\
\text { Wind: }\end{array}$ & 77.1 & 80.5 \\
\hline 68 & $\begin{array}{l}\text { Normal mean hourly velocity, } f_{s} \text { (miles).... } \\
\text { Sunshine: }\end{array}$ & $8-a$ & 9.7 \\
\hline 69 & $\begin{array}{l}\text { Normal total duration, } f s \text { (hours) . . . . . . } \\
\text { Moisture-temperature indices: }\end{array}$ & $2,300+$ & \\
\hline 70 & Normal $P / E \times T, f_{s}$, remainder method. & 1,155 & $1,200+$ \\
\hline 71 & Normal $P / E \times T, f_{s}$, exponential method.......... & 10,877 & $11,000+a$ \\
\hline 72 & Normal $P / E \times T, f_{s}$, physiological method......... & $23,000+a$ & $\ldots$. \\
\hline
\end{tabular}


TABLE 56.-Climatic extremes for region with no species of a selected group of 15 deciduous trees of the southeastern states (North).

\begin{tabular}{|c|c|c|c|}
\hline Plate & Temperature: & Low. & High. \\
\hline 34 & Days in normal frostless season $\left(f_{8}\right) \ldots \ldots \ldots \ldots \ldots$ & 25 & 316 \\
\hline 35 & Hot days, $f_{s} \ldots \ldots \ldots \ldots \ldots \ldots \ldots \ldots$ & 0 & 173 \\
\hline 36 & Cold days, $f_{3} \ldots \ldots \ldots \ldots \ldots$. & 0 & 158 \\
\hline 37 & Remainder summation above $32^{\circ}$, year (thousands). & 10.0 & 11.5 \\
\hline 38 & Remainder summation above $39^{\circ}, f_{8}$ (thousands).. & 2.6 & 10.0 \\
\hline 39 & Exponential summation, $f_{8}$ (hundreds) ............ & 3.0 & 11.8 \\
\hline 40 & Physiological summation, $f s$ (thousands). . & 2.1 & 20.6 \\
\hline 41 & Absolute minimum................. & -59 & +22 \\
\hline 43 & Normal daily mean, coldest 14 days of year $\left({ }^{\circ} \mathrm{F}.\right)$. & 0 & 54 \\
\hline 44 & Normal daily mean, hottest 6 weeks of year $\left({ }^{\circ} \mathrm{F}\right.$.). & $64.4-$ & $78.8+$ \\
\hline 45 & Normal daily mean, year $\left({ }^{\circ} \mathrm{F}.\right) \ldots \ldots \ldots \ldots \ldots$ & & $70+$ \\
\hline & Precipitation: & & \\
\hline 46 & Normal daily mean, $f_{s}$ (inch). & .009 & 199 \\
\hline 47 & Normal No. rainy days (over $0.10 \mathrm{inch}$ ), $f s$. & 0 & 199 \\
\hline 48 & Normal No. dry days ( 0.10 inch or less), $f_{s} .$. & 19 & 294 \\
\hline 49 & Dry days, percentage of total, $f s$ (per cent). & 11 & 100 \\
\hline 50 & Days in longest normal rainy period, $f_{s} .$. & 0 & 172 \\
\hline 51 & Days in longest normal dry period, $f_{s} . .$. & 4 & 299 \\
\hline 52 & Mean total, year (inches). & $10-$ & $60+$ \\
\hline & Evaporation: & & \\
\hline 53 & Daily mean, $1887-88, f_{8}$ (inch).. & .052 & .349 \\
\hline 54 & Total annual, 1887-88 (inches).. & 18.1 & 101.2 \\
\hline 58 & $\begin{array}{l}\text { Monsture ratros: } \\
\text { Normal } P / E \text { fs }\end{array}$ & .04 & \\
\hline 59 & Normal $\pi / E, f_{s} \ldots \ldots \ldots$ & .06 & 1.52 \\
\hline 60 & 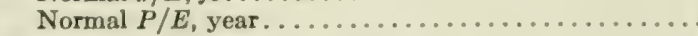 & .03 & 4.90 \\
\hline 63 & $\begin{array}{l}\text { Vapor pressure: } \\
\text { Normal mean, fs (hundredths inch).. } \\
\text { Humidity. }\end{array}$ & 183 & 425 \\
\hline 65 & $\begin{array}{l}\text { Humidity: } \\
\text { Normal mean, } f 8 \text { (per cent)..... }\end{array}$ & 22.6 & 87.5 \\
\hline 66 & $\begin{array}{l}\text { Normal mean, year (per cent).... } \\
\text { Wind: }\end{array}$ & 29.7 & 86.8 \\
\hline 68 & $\begin{array}{l}\text { Normal mean hourly velocity, } f_{s} \text { (miles). . } \\
\text { Sunshine: }\end{array}$ & 4.3 & 17.2 \\
\hline 69 & $\begin{array}{l}\text { Normal total duration, } f_{8} \text { (hours). } \\
\text { Moisture-temperalure indices: }\end{array}$ & 1,134 & 2,995 \\
\hline 70 & Normal $P / E \times T, f_{3}$, remainder method. & 13 & 1,566 \\
\hline 71 & Normal $P / E \times T$, fs, exponential method. & 127 & 11,724 \\
\hline 72 & Normal $P / E \times T, f s$, physiological method. . & 197 & 7,475 \\
\hline
\end{tabular}

TABLE 57.-Climatic extremes for region with 1 to 11 species of a selected group of 16 deciduous trees of the southeastern states.

\begin{tabular}{|c|c|c|c|}
\hline Plate & Temperature: & Low. & High. \\
\hline 34 & Days in normal fiostless season $(f s)$. . & 145 & \\
\hline & Hot days, $f_{s} \ldots \ldots \ldots \ldots \ldots \ldots \ldots$ & 47 & 218 \\
\hline 36 & Cold days, $f_{s} \ldots \ldots \ldots \ldots \ldots \ldots \ldots$ & 0 & 112 \\
\hline 37 & Remainder summation above $32^{\circ}$, year (thousands). & $11.5-$ & $26.0+$ \\
\hline 38 & Remainder summation above $39^{\circ}, f_{s}$ (thousands)... & 4.4 & 10.3 \\
\hline 39 & Exponential summation, $f_{s}$ (hundreds)... & 4.7 & 11.7 \\
\hline 40 & Physiological summation, fs (thousands) & 5.4 & 21.4 \\
\hline 41 & Absolute minimum.................. & -32 & +12 \\
\hline 43 & Normal daily mean, coldest 14 days of year $\left({ }^{\circ}{ }^{\circ}\right.$. $)$. & 18 & 53 \\
\hline 44 & Normal daily mean, hottest 6 weeks of year $\left({ }^{\circ} \mathrm{F}\right.$.). & $71.6-$ & $78.8+$ \\
\hline 45 & Normal daily mean, year $\left({ }^{\circ} \mathrm{F}.\right) \ldots \ldots \ldots \ldots$ & $50-$ & $70+$ \\
\hline & Precipitation: & & \\
\hline 46 & Normal daily mean, $f_{s}$ (inch). & 052 & .170 \\
\hline 47 & Normal No. rainy days (over $0.10 \mathrm{inch}$ ), $f_{s} .$. & $25^{a}$ & 256 \\
\hline 48 & Normal No. dry days (0.10 inch or less), $f_{s}$. & 0 & 259 \\
\hline 49 & Dry days, percentage of total, $f s$ (per cent).... & 0 & $90^{\alpha}$ \\
\hline 50 & Days in longest normal rainy period, $f_{s}, \ldots$ & $25-a$ & 256 \\
\hline 51 & Days in longest normal dry period, $f_{s} . . . .$. & 0 & $100^{\circ}$ \\
\hline 52 & Mean total, year (inches) ............ & $20-$ & $60+$ \\
\hline
\end{tabular}


TABLE 57.-Climatic extremes for region with 1 to 11 species of a selected group of 15 deciduous trees of the southeastern states-Continued.

\begin{tabular}{|c|c|c|c|}
\hline Plate & Evaporation: & Low. & High. \\
\hline 53 & Daily mean, $1887-88, f_{s}$ (inch) $\ldots \ldots \ldots \ldots \ldots \ldots$ & .081 & .200 \\
\hline 54 & $\begin{array}{l}\text { Total annual, } 1887-88 \text { (inches) } \ldots \ldots \ldots \ldots \ldots \ldots \ldots \ldots \\
\text { Moisture ratios: }\end{array}$ & 25.2 & $90.0^{\circ}$ \\
\hline 58 & Normal $P / E, f_{s} \ldots \ldots \ldots \ldots \ldots \ldots \ldots \ldots$ & .34 & 1.76 \\
\hline 59 & Normal $\pi / E, f s \ldots$ & .35 & 1.96 \\
\hline 60 & $\begin{array}{l}\text { Normal } P / E, \text { year. } \ldots \ldots \ldots \ldots \ldots \ldots \ldots \ldots \ldots \ldots \\
\text { Vapor pressure: }\end{array}$ & .24 & 1.94 \\
\hline 63 & $\begin{array}{l}\text { Normal mean, } f s \text { (hundredths inch) ...................... } \\
\text { Humidity: }\end{array}$ & $400-a$ & 573 \\
\hline 65 & Normal mean, $f s$ (per cent) & $50-a$ & 82.7 \\
\hline 66 & $\begin{array}{l}\text { Normal mean, year (per cent) } \ldots \ldots \ldots \ldots \ldots \ldots \ldots \\
\text { Wind: }\end{array}$ & $50-a$ & 82.9 \\
\hline 68 & $\begin{array}{l}\text { Normal mean hourly velocity, } f_{s} \text { (miles)........... } \\
\text { Sunshine: }\end{array}$ & 3.6 & 13.5 \\
\hline 69 & $\begin{array}{l}\text { Normal total duration, } f_{s} \text { (hours)............. } \\
\text { Moisture-temperature indices: }\end{array}$ & 1,626 & 2,625 \\
\hline 70 & Normal $P / E \times T, f_{s}$, remainder method. & $200^{a}$ & 1,418 \\
\hline 71 & 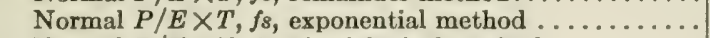 & $3,000+a$ & 13,511 \\
\hline 72 & Normal $P / E \times T, f_{s}$, physiological method.......... & $3,000^{a}$ & 24,265 \\
\hline
\end{tabular}

TABLE 58.-Climatic extremes for region with 12 to 14 species of a selected group of 15 deciduous trees of the southeastern states.

\begin{tabular}{|c|c|c|c|}
\hline Plate & Temperature: & Low. & High. \\
\hline 34 & Days in normal frostless season $(f s) \ldots \ldots \ldots \ldots \ldots$ & 179 & 328 \\
\hline 35 & Hot days, $f_{s} . \ldots \ldots \ldots \ldots \ldots \ldots \ldots \ldots \ldots \ldots \ldots$ & 147 & 192 \\
\hline 36 & Cold days, $f s . \ldots \ldots \ldots \ldots \ldots \ldots \ldots$ & 0 & 0 \\
\hline 37 & Remainder summation above $32^{\circ}$, year (thousands)... & $18.0+$ & $26.0-$ \\
\hline 38 & Remainder summation above $39^{\circ}, f s$ (thousands)..... & 7.3 & 9.9 \\
\hline 39 & 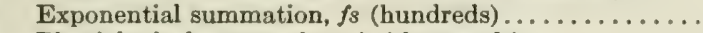 & 7.7 & 10.8 \\
\hline 40 & Physiological summation, $f_{s}$ (thousands) .......... & 13.6 & 19.3 \\
\hline 41 & 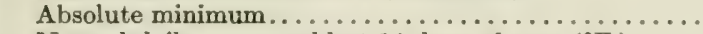 & -18 & +10 \\
\hline 43 & Normal daily mean, coldest 14 days of year $\left({ }^{\circ} \mathrm{F}.\right) \ldots$ & $35^{a}$ & 53 \\
\hline 44 & Normal daily mean, hottest 6 weeks of year $\left({ }^{\circ} \mathrm{F}.\right) \ldots$ & $78.8+$ & $78.8+$ \\
\hline 45 & $\begin{array}{l}\text { Normal daily mean, year }\left({ }^{\circ} \mathrm{F} .\right) \ldots \ldots \ldots \ldots \ldots \ldots \\
\text { Precipitation: }\end{array}$ & $60-$ & $65+$ \\
\hline 46 & Normal daily mean, $f_{s}$ (inch)... & .119 & .159 \\
\hline 47 & Normal No. rainy days (over 0.10 inch), $f s \ldots \ldots \ldots$ & 166 & 284 \\
\hline 48 & Normal No. dry days $(0.10$ inch or less $), f s \ldots \ldots \ldots$ & 26 & 88 \\
\hline 49 & Dry days, percentage of total, $f_{s}$ (per cent).......... & 8 & 34 \\
\hline 50 & Days in longest normal rainy period, $f_{s} \ldots \ldots \ldots \ldots$ & 95 & 196 \\
\hline 51 & Days in longest normal dry period, $f_{s} \ldots \ldots \ldots \ldots \ldots$ & 17 & 34 \\
\hline 52 & $\begin{array}{l}\text { Mean total, year (inches) } \ldots \ldots \ldots \ldots \ldots \ldots \ldots \ldots \\
\text { Evaporation: }\end{array}$ & $50-$ & $60+$ \\
\hline 53 & Daily mean, $1887-88, f_{s}$ (inch). & .117 & .165 \\
\hline 54 & $\begin{array}{l}\text { Total annual, } 1887-88 \text { (inches) ................... } \\
\text { Moisture ratios: }\end{array}$ & 38.4 & 50.0 \\
\hline 58 & Normal $P / E, f_{s}$. & .75 & 1.35 \\
\hline 59 & Normal $\pi / E, f_{3} \ldots \ldots \ldots \ldots \ldots$. & .92 & 1.47 \\
\hline 60 & 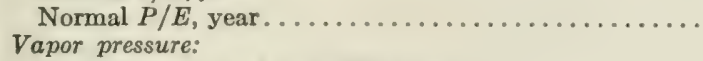 & 1.00 & 1.33 \\
\hline 63 & $\begin{array}{l}\text { Normal mean, } f_{s} \text { (hundredths inch). } \\
\text { Humidity: }\end{array}$ & 545 & 588 \\
\hline 65 & Normal mean, $f_{s}$ (per cent).. & 71.3 & 79.0 \\
\hline 66 & $\begin{array}{l}\text { Normal mean, year (per cent) } \ldots \ldots \ldots \ldots \ldots \ldots \ldots \\
\text { Wind: }\end{array}$ & 71.8 & 80.1 \\
\hline 68 & $\begin{array}{l}\text { Normal mean hourly velocity, fs (miles)............. } \\
\text { Sunshine: }\end{array}$ & 6.3 & 8.0 \\
\hline 69 & $\begin{array}{l}\text { Normal total duration, } f s \text { (hours) } \\
\text { Moisture-temperature indices: }\end{array}$ & 1,895 & 2,301 \\
\hline 70 & Normal $P / E \times T, f s$, remainder method........... & 593 & 1,304 \\
\hline 71 & Normal $P / E \times T, f s$, exponential method.......... & 5,253 & 11,956 \\
\hline 72 & Normal $P / E \times T, f s$, physiological method.......... & 10,837 & 23,381 \\
\hline
\end{tabular}


TABLE 59.-Climatic extremes for region with all species of a selected group of 15 deciduous trees of the southeastern states.

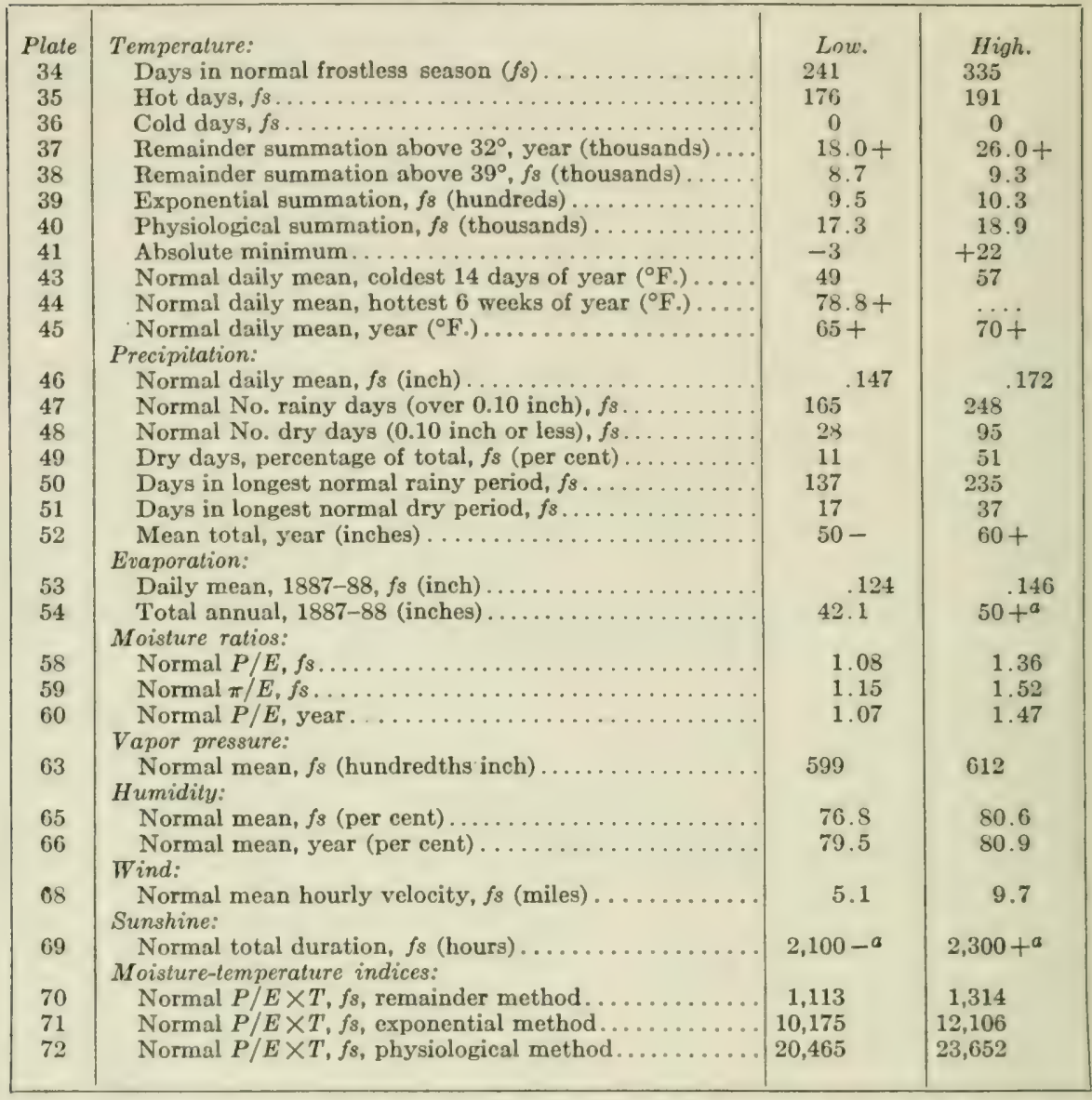

TABLE 60.-Climalic extremes for region with no species of a selected group of 13 deciduous trees of the eastern states (North and West).

\begin{tabular}{|c|c|c|c|}
\hline Plate & Temperature: & Low. & High. \\
\hline 34 & Days in normal frostless season $(f s) \ldots \ldots \ldots \ldots \ldots$ & 25 & 318 \\
\hline 35 & 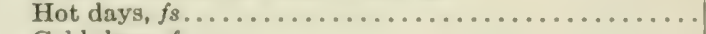 & 4 & 211 \\
\hline 36 & Cold days, $f_{s} \ldots \ldots \ldots \ldots \ldots \ldots \ldots \ldots \ldots$ & 0 & 158 \\
\hline 37 & Remainder summation above $32^{\circ}$, year (thousands).... & $10.0-$ & 26.0 \\
\hline 38 & Remainder summation abovo $39^{\circ}, f_{s}$ (thousands)..... & 2.8 & 10.1 \\
\hline 39 & Exponential summation, $f_{s}$ (hundreds).............. & 291 & 1,184 \\
\hline 40 & Physiological summation, $f_{s}$ (thousands) $\ldots . . . \ldots$ & 1,947 & 20,640 \\
\hline 41 & 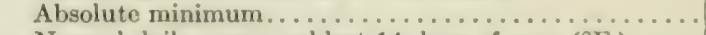 & -49 & +22 \\
\hline 43 & Normal daily mean, coldest 14 days of year ( $\left.{ }^{\circ} \mathrm{F}.\right) \ldots$ & 0 & 54 \\
\hline 44 & Normal daily mean, hottest 6 weeks of year $\left({ }^{\circ} \mathrm{F}.\right) \ldots$ & $64.4-$ & $78.8+$ \\
\hline 45 & $\begin{array}{l}\text { Normal daily mean, year }\left({ }^{\circ} \mathrm{F}_{0}\right) \ldots \ldots \ldots \ldots \ldots \ldots \ldots \\
\text { Precipitation: }\end{array}$ & 35 & $70+$ \\
\hline 46 & Normal daily mean, $f_{s}$ (inch) ..... & .009 & .199 \\
\hline 47 & Normal No. rainy days (over 0.10 inch), $f s \ldots . .$. & 0 & 199 \\
\hline 48 & Normal No. dry days $(0.10$ inch or less $), f s \ldots \ldots \ldots$ & 48 & 294 \\
\hline 49 & Dry days, percentage of total, $f_{s}$ (per cent) .......... & 27 & 100 \\
\hline 50 & Days in longest normal rainy period, $f_{s} \ldots \ldots \ldots \ldots$ & 0 & 99 \\
\hline 51 & Days in longest normal dry period, $f_{s} \ldots \ldots \ldots \ldots$ & 54 & 299 \\
\hline 52 & 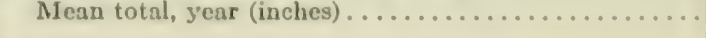 & $10-$ & $30+$ \\
\hline
\end{tabular}


TABLE 60.-Climatic extremes for region with no species of a selected group of 19 deciduous trees of the eastern states (North and West)-Continued.

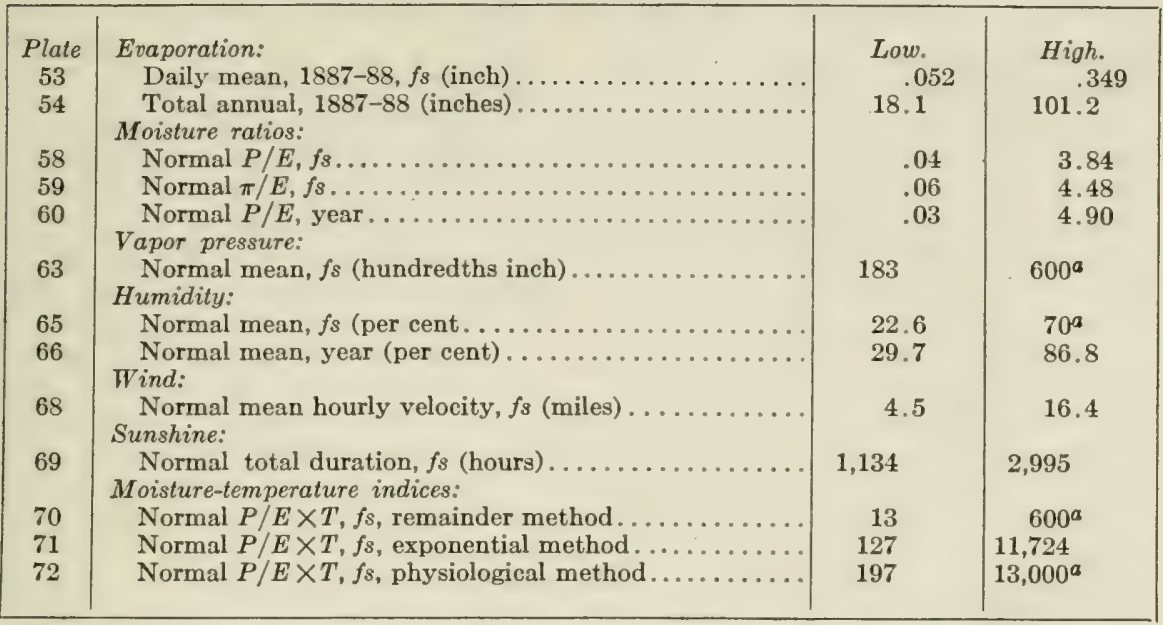

TABLE 61.-Climatic extremes for region with no species of a selected group of 13 deciduous trees of the eastern states (South).

\begin{tabular}{|c|c|c|c|}
\hline Plate & Temperature: & Low. & High. \\
\hline 34 & Days in normal frostless season $(f s) \ldots \ldots \ldots \ldots \ldots$ & 312 & 365 \\
\hline 35 & 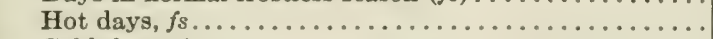 & 285 & 365 \\
\hline 36 & Cold days, $f s \ldots \ldots \ldots \ldots \ldots \ldots \ldots \ldots \ldots \ldots$ & 0 & 0 \\
\hline 37 & Remainder summation above $32^{\circ}$, year (thousands).... & 26.0 & $26.0+$ \\
\hline 38 & Remainder summation above $39^{\circ}, f_{s}$ (thousands)...... & 11.3 & 14.5 \\
\hline 39 & Exponential summation, $f_{s}$ (hundreds) ............ & 1,260 & 1,542 \\
\hline 40 & Physiological summation, $f_{s}$ (thousands) .......... & 24,872 & 31,063 \\
\hline 41 & 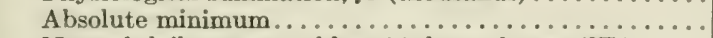 & +19 & +41 \\
\hline 43 & Normal daily mean, coldest 14 days of year $\left({ }^{\circ} \mathrm{F}.\right) . . .$. & $60-$ & 69 \\
\hline 44 & Normal daily mean, hottest 6 weeks of year $\left({ }^{\circ} \mathrm{F}.\right) \ldots$. & $78.8+$ & $78.8+$ \\
\hline 45 & $\begin{array}{l}\text { Normal daily mean, year }\left({ }^{\circ} \mathrm{F} .\right) \ldots \ldots \ldots \ldots \ldots \ldots \\
\text { Precipitation: }\end{array}$ & $70+$ & $75+$ \\
\hline 46 & Normal daily mean, $f_{s}$ (inch) ....... & .106 & .173 \\
\hline 47 & Normal No. rainy days (over $0.10 \mathrm{inch}$ ), $f_{s} \ldots \ldots \ldots \ldots$ & 161 & 234 \\
\hline 48 & Normal No. dry days $(0.10$ inch or less $), f s . . . . .$. & 84 & 201 \\
\hline 49 & Dry days, percentage of total, $f_{s}$ (per cent).......... & 26 & 56 \\
\hline 50 & Days in longest normal rainy period, $f s \ldots \ldots \ldots \ldots$ & 112 & 174 \\
\hline 51 & Days in longest normal dry period, $f_{3} \ldots \ldots \ldots \ldots \ldots$ & 19 & 182 \\
\hline 52 & $\begin{array}{l}\text { Mean total, year (inches) } \ldots \ldots \ldots \ldots \ldots \ldots \ldots \ldots \\
\text { Evaporation: }\end{array}$ & $06-$ & $60+$ \\
\hline 53 & Daily mean, $1887-88, f_{s}$ (inch) $\ldots \ldots \ldots \ldots \ldots \ldots$ & $.140-a$ & .141 \\
\hline 54 & $\begin{array}{l}\text { Total annual, } 1887-88 \text { (inches) } \ldots \ldots \ldots \ldots \ldots \ldots \ldots \\
\text { Moisture ratios: }\end{array}$ & $50-a$ & 51.6 \\
\hline 58 & Normal $P / E, f_{s} \ldots \ldots$ & .75 & $1.00+a$ \\
\hline 59 & 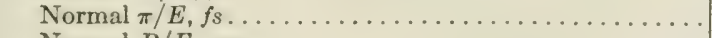 & .75 & $1.20^{a}$ \\
\hline 60 & $\begin{array}{l}\text { Normal } P / E \text {, year. . . . . . . } \ldots \ldots \ldots \ldots \ldots \ldots \\
\text { Vapor pressure: }\end{array}$ & .75 & 1.36 \\
\hline 63 & $\begin{array}{l}\text { Normal mean, } f_{s} \text { (hundredths inch)........ } \\
\text { Humidity: }\end{array}$ & $650-a$ & 707 \\
\hline 65 & 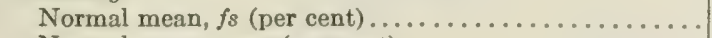 & 77.1 & 80.5 \\
\hline 66 & $\begin{array}{l}\text { Normal mean, year (per cent) } \ldots \ldots \ldots \ldots \ldots \ldots \ldots \\
\text { Wind: }\end{array}$ & 77.1 & 80.5 \\
\hline 68 & $\begin{array}{l}\text { Normal mean hourly velocity, } f s \text { (miles)........... } \\
\text { Sunshine: }\end{array}$ & $8.0-a$ & 9.7 \\
\hline 69 & $\begin{array}{l}\text { Normal total duration, } f s \text { (hours) } \ldots \ldots \ldots \ldots \ldots \ldots \\
\text { Moisture-temperature indices: }\end{array}$ & $2,300+$ & \\
\hline 70 & Normal $P / E \times T, f_{s}$, remainder method... & 1,155 & $1,200+a$ \\
\hline 71 & Normal $P / E \times T, f_{s}$, exponential method...... & 10,877 & $11,000+$ \\
\hline 72 & Normal $P / E \times T, f s$, physiological method...... & $23,000+$ & $\ldots$ \\
\hline
\end{tabular}


TABLE 62.-Climatic cxtremes for region with 1 to 7 species of a selected group of 13 deciduous trees of the eastern slates.

\begin{tabular}{|c|c|c|c|}
\hline Plate & Temperature: & Low. & High. \\
\hline 34 & Days in normal frostless season $\left(f_{8}\right) \ldots$ & 85 & 335 \\
\hline 35 & Hot days, fs.. & 0 & 226 \\
\hline 36 & Cold days, $f s .$. & 0 & 150 \\
\hline 37 & Remainder summation ab $x$ e $32^{\circ}$, year (thousands). & $10.0-$ & $26.0+$ \\
\hline 38 & Remainder summation above $39^{\circ}, f_{8}$ (thousands).. & 2.6 & 10.6 \\
\hline 39 & Exponential summation, $f_{s}$ (hundreds) .......... & 3.0 & 11.7 \\
\hline 40 & Physiological summation, $f_{s}$ (thousands) ........ & 2.1 & 21.4 \\
\hline 41 & Absolute minimum & -59 & +22 \\
\hline 43 & Normal daily mean, coldest 14 days of year $\left({ }^{\circ} \mathrm{F}\right.$.). & 0 & 57 \\
\hline 44 & Normal daily mean, hottest 6 weeks of year $\left({ }^{\circ} \mathrm{F}\right.$ 。). & $64.4-$ & $78.8+$ \\
\hline 45 & Normal daily mean, year $\left({ }^{\circ} \mathrm{F}.\right) \ldots \ldots \ldots \ldots$ & & $70+$ \\
\hline 46 & $\begin{array}{l}\text { Precipitation: } \\
\quad \text { Normal daily mean, } f_{s} \text { (inch).... }\end{array}$ & 077 & 172 \\
\hline 47 & $\begin{array}{l}\text { Normal daily mean, } f_{s} \text { (inch) } \ldots \ldots \ldots \ldots \ldots \\
\text { Normal No. rainy days (over } 0.10 \mathrm{inch}), f_{s} .\end{array}$ & 13 & $284^{.172}$ \\
\hline 48 & Normal No. dry days ( 0.10 inch or less), $f s$. & 28 & 259 \\
\hline 49 & Dry days, percentage of total, $f_{s}$ (per cent). & 8 & 92 \\
\hline 50 & Days in longest normal rainy period, $f s$. & 14 & 235 \\
\hline 51 & Days in longest normal dry period, $f s . .$. & 11 & 88 \\
\hline 52 & Mean total, year (inches) ............ & $20-$ & $60+$ \\
\hline 53 & $\begin{array}{l}\text { Evaporation: } \\
\text { Daily mean, } 1887-88, f_{s} \text { (inch)... }\end{array}$ & 084 & 188 \\
\hline 54 & $\begin{array}{l}\text { Total annual, 188-788 (inches)... } \\
\text { Moisture ratios: }\end{array}$ & 22.1 & 54.4 \\
\hline 58 & Normal $P / E, f_{s}$. . & 39 & 1.36 \\
\hline 59 & Normal $\pi / E, f_{s} \ldots$. . & .43 & 1.52 \\
\hline 60 & 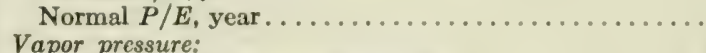 & .38 & 1.72 \\
\hline 63 & $\begin{array}{l}\text { Normal mean, } f_{s} \text { (hundredths inch) ......... } \\
\text { Humidity: }\end{array}$ & 322 & 675 \\
\hline 65 & Normal mean, $f_{s}$ (per cent).. & 53.2 & 81.9 \\
\hline 66 & $\begin{array}{l}\text { Normal mean, year (per cent)........... } \\
\text { Wind: }\end{array}$ & 59.8 & 85.2 \\
\hline 68 & $\begin{array}{l}\text { Normal mean hourly velocity, } f_{s} \text { (miles). . } \\
\text { Sunshine: }\end{array}$ & 5.1 & 12.4 \\
\hline 69 & $\begin{array}{l}\text { Normal total duration, fs (hours)...... } \\
\text { Moisture-temperature indices: }\end{array}$ & 1,225 & 2,650 \\
\hline 70 & Normal $P / E \times T, f_{s}$, remainder method. . & 179 & 1,314 \\
\hline 71 & Normal $P / E \times T, f s$, exponential method.. & 1,711 & 12,106 \\
\hline 72 & Normal $P / E \times T, f_{s}$, physiological method..... & 2,747 & 23,652 \\
\hline
\end{tabular}

TABLE 63.-Climatic extremes for region with 8 to 12 species of a selected group of 18 deciduous trees of the eastern states.

\begin{tabular}{|c|c|c|c|}
\hline Plate & Temperature: & Lou. & High. \\
\hline 34 & Days in normal frostless season $\left(f_{s}\right)$. & 112 & \\
\hline 35 & Hot days, $f_{s} \ldots \ldots \ldots \ldots \ldots \ldots \ldots$ & 0 & 172 \\
\hline 36 & Cold days, $f_{s} \ldots \ldots \ldots \ldots \ldots \ldots$ & 0 & 137 \\
\hline 37 & Remainder summation above $32^{\circ}$, year (thousands). & $10.0-$ & $18.0+$ \\
\hline 38 & Remainder summation above $39^{\circ}, f_{s}$ (thousands) ... & 2.9 & 8.4 \\
\hline 39 & Exponential summation, $f_{s}$ (hundreds) ......... & 3.0 & 9.2 \\
\hline 40 & Physiological summation, $f_{s}$ (thousands) & 4.4 & 17.0 \\
\hline 41 & Absolute minimum.............. & -44 & +12 \\
\hline 43 & Normal daily mean, coldest 14 days of year $\left({ }^{\circ} \mathrm{F}.\right)$. & 11 & 48 \\
\hline 44 & Normal daily mean, hottest 6 weeks of year $\left({ }^{\circ} \mathrm{F}\right.$. ). & $64.4-$ & $78.8+$ \\
\hline 45 & Normal daily mean, year $\left({ }^{\circ} F_{0}\right) \ldots$. & $45-$ & $65+$ \\
\hline & Precipitation: & & \\
\hline 46 & Normal daily mean, $f_{s}$ (inch) & $0 \mathrm{~s} 9$ & 170 \\
\hline 47 & Normal No. rainy days (over 0.10 inch), fs. & 26 & 250 \\
\hline $4 \mathrm{~s}$ & Normal No. dry days (0.10 inch or less), $f_{s}$. & 0 & 154 \\
\hline 49 & Dry days, percentage of total, $f_{s}$ (per cent). & 0 & 83 \\
\hline 50 & Days in longest normal rainy period, $f_{s} . \ldots \ldots$. & 17 & 256 \\
\hline 51 & Days in longest normal dry period, $f_{s} \ldots \ldots \ldots$. & 0 & $\dddot{11}$ \\
\hline 52 & Mean total, year (inches) $\ldots \ldots \ldots \ldots \ldots \ldots \ldots$ & $30-$ & $50+$ \\
\hline
\end{tabular}


TABLE 63.-Climatic extremes for region with 8 to 12 species of a selected group of 13 deciduous trees of the eastern states-Continued.

\begin{tabular}{|c|c|c|c|}
\hline Plate & Evaporation: & Low. & High. \\
\hline 53 & Daily mean, $1887-88, f s$ (inch) $\ldots \ldots \ldots \ldots \ldots \ldots$ & .088 & .195 \\
\hline 54 & $\begin{array}{l}\text { Total annual, } 1887-88 \text { (inches) } \ldots \ldots \ldots \ldots \ldots \ldots \ldots \\
\text { Moisture ratios: }\end{array}$ & 20.3 & 56.6 \\
\hline 58 & Normal $P / E, f s \ldots \ldots \ldots \ldots \ldots \ldots \ldots \ldots \ldots \ldots \ldots \ldots \ldots$ & .58 & 1.76 \\
\hline 59 & Normal $\pi / E, f s, \ldots \ldots \ldots \ldots \ldots \ldots \ldots \ldots$ & .66 & 1.96 \\
\hline 60 & $\begin{array}{l}\text { Normal } P / E \text {, year } \ldots \ldots \ldots \ldots \ldots \ldots \ldots \ldots \ldots \\
\text { Vapor pressure: }\end{array}$ & .71 & 1.94 \\
\hline 63 & $\begin{array}{l}\text { Normal mean, } f_{s} \text { (hundredths inch) } \ldots \ldots \ldots \ldots \ldots \ldots \\
\text { Humidity: }\end{array}$ & 416 & 573 \\
\hline 65 & Normal mean, $f_{8}$ (per cent. & 62.0 & 82.7 \\
\hline 66 & $\begin{array}{l}\text { Normal mean, year (per cent) } \ldots \ldots \ldots \ldots \ldots \ldots \ldots \\
\text { Wind: }\end{array}$ & 69.2 & 82.9 \\
\hline 68 & $\begin{array}{l}\text { Normal mean hourly velocity, } f \text { s (miles)............ } \\
\text { Sunshine: }\end{array}$ & 4.7 & 13.5 \\
\hline 69 & $\begin{array}{l}\text { Normal total duration, } f s \text { (hours)... } \\
\text { Moisture-temperature indices: }\end{array}$ & 1,365 & 1,946 \\
\hline 70 & Normal $P / E \times T, f s$, remainder method. & 301 & 1,418 \\
\hline 71 & Normal $P / E \times T, f s$, exponential method ..... & 2,918 & 13,511 \\
\hline 72 & Normal $P / E \times T, f s$, physiological method....... & 3,819 & 24,265 \\
\hline
\end{tabular}

TABLE 64.-Climatic extremes for region with all species of a selected group of 13 deciduous trees of the eastern states.

\begin{tabular}{|c|c|c|c|}
\hline Plate & Temperature: & Low. & High. \\
\hline 34 & Days in normal frostless season $(f s) \ldots \ldots \ldots \ldots \ldots$ & 124 & 231 \\
\hline 35 & 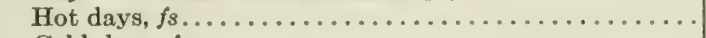 & 47 & 141 \\
\hline 36 & Cold days, $f_{s} \ldots \ldots \ldots \ldots \ldots \ldots \ldots \ldots \ldots \ldots \ldots \ldots \ldots \ldots \ldots \ldots$ & 0 & 109 \\
\hline 37 & Remainder summation above $32^{\circ}$, year (thousands).... & $11.5-$ & $18.0+$ \\
\hline 38 & Remainder summation above $39^{\circ}, f s$ (thousands) ...... & 3.9 & 7.0 \\
\hline 39 & Exponential summation, $f_{s}$ (hundreds)............. & 4.0 & 6.9 \\
\hline 40 & Physiological summation, $f_{s}$ (thousands) $\ldots \ldots \ldots \ldots \ldots$ & 5.4 & 15.0 \\
\hline 41 & Absolute minimum $\ldots \ldots \ldots \ldots \ldots \ldots \ldots \ldots \ldots \ldots$ & -38 & +4 \\
\hline 43 & Normal daily mean, coldest 14 days of year $\left({ }^{\circ} \mathrm{F}.\right) \ldots$ & 16 & 45 \\
\hline 44 & Normal daily mean, hottest 6 weeks of year $\left({ }^{\circ} \mathbf{F}.\right) \ldots$ & 71.6 & $78.8+$ \\
\hline 45 & $\begin{array}{l}\text { Normal daily mean, year }\left({ }^{\circ} \mathrm{F} .\right) \ldots \ldots \ldots \ldots \ldots \ldots \ldots \\
\text { Precipitation: }\end{array}$ & 45 & $60+$ \\
\hline 46 & Normal daily mean, $f_{s}$ (inch) ..... & .095 & .131 \\
\hline 47 & Normal No. rainy days (over 0.10 inch) $f_{s} \ldots \ldots \ldots \ldots$ & 49 & 166 \\
\hline 48 & Normal No. dry days (0.10 inch or less), $f s \ldots \ldots \ldots \ldots$ & 19 & 125 \\
\hline 49 & Dry days, percentage of total, $f s$ (per cent)........... & 11 & 72 \\
\hline 50 & Days in longest normal rainy period, $f_{s} \ldots \ldots \ldots \ldots$ & 23 & 140 \\
\hline 51 & Days in longest normal dry period, $f s \ldots \ldots \ldots \ldots \ldots$ & 4 & 56 \\
\hline 52 & $\begin{array}{l}\text { Mean total, year (inches) } \ldots \ldots \ldots \ldots \ldots \ldots \ldots \ldots \ldots \\
\text { Evaporation: }\end{array}$ & $40-$ & $60+$ \\
\hline 53 & Daily mean, $1887-88, f s$ (inch)... & .108 & .200 \\
\hline 54 & $\begin{array}{l}\text { Total annual, } 1887-88 \text { (inches) } \ldots \ldots \ldots \ldots \ldots \ldots \ldots \ldots \\
\text { Moisture ratios: }\end{array}$ & 25.2 & 54.8 \\
\hline 58 & Normal $P / E, f_{s}, \ldots \ldots$ & .51 & 1.30 \\
\hline 59 & 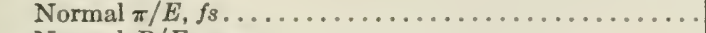 & .60 & 1.50 \\
\hline 60 & $\begin{array}{l}\text { Normal } P / E \text {, year. } \ldots \ldots \ldots \ldots \ldots \ldots \ldots \ldots \ldots \ldots \\
\text { Vapor pressure: }\end{array}$ & .72 & 1.85 \\
\hline 63 & $\begin{array}{l}\text { Normal mean, } f_{s} \text { (hundredths inch)... } \\
\text { Humidity: }\end{array}$ & 405 & 520 \\
\hline 65 & Normal mean, $f_{s}$ (per cent)..... & 65.6 & 84.0 \\
\hline 66 & $\begin{array}{l}\text { Normal mean, year (per cent) } \ldots \ldots \ldots \ldots \ldots \ldots \ldots \\
\text { Wind: }\end{array}$ & 69.0 & 81.4 \\
\hline 68 & $\begin{array}{l}\text { Normal mean hourly velocity, } f s \text { (miles) ............. } \\
\text { Sunshine: }\end{array}$ & 4.2 & 12.9 \\
\hline 69 & $\begin{array}{l}\text { Normal total duration, } f_{s} \text { (hours) } \ldots \ldots \ldots \ldots \ldots \ldots \ldots \\
\text { Moisture-temperature indices: }\end{array}$ & 1,403 & 1,836 \\
\hline 70 & Normal $P / E \times T, f_{s}$, remainder method... & 303 & 707 \\
\hline 71 & Normal $P / E \times T, f_{s}$, exponential method....... & 2,914 & 6,858 \\
\hline 72 & Normal $P / E \times T, f_{s}$, physiological method....... & 4,511 & 10,241 \\
\hline
\end{tabular}


TABLe 65.-Climatic extremes for Pinus toeda, area 1.

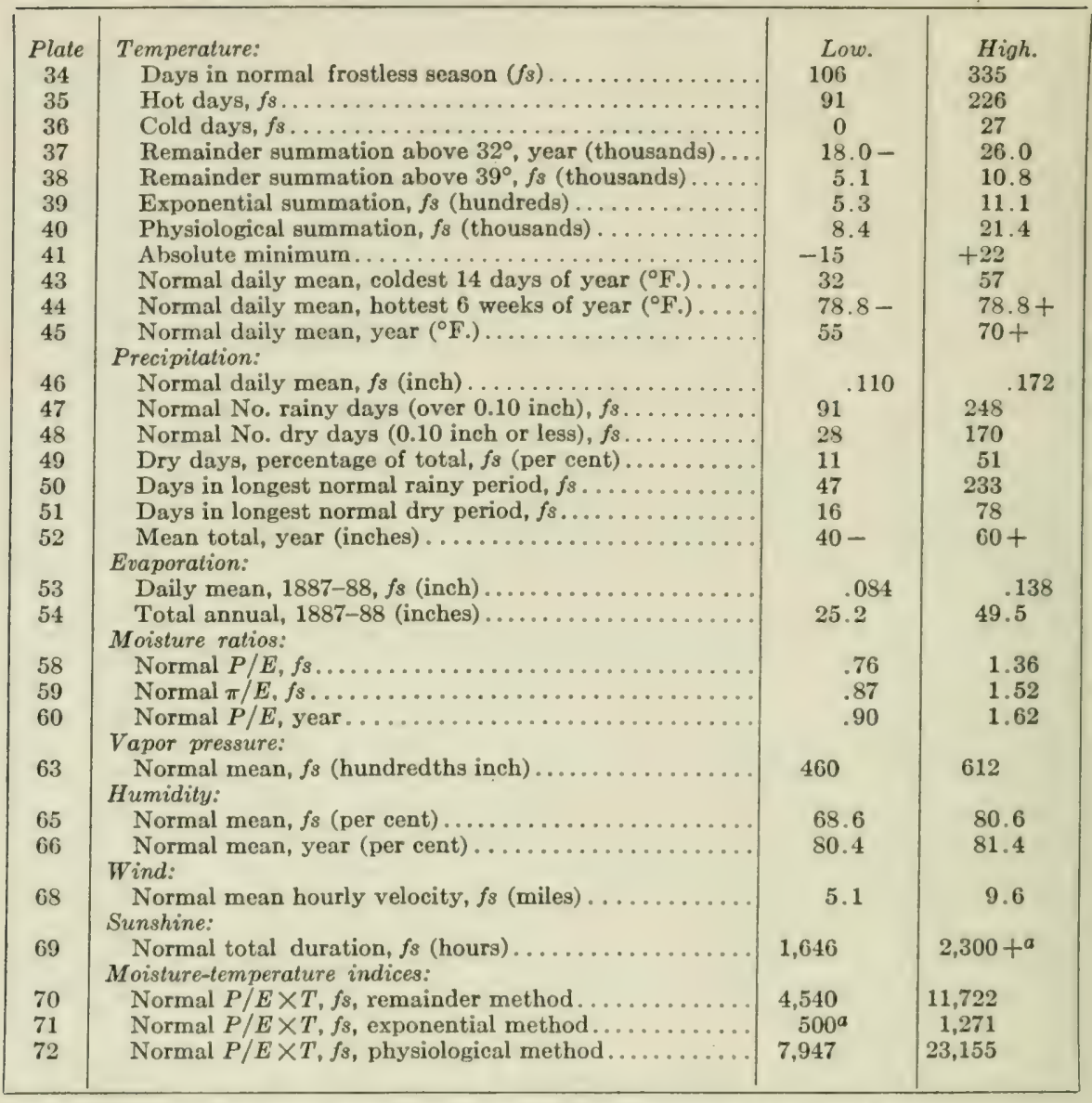

Table 66.-Climatic extremes for Pinus toda, area 2 .

\begin{tabular}{|c|c|c|c|}
\hline Plate & Temperature: & Lou. & High. \\
\hline 34 & Days in normal frostless season $(f s)$. & 117 & \\
\hline & Hot days, $f s \ldots \ldots \ldots \ldots \ldots \ldots$ & 137 & \\
\hline 36 & Cold days, $f_{s} \ldots \ldots \ldots \ldots \ldots \ldots \ldots \ldots \ldots \ldots$ & 0 & 0 \\
\hline 37 & Remainder summation above $32^{\circ}$, year (thousands). & $18.0-$ & $18.0+$ \\
\hline 38 & Remainder summation above $39^{\circ}, f_{s}$ (thousands)... & 6.6 & 10.6 \\
\hline 39 & Exponential summation, $f_{3}$ (hundreds) ........... & 7.0 & 11.7 \\
\hline 40 & Physiological summation, $f_{s}$ (thousands). & 12.3 & 21.2 \\
\hline 41 & Absolute minimum... & -16 & +16 \\
\hline 43 & Normal daily mean, coldest 14 days of year $\left({ }^{\circ} \mathrm{F}.\right)$. & 40 & 52 \\
\hline 44 & Normal daily mean, hottest 6 weeks of year $\left({ }^{\circ} \mathrm{F}\right.$.). & $78.8-$ & $78.8+$ \\
\hline 45 & $\begin{array}{l}\text { Normal daily mean, year }\left({ }^{\circ} \mathrm{F}_{0}\right) \ldots . . . . . \\
\text { Precipitation: }\end{array}$ & $60-$ & \\
\hline 46 & Normal daily mean, $f_{s}$ (inch). & 122 & $15 s$ \\
\hline 47 & Normal No. rainy days (over $0.10 \mathrm{inch}$ ), $f s$.. & 14.4 & 257 \\
\hline 48 & Normal No. dry days ( 0.10 inch or less), $f_{s} \ldots \ldots$ & 33 & 94 \\
\hline 49 & Dry days, percentage of total, $f_{s}$ (per cent) ...... & 16 & 40 \\
\hline 50 & Days in longest normal rainy period, $f_{3} \ldots \ldots \ldots \ldots$. & 114 & 174 \\
\hline 51 & Days in longest normal dry period, $f_{s} \ldots \ldots \ldots \ldots$. & 11 & 46 \\
\hline 52 & Mean total, year (inches) $\ldots \ldots \ldots \ldots \ldots \ldots \ldots$ & $40-$ & $50+$ \\
\hline
\end{tabular}


TABLE 66.-Climatic extremes for Pinus tæda, area 2-Continued.

\begin{tabular}{|c|c|c|c|}
\hline Plate & Evaporation: & Low. & High. \\
\hline 53 & Daily mean, $1887-88, f_{8}$ (inch). & .123 & .172 \\
\hline 54 & $\begin{array}{l}\text { Total annual, 1887-88 (inches).. } \\
\text { Moisture ratios: }\end{array}$ & 37.0 & 56.6 \\
\hline 58 & $\begin{array}{l}\text { Moisture ratios: } \\
\text { Normal } P / E, f s .\end{array}$ & .74 & 1.35 \\
\hline 59 & Normal $\pi / E, f_{s}$. & .88 & 1.47 \\
\hline 60 & Normal $P / E$, year. & .90 & 1.33 \\
\hline 63 & $\begin{array}{l}\text { Vapor pressure: } \\
\text { Normal mean, } f_{s} \text { (hundredths inch)... } \\
\text { Humidity: }\end{array}$ & 500 & 622 \\
\hline 65 & Normal mean, fs (per cent........... & 71.0 & 80.2 \\
\hline 66 & $\begin{array}{l}\text { Normal mean, year (per cent)... } \\
\text { Wind: }\end{array}$ & 71.1 & 85.2 \\
\hline 68 & $\begin{array}{l}\text { Normal mean hourly velocity, } f s \text { (miles).. } \\
\text { Sunshine: }\end{array}$ & 5.5 & 11.0 \\
\hline 69 & $\begin{array}{l}\text { Normal total duration, } f_{s} \text { (hours) } \ldots \ldots \ldots \ldots \\
\text { Moisture-temperature indices: }\end{array}$ & 1,946 & 2,650 \\
\hline 70 & Normal $P / E \times T, f_{s}$, remainder method. & 5,175 & 10,331 \\
\hline 71 & Normal $P / E \times T, f s$, exponential method. & 549 & 1,196 \\
\hline 72 & Normal $P / E \times T, f s$, physiological method. & 9,686 & 20,570 \\
\hline
\end{tabular}

TABLE 67.-Climatic extremes for Pinus toda, area 3.

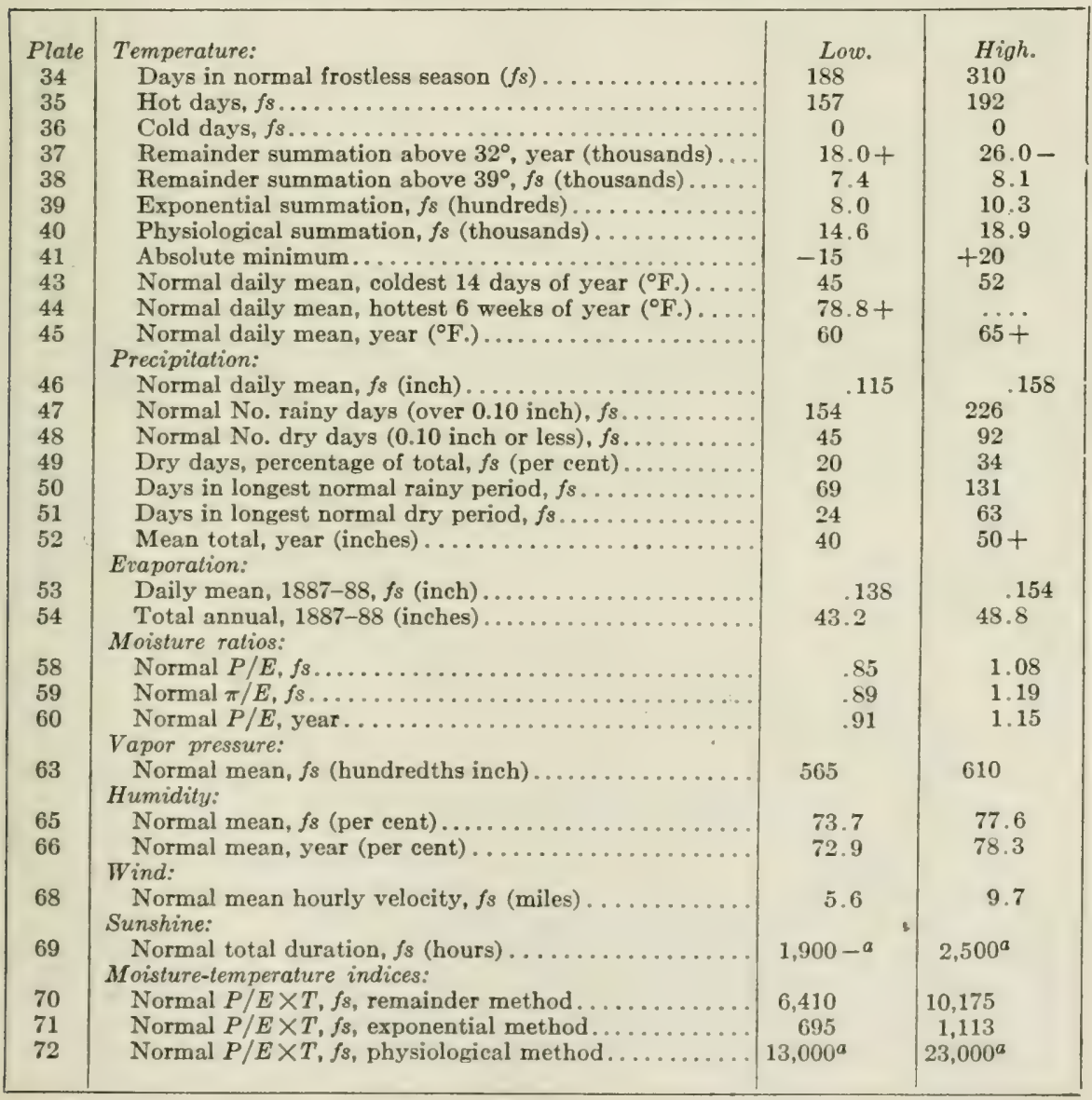


Table 68.-Climatic extremes for Pinus toda, area 4.

\begin{tabular}{|c|c|c|c|}
\hline Plate & Temperature: & Low. & Hioh. \\
\hline 34 & Days in normal frostless season $\left(f_{8}\right) \ldots$ & 184 & 268 \\
\hline 35 & 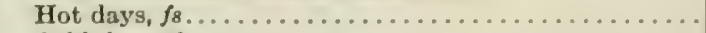 & 128 & 152 \\
\hline 36 & Cold days, $f_{s} \ldots \ldots \ldots \ldots \ldots \ldots \ldots \ldots \ldots \ldots \ldots \ldots$ & $\mathbf{0}$ & 0 \\
\hline 37 & Remainder summation above $32^{\circ}$, year (thousands)... & $8.0-$ & $18.0+$ \\
\hline 38 & Remainder summation above $39^{\circ}, f_{s}$ (thousands) ..... & 6.5 & 7.7 \\
\hline 39 & Exponential summation, $f s$ (hundreds) ............ & 7.0 & 8.0 \\
\hline 40 & Physiological summation, $f_{8}$ (thousands) .......... & 12.3 & 13.8 \\
\hline 41 & 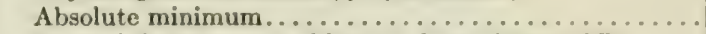 & -16 & +13 \\
\hline 43 & Normal daily mean, coldest 14 days of year $\left({ }^{\circ} \mathrm{F}.\right) \ldots$ & 38 & 46 \\
\hline 44 & Normal daily mean, hottest 6 weeks of year $\left({ }^{\circ} \mathrm{F}\right.$. $) \ldots$ & $78.8+$ & \\
\hline 45 & $\begin{array}{l}\text { Normal daily mean, year }\left({ }^{\circ} \mathrm{F} .\right) \ldots \ldots \ldots \ldots \ldots \ldots \ldots \\
\text { Precipitation: }\end{array}$ & $55+$ & 65 \\
\hline 46 & Normal daily mean, $f_{s}$ (inch)... & .123 & 170 \\
\hline 47 & Normal No. rainy days (over 0.10 inch), $f_{s} \ldots \ldots \ldots$ & 160 & 256 \\
\hline 48 & Normal No. dry days ( 0.10 inch or less), $f s \ldots . .$. & $\mathbf{0}$ & $75^{a}$ \\
\hline 49 & Dry days, percentage of total, $f_{s}$ (per cent) ......... & 0 & 26 \\
\hline 50 & Days in longest normal rainy period, $f_{3} \ldots \ldots \ldots \ldots$ & 121 & 256 \\
\hline 51 & Days in longest normal dry period, $f s \ldots \ldots \ldots \ldots$ & 0 & 19 \\
\hline 52 & $\begin{array}{l}\text { Mean total, year (inches) } \ldots \ldots \ldots \ldots \ldots \ldots \ldots \\
\text { Evaporation: }\end{array}$ & $50-$ & $50+$ \\
\hline 53 & Daily mean, $1887-88, f_{s}$ (inch). & .096 & 123 \\
\hline 54 & $\begin{array}{l}\text { Total annual, } 1887-88 \text { (inches) } \ldots \ldots \ldots \ldots \ldots \ldots \ldots \\
\text { Moisture ratios: }\end{array}$ & 31.3 & 35.6 \\
\hline 58 & Normal $P / E, f_{3} \ldots$ & 1.22 & 1.76 \\
\hline 59 & Normal $\pi / E, f_{8}, \ldots \ldots \ldots$ & 1.37 & 1.96 \\
\hline 60 & 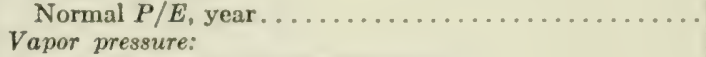 & 1.34 & 1.94 \\
\hline 63 & $\begin{array}{l}\text { Normal mean, } f s \text { (hundredths inch) ........................ } \\
\text { Humidity: }\end{array}$ & 525 & 573 \\
\hline 65 & Normal mean, $f s$ (per cent). & 74.1 & 82.7 \\
\hline 66 & $\begin{array}{l}\text { Normal mean, year (per cent) } \ldots \ldots \ldots \ldots \ldots \ldots \\
\text { Wind: }\end{array}$ & 73.8 & 82.9 \\
\hline 68 & $\begin{array}{l}\text { Normal mean hourly velocity, } f_{s} \text { (miles).... } \\
\text { Sunshine: }\end{array}$ & 6.4 & 13.5 \\
\hline 69 & $\begin{array}{l}\text { Normal total duration, } f s \text { (hours). } \\
\text { Moisture-temperature indices: }\end{array}$ & 1,700 & $2,100+a$ \\
\hline 70 & Normal $P / E \times T, f s$, remainder method... & $6,000^{\alpha}$ & 13,511 \\
\hline 71 & Normal $P / E \times T, f_{s}$, exponential method..... & $600^{a}$ & 1,418 \\
\hline 72 & Normal $P / E \times T, f_{s}$, physiological method..... & $9,000^{a}$ & 24,265 \\
\hline
\end{tabular}

TABLE 69.-Climatic extremes for Liriodendron tulipifera, region of infrequent occurrence (fringe).

\begin{tabular}{|c|c|c|c|}
\hline Plate & Temperalure: & Low. & High. \\
\hline 34 & Days in normal frostless season $(f s)$. & 121 & \\
\hline 35 & Hot days, $f_{s} \ldots \ldots \ldots \ldots \ldots \ldots \ldots$ & 46 & 183 \\
\hline 36 & Cold days, $f_{s} \ldots$. . & 0 & 117 \\
\hline 37 & Remainder summation above $32^{\circ}$, year (thousands) & $11.5-$ & $18.0+$ \\
\hline 38 & Remainder summation above $39^{\circ}, f_{s}$ (thousands)... & 3.7 & 8.7 \\
\hline 39 & Exponential summation, $f_{s}$ (hundreds) ........... & 3.8 & 9.5 \\
\hline 40 & Physiological summation, $f_{3}$ (thousands) & 4.9 & 17.9 \\
\hline 41 & Absolute minimum... & -38 & +16 \\
\hline 43 & Normal daily mean, coldest 14 days of year $\left({ }^{\circ} \mathrm{F}.\right)$. & 21 & 50 \\
\hline 44 & Normal daily mean, hottest 6 weeks of year $\left({ }^{\circ} \mathrm{F}\right.$.). & $64.4-$ & $78.8+$ \\
\hline 45 & Normal daily mean, year $\left({ }^{\circ} \mathrm{F}.\right) \ldots \ldots$ & 45 & 70 \\
\hline 46 & $\begin{array}{l}\text { Precipitation: } \\
\text { Normal daily mean, } f_{s} \text { (inch). }\end{array}$ & .089 & .170 \\
\hline .47 & Normal No. rainy days (over $0.10 \mathrm{inch}$ ), $f s$. & 26 & 256 \\
\hline 48 & Normal No. dry days ( 0.10 inch or less), $f s .$. & 0 & 136 \\
\hline 49 & Dry days, percentago of total, $f_{s}$ (per cent)... & 0 & 83 \\
\hline 50 & Days in longest normal rainy period, $f s \ldots$ & 21 & 256 \\
\hline 51 & Days in longest normal dry period, $f_{s} \ldots . .$. & 0 & \\
\hline 52 & Mean total, year (inches) ........... & $30-$ & $60+$ \\
\hline
\end{tabular}


TABLE 69.-Climatic extremes for Liriodendron tulipifera, region of infrequent occurrence (fringe)-Continued.

\begin{tabular}{|c|c|c|c|}
\hline Plate & Evaporation: & Low. & High. \\
\hline 53 & Daily mean, $1887-88$, fs (inch) $\ldots \ldots \ldots \ldots \ldots \ldots$ & .084 & .172 \\
\hline 54 & $\begin{array}{l}\text { Total annual, } 1887-88 \text { (inches) } \ldots \ldots \ldots \ldots \ldots \ldots \ldots \ldots \\
\text { Moisture ratios: }\end{array}$ & 27.6 & 56.6 \\
\hline 58 & 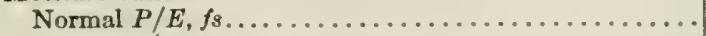 & .62 & 1.76 \\
\hline 59 & Normal $\pi / E, f_{s} \ldots \ldots \ldots \ldots \ldots \ldots . . . \ldots$ & .81 & 1.96 \\
\hline 60 & $\begin{array}{l}\text { Normal } P / E \text {, year. . . . } \ldots \ldots \ldots \ldots \ldots \ldots \ldots \ldots \\
\text { Vapor pressure: }\end{array}$ & .89 & 1.94 \\
\hline 63 & $\begin{array}{l}\text { Normal mean, } f_{s} \text { (hundredths inch)..................... } \\
\text { Humidity: }\end{array}$ & 405 & 600 \\
\hline 65 & Normal mean, $f_{s}$ (per cent) . . . . . . . . . . . . . & 69.0 & 82.7 \\
\hline 66 & $\begin{array}{l}\text { Normal mean, year (per cent) } \ldots \ldots \ldots \ldots \ldots \ldots \ldots \\
\text { Wind: }\end{array}$ & 69.2 & 82.9 \\
\hline 68 & $\begin{array}{l}\text { Normal mean hourly velocity, } f s \text { (miles) } \ldots \ldots \ldots \ldots \ldots \\
\text { Sunshine: }\end{array}$ & 5.1 & 14.9 \\
\hline 69 & $\begin{array}{l}\text { Normal total duration, } f s \text { (hours) } \ldots \ldots \ldots \ldots \ldots \ldots \\
\text { Moisture-temperature indices: }\end{array}$ & 1,418 & 2,300 \\
\hline 70 & Normal $P / E \times T, f s$, remainder method... & 2,914 & $10,000^{a}$ \\
\hline 71 & Normal $P / E \times T, f s$, exponential method... & 301 & 1,418 \\
\hline 72 & Normal $P / E \times T, f s$, physiological method. & 3,819 & 24,265 \\
\hline
\end{tabular}

TABLE 70.-Climatic extremes for Liriodendron tulipifera, region of greatest abundance (center).

\begin{tabular}{|c|c|c|c|}
\hline Plate & Temperature: & Low. & High. \\
\hline 34 & Days in normal frostless season $(f s) \ldots \ldots \ldots \ldots \ldots$ & 145 & 217 \\
\hline 35 & 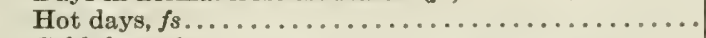 & 63 & 141 \\
\hline 36 & Cold days, $f s . . \ldots \ldots \ldots \ldots \ldots \ldots \ldots \ldots \ldots$ & 0 & 66 \\
\hline 37 & Remainder summation above $32^{\circ}$, year (thousands)... & $11.5+$ & $18.0+$ \\
\hline 38 & Remainder summation above $39^{\circ}, f s$ (thousands)..... & 3.8 & 6.6 \\
\hline 39 & Exponential summation, $f s$ (hundreds) ............. & 3.9 & 7.1 \\
\hline 40 & 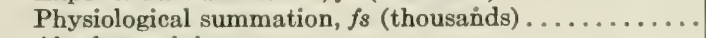 & 5.7 & 12.9 \\
\hline 41 & 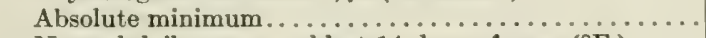 & -28 & -5 \\
\hline 43 & Normal daily mean, coldest 14 days of year $\left({ }^{\circ} \mathrm{F}.\right) \ldots$. & 29 & 40 \\
\hline 44 & Normal daily mean, hottest 6 weeks of year $\left({ }^{\circ} \mathbf{F}\right.$.)..... & 64.4 & $78.8+$ \\
\hline 45 & $\begin{array}{l}\text { Normal daily mean, year }\left({ }^{\circ} \mathrm{F}_{.}\right) \ldots \ldots \ldots \ldots \ldots \ldots \ldots \\
\text { Precipitation: }\end{array}$ & $50-$ & $60+$ \\
\hline 46 & Normal daily mean, $f_{s}($ inch $) \ldots \ldots \ldots \ldots \ldots \ldots$ & .099 & .131 \\
\hline 47 & Normal No. rainy days (over 0.10 inch), $f s \ldots . . .$. & 120 & 166 \\
\hline 48 & Normal No. dry days $(0.10$ inch or less $), f s \ldots . . .$. & 19 & 83 \\
\hline 49 & Dry days, percentage of total, $f s$ (per cent)......... & 11 & 48 \\
\hline 50 & Days in longest normal rainy period, $f_{s} \ldots \ldots \ldots \ldots$ & 72 & 140 \\
\hline 51 & Days in longest normal dry period, $f s \ldots \ldots \ldots \ldots$ & 4 & 56 \\
\hline 52 & $\begin{array}{l}\text { Mean total, year (inches) } \ldots \ldots \ldots \ldots \ldots \ldots \ldots \ldots \\
\text { Evaporation: }\end{array}$ & $40-$ & $60+$ \\
\hline 53 & Daily mean, $1887-88, f_{s}$ (inch) $\ldots \ldots \ldots \ldots \ldots \ldots \ldots$ & .148 & .200 \\
\hline 54 & $\begin{array}{l}\text { Total annual, } 1887-88 \text { (inches) } \ldots \ldots \ldots \ldots \ldots \ldots \ldots \\
\text { Moisture ratios: }\end{array}$ & 45.5 & 54.8 \\
\hline 58 & Normal $P / E, f_{s} \ldots \ldots \ldots \ldots \ldots \ldots$ & .51 & .87 \\
\hline 59 & Normal $\pi / E, f_{s} \ldots \ldots \ldots \ldots \ldots \ldots \ldots \ldots \ldots$ & .60 & 1.01 \\
\hline 60 & $\begin{array}{l}\text { Normal } P / E \text {, year } \ldots \ldots \ldots \ldots \ldots \ldots \ldots \ldots \\
\text { Vapor pressure: }\end{array}$ & .72 & 1.09 \\
\hline 63 & $\begin{array}{l}\text { Normal mean, } f_{s} \text { (hundredths inch) ................ } \\
\text { Humidity: }\end{array}$ & 438 & 520 \\
\hline 65 & 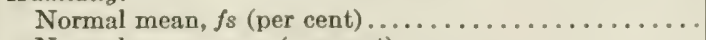 & 65.8 & 73.7 \\
\hline 66 & $\begin{array}{l}\text { Normal mean, year (per cent) ..................... } \\
\text { Wind: }\end{array}$ & 67.5 & 75.7 \\
\hline 68 & $\begin{array}{l}\text { Normal mean hourly velocity, } f s \text { (miles).... } \\
\text { Sunshine: }\end{array}$ & 3.1 & 9.0 \\
\hline 69 & $\begin{array}{l}\text { Normal total duration, } f_{s} \text { (hours) } \ldots \ldots \ldots \\
\text { Moisture-temperature indices: }\end{array}$ & 1,646 & 1,878 \\
\hline 70 & Normal $P / E \times T, f s$, remainder method. & 3,007 & 5,295 \\
\hline 71 & Normal $P / E \times T, f_{s}$, exponential method...... & 319 & 562 \\
\hline 72 & Normal $P / E \times T, f s$, physiological method......... & 5,513 & 10,052 \\
\hline
\end{tabular}


TABLE 71.-Climatic extremes for Bulbilis dactyloides, region of infrequent occurrence (fringe).

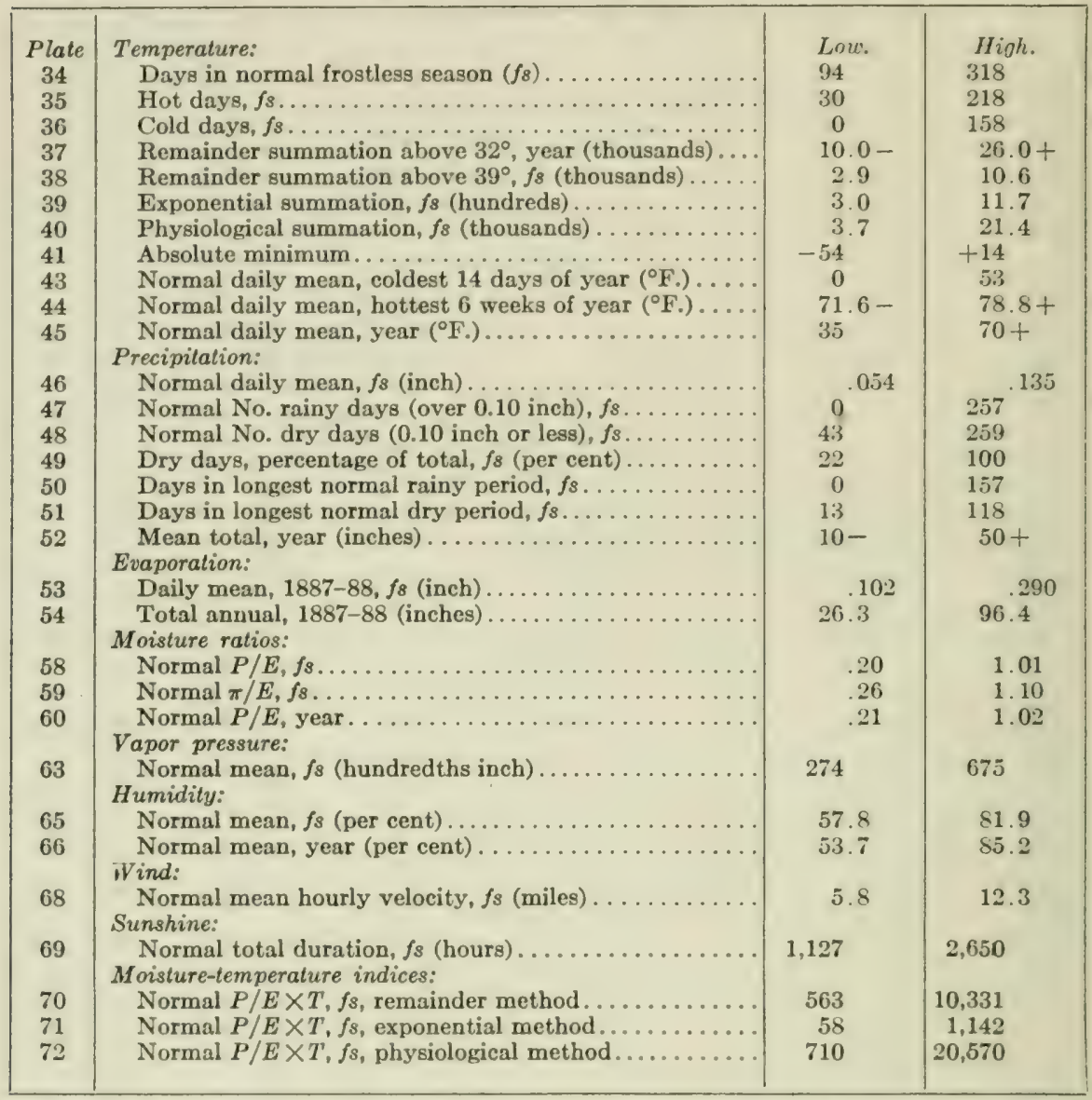

TABLE 72.-Climatic extremes for Bulbilis dactyloides, region of frequent occurrence (subcenter).

\begin{tabular}{|c|c|c|c|}
\hline Plate & Temperature: & Lou: & High. \\
\hline 34 & Days in normal frostless season $(f s)$. & 103 & 229 \\
\hline 35 & Hot days, $f s \ldots \ldots \ldots \ldots \ldots \ldots$. & 4 & $16: 3$ \\
\hline 36 & Cold days, $f_{s}$. & 0 & 140 \\
\hline 37 & Remainder summation above $32^{\circ}$, year (thousands). & $11.5-$ & $18.0+$ \\
\hline 38 & Remainder summation above $39^{\circ}$, fs (thousands).. & 2.9 & 6.1 \\
\hline 39 & Exponential summation, $f_{s}$ (hundreds) .......... & 3.4 & 8. 7 \\
\hline 40 & Physiological summation, $f_{s}$ (thousands). & 4.8 & 16.0 \\
\hline 41 & Absolute minimum.............. & -44 & +3 \\
\hline 43 & Normal daily mean, coldest 14 days of year $\left({ }^{\circ} \mathrm{F}.\right)$. & 6 & 12 \\
\hline 44 & Normal daily mean, hottest 6 weeks of year $\left({ }^{\circ} \mathrm{F}\right.$.). & $71.6-$ & 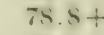 \\
\hline 45 & Normal daily mean, year $\left({ }^{\circ} \mathrm{F}.\right) \ldots \ldots \ldots \ldots \ldots$ & $45-$ & $6 i+$ \\
\hline & Precipitation: & & \\
\hline 46 & Normal daily mean, $f_{8}$ (inch) ....... & .081 & $.11 !$ \\
\hline 47 & Normal No. rainy day's (over 0.10 inch), $f s$. . & 21 & 111 \\
\hline 48 & Normal No. dry days ( 0.10 inch or less), $f s .$. & 55 & 192 \\
\hline 49 & Dry days, percentage of total, $f_{s}$ (per cent) ....... & 36 & $\therefore 4$ \\
\hline 50 & Days in longest normal rainy period, $f_{3} \ldots \ldots \ldots$. & 0 & Sit \\
\hline 51 & Days in longest normal dry period, $f s \ldots \ldots \ldots \ldots \ldots$ & 27 & ss \\
\hline 52 & 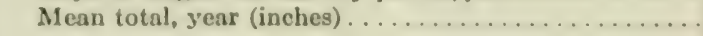 & $20-$ & $30+$ \\
\hline
\end{tabular}


TABLE 72.-Climatic extremes for Bulbilis dactyloides, region of frequent occurrence (subcenter)-Continued.

\begin{tabular}{|c|c|c|c|}
\hline $\begin{array}{l}\text { Plate } \\
53\end{array}$ & $\begin{array}{l}\text { Eraporation: } \\
\text { Daily mean, } 1887-88 \text {, fs (inch)...... }\end{array}$ & Low. & High. \\
\hline 54 & $\begin{array}{l}\text { Total annual, } 1887-88 \text { (inches)..... } \\
\text { Moisture ratios: }\end{array}$ & 31.0 & 55.4 \\
\hline 58 & Normal $P / E, f_{s}$. & .44 & .91 \\
\hline 59 & Normal $\pi / E, f_{s} \ldots \ldots \ldots \ldots \ldots \ldots \ldots$ & .47 & 1.07 \\
\hline 60 & $\begin{array}{c}\text { Normal } P / E \text {, year. } \ldots \ldots \ldots \ldots \ldots \ldots \\
\text { Vapor pressure: }\end{array}$ & .58 & .87 \\
\hline 63 & $\begin{array}{l}\text { Normal mean, } f_{s} \text { (hundredths inch)....... } \\
\text { Humidity: }\end{array}$ & 373 & 466 \\
\hline 65 & Normal mean, $f_{s}$ (per cent). & 59.4 & 67.8 \\
\hline 66 & $\begin{array}{l}\text { Normal mean, year (per cent).......... } \\
\text { Wind: }\end{array}$ & 59.3 & 70.4 \\
\hline 68 & $\begin{array}{l}\text { Normal mean hourly velocity, } f_{s} \text { (miles).... } \\
\text { Sunshine: }\end{array}$ & 7.4 & 14.2 \\
\hline 69 & $\begin{array}{l}\text { Normal total duration, } f s \text { (hours) . . . . . . . } \\
\text { Moisture-temperature indices: }\end{array}$ & 1,127 & 2,057 \\
\hline 70 & Normal $P / E \times T, f s$, remainder method. & 1,590 & 3,781 \\
\hline 71 & Normal $P / E \times T, f s$, exponential method. . & $100-a$ & $500^{3}$ \\
\hline 72 & Normal $P / E \times T, f s$, physiological method. & $1,000-a$ & $10,000^{\alpha}$ \\
\hline
\end{tabular}

TABLE 73.-Climatic extremes for Bulbilis dactyloides, region of greatest abundance (center).

\begin{tabular}{|c|c|c|c|}
\hline Plate & Temperature: & Low. & High. \\
\hline 34 & Days in normal frostless season $\left(f_{s}\right) \ldots \ldots \ldots \ldots$ & 120 & 211 \\
\hline 35 & Hot days, $f_{s} \ldots \ldots \ldots \ldots \ldots \ldots \ldots$ & 85 & \\
\hline 36 & 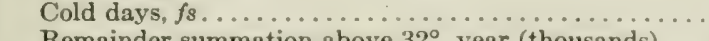 & 0 & 129 \\
\hline 37 & Remainder summation above $32^{\circ}$, year (thousands) & $11.5-$ & 18.0 \\
\hline 38 & Remainder summation above $39^{\circ}, f_{s}$ (thousands) ... & 3.8 & 5.8 \\
\hline 39 & Exponential summation, $f_{s}$ (hundreds) ........... & 4.6 & 6.0 \\
\hline 40 & Physiological summation, $f_{s}$ (thousands). & 7.6 & 10.7 \\
\hline 41 & Absolute minimum............... & -44 & +7 \\
\hline 43 & Normal daily mean, coldest 14 day's of year $\left({ }^{\circ} \mathrm{F}.\right)$. & 14 & 34 \\
\hline 44 & Normal daily mean, hottest 6 weeks of year $\left({ }^{\circ} \mathrm{F}\right.$.). & $71.6-$ & 78.8 \\
\hline 45 & $\begin{array}{l}\text { Normal daily mean, year }\left({ }^{(} \mathrm{F} .\right) \ldots \ldots \ldots \ldots \ldots \ldots \\
\text { Precipitation: }\end{array}$ & $45-$ & $60+$ \\
\hline 46 & Normal daily mean, $f_{s}$ (inch). & .070 & .103 \\
\hline 47 & Normal No. rainy days (over 0.10 inch), $f s$. & 13 & 57 \\
\hline 48 & Normal No. dry days (0.10 inch or less), $f_{s}$. & 60 & 140 \\
\hline 49 & Dry days, percentage of total, $f_{s}$ (per cent). & 46 & 92 \\
\hline 50 & Days in longest normal rainy period, $f_{s}$. & 20 & 59 \\
\hline 51 & Days in longest normal dry period, $f s \ldots$ & 38 & 88 \\
\hline 52 & $\begin{array}{l}\text { Mean total, year (inches)............. } \\
\text { Evaporation: }\end{array}$ & $20-$ & $20+$ \\
\hline 53 & $\begin{array}{l}\text { Evaporanon: } \\
\text { Daily mean, } 1887-88, f_{3} \text { (inch). }\end{array}$ & .166 & .203 \\
\hline 54 & $\begin{array}{l}\text { Total annual, } 1887-88 \text { (inches) } \ldots . \\
\text { Moisture ratios: }\end{array}$ & 41.3 & 54.6 \\
\hline 58 & Normal $P / E, f_{s} \ldots$ & .39 & .60 \\
\hline 59 & Normal $\pi / E, f_{s} \ldots$ & .43 & .73 \\
\hline 60 & $\begin{array}{l}\text { Normal } P / E \text {, year................. } \\
\text { Vapor pressure: }\end{array}$ & .38 & .51 \\
\hline 63 & $\begin{array}{l}\text { Normal mean, fs (hundredths inch).. } \\
\text { Humidity: }\end{array}$ & 370 & 431 \\
\hline 65 & Normal mean, $f_{s}$ (per cent).... & 57.6 & 65.3 \\
\hline 66 & $\begin{array}{l}\text { Normal mean, year (per cent)....... } \\
\text { Wind: }\end{array}$ & 64.7 & 66.9 \\
\hline 68 & $\begin{array}{l}\text { Normal mean hourly velocity, } f_{s} \text { (miles). } \\
\text { Sunshine: }\end{array}$ & 8.8 & 11.5 \\
\hline 69 & $\begin{array}{l}\text { Normal total duration, } f_{s} \text { (hours). } \\
\text { Moisture-temperature indices: }\end{array}$ & $1,300-a$ & $1,900+a$ \\
\hline 70 & Normal $P / E \times T, f_{s}$, remainder method. & 1,711 & $3,000+a$ \\
\hline 71 & Normal $P / E \times T, f s$, exponential method.. & $100-a$ & $400^{a}$ \\
\hline 72 & Normal $P / E \times T, f s$, physiological method. & $2,000-a$ & $7,000^{a}$ \\
\hline
\end{tabular}


TABLE 74.-Climatic extremes for region of cumulative occurrence of four species of grasses common in the great plains.

\begin{tabular}{|c|c|c|c|}
\hline Plate & Temperature: & Low. & High. \\
\hline 34 & Days in normal frostless season $(f s)$.. & 94 & 254 \\
\hline 35 & 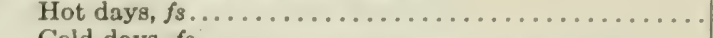 & 4 & 173 \\
\hline 36 & Cold days, $f_{s} \ldots \ldots \ldots \ldots \ldots \ldots \ldots \ldots \ldots \ldots \ldots \ldots \ldots \ldots \ldots \ldots$ & 0 & 158 \\
\hline 37 & Remainder summation above $32^{\circ}$, year (thousands)... & $10.0-a$ & $18.0+a$ \\
\hline 38 & Remainder summation above $39^{\circ}, f_{s}$ (thousands) ..... & $3.0-a$ & $8.0+a$ \\
\hline 39 & 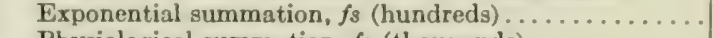 & 3.0 & 9.6 \\
\hline 40 & 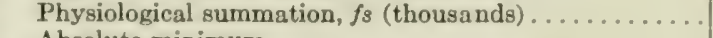 & 3.7 & 17.6 \\
\hline 41 & 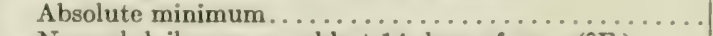 & -54 & +8 \\
\hline 43 & Normal daily mean, coldest 14 days of year $\left({ }^{\circ} \mathrm{F}.\right) \ldots$ & 0 & 44 \\
\hline 44 & Normal daily mean, hottest 6 weeks of year $\left({ }^{\circ} \mathrm{F}.\right) \ldots$ & $71.6-a$ & $78.8+a$ \\
\hline 45 & $\begin{array}{l}\text { Normal daily mean, year }\left({ }^{\circ} \mathrm{F} .\right) \ldots \ldots \ldots \ldots \ldots \ldots \ldots \\
\text { Precipitation: }\end{array}$ & $40-a$ & $65+a$ \\
\hline 46 & Normal daily mean, $f_{s}$ (inch) $\ldots \ldots \ldots \ldots \ldots \ldots$ & .033 & .143 \\
\hline 47 & Normal No. rainy days (over 0.10 inch), $f_{s} \ldots . . .$. & 13 & 141 \\
\hline 48 & Normal No. dry days $(0.10$ inch or less $), f_{3} \ldots \ldots \ldots$ & 48 & $225^{a}$ \\
\hline 49 & Dry days, percentage of total, $f_{3}$ (per cent).......... & 25 & 92 \\
\hline 50 & Days in longest normal rainy period, $f_{s}, \ldots \ldots \ldots \ldots$ & 14 & 125 \\
\hline 51 & Days in longest normal dry period, $f_{s} \ldots \ldots \ldots \ldots \ldots$ & 13 & $100+a$ \\
\hline 52 & $\begin{array}{l}\text { Mean total, year (inches) } \ldots \ldots \ldots \ldots \ldots \ldots \ldots \ldots \\
\text { Evaporation: }\end{array}$ & 10 & $40+$ \\
\hline 53 & Daily mean, $1887-88, f_{s}$ (inch) $\ldots \ldots \ldots \ldots \ldots \ldots \ldots$ & .122 & .290 \\
\hline 54 & $\begin{array}{l}\text { Total annual, } 1887-88 \text { (inches) } \ldots \ldots \ldots \ldots \ldots \ldots \ldots \ldots \\
\text { Moisture ratios: }\end{array}$ & 27.2 & 96.4 \\
\hline 58 & 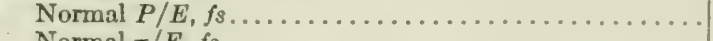 & .23 & 1.03 \\
\hline 59 & 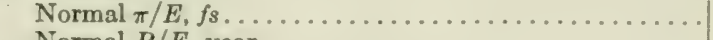 & .27 & 1.10 \\
\hline 60 & $\begin{array}{l}\text { Normal } P / E \text {, year } \ldots \ldots \ldots \ldots \ldots \ldots \ldots \ldots \ldots \ldots \\
\text { Vapor pressure: }\end{array}$ & .21 & 1.04 \\
\hline 63 & $\begin{array}{l}\text { Normal mean, } f_{s} \text { (hundredths inch).......... } \\
\text { Humidity: }\end{array}$ & $300-a$ & $500+a$ \\
\hline 65 & 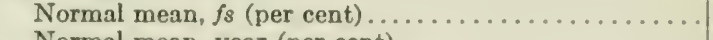 & $40-^{a}$ & 71.5 \\
\hline 66 & $\begin{array}{l}\text { Normal mean, year (per cent) } \ldots \ldots \ldots \ldots \ldots \ldots \ldots \\
\text { Wind: }\end{array}$ & $40^{a}$ & 74.1 \\
\hline 68 & $\begin{array}{l}\text { Normal mean hourly velocity, fs (miles) ............. } \\
\text { Sunshine: }\end{array}$ & 5.8 & 14.2 \\
\hline 69 & $\begin{array}{l}\text { Normal total duration, } f_{s} \text { (hours) ......... } \\
\text { Moisture-temperature indices: }\end{array}$ & 1,127 & $2.100+$ \\
\hline 70 & 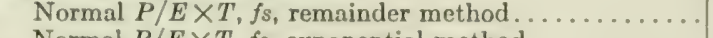 & $1,000-a$ & 5,744 \\
\hline 71 & Normal $P / E \times T, f_{s}$, exponential method.......... & $100-a$ & 611 \\
\hline 72 & Normal $P / E \times T, f_{s}$, physiological method.......... & $1,000-a$ & $12,000^{a}$ \\
\hline
\end{tabular}

TABLE 75.-Climatic extremes for total range of all species of Platyopuntias.

\begin{tabular}{|c|c|c|c|}
\hline Plate & Temperalure: & Low. & High. \\
\hline 34 & Days in normal frostless season $(f s)$ & $5: 3$ & 365 \\
\hline 35 & 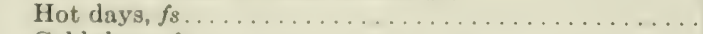 & 4 & 365 \\
\hline 36 & 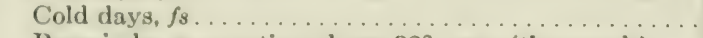 & 0 & $15 \overline{4}$ \\
\hline 37 & Remainder summation above $32^{\circ}$, year (thousands).... & $10.0-$ & $26.0+$ \\
\hline 38 & Remainder summation above $39^{\circ}, f_{s}$ (thousands) ..... & 2.4 & 1.4 .5 \\
\hline 39 & Exponential summation, $f_{s}$ (hundreds) ........... & 2.4 & 15.4 \\
\hline 40 & Physiological summation, $f_{s}$ (thousands) & 26 & 31.1 \\
\hline 41 & Absolute minimum .................. & $-6 . \overline{3}$ & +41 \\
\hline $4: 3$ & Normal daily mean, coldest 14 days of year $\left({ }^{\circ} \mathrm{F}.\right) \ldots$ & 0 & 69 \\
\hline 44 & Normal daily mean, hottest 6 weeks of year $\left({ }^{\circ} \mathrm{F}.\right)$. & $64.4-$ & $78.8+$ \\
\hline 45 & $\begin{array}{l}\text { Normal daily mean, } \operatorname{year}\left({ }^{\circ} \mathrm{F} .\right) \ldots \ldots \ldots \ldots \ldots \ldots \ldots \\
\text { Precipitation: }\end{array}$ & 3.3 & $75+$ \\
\hline 46 & Normal daily mean, $f_{s}$ (inch) . . . . . . & 000 & 173 \\
\hline 47 & Normal No, rainy days (over 0.10 inch), $f_{s}$. . & () & 256 \\
\hline 48 & Normal No. dry days (0.10 inch or less), $f_{s} \ldots \ldots$. & () & 294 \\
\hline 49 & Dry days, percentago of total, $\rho_{s}$ (per cent) ....... & 0 & 10() \\
\hline 50 & Days in longest normal rainy period, $f_{s} \ldots \ldots \ldots$. & 0 & 256 \\
\hline 51 & Days in longest normal dry period, $f_{s} \ldots$ & () & 299 \\
\hline 52 & Mean total, year (inches) .............. & $10-$ & $60+$ \\
\hline
\end{tabular}


TABLe 75.-Climatic extremes for total range of all species of Platyopuntias-Continued.

\begin{tabular}{|c|c|c|c|}
\hline Plate & Evaporation: & Low. & High. \\
\hline 53 & Daily mean, $1887-88, f s$ (inch) $\ldots \ldots \ldots \ldots \ldots \ldots$ & $.080^{-a}$ & .351 \\
\hline 54 & $\begin{array}{l}\text { Total annual, } 1887-88 \text { (inches) } \ldots \ldots \ldots \ldots \ldots \ldots \ldots \\
\text { Moisture ratios: }\end{array}$ & 24.0 & 101.2 \\
\hline 58 & 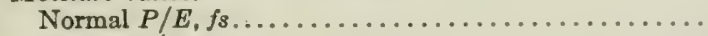 & .04 & 1.76 \\
\hline 59 & Normal $\pi / E, f_{s} \ldots \ldots \ldots \ldots \ldots \ldots \ldots \ldots$ & .06 & 1.96 \\
\hline 60 & 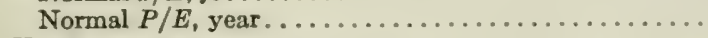 & .06 & $3.00^{a}$ \\
\hline 63 & $\begin{array}{l}\text { Vapor pressure: } \\
\text { Normal mean, } f s \text { (hundredths inch) } \ldots \ldots \ldots \ldots \ldots \ldots \\
\text { Humidity: }\end{array}$ & 183 & 707 \\
\hline 65 & 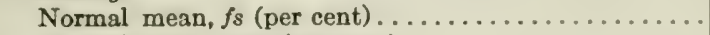 & 22.6 & 84 \\
\hline 66 & $\begin{array}{l}\text { Normal mean, year (per cent) } \ldots \ldots \ldots \ldots \ldots \ldots \ldots \\
\text { Wind: }\end{array}$ & 29.7 & 82.9 \\
\hline 68 & $\begin{array}{l}\text { Normal mean hourly velocity, } f_{s} \text { (miles) . . . . . . . . . } \\
\text { Sunshine: }\end{array}$ & 4.5 & 14.9 \\
\hline 69 & $\begin{array}{l}\text { Normal total duration, } f_{s} \text { (hours)................ } \\
\text { Moisture-temperature indices: }\end{array}$ & 1,134 & 2,995 \\
\hline 70 & Normal $P / E \times T, f_{s}$, remainder method....... & 13 & 1,418 \\
\hline 71 & Normal $P / E \times T, f_{s}$, exponential method......... & 127 & 13,511 \\
\hline 72 & Normal $P / E \times T, f_{3}$, physiological method.... & 197 & 24,265 \\
\hline
\end{tabular}

TABLE 76.-Climatic extremes for total range of all species of Cylindropuntias.

\begin{tabular}{|c|c|c|c|}
\hline Plate & Temperature: & Low. & High. \\
\hline 34 & Days in normal frostless season $\left(f_{s}\right) \ldots \ldots \ldots \ldots \ldots$ & 140 & 311 \\
\hline 35 & Hot days, $f_{s} \ldots \ldots \ldots \ldots \ldots \ldots \ldots$ & 38 & 211 \\
\hline 36 & Cold days, $f_{s} \ldots \ldots \ldots \ldots \ldots \ldots \ldots \ldots \ldots \ldots \ldots \ldots \ldots \ldots \ldots$ & 0 & 88 \\
\hline 37 & Remainder summation above $32^{\circ}$, year (thousands).... & $10.0-$ & $26.0+$ \\
\hline 38 & Remainder summation above $39^{\circ}, f s$ (thousands) ...... & 3.4 & 10.1 \\
\hline 39 & 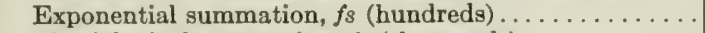 & 3.1 & 11.8 \\
\hline 40 & Physiological summation, $f_{s}$ (thousands) . . . . . . . . & 4.1 & 20.6 \\
\hline 41 & 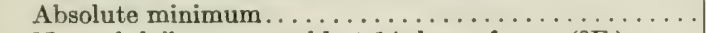 & -32 & +23 \\
\hline 43 & Normal daily mean, coldest 14 days of year $\left({ }^{\circ} \mathrm{F}.\right)$..... & 29 & 54 \\
\hline 44 & Normal daily mean, hottest 6 weeks of year $\left({ }^{\circ} \mathrm{F}.\right) \ldots$. & $64.4-$ & $78.8+$ \\
\hline 45 & $\begin{array}{l}\text { Normal daily mean, year }\left({ }^{\circ} \mathrm{F} .\right) \ldots \ldots \ldots \ldots \ldots \ldots \ldots \\
\text { Precipitation: }\end{array}$ & 55 & $70+$ \\
\hline 46 & Normal daily mean, $f_{s}$ (inch) $\ldots \ldots \ldots \ldots \ldots \ldots$ & 0.20 & .088 \\
\hline 47 & Normal No. rainy days (over 0.10 inch), $f_{s} \ldots \ldots \ldots$ & 0 & 57 \\
\hline 48 & Normal No. dry days $(0.10$ inch or less $), f_{s} \ldots \ldots \ldots \ldots$ & 142 & 283 \\
\hline 49 & Dry days, percentage of total, $f s$ (per cent)........... & 71 & 100 \\
\hline 50 & Days in longest normal rainy period, $f_{s} \ldots \ldots \ldots \ldots$ & 0 & 26 \\
\hline 51 & Days in longest normal dry period, $f s \ldots \ldots \ldots \ldots \ldots$ & 51 & 283 \\
\hline 52 & $\begin{array}{l}\text { Mean total, year (inches) } \ldots \ldots \ldots \ldots \ldots \ldots \ldots \ldots \ldots \\
\text { Evaporation: }\end{array}$ & $10-$ & $20+$ \\
\hline 53 & Daily mean, $1887-88, f_{s}$ (inch) $\ldots \ldots \ldots \ldots \ldots \ldots$ & .201 & .349 \\
\hline 54 & $\begin{array}{l}\text { Total annual, } 1887-88 \text { (inches) } \ldots \ldots \ldots \ldots \ldots \ldots \ldots \ldots \\
\text { Moisture ratios: }\end{array}$ & 32.5 & 101.2 \\
\hline 58 & 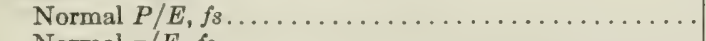 & .01 & .44 \\
\hline 59 & 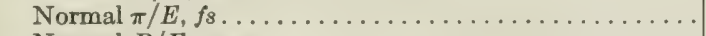 & .06 & .47 \\
\hline 60 & $\begin{array}{l}\text { Normal } P / E \text {, year. . . . . } \ldots \ldots \ldots \ldots \ldots \ldots \ldots \ldots \\
\text { Vapor pressure: }\end{array}$ & .03 & .28 \\
\hline 63 & $\begin{array}{l}\text { Normal mean, } f s \text { (hundredths inch) } \ldots \ldots \ldots \ldots \ldots \ldots \\
\text { Humidity: }\end{array}$ & 233 & $450+a$ \\
\hline 65 & Normal mean, $f_{s}$ (per cent). & 22.6 & 59.4 \\
\hline 66 & $\begin{array}{l}\text { Normal mean, year (per cent) } \ldots \ldots \ldots \ldots \ldots \ldots \ldots \ldots \\
\text { Wind: }\end{array}$ & 29.7 & 59.3 \\
\hline 68 & $\begin{array}{l}\text { Normal mean hourly velocity, fs (miles).. } \\
\text { Sunshine: }\end{array}$ & 4.5 & 14.2 \\
\hline 69 & $\begin{array}{l}\text { Normal total duration, } f s \text { (hours) } \\
\text { Moisture-temperature indices: }\end{array}$ & 1,367 & $2,900^{a}$ \\
\hline 70 & Normal $P / E \times T, f s$, remainder method... & 81 & 262 \\
\hline 71 & Normal $P / E \times T, f s$, exponential method........... & 772 & 2,532 \\
\hline 72 & Normal $P / E \times T, f_{s}$, physiological method.......... & 979 & 4,673 \\
\hline
\end{tabular}


TABLE 77.-Climatic extremes for Tsuga helerophylla.

\begin{tabular}{|c|c|c|c|}
\hline Plate & Temperature: & Low. & High. \\
\hline 34 & Days in normal frostless season $(f s)$. & 25 & \\
\hline 35 & Hot days, $f_{8} \ldots \ldots \ldots \ldots \ldots \ldots \ldots$ & 0 & $30+a$ \\
\hline 36 & Cold days, $f_{s} \ldots \ldots \ldots \ldots \ldots \ldots \ldots$ & 0 & 120 \\
\hline 37 & Remainder summation above $32^{\circ}$, year (thousands) & $10.0-$ & $18.0+$ \\
\hline 38 & Remainder summation above $39^{\circ}, f_{s}$ (thousands)... & 3.5 & 4.6 \\
\hline 39 & Exponential summation, $f s$ (hundreds) ...... & $3.0-a$ & 5.0 \\
\hline 40 & Physiological summation, $f_{8}$ (thousands). & 1.9 & 4.8 \\
\hline 41 & Absolute minimum................. & -46 & +30 \\
\hline 43 & Normal daily mean, coldest 14 days of year $\left({ }^{\circ} \mathrm{F}.\right)$. & $20^{a}$ & 46 \\
\hline 44 & Normal daily mean, hottest 6 weeks of year $\left({ }^{\circ} \mathrm{F}\right.$.) & $64.4-$ & $71.6+$ \\
\hline 45 & Normal daily mean, year $\left({ }^{\circ} \mathrm{F}\right.$.)..... & $45-$ & $55+$ \\
\hline & Precipitation: & & \\
\hline 46 & Normal daily mean, $f_{s}$ (inch) $\ldots \ldots \ldots \ldots$ & $28^{.040^{\circ}}$ & $199^{.199}$ \\
\hline 48 & Normal No. dry days (0.10 inch or less), fs... & 72 & 257 \\
\hline 49 & Dry days, percentage of total, $f_{s}$ (per cent).. & 27 & 90 \\
\hline 50 & Days in longest normal rainy period, $f_{3} \ldots \ldots$ & 21 & 99 \\
\hline 51 & Days in longest normal dry period, $f_{s} .$. & 56 & 187 \\
\hline 52 & Mean total, year (inches). & 30 & $90+$ \\
\hline & Evaporation: & & \\
\hline 53 & Daily mean, $1887-88, f_{8}$ (inch). & 052 & $.180^{\circ}$ \\
\hline 54 & $\begin{array}{l}\text { Total annual, } 1887-88 \text { (inches).... } \\
\text { Moisture ratios: }\end{array}$ & 18.1 & $40+a$ \\
\hline 58 & Normal $P / E, f 8$. & $.20^{a}$ & 3.84 \\
\hline 59 & Normal $\pi / E, f_{s} \ldots$ & 41 & 4.48 \\
\hline 60 & Normal $P / E$, year. . & $.40^{a}$ & 4.90 \\
\hline 63 & $\begin{array}{l}\text { Normal mean, } f_{8} \text { (hundredths inch)... } \\
\text { Humidity: }\end{array}$ & $300-a$ & 329 \\
\hline 65 & Normal mean, $f_{s}$ (per cent).. & $60.0-a$ & 87.5 \\
\hline 66 & $\begin{array}{l}\text { Normal mean, year (per cent)..... } \\
\text { Wind: }\end{array}$ & 74.6 & 86.8 \\
\hline 68 & $\begin{array}{l}\text { Normal mean hourly velocity, } f_{s} \text { (miles)... } \\
\text { Sunshine: }\end{array}$ & 3.5 & 16.4 \\
\hline 69 & $\begin{array}{l}\text { Normal total duration, } f_{s} \text { (hours) ..... } \\
\text { Mfoisture-temperature indices: }\end{array}$ & $1,300-a$ & $2,100+a$ \\
\hline 70 & Normal $P / E \times T, f_{8}$, remainder method. & $100-a$ & 1,566 \\
\hline 71 & Normal $P / E \times T, f_{s}$, exponential method... & $1,000-$ & 11,724 \\
\hline 72 & Normal $P / E \times T, f s$, physiological method.. & 1,313 & 7,475 \\
\hline
\end{tabular}

TABLE 78.-Climalic extremes for Picea sitchensis.

\begin{tabular}{|c|c|c|c|}
\hline Plate & Temperature: & Love. & High. \\
\hline 34 & Days in normal frostless season $(f s)$ & 196 & \\
\hline .35 & Hot days, $f s \ldots \ldots \ldots \ldots \ldots \ldots$. . . . . . & & $30-a$ \\
\hline 36 & Cold days, $f_{s}$. & () & $30-a$ \\
\hline 37 & Remainder summation above $32^{\circ}$, year (thousands) & $10.0-$ & $18.0+$ \\
\hline 38 & Remainder summation above $39^{\circ}, f s$ (thousands) ... & 3.0 & 3.8 \\
\hline 39 & Exponential summation, fs (hundreds). & 4.1 & 5.0 \\
\hline 40 & Physiological summation, $f_{s}$ (thousands) & 1.9 & $3 . \pi$ \\
\hline 41 & Absolute minimum................... & -3 & +30 \\
\hline 43 & Normal daily mean, coldest 14 days of year $\left({ }^{\circ} \mathrm{F}.\right)$ & $35^{a}$ & 46 \\
\hline 4.4 & Normal daily mean, hottest 6 weeks of year $\left({ }^{\circ} \mathrm{F}\right.$. $)$ & (i.4. 4 & \\
\hline 45 & $\begin{array}{l}\text { Normal daily metu, year }\left({ }^{\circ} \mathrm{F} .\right) \ldots \ldots \ldots \ldots \ldots \\
\text { Precipitation: }\end{array}$ & $.50-$ & $55+$ \\
\hline 46 & Normal daily mean, $f_{s}$ (inch). & 070 & 199 \\
\hline 47 & Normal No. rainy days (over $0.10 \mathrm{inch}$ ), $f s$. & is) & 199 \\
\hline 48 & Normal No. dry days ( 0.10 inch or less), $f s \ldots$ & 72 & 200 \\
\hline 49 & Dry days, percentage of total, $f s$ (per cent).. & 27 & si \\
\hline 50 & Days in longest normal rainy period, $f_{s} \ldots$. & 21 & 99 \\
\hline 51 & Days in longest normal dry period, $f s . \ldots$. & itit & 107 \\
\hline 52 & Mean total, year (inches). & 411 & $90+$ \\
\hline
\end{tabular}


TABle 78.-Climatic extremes for Picea sitchensis-Continued.

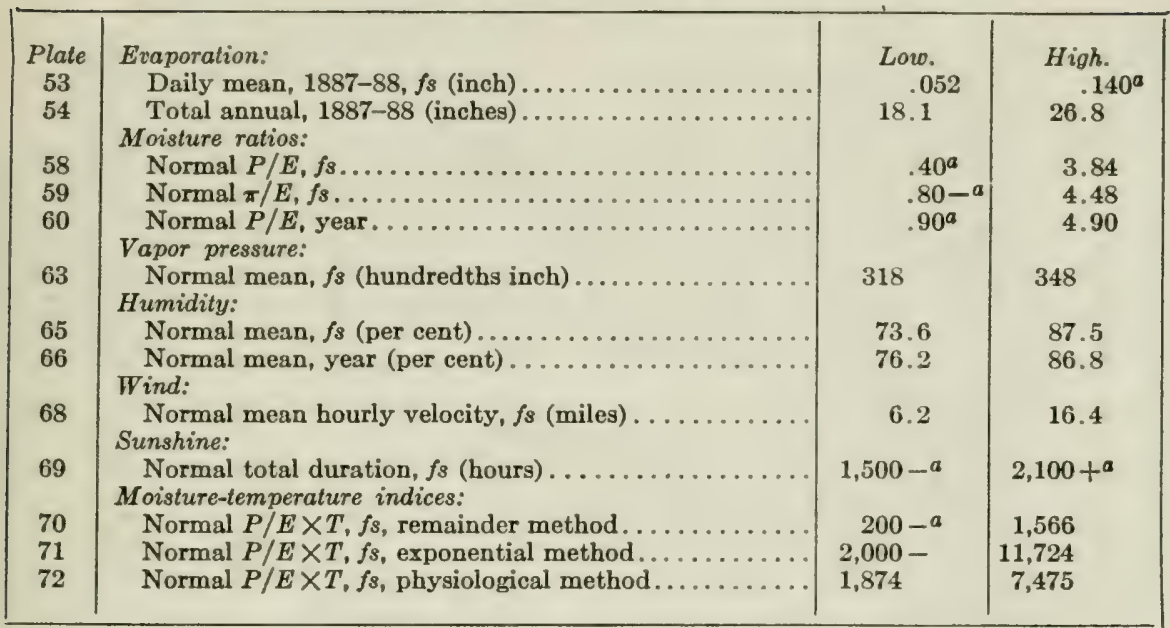

TABLE 79.-Climatic extremes for Pseudotsuga mucronata.

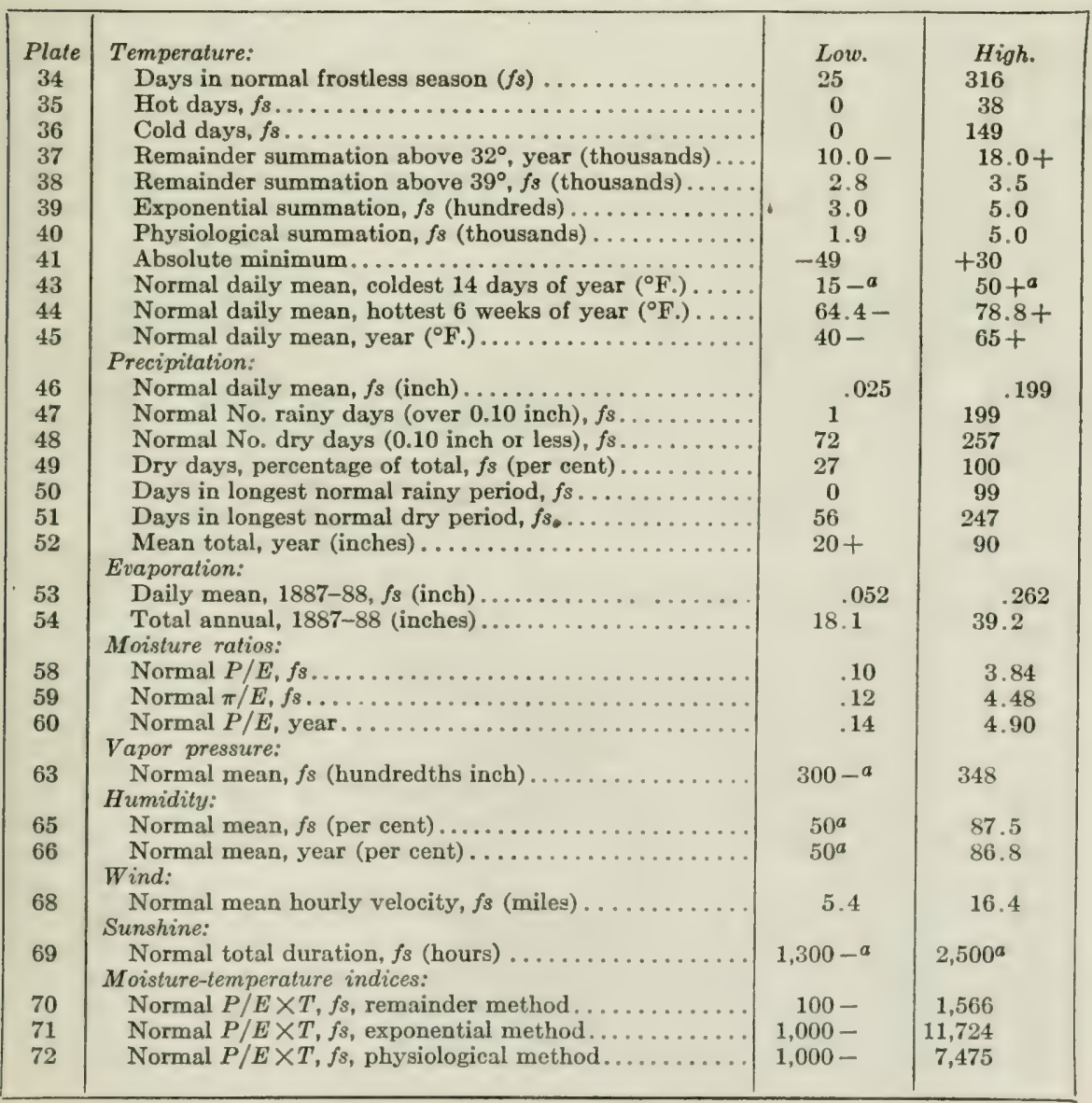


TABLE 80.-Climatic extremes for Pinus ponderosa.

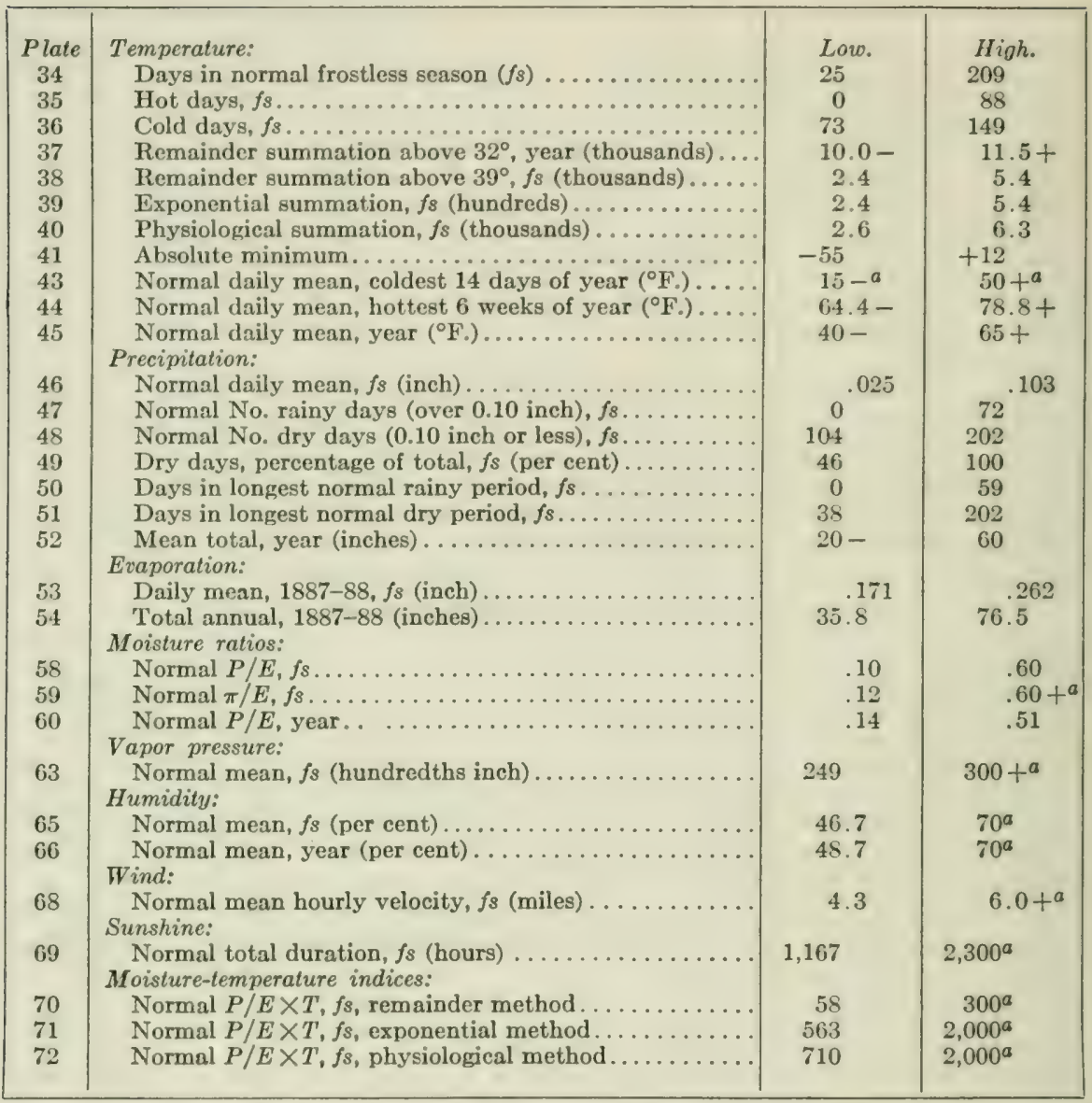

Table 81.-Climatic extremes for Pinus contorta.

\begin{tabular}{|c|c|c|c|}
\hline Plate & Temperature: & Low. & High. \\
\hline 34 & Days in normal frostless season $\left(f_{8}\right) \ldots \ldots \ldots \ldots \ldots$ & & \\
\hline 35 & Hot days, $f_{s} \ldots \ldots \ldots \ldots \ldots \ldots \ldots$ & 0 & 88 \\
\hline 36 & Cold days, $f_{s} \ldots \ldots \ldots \ldots \ldots$ & 0 & 149 \\
\hline 37 & Remainder summation above $32^{\circ}$, year (thousands) & $10.0-$ & $18.0+$ \\
\hline 38 & Remainder summation above $39^{\circ}, f_{s}$ (thousands)... & 2.7 & 5.1 \\
\hline 39 & Exponential summation, $f_{s}$ (hundreds) .............. & 2.8 & 5.4 \\
\hline 40 & Physiological summation, fs (thousands). & 2.4 & 8.1 \\
\hline 41 & Absolute minimum............... & -55 & +30 \\
\hline 43 & Normal daily mean, coldest 14 days of year $\left({ }^{\circ} \mathrm{F}\right.$. $)$. & $20^{a}$ & $50+{ }^{a}$ \\
\hline 44 & Normal daily mean, hottest 6 weeks of year $\left({ }^{\circ} \mathrm{F}\right.$. $)$. & $64.4-$ & $78.8+$ \\
\hline 45 & $\begin{array}{l}\text { Normal daily mean, year }\left({ }^{(} \mathrm{F}_{0}\right) \ldots \ldots \ldots \ldots \ldots \ldots \ldots \ldots \\
\text { Precipitation: }\end{array}$ & $40-$ & $60+$ \\
\hline 46 & Normal daily mean, fs (inch). & .025 & .199 \\
\hline 47 & Normal No, rainy days (over $0.10 \mathrm{inch}$ ), $f_{s}$. & 0 & 199 \\
\hline 48 & Normal No. dry days ( 0.10 inch or less), $f_{s}$. & 104 & $225^{a}$ \\
\hline 49 & Dry days, percentage of total, $f_{s}$ (per cent)......... & 27 & 100 \\
\hline 50 & Days in longest normal rainy period, $f_{s} \ldots \ldots \ldots \ldots$ & 0 & $9 s$ \\
\hline 51 & Days in longest normal dry period, $f s$. . & 56 & 202 \\
\hline 52 & Menn total, year (inches) $\ldots \ldots \ldots \ldots \ldots \ldots \ldots \ldots \ldots$ & $20-$ & 90 \\
\hline
\end{tabular}


Table 81-Climatic extremes for Pinus contorta-Continued.

\begin{tabular}{|c|c|c|c|}
\hline $\begin{array}{c}\text { Plate } \\
53\end{array}$ & $\begin{array}{l}\text { Evaporation: } \\
\text { Daily mean, } 1887-88, f s \text { (inch)... }\end{array}$ & $\begin{array}{l}\text { Low. } \\
.052\end{array}$ & $\begin{array}{l}\text { High. } \\
\quad .262\end{array}$ \\
\hline 54 & $\begin{array}{l}\text { Total annual, } 1887-88 \text { (inches) ........................... } \\
\text { Moisture ratios: }\end{array}$ & 18.1 & 68.3 \\
\hline 58 & Normal $P / E, f s \ldots \ldots \ldots \ldots$ & .10 & 3.84 \\
\hline 59 & Normal $\pi / E, f_{3}$. & .12 & 4.48 \\
\hline 60 & $\begin{array}{l}\text { Normal } P / E \text {, year... } \\
\text { Vapor pressure: }\end{array}$ & .14 & 4.90 \\
\hline 63 & $\begin{array}{l}\text { Normal mean, } f_{s} \text { (hundredths inch)...... } \\
\text { Humidity: }\end{array}$ & 249 & 348 \\
\hline 65 & Normal mean, $f s$ (per cent.......... & 47.9 & 87.5 \\
\hline 66 & $\begin{array}{l}\text { Normal mean, year (per cent)... } \\
\text { Wind: }\end{array}$ & 56.1 & 86.8 \\
\hline 68 & $\begin{array}{l}\text { Normal mean hourly velocity, } f_{s} \text { (miles). } \\
\text { Sunshine: }\end{array}$ & 4.3 & 16.4 \\
\hline 69 & $\begin{array}{l}\text { Normal total duration, } f_{s} \text { (hours) .... } \\
\text { Moisture-temperature indices: }\end{array}$ & 1,167 & $2,300^{a}$ \\
\hline 70 & Normal $P / E \times T, f_{s}$, remainder method. & 72 & 1,566 \\
\hline 71 & Normal $P / E \times T, f s$, exponential method. . & 691 & 11,724 \\
\hline 72 & Normal $P / E \times T, f s$, physiological method. & 809 & 7,475 \\
\hline
\end{tabular}

Table 82.-Climatic extremes for Pinus edulis.

\begin{tabular}{|c|c|c|c|}
\hline Plate & Temperature: & Low. & High. \\
\hline 34 & Days in normal frostless season $(f s) \ldots \ldots \ldots \ldots \ldots$ & 83 & 267 \\
\hline 35 & 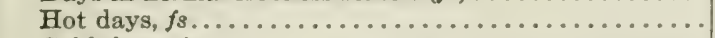 & 0 & 105 \\
\hline 36 & 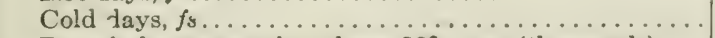 & 0 & 110 \\
\hline 37 & Remainder summation above $32^{\circ}$, year (thousands).... & $10.0-$ & $18.0+$ \\
\hline 38 & Remainder summation above $39^{\circ}, f s$ (thousands)...... & 2.4 & 5.4 \\
\hline 39 & 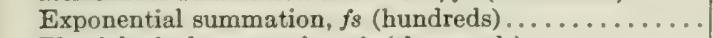 & 2.4 & 5.8 \\
\hline 40 & 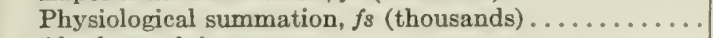 & 2.6 & 9.9 \\
\hline 41 & 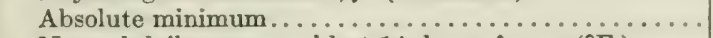 & -37 & -3 \\
\hline 43 & Normal daily mean, coldest 14 days of year $\left({ }^{\circ} \mathrm{F}.\right) \ldots$. & $20-a$ & $50+a$ \\
\hline 44 & Normal daily mean, hottest 6 weeks of year $\left({ }^{\circ} \mathrm{F}.\right) \ldots$. & $64.4-$ & $78.8+$ \\
\hline 45 & $\begin{array}{l}\text { Normal daily mean, year }\left({ }^{\circ} \mathrm{F} .\right) \ldots \ldots \ldots \ldots \ldots \ldots \ldots \\
\text { Precipitation: }\end{array}$ & 40 & $70+$ \\
\hline 46 & Normal daily mean, $f_{s}$ (inch). & $.020^{a}$ & .088 \\
\hline 47 & Normal No. rainy days (over $0.10 \mathrm{inch}$ ), $f_{s} \ldots \ldots \ldots \ldots$ & 0 & 57 \\
\hline 48 & Normal No. dry days (0.10 inch or less), $f s \ldots \ldots \ldots$. & 142 & $275^{a}$ \\
\hline 49 & Dry days, percentage of total, $f s$ (per cent).......... & 71 & 100 \\
\hline 50 & Days in longest normal rainy period, $f_{s} \ldots \ldots \ldots \ldots$ & 0 & 26 \\
\hline 51 & Days in longest normal dry period, $f_{8} \ldots \ldots \ldots \ldots \ldots$ & $75-a$ & $225+a$ \\
\hline 52 & $\begin{array}{l}\text { Mean total, year (inches) } \ldots \ldots \ldots \ldots \ldots \ldots \ldots \ldots \\
\text { Evaporation: }\end{array}$ & $20-$ & $30+$ \\
\hline 53 & Daily mean, $1887-88, f_{s}$ (inch) $\ldots \ldots \ldots \ldots \ldots \ldots$ & $.180^{a}$ & .330 \\
\hline 54 & $\begin{array}{l}\text { Total annual, } 1887-88 \text { (inches) } \ldots \ldots \ldots \ldots \ldots \ldots \ldots \ldots \\
\text { Moisture ratios: }\end{array}$ & 55.4 & 101.2 \\
\hline 58 & 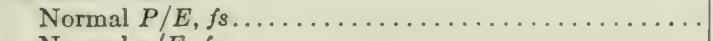 & .10 & .44 \\
\hline 59 & 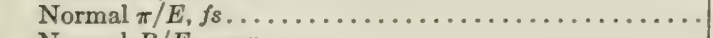 & .13 & .39 \\
\hline 60 & 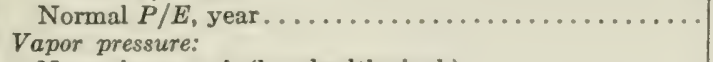 & .12 & .27 \\
\hline 63 & $\begin{array}{l}\text { Normal mean, } f_{s} \text { (hundredths inch) } \ldots \ldots \ldots \ldots \ldots \ldots \ldots \\
\text { Humidity: }\end{array}$ & 233 & $450+a$ \\
\hline 65 & 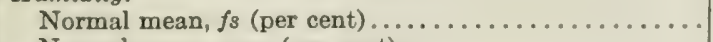 & 37.0 & 46.7 \\
\hline 66 & $\begin{array}{l}\text { Normal mean, year (per cent) } \ldots \ldots \ldots \ldots \ldots \ldots \ldots \ldots \\
\text { Wind: }\end{array}$ & 38.8 & 48.7 \\
\hline 68 & $\begin{array}{l}\text { Normal mean bourly velocity, } f_{s} \text { (miles) ........... } \\
\text { Sunshine: }\end{array}$ & 5.6 & 10.2 \\
\hline 69 & $\begin{array}{l}\text { Normal total duration, } f s \text { (hours) ......... } \\
\text { Moisture-temperature indices: }\end{array}$ & 1,134 & $2,300^{a}$ \\
\hline 70 & Normal $P / E \times T, f_{s}$, remainder method... & 58 & $400^{a}$ \\
\hline 71 & 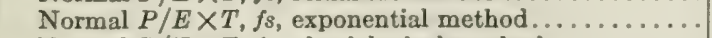 & 563 & $4000^{a}$ \\
\hline 72 & Normal $P / E \times T, f s$, physiological method...... & 710 & $7,000^{a}$ \\
\hline
\end{tabular}


Table 83.-Climatic extremes for Pinus palustris.

\begin{tabular}{|c|c|c|c|}
\hline Plate & Temperature: & Low. & High. \\
\hline 34 & Days in normal frostless season $(f s)$. & 181 & 365 \\
\hline 35 & Hot days, $f_{s} \ldots \ldots \ldots \ldots \ldots \ldots \ldots$ & 137 & 226 \\
\hline 36 & Cold days, $f_{3} \ldots \ldots \ldots$ & 0 & 0 \\
\hline 37 & Remainder summation above $32^{\circ}$, year (thousands) & 11.5 & $26.0+$ \\
\hline 38 & Remainder summation above $39^{\circ}, f_{8}$ (thousands)... & 6.6 & 10.8 \\
\hline 39 & Exponential summation, $f_{s}$ (hundreds). & 6.8 & 11.1 \\
\hline 40 & Physiological summation, $f_{s}$ (thousands) & 12,326 & 21,420 \\
\hline 41 & Absolute minimum.............. & -15 & +24 \\
\hline 43 & Normal daily mean, coldest 14 days of year $\left({ }^{\circ} \mathrm{F}.\right)$ & 40 & 69 \\
\hline 44 & Normal daily mean, hottest 6 weeks of year $\left({ }^{\circ} \mathrm{F}\right.$ ). & $71.6-$ & $78.8+$ \\
\hline 45 & Normal daily mean, year $\left({ }^{\circ} \mathrm{F}.\right) \ldots \ldots \ldots \ldots$ & & $75+$ \\
\hline & Precipitation: & & \\
\hline 46 & Normal daily mean, $f_{s}$ (inch) $\ldots \ldots \ldots \ldots$ & $144^{.119}$ & .170 \\
\hline 47 & & $\begin{array}{r}144 \\
0\end{array}$ & $\begin{array}{l}256 \\
170\end{array}$ \\
\hline $\begin{array}{l}48 \\
49\end{array}$ & $\begin{array}{l}\text { Normal No. dry days }\left(0.10 \text { inch or less), } f_{3} \ldots \ldots \ldots\right. \\
\text { Dry days, percentage of total, } f_{s} \text { (per cent) ....... }\end{array}$ & 0 & 51 \\
\hline $\begin{array}{l}49 \\
50\end{array}$ & Days in longest normal rainy period, $f_{s} \ldots .$. & 92 & 256 \\
\hline 51 & Days in longest normal dry period, $f_{3} .$. & 0 & 78 \\
\hline 52 & $\begin{array}{l}\text { Mean total, year (inches) ........................ } \\
\text { Eraporation: }\end{array}$ & $50-$ & $60+$ \\
\hline 53 & $\begin{array}{l}\text { Eraporation: } \\
\text { Daily mean, } 1887-88, f_{s} \text { (inch). }\end{array}$ & 096 & .172 \\
\hline 54 & $\begin{array}{l}\text { Total annual, 1887-88 (inches)........... } \\
\text { Moisture ratios: }\end{array}$ & 31.3 & 56.6 \\
\hline 58 & Normal $P / E, f_{3} \ldots \ldots \ldots$ & .75 & 1.76 \\
\hline 59 & Normal $\pi / E, f_{s} . .$. & .90 & 1.96 \\
\hline 60 & Normal $P / E$, year. & 1.00 & 1.94 \\
\hline 63 & $\begin{array}{l}\text { Vapor pressure: } \\
\quad \text { Normal mean, } f_{s} \text { (hundredths inch).. }\end{array}$ & 527 & 707 \\
\hline & Humidity: & & 80 \\
\hline 65 & Normal mean, $f s$ (per cent).. & 71.0 & 82.7 \\
\hline 66 & $\begin{array}{l}\text { Normal mean, year (per cent) .......... } \\
\text { Wind: }\end{array}$ & 72.1 & 82.9 \\
\hline 68 & $\begin{array}{l}\text { Normal mean hourly velocity, } f s \text { (miles). } \\
\text { Sunshine: }\end{array}$ & 5.0 & 13.5 \\
\hline 69 & $\begin{array}{l}\text { Sunshine: } \\
\text { Normal total duration, } f_{s} \text { (hours). } \\
\text { Moisture-temperature indices: }\end{array}$ & $1,900-a$ & $2,300+a$ \\
\hline 70 & Normal $P / E \times T, f_{s}$, remainder method. & $600-a$ & 1,314 \\
\hline 71 & Normal $P / E \times T, f_{s}$, exponential method... & 6,114 & 13,511 \\
\hline 72 & Normal $P / E \times T, f s$, physiological method.. & $10,000^{a}$ & 24,265 \\
\hline
\end{tabular}

Table 84.-Climatic extremes for Pinus cchinala.

\begin{tabular}{|c|c|c|c|}
\hline Plale & Temperature: & Low. & High. \\
\hline 34 & Days in normal frostless season $\left(f_{s}\right)$. & 143 & 279 \\
\hline 35 & Hot day's, $f s \ldots \ldots \ldots \ldots \ldots \ldots$. . . . . . . & 63 & \\
\hline 36 & Cold days, fs . . . . & 0 & 55 \\
\hline 37 & Remainder summation above $32^{\circ}$, year (thousands) & $11.5-$ & $18.0+$ \\
\hline 38 & Remainder summation above $39^{\circ}, f_{s}$ (thousands)... & 3.9 & 8.7 \\
\hline 39 & Exponential summation, fs (hundreds). & 3.9 & 9.6 \\
\hline 40 & Physiological summation, $f_{s}$ (thousands) & $5.7-$ & $17.8+$ \\
\hline 41 & Absolute minimum... & -25 & +16 \\
\hline 43 & Normal daily mean, coldest 14 days of year $\left({ }^{\circ} \mathrm{F}.\right)$. & 25 & $50+$ \\
\hline 44 & Normal daily mean, hottest 6 weeks of year $\left({ }^{\circ} \mathrm{F}_{0}\right)$. & $64.4-$ & $78.8+$ \\
\hline 45 & Normal daily mean, year $\left({ }^{\circ} \mathrm{F}.\right) \ldots$ & $50-$ & $65+$ \\
\hline & Precipitation: & & \\
\hline 46 & Normal daily mean, fs (inch). & .112 & .170 \\
\hline 47 & Normal No. rainy days (over 0.10 inch), $f_{s}$. & 102 & 256 \\
\hline 48 & Normal No. dry days (0.10 inch or less), $f s .$. & 0 & 90 \\
\hline 49 & Dry days, percentage of total, $f_{s}$ (per cent).... & 0 & 51 \\
\hline 50 & Days in longest normal rainy period, $f_{s} \ldots \ldots$. & 48 & 250 \\
\hline 51 & Days in longest normal dry period, $f_{3} \ldots \ldots \ldots$. & 0 & .0 \\
\hline 52 & Mean total, year (inches) . . . . . . . . . . . . . & 40 & $60+$ \\
\hline
\end{tabular}


TABLE 84.-Climatic extremes for Pinus echinata-Conlinued.

\begin{tabular}{|c|c|c|c|}
\hline Plate & Evaporation: & Low. & High. \\
\hline 53 & Daily mean, $1887-88, f_{s}$ (inch) $\ldots \ldots \ldots \ldots \ldots \ldots$ & .081 & .172 \\
\hline 54 & $\begin{array}{l}\text { Total annual, } 1887-88 \text { (inches) } \ldots \ldots \ldots \ldots \ldots \ldots \ldots \ldots \\
\text { Moisture ratios: }\end{array}$ & 25.2 & 56.6 \\
\hline 58 & Normal $P / E, f_{s} \ldots \ldots \ldots \ldots \ldots \ldots \ldots \ldots$ & .64 & 1.76 \\
\hline 59 & Normal $\pi / E, f s . . . . . . .$. & .75 & 1.96 \\
\hline 60 & $\begin{array}{l}\text { Normal } P / E \text {, year } \ldots \ldots \ldots \ldots \ldots \ldots \ldots \ldots \ldots \ldots \ldots \ldots \\
\text { Vapor pressure: }\end{array}$ & .85 & 1.94 \\
\hline 63 & $\begin{array}{l}\text { Normal mean, } f s \text { (hundredths inch)......................... } \\
\text { Humidity: }\end{array}$ & 428 & 600 \\
\hline 65 & Normal mean, $f_{s}$ (per cent... & 68.6 & 82.7 \\
\hline 66 & $\begin{array}{l}\text { Normal mean, year (per cent) } \ldots \ldots \ldots \ldots \ldots \ldots \ldots \ldots \\
\text { Wind: }\end{array}$ & 69.5 & 82.9 \\
\hline 68 & $\begin{array}{l}\text { Normal mean hourly velocity, fs (miles)............ } \\
\text { Sunshine: }\end{array}$ & 3.1 & 14.9 \\
\hline 69 & $\begin{array}{l}\text { Normal total duration, } f s \text { (hours) } \ldots \ldots \ldots \ldots \\
\text { Moisture-temperature indices: }\end{array}$ & 1,626 & 2,301 \\
\hline 70 & Normal $P / E \times T, f s$, remainder method... & 441 & 1,418 \\
\hline 71 & Normal $P / E \times T, f_{s}$, exponential method.......... & 4,174 & 13,511 \\
\hline 72 & Normal $P / E \times T, f s$, physiological method........... & $7,000-a$ & 24,265 \\
\hline
\end{tabular}

TaBle 85.-Climatic extremes for Pinus caribca.

\begin{tabular}{|c|c|c|c|}
\hline & 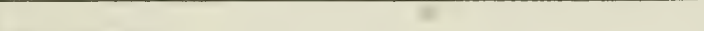 & & \\
\hline Flate & Temperature: & Lou: & High. \\
\hline 34 & Days in normal frostless season $(f s) \ldots \ldots \ldots \ldots$ & 241 & 348 \\
\hline 35 & 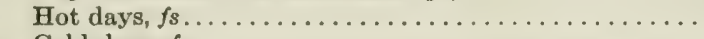 & 168 & $360+$ \\
\hline 36 & Cold days, $f s \ldots \ldots \ldots \ldots \ldots \ldots \ldots \ldots \ldots \ldots \ldots$ & 0 & 0 \\
\hline 37 & Remainder summation above $32^{\circ}$, year (thousands)... & $18.0+$ & $26.0+$ \\
\hline 38 & Remainder summation above $39^{\circ}, f s$ (thousands) ..... & 8.4 & 11.3 \\
\hline 39 & Exponential summation, $f_{s}$ (hundreds) ............. & 9.1 & 12.6 \\
\hline 40 & Physiological summation, $f s$ (thousands) . . . . . . . . & 16.4 & 24.9 \\
\hline 41 & Absolute minimum $\ldots \ldots \ldots \ldots \ldots \ldots \ldots \ldots \ldots \ldots$ & -1 & +30 \\
\hline 43 & Normal daily mean, coldest 14 days of year $\left({ }^{\circ} \mathrm{F}.\right) \ldots$ & 49 & 64 \\
\hline 44 & Normal daily mean, hottest 6 weeks of year $\left({ }^{\circ} \mathrm{F}.\right) \ldots$ & $78.8+$ & \\
\hline 45 & $\begin{array}{l}\text { Normal daily mean, year }\left({ }^{\circ} \mathrm{F} .\right) \ldots \ldots \ldots \ldots \ldots \ldots \ldots \\
\text { Precipitation: }\end{array}$ & $65+$ & 75 \\
\hline 46 & Normal daily mean, $f s$ (inch). & 141 & .173 \\
\hline 47 & Normal No, rainy days (over 0.10 inch), $f s \ldots \ldots \ldots$. & 165 & 248 \\
\hline 48 & Normal No. dry days $(0.10$ inch or less $), f_{s} \ldots \ldots \ldots$ & 28 & $175^{a}$ \\
\hline 49 & Dry days, percentage of total, $f_{s}$ (per cent) ......... & 11 & 51 \\
\hline 50 & Days in longest normal rainy period, $f s \ldots \ldots \ldots \ldots$ & 117 & 235 \\
\hline 51 & Days in longest normal dry period, $f_{s} \ldots \ldots \ldots \ldots \ldots$ & 17 & $100^{a}$ \\
\hline 52 & Mean total, year (inches) $\ldots \ldots \ldots \ldots \ldots \ldots \ldots \ldots$ & $50-$ & $60+$ \\
\hline 53 & Daily mean, $1887-88, f_{s}$ (inch) $\ldots \ldots \ldots \ldots \ldots \ldots$ & .124 & .146 \\
\hline 54 & $\begin{array}{l}\text { Total annual, } 1887-88 \text { (inches) } \ldots \ldots \ldots \ldots \ldots \ldots \ldots \ldots \\
\text { Moisture ratios: }\end{array}$ & 42.1 & 49.5 \\
\hline 58 & Normal $P / E, f_{s} \ldots$ & 1.08 & 1.36 \\
\hline 59 & 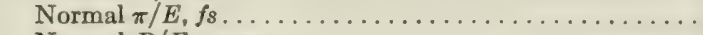 & $1.00^{a}$ & 1.52 \\
\hline 60 & $\begin{array}{l}\text { Normal } P / E \text {, year. } \ldots \ldots \ldots \ldots \ldots \ldots \ldots \ldots \ldots \\
\text { Vapor pressure: }\end{array}$ & 1.09 & 1.47 \\
\hline 63 & $\begin{array}{l}\text { Normal mean, fs (hundredths inch). } \\
\text { Humidity: }\end{array}$ & 599 & 612 \\
\hline 65 & Normal mean, $f s$ (per cent).... & 76.8 & 80.6 \\
\hline 66 & $\begin{array}{l}\text { Normal mean, year (per cent). } \\
\text { Wind: }\end{array}$ & 78.3 & 80.9 \\
\hline 68 & $\begin{array}{l}\text { Normal mean hourly velocity, } f_{s} \text { (miles) .. } \\
\text { Sunshine: }\end{array}$ & 5.1 & 9.7 \\
\hline 69 & $\begin{array}{l}\text { Normal total duration, } f_{s} \text { (hours) } \\
\text { Moisture-temperature indices: }\end{array}$ & 2,026 & $2,300+^{a}$ \\
\hline 70 & 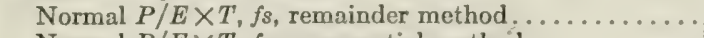 & 1,014 & 1,314 \\
\hline 71 & Normal $P / E \times T, f s$, exponential method........... & 9,385 & 12,106 \\
\hline 72 & Normal $P / E \times T, f s$, physiological method.......... & 18,294 & 23,652 \\
\hline
\end{tabular}


TABLE 86.-Climatic extremes for Pinus strobus.

\begin{tabular}{|c|c|c|c|}
\hline Plate & Temperature: & Low. & High. \\
\hline 34 & Days in normal frostless season $\left(f_{s}\right)$. & 85 & 207 \\
\hline 35 & Hot days, $f_{8} \ldots \ldots \ldots \ldots \ldots \ldots \ldots$ & & \\
\hline 36 & Cold days, $f s \ldots \ldots \ldots \ldots \ldots \ldots \ldots \ldots \ldots \ldots$ & 0 & 150 \\
\hline 37 & Remainder summation above $32^{\circ}$, year (thousands) & 10.0 & $11.5+$ \\
\hline 38 & Remainder summation above $39^{\circ}, f_{3}$ (thousands)... & 2.6 & 6.0 \\
\hline 39 & Exponential summation, $f_{s}$ (hundreds)......... & 3.0 & 5.9 \\
\hline 40 & Physiological summation, fs (thousands). & 2.1 & 10.4 \\
\hline 41 & Absolute minimum............ & -49 & -5 \\
\hline 43 & Normal daily mean, coldest 14 days of year $\left({ }^{\circ} \mathrm{F}\right.$. $)$. & 0 & 37 \\
\hline 44 & Normal daily mean, hottest 6 weeks of year $\left({ }^{\circ} \mathrm{F}\right.$.). & $64.4-$ & 78.8 \\
\hline 45 & Normal daily mean, year $\left({ }^{\circ} \mathrm{F}_{0}\right) \ldots$ & & 60 \\
\hline 46 & $\begin{array}{l}\text { Precipitation: } \\
\quad \text { Normal daily mean, } f_{s} \text { (inch). }\end{array}$ & .091 & 131 \\
\hline 47 & Normal No. rainy days (over $0.10 \mathrm{inch}$ ), $f s$. & & $156^{.101}$ \\
\hline 48 & Normal No. dry days (0.10 inch or less), fs. & 28 & 154 \\
\hline 49 & Dry days, percentage of total, $f_{s}$ (per cent). & 11 & 83 \\
\hline 50 & Days in longest normal rainy period, $f_{s} \ldots$... & 17 & 140 \\
\hline 51 & Days in longest normal dry period, $f_{8} \ldots$ & 9 & 91 \\
\hline 52 & $\begin{array}{l}\text { Mean total, year (inches) } . . \ldots \ldots \ldots \ldots \ldots \\
\text { Evaporation: }\end{array}$ & $30-$ & $60+$ \\
\hline 53 & Daily mean, $1887-88, f_{s}$ (inch)... & .081 & 166 \\
\hline 54 & $\begin{array}{l}\text { Total annual, } 1887-88 \text { (inches)....... } \\
\text { Moisture ratios: }\end{array}$ & 20.3 & 48.1 \\
\hline 58 & Normal $P / E, f_{s} \ldots \ldots \ldots \ldots$ & .66 & 1.39 \\
\hline 59 & Normal $\pi / E, f_{3} \ldots \ldots \ldots \ldots \ldots \ldots \ldots$ & .75 & 1.52 \\
\hline 60 & $\begin{array}{l}\text { Normal } P / E \text {, year.......................... } \\
\text { Vapor pressure: }\end{array}$ & .82 & 1.85 \\
\hline 63 & $\begin{array}{l}\text { Normal mean, } f s \text { (hundredths inch)...... } \\
\text { Humidity: }\end{array}$ & 345 & 505 \\
\hline 65 & Normal mean, $f_{s}$ (per cent)......... & 68.6 & 83.9 \\
\hline 66 & $\begin{array}{l}\text { Normal mean, year (per cent)............ } \\
\text { Wind: }\end{array}$ & 69.5 & 82.1 \\
\hline 68 & $\begin{array}{l}\text { Normal mean hourly velocity, } f_{s} \text { (miles)... } \\
\text { Sunshine: }\end{array}$ & 3.1 & 14.9 \\
\hline 69 & $\begin{array}{l}\text { Normal total duration, } f_{s} \text { (hours) ........ } \\
\text { Moisture-temperature indices: }\end{array}$ & 1,225 & 1,772 \\
\hline 70 & Normal $P / E \times T, f s$, remainder method.. & 301 & 530 \\
\hline 71 & Normal $P / E \times T, f s$, exponential method... & 2,918 & 5,040 \\
\hline 72 & Normal $P / E \times T, f s$, physiological method.. & 2,747 & 8,970 \\
\hline
\end{tabular}

TABLE 87.-Climatic extremes for Tsuga canadensis.

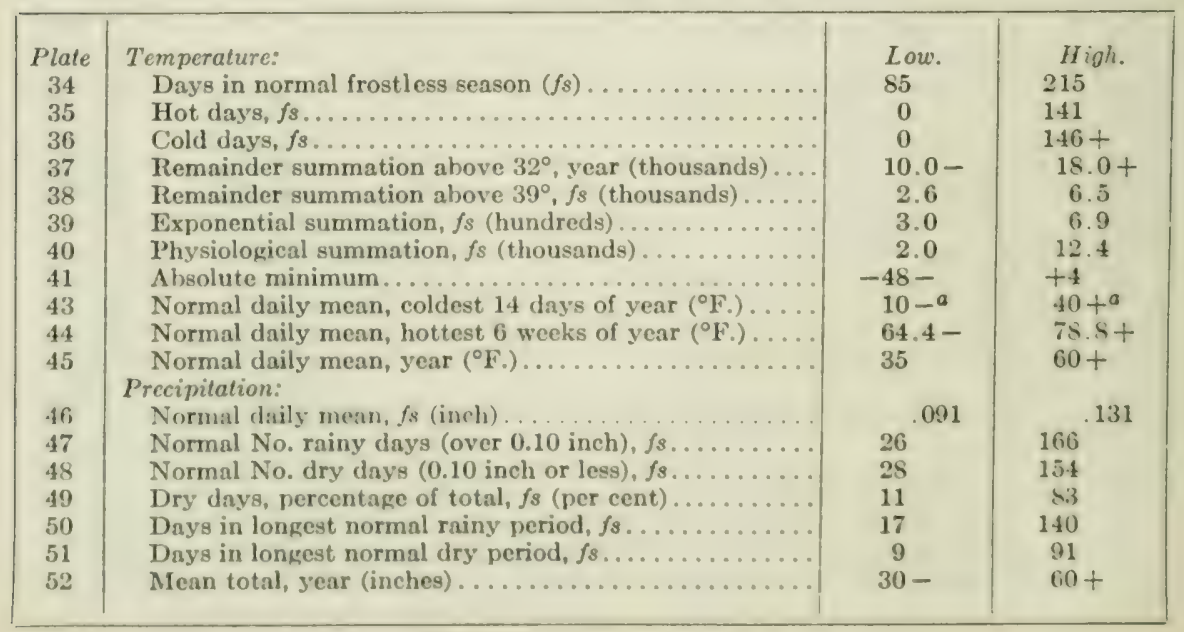


TABLE 87.-Climatic extremes for Tsuga canadensis-Continued.

\begin{tabular}{|c|c|c|c|}
\hline $\begin{array}{c}\text { Plate } \\
53\end{array}$ & $\begin{array}{l}\text { Evaporation: } \\
\text { Daily mean, 1887-88, fs (inch) }\end{array}$ & Low. & High. \\
\hline 54 & $\begin{array}{l}\text { Total annual, } 1887-88 \text { (inches) ............................ } \\
\text { Moisture ratios: }\end{array}$ & 20.3 & 48.1 \\
\hline 58 & Normal $P / E, f_{s} \ldots \ldots \ldots \ldots \ldots \ldots$ & .66 & 1.39 \\
\hline 59 & Normal $\pi / E, f s$. & .75 & 1.52 \\
\hline 60 & $\begin{array}{l}\text { Normal } P / E \text {, year.......... } \\
\text { Vapor pressure: }\end{array}$ & .82 & 1.85 \\
\hline 63 & $\begin{array}{l}\text { Normal mean, } f s \text { (hundredths inch)... } \\
\text { Humidity: }\end{array}$ & 345 & 520 \\
\hline 65 & Normal mean, $f_{3}$ (per cent.... & 68.6 & 83.9 \\
\hline 66 & $\begin{array}{l}\text { Normal mean, year (per cent).... } \\
\text { Wind: }\end{array}$ & 69.5 & 82.1 \\
\hline 68 & $\begin{array}{l}\text { Normal meanh ourly velocity, } f_{s} \text { (miles). } \\
\text { Sunshine: }\end{array}$ & 3.1 & 12.9 \\
\hline 69 & $\begin{array}{l}\text { Normal total duration, } f_{s} \text { (hours)... } \\
\text { Moisture-temperature indices: }\end{array}$ & 1,225 & 1,836 \\
\hline 70 & Normal $P / E \times T, f s$, remainder method. & 301 & 562 \\
\hline 71 & Normal $P / E \times T, f s$, exponential method. & 2,918 & 5,295 \\
\hline 72 & Normal $P / E \times T, f s$, physiological method. & 2,747 & 10,052 \\
\hline
\end{tabular}

TABle 88.-Climatic extremes for Pinus virginiana.

\begin{tabular}{|c|c|c|c|}
\hline Plate & Temperature: & Low. & High. \\
\hline 34 & Days in normal frostless season $(f s)$. & 146 & 227 \\
\hline 35 & Hot days, $f_{s} \ldots \ldots \ldots \ldots \ldots \ldots \ldots$. & 90 & 141 \\
\hline 36 & Cold days, $f s .$. & 0 & 75 \\
\hline 37 & Remainder summation above $32^{\circ}$, year (thousands) & $11.5-$ & $18.0+$ \\
\hline 38 & Remainder summation above $39^{\circ}, f_{s}$ (thousands)... & 4.7 & 6.5 \\
\hline 39 & Exponential summation, $f s$ (hundreds)... & 4.9 & 7.2 \\
\hline 40 & Physiological summation, $f_{s}$ (thousands) ... & 7.9 & 12.8 \\
\hline 41 & Absolute minimum ................ & -29 & +4 \\
\hline 43 & Normal daily mean, coldest 14 days of year $\left({ }^{\circ} \mathrm{F}.\right)$. & 28 & $45^{a}$ \\
\hline 44 & Norma ldaily mean, hottest 6 weeks of year $\left({ }^{\circ} \mathrm{F}\right.$.). & $64.4-$ & $78.8+$ \\
\hline 45 & Normal daily mean, year $\left({ }^{\circ} \mathrm{F}.\right) \ldots \ldots$ & $50-$ & $60+$ \\
\hline & Precipitation: & & \\
\hline 46 & Normal daily mean, $f_{s}$ (inch). & .110 & .131 \\
\hline 47 & Normal No. rainy days (over $0.10 \mathrm{inch}$ ), $f_{s} .$. & 91 & 166 \\
\hline 48 & Normal No. dry days ( 0.10 inch or less), $f_{s} \ldots$ & 19 & 93 \\
\hline 49 & Dry days, percentage of total, $f s$ (per cent). & 11 & 48 \\
\hline 50 & Days in longest normal rainy period, $f_{s} \ldots \ldots$ & 47 & $175^{a}$ \\
\hline 51 & Days in longest normal dry period, $f s . . .$. & 4 & 56 \\
\hline 52 & Mean total, year (inches)............. & $40-$ & $60+$ \\
\hline & Evaporation: & & \\
\hline 53 & Daily mean, $1887-88, f_{s}$ (inch). & .084 & .200 \\
\hline 54 & $\begin{array}{c}\text { Total annual, 1887-88 (inches).......... } \\
\text { Moisture ratios: }\end{array}$ & 45.0 & 54.8 \\
\hline 58 & Normal $P / E, f_{s}$. & .51 & 1.30 \\
\hline 59 & Normal $\pi / E, f_{s} \ldots .$. & .60 & 1.50 \\
\hline 60 & $\begin{array}{l}\text { Normal } P / E \text {, year... } \\
\text { Vapor pressure: }\end{array}$ & .72 & 1.62 \\
\hline 63 & $\begin{array}{l}\text { Normal mean, fs (hundredths inch).... } \\
\text { Humidity: }\end{array}$ & 450 & 513 \\
\hline 65 & Normal mean, $f_{s}$ (per cent).... & 65.8 & 82.3 \\
\hline 66 & $\begin{array}{l}\text { Normal mean, year (per cent). } \\
\text { Wind: }\end{array}$ & 67.5 & 81.4 \\
\hline 68 & Normal mean hourly velocity, $f_{s}$ (miles). & 3.1 & 9.6 \\
\hline 69 & $\begin{array}{l}\text { Sunshine: } \\
\text { Normal total duration, } f_{s} \text { (hours)....... }\end{array}$ & 1,646 & 1,878 \\
\hline 70 & $\begin{array}{l}\text { Morsture-temperature indeces: } \\
\text { Normal } P / E \times T, f s, \text { remainder method. }\end{array}$ & 319 & 707 \\
\hline 71 & Normal $P / E \times T, f_{s}$, exponential method.. & 3,007 & $7,000+a$ \\
\hline 72 & Normal $P / E \times T, f s$, physiological method... & 5,513 & $15,000^{a}$ \\
\hline
\end{tabular}


TABLE 89.-Climalic extremes for Pinus divaricala.

\begin{tabular}{|c|c|c|c|}
\hline Plate & Temperature: & Low. & High. \\
\hline 34 & Days in normal frostless season $(f s)$. & 85 & \\
\hline 35 & Hot days, $f_{s}, \ldots \ldots \ldots \ldots \ldots \ldots$ & 0 & 79 \\
\hline 36 & Cold days, $f_{8} \ldots \ldots \ldots \ldots \ldots$ & $146+$ & 97 \\
\hline 37 & Remainder summation above $32^{\circ}$, year (thousands) & $10.0-$ & $11.5+$ \\
\hline 38 & Remainder summation above $39^{\circ}, f_{8}$ (thousands).. & 2.6 & 4.6 \\
\hline 39 & Exponential summation, $f_{8}$ (hundreds) ........... & 3.0 & 4.8 \\
\hline 40 & Physiological summation, $f_{8}$ (thousands) & 2.1 & 6.9 \\
\hline 41 & Absolute minimum.............. & -59 & -22 \\
\hline 43 & Normal daily mean, coldest 14 days of year ( $\left.{ }^{\circ} \mathrm{F}.\right)$. & 0 & 23 \\
\hline 44 & Normal daily mean, hottest 6 weeks of year $\left({ }^{\circ} \mathrm{F}.\right)$. & $64.4-$ & $71.6+$ \\
\hline 45 & Normal daily mean, year $\left({ }^{\circ} \mathrm{F}.\right) \ldots \ldots \ldots \ldots \ldots$ & & \\
\hline & Precipitation: & & \\
\hline 46 & Normal daily mean, $f s$ (inch). & .099 & .120 \\
\hline 47 & Normal No. rainy days (over $0.10 \mathrm{inch}), f s$.. & 43 & 120 \\
\hline 48 & Normal No. dry days (0.10 inch or less), $f 8 \ldots .$. & 28 & 120 \\
\hline 49 & Dry days, percentage of total, $f_{3}$ (per cent).... & 17 & 72 \\
\hline 50 & Days in longest normal rainy period, $f_{8} \ldots \ldots \ldots$ & 22 & 73 \\
\hline 51 & Days in longest normal dry period, $f_{3} \ldots \ldots \ldots \ldots$ & 9 & 58 \\
\hline 52 & 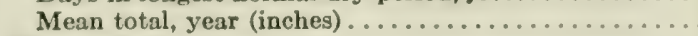 & $30-$ & $50+$ \\
\hline & Evaporation: & & \\
\hline 53 & Daily mean, $1887-88, f_{s}$ (inch).. & .084 & 143. \\
\hline 54 & Total annual, $1887-88$ (inches)... & 24.3 & 29.0 \\
\hline & Moisture ratios: & & \\
\hline 58 & Normal $P / E, f s$. & .74 & 1.31 \\
\hline 59 & Normal $\pi / E, f_{s}$. & .92 & 1.52 \\
\hline 60 & Normal $P / E$, year. & .90 & 1.72 \\
\hline & Vapor pressure: & & \\
\hline 63 & $\begin{array}{l}\text { Normal mean, } f_{s} \text { (hundredths inch). } \\
\text { Humidity: }\end{array}$ & 345 & 438 \\
\hline 65 & Normal mean, $f_{s}$ (per cent).......... & 69.5 & 81.8 \\
\hline 66 & $\begin{array}{l}\text { Normal mean, year (per cent).... } \\
\text { Wind: }\end{array}$ & 71.9 & 80.2 \\
\hline 68 & $\begin{array}{l}\text { Normal mean hourly velocity, } f_{8} \text { (miles) .... } \\
\text { Sunshine: }\end{array}$ & 7.6 & 12.4 \\
\hline 69 & $\begin{array}{l}\text { Normal total duration, } f_{s} \text { (hours) ..... } \\
\text { Moisture-temperature indices: }\end{array}$ & 1,225 & $1,700+a$ \\
\hline 70 & Normal $P / E \times T, f_{s}$, remainder method. & 312 & 442 \\
\hline 71 & Normal $P / E \times T, f_{s}$ exponential method. & 2,997 & 4,269 \\
\hline 72 & Normal $P / E \times T, f_{s}$, physiological method. & 2,747 & 6,423 \\
\hline
\end{tabular}

TABle 90.-Climatic extremes for Abies balsamea.

\begin{tabular}{|c|c|c|c|}
\hline Plate & Temperature: & Lou. & High. \\
\hline 34 & Days in normal frostless season $\left(f_{s}\right) \ldots \ldots \ldots$ & 100 & \\
\hline 35 & Hot days, $f_{s} \ldots \ldots \ldots \ldots \ldots \ldots \ldots$ & 0 & 85 \\
\hline 36 & Cold days, fs... & 0 & $146+$ \\
\hline 37 & Remainder summation above $32^{\circ}$, year (thousands). & $10.0-$ & $11.5+$ \\
\hline 38 & Remainder summation above $39^{\circ}$, fs (thousands) & 2.6 & 4.7 \\
\hline 39 & Exponential summation, $f_{s}$ (hundreds). & 2.5 & 4.6 \\
\hline 40 & Physiological summation, $f_{s}$ (thousands) & 2.1 & 6.7 \\
\hline 41 & Absolute minimum.............. & $-49-$ & -7 \\
\hline 43 & Normal daily mean, coldest 14 days of year $\left({ }^{\circ} \mathrm{F}\right.$. & 0 & $30+a$ \\
\hline 44 & Normal daily mean, hottest 6 weeks of year $\left({ }^{\circ} \mathrm{F}\right.$.). & $61.4-$ & $71.6+$ \\
\hline 45 & Normal daily mean, year $\left({ }^{\circ} \mathrm{F}.\right) . .$. & 35 & 55 \\
\hline & Prccipitation: & & \\
\hline 46 & Normal daily mean, $f s$ (ineh). & . 099 & 131 \\
\hline 47 & Normal No. rainy days (over $0.10 \mathrm{inch}$ ), $f_{s}$. & 43 & 156 \\
\hline 48 & Normal No. dry days $(0.10$ inch or less), fs.. & 19 & 136 \\
\hline 49 & Dry days, percentage of total, $f_{s}$ (per cent). & 11 & so \\
\hline 50 & Drys in longest normal rainy period, $f_{s}$. . & 22 & 140 \\
\hline 51 & Days in longest normal dry period, $f s \ldots$ & 9 & is \\
\hline 52 & Mean total, year (inches) ............... & $30-$ & 60 \\
\hline
\end{tabular}


TABLE 90.-Climatic extremes for Abies balsamea-Continued.

\begin{tabular}{|c|c|c|c|}
\hline $\begin{array}{c}\text { Plate } \\
53\end{array}$ & $\begin{array}{l}\text { Evaporation: } \\
\quad \text { Daily mean, } 1887-88, f_{s} \text { (inch) } \ldots \ldots \ldots \ldots \ldots \ldots \ldots\end{array}$ & $\begin{array}{l}\text { Low. } \\
.084\end{array}$ & $\begin{array}{l}\text { High. } \\
.143\end{array}$ \\
\hline 54 & $\begin{array}{l}\text { Total annual, } 1887-88 \text { (inches) } \ldots \ldots \ldots \ldots \ldots \ldots \ldots \\
\text { Moisture ratios: }\end{array}$ & 24.3 & 34.4 \\
\hline 58 & Normal $P / E, f s \ldots \ldots \ldots \ldots$ & .71 & 1.31 \\
\hline 59 & Normal $\pi / E, f_{s}$. & .81 & 1.52 \\
\hline 60 & $\begin{array}{l}\text { Normal } P / E \text {, year............ } \\
\text { Vapor pressure: }\end{array}$ & .95 & 1.72 \\
\hline 63 & $\begin{array}{l}\text { Normal mean, } f_{s} \text { (hundredths inch).. } \\
\text { Humidity: }\end{array}$ & 345 & 438 \\
\hline 65 & Normal mean, $f_{s}$ (per cent..... & 67.3 & 81.8 \\
\hline 66 & $\begin{array}{l}\text { Normal mean, year (per cent)........... } \\
\text { Wind: }\end{array}$ & 71.9 & 80.2 \\
\hline 68 & $\begin{array}{l}\text { Normal mean hourly velocity, } f_{s} \text { (miles). } \\
\text { Sunshine: }\end{array}$ & 3.1 & 12.4 \\
\hline 69 & $\begin{array}{l}\text { Normal total duration, } f s \text { (hours) .. } \\
\text { Moisture-temperature indices: }\end{array}$ & 1,225 & $1,500+a$ \\
\hline 70 & Normal $P / E \times T, f_{s}$, remainder method. & 312 & $500+{ }^{a}$ \\
\hline 71 & Normal $P / E \times T, f s$, exponential method.. & 2,997 & 4,097 \\
\hline 72 & Normal $P / E \times T, f s$, physiological method. & 2,747 & $7,000^{\alpha}$ \\
\hline
\end{tabular}

TABLE 91.-Climatic extremes for Quercus alba.

\begin{tabular}{|c|c|c|c|}
\hline Plate & Temperature: & Low. & High. \\
\hline 34 & Days in normal frostless season $\left(f_{s}\right) \ldots \ldots \ldots \ldots \ldots$ & 101 & 331 \\
\hline 35 & 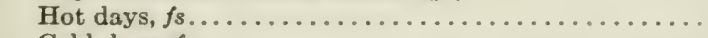 & 0 & 215 \\
\hline 36 & Cold days, $f_{s} \ldots \ldots \ldots \ldots \ldots \ldots \ldots \ldots \ldots \ldots$ & 0 & 137 \\
\hline 37 & Remainder summation above $32^{\circ}$, year (thousands).... & $10.0-$ & $18.0+$ \\
\hline 38 & Remainder summation above $39^{\circ}, f_{s}$ (thousands) ..... & 2.6 & 10.6 \\
\hline 39 & Exponential summation, $f s$ (hundreds) ............. & 3.0 & 11.7 \\
\hline 40 & Physiological summation, $f_{s}$ (thousands) . . . . . . . . & 2.1 & 21.2 \\
\hline 41 & 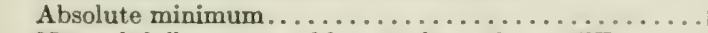 & -48 & +19 \\
\hline 43 & Normal daily mean, coldest 14 days of year $\left({ }^{\circ} \mathrm{F}.\right) \ldots$ & 11 & 53 \\
\hline 44 & Normal daily mean, hottest 6 weeks of year $\left({ }^{\circ} \mathrm{F}\right.$ ) ..... & $64.4-$ & $78.8+$ \\
\hline 45 & $\begin{array}{l}\text { Normal daily mean, year }\left({ }^{\circ} \mathrm{F} .\right) \ldots \ldots \ldots \ldots \ldots \ldots \\
\text { Precipitation: }\end{array}$ & 40 & $65+$ \\
\hline 46 & Normal daily mean, $f_{s}$ (inch). & .091 & .170 \\
\hline 47 & Normal No. rainy days (over 0.10 inch), $f_{8} \ldots \ldots \ldots$ & 26 & 284 \\
\hline 48 & Normal No. dry days $(0.10$ inch or less $), f_{s} \ldots \ldots \ldots$ & 0 & 136 \\
\hline 49 & Dry days, percentage of total, $f_{3}$ (per cent) ......... & 8 & 83 \\
\hline 50 & Days in longest normal rainy period, $f s \ldots \ldots \ldots \ldots$ & 17 & 256 \\
\hline 51 & Days in longest normal dry period, $f_{s} \ldots \ldots \ldots$ & 0 & 91 \\
\hline 52 & $\begin{array}{l}\text { Mean total, year (inches) } \ldots \ldots \ldots \ldots \ldots \ldots \ldots \ldots \\
\text { Evaporation: }\end{array}$ & $30-$ & $60+$ \\
\hline 53 & Daily mean, $1887-88, f_{s}$ (inch) $\ldots \ldots \ldots \ldots \ldots \ldots$ & .081 & .200 \\
\hline 54 & $\begin{array}{l}\text { Total annual, } 1887-88 \text { (inches) .................... } \\
\text { Moisture ratios: }\end{array}$ & 24.3 & 54.8 \\
\hline 58 & Normal $P / E, f s . . . \ldots$. & .51 & 1.76 \\
\hline 59 & Normal $\pi / E, f_{s} \ldots \ldots \ldots \ldots \ldots \ldots \ldots \ldots \ldots$ & .60 & 1.96 \\
\hline 60 & $\begin{array}{l}\text { Normal } P / E \text {, year } \ldots \ldots \ldots \ldots \ldots \ldots \ldots \ldots \ldots \ldots \\
\text { Vapor pressure: }\end{array}$ & .74 & 1.91 \\
\hline 63 & $\begin{array}{l}\text { Normal mean, } f_{s} \text { (hundredths inch) } \ldots \ldots \ldots \ldots \ldots \ldots \\
\text { Humidity: }\end{array}$ & 345 & 622 \\
\hline 65 & Normal mean, fs (per cent).... & 64.5 & 83.9 \\
\hline 66 & $\begin{array}{l}\text { Normal mean, year (per cent) } \ldots \ldots \ldots \ldots \ldots \ldots \ldots \\
\text { Wind: }\end{array}$ & 69.0 & 85.2 \\
\hline 68 & $\begin{array}{l}\text { Normal mean hourly velocity, } f s \text { (miles). } \\
\text { Sunshine: }\end{array}$ & 3.1 & 14.9 \\
\hline 69 & $\begin{array}{l}\text { Normal total duration, } f_{s} \text { (hours). } \\
\text { Moisture-temperature indices: }\end{array}$ & 1,225 & 2,650 \\
\hline 70 & Normal $P / E \times T, f s$, remainder method... & 301 & 1,415 \\
\hline 71 & Normal $P / E \times T, f s$, exponential method........... & 2,914 & 13,511 \\
\hline 72 & Normal $P / E \times T, f_{s}$, physiological method.......... & 2,747 & 24,265 \\
\hline
\end{tabular}


TABLE 92.-Climatic extremes for Fagus atropunicea.

\begin{tabular}{|c|c|c|c|}
\hline Plate & Temperature: & Low. & High. \\
\hline 34 & Days in normal frostless season $(f s)$. & 95 & \\
\hline 35 & Hot days, $f_{s} \ldots \ldots \ldots \ldots \ldots \ldots$ & 0 & 215 \\
\hline 36 & Cold days, fs.. & 0 & 149 \\
\hline 37 & Remainder summation above $32^{\circ}$, year (thousands) & $10.0-$ & $18.0+$ \\
\hline 38 & Remainder summation above $39^{\circ}, f_{s}$ (thousands). & 2.6 & 10.6 \\
\hline 39 & Exponential summation, $f_{s}$ (hundreds)......... & 3.0 & 11.7 \\
\hline 40 & Physiological summation, $f_{s}$ (thousands). & 2.1 & 21.1 \\
\hline 41 & Absolute minimum......... & -44 & +12 \\
\hline 43 & Normal daily mean, coldest 14 days of year $\left({ }^{\circ} \mathrm{F}\right.$.) & 12 & 52 \\
\hline 44 & Normal daily mean, hottest 6 weeks of year $\left({ }^{\circ} \mathrm{F}\right.$. $)$. & $64.4-$ & $78.8+$ \\
\hline 45 & $\begin{array}{l}\text { Normal daily mean, year }\left({ }^{\circ} \mathrm{F} .\right) \ldots \ldots \ldots \ldots \ldots \\
\text { Precipitation: }\end{array}$ & $40-$ & $65+$ \\
\hline 46 & Normal daily mean, $f_{s}$ (inch) & .091 & .172 \\
\hline 47 & Normal No, rainy days (over 0.10 inch), $f s$. & 26 & 256 \\
\hline 48 & Normal No. dry days ( 0.10 inch or less), fs. & 0 & 154 \\
\hline 49 & Dry days, percentage of total, $f_{3}$ (per cent). & 0 & 83 \\
\hline 50 & Days in longest normal rainy period, $f_{s} \ldots$ & 17 & 256 \\
\hline 51 & Days in longest normal dry period, $f_{3} \ldots \ldots$. & 4 & 91 \\
\hline 52 & $\begin{array}{c}\text { Mean total, year (inches)................... } \\
\text { Evaporation: }\end{array}$ & $30-$ & $60+$ \\
\hline 53 & Daily mean, $1887-88, f_{s}$ (inch) $\ldots \ldots \ldots$. & .084 & .200 \\
\hline 54 & $\begin{array}{l}\text { Total annual, } 1887-88 \text { (inches)... } \\
\text { Moisture ratios: }\end{array}$ & 24.3 & 54.8 \\
\hline 58 & Normal $P / E, f_{s} .$. & .62 & 1.76 \\
\hline 59 & Normal $\pi / E, f_{3} \ldots$ & .60 & 1.96 \\
\hline 60 & $\begin{array}{l}\text { Normal } P / E \text {, year. . . . . . . . . . . . . . . } \\
\text { Vapor pressure: }\end{array}$ & .72 & 1.94 \\
\hline 63 & $\begin{array}{l}\text { Normal mean, } f_{s} \text { (hundredths inch)..... } \\
\text { Humidity: }\end{array}$ & 345 & 622 \\
\hline 65 & Normal mean, $f_{s}$ (per cent).. & 65.5 & 84.0 \\
\hline 66 & $\begin{array}{l}\text { Normal mean, year (per cent) ................ } \\
\text { Wind: }\end{array}$ & 67.5 & 85.2 \\
\hline 68 & $\begin{array}{l}\text { Normal mean hourly velocity, } f_{s} \text { (miles)...... } \\
\text { Sunshine: }\end{array}$ & 3.6 & 14.9 \\
\hline 69 & $\begin{array}{l}\text { Normal total duration, } f_{s} \text { (hours) ..... } \\
\text { Moisture-temperature indices: }\end{array}$ & 1,225 & 2,650 \\
\hline 70 & Normal $P / E \times T, f_{s}$, remainder method. & 301 & 1,418 \\
\hline 71 & Normal $P / E \times T, f s$, exponential method. & 2,918 & 13,511 \\
\hline 72 & Normal $P / E \times T, f s$, physiological method. & 2,747 & 24,265 \\
\hline
\end{tabular}

TABLE 93.-Climatic extremes for Castanea dentata.

\begin{tabular}{|c|c|c|c|}
\hline Plate & Temperature: & Loue. & High. \\
\hline 34 & Days in normal frostless season $(f s) \ldots \ldots \ldots \ldots$ & 127 & \\
\hline 35 & Hot days, $f_{s} .$. & 31 & 160 \\
\hline 36 & Cold days, $f_{s}$. & 0 & 117 \\
\hline 37 & Remainder summation above $32^{\circ}$, year (thousands) & $11.5-$ & $15.0+$ \\
\hline 38 & Remainder summation above $39^{\circ}, f_{3}$ (thousands)... & 3.6 & 7.6 \\
\hline 39 & Exponential summation, $f_{s}$ (hundreds) .......... & 3.7 & 7.4 \\
\hline 40 & Physiological summation, $f_{s}$ (thousands). & 5.0 & 15.0 \\
\hline 41 & Absolute minimum. . & -35 & +4 \\
\hline 43 & Normal daily mean, coldest 14 days of year $\left({ }^{\circ} \mathrm{F}.\right)$. & 16 & 45 \\
\hline 44 & Normal daily mean, hottest 6 weeks of year $\left({ }^{\circ} \mathrm{F}\right.$.). & 6it.4- & 75.8 \\
\hline 45 & Normal daily mean, year $\left({ }^{\circ} \mathrm{F}.\right) \ldots \ldots \ldots \ldots \ldots$ & 45 & 65 \\
\hline & Precipitation: & & \\
\hline 46 & Normal daily mean, $f s$ (inch). & 091 & 140 \\
\hline 47 & Normal No. rainy days (over $0.10 \mathrm{inch}$ ), $f_{3} \ldots \ldots$. & 26 & 166 \\
\hline 4s & Normal No. dry days ( 0.10 inch or less), $f_{s} .$. & 32 & 154 \\
\hline 49 & Dry days, percentage of total, $f_{s}$ (per cent)...... & 11 & 83 \\
\hline$\therefore()$ & Days in longest normal rainy period, $f_{s} \ldots \ldots \ldots \ldots$ & 17 & 256 \\
\hline 51 & Days in longest normal dry period, $f_{s} \ldots \ldots \ldots \ldots$ & 4 & 91 \\
\hline 52 & Mean total, year (inches) . . . . . . . . . . . . . . & 30 & $60+$ \\
\hline
\end{tabular}


TABLE 93.-Climatic extremes for Castanea dentata-Continued.

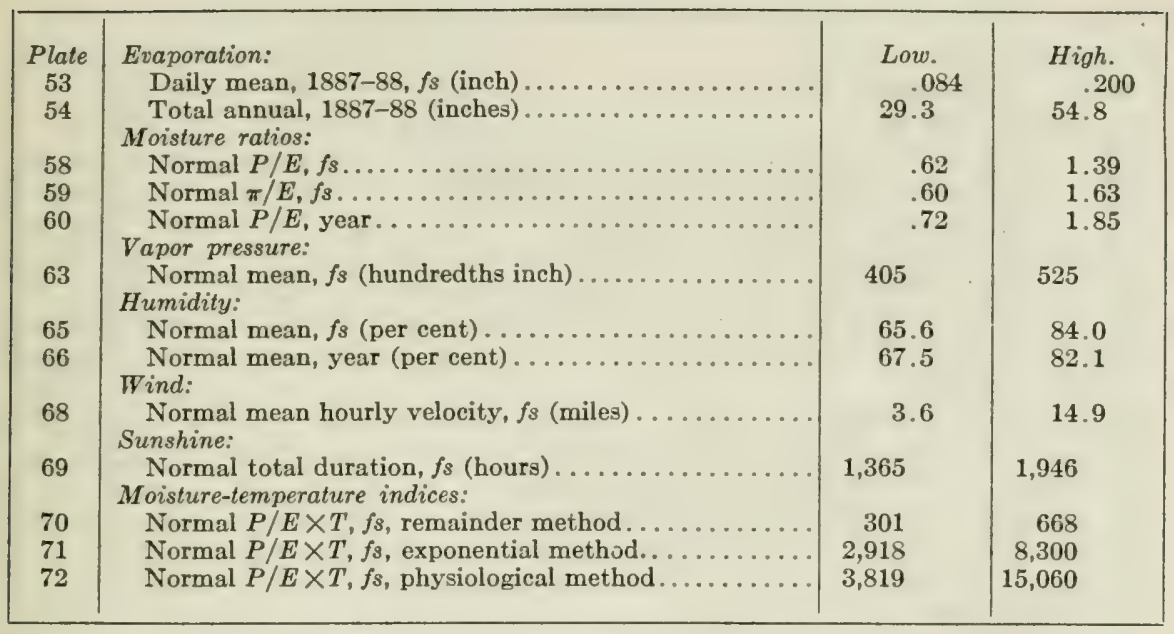

TABLE 94.-Climatic extremes for Acer saccharum.

\begin{tabular}{|c|c|c|c|}
\hline Plate & Temperature: & Low. & High. \\
\hline 34 & Days in normal frostless season $(f s) \ldots$ & 98 & 268 \\
\hline 35 & Hot days, $f s . . . \ldots \ldots \ldots \ldots \ldots \ldots \ldots \ldots \ldots$ & 0 & 172 \\
\hline 36 & Cold days, $f s \ldots \ldots \ldots \ldots \ldots \ldots \ldots \ldots$ & 0 & 149 \\
\hline 37 & Remainder summation above $32^{\circ}$, year (thousands) & $10.0-$ & $18.0+$ \\
\hline 38 & Remainder summation above $39^{\circ}, f s$ (thousands)... & 2.6 & 8.4 \\
\hline 39 & Exponential summation, $f_{s}$ (hundreds) ............ & 3.0 & 9.2 \\
\hline 40 & Physiological summation, $f s$ (thousands). & 2.1 & 17.0 \\
\hline 41 & Absolute minimum... & -49 & +4 \\
\hline 43 & Normal daily mean, coldest 14 days of year $\left({ }^{\circ} \mathrm{F}.\right)$. & 10 & 46 \\
\hline 44 & Normal daily mean, hottest 6 weeks of year $\left({ }^{\circ} \mathrm{F}.\right)$ & $64.4-$ & $78.8+$ \\
\hline 45 & $\begin{array}{l}\text { Normal daily mean, year }\left({ }^{\circ} \mathrm{F} .\right) \ldots \ldots \ldots \ldots \ldots \\
\text { Precipitation: }\end{array}$ & & $65+$ \\
\hline 46 & Normal daily mean, $f_{s}$ (inch). & .091 & .170 \\
\hline 47 & Normal No. rainy days (over $0.10 \mathrm{inch}$ ), $f s$. & & 256 \\
\hline 48 & Normal No. dry days ( 0.10 inch or less), $f s$. & 0 & 154 \\
\hline 49 & Dry days, percentage of total, $f s$ (per cent).... & 0 & 83 \\
\hline 50 & Days in longest normal rainy period, $f_{s} \ldots$. & 17 & 256 \\
\hline 51 & Days in longest normal dry period, $f$ s... & 4 & 91 \\
\hline 52 & Mean total, year (inches) ............ & $30-$ & $60+$ \\
\hline 53 & $\begin{array}{l}\text { Evaporation: } \\
\text { Daily mean, } 1887-88, f_{s} \text { (inch).... }\end{array}$ & 084 & \\
\hline 54 & $\begin{array}{l}\text { Dally mean, } 1887-88, \text { f } 8 \text { (inch) } \ldots \ldots \ldots \ldots \\
\text { Total annual, } 1887-88 \text { (inches) } . . . . . . \\
\text { Moisture ratios: }\end{array}$ & 23.0 & 54.8 \\
\hline 58 & Normal $P / E, f_{3} \ldots$ & .62 & 1.76 \\
\hline 59 & Normal $\pi / E, f_{s} \ldots$ & .60 & 1.96 \\
\hline 60 & $\begin{array}{l}\text { Normal } P / E \text {, year........ } \\
\text { Vapor pressure: }\end{array}$ & .72 & 1.94 \\
\hline 63 & $\begin{array}{l}\text { Normal mean, } f_{s} \text { (hundredths inch)..... } \\
\text { Humidity: }\end{array}$ & 345 & 588 \\
\hline 65 & Normal mean, $f_{s}$ (per cent).. & 62.0 & 84.0 \\
\hline 66 & $\begin{array}{l}\text { Normal mean, year (per cent).. } \\
\text { Wind: }\end{array}$ & 67.5 & 82.9 \\
\hline 68 & $\begin{array}{l}\text { Normal mean hourly velocity, } f_{s} \text { (miles)... } \\
\text { Sunshine: }\end{array}$ & 3.6 & 14.9 \\
\hline 69 & $\begin{array}{l}\text { Normal total duration, } f s \text { (hours)........ } \\
\text { Moisture-temperature indices: }\end{array}$ & 1,225 & 2,166 \\
\hline 70 & Normal $P / E \times T, f s$, remainder method. . & 301 & 1,418 \\
\hline 71 & Normal $P / E \times T, f s$, exponential method.. & 2,918 & 13,511 \\
\hline 72 & Normal $P / E \times T, f s$, physiological method. & 2,747 & 24,265 \\
\hline
\end{tabular}


TABLE 95.-Climatic extremes for Quercus falcata.

\begin{tabular}{|c|c|c|c|}
\hline Plate & Temperature: & Low. & High. \\
\hline 34 & Days in normal frostless season $\left(f_{3}\right)$. & 167 & 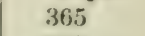 \\
\hline 35 & Hot days, $f_{8} \ldots \ldots \ldots \ldots \ldots \ldots \ldots$ & 90 & 365 \\
\hline 36 & Cold days, $f_{s} \ldots \ldots \ldots \ldots \ldots$ & 0 & 44 \\
\hline 37 & Remainder summation above $32^{\circ}$, year (thousands) & $11.5+$ & $18.0+$ \\
\hline 38 & Remainder summation above $39^{\circ}, f_{8}$ (thousands).. & 5.1 & 14.5 \\
\hline 39 & Exponential summation, $f_{8}$ (hundreds).......... & 5.3 & 15.4 \\
\hline 40 & Physiological summation, fs (thousands) & 7.9 & 31.1 \\
\hline 41 & Absolute minimum .......... & -16 & +41 \\
\hline 43 & Normal daily mean, coldest 14 days of year $\left({ }^{\circ} \mathrm{F}.\right)$. & 32 & 69 \\
\hline 44 & Normal daily mean, hottest 6 weeks of year $\left({ }^{\circ} \mathrm{F}.\right)$ & $71.6-$ & $78.8+$ \\
\hline 45 & $\begin{array}{l}\text { Normal daily mean, year }\left({ }^{\circ} \mathrm{F} .\right) \ldots \ldots \ldots \ldots \ldots \\
\text { Precipitation: }\end{array}$ & $5.5-$ & $75+$ \\
\hline 46 & Normal daily mean, $f_{s}$ (inch). & 106 & .170 \\
\hline 47 & Normal No. rainy days (over $0.10 \mathrm{inch}$ ), $f_{8}$ & 91 & $2 \$ 4$ \\
\hline 48 & Normal No. dry days ( 0.10 inch or less), f8. & 0 & 92 \\
\hline 49 & Dry days, percentage of total, $f_{8}$ (per cent).. & $s$ & 51 \\
\hline 50 & Days in longest normal rainy period, $f 8 \ldots$ & 47 & 256 \\
\hline 51 & Days in longest normal dry period, $f_{3}$.. & 0 & 63 \\
\hline 52 & Mean total, year (inches) . . . . . . . & $40-$ & $60+$ \\
\hline & Evaporation: & & \\
\hline $\begin{array}{l}53 \\
54\end{array}$ & $\begin{array}{l}\text { Daily mean, } 1887-88, \text { fs (inch)... } \\
\text { Total annual, 1887-88 (inches).. }\end{array}$ & 2.084 & 51.7 \\
\hline & Moisture ratios: & & \\
\hline 58 & Normal $P / E, f_{s}$. & .64 & 1.76 \\
\hline 59 & Normal $\pi / E, f_{s}$.. & .75 & 1.96 \\
\hline 60 & $\begin{array}{l}\text { Normal } P / E \text {, year........................... } \\
\text { Vapor pressure: }\end{array}$ & .85 & 1.94 \\
\hline 63 & $\begin{array}{l}\text { Normal mean, fs (hundredths inch).... } \\
\text { Humidity: }\end{array}$ & 460 & 707 \\
\hline 65 & Normal mean, $f_{s}$ (per cent)......... & 69.1 & S2.7 \\
\hline 66 & $\begin{array}{l}\text { Normal mean, year (per cent). } \\
\text { Wind: }\end{array}$ & 69.5 & 85.2 \\
\hline 68 & $\begin{array}{l}\text { Normal mean hourly velocity, } f s \text { (miles). } \\
\text { Sunshine: }\end{array}$ & 3.6 & 13.5 \\
\hline 69 & $\begin{array}{l}\text { Normal total duration, } f s \text { (hours). } \\
\text { Moisture-temperature indices: }\end{array}$ & 1,646 & 2.650 \\
\hline 70 & Normal $P / E \times T, f_{s}$, remainder method. & 446 & 1,418 \\
\hline 71 & Normal $P / E \times T, f s$, exponential method. & 4,174 & 13,511 \\
\hline 72 & Normal $P / E \times T, f_{3}$, physiological method. & 7,010 & 24,265 \\
\hline
\end{tabular}

TABLE 96--Climatic extremes for Sapindus marginatus.

\begin{tabular}{|c|c|c|c|}
\hline Plate & Temperalure: & Lou. & High. \\
\hline 34 & Days in normal frostless season $\left(f_{s}\right)$. & 153 & 328 \\
\hline & Hot days, $f s \ldots \ldots \ldots \ldots \ldots \ldots \ldots$ & 113 & 226 \\
\hline 36 & Cold days, $f_{3} \ldots \ldots \ldots \ldots$ & 0 & 70 \\
\hline 37 & Remainder summation above $32^{\circ}$, year (thousands) & $11.5-$ & $26.0+$ \\
\hline 38 & Remainder summation above $39^{\circ}, f_{s}$ (thousands)... & 5.5 & 10.8 \\
\hline 39 & Exponential summation, $f_{s}$ (hundreds)... & 5.90 & 11.1 \\
\hline 40 & Physiological summation, fs (thousands).. & 10.3 & 21.4 \\
\hline 41 & Absolute minimum................ & -32 & +19 \\
\hline 43 & Normal daily mean, coldest 14 days of year $\left({ }^{\circ} \mathrm{F}\right.$ ) & 27 & 57 \\
\hline 44 & Normal daily mean, hottest 6 weeks of year ( ${ }^{\circ} \mathrm{F}$.). & $71.6-$ & $78.8+$ \\
\hline 45 & Normal daily mean, year $\left({ }^{\circ} \mathbf{F}, \ldots \ldots \ldots \ldots \ldots\right.$ & $55-$ & $75+$ \\
\hline & Prccipitation: & & \\
\hline 46 & Normal daily mean, $f_{s}$ (inch). & .052 & .172 \\
\hline 47 & Normal No. rainy days (over 0.10 inch), fs. . & $25^{a}$ & 254 \\
\hline 48 & Normal No. dry days $(0.10$ inch or less), $f 8 \ldots$. & 263 & $259^{\circ}$ \\
\hline 49 & Dry days, percentage of total, $f$ s (per cent)... & s & $\$ 7$ \\
\hline 50 & Dnys in longest normal rainy period, fs.... & $25-a$ & 235 \\
\hline 51 & Days in longest normal dry period, $f_{s} . . . . .$. & is & $100^{a}$ \\
\hline 52 & Mean total, year (inches)..$\ldots \ldots \ldots \ldots \ldots$ & 20 & $60+$ \\
\hline
\end{tabular}


TABLE 96.-Climatic extremes for Sapindus marginatus-Continued.

\begin{tabular}{|c|c|c|c|}
\hline Plate & Evaporation: & Lovo. & High. \\
\hline 53 & Daily mean, $1887-88, f_{s}$ (inch). & .130 & .203 \\
\hline 54 & $\begin{array}{l}\text { Total annual, } 1887-88 \text { (inches) .......... } \\
\text { Moisture ratios: }\end{array}$ & 42.1 & $80^{a}$ \\
\hline 58 & Normal $P / E, f_{s} \ldots \ldots \ldots \ldots \ldots$ & $.40-a$ & 1.36 \\
\hline 59 & Normal $\pi / E, f_{s}$. & .35 & 1.52 \\
\hline 60 & $\begin{array}{l}\text { Normal } P / E \text {, year. . . . . . . . . . . . } \\
\text { Vapor pressure: }\end{array}$ & .24 & 1.47 \\
\hline 63 & $\begin{array}{l}\text { Normal mean, } f_{s} \text { (hundredths inch)... } \\
\text { Humidity: }\end{array}$ & $350-a$ & 707 \\
\hline 65 & Normal mean, fs (per cent.... & 59.4 & 80.6 \\
\hline 66 & $\begin{array}{l}\text { Normal mean, year }(\text { per cent) } \ldots \ldots \ldots \ldots \ldots \ldots \ldots \\
\text { Wind: }\end{array}$ & 59.3 & 85.2 \\
\hline 68 & $\begin{array}{l}\text { Normal mean hourly velocity, } f_{3} \text { (miles). } \\
\text { Sunshine: }\end{array}$ & 6.3 & 14.2 \\
\hline 69 & $\begin{array}{l}\text { Normal total duration, } f_{s} \text { (hours) . } \\
\text { Moisture-temperature indices: }\end{array}$ & $1,700-a$ & 2,650 \\
\hline 70 & Normal $P / E \times T, f s$, remainder method. & 256 & 1,314 \\
\hline 71 & Normal $P / E \times T, f_{s}$, exponential method & 2,411 & 12,106 \\
\hline 72 & Normal $P / E \times T, f s$, physiological method & 4,673 & 24,265 \\
\hline
\end{tabular}

TABLE 97.-Climatic extremes for Populus balsamifera.

\begin{tabular}{|c|c|c|c|}
\hline Plate & Temperalure: & Low. & High. \\
\hline 34 & Days in normal frostless season $\left(f_{s}\right)$. & & 172 \\
\hline 35 & Hot days, fs... & 0 & 88 \\
\hline 36 & Cold days, $f_{3}$. & 92 & 137 \\
\hline 37 & Remainder summation above $32^{\circ}$, year (thousands) & $10.0-$ & $11.5+$ \\
\hline 38 & Remainder summation above $39^{\circ}, f s$ (thousands)... & 2.6 & 4.5 \\
\hline 39 & Exponential summation, $f_{S}$ (hundreds) ........ & 3.0 & 4.90 \\
\hline 40 & Physiological summation, $f_{s}$ (thousands). & 2.1 & 7.5 \\
\hline 41 & Absolute minimum.............. & -59 & -13 \\
\hline 43 & Normal daily mean, coldest 14 days of year $\left({ }^{\circ} \mathrm{F}\right.$.) & 0 & 24 \\
\hline 44 & Normal daily mean, hottest 6 weeks of year $\left({ }^{\circ} \mathrm{F}.\right)$. & $64.4-$ & $71.6+$ \\
\hline 45 & Normal daily mean, year $\left({ }^{\circ} \mathrm{F}.\right) \ldots \ldots \ldots \ldots \ldots \ldots$ & & \\
\hline 46 & $\begin{array}{l}\text { Precipitation: } \\
\text { Normal daily mean fs (inch) }\end{array}$ & 043 & 126 \\
\hline 47 & Normal No. rainy days (over 0.10 inch), $f_{s}$. & 0.040 & $135^{.00}$ \\
\hline 48 & Normal No. dry days ( 0.10 inch or less), $f s$. & 28 & 140 \\
\hline 49 & Dry days, percentage of total, $f s$ (per cent). & 17 & 100 \\
\hline 50 & Days in longest normal rainy period, $f_{s} . \ldots$ & 0 & 100 \\
\hline 51 & Days in longest normal dry period, $f_{s} .$. & 11 & $175^{a}$ \\
\hline 52 & Mean total, year (inches)... & $20-$ & $50+$ \\
\hline & Evaporation: & & \\
\hline 53 & Daily mean, $1887-88, f_{s}$ (inch)... & $.0 \$ 4$ & $.220+c$ \\
\hline 54 & $\begin{array}{l}\text { Total annual, } 1887-88 \text { (inches. . . . . . . . } \\
\text { Moisture ratios: }\end{array}$ & 22.1 & $50+a$ \\
\hline 58 & Normal $P / E, f_{3} \ldots \ldots \ldots$ & $.20-a$ & 1.23 \\
\hline 59 & Normal $\pi / E, f_{s}$. & $.20+a$ & 1.52 \\
\hline 60 & $\begin{array}{l}\text { Normal } P / E \text {, year } \ldots \ldots \ldots \ldots \ldots \ldots \ldots \\
\text { Vapor pressure: }\end{array}$ & $.20-a$ & 1.72 \\
\hline 63 & $\begin{array}{l}\text { Normal mean, } f_{s} \text { (hundredths inch)..... } \\
\text { Humidity: }\end{array}$ & $300^{a}$ & 438 \\
\hline 65 & Normal mean, $f_{s}$ (per cent). & $50-a$ & 81.8 \\
\hline 66 & $\begin{array}{l}\text { Normal mean, year (per cent)...... } \\
\text { Wind: }\end{array}$ & $60-a$ & 80.2 \\
\hline 68 & $\begin{array}{l}\text { Normal mean hourly velocity, } f_{s} \text { (miles)... } \\
\text { Sunshine: }\end{array}$ & 4.7 & 12.4 \\
\hline 69 & $\begin{array}{l}\text { Normal total duration, } f_{s} \text { (hours). } \\
\text { Moisture-temperature indices: }\end{array}$ & 1,225 & 1,512 \\
\hline 70 & Normal $P / E \times T, f s$, remainder method & $100-a$ & 442 \\
\hline 71 & Normal $P / E \times T, f s$, exponential method. & $1,000-a$ & 4,269 \\
\hline 72 & Normal $P / E \times T, f s$, physiological method. & $1,000-a$ & 6,423 \\
\hline
\end{tabular}


TABLE 98.-Climatic extremes for Quercus macrocarpa.

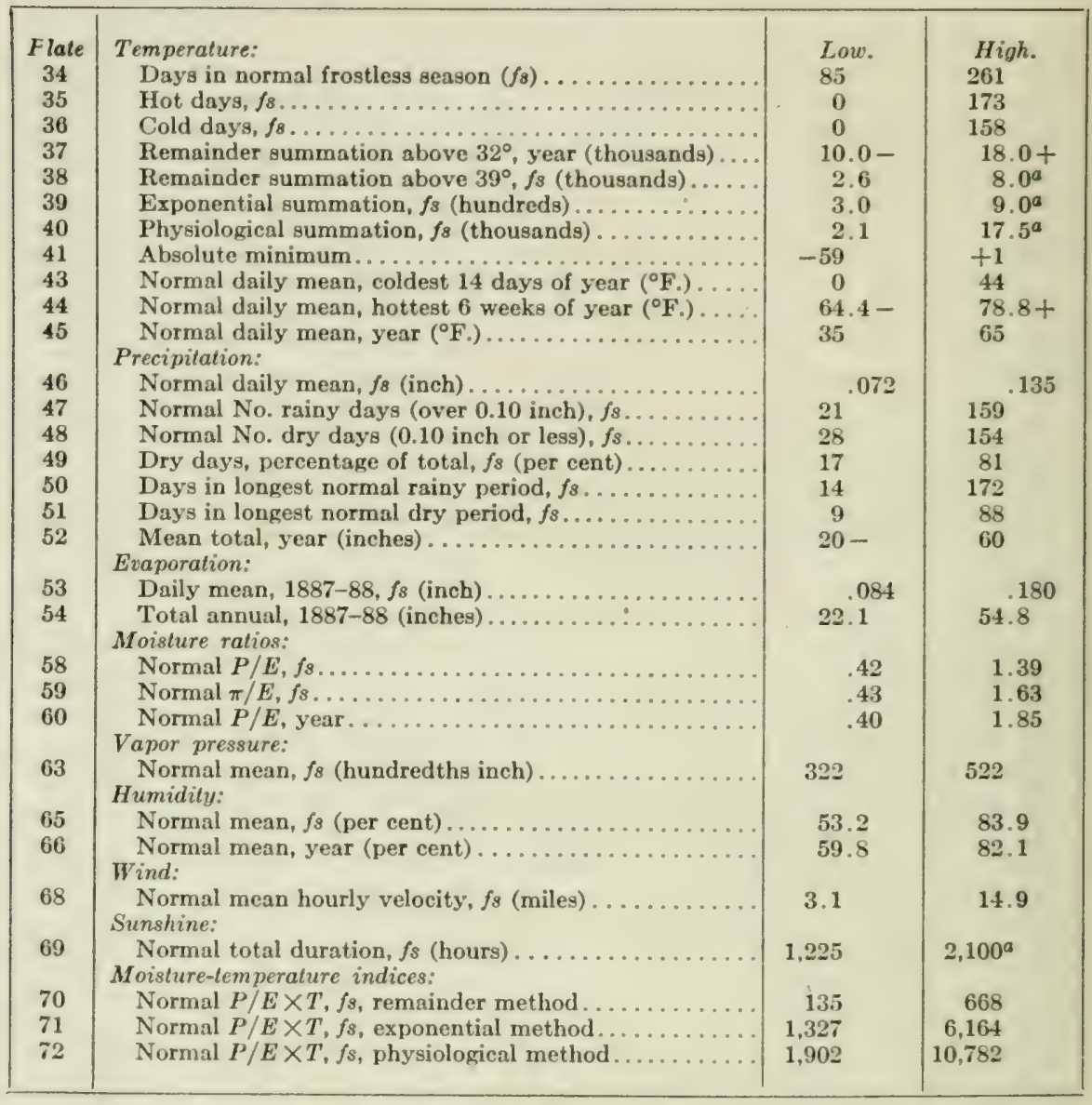

TABLe 99.-Climatic extremes for Ilex opaca.

\begin{tabular}{|c|c|c|c|}
\hline Plate & Temperature: & Low. & High. \\
\hline 34 & Days in normal frostless season $\left(f_{s}\right)$. & 141 & \\
\hline 35 & Hot days, $f_{s} \ldots \ldots \ldots \ldots \ldots \ldots \ldots$. . . . . . & 47 & 365 \\
\hline 36 & Cold days, $f_{3} . .$. & 0 & 55 \\
\hline 37 & Remainder summation above $32^{\circ}$, year (thousands) & $11.5-$ & 26.0 \\
\hline 38 & Remainder summation above $39^{\circ}, f_{s}$ (thousands)... & 4.4 & 14.5 \\
\hline 39 & Exponential summation, $f_{s}$ (hundreds).......... & 4.7 & 15.4 \\
\hline 40 & Physiological summation, $f_{s}$ (thousands). & 6.7 & 31.1 \\
\hline 41 & 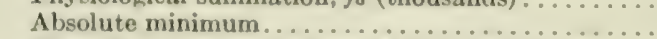 & -15 & +41 \\
\hline 43 & Normal daily mean, coldest 14 days of year $\left({ }^{\circ} \mathrm{F}\right)$ & 27 & 69 \\
\hline 44 & Normal daily mean, hottest 6 weeks of year $\left({ }^{\circ} \mathrm{F}\right.$.). & $71.6-$ & $78.8+$ \\
\hline 45 & Normal daily mean, year $\left({ }^{\circ} \mathrm{F},{ }\right) \ldots \ldots \ldots \ldots \ldots$ & $50-$ & $75+$ \\
\hline 46 & $\begin{array}{l}\text { Precipitation: } \\
\text { Normal daily mean fs (inch) }\end{array}$ & & 173 \\
\hline 47 & $\begin{array}{l}\text { Normal daly mean, } f s \text { (inch) } \ldots \ldots \ldots \ldots \ldots \\
\text { Normal No. rainy days (over } 0.10 \text { inch), } f_{s} \ldots\end{array}$ & $55^{.091}$ & $284^{.163}$ \\
\hline 48 & Normal No. dry days ( 0.10 inch or less), fs.. & 0 & 204 \\
\hline 49 & Dry days, percentage of total, $f_{s}$ (per cent). & 8 & 74 \\
\hline 50 & Days in longest normal rainy period, $f_{8} \ldots$ & 17 & 256 \\
\hline 51 & Days in longest normal dry period, $f_{3} \ldots$ & 0 & 182 \\
\hline 52 & Mean total, year (inches) ............ & $50-$ & $60+$ \\
\hline
\end{tabular}


TABLE 99.-Climatic extremes for Ilex opaca-Continued.

\begin{tabular}{|c|c|c|c|}
\hline Plate & Evaporation: & Low. & High. \\
\hline 53 & Daily mean, $1887-88, f_{8}$ (inch). & .088 & .177 \\
\hline & $\begin{array}{l}\text { Total annual, } 1887-88 \text { (inches).... } \\
\text { Moisture ratios: }\end{array}$ & 20.3 & 51.7 \\
\hline 58 & Normal $P / E, f_{8} \ldots \ldots \ldots \ldots$ & .64 & 1.76 \\
\hline 59 & Normal $\pi / E, f_{3}$. & .75 & 1.96 \\
\hline 60 & Normal $P / E$, year. . & 1.00 & 1.85 \\
\hline 63 & $\begin{array}{l}\text { Normal mean, fs (hundredths inch).... } \\
\text { Humidity: }\end{array}$ & 425 & 707 \\
\hline 65 & Normal mean, $f_{s}$ (per cent)....... & 69.1 & 84.0 \\
\hline 66 & $\begin{array}{l}\text { Normal mean, year (per cent).... } \\
\text { Wind: }\end{array}$ & 69.5 & .82 .9 \\
\hline 68 & $\begin{array}{l}\text { Normal mean hourly velocity, fs (miles)... } \\
\text { Sunshine: }\end{array}$ & 5.2 & 13.5 \\
\hline 69 & $\begin{array}{l}\text { Normal total duration, fs (hours). } \\
\text { Moisture-temperature indices: }\end{array}$ & 1,646 & $2,500^{a}$ \\
\hline 70 & Normal $P / E \times T, f s$, remainder method. & 446 & 1,418 \\
\hline 71 & Normal $P / E \times T, f s$, exponential method. & 4,174 & 13,511 \\
\hline 72 & Normal $P / E \times T, f_{s}$, physiological method. & 5,193 & 24,265 \\
\hline
\end{tabular}

TABLE 100.-Climatic extremes for Magnolia grandiflora.

\begin{tabular}{|c|c|c|c|}
\hline Plate & Temperature: & Low. & High. \\
\hline 34 & Days in normal frostless season $(f s) \ldots$ & $221-$ & 365 \\
\hline 35 & Hot days, $f s . \ldots \ldots \ldots \ldots \ldots \ldots \ldots \ldots$ & 147 & 365 \\
\hline 36 & Cold days, $f_{3} \ldots \ldots \ldots \ldots \ldots \ldots \ldots \ldots \ldots$ & 0 & 0 \\
\hline 37 & Remainder summation above $32^{\circ}$, year (thousands) & $18.0+$ & $26.0+$ \\
\hline 38 & Remainder summation above $39^{\circ}, f_{s}$ (thousands)... & 7.3 & 14.5 \\
\hline 39 & Exponential summation, $f_{s}$ (hundreds)... & 7.7 & 15.4 \\
\hline 40 & Physiological summation, $f s$ (thousands). & 13.6 & 31.1 \\
\hline 41 & Absolute minimum................... & -3 & +41 \\
\hline 43 & Normal daily mean, coldest 14 days of year $\left({ }^{\circ} \mathrm{F}.\right)$. & 45 & 69 \\
\hline 44 & Normal daily mean, hottest 6 weeks of year $\left({ }^{\circ} \mathrm{F}\right.$.). & $78.8-$ & \\
\hline 45 & Normal daily mean, year $\left({ }^{\circ} \mathrm{F}.\right) \ldots \ldots \ldots$ & $65-$ & $75+$ \\
\hline 46 & $\begin{array}{l}\text { Precipitation: } \\
\text { Normal daily mean, fs (inch). }\end{array}$ & .129 & 173 \\
\hline 47 & Normal No. rainy days (over $0.10 \mathrm{inch}$ ), $f_{s}$. & 161 & 284 \\
\hline 48 & Normal No. dry days (0.10 inch or less), fs... & 26 & 204 \\
\hline 49 & Dry days, percentage of total, $f_{s}$ (per cent).... & 8 & 51 \\
\hline 50 & Days in longest normal rainy period, $f s \ldots \ldots$ & 92 & 235 \\
\hline 51 & Days in longest normal dry period, $f_{3} \ldots$ & 14 & 182 \\
\hline 52 & Mean total, year (inches)..$\ldots \ldots \ldots \ldots \ldots$ & $50-$ & $60+$ \\
\hline 53 & $\begin{array}{l}\text { Evaporation: } \\
\text { Daily mean, } 1887-88, f s \text { (inch) }\end{array}$ & 117 & 148 \\
\hline 54 & $\begin{array}{l}\text { Total annual, } 1887-88 \text { (inches)............... } \\
\text { Moisture ratios: }\end{array}$ & 42.1 & 51.6 \\
\hline 58 & Normal $P / E, f_{s}$.. & .75 & 1.36 \\
\hline 59 & Normal $\pi / E, f_{s} .$. & 1.05 & 1.47 \\
\hline 60 & $\begin{array}{l}\text { Normal } P / E \text {, year. } \\
\text { Vapor pressure: }\end{array}$ & 1.02 & 1.47 \\
\hline 63 & $\begin{array}{l}\text { Normal mean, } f_{s} \text { (hundredths inch)....... } \\
\text { Humidity: }\end{array}$ & 569 & 707 \\
\hline 65 & Normal mean, $f s$ (per cent. . & 73.9 & 80.6 \\
\hline 66 & $\begin{array}{l}\text { Normal mean, year (per cent).. } \\
\text { Wind: }\end{array}$ & 73.5 & 85.2 \\
\hline 68 & $\begin{array}{l}\text { Normal mean hourly velocity, } f_{s} \text { (miles)..... } \\
\text { Sunshine: }\end{array}$ & 5.1 & 11.0 \\
\hline 69 & $\begin{array}{l}\text { Normal total duration, } f_{s} \text { (hours) } \ldots \ldots \ldots \ldots \text {. } \\
\text { Moisture-temperature indices: }\end{array}$ & 1,895 & 2,650 \\
\hline 70 & Normal $P / E \times T, f_{s}$, remainder method. & 834 & 1,314 \\
\hline 71 & Normal $P / E \times T, f_{s}$, exponential method.. & 7,663 & 12,106 \\
\hline 72 & Normal $P / E \times T, f 8$, physiological method... & 15,125 & 24,265 \\
\hline
\end{tabular}


TABLE 101.-Climatic extremes for Sabal palmetto.

\begin{tabular}{|c|c|c|c|}
\hline Plate & Temperature: & Low. & High. \\
\hline 34 & Days in normal frostless season $(f s) \ldots$ & 228 & 365 \\
\hline 35 & Hot days, $f_{8} \ldots \ldots \ldots \ldots \ldots \ldots \ldots$ & 168 & 365 \\
\hline 36 & Cold days, $f 8, \ldots \ldots \ldots \ldots \ldots \ldots \ldots \ldots \ldots$ & 0 & 0 \\
\hline 37 & Remainder summation above $32^{\circ}$, year (thousands). . & $18.0+$ & $26.0+$ \\
\hline 38 & Remainder summation above $39^{\circ}, f_{8}$ (thousands).... & 8.4 & 14.5 \\
\hline 39 & Exponential summation, $f_{s}$ (hundreds) ........... & $8.0-a$ & 15.4 \\
\hline 40 & Physiological summation, $f_{s}$ (thousands) . . . . . . . & $15.0-a$ & 31.1 \\
\hline 41 & Absolute minimum.$\ldots \ldots \ldots \ldots \ldots \ldots \ldots$ & +1 & +41 \\
\hline 43 & Normal daily mean, coldest 14 days of year $\left({ }^{\circ} \mathrm{F}.\right) \ldots$ & 45 & 69 \\
\hline 44 & Normal daily mean, hottest 6 weeks of year $\left({ }^{\circ} \mathrm{F}\right.$.)... & $78.8+$ & $78.8+$ \\
\hline 45 & $\begin{array}{l}\text { Normal daily mean, year }\left({ }^{\circ} \mathrm{F} .\right) \ldots \ldots \ldots \ldots \ldots \ldots \\
\text { Precipitation: }\end{array}$ & $65-$ & $75+$ \\
\hline 46 & Normal daily mean, $f s$ (inch). & .106 & .173 \\
\hline 47 & Normal No. rainy days (over 0.10 inch), $f_{s} \ldots \ldots \ldots$ & 206 & 234 \\
\hline 48 & Normal No. dry days (0.10 inch or less), $f 8 \ldots \ldots \ldots$ & 70 & 204 \\
\hline 49 & Dry days, percentage of total, $f 8$ (per cent)....... & $20^{a}$ & 56 \\
\hline 50 & Days in longest normal rainy period, $f_{s} \ldots \ldots \ldots \ldots$ & 112 & 235 \\
\hline 51 & Days in longest normal dry period, $f_{8} \ldots \ldots \ldots \ldots$ & 17 & 182 \\
\hline 52 & $\begin{array}{l}\text { Mean total, year (inches) } \ldots \ldots \ldots \ldots \ldots \ldots \ldots \ldots \\
\text { Evaporation: }\end{array}$ & $60-$ & $60+$ \\
\hline 53 & Daily mean, $1887-88, f_{8}$ (inch) $\ldots \ldots \ldots$ & $.120^{a}$ & .141 \\
\hline 54 & $\begin{array}{l}\text { Total annual, } 1887-88 \text { (inches) } \ldots \ldots \ldots \ldots \ldots \ldots \ldots \\
\text { Moisture ratios: }\end{array}$ & 38.4 & 51.6 \\
\hline 58 & Normal $P / E, f_{8} \ldots$ & .75 & $1.20+a$ \\
\hline 59 & 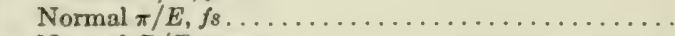 & .75 & $1.40^{a}$ \\
\hline 60 & 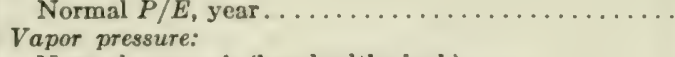 & .75 & 1.36 \\
\hline 63 & $\begin{array}{l}\text { Normal mean, } f_{s} \text { (hundredths inch)..... } \\
\text { Humidity: }\end{array}$ & 586 & 707 \\
\hline 65 & Normal mean, $f_{8}$ (per cent) . . . . . . . . . . & 77.1 & 80.5 \\
\hline 66 & $\begin{array}{l}\text { Normal mean, year (per cent) } \ldots \ldots \ldots \ldots \ldots \ldots \ldots \\
\text { Wind: }\end{array}$ & 77.1 & 80.5 \\
\hline 68 & $\begin{array}{l}\text { Normal mean hourly velocity, } f_{8} \text { (miles) ......... } \\
\text { Sunshine: }\end{array}$ & 6.7 & 9.9 \\
\hline 69 & $\begin{array}{l}\text { Normal total duration, } f s \text { (hours) } \ldots \ldots \ldots \ldots \ldots \ldots \\
\text { Moisture-temperature indices: }\end{array}$ & 2,026 & 2,297 \\
\hline 70 & Normal $P / E \times T, f_{s}$, remainder method $\ldots \ldots \ldots$ & 1,014 & 1,271 \\
\hline 71 & Normal $P / E \times T, f s$, exponential method......... & 9,385 & 11,722 \\
\hline 72 & Normal $P / E \times T, f s$, physiological method......... & 18,294 & 23,265 \\
\hline
\end{tabular}

TABle 102,-Climatic exlremes for Serenoa serullata.

\begin{tabular}{|c|c|c|c|}
\hline Plate & Temperalure: & Lote. & High. \\
\hline 34 & Days in normal frostless season $\left(f_{s}\right) \ldots \ldots \ldots \ldots \ldots$ & 231 & 365 \\
\hline 35 & 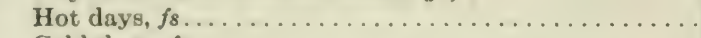 & 168 & 365 \\
\hline 36 & 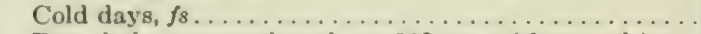 & 0 & 0 \\
\hline 37 & Remainder summation above $32^{\circ}$, year (thousands)... . & $18.0+$ & $26.0+$ \\
\hline 38 & Remainder summation above $39^{\circ}, f_{s}$ (thousands)..... & 8.4 & 14.5 \\
\hline 39 & Exponential summation, $f_{s}$ (hundreds) ............. & 9.1 & 15.4 \\
\hline 40 & Physiological summation, fs (thousands) . . . . . . . . & 16.4 & 31.1 \\
\hline 41 & Absolute minimum . . . . . . . . . . . . . . . . . & -2 & +41 \\
\hline 43 & Normal daily mean, coldest 14 days of year $\left({ }^{\circ} \mathrm{F}.\right) \ldots$ & 49 & 69 \\
\hline 44 & Normal daily mean, hottest 6 weeks of year $\left({ }^{\circ} \mathrm{F}.\right), \ldots$ & $78.5+$ & $78.8+$ \\
\hline 45 & $\begin{array}{l}\text { Normal daily mean, year }\left({ }^{\circ} \mathrm{F} .\right) \ldots \ldots \ldots \ldots \ldots \ldots \ldots \\
\text { Precipilation: }\end{array}$ & $65+$ & $75+$ \\
\hline 46 & Normal daily mean, $f_{s}$ (inch). & .106 & .173 \\
\hline 47 & Normal No. rainy days (over 0.10 inch), $f s \ldots \ldots \ldots$ & 206 & 254 \\
\hline 48 & Normal No. dry days $(0.10$ inch or less $), f_{s} \ldots \ldots \ldots \ldots$ & 26 & $20-4$ \\
\hline 49 & Dry days, percentage of total, $f_{s}$ (per cent) ........ & 8 & 56 \\
\hline 50 & Days in longest normal rainy period, $f_{s} \ldots \ldots \ldots \ldots$ & 112 & 235 \\
\hline 51 & Days in longest normal dry period, $f 8 \ldots \ldots \ldots \ldots \ldots$ & 14 & 182 \\
\hline 52 & Mean total, year (inches) . . . . . . . . . . . . . . & $50-$ & $60+$ \\
\hline
\end{tabular}


TABLE 102.-Climatic extremes for Serenog serullata-Continued.

\begin{tabular}{|c|c|c|c|}
\hline Plate & Evaporation: & Low. & High. \\
\hline 53 & Daily mean, $1887-88, f_{s}$ (inch). & .124 & .146 \\
\hline 54 & $\begin{array}{l}\text { Total annual, } 1887-88 \text { (inches).......... } \\
\text { Moisture ratios: }\end{array}$ & 42.1 & 51.6 \\
\hline 58 & Normal $P / E, f_{s} \ldots \ldots \ldots \ldots \ldots$ & .75 & 1.36 \\
\hline 59 & Normal $\pi / E, f_{3} \ldots$ & .75 & 1.52 \\
\hline 60 & $\begin{array}{l}\text { Normal } P / E \text {, year... } \\
\text { Vapor pressure: }\end{array}$ & .75 & 1.47 \\
\hline 63 & $\begin{array}{l}\text { Normal mean, fs (hundredths inch).. } \\
\text { Humidity: }\end{array}$ & 585 & 707 \\
\hline 65 & Normal mean, $f_{s}$ (per cent).. & 77.1 & 80.6 \\
\hline 66 & $\begin{array}{l}\text { Normal mean, year (per cent)... } \\
\text { Wind: }\end{array}$ & 77.1 & 80.5 \\
\hline 68 & $\begin{array}{l}\text { Normal mean hourly velocity, } f_{s} \text { (miles). } \\
\text { Sunshine: }\end{array}$ & 5.1 & 11 \\
\hline 69 & $\begin{array}{l}\text { Normal total duration, } f_{s} \text { (hours) } \ldots . \\
\text { Moisture-temperature indices: }\end{array}$ & 2,026 & 2,650 \\
\hline 70 & Normal $P / E \times T, f s$, remainder method. & 1,014 & 1,314 \\
\hline 71 & Normal $P / E \times T, f_{s}$, exponential method. & 9,385 & 12,106 \\
\hline 72 & Normal $P / E \times T, f_{s}$, physiological method & 18,294 & 26,652 \\
\hline
\end{tabular}

TABLE 103.-Climatic extremes for Washingtonia filamentosa.

\begin{tabular}{|c|c|c|c|}
\hline Plate & Temperature: & Low. & High. \\
\hline 34 & Days in normal frostless season $(f s)$. & $240-a$ & $240+a$ \\
\hline 35 & Hot days, $f s \ldots \ldots \ldots \ldots \ldots \ldots \ldots \ldots$. . . . . . . & $120-$ & $150+$ \\
\hline 36 & Cold days, $f s \ldots \ldots \ldots \ldots \ldots \ldots$ & 0 & 0 \\
\hline 37 & Remainder summation above $32^{\circ}$, year (thousands) & 18.0 & 26.0 \\
\hline 38 & Remainder summation above $39^{\circ}, f_{s}$ (thousands). & $8.0-a$ & $10.0+a$ \\
\hline 39 & Exponential summation, $f_{s}$ (hundreds) ......... & $8.0-a$ & $10.0+{ }^{a}$ \\
\hline 40 & Physiological summation, $f s$ (thousands). & $12.5+^{a}$ & $15.0+a$ \\
\hline 41 & Absolute minimum........... & $-4+a$ & $+14+a$ \\
\hline 43 & Normal daily mean, coldest 14 days of year $\left({ }^{\circ} \mathrm{F}.\right)$. & $50+a$ & $50+a$ \\
\hline 44 & Normal daily mean, hottest 6 weeks of year $\left({ }^{\circ} \mathrm{F}\right.$.). & $71.6-$ & $78.8+$ \\
\hline 45 & $\begin{array}{l}\left.\text { Normal daily mean, year }{ }^{\circ} \mathrm{F} .\right) \ldots \ldots \ldots \ldots \ldots \\
\text { Precipitation: }\end{array}$ & $70-$ & $70+$ \\
\hline 46 & Normal daily mean, $f_{s}$ (inch). & $.020-a$ & $.020+a$ \\
\hline 47 & Normal No. rainy days (over $0.10 \mathrm{inch}$ ), $f_{s}$. & & \\
\hline 48 & Normal No. dry days ( 0.10 inch or less), $f_{s}$. & $250+a$ & $275+a$ \\
\hline 49 & Dry days, percentage of total, $f_{s}$ (per cent). & $90+a$ & 100 \\
\hline 50 & Days in longest normal rainy period, $f_{s} .$. & 0 & 0 \\
\hline 51 & Days in longest normal dry period, $f_{s} \ldots \ldots \ldots$ & $250+a$ & $250+a$ \\
\hline 52 & Mean total, year (inches) ............. & $10-$ & $10+$ \\
\hline & Evaporation: & $180-a$ & $180+a$ \\
\hline $\begin{array}{l}53 \\
54\end{array}$ & $\begin{array}{l}\text { Daily mean, } 1887-88, f_{s} \text { (inch)... } \\
\text { Total annual, 1887-88 (inches).. }\end{array}$ & $80-a$ & $90+a$ \\
\hline & Moisture ratios: & & \\
\hline 58 & Normal $P / E, f_{s}$. & $.20-a$ & $.20+{ }^{a}$ \\
\hline 59 & Normal $\pi / E, f_{s}$. & $.20-a$ & $.20+a$ \\
\hline 60 & $\begin{array}{l}\text { Normal } P / E \text {, year. } \\
\text { Vapor pressure: }\end{array}$ & $.10-a$ & $.10+a$ \\
\hline 63 & $\begin{array}{l}\text { Normal mean, } f_{s} \text { (hundredths inch)... } \\
\text { Humidity: }\end{array}$ & $300+a$ & $300+a$ \\
\hline 65 & Normal mean, $f_{s}$ (per cent). & $40^{\alpha}$ & $50^{a}$ \\
\hline 66 & $\begin{array}{l}\text { Normal mean, year (per cent).. } \\
\text { Wind: }\end{array}$ & $40++^{a}$ & $40+a$ \\
\hline 68 & $\begin{array}{l}\text { Normal mean hourly velocity, } f_{s} \text { (miles)... } \\
\text { Sunshine: }\end{array}$ & $6^{a}$ & \\
\hline 69 & $\begin{array}{l}\text { Normal total duration, } f_{s} \text { (hours) .. } \\
\text { Moisture-temperature indices: }\end{array}$ & $2,500^{a}$ & $2,700^{a}$ \\
\hline 70 & Normal $P / E \times T, f_{s}$, remainder method. & $100^{\alpha}$ & $100+a$ \\
\hline 71 & Normal $P / E \times T, f_{s}$, exponential method & $1,000^{a}$ & $1,000+a$ \\
\hline 72 & Normal $P / E \times T, f s$, physiological method. & $1,000^{a}$ & $1,000+a$ \\
\hline
\end{tabular}


TABLE 104.-Climatic extremes for Cephalanthus occidentalis.

\begin{tabular}{|c|c|c|c|}
\hline Plate & Temperalure: & Low. & High. \\
\hline 34 & Days in normal frostless season $(f s)$. & 111 & \\
\hline 35 & Hot days, $f_{3} \ldots \ldots \ldots \ldots \ldots \ldots \ldots$. & 0 & 365 \\
\hline 36 & Cold days, $f_{s} \ldots \ldots \ldots \ldots \ldots \ldots \ldots$ & 0 & 137 \\
\hline 37 & Remainder summation above $32^{\circ}$, year (thousands). & $10.0-$ & $26.0+$ \\
\hline 38 & Remainder summation above $39^{\circ}$, fs (thousands).. & 2.6 & 14.5 \\
\hline 39 & Exponential summation, $f s$ (hundreds) ..... & 3.0 & 15.4 \\
\hline 40 & Physiological summation, $f_{s}$ (thousands) & 2.1 & 31.1 \\
\hline 41 & 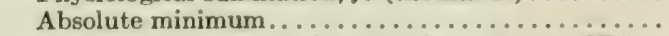 & -43 & +41 \\
\hline 43 & Normal daily mean, coldest 14 days of year $\left({ }^{\circ} \mathrm{F}\right.$. $)$ & 11 & 69 \\
\hline 44 & Normal daily mean, hottest 6 weeks of year $\left({ }^{\circ} \mathrm{F}\right.$. $)$ & $64.4-$ & $78.8+$ \\
\hline 45 & Normal daily mean, year $\left({ }^{\circ} \mathrm{F}.\right) \ldots$ & $40-$ & $75+$ \\
\hline & Precipilation: & & \\
\hline 46 & 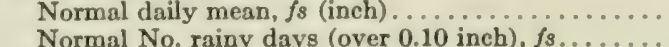 & $0^{.017}$ & $284^{.172}$ \\
\hline 48 & $\begin{array}{l}\text { Normal No. rainy days (over } 0.10 \text { inch), } f_{s} \text {. } \\
\text { Normal No. dry days }\left(0.10 \text { inch or less), } f_{8} \text {. }\right.\end{array}$ & $\begin{array}{l}0 \\
0\end{array}$ & 283 \\
\hline 49 & Dry days, percentage of total, $f_{8}$ (per cent). & 0 & 100 \\
\hline 50 & Days in longest normal rainy period, $f_{s} \ldots$ & 0 & 256 \\
\hline 51 & Days in longest normal dry period, $f_{8} \ldots \ldots$ & 4 & 283 \\
\hline 52 & Mean total, year (inches)............ & $10-$ & $60+$ \\
\hline & Evaporation: & & \\
\hline 53 & Daily mean, $1887-88, f_{8}$ (inch). & .081 & $.240+6$ \\
\hline 54 & $\begin{array}{l}\text { Total annual, } 1887-88 \text { (inches)..... } \\
\text { Moisture ratios: }\end{array}$ & 24.3 & 101.2 \\
\hline 58 & Normal $P / E, f_{s} \ldots$ & .08 & 1.76 \\
\hline 59 & Normal $\pi / E, f_{8} .$. & .10 & 1.96 \\
\hline 60 & Normal $P / E$, year........ & .03 & 1.94 \\
\hline 63 & $\begin{array}{l}\text { Normal mean, } f_{s} \text { (hundredths inch). } \\
\text { Humidity: }\end{array}$ & 279 & 707 \\
\hline 65 & Normal mean, $f_{8}$ (per cent).... & 48.9 & 82.7 \\
\hline 66 & $\begin{array}{l}\text { Normal mean, year (per cent)................... } \\
\text { Wind: }\end{array}$ & 57.2 & 85.2 \\
\hline 68 & $\begin{array}{l}\text { Normal mean hourly velocity, fs (miles)..... } \\
\text { Sunshiné: }\end{array}$ & 3.1 & 13.5 \\
\hline 69 & $\begin{array}{l}\text { Normal total duration, } f_{s} \text { (hours).... } \\
\text { Moisture-temperature indices: }\end{array}$ & 1,225 & 2,650 \\
\hline 70 & Normal $P / E \times T, f_{s}$, remainder method. & 68 & 1,418 \\
\hline 71 & Normal $P / E \times T, f 8$, exponential method.. & 625 & 13,511 \\
\hline 72 & Normal $P / E \times T, f_{s}$, physiological method. & 1,186 & 24,265 \\
\hline
\end{tabular}

TABLE 105.-Climatic extremes for Adelia acuminala.

\begin{tabular}{|c|c|c|c|}
\hline Plate & Temperature: & Lotr. & High. \\
\hline 34 & Days in normal frostless season $(f s)$ & 180 & \\
\hline & Hot days, $f s \ldots \ldots \ldots \ldots \ldots \ldots$ & 129 & 226 \\
\hline 36 & Cold days, fs............. & 0 & 22 \\
\hline 37 & Remainder summation above $32^{\circ}$, year (thousands).. & $18.0-$ & $26.0+$ \\
\hline 38 & Remainder summation above $39^{\circ}, f_{s}$ (thousands).... & 6.3 & 10.8 \\
\hline 39 & Exponential summation, $f_{s}$ (hundreds) ........ & 6.7 & 11.7 \\
\hline 40 & Physiological summation, $f_{8}$ (thousands). & 11.9 & 21.2 \\
\hline 41 & Absolute minimum.... & -29 & +20 \\
\hline 43 & Normal daily mean, coldest 14 days of year $\left({ }^{\circ} \mathrm{F}.\right)$. & 32 & 57 \\
\hline 44 & Normal daily mean, hottest 6 weeks of year $\left({ }^{\circ} \mathrm{F}\right.$.). & $78.8-$ & $78.8+$ \\
\hline 45 & Normal daily mean, year $\left({ }^{\circ} \mathrm{F}\right.$.)... & 55 & $70+$ \\
\hline 46 & $\begin{array}{l}\text { Precipitation: } \\
\text { Normal drily mean, } f_{s} \text { (inch). }\end{array}$ & .109 & .172 \\
\hline 47 & Normal No. rainy days (over $0.10 \mathrm{inch}$ ), fs. & 120 & $2 \$ 4$ \\
\hline 48 & Normal No. dry days (0.10 inch or less), $f_{s}$. & 26 & $200^{a}$ \\
\hline 49 & Dry days, pereentage of total, $f_{s}$ (per cent).. & 8 & 54 \\
\hline 50 & Days in longest normal rainy period, $f s \ldots \ldots$. & 72 & 157 \\
\hline 51 & Days in longest normal dry period, $f s \ldots \ldots \ldots \ldots$ & 14 & 7s \\
\hline 52 & Mean total, year (inches)..... & $50-$ & $60+$ \\
\hline
\end{tabular}


TABLE 105.-Climatic extremes for Adelia acuminata-Continued.

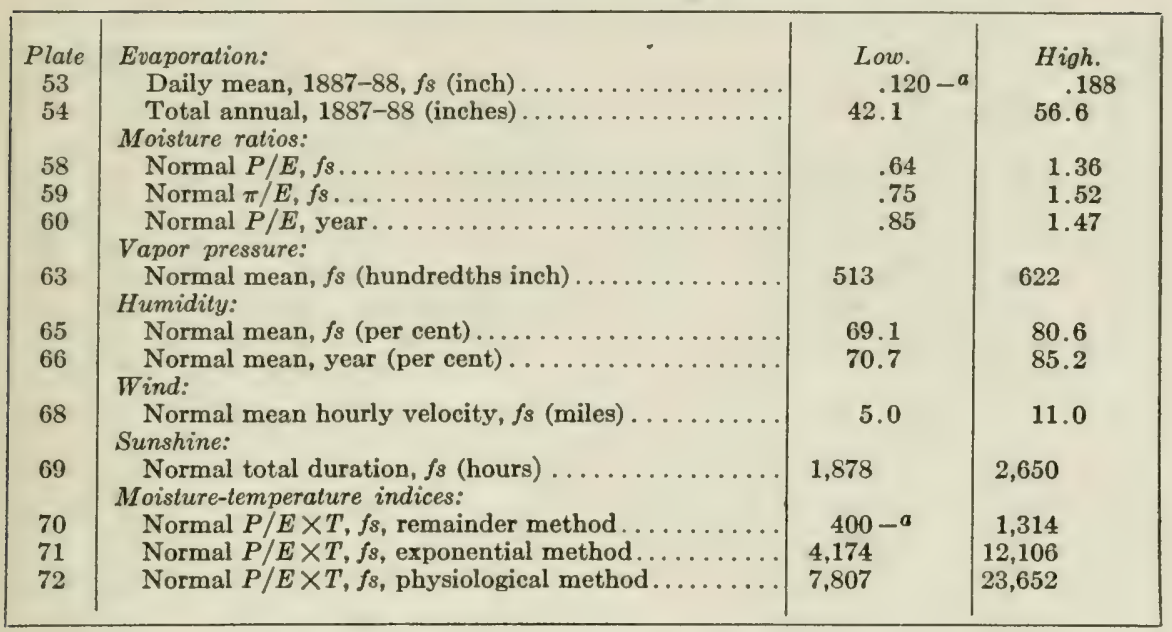

TABLE 106.-Climatic extremes for Decodon verticillatus.

\begin{tabular}{|c|c|c|c|}
\hline Plate & Temperature: & Low. & High. \\
\hline 34 & Days in normal frostless season $(f s)$. . & 111 & \\
\hline 35 & Hot days, $f_{s} \ldots \ldots \ldots \ldots \ldots \ldots \ldots$ & 0 & $240+a$ \\
\hline 36 & Cold days, $f_{8}, \ldots \ldots \ldots \ldots \ldots$ & 0 & 149 \\
\hline 37 & Remainder summation above $32^{\circ}$, year (thousands) & $10.0-$ & $26.0+$ \\
\hline 38 & Remainder summation above $39^{\circ}$, fs (thousands).. & 2.6 & 10.8 \\
\hline 39 & Exponential summation, $f_{s}$ (hundreds) .......... & 3.0 & 11.1 \\
\hline 40 & Physiological summation, fs (thousands). & 2.1 & 21.4 \\
\hline 41 & Absolute minimum............. & -43 & +22 \\
\hline 43 & Normal daily mean, coldest 14 days of year $\left({ }^{\circ} \mathrm{F}.\right)$ & 12 & 57 \\
\hline 44 & Normal daily mean, hottest 6 weeks of year $\left({ }^{\circ} \mathrm{F}\right.$.) & $64.4-$ & $78.8+$ \\
\hline 45 & $\begin{array}{l}\text { Normal daily mean, year }\left({ }^{\circ} \mathrm{F} .\right) \ldots \ldots \ldots \ldots \ldots \\
\text { Precipitation }\end{array}$ & & $70+$ \\
\hline 46 & $\begin{array}{l}\text { Prectpitation: } \\
\text { Normal daily mean, } f_{s} \text { (inch). }\end{array}$ & 089 & 172 \\
\hline 47 & Normal No. rainy days (over $0.10 \mathrm{inch}$ ), $f_{s}$. & $55^{.003}$ & 284 \\
\hline 48 & Normal No. dry days ( 0.10 inch or less), $f_{8}$. & 0 & 170 \\
\hline 49 & Dry days, percentage of total, $f_{s}$ (per cent). & 0 & 83 \\
\hline 50 & Days in longest normal rainy period, $f_{s} \ldots$. & 17 & 256 \\
\hline 51 & Days in longest normal dry period, $f_{s} \ldots \ldots$ & 4 & 78 \\
\hline 52 & Mean total, year (inches)............ & $30-$ & $60+$ \\
\hline & Evaporation: & & \\
\hline 53 & Daily mean, $1887-88, f_{8}$ (inch). & .081 & .200 \\
\hline 54 & $\begin{array}{l}\text { Total annual, } 1887-88 \text { (inches)........... } \\
\text { Moisture ratios: }\end{array}$ & 24.3 & 56.6 \\
\hline 58 & Normal $P / E, f_{s} \ldots \ldots \ldots \ldots \ldots$ & .51 & 1.76 \\
\hline 59 & Normal $\pi / E, f_{s}$... & .60 & 1.96 \\
\hline 60 & Normal $P / E$, year. & .71 & 1.94 \\
\hline & Vapor pressure: & & \\
\hline 63 & $\begin{array}{l}\text { Normal mean, } f_{\beta} \text { (hundredths inch). } \\
\text { Humidity: }\end{array}$ & 345 & 612 \\
\hline 65 & Normal mean, $f_{s}$ (per cent) & 64.5 & 82.7 \\
\hline 66 & $\begin{array}{l}\text { Normal mean, year (per cent)............... } \\
\text { Wind: }\end{array}$ & 67.5 & 82.9 \\
\hline 68 & $\begin{array}{l}\text { Normal mean hourly velocity, } f_{s} \text { (miles). } \\
\text { Sunshine: }\end{array}$ & 3.1 & 13.5 \\
\hline 69 & Normal total duration, $f_{s}$ (hours) .... & 1,225 & 2,301 \\
\hline 70 & $\begin{array}{l}\text { Moisture-temperature indices: } \\
\text { Normal } P / E \times T, f_{3} \text {, remainder method. }\end{array}$ & 301 & 1,418 \\
\hline 71 & Normal $P / E \times T, f_{s}$, exponential method. . & 2,914 & 13,511 \\
\hline 72 & Normal $P / E \times T, f s$, physiological method. & 2,747 & 24,265 \\
\hline
\end{tabular}


TABLE 107.-Climatic extremes for Itea virginica.

\begin{tabular}{|c|c|c|c|}
\hline Plate & Temperature: & Low. & High. \\
\hline 34 & Days in normal frostiess season $\left(f_{8}\right)$ & 158 & 365 \\
\hline 35 & Hot days, $f_{3} \ldots \ldots \ldots \ldots \ldots \ldots$ & 91 & 365 \\
\hline 36 & Cold days, fs.. & 0 & 42 \\
\hline 37 & Remainder summation above $32^{\circ}$, year (thousands). & $11.5-$ & $26.0+$ \\
\hline 38 & Remainder summation above $39^{\circ}, f_{8}$ (thousands).. & 5.1 & 14.5 \\
\hline 39 & Exponential summation, $f_{8}$ (hundreds)........... & 5.3 & 15.4 \\
\hline 40 & Physiological summation, $f_{s}$ (thousands). & 7.9 & 31.1 \\
\hline 41 & Absolute minimum................ & -23 & +41 \\
\hline 43 & Normal daily mean, coldest 14 days of year $\left({ }^{\circ} \mathrm{F}.\right)$. & 26 & 69 \\
\hline 44 & Normal daily mean, hottest 6 weeks of year $\left({ }^{\circ} \mathrm{F}\right.$.). & $71.6-$ & $78.8+$ \\
\hline 45 & Normal daily mean, year $\left({ }^{\circ} \mathrm{F}.\right) \ldots \ldots \ldots \ldots \ldots$ & $50-$ & \\
\hline 46 & $\begin{array}{l}\text { Precipiation: } \\
\text { Normal daily mean, } f_{8} \text { (inch). }\end{array}$ & 110 & .17 \\
\hline 47 & Normal No. rainy days (over $0.10 \mathrm{inch}$ ), $f_{8}$. & & 284 \\
\hline 48 & Normal No. dry days (0.10 inch or less), $f s$. & 26 & 204 \\
\hline 49 & Dry days, percentage of total, $f_{s}$ (per cent). & 0 & 48 \\
\hline 50 & Days in longest normal rainy period, $f_{s} \ldots$. & 48 & 256 \\
\hline 51 & Days in longest normal dry period, $f_{s} \ldots$ & 5 & 78 \\
\hline 52 & Mean total, year (inches) . . . . . . . . . & $40-$ & $60+$ \\
\hline & Evaporation: & & \\
\hline 53 & Daily mean, $1887-88, f_{8}$ (inch). & .084 & .195 \\
\hline 54 & Total annual, 1887-88 (inches) .... & 25.2 & 56.6 \\
\hline 58 & Normal $P / E, f_{8} \ldots$ & .58 & 1.76 \\
\hline 59 & Normal $\pi / E, f_{s} \ldots$ & .66 & 1.96 \\
\hline 60 & Normal $P / E$, year. & .71 & 1.94 \\
\hline 63 & $\begin{array}{l}\text { Vapor pressure: } \\
\text { Normal mean, } f_{s} \text { (hundredths inch). } \\
\text { Humidity: }\end{array}$ & $450^{3}$ & 707 \\
\hline 65 & Normal mean, $f_{s}$ (per cent). & 67.3 & 82.7 \\
\hline 66 & $\begin{array}{l}\text { Normal mean, year (per cent)....... } \\
\text { Wind: }\end{array}$ & 69.5 & 80.9 \\
\hline 68 & $\begin{array}{l}\text { Normal mean hourly velocity, } f s \text { (miles)... } \\
\text { Sunshine: }\end{array}$ & 3.6 & 13.5 \\
\hline 69 & $\begin{array}{l}\text { Normal total duration, } f_{s} \text { (hours). } \\
\text { Moisture-temperature indices: }\end{array}$ & $1,700^{a}$ & 2,301 \\
\hline 70 & Normal $P / E \times T, f_{8}$, remainder method. & 390 & 1,418 \\
\hline 71 & Normal $P / E \times T, f_{s}$, exponential method. & 3,635 & 13,511 \\
\hline 72 & Normal $P / E \times T, f_{s}$, physiological method. & 6,824 & 24,265 \\
\hline
\end{tabular}

Table 108.-Climatic extremes for Artemisia tridentata.

\begin{tabular}{|c|c|c|c|}
\hline Plate & T'emperature: & Low. & High. \\
\hline 34 & Days in normal frostless season $\left(f_{s}\right) \ldots$ & $39-$ & 334 \\
\hline 35 & Hot days, $f_{s} \ldots \ldots \ldots \ldots \ldots \ldots \ldots$ & 0 & $11 \mathrm{~s}$ \\
\hline 36 & Cold days, $f_{3}$.. & 0 & 134 \\
\hline 37 & Remainder summation above $32^{\circ}$, year (thousands) & $10.0-$ & $15.0+$ \\
\hline 38 & Remainder summation above $39^{\circ}$, fs (thousands).. & 2.8 & 7.3 \\
\hline 39 & Exponential summation, $f_{s}$ (hundreds).......... & 3.0 & 7.6 \\
\hline 40 & Physiological summation, $f s$ (thousands). & 2.8 & 8.4 \\
\hline 41 & Absolute minimum.... & -59 & +32 \\
\hline 43 & Normal daily mean, coldest 14 days of year $\left({ }^{\circ} \mathrm{F}_{\text {. }}\right.$ ). & 17 & 54 \\
\hline 44 & Normal daily mean, hottest 6 weeks of year $\left({ }^{\circ} \mathrm{F}\right.$.). & $64.4-$ & $7 s .5+$ \\
\hline 45 & Normal daily mean, year $\left({ }^{\circ} \mathrm{F},{ }^{\circ}, \ldots \ldots \ldots \ldots \ldots . .\right.$. & $40-$ & $70+$ \\
\hline & Precipitation: & & \\
\hline 46 & Normal daily mean, $f_{3}$ (inch). & .009 & $.07 \mathrm{~s}$ \\
\hline 47 & Normal No. rainy days (over $0.10 \mathrm{inch}$ ), $f s$. & 0 & 40 \\
\hline 48 & Normal No. dry days (0.10 inch or less), fs.. & 104 & 294 \\
\hline 49 & Dry days, percentage of total, $f_{s}$ (per cent).. & ss & 100 \\
\hline 50 & Days in longest normal rainy period, $f_{s} . .$. & 0 & 23 \\
\hline 51 & Days in longest normal dry period, $f_{3} \ldots \ldots$ & 101 & 2999 \\
\hline 52 & Mean total, year (inches)................. & $10-$ & $30+$ \\
\hline
\end{tabular}


TABLE 108.-Climatic extremes for Artemisia tridentata-Continued.

\begin{tabular}{|c|c|c|c|}
\hline Plate & Evaporation: & Low. & High. \\
\hline $\begin{array}{l}53 \\
54\end{array}$ & $\begin{array}{l}\text { Daily mean, } 1887-88, f_{s} \text { (inch)..... } \\
\text { Total annual, } 1887-88 \text { (inches).... }\end{array}$ & 35.8 & 100.6 \\
\hline & Moisture ratios: & & \\
\hline 58 & Normal $P / E, f_{s}$. & .04 & .37 \\
\hline 59 & Normal $\pi / E, f s$. & .06 & .62 \\
\hline 60 & $\begin{array}{l}\text { Normal } P / E \text {, year............ } \\
\text { Vapor pressure: }\end{array}$ & .03 & .24 \\
\hline 63 & $\begin{array}{l}\text { Normal mean, } f s \text { (hundredths inch)..... } \\
\text { Humidity: }\end{array}$ & 183 & 378 \\
\hline 65 & Normal mean, $f s$ (per cent). & 22.6 & 72.2 \\
\hline 66 & $\begin{array}{l}\text { Normal mean, year (per cent)... } \\
\text { Wind: }\end{array}$ & 29.7 & 71.7 \\
\hline 68 & $\begin{array}{l}\text { Normal mean hourly velocity, } f_{s} \text { (miles).. } \\
\text { Sunshine: }\end{array}$ & 4.3 & 11.4 \\
\hline 69 & $\begin{array}{l}\text { Normal total duration, } f_{s} \text { (hours) } \ldots \ldots \ldots \ldots \\
\text { Moisture-temperature indices: }\end{array}$ & 1,127 & 2,995 \\
\hline 70 & Normal $P / E \times T, f s$, remainder method & 13 & 283 \\
\hline 71 & Normal $P / E \times T, f s$, exponential method. & 127 & 2,721 \\
\hline 72 & Normal $P / E \times T, f s$, physiological method. & 197 & 3,127 \\
\hline
\end{tabular}

TABLE 109.-Climatic extremes for Covillea tridentata.

\begin{tabular}{|c|c|c|c|}
\hline Plate & Temperature: & Low. & High. \\
\hline 34 & Days in normal frostless season $\left(f_{s}\right)$. & 172 & 283 \\
\hline 35 & Hot days, $f s \ldots \ldots \ldots \ldots \ldots \ldots$ & & $211+$ \\
\hline 36 & Cold days, fs................ & 0 & 0 \\
\hline 37 & Remainder summation above $32^{\circ}$, year (thousands) & $18.0-$ & $26.0+$ \\
\hline 38 & Remainder summation above $39^{\circ}, f_{s}$ (thousands).. & 6.5 & 10.1 \\
\hline 39 & Exponential summation, $f_{s}$ (hundreds)... & 6.8 & 11.8 \\
\hline 40 & Physiological summation, $f_{s}$ (thousands). & 12.4 & 20.6 \\
\hline 41 & Absolute minimum................ & -29 & +23 \\
\hline 43 & Normal daily mean, coldest 14 days of year $\left({ }^{\circ} \mathrm{F}.\right)$. & $30-$ & 54 \\
\hline 44 & Normal daily mean, hottest 6 weeks of year $\left({ }^{\circ} \mathrm{F}.\right)$. & $71.6-$ & $78.8+$ \\
\hline 45 & Normal daily mean, year $\left({ }^{\circ} \mathbf{F}.\right) \ldots \ldots \ldots$ & & $70+$ \\
\hline 46 & $\begin{array}{l}\text { Precipitation: } \\
\text { Normal daily mean, } f_{s} \text { (inch). }\end{array}$ & .014 & .072 \\
\hline 47 & Normal No rainy days (over $0.10 \mathrm{inch}$ ), fs. & & $25+a$ \\
\hline 48 & Normal No. dry days ( 0.10 inch or less), $f s$. & $150-a$ & 283 \\
\hline 49 & Dry days, percentage of total, $f_{s}$ (per cent). & $91-a$ & 100 \\
\hline 50 & Days in longest normal rainy period, $f_{s} . \ldots$. & 0 & 2 \\
\hline 51 & Days in longest normal dry period, $f_{3} \ldots$ & $100-a$ & 283 \\
\hline 52 & Mean total, year (inches)... & $10-$ & $20+$ \\
\hline 53 & $\begin{array}{l}\text { Evaporation: } \\
\text { Daily mean, } 1887-88, f_{s} \text { (inch). }\end{array}$ & $160^{a}$ & .349 \\
\hline 54 & $\begin{array}{l}\text { Total annual, } 1887-88 \text { (inches)......... } \\
\text { Moisture ratios: }\end{array}$ & $60-$ & 101.2 \\
\hline 58 & Normal $P / E, f s$. & .04 & $.20+{ }^{a}$ \\
\hline 59 & Normal $\pi / E, f_{s}$. & .06 & $.20+a$ \\
\hline 60 & $\begin{array}{l}\text { Normal } P / E \text {, year..... } \\
\text { Vapor pressure: }\end{array}$ & .03 & .24 \\
\hline 63 & $\begin{array}{l}\text { Normal mean, } f_{s} \text { (hundredths inch)..... } \\
\text { Humidity: }\end{array}$ & $300-a$ & $500^{a}$ \\
\hline 65 & Normal mean, fs (per cent) & 22.6 & $60^{a}$ \\
\hline 66 & $\begin{array}{l}\text { Normal mean, year (per cent)......... } \\
\text { Wind: }\end{array}$ & 29.7 & $60^{a}$ \\
\hline 68 & $\begin{array}{l}\text { Normal mean hourly velocity, } f_{s} \text { (miles)... } \\
\text { Sunshine: }\end{array}$ & 4.5 & 10.2 \\
\hline 69 & $\begin{array}{l}\text { Normal total duration, } f s \text { (hours) } \ldots \ldots \ldots \ldots \\
\text { Moisture-temperature indices: }\end{array}$ & 1,900 & 2,300 \\
\hline 70 & Normal $P / E \times T, f_{s}$, remainder method. . & 27 & $400^{a}$ \\
\hline 71 & Normal $P / E \times T, f s$, exponential method... & 254 & $4,000^{a}$ \\
\hline 72 & Normal $P / E \times T, f s$, physiological method.. & 449 & $8,000^{a}$ \\
\hline
\end{tabular}


TABLE 110.-Climatic extremes for Opuntia polyacantha.

\begin{tabular}{|c|c|c|c|}
\hline Plate & Temperature: & Lou: & High. \\
\hline 34 & Days in normal frostless season $\left(f_{8}\right)$. & 53 & 236 \\
\hline 35 & Hot days, $f_{8} \ldots \ldots \ldots \ldots \ldots \ldots \ldots . . . . .$. & & 149 \\
\hline 36 & Cold days, $f_{s} \ldots . . .$. & 0 & 152 \\
\hline 37 & Remainder summation above $32^{\circ}$, year (thousands). & $10.0-$ & $18.0+$ \\
\hline 38 & Remainder summation above $39^{\circ}, f_{8}$ (thousands)... & 3.0 & 7.3 \\
\hline 39 & Exponential summation, $f_{s}$ (hundreds)... & 3.1 & 7.9 \\
\hline 40 & Physiological summation, $f 8$ (thousands). & 4.0 & 14.2 \\
\hline 41 & Absolute minimum............... & -65 & +4 \\
\hline 43 & Normal daily mean, coldest 14 days of year $\left({ }^{\circ} \mathrm{F}\right.$.). & 0 & \\
\hline 44 & Normal daily mean, hottest 6 weeks of year $\left({ }^{\circ} \mathrm{F}_{0}\right)$. & $64.4-$ & $78.8+$ \\
\hline 45 & $\begin{array}{l}\text { Normal daily mean, year }\left({ }^{\circ} \mathrm{F} .\right) \ldots \ldots . \\
\text { Precipitation: }\end{array}$ & & $60+$ \\
\hline 46 & $\begin{array}{l}\text { Precipitation: } \\
\quad \text { Normal daily mean, } f_{8} \text { (inch). }\end{array}$ & .025 & .199 \\
\hline 47 & Normal No. rainy days (over $0.10 \mathrm{inch}$ ), $f 8$. & 0 & 199 \\
\hline 48 & Normal No. dry days ( 0.10 inch or less), $f_{8}$. & 26 & 216 \\
\hline 49 & Dry days, percentage of total, $f_{s}$ (per cent). & 14 & 100 \\
\hline 50 & Days in longest normal rainy period, $f_{8} \ldots \ldots$ & 0 & 172 \\
\hline 51 & Days in longest normal dry period, $f_{8} .$. & 15 & 153 \\
\hline 52 & $\begin{array}{c}\text { Mean total, year (inches) } \ldots \ldots \ldots \ldots \ldots \ldots \ldots \ldots \\
\text { Eraporation: }\end{array}$ & $10-$ & $40+$ \\
\hline 53 & $\begin{array}{l}\text { Evaporation: } \\
\text { Daily mean, } 1887-88, f s \text { (inch).. }\end{array}$ & .052 & \\
\hline 54 & $\begin{array}{l}\text { Total annual, } 1887-88 \text { (inches)....... } \\
\text { Moisture ratios: }\end{array}$ & 27.2 & 76.0 \\
\hline 58 & Normal $P / E, f s . . .$. & .10 & 3.84 \\
\hline 59 & Normal $\pi / E, f_{3} \ldots .$. & .12 & 4.48 \\
\hline 60 & Normal $P / E$, year. & .21 & 4.90 \\
\hline 63 & $\begin{array}{l}\text { Vapor pressure: } \\
\text { Normal mean, } f s \text { (hundredths inch).. } \\
\text { Humidity: }\end{array}$ & 233 & 522 \\
\hline 65 & Normal mean, $f_{s}$ (per cent)..... & 41.5 & 73.6 \\
\hline 66 & $\begin{array}{l}\text { Normal mean, year (per cent)............. } \\
\text { Wind: }\end{array}$ & 45.5 & 76.2 \\
\hline 68 & $\begin{array}{l}\text { Normal mean hourly velocity, } f_{s} \text { (miles). } \\
\text { Sunshine: }\end{array}$ & 5.6 & 14.2 \\
\hline 69 & $\begin{array}{l}\text { Normal total duration, } f s \text { (hours) . } \\
\text { Moisture-temperature indices: }\end{array}$ & 1,127 & $2,100^{\alpha}$ \\
\hline 70 & Normal $P / E \times T, f_{s}$, remainder method. . & 58 & 1,566 \\
\hline 71 & Normal $P / E \times T$. $f s$, exponential method.... & 563 & 11,724 \\
\hline 72 & Normal $P / E \times T, f s$, physiological method..... & 710 & 10,782 \\
\hline
\end{tabular}

TABLE 111.-Climatic extremes for Carnegiea gigantea.

\begin{tabular}{|c|c|c|c|}
\hline Plate & Temperalure: & Low. & High. \\
\hline 34 & Days in normal frostless season $(f s)$. & 262 & \\
\hline 35 & Hot days, $f_{s} \ldots \ldots \ldots \ldots \ldots \ldots \ldots$ & 186 & 211 \\
\hline 36 & Cold days, fs................ & 0 & 0 \\
\hline 37 & Remainder summation above $32^{\circ}$, year (thousands). & $11.5+$ & $26.0+$ \\
\hline 38 & Remainder summation above $39^{\circ}, f_{s}$ (thousands)... & $6.0 \pm$ & 10.1 \\
\hline 39 & Exponential summation, $f_{s}$ (hundreds)........... & $6.0 \pm$ & 11.8 \\
\hline 40 & Physiological summation, $f s$ (thousands). . & $12.5 \pm$ & 20.6 \\
\hline 41 & Absolute minimum. . & +10 & +22 \\
\hline 43 & Normal daily mean, coldest 14 days of year $\left({ }^{\circ} \mathrm{F}_{*}\right)$. & 45 & $50+$ \\
\hline 44 & Normal daily mean, hottest 6 weeks of year $\left({ }^{\circ} \mathrm{F}.\right)$. & 71.6 & $78.8+$ \\
\hline 45 & $\begin{array}{l}\text { Normal daily mean, year }\left({ }^{\circ} \mathrm{F} .\right) \ldots \ldots \ldots \ldots \ldots \ldots \\
\text { Precimitation: }\end{array}$ & $65-$ & $70+$ \\
\hline 46 & $\begin{array}{l}\text { Precipitation: } \\
\text { Normal daily mean, fs (inch). }\end{array}$ & $020-a$ & $040 t^{\circ}$ \\
\hline 47 & Normal No. rainy days (over 0.10 inch), fs... & 0 & 0 \\
\hline 48 & Normal No. dry days $(0.10$ inch or less), fs... & $225^{a}$ & 283 \\
\hline 49 & Dry days, percentage of total, $f s$ (per cent). & 100 & ... \\
\hline 50 & Days in longest normal rainy period, $f_{s} \ldots \ldots$ & 0 & \\
\hline 51 & Days in longest normal dry period, $f_{s} .$. & $175^{a}$ & 283 \\
\hline 52 & Mean total, year (inches)............ & $10-$ & $10+$ \\
\hline
\end{tabular}


TABLE 111.-Climatic extremes for Carnegiea gigantea-Continued.

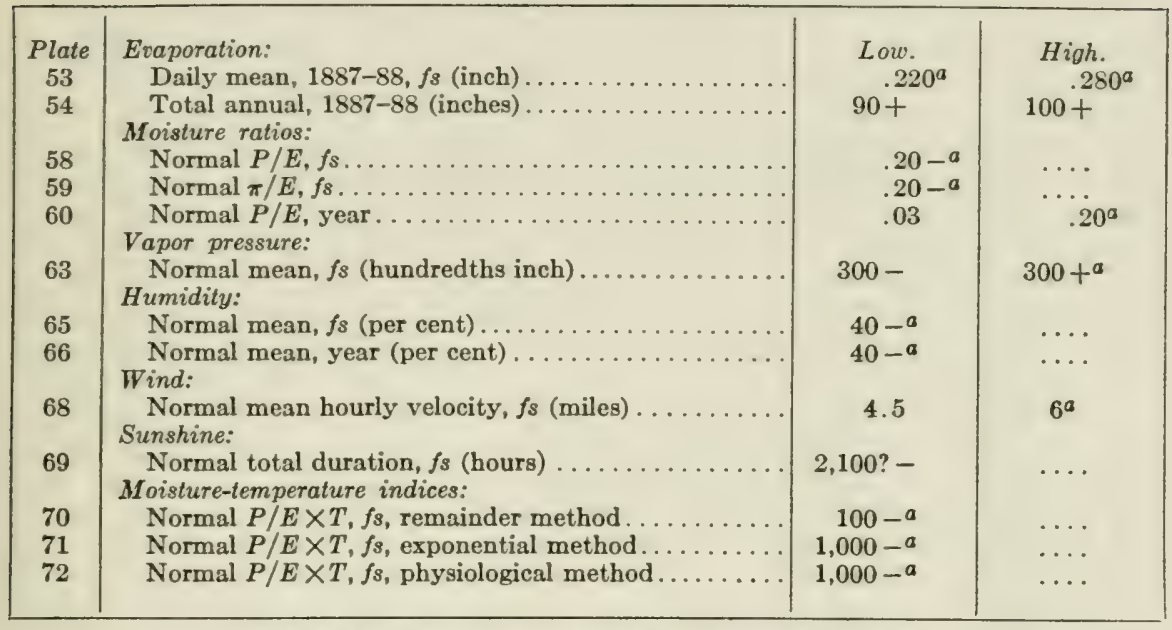

TABLE 112.-Climatic extremes for Silphium laciniatum.

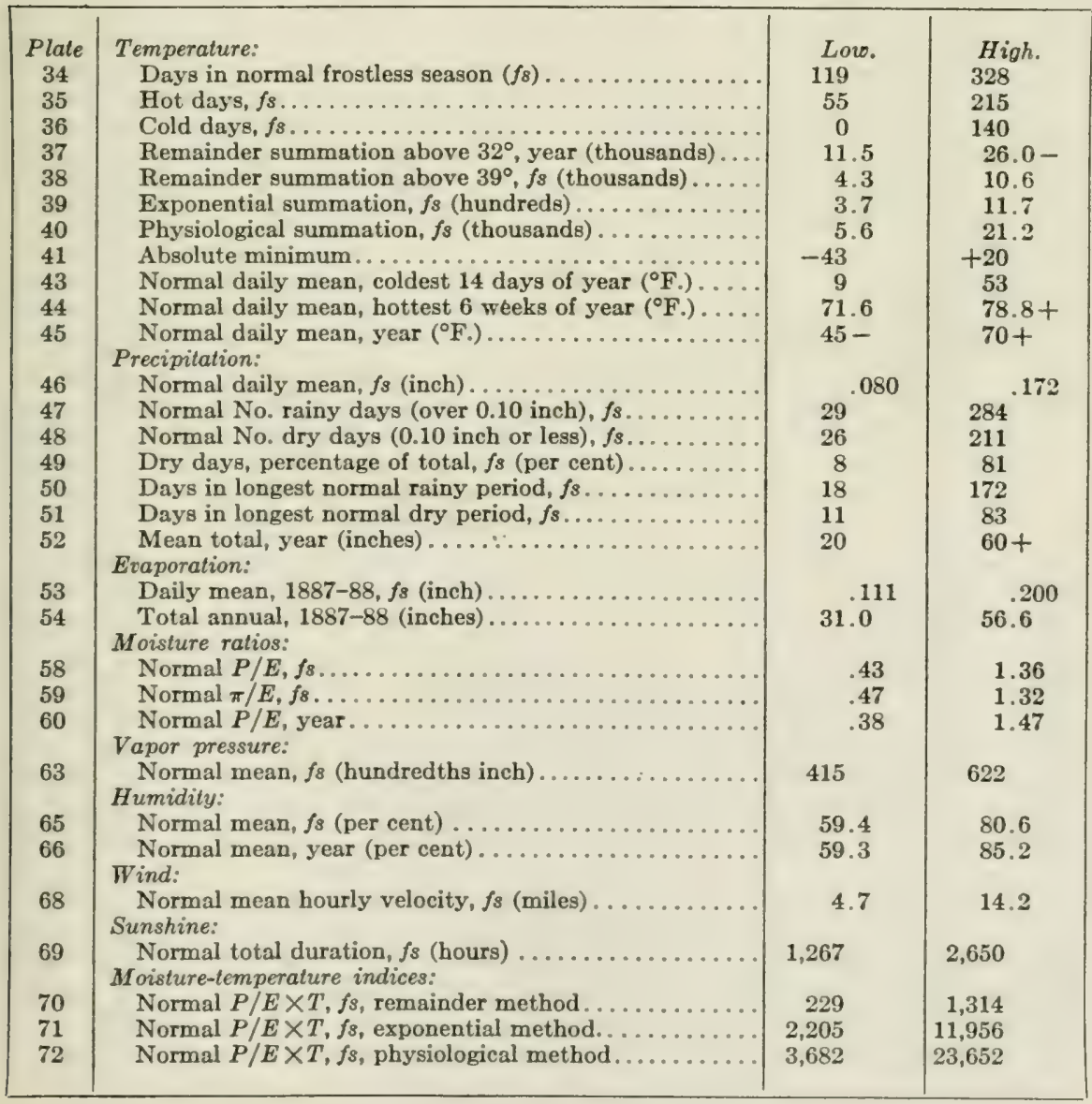


Table 113.-Climatic extremes for Solidago missouriensis.

\begin{tabular}{|c|c|c|c|}
\hline Plate & Temperature: & Low. & High. \\
\hline 34 & Days in normal frostless season $\left(f_{s}\right) \ldots \ldots \ldots \ldots \ldots$ & 53 & 323 \\
\hline 35 & Hot days, $f_{s} \ldots \ldots \ldots \ldots \ldots \ldots \ldots \ldots \ldots \ldots \ldots$ & 0 & 215 \\
\hline 36 & Cold days, $f_{s} \ldots \ldots \ldots \ldots \ldots \ldots \ldots \ldots \ldots \ldots$ & 0 & 158 \\
\hline 37 & Remainder summation above $32^{\circ}$, year (thousands).... & $10.0-$ & $18.0+$ \\
\hline 38 & Remainder summation above $39^{\circ}, f s$ (thousands)...... & 2.7 & 10.6 \\
\hline 39 & Exponential sumpaation, $f_{s}$ (hundreds)............. & 3.0 & 11.7 \\
\hline 40 & Physiological summation, $f_{s}$ (thousands)........... & 4.0 & 21.2 \\
\hline 41 & 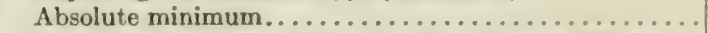 & -59 & +15 \\
\hline 43 & Normal daily mean, coldest 14 days of year $\left({ }^{\circ} \mathrm{F}.\right) \ldots$ & 0 & 53 \\
\hline 44 & Normal daily mean, hottest 6 weeks of year $\left({ }^{\circ} \mathrm{F},\right) \ldots$. & $64.4-$ & $78.8+$ \\
\hline 45 & $\begin{array}{l}\text { Normal daily mean, year }\left({ }^{\circ} \mathrm{F} .\right) \ldots \ldots \ldots \ldots \ldots \\
\text { Precipitation: }\end{array}$ & 45 & 70 \\
\hline 46 & Normal daily mean, $f s$ (inch). .... & .029 & .147 \\
\hline 47 & Normal No. rainy days (over 0.10 inch), fs......... & 0 & 257 \\
\hline 48 & Normal No. dry days $(0.10$ inch or less $), f_{8} \ldots \ldots \ldots \ldots$ & 26 & 211 \\
\hline 49 & Dry days, percentage of total, $f_{s}$ (per cent).......... & 14 & 100 \\
\hline 50 & Days in longest normal rainy period, $f_{s} \ldots \ldots \ldots \ldots \ldots$ & 0 & 172 \\
\hline 51 & Days in longest normal dry period, $f_{s} \ldots \ldots \ldots \ldots \ldots$ & 11 & 202 \\
\hline 52 & 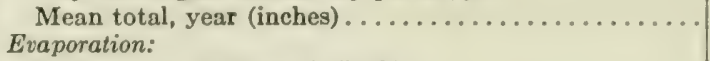 & $10-$ & $40+$ \\
\hline 53 & Daily mean, $1887-88, f_{3}$ (inch). & .118 & .275 \\
\hline 54 & $\begin{array}{l}\text { Total annual, } 1887-88 \text { (inches) } \ldots \ldots \ldots \ldots \ldots \ldots \ldots \\
\text { Moisture ratios: }\end{array}$ & 27.2 & 76.5 \\
\hline 58 & Normal $P / E, f_{s} \ldots$. & .21 & 1.01 \\
\hline 59 & Normal $\pi / E, f_{s} \ldots \ldots \ldots \ldots \ldots \ldots \ldots \ldots \ldots$ & .26 & 1.05 \\
\hline 60 & 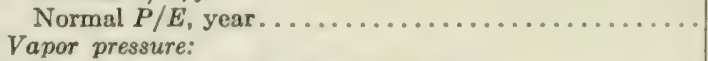 & .18 & 1.16 \\
\hline 63 & $\begin{array}{l}\text { Normal mean, fs (hundredths inch)..... } \\
\text { Humidity: }\end{array}$ & 253 & 622 \\
\hline 65 & 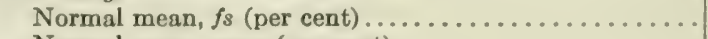 & 47.9 & 80.2 \\
\hline 66 & $\begin{array}{l}\text { Normal mean, year (per cent) } \ldots \ldots \ldots \ldots \ldots \ldots \ldots \ldots \\
\text { Wind: }\end{array}$ & 53.7 & 85.2 \\
\hline 68 & $\begin{array}{l}\text { Normal mean hourly velocity, } f_{s} \text { (miles). } \\
\text { Sunshine: }\end{array}$ & 6.0 & 14.2 \\
\hline 69 & $\begin{array}{l}\text { Normal total duration, } f_{s} \text { (hours)..... } \\
\text { Moisture-temperature indices: }\end{array}$ & 1,127 & 2,650 \\
\hline 70 & 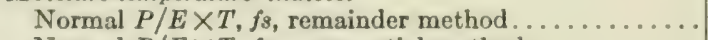 & 58 & 114.2 \\
\hline 71 & Normal $P / E \times T, f s$, exponential method.......... & 563 & 10,331 \\
\hline 72 & Normal $P / E \times T, f s$, physiological method.......... & 710 & 20,570 \\
\hline
\end{tabular}

TABLE 114.-Climatic extremes for Gutierrezia sarothro.

\begin{tabular}{|c|c|c|c|}
\hline Plate & Temperalure: & Low. & High. \\
\hline 34 & Days in normal frostless season $\left(f_{s}\right)$ & 53 & 331 \\
\hline 35 & 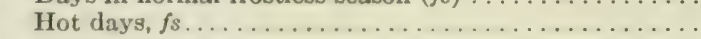 & 4 & 211 \\
\hline 36 & 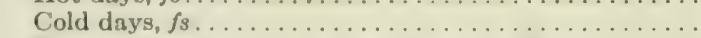 & 0 & 155 \\
\hline 37 & Remainder summation above $32^{\circ}$, year (thousands).... & $10.0-$ & $18.0+$ \\
\hline 38 & Remainder summation above $39^{\circ}, f s$ (thousands)...... & 2.4 & 10.1 \\
\hline 39 & Exponential summation, $f s$ (hundreds) . . . . . . . . . & 2.4 & 11.5 \\
\hline 40 & Physiological summation, $f s$ (thousands) ........... & 2.6 & 20.6 \\
\hline 41 & Absolute minimum. . . . . . . . & -6.5 & +23 \\
\hline 43 & Normal daily mean, coldest 14 days of year $\left({ }^{\circ} \mathrm{F}.\right) \ldots$. & 0 & 54 \\
\hline 44 & Normal daily mean, hottest 6 weeks of year ( $\left.{ }^{\circ} \mathrm{F}.\right)$.... & $64.4-$ & $78.8+$ \\
\hline 45 & Normal daily mean, year $\left({ }^{\circ} \mathrm{F}_{0}\right) \ldots \ldots \ldots \ldots \ldots \ldots$ & 35 & $65+$ \\
\hline 46 & Precipitation: & 01.4 & 096 \\
\hline 47 & Normal No. rainy days (over 0.10 inch), fs $\ldots \ldots \ldots \ldots$ & $0^{.018}$ & $99^{.000}$ \\
\hline 48 & Normal No. dry days $(0.10$ inch or less $), f_{s} \ldots \ldots \ldots \ldots$ & 55 & $2 !) 4$ \\
\hline 49 & Dry days, percentage of total, $f s$ (per cent)......... & 36 & 100 \\
\hline 50 & Days in longest normal rainy period $f s \ldots \ldots \ldots \ldots$ & 0 & 75 \\
\hline 51 & Days in longest normal dry period, $f_{3} \ldots \ldots \ldots \ldots \ldots$ & 37 & 2999 \\
\hline 52 & Menn total, year (inches) $\ldots \ldots \ldots \ldots \ldots \ldots \ldots$ & $10-$ & $30+$ \\
\hline
\end{tabular}


TABLE 114.-Climatic extremes for Gutierrezia sarothro-Continued.

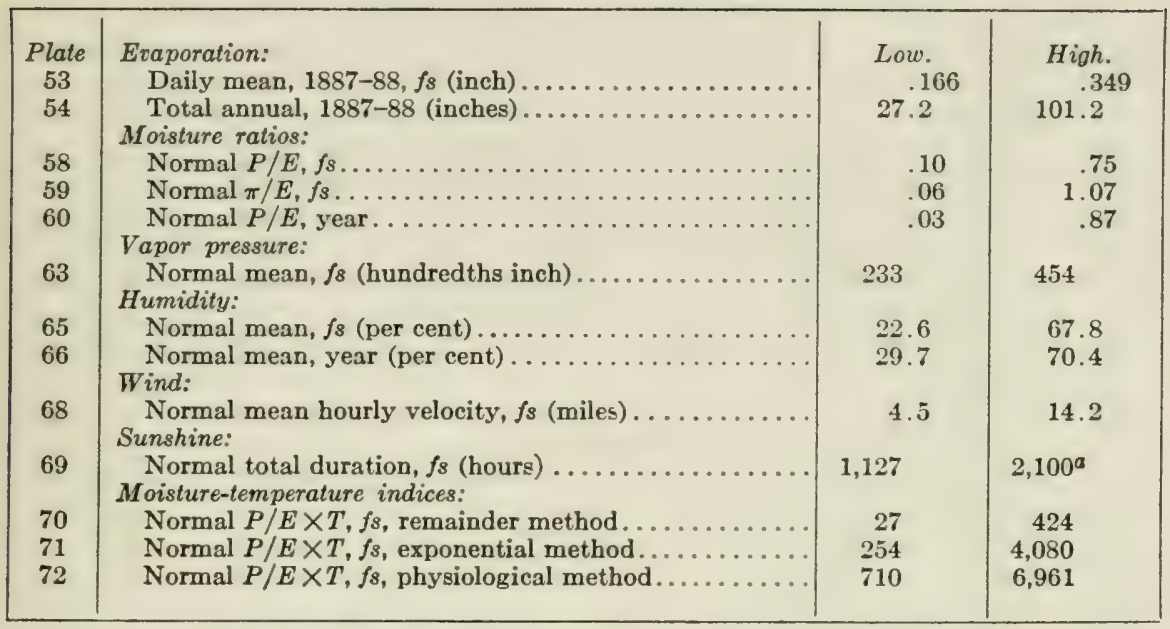

TABLE 115.-Climatic extremes for Bouteloua oligostachya.

\begin{tabular}{|c|c|c|c|}
\hline Plate & Temperature: & Low. & High. \\
\hline 34 & Days in normal frostless season $(f s)$. & 53 & 305 \\
\hline 35 & Hot days, $f_{3} .$. & 0 & 211 \\
\hline 36 & Cold days, $f s$. & 0 & 158 \\
\hline 37 & Remainder summation above $32^{\circ}$, year (thousands) & $10.0-$ & $26.0+$ \\
\hline 38 & Remainder summation above $39^{\circ}, f_{s}$ (thousands)... & 2.9 & 10.1 \\
\hline 39 & Exponential summation, $f_{s}$ (hundreds) ............. & 3.0 & 11.8 \\
\hline 40 & Physiological summation, $f_{s}$ (thousands). & 2.6 & 20.6 \\
\hline 41 & Absolute minimum.. & -59 & +23 \\
\hline 43 & Normal daily mean, coldest 14 days of year $\left({ }^{\circ} \mathrm{F}\right.$ ) & 0 & 51 \\
\hline 44 & Normal daily mean, hottest 6 weeks of year $\left({ }^{\circ} \mathrm{F}\right.$.). & $64.4-$ & $78.8+$ \\
\hline 45 & $\begin{array}{l}\text { Normal daily mean, year }\left({ }^{\circ} \mathrm{F} .\right) \ldots \ldots \ldots \ldots \ldots \\
\text { Precipitation: }\end{array}$ & & $70+$ \\
\hline 46 & Normal daily mean, fs (inch). & .015 & .143 \\
\hline 47 & Normal No. rainy days (over $0.10 \mathrm{inch}$ ), $f_{s}$. & 0 & 141 \\
\hline 48 & Normal No. dry days ( 0.10 inch or less), $f_{s}$. & 41 & 283 \\
\hline 49 & Dry days, percentage of total, $f_{3}$ (per cent). & 23 & 100 \\
\hline 50 & Days in longest normal rainy period, $f_{3} \ldots \ldots$ & 0 & 136 \\
\hline 51 & Days in longest normal dry period, $f_{8} \ldots$. & 13 & 283 \\
\hline 52 & $\begin{array}{l}\text { Mean total, year (inches) .......................... } \\
\text { Evaporation: }\end{array}$ & $10-$ & $40+$ \\
\hline 53 & Daily mean, $1887-88$, fs (inch). . & .101 & 330 \\
\hline 54 & $\begin{array}{l}\text { Total annual, 1887-88 (inches) ................. } \\
\text { Moisture ratios: }\end{array}$ & 22.1 & 101.2 \\
\hline 58 & Normal $P / E, f_{8}$. . & 12 & 1.03 \\
\hline 59 & 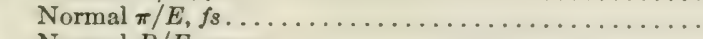 & .13 & 1.15 \\
\hline 60 & $\begin{array}{l}\text { Normal } P / E \text {, year............. } \\
\text { Vapor pressure: }\end{array}$ & .03 & 1.02 \\
\hline 63 & $\begin{array}{l}\text { Normal mean, } f s \text { (hundredths inch)... } \\
\text { Humidity: }\end{array}$ & 249 & 540 \\
\hline 65 & Normal mean, $f_{s}$ (per cent) & 37.0 & 71.5 \\
\hline 66 & $\begin{array}{l}\text { Normal mean, year (per cent).... } \\
\text { Wind: }\end{array}$ & 38.7 & 74.1 \\
\hline 68 & $\begin{array}{l}\text { Normal mean hourly velocity, } f_{s} \text { (miles)...... } \\
\text { Sunshine: }\end{array}$ & 5.6 & 14.2 \\
\hline 69 & $\begin{array}{l}\text { Normal total duration, } f_{8} \text { (hours) } \ldots \ldots \ldots \ldots \\
\text { Moisture-temperature indices: }\end{array}$ & 1,127 & 2,343 \\
\hline 70 & Normal $P / E \times T, f \delta$, remainder method. & 58 & 611 \\
\hline 71 & Normal $P / E \times T, f s$, exponential method. . & 563 & 5,744 \\
\hline 72 & Normal $P / E \times T, f s$, physiological method.... & 710 & $12,000^{a}$ \\
\hline
\end{tabular}


TABLE 116.-Climatic extremes for Bulbilis dactyloides.

\begin{tabular}{|c|c|c|c|}
\hline Plate & Temperature: & Low. & Hioh. \\
\hline 34 & Days in normal frostless season $\left(f_{s}\right)$. & 94 & 323 \\
\hline 35 & Hot days, $f_{s} \ldots \ldots \ldots \ldots \ldots \ldots$ & & 218 \\
\hline 36 & Cold days, fs. . & 0 & 158 \\
\hline 37 & Remainder summation above $32^{\circ}$, year (thousands) & $10.0-$ & $26.0+$ \\
\hline 38 & Remainder summation above $39^{\circ}, f_{s}$ (thousands).. & 2.9 & 10.3 \\
\hline 39 & Exponential summation, $f_{s}$ (hundreds) .......... & 3.0 & 11.3 \\
\hline 40 & Physiological summation, fs (thousands). & 3.7 & 21.4 \\
\hline 41 & Absolute minimum................ & -54 & +12 \\
\hline 43 & Normal daily mean, coldest 14 days of year $\left({ }^{\circ} \mathrm{F}.\right)$. & 0 & 53 \\
\hline 44 & Normal daily mean, hottest 6 weeks of year $\left({ }^{\circ} \mathrm{F}\right.$.). & $64.4-$ & $78.8+$ \\
\hline 45 & Normal daily mean, year $\left({ }^{\circ} \mathrm{F}.\right) \ldots \ldots \ldots$ & & \\
\hline & Precipitation: & & \\
\hline 46 & Normal daily mean, $f_{s}$ (inch) . ............ & .033 & .143 \\
\hline 47 & Normal No. rainy days (over 0.10 inch), $f s .$. & 0 & 159 \\
\hline 48 & Normal No. dry days (0.10 inch or less), fs... & 48 & 259 \\
\hline 49 & Dry days, percentage of total, $f s$ (per cent).. & 25 & 100 \\
\hline 50 & Days in longest normal rainy period, $f_{3} \ldots \ldots$ & 0 & 125 \\
\hline 51 & Days in longest normal dry period, $f_{s} \ldots \ldots \ldots$ & 21 & $150^{a}$ \\
\hline 52 & 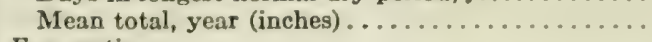 & $10+$ & $40+$ \\
\hline & Evaporation: & & \\
\hline 53 & Daily mean, $1887-88$, $f_{s}$ (inch). & 102 & 293 \\
\hline 54 & $\begin{array}{l}\text { Total annual, 1887-88 (inches)..... } \\
\text { Moisture ratios: }\end{array}$ & 26.3 & 96.4 \\
\hline 58 & Normal $P / E, f_{s}$ & .12 & 94 \\
\hline 59 & Normal $\pi / E, f_{s} \ldots$ & .13 & 1.10 \\
\hline 60 & Normal $P / E$, year. & .12 & .94 \\
\hline 63 & $\begin{array}{l}\text { Vapor pressure: } \\
\text { Normal mean, } f_{s} \text { (hundredths inch) }\end{array}$ & 233 & 540 \\
\hline & Humidity: & & \\
\hline 65 & Normal mean, fs (per cent)....... & 37.0 & 71.5 \\
\hline 66 & $\begin{array}{l}\text { Normal mean, year (per cent).......... } \\
\text { Wind: }\end{array}$ & 38.8 & 74.1 \\
\hline 68 & Normal mean hourly velocity, $f_{8}$ (miles). & 5.8 & 14.2 \\
\hline 69 & $\begin{array}{l}\text { Normal total duration, } f_{s} \text { (hours)... } \\
\text { Moisture-temperature indices: }\end{array}$ & 1,127 & 2,343 \\
\hline 70 & Normal $P / E \times T, f s$, remainder method. & 58 & 611 \\
\hline 71 & Normal $P / E \times T, f_{s}$, exponential method. & 563 & 5,744 \\
\hline 72 & Normal $P / E \times T, f s$, physiological method.. & 710 & $13,000^{a}$ \\
\hline
\end{tabular}

TABLE 117.-Climatic extremes for Koeleria cristata.

\begin{tabular}{|c|c|c|c|}
\hline Plate & Temperature: & Low. & High. \\
\hline 34 & Days in normal frostless season $\left(f_{s}\right)$ & 25 & \\
\hline 35 & Hot days, $f_{s} \ldots \ldots \ldots \ldots \ldots \ldots$. & & 173 \\
\hline 36 & Cold days, $f s . . . \ldots \ldots$ & 0 & $155+$ \\
\hline 37 & Remainder summation above $32^{\circ}$, year (thousands) & $10.0-$ & $18.0+$ \\
\hline 38 & Remainder summation above $39^{\circ}, f_{3}$ (thousands)... & 2.6 & 8.6 \\
\hline 39 & Exponential summation, $f_{s}$ (hundreds).. & 2.8 & 9.6 \\
\hline 40 & Physiological summation, $f s$ (thousands). & 2.1 & 17.6 \\
\hline 41 & Absolute minimum.............. & -59 & +32 \\
\hline 43 & Normal daily mean, coldest 14 days of year $\left({ }^{\circ} \mathrm{F}\right.$. $)$. & 0 & 46 \\
\hline 44 & Normal daily mean, hottest 6 weeks of year $\left({ }^{\circ} \mathrm{F}\right.$. $)$. & $64.4-$ & 78.8 \\
\hline 45 & Normal daily mean, year $\left({ }^{\circ} \mathrm{F}.\right) .$. & 35 & 70 \\
\hline & Precipilation: & & \\
\hline 46 & Normal daily mean, $f_{s}$ (inch). & .009 & 199 \\
\hline 47 & Normal No. rainy days (over 0.10 inch), fs & 0 & 199 \\
\hline 48 & Normal No. dry days (0.10 inch or less), fs.. & 26 & 294 \\
\hline .19 & Dry days, percentage of total, $f_{s}$ (per cent). & 18 & 100 \\
\hline 50 & Days in longest normal rainy period, $f_{s} \ldots$ & 0 & 172 \\
\hline 51 & Days in longest normal dry period, $f_{s} \ldots$ & 9 & 299 \\
\hline 52 & Mean total, year (inches)............. & $10-$ & 50 \\
\hline
\end{tabular}


TABLE 117.-Climatic extremes for Koleria cristata-Continued.

\begin{tabular}{|c|c|c|c|}
\hline Plate & Evaporation: & Low. & High. \\
\hline 53 & Daily mean, 1887-88, fs (inch) & .052 & .349 \\
\hline 54 & Total annual, $1887-88$ (inches) & & \\
\hline & Moisture ratios: & & 3.84 \\
\hline 58 & Normal $P / E, f_{s}$. & .04 & $\begin{array}{l}3.84 \\
4.48\end{array}$ \\
\hline 59 & $\begin{array}{l}\text { Normal } \pi / E, f 3 \ldots \ldots \ldots \\
\text { Normal } P / E \text {, year } \ldots\end{array}$ & $\begin{array}{l}.06 \\
.09\end{array}$ & $\begin{array}{l}4.48 \\
4.90\end{array}$ \\
\hline & $\begin{array}{l}\text { Normal } P / E, \text { year............ } \\
\text { Vapor pressure: }\end{array}$ & & \\
\hline 63 & $\begin{array}{l}\text { Normal mean, } f_{s} \text { (hundredths inch). } \\
\text { Humidity: }\end{array}$ & 279 & 567 \\
\hline 65 & Normal mean, $f_{s}$ (per cent). & 22.6 & 87.5 \\
\hline 66 & $\begin{array}{l}\text { Normal mean, year (per cent)... } \\
\text { Wind: }\end{array}$ & 29.7 & 86.8 \\
\hline 68 & $\begin{array}{l}\text { Normal mean hourly velocity, } f s \text { (miles).. } \\
\text { Sunshine: }\end{array}$ & 4.5 & 16.4 \\
\hline 69 & $\begin{array}{l}\text { Normal total duration, } f_{s} \text { (hours) . } \\
\text { Moisture-temperature indices: }\end{array}$ & 1,225 & 2,995 \\
\hline 70 & Normal $P / E \times T, f s$, remainder method & 13 & 1,566 \\
\hline 71 & Normal $P / E \times T, f_{s}$, exponential method. & 127 & 11.724 \\
\hline 72 & Normal $P / E \times T, f s$, physiological method. & 197 & 7,869 \\
\hline
\end{tabular}

TABLE 118.-Climatic extremes for Agropyron spicatum.

\begin{tabular}{|c|c|c|c|}
\hline Plate & Temperature: & Low. & High. \\
\hline 34 & Days in normal frostless season $(f s)$. & 25 & 198 \\
\hline 35 & Hot days, $f$ s.................... & 0 & 125 \\
\hline 36 & Cold days, fs. & 0 & 158 \\
\hline 37 & Remainder summation above $32^{\circ}$, year (thousands). & $10.0-$ & $18.0+$ \\
\hline 38 & Remainder summation above $39^{\circ}, f_{s}$ (thousands).. & 2.7 & 6.1 \\
\hline 39 & Exponential summation, $f_{s}$ (hundreds) ............ & 2.8 & 6.5 \\
\hline 40 & Physiological summation, $f_{s}$ (thousands). & 3.5 & 11.6 \\
\hline 41 & Absolute minimum.................. & -59 & +8 \\
\hline 43 & Normal daily mean, coldest 14 days of year $\left({ }^{\circ} \mathrm{F}.\right)$. & 0 & $35^{a}$ \\
\hline 44 & Normal daily mean, hottest 6 weeks of year $\left({ }^{\circ} \mathrm{F}\right.$. $)$. & $64.4-$ & $78.8+$ \\
\hline 45 & $\begin{array}{c}\text { Normal daily mean, year }\left({ }^{\circ} \mathrm{F} .\right) \ldots \ldots \ldots \ldots \ldots \ldots \\
\text { Precimitation. }\end{array}$ & & \\
\hline 46 & $\begin{array}{l}\text { Precipitation: } \\
\text { Normal daily mean, } f_{s} \text { (inch). }\end{array}$ & .025 & .135 \\
\hline 47 & Normal No. rainy days (over $0.10 \mathrm{inch}$ ), fs. & 0 & 141 \\
\hline 48 & Normal No. dry days ( 0.10 inch or less), $f_{s}$. & 41 & 216 \\
\hline 49 & Dry days, percentage of total, $f_{s}$ (per cent). & 23 & 100 \\
\hline 50 & Days in longest normal rainy period, $f_{s} \ldots$. & 0 & 136 \\
\hline 51 & Days in longest normal dry period, $f s .$. & 13 & 216 \\
\hline 52 & Mean total, year (inches) . . . . . . . . & $10-$ & $30+$ \\
\hline & Evaporation: & & \\
\hline 53 & Daily mean, $1887-88, f_{s}$ (inch). & .115 & .293 \\
\hline 54 & Total annual, $1887-88$ (inches). & 22.1 & 79.8 \\
\hline & Moisture ratios: & & 103 \\
\hline 58 & Normal $P / E, f_{s}$.. & .10 & 1.03 \\
\hline 59 & Normal $\pi / E, f_{s}$. . & .12 & 1.16 \\
\hline 60 & Normal $P / E$, year... & .14 & 1.02 \\
\hline 63 & $\begin{array}{l}\text { Vapor pressure: } \\
\text { Normal mean, } f s \text { (hundredths inch). } \\
\text { Humidity: }\end{array}$ & 233 & 466 \\
\hline 65 & Normal mean, $f_{3}$ (per cent) & 40.9 & 68.4 \\
\hline 66 & $\begin{array}{l}\text { Normal mean, year (per cent) ............... } \\
\text { Wind: }\end{array}$ & 45.4 & 74.1 \\
\hline 68 & Normal mean hourly velocity, $f_{s}$ (miles). & 6.2 & 14.2 \\
\hline 69 & Normal total duration, $f_{s}$ (hours) & 1,127 & 1,927 \\
\hline 70 & $\begin{array}{l}\text { Moisture-temperature indices: } \\
\text { Normal } P / E \times T, f_{3} \text { remainder method. }\end{array}$ & & 611 \\
\hline 71 & $\begin{array}{l}\text { Normal } P / E \times T \text {, } f s \text {, remainder method... } \\
\text { Normal } P / E \times T, f_{s} \text {, exponential method.. }\end{array}$ & $\begin{array}{r}58 \\
563\end{array}$ & 5,744 \\
\hline 72 & Normal $P / E \times T, f_{3}$, physiological method. & 710 & 10,599 \\
\hline
\end{tabular}


TABle 119.-Climatic extremes for Hilaria jamesii.

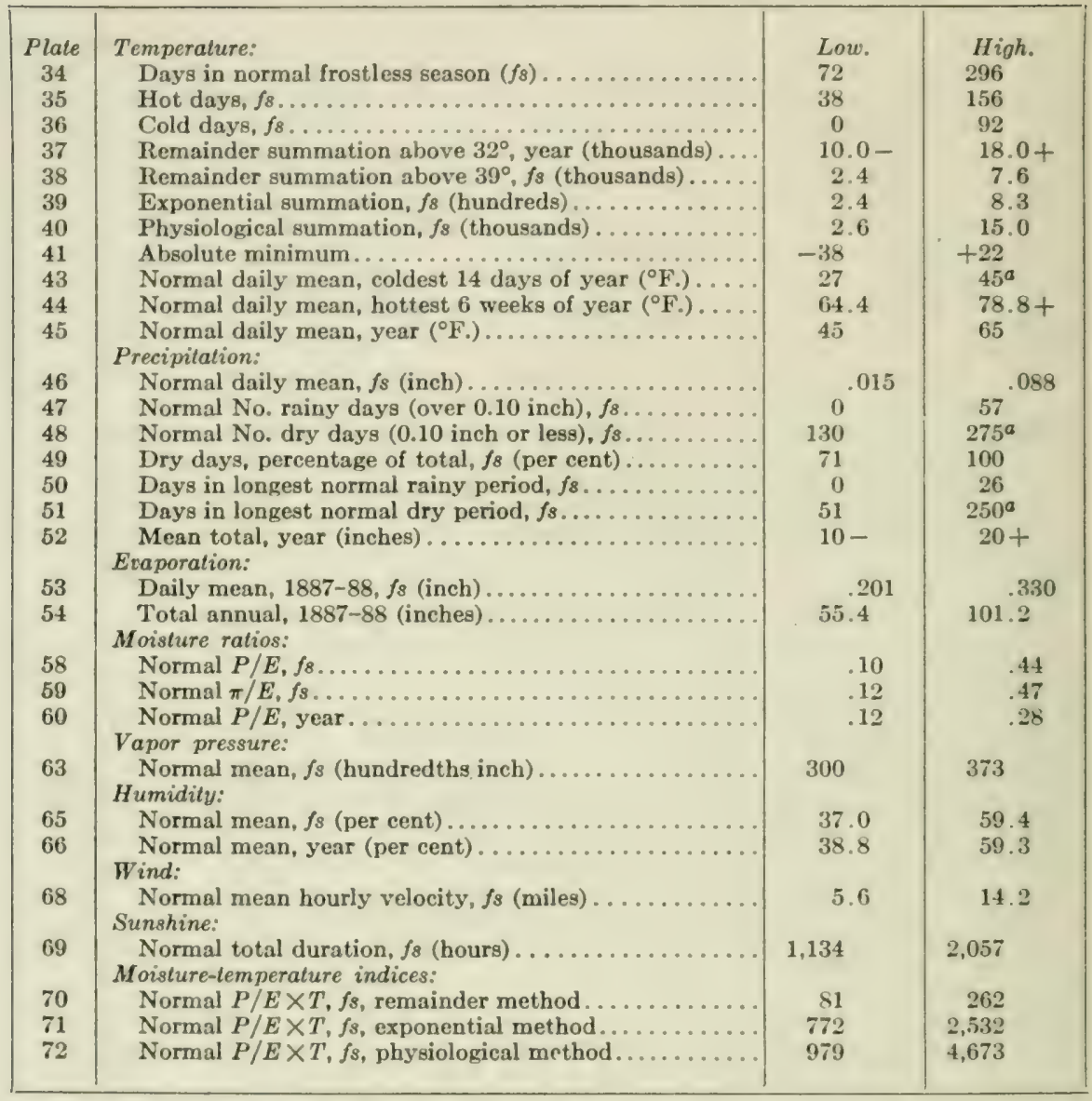

TABLE 120.-Climatic extremes for Andropogon virginicus.

\begin{tabular}{|c|c|c|c|}
\hline Plate & Temperature: & Low. & High. \\
\hline 34 & Days in normal frostless season $(f s)$ & 108 & \\
\hline 35 & Hot days $f_{s} \ldots \ldots \ldots \ldots \ldots \ldots$ & 0 & 365 \\
\hline 36 & Cold days, $f_{s}$. & () & 137 \\
\hline 37 & Remainder summation above $32^{\circ}$, year (thousands) & $10.0-$ & $26.0+$ \\
\hline 38 & Remainder summation above $39^{\circ}, f_{s}$ (thousands)... & 2.6 & 14.5 \\
\hline 39 & Exponential summation, $f_{8}$ (hundreds) ........... & 3.0 & 15.4 \\
\hline 40 & Physiological summation, $f_{s}$ (thousands) ... & 2.1 & 31.1 \\
\hline 41 & Absolute minimum................ & -41 & +41 \\
\hline 43 & Normal daily mean, coldest 14 days of year $\left({ }^{\circ} \mathrm{F}\right)$. & 1.5 & 69 \\
\hline 44 & Normal daily mean, hottest 6 weeks of year $\left({ }^{\circ} \mathrm{F}\right.$ ) . & (i.4.4- & $7 s . s+$ \\
\hline 45 & $\begin{array}{l}\text { Normal daily mean, year }\left({ }^{\circ} F_{0}\right) \ldots \ldots \ldots \ldots \ldots \ldots \\
\text { Precipitation: }\end{array}$ & 35 & $75+$ \\
\hline 46 & Normal daily mesn, $f_{8}$ (inch) & $(1 \leqslant 9)$ & 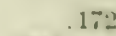 \\
\hline 47 & Normal No, rainy days (over $0.10 \mathrm{inch}$ ), $f_{s} \ldots \ldots$ & is & $2 \times-4$ \\
\hline 48 & Normal No. dry days (0.10 inch or less), $f_{s}$. & 26 & 20.3 \\
\hline 49 & Dry days, percentage of total, $f_{s}$ (per cent)....... & $s$ & is \\
\hline 50 & Days in longest normal rainy period, $f_{s} \ldots \ldots \ldots \ldots$ & 21 & 256 \\
\hline 51 & Days in longest normal dry period, $f_{s} \ldots \ldots \ldots \ldots \ldots$ & 4 & 152 \\
\hline 52 & 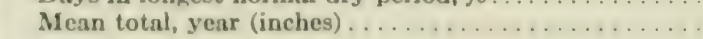 & $30-$ & $60 \div$ \\
\hline
\end{tabular}


TABLE 120.-Climatic extremes for Andropogon virginicus-Continued.

\begin{tabular}{|c|c|c|c|}
\hline Plate & Evaporation: & Low. & High. \\
\hline 53 & Daily mean, $1887-88, f_{s}$ (inch) $\ldots \ldots \ldots \ldots \ldots \ldots$ & .097 & .200 \\
\hline 54 & $\begin{array}{l}\text { Total annual, } 1887-88 \text { (inches) } \ldots \ldots \ldots \ldots \ldots \ldots \ldots \\
\text { Moisture ratios: }\end{array}$ & 20.3 & 56.6 \\
\hline 58 & 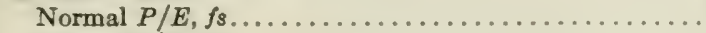 & .51 & 1.76 \\
\hline 59 & Normal $\pi / E, f_{s} \ldots \ldots \ldots \ldots \ldots \ldots \ldots \ldots$ & .60 & 1.96 \\
\hline 60 & $\begin{array}{l}\text { Normal } P / E \text {, year. . . . . . . . . . . . . . . . . . . . } \\
\text { Vapor pressure: }\end{array}$ & .71 & 1.47 \\
\hline 63 & $\begin{array}{l}\text { Normal mean, } f s \text { (hundredths inch) ..................... } \\
\text { Humidity: }\end{array}$ & 345 & 707 \\
\hline 65 & Normal mean, $f_{s}$ (per cent). & 65.6 & 82.7 \\
\hline 66 & $\begin{array}{l}\text { Normal mean, year (per cent) .................. } \\
\text { Wind: }\end{array}$ & 67.5 & 85.2 \\
\hline 68 & $\begin{array}{l}\text { Normal mean hourly velocity, fs (miles) } \ldots \ldots \ldots \ldots \\
\text { Sunshine: }\end{array}$ & 4.2 & 13.5 \\
\hline 69 & $\begin{array}{l}\text { Normal total duration, } f_{s} \text { (hours) } \ldots \ldots \ldots \ldots \ldots \ldots \\
\text { Moisture-temperature indices: }\end{array}$ & 1,225 & 2,650 \\
\hline 70 & Normal $P / E \times T, f s$, remainder method. & 301 & 1,418 \\
\hline 71 & Normal $P / E \times T, f_{s}$, exponential method ...... & 2,918 & 13,511 \\
\hline 72 & Normal $P / E \times T, f s$, physiological method..... & 2,747 & 24,265 \\
\hline
\end{tabular}

TABLE 121.-Climatic extremes for Bouteloua hirsuta.

\begin{tabular}{|c|c|c|c|}
\hline Plate & Temperature: & Lou. & High. \\
\hline 34 & Days in normal frostless season $\left(f_{8}\right)$. & 94 & 323 \\
\hline 35 & 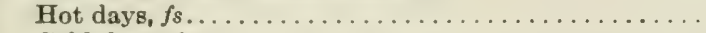 & 0 & 218 \\
\hline 36 & Cold days, $f_{s} \ldots \ldots \ldots \ldots \ldots \ldots \ldots \ldots \ldots \ldots$ & 0 & 158 \\
\hline 37 & Remainder summation above $32^{\circ}$, year (thousands)... & $10.0-a$ & $26.0+a$ \\
\hline 38 & Remainder summation above $39^{\circ}, f_{s}$ (thousands)..... & $3.0-a$ & $10.0+a$ \\
\hline 39 & Exponential summation, $f s$ (hundreds) $\ldots \ldots \ldots \ldots \ldots$ & 3.0 & 11.3 \\
\hline 40 & Physiological summation, $f_{s}$ (thousands) $\ldots \ldots \ldots \ldots$ & 3.7 & 21.4 \\
\hline 41 & 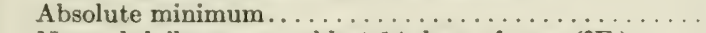 & -59 & +12 \\
\hline 43 & Normal daily mean, coldest 14 days of year $\left({ }^{\circ} \mathrm{F}.\right) \ldots$ & 0 & 53 \\
\hline 44 & Normal daily mean, hottest 6 weeks of year $\left({ }^{\circ} \mathbf{F}\right.$.) .... & $64.4-a$ & $78.8+a$ \\
\hline 45 & $\begin{array}{l}\text { Normal daily mean, year }\left({ }^{\circ} \mathrm{F} .\right) \ldots \ldots \ldots \ldots \ldots \ldots \ldots \\
\text { Precipitation: }\end{array}$ & $35^{a}$ & $70+a$ \\
\hline 46 & Normal daily mean, $f_{s}$ (inch). & .033 & 143 \\
\hline 47 & Normal No. rainy days (over 0.10 inch),$f_{s} \ldots \ldots \ldots$ & 2 & 161 \\
\hline 48 & Normal No. dry days ( 0.10 inch or less), fs......... & $2 x$ & $275^{a}$ \\
\hline 49 & Dry days, percentage of total, $f s$ (per cent)........ & 17 & 99 \\
\hline 50 & Days in longest normal rainy period, $f s \ldots \ldots \ldots \ldots$ & 2 & 172 \\
\hline 51 & Days in longest normal dry period, $f_{s} \ldots \ldots \ldots \ldots$ & 11 & 88 \\
\hline 52 & $\begin{array}{l}\text { Mean total, year (inches) } \ldots \ldots \ldots \ldots \ldots \ldots \ldots \ldots \ldots \\
\text { Evaporation: }\end{array}$ & $10-a$ & $40+$ \\
\hline 53 & Daily mean, $1887-88, f s$ (inch) $\ldots \ldots \ldots \ldots \ldots \ldots$ & .101 & .330 \\
\hline 54 & $\begin{array}{l}\text { Total annual, } 1887 \text { I } 88 \text { (inches) } \ldots \ldots \ldots \ldots \ldots \ldots \ldots \ldots \\
\text { Moisture ratios: }\end{array}$ & 22.1 & 101.2 \\
\hline 58 & Normal $P / E, f s \ldots$ & .12 & 1.23 \\
\hline 59 & 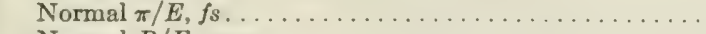 & .13 & 1. 16 \\
\hline 60 & $\begin{array}{l}\text { Normal } P / E \text {, year } \ldots \ldots \ldots \ldots \ldots \ldots \ldots \ldots \ldots \ldots \\
\text { Vapor pressure: }\end{array}$ & .12 & 1.02 \\
\hline 63 & $\begin{array}{l}\text { Normal mean, } f_{s} \text { (hundredths inch)............ } \\
\text { Humidity: }\end{array}$ & 300 & 675 \\
\hline 65 & Normal mean, $f_{s}$ (per cent) & 37.0 & 71.9 \\
\hline 66 & $\begin{array}{l}\text { Normal mean, year (per cent).. } \\
\text { Wind: }\end{array}$ & 35.8 & 75.0 \\
\hline 68 & $\begin{array}{l}\text { Normal mean hourly velocity, fs (miles).... } \\
\text { Sunshine: }\end{array}$ & 5.8 & 14.2 \\
\hline 69 & $\begin{array}{l}\text { Normal total duration, } f_{s} \text { (hours) .. } \\
\text { Moisture-temperature indices: }\end{array}$ & 1,127 & 2,343 \\
\hline 70 & Normal $P / E \times T, f_{s}$, remainder method. & 906 & 6,690 \\
\hline 71 & Normal $P / E \times T, f s$, exponential method....... & 98 & $1,100^{a}$ \\
\hline 72 & Normal $P / E \times T, f s$, physiological method.......... & $1,000-a$ & $20,000^{a}$ \\
\hline
\end{tabular}


TABLE 122.-Climatic extremes for Sparganium americanum.

\begin{tabular}{|c|c|c|c|}
\hline Plate & Temperature: & Low. & High. \\
\hline 34 & Days in normal frostless season $\left(f_{3}\right) \ldots$ & 85 & \\
\hline 35 & Hot days, $f_{8} \ldots \ldots$ & 0 & 191 \\
\hline 36 & Cold days, $f 8 \ldots \ldots \ldots \ldots \ldots \ldots$ & 0 & 149 \\
\hline 37 & Remainder summation above $32^{\circ}$, year (thousands) & $10.0-$ & $18.0+$ \\
\hline 38 & Remainder summation above $39^{\circ}$, $f_{s}$ (thousands).. & 2.6 & 9.4 \\
\hline 39 & Exponential summation, $f_{s}$ (hundreds) ........... & 3.0 & 10.3 \\
\hline 40 & Physiological summation, $f_{8}$ (thousands). & 2.1 & 18.9 \\
\hline 41 & Absolute minimum............... & -48 & +20 \\
\hline 43 & Normal daily mean, coldest 14 days of year $\left({ }^{\circ}{ }^{\circ} \mathrm{F}.\right)$. & 0 & 54 \\
\hline 44 & Normal daily mean, hottest 6 weeks of year $\left({ }^{\circ} \mathrm{F}.\right)$. & $64.4-$ & $78.8+$ \\
\hline 45 & Normal daily mean, year $\left({ }^{\circ} \mathrm{F}.\right) \ldots \ldots \ldots \ldots \ldots$ & & \\
\hline 46 & $\begin{array}{l}\text { Precipitation: } \\
\text { Normal daily mean, } f_{s} \text { (inch). }\end{array}$ & .037 & 199 \\
\hline 47 & Normal No. rainy days (over 0.10 inch), $f_{s}$. & 0 & 248 \\
\hline 48 & Normal No. dry days (0.10 inch or less), $f s$. & 0 & 216 \\
\hline 49 & Dry days, percentage of total, $f_{s}$ (per cent). & 11 & 100 \\
\hline 50 & Days in longest normal rainy period, $f_{s} . .$. & 0 & 256 \\
\hline 51 & Days in longest normal dry period, $f_{3} .$. & 0 & 216 \\
\hline 52 & Mean total, year (inches)........... & $10-$ & $60+$ \\
\hline & Evaporation: & & \\
\hline 53 & Daily mean, $1887-88, f_{s}$ (inch)... & .052 & .212 \\
\hline 54 & $\begin{array}{l}\text { Total annual, } 1887-88 \text { (inches)... } \\
\text { Moisture ratios: }\end{array}$ & 18.1 & 57.7 \\
\hline 58 & Normal $P / E, f_{8}, \ldots \ldots \ldots$ & .18 & 3.84 \\
\hline 59 & Normal $\pi / E, f_{s} . .$. & .22 & 4.48 \\
\hline 60 & $\begin{array}{l}\text { Normal } P / E \text {, year........................... } \\
\text { Vapor pressure: }\end{array}$ & .31 & 4.90 \\
\hline 63 & $\begin{array}{l}\text { Normal mean, fs (hundredths inch).... } \\
\text { Humidity: }\end{array}$ & 297 & 610 \\
\hline 65 & Normal mean, $f_{s}$ (per cent). & 54.6 & 82.7 \\
\hline 66 & $\begin{array}{l}\text { Normal mean, year (per cent).............. } \\
\text { Wind: }\end{array}$ & 64.8 & 82.9 \\
\hline 68 & $\begin{array}{l}\text { Normal mean hourly velocity, } f_{3} \text { (miles)...... } \\
\text { Sunshine: }\end{array}$ & 3.1 & 13.5 \\
\hline 69 & $\begin{array}{l}\text { Normal total duration, } f_{s} \text { (hours) ..... } \\
\text { Moisture-temperature indices: }\end{array}$ & 1,225 & 2,297 \\
\hline 70 & Normal $P / E \times T, f_{s}$, remainder method. & 101 & 1,418 \\
\hline 71 & Normal $P / E \times T, f s$, exponential method. & 950 & 13,511 \\
\hline 72 & Normal $P / E \times T, f_{s}$, physiological method.. & 1,475 & 24,265 \\
\hline
\end{tabular}

TABLE 123.-Climatic extremes for Dianthera americana.

\begin{tabular}{|c|c|c|c|}
\hline Plate & Temperature: & Lou. & High. \\
\hline 34 & Days in normal frostless season $\left(f_{s}\right)$ & 108 & \\
\hline 35 & Hot days, $f s \ldots \ldots \ldots \ldots \ldots \ldots$. & & \\
\hline 36 & Cold days, $f_{s}$. & 0 & 137 \\
\hline 37 & Remainder summation above $32^{\circ}$, year (thousands) & $10.0-$ & 26.0 \\
\hline 38 & Remainder summation above $39^{\circ}, f_{s}$ (thousands) ... & 2.6 & 10.6 \\
\hline 39 & Exponential summation, $f_{s}$ (hundreds) .......... & 3.0 & 11.7 \\
\hline 40 & Physiological summation, fs (thousands) & 2.1 & 21.4 \\
\hline 41 & Absolute minimum.................. & $-4: 3$ & +19 \\
\hline 43 & Normal daily mean, coldest 14 days of year $\left({ }^{\circ} \mathrm{F}_{0}\right)$ & 15 & 69 \\
\hline 44 & Normal daily mean, hottest 6 weeks of year $\left({ }^{\circ} \mathrm{F}\right.$.). & $6.4 .4-$ & $\pi s . s+$ \\
\hline 45 & $\begin{array}{l}\text { Normal daily mean, year }\left({ }^{\circ} \mathrm{F} .\right) \ldots \ldots \ldots \ldots \ldots \ldots \\
\text { Precipitation: }\end{array}$ & & $70+$ \\
\hline 46 & Normal daily mean, $f_{8}$ (inch). & 052 & .172 \\
\hline 47 & Normal No. rainy days (over 0.10 inch), fs. & 26 & 254 \\
\hline 48 & Normal No. dry days (0.10 inch or less), fs. & 26 & 259 \\
\hline 49 & Dry days, percentage of total, $f_{s}$ (per cent). & $s$ & 87 \\
\hline 50 & Days in longest normal rainy period, $f s \ldots \ldots$ & $25-$ & $157+$ \\
\hline 51 & Days in longest normal dry period, $f_{s} \ldots \ldots \ldots$ & 4 & 91 \\
\hline 52 & Mean total, year (inches) . . . . . . . . . . & $30-$ & $60+$ \\
\hline
\end{tabular}


TABLe 123.-Climatic extremes for Dianthera americana-Continued.

\begin{tabular}{|c|c|c|c|}
\hline Plate & Evaporation: & Low. & High. \\
\hline & $\begin{array}{l}\text { Daily mean, } 1887-88, f_{s} \text { (inch)... } \\
\text { Total annual, } 1887-88 \text { (inches) .. }\end{array}$ & 20.3 & 56.6 \\
\hline & Moisture ratios: & & \\
\hline 58 & Normal $P / E, f_{3}$. & .34 & 1.36 \\
\hline 59 & Normal $\pi / E, f_{s}$. & .35 & 1.96 \\
\hline 60 & Normal $P / E$, year. . & .51 & 1.47 \\
\hline 63 & $\begin{array}{l}\text { Vapor pressure: } \\
\text { Normal mean, fs (hundredths inch)... }\end{array}$ & 345 & 675 \\
\hline 65 & Normal mean, $f_{s}$ (per cent).......... & 64.5 & 84.0 \\
\hline 66 & $\begin{array}{l}\text { Normal mean, year (per cent)....... } \\
\text { Wind: }\end{array}$ & 66.6 & 85.2 \\
\hline 68 & $\begin{array}{l}\text { Normal mean hourly velocity, } f_{s} \text { (miles) } \\
\text { Sunshine: }\end{array}$ & 3.1 & 14.9 \\
\hline 69 & $\begin{array}{l}\text { Normal total duration, } f_{s} \text { (hours) } \\
\text { Moisture-temperature indices: }\end{array}$ & 1,225 & 2,650 \\
\hline 70 & Normal $P / E \times T, f s$, remainder method. & 301 & 1,418 \\
\hline 71 & Normal $P / E \times T, f s$, exponential method. & 2,914 & 13,511 \\
\hline 72 & Normal $P / E \times T, f_{s}$, physiological method & 2,747 & 24,265 \\
\hline
\end{tabular}

TABLE 124.-Climatic extremes for Sium cicutofolium.

\begin{tabular}{|c|c|c|c|}
\hline Plate & Temperature: & Low. & High. \\
\hline 34 & Days in normal frostless season $(f s) \ldots \ldots \ldots$ & 25 & \\
\hline 35 & Hot days, $f_{s} \ldots \ldots \ldots \ldots \ldots \ldots \ldots \ldots \ldots$ & & 215 \\
\hline 36 & Cold days, $f_{3} \ldots \ldots \ldots \ldots \ldots \ldots \ldots \ldots$ & 0 & 158 \\
\hline 37 & Remainder summation above $32^{\circ}$, year (thousands) & $10.0-$ & $18.0+$ \\
\hline 38 & Remainder summation above $39^{\circ}, f s$ (thousands).. & 2.6 & 10.6 \\
\hline 39 & Exponential summation, $f_{s}$ (hundreds) .......... & 2.8 & 11.7 \\
\hline 40 & Physiological summation, $f_{s}$ (thousands). & 2.1 & 21.2 \\
\hline 41 & Absolute minimum............... & -65 & +19 \\
\hline 43 & Normal daily mean, coldest 14 days of year $\left({ }^{\circ} \mathrm{F}.\right)$. & 0 & 54 \\
\hline 44 & Normal daily mean, hottest 6 weeks of year $\left({ }^{\circ} \mathrm{F}.\right) \ldots$ & $64.4-$ & $78.8+$ \\
\hline 45 & $\begin{array}{l}\text { Normal daily mean, year }\left({ }^{(} \mathbf{F} .\right) \ldots \ldots \ldots \ldots \ldots \ldots \ldots \\
\text { Precipitation: }\end{array}$ & 35 & 70 \\
\hline 46 & Normal daily mean, $f_{s}$ (inch). & .022 & .199 \\
\hline 47 & Normal No. rainy days (over $0.10 \mathrm{inch}$ ), $f s$. & & 199 \\
\hline 48 & Normal No. dry days ( 0.10 inch or less), $f_{s}$. & 19 & 294 \\
\hline 49 & Dry days, percentage of total, $f_{s}$ (per cent).. & 8 & 100 \\
\hline 50 & Days in longest normal rainy period, $f_{s}, \ldots$ & 0 & 256 \\
\hline 51 & Days in longest normal dry period, $f_{8} . .$. & 4 & 299 \\
\hline 52 & Mean total, year (inches) ............ & $10-$ & $60+$ \\
\hline & Evaporation: & & \\
\hline 53 & Daily mean, $1887-88, f_{s}$ (inch). & .052 & .268 \\
\hline 54 & $\begin{array}{l}\text { Total annual, 1887-88 (inches).. } \\
\text { Moisture ratios: }\end{array}$ & 18.1 & 79.8 \\
\hline 58 & Normal $P / E, f_{s}$. . & .08 & 3.84 \\
\hline 59 & Normal $\pi / E, f_{s} .$. & .10 & 1.96 \\
\hline 60 & $\begin{array}{l}\text { Normal } P / E \text {, year....... } \\
\text { Vapor pressure: }\end{array}$ & .15 & 4.90 \\
\hline 63 & $\begin{array}{l}\text { Normal mean, } f s \text { (hundredths inch)... } \\
\text { Humidity: }\end{array}$ & 233 & 622 \\
\hline 65 & Normal mean, $f s$ (per cent... & 41.5 & 87.5 \\
\hline 66 & $\begin{array}{l}\text { Normal mean, year (per cent).......... } \\
\text { Wind: }\end{array}$ & 48.1 & 86.8 \\
\hline 68 & $\begin{array}{l}\text { Normal mean hourly velocity, fs (miles). } \\
\text { Sunshine: }\end{array}$ & 3.1 & 16.4 \\
\hline 69 & $\begin{array}{l}\text { Normal total duration, } f_{8} \text { (hours) } \\
\text { Moisture-temperature indices: }\end{array}$ & 1,127 & 2,650 \\
\hline 70 & $\begin{array}{l}\text { Moisture-temperature indices: } \\
\text { Normal } P / E \times T, f s \text {, remainder method. . }\end{array}$ & 42 & 1,566 \\
\hline 71 & Normal $P / E \times T, f_{3}$, exponential method.. & 405 & 13,511 \\
\hline 72 & Normal $P / E \times T, f s$, physiological method... & 598 & 24,265 \\
\hline
\end{tabular}


TABLE 125.-Climatic extremes for Arundinaria tecta.

\begin{tabular}{|c|c|c|c|}
\hline Plate & Temperature: & Loik. & High. \\
\hline 34 & Days in normal frostless season $\left(f_{8}\right) \ldots$ & 178 & 365 \\
\hline 35 & Hot days, $f_{8} \ldots \ldots \ldots \ldots \ldots \ldots \ldots \ldots \ldots \ldots \ldots \ldots \ldots \ldots$ & 116 & \\
\hline 36 & Cold days, $f_{8} \ldots \ldots \ldots \ldots \ldots \ldots \ldots \ldots \ldots$ & 0 & 22 \\
\hline 37 & Remainder summation above $32^{\circ}$, year (thousands) & $11.5-$ & $26.0+$ \\
\hline 38 & Remainder summation above $39^{\circ}, f_{8}$ (thousands)... & 5.7 & 14.5 \\
\hline 39 & Exponential summation, $f_{8}$ (hundreds). & 6.0 & 15.4 \\
\hline 40 & Physiological summation, $f_{8}$ (thousands). & 10.0 & 31.1 \\
\hline 41 & Absolute minimum.............. & -15 & +41 \\
\hline 43 & Normal daily mean, coldest 14 days of year $\left({ }^{\circ} \mathrm{F}\right.$.) & 32 & 69 \\
\hline 44 & Normal daily mean, hottest 6 weeks of year $\left({ }^{\circ} \mathrm{F}\right.$. ). & 71.6 & $78.8+$ \\
\hline 45 & $\begin{array}{l}\text { Normal daily mean, year }\left({ }^{\circ} \mathrm{F} .\right) \ldots \ldots \ldots \ldots \ldots \ldots \\
\text { Precipitation: }\end{array}$ & 55 & $75+$ \\
\hline 46 & Normal daily mean, $f_{8}$ (inch) ..... & 103 & .173 \\
\hline 47 & Normal No. rainy days (over $0.10 \mathrm{inch}$ ), fs. & 120 & 284 \\
\hline 48 & Normal No. dry days (0.10 inch or less), $f s$. & 0 & 90 \\
\hline 49 & Dry days, percentage of total, $f_{8}$ (per cent). & 8 & 56 \\
\hline 50 & Days in longest normal rainy period, $f_{s} \ldots$. & 72 & 256 \\
\hline 51 & Days in longest normal dry period, $f_{s} .$. & 0 & 50 \\
\hline 52 & $\begin{array}{l}\text { Mean total, year (inches) . . . . . . . . . . . } \\
\text { Eraporation: }\end{array}$ & $50-$ & $60+$ \\
\hline 53 & $\begin{array}{l}\text { Eraporation: } \\
\text { Daily mean, } 1887-88, f_{8} \text { (inch). }\end{array}$ & .130 & .177 \\
\hline 54 & $\begin{array}{l}\text { Total annual, } 1887-88 \text { (inches) ..... } \\
\text { Moisture ratios: }\end{array}$ & 31.3 & 56.6 \\
\hline 58 & Normal $P / E, f_{s} \ldots$ & 64 & 1.76 \\
\hline 59 & Normal $\pi / E, f_{3} \ldots$ & .75 & 1.96 \\
\hline 60 & $\begin{array}{l}\text { Normal } P / E \text {, year................ } \\
\text { Vapor pressure: }\end{array}$ & .85 & 1.94 \\
\hline 63 & $\begin{array}{l}\text { Normal mean, fs (hundredths inch).. } \\
\text { Humidity: }\end{array}$ & $4 \times 8$ & 707 \\
\hline 65 & Normal mean, $f_{s}$ (per cent). & 69.1 & 82.7 \\
\hline 66 & $\begin{array}{l}\text { Normal mean, year (per cent) ......... } \\
\text { Wind: }\end{array}$ & 70.7 & 85.2 \\
\hline 68 & $\begin{array}{l}\text { Normal mean hourly velocity, } f_{s} \text { (miles) } \\
\text { Sunshine: }\end{array}$ & 5.1 & 13.5 \\
\hline 69 & $\begin{array}{l}\text { Normal total duration, } f_{s} \text { (hours). } \\
\text { Moisture-temperature indices: }\end{array}$ & 1,646 & 2,650 \\
\hline 70 & Normal $P / E \times T, f_{s}$, remainder method. & 446 & 1,418 \\
\hline 71 & Normal $P / E \times T, f_{8}$, exponential method. & 4,174 & 13,511 \\
\hline 72 & Normal $P / E \times T, f s$, physiological method. & 1,807 & 24,265 \\
\hline
\end{tabular}

TABLE 126.-Climatic extremes for Dulichium arundinaceum.

\begin{tabular}{|c|c|c|c|}
\hline Plate & Temperature: & Low. & Hioh. \\
\hline 34 & Days in normal frostless season $(f s)$ & 85 & \\
\hline 35 & Hot days, $f s . . . \ldots \ldots \ldots . . .$. & 0 & 189 \\
\hline 36 & Cold days, $f_{s}$. & 0 & 149 \\
\hline 37 & Remainder summation above $32^{\circ}$, year (thousands) & $10.0-$ & $18.0+$ \\
\hline 38 & Remainder summation above $39^{\circ}, f_{s}$ (thousands).. & 2.6 & 9.4 \\
\hline 39 & Exponential summation, $f_{s}$ (hundreds) ......... & 3.0 & 10.3 \\
\hline 40 & Physiological summation, $f_{8}$ (thousands). & 2.1 & 15.9 \\
\hline 41 & 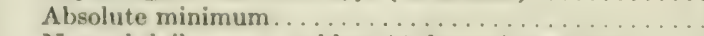 & -59 & +20 \\
\hline 43 & Normal daily mean, coldest 14 days of year $\left({ }^{\circ} \mathrm{F}.\right)$. & 0 & 52 \\
\hline 44 & Normal daily mean, hottest 6 weeks of year $\left({ }^{\circ} \mathrm{F}.\right)$. & $64.4-$ & $75.8+$ \\
\hline 45 & $\begin{array}{l}\text { Normal daily mean, year }\left({ }^{\circ} \mathrm{F}_{0}\right) \ldots \ldots \ldots \ldots \ldots \ldots \\
\text { Precipitation: }\end{array}$ & 35 & $70-$ \\
\hline 46 & Normal daily mean, $f_{s}$ (inch). & 074 & .199 \\
\hline 47 & Normal No. rainy days (over $0.10 \mathrm{inch}$ ), $f s$. & 26 & 245 \\
\hline 48 & Normal No. dry days (0.10 inch or less), $f_{s}$. & 0 & 200 \\
\hline 49 & Dry days, percentage of total, $f_{s}$ (per cent) ..... & 11 & 8.3 \\
\hline 50 & Days in longest normal rainy period, $f_{s} \ldots$... & 21 & 256 \\
\hline 51 & Days in longest normal dry period, $f_{s}$. . & 0 & 157 \\
\hline 52 & Mean total, year (inches) ............. & 20 & 90 \\
\hline
\end{tabular}


TABLE 126.-Climatic extremes for Dulichium arundinaceum-Continued.

\begin{tabular}{|c|c|c|c|}
\hline Plate & Evaporation: & Low. & High. \\
\hline 53 & Daily mean, $1887-88, f s$ (inch) $\ldots \ldots \ldots \ldots \ldots \ldots \ldots$ & .052 & .200 \\
\hline 54 & $\begin{array}{l}\text { Total annual, } 1887-88 \text { (inches) } \ldots \ldots \ldots \ldots \ldots \ldots \ldots \ldots \\
\text { Moisture ratios: }\end{array}$ & 18.1 & 56.6 \\
\hline 58 & 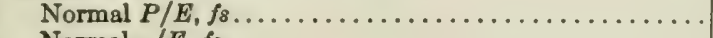 & .51 & 3.84 \\
\hline 59 & 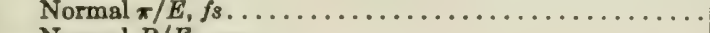 & .60 & 4.48 \\
\hline 60 & $\begin{array}{l}\text { Normal } P / E \text {, year. } \ldots \ldots \ldots \ldots \ldots \ldots \ldots \ldots \ldots \ldots \\
\text { Vapor pressure: }\end{array}$ & .71 & 4.90 \\
\hline 63 & $\begin{array}{l}\text { Normal mean, } f_{s} \text { (hundredths inch) } \ldots \ldots \ldots \ldots \ldots \ldots \\
\text { Humidity: }\end{array}$ & 318 & 610 \\
\hline 65 & 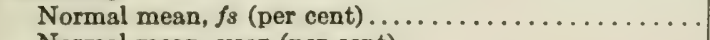 & 64.5 & 82.7 \\
\hline 66 & $\begin{array}{l}\text { Normal mean, year (per cent) } \ldots \ldots \ldots \ldots \ldots \ldots \ldots \ldots \\
\text { Wind: }\end{array}$ & 67.5 & 82.9 \\
\hline 68 & $\begin{array}{l}\text { Normal mean hourly velocity, } f s \text { (miles) } \ldots \ldots \ldots \ldots \ldots \\
\text { Sunshine: }\end{array}$ & 3.1 & 13.5 \\
\hline 69 & $\begin{array}{l}\text { Normal total duration, } f_{s} \text { (hours) } \ldots \ldots \ldots \ldots \ldots \ldots \ldots \\
\text { Moisture-temperature indices: }\end{array}$ & 1,225 & 2,026 \\
\hline $\begin{array}{l}70 \\
71\end{array}$ & Normal $P / E \times T, f s$, remainder method............... & $\begin{array}{r}301 \\
2,918\end{array}$ & $\begin{array}{r}1,418 \\
13,511\end{array}$ \\
\hline 72 & 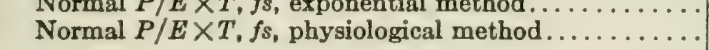 & $\begin{array}{l}2,918 \\
2,747\end{array}$ & 24,265 \\
\hline
\end{tabular}

TABLE 127.-Climatic extremes for Spartina michauxiana.

\begin{tabular}{|c|c|c|c|}
\hline Plate & Temperature: & Low. & High. \\
\hline 34 & Days in normal frostless season $\left(f_{8}\right) \ldots \ldots \ldots \ldots \ldots$ & 53 & 231 \\
\hline 35 & 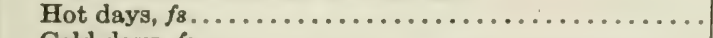 & 0 & 153 \\
\hline 36 & Cold days, $f_{s}, \ldots \ldots \ldots$. & 0 & 158 \\
\hline 37 & Remainder summation above $32^{\circ}$, year (thousands).... & $10.0-$ & $18.0+$ \\
\hline 38 & Remainder summation above $39^{\circ}, f s$ (thousands) ...... & 2.6 & 7.5 \\
\hline 39 & Exponential summation, $f_{s}$ (hundreds)..$\ldots \ldots \ldots \ldots$ & 2.8 & 8.1 \\
\hline 40 & Physiological summation, $f s$ (thousands) ............ & 2.1 & 14.6 \\
\hline 41 & Absolute minimum.......... & -65 & +4 \\
\hline 43 & Normal daily mean, coldest 14 days of year $\left({ }^{\circ} \mathrm{F}.\right) . . .$. & $\mathbf{0}$ & 42 \\
\hline 44 & Normal daily mean, hottest 6 weeks of year $\left({ }^{\circ} \mathrm{F}.\right)$.... & $64.4-$ & $78.8+$ \\
\hline 45 & $\begin{array}{l}\text { Normal daily mean, year }\left({ }^{\circ} \mathrm{F} .\right) \ldots \ldots \ldots \ldots \ldots \ldots \ldots \\
\text { Precipitation: }\end{array}$ & 35 & $60+$ \\
\hline 46 & Normal daily mean, $f_{s}$ (inch) $\ldots \ldots \ldots \ldots \ldots \ldots$ & .045 & .136 \\
\hline 47 & Normal No. rainy days (over 0.10 inch), $f s \ldots . . .$. & 0 & 182 \\
\hline 48 & Normal No. dry days $(0.10$ inch or less $), f s . . . \ldots \ldots$ & 19 & 140 \\
\hline 49 & Dry days, percentage of total, $f_{8}$ (per cent).......... & 11 & 100 \\
\hline 50 & Days in longest normal rainy period, $f_{s} \ldots \ldots \ldots \ldots$ & 0 & 144 \\
\hline 51 & Days in longest normal dry period, $f s . . . \ldots \ldots \ldots$ & 4 & 144 \\
\hline 52 & $\begin{array}{l}\text { Mean total, year (inches) } \ldots \ldots \ldots \ldots \ldots \ldots \ldots \ldots \\
\text { Evaporation: }\end{array}$ & $20-$ & $60+$ \\
\hline 53 & Daily mean, $1887-88, f_{s}$ (inch) .... & .084 & .234 \\
\hline 54 & $\begin{array}{l}\text { Total annual, } 1887-88 \text { (inches) } \ldots \ldots \ldots \ldots \ldots \ldots \ldots \ldots \\
\text { Moisture ratios: }\end{array}$ & 20.3 & 54.8 \\
\hline 58 & 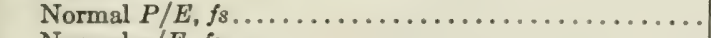 & .21 & 1.39 \\
\hline 59 & 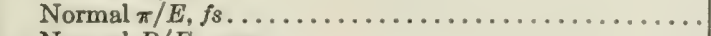 & .26 & 1.63 \\
\hline 60 & 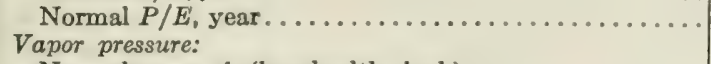 & .24 & 1.72 \\
\hline 63 & $\begin{array}{l}\text { Normal mean, fs (hundredths inch)....... } \\
\text { Humidity: }\end{array}$ & 249 & 545 \\
\hline 65 & Normal mean, $f s$ (per cent) & 48.0 & 84.0 \\
\hline 66 & $\begin{array}{l}\text { Normal mean, year (per cent) } \ldots \ldots \ldots \ldots \ldots \ldots \ldots \ldots \\
\text { Wind: }\end{array}$ & 56.1 & 82.1 \\
\hline 68 & $\begin{array}{l}\text { Normal mean hourly velocity, } f_{s} \text { (miles) } \ldots \ldots \ldots \ldots \ldots \\
\text { Sunshine: }\end{array}$ & 3.1 & 14.9 \\
\hline 69 & $\begin{array}{l}\text { Normal total duration, } f_{s} \text { (hours) } \\
\text { Moisture-temperature indices: }\end{array}$ & 1,225 & 2,166 \\
\hline 70 & 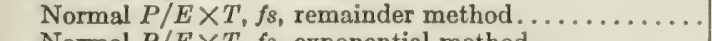 & 72 & 707 \\
\hline 71 & Normal $P / E \times T, f s$, exponential method.................... & 691 & 6,858 \\
\hline 72 & Normal $P / E \times T, f s$, physiological method.......... & 809 & 11,246 \\
\hline
\end{tabular}


TABLE 128.-Climatic extremes for Arceuthobium cryplopodum.

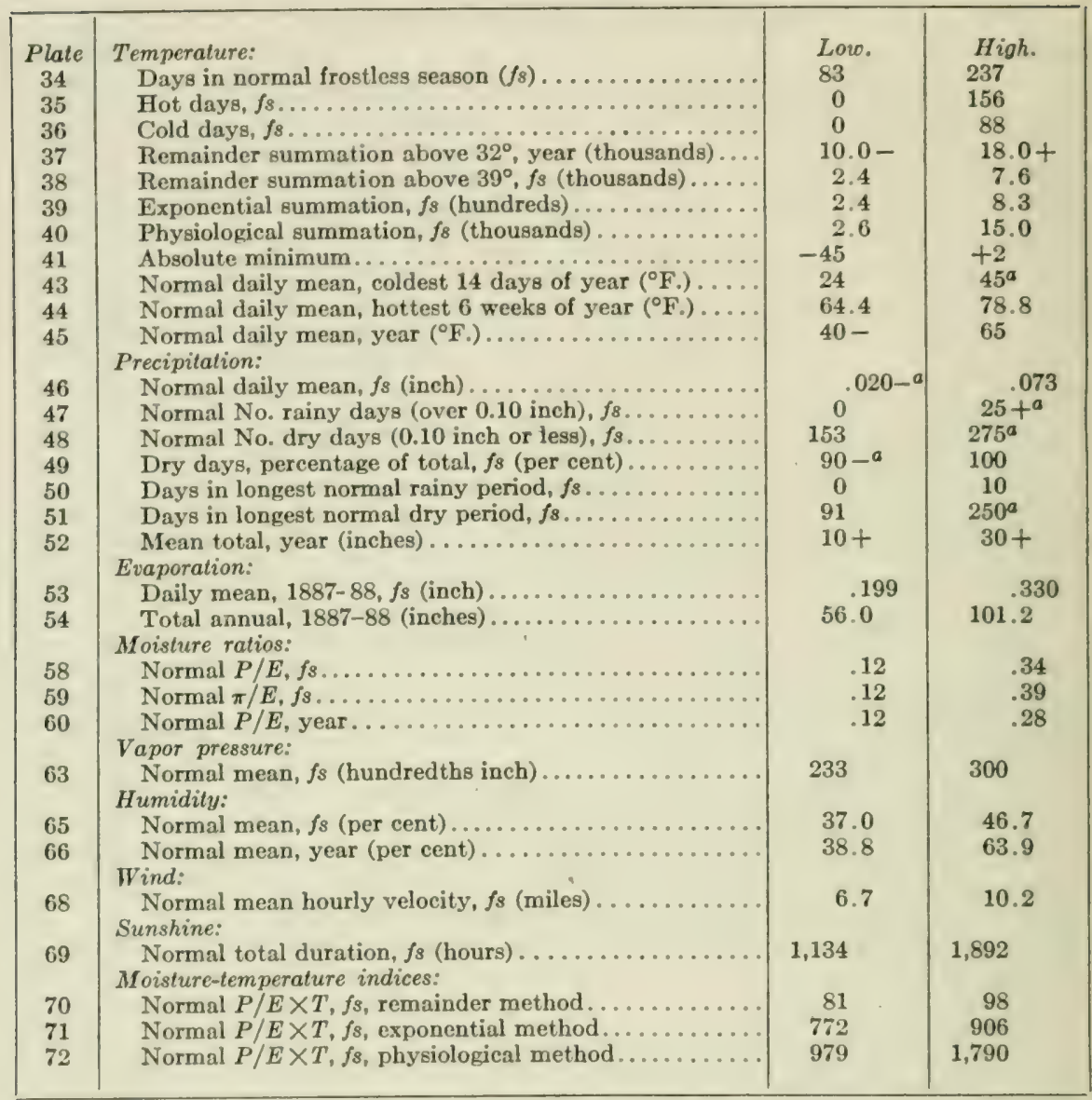

TABLE 129.-Climatic extremes for Arceuthobium americanum.

\begin{tabular}{|c|c|c|c|}
\hline Plate & Temperature: & Low. & High. \\
\hline 34 & Days in normal frostless season $\left(f_{s}\right)$. & 25 & \\
\hline 35 & Hot days, $f_{8} \ldots \ldots \ldots \ldots \ldots \ldots . . . .$. & 0 & 105 \\
\hline 36 & Cold days, $f s . . . \ldots \ldots \ldots \ldots \ldots$. & 26 & 121 \\
\hline 37 & Remainder summation above $32^{\circ}$, year (thousands). & 10.0 & $18.0+$ \\
\hline 38 & Remainder summation above $39^{\circ}, f_{3}$ (thousands).... & 2.4 & 5.4 \\
\hline 39 & Exponential summation, $f_{s}$ (hundreds).. & 2.4 & 5.7 \\
\hline 40 & Physiological summation, $f_{s}$ (thousands). & 2.6 & 8.4 \\
\hline 41 & 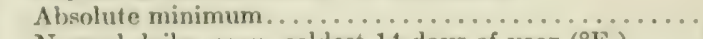 & -49 & +11 \\
\hline 43 & Normal daily mean, coldest 14 days of year $\left({ }^{\circ} \mathrm{F}_{0}\right)$. & 17 & \\
\hline 44 & Normal daily mean, hottest 6 weeks of year $\left({ }^{\circ} \mathrm{F}\right.$.). & $64.4-$ & $78.8+$ \\
\hline 45 & Normal daily mean, year $\left({ }^{\circ} \mathrm{F}, \ldots . \ldots \ldots\right.$ & $40-$ & 60 \\
\hline 46 & $\begin{array}{l}\text { Precipitation: } \\
\text { Normal daily mean } f_{s} \text { (inch).... }\end{array}$ & & \\
\hline 47 & $\begin{array}{l}\text { Normal daily mean, } f_{s} \text { (inch) } \ldots \ldots \ldots \ldots \ldots \ldots \\
\text { Normal No. rainy days (over } 0.10 \text { inch), } f_{s} . .\end{array}$ & $0^{.009}$ & $25+067$ \\
\hline 48 & Normal No. dry days (0.10 inch or less), fs........ & $10 \%$ & 216 \\
\hline 49 & Dry days, percentnge of total, $f$ s (per cent).... . & $90-a$ & 100 \\
\hline 50 & Days in longest normal rainy period, $f_{s} \ldots \ldots$. & 0 & 7 \\
\hline 51 & Days in longest normal dry period, $f_{3} \ldots \ldots \ldots$ & $100-a$ & 210 \\
\hline 52 & Menn total, year (inches)....... & $10+$ & $50+$ \\
\hline
\end{tabular}


TABLE 129.-Climatic extremes for Arceuthobium americanum-Continued.

\begin{tabular}{|c|c|c|c|}
\hline Plate & Evaporation: & Low. & High. \\
\hline 53 & Daily mean, $1887-88, f s$ (inch) $\ldots \ldots \ldots \ldots \ldots \ldots$ & .100 & .349 \\
\hline 54 & $\begin{array}{l}\text { Total annual, } 1887-88 \text { (inches) } \ldots \ldots \ldots \ldots \ldots \ldots \ldots \ldots \\
\text { Moisture ratios: }\end{array}$ & 42.8 & 100.6 \\
\hline 58 & 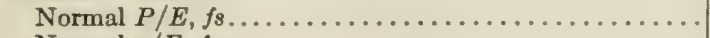 & .04 & .34 \\
\hline 59 & Normal $\pi / E, f s \ldots \ldots \ldots \ldots \ldots \ldots \ldots \ldots \ldots$ & .06 & .39 \\
\hline 60 & 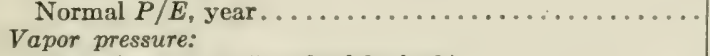 & .09 & $1.30^{a}$ \\
\hline 63 & $\begin{array}{l}\text { Normal mean, } f_{s} \text { (hundredths inch) ................ } \\
\text { Humidity: }\end{array}$ & 183 & 285 \\
\hline 65 & Normal mean, $f s$ (per cent).......... & 22.6 & 54.6 \\
\hline 66 & $\begin{array}{l}\text { Normal mean, year (per cent) } \ldots \ldots \ldots \ldots \ldots \ldots \ldots \ldots \\
\text { Wind: }\end{array}$ & 29.7 & 64.8 \\
\hline 68 & $\begin{array}{l}\text { Normal mean hourly velocity, fs (miles) } \ldots \ldots \ldots \ldots \ldots \\
\text { Sunshine: }\end{array}$ & 4.9 & 11.4 \\
\hline 69 & $\begin{array}{l}\text { Normal total duration, } f_{s} \text { (hours) } \ldots \ldots \ldots \ldots \ldots \ldots \\
\text { Moisture-temperature indices: }\end{array}$ & 1,127 & 1,927 \\
\hline 70 & 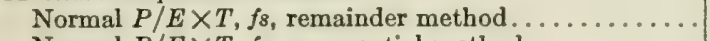 & 13 & $300^{a}$ \\
\hline 71 & Normal $P / E \times T, f s$, exponential method.......... & 127 & 950 \\
\hline 72 & Normal $P / E \times T, f s$, physiological method.......... & 197 & 1,475 \\
\hline
\end{tabular}

TABLE 130.-Climatic extremes for Phoradendron flavescens and varieties.

\begin{tabular}{|c|c|c|c|}
\hline Plate & Temperature: & Low. & High. \\
\hline 34 & Days in normal frostless season $(f s) \ldots \ldots \ldots \ldots$ & 148 & 365 \\
\hline 35 & 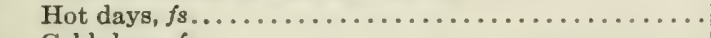 & 0 & 365 \\
\hline 36 & Cold days, $f s \ldots \ldots \ldots \ldots \ldots \ldots \ldots \ldots \ldots \ldots \ldots$ & 0 & 44 \\
\hline 37 & Remainder summation above $32^{\circ}$, year (thousands).... & $11.5-$ & $26.0+$ \\
\hline 38 & Remainder summation above $39^{\circ}, f_{s}$ (thousands)...... & 3.5 & 14.5 \\
\hline 39 & 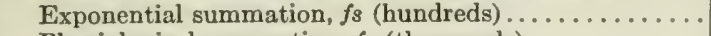 & 4.1 & 15.4 \\
\hline 40 & Physiological summation, $f s$ (thousands) ............ & 2.4 & 31.1 \\
\hline 41 & 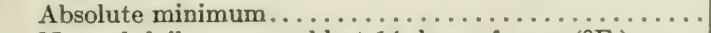 & -34 & +41 \\
\hline 43 & Normal daily mean coldest 14 days of year $\left({ }^{\circ} \mathrm{F}.\right) \ldots$ & 28 & 69 \\
\hline 44 & Normal daily mean, hottest 6 weeks of year $\left({ }^{\circ} \mathrm{F}.\right) \ldots$ & $64.4-$ & $78.8+$ \\
\hline 45 & $\begin{array}{l}\text { Normal daily mean, year }\left({ }^{\circ} \mathrm{F} .\right) \ldots \ldots \ldots \ldots \ldots \ldots \ldots \\
\text { Precipitation: }\end{array}$ & $55-$ & $75+$ \\
\hline 46 & 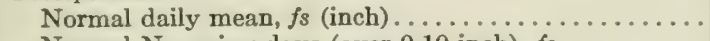 & .020 & .173 \\
\hline 47 & Normal No. rainy days (over 0.10 inch), $f_{s} \ldots \ldots \ldots$ & 0 & 284 \\
\hline 48 & Normal No. dry days (0.10 inch or less), $f$ s.......... & 26 & 294 \\
\hline 49 & Dry days, percentage of total, $f_{s}$ (per cent)......... & 8 & 100 \\
\hline 50 & Days in longest normal rainy period, $f s, \ldots \ldots \ldots \ldots$ & 0 & 235 \\
\hline 51 & Days in longest normal dry period, $f 8 \ldots \ldots \ldots \ldots \ldots$ & 9 & 299 \\
\hline 52 & $\begin{array}{l}\text { Mean total, year (inches) } \ldots \ldots \ldots \ldots \ldots \ldots \ldots \ldots \\
\text { Evaporation: }\end{array}$ & $10+$ & $70+$ \\
\hline 53 & Daily mean, $1887-88, f_{s}$ (inch) $\ldots \ldots \ldots \ldots \ldots \ldots$ & .084 & .330 \\
\hline 54 & $\begin{array}{l}\text { Total annual, } 1887-88 \text { (inches) } \ldots \ldots \ldots \ldots \ldots \ldots \ldots \\
\text { Moisture ratios: }\end{array}$ & 25.2 & 101.2 \\
\hline 58 & 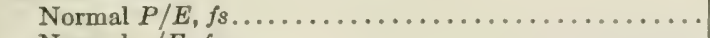 & .08 & 1.76 \\
\hline 59 & 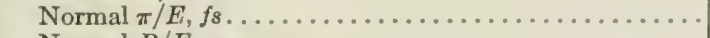 & .10 & 1.96 \\
\hline 60 & 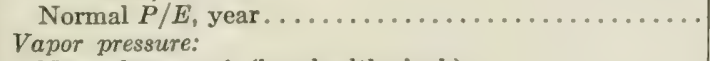 & .03 & $2.00^{a}$ \\
\hline 63 & $\begin{array}{l}\text { Normal mean, } f_{s} \text { (hundredths inch)....................... } \\
\text { Humidity: }\end{array}$ & 279 & 707 \\
\hline 65 & Normal mean, $f_{s}$ (per cent) $\ldots \ldots \ldots \ldots \ldots \ldots \ldots$ & 36.3 & 87.5 \\
\hline 66 & $\begin{array}{l}\text { Normal mean, year (per cent) ..................... } \\
\text { Wind: }\end{array}$ & 38.1 & 86.8 \\
\hline 68 & $\begin{array}{l}\text { Normal mean hourly velocity, } f_{s} \text { (miles).... } \\
\text { Sunshine: }\end{array}$ & 3.5 & 14.2 \\
\hline 69 & $\begin{array}{l}\text { Normal total duration, } f_{s} \text { (hours) ...... } \\
\text { Moisture-temperature indices: }\end{array}$ & 1,651 & 2,650 \\
\hline 70 & 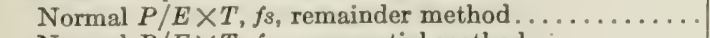 & 68 & 1,418 \\
\hline 71 & 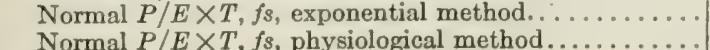 & $\begin{array}{r}625 \\
1,186\end{array}$ & $\begin{array}{l}13,511 \\
24,265\end{array}$ \\
\hline 72 & Normal $P / E \times T, f s$, physiological method.......... & 1,180 & 24,200 \\
\hline
\end{tabular}


TABLE 131.-Climatic extremes for Phoradendron juniperinum.

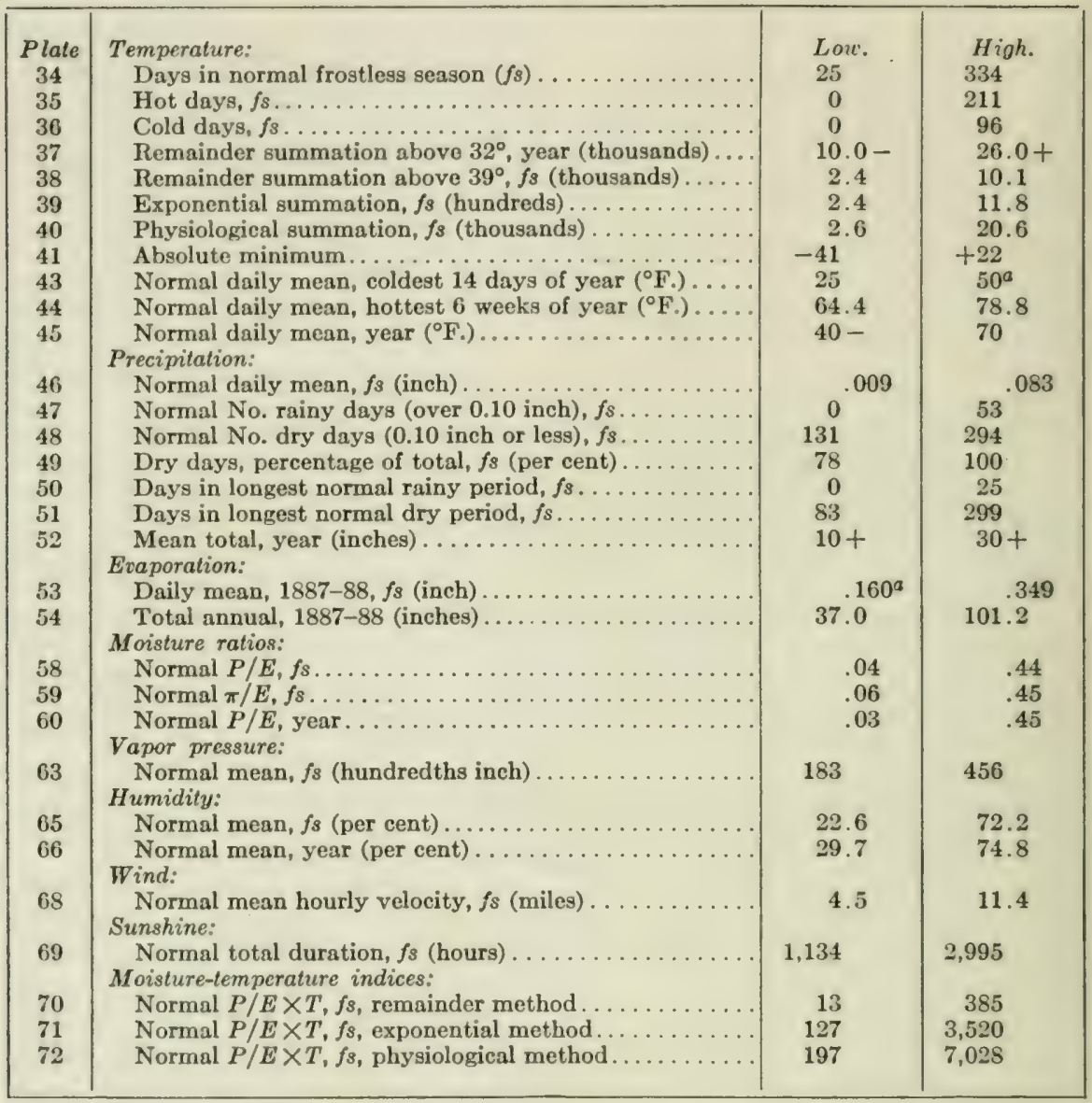

Table 132.-Climatic extremes for Arenaria lateriflora.

\begin{tabular}{|c|c|c|c|}
\hline Plate & Temperature: & Low. & High. \\
\hline 34 & Days in normal frostless season $(f s)$ & & \\
\hline & Hot days, $f s \ldots \ldots \ldots \ldots \ldots \ldots . . . .$. & 0 & 127 \\
\hline 36 & Cold days, fs ...... & 0 & 158 \\
\hline 37 & Remainder summation above $32^{\circ}$, year (thousands) & $10.0-$ & $11.5+$ \\
\hline 38 & Remainder summation above $39^{\circ}$, fs (thousands).. & 2.6 & 5.7 \\
\hline 39 & Exponential summation, $f_{s}$ (hundreds). & 2.8 & 6.8 \\
\hline 40 & Physiological summation, $f_{s}$ (thousands). & 2.1 & 11.9 \\
\hline 41 & Absolute minimum............... & -65 & +30 \\
\hline 43 & Normal daily mean, coldest 14 days of year $\left({ }^{\circ} \mathrm{F}\right.$. $)$ & 0 & 42 \\
\hline 44 & Normal daily mean, hottest 6 weelis of year $\left({ }^{\circ} \mathrm{F}\right.$.). & $64.4-$ & 78.8 \\
\hline 45 & Normal daily mean, year $\left({ }^{\circ} \mathrm{F}.\right) \ldots$ & 35 & 55 \\
\hline & Precipitation: & & \\
\hline 46 & Normal daily mean, $f_{s}$ (inch). & .022 & 199 \\
\hline 47 & Normal No. rainy days (over $0.10 \mathrm{inch}$ ), $f_{s}$. & 0 & 199 \\
\hline 48 & Normal No. dry days ( 0.10 inch or less), $f s$. & 28 & 216 \\
\hline 49 & Dry days, percentage of total, $f_{s}$ (per cent). & 17 & 100 \\
\hline 50 & Days in longest normal rainy period, fs..... & 0 & 132 \\
\hline 51 & Days in longest normal dry period, $f_{s} . . . \ldots \ldots$ & 9 & 216 \\
\hline 52 & Mean total, year (inches) ............ & $10+$ & 90 \\
\hline
\end{tabular}


TABLE 132.-Climatic extremes for Arenaria lateriflora-Continued.

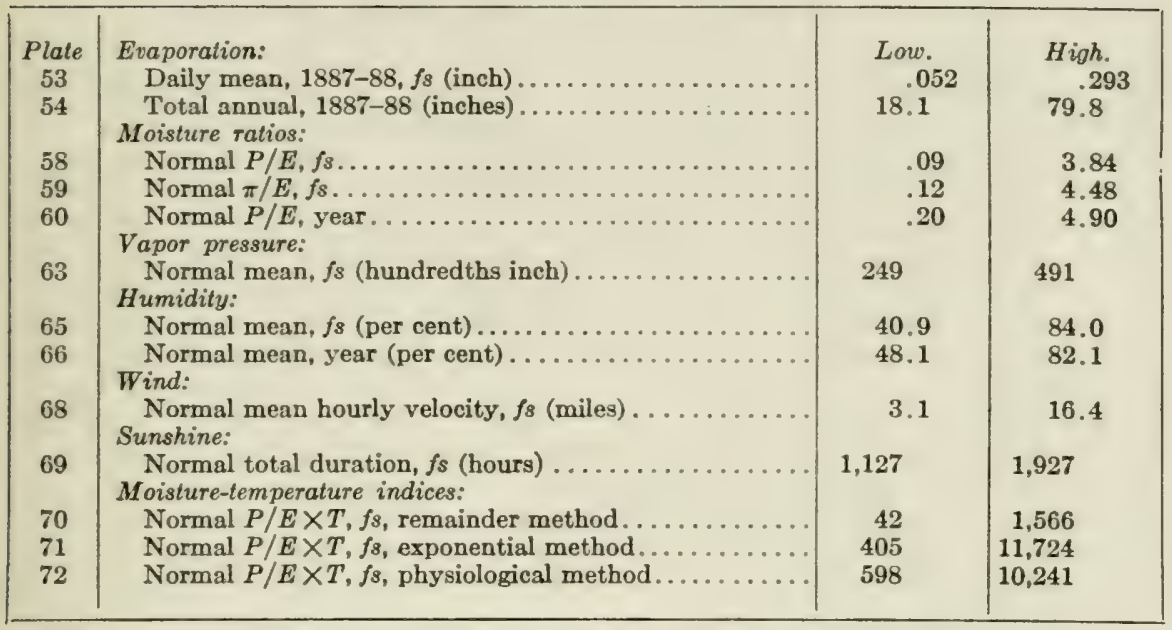

TABle 133.-Climatic extremes for Parietaria pennsylvanica.

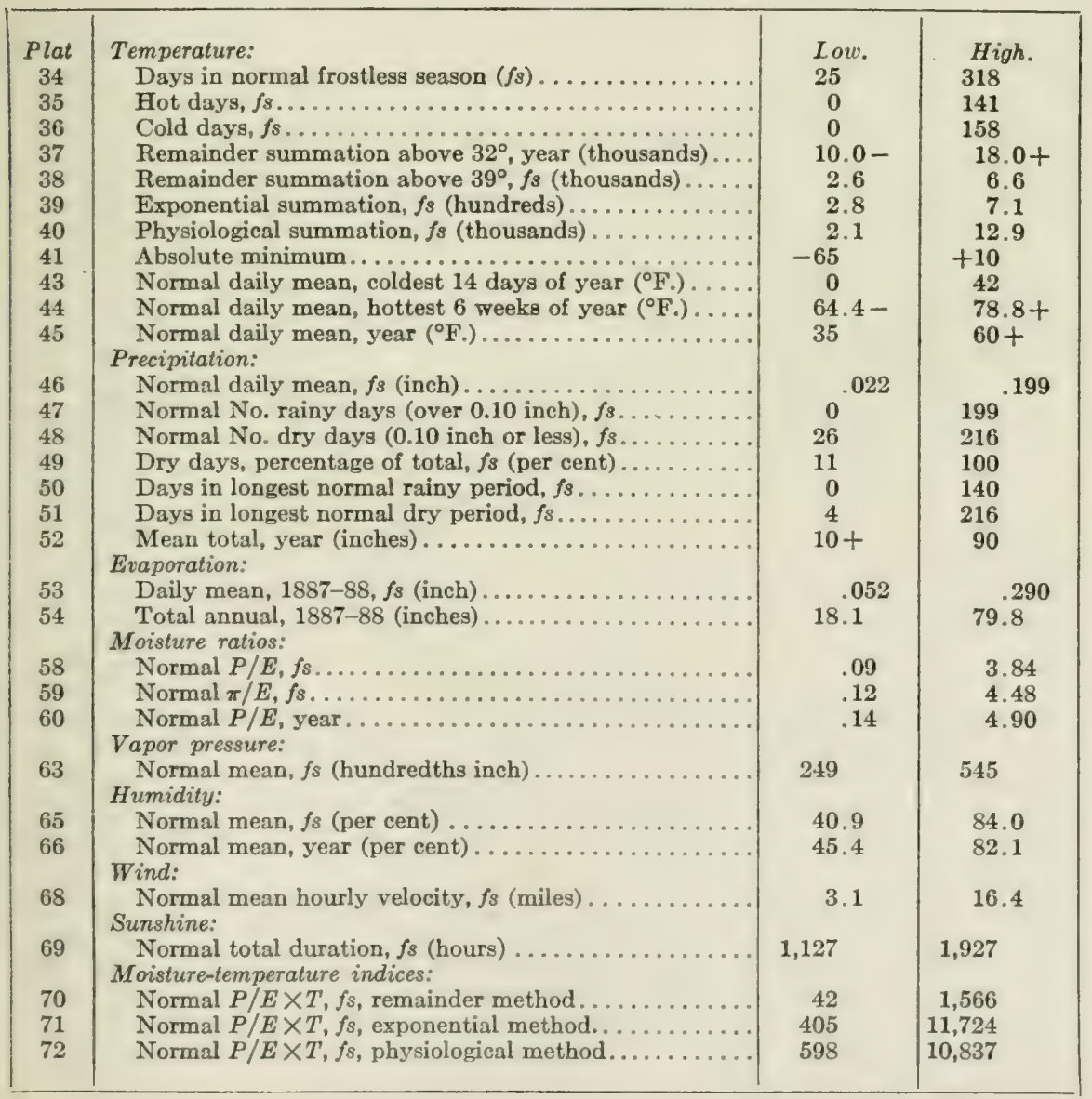


TABLE 134.-Climatic extremes for Cornus canadensis.

\begin{tabular}{|c|c|c|c|}
\hline Plate & Temperature: & Low. & High. \\
\hline 34 & Days in normal frostless season $(f s) \ldots \ldots \ldots \ldots \ldots$ & 25 & 318 \\
\hline 35 & 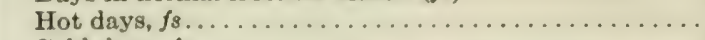 & 0 & 88 \\
\hline 36 & 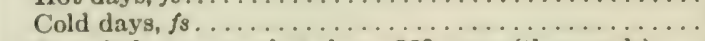 & 0 & 158 \\
\hline 37 & Remainder summation above $32^{\circ}$, year (thousands).... & $10.0-$ & $18.0+$ \\
\hline 38 & Remainder summation above $39^{\circ}, f_{8}$ (thousands)...... & 2.6 & 5.1 \\
\hline 39 & Exponential summation, $f_{8}$ (hundreds) . . . . . . . . & 2.8 & 5.4 \\
\hline 40 & Physiological summation; $f_{s}$ (thousands) . . . . . . . . & 2.1 & 8.0 \\
\hline 41 & 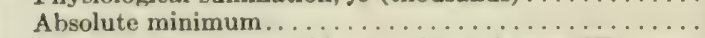 & -59 & +30 \\
\hline 43 & Normal daily mean, coldest 14 days of year $\left({ }^{\circ} \mathrm{F}.\right) \ldots$ & 0 & 46 \\
\hline 44 & Normal daily mean, hottest 6 weeks of year ( $\left.{ }^{\circ} \mathrm{F}.\right) . .$. & $64.4-$ & 78.8 \\
\hline 45 & $\begin{array}{l}\text { Normal daily mean, year }\left({ }^{\circ} \mathbf{F}\right) \ldots \ldots \ldots \ldots \ldots \ldots \ldots \\
\text { Precipitation: }\end{array}$ & 35 & 60 \\
\hline 46 & Normal daily mean, $f_{s}$ (inch) & .037 & .199 \\
\hline 47 & Normal No. rainy days (over 0.10 inch), $f_{s} \ldots \ldots \ldots$ & 0 & 199 \\
\hline 48 & Normal No. dry days $\left(0.10\right.$ inch or less), $f_{8} \ldots \ldots \ldots$ & 28 & 202 \\
\hline 49 & Dry days, percentage of total, $f_{8}$ (per cent)........... & 17 & 100 \\
\hline 50 & Days in longest normal rainy period, $f_{8} \ldots \ldots \ldots \ldots$ & 0 & 106 \\
\hline 51 & Days in longest normal dry period, $f s \ldots \ldots \ldots \ldots \ldots$ & 9 & 202 \\
\hline 52 & $\begin{array}{l}\text { Mean total, year (inches) } \ldots \ldots \ldots \ldots \ldots \ldots \ldots \ldots \\
\text { Evaporation: }\end{array}$ & $20-$ & 90 \\
\hline 53 & Daily mean, $1887-88, f s$ (inch) $\ldots \ldots \ldots \ldots \ldots \ldots$ & .052 & .275 \\
\hline 54 & $\begin{array}{l}\text { Total annual, } 1887-88 \text { (inches) } \ldots \ldots \ldots \ldots \ldots \ldots \ldots \ldots \\
\text { Moisture ratios: }\end{array}$ & 18.1 & 76.5 \\
\hline 58 & Normal $P / E, f s \ldots$ & .19 & 3.84 \\
\hline 59 & 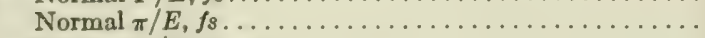 & .25 & 4.48 \\
\hline 60 & 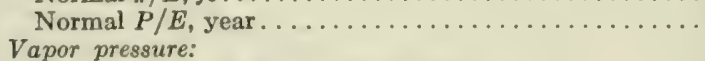 & .20 & 4.90 \\
\hline 63 & $\begin{array}{l}\text { Normal mean, } f_{s} \text { (hundredths inch) } \ldots \ldots \ldots \ldots \ldots \ldots \\
\text { Humidity: }\end{array}$ & 249 & 447 \\
\hline 65 & Normal mean, $f_{8}$ (per cent). & 46.7 & 84.0 \\
\hline 66 & $\begin{array}{l}\text { Normal mean, year (per cent). } \\
\text { Wind: }\end{array}$ & 48.7 & 82.1 \\
\hline 68 & $\begin{array}{l}\text { Normal mean hourly velocity, } f_{s} \text { (miles)... } \\
\text { Sunshine: }\end{array}$ & 3.5 & 16.4 \\
\hline 69 & $\begin{array}{l}\text { Normal total duration, } f_{s} \text { (hours) . . . . . } \\
\text { Moisture-temperature indices: }\end{array}$ & 1,127 & 1,927 \\
\hline 70 & 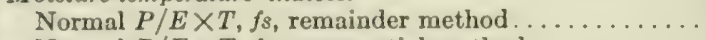 & 58 & 1,566 \\
\hline 71 & Normal $P / E \times T, f_{s}$, exponential method.......... & 563 & 11,724 \\
\hline 72 & 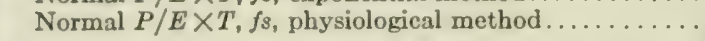 & 710 & 7,569 \\
\hline
\end{tabular}

TABLE 135.-Climatic extremes for Spermolepis echinata.

\begin{tabular}{|c|c|c|c|}
\hline Plate & Temperature: & Lou. & High. \\
\hline 34 & Days in normal frostless season $(f s)$ & 167 & 334 \\
\hline 35 & 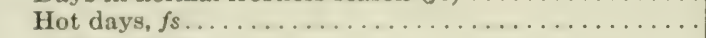 & 0 & 365 \\
\hline 36 & 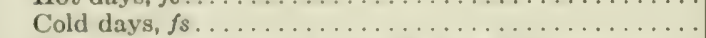 & 0 & 43 \\
\hline 37 & Remainder summation above $32^{\circ}$, year (thousands).... & $18.0-$ & $26.0+$ \\
\hline 38 & Remainder summation above $39^{\circ}, f_{s}$ (thousands)....... & 5.6 & 10.6 \\
\hline 39 & 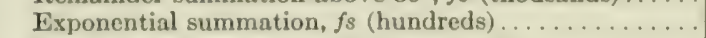 & 5.0 & 11.8 \\
\hline 40 & Physiological summation, $f_{s}$ (thousands) . . . . . . & 4.1 & 21.4 \\
\hline 41 & 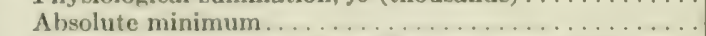 & -32 & +32 \\
\hline 43 & Normal daily mean, coldest 14 days of year $\left({ }^{\circ} \mathrm{F}\right.$ )... & 34 & 54 \\
\hline 44 & Normal daily mean, hottest 6 weeks of year $\left({ }^{\circ} \mathrm{F}_{0}\right) \ldots$. & $64.4-$ & $7 S .8+$ \\
\hline 45 & $\begin{array}{l}\text { Normal daily mean, year }\left({ }^{\circ} \mathrm{F}_{0}\right) \ldots \ldots \ldots \ldots \ldots \ldots \ldots \\
\text { Precipitation: }\end{array}$ & $60-$ & $70+$ \\
\hline 46 & Normal daily mean, $f s$ (inch). & 020 & 172 \\
\hline 47 & Normal No. rainy days (over 0.10 inch), $f s \ldots \ldots \ldots$ & 0 & 254 \\
\hline 48 & Normal No. dry days (0.10 inch or less), $f_{s} \ldots \ldots \ldots$ & 26 & 294 \\
\hline 49 & Dry days, percentage of total, $f_{s}$ (per cent)....... & s & 100 \\
\hline 50 & Days in longest normal rainy period, $f_{s} \ldots \ldots \ldots \ldots$ & 0 & $15 \pi$ \\
\hline 51 & Days in longest normal dry period, $f s \ldots \ldots \ldots \ldots \ldots$ & 14 & 299 \\
\hline 52 & Mean total, year (inches) . . . . . . . . . . . . . . & $10-$ & $60-$ \\
\hline
\end{tabular}


TABLE 135.-Climatic extremes for Spermolepis echinata-Continued.

\begin{tabular}{|c|c|c|c|}
\hline Plate & Evaporation: & Low. & High. \\
\hline 53 & Daily mean, $1887-88, f_{s}$ (inch). & .102 & .330 \\
\hline 54 & $\begin{array}{l}\text { Total annual, } 1887-88 \text { (inches)............ } \\
\text { Moisture ratios: }\end{array}$ & 36.7 & 101.2 \\
\hline 58 & Normal $P / E, f_{s} \ldots \ldots$ & .12 & 1.36 \\
\hline 59 & Normal $\pi / E, f_{s} \ldots$ & .13 & 1.52 \\
\hline 60 & $\begin{array}{l}\text { Normal } P / E \text {, year..... } \\
\text { Vapor pressure: }\end{array}$ & .03 & 1.47 \\
\hline 63 & $\begin{array}{l}\text { Normal mean, } f_{s} \text { (hundredths inch)... } \\
\text { Humidity: }\end{array}$ & 300 & 675 \\
\hline 65 & Normal mean, $f_{s}$ (per cent)... & 36.3 & 81.9 \\
\hline 66 & $\begin{array}{l}\text { Normal mean, year (per cent)... } \\
\text { Wind: }\end{array}$ & 38.8 & 85.2 \\
\hline 68 & $\begin{array}{l}\text { Normal mean hourly velocity, } f_{s} \text { (miles). . } \\
\text { Sunshine: }\end{array}$ & 4.5 & 12.3 \\
\hline 69 & $\begin{array}{l}\text { Normal total duration, } f_{s} \text { (hours) ....... } \\
\text { Moisture-temperature indices: }\end{array}$ & 1,895 & 2,995 \\
\hline 70 & Normal $P / E \times T, f_{s}$, remainder method... & 98 & 1,314 \\
\hline 71 & Normal $P / E \times T, f s$, exponential method. & 625 & 12,106 \\
\hline 72 & Normal $P / E \times T, f_{s}$, physiological method. & 1,186 & 23,652 \\
\hline
\end{tabular}

TABLE 136.-Climatic extremes for Daucus pusillus.

\begin{tabular}{|c|c|c|c|}
\hline Plate & Temperature: & Low. & High. \\
\hline 34 & Days in normal frostless season $(f s)$. & 166 & 365 \\
\hline 35 & Hot days, $f_{3} \ldots \ldots \ldots \ldots \ldots \ldots \ldots$ & 0 & 365 \\
\hline 36 & Cold days, $f_{s} \ldots$ & 0 & 43 \\
\hline 37 & Remainder summation above $32^{\circ}$, year (thousands) & 10.0 & $26.0+$ \\
\hline 38 & Remainder summation above $39^{\circ}, f_{3}$ (thousands). & 3.5 & 14.5 \\
\hline 39 & Exponential summation, $f s$ (hundreds)............ & 4.1 & 15.4 \\
\hline 40 & Physiological summation, $f_{s}$ (thousands) ......... & 23.9 & 31.1 \\
\hline 41 & Absolute minimum ............ & -30 & +41 \\
\hline 43 & Normal daily mean, coldest 14 days of year $\left({ }^{\circ} \mathrm{F}.\right)$ & 31 & 69 \\
\hline 44 & Normal daily mean, hottest 6 weeks of year $\left({ }^{\circ} \mathrm{F}\right.$.).... & 64.4 & 78.8 \\
\hline 45 & $\begin{array}{l}\text { Normal daily mean, year }\left({ }^{\circ} F_{0}\right) \ldots \ldots \ldots \ldots \ldots \ldots \ldots \\
\text { Precipitation: }\end{array}$ & $50-$ & $75+$ \\
\hline 46 & Normal daily mean, $f_{s}$ (inch) & .020 & 199 \\
\hline 47 & Normal No. rainy days (over $0.10 \mathrm{inch}$ ), $f_{s}$. & 0 & 284 \\
\hline 48 & Normal No. dry days ( 0.10 inch or less), $f s$. & 0 & 294 \\
\hline 49 & Dry days, percentage of total, $f_{s}$ (per cent).. & 0 & 100 \\
\hline 50 & Days in longest normal rainy period, $f_{s} . .$. & 0 & 256 \\
\hline 51 & Days in longest normal dry period, $f_{s} \ldots \ldots \ldots \ldots \ldots$ & 0 & 299 \\
\hline 52 & 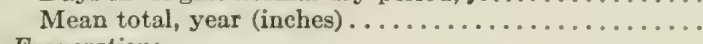 & $10-$ & 90 \\
\hline & Evaporation: & & \\
\hline 53 & Daily mean, $1887-88, f s$ (inch). & .052 & .330 \\
\hline 54 & $\begin{array}{l}\text { Total annual, } 1887-88 \text { (inches).................... } \\
\text { Moisture ratios: }\end{array}$ & 18.1 & 101.2 \\
\hline 58 & Normal $P / E, f_{s} \ldots \ldots \ldots \ldots$ & .12 & 3.84 \\
\hline 59 & Normal $\pi / E, f_{s} \ldots$ & .13 & 4.48 \\
\hline 60 & $\begin{array}{l}\text { Normal } P / E \text {, year........................... } \\
\text { Vapor pressure: }\end{array}$ & .03 & 4.90 \\
\hline 63 & $\begin{array}{l}\text { Normal mean, } f_{s} \text { (hundredths inch) ...... } \\
\text { Humidity: }\end{array}$ & 300 & 707 \\
\hline 65 & Normal mean, $f_{s}$ (per cent). & 36.3 & 87.5 \\
\hline 66 & $\begin{array}{l}\text { Normal mean, year (per cent)............. } \\
\text { Wind: }\end{array}$ & 38.7 & 86.8 \\
\hline 68 & $\begin{array}{l}\text { Normal mean hourly velocity, } f_{s} \text { (miles). } \\
\text { Sunshine: }\end{array}$ & 3.5 & 16.4 \\
\hline 69 & $\begin{array}{l}\text { Normal total duration, } f_{s} \text { (hours) } \ldots \ldots \ldots \ldots \\
\text { Moisture-temperature indices: }\end{array}$ & 1,578 & 2,995 \\
\hline 70 & Normal $P / E \times T, f_{s}$, remainder method. & 68 & 1,418 \\
\hline 71 & Normal $P / E \times T, f s$, exponential method. . & 625 & 13,511 \\
\hline 72 & Normal $P / E \times T, f s$, physiological method. & 1,186 & 24,265 \\
\hline
\end{tabular}


TAble 137.-Climatic extremes for Parielaria debilis.

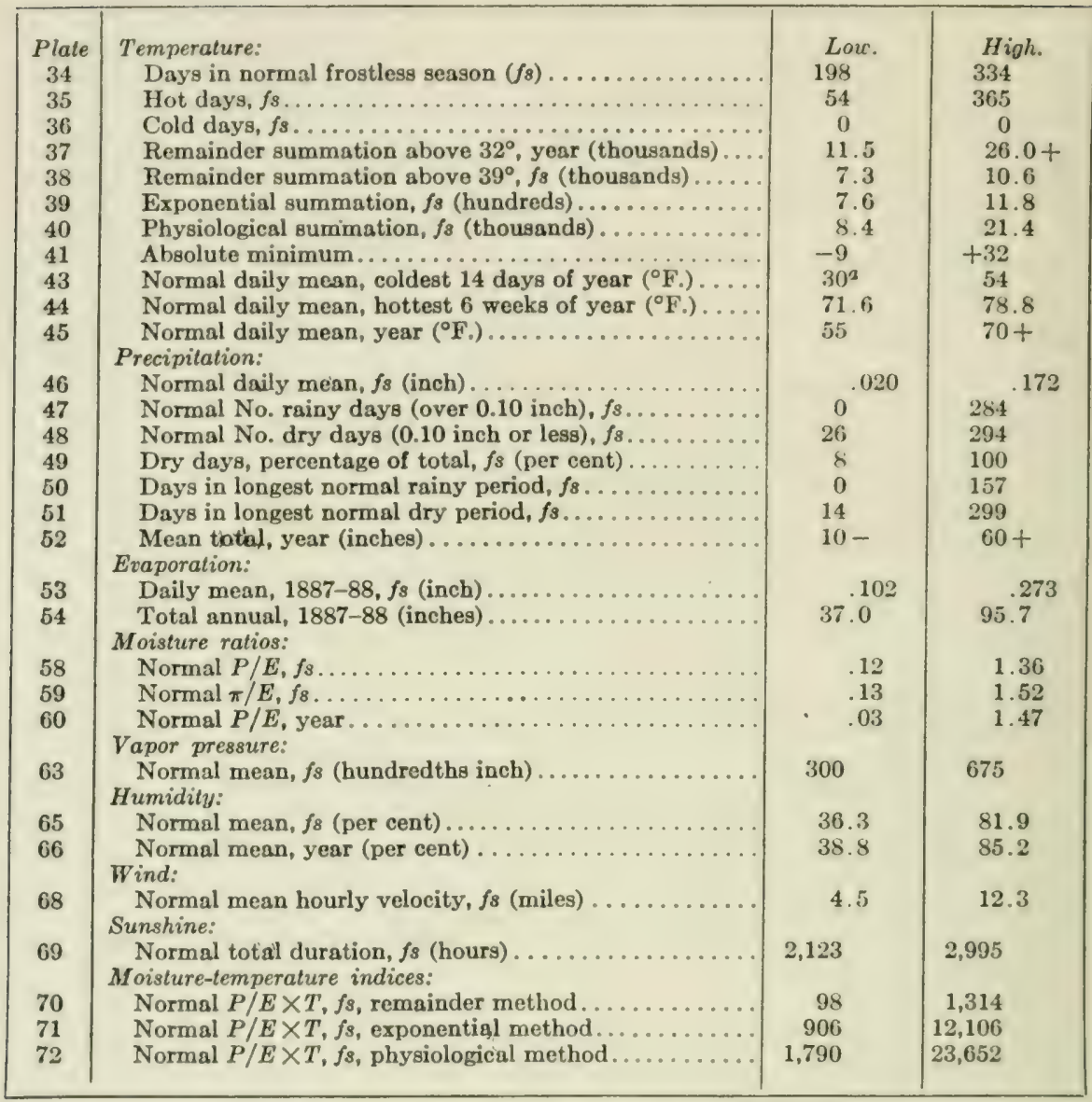

TABLE 138.-Climatic extremes for Kallstromia grandiflora.

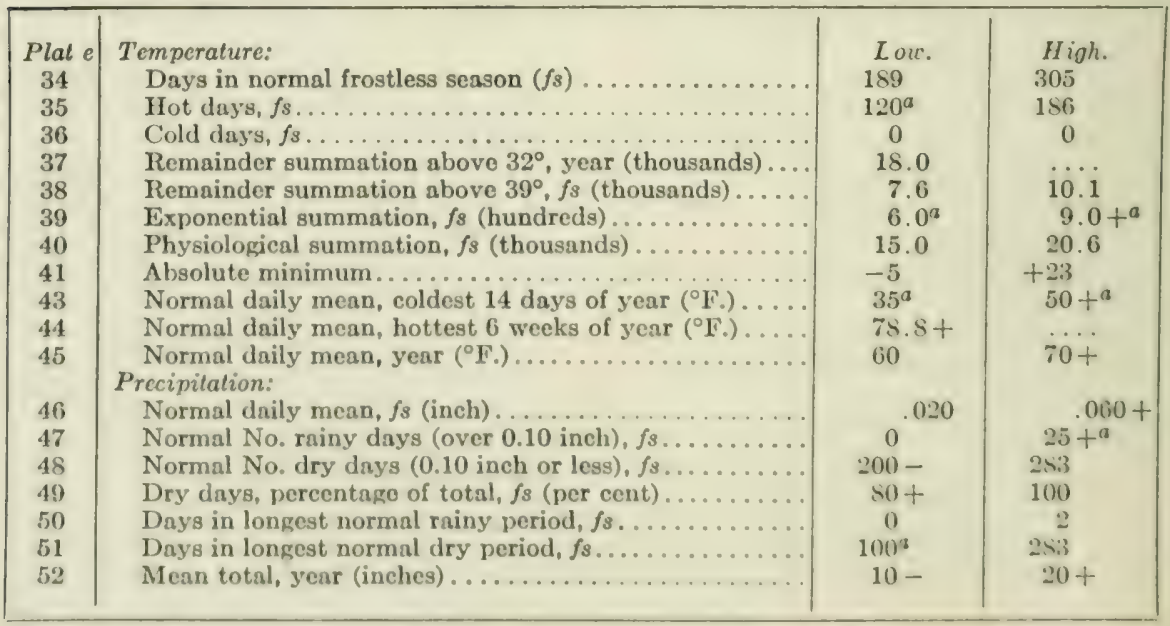


TABLE 138.-Climatic extremes for Kallstrœmia grandiflora-Continued.

\begin{tabular}{|c|c|c|c|}
\hline Plate & Evaporation: & Low. & High. \\
\hline 53 & Daily mean, $1887-88, f_{s}$ (inch) $\ldots \ldots \ldots \ldots \ldots \ldots$ & $.180^{a}$ & .330 \\
\hline 54 & Total annual, $1887-88$ (inches) $\ldots \ldots \ldots \ldots \ldots \ldots \ldots$ & $70^{a}$ & 101.2 \\
\hline 58 & $\begin{array}{l}\text { Moisture ratios: } \\
\text { Normal } P / E, f s .\end{array}$ & .12 & $.20+^{a}$ \\
\hline 59 & Normal $\pi / E, f_{8} \ldots \ldots \ldots \ldots \ldots \ldots \ldots \ldots \ldots$ & .13 & .15 \\
\hline 60 & Normal $P / E$, year. . . . . . . . . . . . . . & .12 & $.20+a$ \\
\hline 63 & $\begin{array}{l}\text { Vapor pressure: } \\
\text { Normal mean, } f s \text { (hundredths inch)..................... } \\
\text { Humidity: }\end{array}$ & 300 & $450^{3}$ \\
\hline 65 & Normal mean, $f_{s}$ (per cent)....... & 36.3 & $50^{a}$ \\
\hline 66 & $\begin{array}{l}\text { Normal mean, year (per cent) } \ldots \ldots \ldots \ldots \ldots \ldots \ldots \ldots \\
\text { Wind: }\end{array}$ & 38.7 & $50+^{a}$ \\
\hline 68 & $\begin{array}{l}\text { Normal mean hourly velocity, } f_{8} \text { (miles) ........... } \\
\text { Sunshine: }\end{array}$ & 4.5 & 10.2 \\
\hline 69 & $\begin{array}{l}\text { Normal total duration, } f_{s} \text { (hours) } \ldots \ldots \ldots \ldots \ldots \ldots \ldots \\
\text { Moisture-temperature indices: }\end{array}$ & $\ldots$ & \\
\hline 70 & Normal $P / E \times T, f_{8}$, remainder method. & 98 & $300^{a}$ \\
\hline 71 & 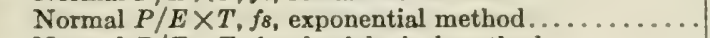 & 906 & $3,000+a$ \\
\hline 72 & Normal $P / E \times T, f_{s}$, physiological method.......... & 1,790 & $4,000+\infty$ \\
\hline
\end{tabular}

TABLe 139.-Climatic extremes for Cladothrix lanuginosa.

\begin{tabular}{|c|c|c|c|}
\hline Plate & Temperature: & Low. & High. \\
\hline 34 & Days in normal frostless season $(f s) \ldots \ldots \ldots \ldots$ & 142 & 323 \\
\hline 35 & 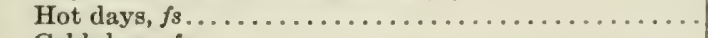 & 113 & 208 \\
\hline 36 & Cold days, $f 8, \ldots \ldots \ldots \ldots \ldots \ldots \ldots \ldots \ldots \ldots \ldots \ldots \ldots \ldots \ldots \ldots$ & 0 & 70 \\
\hline 37 & Remainder summation above $32^{\circ}$, year (thousands).... & 11.5 & $26.0+$ \\
\hline 38 & Remainder summation above $39^{\circ}, f_{8}$ (thousands)...... & 5.5 & 10.3 \\
\hline 39 & 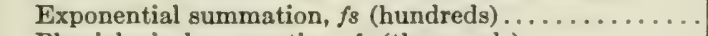 & 5.9 & 11.8 \\
\hline 40 & Physiological summation, fs (thousands) ............ & 10.3 & 23.9 \\
\hline 41 & 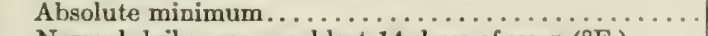 & -29 & +23 \\
\hline 43 & Normal daily mean, coldest 14 days of year $\left({ }^{\circ} \mathrm{F}.\right) \ldots$ & 27 & 53 \\
\hline 44 & Normal daily mean, hottest 6 weeks of year $\left({ }^{\circ} \mathrm{F}.\right) . .$. & 71.6 & $78.8+$ \\
\hline 45 & $\begin{array}{l}\text { Normal daily mean, year }\left({ }^{\circ} \mathrm{F} .\right) . \therefore \ldots \ldots \ldots \ldots \ldots \ldots \\
\text { Precipitation: }\end{array}$ & $55-$ & $70+$ \\
\hline 46 & Normal daily mean, $f_{8}$ (inch) $\ldots \ldots \ldots \ldots \ldots \ldots$ & .020 & .088 \\
\hline 47 & Normal No. rainy days (over $0.10 \mathrm{inch}$ ), $f_{s} \ldots . . . .$. & 0 & $100^{a}$ \\
\hline 48 & Normal No. dry days $(0.10$ inch or less), $f 8 \ldots \ldots \ldots$ & 133 & 283 \\
\hline 49 & Dry days, percentage of total, $f s$ (per cent)........... & 77 & 100 \\
\hline 50 & Days in longest normal rainy period, $f_{s} . . . \ldots \ldots \ldots$ & 0 & $50^{a}$ \\
\hline 51 & Days in longest normal dry period, $f s, \ldots \ldots \ldots \ldots$ & 53 & 283 \\
\hline 52 & $\begin{array}{l}\text { Mean total, year (inches)............................. } \\
\text { Evaporation: }\end{array}$ & $10-$ & 40 \\
\hline 53 & Daily mean, $1887-88, f_{s}$ (inch) $\ldots \ldots \ldots \ldots \ldots$ & .102 & .330 \\
\hline 54 & $\begin{array}{l}\text { Total annual, } 1887-88 \text { (inches) } \ldots \ldots \ldots \ldots \ldots \ldots \ldots \\
\text { Moisture ratios: }\end{array}$ & 38.8 & 101.2 \\
\hline 58 & 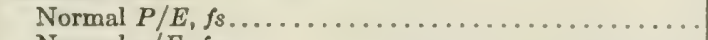 & .12 & .77 \\
\hline 59 & Normal $\pi / E, f s \ldots \ldots \ldots \ldots \ldots \ldots \ldots \ldots \ldots \ldots \ldots \ldots \ldots$ & .13 & .81 \\
\hline 60 & 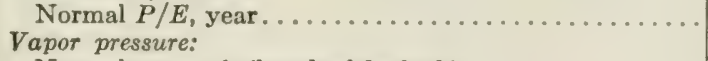 & .12 & .76 \\
\hline 63 & $\begin{array}{l}\text { Normal mean, } f_{s} \text { (hundredths inch)................. } \\
\text { Humidity: }\end{array}$ & 300 & 675 \\
\hline 65 & Normal mean, $f s$ (per cent) & 36.3 & 81.9 \\
\hline 66 & $\begin{array}{l}\text { Normal mean, year (per cent) } \ldots \ldots \ldots \ldots \ldots \ldots \ldots \\
\text { Wind: }\end{array}$ & 38.7 & 82.1 \\
\hline 68 & $\begin{array}{l}\text { Normal mean hourly velocity, } f_{3} \text { (miles).. } \\
\text { Sunshine: }\end{array}$ & 4.5 & 14.2 \\
\hline 69 & $\begin{array}{l}\text { Normal total duration, } f_{s} \text { (hours) } \\
\text { Moisture-temperature indices: }\end{array}$ & $1,700^{a}$ & 2,343 \\
\hline 70 & Normal $P / E \times T, f s$, remainder method.... & 98 & 737 \\
\hline 71 & Normal $P / E \times T, f s$, exponential method........... & 906 & 6,690 \\
\hline 72 & Normal $P / E \times T, f 8$, physiological method......... & 1,790 & 13,926 \\
\hline
\end{tabular}


TABLE 140.-Climatic extremes for Pectis paposa.

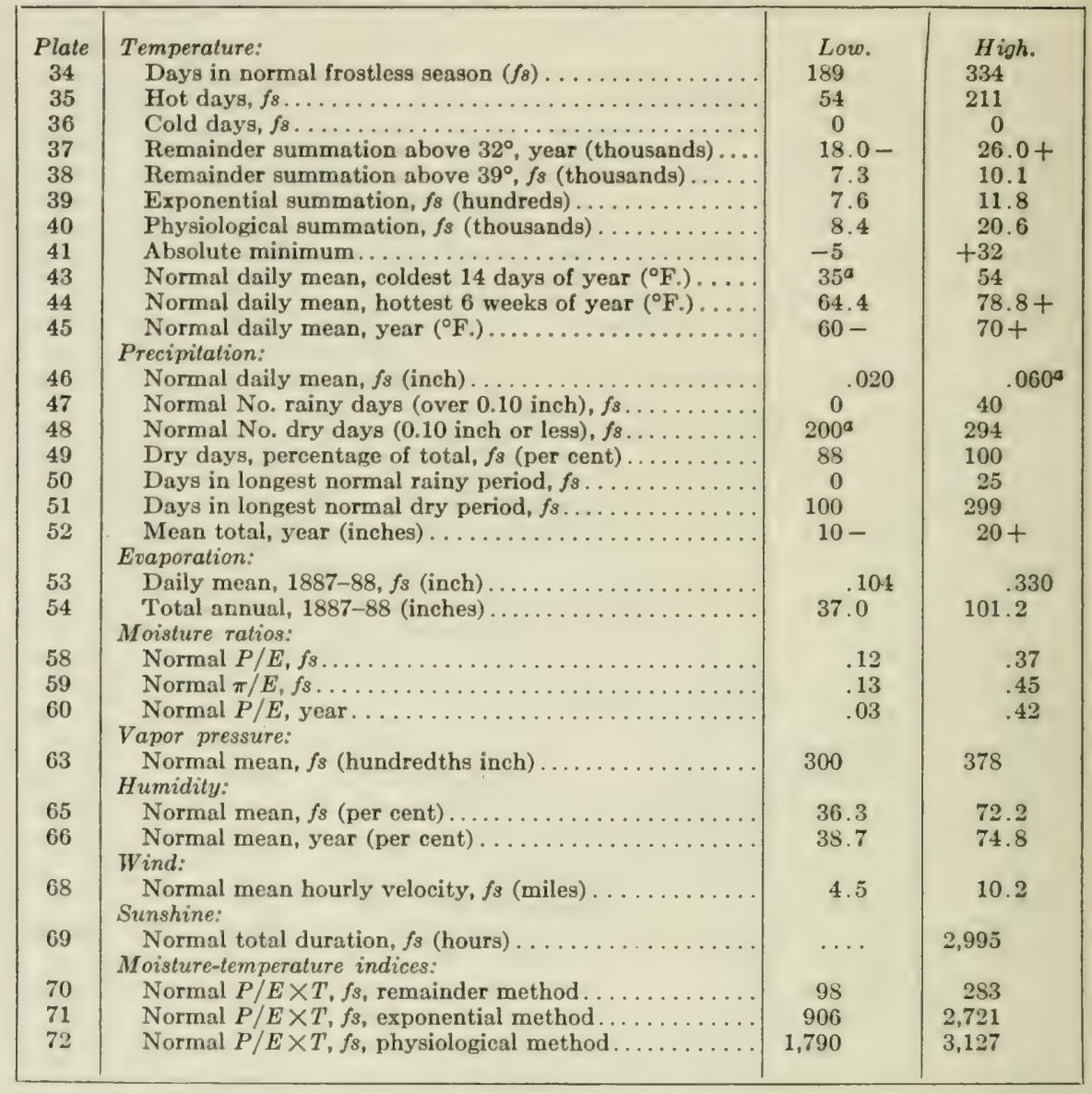

TABLE 141.-Climatic extremes for Euphorbia serpyllifolia.

\begin{tabular}{|c|c|c|c|}
\hline Plate & Temperalure: & Low. & High. \\
\hline 34 & Days in normal frostless season $\left(f_{s}\right)$ & 25 & 334 \\
\hline 35 & Hot days, $f_{s} \ldots \ldots \ldots \ldots \ldots \ldots \ldots$ & 0 & 218 \\
\hline 36 & Cold days, $f_{s}$. . & 0 & 158 \\
\hline 37 & Remainder summation above $32^{\circ}$, year (thousands). & $10.0-$ & $26.0+$ \\
\hline 38 & Remainder summation above $39^{\circ}, f_{s}$ (thousands)... & 2.4 & 10.3 \\
\hline 39 & Exponential summation, $f_{3}$ (hundreds)......... & 2.5 & 11.3 \\
\hline 40 & Physiological summation, $f_{3}$ (thousands). & 2.6 & 21.3 \\
\hline 41 & Absolute minimum................. & -65 & +32 \\
\hline 43 & Normal daily mean, coldest 14 days of year $\left({ }^{\circ} \mathrm{F}\right)$. & 0 & 53 \\
\hline 44 & Normal daily mean, hottest 6 weeks of year $\left({ }^{\circ} \mathrm{F}\right.$. $)$. & $64.4-$ & $78.8+$ \\
\hline 45 & Normal daily mean, year $\left({ }^{\circ} \mathrm{F}_{0}\right) \ldots \ldots \ldots \ldots$. & 35 & $70+$ \\
\hline & Precipilation: & & \\
\hline 46 & Normal daily mean, $f_{s}$ (inch). & .009 & 199 \\
\hline 47 & Normal No. rainy days (over 0.10 inch), fs. & 0 & $200^{\circ}$ \\
\hline 48 & Normal No. dry days $(0.10$ inch or less), fs... & 26 & 294 \\
\hline 49 & Dry days, percentage of total, $f_{s}$ (per cent). & 14 & 100 \\
\hline 50 & Days in longest normal rainy period, $f_{s}, \ldots$. & 0 & 172 \\
\hline 51 & Days in longest normal dry period, $f_{8} \ldots \ldots$ & 11 & 299 \\
\hline 52 & Mean total, year (inches)........... & $10-$ & $40+$ \\
\hline
\end{tabular}


TABLE 141.-Climatic extremes for Euphorbia serpyllifolia-Continued.

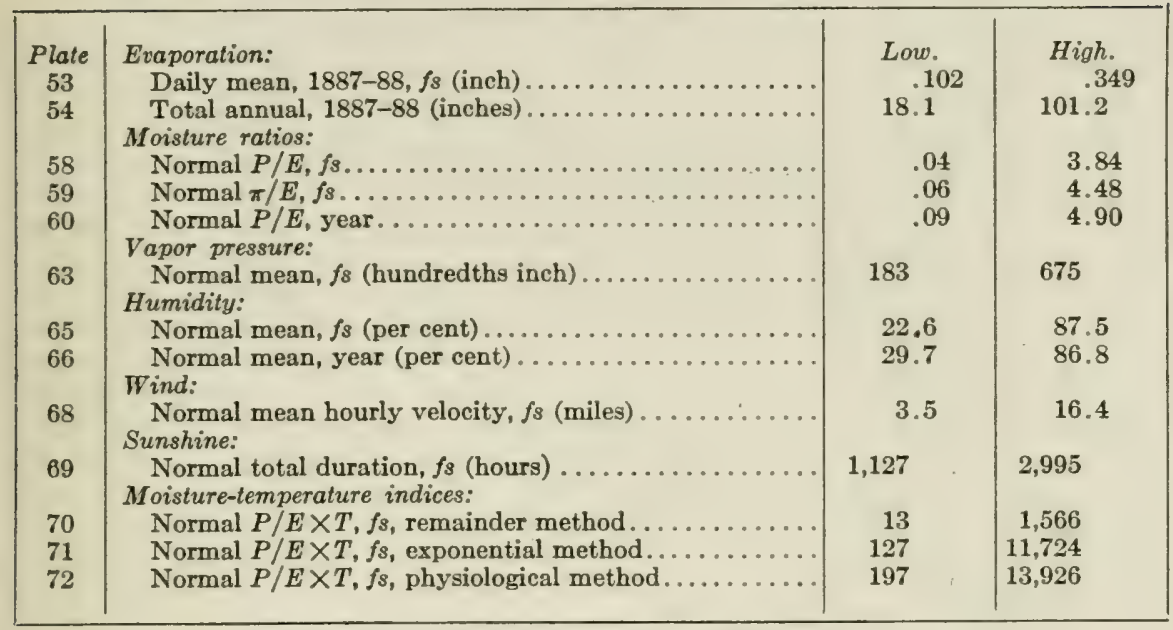

TABLE 142.-Climatic extremes for Bœrhaavia erecta.

\begin{tabular}{|c|c|c|c|}
\hline Plate & Temperature: & Low. & High. \\
\hline 34 & Days in normal frostless season $(f s) \ldots \ldots \ldots \ldots \ldots$ & 198 & 365 \\
\hline 35 & 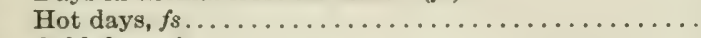 & 147 & 365 \\
\hline 36 & Cold days, $f_{s} \ldots \ldots \ldots \ldots \ldots \ldots \ldots \ldots \ldots$ & 0 & 0 \\
\hline 37 & Remainder summation above $32^{\circ}$, year (thousands).... & 18.0 & $26.0+$ \\
\hline 38 & Remainder summation above $39^{\circ}, f_{s}$ (thousands)...... & 7.3 & 14.5 \\
\hline 39 & Exponential summation, $f_{s}$ (hundreds) ............ & 7.4 & 15.4 \\
\hline 40 & Physiological summation, $f_{s}$ (thousands)........... & 13.6 & 31.1 \\
\hline 41 & 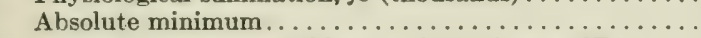 & -13 & +41 \\
\hline 43 & Normal daily mean, coldest 14 days of year $\left({ }^{\circ} \mathrm{F}.\right) \ldots$ & 42 & 69 \\
\hline 44 & Normal daily mean, hottest 6 weeks of year $\left({ }^{\circ} \mathbf{F}\right.$.).... & $78.8+$ & \\
\hline 45 & $\begin{array}{l}\text { Normal daily mean, year }\left({ }^{\circ} \mathrm{F} .\right) \ldots \ldots \ldots \ldots \ldots \ldots \ldots \\
\text { Precipitation: }\end{array}$ & $65-$ & $75+$ \\
\hline 46 & Normal daily mean, $f_{s}$ (inch) $\ldots \ldots \ldots \ldots \ldots \ldots$ & .020 & .172 \\
\hline 47 & Normal No. rainy days (over 0.10 inch), $f_{s} \ldots . . .$. & 0 & 284 \\
\hline 48 & Normal No. dry days (0.10 inch or less), fs......... & 44 & 283 \\
\hline 49 & Dry days, percentage of total, $f_{s}$ (per cent) .......... & 8 & 100 \\
\hline 50 & Days in longest normal rainy period, $f_{s} \ldots \ldots \ldots \ldots$ & 0 & 187 \\
\hline 51 & Days in longest normal dry period, $f_{s} \ldots \ldots \ldots \ldots \ldots$ & 17 & 283 \\
\hline 52 & $\begin{array}{l}\text { Mean total, year (inches) } \ldots \ldots \ldots \ldots \ldots \ldots \ldots \ldots \\
\text { Evaporation: }\end{array}$ & $10-$ & $60+$ \\
\hline 53 & Daily mean, $1887-88, f_{s}$ (inch) $\ldots \ldots \ldots \ldots \ldots \ldots$ & .102 & .330 \\
\hline 54 & $\begin{array}{l}\text { Total annual, } 1887-88 \text { (inches) } \ldots \ldots \ldots \ldots \ldots \ldots \ldots \ldots \\
\text { Moisture ratios: }\end{array}$ & 37.0 & 101.2 \\
\hline 58 & Normal $P / E, f s .$. & .12 & 1.20 \\
\hline 59 & Normal $\pi / E, f_{s} . .$. & .15 & 1.47 \\
\hline 60 & $\begin{array}{l}\text { Normal } P / E \text {, year. . . . } \ldots \ldots \ldots \ldots \ldots \ldots \ldots \ldots \ldots \\
\text { Vapor pressure: }\end{array}$ & .03 & 1.47 \\
\hline 63 & $\begin{array}{l}\text { Normal mean, } f_{s} \text { (hundredths inch) } \ldots \ldots \ldots \ldots \ldots \\
\text { Humidity: }\end{array}$ & 300 & 707 \\
\hline 65 & Normal mean, $f s$ (per cent) .. & 36.3 & 81.9 \\
\hline 66 & $\begin{array}{l}\text { Normal mean, year (per cent) . . . . . . . . . . . } \\
\text { Wind: }\end{array}$ & 38.7 & 85.2 \\
\hline 68 & $\begin{array}{l}\text { Normal mean hourly velocity, } f_{s} \text { (miles).. } \\
\text { Sunshine: }\end{array}$ & 4.5 & 12.3 \\
\hline 69 & $\begin{array}{l}\text { Normal total duration, } f_{s} \text { (hours) } \ldots \ldots \\
\text { Moisture-temperature indices: }\end{array}$ & $2,100-a$ & 2,650 \\
\hline 70 & Normal $P / E \times T, f_{s}$, remainder method... & 98 & 1,314 \\
\hline 71 & 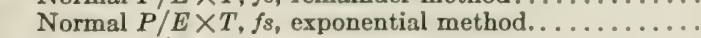 & 906 & 12,106 \\
\hline 72 & 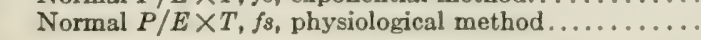 & $1,000-a$ & 23,652 \\
\hline
\end{tabular}


TABLE 143.-Climatic extremes for Oxybaphus nyctagineus.

\begin{tabular}{|c|c|c|c|}
\hline Plate & Temperature: & Low. & High. \\
\hline 34 & Days in normal frostless season $\left(f_{8}\right) \ldots \ldots \ldots \ldots \ldots$ & 103 & 331 \\
\hline 35 & 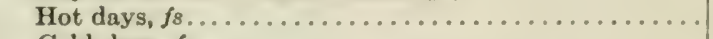 & 30 & 215 \\
\hline 36 & Cold days, $f_{8}, \ldots \ldots \ldots \ldots \ldots \ldots \ldots \ldots \ldots \ldots$ & 0 & 158 \\
\hline 37 & Remainder summation above $32^{\circ}$, year (thousands).... & $10.0-$ & $18.0+$ \\
\hline 38 & Remainder summation above $39^{\circ}, f_{8}$ (thousands) ...... & 2.9 & 10.6 \\
\hline 39 & Exponential summation, $f s$ (hundreds)............. & 3.0 & 11.7 \\
\hline 40 & Physiological summation, $f_{8}$ (thousands) ........... & 3.7 & 21.2 \\
\hline 41 & 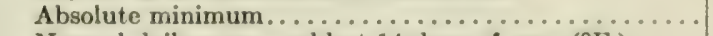 & -59 & +19 \\
\hline 43 & Normal daily mean, coldest 14 days of year $\left({ }^{\circ} \mathrm{F}.\right) \ldots$. & 0 & 53 \\
\hline 44 & Normal daily mean, hottest 6 weeks of year $\left({ }^{\circ} \mathrm{F}.\right) \ldots$ & 64.4 & 78.8 \\
\hline 45 & $\begin{array}{l}\text { Normal daily mean, year }\left({ }^{\circ} \mathrm{F} .\right) \ldots \ldots \ldots \ldots \ldots \ldots \ldots \\
\text { Precipitation: }\end{array}$ & 35 & 70 \\
\hline 46 & Normal daily mean, $f_{8}$ (inch).. & .070 & .159 \\
\hline 47 & Normal No. rainy days (over $0.10 \mathrm{inch}$ ), $f_{s} \ldots . . . .$. & 0 & 284 \\
\hline 48 & Normal No. dry days (0.10 inch or less), $f_{8} \ldots . . .$. & 28 & $250^{a}$ \\
\hline 49 & Dry days, percentage of total, fs (per cent) .......... & 8 & 100 \\
\hline 50 & Days in longest normal rainy period, $f s \ldots \ldots \ldots \ldots$ & 14 & 172 \\
\hline 51 & Days in longest normal dry period, $f_{8} \ldots \ldots \ldots \ldots \ldots$ & 12 & 88 \\
\hline 52 & $\begin{array}{l}\text { Mean total, year (inches) } \ldots \ldots \ldots \ldots \ldots \ldots \ldots \ldots \\
\text { Evaporation: }\end{array}$ & $20-$ & 60 \\
\hline 53 & Daily mean, $1887-88, f_{s}$ (inch) $\ldots \ldots \ldots \ldots \ldots \ldots$ & .101 & .195 \\
\hline 54 & $\begin{array}{l}\text { Total annual, } 1887-88 \text { (inches) } \ldots \ldots \ldots \ldots \ldots \ldots \ldots \ldots \\
\text { Moisture ratios: }\end{array}$ & 22.1 & 76.5 \\
\hline 58 & Normal $I^{2} / E, f_{s}$. & .39 & 1.21 \\
\hline 59 & Normal $\pi / E, f_{s} \ldots \ldots \ldots \ldots \ldots \ldots \ldots \ldots \ldots$ & .26 & 1.32 \\
\hline 60 & $\begin{array}{l}\text { Normal } P / E \text {, year } \ldots \ldots \ldots \ldots \ldots \ldots \ldots \ldots \ldots \\
\text { Vapor pressure: }\end{array}$ & .38 & 1.26 \\
\hline 63 & $\begin{array}{l}\text { Normal mean, } f_{s} \text { (hundredths inch).............. } \\
\text { Humidity: }\end{array}$ & 274 & 675 \\
\hline 65 & Normal mean, $f s$ (per cent). & 36.3 & S0.2 \\
\hline 66 . & $\begin{array}{l}\text { Normal mean, year (per cent) } \ldots \ldots \ldots \ldots \ldots \ldots \ldots \ldots \\
\text { Wind: }\end{array}$ & 48.1 & 85.2 \\
\hline 68 & $\begin{array}{l}\text { Normal mean hourly velocity, fs (miles).... } \\
\text { Sunshine: }\end{array}$ & 6.0 & 14.2 \\
\hline 69 & $\begin{array}{l}\text { Normal total duration, } f s \text { (hours) ..... } \\
\text { Moisture-temperature indices: }\end{array}$ & 1,127 & 2,650 \\
\hline 70 & 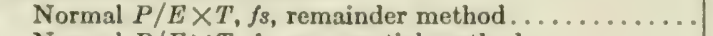 & 58 & 1,304 \\
\hline 71 & Normal $P / E \times T, f_{s}$, exponential method......... & 563 & 11,956 \\
\hline 72 & Normal $P / E \times T, f s$, physiological method.......... & 710 & 23,381 \\
\hline
\end{tabular}

TABLE 144.-Climatic extremes for Oxybaphus angustifolius.

\begin{tabular}{|c|c|c|c|}
\hline Plate & Tcmperature: & Iow. & High. \\
\hline 34 & Days in normal frostless season $(f s) \ldots$ & 83 & 331 \\
\hline 35 & 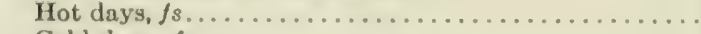 & 0 & 218 \\
\hline 36 & Cold days, $f_{s} \ldots \ldots \ldots \ldots \ldots \ldots \ldots$ & 0 & 140 \\
\hline 37 & Remainder summation above $32^{\circ}$, year (thousands)... & $10.0-$ & $26.0+$ \\
\hline 38 & Remainder summation above $39^{\circ}, f_{s}$ (thousands) ..... & 2.4 & 10.6 \\
\hline 39 & Exponential summation, $f s$ (hundreds) ............ & 2.4 & 11.7 \\
\hline 40 & Physiological summation, $f_{s}$ (thousands) .......... & 2.6 & 21.4 \\
\hline 41 & 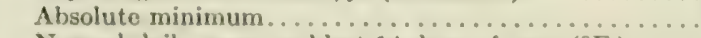 & -51 & +22 \\
\hline 43 & Normal daily mean, coldest 14 days of year $\left({ }^{\circ} \mathrm{F}.\right) \ldots$ & 9 & 53 \\
\hline 44 & Normal daily mean, hottest 6 weeks of year $\left({ }^{\circ} \mathrm{F}.\right) \ldots$ & 64.4 & $78.8+$ \\
\hline 45 & $\begin{array}{l}\text { Normal daily mean, year }\left({ }^{\circ} \mathrm{F} .\right) \ldots \ldots \ldots \ldots \ldots \ldots \\
\text { Precipitation: }\end{array}$ & $45-$ & $70+$ \\
\hline 46 & Normal daily mean, $f s$ (inch) .... & .025 & $.12 ?$ \\
\hline 47 & Normal No. rainy days (over 0.10 inch), fs... & 0 & 257 \\
\hline 48 & Normal No. dry days $(0.10$ inch or less $), f s \ldots$ & 32 & 259 \\
\hline 49 & Dry days, pereentage of total, $f_{s}$ (per cent).......... & 14 & 100 \\
\hline 50 & Days in longest normal rainy period, $f s \ldots . . . \ldots$ & 0 & 157 \\
\hline 51 & Days in longest normal dry period, $f_{s} \ldots \ldots \ldots \ldots \ldots$ & 15 & $250^{a}$ \\
\hline 52 & 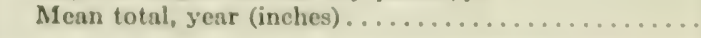 & 10 & 50 \\
\hline
\end{tabular}


TABLE 144.-Climatic extremes for Oxybaphus angustifolius-Continued.

\begin{tabular}{|c|c|c|c|}
\hline Plate & Evaporation: & Low. & High. \\
\hline 53 & Daily mean, $1887-88, f_{s}$ (inch) & 102 & 330 \\
\hline 54 & $\begin{array}{l}\text { Total annual, } 1887-88 \text { (inches).... } \\
\text { Moisture ratios: }\end{array}$ & 37.0 & 101.2 \\
\hline 58 & Normal $P / E, f_{s} \ldots \ldots \ldots \ldots$ & 10 & 1.01 \\
\hline 59 & Normal $\pi / E, f_{3} \ldots$ & 12 & 1.05 \\
\hline 60 & Normal $P / E$, year.... & .12 & 1.16 \\
\hline 63 & $\begin{array}{l}\text { Vapor pressure: } \\
\text { Normal mean, } f_{s} \text { (hundredths inch)... } \\
\text { Humidity: }\end{array}$ & 300 & 675 \\
\hline 65 & Normal mean, $f_{s}$ (per cent)........ & 37.0 & 73.5 \\
\hline 66 & $\begin{array}{l}\text { Normal mean, year (per cent) } \ldots \ldots \ldots \ldots \ldots \ldots \\
\text { Wind: }\end{array}$ & 38.8 & 85.2 \\
\hline 68 & $\begin{array}{l}\text { Normal mean hourly velocity, fs (miles)...... } \\
\text { Sunshine: }\end{array}$ & 4.5 & 14.2 \\
\hline 69 & $\begin{array}{l}\text { Normal total duration, } f_{s} \text { (hours) ....... } \\
\text { Moisture-temperature indices: }\end{array}$ & 1,127 & 2,650 \\
\hline 70 & Normal $P / E \times T, f_{s}$, remainder method. & 58 & 1,142 \\
\hline 71 & Normal $P / E \times T, f s$, exponential method.. & 563 & 10,331 \\
\hline 72 & Normal $P / E \times T, f s$, physiological method. & 710 & 20,570 \\
\hline
\end{tabular}

TABLE 145.-Climatic extremes for Oxybaphus floribundus.

\begin{tabular}{|c|c|c|c|}
\hline Plate & Temperature: & Low. & High. \\
\hline 34 & Days in normal frostless season $\left(f_{s}\right)$. & 94 & 261 \\
\hline 35 & Hot days, $f_{s} \ldots \ldots \ldots \ldots \ldots \ldots \ldots \ldots$ & 30 & 173 \\
\hline 36 & Cold days, fs............. & 0 & 158 \\
\hline 37 & Remainder summation above $32^{\circ}$, year (thousands) & $10.0-$ & $18.0+$ \\
\hline 38 & Remainder summation above $39^{\circ}, f_{s}$ (thousands). . & 2.9 & 8.6 \\
\hline 39 & Exponential summation, $f_{8}$ (hundreds) .......... & 3.0 & 9.6 \\
\hline 40 & Physiological summation, $f_{s}$ (thousands). & 3.7 & 17.6 \\
\hline 41 & 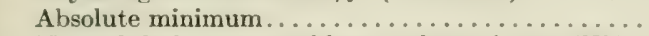 & -65 & +19 \\
\hline 43 & Normal daily mean, coldest 14 days of year ( ${ }^{\circ} \mathrm{F}$. .) & 0 & 46 \\
\hline 44 & Normal daily mean, hottest 6 weeks of year $\left({ }^{\circ} \mathrm{F}\right.$.). & 64.4 & 78.8 \\
\hline 45 & Normal daily mean, year $\left({ }^{\circ} \mathbf{F}_{0}\right) \ldots \ldots \ldots \ldots \ldots$ & 35 & $65+$ \\
\hline 46 & $\begin{array}{l}\text { Precipitation: } \\
\text { Normal daily mean, fs (inch). }\end{array}$ & 049 & 128 \\
\hline 47 & Normal No. rainy days (over $0.10 \mathrm{inch}$ ), $f_{s}$. & 0 & 182 \\
\hline 48 & Normal No. dry days ( 0.10 inch or less), $f s$. & 28 & 203 \\
\hline 49 & Dry days, percentage of total, $f s$ (per cent). & 14 & 100 \\
\hline 50 & Days in longest normal rainy period, $f_{s} \ldots$. & 0 & 172 \\
\hline 51 & Days in longest normal dry period, $f_{s} \ldots$ & 11 & 163 \\
\hline 52 & Mean total, year (inches) .......... & 20 & $50+$ \\
\hline & Evaporation: & & \\
\hline 53 & Daily mean, $1887-88, f_{s}$ (inch). & .101 & .200 \\
\hline 54 & $\begin{array}{l}\text { Total annual, } 1887-88 \text { (inches) ............. } \\
\text { Moisture ratios: }\end{array}$ & 22.1 & 76.5 \\
\hline 58 & Normal $P / E, f s \ldots \ldots \ldots$ & .19 & 1.23 \\
\hline 59 & Normal $\pi / E, f_{s} \ldots$ & .25 & .89 \\
\hline 60 & $\begin{array}{c}\text { Normal } P / E \text {, year. } \ldots \ldots \ldots \ldots \ldots \ldots \\
\text { Vapor pressure: }\end{array}$ & .18 & 1.30 \\
\hline 63 & $\begin{array}{l}\text { Normal mean, } f_{s} \text { (hundredths inch)... } \\
\text { Humidity: }\end{array}$ & 253 & 545 \\
\hline 65 & Normal mean, $f s$ (per cent). . & 45.5 & 73.5 \\
\hline 66 & $\begin{array}{l}\text { Normal mean, year (per cent) ............ } \\
\text { Wind: }\end{array}$ & 48.1 & 74.2 \\
\hline 68 & $\begin{array}{l}\text { Normal mean hourly velocity, } f_{s} \text { (miles). } \\
\text { Sunshine: }\end{array}$ & 6.0 & 14.2 \\
\hline 69 & $\begin{array}{l}\text { Normal total duration, } f s \text { (hours) ... } \\
\text { Moisture-temperature indices: }\end{array}$ & 1,127 & 2,166 \\
\hline 70 & Normal $P / E \times T, f_{s}$, remainder method. & 58 & 700 \\
\hline 71 & Normal $P / E \times T, f_{s}$, exponential method.. & 563 & 6,410 \\
\hline 72 & Normal $P / E \times T, f s$, physiological method. & 710 & 12,977 \\
\hline
\end{tabular}


TABLE 146.-Climatic extremes for Florkea occidentalis.

\begin{tabular}{|c|c|c|c|}
\hline Plate & Temperature: & Low. & High. \\
\hline 34 & Days in normal frostless season $(f s)$. & 25 & 224 \\
\hline 35 & Hot days, $f_{s} \ldots \ldots \ldots \ldots \ldots \ldots \ldots \ldots \ldots$ & 0 & 105 \\
\hline 36 & Cold days, $f_{3} \ldots \ldots \ldots \ldots \ldots \ldots \ldots$ & 0 & 134 \\
\hline 37 & Remainder summation above $32^{\circ}$, year (thousands) & $10.0-$ & $11.5+$ \\
\hline 38 & Remainder summation above $39^{\circ}, f_{3}$ (thousands). & 2.7 & 5.4 \\
\hline 39 & Exponential summation, $f_{s}$ (hundreds)......... & $2.8^{\circ}$ & 5.7 \\
\hline 40 & Physiological summation, $f_{s}$ (thousands). & 3.1 & 9.9 \\
\hline 41 & Absolute minimum......... & -48 & -2 \\
\hline 43 & Normal daily mean, coldest 14 days of year $\left({ }^{\circ} \mathrm{F}.\right)$ & 17 & \\
\hline 44 & Normal daily mean, hottest 6 weeks of year $\left({ }^{\circ} \mathrm{F}\right.$. $)$ & $64.4-$ & $71.6+$ \\
\hline 45 & Normal daily mean, year $\left({ }^{\circ} \mathrm{F}.\right) \ldots \ldots \ldots$ & $40-$ & $50+$ \\
\hline 46 & $\begin{array}{l}\text { Precipilation: } \\
\text { Normal daily mean, } f_{s} \text { (inch). }\end{array}$ & .025 & .079 \\
\hline 47 & Normal No, rainy days (over $0.10 \mathrm{inch}$ ), $f s$. & 0 & 73 \\
\hline 48 & Normal No. dry days ( 0.10 inch or less), $f s$. & 104 & 216 \\
\hline 49 & Dry days, percentage of total, $f_{s}$ (per cent). & 70 & 100 \\
\hline 50 & Days in longest normal rainy period, $f_{3} . .$. & 0 & 44 \\
\hline 51 & Days in longest normal dry period, $f_{3} \ldots$ & 101 & 216 \\
\hline 52 & $\begin{array}{c}\text { Mean total, year (inches)............... } \\
\text { Evaporation: }\end{array}$ & $10+$ & $50+$ \\
\hline 53 & Daily mean, $1887-88, f s$ (inch). & .120 & $.260+^{a}$ \\
\hline 54 & $\begin{array}{l}\text { Total annual, } 1887-88 \text { (inches) ..... } \\
\text { Moisture ratios: }\end{array}$ & 34.7 & 69.0 \\
\hline 58 & Normal $P / E, f s \ldots \ldots \ldots$ & .18 & .66 \\
\hline 59 & Normal $\pi / E, f_{3} \ldots \ldots \ldots \ldots \ldots \ldots$ & .12 & .85 \\
\hline 60 & $\begin{array}{l}\text { Normal } P / E \text {, year }, \ldots \ldots \ldots \ldots \ldots \\
\text { Vapor pressure: }\end{array}$ & .23 & 1.30 \\
\hline 63 & $\begin{array}{l}\text { Normal mean, } f_{s} \text { (hundredths inch)... } \\
\text { Humidity: }\end{array}$ & 236 & 329 \\
\hline 65 & Normal mean, $f_{s}$ (per cent)........ & 45.8 & 71.4 \\
\hline 66 & $\begin{array}{l}\text { Normal mean, year (per cent)............ } \\
\text { Wind: }\end{array}$ & 48.1 & 75.5 \\
\hline 68 & $\begin{array}{l}\text { Normal mean hourly velocity, fs (miles). } \\
\text { Sunshine: }\end{array}$ & 4.3 & 7.5 \\
\hline 69 & $\begin{array}{l}\text { Normal total duration, } f_{s} \text { (hours)... } \\
\text { Moisture-temperature indices: }\end{array}$ & 1,167 & 1,578 \\
\hline 70 & Normal $P / E \times T, s$, remainder method. & 66 & 332 \\
\hline 71 & Normal $P / E \times T, f_{s}$, exponential method.. & 623 & 3,052 \\
\hline 72 & Normal $P / E \times T, f s$, physiological method.. & 1,204 & 3,160 \\
\hline
\end{tabular}

TABLe 147.-Climatic extremes for Flarka proserpinacoides.

\begin{tabular}{|c|c|c|c|}
\hline Plate & Temperature: & Low. & High. \\
\hline 34 & Days in normal frostless season $(f s)$. & 108 & 224 \\
\hline 35 & Hot days, $f_{s} \ldots \ldots \ldots \ldots \ldots \ldots$ & 0 & 153 \\
\hline 36 & Cold days, fs....... & 0 & 137 \\
\hline 37 & Remainder summation above $32^{\circ}$, year (thousands) & $10.0-$ & $18.0+$ \\
\hline 38 & Remainder summation above $39^{\circ}$, 8 (thousands). & 2.6 & 7.0 \\
\hline 39 & Exponential summation, $f_{s}$ (hundreds).......... & 3.0 & 7.9 \\
\hline 40 & Physiological summation, fs (thousands) .... & 2.1 & 14.4 \\
\hline 41 & Absolute minimum............... & -40 & -5 \\
\hline 43 & Normal daily mean, coldest 14 days of year $\left({ }^{\circ} \mathrm{F}\right.$ ) & 15 & 40 \\
\hline 44 & Normal daily mean, hottest 6 weeks of year $\left({ }^{\circ} \mathrm{F}\right.$ ). & $64.4-$ & $78.8+$ \\
\hline 45 & Normal daily mean, year $\left({ }^{\circ} \mathrm{F}.\right) \ldots \ldots \ldots \ldots \ldots$ & 35 & $60+$ \\
\hline & Precipitation: & & \\
\hline 46 & Normal daily mean, $f_{s}$ (inch). & $0 \$ 9$ & 131 \\
\hline 47 & Normal No. rainy days (over 0.10 inch), $f_{s}$. & 26 & 161 \\
\hline 48 & Normal No. dry days $(0.10$ inch or less $) f_{s}$. & 19 & 136 \\
\hline 49 & Dry days, percentage of total, $f_{s}$ (per cent). & 11 & 83 \\
\hline 50 & Days in longest normal rainy period, $f s, \ldots$. & 21 & 117 \\
\hline 51 & Days in longest normal dry period, $f s \ldots \ldots$ & 4 & 91 \\
\hline 52 & Mlean total, year (inches)........... & $30-$ & $60+$ \\
\hline
\end{tabular}


TABLE 147.-Climatic extremes for Floerka proserpinacoides-Continued.

\begin{tabular}{|c|c|c|c|}
\hline Plate & Evaporation: & Low. & High. \\
\hline 53 & Daily mean, $1887-88, f_{s}$ (inch)... & .084 & .200 \\
\hline 54 & $\begin{array}{l}\text { Total annual, } 1887-88 \text { (inches) } \ldots \ldots \ldots \ldots \ldots \ldots \ldots \\
\text { Moisture ratios: }\end{array}$ & 20.3 & 54.8 \\
\hline 58 & 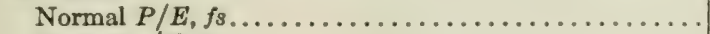 & .51 & 1.39 \\
\hline 59 & 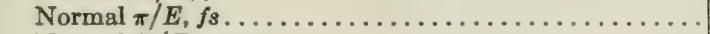 & .60 & 1.63 \\
\hline 60 & 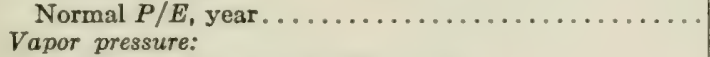 & .72 & 1.72 \\
\hline 63 & $\begin{array}{l}\text { Normal mean, } f_{s} \text { (hundredths inch)...................... } \\
\text { Humidity: }\end{array}$ & 345 & 545 \\
\hline 65 & 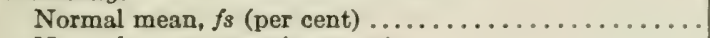 & 65.6 & 84.0 \\
\hline 66 & $\begin{array}{l}\text { Normal mean, year (per cent) } \ldots \ldots \ldots \ldots \ldots \ldots \ldots \ldots \\
\text { Wind: }\end{array}$ & 67.5 & 82.1 \\
\hline 68 & $\begin{array}{l}\text { Normal mean hourly velocity, } f_{s} \text { (miles)........... } \\
\text { Sunshine: }\end{array}$ & 3.1 & 14.9 \\
\hline 69 & $\begin{array}{l}\text { Normal total duration, } f s \text { (hours) } \ldots \ldots \ldots \ldots \ldots \ldots \ldots \\
\text { Moisture-temperature indices: }\end{array}$ & 1,225 & 1,878 \\
\hline 70 & 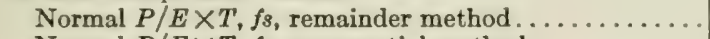 & 301 & 593 \\
\hline 71 & Normal $P / E \times T, f s$, exponential method.......... & 2,914 & 1,592 \\
\hline 72 & Normal $P / E \times T, f_{8}$, physiological method......... & 2,747 & 10,837 \\
\hline
\end{tabular}

TABLE 148.-Climatic extremes for Trautvetteria grandis.

\begin{tabular}{|c|c|c|c|}
\hline Plate & Temperature: & Low. & High. \\
\hline 34 & Days in normal frostless season $(f s) \ldots \ldots \ldots \ldots$ & 25 & 318 \\
\hline 35 & 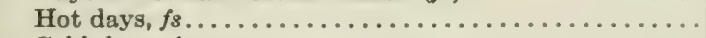 & 0 & 57 \\
\hline 36 & 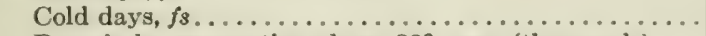 & 0 & 149 \\
\hline 37 & Remainder summation above $32^{\circ}$, year (thousands).... & $10.0-$ & 18.0 \\
\hline 38 & Remainder summation above $39^{\circ}, f_{s}$ (thousands)..... & 2.7 & 5.4 \\
\hline 39 & Exponential summation, $f s$ (hundreds) ............. & 2.8 & 5.7 \\
\hline 40 & Physiological summation, $f_{s}$ (thousands)........... & 1.9 & 8.4 \\
\hline 41 & Absolute minimum $\ldots \ldots \ldots \ldots \ldots \ldots \ldots \ldots \ldots \ldots \ldots$ & -51 & +30 \\
\hline 43 & Normal daily mean, coldest 14 days of year $\left({ }^{\circ} \mathrm{F}.\right) \ldots$ & 17 & 42 \\
\hline 44 & Normal daily mean, hottest 6 weeks of year $\left({ }^{\circ} \mathrm{F}\right.$.).... & $64.4-$ & $71.6+$ \\
\hline 45 & $\begin{array}{l}\text { Normal daily mean, year }\left({ }^{\circ} \mathbf{F} .\right) \ldots \ldots \ldots \ldots \ldots \ldots \ldots \\
\text { Precipitation: }\end{array}$ & $40-$ & 60 \\
\hline 46 & Normal daily mean, $f_{s}$ (inch) $\ldots \ldots \ldots \ldots \ldots \ldots \ldots$ & .025 & .199 \\
\hline 47 & Normal No. rainy days (over 0.10 inch),$f s \ldots \ldots \ldots$ & 0 & 199 \\
\hline 48 & Normal No. dry days ( 0.10 inch or less) $f s \ldots \ldots \ldots$ & 72 & 179 \\
\hline 49 & Dry days, percentage of total, $f_{s}$ (per cent)......... & 27 & 100 \\
\hline 50 & Days in longest normal rainy period, $f s . \ldots \ldots \ldots$ & 0 & 99 \\
\hline 51 & Days in longest normal dry period, $f_{s} \ldots \ldots \ldots \ldots$ & 56 & 216 \\
\hline 52 & $\begin{array}{l}\text { Mean total, year (inches).......................... } \\
\text { Evaporation: }\end{array}$ & $20+$ & 90 \\
\hline 53 & Daily mean, $1887-88, f_{s}$ (inch) $\ldots \ldots \ldots \ldots \ldots$ & .052 & .293 \\
\hline 54 & $\begin{array}{l}\text { Total annual, 1887-88 (inches) } \ldots \ldots \ldots \ldots \ldots \ldots \ldots \ldots \\
\text { Moisture ratios: }\end{array}$ & 18.1 & 79.8 \\
\hline 58 & 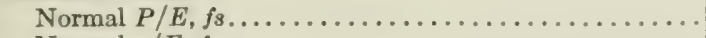 & .18 & 3.84 \\
\hline 59 & Normal $\pi / E, f s \ldots \ldots \ldots \ldots \ldots \ldots \ldots \ldots$ & .20 & 4.48 \\
\hline 60 & 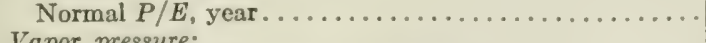 & .18 & 4.90 \\
\hline 63 & $\begin{array}{l}\text { Vapor pressure: } \\
\text { Normal mean, } f s \text { (hundredths inch).. } \\
\text { Humidity: }\end{array}$ & 233 & 329 \\
\hline 65 & Normal mean, $f_{s}$ (per cent)........ & 41.5 & 73.6 \\
\hline 66 & $\begin{array}{l}\text { Normal mean, year (per cent) } \ldots \ldots \ldots \ldots \ldots \ldots \ldots \\
\text { Wind: }\end{array}$ & 45.4 & 76.2 \\
\hline 68 & $\begin{array}{l}\text { Normal mean hourly velocity, } f_{s} \text { (miles).. } \\
\text { Sunshine: }\end{array}$ & 3.5 & 16.4 \\
\hline 69 & $\begin{array}{l}\text { Normal total duration, } f s \text { (hours).. } \\
\text { Moisture-temperature indices: }\end{array}$ & 1,167 & 1,578 \\
\hline 70 & 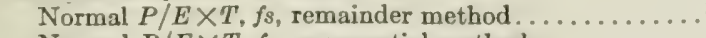 & 81 & 1,566 \\
\hline 71 & Normal $P / E \times T, f s$, exponential method........... & 691 & 11,724 \\
\hline 72 & Normal $P / E \times T, f_{s}$, physiological method.......... & 809 & 7,475 \\
\hline
\end{tabular}


TABle 149.-Climatic extremes for Trautvelleria carolinensis.

\begin{tabular}{|c|c|c|c|}
\hline $\begin{array}{l}\text { Plate } \\
34\end{array}$ & Temperature: & Low. & High. \\
\hline $\begin{array}{l}34 \\
35\end{array}$ & $\begin{array}{l}\text { Days in normal frostless season }(f s) \ldots \ldots \ldots \\
\text { Hot days, } f_{s} \ldots \ldots \ldots \ldots \ldots \ldots \ldots\end{array}$ & $\begin{array}{r}145 \\
63\end{array}$ & $\begin{array}{l}231 \\
160\end{array}$ \\
\hline 36 & Cold days, $f_{3} \ldots \ldots \ldots \ldots \ldots \ldots \ldots \ldots \ldots$ & 0 & 66 \\
\hline 37 & Remainder summation above $32^{\circ}$, year (thousands) & $11.5-$ & $18.0+$ \\
\hline 38 & Remainder summation above $39^{\circ}, f_{8}$ (thousands). & 3.9 & 7.0 \\
\hline 39 & Exponential summation, $f_{8}$ (hundreds) .......... & 3.9 & 8.2 \\
\hline 40 & Physiological summation, $f_{s}$ (thousands) . . . . . & 5.7 & 15.0 \\
\hline 41 & Absolute minimum................... & -35 & +4 \\
\hline 43 & Normal daily mean, coldest 14 days of year $\left({ }^{\circ} \mathrm{F}.\right)$. & 16 & 45 \\
\hline 44 & Normal daily mean, hottest 6 weeks of year $\left({ }^{\circ} \mathrm{F}\right.$.). & $64.4-$ & $78.8+$ \\
\hline 45 & $\begin{array}{l}\text { Normal daily mean, year }\left({ }^{\circ} \mathrm{F} .\right) \ldots \ldots \ldots \ldots \ldots \ldots \\
\text { Precipitation: }\end{array}$ & $50-$ & $60+$ \\
\hline 46 & Normal daily mean, $f_{s}$ (inch). & .099 & .147 \\
\hline 47 & Normal No. ra ny days (over 0.10 inch), $f_{s} .$. & & 161 \\
\hline 48 & Normal No. dry days ( 0.10 inch or less), $f_{s}$. & 19 & 93 \\
\hline 49 & Dry days, percentage of total, $f s$ (per cent).. & 11 & 49 \\
\hline 50 & Days in longest normal rainy period, $f s \ldots \ldots$. & 52 & 172 \\
\hline 51 & Days in longest normal dry period, $f_{s} . . . \ldots \ldots$ & 4 & 56 \\
\hline 52 & $\begin{array}{l}\text { Mean total, year (inches) .................... } \\
\text { Eraporation: }\end{array}$ & $40-$ & $60+$ \\
\hline 53 & Daily mean, $1887-88, f_{s}$ (inch). & $.140-a$ & .200 \\
\hline 54 & $\begin{array}{l}\text { Total annual, } 1887-88 \text { (inches).... } \\
\text { Moisture ratios: }\end{array}$ & 38.3 & 54.8 \\
\hline 58 & Normal $P / E, f_{s} \ldots$ & .51 & 1.00 \\
\hline 59 & Normal $\pi / E, f_{s} \ldots$ & .60 & 1.25 \\
\hline 60 & $\begin{array}{l}\text { Normal } P / E \text {, year ................ } \\
\text { Vapor pressure: }\end{array}$ & .72 & 1.16 \\
\hline 63 & $\begin{array}{l}\text { Normal mean, } f_{s} \text { (hundredths inch)... } \\
\text { Humidity: }\end{array}$ & 345 & 545 \\
\hline 65 & Normal mean, $f s$ (рег cent)........ & 65.6 & 73.7 \\
\hline 66 & $\begin{array}{l}\text { Normal mean, year (per cent)............ } \\
\text { Wind: }\end{array}$ & 67.5 & 75.7 \\
\hline 68 & $\begin{array}{l}\text { Normal mean hourly velocity, } f_{s} \text { (miles)... } \\
\text { Sunshine: }\end{array}$ & 3.1 & 9.4 \\
\hline 69 & $\begin{array}{l}\text { Normal total duration, } f_{s} \text { (hours) .... } \\
\text { Moisture-temperature indices: }\end{array}$ & 1,403 & 1,878 \\
\hline 70 & Normal $P / E \times T, f_{s}$, remainder method. & 316 & 593 \\
\hline 71 & Normal $P / E \times T, f s$, exponential method. & 3,007 & 5,631 \\
\hline 72 & Normal $P / E \times T, f_{s}$, physiological method..... & 5,112 & 10,061 \\
\hline
\end{tabular}

TABle 150.-Climatic extremes for Cebatha diversifolia.

\begin{tabular}{|c|c|c|c|}
\hline Plate & Temperature: & Low. & High. \\
\hline 34 & Days in normal frostless season $(f s)$. & & \\
\hline & Hot days, $f_{s} \ldots \ldots \ldots \ldots \ldots$. & $150-a$ & $180+a$ \\
\hline 36 & (oold days, $f_{3} \ldots \ldots \ldots \ldots \ldots \ldots \ldots \ldots \ldots \ldots \ldots$ & 0 & \\
\hline & Remainder summation above $32^{\circ}$, year (thousands) & & $18.0+$ \\
\hline 38 & Remainder summation above $39^{\circ}$, fs (thousands)... & $7.0-a$ & $9.0+a$ \\
\hline 39 & Exponential summation, $f_{s}$ (hundreds) ........... & $8.0^{a}$ & $10.0+a$ \\
\hline 40 & Physiological summation, $f_{s}$ (thousands). & $15.0^{8}$ & $17.5+a$ \\
\hline 41 & Absolute minimum................. & -3 & +17 \\
\hline 43 & Normal daily mean, coldest 14 days of year $\left({ }^{\circ} \mathrm{F}.\right)$. . & 42 & $50+a$ \\
\hline 44 & Normal daily mean, hottest 6 weeks of year $\left({ }^{\circ} \mathrm{F}\right.$. $) \ldots$. & 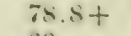 & \\
\hline 45 & $\begin{array}{l}\text { Normal daily mean, year }\left({ }^{\circ} \mathrm{F} .\right) \ldots \ldots \ldots \ldots \ldots \ldots \ldots \\
\text { Precipitation: }\end{array}$ & 60 & $70+$ \\
\hline 46 & Normal daily mean, $f_{s}$ (inch) & .033 & .083 \\
\hline 47 & Normal No. rainy days (over 0.10 inch), $f_{s} \ldots$ & 0 & 53 \\
\hline 48 & Normal No. dry days ( 0.10 inch or less), fs...... & 192 & $275+$ \\
\hline 49 & Dry days, percentage of total, $f_{s}$ (per cent) ............. & is & $1(0)$ \\
\hline 50 & Days in longest normal rainy period, $f s \ldots \ldots \ldots \ldots$. & 0 & 25 \\
\hline 51 & Days in longest normal dry period, $f_{8} \ldots \ldots \ldots \ldots \ldots$ & $75^{a}$ & $250+$ \\
\hline 52 & 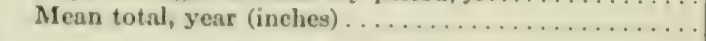 & $10-$ & $20+$ \\
\hline
\end{tabular}


TABLE 150.-Climatic extremes for Cebatha diversifolia-Continued.

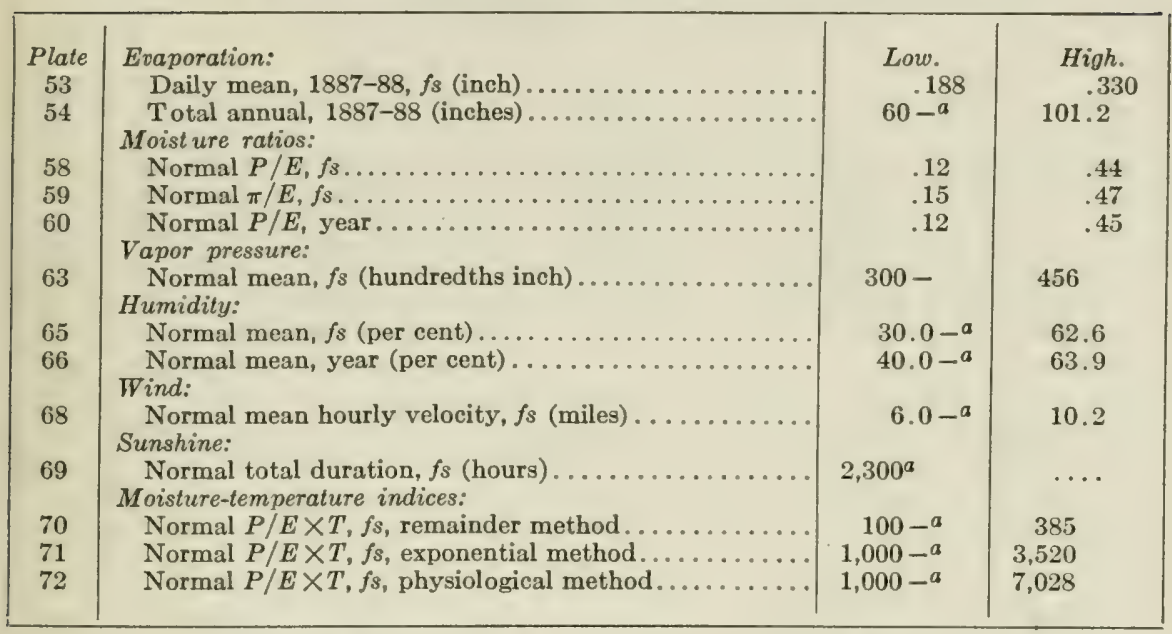

TABLE 151.-Climatic extremes for Cebatha carolina.

\begin{tabular}{|c|c|c|c|}
\hline Plate & Temperature: & Low. & High. \\
\hline 34 & Days in normal frostless season $\left(f_{3}\right)$. & 172 & \\
\hline 35 & Hot days, $f_{s} \ldots \ldots \ldots \ldots \ldots \ldots \ldots$ & 129 & 365 \\
\hline 36 & Cold days, $f_{s} \ldots \ldots \ldots \ldots \ldots$ & 0 & 22 \\
\hline 37 & Remainder summation above $32^{\circ}$, year (thousands). & 11.5 & $26.0+$ \\
\hline 38 & Remainder summation above $39^{\circ}, f_{s}$ (thousands). & 6.3 & 14.5 \\
\hline 39 & Exponential summation, $f_{s}$ (hundreds). & 6.7 & 15.4 \\
\hline 40 & Physiological summation, $f_{s}$ (thousands). & 11.9 & 31.1 \\
\hline 41 & Absolute minimum................. & -32 & +41 \\
\hline 43 & Normal daily mean, coldest 14 days of year $\left({ }^{\circ} \mathrm{F}.\right)$. & 32 & 69 \\
\hline 44 & Normal daily mean, hottest 6 weeks of year $\left({ }^{\circ} \mathrm{F}.\right)$ & 71.6 & $78.8+$ \\
\hline 45 & Normal daily mean, year $\left({ }^{\circ} \mathrm{F}.\right) . . .$. . & $55+$ & $75+$ \\
\hline & Precipitation: & & \\
\hline 46 & Normal daily mean, $f_{3}$ (inch). . & .052 & .173 \\
\hline 47 & Normal No. rainy days (over 0.10 inch), $f s$. & & 284 \\
\hline 48 & Normal No. dry days ( 0.10 inch or less), $f s$. & 26 & 259 \\
\hline 49 & Dry days, percentage of total, $f s$ (per cent). & 8 & $87+$ \\
\hline 50 & Days in longest normal rainy period, $f s$. & $25-$ & 235 \\
\hline 51 & Days in longest normal dry period, $f s . .$. & 14 & 182 \\
\hline 52 & Mean total, year (inches). & 20 & $60+$ \\
\hline & Evaporation: & & \\
\hline 53 & Daily mean, $1887-88, f_{s}$ (inch). . & .102 & .188 \\
\hline 54 & Total annual, 1887-88 (inches). & 38.4 & $70.0^{\alpha}$ \\
\hline 58 & $\begin{array}{l}\text { Moisture ratios: } \\
\text { Normal } P / E, f_{3} . .\end{array}$ & .34 & 1.36 \\
\hline 59 & Normal $\pi / E, f_{3}$. . & .47 & 1.47 \\
\hline 60 & $\begin{array}{l}\text { Normal } P / E \text {, year...................... } \\
\text { Vapor pressure: }\end{array}$ & .24 & 1.47 \\
\hline 63 & $\begin{array}{l}\text { Normal mean, } f_{s} \text { (hundredths inch)... } \\
\text { Humidity: }\end{array}$ & $400^{a}$ & 707 \\
\hline 65 & Normal mean, $f_{s}$ (per cent). & $50^{a}$ & 80.4 \\
\hline 66 & $\begin{array}{l}\text { Normal mean, year (per cent) } \ldots \ldots \ldots \ldots \\
\text { Wind: }\end{array}$ & 63.9 & 85.2 \\
\hline 68 & $\begin{array}{l}\text { Normal mean hourly velocity, } f_{s} \text { (miles)... } \\
\text { Sunshine: }\end{array}$ & 5.1 & 12.3 \\
\hline 69 & $\begin{array}{l}\text { Normal total duration, } f s \text { (hours). } \\
\text { Moisture-temperature indices: }\end{array}$ & 1,878 & 2,650 \\
\hline 70 & Normal $P / E \times T, f_{s}$, remainder method. & $300-a$ & 1,314 \\
\hline 71 & Normal $P / E \times T$ fs, exponential method. & $3,000-a$ & 12,106 \\
\hline 72 & Normal $P / E \times T, f s$, physiological method..... & $4,000^{a}$ & 23,652 \\
\hline
\end{tabular}




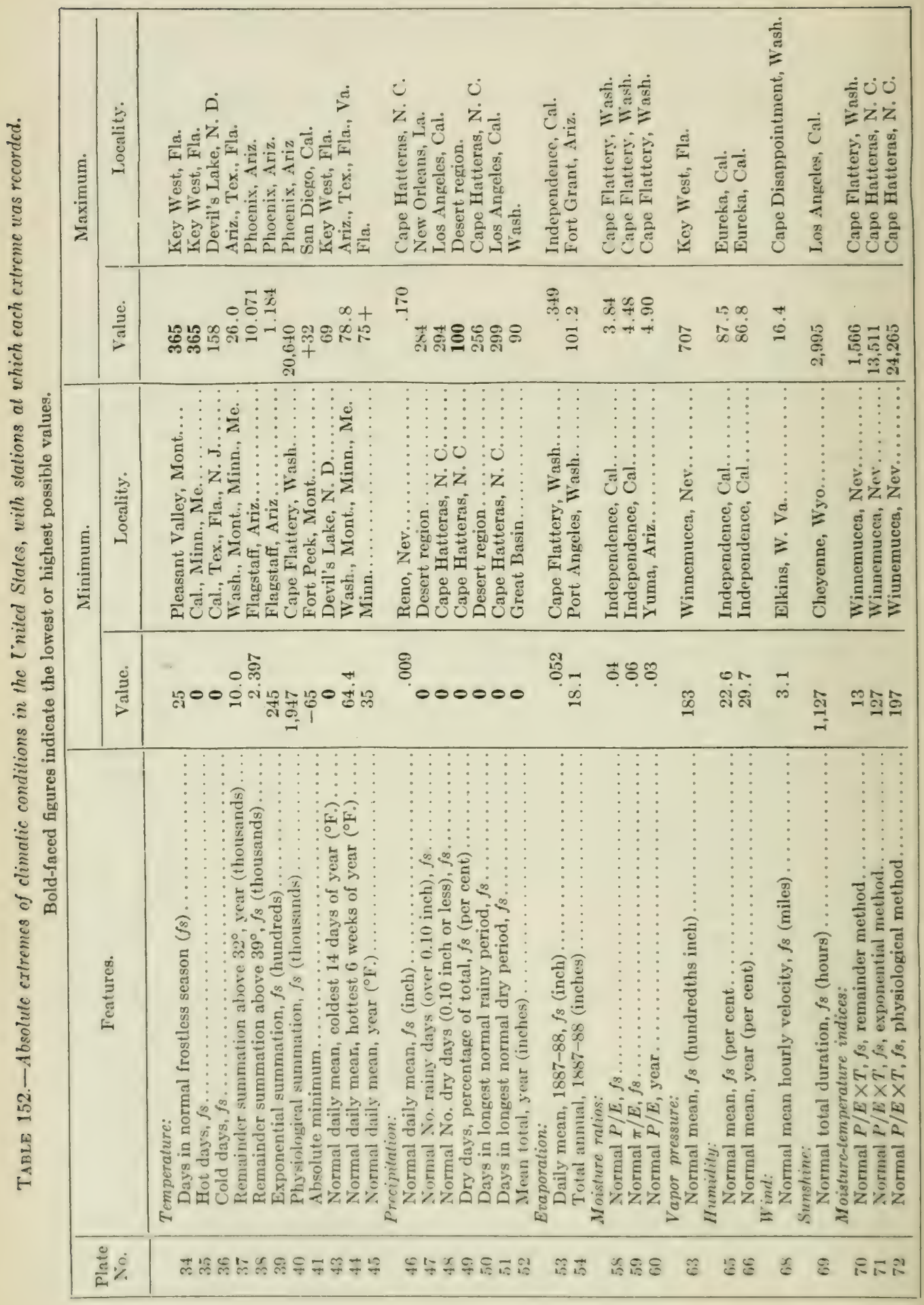




\section{DISCUSSION AND PRELIMINARY INTERPRETATIONS OF THE CORRELATION DATA.}

\section{CORRELATIONS AS INDICATING CONTROLLING CONDITIONS.}

An adequate discussion of the extreme values of each of the climatic conditions which we have used, and of the probable importance of each of them in controlling the distribution of the various vegetations and species, would lead beyond the bounds of practicality if extended to all of the 3,906 readings comprised in the tables just given. It has seemed desirable, therefore, to discuss the controls for the vegetational areas and groups of growth-forms, and for only a selected series of the indivirlual species. Such a discussion involves repeated reference to the tables of climatic extremes and to the graphs in which selected extremes are shown, as well as frequent direct comparison of the plates showing the isoclimatic lines and those showing the distributional limits in question.

We are far from taking the position that it is possible to point out a single climatic condition which may be regarded as acting alone in the control of any distributional feature. Enough has been said in preceding sections to indicate the importance that we attribute to the combined operation of the entire constellation of climatic conditions in determining the distribution of species and vegetations, as well as in controlling their physiological processes. Our desire to consider the climatic conditions collectively, so far as possible, has been responsible for the elaboration of the data of the moisture ratio and of the moisturetemperature indices. The evaporation data may also be regarded as a collective expression of separate climatic elements, although the plant is influenced by these elements collectively just as the atmometer is, in addition to whatever other separate effects they may have.

The tables of climatic extremes show a very large number of cases in which species or vegetations are subjected to at least one-third of the entire gamut of difference exhibited by the climatic conditions, at least in the United States, and there is a large number of cases in which they are subjected to more than half of it. The endurance of such widely differing conditions is partly real and partly such as to require qualification. Widely dissimilar temperature conditions are encountered by those grasses of the Great Plains region which extend from the Canadian boundary to the Rio Grande, and widely unlike conditions of evaporation and the moisture ratio are encountered by plants of southern transcontinental distribution, such as Daucus pusillus or Cephalanthus occidentalis. In the case of Daucus, however, the seasonal habits of the plant are entirely different in the three sections of its range, so that a comparison of the moisture conditions of 
the entire frostless season throughout the range is meaningless. In the Desert area the entire life-history of this annual plant is passed in the earliest weeks of the frostless season and it escapes the more arid conditions of the late spring and early summer by existing only as dormant seeds during that season. Cephalanthus is in foliage throughout its range during the entire frostless season, but, like several other plants that we have used, it is found only in palustrine situations, and its ability to withstand high intensities of evaporation is entirely different from the ability of Covillea or of Artemisia tridentata to withstand the same intensities. In short, the conditions expressed by the moisture ratio are not applicable to Cephalanthus nor to any other aquatic or palustrine plant, since conditions of precipitation in the distributional areas of such forms have no immediate relation to their water-supply.

The fact that a given plant is able to withstand a particular climatic condition of high or low intensity does not mean that the plant is able to withstand it in all parts of the distributional range of that intensity. The eastern deciduous trees are unable to follow westward the temperatures which are favorable to them, and Covillea tridentata is unable to follow northward the moisture conditions which favor it. This is simply another way of stating the fact, already emphasized, that the influence of a given climatic intensity is determined by the accompanying intensities of other climatic conditions. And, furthermore, the manner in which a given climatic intensity is modified by other features of the climate is different for almost every two plants that may be compared.

In the tables of climatic extremes may be noted very many cases in which plants or vegetations range through only a small part of the total gamut of a particular climatic condition. It may be seen more readily in the diagrams (figs. 21 to 74 ) that there are numerous extremely short blocks, indicating that the plant in question meets only a small part of the nation-wide scale of intensities of this condition. It stands to reason that a narrow amplitude of a climatic condition, represented in the graphs by a short block, indicates that the condition in question is more critical for this plant than the conditions which show a wide amplitude. If, for example, a plant is limited on the south by a boundary which corresponds closely with the isotherm of $60^{\circ}$ normal daily mean temperature and is bounded on the north by the corresponding isotherm of $50^{\circ}$, the dimensions for this element of the climate will be narrow. Such a distribution, however, would cross nearly all of the isoclimatic lines indicating differences in the normal daily mean precipitation, so that the dimensions for that element would be very broad. It is obvious that such a distribution would be controlled by the daily mean temperature of $50^{\circ}$ and $60^{\circ}$, and would have no control from the daily mean precipitation. The narrow and broad dimensions, or amplitudes, indicated respectively by short and 
long blocks in the figures, would therefore give a suggestion as to the more important of these two climatic conditions in the control of the distribution of such a hypothetical plant.

Although the comparative amplitudes of two climatic conditions may serve as indicators of their relative importance in controlling a stated case of distribution in an area as large as the United States, it needs always to be borne in mind that we are dealing with only a part of the total possible amplitude of the conditions for the entire globe. Our method would be much more useful if we were studying the vegetation of an area sufficiently large to comprise the greatest known extremes under which plants occur, and it would be of little use in investigating the controlling conditions of a small area.

It must also be kept in mind that the minimum and maximum values of a condition which shows wide amplitude in a particular distributional area are just as truly the limiting intensities as are those of a condition exhibiting narrow amplitude in the same area. The desert grass Hilaria jamesii, for example, encounters a range of length of frostless season from 72 to 296 days, which is a rather wide amplitude, being nearly two-thirds that for the United States. Although this perennial grass is able to accommodate its vegetative activities to a frostless season which is twice as long in some parts of its range as it is in others, there is still every reason to believe that Hilaria is unable to carry through its development with a frostless season of less than 72 days, at least under the conditions which accompany a season of that length in the region in which Hilaria encounters them. It is unable, likewise, to spread into regions with a frostless season of more than 296 days, due undoubtedly to some accompanying adverse conditions rather than to too long a season favorable for growth. Although there is a wide range of differences in length of growing-season which have no apparent restricting influence on the distributional movements of Hilaria, we must not allow this fact to obscure the possibility that the ultimate extremes which it encounters are indeed of importance to its limitation. While Hilaria is able to accommodate itself to frostless seasons of widely differing length, it is able to grow only within narrowly restricted limits with respect to the values of the moisture ratios; it encounters extremely narrow amplitudes of all of these ratios. Its limitation, at different parts of its distributional edge, by frostless seasons of very different lengths, indicates that associated conditions have much to do with whatever influence the length of season may be able to exert. Its limitation by moisture ratios of so nearly the same value means that the influence of this compound condition is but little affected by the various values of associated factors that are to be found on different parts of the distributional limit. This is as much as it is possible to infer from the statement that one environmental condition is more important that another in limiting plant distribution. 
In order to make a thoroughgoing and entirely satisfactory investigation into the nature of the conditions which limit a plant at the various portions of its distributional edge, we should ascertain the entire complex of conditions for numerous localities along this edge. We should know at the outset the amplitude of each condition for the entire range of the plant or vegetation, and should know the localities (usually on the edge of the geographic range) at which the extreme values are encountered. For each station at which a maximum or minimum value of any condition was found we should then ascertain the entire constellation of other conditions. Since no extreme condition operates in any other way than in conjunction with the associated conditions of the same locality, we might thus be able to ascertain the controlling complex of conditions for the given locality, and, in turn, for all other localities in the edge of the distributional area. But the problems with which we are dealing are too new, and the available quantitative data and precise information pertaining to them are too limited at present, to warrant serious attempts to pass beyond the limits of very general considerations.

\section{COMPARATIVE CLIMATIC FEATURES OF THE NINE GENERAL VEGETATIONAL AREAS.}

Before considering the complexes or constellations of climatic conditions which characterize each of our various vegetational areas, it will be instructive to see how the extreme values of several of the leading features of the climate compare in these areas. Our later discussions will concern the whole climatic character of each botanical area, whereas we now wish to compare each climatic element singly and as differing in intensity among the nine general vegetational areas. For this purpose 12 of the climatic charts have been selected (plates 34, $35,36,40,43,46,52,53,59,65,69$, and 72 ), and their climatic dimensions are presented in figures 21 to 26 . These charts will now be considered in order.

Number of days in normal frostless season (plate 34, fig. 21). - A comparison of the blocks in this graph shows that the longest frostless seasons are found in the Desert and the western section of the Northern Mesophytic Evergreen Forest, and that nearly as short a season is found in the Grassland. The first two of these have maximum values which nearly coincide, meaning that there is almost exactly the same amplitude ${ }^{1}$ in the length of the frostless season in these two very dis-

\footnotetext{
1 "lhe word "amplitude" is used throughout the succeding pages to express the degree of dissimilarity between the values of a climatic condition in the different parts of a botanical area. Numerically it is the difference between the maximum and the minimum value for the area. This is done in order to avoid the use of the word "variation," which might be taken to indicate the seasonal differences, or the differences from year to year, at the same climatological station. We are not here concerned with the seasonal or annual march of any of the climatic conditions, nor with any of their other rariations, but solely with the differences which their index values exhibit from place to place, and with the broad or narrow amplitude of these differences in given areas.
} 
similar vegetations. The longest frostless season is found in the Southeastern Mesophytic Evergreen Forest, and the amplitude for that vegetation is approximated by the Semidesert. Aside from the cases mentioned, there is considerable dissimilarity between the amplitudes of this condition in the various vegetations, particularly between the Evergreen Forest areas. The amplitudes of the other areas overlap to such an extent that in all five of them it is possible to find localities with frostless seasons ranging from 197 to 245 days in length, that is, from the minimum for Semidesert to the maximum for Grassland. This same range of seasonal length may also be found in all of the Evergreen Forest areas except the eastern section of the Northern Mesophytic Evergreen Forest.

Hot days (plate 35 , fig. 21).- The number of days with a normal daily mean temperature of $68^{\circ}$ or above is relatively uniform for the first group of vegetations (group A), although reaching slightly lower maximum values for Grassland and Grassland Deciduous-Forest Transition. The Northwestern Hygrophytic Evergreen Forest has no hot days in any part of its range, and portions of four other vegetational areas are also without hot days in this sense. The greatest number of hot days is encountered in the Southeastern Mesophytic Evergreen Forest, where the range from 120 to 180 days, which is characteristic of a large area in this vegetation, is raised to 365 days for Key West.

Cold days (plate 36 , fig. 22). - The number of days with a normal daily mean temperature of $32^{\circ}$ or below is a climatic feature of great amplitude for several of the second group of vegetations (group B). The

\begin{tabular}{|c|}
\hline VEGETATION \\
\hline Desest \\
\hline SEMI-DESERT \\
\hline Gerssunio \\
\hline GRASSLAND-DECIDUOUS-FORESTTRANSITION \\
\hline DECIDUOUS FOREST \\
\hline NORTHWESTERV HTGROFHTTIC EVERGREEN FOSEST \\
\hline SOUTHEASTERN MESOPHYTIC EVEFGREEN FOREST \\
\hline NDFTHERN MESOPHTIC EVERGREEN FOREST (WLST) \\
\hline NORTHERN MESOPWITIC EVERGREEN FOREST (EAST) \\
\hline
\end{tabular}
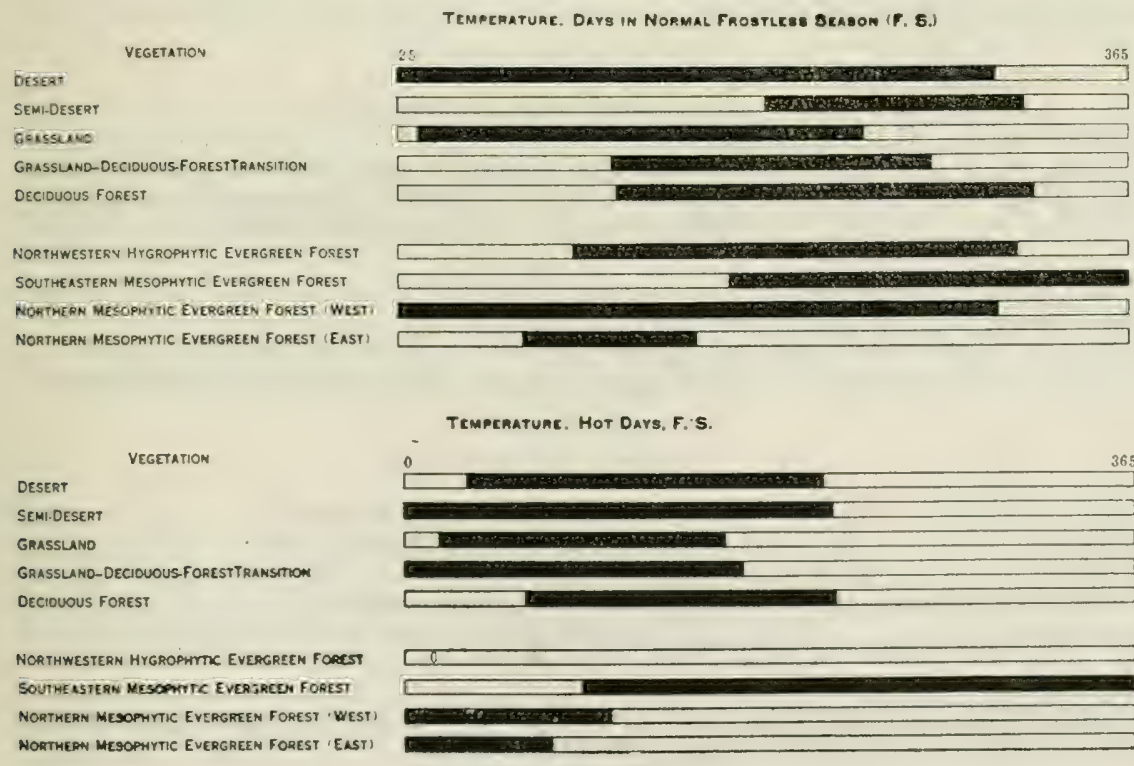

Tempenatune. Hot Dars, F.'S.

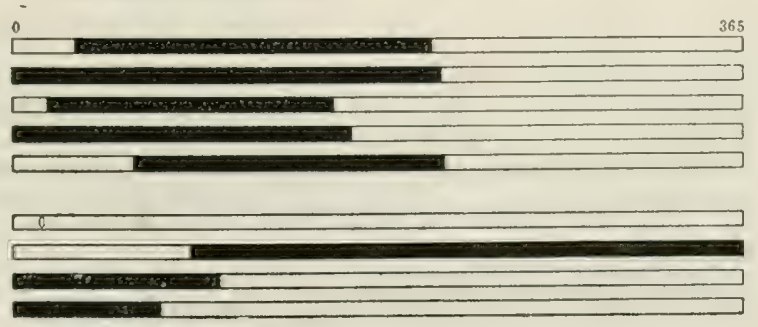

FIG. 21. 
Grassland area exhibits the total range for the United States, from no cold days in the south to 158 at the Canadian boundary, and the Grassland Deciduous-Forest Transition shows nearly as great an amplitude with respect to this condition. The two Semidesert areas are without cold days, and the Desert area is surprisingly like the Deciduous Forest in the amplitude and extremes of this condition. The Northwestern Hygrophytic Evergreen Forest is also without cold days, and the Southeastern Mesophytic Evergreen Forest ranges from none to only 27 days at the coldest station. The two sections of the Northern Mesophytic Evergreen Forest are very similar with respect to this condition.

Physiological temperature summation (plate 40, fig. 22).--The physiological summation of temperature (expressed in thousands) exhibits wide amplitudes for the vegetations of group A (Desert to Deciduous Forest). The most striking feature brought out by a comparison of the blocks is the relative similarity of the climatic dimensions for the vegetations of group A as compared with the unlikeness of the blocks for those of group B (the Evergreen Forest regions). The minimum value for the United States and the smallest amplitude are exhibited by the Northwestern Hygrophytic Evergreen Forest. The eastern and western sections of the Northern Mesophytic Evergreen Forest are also characterized by low summations, but they both reach much higher maximum values than does the Northwestern Forest. The Southeastern Mesophytic Evergreen Forest reaches the highest values of any of the vegetations and also exhibits the greatest amplitude of this condition.

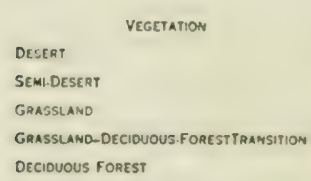

NORTMWESTRK HYGROPHYTIC EVERGREEN FOREST SOUTHEASTERM MESOPHITIC EVERGaEEM FOREST NORTMEAN MESOPHITC EVERGRECM FOREST (WEST) NORTMERN MESOPHTIC EVERGREEN FOREST (EAST)

vegrtation

Descat

SEMI OESERT

GaRSLUND

GARSLAHD DECIOUOUG FOACSTTRANSTONON DeClDUOUS FOREST

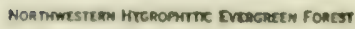

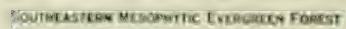
NOATMEAM ME , ONATIC EVIRGRCEN FORES? IWEST)

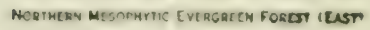

TEmpeantuac. Colo Dare, F. S
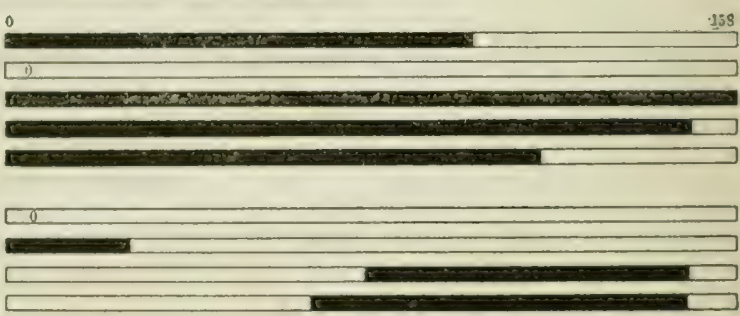

Tempearune. Paysiological SUmmation, F. S.

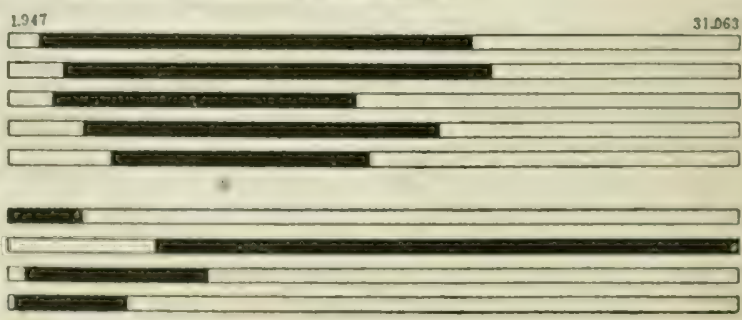

FiG. 22. 
Normal daily mean temperature of coldest 14 days of the year (plate 43, fig. 23).-The daily mean temperature of the coldest period of the year is a climatic factor which varies greatly in the various vegetational areas. The lowest value, $0^{\circ}$, is found in the northernmost part of the Grassland area, and minimum values of $5^{\circ}$ are found in the Grassland Deciduous-Forest Transition and in the eastern section of the Northern Mesophytic Evergreen Forest. The highest mean temperature of this coldest period, $69^{\circ}$, is found in the Southeastern Mesophytic Evergreen Forest, and the nearest maximum values approaching it in other vegetations are found in the Desert and Semidesert, where maxima of $54^{\circ}$ occur.

There is apparently no temperature factor which exhibits greater diversity in its amplitude and extremes in the several vegetational areas than does the mean temperature of the coldest fortnight. The normal daily mean of the hottest six weeks, for which we have used the data elaborated by Merriam, fails to show such a diversity for the vegetational areas (see tables 32 to 40 ).

Normal daily mean precipitation of frostless season (plate 46, fig. 23).This climatic index exhibits a well-graduated series of differences in the 9 vegetational areas, from its lowest values for the Desert $(0.009 \mathrm{inch})$ and the Semidesert (0.017 inch) to its highest value for the Northwestern Hygrophytic Evergreen Forest (0.199 inch). Here again the Evergreen Forest areas of group B exhibit greater differences than do the areas of group A. Pronounced similarities exist between the Desert and Semidesert, and between the Grassland Deciduous-Forest Transition and the Deciduous Forest. Similarities which are less
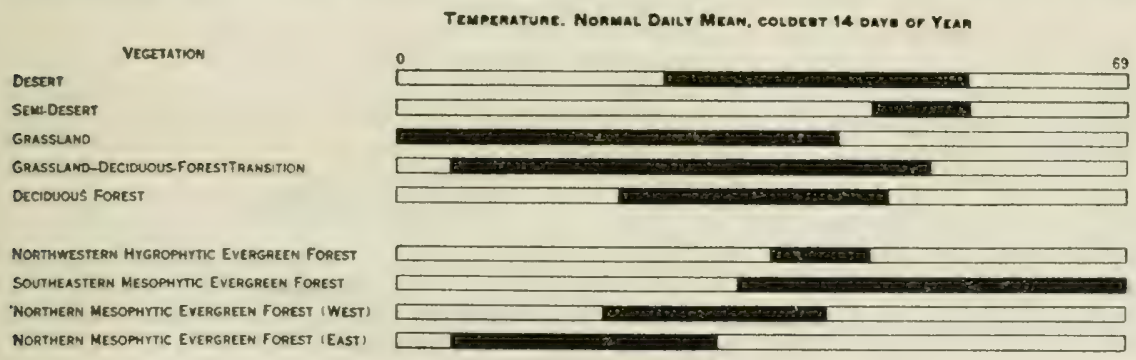

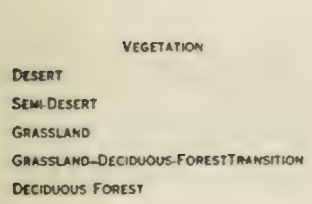

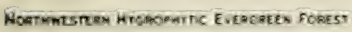
SOUTHEASTERN MESOPAYTIC EVERGAEEN FOREST NORTHERN MESOPHYTC EVERGREEN FOREST IWEST NORTRERN MESOFHTTC EVERGREEN FOREST IEAST)

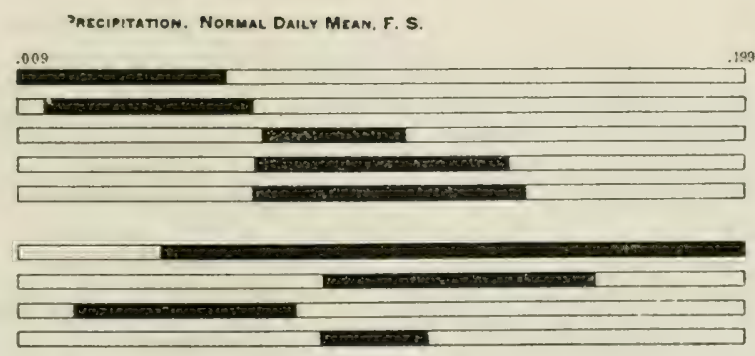

Fig. 23. 
marked but more surprising exist between the Semidesert and the western section of the Northern Mesophytic Evergreen Forest, and between the Grassland and the eastern section of the Northern Mesophytic Evergreen Forest. These incongruities probably find their explanation in the differences in the seasonal distribution of rainfall and in the compensating influences of other conditions. The western section of the Northern Mesophytic Evergreen Forest (which is much drier than the eastern section) does not include markedly higher values than does the Desert, a fact which must be considered in connection with the temperature and evaporation differences between these two vegetations.

Mean total annual precipitation (plate 52, fig. 24).-The graph representing these data is given a conventionalized appearance from the fact that we have used the map prepared by Gannett and have not drawn the extremes from the readings of individual stations, as in the case of most of the other climatic conditions. This graph bears a generic resemblance to the one showing the normal daily mean precipitation for the frostless season. Owing to the smoothed nature of the data on which it is based, it shows an even more pronounced gradation between the several vegetational areas. No new features are brought out in this figure, as compared with the one just discussed, and indeed some of the indications of the latter are partially contradicted by this one. Owing to the fact that the data from plate 52 are not the readings of individual stations, it is impossible to tell in how far the differences between this figure and the preceding are due to this circumstance and in how far they are due to the fact that the

\begin{tabular}{|c|}
\hline VEGETATKN \\
\hline DESER: \\
\hline SEM.DESERY \\
\hline GRASSLAND \\
\hline GRASSLAND-DECIOUOUS-FORESTTRAMITIOV \\
\hline DECIDUCUS FOREST \\
\hline
\end{tabular}

NOATWAESTERN HYGROPHYTIC EVEAGAEEN FOREST SOUTHEASTERY MESOPMYTIC EVEAGREEN FOREST NORTHERN MESOPHYTIC EYERGREEN FOAEST I WEST: NORTMERN MESOPHYIC EVEPGREEN FOREST IEMST

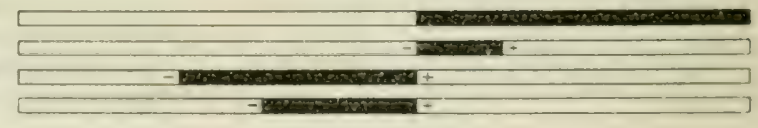

VRGETATION

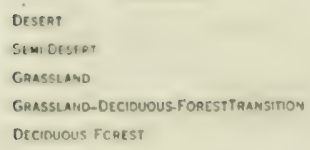

NONTHWLSTEAN HYGROPMYTIC EvEagaEen FOREST SOUTHCASTERM MLSCPMITIC EVERGAECN Fopest

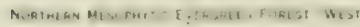

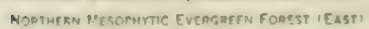

Evaponation. DaILY Mina, 1887-0, F, S

PRceipitation, MEan TOTAL YEAA
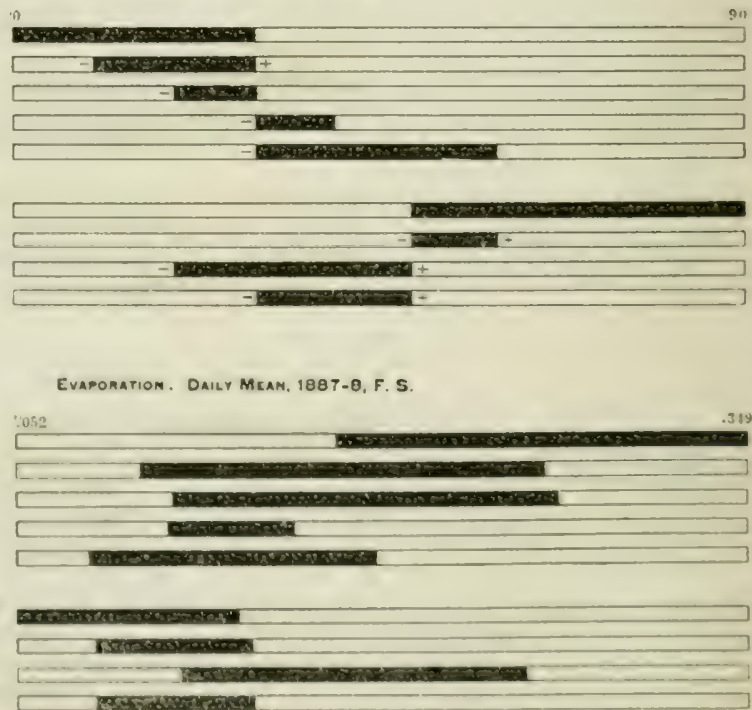

Firs. 24 
former is based on the precipitation data of the frostless season and the latter on those of the entire year.

Evaporation (plate 53, fig. 24). - The daily mean evaporation for the frostless season reaches its highest values in the Desert region, although some of the stations situated in this vegetation have readings so low as to give the Desert an amplitude of evaporation conditions which is half that for the entire country. The Semidesert and Grassland have limits and amplitudes which are closely similar. The Grassland Deciduous-Forest Transition exhibits a minimum which is the same as that of the Grassland, but it has a much smaller amplitude of evaporation conditions, nowhere reaching values as high as the lowest ones for the Desert. The Deciduous Forest has a much greater amplitude than the transition region between it and the Grassland, and its highest values are as great as the minimum values for the Desert, corresponding to the southward prolongation of the Deciduous Forest into Texas. The lowest evaporation values are found in the Northwestern Hygrophytic Evergreen Forest, and relatively low minimum values are found in the Southwestern Mesophytic Evergreen Forest and in the eastern section of the Northern Mesophytic Evergreen Forest. The maximum values of the three areas just mentioned are remarkably similar. The limits and amplitude of evaporation conditions for the western section of the Northern Mesophytic Forest are higher than for any of the other evergreen forest areas, and closely similar to the Semidesert and Grassland values.

Moisture ratio (plate 59, fig. 25.) - The data here considered are those derived from the precipitation index for the frostless season and the 30

VEGETATION
OESERT
SEMF-DESERT
GRASSLAND
GRASSLUNO-DECIDUOUS.FORESTTRANSIIION
DECIDUOUS FOREST

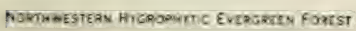
SOUTHEASTERN MESOPHTTIC EVERGREEN FOREST NORTMERN MESOPHYYIC EVERGREEN FOREST (WEST) NORTHERN MESOPHYTIC EVERGREEN FOREST IEASTI

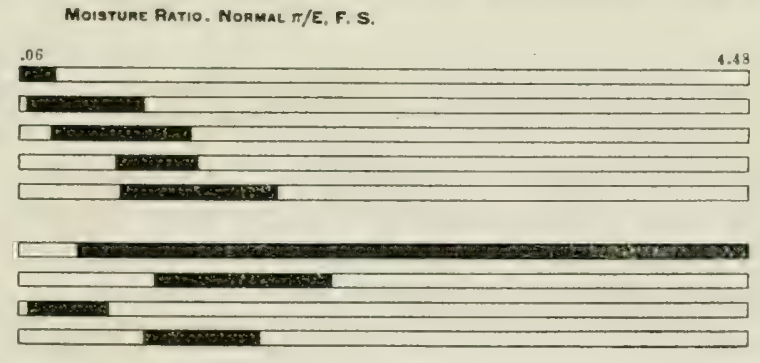

HUMIOITY. NOAMAL MCAN, F. S.

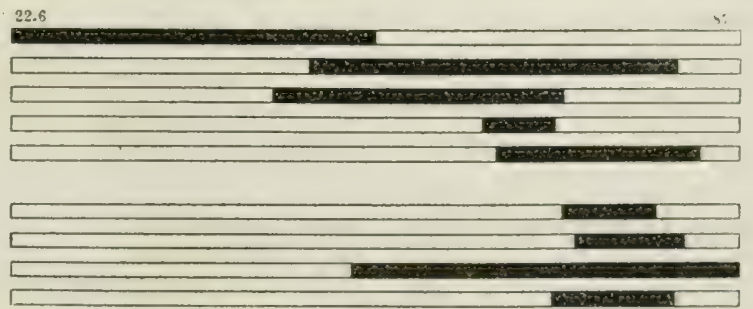

FI 1 . 25. 
days preceding its commencement, and from the evaporation data for the frostless season. The differentiation of the values of this ratio for the vegetational areas is restricted by reason of the extremely high ratios for the Northwestern Hygrophytic Evergreen Forest. The collective amplitudes of the other vegetations cover less than half the total range for the United States. The narrow amplitudes of this climatic feature for all the vegetations except the Hygrophytic Forest are an indication of the importance of the moisture ratio as an expression of the conditions which are critical in determining the distribution of the vegetation of the United States as we have charted it.

The five vegetations in group $\mathrm{A}$ exhibit progressively higher limits for their values of the moisture ratio. The evergreen forest areas of group B are very dissimilar. The western and eastern sections of the Northern Mesophytic Evergreen Forest stand well apart, the former overlapping with the Northwestern Hygrophytic Evergreen Forest, just as these vegetations merge into one another in their actual occurrence. The Southeastern Mesophytic Evergreen Forest possesses a greater amplitude than the eastern section of the Northern Mesophytic Evergreen Forest, but embraces almost the whole scale of values found in the latter vegetation. The amplitude of the western section of the Northern Evergreen Mesophytic Forest is covered by that of the Semidesert, again emphasizing the relative aridity of this forest region. The amplitude of the eastern section of the Northern Mesophytic Evergreen Forest is also covered by that of the Deciduous Forest, which is accordant with the overlapping and intermixture of these two forests.

Normal mean relative humidity (plate 65, fig. 25).-The relative humidity values for the Desert are so low that they have the effect of restricting the differentiation of the values for the remainder of the country, just as the Hygrophytic Forest does with respect to the moisture ratios. The lowest humidity is to be expected in the Desert, but the highest readings would be looked for in the Northwestern Hygrophytic Evergreen Forest rather than in the western section of the Northern Mesophytic Forest, as is the actual case for our data. This is doubtless due to the unfortunate circumstance that there are no humidity records available for localities in the most humid portions of the coast or mountains of Washington and Oregon. The maximum value is recorded for Eureka, California, which is situated in the redwood type of mesophytic forest. The Semidesert greatly exceeds the Desert in the range of its humidities, as would be expected in the contrasting of arid coastal regions with an arid interior region. The blocks showing the range and amplitude of humidity for the Grassland, Grassland Deciduous-Forest, and Deciduous Forest overlap in a manner which is quite analogous to the occurrence of these vegetations. The transition region possesses an amplitude of humidities which is nearly 
the same as the overlapping of the Grassland and Deciduous Forest. The total amplitude of humidity conditions in the western section of the Northern Mesophytic Evergreen Forest is half that of the entire United States. The other three evergreen-forest areas have amplitudes and limits which are extremely similar, and fall within the range for the western section of the northern forest. The range of humidity conditions which appears to favor the evergreen type of forest also falls within the range for the Deciduous Forest. This indicates that the forest regions of the United States as a whole are to be found under very similar humidity conditions (about 70 to 80 per cent) for the frostless season, and that the diversified types of forest comprised in the western section of the Northern Mesophytic Evergreen Forest extend into regions of both lower and higher humidity, overlapping slightly with the highest values of the Desert Region.

Normal daily duration of sunshine (plate 69, fig. 26).- The sunshine conditions of the frostless season are imperfectly known for the Desert region, where they might be expected to possess their highest values. Much lower minimum values are found in the Desert than in the Semidesert, and in the latter region are the highest known values. The sunshine conditions are very similar throughout the Grassland, Grassland-Deciduous Forest, and Deciduous Forest regions, reaching the lowest value for the United States in the first-named of these regions. The sunshine conditions are very dissimilar in the four evergreen forest areas, reaching a wide amplitude in the Southeastern Mesophytic and western section of the Northern Mesophytic Evergreen Forests.

SUnSmine. NORMal DallY DUMation, F, S.

VEGETATION

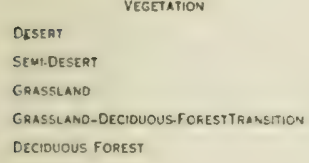

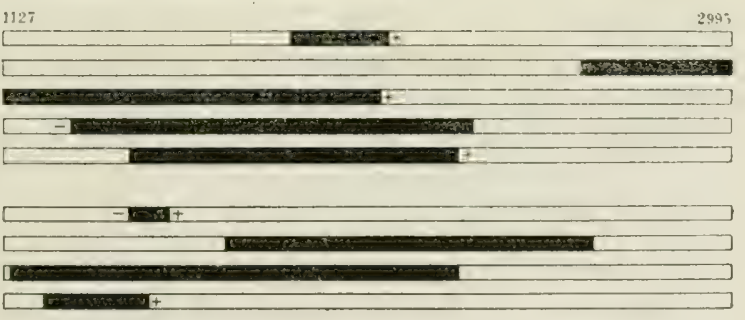

NORTHWESYERN HYGROPHYTIC EVERGREEN FOREST SOUTHEASTERN MESOPHYTKC EVERGREEN FOREST NORTHERN MESOPHYTIC EVERGREEN FOREST i WEST NORTHERN MESOPHYTLC EVERGREEN FOREST IEAST

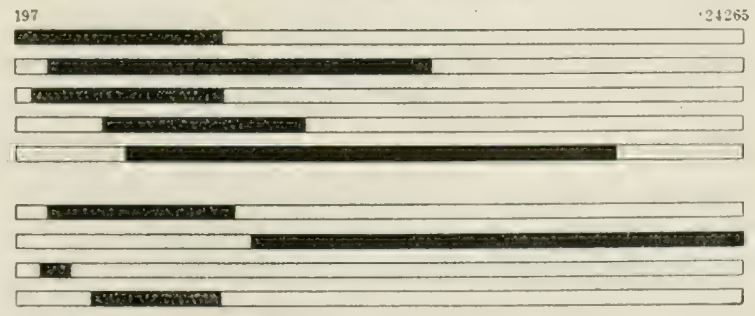


Normal moisture-temperature index for the growing-season (plate 72 , fig. 26).- This composite climatic feature is remarkable in the fact that it possesses a steep gradient of change on passing from the central to the southeastern part of the United States, and possesses a relative uniformity over the western third of the country. The highest values are consequently to be found in the Southeastern Mesophytic Evergreen Forest and the second highest in the Deciduous Forest, both of which areas show wide amplitudes of this condition. The lowest value is found in the Desert, which is closely approached by the Grassland and the western section of the Northern Mesophytic Evergreen Forest. The last-named vegetation, by reason of its western position, possesses a very narrow amplitude of the conditions expressed by this index. The distribution of the various values of the moisture-temperature index is such as to give closely similar limits and amplitudes to such dissimilar vegetations as Desert, Grassland, Northwestern Hygrophytic Evergreen Forest, and the eastern section of the Northern Mesophytic Evergreen Forest, with the Deciduous Forest overlapping into this range of conditions.

\section{CONDITIONS THAT PROBABLY DETERMINE THE GENERAL VEGETATIONAL AREAS.}

\section{OBSERVATIONS FROM THE CHARTS.}

The foregoing review of the comparative ranges and intensities of 12 of the leading climatic conditions for the 9 vegetational areas of the United States has served to throw some light on the question as to which of these conditions are most important in controlling the limits of the vegetations. The wide amplitude of all of the temperature conditions has indicated that in the case of many of the regetations, and particularly those with a wide north-and-south extent, there may be found parallel series of temperature conditions in two or more vegetations. The moisture-temperature index also fails to exhibit differences between the several vegetational areas such as to give it importance as representing a controlling factor. The precipitation and evaporation data fall much more nearly into groups of intensities and amplitudes which show dissimilarity throughout the series of vegetational areas. The moisture ratio and the relative humidity also show striking differences between the rarious regetations.

Figures 27 to 35 give, in diagrammatie form, the limits and amplitudes of 17 selected climatic factors for each of the 9 generalized regetational areas. These diagrams make it possible to riew the correlation of climate and vegetation from a different angle to that employed in the immediately preceding pages. Here it is possible to see a diagrammatic picture of the climate of each of the vegetations, to note whether each of the conditions ranges through a series of values which 
are high or low as contrasted with conditions in other parts of the United States, and to observe whether the amplitude of each condition is wide or narrow. The last has already been mentioned as a valuable means of discovering the climatic conditions which are most critical in controlling the distribution of a given vegetation or plant.

It will be fruitful to discuss these diagrams in connection with a comparison between the vegetational boundaries and the isoclimatic lines of the corresponding plates. In this manner it will be possible to test out the indications given by narrow amplitudes of the conditions (short blocks in the diagrams), and not only to discover which of the various conditions appear to be the most potent in controlling distribution, but also to find the particular intensity of the condition which seems to be critical in each case.

Desert (fig. 27).-Wide amplitudes are exhibited by the Desert with respect to all of the temperature conditions, the number of days in the longest normal dry period of the frostless season, and also the daily mean evaporation for the frostless season. Narrow amplitudes are exhibited by the number of days in the longest normal rainy period of the frostless season and by the moisture ratios and sunshine duration (plate 69).

An examination of the 6 plates showing the isoclimatic lines for the temperature conditions used on this diagram (plates 34, 35, 36, 40, $43,45)$ will discover that numerous lines cross the Desert region in a northwest-southeast direction, indicating, as we have already been prepared to find, from the wide amplitudes in figures 21 and 22, that the Desert possesses a wide range of temperature conditions, which may also be found to the eastward in three or four other vegetations.

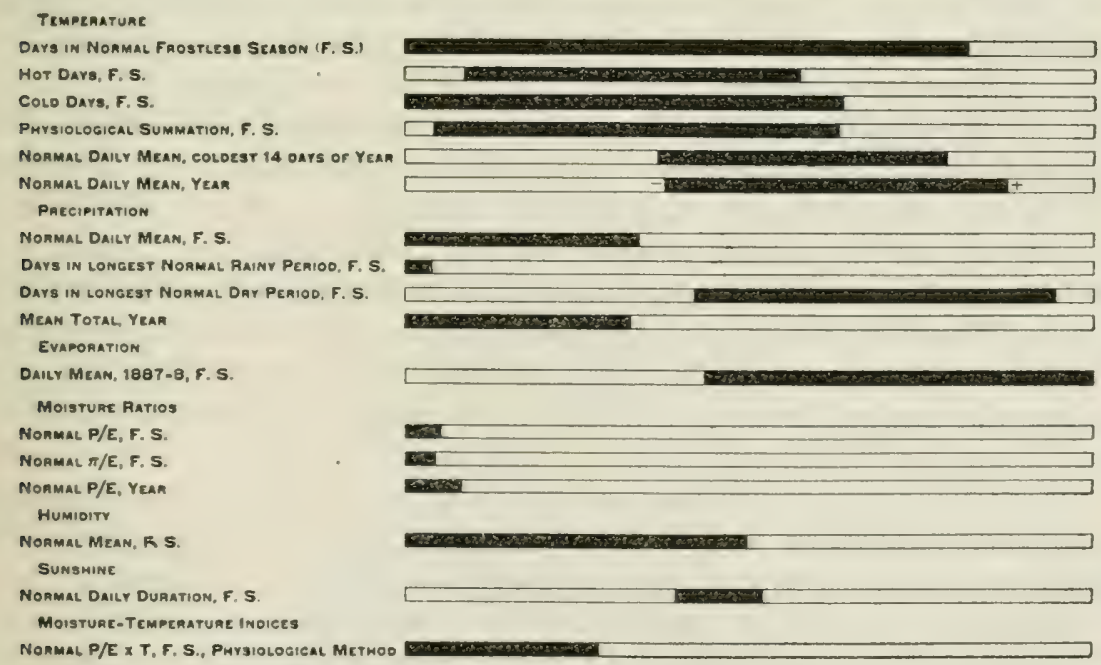

FIG. 27. Climatic extremes for the Desert. 
We may not, therefore, look for any of the factors controlling the distribution of the Desert region in these features of the temperature.

The amplitude of the number of days in the longest normal dry period of the frostless season is also great for the Desert (from 127 to 283). This is a case, however, in which even the minimum value for the Desert indicates dry conditions, and the maximum value signifies conditions of extreme aridity. The lower values of this condition may be of importance elsewhere in differentiating vegetation, and the various values within the Desert area may be of importance in connection with the minor vegetational differences of that diversified region. The position of the isoclimatic lines of plate 51 does not indicate that any particular intensity of this condition is critical in limiting the Desert region, which is in accord with the evidence of figure 27. The case of the daily mean evaporation is a very similar one.

The number of days in the longest normal rainy period in the frostless season ranges, in the Desert region, from its minimum value of no days at several stations to a maximum of 10 days. The isoclimatic line of 25 days embraces the entire Desert region and portions of the Semidesert, the Grassland, and the western section of the Northern Mesophytic Evergreen Forest. This factor appears to be an important one and it is possible that numerous and adequately distributed stations would show that the isoclimatic line of 10 days lies near the boundary of the Desert area.

The three derivations of the moisture ratio (plates 58, 59,60) are very similar here, as they are in all of the vegetations and other botanical areas. The narrow amplitude of this condition for the Desert area suggests an importance which is borne out by an examination of the isoclimatic lines. The line representing values of 0.20 for the moisture ratio is roughly similar to the outline of the Desert, and suggests that the isoclimatic line of the ratio 0.25 might be a still closer approximation to the limits of the Desert. The area with values below 0.25 would then include more of western Texas, would separate the Mogollon Plateau of Arizona from the Rocky Mountains, and would extend somewhat further north, thereby coming into still closer agreement with the outlines of the map of generalized vegetation. The bend of the isoclimatic line for 0.20 which extends through the Tehachapi Pass into the San Joaquin Valley of California is in accord with the pronouncedly desert character of this valley, which has been included in the composite Semidesert Area.

All the evidence which we have been able to bring out in this case points to the moisture ratio as being the climatic condition of most importance in determining the boundaries of the Desert region.

Scmidesert (fig. 28). - The Semidesert embraces two areas-one in California and one in Texas. These areas are not only diversified within themselves, but are somewhat dissimilar in their vegetation and 
still more unlike in the general character of their climates, particularly with respect to the seasonal distribution of precipitation and other moisture conditions. In a more detailed study of climatic correlations these two areas would repay separate investigation.

The evidence of the amplitudes and also of the position of the isoclimatic lines indicates that temperature conditions are of more importance in limiting the Semidesert than is the case with the Desert. The normal daily mean of the coldest 14 days of the year appears particularly to be of importance, ranging only from values slightly below $45^{\circ}$ to $54^{\circ}$ for different sections of the region. The amplitudes of the moisture ratios are greater than in the case of the Desert, and the position of the isoclimatic lines corroborates this indication that the conditions expressed by the ratios are not so important in the limitation of the Semidesert. In passing from the coastal to the interior portion of the Texas section of the Semidesert there is a rapid fall in the values of the moisture ratio (plate 59) from 0.81 (Brownsville) to 0.35 (Fort Ringgold). A similar diversity in the California section of the area is indicated by the value 0.10 for Fresno, as compared with the value 0.45 for Los Angeles. The value for Fresno is well above the minimum value for the Desert and the maximum values in each section of the area are well above the maximum (0.27) for the Desert.

Grassland (fig. 29).- - The longest axis of the Grassland region runs in a north-and-south direction nearly across the United States, with the result that all of the leading temperature conditions exhibit wide amplitudes within its boundaries. The number of cold days in the frostless season runs through the entire gamut for the United States,

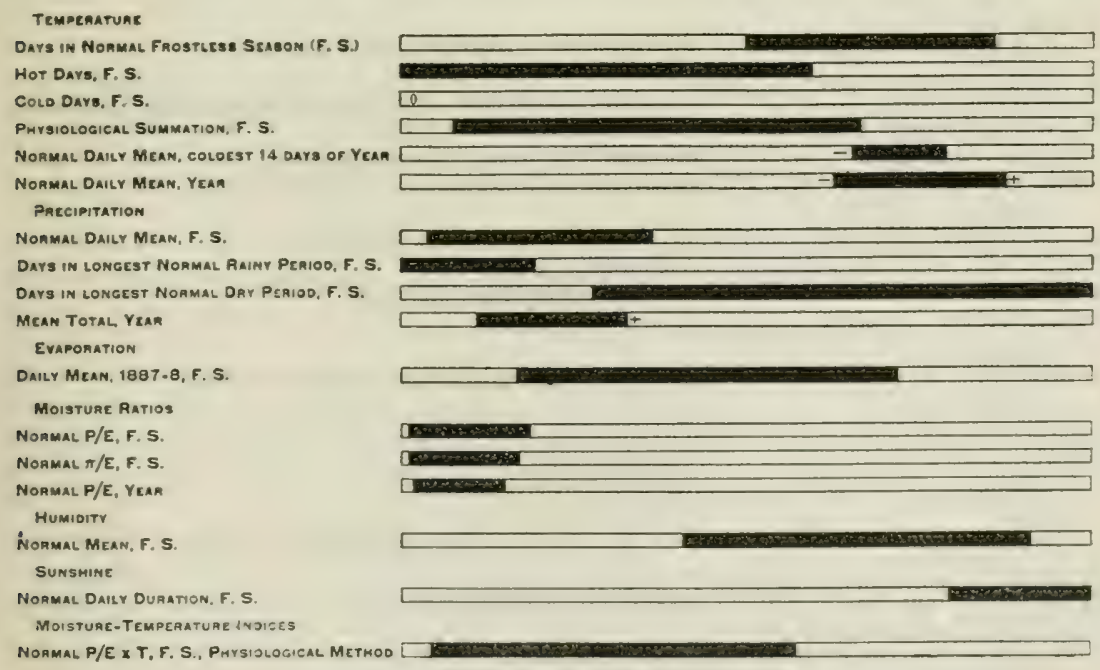

FIG. 28. Climatic extremes for Semidesert. 
and the other five conditions shown in figure 29 run through about half the gamut for the country. Although the mean total precipitation is both low in amount and narrow in its amplitude for this region, the normal daily mean precipitation ranges through a greater amplitude of values and reaches maximum values which are slightly more than half as great as the maximum values for the Northwestern Hygrophitic Evergreen Forest. This difference points to the importance of the seasonal distribution of rainfall in the Grassland region and to the heavier precipitation of the frostless season. The evaporation index for the frostless season ranges from a daily mean of 0.117 inch, at Moorhead, Minnesota, to $0.275 \mathrm{inch}$, at Cheyenne, Wyoming, which is nearly half the amplitude for the United States. Numerous isoclimatic lines cross the central portion of the Grassland, as this would indicate.

A comparison of the position of the Grassland (plate 2) with the location of the isoclimatic lines showing the values of the moisture ratio $\pi / \mathrm{E}$ for the frostless season (plate 59) indicates a correspondence as close as that exhibited for the Desert. The isoclimatic line for the value 0.40 lies slightly to the east of the western edge of the Grassland in Montana, South Dakota, and Nebraska, although very close to it in Texas. The line for the value 0.30 would lie much nearer this boundary along the northwestern edge of the area. The eastern edge follows closely the line for the value 0.60 in Texas and Oklahoma, but crosses rapidly in Kansas and Nebraska to the lines for higher values, and reaches its maximum of 0.117 in western Minnesota. A correspondence almost as close is shown between the Grassland and the area comprising normal mean relative humidities between values of 50 per cent and 65 per cent for the frostless season (plate 65). Along

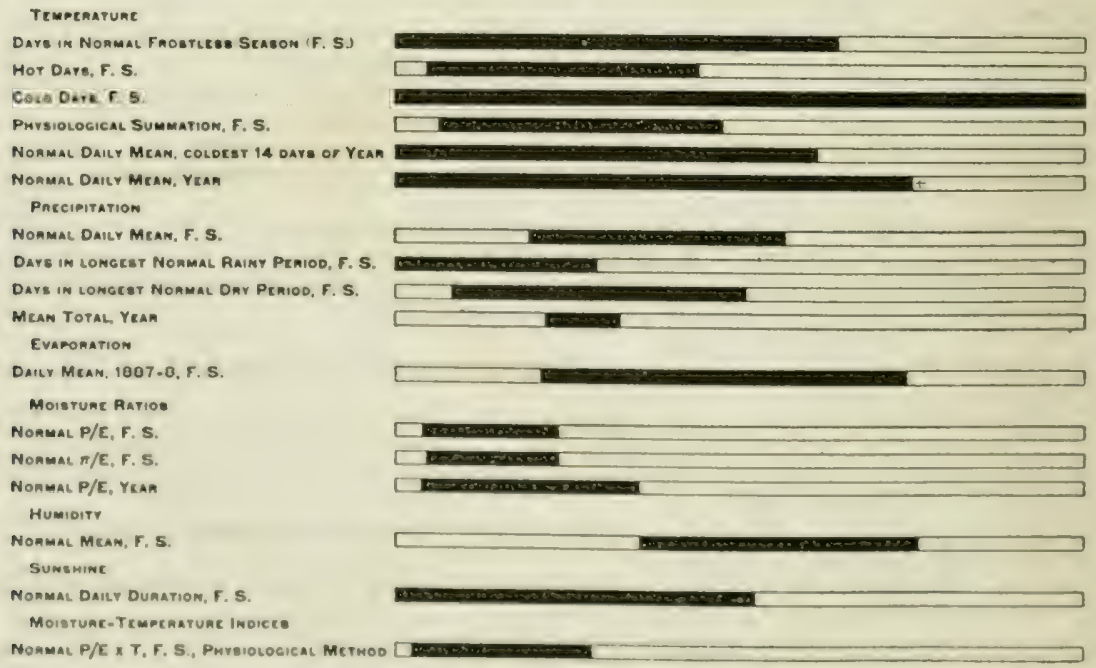

Fro. 29. Climatic extremes for Grassland. 
both edges of a region with such great north-and-south extent as the Grassland there is abundant opportunity for the interaction of conditions of critical importance, or for the influencing of the effects of a critical condition by one that is nowhere of primary critical importance itself. This is well exemplified in the changing values of the moisture ratio which are found to coincide with the western edge of the region. If this composite condition is as critical as its general distributional relations seem to indicate, we have here a case in which the vegetation of the Grassland is able to endure lower and more exacting values of this condition at the north than it is at the south, owing to the difference between the temperature conditions at north and south. On the eastern edge of the Grassland there is a similar change in the apparent controlling condition, but in this case we have the Grassland extending into the region possessing the same values for the moisture ratio that are found in the forested regions. It is possible that this is due to the inter-operation of temperature conditions and those expressed by the moisture ratio. Such a possibility is indicated by the fact that the values of the moisture-temperature index which are found in the Grassland also extend eastward in the Northern States.

Grassland Deciduous-Forest Transition (fig. 30). -A comparison of the temperature blocks in figures 29 and 30 will show that the Transition region is very similar to the Grassland in the limits and amplitudes of its temperature conditions. The principal divergence lies in the fact that the frostless season is slightly narrower in its amplitude in the former area, and does not reach such low values. A comparison of the precipitation conditions shows a difference in each case in the direction of more moist conditions for the Transition region, although

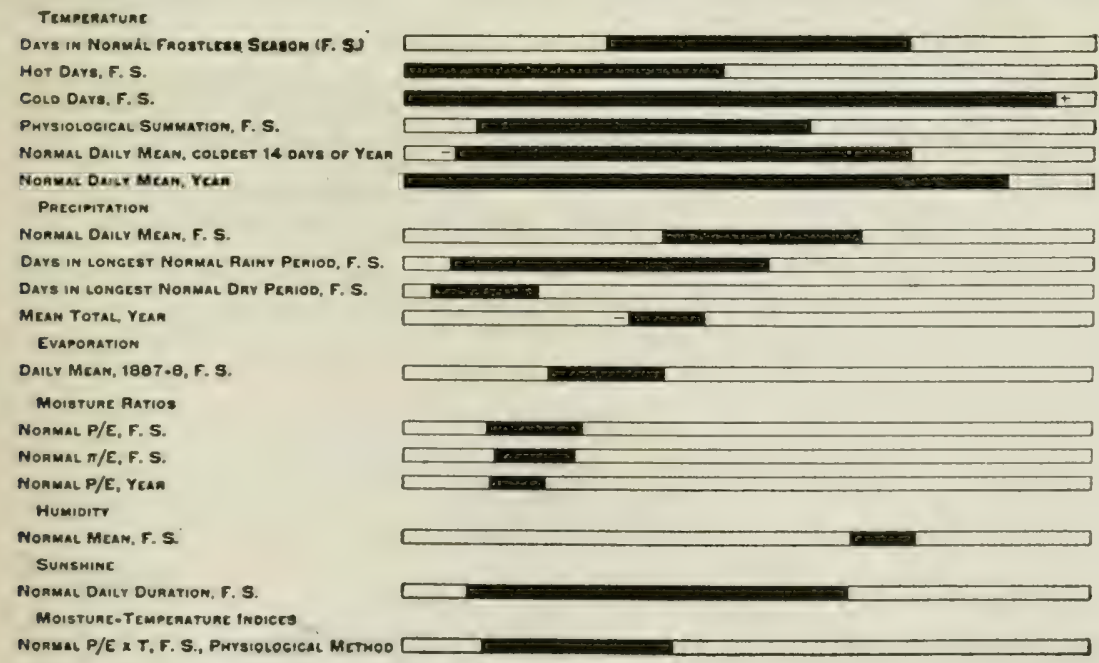

Frg. 30. Climatic extremes for the Grassland Deciduous-Forest-Transition. 
the amplitude of its normal daily mean is less, and also that of its number of days in the longest normal dry period. The lowest values for the daily mean evaporation are very similar in the Grassland and the Transition, but the much narrower amplitude in the Transition region gives it much lower maximum values ( 0.166 inch as compared with 0.275 inch).

The moisture ratios show a narrower amplitude in the Transition region than they do in either the Grassland or the Deciduous Forest, falling, in general, between the values for these regions, as has been shown in the earlier discussion. The narrow amplitude of the moisture ratios and of relative humidity would point to these corrditions as having a strong controlling importance for the Transition region. Its western edge coincides with the eastern edge of the Grassland and the conditions with regard to the moisture ratio along that line have just been mentioned. Along the eastern edge of the Transition region there is not a close correspondence with any of the isoclimatic lines of the moisture ratio, although there is a good agreement with the line for 0.110 in the north and an approximation to the interpolated line for 0.90 in the south. The Transition region lies almost precisely over the area that is comprised between the lines for 65 per cent and 70 per cent normal mean relative humidity for the frostless season, pointing to a strong controlling importance in this condition.

Deciduous Forest (fig. 31).-The leading temperature conditions of the Deciduous Forest are of an intermediate character as compared with those of the entire country, reaching an extreme value only in the case of the minimum number of cold days. The amplitude of these conditions is, in general, similar to that found in the Grassland and the Grassland Deciduous-Forest Transition, although the north-south

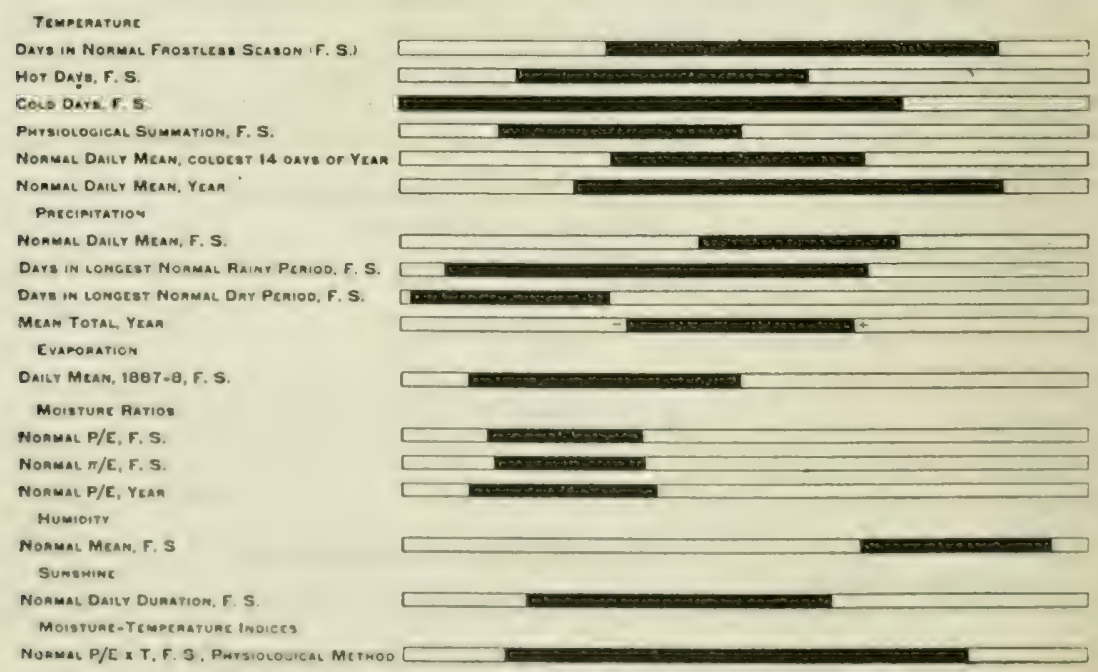

Fis. 31. Climatic extromes for Deciduous Forest. 
extent of the Deciduous Forest is somewhat less than that of the two vegetations just mentioned. The most significant changes of amplitude that may be observed in comparing these three vegetations are found in the normal daily mean for the 14 coldest days and in the normal daily mean for the year. Both of these conditions are much narrower in their amplitude for the Deciduous Forest than they are for the Grassland and the Transition region, indicating that these conditions are of increasing importance as we pass from pure grass to pure forest.

It has already been shown that a comparison of Grassland, Transition, and Deciduous Forest exhibits respective increase in the values for all of the moisture conditions, except, of course, that a reciprocal relation exists with regard to the number of dry days. While this fact might well be anticipated, it is somewhat surprising to find that the amplitude of several of the moisture conditions is greater for the Deciduous Forest than for either of the other two vegetations mentioned. The amplitude of the normal daily mean precipitation for the frostless season is slightly greater for the Grassland than for either of the other areas, and the number of days in the longest normal dry period for the frostless season is greater for the Grassland than it is for the Deciduous Forest, although it is less for the Transition area than for either of these. The mean total precipitation for the year and the number of days in the longest normal rainy period of the frostless season are both conditions that show the widest amplitude in the Deciduous Forest region.

The evaporation conditions, which show such wide amplitude in the Grassland and such narrow amplitude in the Transition region, again show a relatively wide amplitude in the Deciduous Forest. This fact determines the great amplitude of the moisture ratios in the Deciduous Forest as compared with the Transition region. While the moisture ratio appears to be of great importance in controlling the limits of the Desert and Grassland, and also those of the Deciduous Forest, the evidence shows it to be of even more critical importance in controlling the boundaries of the Grassland Deciduous-Forest Transition.

To continue our comparison of the three vegetations which have already been contrasted, we find that the value for relative humidity becomes progressively greater from Grassland to Deciduous Forest, and that its amplitude is very narrow for the Transition region, while it is relatively broad for the Grassland and Forest, ranging through a third to a fourth of the total amplitude for the United States. The evaporation conditions bear a reciprocal relation to relative humidity, but show the same narrow amplitude for the Transition region and wider amplitudes for the adjacent vegetations. It appears from this circumstance that relative humidity is the strongest determinant of 
the rate of evaporation for these areas. The influence which it exerts in this respect is therefore embraced in the moisture ratios, and additional evidence is supplied to the view that the moisture ratios are of particular importance in controlling the bounds of the Transition region.

While the moisture-temperature index shows little difference when the Grassland and Transition areas are contrasted, it exhibits a very wide amplitude for the Deciduous Forest, indicating that the interplay of moisture and temperature differences causes a wide diversity of conditions in the Deciduous Forest region.

Northwestern Hygrophytic Evergreen Forest (fig. 32).-There are many respects in which this region possesses the most marked set of climatic conditions of any of the vegetational areas of the United States. Although the Hygrophytic Forest merges into the western section of the Northern Mesophytic Forest, both southward along the California coast and in isolated areas on the western slopes of the Rocky Mountains, nevertheless the climatic characteristics of the most pronouncedly hygrophytic region, as indicated on plate 2 , cause it to stand out in sharp contrast with the Mesophytic Forest.

The length of the frostless season in the Hygrophytic Forest varies from 103 days at McKenzie Bridge, Oregon, to 316 days at Cape Disappointment, the greatest amplitude to be found in any vegetation in the country. There are no hot days and no cold days, in our sense of these terms, in any part of the region. The physiological summation of temperature for the frostless season is both low in its values and small in amplitude, resembling closely the eastern section of the Northern Mesophytic Evergreen Forest. The normal daily mean temperature of the coldest 14 days of the year ranges from about $35^{\circ}$

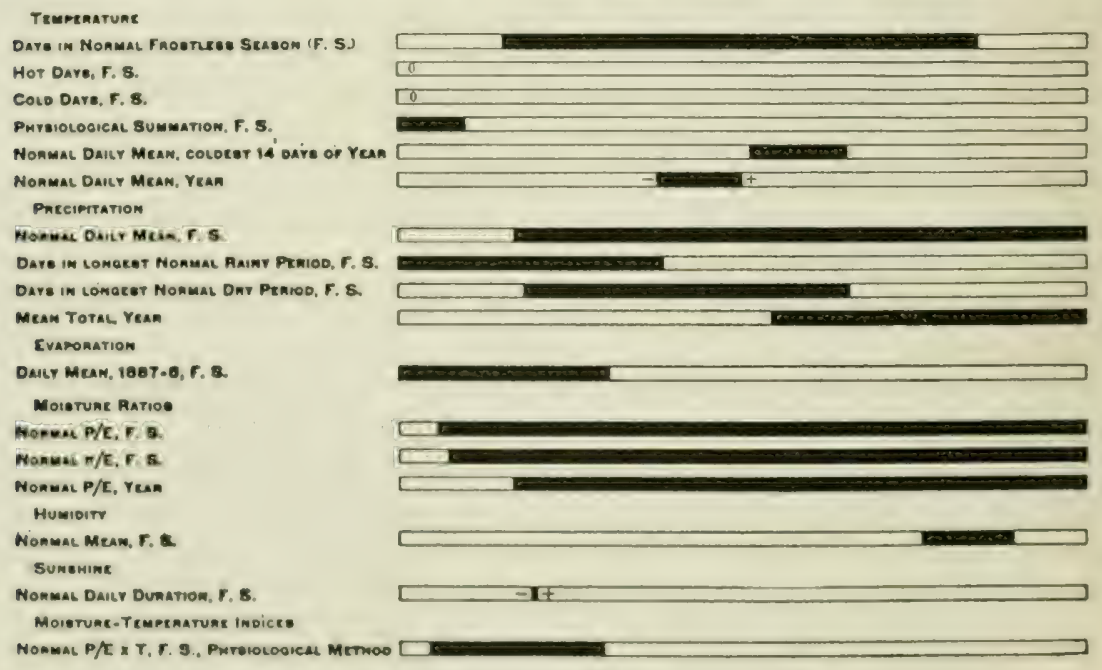

Fra. 32. Climatic extremes for Hygrophytic Forest. 
to about $45^{\circ}$. Inasmuch as the coldest portion of the year is coincident with the moist portion, this circumstance is of great importance, as indicating that the highest temperatures of the coldest days of the year are still well above frost, while the winter days outside the coldest period present conditions which are favorable for photosynthesis and growth. The normal daily mean temperature of the year ranges from below $50^{\circ}$ for the coldest stations to above $55^{\circ}$ for the warmest.

The whole series of moisture conditions, including evaporation and the moisture ratios, is remarkable in this region for the extremely wide amplitudes exhibited. The highest values for all of these conditions are secured from Cape Disappointment, and the lowest values from Roseburg, Oregon, a town located in the valley of the Umpqua River, in the driest conditions that are to be found in the region. The normal daily mean precipitation exhibits an amplitude which is about fivesixths of that for the entire United States. The values and amplitude of the number of days in the longest normal rainy period of the frostless season are here very similar to these conditions for the Grassland, while the greatest number of days in the longest normal dry period of the frostless season (198) is greatly in excess of the smallest number of days in this period (127) for the Desert region. The mean total precipitation for the year is not only higher for this vegetation than for any other, but it also shows a greater amplitude than elsewhere. The daily mean evaporation for the frostless season ranges from the lowest value in the United States, 0.052 inch at Cape Flattery, Washington, to a value of 0.143 inch at Roseburg. The values and amplitudes of evaporation conditions in the Hygrophytic Forest are closely similar to those in the Southeastern Mesophytic Evergreen Forest and in the eastern section of the Northern Mesophytic Evergreen Forest. The maximum values for the three vegetations are nearly the same, but the very low values for the Hygrophytic Forest are not found in the two latter regions (see fig. 24). There is a slight overlapping of the evaporation conditions with those of the western section of the Northern Mesophytic Evergreen Forest, into which the Hygrophytic Forest merges to the east and south, and with those of the Pacific Semidesert region, the conditions of which are approached in all of the broad valleys of coastal Washington and Oregon which lie in the lee of the mountains.

The values for all three forms of the moisture ratio are remarkable, in the Hygrophytic Forest, for their great amplitude. It has already been seen (fig. 25) that the highest values of the ratios for this region far exceed the maximum values recorded for any of the other vegetations of the United States. The relatively low values of the Southeastern Evergreen Forest and of the eastern section of the Northern Evergreen Forest are completely overlapped in the Northwestern Forest, and the moisture conditions of such localities as Astoria and 
Cape Disappointment are far exceeded by the still more moist conditions of Cape Flattery. In short, the difference between the values of the moisture ratios in moderately moist localities in the Northwestern Forest and in the most moist localities is as great as the differences which have already been seen to play such an important rôle in determining the distribution of the vegetation in the remainder of the United States.

It is unfortunate that the inadequacy of the climatological data gives this region the appearance of being exceeded in the normal mean relative humidity of the frostless season by the western section of the Northern Mesophytic Evergreen Forest, an appearance that is probably misleading. The sunshine conditions are also imperfectly known for this area.

The moisture-temperature index for the frostless season has already been shown to be closely similar in values and in amplitude for this most hygrophytic of the vegetations of the United States and for the Desert. In forming the product by which this compound condition has been secured, the high temperature summation of the desert, together with the low moisture indices, and the low temperature summations of the Hygrophytic Forest, together with its high moisture indices, have given results which are closely identical.

Southeastern Mesophytic Evergreen Forest (fig. 33). - In viewing the entire series of blocks drawn to represent the chief climatic conditions of this region, it will be noted that there is, in general, a wide amplitude of nearly all conditions, including those of temperature and precipitation, and excepting only the imperfectly exhibited mean annual precipitation and the relative humidity. The amplitude of the moisture ratios is no greater than in the case of the Deciduous Forest

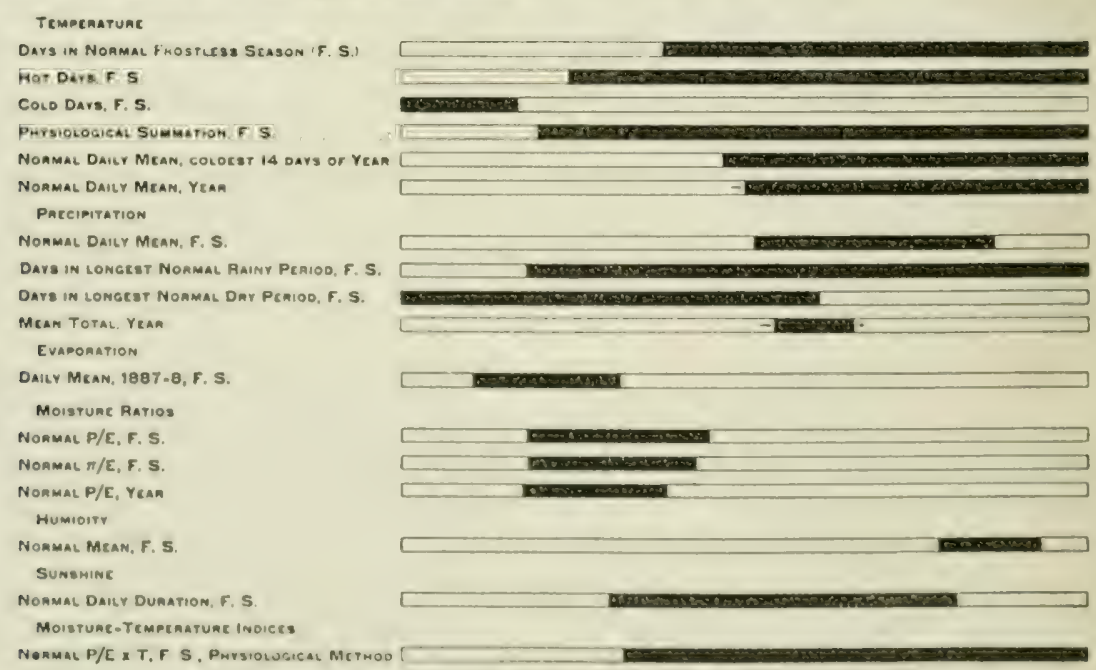

Fin. 33. Climntic extremes for Southenstern Evergreen Forest. 
and but little in excess of the corresponding amplitude for the Grassland. The wide amplitude of the temperature and precipitation conditions, taken in themselves, might be held to indicate that there is not a close correlation between the distribution of this vegetation and that of any of the important controlling physical conditions. Such a view would have in its support the fact that this vegetation is one that is well known to be closely correlated in its distribution with the extent of the Atlantic Coastal Plain and with the series of soils typical of that physiographic province. However, the types of vegetation which seem to be strictly controlled by topographic features and by soils in the northern part of the Coastal Plain are not so controlled in the southern part and in the lower Mississippi Valley, where the Coastal Plain is not so sharply defined. In spite of the wide amplitude of many other conditions, the moisture ratios are of a significance with respect to this region which must not be omitted from consideration.

In the Southeastern Mesophytic Evergreen Forest all of the leading temperature conditions of the country reach their maximum values, except the number of cold days, which, reciprocally, reaches its minimum value. Five of the six temperature conditions represented in figure 33 range in this vegetation through more than half of the amplitude found in the United States as a whole, due to the far northward extension of the region along the Atlantic coast. All of these conditions overlap with those of the Deciduous Forest region, which is quite to be expected in view of the overlapping and admixture of the vegetations themselves. A transition region between the Deciduous Forest and the Southeastern Evergreen Forest has been outlined in the detailed map of vegetation (plate 1 ), and there is considerable evidence (some of which will be discussed) that portions of the Southeastern Evergreen area are of such a character climatically as to support a deciduous forest.

The amplitude of the normal daily mean precipitation is nearly equal to that of the Deciduous Forest, but the minimum and maximum values are somewhat higher. The number of days in the longest normal rainy period of the frostless season exceeds even the great amplitude exhibited by this condition in the Deciduous Forest, and reaches, at Cape Hatteras, the highest value for the United States. The number of days in the longest normal dry period exceeds the amplitude of this condition for any of the other forest regions, and is even greater than that for the Grassland and the Desert, although the actual range of this condition is from the lowest value for the country, no days at Cape Hatteras, to 182 days at Key West.

The amplitude of evaporation conditions for the Southeastern Evergreen Forest is narrow, and the values are relatively low, being almost equal, as already shown, to the evaporation values for the eastern section of the Northern Evergreen Forest. The amplitudes of the 
moisture ratios have also been seen to be closely similar for these two forests, and their minimum values are almost identical (see fig. 25). There is also little difference between the amplitudes and extremes of the humidity conditions for these two forests and for the Northwestern Hygrophytic Forest as well. The narrowest amplitudes for the Southeastern Evergreen Forest are found in the evaporation, relative humidity, and moisture ratios, and these must be regarded, therefore, as the most important of the various conditions in controlling the distribution of this vegetation, in so far as its control is a matter of climate. A comparison of the position of the isoclimatic lines for the values 0.100 and 0.110 of the moisture ratio based on the conditions of the frostless season and the preceding 30 days (plate 59), with the position of the boundary of the Southeastern Mesophytic Evergreen Forest, shows a close correspondence. This is least satisfactory in the vicinity of Arkansas, where the Southeastern Forest extends into a region with lower values for the ratio. This region, however, is the one in which there are the largest areas of mixed forest forming a transition to the Deciduous Forest region (see plate 1).

The moisture-temperature index reaches its highest values in this vegetation, and has an amplitude almost exactly equal to that for the Deciduous Forest. The high values of the physiological summation of temperature are responsible for the high values of this index, when the relatively low values of the moisture ratios are taken into account. The very high values which are rapidly attained by this form of the moisture-temperature index on approaching the southeastern corner of the United States may be taken to signify that this region presents the optimum conditions for plant activity in the entire area studied, as far as climate is concerned.

Northern Mesophytic Evergreen Forest, western section (fig. 34).-The Northern Mesophytic Evergreen Forest, when considered as a whole, is so widely distributed and so varied in its character and specific composition that it assumes a unity only when contrasted with the other evergreen-forest areas of the country. The only natural subdivision of this region is that which is made possible in the United States by the geographical separation of the eastern and western portions. In the study of the correlation of this regetation with the climatic conditions it has seemed desirable to determine the climatic extremes separately for the eastern and western sections, which are sharply separated by the northern arms of the Grassland and the Grassland Deciduous-Forest Transition.

The most striking feature of the diagram which shows the leading climatic features of the western section of the Northern Mesophytic Evergreen Forest (fig. 34) is the relatively narrow amplitude of the majority of the conditions which acempany this widely and irregularly distributed forest area. There is a particularly strong contrast in this 
respect with the conditions for the Southeastern Evergreen Forest and for the Deciduous Forest, indicating that the western section of the Northern Evergreen Forest is confined in its distribution to a region in which there is a relatively narrow range of physical conditions, in spite of its wide geographical extent. Owing to the mountainous and thinly settled character of most of this region, there is an inadequate series of climatological stations from which data may be obtained. This is particularly true of the southernmost portions in New Mexico, Arizona, and California, and of the portions which face the Desert region on all sides of the Great Basin. The climatic conditions of the western Xerophytic Evergreen Forest (see plate 1) are even more poorly known, and this has been a strong consideration in omitting that vegetation from the generalized map. Its conditions would doubtless be found to be generally intermediate between those of the Desert and those of the western section of the Northern Evergreen Forest.

The length of average frostless season ranges from the minimum for the United States to 307 days, a remarkably great amplitude, which is matched only for the Desert region. The lowest values of this condition are recorded for several stations in the Klamath Lake region, where frost has been observed on every day in the year. The shortest normal frostless season outside these stations is 25 days in length, and this has been taken as the minimum for the United States in view of the fact that there is actually, in any given year, a growing-season for plants even in localities where frost is of average daily occurrence, and that the length of this season is at least 25 days even for Klamath Lake stations. The maximum length of frostless season for this vege-

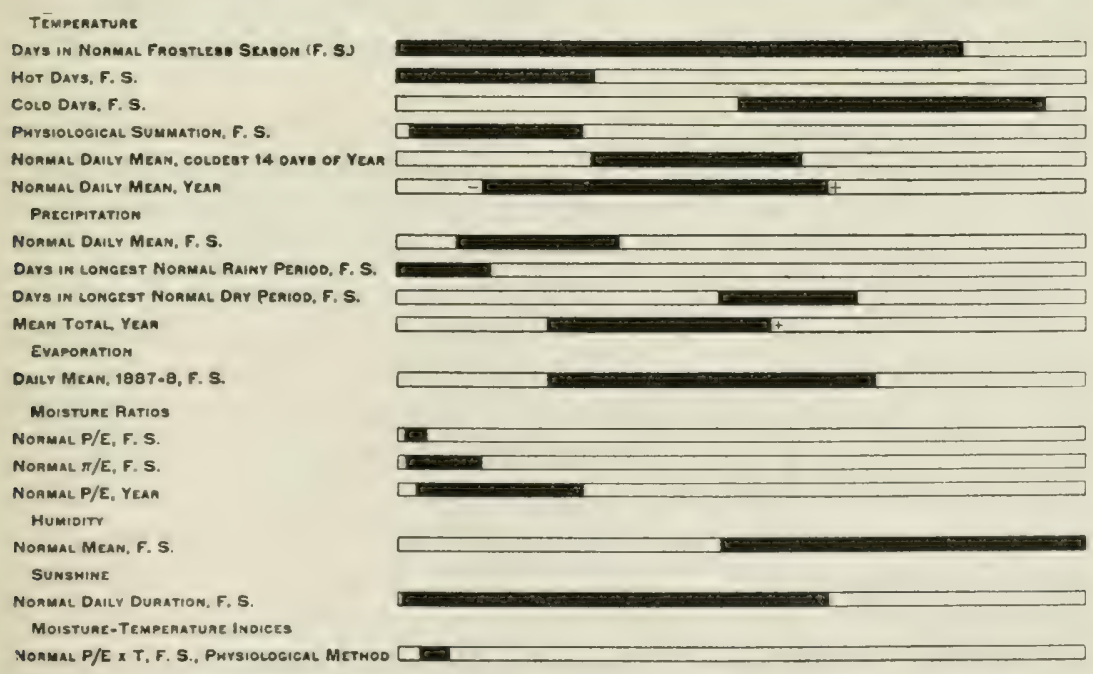

FIG. 34. Climatic extremes for western section of Northern Mesophytic Evergreen Forest. 
tation has been recorded at Fort Bragg, California, where the conditions and vegetation of the ocean front are more nearly those of the Hygrophytic Forest than of the western section of the Mesophytic Forest. At stations just inland from Fort Bragg the frestless season is about 100 days shorter. The number of hot days ranges from 0 to 105, which is about one-third of the amplitude for the entire country, and the number of cold days ranges from 78 to $149+$, closely approaching the maximum values, which occur in the Grassland. The physiological summation of temperature has a relatively narrow amplitude, from values approaching the minimum to 9,921 at Grand Junction, Colorado, a station which must be regarded as approximating the conditions of the forests in that vicinity. Much higher values of the physiological summation might be expected for stations located in the more southern extensions of the Mesophytic Evergreen Forest, but our data fail to bear out such an expectation. Santa Fe, New Mexico, is located close to bodies of Mesophytic Evergreen Forest, and is so situated as to enjoy higher temperatures than the forest, yet the summation for Santa Fe is only 5,350, a figure considerably below the maximum at Grand Junction. Flagstaff, Arizona, which is situated in the midst of forest, has the very low summation of 2,652 . The cold nights of the mountain altitudes at which the Mesophytic Forest of New Mexico and Arizona occurs are responsible for low daily mean temperatures, and consequently for low values of the summations for the frostless season.

The normal daily mean for the coldest 14 consecutive days of the year is a condition ranging through a moderate amplitude and reaching extreme values which are neither very high nor very low, overlapping with the conditions in the Hygrophytic Forest. The normal daily mean temperature for the year is of wide amplitude, but also fails to attain the extremes for the country.

When the climatic extremes for the precipitation conditions are compared, as a whole, with those for the temperature conditions, the former are seen to be of narrower amplitude, which points to the limitation of the western section of the Mesophytic Evergreen Forest. as being due more largely to moisture conditions than to those of temperature. This is confirmed by the narrow amplitude of the moisture ratios, at least of those which relate to the frostless season only. Although the actual extremes of the moisture ratio $\pi / \mathrm{E}$ for the frostless season are 0.12 and 0.60 , it will be seen by an examination of plate 59 that the major portion of the western Mesophytic Forest is comprised between the lines for values of 0.20 and 0.40 . The lowest. evaporation values and the highest relative humidities for this region are recorded for the extreme coastal stations of northern California, and they are not typical of the great bulk of the forest, although they permit its occurrence in a form approaching the character of the Northwestern Hygrophytic Forest. 
No vegetational region exhibits a narrower amplitude of the moisture-temperature index than does the western section of the Mesophytic Forest. This derived and complex expression of climatic conditions has been shown to differentiate the evergreen-forest regions of the United States, in spite of its failure to bring out consistent differences between the other regions. Its extremely narrow amplitude for the western section of the Mesophytic Evergreen Forest points to its importance as an expression of the conditions controlling this vegetation.

Northern Mesophytic Evergreen Forest, eastern section (fig. 35).-A comparison of the climatic extremes of the eastern and western sections of this forest shows a general similarity of temperature conditions, and a dissimilarity of moisture conditions and the expressions derived from them. The length of frostless season is much narrower in its amplitude in the eastern section, ranging from a length of 85 days at Thomaston, Michigan, to 167 days at Fitchburg, Massachusetts, values which lie well within those of the western section. The normal daily mean temperature for the coldest 14 days of the year ranges through lower values in the eastern section, as does also the normal daily mean temperature for the year.

The only precipitation condition in regard to which the eastern and western sections of the Northern Mesophytic Evergreen Forest are at all alike is the normal annual total. The normal daily mean for the frostless season ranges in the eastern section through values which are well above those of the western section, the maximum of the latter being 0.070 inch and the minimum of the former being 0.091 inch. In accordance with this fact the number of days in the longest normal rainy period of the frostless season is greater in the eastern section than in the western, and the number of days in the longest normal dry period is pronouncedly less in the eastern section.

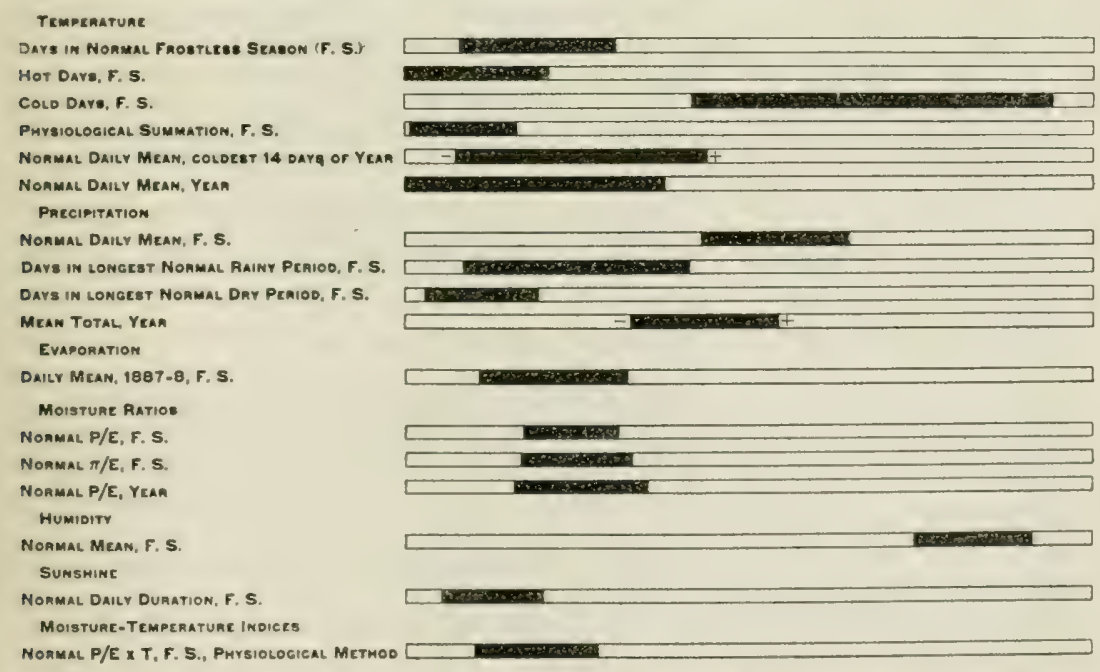

FIG. 35. Climatic extremes for castern section of Northern Mesophytic Evergreen Forest. 
The amplitude of evaporation conditions is much narrower in the eastern section than in the western, agreeing closely, as already stated, with the amplitude and values for the Southeastern Mesophytic Evergreen Forest. The humidity conditions also exhibit a much narrower amplitude, although they nowhere reach in the East the maximum values that are to be found at the Pacific coast stations of the western section. The moisture ratios all exhibit higher values for the eastern section, and the ratio $\pi / \mathrm{E}$ for the frostless season fails to overlap with the range of this condition in the western section (see fig. 25). Here again there is a close correspondence between the Evergreen Mesophytic Forests of the Northeastern and the Southeastern States. The sunshine values are low and of narrow amplitude for the Northeastern Evergreen Forest, the maximum value for this region being lower than the maximum in any of the other vegetational areas.

The moisture-temperature index for the eastern section ranges from a minimum value which is slightly above the maximum for the western section to a maximum which nearly coincides with that for the Northwestern Hygrophytic Evergreen Forest. The close correspondence between the range of this condition in the last-named region and in the eastern section of the mesophytic forest doubtless affords a means of explaining the features of resemblance between these two regetations, which are so widely separated in the United States but have a narrow strip of connecting forest in Canada.

The Climatic Conditions for Evergreen and Deciduous Forests.-In connection with the inquiry into the climatic conditions characterizing the regetational areas, a comparison has been made of the climatic extremes for the Deciduous Forest and for the four erergreen forest areas considered collectively. In figure 36 are shown graphs for the

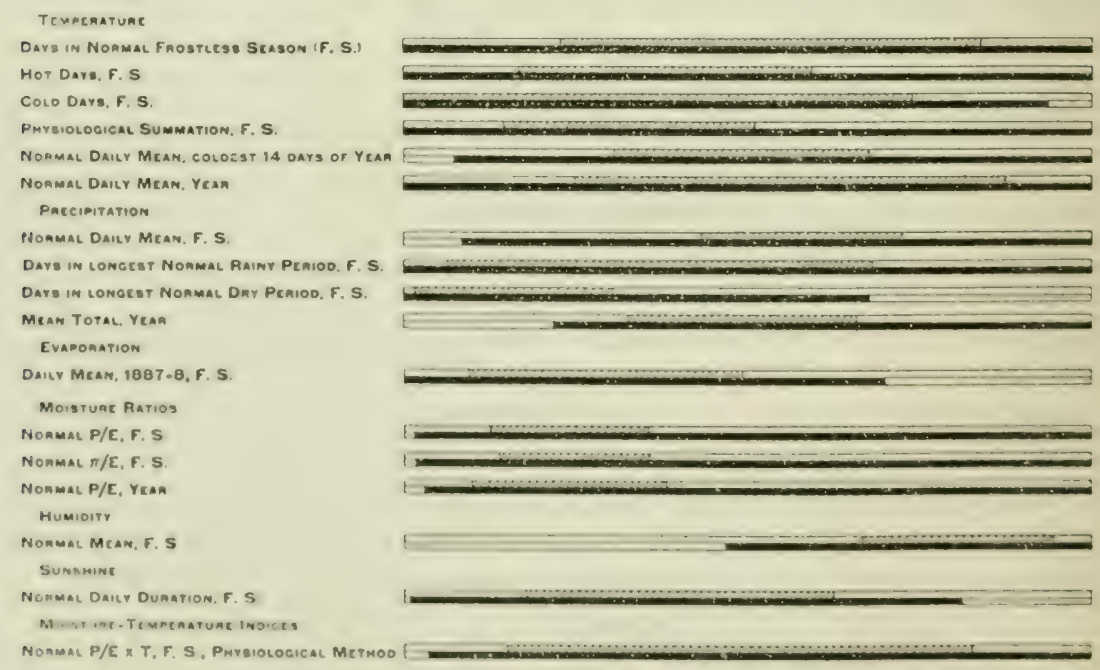

Fic. 36. Contrasted climatic extremes for Evergreen (black) and Deciduous (shaded) Forests. 
principal features of the climate for these two regions, brought together for ready comparison. The widespread occurrence of evergreen forest, extending into the northwestern, northeastern, and southeastern corners of the United States, gives a very wide amplitude of conditions for this type of forest. In 5 cases the amplitudes of conditions are as great for the collective evergreen regions as for the entire United States, and in 6 cases they are nearly as great. These wide amplitudes are found among temperature and moisture conditions alike. The narrowest amplitude is that of relative humidity, which ranges through the upper half of its scale for the entire country. The importance which humidity is here indicated to have for evergreen forest is borne out by the detailed humidity extremes for each of the evergreen areas (see fig. 25).

The amplitude of the conditions found in the Deciduous Forest region is narrower in every case than that of the collective evergreen regions, and for none of the conditions does it approach the entire amplitude for the country. It is also to be noted that the extremes for the Deciduous Forest are, in nearly every case, well within the extremes for the evergreen regions. In the number of cold days in the frostless season the two have the same minimum, and in the number of days in the longest normal dry period of the frostless season the two have nearly the same minimum. The only case in which the two maxima approach each other is that of humidity.

If we disregard the diversities in the evergreen forests which have led us to their separate treatment, it is possible to say that this type of forest in general is capable of withstanding a much wider range of climatic conditions than is the deciduous type.

\section{DISCUSSION OF THE OBSERVATIONS.}

We have now reviewed the observed correlations between the general vegetation areas and some of the leading climatic conditions, both from the point of view of the vegetation and from that of the conditions. In the comparison of the amplitudes and extremes of each single condition, as shown for each of the vegetational areas, it has been possible to see to what extent that condition is unlike in the several vegetations. It is obvious that a condition which ranges through nearly the same values in two vegetations can not be looked upon as one that is important in determining the optimum conditions for each of these vegetations, nor as one that plays an important rôle in controlling the limit between the two. It is evident, for example, that the physiological summation of temperatures can not be held to play a primary part in establishing the optimum conditions for Grassland, Grassland Deciduous-Forest Transition, and Deciduous Forest (see fig. 22). If, on the other hand, a given condition exhibits a sliding scale of values for several adjacent vegetations, it is evident that such 
a correlation points to this condition as playing an important part in the existence and distributional limits of these vegetations. This should be true even if there is some overlapping of the values of the condition between the adjacent vegetations, for there is always such overlapping of the vegetations themselves. A comparison of the humidity conditions for Grassland, Grassland Deciduous-Forest Transition, and Deciduous Forest, or a comparison of the moisture ratios for these vegetations and for Desert and Semidesert, shows a progressive change of position of the climatic extremes with respect to the extremes for the entire country (see fig. 25).

A comparison of the amplitudes and extremes of all conditions for a single vegetational area makes it possible to discover which conditions tend to exhibit great differences in the various parts of the area and which ones tend to show a relative uniformity throughout the area. We have already seen that this method of evaluating the conditions makes it possible to use their relative amplitude as a measure of their comparative importance in establishing the limiting conditions for the vegetation in question.

A cartographic representation of the distribution of vegetation and of the distribution of the various intensities of the climatic conditions makes it possible to compare distributional limits and isoclimatic lines, and to search for correspondence between the two. But such a search is best carried out by the aid of suggestions from the graphs showing the climatic extremes.

The use of the three methods of correlation has shown them to be consistent in their indications. If a condition shows a sliding scale of values for a given series of vegetations, it is also found to show amplitudes, in each of the vegetations, which are narrow as compared with those of other conditions, and the isoclimatic lines showing the distribution of the intensities of this condition are found to approximate the distributional lines between the series of vegetations. The first two methods serve for the discovery of the relative importance of various conditions in determining distribution, and the third method serves to show the eritical intensities of the condition which appear to be important.

In a general review of our examination into the correlations between climatic conditions and the general regetational areas we find the most salient fact to be the great controlling importance of moisture conditions, embracing precipitation, exaporation, relative humidity, and the moisture ratio, as compared with the small controlling importance of temperature conditions, embracing length of frostless season, number of hot and of cold days, and the temperature summations. The moisture-temperature index partakes strongly of the character of a tenperafure condition when it is brought into this comparison.

In the regetations of group A (including our series from Desert to Deciduous Forest; see figs. 21 to 26) the temperature conditions show 
particularly wide amplitudes and a pronounced tendency to range through about the same values, except in the case of the length of frostless season and the normal daily mean of the coldest 14 days of the year, in both of which conditions the Semidesert departs from the conditions of the other vegetations of the group. A comparison of the extremes of the temperature conditions for the vegetations of group $\mathrm{B}$ (the evergreen forests) shows a much greater dissimilarity, indicating that temperature conditions play a more important rôle in differentiating our evergreen-forest areas than they do in differentiating the other vegetations of the country. This applies to the moisture-temperature index, as well as to the purely temperature conditions.

It is in the vegetations of group A that the moisture conditions show their greatest differentiation and appear to exert their strongest control. This is shown with diagrammatical clearness by the smoothed data for the mean total precipitation of the year (fig. 24), and it is also shown by the moisture ratio and by relative humidity (fig. 25). There is a much less marked differentiation of moisture conditions among the evergreen-forest areas, as will be seen in the close agreement of the extremes of daily mean evaporation for the Southeastern Mesophytic Evergreen Forest and for the eastern section of the Northern Mesophytic Evergreen Forest (fig. 24), and also in the similarity of humidity conditions for the above forests and for the Northwestern Hygrophytic Evergreen Forest (fig. 25).

The preceding pages have brought out the fact that the moisture ratio $\pi / E$ is the most important single expression of climatic conditions with respect to the vegetation as a whole. The narrow amplitude of this condition in all of the vegetations except the Northwestern Hygrophytic Evergreen Forest, and the distinctness of its extremes for all of the vegetations give an indication of its importance which is well borne out by a comparison of the climatic and vegetational lines of plate 2 and plate 59. The isoclimatic line for the ratio value 0.110 closely follows the limit of the Southeastern Mesophytic Evergreen Forest from Alabama to New Jersey, and then swings westward in such a manner as to approximate closely the southern limit of the eastern section of the Northern Mesophytic Evergreen Forest, failing to dip with the vegetation along the Alleghenies (where there are no data for this climatic map), but closely following the vegetational line to Minnesota. The similarity of the conditions expressed by the moisture ratio for the evergreen forests of the Southeastern and Northeastern States is indicated in figure 25, but no indication is there given of the closeness with which the inner limits of these vegetations follow a single isoclimatic line. The line for the value 0.80 is an equally close approximation of the inner limit of the Northwestern Hygrophytic Evergreen Forest. The line separating the Grassland and the Grassland Deciduous-Forest Transition is closely followed by the isoclimatic line of 0.60 in the South and by that 
of 0.80 in the North. The area lying inside the line for values of 0.20 is entirely occupied by Desert, which exceeds the line only to short distances in Texas and Washington.

The importance of the moisture ratio in controlling the leading vegetations was shown by Transeau for the eastern United States, ${ }^{1}$ and our investigation has served to confirm his deductions, as well as to extend their application to the entire country. The comparisons which we have made between the vegetational areas and the various other climatic conditions have served to emphasize the importance of the moisture ratio even more than was done by Transeau, since no other single datum has been found in our work to approach it as an expression of the controlling conditions for forest, grassland, and desert.

The importance of the moisture ratio is due partly to the fact that it is a combined expression of several other conditions, and still more to the fact that these conditions are ones which have a combined effect upon the physiological processes of the plant. The moisture ratio is, in brief, an expression of the relation existing between the water available for plants and the amount of water lost as a result of atmospheric conditions. The moisture ratio gives us a single numerical expression for the group of conditions which control a single important physiological condition of the plant, namely, its maintenance of a balance between intake and outgo of water. When we secure the product of the moisture ratio and the temperature summation we have a still more comprehensive expression of conditions, the moisturetemperature index. In this index, however, we have no such succinct expression of a set of conditions that are closely coordinated in their relation to the physiology of the plant, in spite of the individual importance of each factor in the product. The moisture-temperature index is correspondingly of less value in interpreting distributional etiology than is the moisture ratio itself.

$A$ much more ideal derivation of the moisture ratio is one employing the soil-moisture rather than the precipitation, since it is the former rather than the latter condition that is of immediate relation to the activities of the plant. Shreve has used the ratio of soil-moisture to evaporation in a discussion of the annual march of moisture conditions at Tueson, Arizona, ${ }^{2}$ and also in describing the gradient of conditions from the base to the summit of the Santa Catalina Mountains, in southern Arizona, a range surrounded by desert and capped by heary forest. For detailed work, and particularly in arid regions or regions with pronounced periodicity of rainfall, the ratio of soil-moisture to evaporation will be found to express the prevailing conditions for

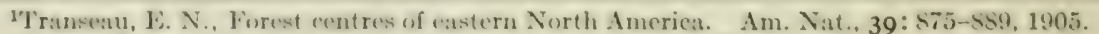

${ }^{2}$ Shreve, forrest, Rainfall as a determinant of soil moisture, The Plant World, 17:9-26, 1914.Idcm, 1915 .
} 
plants more precisely than the ratio of precipitation to evaporation. In dealing with large areas, however, the soil-moisture is so closely a function of the precipitation that the two expressions frequently approach identity, or at least proportionality. Of course, it is understood that in all cases where the soil-moisture content (percentage of soilmoisture on a volume or weight basis) is employed as the index of the soil-moisture condition, great differences in the water-retaining power of the soil in different areas must upset this clear relation. If the soils compared are all clay or all sands, etc., the soil-moisture content itself is probably a relatively good index of the water-supplying power of the soil, but this is not true when sands are to be compared with clays, for a 10-per cent water-content in a sand may be physiologically equivalent to a 50-per cent content in a clay, other conditions being considered as alike.

\section{CONDITIONS THAT PROBABLY DETERMINE THE LIFE-ZONES OF MERRIAM.}

\section{OBSERVATIONS FROM THE CHARTS.}

In a discussion of the climatic extremes of the life-zones of the United States, as outlined by Merriam, it is necessary for us to disregard the Boreal and Tropical Regions, and also the Gulf Strip of the Lower Austral Zone, on account of the very small number of climatological stations comprised in those areas. As originally drawn by Merriam, the life-zone map of the United States was in actuality a climatological map, based on a summation of temperatures and slightly modified, particularly on the Pacific coast, by data on the temperature of the hottest 6 weeks of the summer. It is not necessary, therefore, to make correlations of the range of the life-zones with the distribution of the different intensities of the remainder summation of temperatures above $32^{\circ}$ for the year (plate 37, which is Merriam's chart), nor with the normal daily mean of the hottest 6 weeks of the year (plate 44, which is also Merriam's).

In figures 37 to 42 are shown the graphs for the leading climatic dimensions given in tables 33 to 38 for the Transition, Alleghanian, Upper Sonoran, Carolinian, Lower Sonoran, and Austroriparian zones. These 6 subdivisions comprise the western or arid and the eastern or humid subdivisions of the transcontinental zones based on temperature conditions. They represent, in other words, an exact but special subdivision of the country on a basis of certain temperature conditions, together with a rough subdivision on the basis of moisture conditions. The line separating the arid and humid divisions of these zones is drawn by Merriam along the one-hundredth meridian from Oklahoma to South Dakota, departing a little to the eastward at the north and south. This line corresponds rather closely with the isoclimatic line of a value of 0.60 for the moisture ratio $\pi / E$. It does not take account, 
however, of the fact that humid conditions exist in the extreme Northwest, as shown by the return of the isoclimatic line of 0.60 for the moisture ratio, running from the Upper Columbia River to San Francisco Bay (see plate 59).

Transition Zone (fig. 37). - The dimensions of nearly all of the leading climatic conditions exhibit a very wide amplitude in this zone, which embraces the northern Great Plains, portions of the humid Pacific Northwest, and mountain areas throughout the Western States. The length of frostless season and the number of cold days in the frostless season both range through nearly their entire amplitude for the United States. The normal daily mean precipitation and the mean total precipitation of the year likewise range through very wide amplitudes. The amplitude in the number of hot days is not great, and the extremes for this condition are low, ranging from 0 to 105 . The physiological temperature summation exhibits a narrow range, as might be expected from the similarity of its derivation to that of the remainder summation above $32^{\circ}$, the use of which in outlining these zones has been mentioned. Eraporation and humidity both show a much wider amplitude in this zone than in any of the vegetational areas that have been discussed, exceeding greatly the amplitude for the western section of the Northern Mesophytic Evergreen Forest, with which this zone has some distributional features in common. The moisture ratios all show wide amplitude for the Transition Zone, $P / E$ and $\pi / E$ ranging through about half the total amplitude and $P / E$ for the year through a still greater amplitude of conditions. The Northwestern Hygrophytic Evergreen Forest is the only one of our vegetational areas that exceeds the Transition Zone in the amplitude of the second of these ratios. The entire gamut of sunshine conditions for the United States is to be

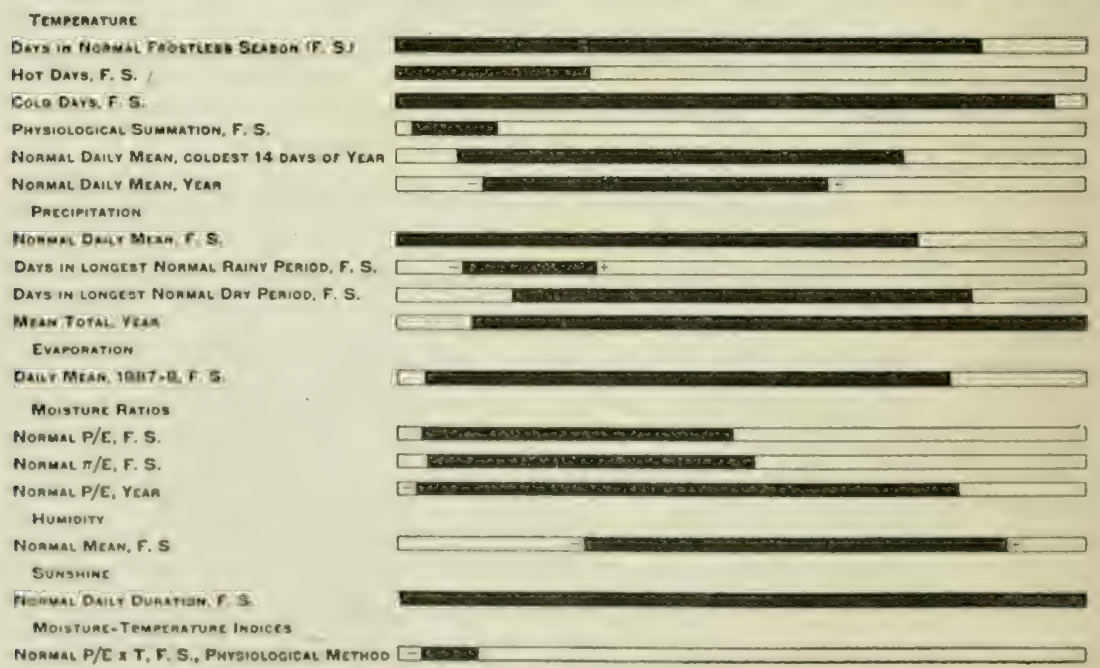

Fic. 37. Climatio extremes for the Transition \%one. 
encountered in the Transition Zone. The moisture-temperature index possesses almost as narrow an amplitude as that characteristic of the western section of the Northern Forest region.

Alleghanian Zone (fig. 38).-The temperature conditions of this zone, excepting the length of the frostless season, are very similar to those of the Transition Zone, while the precipitation and other moisture conditions are quite unlike. The frostless season ranges from a length of 106 days to one of 211 days, extremes which lie well within those of the Transition Zone. The amplitude and extremes in the number of hot days and the number of cold days are very similar, while the normal daily mean of the coldest 14 days of the year is somewhat higher in its extremes, although very similar in its amplitude.

In all of the precipitation and other moisture data the Alleghanian Zone exhibits much narrower amplitudes than those of the Transition Zone, because of the high values characteristic of the portions of the latter zone which lie in the extreme Northwest. In nearly all cases extremes for the Alleghanian Zone lie within those of the Transition Zone, the exceptions being the number of days in the longest normal rainy and dry periods in the frostless season. Owing to the winter occurrence of rainfall in the Northwest, these features indicate more moist conditions for the Alleghanian than for the Transition Zone. The amplitude of sunshine duration for the Alleghanian Zone is much less than that for the Transition, ranging from low values upward through about one-third of the entire amplitude for the country. The moisture-temperature index has a much wider amplitude in the former than in the latter zone, slightly exceeding the amplitude for the eastern section of the Northern Mesophytic Evergreen Forest.

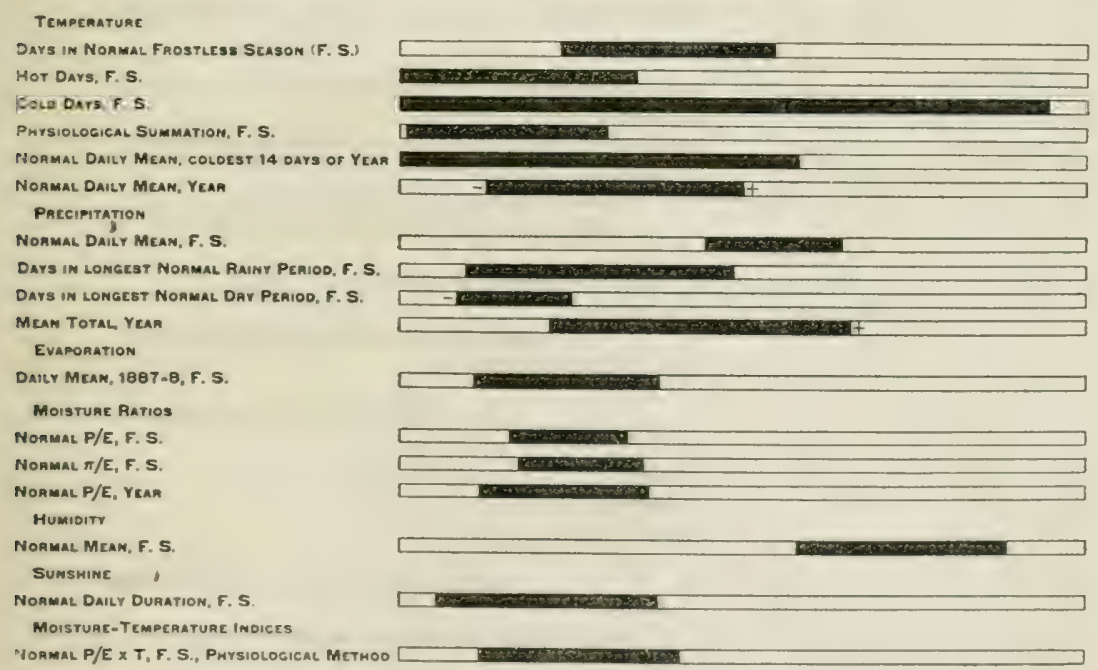

Fig. 38. Climatic extremes for the Alleghanian Zone. 
Upper Sonoran Zone (fig. 39).-In this zone are comprised portions of the Desert and more than half of the Grassland region. The amplitude of the conditions is not, in general, as great as it is for either of these two vegetations, since the zone does not include the more moist half of the Grassland nor the extremely arid portions of the Desert. This zone is largely one of arid grassland and comprises the DesertGrassland region of our detailed vegetation map. There is a wide amplitude in length of frostless season and in number of cold days, both seen to be characteristic of the Grassland, and there is a wide amplitude in the number of days in the longest normal dry period. Both the evaporation and humidity conditions range through wide amplitudes, the former from 0.166 to 0.330 inch and the latter from 40 per cent to 80 per cent. The most moist conditions in this zone are to be found in the narrow strip which follows the coast of southern California.

Both the physiological temperature summation for the frostless season and the moisture-temperature index show relatively narrow amplitudes for this zone. The narrowest ones, however, are those exhibited by the number of days in the longest normal rainy period of the frostless season and by the moisture ratios. The latter criterion appears to express the conditions which are critical in the limitations of this zone just as it does in the case of the Desert and Grassland.

Carolinian Zone (fig. 40).--This zone bears about the same relation to the Upper Sonoran that the Alleghanian does to the Transition. That is to say, the temperature conditions are generally similar in the two, while the moisture conditions are dissimilar. The temperature similarity does not hold with respect to the length of frostless season, as it did not in the case of the Transition and Alleghanian Zones. The

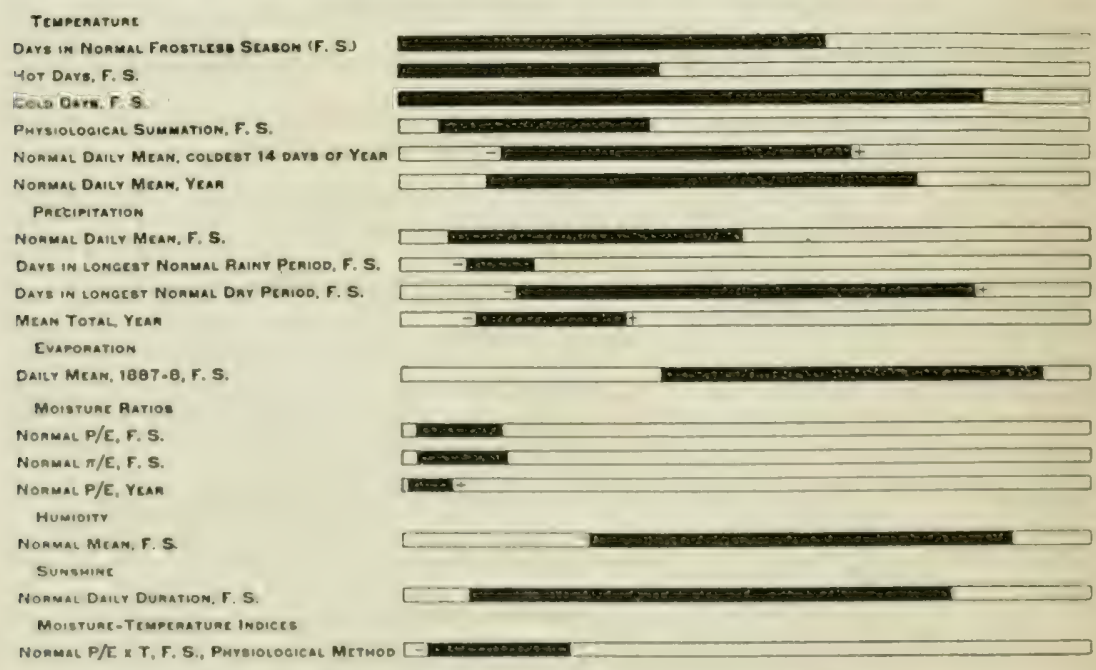

Fro. 39. Climatic extremes for the Upper Sonoran 'Zone. 
maximum length is very similar in the two, being 237 days for the Upper Sonoran and 241 days for the Carolinian, but the minimum value for the former is 25 days and for the latter 127 days. Whereas the moisture conditions of the Alleghanian Zone were found to lie well within the extremes for the Transition Zone we have in the case of the Upper Sonoran and Carolinian Zones a more sharp separation of the ranges of these conditions. In every case there is an overlapping of moisture conditions, by which the minimum values of the Carolinian are seen to be lower than the maximum values for the Upper Sonoran. This circumstance is due to the fact that the highest moisture values of the Upper Sonoran are registered on the Pacific coast, while the lowest values of the Carolinian are experienced along the one-hundredth meridian.

The minimum sunshine values for the Carolinian and Upper Sonoran Zones are very similar, but the amplitude of the former is only half that of the latter. The moisture-temperature index is higher in its extreme values and wider in its amplitude in the Carolinian Zone, reaching a maximum which is about midway of the amplitude for the United States.

Among the relatively narrow amplitudes for this zone should be noted the normal daily mean precipitation for the average frostless season and the number of days in the longest normal dry period within that season. It is of interest to $\mathrm{no}^{+} \mathrm{e}$ that the length of the dry period shows a narrow amplitude in the Carolinian Zone, indicating its critical limiting importance, whereas the longest rainy periods are demonstrated to have a critical value for the Upper Sonoran. Conversely, the length of the rainy period shows a wide, but imperfectly

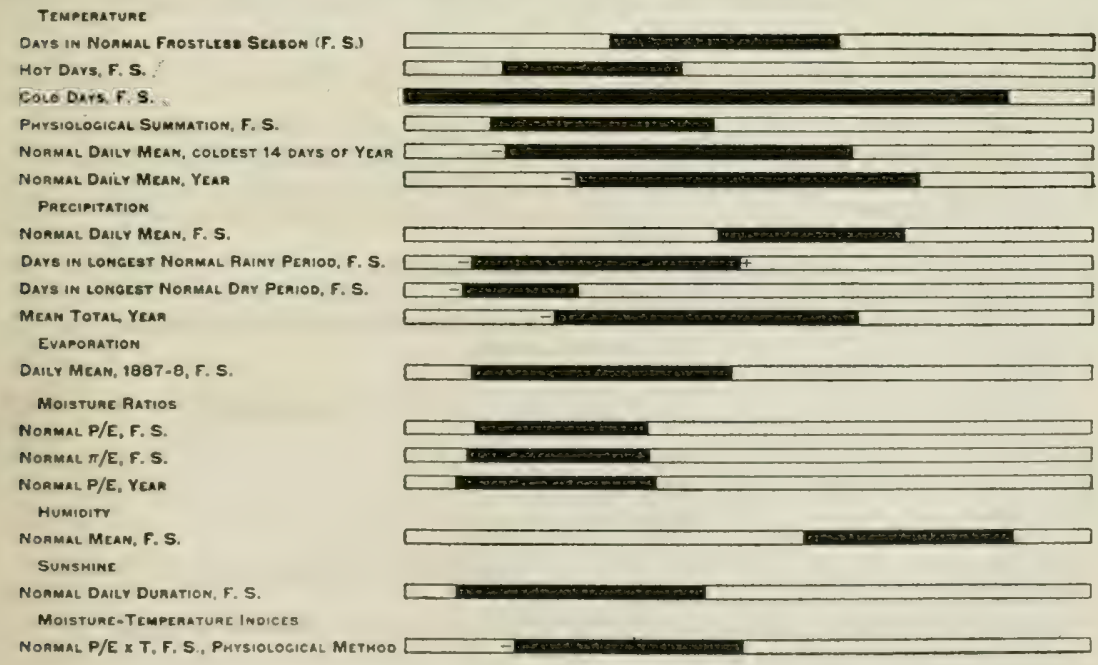

FIG. 40. Climatic extremes for the Caroliniar Zone. 
determined, amplitude for the Carolinian, and the length of the dry period shows an even greater, but also inaccurately determined, amplitude for the Upper Sonoran.

The moisture ratios show higher values and wider amplitudes for the Carolinian than for the Upper Sonoran, as was true of their more northern analogues, the indication being that the conditions expressed by these ratios are somewhat less critical in the moister Carolinian Zone than in the Lower Sonoran.

Lower Sonoran Zone (fig. 41). - When a comparison is made between the zones which form the eastern and western halves of a given region, the temperature conditions are found to be similar in the two and the moisture conditions dissimilar. When a comparison is made between the temperature conditions of the Upper Sonoran and Lower Sonoran Zones, the values and amplitudes are found to be dissimilar, whereas the moisture conditions in the two are very much alike throughout, with the exception of the higher range of evaporation in the Upper Sonoran.

The length of frostless season in the Lower Sonoran ranges from 106 to 331 days, which is both a wider amplitude and a higher series of values than those found in the Upper Sonoran. Other very wide amplitudes are those of the normal daily mean temperature for the year, the number of days in the longest normal dry period within the frostless season, and the daily mean evaporation for the frostless season. These are all conditions which are likewise of wide amplitude in the Desert region.

One of the narrowest amplitudes of this zone is the normal daily mean for the coldest 14 days of the year, for which the amplitude in the Upper Sonoran is great. This emphasizes the importance which
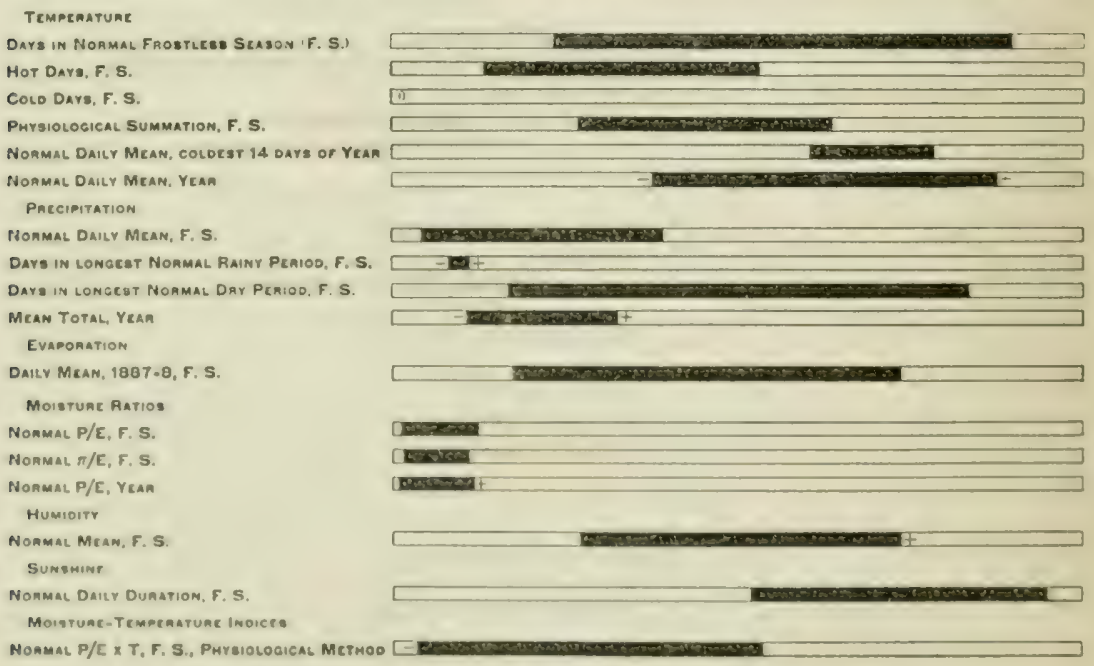

Frc. 41. Climntio extremes for tho Lower Sonoran Zone. 
the temperatures of the coldest periods of winter assume on passing to a warm subtropical zone in which there are no cold days in our sense. The number of days in the longest normal rainy period of the frostless season appears to be of narrow amplitude in the Lower Sonoran Zone, although this is not accurately determinable. Its values and amplitudes are doubtless very similar to those of the Upper Sonoran Zone, as indicated by a comparison of figure 39 and figure 41 . While, in other words, there is a sharp contrast between the conditions in the Upper and Lower Sonoran Zones with respect to one of the most critical temperature conditions of the latter, there is a similarity with respect to a moisture condition which is not critical in separating these zones, but is critical in separating them from their eastern humid analogues. The moisture ratios, which show narrow amplitudes for this zone, are also very similar in range and amplitude to the moisture ratios of the Upper Sonoran Zone.

Austroriparian Zone (fig. 42).--The temperature conditions of this zone show less similarity to those of the Lower Sonoran Zone than is shown by a comparison of Upper Sonoran with Carolinian or Transition with Alleghenian. The amplitude of the length of the frostless season is relatively narrow, and that of all the other temperature conditions is narrower than in the Lower Sonoran. There are rather wide limits, however, within which the temperature conditions of these two zones overlap.

The normal daily mean precipitation ranges through a wide amplitude in the Austroriparian Zone, exceeding its amplitude in the Carolinian. The number of days in the longest normal rainy period of the frostless season also reaches much higher maximum values in the former zone than in the latter. With these exceptions, there is a general

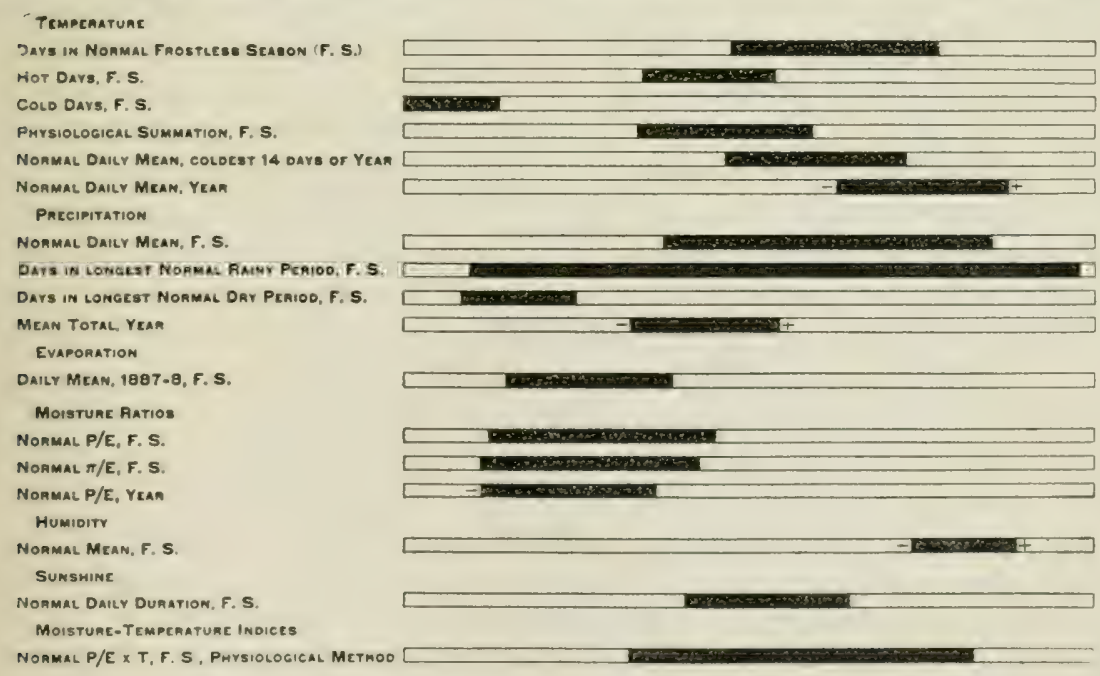

FIG. 42. Climatic extremes for the Austroriparian Zone. 
correspondence of limiting values for the moisture conditions in the Austroriparian and Carolinian. There is a very strong dissimilarity between the moisture conditions for the Austroriparian and the Lower Sonoran. The daily mean evaporation of the frostless season ranges from 0.96 to 1.69 (?) in the former zone and from 104 to 273 in the latter, thereby overlapping to a considerable extent.

Several of the temperature conditions exhibit narrow amplitudes in this zone, notably the number of cold days and the number of hot days. A narrow amplitude is also exhibited by the number of days in the longest normal dry period of the frostless season, and in the imperfectly determined data for the mean total precipitation for the year and the normal mean humidity for the frostless season. It is apparent from this evidence that the Austroriparian Zone is differentiated from the adjacent zone on the north by temperature condi-

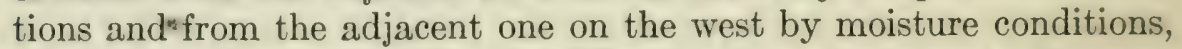
the greatest importance in this differentiation attaching to the conditions of narrow amplitude which have been mentioned.

\section{DISCUSSION OF THE OBSERVATIONS.}

It is impossible to undertake a logical discussion of the correlation of climatic conditions with the areas occupied by the life-zones, because of the climatic basis on which these zones were originally outlined by Merriam. The boundaries running, in general, in an east-and-west direction were determined by the remainder temperature summation above $32^{\circ}$, and the boundary running north and south along the onehundredth meridian was selected because it is a pronounced climatic line, separating what would otherwise be very irreconcilable faunal and floristic regions. From these considerations it may be seen that we should be able to predict the nature of the conditions which limit these areas. An examination of figures 37 to 42, indeed, shows that we encounter marked differences in all of the temperature conditions on passing southward from the Transition Zone, through the Upper Sonoran to the Lower Sonoran, or in passing from the Alleghanian Zone down through the Carolinian and Austroriparian, barring a rather strong similarity between these conditions for the Alleghanian and Carolinian Zones. A comparison of Transition Zone with Alleghanian, of Tpper sonoran with Carolinian, and of Lower Sonoran with dustroriparian, exhibits a similar marked difference of moisture conditions. If the humid Transition of Washington and Oregon is considered separately from the remainder of the Transition, this contrast becomes more striking in the first of these comparisons.

As far as possible we have made use of Merriam's maps of the remainder summation of temperatures above $32^{\circ} \mathrm{F}$. and of the normal daily mean temperature for the hottest 6 weeks of the year, but we have been prevented from making as full use of these maps as their 
importance warrants on account of Merriam's failure to publish the numerical data on which they were based. The climatic extremes have been read from these maps, however, for all of our botanical areas. A consultation of the tables of climatic extremes will show that the absence of the readings for individual stations, and the mere possession of the values for the isoclimatic lines, has made the climatic extremes for these maps (plates 37 and 44) very general in their nature, and has resulted in giving the same extremes for areas which are widely separated or very unlike.

The physiological summation of temperatures appears to be a more natural method of securing a measure of the cumulative effects of this factor on plants than either of the remainder summations which we have used, and its resulting figures should bear a closer relation to distributional facts than the figures derived from any other mode of summation thus far suggested. The four charts showing summations by the different methods (plates 37, 38, 39, and 40) have a generic resemblance so far as concerns the general sweep of their isoclimatic lines, although the actual values represented by the lines differ. The amplitude of the conditions expressed by the physiological summation is not great for any one of the 6 life zones, although it is not so narrow, in any case except the Transition Zone, as to indicate that it expresses one of the conditions of most vital importance in controlling the position of the limits between the life-zones. When the remainder summation above $32^{\circ}$ has been found to have such apparent importance as a controlling condition as to form a leading basis for the delineation of life-zones, it is a matter of surprise that the more logical physiological summation does not show a high degree of importance when correlated with the life-zones. This discrepancy might be attributed to the fact that the boundaries of the zones were partly determined by the temperature of the hottest 6 weeks, particularly in the western or arid zones, if it were not for the fact that it is these zones in which there is the strongest indication that the physiological summation is of importance as a controlling condition. It is at least true, from the indications of our data, that the physiological summation appears to be more important in controlling the zones than is any other temperature factor that we have used, except in the Austroriparian Zone, where several others are of equally narrow amplitude. As we proceed from the northern toward southern zones the mean temperature of the coldest 14 days of the year is seen to be a controlling condition of increasing importance.

With a single exception, the moisture conditions of the western and eastern subdivisions of Merriam's transcontinental zones show a very different range of values, as would be expected from the fact that the subdivision was made on a basis of the differences in these conditions. The one exception is the case of the Transition Zone, in which the 
humid northwestern region gives the moisture conditions both higher and lower extreme values in the Transition than they exhibit in the Alleghanian. With the same exception the moisture ratios have a much narrower amplitude in the western zones than in the easte" $n$ ones. They are clearly to be looked upon as expressing the most. important controlling condition in the Upper Sonoran and Lower Sonoran Zones, but their amplitudes are relatively wide in the eastern zones.

The moisture-temperature index is also of narrower amplitude in the western zones than in the eastern ones. While it is sufficiently narrow in the Transition Zone to be regarded as an expression of important conditions in the limitation of this zone, ihis is not the case with the other zones. In the three western zones this index has the same minimum value, but increases in amplitude on passing southward, so that its maximum values are progressively higher and consequently its amplitudes increasingly wider. In the eastern zones the extremes become progressively higher on passing from north to south, and the amplitudes become greater.

From the preceding discussion, and from considerations presented in Part II, it appears that the system of life-zones worked out by Merriam and now rather widely used in a descriptive way, especially by the United States Biological Survey, will require much modification before it may become at all satisfactory to a serious student of etiological plant geography. It is extremely unfortunate that the actual data on which this system was originally based, and on which its applications are based in current descriptions, do not exist in the published literature. Neither Merriam nor any of his followers has thus far attempted to present the actual basis for the system in form such that a critical study of its good and bad features may be undertaken. Perhaps this may be a main reason why the whoce subject of the climatic relations of floral and faunal areas has received so little attention at the hands of students who are able and willing to undertake the complex analyses which are involved in such a subject. The publication of the charts without the data on which they were based, together with the general and official adoption of the system by the United States Biological Survey, have given this important problem the appearance of having been satisfactorily solved-- of being a closed subject. Those who have employed this zone system have either refrained from any discussion of its good and bad characteristics, or else they have merely taken the standpoint of adrocates, and the lack of the numerical data that are absolutely necessary for a critical study has tended strongly to discourage such inquiries. Also, a sort of authoritative atmosphere that seems to hang over government. publications in general, together with the apparent authority and dogmatism that invariably go with well-printed (and especially colored) 
charts, to the exoteric reader, tend in the same direction, to retard real progress. Ecological students should realize that this is not by any means a closed subject, but that it is in a very early, formative stage, and that it requires vastly more critical and original study than has ever been accorded it. Merriam's woik formed an excellent beginning and he opened up a new and very important field, but his presentation of the matter was hurried and incomplete, and the later work of his followers has consisted in drawing zonal boundaries on a finer scale on biological evidence without any effort to extend the investigation of the climatic basis of the scheme. The work of Merriam should be regarded as a beginning and the whole field opened by him assuredly deserves elaborate critical study at the hands of ecologists. We do not wish to attempt to substitute any other dogmatic scheme of climatic provinces in place of this one, but we do wish to emphasize the fact that some other and much better scheme is to be expected when this subject receives attention such as it deserves.

\section{CONDITIONS THAT PROBABLY DETERMINE THE DISTRIBUTION OF GROWTH-FORMS AND THE ECOLOGICAL DISTRIBUTION OF INDIVIDUAL SPECIES.}

\section{GROWTH-FORMS.}

The fundamental vegetational data for use in any study of climatic conditions in relation to the relative abundance of a particular growthform should be based on a knowledge of the rôle played by this growthform in the regetation of the region involved. Such knowledge is in hand for certain small areas, but is lacking for the great bulk of our region. We have therefore fallen back upon the best obtainable substitute, namely, the securing of distributional data for each of the various species belonging to these growth-forms, and the plotting of their cumulative distribution.

The distribution of all evergreen broad-leaved trees and of all microphyllous trees of the United States have been superposed so as to show the regions in which these growth-forms are represented by the greatest number of species. The resulting maps doubtless come near to showing the relative importance of these forms in the vegetation, as well as their numerical representation in the flora. The distribution of the eastern deciduous trees has been treated by superposing the ranges of a group of the most common and widespread species, rather than by an attempt to use all of the very numerous trees of this growthform. The resulting area does not coincide with the Deciduous Forest region of plates 1 and 2 , but it comprises the region in which deciduous trees are known to reach their maximum abundance, size, rate of growth, and speed of reproduction, as well as the regions in which they are abundant and successful in the optimum habitats. 
The ecological distribution of two species, Liriodendron tulipifera and Bulbitis dactyloides, has been investigated, as exemplifying the methods that it would be highly desirable to extend to a much larger number of species if the data were available for doing so. The aim of securing the climatic data for the areas of relative abundance has been to determine the optimum conditions for these species, as contrasted with the conditions existing where they are not so well represented in the vegetation. The relatively narrow amplitude of conditions exhibited by the central areas of ecological distribution points in each case to the conditions of these areas as the optimal ones.

In all five of the following cases our effort has been the same, whether concerned with the ecological centers for growth-forms or for individual species. The former have been determined entirely from floristic data in the case of the evergreen broad-leaved and microphyllous trees, from floristic data on ecologically important species in the case of the deciduous trees, and from purely vegetational data in the case of Liriodendron and Bulbilis.

Evergreen broad-leaved trees.-Owing to the complicated nature of the areas in which different numbers of trees of this group are found, it has been impossible to construct a satisfactory figure to represent graphically the limiting conditions for the several areas. By a comparison of the map showing the cumulative distribution of this group of trees (plate 3 ) with the various climatic maps, it is possible, however, to determine some of the conditions upon which an abundant representation of evergreen broad-leaved trees is apparently dependent.

Both in the West and the Southeast these trees are seen to be almost. wholly confined to the region with an average frostless season of more than 180 days, and with no cold days in our sense. The Desert region between Texas and southern California is nowhere occupied by more than 10 species of evergreen broad-leaved trees, and extensive stretches of it are occupied by less than 5 species or by none at all, although the temperature conditions are analogous to those of the adjacent regions to the east and west in which there are 10 or more species. The eastern boundary of this group of trees is mainly formed by the limit of Ilet opaca, while the boundary for o or more species is formed by sereral intersecting limits. The position of the latter boundary corresponds roughly with the line for a frostless season of 240 days, while in the West and the East the areats with 5 or more species are so situated as to have a daily mean of $40^{\circ}$ or more for the coldest 14 days of the year.

The physiological summation of temperature appears to have a slight correlation with the cumulative distribution of this group of trees in the Southeastern States, but such correlation is not borne out on the Parific eoast, where the region with 10 or more species encounters the same values of the summation as those found in the Northeastern 
States, where no evergreen broad-leaved species occur. Although the length of the frostless season is evidently a condition of great importance for the rich representation of trees of this type, it is apparent that the temperature conditions of the frostless season itself are not so important as are the conditions insuring a mild winter. In parts of the California coast with a physiological summation of 5,000 to 7,500 there are over 10 species of evergreen broad-leaved trees, while in Georgia and Florida the same number of species are to be found in a region with summations of 15,000 to 17,500 . In each of these cases the frostless season is between 240 and 300 days in length. In spite of such great differences in temperature summation between regions with frostless seasons of so nearly the same length, we have, on the other hand, an absence of cold days in both regions and daily mean temperatures in both places that are above $45^{\circ}$, or even above $50^{\circ}$, for the coldest 14 days of the year.

The rapid increase in the total number of evergreen broad-leaved trees encountered in passing from the central Eastern States into peninsular Florida is paralleled by a rapid increase in the number of hot days, by an increase in the physiological temperature summation, by an elevation in the mean temperature of the coldest fortnight to $60^{\circ}$ and above, and by increasing values for the moisture-temperature index. In none of these conditions does the coast of California approach the high values of southern Florida, except in the case of the mean temperature of the coldest 14 days.

The long frostless season and the mild winter, which favor the abundance of broad-leaved evergreens, do so only in regions of high moisture conditions. In the Southeastern States the region with 5 or more species exhibits moisture ratios of 1.00 or above, except in extreme southern Florida. On the Pacific coast the greatest abundance of evergreen broad-leaved trees is in a region with moisture ratios of 0.40 to 0.60 . This marked difference must be interpreted in connection with the much lower summations of temperature for the frostless season which characterize the Pacific coast. Between coastal California and eastern Texas the number of evergreens rises above 5 only in the mountain ranges of southern Arizona and western Texas, where the local conditions are not elucidated by our climatic data.

In the correlation of moisture conditions with the cumulative distribution of the evergreen broad-leaved trees, it should be borne in mind that our moisture data are chiefly elaborated for the frostless season, and that the moisture conditions of the winter (even where it is reduced to a length of less than 9 weeks) are surely of great importance to these trees. The low moisture ratios of the frostless season on the coast of California must be interpreted in the light of the fact that the short frost season is there the time of the principal rainfall.

Microphyllous trees.-The cumulative occurrence of this small group of trees characteristic of the subtropical desert regions has been 
shown on the same map with the cumulative occurrence of the evergreen broad-leaved trees (plate 3), in order to demonstrate the manner in which the former group fills the break in the distribution of the latter. The region of maximum occurrence is in extreme southern Texas, while 5 or more species are found in the Texas Semidesert, along the lower Rio Grande, and in southern Arizona.

The maximum occurrence of microphyllous trees is in a region with a frostless season of 300 days or more, and the areas with 5 or more species are confined to regions with a season of from 240 to 300 days. Nowhere does the occurrence of as many as 5 species encounter any cold days nor a mean temperature for the coldest fortnight that is lower than $40^{\circ}$ (or for the largest areas, $50^{\circ}$ ). The physiological summation of temperature is above 15,000 for 5 or more species and above 20,000 for 10 or more.

The moisture ratio for the region of maximum occurrence of microphyllous trees falls rapidly from 0.80 on the Gulf coast to 0.40 in the interior, and for the region of 5 or more species it falls from values above 0.60 to values below 0.20 . Dry periods of 75 days are experienced on the Texas coast, of 100 days and more along the Rio Grande, and of 250 days and more in southern Arizona.

In spite of the occurrence of the maximum number of trees of this type in the relatively moist climate of extreme southern Texas, as many as 5 species of the group are able to withstand the extremely arid conditions of the desert near the mouth of the Colorado River. The encountering of rainy periods of 25 days (or of 50 days in Texas) appears to limit the western and eastern occurrence of 5 or more species.

While the microphyllous trees are confined longitudinally by moisture conditions, their latitudinal range is restricted by temperature conditions. The continuity of the region of 5 or more species from California to Texas is broken only by the highlands of the Continental Divide near the Arizona-New Mexico boundary, where all temperature conditions are relatively severe.

Eastern Deciduous trees (fig. 43).- The 13 most common and widespread deciduous trees of the eastern United States are all found in a region stretching from Massachusetts and New York to Delaware and Ohio, and southward to northern Alabama. The region with 8 or more of the 13 cmbraces southern New England, southern Michigan, eastern Iowa, the whole of Arkansas, and nearly the whole of South Carolina. The region with from 1 to 7 species embraces all the remaining States east of the one-hundredth meridian, barring southern Florida (see plate 5).

An effort has been made to show the climatic extremes of these three areas graphically and in such a way as to make their direct comparison easy, and the result is shown in figure 43 . In this figure we have a rough means of determining the optimum conditions for deciduous 
trees, not on the ideal basis of the ecological distribution of all species of that type, but on the only basis which is now practicable-the geographical distribution of the most abundant species.

It will be noted that there are a number of cases, particularly among the temperature conditions, in which the upper climatic extreme grows higher as we pass from the center (with 13 species) through the subcenter (with 8 or more species) into the fringe (with 1 to 7 species). Thus, the number of species of deciduous trees grows less as the frostless season grows longer, as the number of hot days increases, as the number of cold days increases, etc. In several cases the extremes are nearly the same for the center and the subcenter, or for the subcenter and the fringe. In the case of humidity we have no deciduous trees growing in the lower half of the gamut of this condition; with increasing humidities above 53.2 per cent we have an increasing number of deciduous species; on approaching the region with highest humidities we first leave the fringe, then the subcenter, and finally the center.

Rather wide amplitudes characterize all of the temperature conditions, and in most cases the conditions of the subcenter and fringe shade off very gradually from the conditions of the center. The minimum number of cold days is the same for all three areas, inasmuch as all of them range into the region with no cold days. A much more irregular set of relations is exhibited between the extremes for the moisture conditions of the three areas. The shortening of the longest rainy period brings us rapidly from the center to the limit of the entire group. Increasing evaporation also brings us, within very narrow limits, from the center to the edge.

\section{TEMPERATURE}

Days in Normal Faostless Season (F. S.)

Hot OAYs, F. S.

Colo Days, F. S.

Physiological Summation, F. S.

NORMAL DaILY MEAN, COLLest 14 DaYs OF YEAR

Normal Dailly Mean, Year

Precipitation

Notmal Daily MEAN, F. S.

Days in longest Normal Rainy Period, F. S.

Days in longest Normal Day Period, $F$. $S$.

MEAN TOTAL, YEAR

Evapoaation

DAILY MEAN, 1887-8, F. S.,

Moisture Ratios

Normal P/E, F.S.

NORMAL $\pi / E$, F.S.

NORMaL P/E, YEAA

HuMIOITY

NORMal MEAN, F. S.

SUNSHINE

NORMAL DaILY DURATION, F. S.

MOISTURE-TEMPERATURE INDICES

Normal P/E X T, F. S., Physiological Method
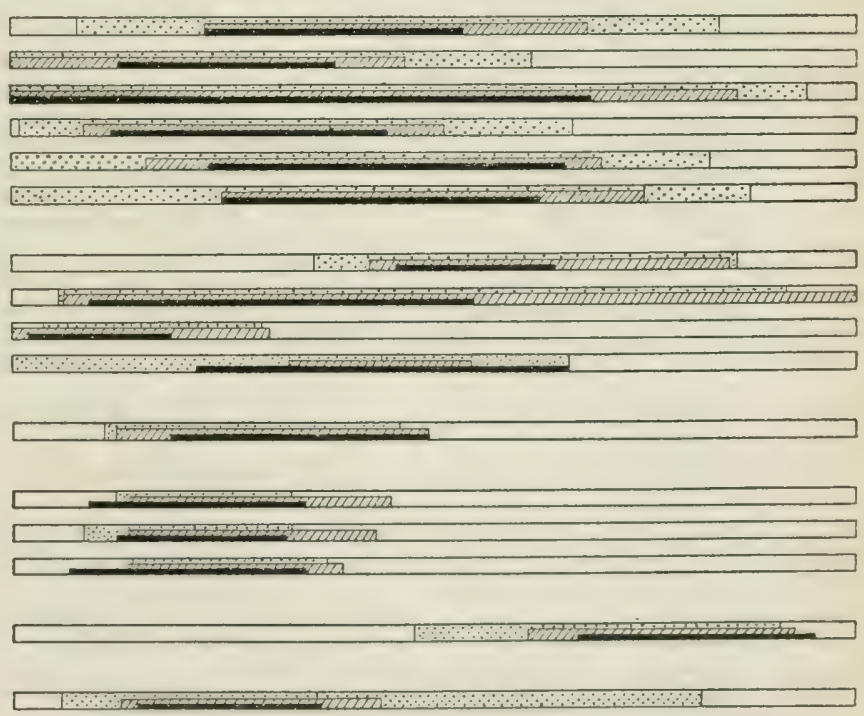

FIG. 43. Climatic extremes for eastern Deciduous trees; center of distribution (black), sulseenter (shaded), fringe (dotted). 
The narrowest amplitudes for the center are in the three moisture ratios and in the number of days in the longest dry period; and in all four of these cases a slightly wider amplitude of conditions brings us to the extremes for the fringe. 1 comparison of plate 5 with plate 59 shows that the whole region occupied by the center, subcenter, and fringe is very nearly confined between the isoclimatic lines for moisture-ratio values of 0.60 and 1.10 , although the fringe enters regions with higher values. The range from 0.80 to 1.10 is scarcely exceeded by the center. The isoclimatic line for dry periods of 50 days is close to marking the western limit of the fringe of deciduous trees. In the center the extreme range is from 4 to 56 days, values above 50 being extremely local in this region, however.

Figure 43 should be compared with figure 31 , which shows the climatic extremes for the Deciduous Forest region. The extremes for the Deciduous Forest lie, in general, outside those of the center of the 13 common species and inside those of the fringe.

Liriodendron tulipifera (fig. 44).-The area in which this tree is of commercial importance may well be regarded as its ecological center, while the region in which it occurs too infrequently to have such importance may be designated as its fringe (plate 9). The center for Liriodendron lies almost wholly within the center for the 13 deciduous trees just treated, and its distributional limit is similar to that of the subcenter of the deciduous trees, although not extending quite so far west. The conditions for the center and fringe of Liriodendron have been shown by pairs of graphs in figure 44 .

The region of greatest abundance for Liriodendron is one of the very few botanical areas investigated in which the edge lies entirely within the United States and is nowhere formed by a coast-line. The fact
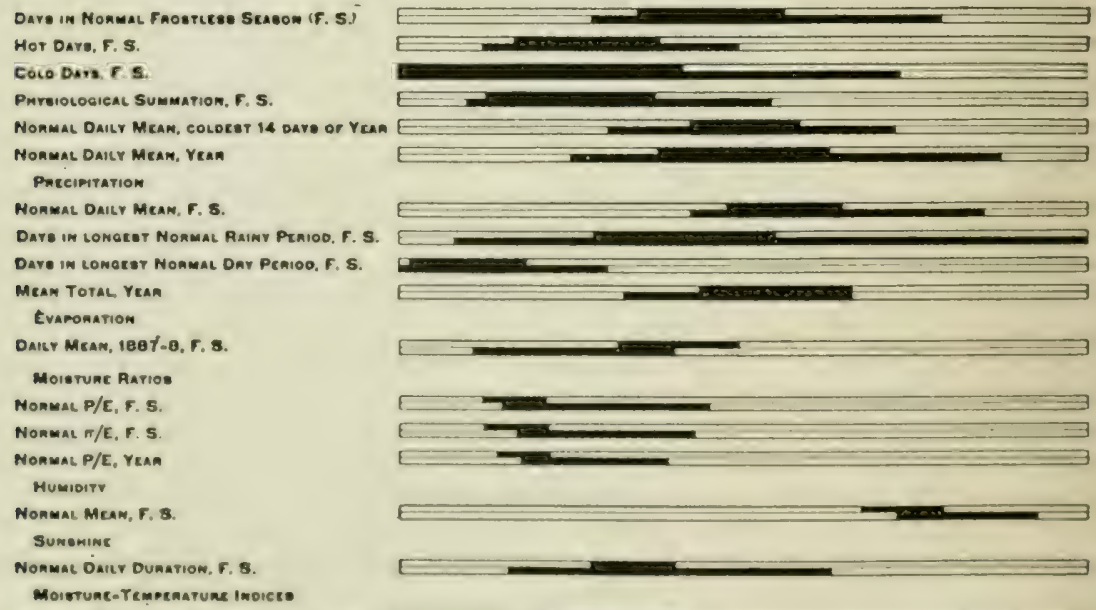

nommat P/E a Y, F. 8., Pursiolosical Mrtroo

Fig. 44. Climatic extremes for Liriodendron; upper blocks for center, lower for fringe. 
that the fringe completely surrounds the region of greatest abundance makes this a case in which it is of interest to compare the character of the two sets of amplitudes. The amplitudes of conditions for the center are narrow in all cases, except the number of cold days. The amplitudes for the fringe are wide in a number of cases, notably the number of cold days, the annual daily mean temperature, the length of the longest rainy period, and the moisture-temperature index. In every case the amplitude of the conditions for the center is less than that for the fringe, and the extremes for the center lie within those for the fringe in all cases except those of evaporation, humidity, and the moisture ratios. The geometrical centers of the blocks representing the extremes for the region of greatest abundance lie within the blocks for the extremes of the fringe in every case except that of evaporation. This means that for all of the conditions except evaporation it is possible to find a locality in the fringe which possesses climatic values that are near those of the absolute ecological optimum of the species.

The fact that a straight line laid down across any portion of the map of the United States passes through localities showing for long distances a progressive change in the values of each climatic condition is responsible for the maximum and minimum values of so many conditions being respectively greater and less in the fringe than in the center. The conditions which are favorable to Liriodendron in the region of greatest abundance are, in some cases, still more favorable to it in the northern part of the fringe, and in other cases still more favorable in the southern part of the fringe. The center exhibits the favorable constellation of conditions designated as the ecological optimum. When a given condition shows minimum and maximum values for the fringe which are not respectively lower and higher than the extremes for the center (fig. 44 , plates $53,57,59,60$, and 65 ), it is probably an indication that the condition involved is not an important one in determining the location of the center and fringe, however narrow the amplitudes involved may be.

The comparative uniformity in the amplitudes of all temperature conditions for the distributional center of Liriodendron indicates that these conditions are of nearly equal weight in determining the limits of the center, with a slight indication of preponderant importance for the mean temperature of the coldest 14 days. The daily mean precipitation and the number of days in the longest normal dry period appear also to be conditions of importance in limiting the center. The narrow amplitude of the moisture ratios for the center is largely to be attributed to the low values which characterize the Ohio Valley (see plate 59). An adequate series of evaporation and precipitation stations in the heart of the southern Alleghenies would doubtless give maxima for the center nearly or quite as high as those for the fringe (see values for Pisgah Forest, North Carolina, table 16). The west- 
ward extension of Liriodendron does not carry it far beyond a mean annual rainfall of 40 inches, nor into the region with more than 25 days in the longest dry period.

Bulbitis dactyloides (fig. 45). - The areas of relative abundance for Bulbilis (plate 10) have been charted in an effort to depict the virgin conditions of the distribution of this grass, using all available sources of information, but the resulting map is probably less faithful to the facts than the map of Liriodendron or the map of the relative abundance of Pinus tada. The areas of the latter are unfortunately so small that they frequently comprise no climatic stations whatever, thereby rendering an adequate discussion of them impossible.

The range of Bulbilis crosses the United States in a broad belt between the ninety-fifth and one hundredth and sixth meridians, extending from the Canadian boundary in North Dakota to southern Texas. In the North the distributional area is narrower than the Grassland, not reaching to its western edge; in the South the distribution is more extended than that of the Grassland, reaching eastward to Louisiana and westward into the Desert-Grassland Transition region. The only limits of the geographical distribution of this species that fall within the United States are the eastern and western ones. The center of ecological distribution extends from South Dakota to northern Texas, and the stibcenter of ecological distribution is only from 100 to 200 miles in width, surrounding the center. Both of these areas lie entirely within the United States (see plate 10).

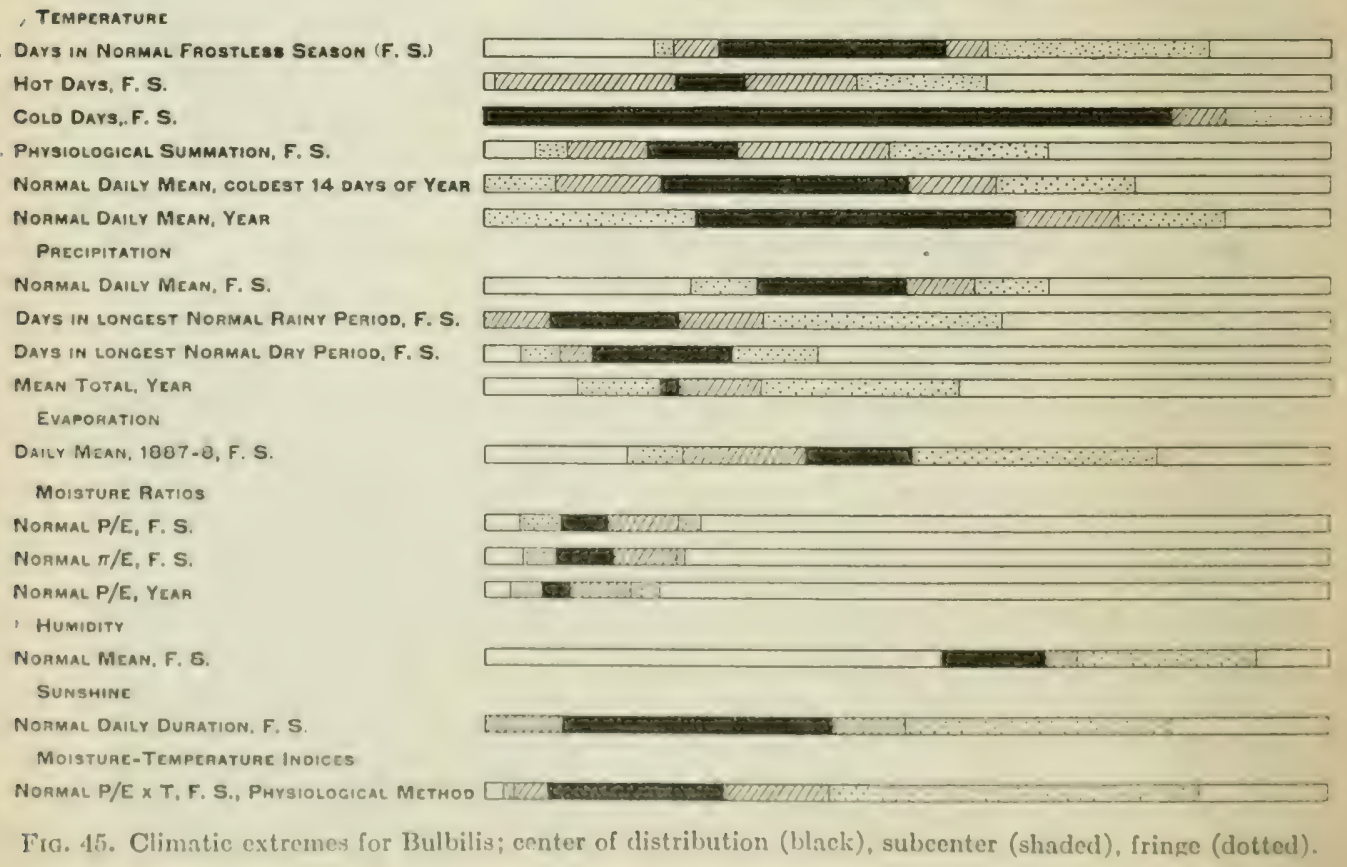


The graphs showing the climatic extremes for Bulbilis (fig. 45) have been drawn in such a way as to show the maximum and minimum values for the center, only such maximum and minimum values for the subcenter as lie outside those for the center itself, and only such extreme values for the fringe as lie outside those for the subcenter.

The ecological center exhibits several climatic conditions with narrow amplitudes, notably the number of hot days, the physiological summation, mean annual precipitation, evaporation, humidity, and the moisture ratios. The position of the isoclimatic lines for the physiological summation would indicate a somewhat wider amplitude $(5,000$ to 15,000$)$ for this condition than that based upon the readings of the minimum and maximum stations and given in table 71 . The widest amplitudes are those of the number of cold days, the daily mean temperature, and the daily duration of sunshine. For the ecological center, then, we may state that the amplitudes are narrow for all of the moisture conditions and are partially narrow and partially wide for the temperature conditions.

An examination of the graphs for the climatic extremes of the subcenter and fringe shows that the amplitudes are wide for all of the temperature conditions, even for those that show narrow amplitudes for the center. With respect to the moisture conditions, however, the amplitudes of the subcenter and fringe are not so greatly in excess of those for the center, except in the cases of evaporation and humidity. The moisture ratios show narrow amplitudes even for the fringe. These facts indicate that the location of the Bulbilis areas as a whole is chiefly determined by moisture conditions, and this is particularly true of the center. The position of the zones of abundance is determined on the east and west by small but significant differences in the moisture conditions, and on the north and south by temperature conditions.

\section{SPECIES.}

The climatic extremes for all of the individual species treated in the following pages will be found in tables 77 to 151 . Some 31 species have been selected from a total of 75 for the presentation of the climatic extremes in graphic form (figs. 46 to 74). These have been chosen so as to represent all types of plants and all types of distribution represented among the larger number. The relation of climatic conditions to the distribution of these species will now be discussed.

Tsuga heterophylla (fig. 46). - This tree occupies an area in which the conditions are similar to those of the Northwestern Evergreen Hygrophytic Forest, with differences due to the extension of the limits of T suga into northern Idaho and Montana, well to the east of the Hygrophytic Forest. The number of cold days in the frostless season endured by the easternmost individuals of this species reaches a maximum of 
120, whereas no cold days are experienced within the Hygrophytic Forest. The normal daily mean temperature also ranges to lower values for the tree than for the vegetation in which it is most characteristically developed. The temperature conditions encountered by Tsuga in northern Idaho and Montana are otherwise very similar to those in coastal Washington and Oregon. The precipitation conditions for the area occupied by Tsuga are very similar to those of the Hygrophytic Forest, at least with respect to the frostless season. Higher intensities of evaporation are encountered in Idaho and Montana and higher values for humidity in northern California, making the amplitudes for both of these conditions somewhat greater than they are for the Hygrophytic Forest. The remarkably wide amplitude of the moisture ratios which is characteristic of the last-named forest is also shown for the area of Tsuga.

The narrow amplitude in the number of hot days in the frostless season and in the physiological summation of temperature would indicate that these conditions are important in the limitation of Tsuga heterophylla, and the position of the isoclimatic lines also suggest that the precipitation conditions are of critical importance, in spite of the wide amplitude which they exhibit within the distributional area of this tree.

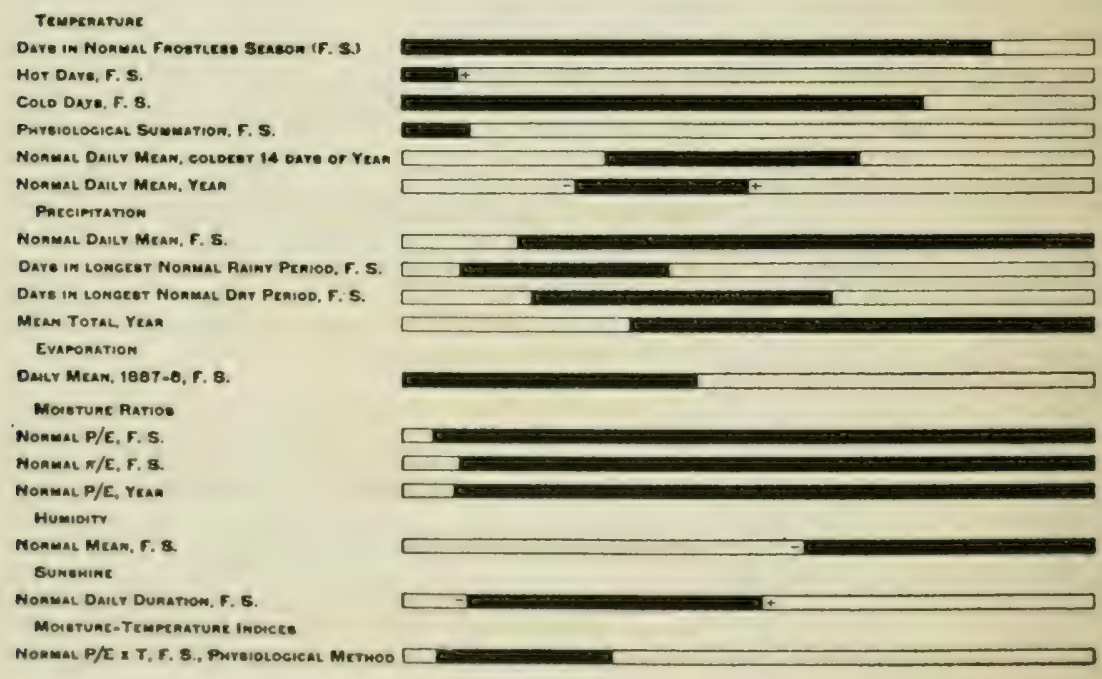

Fra. 46. Climatic extremes for Tsuga heterophylla.

Pseudotsuga mucronata (fig. 47).-The range of this tree covers all of the Northwestern Hygrophytic Evergreen Forest and a large part of the western section of the Northern Mesophytic Evergreen Forest. The climatic extremes for it exhibit some of the features of each of the vegetations with which it is coextensive, and in several cases the 
graphs exhibiting these extremes are such as would be secured by a superposition of the graphs for the two vegetations.

In spite of the great north-and-south extension of the range of Pseudotsuga, it encounters a narrow amplitude of conditions in the number of hot days in the frostless season and in the physiological summation of temperature. Further climatological data from stations situated within the range of this tree at some of its most southerly localities might broaden the amplitude of these conditions, giving values more nearly like those for the western section of the Northern Evergreen Forest, which area is drawn in a more generalized manner.

With respect to all of the conditions involving precipitation or atmospheric moisture, the amplitudes are very wide, being in many cases a superposition of the amplitudes for the two forest areas mentioned. The fact that this tree is so nearly coextensive with the southernmost areas of Mesophytic Evergreen Forest may be taken to mean that the constellations of conditions by which its range is limited are very similar to those limiting this forest. The lowest normal daily mean precipitation, the highest values for evaporation, and the lowest ones for the moisture ratios are all to be regarded as important in limiting the southward range of this tree. Although the lower limit of Pseudotsuga in the mountains of Arizona and New Mexico is slightly higher than the lower limit of the pines which form the edge of the Mesophytic Forest, the data from the few stations in that region show a close correspondence in the extremes just mentioned. The number of hot days and the value of the physiological summation of tempera-

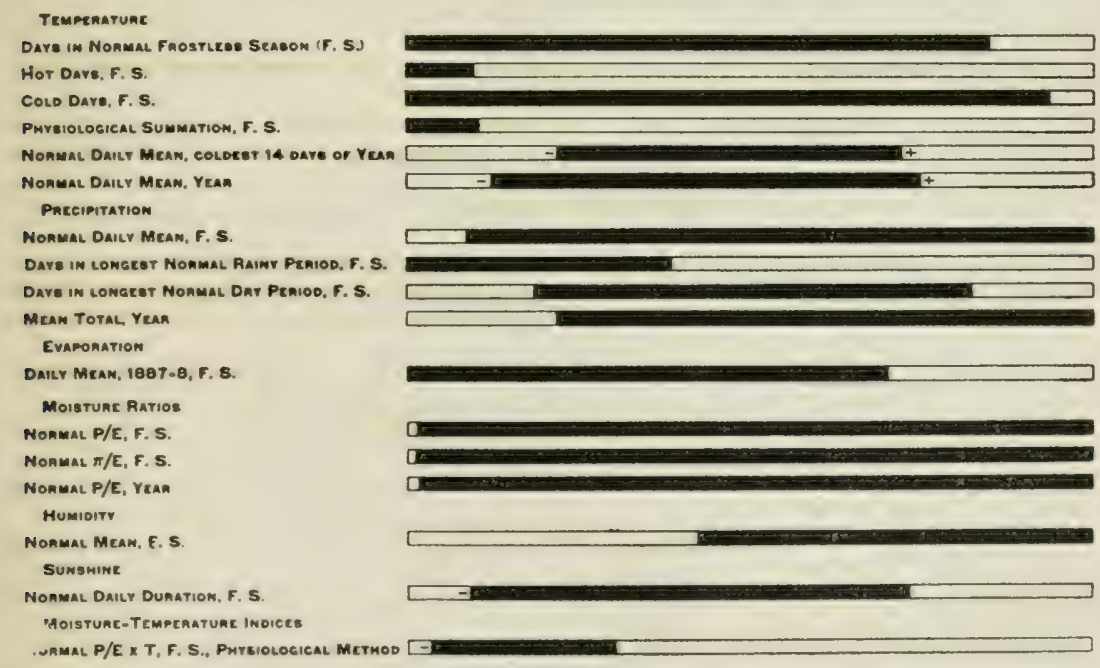

FIG. 47. Climatic extremes for Pseudotsuga mucronata. 
ture are the two conditions which appear to be most critical in the limitation of this species with respect to its entire range. There are few localities in its distributional edge, however, in which the moisture conditions would fail to be of importance in connection with the temperature conditions just mentioned.

Pinus ponderosa (fig. 48). - The range of Pinus ponderosa is similar to that of Pseudotsuga, but is somewhat more extended from Colorado northward to the Canadian boundary, where it exceeds the eastern limit of the western section of the Northern Mesophytic Evergreen Forest, and is less extended in the extreme northwest, where it fails to enter the Northwestern Evergreen Hygrophytic Forest.

Except for the slightly narrower amplitude in length of frostless season, the temperature conditions are similar for Pinus ponderosa and for the western section of the Northern Mesophytic Forest. The number of hot days and the physiological summation of temperature again appear to be factors of importance in limiting this tree.

On account of its extension into the western edge of the Grassland, the moisture values of Pinus ponderosa are of somewhat wider amplitude than those of the forested area in which it is so abundant. This species does not, however, encounter such low evaporation values nor such a high range of humidities as does the forest itself. It is doubtful if the former of these facts would be confirmed by data from a larger number of critically located stations; the latter is due to the absence of Pinus from the extreme coast of northern California.

The conditions expressed by the moisture-temperature index appear to be of considerable importance in limiting this tree, together with the moisture ratios, the number of hot days, and the physiological summation of temperatures.

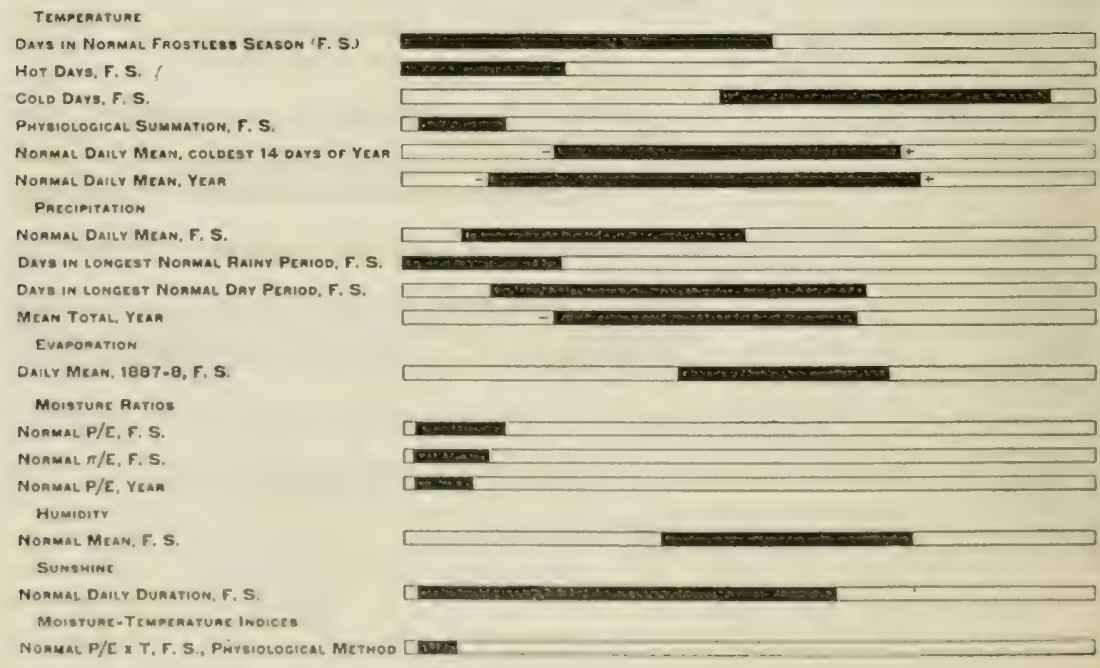

Fig. 48. Climntic extremes for Pinus ponderosn. 
Pinus contorta (fig. 49).-The range given for this tree is based on the view that it is identical with Pinus murrayana, and comprises the regions that are occupied by the two forms-the northern Pacific coast, the Sierra Nevada, the northern Rocky Mountains, and the Black Hills.

The extremes and amplitudes of conditions for this tree are very similar to those for Pseudotsuga mucronata. It encounters a slightly greater number of hot days, and a greater value for the physiological temperature summation, with a lower maximum value for the number of days in the longest dry period. Pinus contorta is like Pseudotsuga in encountering a wide amplitude of nearly all of the conditions here studied, its strongest control appearing to lie in the number of hot days and the values of the physiological summation of temperatures.

It will be noted that both the eastern and western evergreen needleleaved trees are confined to ranges which exhibit a narrow amplitude in the number of hot days. The amplitude of the physiological summation is narrow for all except the species that are found in the Southeastern Evergreen Mesophytic Forest (for example Pinus echinata). With respect to both of these conditions there is a marked contrast between the evergreen needle-leaved and the deciduous broad-leaved trees.

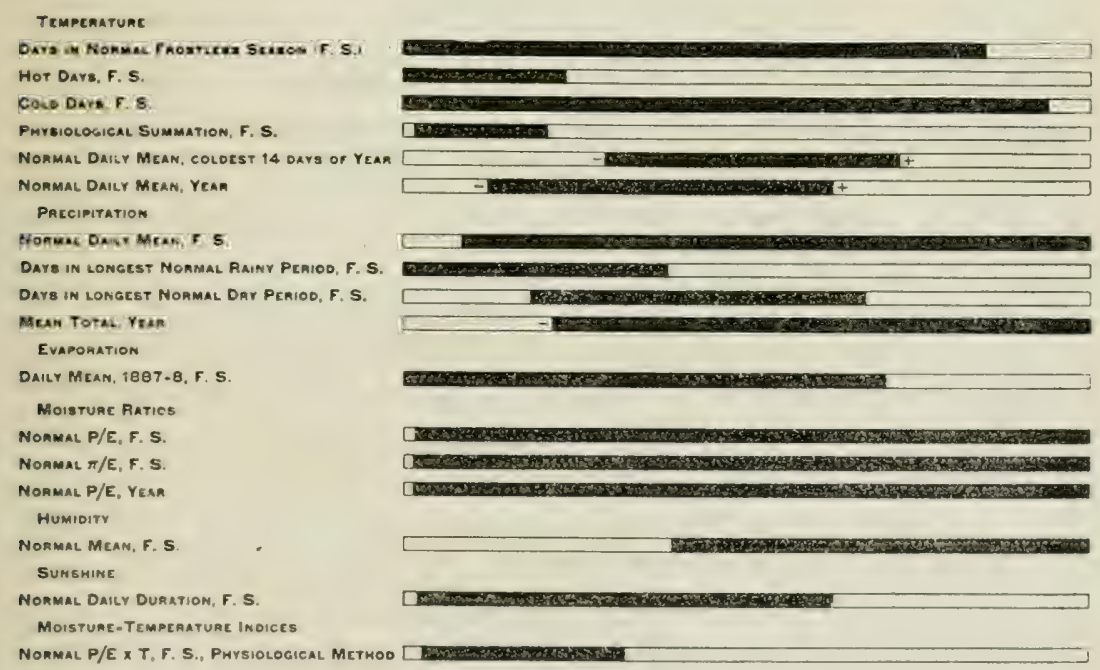

FIG. 49. Climatic extremes for Pinus contorta.

Pinus echinata (fig. 50).--The range of this tree occupies all of the Southeastern Evergreen Mesophytic Forest except a strip along the Gulf of Mexico and peninsular Florida, and also the southern half of the Deciduous Forest region. It therefore exhibits amplitudes and extremes which lie between those of the two vegetations in which it 
occurs, and is remarkable in having a number of relatively narrow amplitudes for a tree of such wide distribution. Its widest amplitudes are in the number of days in the longest normal rainy period and in the moisture-temperature index. The number of hot days and the number of cold days appear to be of about equal importance in limiting the range of Pinus echinata. The number of days in the longest normal dry period and the mean total precipitation of the year appear to be still more important as limiting conditions, while the amplitude of relative humidity is also comparatively narrow.

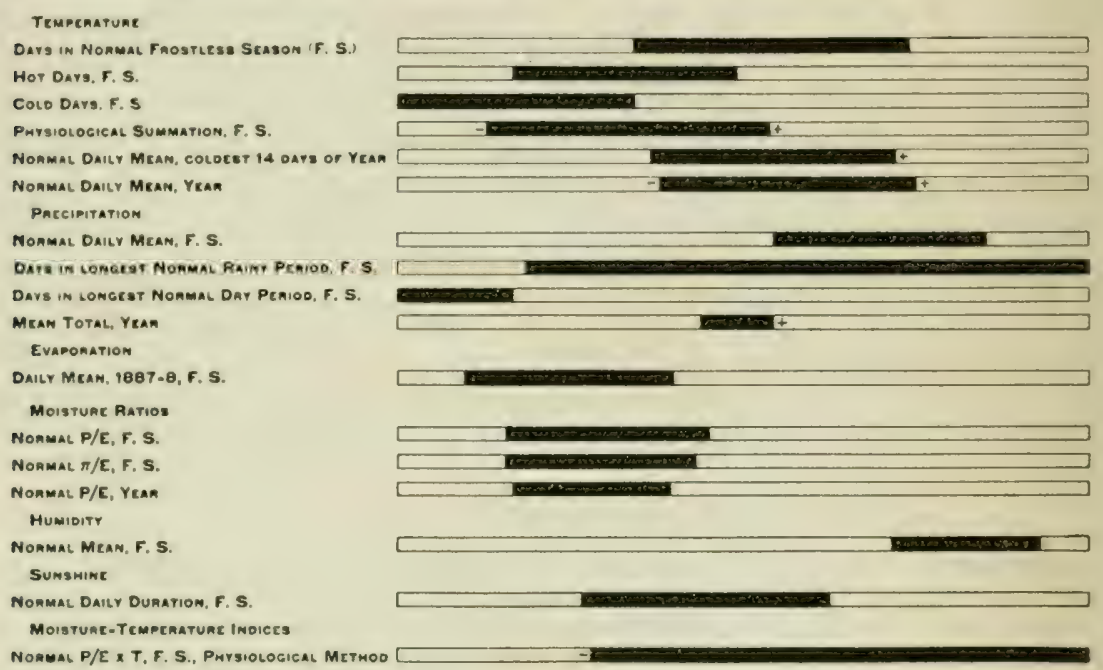

Frg. 50. Climatic extremes for Pinus echinata.

Pinus strobus (fig. 51).-The distribution of Pinus strobus is coextensive with that of the eastern section of the Northern Mesophytic Evergreen Forest, and exceeds it to some extent toward the south and west, carrying the tree into the Deciduous Forest region and into the Grassland-Deciduous Forest Transition region. The conditions in the area of Pinus strobus are therefore similar to those of the vegetational area in which it reaches its greatest abundance. The southernmost extension of the tree carries it into a region with longer frostless season and with no cold days, in our sense of this term. A slightly greater value for the physiological temperature summation and a greater normal daily mean temperature are also encountered by Pinus strobus in its extension toward the Atlantie Coastal Plain and southward to (ieorgia. The number of days in the longest normal rainy period and the number in the longest normal dry period both reach maximum values which are greater for this tree than for the Evergreen Forest, although the minimum values are the same for the two.

There are no very narrow amplitudes for this pine. The narrowest, however, are those for the moisture ratios and for relative humidity, 
and those for the number of hot days, the normal daily mean precipitation, the physiological temperature summation, and the number of days in the longest normal dry period.

From a comparison of the distributional limit with the positions of various isoclimatic lines, the southward range of Pinus strobus appears to be determined by temperature conditions, of which the physiological summation and the number of hot days in the frostless season are the most important, while its westward range appears to be determined by moisture conditions, of which the normal daily mean precipitation and the number of days in the longest normal dry period are the most important. The position of the isoclimatic lines for the moisture ratio, $\pi / E$, would indicate that this compound factor is one of strong importance in determining both the southern and the western limits of this tree.

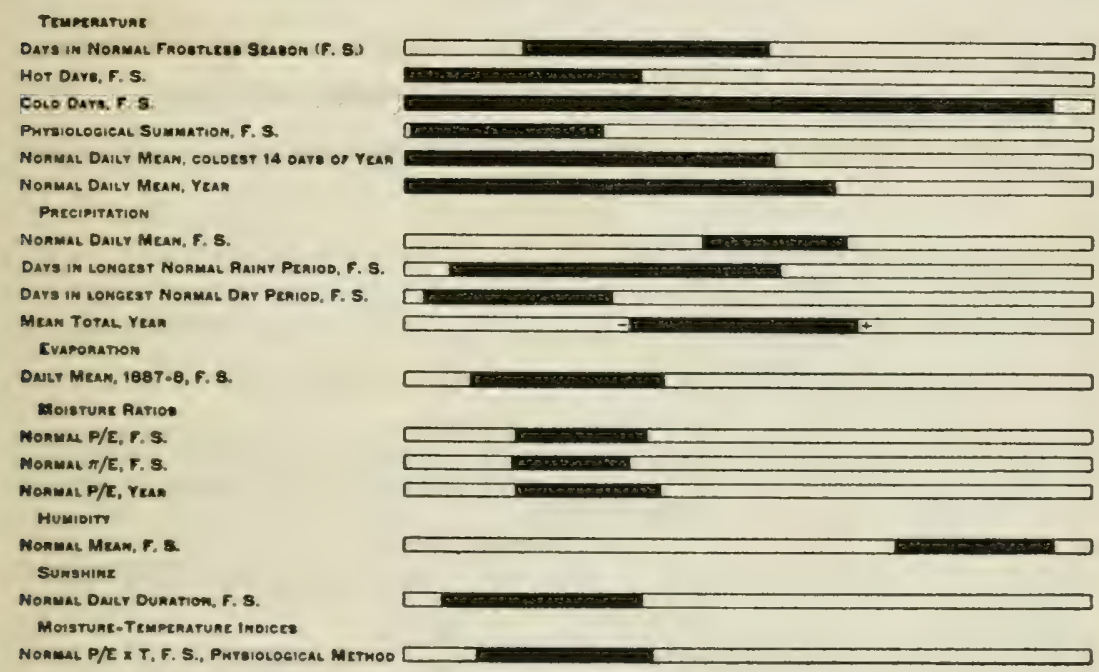

Fig. 51. Climatic extremes for Pinus strobus.

Quercus alba (fig. 52).-This oak is found throughout the eastern United States, with the exception of northern Minnesota and Michigan and peninsular Florida. In keeping with its wide distribution it encounters a very wide range of practically all of the climatic conditions, exceeding in a number of cases the extremes for the Deciduous Forest region, in which it finds its greatest derelopment. The narrowest amplitudes for Quercus alba are those of the number of days in the longest normal dry period, the normal mean relative humidity of the frostless season, and the moisture ratios. The first and last of these conditions appear to be responsible for the western limit of distribution. This edge is roughly paralleled by the line for 25 days in the longest dry period and by the line for a value of 0.60 for the moisture 
ratio. Owing to the extension of the northern edge into Canada it is impossible to speak regarding its probable controls in that direction.

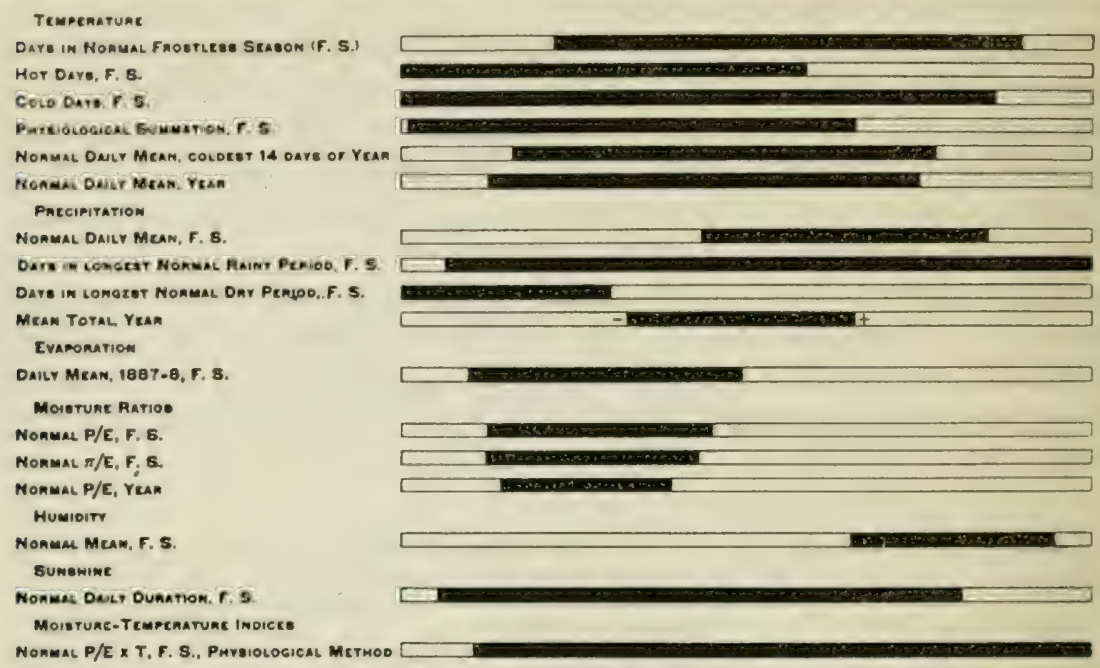

FIG. 52. Climatic extremes for Quercus alba.

Quercus falcata (fig. 53). - This tree is found throughout the Atlantic Coastal Plain and the southern Mississippi Valley, reaching its greatest abundance in the Southeastern Mesophytic Evergreen Forest, but extending well into the Deciduous Forest region. It encounters extremely
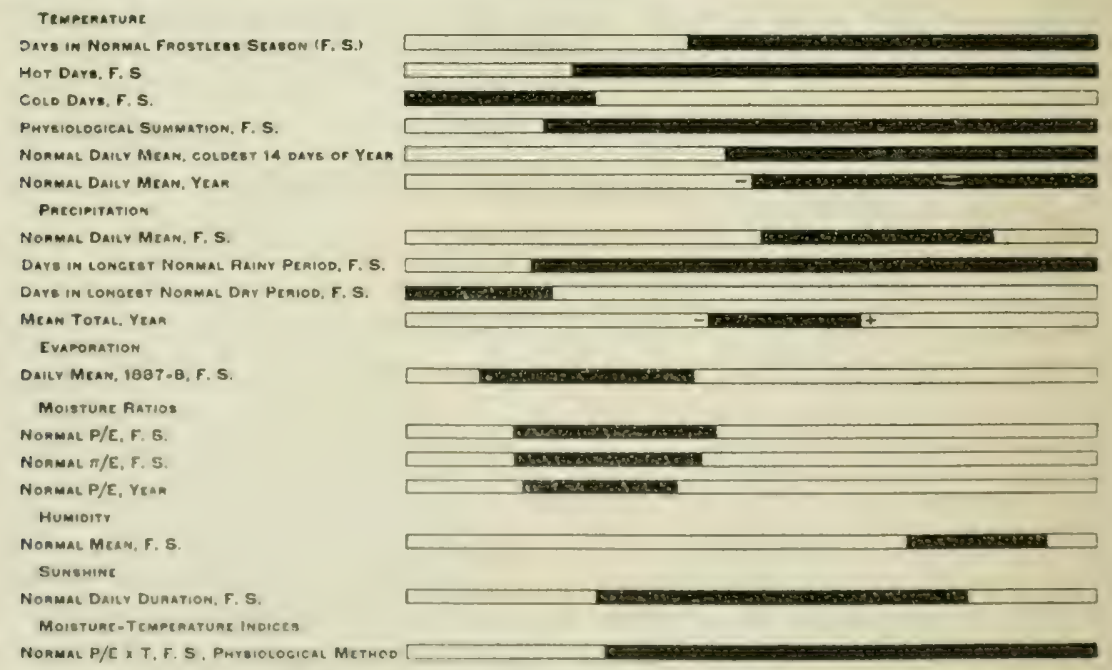

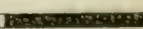

Fig. 53. Climatic extremes for Quercus falenta.

wide amplitudes of all but one of the temperature conditions here dealt with, and its diminishing oceurrence in southern Florida is coincident with the maximum values for these conditions. This is one 
of the cases already mentioned in which the southernmost limit of plants in Florida is not well known, so that it is impossible to state just how far Quercus falcata may fall short of extending into the region of the temperature conditions represented by Key West. The one temperature condition for which Quercus falcata shows a narrow amplitude is the number of cold days, due to the fact that its range barely extends into the region in which any cold days are experienced.

The moisture conditions in the area of this oak are very similar to those of the Southeastern Evergreen Forest, except in the case of the number of days in the longest normal dry period. While the Evergreen Forest withstands a maximum of 182 days (the reading for Key West), Quercus falcata has its maximum at 63 days, at the southwestern edge of its range. The normal dry period, the moisture ratios, and the relative humidity of the frostless season appear to be important conditions for the limitation of Quercus falcata. Its distributional area may be defined as the region in which the frostless season is more than 180 days in length, the moisture ratio greater than 0.80 , the humidity greater than 70 per cent, the normal number of cold days in the year less than 30, and the number of days in the longest normal dry period not more than 63 .

Quercus macrocarpa (fig. 54).-The area occupied by this oak covers the Northeastern States, omitting the Coastal Plain, and extends as far west as eastern Montana and central Oklahoma. Its southern limit is roughly coincident with the northern limit of Quercus falcata, and its northern limit is in Canada. Quercus macrocarpa is like Q. alba in exhibiting broad amplitudes for nearly all of the climatic conditions. It encounters approximately the lower half of the scale of

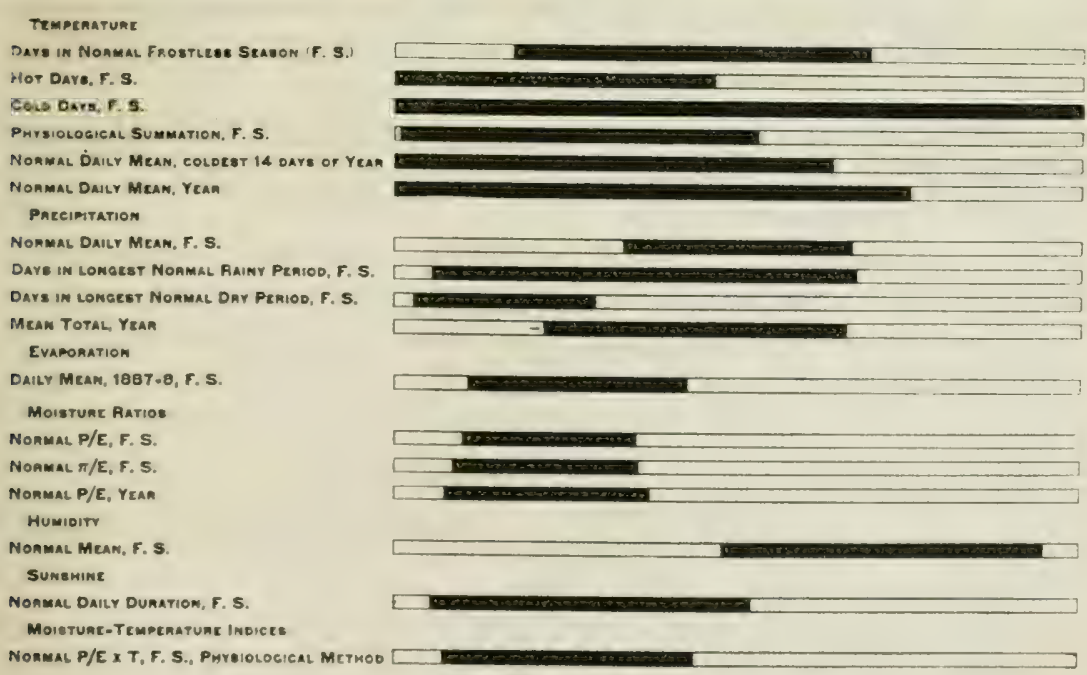

FIG. 54. Climatic extremes for Quercus macrocarpa. 
amplitudes of temperature conditions for the United States, but reaches areas with the maximum number of cold days. One of the narrowest amplitudes among the moisture conditions is found in the case of the number of days in the longest normal dry period, for which the extreme values are 9 and 88 days. A previous allusion was made to the western limit of this tree as showing the manner in which wholly distinct conditions cooperate in controlling the ranges of plants. It will be seen by a comparison of plate 52 (dry days) with plate 18 (distribution of Quercus macrocarpa) that the longest dry period encountered at the western edge of this tree in Oklahoma is less than 50 days, whereas the maximum number encountered near the Canadian boundary is 88 days. The potence of this moisture condition is evidently modified by the differences in temperature conditions which are encountered along the western limit of the tree. The values of the moisture ratio $(\pi / E)$ encountered in Oklahoma are about 0.60 and those encountered in Montana are about 0.40. An ability on the part of Quercus macrocarpa to withstand the same values for these two conditions in the latitude of Oklahoma that it does in Montana would carry the tree to the eastern borders of New Mexico with respect to the moisture ratio, and well into the borders of that State with respect to the longest dry period. These two conditions, as modified in their influence by temperature conditions, may be regarded as setting the limit of the westernmost occurrences of this oak, which (like the western limits of so many deciduous trees) are to be found in alluvial bottoms characterized by moisture conditions which are higher than those of the adjacent upland.

The southern limit of Quercus macrocarpa corresponds roughly with the isotherm of 120 hot days (see plate 36 ), and this condition, possibly in conjunction with closely related conditions, may be regarded as probably controlling the southern edge of the distributional area. The mean temperature of the hottest 6 weeks is apparently one of the most important of these related conditions, as the isotherm of $78.8^{\circ}$ lies near the southern limit (see fig. 45).

Ilex opaca (fig. 55),- - The occurrence of this Ilex is rather closely confined to the Atlantic Coastal Plain throughout all but a small part of its range, where it extends into the Piedmont and mountain sections of (ieorgia, Mlabuma, and Tennessee. This range is characterized by wide amplitudes of the temperature conditions, reaching the maximum values in all cases exerpt the number of cold days. With respect to the latter condition, the amplitude is relatively narrow and the maximum is 5.5 days, encountered only at the northernmost attenuated limit of oceurrence, in Massachusetts.

The moisture eonditions for the area of Ilex are nearly those of the Southeastern Mesophytic Evergreen Forest, the amplitudes being 
wide in all cases except the imperfectly determined mean annual precipitation. Although this plant encounters a wide range of the conditions expressed in the moisture-temperature index, there is still a close correspondence between its limit and the isoclimatic line of 11,000 for this index. At the localities where it occurs in southern Illinois and Indiana it encounters index values of only 7,000, and at its northern limit in Massachusetts it encounters its minimum value of 5,193.

Numerous isoclimatic lines have such a direction as to indicate that there are belts of relatively similar climatic conditions extending parallel to the Atlantic coast for long distances (see plates 46, 50, 53, 59,65 , and 72). The conditions of the southern part of the Mississippi Valley, which is technically a part of the Coastal Plain, are almost always different from those of the coastal strip of this physiographic province. Numerous plants occur throughout the Coastal Plain from New Jersey or Virginia to Georgia or Mississippi, but fail to range coextensively with it in the southern Mississippi Valley, and terminate their distribution before reaching the mouth of the Rio Grande. Ilex opaca, Pinus toeda, Itea virginica, and Quercus falcata are all examples of this type of distribution. In all of these cases we undoubtedly have to do with three sets of limiting conditions; those operating in the Atlantic coast region, those in the Mississippi Valley, and those determining the extreme southern limit in Texas. In at least the first of these regions we have to reckon with the modifications of climatic conditions which are due to the soil.

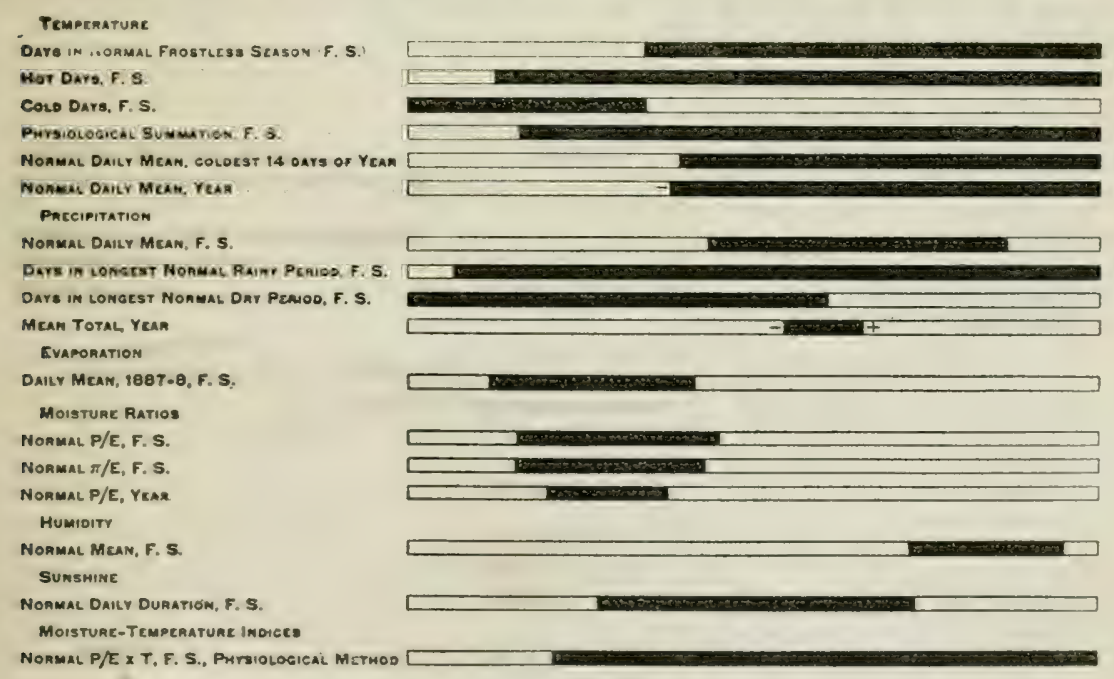

Fig. 55. Climatic extremes for Ilex opaca. 
Magnolia grandiflora (fig. 56).--The distribution of this magnolia covers only the portion of the Coastal Plain between Cape Fear and the Trinity River, and south of the northern boundary of Louisiana. This is a region with no cold days, in our sense, and with high ranges for all of the temperature conditions studied. It is also a region with a high normal daily mean precipitation, although the amplitude of this condition is not great. The amplitude in number of days of both the wet and the dry periods is very wide, and those of evaporation, humidity, and the moisture ratios are narrow.

The range of Magnolia is remarkable from the fact that its limit corresponds with a large number of isoclimatic lines, giving the following indications regarding the conditions which it encounters: a frostless season of 240 days or more, a normal daily mean temperature of $45^{\circ}$ or more for the coldest 14 days of the year, an annual mean temperature of $65^{\circ}$ or more, a mean relative humidity in the frostless season of 65 per cent or more, and a value for the moisture-temperature index of 15,000 or more. None of the isoclimatic lines for moisture conditions coincide, even roughly, with the limit of Magnolia, although the narrow amplitudes of evaporation, moisture ratios, and humidity indicate that this tree encounters only a small part of the total range of these conditions for the United States. The limit of Magnolia corresponds rather closely with the line to the south of which are found 5 or more species of evergreen broad-leaved trees (see plate 3 ), and the climatic characteristics just mentioned may be taken as defining the region which is favorable for the abundant occurrence of trees of this type.

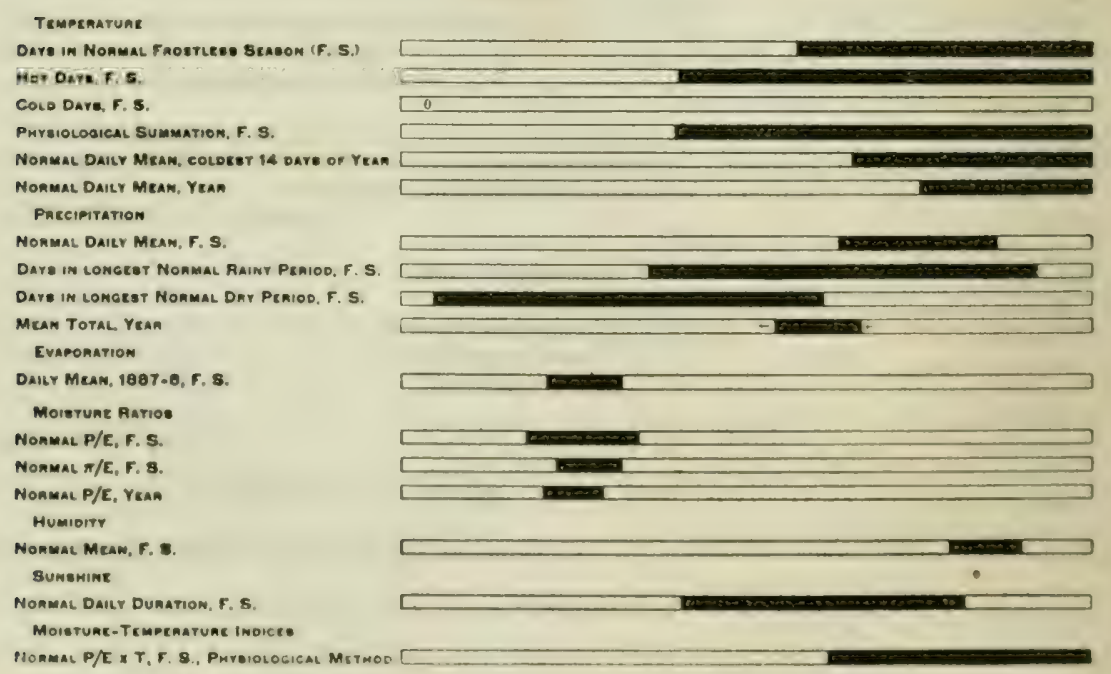

Fig. 56. Climatic extremes for Magnolia grandifora. 
Serenoa serrulata (fig. 57).-Only the extreme edge of the southern Atlantic Coastal Plain is occupied by this palm, from southern South Carolina to the eastern border of Texas. It occupies the warmest and moistest portion of the area which has just been stated to be favorable for the development of evergreen broad-leaved trees, with which it may be classed. Considering the small area occupied by Serenoa, it encounters a wide range of conditions in both temperature and precipitation, together with narrow ranges of evaporation, humidity, and the moisture ratios. It encounters a frostless season of 231 days or more and no cold days, in our sense. Its limit coincides closely with the line of $50^{\circ}$ for the normal mean temperature of the coldest 14 days of the year, although the palm does not follow the region of these temperature conditions into southern Texas. The encountering of the conditions expressed by a moisture ratio of 1.00 appears to be responsible for the westward limitation of a plant which is elsewhere controlled by temperature conditions.

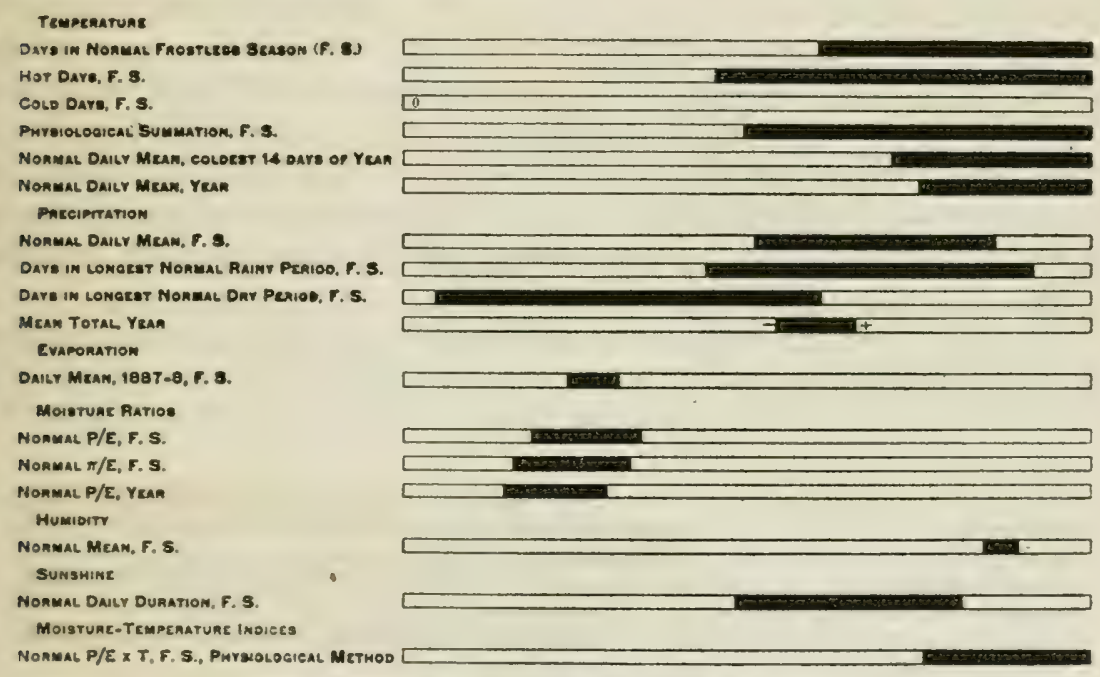

FIG. 57. Climatic extremes for Serenoa serrulata.

Cephalanthus occidentalis (fig. 58). - The distribution of Cephalanthus is remarkable from the fact that it is one of the very few woody perennials of the southern United States which has a nearly transcontinental distribution. It is very infrequent west of the one-hundredth meridian, apparently being absent from New Mexico, but appearing again in southern Arizona and in the San Joaquin Valley of California. A distribution which is so extensive both latitudinally and longitudinally naturally encounters a wide amplitude of conditions, both with respect to temperature and moisture. None of the amplitudes of conditions for Cephalanthus are sufficiently narrow to give any 
suggestion of their importance as limiting the plant. Its distributional edge, extending from central Michigan to the mouth of the Colorado River, is 2,300 miles in length, which in itself suggests that very dissimilar constellations of conditions are involved in its limitation in different sections of this line. In a plant of palustrine habitat it is not surprising to find that the normal moisture conditions of upland habitats have no apparent importance. We find Cephalanthus occurring in localities where the moisture ratios approach their minimum values for the United States, and extending from there halfway through the gamut of values for this compound condition. It also encounters extremely low values for the normal daily precipitation and high values for the number of days in the longest dry periods. These conditions, however, have no apparent influence on the plant in the habitats where it occurs, although they are probably responsible for the fact that there are very few favorable habitats for it in the localities where these extremes are registered. In the San Joaquin Valley the atmospheric conditions are extremely arid, but there are numerous areas of moist soil, and Cephalanthus is there abundant. It is by no means true that all palustrine or swamp plants are able to withstand extremely arid atmospheric conditions if they are supplied with an abundance of soil-moisture, and only a relatively small number of the plants associated with Cephalanthus in the southeastern United States are found growing with it in southern Arizona and the San Joaquin Valley.

It is in the eastern half of its range that Cephalanthus encounters the greatest amplitude of temperature conditions. It is there found in localities with no hot days, as many as 137 cold days, where the

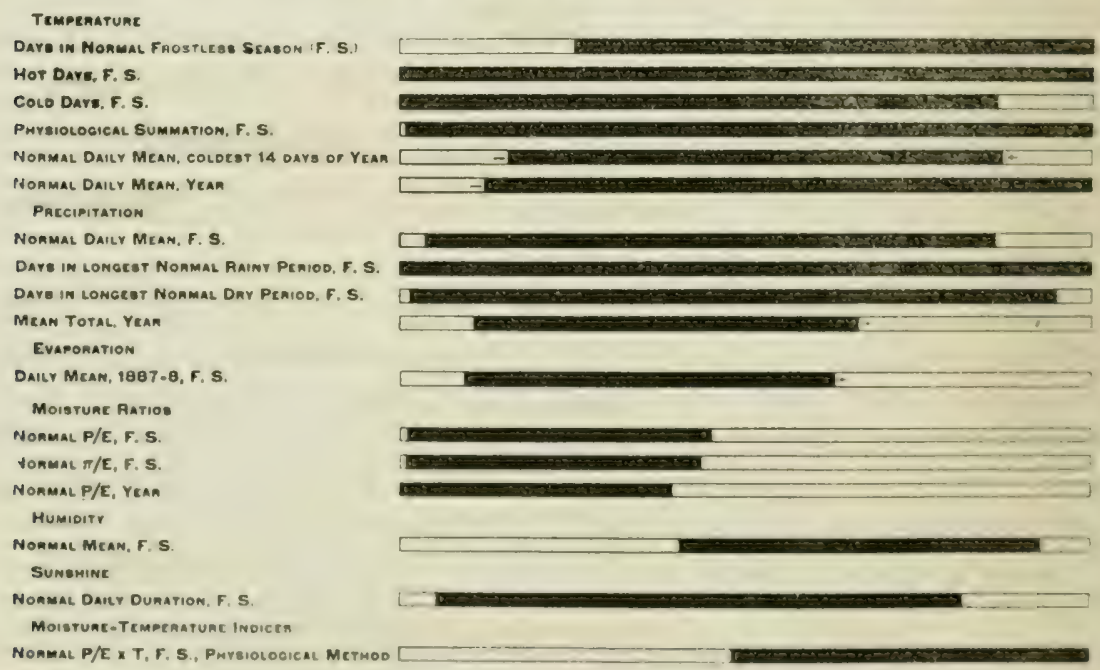

Fig. 58. Climatio extremes for Cephalanthus occidentalis. 
physiological summation is as low as 2,100 , and where the normal daily mean of the coldest 14 days of the year reaches $11^{\circ}$. Some of these conditions may, indeed, be exceeded at the extreme limit of this plant in Canada.

Much more complete information regarding Cephalanthus will be needed before it is possible even so much as to suggest some of the conditions that may be keeping it from spreading into other parts of the United States, if indeed it is not now making secular movements to the west and north. A knowledge of its relative abundance in different parts of its area and of the character of the habitats which it occupies throughout the edge of its distribution might aid in solving the problem which it presents. It seems to be a plant that would be well worthy of a thorough ecological study.

Decodon verticillatus (fig. 59).- - This is an aquatic or palustrine shrub found throughout the States east of Wisconsin, Missouri, and Louisiana, with the exception of southern Florida. The temperature conditions which it encounters are almost as wide in amplitude as those encountered by Cephalanthus. The amplitudes of the moisture conditions are somewhat narrower than in the case of that plant, but the only condition that can be regarded as having a significantly narrow amplitude is the number of days in the longest dry period, which reaches a maximum value of 78 days. The isoclimatic lines for the latter condition indicate that the area of Decodon is roughly limited by the line for 25 days in the longest dry period. Another line approximating the limit of Decodon is that for a mean annual precipitation of 30 inches, the distribution of the plant extending over the region in which the rainfall is greater than that amount.

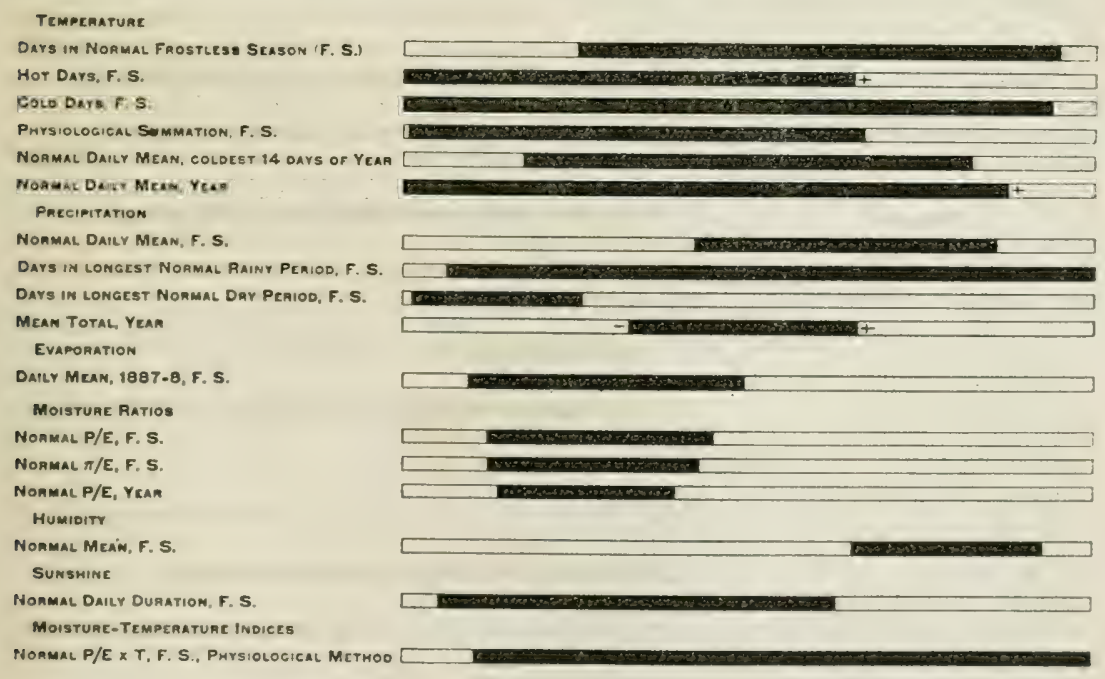

FIg. 59. Climatic extremes for Decodon verticillatus. 
The palustrine and semipalustrine shrub Itea virginica (see plate 23) has a more restricted range than Decodon, very similar in its general outlines to that of Ilex opaca, and apparently limited, like the latter, by high values for temperature and moisture conditions.

Artemisia tridentata (fig. 60).- This plant is the dominant element of the vegetation of the Great Basin, and it extends in diminished abundance eastward to the edge of the Great Plains, upward into the mountains, southward to northern New Mexico and Arizona, and still more sparingly into southern California. The map of its distribution (plate 22) is not drawn to indicate the mountain areas from which it is absent. When these breaks in the distribution are taken into account the shrub is found to occupy an area which is much more homogenous in its climatic conditions than its wide extent would seem to indicate. The amplitude for Artemisia is wide with respect to the number of days in the average frostless season, the number of cold days in the year, the normal daily mean for the coldest 14 days, and the normal daily mean for the year. With respect to the number of hot days and the physiological summation of temperature the amplitudes are narrow, however. The precipitation conditions also exhibit narrow amplitudes, with the exception of the number of days in the longest dry period. The amplitude of evaporation and humidity conditions is made to appear wide because of the extension of its area to the Pacific coast, where the plant is extremely rare, its most westward abundant occurrence being in the Cuyamaca Mountains, 40 miles from the coast. The values for the moisture ratios exhibit narrow amplitudes, reaching, in two cases, the lowest values for the country.

It is manifest that the southern limitation of Artemisia is not solely a matter of its inability to withstand extremely arid conditions, since

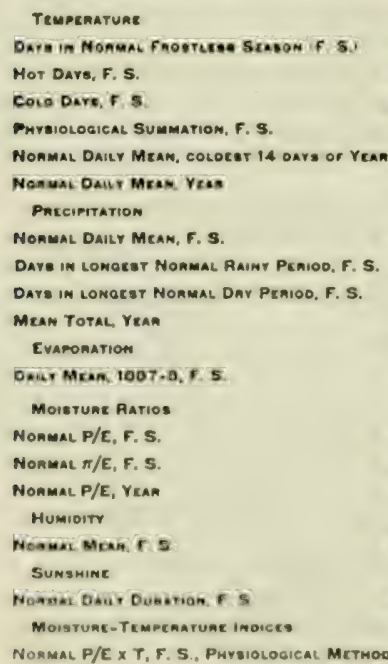

MEan TOTAL YE

Molstune Patios

Nommal P/E, F, S.

Nonual $\pi / E, F, s$.

NOAMaL P/E, YRAR

Humiditr

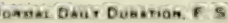

Normal P/E XT, F. S. Physlolocieal Mrtmoo
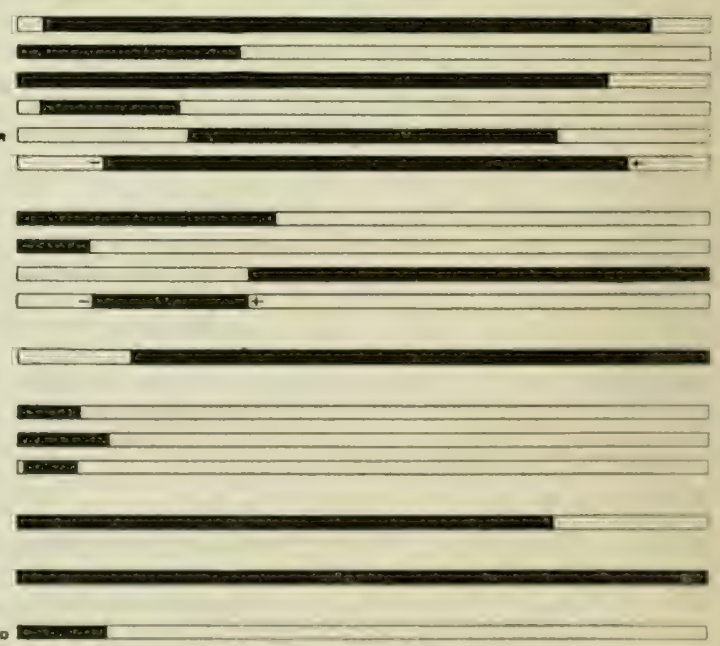

Frg. 60. Climatic extremes for Artemisia tridentata. 
it encounters the lowest normal daily mean precipitation (Reno, Nevada), the highest evaporation (Winnemucca, Nevada), and the lowest moisture ratio (Winnemucca, Nevada). The most trying conditions with respect to these three important moisture conditions are, therefore, not found near the southern edge of the distribution of Artemisia, but well within the region of its greatest abundance. The narrow amplitude of the number of hot days suggests that this may be a condition of importance in limiting this plant at the south, and the maximum of 118 hot days for its area is close to the value of the isoclimatic line of 120 days, which is seen to approximate the distributional limit in Arizona and Nevada. The amplitude of the physiological summation of temperature is also narrow, and the maximum value encountered by Artemisia is 8,400. The evidence would indicate that these and associated temperature conditions are responsible for the southern limit, or else that they cooperate with the low moisture conditions in rendering the deserts along the lower Colorado and Gila Rivers untenable for this plant.

The eastern limit of Artemisia appears to be set by some of the several moisture conditions which present isoclimatic lines closely paralleling its course. The indications of these correlations are that the plant nowhere encounters a mean annual rainfall of more than 20 inches, reaches no areas in which the longest normal rainy period is more than 25 days, nor the moisture ratio more than 0.40 , and that it is accustomed to normal longest dry periods of at least 75 days in length.

It is more than probable that the northern limit of Artemisia is set by conditions similar to those that appear to be responsible for its eastern boundary, with the possible cooperation of low temperature conditions. Low temperatures accompanied by arid atmospheric conditions appear to permit the northward extension of the plant into Canada, but low temperatures accompanied by more humid conditions appear to keep it from the northern Rockies and the Coast Range, as well as from the higher slopes of the Sierra Nevada.

Covillea tridentata (fig. 61).- The range of Covillea extends from southern Nevada and interior California through southern Arizona and New Mexico to the lower part of the valley of the Rio Grande. The area which it occupies in the United States is less than half of its total range in North America, which extends southward through the deserts of central Mexico.

No cold days, in our sense, are encountered by Covillea, and the amplitude of the normal daily mean temperature of its area is relatively wide. In other respects the temperature conditions present narrow amplitudes, especially when compared with those for Artemisia. The data for moisture conditions reveal the extremely arid conditions under which Covillea exists, showing that it also extends into regions 
with much more favorable conditions, particularly with respect to the longest dry periods, daily mean evaporation, and normal humidity. The distributional area of this plant contains few climatological stations, and it is certain that the conditions actually met by it include the lowest values of normal daily mean precipitation, the longest normal dry periods, and the lowest mean total precipitation o the year. In the vicinity of Death Valley and in the arm of the Mojave Desert which stretches southeast toward the Colorado River are to be encountered the most arid areas in the United States, and in them Covillea is one of the most ubiquitous plants.

The narrow amplitude of the moisture ratios indicates here, as in the case of Artemisia, that conditions of greater general favorableness with respect to this condition are either directly inimical to Covillea, or else that they are accompanied by associated conditions which are of importance in limiting its range. The extremely narrow amplitude in number of days in the longest rainy period signifies that there is a great importance in this factor, probably having to do with the effect of prolonged wet periods in making the conditions of soil aeration injurious to Covillea. The distributional boundary lies close to the northern limit of the area in which there are no cold days, and this condition, with its associated conditions of low winter temperatures, is undoubtedly of great importance in controlling the northward limitation of the plant. Covillea appears, in brief, to be confined to a region in which there are no prolonged periods of rain and no severe periods of cold. The narrow amplitude of the physiological summation of temperatures for the frostless season indicates importance for this condition, but the fact that Covillea is an evergreen points to the low temperatures of winter having a greater significance in its limitation than do the summations for the growing season.

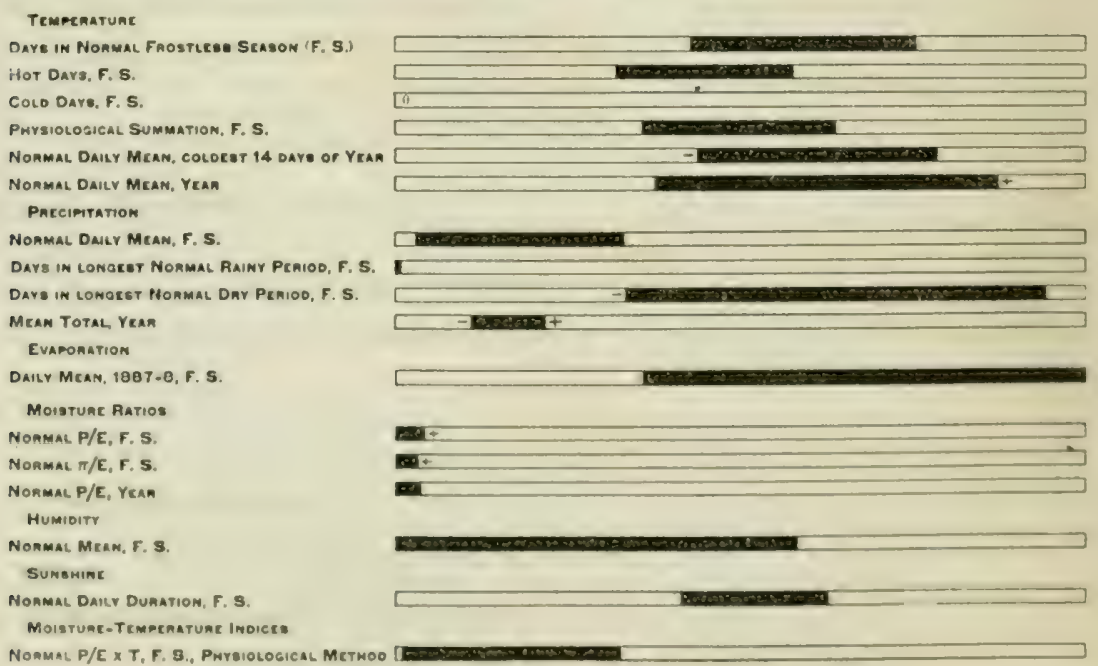

Fro. 61. Climatic extremes for Covillen tridentata. 
Silphium laciniatum (fig. 62).-The range of this plant extends from Texas to South Dakota and from Alabama to Pennsylvania, but it is mostabundant in the Grassland Deciduous-Forest Transition and in the Grassland. The distributional area is such that the plant encounters only 140 cold days in the frostless season. The amplitudes are relatively wide in all of the temperature conditions and are also wide in all of the moisture conditions except the number of days in the longest normal dry period. The moisture ratios are also relatively narrow in amplitude, the extremes for $\pi / E$ in the frostless season being, minimum 0.47 , maximum 1.32 .

The eastern limit of Silphium is very roughly approximated by the isoclimatic line of 0.80 for the moisture ratio $\pi / E$, isotherms of $45^{\circ}$ and $50^{\circ}$ for the annual daily mean temperature, and is closely followed by the isotherm for a physiological summation of $7,500^{\circ}$. The western limit is closely approximated, at least in part, by the isoclimatic lines of 20 inches annual mean total precipitation, 75 days in the longest normal dry period, 25 days in the longest normal rainy period, and a moisture ratio of 0.40 . Although the extreme values of the moisture ratio for Silphium are 0.47 and 1.32, nevertheless the location of its entire range with respect to the isoclimatic lines for this condition indicates that the plant is found mainly where the moisture ratio is between 0.40 and 0.80 . The northward extension in the area presenting this range of conditions is apparently controlled by temperature conditions, among which the physiological summation is most important.

The central location of the range of Silphium laciniatum gives it a distributional edge about 3,300 miles long, with only its southern limitation formed by the ocean. These circumstances make it a par-

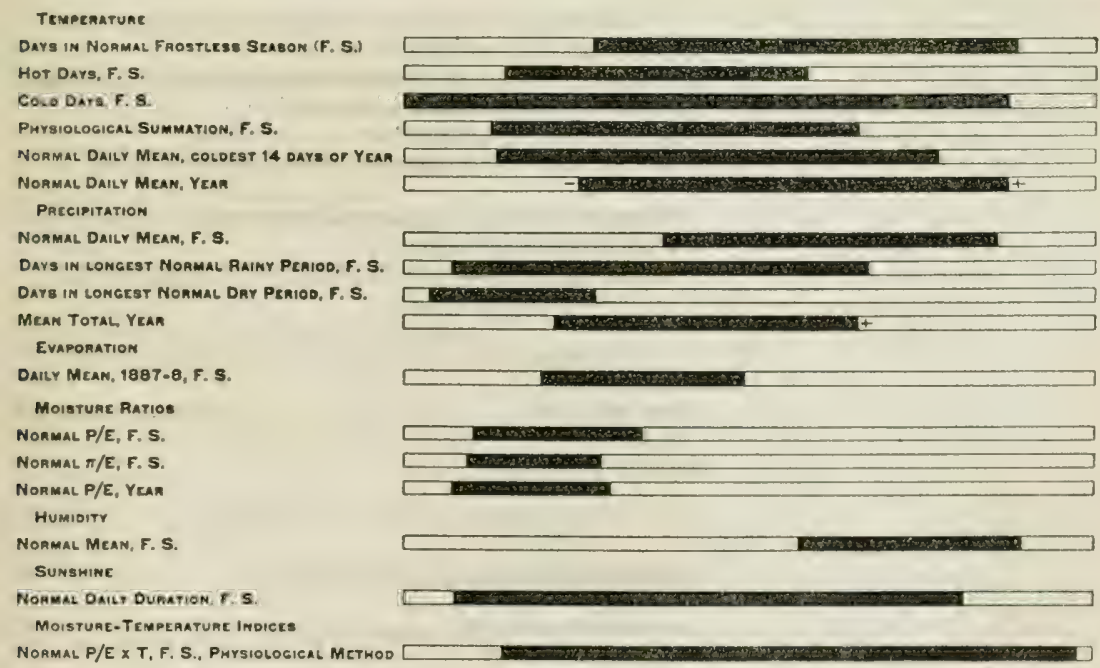

FIG. 62. Climatic extremes for Silphium laciniatum. 
ticularly favorable subject for fuller investigation. Its ability to withstand a considerable range of temperature conditions between Mississippi and South Dakota and its ability to range through dissimilar moisture conditions from Ohio to Texas should be investigated in terms of the habitat requirements of the plant in the various portions of its range. From such information as is available, this plant seems to be confined to the most arid situations on the eastern edge of its area and to relatively moist situations or seasons on the western edge, so that it would be particularly valuable to have parallel series of data for the local conditions met by the most widely separated colonies.

Bouteloua oligostachya (fig. 63).-This abundant and characteristic grass of the Great Plains is found throughout the Grassland, in the western part of the Grassland Deciduous-Forest Transition, and in the southeastern part of the Desert. It is most abundant in the Grassland and the Desert-Grassland Transition, becoming infrequent at the southeastern and southwestern corners of its area.

The temperature conditions encountered by Bouteloua are those of the Grassland, but with slightly higher maxima in each case (except number of cold days). The amplitude of the precipitation conditions is much greater for Bouteloua than for the Grassland, the maxima being higher and the minima lower. With respect to the length of the longest normal dry period particularly, Bouteloua exhibits its ability to range from the conditions of the Grassland far into those of the Desert, enduring 283 days in the vicinity of Phoenix, Arizona. Like many other perennial grasses, it is able to withstand prolonged and severe conditions of drought in its resting condition and to take advantage of moist periods of relative infrequency.

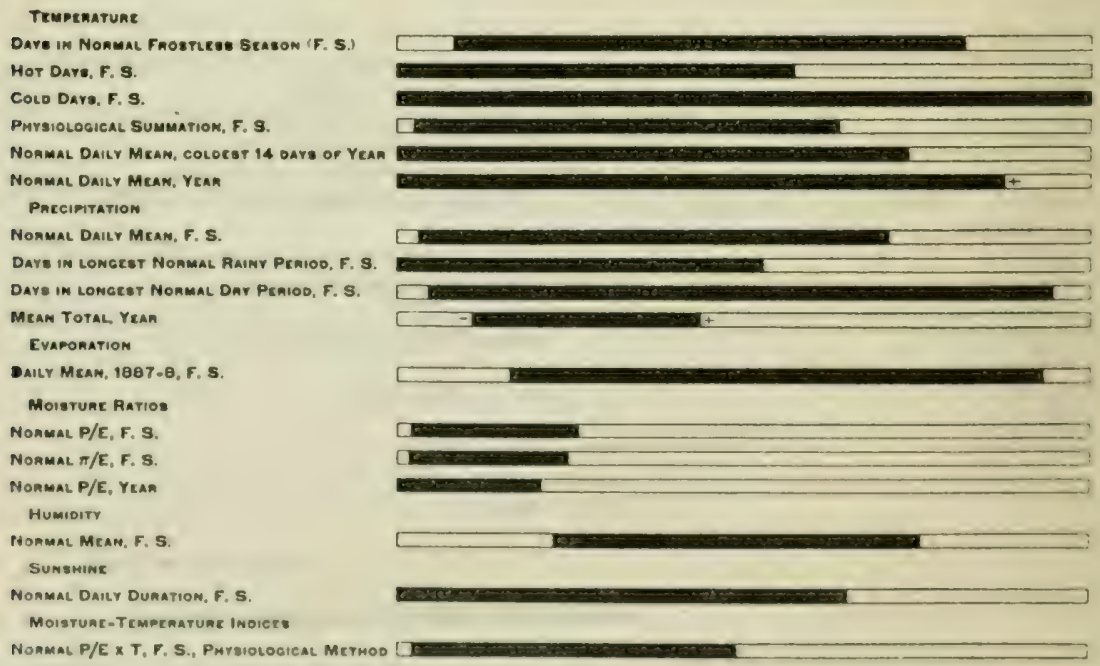

Fra. 63. Climatio ertremes for Bouteloun oligostachya. 
The minimum conditions of evaporation and the maximum conditions of humidity are very similar for Bouteloua and the Grassland region, indicating that conditions which determine the eastern limit of the Grassland also limit one of its most characteristic plants, and this is also true of Bulbilis dactyloides and Bouteloua hirsuta. The maximum conditions of evaporation and the minimum conditions of humidity, however, are respectively higher and lower for Bouteloua than for the Grassland. The moisture ratios for the frostless season are very similar for this plant and for the Grassland as a whole, although the minimum values for Bouteloua are lower.

The eastern limit of Bouteloua, like that of many of the grasses associated with it, is apparently set by some one of the moisture conditions, or by a combined operation of several of them. Neither the area of Grassland nor that of Bouteloua extends very far into the region with more than a daily mean precipitation of 0.100 inch with more than 75 days in the longest rainy period, or with a mean annual precipitation of more than 25 inches. The position of the western boundary of Bouteloua indicates that it is there again limited by moisture conditions. Although we have presented no data bearing directly on the seasonal distribution of precipitation, it is apparent that this grass is unable to penetrate far into the portion of the Desert, in which the summer rainfall is light. The ability to withstand dry periods of as much as 283 days has enabled it to range as far as the area of uncertain summer rains in the lower Colorado Valley. It is not able, however, to extend its area into the region in which there is frequently no summer rain for many successive years.

Agropyron spicatum (fig. 64).--This grass is found throughout the Grassland north of Oklahoma and New Mexico and in the outlying portions of that vegetation which fringe the northern edge of the Great Basin Microphyll Desert. Although withstanding the entire amplitude of cold days this grass encounters lower and narrower amplitudes of the other temperature conditions. It has a relatively low maximum (125) for the number of hot days and a low maximum $(11,600)$ for the physiological temperature summation. With respect to precipitation it shows wider amplitudes than those of the Grassland, and this is true of evaporation and the moisture ratios for the frostless season.

Agropyron appears to have its eastern limit set by the same constellation of moisture conditions that controls the Grassland and other grasses, but it does not follow the conditions favoring Grassland as far as the southern limit of that vegetation. Nowhere does it encounter more than 100 to 120 hot days, nor does it range into regions with a physiological summation of temperature greater than 12,500. These and associated temperature conditions appear to be responsible for its southern limitation. 
The failure of Agropyron to extend farther into the northern part of the Great Basin Desert is apparently due to the moisture conditions of that region. A more precise correlation of its distribution with that of the moisture ratio $(\pi / E)$ would probably show that it does not occur where the values of this ratio are lower than 0.20 , the same value that limits it in Arizona and New Mexico. The actual moisture-ratio conditions of the areas occupied by Agropyron in Utah and northern Nevada are poorly exhibited by our data, which are from stations located in the valleys.

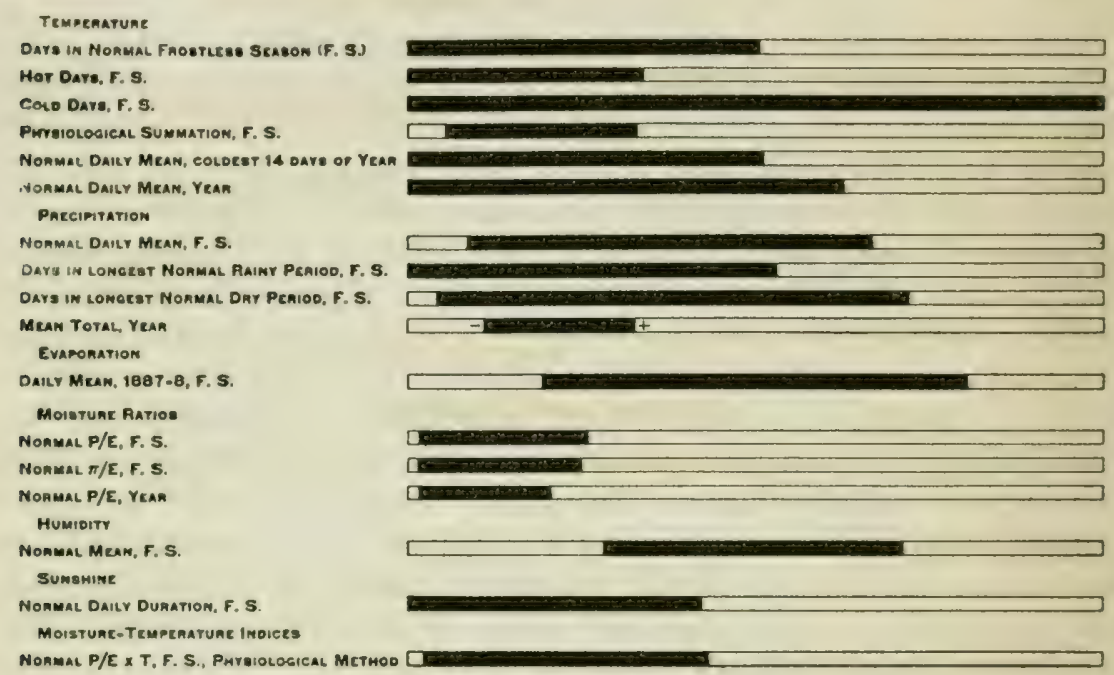

FIG. 64. Climatic extremes for Agropyron spicatum.

Hilaria jamesii (fig. 65).-This is a characteristic grass of the DesertGrassland Transition region, ranging from western Texas to southern Nevada and northward to extreme southwestern Wyoming. All of the climatic conditions exhibit narrower amplitudes for Hilaria than for the Grassland, with the exception of the number of days in the longest normal dry period. The narrowest amplitude among the temperature conditions is that of the normal daily mean for the coldest 14 days of the year, which ranges from $27^{\circ}$ to $45^{\circ}$. At few points does the limit of Irilaria extend north of the isoclimatic line for $25^{\circ}$ as the daily mean of the coldest fortnight, and the correspondence of these lines would indicate that this condition is an important one in limiting the northward distribution of the grass.

The eastward extension of Iilaria in Texas is such that it nowhere encounters moisture ratios $(\pi / E)$ higher than 0.40 , nor rainy periods of more than 25 days. These are the same conditions that appear to limit the eastward range of other grasses, but different intensities are involved in the case of IItaria from those mentioned in connection with Boutelour. 
With respect to the westward range of Hilaria, it appears that the same limiting conditions are operative that have been mentioned in connection with Bouteloua oligostachya. Extremely long normal dry periods, in excess of the 250-day maximum for Hilaria, are inimical to it as to all other perennial grasses.

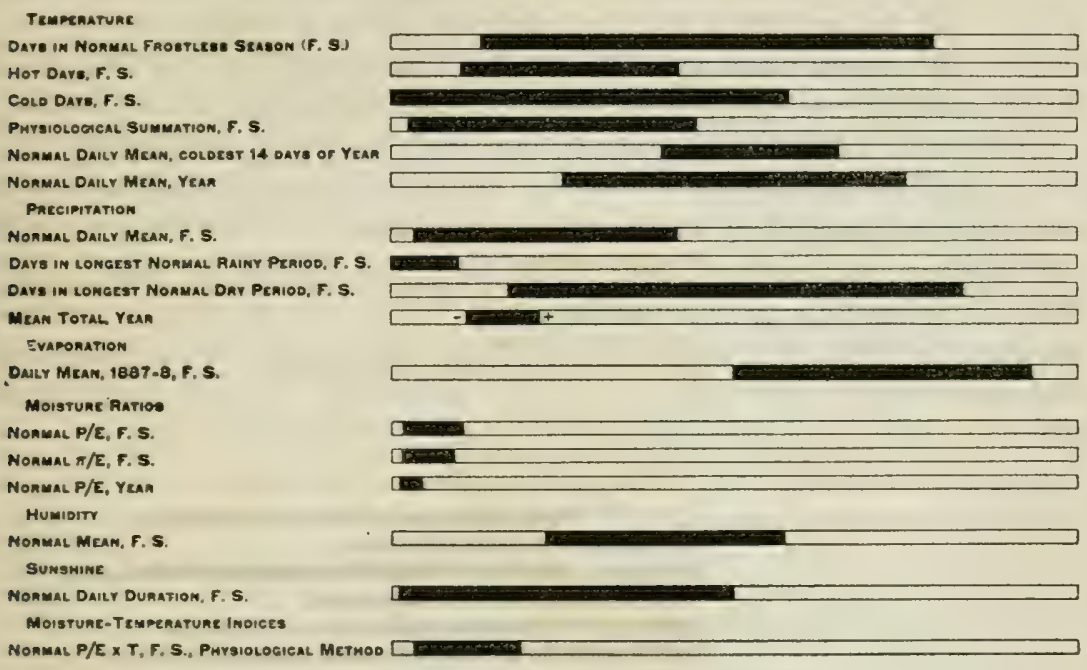

FIG. 65. Climatic extremes for Hilaria jamesii.

Sparganium americanum (fig. 66).-Sparganium is widely distributed throughout the eastern United States, except in peninsular Florida, and from its westward extension in Canada it reappears within our limits in Washington. A considerable number of northern plants exhibit distributions of this type, Dulichium arundinaceum being another example (see plate 27), with a range closely like that of Sparganium. The range of another palustrine plant, Sium cicutoefolium, is of an analogous character (see plate 28), its limits being far enough south, however, for it to present a continuous area within the United States. The distribution of each of these palustrine plants is such that they are absent from the arid and semiarid regions, where they might find localities with suitable soil-moisture conditions, although much more widely separated than in the moist regions. The western edges of the ranges of Sparganium and Dulichium in the Eastern States run parallel to the isoclimatic lines for moisture conditions. Although these are plants of wet habitats, there is here a suggestion of their inability to extend into the regions with very high evaporation. In other words, the conditions expressed by the moisture ratio are of importance to them even when the numerator of the ratio is constant and of high value. Their ability to withstand lower moisture ratios in eastern Washington than they endure along their western edge in the Central States is doubtless due to the interaction of temperature conditions. 
Wide ranges are exhibited by Sparganium for both temperature and moisture conditions, due to its extended north-and-south range in the Eastern States, and to its occurrence from the Atlantic coast to the arid interior of Washington. Its climatic extremes are of interest in comparison with those of Sium, which shows the broadest amplitudes of any of the plants that we have selected for investigation. The distribution of Sium indicates that it is able to withstand the entire gamut of temperature conditions for the United States, excepting those encountered in peninsular Florida, and that it is excluded only by the lowest conditions expressed by the moisture ratio ( 0.40 or lower). Sium, Dulichium, and Sparganium are apparently alike in being unable to withstand the highest intensities of evaporation, in spite of the saturated substrata in which they are invariably found, apparently belonging to that already well-known group of plants in which the transfer of water from absorbing to transpiring organs is internally limited.

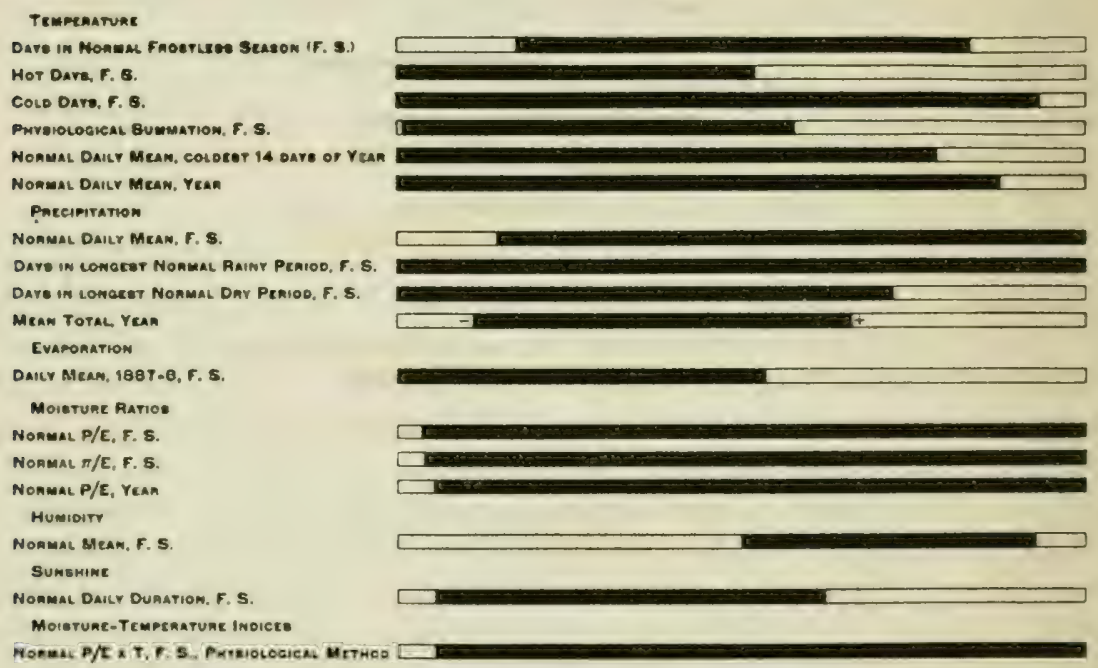

Fig. 66. Climatic extremes for Sparganium americanum.

Arceuthobium americanum (fig. 67). - This mistletoe is found throughout the Rocky Mountains and the mountains of the Great Basin and its western edge. Its actual occurrence is limited to the forested portions of the area indicated for it in plate 29 (see plate 1). A number of the climatological stations located within the area credited to it are not in situations actually occupied by Arceuthotrum, and consequently some of the moisture conditions, in particular, are higher than indicated in figure 67 .

Arceuthobium americamum is chiefly confined to Pinus contortn (including $P$. murrayana) as a host, but appears to be absent from it. in Washington, Oregon, and California east of the Cascade Mountains. 
The area of Arceuthobium is therefore closely similar to that of Pinus contorta, with the exception of its absence from the northern Pacific coast. The area of the mistletoe (plate 29) has been drawn in a more generalized manner than has that of Pinus contorta (plate 16), because less information is available regarding the occurrence of the former.

The climatic extremes for Arceuthobium are very similar to those for Pinus contorta, except with respect to the high conditions of precipitation, moisture ratios, and humidity encountered by the host in the Pacific-coast part of its range. This is a case in which a sap parasite is not able to accompany its host throughout the entire range of the latter, apparently as a result of the operation of limiting climatic conditions upon the parasite. The well-known xerophytic character of the mistletoes apparently brings their distributional behavior into accordance with that of other xerophytic plants in their inability to invade regions of high moisture conditions. The only mistletoe that is found in Washington west of the Cascade Mountains is a locally abundant form of Arceuthobium douglasii growing on Tsuga heterophylla.

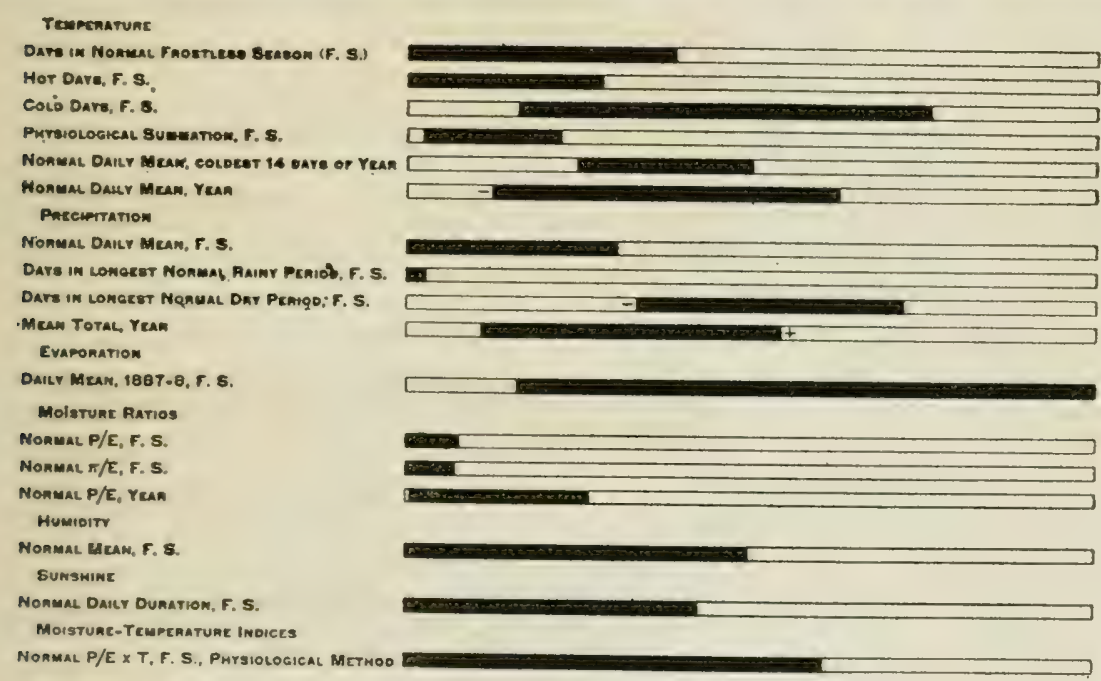

Fig. 67. Climatic extremes for Arceuthobium americanum

Phoradendron flavescens (fig. 68).-A remarkably wide range is exhibited by this plant and its varieties, extending from coastal Oregon, through California and the extreme southwest, to Texas, Florida, Indiana, and New Jersey. The species itself is found from New Jersey to Louisiana, variety orbiculatum in Arkansas, Oklahoma, and Texas, variety pubescens in Texas, variety macrophyllum in Arizona, and variety villosum in California and Oregon.

Both the species and the varieties of this mistletoe are found on at number of different host trees, so there is no such restriction of its range as that shown for Arceuthobium americanum. Little is known 
regarding the water-supply of sap parasites and the relation between the seasonal conditions of the host and the maintenance of the transpiration-stream in the parasite. It is safe to assume, however, that the water-supply for mistletoe is not subject to as sharp nor as pronounced fluctuations as that of most autonomous plants rooted in the soil. The influence of precipitation and soil-moisture conditions is exerted very indirectly on the mistletoes, and we may regard them, for the purposes of our investigation, as somewhat analogous to palustrine plants.

Phoradendron encounters wide ranges of all temperature conditions, except in regard to the number of cold days. The wide range of moisture conditions which it meets is to be anticipated from its independence of these conditions as they affect autonomous plants. The extremely wide amplitudes of evaporation conditions through which it ranges apparently indicate that it is able to secure supplies of water sufficient for the maintenance of high rates of transpiration. In the most arid parts of its range, however, Phoradendron flavescens var. macrophyllum is found only on trees that occur in relatively moist situations, and not on the small microphyllous trees, in which the maintenance of the transpiration-stream is precarious.

The temperature condition which appears to be most potent in limiting the range of Phoradendron is the number of cold days. A very small part of its area lies inside the region in which cold days are encountered, and in this part (the Ohio Valley) it reaches the maximum of 44 days for this condition.

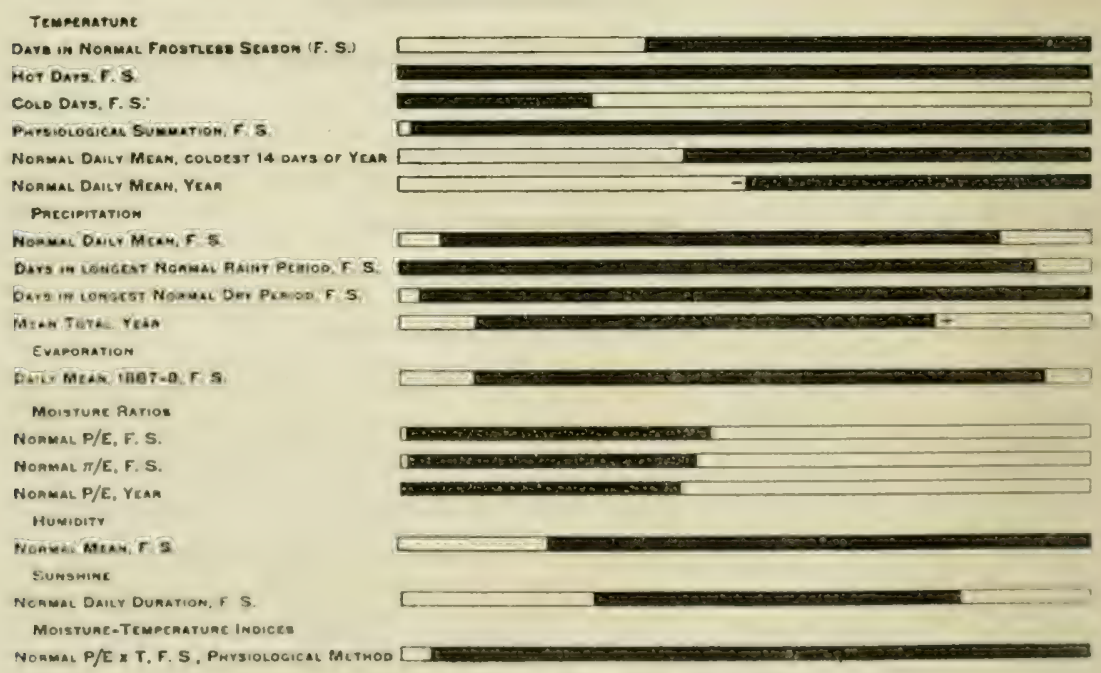

FIfi. 6S. C'limatic extremes for Phoradendron flavescens.

Daucus pusillus (fig. 69). - This annual herbaceous plant ranges from the coastal region of Washington and Oregon through California 
and the extreme Southwest to Oklahoma, Mississippi, Florida, and North Carolina (see plate 31 ).

The relation of Daucus to the climatic conditions of its wide range requires interpretation in terms of its seasonal behavior in different sections of the range. In the States east of Texas it is an early summer plant, reaching maturity in July or later; in the Desert region it is an early spring plant, reaching maturity in March or April; on the Pacific coast it is a late spring or early summer plant, reaching maturity from May (in southern California) to July (in Washington). In order to evaluate properly the conditions under which it actually lives in these sections of its range, we should take into account only those climatological values in each section that refer to the period of its activity. A separate investigation of Daucus and other plants of the same facultative seasonal habits would yield results of great value. The wide amplitudes of moisture conditions shown in figure 69 would thus doubtless be greatly narrowed and the temperature amplitudes would be made somewhat narrower also. Whereas this plant appears at first to encounter a remarkable gamut of conditions through its range, nearly 4,000 miles in length, a study of the conditions in its particular seasons and in the habitats which it occupies would undoubtedly show that it grows only under a relatively limited set of conditions.

The northern (and eastern) limit of Daucus follows certain of the isothermal lines so closely as to indicate that its controlling conditions are to be looked for in the temperature series. The danger of attempting a final explanation of distributional limits by correlational methods alone is shown very clearly in the case of this plant. The distributional limit is closely parallel to the line for a length of growing-season of 240 days, but the length of this season is obviously of no direct importance

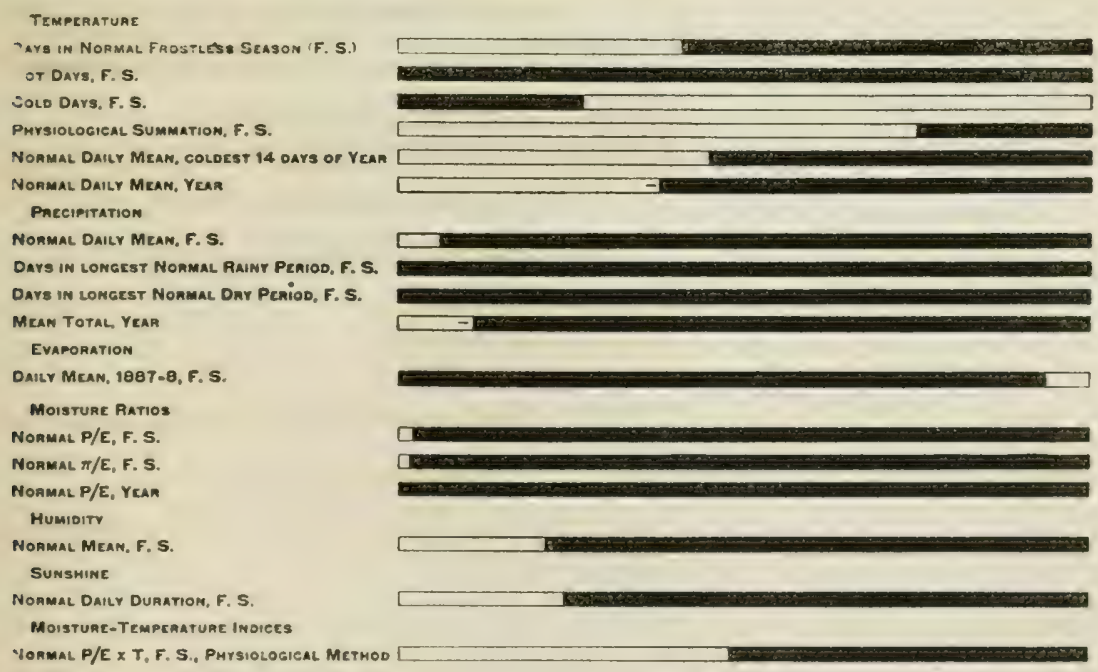

Frg. 69. Climatic extremes for Daucus pusillus. 
to an annual plant which uses only a portion of the season in any part of its range. There is also a close correspondence between the distributional limit of Daucus and the line for a daily mean temperature of $40^{\circ}$ for the coldest 14 days of the year, but here again there is obviously no significance for this plant in the temperatures that supervene while the entire race is represented only by seeds. There is nevertheless a parallelism between the different phases of any climatic condition, as has been remarked before, and this may well mean that there are other indices of temperature conditions critical for Daucus which have not been elaborated in our work, and the isotherms for these may be parallel to the ones mentioned above. It may also be true that there is an unsuspected importance for this plant in the length of the total frostless season and in the low temperatures of winter.

Spermolepis echinatus and Parietaria debilis have ranges which are similar to that of Daucus, although not so extended either to the northwest or the east. These plants are also annuals which have different seasonal habits in the different sections of their areas, and the remarks made about Daucus will apply also to their relation to climatic conditions.

Oxybaphus floribundus (fig. 70).-This herbaceous root-perennial ranges throughout the central United States between the Deciduous Forest region and the Rocky Mountains and north of central Texas. It encounters the entire amplitude for the country, of cold days, and relatively wide amplitudes of the other conditions, both of temperature and moisture. The narrowest amplitude is that of the moisture ratio $(\pi / E)$, which ranges from 0.25 to 0.89 .

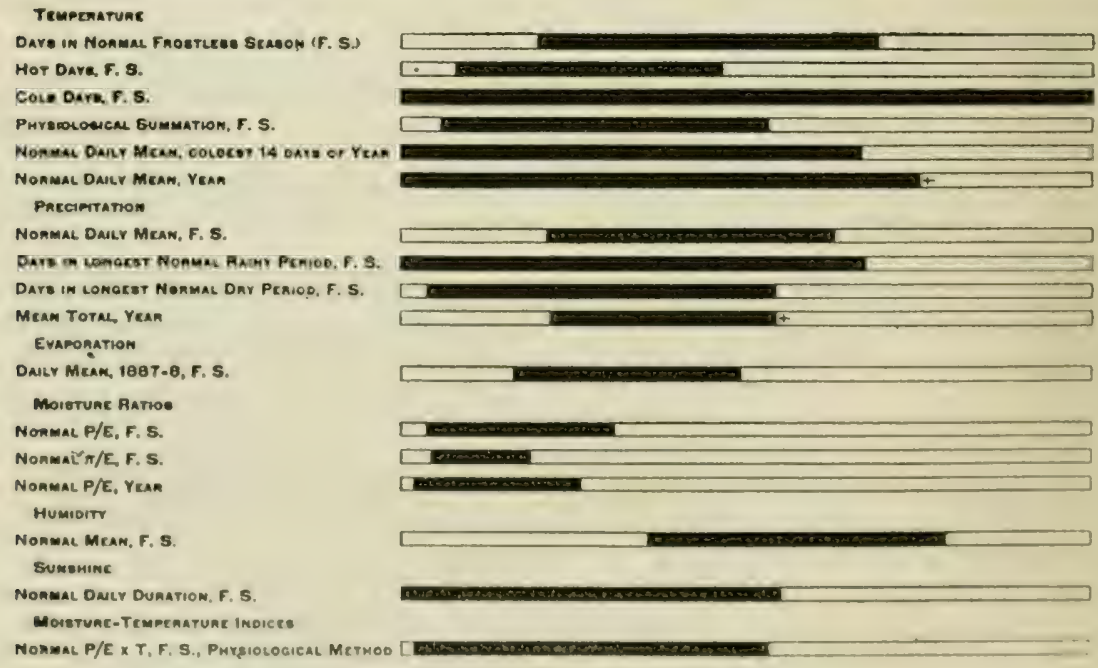

Fo. 70. Climatic extremes for Oxylnphus floribundue. 
The area of Oxybaphus is somewhat similar to that of Silphium laciniatum with respect to eastern and western limits, but the latter plant is definitely limited at the north, failing to reach the Canadian boundary, while Oxybaphus is limited at the south, failing to reach the Gulf coast. The eastern and western limits of Oxybaphus, like those of Silphium, appear to be set by definite constellations of moisture conditions, while the southern limit must apparently be sought in the temperature conditions.

The western edge of the distribution of Oxybaphus is set by the lines for dry periods of 75 to 125 days, according to the latitude, and nowhere does this plant enter regions with a moisture ratio of less than 0.20 . Its eastern limit is like that of Solidago missouriensis (see plate 25), in being located along the western boundary of the Deciduous Forest more nearly than along any of the climatic lines. The actual ecological conditions for these plants of the open prairies are more radically changed on passing from the Grassland Deciduous-Forest region into the Deciduous Forest than the data of ordinary climatological stations are capable of showing. A normal mean relative humidity above 70 per cent and rainy periods of more than 100 to 125 days doubtless serve as limiting intensities of important conditions for these plants in a much more precise manner than is indicated by the positions of the isoclimatic lines as shown on our charts.

At its southern edge Oxybaphus does not enter the regions with more than 200 to 240 days in the frostless season, does not encounter more than 180 hot days, and grows at no place with a physiological summation of more than 17,500 .

Trautvetteria grandis (fig. 71) and Trautvetteria carolinensis (fig. 72).These closely related species are the only North American representa-

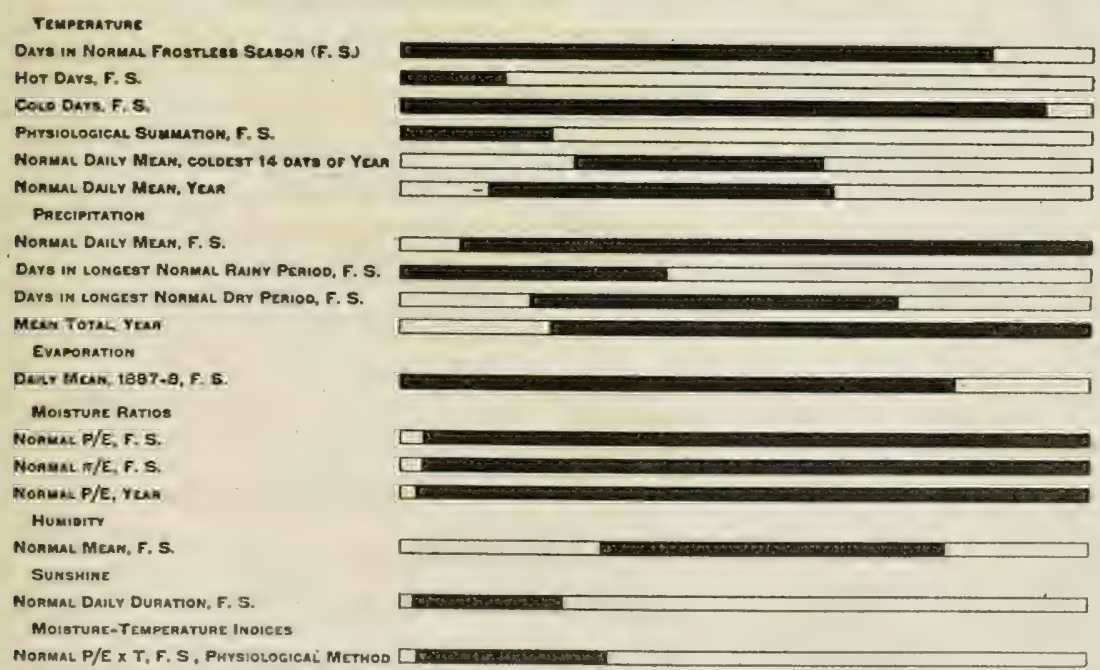

FIG. 71. Climatic extremes for Trautvetteria grandis. 
tives of a genus of Ranunculace which is found elsewhere only in eastern Asia. They are herbaceous root-perennials of moist situations, and neither of them is common nor characteristic of very extensive plant communities. The ranges of both the eastern and western species are based on a very small number of known localities of occurrence, and are so broadly drawn that they comprise climatological stations near which the plants are doubtless absent. Here, as in many other cases, a much more detailed study should be given to the distribution of the plants involved and to the nature of the climatic conditions before attempting to apply the methods of a general study of this character to an investigation of the climatic controls involved. It is, nevertheless, of interest to make even a broad comparison between the conditions under which these related but geographically segregated species exist.

The amplitude of the climatic conditions for the area of Trautvetteria grandis is wider than for that of $T$. carolinensis in all cases except the number of hot days, physiological summation, and the number of days in the longest normal rainy period. The former species exhibits a very narrow range in number of hot days, from 0 on the coast of Washington to 57 at Sante Fe, New Mexico (near one of the southernmost stations for the plant in the Sante Fe Mountains). This condition appears to be an important one in determining the limits of the western species. Trautvetteria carolinensis encounters from 63 to 160 hot days, and also has a relatively narrow amplitude of conditions with respect to the length of the frostless season, 145 to 231 days.

The amplitudes of the daily mean precipitation and of the mean annual precipitation are much narrower for Trautvetteria carolinensis

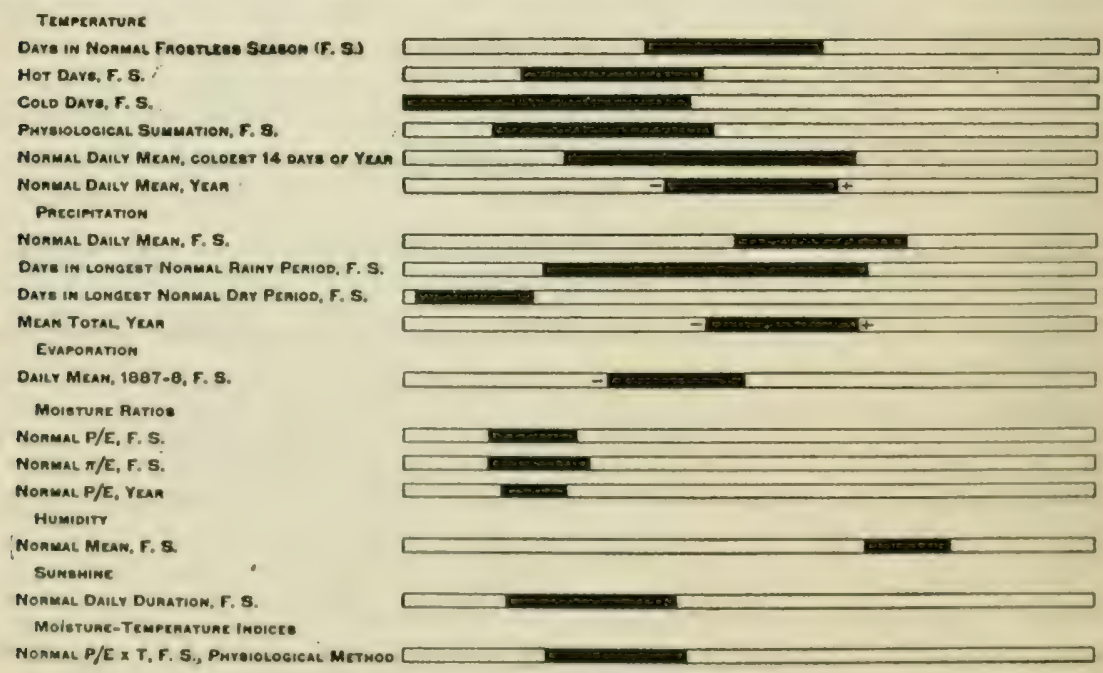

Fra. 72. Climatic extremes for Trautvetterin carolinensis. 
than for the western species, but are comprised in the wide amplitudes of the latter. The amplitudes of the rainy periods and the dry periods, on the other hand, are such as to indicate that the two species have almost nothing in common with respect to these conditions.

The occurrence of Trautvetteria grandis over both interior and coastal regions in the Northwest is responsible for wide amplitudes of evaporation, humidity, and moisture ratios, for all of which the amplitudes are narrow for the eastern species. Climatological data from such localities as Helena, Boise, Walla Walla, and Spokane are not suited, however, to giving an accurate conception of the conditions for this plant. The moisture conditions of those stations are doubtless much more severe than those of the southernmost mountain localities for Trautvelteria grandis in New Mexico. Even though our graphs may indicate conditions of evaporation that are too high for Trautvetteria grandis and conditions of humidity and moisture ratio that are too low, there remains, nevertheless, a marked difference between the amplitudes of these conditions for the eastern and western species, since the low range of evaporation and the high range of humidity and moisture ratios are the conditions in which the western species is most abundant. In this case our climatological stations are located in the midst of the conditions in which it actually grows.

The differences in the extremes and amplitudes of the principal climatic conditions for the two species of Trautvetteria are sufficiently great to indicate that it would be difficult to grow either of them throughout the range of the other. The distinctive conditions under which the two species now grow, together with their complete geographical segregation, must be taken to mean that they are neither recent nor immediate derivatives from a common ancestral stock.

Populus balsamifera and Sapindus marginatus (fig. 73). - A ready comparison of climatic extremes for these two trees has been made possible by placing the blocks for the two on the same diagram. Sapindus is a tree of southern range, extending from central Texas and southern Kansas to Florida. Populus is a tree of northern range, extending from Connecticut to North Dakota, and through Canada to the northern Rocky Mountains, where it occurs in a form which has been recently regarded as a distinct species (see plate 19).

Since the distributional areas of these two trees are quite separate and yet are nowhere less than 400 miles apart, it is not surprising that the ranges of temperature conditions are so unlike as to overlap only in the case of the length of the frostless season, where the maximum for Populus is slightly in excess of the minimum for Sapindus. The precipitation conditions for these two trees are such that there is a considerable range of precipitation values common to both of them, although the extremes are by no means the same. The amplitudes of evaporation and humidity are much greater for Populus than for 
Sapindus, but the values of all three of the moisture ratios are nearly the same. The amplitudes of sunshine duration do not overlap at all, and those of the moisture-temperature index are very dissimilar.

The southern limit of Populus balsamifera and the northern limit of Sapindus marginatus have such a direction as to indicate that both trees are controlled by temperature. The edge of the area of the former corresponds closely to the line for 30 hot days in the normal year, although the tree extends far enough south to encounter 88 days at the edge of its range, at Toledo, Ohio. The area of Sapindus is limited in central Kansas by an average frostless season of slightly less than 180 days, by slightly more than 60 cold days, and by a physiological summation of 10,000 . The position of the northern limit of Sapindus is so placed, however, as to indicate very clearly that its range is determined by the interaction of temperature and moisture conditions in such a manner as to require a detailed investigation based on a more accurate knowledge of the distribution of the tree than is yet available. The arid conditions of the Grassland and Desert regions apparently limit the western extension of both Populus and Sapindus.

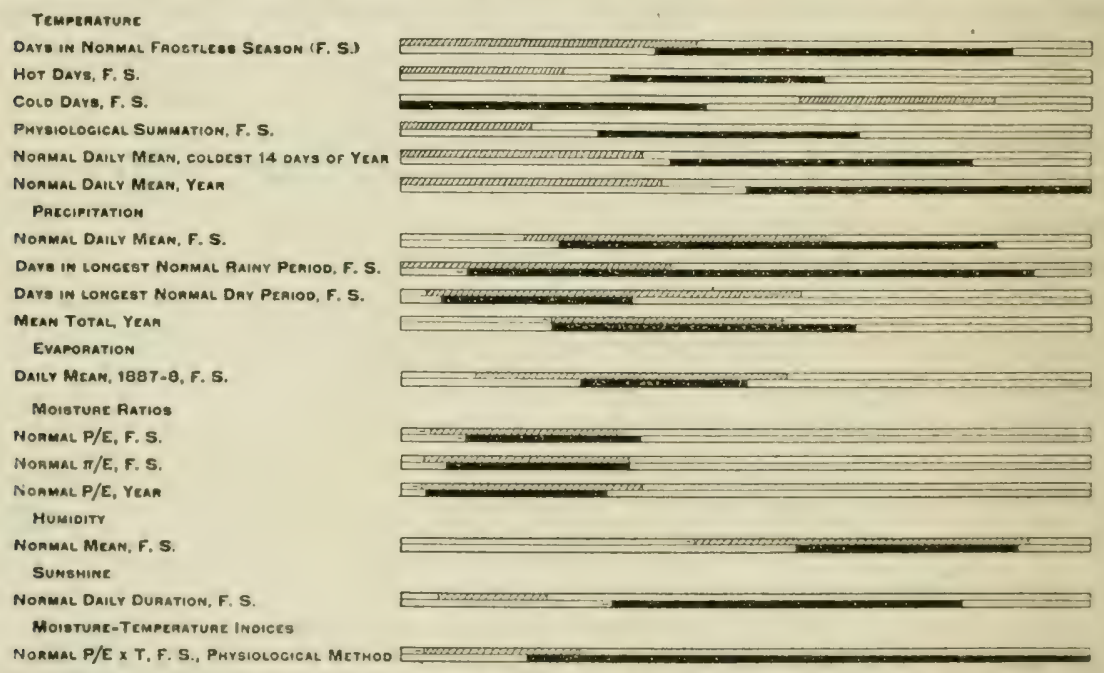

I'Ici. 73. Climatic extremes for Populus balsamifera (shaded) and Sapindus marginatus (black)

Cornus canadensis and Spermolepis echinatus (fig. 74).-These plants are examples of species with northern and southern transcontinental distributions, respectively, and their climatic extremes have been placed together in the same diagram for comparison. Cornus ranges from northern California and the Sierra Nevada through the Rocky Mountains and the Black Hills to the extreme Northeastern States, heing nearly coextensive with the Northern Mesophytic Evergreen Forest (plate 30). Spermolepis ranges from central California through southern Arizona and western Texas to Arkansas, western Tennessee, and western Florida (plate 31). 
Wide amplitudes are exhibited by both of these plants for a number of the temperature conditions. Cornus shows a narrow amplitude for the number of hot days and the physiological temperature summation, and Spermolepis shows narrow ones for the number of cold days and for the annual daily mean temperature. The region with less than 60 hot days coincides roughly with the area of Cornus, except in the Great Basin region, where the plant is absent. The isotherm for a physiological summation of 5,000 corresponds in a striking way with the limit of Cornus, from the Pacific to the Atlantic. Spermolepis nowhere encounters a daily mean temperature of less than $55^{\circ}$, and barely enters the region with cold days. These temperature conditions are manifestly the strongest determinants operating to limit the ranges of these two plants.

Both of the plants under comparison exhibit wide ranges for all of the moisture conditions, and in most cases their amplitudes overlap to a considerable extent, or even show closely similar extremes. The moisture ratios for Spermolepis show much narrower amplitudes, and much lower maxima, than those for Cornus. The sunshine conditions are more nearly wholly dissimilar than any other condition, even than those of the temperature series.

These plants are of particular interest as exhibiting the influence of temperature conditions in controlling the distribution of individual species. Such plants as these and Arenaria lateriflora, Parietaria pennsylvanica, and others of transcontinental distribution are able to range through widely diversified conditions of precipitation, evaporation, humidity, and moisture ratio at the same time that they are strongly controlled by temperature conditions.

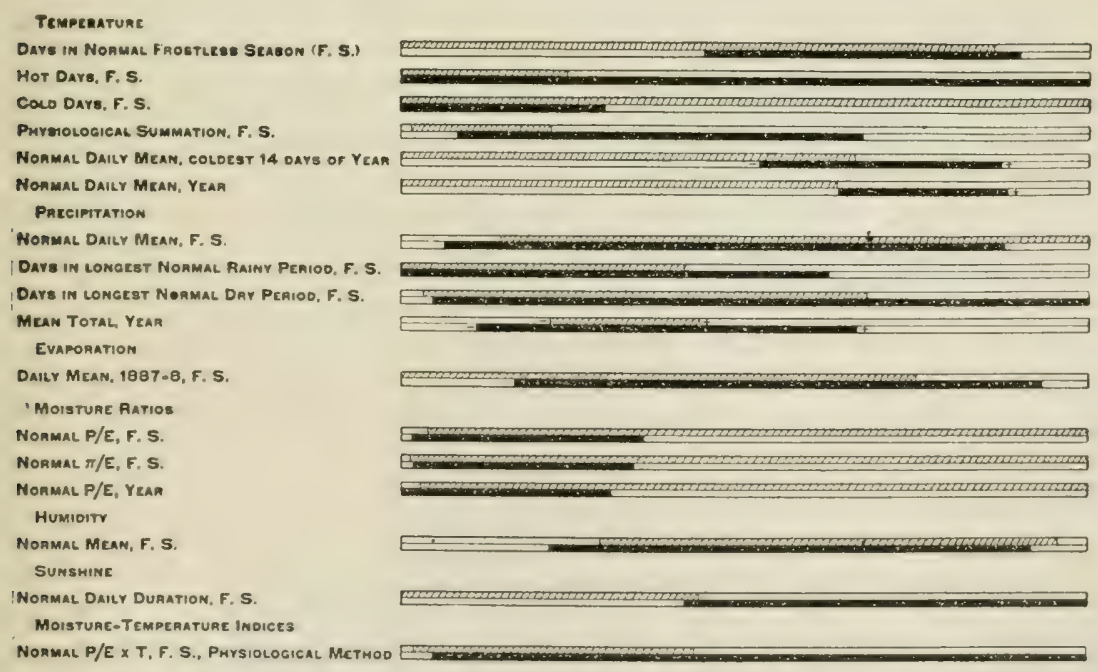

Fra. 74. Climatic extremes for Cornus canadensis (shaded) and Spermolepis echinatus (black). 
Among such species of northern transcontinental range we find chiefly herbaceous and shrubby plants of the evergreen forests, while among those of southern range we find herbaceous plants of facultative seasonal habits, or else palustrine and aquatic forms. We see, therefore, in each group a set of circumstances which apparently tend to equalize the moisture conditions for these plants - the northern species are subordinate associates of the evergreen forests; the southern species are active in different portions of the frostless season, according to the seasonal distribution of rainfall, or else they occupy perpetually moist situations.

\section{CORRELATION OF VEGETATIONAL AREAS WITH GENERALIZED CLIMATIC PROVINCES.}

\section{INTRODUCTORY.}

The generalized climatic provinces roughly defined from the results of our climatic studies should be of value in comparing the geographic distribution of vegetation with the distribution of different degrees of intensity and duration of climatic conditions throughout the country, and we have therefore carried out such comparisons between the vegetation charts (plates 1 to 33) and the generalized climatic graphs of figures 18 to 28 . Of course, it is not to be expected that any vegetational area will be found to correspond perfectly with any climatic area. Probably the only method by which close areal correlations may be attained lies in the employment of several climatic conditions, as in our two-dimensional systems of climatic provinces. A number of cases have been discovered, however, in which the correspondence between areas of plant distribution and simple climatic provinces is very good. The mention of these will be valuable in the formation of a conception of what sort of plants may be expected to occur in the various climatic provinces. Owing to the complexity of the conditions to be compared and to the varying degrees of precision with which the climatic zones can now be defined-as well as to our own limitations, no doubt - these correlations are but preliminary and very tentative.

The first observation to be made in beginning these comparisons is one that might have been expected on general grounds, namely, that relatively few of the vegetational areas show any pronounced correspondence with any single climatic province, defined by whatever method. The second observation is perhaps a little surprising, after all, considering how crude are our climatic charts, namely, that a number of good agreements have been found. As has been previously mentioned, it appears that correlations between moisture provinces and vegetational areas are more frequent than those between temperature provinces and the same areas. This may perhaps be due to the fact that the range of moisture conditions in the United States is very great (from very arid to very humid), while the range of temperature 
conditions is relatively not nearly as great. If the humidity of our most humid areas were to be increased (within the limits set by world climate), little or no alteration in the vegetation would be expected. Nor would any great alteration in the vegetation of our most arid areas be expected from increased aridity, excepting that vegetation would finally be prohibited altogether. On the other hand, if the intensity or duration factor of the temperature conditions of our warmest provinces were increased, or if that of our coldest provinces were decreased, great changes in vegetation would be expected. Both north and south of the area of the United States the same humidity conditions are concomitant with very different vegetation characters, and this difference is to be related mainly or entirely to temperature differences.

We present below some of the most definite cases of concomitancy, considering first the temperature provinces, then the moisture provinces, then the provinces based on the temperature-moisture product, and finally the two-dimensional provinces based on temperature and moisture.

\section{TEMPERATURE PROVINCES.}

Two charts of temperature provinces have been employed for these comparisons, the one based on the average frostless season (plate 34) and the one based on physiological summation indices (plate 40). It will be convenient to consider the comparisons in the order of the vegetation features as these have been presented in plates 1 to 33 .

As has been mentioned several times, there is no primary correlation between temperature conditions and the general types of vegetation as shown by plates 1 and 2 , and a comparison of these plates with those of the temperature provinces emphasizes this statement once more.

In the case of plate 3 , if the evergreen broad-leaved trees and the microphyllous trees are taken together as a single group (characterized by relatively low transpiring power), it is found that the geographic area occupied by this group very nearly corresponds with the area of the very warm, warm, and medium temperature provinces as brought out by the chart of the average frostless season. Near the Pacific and Atlantic coasts the correspondence is not good when the chart of physiological summations is employed.

The two eastern palms, Sabal palmetto and Serenoa serrulata, occupy nearly the same area as does the very warm temperature province on either of the two temperature charts here employed.

On plate 29, Phorodendron flavescens shows an area of distribution that closely agrees with the form and extent of the combined very warm, warm, and medium temperature provinces, as shown on the frostless-season chart. As in the case of the broad-leaved and microphyllous group of trees, the correlation is not good with the physiological summation chart. 
Parietaria pennsylvanica, and, to a less extent, Arenaria lateriflora (plate 30 ), generally agree in their distributional area with the area of the very cool and cool temperature provinces, based on the length of the average frostless-season.

On plate 31 , Daucus pusillus is shown to occupy an area that corresponds, in a very satisfactory manner, with an area composed of the very warm and warm climatic provinces, taken with the warmer half of the medium province, as shown on the frostless-season chart.

Other correspondences are suggested by our charts, but thesr are the most sabisfactory. The generalization is at once suggested that the length of the period of the average frostless season (plate 34 ) exhibits much more striking correlations to vegetational areas than does the chart based on physiological summations (plate 40). The results of these comparisons are shown graphically by the following scheme:

Temperature provinces based on frostless season.

\begin{tabular}{|c|c|c|c|c|c|}
\hline & $\begin{array}{l}\text { Very } \\
\text { warm. }\end{array}$ & Warm. & Medium. & Cool. & $\begin{array}{l}\text { Very } \\
\text { cool. }\end{array}$ \\
\hline $\begin{array}{l}\text { Sabal palmetto........ } \\
\text { Serenoa serrulata.... }\end{array}$ & & & & & \\
\hline $\begin{array}{l}\text { Daucus pusillus....................... } \\
\text { Broad-leaved and microphyllous }\end{array}$ & & & & & \\
\hline 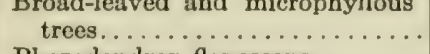 & & & & & \\
\hline $\begin{array}{l}\text { Phorodendron flavescens. } \\
\text { Parietaria pennsylvanica }\end{array}$ & & & & & \\
\hline
\end{tabular}

\section{MOISTURE PROVINCES.}

For these comparisons with the vegetation charts we have employed four charts showing moisture provinces: (1) mean daily normal precipitation $(P$, plate 46$)$; (2) mean daily evaporation, $1887-88(E$, plate $53)$; (3) precipitation-evaporation ratio $(P / E$, plate 57$)$; and (4) mean normal relative humidity ( $H$, plate 65$)$. The main cases of agreement brought out by these comparisons are given below.

The generalized vegetation chart of plate 2 shows many coordinations with the charts of moisture provinces. The best correlation occurs with the moisture-ratio chart $(P / E)$, which alone will be considered here, although a study of the other moisture charts is well worth while in this connection. Desert occupies approximately the arid province. Northwestern Evergreen Forest occupies about the humid and semihumid provinces, western subdivision. Western Evergreen Forest occupies the western and northern subdivisions of the semiarid and semihumid provinces. Grassland occupies most of the eastern subdivision of the semiarid province, extending eastward approximately to the line joining western Hudson Bay with western Gulf of Mexico. Deciduous-Forest Grassland Transition (the so-called prairie type of vegctation) occupies the more arid portion of the eastern subdivision of the semihumid province, merging imperceptibly into the next type. 
Deciduous Forest occupies the remainder of the eastern subdivision of the semihumid province, so that if the two last-named vegetation types are grouped together they occupy the whole eastern subdivision of the semihumid province. Northeastern Evergreen Forest occupies very nearly the northern portion of the eastern subdivision of the humid province, but this vegetation type extends southward in the Appalachians, which is not shown for the corresponding climatic area by the precipitation-evaporation ratio; but this southward extension of the northeastern humid conditions is shown by the line for 140 on the evaporation chart (plate 53, figs. 3 and 22). Southeastern Evergreen Forest occupies nearly the same area as the southern portion of the eastern subdivision of the humid province.

This agreement is about as close as could be hoped for in work of this kind, and the correlations are very nearly what might have been expected. Two apparently important features require brief mention: (a) the relation of the Deciduous Forest area to that of the DeciduousForest Grassland Transition, and $(b)$ the relation between the climatic conditions concomitant with the Northeastern and Southeastern Evergreen Forests.

(a) It will be noticed that no one of the climatic maps shows any line that may be considered as approximating the position of the boundary between the deciduous forest and the prairie. While this boundary, like the other lines of plate 2, does not represent a sharp line of demarcation, nevertheless it is one of the most pronounced and clearly recognizable vegetational boundaries presented by the United States. It is actually a simple matter-for example, in Minnesota, Indiana, or Illinois-for an observer to step, within a very few meters, from what is clearly and unequivocally deciduous forest into what is just as unquestionably prairie. This is not nearly so easy in the case of the other vegetation boundaries as actually encountered in the field; deciduous and evergreen forest usually mingled near their common margins, and desert, grassland, and prairie usually intergrade quite imperceptibly, so that their boundaries frequently have to be regarded as bands or zones many kilometers wide, even by an observer in the field. Furthermore, various species of trees have recently been introduced upon the upland of the prairie region, which originally was forested only on the flood-plains of the streams, while the deciduous forest of Pennsylvania, Ohio, etc., has been largely removed, so that the general aspect of the country is now much the same as in Iowa or eastern Kansas and Nebraska. This fact has led many students to regard the prairie region as potentially a deciduous-forest region, as far as climatic conditions go, and various non-climatic conditions have been suggested as explaining the original absence of trees from the prairie uplands.

Looked at from the dynamic standpoint, it seems clear to us that the difference in environmental conditions that has to be postulated as 
related to the difference in vegetation here considered must be rather recondite and subtile in its nature. We are, however, strongly inclined to maintain that this environmental difference will prove to be a climatic one, though probably not measurable in terms of any of the simpler climatic indices. Here is a problem that is well worthy of much deeper study than we have been able to give it. We wish to suggest one possible dynamic explanation on climatic grounds.

If our charts showing mean daily evaporation (plate 53, figs. 3 and 22 ) be once more examined, it will be recalled that the eastern subdivision of the semiarid moisture province here exhibits a great eastern lobe reaching from Oklahoma to Pennsylvania. This general phenomenon is shown or suggested on other moisture charts, and may be tentatively regarded as of climatic significance, until more thoroughgoing studies of the aerial moisture conditions become possible. Now, this penetration of semiarid conditions into the center of the great eastern area of the semihumid province suggests that the explanation of the vegetational transition before us is probably largely related to evaporation. The same conclusion is suggested by the relative airhumidity chart (plate 65, figs. 17 and 24). Just how atmometric or air-humidity data should be treated in order to obtain a moisture index that may bring this point out in a satisfactory way, if it be true, can not, of course, be predicted. In support of the general probability that evaporation is the main climatic feature to be called upon to explain this vegetational transition, it should be remarked that small local prairies were of frequent occurrence in Indiana and Ohio when these regions were still under forest, so that the tension zone between forest and prairie was apparently very broad in the region south of the Great Lakes. It should also be mentioned that the evaporation data used for these studies all refer to a single year (Russell's data, 1887-88) and it is suggested that a normal evaporation chart may show an isoatmic line approximating the position of the prairic-forest boundary here in question.

(b) The Northeastern and Southeastern Evergreen Forest types of vegetation are well known to be very distinct, at least floristically; yet they are to be regarded (along with the Western and Northwestern Evergreen Forests) as physiologically or ecologically rather similar, being dominated by evergreen, needle-leaved trees. It is therefore important to note that the climatic conditions that seem to correspond to the Northeastern Evergreen Forest are no more continuous with those corresponding to the Southeastern Forest than is the actual distribution area of the former forest itself with that of the latter. This point is clearly shown on the three charts for $E, P / E$, and $H$ (plates 53,57 , and 65 ; figs. 2,3 , and 17 , and 21,22 , and 24 ), and a still more 
marked climatic difference is shown between these two areas on the chart for $P$ (plate 57, figs. 16 and 23).

Turning now to the more detailed vegetation charts, the area occupied by Pinus palustris (plate 6) nearly corresponds to the southeastern humid province, as shown by $P$ (plate 46, figs. 2 and 21 ).

Pinus divaricata (plate 7) occupies about the same area as the northeastern humid province, as shown by $E$ (plate 53, figs. 3 and 22); its area also somewhat nearly corresponds to the same province on the charts for $P / E$ and $H$ (plates 57 and 65, figs. 16 and 23, 17 and 24).

Bulbitis dactyloides (plate 11) shows an area of distribution nearly corresponding with the eastern subdivision of the semiarid province and the most arid portion of the eastern subdivision of the semihumid, as shown by $P / E$ and $H$. The line from Hudson Bay to the Gulf of Mexico passes nearly through the north-south axis of its area.

Pinus edulis (plate 14) occupies nearly the arid province, as shown by $E$.

Picea sitchensis (plate 14) occupies about the northwest humid province, by $P / E$, and Tsuga heterophylla (plate 14) covers about the northwestern humid and semihumid provinces by the same index.

Quercus falcata (plate 18) has an area of distribution nearly corresponding to the southeastern humid province, as shown by $P$. Less satisfactory agreements with this same province are exhibited by Sapindus marginatus (plate 19) and Itea virginica (plate 23). In all these cases the distribution area extends farther north in the Mississippi Valley than does the climatic province as shown.

Populus balsamifera (plate 19) occupies the northern part of the eastern subdivision of the humid province, by $E$. It extends farther south than this climatic area, as shown by $P / E$. The climatic charts are not sufficiently detailed in the Northwest to show a correspondence to the northwestern area of the species.

Decodon verticillatus (plate 23) has an area of distribution nearly conforming with that of the eastern subdivision of the humid province, together with all but the most arid portion of the eastern subdivision of the semihumid province, as shown by $P / E$ or $H$.

Phorodendron juniperinum (plate 29) has an area of distribution roughly corresponding to the arid province, by $P / E$ and $H$.

Oxybaphus nyctagineus (plate 33) occupies the more humid part of the eastern semiarid province and the more arid part of the eastern semihumid province, as shown by $P / E$, the distribution area of this form being much like that of Bulbilis dactyloides (plate 11).

The schematic presentation on page 576 shows the relations just described, for the individual species considered. 
Moisture provinces, by $P, E, P / E$ and $H$.

\begin{tabular}{|c|c|c|c|c|c|c|c|c|}
\hline & \multicolumn{3}{|c|}{ Humid. } & \multicolumn{2}{|c|}{ Semihumid. } & \multicolumn{2}{|c|}{ Semiarid. } & \multirow[b]{2}{*}{ Arid. } \\
\hline & Western. & $\begin{array}{l}\text { North- } \\
\text { eastern. }\end{array}$ & $\begin{array}{l}\text { South- } \\
\text { eastern. }\end{array}$ & Western. & Eastern. & Western. & Eastern. & \\
\hline $\begin{array}{l}\text { Picea sitchensis } \\
(P / E) \ldots \ldots \ldots\end{array}$ & & & & & & & & \\
\hline $\begin{array}{r}\text { Pinus palustris } \\
(P) \ldots \ldots \ldots \ldots\end{array}$ & & & & & & & & \\
\hline $\begin{array}{r}\text { Pinus divaricata } \\
(E, P / E, H) .\end{array}$ & & & & & & , & & \\
\hline $\begin{array}{l}\text { Tsuga hetero- } \\
\text { phylla }(P / E) .\end{array}$ & & & & & & & & \\
\hline $\begin{array}{l}\text { Quercus falcata } \\
(P) \ldots \ldots \ldots\end{array}$ & & & & & & & & \\
\hline $\begin{array}{r}\text { Populus balsam- } \\
\text { ifera }(E) \ldots \ldots\end{array}$ & & & & & & & & \\
\hline $\begin{array}{c}\text { Decodon verti- } \\
\text { cillatus }(P / E, \\
H) \ldots \ldots\end{array}$ & & & & & & & & \\
\hline $\begin{array}{c}\text { Bulbilis dactyl- } \\
\text { oides }(P / E \\
H) \ldots \ldots\end{array}$ & & & & & & & & \\
\hline $\begin{array}{r}\text { Oxybaphus nyc- } \\
\text { tagineus }(P / E \\
H) \ldots \ldots \ldots \ldots\end{array}$ & & & & & & & & \\
\hline $\begin{array}{c}\text { Phorodendron } \\
\text { juniperinum } \\
(P / E) \ldots . . .\end{array}$ & & & & & & & & \\
\hline Pinus edulis $(E)$ & & & & & & & & \\
\hline
\end{tabular}

\section{TEMPERATURE-MOISTURE PROVINCES BASED ON THE PRODUCT INDEX.}

It will be recalled that our three moisture-temperature charts (plates 69 to 72 ) show a form of climatic zonation that is very similar to that shown by the chart based on the mean aqueous-vapor pressure for the period of the average frostless season. For comparison with the vegetational areas, only the generalized chart of moisture-temperature indices using the physiological temperature summation (plate 72 , figs. 18 and 25) has been employed.

To avoid confusion, it is first necessary to point out that certain climatic provinces are shown as practically the same by the moistureratio chart (plate 58, figs. 16 and 23) and by the one here considered (plate 72, figs. 18 and 25). The arid province corresponds very well with the province of very low moisture-temperature values. The semiarid province represents much the same area as the province of low product values, but the north-south boundary of the latter province lies much farther west at its southern end (Texas), and farther east at. its northern end (South Dakota). This line on the moisture-ratio chart is practically the Hudson Bay-Gulf of Mexico line, as has been noted, while the north-south line just mentioned has a very different. position. Also, the eastern projection of the province of lou product values is not represented on the chart of moisture-ratios; it roughly 
corresponds to the northeastern portion of the humid province, as shown on the latter chart. The northwestern areas of medium and high product values correspond, to a degree, with the similar semihumid and humid provinces on the moisture chart.

The eastern half of the product chart resembles a temperature chart in its zonation, as has been mentioned, and shows no clear relation to the moisture-ratio chart, excepting that the southeastern area of very high moisture-temperature values may be considered as corresponding to the southeastern humid area in the latter case. Only this eastern half needs, therefore, to be specially compared with the vegetation charts, and in this comparison the southeastern area (of very high products and of high moisture ratios) may be left out of account. The comparison brings out the fact that there are but two cases where any striking agreement in form and position of areas is to be detected.

Quercus alba (plate 18) occupies, roughly, the provinces of medium, high, and very high product values, but this species does not extend nearly as far westward in the southwestern part of its area as does the province of medium moisture-temperature products. Also, this tree does not occupy peninsular Florida, which includes the highest product values. Quercus alba may be said mainly to correspond, in its distributional area, with the region having moisture-temperature indices ranging from 4 to 22 , but in the southwestern portion of its area it extends westward only about as far as index-value 17. This is not to be considered a very satisfactory agreement.

The other case where an apparent agreement between moisturetemperature product zones and vegetation areas is to be detected is that of the cumulative distribution of southeastern deciduous trees (plate 4). In this case the agreement is more nearly perfect than for Quercus alba, but here, also, the vegetation area does not extend south in Florida far enough to include the very highest product value. These 15 trees, considered together, occupy the provinces of high and very high product indices, except the very highest, and with the further exception that they extend much farther westward in Texas than does the province of high index values. These trees may be considered as occupying the region having moisture-temperature indices ranging from 7 to 23 , but they extend to index-value 2 in Texas.

On the whole, we are once more led to the conclusion that the moisture-temperature products do not furnish a criterion of great general value, as far as the discovery of distributional correlations is concerned, at least for the vegetation areas that we have charted. Of course, many areal correlations not here mentioned are to be found between our vegetation charts and the chart here considered, but most of these represent cases where this chart agrees, in its zonation, with the moisture charts, and a number of such correlations have been noted in connection with those. 


\section{TWO-DIMENSIONAL CLIMATIC PROVINCES.}

Although a number of the various vegetational areas shown on the charts of plates 2 to 33 have been shown to be more or less precisely comparable with geographically corresponding climatic areas, it nevertheless appears that such satisfactory correspondence is the exception rather than the rule. The climatic conditions concomitant with the vegetation areas that fail to show such simple correlations as have been mentioned in the preceding paragraphs require a more complex mode of description, at least until the proper simple climatic indices may be discovered. The most thoroughgoing subdivision of the country into climatic provinces, which has been attempted in our studies, is that based on the two-dimensional provinces. The use of these smaller climatic areas makes it possible to describe any vegetation area not simply correlated with either moisture or temperature provinces alone, in terms of both moisture and temperature conditions together. Such a description is clearly climatic and may lead to further correlations, but this method soon encounters limitations, since, as has been pointed out, there are frequently several two-dimensional provinces with the same dimensions or characteristics, and these can not as yet be simply distinguished on a climatic basis alone. For the present, and in the comparisons mentioned below for illustration, it seems best to fall back upon geographical terms, in order to distinguish such climatically similar but geographically distinct areas. This method frankly begs the entire question of correlations; it furnishes, wherever it is employed, nothing more than a geographical description of the details of configuration with which it deals. It is, however, not to be resorted to until the climatic description of the vegetational area in question is as complete as possible, so that the resulting description always bears much more climatic information than would a purely geographic description. The latter sort of description is quite useless, as far as our purposes are concerned, for it merely states that the given plant or vegetation type occurs where it is.

In the following paragraph we present descriptions of several vegetational areas, following the method just outlined. For the twodimensional climatic provinces we shall here employ only the chart formed from the length of the average frostless season and from the precipitation-evaporation ratio (fig. 19). The cases considered are set forth here simply to illustrate the use of this method of interpretation; we have not yet proceeded far enough with this more complicated aspect of the subject to be able to arrive at any very promising generalization. The distribution areas chosen for discussion here are taken from eastern forms among those that fail to show intelligible relations to the simple climatic provinces of moisture and temperature.

In the case of the evergreen broad-leaved and microphyllous trees (plate 3), attention has been called to the fact that the distribution of 
this group may be very satisfactorily described in terms of the temperature provinces alone, but neither temperature nor moisture conditions alone are adequate to bring out any climatic correlations that may suggest an explanation as to why the broad-leaved trees occur only west and east, while the microphyllous ones occur in an intermediate region.

Comparing plate 3 with figure 19, it becomes at once apparent, however, that the broad-leaved trees occur mainly in the very warm humid province (Florida and Louisiana) and that they occur in smaller number of species in the warm and medium humid and in the warm and medium semihumid provinces. Especially in California they occur in the warm and medium semihumid provinces. The microphyllous trees occur mainly in the warm and medium arid, and they occur as fewer species, especially in Texas, in the warm and medium semiarid. The two-dimensional provinces are thus fairly satisfactory in correlating the distribution of these two groups of trees with the two primary groups of climatic conditions.

The distribution of the eastern deciduous trees (plate 5) must be described, first, in geographic terms. They occur east of the line joining Hudson Bay with the Gulf of Mexico, and they are absent from all climatic provinces west of this line. In the area thus demarked their area of greatest density lies within the cool and medium semihumid provinces. This area does not correspond to all of the area of these provinces, but it does not significantly overlap any of the humid provinces. It occupiesabout the eastern half of the cool semihumid province (Kentucky to Massachusetts), and the northern marginal portion of the eastern half of the medium semihumid province. For the most part, these trees may be said to occur in greatest number of species with the more humid and warmer conditions of the cool semihumid province.

The distributional area of Liriodendron tulipifera, one of the eastern deciduous trees, is also not possible of description in terms of our climatic provinces alone. It must first be stated that this tree occurs only in the East. Its area occupies most of the eastern half of the cool semihumid province, not reaching the boundary of the cool humid on the north and extreme northeast. It occupies about the eastern two-thirds of the medium semihumid and all of the medium humid provinces. It also occupies the eastern lobe of the warm semihumid and a portion of the warm humid (Georgia, etc.). It does not extend into the very warm temperature province.

The area of greatest frequency for this tree, which may be considered as its geographic and climatic center of distribution, lies practically in the center of the distributional area just described. This smaller area may be defined as occupying the southeastern triangular lobe of the cool semihumid province and the northern half of the central portion of the medium semihumid, which adjoins that triangular lobe at the southwest. Thus, this center of distribution occurs with cool- 
medium temperature conditions and with the more humid conditions of the semihumid moisture province. It does not extend to the boundary of the humid province at any point.

Silphium laciniatum (plate 25) shows a clearly outlined geographical area of distribution, occupying the Missouri-Mississippi-Ohio Valley as far north as the Grand River in Michigan, as far west as western Kansas, and as far east as the Appalachian Mountains. It does not occur either in the West or east of the Appalachians. Within these geographical limits the distributional area of this plant corresponds to the following two-dimensional climatic provinces:

(a) Warmer two-thirds of the cool semihumid, west of Appalachians.

(b) Small portion of cool semiarid, the warmer, more humid part of this province.

(c) Medium semihumid, west of Appalachians.

(d) Eastern (more humid) half of medium semiarid.

(e) Most of warm semihumid (all but a small area in Georgia).

(f) Coolest portion of warm semiarid (Texas).

(g) Western half of warm humid (Louisiana to Florida).

This Silphium appears not to extend into the very warm temperature province to any considerable extent. It occupies the more arid part of the semihumid and the more huniid part of the semiarid, within the warm and medium provinces and the warmer part of the cool province.

Many other examples might be given showing the use of two-dimensional climatic provinces, supplemented by geographical data, in climatically describing vegetational areas for purposes of comparison. Indeed, any vegetational area may be so described after the requisite two-dimensional chart has been once prepared. But the four cases considered above should be sufficient to demonstrate the investigational value of this general method. If the relations holding between climatic conditions and plant activity receive the attention that they deserve from ecologists and climatologists, this method, with improvements, should prove very useful. Especially should this be true for studies of agricultural and forest climatology, which is just beginning to attract serious attention in this country. 


\section{CONCLUSION.}

The work presented in this publication has fallen under three heads: (1) giving the facts as to the distribution of certain types of vegetation and certain species of plants of the United States; (2) giving the data to show the intensities of the leading climatic conditions in the United States; (3) correlating these two bodies of facts in such a manner as to learn the exact range of conditions under which each plant or vegetation lives with respect to each of the climatic elements.

The botanical facts lead to the subdivision of the vegetation into a small number of natural areas, delimited on a purely vegetational basis, to the outlining of regions in which particular ecological types are most abundant, and to the presentation of the distributional areas of a number of important species in the vegetation. The climatological data have been selected or elaborated with respect to the conditions which are of most importance to plants, with the aim, wherever possible, of devising new expressions for the climatic conditions that might be suited to the botanical problems in hand. The correlation of the distribution of plants with the distribution of various numerical values or indices of the several climatic conditions has been carried out with the full realization that such correlations do not carry conclusive proof of the existence of causal connection. It is only by careful elimination of possibilities and by comparison of results, that these correlations can be used as more than a source of suggestions.

The existence of a causal relation between the climatic conditions and the vegetation of any given region is so well known as to have become practically axiomatic. A relation between climate and the distribution of the common species which dominate the principal vegetations is likewise well-established fact. But the relations between climate and the distribution of the generality of individual species is indirect and is obscured by many considerations.

In an investigation of the rôle that is played by the various climatic conditions in determining the optimum activity of a plant or the limitation of its distribution, it is necessary to bear in mind that the conditions operate collectively and that their influences are often interdependent. The rôle of each condition changes with the changed values of the other conditions. In attempting to determine the relative importance of several climatic conditions as determinants of a given distributional phenomenon, it is seldom possible to do more than speak in general terms. It may be possible to state, for example, that temperature conditions are more important than moisture conditions in a given case, without its being possible to determine, on the same evidence, which of the several aspects of temperature is most important.

The problem of the rôle of climatic conditions in determining plant distribution is essentially a physiological one, since it rests, in ultimate analysis, upon the influence exerted by environmental conditions on the 
activities of individual plants. The attack upon this problem must, however, be made by methods quite different from those employed in purely physiological investigations. The conditions must be measured rather than controlled, and the plant material must be examined throughout its range of occurrence, much as a large series of experimental cultures is scrutinized for the discovery of the effect produced by controlled conditions. The methods that must be employed hinge very largely upon the interpretation of a vast series of uncontrolled experiments under the varying conditions of natural environment. It is to the geographical aspects of the problem that we must ascribe many of its complexities and much of its difficult nature.

Although the results secured in this investigation are only general in their applicability, we have endeavored to develop and make use of methods which are specific and definite enough to warrant more extended use. The basic data, both as to climate and vegetation, are scanty in many cases, and the methods used could well be employed to greater advantage with fuller data, or for the investigation of similar problems in smaller areas.

The presentation of vegetational data that has been given takes no account of the minor plant communities that occupy relatively small areas in all plant formations, and owe their existence to the modification of the fundamental conditions of climate through differences in what might be designated as the response of soils to the climatic conditions. No account has been taken of the developmental changes of vegetation in regions with rapidly shifting topography, since these changes depend mainly on differences in the character of the soil, or on changes of environment due to the plant covering. All developmental changes in vegetation are due to changes of environmental conditions, and it is only rarely, or over very long periods of time, that these changes are in the nature of definite alterations of the general climate of the region.

Our presentation of climatic conditions has been limited chiefly to those elements of the climate that are commonly measured, as it is impossible to secure well-distributed series of data for other conditions, although many of these are well known to be of great importance to plants. A departure from the customary climatological procedure has been made in securing many of the data on temperature and moisture conditions for the period of the average frostless season as well as for the calendar year. The length of the average frostless season has been determined for each of the stations from which other climatic data have been used. The data for the summation of temperature have been worked out by three methods, in addition to the one used by Merriam. It seemed advisable to give this promising means of securing additive temperature data a thorough test, and to attempt to arrive at a method with fewer objections from a physiological standpoint than could be urged against the older methods. 
The determination of the ratio of precipitation to evaporation, which was first applied to distributional problems by Transeau, has been made for the entire United States, and has been derived by three methods. So great is the importance of the "moisture ratio," as this has been designated, that it is greatly to be hoped that our maps showing the distribution of the ratios may soon be redrawn upon the basis of much fuller evaporation data. A further attempt has been made to secure composite expressions of groups of important climatic conditions by determining the products of the moisture ratios and the summations of temperature. A cartographic method of approaching the same end has been employed for the determination of the areas included between the isoclimatic lines for the moisture ratio and those for the physiological summation of temperature. The result is a series of climatic provinces which are based upon the two expressions of climatic conditions that are probably the most fundamental ones dealt with in this work.

The correlation of climate and vegetation has been carried out in three ways: The maximum and minimum values of each climatic condition have been determined for each vegetational area or for the distributional area of each species. A comparison has been made between the amplitude of the conditions in each botanical area and the amplitude in the United States as a whole, in order to discover how small or how great a portion of the whole range of climatic conditions is occupied by the vegetation or plant in question. Comparisons have been made between the positions of isoclimatic lines and the lines drawn to show the limits of botanical areas, for the purpose of discovering close correspondences. The detailed results of these methods of correlation are given in the preceding pages; they do not lend themselves to being summarized.

The parallelism that exists between the distribution of many of the closely related climatic conditions makes it difficult in some cases to determine which of the several aspects of a given condition is of the greatest importance in controlling a particular plant or vegetation. The methods used rarely fail, however, to demonstrate whether it is the temperature group of conditions or the moisture group that possesses the greater importance.

With respect to the generalized vegetation areas of the United States, one of the most clear-cut evidences of a fundamental correlation exists in the correspondence between the position of the vegetational boundaries and the position of the isoclimatic lines expressing certain values of the moisture ratio for the average frostless season. The composite character of the moisture ratio, and the fact that it is derived from such an important group of climatic conditions, give it a value of the first rank in dealing with the physical conditions determining the distribution of vegetation. 
With respect to the distribution of individual species it is to be noted that most of those which are characteristic and abundant in important vegetations are, like the vegetation itself, controlled by moisture conditions. In fact, the distributional limits of such species frequently lie just within or without the limits of the vegetation in which they are dominant. The limits of distribution of many herbaceous and palustrine plants lie parallel to the isoclimatic lines for temperature conditions. For palustrine plants the topographic conditions make the soil-moisture nearly alike at all times and in all places, and the distribution of these plants is therefore subject to temperature control.

The aim of our studies has been to bring forward certain types of the methods that may be employed in studying the etiology of plant distribution and to present some of the climatological data necessary to such work in the United States. The subject is large and complex, but it offers promising fields for further investigation, and it is to be hoped that many more workers will be attracted to it in the early future.

The growth of our knowledge of plant physiology will bring with it the need of obtaining measurements of new features of the environmental complex, or the need of determining new phases of the climatic elements for which we already have data.

The progress which is being made in the study of light and its influences upon plants may well lead to the discovery that this group of conditions plays a more important rôle in the distribution of plants than has heretofore been suspected. The whole field of the influence of temperature on plant distribution needs to be approached with regard to the temperature requirements of each phase of the life-history of the plant. A much more detailed analysis of temperature effects is needed, and a much more elaborate system of recording temperature data.

The physical conditions of the soil need much more detailed investigation from the point of view of their dependence on general climatic conditions. The geographical aspects of soil-moisture and soil-temperature conditions have been neglected by reason of the local complexities that they present. There is great need of the investigation of these and other soil conditions at a large number of well-distributed localities. An elucidation of the local conditions of each place would throw light on the relation of climatic conditions to the conditions of the soil, would increase our knowledge of important aspects of the soil, and would give a basis for learning the geographical range of the intensities of these conditions.

The methods used in this publication and the climatic data presented may be used to investigate the controlling conditions for other plants than those we have taken up. A marked improvement in methods would doubtless follow a truly thorough investigation of the ecological distribution and controlling conditions of any one plant. To have its greatest value, such an investigation should be made with reference to the ecologieal center for the plant and with reference to all parts of the edge of its distributional area. 
The work of distributional etiology must be carried on in close coordination with the work of plant physiology. A knowledge of the fundamental physiological features of a plant should go hand in hand with an efiort to investigate the features of physiological and ecological behavior that do not lend themselves to laboratory experimentation. A substantial advance in the investigation of the etiology of plant distribution would provide facts and methods of inestimable value in the practice of agriculture, horticulture, and forestry. 
$4^{6}$ 


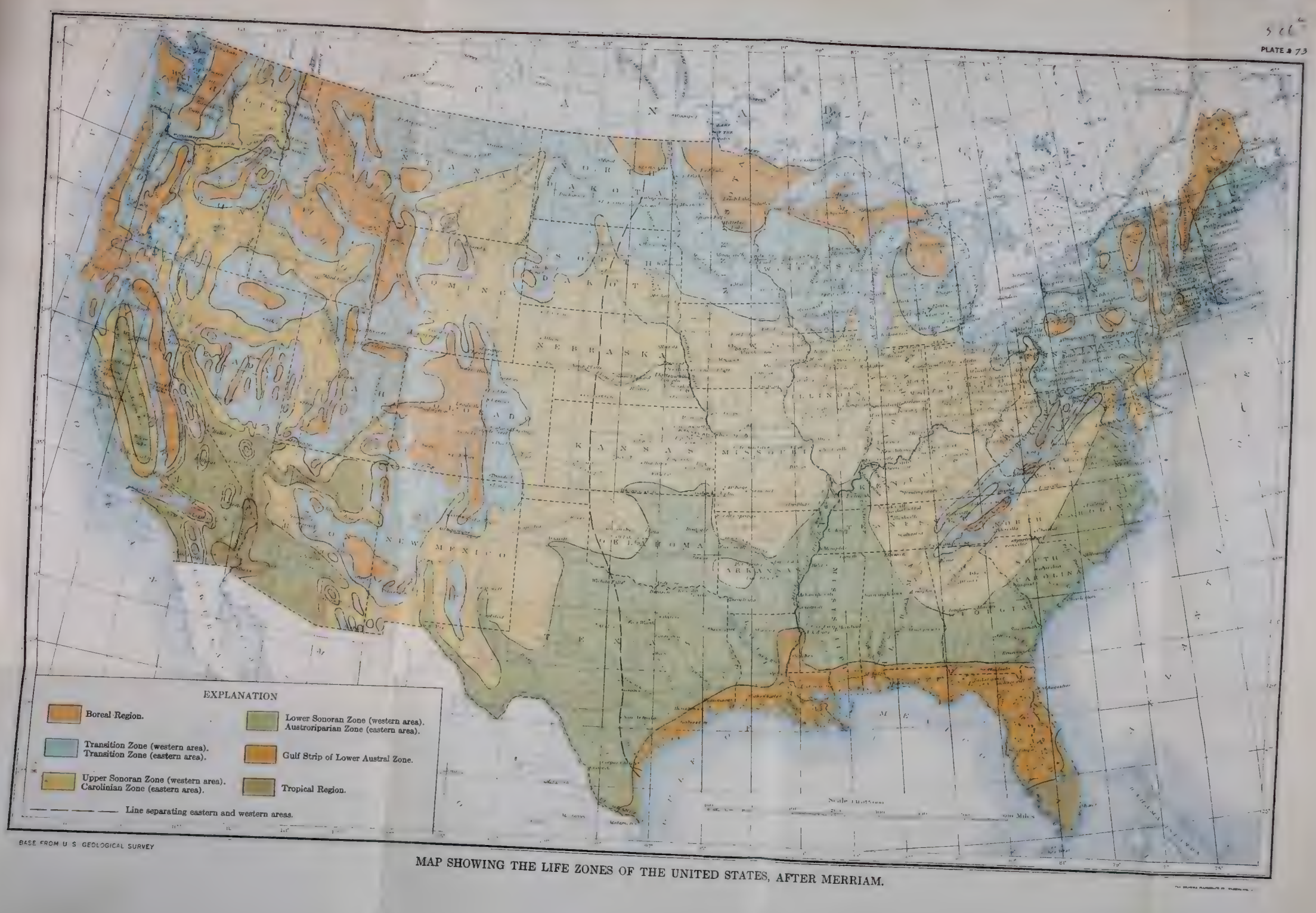





\section{LITERATURE CITED.}

Abbe, Cleveland. 1905. A first report on the relations between climates and crops. U. S. Dept. Agric., Weather Bur. Bull. 36.

Adams, C. C. 1902. Southeastern United States as a center of geographical distribution of flora and fauna. Biol. Bull. 3: 115-132.

BABCOCK, S. M. 1912. Metabolic water: Its production and rôle in vital phenomena. Wiseonsin Agric. Exp. Sta. Bull. 22.

BAKKE, A. L. 1914. Studies on the transpiring power of plants as indicated by the method of standardized hygrometric paper. Jour. Ecol. 2: 145-173.

Berry, Edward W. 1916. The lower eocene floras of southeastern North America. U. S. Geol. Surv. Prof. Paper 91, 73.

Braloblocki, J. 1870. Ueber den Einfluss der Bodenwärme auf die Entwicklung einiger Culturpflanzen. Landw. Versuchsst. 13: 424-472.

BigeLow, F. H. 1908. The daily normal temperature and the daily normal precipitation of the United States. U. S. Dept. Agric., Weather Bur. Bull. R.

1909. Report on the temperatures and vapor tensions of the United States, reduced to a homogeneous system of 24 hourly observations for the 33-year interval, 1873-1905. U. S. Dept. Agric., Weather Bur. Bull. S.

Blackman, F. F. 1905. Optima and limiting factors. Ann. Bot. 19: 281-295.

1908. The metabolism of the plant considered as a catalytic reaction. Science 28: $628-636$.

Briggs, L. J., and H. L. Shantz. 1912. The wilting coefficient for different plants and its indirect determination. U. S. Dept. Agric., Bur. Plant Ind. Bull. 230.

1913. The water requirement of plants. II: A review of the literature. U. S. Dept. Agric., Bur. Plant Ind. Bull. 285.

- 1916. Hourly transpiration rate on clear days as determined by cyclic environmental factors. U. S. Dept. Agric., Jour. Agric. Res. 5: 583-649.

Brown, W. H. 1912. The relation of evaporation to the water-content of the soil at the time of wilting. Plant World 15: 121-134.

CALDWELl, J. S. 1913. The relation of environmental conditions to the phenomenon of permanent wilting in plants. Physiol. Res. 1: 1-56.

Cameron, F. K. 1911. The soil solution, the nutrient medium for plant growth.

Clausen, H. 1890. Beiträge zur Kenntnis der Athmung der Gervachse und des pflanzliches Stoffwechsels. Landw. Jahrb. 19: 893-930.

Clements, F. E. 1905. Research methods in ecology.

DAY, F. C. 1911. Frost data of the United States, and the length of the crop-growing season, as determined from the average of the latest and earliest dates of killing frost. U. S. Dept. Agric., Weather Bur. Bull. V.

Drude, O. 1896. Deutschlands Pflanzengeographie.

1913. Die Ókologie der Pflanzen.

FAssig, O. L. 1907. Report on the climate and weather of Baltimore. Maryland Weather Service 2: 29-312.

1914. The period of safe plant growth in Maryland and Delaware. Monthly Weather Rev, 42: 152-158.

FAwCETt, H. S. 1921. The temperature relations of growth in certain parasitic fungi. Univ. Calif. Publ.; Agric. Sci. 4: 183-232.

Fitring, Hans. 1911. Die Wasserversorgung und die osmotischen Druckverhaltnisse der Wusterpflanzen. Zeitschr. Bot. 3: 209-275.

Fredndich, H. 1909. Kapillarchemie.

Gannetr, Henry. 1909. Distribution of rainfall. U. S. Geol. Surv. Water Supply Paper No. 234, reprinted from report of National Conservation Commission.

Grisebach, A. R. H. 1872. Die Vegetation der Erde.

Harshberger, J. W. 1911. Phytogeographic Survey of North America. Die Vegetation der Erde, vol. 13.

Henry, A. J. 1906. Climatology of the United States. U. S. Dept. Agric., Weather Bur. Bull. Q. 
Hilderbrandt, F. M. 1917. A method for approximating sunshine intensity from ocular observations of cloudiness. Johns Hopkins Univ. Circ., 205-208.

1921. A physiological study of the climatic conditions of Maryland as measured by plant growth. Physiol. Res. 2: 341-405.

Humboldt, A. von. 1805. Essai sur la géographie des plants.

Krmball, H. H. 1905. Evaporation observations in the United States. Monthly Weather Rev. 32: 556-559.

Kincer, J. B. 1917. Average annual precipitation in inches (for the United States), based on records of about 1,600 stations for the 20-year period 1895-1914, and 2,000 additional records, from 5 to 10 years in length, uniformly adjusted to the same period. Advance sheet 1, Atlas of Amer. Agric., U. S. Dept. Agric., Weather Bur.

Krause, E. H. S. 1891. Die Eintheilung der Pflanzen nach ihrer Dauer. Ber. deutsch. Bot. Ges. 9: 223-227.

Lemenbauer, P. A. 1914. Growth of maize seedlings in relation to temperature. Phytiol. Res. $1: 247-288$.

Livingston, B. E. 1906. I. Note on the relation between the growth of roots and of tops in wheat. Bot. Gaz. 41: 139-143.

1906. II. The relation of desert plants to soil moisture and to evaporation. Carnegie Inst. Wash. Pub. No. 50.

1907 I. Evaporation and plant development. Plant World 10: 269-276.

1907 II. Further studies on the properties of unproductive soils. U. S. Dept. Agric., Bur. Soils Bull. 36.

1908. A simple atmometer. Science 28: 319-330.

1909 I. Present problems of physiological plant ecology. Amer. Nat. 43: 369-378.

1909 II. Present problems of physiological plant ecology. Plant World 12: $41-46$.

1910 I. Evaporation as a climatic factor influencing vegetation. Mem. Hortic. Soc. New York 2: 43-54.

1910 II. A rain-correcting atmometer for ecological instrumentation. Plant World 13: 79-82.

1911 I. Ijght intensity and transpiration. Bot. Gaz. 52: 418-438.

1911 II. A radio-atmometer for measuring light intensity. Plant World 14: 96-99.

1911 III. The relation of the osmotic pressure of the cell sap in plants to arid habitats. Plant World 14:153-164.

1911 IV. A study of the relation between summer evaporation intensity and centers of plant distribution in the United States. Plant World 14: 205-222.

1912 I. Incipient drying in plants. Science 35: 394-395.

1912 II. Present problems of soil physies as related to plant activities. Amer. Nat. 46: 294-301.

1912 III. A schematic representation of the water-relation of plants, a pedagogical suggestion. Plant World 15:214-218.

1913 I. Climatic areas of the United States as related to plant growth. Invitation paper read before Amer. Philos. Soc. Phila.; Proc. Amer. Phil. Soc. 52: $257-275$.

1913 II. The persistence offered by leaves to transpirational water-loss. Plant World 16: 1-35.

$1915 \mathrm{I}$. Atmometry and the porous cup atmometer. Plant World 18: 21-30, 51-74, 95-111, 143-149.

1915 II. Atmospheric influence upon evaporation and its direct measurement. Monthly Weather Rev. 43: 126-131.

1915 III. A modification of the Bellani porous plate atmometer. Science 41: 872-874.

1916 I. Physiological temperature indices for the study of plant growth in relation to climatic conditions. Physiol. Res. 1:399-420.

1916 II. A single climatic index to represent both moisture and temperature conditions asr elated to plants. Physiol. Res. 1: 421-440.

1917 I. Atmometric units. Johns Hopkins Univ. Circ. 160-170.

1917 II. The vapor tension deficit as an index of the moisture condition of the air. Johns Hopkins Univ. Circ. 170-175. 
Livingston, B. E., J. C. Britton, and F. E. Reid. 1905. Studies on the properties of an unproductive soil. U. S. Dept. Agric., Bur. Soils Bull. 28.

and W. H. Brown. 1912. Relation of the daily march of transpiration to variations in the water-content of foliage leaves. Bot. Gaz. 53:311-330.

and A. H. Estabrook. 1912. Observations on the degree of stomatal movement in certain plants. Bull. Torrey Bot. Club 39: 15-22.

and L. A. HAwkins. 1915. The water-relation between plant and soil. Carnegie Inst. Wash. Pub. No. 204: 3-48.

and G. H. Jensen. 1904. An experiment on the relation of soil physics to plant growth. Bot. Gaz. 38: 67-71.

, and R. Kокетво. 1920. The water-supplying power of the soil as related to the wilting of plants. Soil Science 9:469-485.

-, and G. J. Livingston. 1913. Temperature coefficients in plant geography and climatology. Bot. Gaz. 56:348-375.

Livingston, G. J. 1909. An annotated bibliography of evaporation. Monthly Weather Rev. 36: 181-186, 301-306, 375-381; 37: 68-72, 103-109, 157-160, 193-199, 248-253.

Loew, O. 1892. Die Bedeutung der Kalk-Magnesiasalze in der Landweirtschaft. Landw. Versuchsst. $41: 467-475$.

, and D. W. MAY. 1901. The relation of lime and magnesia to plant growth. U. S. Dept. Agric., Bur. Plant Ind. Bull. 1.

Marvin, C. F. 1905. Sunshine tables of 1905, giving the times of sunrise and sunset in mean solar time and the total duration of sunshine for every day in the year, latitudes $20^{\circ}$ to $50^{\circ}$ north. U. S. Dept. Agric., Weather Bur. (numbered "W. B. No. 320").

Matthaei, G. S. C. 1904. Experimental researches on vegetable assimilation and respiration. III: On the effect of temperature on carbon-dioxide assimilation. Phil. Trans. Roy. Soc. London, B, 197:47-105.

MacDougal, D. T., E. R. Long, and J. G. Brown. 1915. End results of desiccation and respiration in sueculent plants. Physiol. Res. 1: 289-325.

McGee, W. J. 1913. Wells and sub-soil water. U. S. Dept. Agric., Bur. Soils Bull. 92.

MCLean, F. T. 1917. A preliminary study of elimatic conditions in Maryland, as related to the growth of soy-bean seedlings. Physiol. Res. 2: 129-208.

Merrias, C. H. 1894. Laws of temperature control of the geographic distribution of terrestrial animals and plants. Nation. Geog. Mag. 6:229-238, 3 maps.

-. 1898. Life zones and crop zones. U. S. Dept. Agric., Biol. Surv. Bull. 10

Mrtscherlich, E. A. 1909. Das Gesetz des Minimums und das Gesetz des abnehmenden Badenertragles. Landw. Jahrb. 38: 537-552.

1911. Ueber das Gesetz des Minimums und die sich aus diesem ergebendem Schlussfolgerunglu. Landw. Versuchsst. 75:231-263.

1913. Bodenkunde für Land und Forst-Wirte.

Mонг, C. 1896. Timber pines of the southern United States. U. S. Dept. Agric., Bur. For. Bull. 3.

Notring, P. G. 1912. Outlines of applied topics.

Osterhout, W. J. V. 1907. On the importance of physiologically balanced solutions for plants. II: Fresh water and terrestrial plants. Bot. Gaz. 44:259-292.

Pfeffer, W. 1903. Physiology of plants. Translated by A. J. Ewart 2:121.

Pound, R., and F. E. Clements. 1898. The phytogeography of Nebraska.

PrICE, H. L. 1911. The application of meteorological data in the study of physiological constants. Ann. Dept. Virginia Agric. Exp. Sta. 1909-1910: 206-212.

Pulling, H. E. 1917. The rate of water movement in aerated soils. Soil Science 4: 239-268.

1919. Sunlight and its measurement. Plant World 22: 151-171, 187-209.

- and B. E. Lrvingston. 1915. The water supplying power of the soil as indicated by atmometers. Carnegie Inst. Wash. Pub. No. 204:49-94.

RadNkiär, C. 1905. Types biologique pour la géographie botanique. Bull. Acad. Roy. Soc., Denmark. 
RadNKIÄr C. 1908. Livsformernes Statistik Som Grundlag for Biologisk Plantegeografi. Bot. Tidsskr. 29. [German translation in Beih. Bot. Centralbl. 87. 1910.]

1909. Formationsundersogelse og Formationsstatistik. Bot. Tidsskr. 30. [English abstract in Bot. Centralb. 113:662. 1910.]

1916. Om Bladstorrelsens Avvendelse i der biologiske Plantegeografi. Bot. Tidsskr. 33: 225-240. [English translation by G. D. Fuller and A. L. Bakke, in Plant World 21:25-37. 1918.]

Russelx, E. J. 1917. Soil conditions and plant growth. Third ed.

Russell, T. 1888. Depth of evaporation in the United States. Monthly Weather Rev. $16: 235-239$.

Schreiner, O. 1911. Organic compounds and fertilizer action. U. S. Dept. Agric., Bur. Soils Bull. 77.

Amer. Chem. Soc. 33: 1412-1417.

Surve, J. W. 1915 I. An improved non-absorbing porous cup atmometer. Plant World 18: 7-10.

- 1915 II. A study of physiological balance in nutrient media. Physiol. Res. 1: $327-397$.

- and B. E. Livingston. 1914. The relation of atmospheric evaporating power to soil-moisture content at permanent wilting in plants. Plant World 17: 81-121.

Shreve, Edith B. 1914. The daily march of transpiration in a desert perennial. Carnegie Inst. Wash. Pub. No. 194.

Sureve, Forrest. 1914. Rainfall as a determinant of soil moisture. Plant World 17: 9-26.

1915. The vegetation of a desert mountain range as conditioned by climatic factors. Carnegie Inst. Wash. Pub. No. 217.

1917. A map of the vegetation of the United States. Geog. Rev. 3:119-125, with map.

Spozer, H. A. 1914. Periodic Variations of Respiratory Activity. Carnegie Inst. Wash. Year Book 13: 87-88.

Stockman, W. B. 1905. Temperature and relative humidity data. U. S. Dept. Agric., Weather Bur. Bull. O.

StoklasA, J., and A. Ernst. 1908. Die chemische charakter der Wurzelans Scheidung ver Schiedenartiger Kulturpflanzen. Jahrb. wiss. Bot. 46:52-102.

TAYLOR, N. 1915. Flora of the vicinity of New York, a contribution to plant geography. Mem. New York Bot. Gard. V. vr. +683 p.

Tottinghar, W. E. 1914. A quantitative chemical and physiological study of nutrient solutions for plant cultures. Physiol. Res. 1:133-245.

Transead, E. N. 1905. Forest centers of eastern America. Amer. Nat. 39:875-889.

U. S. Weather Bureau. 1914. Chart of lowest temperatures ever observed. [To and including 1914. Letter from Professor C. F. Marvin.]

- Summary of the climatological data for the United States, by section. [No dates of publ.]

VAN'T HoFf, J. H. 1898. Leetures on theoretical and physical chemistry, translated by R. A. Lehfeldt. [No date; author's preface dated 1898.]

Warmina, E. 1908. Om Planterigets Livsformer. Festskr. udg. af Universitet.

WAtson, J. B., and R. M. Yerkes. 1910. Methods of studying vision in animals. Behavior Monographs, Serial No. 2.

Wiesner, J. 1907. Der Lichtgenuss der Pflanzen.

WooDwand, J. 1699. Some thoughts and experiments concerning vegetation. Phil. Trans. Roy. Soc. London 21:193-227. 




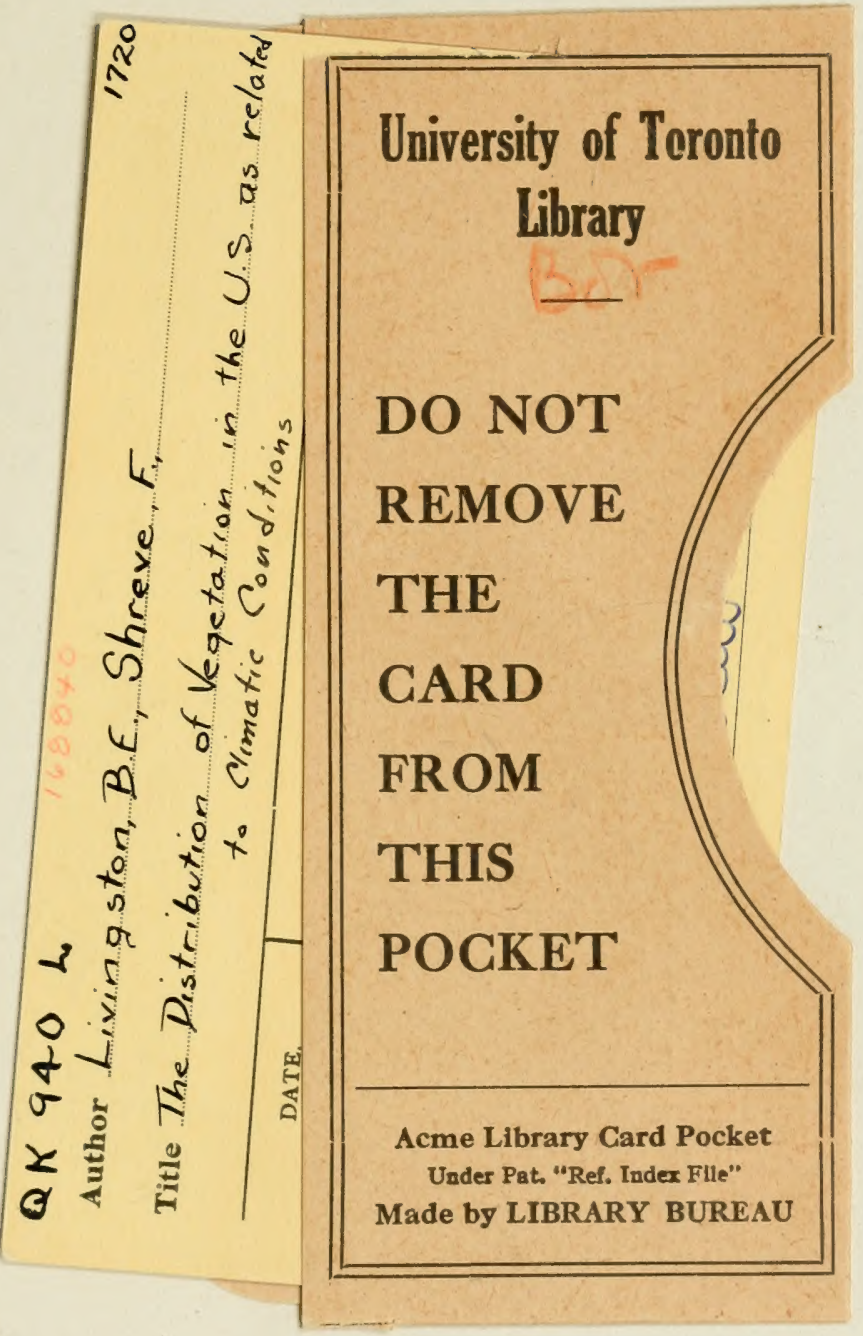




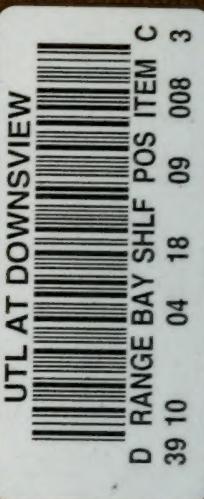

